\title{
A LIPSCHITZ METRIC FOR THE CAMASSA-HOLM EQUATION
}

\author{
JOSÉ A. CARRILLO ${ }^{\circledR 1}$, KATRIN GRUNERT ${ }^{\circledR 2}$ and HELGE HOLDEN ${ }^{\circledR 2}$ \\ ${ }^{1}$ Mathematical Institute, University of Oxford, Oxford OX2 6GG, UK; \\ email: carrillo@maths.ox.ac.uk \\ ${ }^{2}$ Department of Mathematical Sciences, NTNU Norwegian University of Science and Technology, \\ NO-7491 Trondheim, Norway; \\ email: katrin.grunert@ntnu.no, helge.holden@ntnu.no
}

Received 30 November 2019; accepted 7 April 2020

\begin{abstract}
We analyze stability of conservative solutions of the Cauchy problem on the line for the CamassaHolm $(\mathrm{CH})$ equation. Generically, the solutions of the $\mathrm{CH}$ equation develop singularities with steep gradients while preserving continuity of the solution itself. In order to obtain uniqueness, one is required to augment the equation itself by a measure that represents the associated energy, and the breakdown of the solution is associated with a complicated interplay where the measure becomes singular. The main result in this paper is the construction of a Lipschitz metric that compares two solutions of the $\mathrm{CH}$ equation with the respective initial data. The Lipschitz metric is based on the use of the Wasserstein metric.
\end{abstract}

2010 Mathematics Subject Classification: 35Q53, 35B35 (primary); 35B60 (secondary)

\section{Introduction}

We study the Cauchy problem for weak conservative solutions of the CamassaHolm $(\mathrm{CH})$ equation, which reads as

$$
\begin{aligned}
u_{t}+u u_{x}+p_{x} & =0, \\
\mu_{t}+(u \mu)_{x} & =\left(u^{3}-2 p u\right)_{x},
\end{aligned}
$$

where $p(t, x)$ is given by

$$
p(t, x)=\frac{1}{4} \int_{\mathbb{R}} e^{-|x-y|} u^{2}(t, y) d y+\frac{1}{4} \int_{\mathbb{R}} e^{-|x-y|} d \mu(t, y),
$$

(c) The Author(s) 2020. This is an Open Access article, distributed under the terms of the Creative Commons Attribution licence (http://creativecommons.org/licenses/by/4.0/), which permits unrestricted re-use, distribution, and reproduction in any medium, provided the original work is properly cited. 
with initial data $\left.(u, \mu)\right|_{t=0}=\left(u_{0}, \mu_{0}\right)$. In this case, the natural solution space consists of all pairs $(u, \mu)$ such that

$$
u(t, \cdot) \in H^{1}(\mathbb{R}), \quad \mu(t, \cdot) \in \mathcal{M}_{+}(\mathbb{R}), \quad \text { and } \quad d \mu_{\mathrm{ac}}=\left(u^{2}+u_{x}^{2}\right) d x,
$$

where $\mathcal{M}_{+}(\mathbb{R})$ denotes the set of all positive and finite Radon measures on $\mathbb{R}$. Our main goal is to prove the existence of a metric $d$ such that

$$
d\left(\left(u_{1}(t), \mu_{1}(t)\right),\left(u_{2}(t), \mu_{2}(t)\right)\right) \leqslant \alpha(t) d\left(\left(u_{1,0}, \mu_{1,0}\right),\left(u_{2,0}, \mu_{2,0}\right)\right),
$$

for two weak conservative solutions $\left(u_{j}(t), \mu_{j}(t)\right) j=1,2$ of (1.1) with initial data $\left(u_{j, 0}, \mu_{j, 0}\right) j=1,2$. Here $\alpha(t)$ depends on the total energy of the solutions and $\alpha(0)=1$.

The $\mathrm{CH}$ equation has been introduced in the seminal paper [10]; see also [11]. Originally derived in the context of models for shallow water (see also $[27,58]$ ), it also turns up in models for hyperelastic rods [24, 28, 55]. Since it captures the nonlinear effects that give insight into important phenomena such as breaking waves and breaking rods, the $\mathrm{CH}$ equation has been intensively studied. The intricate behavior of solutions of the Cauchy problem will be the focus of this paper. We will not discuss further properties of the $\mathrm{CH}$ equation, for example, the fact that the equation is completely integrable and allows for a geometric interpretation. With the latter, we mean that the $\mathrm{CH}$ equation is a re-expression of a geodesic flow on the diffeomorphism group of the line or the circle [26, 31, 60]. Several extensions and generalizations exist, but we will focus on (1.1).

The intriguing aspect of solutions to the Cauchy problem is the generic development of singularities in finite time, irrespective of the smoothness of the initial data. A solution may develop steep gradients, but in contrast to, say, hyperbolic conservation laws, the solution itself remains continuous. A finer analysis reveals that the energy density $u^{2}+u_{x}^{2}$ develops singularities, and at breakdown, which is often referred to as wave breaking, energy concentrates on sets of measure zero. Thus it becomes useful to introduce a measure, here denoted by $\mu$, that encodes the energy, and away from breakdowns this measure should coincide with the energy density $u^{2}+u_{x}^{2}$. In technical terms, we consider a nonnegative Radon measure $\mu$ with an absolutely continuous part $d \mu_{\mathrm{ac}}=\left(u^{2}+\right.$ $\left.u_{x}^{2}\right) d x$. This measure $\mu$ satisfies equation (1.1b) (and as can easily be verified, $\mu=u^{2}+u_{x}^{2}$ will satisfy the same equation in the case of smooth solutions). An illustrating example of how intricate the structure of the points of wave breaking may be can be found in [40]. The behavior in the proximity of the point of wave breaking, and, in particular, the prolongation of the solution past wave breaking, has been extensively studied. See, for example, [6, 7, 21, 22, 25, 32, 33, 39, 43$\mathbf{5 4}, \mathbf{5 6}, \mathbf{5 7}]$ and references therein. The key point here is that past wave breaking 
uniqueness fails, and there is a continuum of distinct solutions [47], with two extreme cases called dissipative and conservation solutions. To understand this conundrum, it turns out to be advantageous to rewrite the equation in a different set of variables where the solution remains smooth.

The explicit peakon-antipeakon solution [41], which illustrates this problem, is given by

$$
u(t, x)= \begin{cases}-\alpha(t) e^{x}, & x \leqslant-\gamma(t), \\ \beta(t) \sinh (x), & -\gamma(t)<x<\gamma(t), \\ \alpha(t) e^{-x}, & \gamma(t) \leqslant x\end{cases}
$$

where

$$
\alpha(t)=\frac{E}{2} \sinh \left(\frac{E}{2} t\right), \quad \beta(t)=E \frac{1}{\sinh \left(\frac{E}{2} t\right)}, \quad \gamma(t)=\ln \left(\cosh \left(\frac{E}{2} t\right)\right),
$$

where $E=\|u(t)\|_{H^{1}}$ for all $t \neq 0$. This function is a weak conservative solution which consists of a 'peak' moving to the right and an 'antipeak' moving to the left; see Figure B.3. At $t=0$ the 'peak' and 'antipeak' collide, and the solution vanishes, yet the solution is highly nontrivial before and after the collision time. Clearly the trivial solution will coincide with this solution at $t=0$, yet the trivial solution and (1.3) are very different at any other time. Thus it is not clear how to derive a metric comparing two solutions, that is stable under the time evolution. This is the task of the present work.

To be more precise, we are here presenting a metric $d$ with the property that

$$
d\left(\left(u_{1}(t), \mu_{1}(t)\right),\left(u_{2}(t), \mu_{2}(t)\right)\right) \leqslant \alpha(t) d\left(\left(u_{1,0}, \mu_{1,0}\right),\left(u_{2,0}, \mu_{2,0}\right)\right),
$$

for two weak conservative solutions $\left(u_{j}(t), \mu_{j}(t)\right), j=1,2$, of (1.1) with initial data $\left(u_{j, 0}, \mu_{j, 0}\right), j=1,2$. Here $\alpha(t)$ is a continuous function with $\alpha(0)=1$, which may depend on the total energies involved, but not on the particular solutions. We stress that no standard Sobolev norm nor Lebesgue space norm will work. There exist alternative metrics for solutions of the $\mathrm{CH}$ equation; see [8, 42, 43]. In [8] the periodic case is treated by approximating the solution by multipeakons. The metric is defined by optimizing over a class of functions. The approach in $[42,43]$ depends on a reformulation of the $\mathrm{CH}$ equation in terms of Lagrangian variables. An intrinsic problem in this formulation is that of relabeling, where there will be many different parametrizations in Lagrangian coordinates, corresponding to one and the same solution $(u(t), \mu(t))$ in Eulerian variables. Thus one has to compute the distance between equivalence classes, which is not transparent. In the present approach, the key idea is to introduce a new set of variables, where one variable plays a role similar to a characteristic, while the remaining variables are linked to $(u, \mu)$ with the help of the 'characteristic'. As we will outline next, there is no 
need to resort to equivalence classes or to optimize over classes of functions, and in spite of this proof being longer, we consider this approach to be more natural.

Our approach is based on the fact that a natural metric for measuring distances between Radon measures (with the same total mass) is given through the Wasserstein (or Monge-Kantorovich) distance $d_{W}$, which in one dimension is defined with the help of pseudoinverses; see [67]. Given a nonnegative measure $\mu$ of finite mass $M>0$, we define the cumulative distribution function associated to $\mu$ as

$$
F(x)=\mu((-\infty, x)),
$$

which is a nondecreasing function from $\mathbb{R}$ onto $[0, M]$, left continuous and with limit from the right at any point $x \in \mathbb{R}$. The pseudoinverse associated to $\mu$ denoted by $\mathcal{X}$ is the function from $[0, M]$ onto $\mathbb{R}$ given by

$$
\mathcal{X}(\eta)=\sup \{x \mid F(x)<\eta\} .
$$

The pseudoinverse of $F$ is a nondecreasing function from $[0, M]$ onto $\mathbb{R}$, left continuous and with limit from the right (caglad) at any point $x \in \mathbb{R}$. Notice the different convention adopted here with respect to the usual one in probability theory defining cumulative distribution functions continuous from the right and with limits from the left (cadlag) at every point. We prefer to have caglad instead of cadlag functions due to the use of the methods from [54] developed under the present convention. Wasserstein distances between nonnegative measures with the same mass can be defined via $L^{p}$-norms of the difference between their associated pseudoinverses; see $[19,20,61,67]$ and the references therein.

The approach of using Wasserstein distances to control the expansion of solutions of evolutionary PDEs leading to curves of probability measures goes back to the proofs of the mean-field limit of McKean-Vlasov and Vlasov equations in the late seventies and eighties of the last century. We refer to the classical references [5, 29, 63-65] proving these large particle limits by means of the bounded Lipschitz distance and the coupling method. See the recent results and surveys in $[3,12,13,35,36]$. The optimal transport viewpoint for one-dimensional models was developed using pseudoinverse distributions for nonlinear aggregation and diffusion equations in $[15,19,20,61,66]$ and the references therein, showing the contractivity of the Wasserstein distance in one dimension without the heavy machinery of optimal transport developed for general gradient flows in [1]. More recently, these metrics have been used with success to show uniqueness past the blow-up time for multidimensional aggregation equations [14] using gradient flow solutions. It is also interesting to point out that gradient flow solutions of the aggregation equation in one dimension with particular potentials are equivalent to entropy solutions of the Burgers 
equation as proven in [4]. Another strategy using unbalanced optimal transport tools has been recently analyzed in [34] with the objective of understanding the relation between the incompressible Euler equations and the $\mathrm{CH}$ equations.

Finally, it is worth mentioning that there have been several works $[2,17,18,30$, $37,38,59,62,68$ ] making use of this change of variables to produce numerical schemes capable of going over blow-up of solutions to nonlinear aggregations and being able to capture the blow-up of solutions of aggregation-diffusion models in one dimension such as toy versions of the Keller-Segel model for chemotaxis. It is a nice avenue of research to use this approach to produce numerical schemes for conservative solutions of the $\mathrm{CH}$ equation; see [23] for related particle methods.

In the present work, we will adapt this strategy of defining suitable distances between measures to the present problem of finding good metrics for solutions of the $\mathrm{CH}$ equation. Let $(u(t, \cdot), \mu(t, \cdot))$ be a weak conservative solution to the $\mathrm{CH}$ equation with total energy $\mu(t, \mathbb{R})=C>0$ (which for simplicity here is assumed to be smooth). Let

$$
F(t, x)=\mu(t,(-\infty, x))=\int_{-\infty}^{x} d \mu(t)
$$

due to the smoothness, and introduce the basic quantity

$$
G(t, x)=\int_{-\infty}^{x}\left(2 p-u^{2}\right)(t, y) d y+F(t, x)=2 p_{x}(t, x)+2 F(t, x) .
$$

The key function here is the (spatial) inverse of the strictly increasing function $G$ for fixed time $t$. To that end, we define

$$
\mathcal{Y}(t, \eta)=\sup \{x \mid G(t, x)<\eta\} .
$$

Formally we have that $G(t, \mathcal{Y}(t, \eta))=\eta$ for all $\eta \in(0,2 C)$ and $\mathcal{Y}(t, G(t, x))=x$ for all $x \in \mathbb{R}$. Here it is important to note that the domain of $\mathcal{Y}$ depends on the total energy $C$. Next, we want to determine the time evolution of $\mathcal{Y}$. Direct formal calculations yield that

$$
\begin{aligned}
\mathcal{Y}_{t}(t, G(t, x))+\mathcal{Y}_{\eta}(t, G(t, x)) G_{t}(t, x) & =0, \\
\mathcal{Y}_{\eta}(t, G(t, x)) G_{x}(t, x) & =1 .
\end{aligned}
$$

Thus we need to compute the time evolution of $G(t, x)$ before being able to compute the time evolution of $\mathcal{Y}(t, \eta)$. To that end, we find, after some computations, that

$$
G_{t}(t, x)+u G_{x}(t, x)=\frac{2}{3} u^{3}(t, x)+S(t, x),
$$


where

$$
S(t, x)=\int_{\mathbb{R}} e^{-|x-y|}\left(\frac{2}{3} u^{3}-u_{x} p_{x}-2 p u\right)(t, y) d y .
$$

Introducing $\eta=G(t, x), \mathcal{S}(t, \eta)=S(t, \mathcal{Y}(t, \eta))$, and

$$
\mathcal{U}(t, \eta)=u(t, \mathcal{Y}(t, \eta))
$$

we find by combining (1.4) and (1.5) that

$$
\mathcal{Y}_{t}(t, \eta)+\left(\frac{2}{3} \mathcal{U}^{3}+\mathcal{S}\right)(t, \eta) \mathcal{Y}_{\eta}(t, \eta)=\mathcal{U}(t, \eta),
$$

where we used that $\mathcal{Y}(t, G(t, x))=x$ for all $x \in \mathbb{R}$. As far as the time evolution of $\mathcal{U}(t, \eta)$ is concerned, we find

$$
\mathcal{U}_{t}(t, \eta)=-\mathcal{Q}(t, \eta)-\left(\frac{2}{3} \mathcal{U}^{3}+\mathcal{S}\right) \mathcal{U}_{\eta}(t, \eta)
$$

where we introduced $\mathcal{Q}(t, \eta)=p_{x}(t, \mathcal{Y}(t, \eta))$. Thus, formally we end up with the system

$$
\begin{aligned}
& \mathcal{Y}_{t}(t, \eta)+\left(\frac{2}{3} \mathcal{U}^{3}+\mathcal{S}\right) \mathcal{Y}_{\eta}(t, \eta)=\mathcal{U}(t, \eta) \\
& \mathcal{U}_{t}(t, \eta)+\left(\frac{2}{3} \mathcal{U}^{3}+\mathcal{S}\right) \mathcal{U}_{\eta}(t, \eta)=-\mathcal{Q}(t, \eta)
\end{aligned}
$$

However, this system is not closed, and we need to introduce the function

$$
\mathcal{P}(t, \eta)=p(t, \mathcal{Y}(t, \eta))
$$

and determine its time evolution. We find, after some computations, that

$$
\mathcal{P}_{t}(t, \eta)+\left(\frac{2}{3} \mathcal{U}^{3}+\mathcal{S}\right) \mathcal{P}_{\eta}(t, \eta)=\mathcal{Q U}(t, \eta)+\mathcal{R}(t, \eta)
$$

where

$$
\begin{aligned}
\mathcal{R}(t, \eta)= & \frac{1}{4} \int_{0}^{2 C} \operatorname{sign}(\eta-\theta) e^{-|\mathcal{Y}(t, \eta)-\mathcal{Y}(t, \theta)|}\left(\frac{2}{3} \mathcal{U}^{3} \mathcal{Y}_{\eta}+\mathcal{U}\right)(t, \theta) d \theta \\
& -\frac{1}{2} \int_{0}^{2 C} e^{-|\mathcal{Y}(t, \eta)-\mathcal{Y}(t, \theta)|} \mathcal{U} \mathcal{Q Y}_{\eta}(t, \theta) d \theta
\end{aligned}
$$

To summarize, we have established the following system of differential equations

$$
\begin{aligned}
& \mathcal{Y}_{t}(t, \eta)+\left(\frac{2}{3} \mathcal{U}^{3}+\mathcal{S}\right) \mathcal{Y}_{\eta}(t, \eta)=\mathcal{U}(t, \eta) \\
& \mathcal{U}_{t}(t, \eta)+\left(\frac{2}{3} \mathcal{U}^{3}+\mathcal{S}\right) \mathcal{U}_{\eta}(t, \eta)=-\mathcal{Q}(t, \eta) \\
& \mathcal{P}_{t}(t, \eta)+\left(\frac{2}{3} \mathcal{U}^{3}+\mathcal{S}\right) \mathcal{P}_{\eta}(t, \eta)=\mathcal{Q} \mathcal{U}(t, \eta)+\mathcal{R}(t, \eta)
\end{aligned}
$$


where

$$
\begin{aligned}
\mathcal{Q}(t, \eta)= & -\frac{1}{4} \int_{0}^{2 C} \operatorname{sign}(\eta-\theta) e^{-|\mathcal{Y}(t, \eta)-\mathcal{Y}(t, \theta)|}\left(2\left(\mathcal{U}^{2}-\mathcal{P}\right) \mathcal{Y}_{\eta}(t, \theta)+1\right) d \theta \\
\mathcal{S}(t, \eta)= & \int_{0}^{2 C} e^{-|\mathcal{Y}(t, \eta)-\mathcal{Y}(t, \theta)|}\left(\frac{2}{3} \mathcal{U}^{3} \mathcal{Y}_{\eta}-\mathcal{U}_{\eta} \mathcal{Q}-2 \mathcal{P} \mathcal{U} \mathcal{Y}_{\eta}\right)(t, \theta) d \theta \\
\mathcal{R}(t, \eta)= & \frac{1}{4} \int_{0}^{2 C} \operatorname{sign}(\eta-\theta) e^{-|\mathcal{Y}(t, \eta)-\mathcal{Y}(t, \theta)|}\left(\frac{2}{3} \mathcal{U}^{3} \mathcal{Y}_{\eta}+\mathcal{U}\right)(t, \theta) d \theta \\
& -\frac{1}{2} \int_{0}^{2 C} e^{-|\mathcal{Y}(t, \eta)-\mathcal{Y}(t, \theta)|} \mathcal{U} \mathcal{Q Y}_{\eta}(t, \theta) d \theta
\end{aligned}
$$

Derived under assumptions of smoothness of the functions involved, the same system is valid also in the general case of weak conservation solutions. However, that requires considerable analysis, and Section 3 is devoted to that. The next step is to estimate the time evolution of these quantities $(\mathcal{Y}, \mathcal{U}, \mathcal{P})$. It turns out that the natural functional space is the space of square integrable functions for the unknowns $\left(\mathcal{Y}, \mathcal{U}, \mathcal{P}^{1 / 2}\right)$. For this reason, we prefer to work with $\mathcal{P}^{1 / 2}$ rather than $\mathcal{P}$. Section 3 focuses on the first qualitative properties of the time evolution of the solutions of (1.9) for weak conservative solutions of the $\mathrm{CH}$ equation (1.1) as well as the propagation in time of the $L^{2}$-norm of the unknowns.

The main aim of our work is to identify the right distance between two general conservative solutions of the $\mathrm{CH}$ equation (1.1), or equivalently, between two general $L^{2}$ solutions $\left(\mathcal{Y}, \mathcal{U}, \mathcal{P}^{1 / 2}\right)$ of system (1.9) with possibly different energies. In order to compare solutions with different energies, we need to rescale the solutions of (1.9) in such a way that they are defined on the same interval. Since the natural functional space for our unknowns $\left(\mathcal{Y}, \mathcal{U}, \mathcal{P}^{1 / 2}\right)$ was identified as the $L^{2}$-functional space, it seems natural to do a scaling conserving the $L^{2}$-norms of the unknowns $\left(\mathcal{Y}, \mathcal{U}, \mathcal{P}^{1 / 2}\right)$, but leading to the domain being independent of the total energy $C$.

Let us define the scaled unknowns $\left(\tilde{\mathcal{Y}}, \tilde{\mathcal{U}}, \tilde{\mathcal{P}}^{1 / 2}\right)$ associated to a conservative solution $(u(t), \mu(t))$ with energy $C=\mu(t, \mathbb{R})$ of the $C H$ equation (1.1) as $\tilde{\mathcal{Y}}(t$, $\eta)=\sqrt{2 C} \mathcal{Y}(t, 2 C \eta), \tilde{\mathcal{U}}(t, \eta)=\sqrt{2 C} \mathcal{U}(t, 2 C \eta)$, and $\tilde{\mathcal{P}}^{1 / 2}(t, \eta)=\sqrt{2 C} \mathcal{P}^{1 / 2}(t$, $2 C \eta)$, where $\left(\mathcal{Y}, \mathcal{U}, \mathcal{P}^{1 / 2}\right)$ is the solution of (1.9). This scaling allows also for the zero solution to (1.1) to be included in our considerations, as outlined in Section 4. A similar system to (1.9) can be written for $\left(\tilde{\mathcal{Y}}, \tilde{\mathcal{U}}, \tilde{\mathcal{P}}^{1 / 2}\right)$, but this is postponed to Section 4. With this new set of unknowns in place, we can now define a metric to compare two general conservative solutions $\left(u_{i}, \mu_{i}\right), i=1,2$, of (1.1) with total 
energy $C_{i}=\mu_{i}(\mathbb{R})$. We define it as

$$
\begin{aligned}
d\left(\left(u_{1}, \mu_{1}\right),\left(u_{2}, \mu_{2}\right)\right)= & \left\|\tilde{\mathcal{Y}}_{1}-\tilde{\mathcal{Y}}_{2}\right\|_{L^{2}([0,1])}+\left\|\tilde{\mathcal{U}}_{1}-\tilde{\mathcal{U}}_{2}\right\|_{L^{2}([0,1])} \\
& +\left\|\tilde{\mathcal{P}}_{1}^{1 / 2}-\tilde{\mathcal{P}}_{2}^{1 / 2}\right\|_{L^{2}([0,1])}+\left|\sqrt{2 C_{1}}-\sqrt{2 C_{2}}\right| .
\end{aligned}
$$

Our main result reads as follows.

TheOREM 1.1. Consider initial data $u_{i, 0} \in H^{1}(\mathbb{R}), \mu_{i, 0} \in \mathcal{M}_{+}(\mathbb{R})$ such that $d\left(\mu_{\mathrm{ac}}\right)_{i, 0}=\left(u_{i}^{2}+u_{i, x}^{2}\right) d x$ and $C_{i}=\mu_{i}(\mathbb{R})$, and let $\left(u_{i}, \mu_{i}\right)$ for $i=1,2$ denote the corresponding weak conservative solutions of the $\mathrm{CH}$ equation (1.1). Then we have that

$$
d\left(\left(u_{1}(t), \mu_{1}(t)\right),\left(u_{2}(t), \mu_{2}(t)\right)\right) \leqslant e^{\mathcal{O}(1) t} d\left(\left(u_{1,0}, \mu_{1,0}\right),\left(u_{2,0}, \mu_{2,0}\right)\right),
$$

where $\mathcal{O}(1)$ denotes a constant depending only on $\max _{j}\left(C_{j}\right)$ remaining bounded as $\max _{j}\left(C_{j}\right) \rightarrow 0$.

The main core of this work lies in estimating the Lipschitz property of the right-hand side of the equivalent system to (1.9) in the $L^{2}$-sense for the unknowns $\left(\tilde{\mathcal{Y}}, \tilde{\mathcal{U}}, \tilde{\mathcal{P}}^{1 / 2}\right)$. This is much easier in case we compare to the zero solution as it coincides with the propagation of the $L^{2}$-norms of the unknowns. Due to the intricate nonlinearities of the right-hand sides of (1.9), this leads in the general case to long detailed technical estimates that are displayed in full in Subsections 5.1, 5.2, and 5.3. In the case of peakon-antipeakon solutions, as solution (1.3) is denoted, all quantities described in this paper can be computed explicitly. The details are to be found in Appendix B.

A notational comment is in order. We decided to denote by $\mathcal{O}(1)$ constants depending on $\max _{j}\left(C_{j}\right)$ that may change from line to line along the proofs, but remain bounded as $\max _{j}\left(C_{j}\right) \rightarrow 0$. Explicit tracking of the constants could be possible but it is highly cumbersome and avoided for the sake of the reader.

\section{Formal ideas: transformations with smoothness}

Let us start by explaining all the mathematical details for the transformation in the case of smooth solutions as outlined in the introduction. Let $(u(t, \cdot)$, $\mu(t, \cdot))$ be a weak conservative solution to the $\mathrm{CH}$ equation with total energy $\mu(t, \mathbb{R})=C>0$. We assume that $F(t, x)$, given by

$$
F(t, x)=\int_{-\infty}^{x} d \mu(t)
$$


is increasing and smooth, and, in particular, that $\mu=\mu_{\mathrm{ac}}=\left(u^{2}+u_{x}^{2}\right) d x$ for all $t$. Introduce the function

$$
\begin{aligned}
G(t, x) & =\int_{-\infty}^{x}\left(2 p-u^{2}\right)(t, y) d y+F(t, x) \\
& =2 p_{x}(t, x)+2 F(t, x),
\end{aligned}
$$

where we used integration by parts and (1.2). First of all, note that the function $G(t, x)$ satisfies

$$
\lim _{x \rightarrow-\infty} G(x)=0 \text { and } \lim _{x \rightarrow \infty} G(x)=2 C,
$$

since $\left|p_{x}(t, x)\right| \leqslant p(t, x)$ and $p$ is an $H^{1}$ function on the line due to (1.2). Moreover, the function $\left(2 p-u^{2}\right)(t, x) \geqslant 0$ for all $(t, x) \in \mathbb{R}^{2}$ as the following computation shows,

$$
\begin{aligned}
\left(2 p-u^{2}\right)(t, x)= & \frac{1}{2} \int_{\mathbb{R}} e^{-|x-y|}\left(u^{2}+F_{x}\right)(t, y) d y-u^{2}(t, x) \\
= & \frac{1}{2} \int_{-\infty}^{x} e^{y-x} u^{2}(t, y) d y+\frac{1}{2} \int_{x}^{\infty} e^{x-y} u^{2}(t, y) d y \\
& +\frac{1}{2} \int_{\mathbb{R}} e^{-|x-y|} F_{x}(t, y) d y-u^{2}(t, x) \\
= & \frac{1}{2} u^{2}(t, x)-\frac{1}{2} \int_{-\infty}^{x} e^{y-x} 2 u u_{x}(t, y) d y \\
& +\frac{1}{2} u^{2}(t, x)+\frac{1}{2} \int_{x}^{\infty} e^{x-y} 2 u u_{x}(t, y) d y \\
& +\frac{1}{2} \int_{\mathbb{R}} e^{-|x-y|} F_{x}(t, y) d y-u^{2}(t, x) \\
\geqslant & \frac{1}{2} \int_{\mathbb{R}} e^{-|x-y|}\left(F_{x}(t, y)-2\left|u u_{x}\right|(t, y)\right) d y \\
= & \frac{1}{2} \int_{\mathbb{R}} e^{-|x-y|}\left(|u(t, y)|-\left|u_{x}(t, y)\right|\right)^{2} d y \geqslant 0 .
\end{aligned}
$$

Thus the function $G(t, x)$ is nondecreasing and, in our case, since the function $F(t, x)$ is smooth, also $G(t, x)$ is smooth.

REMARK 2.1. Estimate (2.2), that is, $2 p-u^{2} \geqslant 0$, remains valid also in the case where the functions are nonsmooth.

Last but not least, we want to make sure that $G(t, x)$ is strictly increasing, so that its pseudoinverse will have no jumps. $F(t, x)$ is constant if and only if $d \mu$, 
$u$, and $u_{x}$ are equal to zero. Therefore assume that there exists (for fixed $t$ ) some interval $[b, c]$ such that $d \mu(t, x)=u(t, x)=u_{x}(t, x)=0$ for all $x \in[b, c]$. Then the only term that can save us is $p(t, x)$, which in general satisfies $p(t, x) \geqslant 0$ for all $(t, x) \in \mathbb{R}^{2}$. However, whenever $\mu(t, \mathbb{R}) \neq 0$, one has by its definition in (1.2) that $p(t, x)>0$ and the claim follows.

Thus the function $G(t, x)$ is strictly increasing and continuous, and we can consider its pseudoinverse $\mathcal{Y}:[0,2 C] \rightarrow \mathbb{R}$, which in this case coincides with its inverse and which is given by

$$
\mathcal{Y}(t, \eta)=\sup \{x \mid G(t, x)<\eta\} .
$$

Since $G(t, x)$ is strictly increasing and continuous, we have that $G(t, \mathcal{Y}(t, \eta))=\eta$ for all $\eta \in(0,2 C)$ and $\mathcal{Y}(t, G(t, x))=x$ for all $x \in \mathbb{R}$. By the smoothness assumption on $F$, direct calculations yield that

$$
\begin{aligned}
\mathcal{Y}_{t}(t, G(t, x))+\mathcal{Y}_{\eta}(t, G(t, x)) G_{t}(t, x) & =0, \\
\mathcal{Y}_{\eta}(t, G(t, x)) G_{x}(t, x) & =1 .
\end{aligned}
$$

Thus we need to compute the time evolution of $G(t, x)$ before being able to compute the time evolution of $\mathcal{Y}(t, \eta)$. The following calculations are only valid in the case of smooth solutions, but we will show in the next section how to overcome this issue for weak conservative solutions. Since $e^{-|x-y|} / 2$ is the integral kernel of $\left(-\partial_{x}^{2}+1\right)^{-1}$, we observe from (1.2) that $p$ is the solution to

$$
p-p_{x x}=\frac{1}{2} u^{2}+\frac{1}{2} \mu
$$

and hence

$$
\begin{aligned}
p_{t}-p_{t x x} & =u u_{t}+\frac{1}{2} F_{x t} \\
& =-u^{2} u_{x}-u p_{x}-\frac{1}{2}\left(u F_{x}\right)_{x}+\frac{1}{2}\left(u^{3}\right)_{x}-(p u)_{x} \\
& =\frac{1}{6}\left(u^{3}\right)_{x}-(p u)_{x}-\frac{1}{2}\left(u F_{x}\right)_{x}-u p_{x},
\end{aligned}
$$

where we used the abbreviation $\mu=F_{x}$. Thus we end up with

$$
\begin{aligned}
p_{t}(t, x)= & -\frac{1}{2} \int_{\mathbb{R}} \operatorname{sign}(x-y) e^{-|x-y|}\left(\frac{1}{6} u^{3}-p u-\frac{1}{2} u F_{x}\right)(t, y) d y \\
& -\frac{1}{2} \int_{\mathbb{R}} e^{-|x-y|} u p_{x}(t, y) d y .
\end{aligned}
$$

Similar calculations yield that

$$
p_{x t}(t, x)=-\frac{1}{6} u^{3}+p u+\frac{1}{2} u F_{x}+\frac{1}{2} \int_{\mathbb{R}} e^{-|x-y|}\left(\frac{2}{3} u^{3}-u_{x} p_{x}-2 p u\right)(t, y) d y .
$$


Thus we get for the time evolution of $G(t, x)$ that

$$
G_{t}(t, x)+u G_{x}(t, x)=\frac{2}{3} u^{3}(t, x)+S(t, x),
$$

where

$$
S(t, x)=\int_{\mathbb{R}} e^{-|x-y|}\left(\frac{2}{3} u^{3}-u_{x} p_{x}-2 p u\right)(t, y) d y .
$$

Combining (2.4) and (2.5), we end up with

$$
\mathcal{Y}_{t}(t, G(t, x))+\left(\frac{2}{3} u^{3}(t, x)+S(t, x)\right) \mathcal{Y}_{\eta}(t, G(t, x))=u(t, x) .
$$

Introducing $\eta=G(t, x)$, we deduce

$$
\mathcal{Y}_{t}(t, \eta)+\left(\frac{2}{3} u^{3}+S\right)(t, \mathcal{Y}(t, \eta)) \mathcal{Y}_{\eta}(t, \eta)=u(t, \mathcal{Y}(t, \eta)),
$$

where we used that $\mathcal{Y}(t, G(t, x))=x$ for all $x \in \mathbb{R}$. As far as the time evolution of

$$
\mathcal{U}(t, \eta)=u(t, \mathcal{Y}(t, \eta))
$$

is concerned, we have

$$
\begin{aligned}
\mathcal{U}_{t}(t, \eta) & =u_{t}(t, \mathcal{Y}(t, \eta))+u_{x}(t, \mathcal{Y}(t, \eta)) \mathcal{Y}_{t}(t, \eta) \\
& =u_{t}(t, \mathcal{Y}(t, \eta))+u u_{x}(t, \mathcal{Y}(t, \eta))-\left(\frac{2}{3} u^{3}+S\right) u_{x}(t, \mathcal{Y}(t, \eta)) \mathcal{Y}_{n}(t, \eta) \\
& =-p_{x}(t, \mathcal{Y}(t, \eta))-\left(\frac{2}{3} u^{3}+S\right) u_{x}(t, \mathcal{Y}(t, \eta)) \mathcal{Y}_{n}(t, \eta) \\
& =-\mathcal{Q}(t, \eta)-\left(\frac{2}{3} \mathcal{U}^{3}+\mathcal{S}\right) \mathcal{U}_{\eta}(t, \eta),
\end{aligned}
$$

where we introduced $\mathcal{Q}(t, \eta)=p_{x}(t, \mathcal{Y}(t, \eta))$ and $\mathcal{S}(t, \eta)=S(t, \mathcal{Y}(t, \eta))$. Thus, formally we end up with the system

$$
\begin{aligned}
& \mathcal{Y}_{t}(t, \eta)+\left(\frac{2}{3} \mathcal{U}^{3}+\mathcal{S}\right) \mathcal{Y}_{\eta}(t, \eta)=\mathcal{U}(t, \eta), \\
& \mathcal{U}_{t}(t, \eta)+\left(\frac{2}{3} \mathcal{U}^{3}+\mathcal{S}\right) \mathcal{U}_{\eta}(t, \eta)=-\mathcal{Q}(t, \eta),
\end{aligned}
$$

where $\mathcal{Q}(t, \eta)$ and $\mathcal{S}(t, \eta)$ can be written as

$$
\begin{aligned}
\mathcal{Q}(t, \eta) \\
\quad=-\frac{1}{4} \int_{\mathbb{R}} \operatorname{sign}(\mathcal{Y}(t, \eta)-y) e^{-|\mathcal{Y}(t, \eta)-y|}\left(u^{2}(t, y)+F_{x}(t, y)\right) d y \\
\quad=-\frac{1}{4} \int_{\mathbb{R}} \operatorname{sign}(\mathcal{Y}(t, \eta)-y) e^{-|\mathcal{Y}(t, \eta)-y|}\left(2\left(u^{2}(t, y)-p(t, y)\right)+G_{x}(t, y)\right) d y \\
\quad=-\frac{1}{4} \int_{0}^{2 C} \operatorname{sign}(\eta-\theta) e^{-|\mathcal{Y}(t, \eta)-\mathcal{Y}(t, \theta)|}\left(2\left(\mathcal{U}^{2}-\mathcal{P}\right) \mathcal{Y}_{\eta}(t, \theta)+1\right) d \theta,
\end{aligned}
$$


and

$$
\begin{aligned}
\mathcal{S}(t, \eta) & =\int_{\mathbb{R}} e^{-|\mathcal{Y}(t, \eta)-y|}\left(\frac{2}{3} u^{3}-u_{x} p_{x}-2 p u\right)(t, y) d y \\
& =\int_{0}^{2 C} e^{-|\mathcal{Y}(t, \eta)-\mathcal{Y}(t, \theta)|}\left(\frac{2}{3} \mathcal{U}^{3} \mathcal{Y}_{\eta}-\mathcal{U}_{\eta} \mathcal{Q}-2 \mathcal{P} \mathcal{U} \mathcal{Y}_{\eta}\right)(t, \theta) d \theta,
\end{aligned}
$$

with

$$
\mathcal{P}(t, \eta)=p(t, \mathcal{Y}(t, \eta))
$$

It is then natural, in order to close system (2.7), that besides the quantities $u$ and $\mu, p$ in the new variables must be considered. One main reason is that these three quantities turn up in the definition of $G$. We already computed before that

$$
\begin{aligned}
p_{t}(t, x)= & -\frac{1}{2} \int_{\mathbb{R}} \operatorname{sign}(x-y) e^{-|x-y|}\left(\frac{1}{6} u^{3}-p u-\frac{1}{2} u F_{x}\right)(t, y) d y \\
& -\frac{1}{2} \int_{\mathbb{R}} e^{-|x-y|} u p_{x}(t, y) d y \\
= & \frac{1}{2} \int_{\mathbb{R}} \operatorname{sign}(x-y) e^{-|x-y|}\left(\frac{1}{3} u^{3}+\frac{1}{2} u G_{x}\right)(t, y) d y \\
& -\frac{1}{2} \int_{\mathbb{R}} e^{-|x-y|} u p_{x}(t, y) d y,
\end{aligned}
$$

where we used that

$$
G_{x}(t, x)=2 p(t, x)-u^{2}(t, x)+F_{x}(t, x) .
$$

Thus direct computations yield the additional equation

$$
\mathcal{P}_{t}(t, \eta)+\left(\frac{2}{3} \mathcal{U}^{3}+\mathcal{S}\right) \mathcal{P}_{\eta}(t, \eta)=\mathcal{Q U}(t, \eta)+\mathcal{R}(t, \eta)
$$

where

$$
\begin{aligned}
\mathcal{R}(t, \eta)= & \frac{1}{4} \int_{\mathbb{R}} \operatorname{sign}(\mathcal{Y}(t, \eta)-y) e^{-|\mathcal{Y}(t, \eta)-y|}\left(\frac{2}{3} u^{3}+u G_{x}\right)(t, y) d y \\
& -\frac{1}{2} \int_{\mathbb{R}} e^{-|\mathcal{Y}(t, \eta)-y|} u p_{x}(t, y) d y \\
= & \frac{1}{4} \int_{0}^{2 C} \operatorname{sign}(\eta-\theta) e^{-|\mathcal{Y}(t, \eta)-\mathcal{Y}(t, \theta)|}\left(\frac{2}{3} \mathcal{U}^{3} \mathcal{Y}_{\eta}+\mathcal{U}\right)(t, \theta) d \theta \\
& -\frac{1}{2} \int_{0}^{2 C} e^{-|\mathcal{Y}(t, \eta)-\mathcal{Y}(t, \theta)|} \mathcal{U} \mathcal{Q Y}_{\eta}(t, \theta) d \theta
\end{aligned}
$$


We summarize the result in the following proposition.

Proposition 2.2. Let $(u, \mu)$ denote a smooth solution of (1.1). Define $\mathcal{Y}$ by (2.3), $\mathcal{U}$ by (2.6), and $\mathcal{P}$ by (2.8). Then the following system of differential equations holds:

$$
\begin{aligned}
& \mathcal{Y}_{t}(t, \eta)+\left(\frac{2}{3} \mathcal{U}^{3}+\mathcal{S}\right) \mathcal{Y}_{\eta}(t, \eta)=\mathcal{U}(t, \eta) \\
& \mathcal{U}_{t}(t, \eta)+\left(\frac{2}{3} \mathcal{U}^{3}+\mathcal{S}\right) \mathcal{U}_{\eta}(t, \eta)=-\mathcal{Q}(t, \eta) \\
& \mathcal{P}_{t}(t, \eta)+\left(\frac{2}{3} \mathcal{U}^{3}+\mathcal{S}\right) \mathcal{P}_{\eta}(t, \eta)=\mathcal{Q U}(t, \eta)+\mathcal{R}(t, \eta)
\end{aligned}
$$

where

$$
\begin{aligned}
\mathcal{Q}(t, \eta)= & -\frac{1}{4} \int_{0}^{2 C} \operatorname{sign}(\eta-\theta) e^{-|\mathcal{Y}(t, \eta)-\mathcal{Y}(t, \theta)|}\left(2\left(\mathcal{U}^{2}-\mathcal{P}\right) \mathcal{Y}_{\eta}(t, \theta)+1\right) d \theta \\
\mathcal{S}(t, \eta)= & \int_{0}^{2 C} e^{-|\mathcal{Y}(t, \eta)-\mathcal{Y}(t, \theta)|}\left(\frac{2}{3} \mathcal{U}^{3} \mathcal{Y}_{\eta}-\mathcal{U}_{\eta} \mathcal{Q}-2 \mathcal{P} \mathcal{U} \mathcal{Y}_{\eta}\right)(t, \theta) d \theta \\
\mathcal{R}(t, \eta)= & \frac{1}{4} \int_{0}^{2 C} \operatorname{sign}(\eta-\theta) e^{-|\mathcal{Y}(t, \eta)-\mathcal{Y}(t, \theta)|}\left(\frac{2}{3} \mathcal{U}^{3} \mathcal{Y}_{\eta}+\mathcal{U}\right)(t, \theta) d \theta \\
& -\frac{1}{2} \int_{0}^{2 C} e^{-|\mathcal{Y}(t, \eta)-\mathcal{Y}(t, \theta)|} \mathcal{U} \mathcal{Q} \mathcal{Y}_{\eta}(t, \theta) d \theta
\end{aligned}
$$

In the next section we will derive this system of equations also in the general case without assuming smoothness of the quantities involved; see (3.28).

Let us finish this section by checking some properties of system (2.9), which will also hold in the case of weak conservative solutions as we will see in the next section.

The quantity $\frac{2}{3} \mathcal{U}^{3}+\mathcal{S}$ is the velocity field of the three equations in (2.9). Instead of applying a characteristic method to estimate the solutions to this system, we will perform integration by parts by which the $\eta$-derivative of this quantity will naturally appear.

LEMMA 2.3. Given $(u, \mu)$ a smooth solution of (1.1), then the solution to (2.9) satisfies

$$
\left|\left(\frac{2}{3} \mathcal{U}^{3}+\mathcal{S}\right)_{\eta}\right| \leqslant \mathcal{O}(1)
$$

Proof. Since $G(t, \mathcal{Y}(t, \eta))=2 p_{x}(t, \mathcal{Y}(t, \eta))+2 F(t, \mathcal{Y}(t, \eta))=\eta$, we have due to the smoothness that

$$
G_{x}(t, \mathcal{Y}(t, \eta)) \mathcal{Y}_{\eta}(t, \eta)=\left(2 p-u^{2}+F_{x}\right)(t, \mathcal{Y}(t, \eta)) \mathcal{Y}_{\eta}(t, \eta)=1 .
$$


Since $\left(2 p-u^{2}\right)(t, x) \geqslant 0$ due to (2.2) and $\left(F_{x}-u^{2}\right)(t, x) \geqslant 0$ due to (2.1), we have that

$$
\begin{aligned}
2 \mathcal{P} \mathcal{Y}_{\eta}(t, \eta) & \leqslant\left(2 p-u^{2}+F_{x}\right)(t, \mathcal{Y}) \mathcal{Y}_{\eta}(t, \eta)=1 \\
\mathcal{U}^{2} \mathcal{Y}_{\eta}(t, \eta) & \leqslant 2 \mathcal{P} \mathcal{Y}_{\eta}(t, \eta) \leqslant 1 \\
2\left|\mathcal{U} \mathcal{U}_{\eta}(t, \eta)\right| & =2\left|u u_{x}(t, \mathcal{Y}) \mathcal{Y}_{\eta}(t, \eta)\right| \leqslant\left(u^{2}+u_{x}^{2}\right)(t, \mathcal{Y}) \mathcal{Y}_{\eta}(t, \eta) \\
& =F_{x}(t, \mathcal{Y}) \mathcal{Y}_{\eta}(t, \eta) \leqslant\left(2 p-u^{2}+F_{x}\right)(t, \mathcal{Y}) \mathcal{Y}_{\eta}(t, \eta)=1 .
\end{aligned}
$$

We conclude that

$$
\mathcal{P} \mathcal{Y}_{\eta}(t, \eta) \leqslant \frac{1}{2}, \quad\left|\mathcal{U} \mathcal{U}_{\eta}(t, \eta)\right| \leqslant \frac{1}{2}, \quad \text { and } \quad \mathcal{U}^{2} \mathcal{Y}_{\eta}(t, \eta) \leqslant 1
$$

From the fact that the energy is conserved, it follows that $u(t) \in H^{1}(\mathbb{R})$ for all $t \geqslant 0$ and

$$
\|u(t, \cdot)\|_{L^{\infty}(\mathbb{R})} \leqslant \sqrt{C} .
$$

Therefore, the term $\mathcal{U}^{2} \mathcal{U}_{\eta}$ is bounded by $\mathcal{O}(1)$.

Furthermore, $\mathcal{S}_{\eta}(t, \eta)$ is bounded. In particular, one can establish that

$$
\mathcal{S}_{\eta}(t, \eta) \leqslant \mathcal{O}(1) \mathcal{P} \mathcal{Y}_{\eta}(t, \eta)
$$

which is going to play a key role. Indeed, by definition one has

$$
S(t, x)=\int_{\mathbb{R}} e^{-|x-y|}\left(\frac{2}{3} u^{3}-u_{x} p_{x}-2 p u\right)(t, y) d y,
$$

and hence

$$
S_{x}(t, x)=-\int_{\mathbb{R}} \operatorname{sign}(x-y) e^{-|x-y|}\left(\frac{2}{3} u^{3}-u_{x} p_{x}-2 p u\right)(t, y) d y .
$$

Our aim is to show that

$$
S_{x}(t, x) \leqslant \mathcal{O}(1) p(t, x) .
$$

First of all, note that we have

$$
\begin{aligned}
\left|\int_{\mathbb{R}} \operatorname{sign}(x-y) e^{-|x-y|} \frac{2}{3} u^{3}(t, y) d y\right| & \leqslant \frac{8}{3}\|u(t, \cdot)\|_{L^{\infty}(\mathbb{R})} \frac{1}{4} \int_{\mathbb{R}} e^{-|x-y|} u^{2}(t, y) d y \\
& \leqslant \mathcal{O}(1) p(t, x) .
\end{aligned}
$$


Moreover,

$$
\begin{aligned}
& \left|\int_{\mathbb{R}} \operatorname{sign}(x-y) e^{-|x-y|}\left(u_{x} p_{x}+2 p u\right)(t, y) d y\right| \\
& \quad \leqslant \frac{1}{2} \int_{\mathbb{R}} e^{-|x-y|}\left(2 u^{2}+u_{x}^{2}+3 p^{2}\right)(t, y) d y \\
& \quad \leqslant 2 p(t, x)+\frac{3}{2} \int_{\mathbb{R}} e^{-|x-y|} p^{2}(t, y) d y,
\end{aligned}
$$

since $\left|p_{x}(t, x)\right| \leqslant p(t, x)$. Thus it remains to show that the last term can be bounded by a multiple of $p(t, x)$. Our reasoning will be based on integration by parts and the fact that

$$
p(t, x)-p_{x x}(t, x)=\frac{1}{2} u^{2}(t, x)+\frac{1}{2} F_{x}(t, x) .
$$

Indeed, first direct computations yield

$$
\begin{aligned}
& \int_{\mathbb{R}} e^{-|x-y|} p^{2}(t, y) d y \\
& \quad=\int_{\mathbb{R}} e^{-|x-y|} p\left(\frac{1}{2} u^{2}+\frac{1}{2} F_{x}\right)(t, y) d y+\int_{\mathbb{R}} e^{-|x-y|} p p_{x x}(t, y) d y \\
& \quad=I_{1}(t, x)+I_{2}(t, x) .
\end{aligned}
$$

Since $p(t, x) \leqslant \frac{1}{2} \int_{\mathbb{R}} F_{x}(t, y) d y \leqslant \frac{1}{2} C$, we have that $I_{1}(t, x) \leqslant\|p(t, \cdot)\|_{L^{\infty}(\mathbb{R})} \frac{1}{2} \int_{\mathbb{R}} e^{-|x-y|}\left(u^{2}+F_{x}\right)(t, y) d y \leqslant 2\|p(t, x)\|_{L^{\infty}(\mathbb{R})} p(t, x)$.

As far as $I_{2}$ is concerned, we have

$$
\begin{aligned}
& \int_{-\infty}^{x} e^{y-x} p p_{x x}(t, y) d y \\
& \quad=p p_{x}(t, x)-\int_{-\infty}^{x} e^{y-x}\left(p p_{x}+p_{x}^{2}\right)(t, y) d y \\
& \quad=p p_{x}(t, x)-\frac{1}{2} p^{2}(t, x)+\int_{-\infty}^{x} e^{y-x}\left(\frac{1}{2} p^{2}-p_{x}^{2}\right)(t, y) d y
\end{aligned}
$$

and

$$
\begin{aligned}
\int_{x}^{\infty} & e^{x-y} p p_{x x}(t, y) d y \\
& =-p p_{x}(t, x)-\int_{x}^{\infty} e^{x-y}\left(p_{x}^{2}-p p_{x}\right)(t, y) d y \\
& =-p p_{x}(t, x)-\frac{1}{2} p^{2}(t, x)+\int_{x}^{\infty} e^{x-y}\left(\frac{1}{2} p^{2}-p_{x}^{2}\right)(t, y) d y
\end{aligned}
$$


Thus

$$
I_{2}(t, x)=-p^{2}(t, x)+\int_{\mathbb{R}} e^{-|x-y|}\left(\frac{1}{2} p^{2}-p_{x}^{2}\right)(t, y) d y
$$

and subsequently

$$
\begin{aligned}
& \int_{\mathbb{R}} e^{-|x-y|} p^{2}(t, y) d y \\
& \quad \leqslant 2\|p(t, \cdot)\|_{L^{\infty}(\mathbb{R})} p(t, x)-p^{2}(t, x)+\int_{\mathbb{R}} e^{-|x-y|}\left(\frac{1}{2} p^{2}-p_{x}^{2}\right)(t, y) d y \\
& \quad \leqslant 2\|p(t, \cdot)\|_{L^{\infty}(\mathbb{R})} p(t, x)+\int_{\mathbb{R}} e^{-|x-y|}\left(\frac{1}{2} p^{2}-p_{x}^{2}\right)(t, y) d y .
\end{aligned}
$$

Reshuffling the terms, we end up with

$$
\begin{aligned}
\frac{1}{2} \int_{\mathbb{R}} e^{-|x-y|} p^{2}(t, y) d y & \leqslant \frac{1}{2} \int_{\mathbb{R}} e^{-|x-y|}\left(p^{2}+p_{x}^{2}\right)(t, y) d y \\
& \leqslant 2\|p(t, \cdot)\|_{L^{\infty}(\mathbb{R})} p(t, x) \leqslant C p(t, x),
\end{aligned}
$$

showing the desired estimate.

Next, we show that all properties seen in this section for smooth solutions remain true for weak conservative solutions to (1.1).

\section{Rigorous transformation: weak conservative solutions}

To accommodate for the wave breaking of the solutions, it has turned out to be advantageous to rewrite the $\mathrm{CH}$ equation from the original Eulerian variables into Lagrangian variables; see $[6,54]$. We will show that the system of equations obtained in Proposition 2.2 holds for the weak conservative solutions introduced in [54]. With this aim in mind let us start by summarizing their approach, which uses the different adopted convention followed in this work for cumulative distribution functions to be continuous from the left and with limit from the right (caglad) at all points $x \in \mathbb{R}$. Therefore, both $F(t, \cdot)$ and $G(t, \cdot)$ are nondecreasing and caglad functions.

Given some initial data $\left(u_{0}, \mu_{0}\right)$, the corresponding initial data in Lagrangian coordinates is then given by

$$
\begin{aligned}
y(0, \xi) & =\sup \left\{x \mid x+F_{0}(x)<\xi\right\} \\
H(0, \xi) & =\xi-y(0, \xi), \\
U(0, \xi) & =u(0, y(0, \xi))
\end{aligned}
$$


and $(y(t, \cdot), U(t, \cdot), H(t, \cdot))$ are the solutions of

$$
\begin{aligned}
y_{t}(t, \xi) & =U(t, \xi) \\
U_{t}(t, \xi) & =-Q(t, \xi) \\
H_{t}(t, \xi) & =\left(U^{3}-2 P U\right)(t, \xi),
\end{aligned}
$$

where

$$
\begin{aligned}
& P(t, \xi)=\frac{1}{4} \int_{\mathbb{R}} e^{-|y(t, \xi)-y(t, \sigma)|}\left(U^{2} y_{\xi}+H_{\xi}\right)(t, \sigma) d \sigma \\
& Q(t, \xi)=-\frac{1}{4} \int_{\mathbb{R}} \operatorname{sign}(\xi-\sigma) e^{-|y(t, \xi)-y(t, \sigma)|}\left(U^{2} y_{\xi}+H_{\xi}\right)(t, \sigma) d \sigma .
\end{aligned}
$$

Moreover, the relation between $P$ and $Q$ is given by

$$
\begin{aligned}
P_{\xi}(t, \xi) & =Q(t, \xi) y_{\xi}(t, \xi), \\
Q_{\xi}(t, \xi) & =\left(P-\frac{1}{2} U^{2}\right) y_{\xi}(t, \xi)-\frac{1}{2} H_{\xi}(t, \xi) .
\end{aligned}
$$

Introduce the function

$$
\begin{aligned}
I(t, \xi) & =\int_{-\infty}^{\xi}\left(2 P-U^{2}\right) y_{\xi}(t, \sigma) d \sigma=\int_{-\infty}^{\xi}\left(2 Q_{\xi}+H_{\xi}\right)(t, \sigma) d \sigma \\
& =2 Q(t, \xi)+H(t, \xi),
\end{aligned}
$$

where we used that

$$
\lim _{\xi \rightarrow-\infty} Q(t, \xi)=0=\lim _{\xi \rightarrow-\infty} H(t, \xi),
$$

which follows from the definition of $H(t, \xi)$. The relation between $H$ and $F$ is given by

$$
F(t, y(t, \xi)) \leqslant H(t, \xi) \leqslant F(t, y(t, \xi)+) .
$$

Here we have introduced the common notation

$$
\Phi(x \pm)=\lim _{\epsilon \downarrow 0} \Phi(x \pm \epsilon) .
$$

Notice that $I(t, \xi)+H(t, \xi)$ is the Lagrangian counterpart to the function $G(t, x)$. To convince oneself that this is really the case, one should take a quick look back first. The function $G(t, x)$ was defined as

$$
G(t, x)=\int_{-\infty}^{x}\left(2 p-u^{2}\right)(t, y) d y+F(t, x) .
$$


Thus, whenever $F(t, x)$ has a jump of height $\alpha$ at a point $\bar{x}$, that is, $\mu(t,\{\bar{x}\})=\alpha$, then also $G(t, x)$ has a jump of height $\alpha$ at $\bar{x}$, since the function $2 p-u^{2}$ is continuous. Furthermore, the point $\bar{x}$ in Eulerian coordinates is mapped to some maximal interval $\left[\xi_{l}, \xi_{r}\right]$ in Lagrangian coordinates, on which $y_{\xi}(t, \xi)=0$ and $H_{\xi}(t, \xi)=1$ for a specific choice of a relabeling function. In fact, $H_{\xi}(t, \xi)>0$ for all $\xi \in\left[\xi_{l}, \xi_{r}\right]$ as proven in [54, Theorem 4.2] and [54, Definition 2.6]. Thus a close look at $Q(t, \xi)$ reveals that $Q(t, \xi)=Q\left(t, \xi_{l}\right)-\frac{1}{2} \int_{\xi_{l}}^{\xi} H_{\xi}(t, \sigma) d \sigma$ for all $\xi \in\left[\xi_{l}, \xi_{r}\right]$ and hence

$$
\begin{aligned}
I(t, \xi) & =2 Q(t, \xi)+H(t, \xi) \\
& =2 Q\left(t, \xi_{l}\right)-\int_{\xi_{l}}^{\xi} H_{\xi}(t, \sigma) d \sigma+H\left(t, \xi_{l}\right)+\int_{\xi_{l}}^{\xi} H_{\xi}(t, \sigma) d \sigma \\
& =2 Q\left(t, \xi_{l}\right)+H\left(t, \xi_{l}\right)=I\left(t, \xi_{l}\right) .
\end{aligned}
$$

In short, we have that $I(t, \xi)=I\left(t, \xi_{l}\right)$ for all $\xi \in\left[\xi_{l}, \xi_{r}\right]$. This allows us now to follow a similar approach as for the Hunter-Saxton (HS) equation in $[9,16]$. Therefore introduce

$$
J(t, \xi)=I(t, \xi)+H(t, \xi)=\int_{-\infty}^{\xi}\left(2 P-U^{2}\right) y_{\xi}(t, \sigma) d \sigma+H(t, \xi),
$$

and observe that for all solutions except the zero solution $J(t, \xi)$ is strictly increasing and continuous. In more detail, one has for all solutions except the zero solution that $P(t, \xi) \neq 0$ for all $\xi \in \mathbb{R}$, since $y_{\xi}+H_{\xi}>0$ almost everywhere due to [54, Definition 2.6]. Moreover, if $H_{\xi}(t, \bar{\xi})=0$ for some $\bar{\xi}$ one has that $U(t, \bar{\xi})=0$ and $y_{\xi}(t, \bar{\xi}) \neq 0$, since $y_{\xi} H_{\xi}(t, \bar{\xi})=U^{2} y_{\xi}(t, \bar{\xi})+U_{\xi}^{2}(t, \bar{\xi})$ almost everywhere due to [54, Definition 2.6], and hence the $\xi$-derivative of $J(t, \xi)$ is strictly positive at the point $\bar{\xi}$.

LemMA 3.1. Given $\mathcal{Y}(t, \eta)=\sup \{x \mid G(t, x)<\eta\}$, then

$$
\mathcal{Y}(t, \eta)=y(t, l(t, \eta)),
$$

where we have introduced $l(t, \cdot):[0,2 C] \rightarrow \mathbb{R}$ by

$$
l(t, \eta)=\sup \{\xi \mid J(t, \xi)<\eta\} .
$$

Proof. For each time $t$ we have

$$
y(t, \xi)=\sup \{x \mid x+F(t, x)<y(t, \xi)+H(t, \xi)\},
$$

which implies that

$$
y(t, \xi)+F(t, y(t, \xi)) \leqslant y(t, \xi)+H(t, \xi) \leqslant y(t, \xi)+F(t, y(t, \xi)+) .
$$


Moreover, one has that $G(t, x)-F(t, x)$ is continuous, and, in particular,

$$
\begin{aligned}
G(t, y(t, \xi))-F(t, y(t, \xi)) & =\int_{-\infty}^{y(t, \xi)}\left(2 p-u^{2}\right)(t, y) d y \\
& =\int_{-\infty}^{\xi}\left(2 P-U^{2}\right) y_{\xi}(t, \sigma) d \sigma=I(t, \xi) .
\end{aligned}
$$

Thus one has

$$
y(t, \xi)+G(t, y(t, \xi)) \leqslant y(t, \xi)+J(t, \xi) \leqslant y(t, \xi)+G(t, y(t, \xi)+) .
$$

Subtracting $y(t, \xi)$ in the above inequality, we end up with

$$
G(t, y(t, \xi)) \leqslant J(t, \xi) \leqslant G(t, y(t, \xi)+) \quad \text { for all } \xi \in \mathbb{R} .
$$

Comparing the last equation and (3.8), we have

$$
G(t, y(t, l(t, \eta))) \leqslant J(t, l(t, \eta))=\eta \leqslant G(t, y(t, l(t, \eta))+) .
$$

Since $y(t, \cdot)$ is surjective and nondecreasing, we end up with

$$
\mathcal{Y}(t, \eta)=\sup \{x \mid G(t, x)<\eta\}=y(t, l(t, \eta)),
$$

thereby proving (3.7).

In the next step we want to establish rigorously the corresponding system of differential equations. Hence we first have to establish that the function $l(t, \eta)$ is differentiable, both with respect to time and space.

3.1. The differentiability of $Q(t, \xi)$. The differentiability of $Q(t, \xi)$ with respect to $\xi$ has been proven in [54] and $Q_{\xi}(t, \xi)$ is given by (3.4b). Thus it is left to establish the differentiability with respect to time of $Q(t, \xi)$. To be more precise, we are going to establish the Lipschitz continuity of $Q(t, \xi)$ with respect to time.

Let us recall that since $U(t, \cdot) \in H^{1}(\mathbb{R})$, one has in particular that $U(t, \cdot) \in$ $L^{\infty}(\mathbb{R})$. Moreover, direct calculations yield

$$
\begin{aligned}
U^{2}(t, \xi) & =U^{2}(t, \xi)-U^{2}(t,-\infty)=\int_{-\infty}^{\xi} 2 U U_{\xi}(t, \sigma) d \sigma \\
& \leqslant \int_{-\infty}^{\xi} H_{\xi}(t, \sigma) d \sigma \leqslant H(t, \infty)=C,
\end{aligned}
$$

and we end up with

$$
\|U(t, \cdot)\|_{L^{\infty}(\mathbb{R})} \leqslant \sqrt{C} \text { for all } t \in \mathbb{R} .
$$


Here we used Xavier's relation from [54] which asserts that

$$
U^{2} y_{\xi}^{2}(t, \xi)+U_{\xi}^{2}(t, \xi)=y_{\xi} H_{\xi}(t, \xi)
$$

and hence

$$
\begin{aligned}
\left|U_{\xi}(t, \xi)\right| & \leqslant \sqrt{y_{\xi} H_{\xi}}(t, \xi) \leqslant \frac{1}{2}\left(y_{\xi}+H_{\xi}\right)(t, \xi), \\
\left|U y_{\xi}(t, \xi)\right| & \leqslant \sqrt{y_{\xi} H_{\xi}}(t, \xi) \leqslant \frac{1}{2}\left(y_{\xi}+H_{\xi}\right)(t, \xi), \\
\left|U U_{\xi}(t, \xi)\right| & \leqslant \frac{1}{2} H_{\xi}(t, \xi) .
\end{aligned}
$$

Similar considerations apply for $P(t, \xi)$. Indeed one has

$$
\begin{aligned}
0 \leqslant P(t, \xi) & =\frac{1}{4} \int_{\mathbb{R}} e^{-|y(t, \sigma)-y(t, \xi)|}\left(U^{2} y_{\xi}+H_{\xi}\right)(t, \sigma) d \sigma \\
& \leqslant \frac{1}{4} \int_{\mathbb{R}} 2 H_{\xi}(t, \sigma) d \sigma=\frac{1}{2} C .
\end{aligned}
$$

Since $|Q(t, \xi)| \leqslant P(t, \xi)$, we end up with

$$
\|Q(t, \cdot)\|_{L^{\infty}(\mathbb{R})} \leqslant \frac{1}{2} C, \quad\|P(t, \cdot)\|_{L^{\infty}(\mathbb{R})} \leqslant \frac{1}{2} C \quad \text { for all } t \in \mathbb{R} .
$$

Direct calculations, using (3.11) and (3.12), yield

$$
\begin{aligned}
- & \mathcal{O}(1)\left(y_{\xi}(t, \xi)+H_{\xi}(t, \xi)\right) \\
& \leqslant\left(y_{\xi}(t, \xi)+H_{\xi}(t, \xi)\right)_{t} \\
& =U_{\xi}(t, \xi)+3 U^{2} U_{\xi}(t, \xi)-2 Q U y_{\xi}(t, \xi)-2 P U_{\xi}(t, \xi) \\
& \leqslant \mathcal{O}(1)\left(y_{\xi}(t, \xi)+H_{\xi}(t, \xi)\right),
\end{aligned}
$$

since $U(t, \xi), P(t, \xi)$, and $Q(t, \xi)$ are uniformly bounded with respect to both space and time due to (3.10), (3.13), and (3.14). Thus, we have for $s<t$ that

$$
\begin{aligned}
& \left(y_{\xi}(s, \xi)+H_{\xi}(s, \xi)\right) e^{-\mathcal{O}(1)(t-s)} \\
& \quad \leqslant y_{\xi}(t, \xi)+H_{\xi}(t, \xi) \leqslant\left(y_{\xi}(s, \xi)+H_{\xi}(s, \xi)\right) e^{\mathcal{O}(1)(t-s)}
\end{aligned}
$$

or equivalently

$$
\begin{aligned}
\left(y_{\xi}(t, \xi)+H_{\xi}(t, \xi)\right) e^{-\mathcal{O}(1)(t-s)} & \leqslant\left(y_{\xi}(s, \xi)+H_{\xi}(s, \xi)\right) \\
& \leqslant\left(y_{\xi}(t, \xi)+H_{\xi}(t, \xi)\right) e^{\mathcal{O}(1)(t-s)} .
\end{aligned}
$$


Recall that

$$
\begin{aligned}
Q(t, \xi)= & -\frac{1}{4} \int_{-\infty}^{\xi} e^{y(t, \sigma)-y(t, \xi)}\left(U^{2} y_{\xi}+H_{\xi}\right)(t, \sigma) d \sigma \\
& +\frac{1}{4} \int_{\xi}^{\infty} e^{y(t, \xi)-y(t, \sigma)}\left(U^{2} y_{\xi}+H_{\xi}\right)(t, \sigma) d \sigma \\
= & : \frac{1}{4} Q_{1}(t, \xi)+\frac{1}{4} Q_{2}(t, \xi) .
\end{aligned}
$$

We are only establishing the Lipschitz continuity with respect to time for $Q_{1}(t, \xi)$, since the argument for $Q_{2}(t, \xi)$ follows the same lines. Let $t<\hat{t}$. Then one has

$$
\begin{aligned}
& \left|Q_{1}(t, \xi)-Q_{1}(\hat{t}, \xi)\right| \\
& \leqslant \int_{-\infty}^{\xi}\left|e^{y(t, \sigma)-y(t, \xi)}-e^{y(\hat{t}, \sigma)-y(\hat{t}, \xi)}\right|\left(U^{2} y_{\xi}+H_{\xi}\right)(t, \sigma) d \sigma \\
& \quad+\int_{-\infty}^{\xi} e^{y(\hat{t}, \sigma)-y(\hat{t}, \xi)}\left|\left(U^{2} y_{\xi}+H_{\xi}\right)(t, \sigma)-\left(U^{2} y_{\xi}+H_{\xi}\right)(\hat{t}, \sigma)\right| d \sigma \\
& =: I_{1}+I_{2} .
\end{aligned}
$$

As far as $I_{1}$ is concerned, we observe, using Lemma A.1(i), that

$$
\begin{aligned}
\left|e^{y(t, \sigma)-y(t, \xi)}-e^{y(\hat{t}, \sigma)-y(\hat{t}, \xi)}\right| & \leqslant|y(t, \sigma)-y(\hat{t}, \sigma)|+|y(t, \xi)-y(\hat{t}, \xi)| \\
& \leqslant \int_{t}^{\hat{t}}(|U(s, \sigma)|+|U(s, \xi)|) d s \leqslant \mathcal{O}(1)|t-\hat{t}|,
\end{aligned}
$$

where we used that both $e^{y(\hat{t}, \sigma)-y(\hat{t}, \xi)}$ and $e^{y(t, \sigma)-y(t, \xi)}$ are bounded from above by one, and that $U$ can be uniformly bounded both with respect to space and time due to (3.10). Thus we end up with

$$
\begin{aligned}
I_{1} & =\int_{-\infty}^{\xi}\left|e^{y(t, \sigma)-y(t, \xi)}-e^{y(\hat{t}, \sigma)-y(\hat{t}, \xi)}\right|\left(U^{2} y_{\xi}+H_{\xi}\right)(t, \sigma) d \sigma \\
& \leqslant \mathcal{O}(1)|t-\hat{t}| \int_{-\infty}^{\xi} 2 H_{\xi}(t, \sigma) d \sigma \leqslant \mathcal{O}(1)|t-\hat{t}|,
\end{aligned}
$$

where we used that $U^{2} y_{\xi} \leqslant H_{\xi}$.

Thus it remains to establish a similar estimate for $I_{2}$. The idea is to use a similar strategy combined with (3.12a) and (3.15). We have

$$
\begin{aligned}
I_{2} & =\int_{-\infty}^{\xi} e^{y(\hat{t}, \sigma)-y(\hat{t}, \xi)}\left|\left(U^{2} y_{\xi}+H_{\xi}\right)(t, \sigma)-\left(U^{2} y_{\xi}+H_{\xi}\right)(\hat{t}, \sigma)\right| d \sigma \\
& \leqslant \int_{-\infty}^{\xi} e^{y(\hat{t}, \sigma)-y(\hat{t}, \xi)} \int_{t}^{\hat{t}}\left|4 U^{2} U_{\xi}-4 Q U y_{\xi}-2 P U_{\xi}\right|(s, \sigma) d s d \sigma
\end{aligned}
$$




$$
\begin{aligned}
& \leqslant \int_{-\infty}^{\xi} e^{y(\hat{t}, \sigma)-y(\hat{t}, \xi)} \mathcal{O}(1) \int_{t}^{\hat{t}}\left(y_{\xi}+H_{\xi}\right)(s, \sigma) d s d \sigma \\
& \leqslant \mathcal{O}(1) \int_{-\infty}^{\xi} e^{y(\hat{t}, \sigma)-y(\hat{t}, \xi)} \int_{t}^{\hat{t}} e^{\mathcal{O}(1)(\hat{t}-s)}\left(y_{\xi}+H_{\xi}\right)(\hat{t}, \sigma) d s d \sigma \\
& \leqslant \mathcal{O}(1) \int_{-\infty}^{\xi} e^{y(\hat{t}, \sigma)-y(\hat{t}, \xi)}\left(y_{\xi}+H_{\xi}\right)(\hat{t}, \sigma) \int_{t}^{\hat{t}} e^{\mathcal{O}(1)(\hat{t}-s)} d s d \sigma \\
& \leqslant \mathcal{O}(1) e^{\mathcal{O}(1)(\hat{t}-t)}|\hat{t}-t| \leqslant \mathcal{O}(1)|\hat{t}-t|,
\end{aligned}
$$

under the assumption that $|\hat{t}-t| \leqslant 1$ (or in general bounded). Thus we established that

$$
\left|Q_{1}(t, \xi)-Q_{1}(\hat{t}, \xi)\right| \leqslant \mathcal{O}(1)|\hat{t}-t|
$$

for all pairs $t, \hat{t}$, such that $|\hat{t}-t| \leqslant 1$. Furthermore, one has

$$
|Q(t, \xi)-Q(\hat{t}, \xi)| \leqslant \mathcal{O}(1)|\hat{t}-t|
$$

for all pairs $t, \hat{t}$, such that $|\hat{t}-t| \leqslant 1$. Thus for fixed $\xi$, the function $Q$ is locally Lipschitz with respect to time, and hence differentiable almost everywhere by Rademacher's theorem.

A close look at the above argument reveals that we cannot only differentiate $Q(t, \xi)$ with respect to time, but also that we can apply the dominated convergence theorem, which yields

$$
\begin{aligned}
Q_{t}(t, \xi)= & -\frac{1}{4} \int_{\mathbb{R}} \frac{d}{d t}\left(\operatorname{sign}(\xi-\sigma) e^{-|y(t, \xi)-y(t, \sigma)|}\left(U^{2} y_{\xi}+H_{\xi}\right)(t, \sigma)\right) d \sigma \\
= & -\frac{2}{3} U^{3}(t, \xi)+2 P U(t, \xi) \\
& +\frac{1}{2} \int_{\mathbb{R}} e^{-|y(t, \xi)-y(t, \sigma)|}\left(\frac{2}{3} U^{3} y_{\xi}-2 P U y_{\xi}-Q U_{\xi}\right)(t, \sigma) d \sigma
\end{aligned}
$$

after some integrations by parts.

Next we want to show that $Q_{t}(t, \xi)$ can be uniformly bounded by a constant $\mathcal{O}(1)$. Observe that (3.10) and (3.13) imply

$$
\begin{aligned}
& \left|\int_{\mathbb{R}} e^{-|y(t, \sigma)-y(t, \xi)|}\left(\frac{2}{3} U^{3} y_{\xi}-2 P U y_{\xi}-Q U y_{\xi}\right)(t, \sigma) d \sigma\right| \\
& \quad \leqslant \mathcal{O}(1)\left(\int_{\mathbb{R}} e^{-|y(t, \sigma)-y(t, \xi)|} y_{\xi}(t, \sigma) d \sigma\right) \leqslant \mathcal{O}(1) .
\end{aligned}
$$

Thus recalling (3.16) and combining (3.10), (3.13), and (3.17), we finally end up with

$$
\left\|Q_{t}(t, \cdot)\right\|_{L^{\infty}(\mathbb{R})} \leqslant \mathcal{O}(1) \quad \text { for all } t \in \mathbb{R} .
$$


This completes the argument regarding the differentiability of $Q$. Notice that a direct application of the dominated convergence theorem using formula (3.16) shows that $Q_{t}$ is a continuous function in time, under the assumption that $P_{t}$ exists and satisfies an estimate similar to (3.18).

Next we focus on the differentiability with respect to time of $P$. An analogous argument to the one for $Q$ leads to the differentiability in time of $P$. Let us show that the derivative of $P$ with respect to time exists by applying the dominated convergence theorem to

$$
\lim _{s \rightarrow t} \frac{P(t, \xi)-P(s, \xi)}{t-s},
$$

where $\xi$ is chosen such that $y$ is differentiable with respect to time. Since

$$
\frac{d}{d t}\left(e^{-|y(t, \xi)-y(t, \sigma)|}\left(U^{2} y_{\xi}+H_{\xi}\right)(t, \sigma)\right)
$$

exists almost everywhere, it is left to show that we can find a function in $L^{1}(\mathbb{R})$, which bounds the integrand of (3.19) uniformly in $s$. Therefore observe that we can write, using (3.2), for $s<t$, that

$$
\begin{aligned}
P(t, \xi)-P(s, \xi) & \\
= & \frac{1}{4} \int_{-\infty}^{\xi} \int_{s}^{t} e^{y(l, \sigma)-y(l, \xi)}(U(l, \sigma)-U(l, \xi))\left(U^{2} y_{\xi}+H_{\xi}\right)(l, \sigma) d l d \sigma \\
& +\frac{1}{4} \int_{\xi}^{\infty} \int_{s}^{t} e^{y(l, \xi)-y(l, \sigma)}(U(l, \xi)-U(l, \sigma))\left(U^{2} y_{\xi}+H_{\xi}\right)(l, \sigma) d l d \sigma \\
& +\frac{1}{4} \int_{\mathbb{R}} \int_{s}^{t} e^{-|y(l, \sigma)-y(l, \xi)|}\left(4 U^{2} U_{\xi}-4 U Q y_{\xi}-2 P U_{\xi}\right)(l, \sigma) d l d \sigma \\
= & I_{1}+I_{2}+I_{3} .
\end{aligned}
$$

As far as the first term is concerned, observe that (3.15) implies that

$$
\begin{aligned}
& \left|\int_{s}^{t} e^{y(l, \sigma)-y(l, \xi)}(U(l, \sigma)-U(l, \xi))\left(U^{2} y_{\xi}+H_{\xi}\right)(l, \sigma) d l\right| \\
& \quad \leqslant \mathcal{O}(1) \int_{s}^{t} e^{y(l, \sigma)-y(l, \xi)}\left|\left(U^{2} y_{\xi}+H_{\xi}\right)(l, \sigma)\right| d l \\
& \quad \leqslant \mathcal{O}(1) e^{\mathcal{O}(1)|t-s|} e^{y(t, \sigma)-y(t, \xi)}\left(y_{\xi}+H_{\xi}\right)(t, \sigma)|t-s| \\
& \quad \leqslant \mathcal{O}(1) e^{y(t, \sigma)-y(t, \xi)}\left(y_{\xi}+H_{\xi}\right)(t, \sigma)|t-s|,
\end{aligned}
$$

if we assume that $|t-s| \leqslant 1$ or any other fixed constant. Moreover, the function in the last line belongs to $L^{1}(\mathbb{R})$. The remaining two terms can be bounded by a function, which is of the same form and belongs to $L^{1}(\mathbb{R})$ uniformly in $s$. 
Thus all assumptions of the dominated convergence theorem are fulfilled, and we end up with

$$
\begin{aligned}
P_{t}(t, \xi)= & U Q(t, \xi)+\frac{1}{4} \int_{\mathbb{R}} \operatorname{sign}(\xi-\sigma) e^{-|y(t, \xi)-y(t, \sigma)|} U\left(U^{2} y_{\xi}+H_{\xi}\right)(t, \sigma) d \sigma \\
& +\frac{1}{4} \int_{\mathbb{R}} e^{-|y(t, \xi)-y(t, \sigma)|}\left(4 U^{2} U_{\xi}-4 U Q y_{\xi}-2 P U_{\xi}\right)(t, \sigma) d \sigma \\
= & U Q(t, \xi)+\frac{1}{2} \int_{\mathbb{R}} \operatorname{sign}(\xi-\sigma) e^{-|y(t, \xi)-y(t, \sigma)|} \\
& \times U\left(\frac{1}{3} U^{2} y_{\xi}+Q_{\xi}+H_{\xi}\right)(t, \sigma) d \sigma \\
& -\frac{1}{2} \int_{\mathbb{R}} e^{-|y(t, \xi)-y(t, \sigma)|} U Q y_{\xi}(t, \sigma) d \sigma
\end{aligned}
$$

where we used integration by parts together with (3.4b) in the last step. Moreover, we have that

$$
\begin{aligned}
\left|P_{t}(t, \xi)\right| \leqslant & |U Q(t, \xi)|+\frac{1}{4} \int_{\mathbb{R}} e^{-|y(t, \xi)-y(t, \sigma)|}|U|\left(U^{2} y_{\xi}+H_{\xi}\right)(t, \sigma) d \sigma \\
& +\frac{1}{4} \int_{\mathbb{R}} e^{-|y(t, \xi)-y(t, \sigma)|}\left(4 U^{2}\left|U_{\xi}\right|+4|U Q| y_{\xi}+2 P\left|U_{\xi}\right|\right)(t, \sigma) d \sigma \\
\leqslant & \|U(t, \cdot)\|_{L^{\infty}} P(t, \xi) \\
& +\frac{1}{4}\|U(t, \cdot)\|_{L^{\infty}} \int_{\mathbb{R}} e^{-|y(t, \xi)-y(t, \sigma)|}\left(U^{2} y_{\xi}+H_{\xi}\right)(t, \sigma) d \sigma \\
& +\frac{1}{4} \int_{\mathbb{R}} e^{-|y(t, \xi)-y(t, \sigma)|}\left(2 U^{4} y_{\xi}+3 H_{\xi}+2 U^{2} y_{\xi}+3 P^{2} y_{\xi}\right)(t, \sigma) d \sigma \\
\leqslant & \mathcal{O}(1) P(t, \xi)+\frac{3}{4} \int_{\mathbb{R}} e^{-|y(t, \xi)-y(t, \sigma)|} P^{2} y_{\xi}(t, \sigma) d \sigma \\
& +\frac{1}{4} \int_{\mathbb{R}} e^{-|y(t, \xi)-y(t, \sigma)|}\left(2\|U(t, \cdot)\|_{L^{\infty}}^{2} U^{2} y_{\xi}+3 H_{\xi}+2 U^{2} y_{\xi}\right)(t, \sigma) d \sigma \\
\leqslant & \mathcal{O}(1) P(t, \xi)+\frac{3}{4} \int_{\mathbb{R}} e^{-|y(t, \xi)-y(t, \sigma)|} P^{2} y_{\xi}(t, \sigma) d \sigma \\
\leqslant & \mathcal{O}(1)\left(P(t, \xi)+\int_{\mathbb{R}} e^{-|y(t, \xi)-y(t, \sigma)|} P^{2} y_{\xi}(t, \sigma) d \sigma\right)
\end{aligned}
$$

due to (3.10). It remains to show that

$$
\int_{\mathbb{R}} e^{-|y(t, \xi)-y(t, \sigma)|} P^{2} y_{\xi}(t, \sigma) d \eta \leqslant \mathcal{O}(1) P(t, \xi) .
$$

The proof follows the same lines as the one of (2.12) in Eulerian coordinates. 
3.2. The differentiability of $l(t, \eta)$. Finally, we can start thinking about the differentiability with respect to space and time of $l(t, \eta)$. Therefore, recall that the function $l(t, \eta)$ is defined as

$$
l(t, \eta)=\sup \{\xi \in \mathbb{R} \mid J(t, \xi)<\eta\} .
$$

Differentiability with respect to $\eta$ : For every $t \in \mathbb{R}$, the function $J(t, \cdot)=2 Q$ $(t, \cdot)+2 H(t, \cdot)$ is strictly increasing and continuous, and hence differentiable almost everywhere with respect to $\xi$. Its inverse $J^{-1}(t, \cdot):[0,2 C] \rightarrow \mathbb{R}$ is therefore also strictly increasing and continuous, and hence differentiable almost everywhere. Since we have by definition that $J(t, l(t, \eta))=\eta$ for all $\eta$, it follows immediately that $J(t, l(t, \cdot))$ is Lipschitz continuous with Lipschitz constant one and hence differentiable almost everywhere with respect to $\eta$. Since

$$
J^{-1}(t, J(t, l(t, \eta)))=l(t, \eta) \text { for all } \eta,
$$

we can finally conclude that $l(t, \eta)$ is differentiable almost everywhere with respect to $\eta$. In particular, one has (cf. (3.6)) that

$$
J_{\xi}(t, l(t, \eta)) l_{\eta}(t, \eta)=2 \mathcal{P} \mathcal{Y}_{\eta}(t, \eta)-\mathcal{U}^{2} \mathcal{Y}_{\eta}(t, \eta)+\mathcal{H}_{\eta}(t, \eta)=1,
$$

with

$$
\begin{gathered}
\mathcal{H}(t, \eta)=H(t, l(t, \eta)), \quad \mathcal{P}(t, \eta)=P(t, l(t, \eta)), \\
\text { and } \mathcal{U}(t, \eta)=U(t, l(t, \eta)) .
\end{gathered}
$$

These identities follow from equality (3.9) since $\mathcal{P}(t, \eta)=p(t, \mathcal{Y}(t, \eta))$ and $P(t$, $\xi)=p(t, y(t, \xi))$, and analogously $\mathcal{U}(t, \eta)=u(t, \mathcal{Y}(t, \eta))$ and $U(t, \xi)=u(t$, $y(t, \xi))$.

Following the same argument as in the smooth case (see (2.11)), we end up with

$$
0 \leqslant 2 \mathcal{P} \mathcal{Y}_{\eta}(t, \eta) \leqslant 1, \quad 2\left|\mathcal{U} \mathcal{U}_{\eta}(t, \eta)\right| \leqslant 1, \quad \text { and } \quad 0 \leqslant \mathcal{U}^{2} \mathcal{Y}_{\eta}(t, \eta) \leqslant 1
$$

Differentiability with respect to $t$ : As far as the differentiability of $l(t, \eta)$ with respect to time is concerned, the argument is a bit more involved. First of all, note that for any time $t$ we have $J(t, l(t, \eta))=\eta$ and hence

$$
J(t, l(t, \eta))=J(\tilde{t}, l(\tilde{t}, \eta)) \quad \text { for all } \eta \text { and } \tilde{t} .
$$


In particular, we can conclude that

$$
\begin{aligned}
J(t, l(t, \eta))-J(t, l(\tilde{t}, \eta)) & =J(\tilde{t}, l(\tilde{t}, \eta))-J(t, l(\tilde{t}, \eta)) \\
& =\int_{t}^{\tilde{t}} J_{t}(s, l(\tilde{t}, \eta)) d s \\
& =2 \int_{t}^{\tilde{t}}\left(Q_{t}(s, l(\tilde{t}, \eta))+H_{t}(s, l(\tilde{t}, \eta))\right) d s
\end{aligned}
$$

We already showed that $Q_{t}(t, \xi)$ exists and is given by (3.16). However, a close look reveals that $Q_{t}(t, \xi)$ is uniformly bounded both with respect to space and time and $Q_{t}(t, \xi)$ is continuous with respect to space for fixed $t$. The same holds for

$$
H_{t}(t, \xi)=U^{3}(t, \xi)-2 P U(t, \xi)
$$

Thus we have that

$$
\lim _{\tilde{t} \rightarrow t} \frac{J(t, l(t, \eta))-J(t, l(\tilde{t}, \eta))}{t-\tilde{t}}=\lim _{\tilde{t} \rightarrow t} 2 \int_{t}^{\tilde{t}} \frac{Q_{t}(s, l(\tilde{t}, \eta))+H_{t}(s, l(\tilde{t}, \eta))}{t-\tilde{t}} d s
$$

from a formal point of view. Thus it is left to establish that the limit exists and is finite.

Since $J_{t}(t, \xi)=2 Q_{t}(t, \xi)+2 H_{t}(t, \xi)$ and both $Q_{t}(t, \xi)$ and $H_{t}(t, \xi)$ can be bounded uniformly both in space and time by a constant only dependent on the total energy $C$, it follows that there exists a constant $\mathcal{O}(1)$ such that

$$
\left|J_{t}(t, \xi)\right| \leqslant \mathcal{O}(1) \quad \text { for all } t \text { and } \xi .
$$

Thus it follows that for all $s \in[t-|t-\tilde{t}|, t+|t-\tilde{t}|]$, we have

$$
J(s, \xi) \in[J(t, \xi)-\mathcal{O}(1)|\tilde{t}-t|, J(t, \xi)+\mathcal{O}(1)|\tilde{t}-t|]
$$

Accordingly we can conclude, since $J(t, l(t, \eta))=\eta$ for all $\eta$, that

$$
J(s, l(t, \eta)) \in[\eta-\mathcal{O}(1)|\tilde{t}-t|, \eta+\mathcal{O}(1)|\tilde{t}-t|],
$$

and hence

$$
l(t, \eta)=l(s, \eta(s)) \quad \text { for some } \eta(s) \in[\eta-\mathcal{O}(1)|\tilde{t}-t|, \eta+\mathcal{O}(1)|\tilde{t}-t|],
$$

and, in particular,

$$
|\eta-\eta(s)| \leqslant \mathcal{O}(1)|\tilde{t}-t| \quad \text { for all } s \in[t-|t-\tilde{t}|, t+|t-\tilde{t}|] .
$$


Similar considerations yield that for all $s \in[\tilde{t}-|t-\tilde{t}|, \tilde{t}+|t-\tilde{t}|]$, we can find $\tilde{\eta}(s)$ such that

$$
l(\tilde{t}, \eta)=l(s, \tilde{\eta}(s)) \quad \text { and } \quad|\eta-\tilde{\eta}(s)| \leqslant \mathcal{O}(1)|t-\tilde{t}| .
$$

Let us return to the integral we are actually interested in. Namely,

$$
\begin{aligned}
\int_{t}^{\tilde{t}} J_{t}(s, l(\tilde{t}, \eta)) d s= & 2 \int_{t}^{\tilde{t}}\left(Q_{t}(s, l(\tilde{t}, \eta))+H_{t}(s, l(\tilde{t}, \eta))\right) d s \\
= & 2 \int_{t}^{\tilde{t}}\left(Q_{t}(s, l(t, \eta))+H_{t}(s, l(t, \eta))\right) d s \\
& +2 \int_{t}^{\tilde{t}}\left(\left(Q_{t}(s, l(\tilde{t}, \eta))+H_{t}(s, l(\tilde{t}, \eta))\right)\right. \\
& \left.-\left(Q_{t}(s, l(t, \eta))+H_{t}(s, l(t, \eta))\right)\right) d s \\
= & \tilde{I}_{1}+\tilde{I}_{2} .
\end{aligned}
$$

What we are hoping for is that the second term $\tilde{I}_{2}$ is of order $o(|t-\tilde{t}|)$, and hence plays no role when computing (3.23). Therefore observe that

$$
\begin{aligned}
H_{t \xi}(t, \xi)= & 3 U^{2} U_{\xi}(t, \xi)-2 Q U y_{\xi}(t, \xi)-2 P U_{\xi}(t, \xi), \\
Q_{t \xi}(t, \xi)= & -2 U^{2} U_{\xi}(t, \xi)+2 Q U y_{\xi}(t, \xi)+2 P U_{\xi}(t, \xi) \\
& -\frac{1}{2} \int_{\mathbb{R}} \operatorname{sign}(\xi-\sigma) e^{-|y(t, \xi)-y(t, \sigma)|} \\
& \times\left(\frac{2}{3} U^{3} y_{\xi}-2 P U y_{\xi}-Q U_{\xi}\right)(t, \sigma) d \sigma y_{\xi}(t, \xi) .
\end{aligned}
$$

Notice that the second derivative $Q_{t \xi}$ is well defined by a dominated convergence argument as before for $Q_{t}$. Recalling (3.24), we have

$$
\begin{aligned}
\tilde{I}_{2}= & \int_{t}^{\tilde{t}} J_{t}(s, l(\tilde{t}, \eta))-J_{t}(s, l(t, \eta)) d s \\
= & 2 \int_{t}^{\tilde{t}}\left(\left(Q_{t}(s, l(\tilde{t}, \eta))+H_{t}(s, l(\tilde{t}, \eta))\right)-\left(Q_{t}(s, l(t, \eta))+H_{t}(s, l(t, \eta))\right)\right) d s \\
= & 2 \int_{t}^{\tilde{t}} \int_{l(t, \eta)}^{l(\tilde{t}, \eta)}\left(Q_{t, \xi}+H_{t, \xi}\right)(s, z) d z d s \\
= & 2 \int_{t}^{\tilde{t}} \int_{l(t, \eta)}^{l(\tilde{t}, \eta)}\left[U^{2} U_{\xi}(s, z)\right. \\
& -\frac{1}{2} \int_{\mathbb{R}}^{\operatorname{sign}(z-\sigma) e^{-|y(s, z)-y(s, \sigma)|}}
\end{aligned}
$$




$$
\begin{aligned}
& \left.\times\left(\frac{2}{3} U^{3} y_{\xi}-2 P U y_{\xi}-Q U_{\xi}\right)(s, \sigma) d \sigma y_{\xi}(s, z)\right] d z d s \\
= & 2 \int_{t}^{\tilde{t}} \int_{l(s, \eta(s))}^{l(s, \tilde{\eta}(s))}\left[U^{2} U_{\xi}(s, z)\right. \\
& -\frac{1}{2} \int_{\mathbb{R}} \operatorname{sign}(z-\sigma) e^{-|y(s, z)-y(s, \sigma)|} \\
& \left.\times\left(\frac{2}{3} U^{3} y_{\xi}-2 P U y_{\xi}-Q U_{\xi}\right)(s, \sigma) d \sigma y_{\xi}(s, z)\right] d z d s \\
= & 2 \int_{t}^{\tilde{t}} \int_{\eta(s)}^{\tilde{\eta}(s)} \mathcal{U}^{2} \mathcal{U}_{\eta}(s, m) d m d s \\
& -\int_{t}^{\tilde{t}} \int_{\eta(s)}^{\tilde{\eta}(s)} \int_{0}^{2 C} \operatorname{sign}(m-n) e^{-|\mathcal{Y}(s, m)-\mathcal{Y}(s, n)|} \\
& \times\left(\frac{2}{3} \mathcal{U}^{3} \mathcal{Y}_{\eta}-2 \mathcal{P} \mathcal{U}_{\eta}-\mathcal{Q} \mathcal{U}_{\eta}\right)(s, n) d n \mathcal{Y}_{\eta}(s, m) d m d s,
\end{aligned}
$$

where $\eta, \eta(s)$, and $\tilde{\eta}(s)$ satisfy

$$
l(t, \eta)=l(s, \eta(s)) \quad \text { and } \quad l(\tilde{t}, \eta)=l(s, \tilde{\eta}(s))
$$

and

$$
|\eta-\eta(s)| \leqslant \mathcal{O}(1)|t-\tilde{t}| \quad \text { and } \quad|\eta-\tilde{\eta}(s)| \leqslant \mathcal{O}(1)|t-\tilde{t}| \text {. }
$$

As far as the first term is concerned, we can apply (3.10), (3.22) and (3.25) as follows:

$$
\begin{aligned}
& \left|2 \int_{t}^{\tilde{t}} \int_{\eta(s)}^{\tilde{\eta}(s)} \mathcal{U}^{2} \mathcal{U}_{\eta}(s, m) d m d s\right| \\
& \quad \leqslant \int_{t}^{\tilde{t}}\|\mathcal{U}(s, \cdot)\|_{L^{\infty}([0,2 C])}|\eta(s)-\tilde{\eta}(s)| d s \leqslant \mathcal{O}(1)|t-\tilde{t}|^{2} .
\end{aligned}
$$

As far as the second and last term is concerned, we want to apply the CauchySchwarz inequality to the integral term at first. Indeed,

$$
\begin{aligned}
& \mid \int_{\eta(s)}^{\tilde{\eta}(s)} \int_{0}^{2 C} \operatorname{sign}(m-n) e^{-|\mathcal{Y}(s, m)-\mathcal{Y}(s, n)|} \\
& \quad \times\left(\frac{2}{3} \mathcal{U}^{3} \mathcal{Y}_{\eta}-2 \mathcal{P} \mathcal{U} \mathcal{Y}_{\eta}-\mathcal{Q} \mathcal{U}_{\eta}\right)(s, n) d n \mathcal{Y}_{\eta}(s, m) d m \mid \\
& \leqslant \mid \int_{\eta(s)}^{\tilde{\eta}(s)} \int_{0}^{2 C} e^{-|\mathcal{Y}(s, m)-\mathcal{Y}(s, n)|}\left[\frac{2}{3} \mathcal{U}^{2} \sqrt{\mathcal{Y}_{\eta}}\left(\mathcal{U}^{2} \mathcal{Y}_{\eta}\right)^{1 / 2}\right.
\end{aligned}
$$




$$
\begin{aligned}
& \left.+2 \mathcal{P} \sqrt{\mathcal{Y}_{\eta}}\left(\mathcal{U}^{2} \mathcal{Y}_{\eta}\right)^{1 / 2}+\mathcal{P} \sqrt{\mathcal{Y}_{\eta}} \sqrt{\mathcal{H}_{\eta}}\right](s, n) d n \mathcal{Y}_{\eta}(s, m) d m \mid \\
& \leqslant\left|\int_{\eta(s)}^{\tilde{\eta}(s)} \int_{0}^{2 C} e^{-|\mathcal{Y}(s, m)-\mathcal{Y}(s, n)|}\left(\frac{2}{3} \mathcal{U}^{2} \sqrt{\mathcal{Y}_{\eta}}+3 \mathcal{P} \sqrt{\mathcal{Y}_{\eta}}\right) \sqrt{\mathcal{H}_{\eta}}(s, n) d n \mathcal{Y}_{\eta}(s, m) d m\right| \\
& \leqslant \int_{\eta(s)}^{\tilde{\eta}(s)}\left(\int_{0}^{2 C} e^{-|\mathcal{Y}(s, m)-\mathcal{Y}(s, n)|}\left(\frac{2}{3} \mathcal{U}^{2}+3 \mathcal{P}\right)^{2} \mathcal{Y}_{\eta}(s, n) d n\right)^{1 / 2} \\
& \times\left(\int_{0}^{2 C} e^{-|\mathcal{Y}(s, m)-\mathcal{Y}(s, n)|} \mathcal{H}_{\eta}(s, n) d n\right)^{1 / 2} \mathcal{Y}_{\eta}(s, m) d m \\
& \leqslant \int_{\eta(s)}^{\tilde{\eta}(s)}\left(\int_{0}^{2 C} e^{-|\mathcal{Y}(s, m)-\mathcal{Y}(s, n)|}\left(\frac{8}{9} \mathcal{U}^{4}+18 \mathcal{P}^{2}\right) \mathcal{Y}_{\eta}(s, n) d n\right)^{1 / 2} \\
& \times\left(\int_{0}^{2 C} e^{-|\mathcal{Y}(s, m)-\mathcal{Y}(s, n)|} \mathcal{H}_{\eta}(s, n) d n\right)^{1 / 2} \mathcal{Y}_{\eta}(s, m) d m \\
& \leqslant \mathcal{O}(1)\left(\int_{\eta(s)}^{\tilde{\eta}(s)} \int_{0}^{2 C} e^{-|\mathcal{Y}(s, m)-\mathcal{Y}(s, n)|}\left(\mathcal{U}^{2}+\mathcal{P}\right) \mathcal{Y}_{\eta}(s, n) d n \mathcal{Y}_{\eta}(s, m) d m\right)^{1 / 2} \\
& \times\left(\int_{\eta(s)}^{\tilde{\eta}(s)} \int_{0}^{2 C} e^{-|\mathcal{Y}(s, m)-\mathcal{Y}(s, n)|} \mathcal{H}_{\eta}(s, n) d n \mathcal{Y}_{\eta}(s, m) d m\right)^{1 / 2} \\
& \leqslant \mathcal{O}(1)\left(\int_{\eta(s)}^{\tilde{\eta}(s)} \int_{0}^{2 C} e^{-|\mathcal{Y}(s, m)-\mathcal{Y}(s, n)|}\left(\mathcal{U}^{2}+\mathcal{P}\right) \mathcal{Y}_{\eta}(s, n) d n \mathcal{Y}_{\eta}(s, m) d m\right)^{1 / 2} \\
& \times\left(\int_{\eta(s)}^{\tilde{\eta}(s)} 4 \mathcal{P} \mathcal{Y}_{\eta}(s, m) d m\right)^{1 / 2} \\
& \leqslant \mathcal{O}(1)\left(\int_{\eta(s)}^{\tilde{\eta}(s)} \int_{0}^{2 C} e^{-|\mathcal{Y}(s, m)-\mathcal{Y}(s, n)|}\left(\mathcal{U}^{2}+\mathcal{P}\right) \mathcal{Y}_{\eta}(s, n) d n \mathcal{Y}_{\eta}(s, m) d m\right)^{1 / 2} \\
& \times \sqrt{|\eta(s)-\tilde{\eta}(s)|} \\
& \leqslant \mathcal{O}(1)\left(|t-\tilde{t}| \int_{\eta(s)}^{\tilde{\eta}(s)} \int_{0}^{2 C} e^{-|\mathcal{Y}(s, m)-\mathcal{Y}(s, n)|}\left(\mathcal{U}^{2}+\mathcal{P}\right) \mathcal{Y}_{\eta}(s, n) d n \mathcal{Y}_{\eta}(s, m) d m\right)^{1 / 2} .
\end{aligned}
$$

Thus it is left to show that

$$
\left|\int_{\eta(s)}^{\tilde{\eta}(s)} \int_{0}^{2 C} e^{-|\mathcal{Y}(s, m)-\mathcal{Y}(s, n)|}\left(\mathcal{U}^{2}+\mathcal{P}\right) \mathcal{Y}_{\eta}(s, n) d n \mathcal{Y}_{\eta}(s, m) d m\right|
$$

is bounded uniformly with respect to both space and time. Therefore observe that, since all the involved terms are positive, we have

$$
\begin{aligned}
& \left|\int_{\eta(s)}^{\tilde{\eta}(s)} \int_{0}^{2 C} e^{-|\mathcal{Y}(s, m)-\mathcal{Y}(s, n)|}\left(\mathcal{U}^{2}+\mathcal{P}\right) \mathcal{Y}_{\eta}(s, n) d n \mathcal{Y}_{\eta}(s, m) d m\right| \\
& \quad \leqslant \int_{0}^{2 C} \int_{0}^{2 C} e^{-|\mathcal{Y}(s, m)-\mathcal{Y}(s, n)|}\left(\mathcal{U}^{2}+\mathcal{P}\right) \mathcal{Y}_{\eta}(s, n) d n \mathcal{Y}_{\eta}(s, m) d m
\end{aligned}
$$




$$
\begin{aligned}
& =\int_{0}^{2 C} \int_{0}^{2 C} e^{-|\mathcal{Y}(s, m)-\mathcal{Y}(s, n)|} \mathcal{Y}_{\eta}(s, m) d m\left(\mathcal{U}^{2}+\mathcal{P}\right) \mathcal{Y}_{\eta}(s, n) d n \\
& \leqslant 2 \int_{0}^{2 C}\left(\mathcal{U}^{2}+\mathcal{P}\right) \mathcal{Y}_{\eta}(s, n) d n \leqslant 6 C
\end{aligned}
$$

where we used (3.22) in the last step. Thus we obtain

$$
\begin{aligned}
& \mid \int_{\eta(s)}^{\tilde{\eta}(s)} \int_{0}^{2 C} \operatorname{sign}(m-n) e^{-|\mathcal{Y}(s, m)-\mathcal{Y}(s, n)|} \\
& \quad \times\left(\frac{2}{3} \mathcal{U}^{3} \mathcal{Y}_{\eta}-2 \mathcal{P} \mathcal{U} \mathcal{Y}_{\eta}-\mathcal{Q} \mathcal{U}_{\eta}\right)(s, n) d n \mathcal{Y}_{\eta}(s, m) d m \mid \\
& \quad \leqslant \mathcal{O}(1)\left(|t-\tilde{t}| \int_{\eta(s)}^{\tilde{\eta}(s)} \int_{0}^{2 C} e^{-|\mathcal{Y}(s, m)-\mathcal{Y}(s, n)|}\left(\mathcal{U}^{2}+\mathcal{P}\right) \mathcal{Y}_{\eta}(s, n) d n \mathcal{Y}_{\eta}(s, m) d m\right)^{1 / 2} \\
& \quad \leqslant \mathcal{O}(1)|t-\tilde{t}|^{1 / 2}
\end{aligned}
$$

and

$$
\begin{aligned}
& \mid \int_{t}^{\tilde{t}} \int_{\eta(s)}^{\tilde{\eta}(s)} \int_{0}^{2 C} \operatorname{sign}(m-n) e^{-|\mathcal{Y}(s, m)-\mathcal{Y}(s, n)|} \\
& \quad \times\left(\frac{2}{3} \mathcal{U}^{3} \mathcal{Y}_{\eta}-2 \mathcal{P} \mathcal{U} \mathcal{Y}_{\eta}-\mathcal{Q} \mathcal{U}_{\eta}\right)(s, n) d n \mathcal{Y}_{\eta}(s, m) d m d s \mid \\
& \quad \leqslant \mathcal{O}(1)|t-\tilde{t}|^{3 / 2}
\end{aligned}
$$

Combining (3.26) and (3.27), we end up with

$$
\begin{aligned}
\left|\tilde{I}_{2}\right| & =\left|2 \int_{t}^{\tilde{t}}\left(Q_{t}(s, l(\tilde{t}, \eta))+H_{t}(s, l(\tilde{t}, \eta))\right)-\left(Q_{t}(s, l(t, \eta))+H_{t}(s, l(t, \eta))\right) d s\right| \\
& \leqslant \mathcal{O}(1)\left(|t-\tilde{t}|^{3 / 2}+|t-\tilde{t}|^{2}\right) .
\end{aligned}
$$

As far as $\tilde{I}_{1}$ is concerned, we would like to apply the mean-value theorem. Note therefore that we showed before that the function $\left(Q_{t}+H_{t}\right)(t, \xi)$ is continuous with respect to time, and hence the function $\left(Q_{t}+H_{t}\right)(s, l(t, \eta))$, considered as a function of $s$, is continuous with respect to $s$. Thus we end up with

$$
\tilde{I}_{1}=2 \int_{t}^{\tilde{t}}\left(Q_{t}+H_{t}\right)(s, l(t, \eta)) d s=2(\tilde{t}-t)\left(Q_{t}+H_{t}\right)(\tilde{s}, l(t, \eta))
$$

for some $\tilde{s}$ between $t$ and $\tilde{t}$. 
Last but not least, we therefore have

$$
\begin{aligned}
2 \int_{t}^{\tilde{t}}\left(Q_{t}+H_{t}\right)(s, l(\tilde{t}, \eta)) d s= & \tilde{I}_{1}+\tilde{I}_{2} \\
= & 2(\tilde{t}-t)\left(Q_{t}+H_{t}\right)(\tilde{s}, l(t, \eta))+\mathcal{O}(1)|\tilde{t}-t|^{3 / 2} \\
& +\mathcal{O}(1)|\tilde{t}-t|^{2},
\end{aligned}
$$

for some $\tilde{s}$ between $t$ and $\tilde{t}$, which implies (cf. (3.23)) that

$$
\begin{aligned}
\lim _{\tilde{t} \rightarrow t} \frac{J(t, l(t, \eta))-J(t, l(\tilde{t}, \eta))}{t-\tilde{t}} & =\lim _{\tilde{t} \rightarrow t} 2 \int_{t}^{\tilde{t}} \frac{\left(Q_{t}+H_{t}\right)(s, l(\tilde{t}, \eta))}{t-\tilde{t}} d s \\
& =2\left(Q_{t}+H_{t}\right)(t, l(t, \eta)) .
\end{aligned}
$$

Recalling that $J(t, l(t, \eta))=\eta$ for all $t$ and $\eta$, we have that

$$
J^{-1}(t, J(t, l(t, \eta)))=l(t, \eta)
$$

if we, as before, denote the inverse to $J(t, \cdot)$ by $J^{-1}(t, \cdot)$. Thus we have

$$
\begin{aligned}
l_{t}(t, \eta)= & \lim _{\tilde{t} \rightarrow t} \frac{l(t, \eta)-l(\tilde{t}, \eta)}{t-\tilde{t}} \\
= & \lim _{\tilde{t} \rightarrow t} \frac{J^{-1}(t, J(t, l(t, \eta)))-J^{-1}(t, J(t, l(\tilde{t}, \eta)))}{t-\tilde{t}} \\
= & \lim _{\tilde{t} \rightarrow t} \frac{J^{-1}(t, J(t, l(t, \eta)))-J^{-1}(t, J(t, l(\tilde{t}, \eta))}{J(t, l(t, \eta))-J(t, l(\tilde{t}, \eta))} \\
& \times \lim _{\tilde{t} \rightarrow t} \frac{J(t, l(t, \eta))-J(t, l(\tilde{t}, \eta))}{t-\tilde{t}} .
\end{aligned}
$$

We have established that the last limit on the right-hand side exists and the existence of the first one follows from the fact that $J^{-1}(t, \cdot)$ is differentiable almost everywhere.

REMARK 3.2. The above argument relies heavily on the fact that the function $l(t, \cdot):[0,2 C] \rightarrow \mathbb{R}$ is continuous and strictly increasing, the reason being that $J(t, \cdot)$ is continuous and strictly increasing. This is in contrast to the HS equation in [16], where the function $H(t, \cdot)$ is increasing, but not necessarily strictly increasing and its inverse may have jumps. In the HS equations, if $H(t, \cdot)$ is constant on some interval $I$, then $H(s, \cdot)$ will be constant on $I$ for any $s$, since $H$ is independent of time. Furthermore, this means that the jumps in the new coordinates might change in height with respect to time, but never change their position. This is the essential difference to the $\mathrm{CH}$ equation where the jumps would turn up and disappear again immediately, which motivates the choice of $G(t, x)$ and $J(t, \xi)$. 
3.3. New system: the right quantities. Since we have shown that $l(t, \eta)$ is differentiable almost everywhere with respect to both space and time, we can now establish rigorously the system of differential equations in our new coordinates. Recall that $J(t, l(t, \eta))=\eta$ and hence direct calculations yield

$$
\begin{aligned}
J_{t}(t, l(t, \eta))+J_{\xi}(t, l(t, \eta)) l_{t}(t, \eta) & =0, \\
J_{\xi}(t, l(t, \eta)) l_{\eta}(t, \eta) & =1 .
\end{aligned}
$$

According to the time evolution in Lagrangian coordinates, we thus end up with

$$
\begin{aligned}
& l_{\eta}(t, \eta)=\frac{1}{J_{\xi}(t, l(t, \eta))}, \\
& l_{t}(t, \eta)=-\frac{J_{t}(t, l(t, \eta))}{J_{\xi}(t, l(t, \eta))}=-J_{t}(t, l(t, \eta)) l_{\eta}(t, \eta) .
\end{aligned}
$$

As far as the new coordinates are concerned, we recall that

$$
\begin{gathered}
\mathcal{Y}(t, \eta)=y(t, l(t, \eta)), \quad \mathcal{U}(t, \eta)=U(t, l(t, \eta)), \\
\text { and } \mathcal{P}(t, \eta)=P(t, l(t, \eta)),
\end{gathered}
$$

and direct calculations using the differentiabilities proved for $Q, P$, and $l$ in Subsections 3.1 and 3.2 yield the following differential equations for the unknowns $(\mathcal{Y}, \mathcal{U}, \mathcal{P})$.

THEOREM 3.3. Let $(u, \mu)$ denote a weak conservative solution of (1.1). Define $\mathcal{Y}$ by (2.3), $\mathcal{U}$ by (2.6), and $\mathcal{P}$ by (2.8). Then the following system of differential equations holds:

$$
\begin{aligned}
& \mathcal{Y}_{t}(t, \eta)+\left(\frac{2}{3} \mathcal{U}^{3}+\mathcal{S}\right) \mathcal{Y}_{\eta}(t, \eta)=\mathcal{U}(t, \eta), \\
& \mathcal{U}_{t}(t, \eta)+\left(\frac{2}{3} \mathcal{U}^{3}+\mathcal{S}\right) \mathcal{U}_{\eta}(t, \eta)=-\mathcal{Q}(t, \eta), \\
& \mathcal{P}_{t}(t, \eta)+\left(\frac{2}{3} \mathcal{U}^{3}+\mathcal{S}\right) \mathcal{P}_{\eta}(t, \eta)=\mathcal{Q U}(t, \eta)+\mathcal{R}(t, \eta),
\end{aligned}
$$

where

$$
\begin{aligned}
& \mathcal{Q}(t, \eta)=-\frac{1}{4} \int_{0}^{2 C} \operatorname{sign}(\eta-\theta) e^{-|\mathcal{Y}(t, \eta)-\mathcal{Y}(t, \theta)|}\left(2\left(\mathcal{U}^{2}-\mathcal{P}\right) \mathcal{Y}_{\eta}(t, \theta)+1\right) d \theta \\
& \mathcal{S}(t, \eta)=\int_{0}^{2 C} e^{-|\mathcal{Y}(t, \eta)-\mathcal{Y}(t, \theta)|}\left(\frac{2}{3} \mathcal{U}^{3} \mathcal{Y}_{\eta}-\mathcal{U}_{\eta} \mathcal{Q}-2 \mathcal{P} \mathcal{U} \mathcal{Y}_{\eta}\right)(t, \theta) d \theta
\end{aligned}
$$




$$
\begin{aligned}
\mathcal{R}(t, \eta)= & \frac{1}{4} \int_{0}^{2 C} \operatorname{sign}(\eta-\theta) e^{-|\mathcal{Y}(t, \eta)-\mathcal{Y}(t, \theta)|}\left(\frac{2}{3} \mathcal{U}^{3} \mathcal{Y}_{\eta}+\mathcal{U}\right)(t, \theta) d \theta \\
& -\frac{1}{2} \int_{0}^{2 C} e^{-|\mathcal{Y}(t, \eta)-\mathcal{Y}(t, \theta)|} \mathcal{U} \mathcal{Q Y}_{\eta}(t, \theta) d \theta
\end{aligned}
$$

REMARK 3.4. This system is identical to the system derived in the smooth case (cf. (2.9)) as expected. We also remind the reader that $\mathcal{Q}(t, \eta)=Q(t, l(t, \eta))$, $\mathcal{S}(t, \eta)=J_{t}(t, l(t, \eta))-\frac{2}{3} \mathcal{U}^{3}(t, \eta)$, and $\mathcal{R}(t, \eta)=P_{t}(t, l(t, \eta))-\mathcal{Q U}(t, \eta)$.

\subsection{Functional setting: consistency of the new coordinates. The next main} question is which functional space we should work in such that the right-hand side of system (3.28) can be regarded as a Lipschitz function of the chosen unknowns. For instance, it is difficult or rather impossible to establish the Lipschitz continuity of $\mathcal{Q}$ with respect to $\mathcal{P}, \mathcal{U}$, and $\mathcal{Y}$. However, we will see that one can establish that $\mathcal{Q}$ is Lipschitz continuous with respect to $\mathcal{P}^{1 / 2}, \mathcal{U}$, and $\mathcal{Y}$. At first sight this seems to be surprising, but on the other hand we established in Eulerian coordinates that $2 p(t, x) \geqslant u^{2}(t, x)$, which rewrites in our new coordinates as

$$
2 \mathcal{P}(t, \eta) \geqslant \mathcal{U}^{2}(t, \eta)
$$

Thus it seems somehow natural that $\mathcal{P}(t, \eta)^{1 / 2}$ and $|\mathcal{U}(t, \eta)|$ will behave similarly.

The aim of this subsection is to derive the system of differential equations for $\mathcal{Y}, \mathcal{U}$, and $\mathcal{P}^{1 / 2}$ and subsequently to establish that all terms turning up in this system are well defined by assuming that each of the new variables $\left(\mathcal{Y}, \mathcal{U}, \mathcal{P}^{1 / 2}\right)$ is in $L^{2}([0,2 C])$.

Replacing the equation for $\mathcal{P}$ with the corresponding one for $\mathcal{P}^{1 / 2}$, we find that system (3.28) reads as

$$
\begin{aligned}
\mathcal{Y}_{t}+\left(\frac{2}{3} \mathcal{U}^{3}+\mathcal{S}\right) \mathcal{Y}_{\eta} & =\mathcal{U} \\
\mathcal{U}_{t}+\left(\frac{2}{3} \mathcal{U}^{3}+\mathcal{S}\right) \mathcal{U}_{\eta} & =-\mathcal{Q} \\
\left(\mathcal{P}^{1 / 2}\right)_{t}+\left(\frac{2}{3} \mathcal{U}^{3}+\mathcal{S}\right)\left(\mathcal{P}^{1 / 2}\right)_{\eta} & =\frac{\mathcal{Q U}}{2 \mathcal{P}^{1 / 2}}+\frac{\mathcal{R}}{2 \mathcal{P}^{1 / 2}}
\end{aligned}
$$

where $\mathcal{Q}, \mathcal{S}$, and $\mathcal{R}$ are given by (3.29).

Assume for the moment that $\mathcal{P}^{1 / 2}, \mathcal{U}$, and $\mathcal{Y}$ belong to $L^{2}([0,2 C])$. Then we want to show that all terms appearing in the above system also belong to $L^{2}$ ([0, $2 C]$ ). Therefore it is important to keep in mind, in addition to (3.30), that

$$
2 \mathcal{P} \mathcal{Y}_{\eta}(t, \eta)-\mathcal{U}^{2} \mathcal{Y}_{\eta}(t, \eta)+\mathcal{H}_{\eta}(t, \eta)=1
$$


and, in particular (cf. (3.12), (3.21), and (3.22)),

$$
\begin{aligned}
2\left|\mathcal{U} \mathcal{U}_{\eta}(t, \eta)\right| & \leqslant \mathcal{H}_{\eta}(t, \eta) \leqslant 1, \\
\mathcal{U}^{2} \mathcal{Y}_{\eta}(t, \eta) & \leqslant \mathcal{H}_{\eta}(t, \eta) \leqslant 1, \\
2|\mathcal{Q}| \mathcal{Y}_{\eta}(t, \eta) & \leqslant 2 \mathcal{P} \mathcal{Y}_{\eta}(t, \eta) \leqslant 1 .
\end{aligned}
$$

As an immediate consequence we obtain that

$$
\mathcal{U}^{3} \mathcal{Y}_{\eta}(t, \eta) \text { and } \mathcal{U}^{3} \mathcal{U}_{\eta}(t, \eta)
$$

are uniformly bounded. The last term of this form can be estimated as follows:

$$
\left|\mathcal{U}^{3}\left(\mathcal{P}^{1 / 2}\right)_{\eta}(t, \eta)\right|=\left|\frac{\mathcal{U}^{3} \mathcal{Q} \mathcal{Y}_{\eta}}{2 \mathcal{P}^{1 / 2}}(t, \eta)\right| \leqslant\left|\frac{\mathcal{U}^{3}}{2 \mathcal{P}^{1 / 2}}(t, \eta)\right| \leqslant \mathcal{U}^{2}(t, \eta),
$$

and is therefore uniformly bounded. Here we used (3.30).

As far as $\mathcal{S}$ is concerned, we want to establish that

$$
|\mathcal{S}(t, \eta)| \leqslant \mathcal{O}(1) \mathcal{P}(t, \eta)
$$

implying that

$$
\begin{aligned}
\left|\mathcal{S} \mathcal{Y}_{\eta}(t, \eta)\right| & \leqslant \mathcal{O}(1) \mathcal{P} \mathcal{Y}_{\eta}(t, \eta) \leqslant \mathcal{O}(1), \\
\left|\mathcal{S U}_{\eta}(t, \eta)\right| & \leqslant \mathcal{S}^{2} \mathcal{Y}_{\eta}(t, \eta)+\mathcal{H}_{\eta}(t, \eta) \leqslant \mathcal{O}(1), \\
\left|\mathcal{S}\left(\mathcal{P}^{1 / 2}\right)_{\eta}(t, \eta)\right| & \leqslant \mathcal{O}(1) \mathcal{P}^{3 / 2} \mathcal{Y}_{\eta}(t, \eta) \leqslant \mathcal{O}(1) .
\end{aligned}
$$

Indeed, by the definition of $\mathcal{S}$ and since $|\mathcal{Q}| \leqslant \mathcal{P}$ and (cf. (3.12a))

$$
\mathcal{U}_{\eta}^{2}(t, \eta) \leqslant \mathcal{H}_{\eta} \mathcal{Y}_{\eta}(t, \eta)
$$

we have

$$
\begin{aligned}
|\mathcal{S}(t, \eta)|= & \left|\int_{0}^{2 C} e^{-|\mathcal{Y}(t, \eta)-\mathcal{Y}(t, \theta)|}\left(\frac{2}{3} \mathcal{U}^{3} \mathcal{Y}_{\eta}-\mathcal{U}_{\eta} \mathcal{Q}-2 \mathcal{P} \mathcal{U} \mathcal{Y}_{\eta}\right)(t, \theta) d \theta\right| \\
\leqslant & \|\mathcal{U}(t, \cdot)\|_{L^{\infty}([0,2 C])} \int_{0}^{2 C} e^{-|\mathcal{Y}(t, \eta)-\mathcal{Y}(t, \theta)|} \mathcal{U}^{2} \mathcal{Y}_{\eta}(t, \theta) d \theta \\
& +2\left(\int_{0}^{2 C} e^{-|\mathcal{Y}(t, \eta)-\mathcal{Y}(t, \theta)|} \mathcal{P}^{2} \mathcal{Y}_{\eta}(t, \theta) d \theta\right)^{1 / 2} \\
& \times\left(\int_{0}^{2 C} e^{-|\mathcal{Y}(t, \eta)-\mathcal{Y}(t, \theta)|} \mathcal{U}^{2} \mathcal{Y}_{\eta}(t, \theta) d \theta\right)^{1 / 2}
\end{aligned}
$$




$$
\begin{aligned}
& +\left(\int_{0}^{2 C} e^{-|\mathcal{Y}(t, \eta)-\mathcal{Y}(t, \theta)|} \mathcal{P}^{2} \mathcal{Y}_{\eta}(t, \theta) d \theta\right)^{1 / 2} \\
& \times\left(\int_{0}^{2 C} e^{-|\mathcal{Y}(t, \eta)-\mathcal{Y}(t, \theta)|} \mathcal{H}_{\eta}(t, \theta) d \theta\right)^{1 / 2} \\
& \leqslant 4 \sqrt{C} \mathcal{P}(t, \eta)+6\left(\int_{0}^{2 C} e^{-|\mathcal{Y}(t, \eta)-\mathcal{Y}(t, \theta)|} \mathcal{P}^{2} \mathcal{Y}_{\eta}(t, \theta) d \theta\right)^{1 / 2} \mathcal{P}^{1 / 2}(t, \eta) .
\end{aligned}
$$

Thus it is left to show that

$$
\int_{0}^{2 C} e^{-|\mathcal{Y}(t, \eta)-\mathcal{Y}(t, \theta)|} \mathcal{P}^{2} \mathcal{Y}_{\eta}(t, \theta) d \theta \leqslant \mathcal{O}(1) \mathcal{P}(t, \eta)
$$

As several times before, the main tool will be integration by parts together with

$$
\mathcal{P}(t, \eta)=\frac{1}{4} \int_{0}^{2 C} e^{-|\mathcal{Y}(t, \eta)-\mathcal{Y}(t, \theta)|}\left(2\left(\mathcal{U}^{2}-\mathcal{P}\right) \mathcal{Y}_{\eta}(t, \theta)+1\right) d \theta
$$

Direct computations yield

$$
\begin{aligned}
\int_{0}^{\eta} & e^{\mathcal{Y}(t, \theta)-\mathcal{Y}(t, \eta)} \mathcal{P}^{2} \mathcal{Y}_{\eta}(t, \theta) d \theta \\
= & \mathcal{P}^{2}(t, \eta)-\int_{0}^{\eta} e^{\mathcal{Y}(t, \theta)-\mathcal{Y}(t, \eta)} 2 \mathcal{P} \mathcal{Q} \mathcal{Y}_{\eta}(t, \theta) d \theta \\
= & \mathcal{P}^{2}(t, \eta)-2 \mathcal{P} \mathcal{Q}(t, \eta) \\
& +\int_{0}^{\eta} e^{\mathcal{Y}(t, \theta)-\mathcal{Y}(t, \eta)}\left(2 \mathcal{Q}^{2} \mathcal{Y}_{\eta}+2 \mathcal{P} \mathcal{Q}_{\eta}\right)(t, \theta) d \theta \\
= & \mathcal{P}^{2}(t, \eta)-2 \mathcal{P} \mathcal{Q}(t, \eta) \\
& +\int_{0}^{\eta} e^{\mathcal{Y}(t, \theta)-\mathcal{Y}(t, \eta)} 2\left(\mathcal{P}^{2}+\mathcal{Q}^{2}\right) \mathcal{Y}_{\eta}(t, \theta) d \theta \\
& +\int_{0}^{\eta} e^{\mathcal{Y}(t, \theta)-\mathcal{Y}(t, \eta)}\left(2\left(\mathcal{P}-\mathcal{U}^{2}\right) \mathcal{Y}_{\eta}(t, \theta)-1\right) \mathcal{P}(t, \theta) d \theta
\end{aligned}
$$

Here we have used (3.4b) and (3.32). Thus, rearranging the terms, we end up with

$$
\begin{aligned}
& \int_{0}^{\eta} e^{\mathcal{Y}(t, \theta)-\mathcal{Y}(t, \eta)} \mathcal{P}^{2} \mathcal{Y}_{\eta}(t, \theta) d \theta \\
& \quad \leqslant \int_{0}^{\eta} e^{\mathcal{Y}(t, \theta)-\mathcal{Y}(t, \eta)}\left(\mathcal{P}^{2}+2 \mathcal{Q}^{2}\right) \mathcal{Y}_{\eta}(t, \theta) d \theta \\
& \quad=2 \mathcal{P} \mathcal{Q}(t, \eta)-\mathcal{P}^{2}(t, \eta)
\end{aligned}
$$




$$
\begin{aligned}
& +\int_{0}^{\eta} e^{\mathcal{Y}(t, \theta)-\mathcal{Y}(t, \eta)}\left(2\left(\mathcal{U}^{2}-\mathcal{P}\right) \mathcal{Y}_{\eta}(t, \theta)+1\right) \mathcal{P}(t, \theta) d \theta \\
\leqslant & 2 \mathcal{P}^{2}(t, \eta)+4\|\mathcal{P}(t, \cdot)\|_{L^{\infty}([0,2 C])} \mathcal{P}(t, \eta) \\
\leqslant & 6\|\mathcal{P}(t, \cdot)\|_{L^{\infty}([0,2 C])} \mathcal{P}(t, \eta) .
\end{aligned}
$$

Similar computations yield that

$$
\begin{aligned}
\int_{\eta}^{2 C} e^{\mathcal{Y}(t, \eta)-\mathcal{Y}(t, \theta)} \mathcal{P}^{2} \mathcal{Y}_{\eta}(t, \theta) d \theta \leqslant & \int_{\eta}^{2 C} e^{\mathcal{Y}(t, \eta)-\mathcal{Y}(t, \theta)}\left(\mathcal{P}^{2}+2 \mathcal{Q}^{2}\right) \mathcal{Y}_{\eta}(t, \theta) d \theta \\
= & -\mathcal{P}^{2}(t, \eta)-2 \mathcal{P} \mathcal{Q}(t, \eta) \\
& +\int_{\eta}^{2 C} e^{\mathcal{Y}(t, \eta)-\mathcal{Y}(t, \theta)} \\
& \times\left(2\left(\mathcal{U}^{2}-\mathcal{P}\right) \mathcal{Y}_{\eta}(t, \theta)+1\right) \mathcal{P}(t, \theta) d \theta \\
\leqslant & 2 \mathcal{P}^{2}(t, \eta)+4\|\mathcal{P}(t, \cdot)\|_{L^{\infty}([0,2 C])} \mathcal{P}(t, \eta) \\
\leqslant & 6\|\mathcal{P}(t, \cdot)\|_{L^{\infty}([0,2 C])} \mathcal{P}(t, \eta)
\end{aligned}
$$

This proves (3.34) due to (3.13).

There are two terms left to investigate. Namely, the term $\mathcal{Q U} / \mathcal{P}^{1 / 2}$, and we easily find that

$$
\left|\frac{\mathcal{Q U}}{\sqrt{2} \mathcal{P}^{1 / 2}}(t, \eta)\right| \leqslant \mathcal{P}(t, \eta),
$$

and hence it is uniformly bounded. The last term $\mathcal{R} / \mathcal{P}^{1 / 2}$ is a bit more involved. We have

$$
\begin{aligned}
|\mathcal{R}(t, \eta)| & \left|\frac{1}{4} \int_{0}^{2 C} \operatorname{sign}(\eta-\theta) e^{-|\mathcal{Y}(t, \eta)-\mathcal{Y}(t, \theta)|}\left(\frac{2}{3} \mathcal{U}^{3} \mathcal{Y}_{\eta}+\mathcal{U}\right)(t, \theta) d \theta\right| \\
& +\left|\frac{1}{2} \int_{0}^{2 C} e^{-|\mathcal{Y}(t, \eta)-\mathcal{Y}(t, \theta)|} \mathcal{U} \mathcal{Q Y}_{\eta}(t, \theta) d \theta\right| \\
\leqslant & \frac{1}{4} \int_{0}^{2 C} e^{-|\mathcal{Y}(t, \eta)-\mathcal{Y}(t, \theta)|}\left(2\left(\mathcal{U}^{2}-\mathcal{P}\right) \mathcal{Y}_{\eta}(t, \theta)+1\right)|\mathcal{U}|(t, \theta) d \theta \\
& +\frac{1}{4} \int_{0}^{2 C} e^{-|\mathcal{Y}(t, \eta)-\mathcal{Y}(t, \theta)|} 2 \mathcal{P}|\mathcal{U}| \mathcal{Y}_{\eta}(t, \theta) d \theta \\
& +\frac{1}{2} \int_{0}^{2 C} e^{-|\mathcal{Y}(t, \eta)-\mathcal{Y}(t, \theta)|}|\mathcal{U} \mathcal{Q}| \mathcal{Y}_{\eta}(t, \theta) d \theta \\
\leqslant & || \mathcal{U}(t, \cdot) \|_{L^{\infty}([0,2 C])} \mathcal{P}(t, \eta)+\frac{1}{2} \int_{0}^{2 C} e^{-|\mathcal{Y}(t, \eta)-\mathcal{Y}(t, \theta)|}\left(\mathcal{P}^{2}+\mathcal{U}^{2}\right) \mathcal{Y}_{\eta}(t, \theta) d \theta \\
\leqslant & \mathcal{O}(1) \mathcal{P}(t, \eta) .
\end{aligned}
$$


Thus

$$
\left|\frac{\mathcal{R}}{\mathcal{P}^{1 / 2}(t, \eta)}\right| \leqslant \mathcal{O}(1) \mathcal{P}^{1 / 2}(t, \eta),
$$

and hence belongs to $L^{2}([0,2 C])$.

Later on, we will need in some of our estimates (cf. (3.3a)) that

$$
\mathcal{P}(t, \eta)=\frac{1}{4} \int_{0}^{2 C} e^{-|\mathcal{Y}(t, \eta)-\mathcal{Y}(t, \theta)|}\left(\mathcal{U}^{2} \mathcal{Y}_{\eta}+\mathcal{H}_{\eta}\right)(t, \theta) d \theta,
$$

which implies the estimate

$$
\int_{0}^{\eta} e^{-(\mathcal{Y}(t, \eta)-\mathcal{Y}(t, \theta))}\left(\mathcal{U}^{2} \mathcal{Y}_{\eta}+\mathcal{H}_{\eta}\right)(t, \theta) d \theta \leqslant 4 \mathcal{P}(t, \eta) .
$$

3.5. The choice of the distance. In order to motivate the choice of the distance between two solutions with the same energy and to outline the strategy for proving the Lipschitz continuity, let us come back to system (3.31). We observe that the unknowns $\left(\mathcal{Y}, \mathcal{U}, \mathcal{P}^{1 / 2}\right)$ are advected by the same velocity field in the $\eta$ variable. Moreover, since all terms make sense under the assumption that the unknowns belong to $L^{2}([0,2 C])$, as checked in the previous subsection, we consider a toy problem of the form

$$
f_{t}+\mathcal{A}(f) f_{\eta}=\mathcal{B}(f),
$$

where $f$ might be a vector valued function in $L^{2}([0,2 C])$. This toy problem resembles our situation with the complicated operator dependencies on the unknowns. Within this functional framework, it is natural to look at the evolution of the $L^{2}$-norm of the unknown $f$. Given two solutions $f_{1}$ and $f_{2}$ to $f_{t}+\mathcal{A}(f) f_{\eta}=$ $\mathcal{B}(f)$, direct computations yield

$$
\begin{aligned}
\frac{d}{d t}\left\|f_{1}-f_{2}\right\|_{L^{2}([0,2 C])}^{2}= & 2 \int_{0}^{2 C}\left(f_{1}-f_{2}\right)\left(f_{1, t}-f_{2, t}\right)(t, \theta) d \theta \\
= & -2 \int_{0}^{2 C}\left(\mathcal{A}\left(f_{1}\right) f_{1, \eta}-\mathcal{A}\left(f_{2}\right) f_{2, \eta}\right)\left(f_{1}-f_{2}\right)(t, \theta) d \theta \\
& +2 \int_{0}^{2 C}\left(\mathcal{B}\left(f_{1}\right)-\mathcal{B}\left(f_{2}\right)\right)\left(f_{1}-f_{2}\right)(t, \theta) d \theta \\
= & -2 \int_{0}^{2 C} \mathcal{A}\left(f_{1}\right)\left(f_{1, \eta}-f_{2, \eta}\right)\left(f_{1}-f_{2}\right)(t, \theta) d \theta \\
& -2 \int_{0}^{2 C} f_{2, \eta}\left(\mathcal{A}\left(f_{1}\right)-\mathcal{A}\left(f_{2}\right)\right)\left(f_{1}-f_{2}\right)(t, \theta) d \theta
\end{aligned}
$$




$$
\begin{aligned}
& +2 \int_{0}^{2 C}\left(\mathcal{B}\left(f_{1}\right)-\mathcal{B}\left(f_{2}\right)\right)\left(f_{1}-f_{2}\right)(t, \theta) d \theta \\
= & \mathcal{A}\left(f_{1}\right)\left(f_{1}-f_{2}\right)^{2}(t, 0)-\mathcal{A}\left(f_{1}\right)\left(f_{1}-f_{2}\right)^{2}(t, 2 C) \\
& +\int_{0}^{2 C} \mathcal{A}^{\prime}\left(f_{1}\right) f_{1, \eta}\left(f_{1}-f_{2}\right)^{2}(t, \theta) d \theta \\
& -2 \int_{0}^{2 C} f_{2, \eta}\left(\mathcal{A}\left(f_{1}\right)-\mathcal{A}\left(f_{2}\right)\right)\left(f_{1}-f_{2}\right)(t, \theta) d \theta \\
& +2 \int_{0}^{2 C}\left(\mathcal{B}\left(f_{1}\right)-\mathcal{B}\left(f_{2}\right)\right)\left(f_{1}-f_{2}\right)(t, \theta) d \theta,
\end{aligned}
$$

where the first two terms in the last line are going to vanish if we impose the correct boundary conditions or rather if we have a good behavior of the solutions at both boundaries. Under this assumption, we can then use norm estimates if we know that $\mathcal{A}^{\prime}\left(f_{1}\right) f_{1, \eta}$ and $f_{2, \eta}$ are uniformly bounded by a constant $\mathcal{O}(1)$ and that $\mathcal{A}$ and $\mathcal{B}$ are Lipschitz continuous with Lipschitz constant $\mathcal{O}(1)$ with respect to $f$ to conclude that

$$
\frac{d}{d t}\left\|f_{1}-f_{2}\right\|_{L^{2}([0,2 C])}^{2} \leqslant \mathcal{O}(1)\left\|f_{1}-f_{2}\right\|_{L^{2}([0,2 C])}^{2},
$$

and Gronwall's lemma then implies

$$
\left\|\left(f_{1}-f_{2}\right)(t)\right\|_{L^{2}([0,2 C])} \leqslant\left\|\left(f_{1}-f_{2}\right)(0)\right\|_{L^{2}([0,2 C])} e^{\mathcal{O}(1) t} .
$$

Hence the strategy consists in proving first a propagation in time for the $L^{2}$ norm of $f$ in order to get bounds on the unknowns and check the validity of the approach. Then the second, and the most important and technical point, is to establish the Lipschitz estimates. We will come back to this point in Section 4, where we will also fix the following problem in the definition of our metric: the domain of definition of our unknowns depends on the total energy. This is unsatisfactory, if we want to compare solutions with different total energies.

3.6. Propagation of $\boldsymbol{L}^{\mathbf{2}}$ bounds: moment conditions. Consider $(u, \mu)$ a weak conservative solution of (1.1) and $f=\left(\mathcal{Y}, \mathcal{U}, \mathcal{P}^{1 / 2}\right)$, defined by (2.3), (2.6), and (2.8), the solution to system (3.31). We want to show that the $L^{2}$-norm of $f$ is propagated in time. Since both $\mathcal{U}$ and $\mathcal{P}$ are bounded functions due to (3.10) and (3.13), we are reduced to show the propagation of the $L^{2}$-norm of $\mathcal{Y}$. First of all, note that we have

$$
\mathcal{Y}(t, G(t, x))=x \quad \text { for all } x \in \mathbb{R},
$$


where $G(t, x)$ is given by

$$
G(t, x)=\int_{-\infty}^{x}\left(2 p-u^{2}\right)(t, y) d y+F(t, x) .
$$

Notice that the $x$-distributional derivative of $G$ is a measure that we denoted by $v(t, \cdot) \in \mathcal{M}_{+}(\mathbb{R})$ given by

$$
d v(t, x)=\left(2 p-u^{2}\right)(t, x)+d \mu(t, x) .
$$

Since $\mathcal{Y}(t, \eta)$ is the pseudoinverse to $G(t, x)$, then $\mathcal{Y}$ pushes forward the uniform distribution on $[0,2 C]$ to $v$ (see [67]) and then

$$
\int_{\mathbb{R}} x^{2} d v=\int_{0}^{2 C} \mathcal{Y}^{2}(t, \eta) d \eta .
$$

Thus $\mathcal{Y} \in L^{2}([0,2 C])$ is equivalent to $v(t, \cdot)$ having a finite second moment.

Proposition 3.5. Let $(u, \mu)$ denote a weak conservative solution of (1.1), and by $f=\left(\mathcal{Y}, \mathcal{U}, \mathcal{P}^{1 / 2}\right)$ the solution to system (3.31); then

$$
\int_{0}^{2 C} \mathcal{Y}^{2}(t, \eta) d \eta \leqslant e^{\mathcal{O}(1) t}\left(1+\int_{0}^{2 C} \mathcal{Y}^{2}(0, \eta) d \eta\right) .
$$

Proof. We show the propagation of the second moment in time using the Lagrangian coordinates. Note first that

$$
\begin{aligned}
\int_{\mathbb{R}} x^{2} d \mu(t, x) & =\int_{\mathbb{R}} y^{2} H_{\xi}(t, \xi) d \xi \\
\int_{\mathbb{R}} x^{2} u^{2}(t, x) d x & =\int_{\mathbb{R}} y^{2} U^{2} y_{\xi}(t, \xi) d \xi, \\
\int_{\mathbb{R}} x^{2} p(t, x) d x & =\int_{\mathbb{R}} y^{2} P y_{\xi}(t, \xi) d \xi
\end{aligned}
$$

We start by computing the derivatives of $y^{2} H_{\xi}, y^{2} U^{2} y_{\xi}$, and $y^{2} P y_{\xi}$ with respect to time. Direct computations using the formulas in Section 3.1 then yield

$$
\begin{aligned}
\left|\left(y^{2} H_{\xi}\right)_{t}\right| & =\left|2 y U H_{\xi}+y^{2}\left(3 U^{2} U_{\xi}-2 P U_{\xi}-2 Q U y_{\xi}\right)\right| \\
& \leqslant y^{2} H_{\xi}+U^{2} H_{\xi}+3 y^{2}|U| H_{\xi}+2 y^{2} P^{2} y_{\xi}+2 y^{2} H_{\xi} \\
& \leqslant \mathcal{O}(1)\left(y^{2} H_{\xi}+y^{2} P y_{\xi}+H_{\xi}\right)
\end{aligned}
$$


and

$$
\begin{aligned}
\left|\left(y^{2} P y_{\xi}\right)_{t}\right| & =\left|2 y U P y_{\xi}+y^{2} P_{t} y_{\xi}+y^{2} P U_{\xi}\right| \\
& \leqslant y^{2} P^{2} y_{\xi}+U^{2} y_{\xi}+y^{2}\left|P_{t}\right| y_{\xi}+y^{2} P\left(y_{\xi}+H_{\xi}\right) \\
& \leqslant \mathcal{O}(1)\left(y^{2} H_{\xi}+y^{2} P y_{\xi}+H_{\xi}\right)
\end{aligned}
$$

where we used (3.20). Thus

$$
\frac{d}{d t}\left(\left\|y^{2} P y_{\xi}\right\|_{L^{1}}+\left\|y^{2} H_{\xi}\right\|_{L^{1}}\right) \leqslant \mathcal{O}(1)\left(\left\|y^{2} P y_{\xi}\right\|_{L^{1}}+\left\|y^{2} H_{\xi}\right\|_{L^{1}}+C\right)
$$

and

$$
\int_{\mathbb{R}}\left(y^{2} P y_{\xi}+y^{2} H_{\xi}\right)(t, \xi) d \xi \leqslant e^{\mathcal{O}(1) t}\left(\int_{\mathbb{R}}\left(y^{2} P y_{\xi}+y^{2} H_{\xi}\right)(0, \xi) d \xi+C\right) .
$$

Since $y^{2} U^{2} y_{\xi}(t, \xi) \leqslant y^{2} H_{\xi}(t, \xi)$, it follows that also

$$
\int_{\mathbb{R}} y^{2} U^{2} y_{\xi}(t, \xi) d \xi
$$

remains finite for all times, which proves the desired estimate since

$$
\int_{\mathbb{R}} x^{2} d v(t, x)=\int_{\mathbb{R}} x^{2}\left(2 p-u^{2}\right)(t, x) d x+\int_{\mathbb{R}} x^{2} d \mu(t, x) .
$$

The propagation of moments not only implies the feasibility of the strategy illustrated in Section 3.5 but also gives us a control on the 'boundary terms'. In fact, since $\left(u^{2}+u_{x}^{2}\right)(t, x) \leqslant d \mu(t, x)$ and $\left(2 p-u^{2}\right)(t, x) \geqslant 0$, the previous result implies that

$$
\int_{\mathbb{R}} x^{2} p(t, x) d x, \quad \int_{\mathbb{R}} x^{2} u^{2}(t, x) d x, \quad \int_{\mathbb{R}} x^{2} u_{x}^{2}(t, x) d x, \quad \text { and } \quad \int_{\mathbb{R}} x^{2} d \mu(t, x)
$$

are all finite. We show next that (3.39) implies that

$$
x u(t, x) \rightarrow 0 \quad \text { and } \quad x^{2} p(t, x) \rightarrow 0 \quad \text { as } x \rightarrow \pm \infty .
$$

Indeed, (3.39) and $u \in H^{1}(\mathbb{R})$ imply that

$$
x u(t, x) \in L^{2}(\mathbb{R}) \quad \text { and } \quad(x u(t, x))_{x}=u(t, x)+x u_{x}(t, x) \in L^{2}(\mathbb{R}) .
$$

Hence $x u(t, x)$ belongs to $H^{1}(\mathbb{R})$ for any fixed $t$ and in particular $x u(t, x) \rightarrow 0$ as $x \rightarrow \pm \infty$ for any fixed $t$. 
The argument for $x p(t, x)$ is a bit more involved, but follows the same lines. To be more precise, $p(t, x) \in L^{2}(\mathbb{R}),\left|p_{x}(t, x)\right| \leqslant p(t, x)$, and

$$
\int_{\mathbb{R}} x^{2} p^{2}(t, x) d x \leqslant \mathcal{O}(1) \int_{\mathbb{R}} x^{2} p(t, x) d x<\infty,
$$

imply that

$$
x p(t, x) \in L^{2}(\mathbb{R}) \quad \text { and } \quad(x p(t, x))_{x}=p(t, x)+x p_{x}(t, x) \in L^{2}(\mathbb{R}) .
$$

Hence $x p(t, x)$ belongs to $H^{1}(\mathbb{R})$ for any fixed $t$ and in particular $x p(t, x) \rightarrow 0$ as $x \rightarrow \pm \infty$.

Finally, it is left to show that $x \sqrt{p(t, x)} \rightarrow 0$ as $x \rightarrow \pm \infty$. Therefore note that $p(t, x) \in L^{1}(\mathbb{R}) \cap L^{2}(\mathbb{R})$ and

$$
\left|(x \sqrt{p(t, x)})_{x}\right|=\left|\sqrt{p(t, x)}+x \frac{p_{x}(t, x)}{2 \sqrt{p(t, x)}}\right| \leqslant \sqrt{p(t, x)}+|x| \sqrt{p(t, x)},
$$

which together with (3.39) implies that

$$
x \sqrt{p(t, x)} \in L^{2}(\mathbb{R}) \quad \text { and } \quad(x \sqrt{p(t, x)})_{x} \in L^{2}(\mathbb{R}) .
$$

Hence $x \sqrt{p(t, x)}$ belongs to $H^{1}(\mathbb{R})$ for any fixed $t$ and in particular $x^{2} p(t, x) \rightarrow$ 0 as $x \rightarrow \pm \infty$. These properties will allow us to carry out several integrations by parts when deriving the anticipated Lipschitz estimate.

\section{The right metric: solutions with different energies}

For the remaining part of this paper, we will consider two distinct solutions $\left(u_{j}, \mu_{j}\right)$ for $j=1,2$ of the $\mathrm{CH}$ equation (1.1) and estimate their difference using a carefully selected norm based on our new variables $\left(\mathcal{Y}_{j}, \mathcal{U}_{j}, \mathcal{P}_{j}^{1 / 2}\right)$ for $j=1$, 2 that yields a Lipschitz metric. The main idea remains to a large extent the one from the HS equation in [16], where a Lipschitz metric for measuring the distance between solutions with nonzero energy has been constructed.

We want to define a Lipschitz metric, which can measure the distance between solutions with different total energy based on our new coordinates. At first sight this seems to be impossible for two reasons:

1. The support of the new variables depends on the total energy since

$$
\mathcal{Y}, \mathcal{U}, \mathcal{P}^{1 / 2}:[0,2 C] \rightarrow \mathbb{R} .
$$

2. In the case of the zero solution, that is, $(u, \mu)=(0,0)$, the function $\mathcal{Y}$ is not defined and the same applies to the new variables $\left(\mathcal{Y}, \mathcal{U}, \mathcal{P}^{1 / 2}\right)$. 
The key idea is to use a rescaling; more precisely, given $\left(\mathcal{Y}, \mathcal{U}, \mathcal{P}^{1 / 2}\right)$, where $\mathcal{Y}:[0,2 C] \rightarrow \mathbb{R}$ with $C \neq 0$. Then we can introduce

$$
\begin{aligned}
\tilde{\mathcal{Y}}(t, \eta) & =A \mathcal{Y}\left(t, A^{2} \eta\right), \quad \tilde{\mathcal{U}}(t, \eta)=A \mathcal{U}\left(t, A^{2} \eta\right), \\
\tilde{\mathcal{P}}^{1 / 2}(t, \eta) & =A \mathcal{P}^{1 / 2}\left(t, A^{2} \eta\right), \quad \tilde{\mathcal{H}}(t, \eta)=A^{3} \mathcal{H}\left(t, A^{2} \eta\right),
\end{aligned}
$$

with

$$
A=\sqrt{2 C}
$$

for notational purposes.

This rescaling has three main properties that motivate our choice:

1. The support of the variables $\left(\tilde{\mathcal{Y}}, \tilde{\mathcal{U}}, \tilde{\mathcal{P}}^{1 / 2}\right)$ is independent of the energy, which allows us to compare arbitrary solutions with nonzero energy.

2. In the last section, we discussed that the natural solution space for the unknowns $\left(\mathcal{Y}, \mathcal{U}, \mathcal{P}^{1 / 2}\right)$ is $L^{2}([0,2 C])$. Since our rescaling preserves the $L^{2}$-norm, the natural solution space for the unknowns $\left(\tilde{\mathcal{Y}}, \tilde{\mathcal{U}}, \tilde{\mathcal{P}}^{1 / 2}\right)$ is $L^{2}([0,1])$.

3. The most important property is that this rescaling allows us to set

$$
\left(\tilde{\mathcal{Y}}, \tilde{\mathcal{U}}, \tilde{\mathcal{P}}^{1 / 2}\right)=(0,0,0) \quad \text { for }(u, \mu)=(0,0) .
$$

A close look at the definition of $\tilde{\mathcal{Y}}(t, \eta)$ reveals that $\tilde{\mathcal{Y}}(t, \eta)$ is the pseudoinverse to $\tilde{G}(t, x)$, which is given by

$$
\tilde{G}(t, x)=\frac{1}{A^{2}} G\left(t, \frac{x}{A}\right) \text { for } A \neq 0 .
$$

If one compares $\tilde{G}(t, x)$ with $G(t, x)$ for small $A$, then the graph of $G(t, x)$ is squeezed in the $x$ direction, but stretched in the $y$ direction (cf. Figure 1). Taking a sequence of functions $G_{n}(t, x)$ with $A_{n}$ tending to zero, then the corresponding sequence of functions $\tilde{G}_{n}(t, x)$ tends to the Heaviside function, which has as pseudoinverse $\tilde{\mathcal{Y}}(t, \eta)=0$ (cf. Figure 2).

Direct calculation yields the following theorem.

THEOREM 4.1. Let $(u, \mu)$ denote a weak conservative solution of (1.1). Define $\tilde{\mathcal{Y}}, \tilde{\mathcal{U}}$ and $\tilde{\mathcal{P}}^{1 / 2}$ by (4.1). Then the following system of differential equations holds:

$$
\begin{aligned}
& \tilde{\mathcal{Y}}_{t}+\left(\frac{2}{3} \frac{1}{A^{5}} \tilde{\mathcal{U}}^{3}+\frac{1}{A^{6}} \tilde{\mathcal{S}}\right) \tilde{\mathcal{Y}}_{\eta}=\tilde{\mathcal{U}}, \\
& \tilde{\mathcal{U}}_{t}+\left(\frac{2}{3} \frac{1}{A^{5}} \tilde{\mathcal{U}}^{3}+\frac{1}{A^{6}} \tilde{\mathcal{S}}\right) \tilde{\mathcal{U}}_{\eta}=-\frac{1}{A^{2}} \tilde{\mathcal{Q}},
\end{aligned}
$$




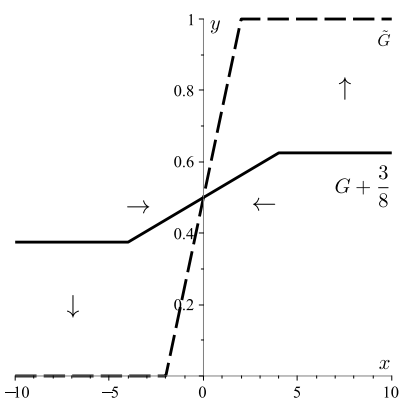

Figure 1. An example of how the rescaling changes the graph of a function $G$ : $\mathbb{R} \rightarrow \frac{1}{4}$. The picture shows the function $G+\frac{3}{8}$ (solid) and its rescaled version $\tilde{G}$ (dash).
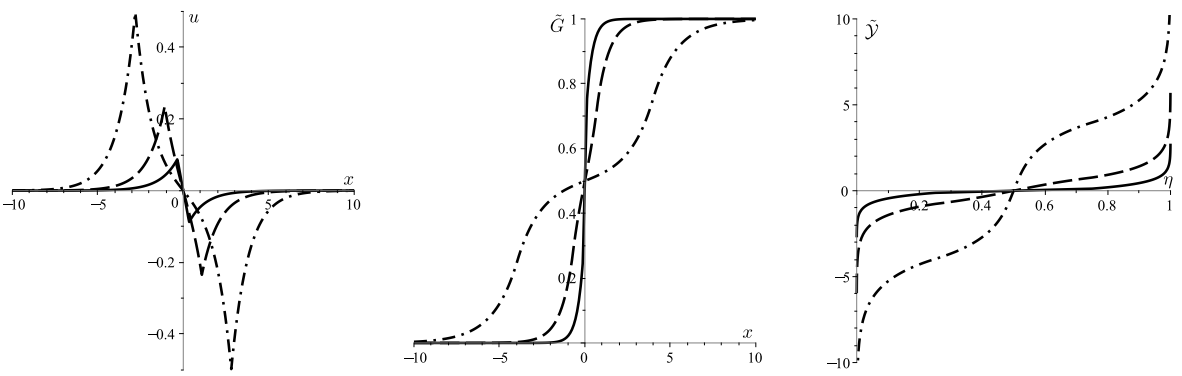

Figure 2. Three different peakon-antipeakon solutions $u$ (left) with total energy 1 (dash-dot), 0.5 (dash), and 0.25 (solid) and the corresponding functions $\tilde{G}$ (middle) and $\tilde{\mathcal{Y}}$ (right).

$$
\left(\tilde{\mathcal{P}}^{1 / 2}\right)_{t}+\left(\frac{2}{3} \frac{1}{A^{5}} \tilde{\mathcal{U}}^{3}+\frac{1}{A^{6}} \tilde{\mathcal{S}}\right)\left(\tilde{\mathcal{P}}^{1 / 2}\right)_{\eta}=\frac{1}{2 A^{2}} \frac{\tilde{\mathcal{Q}} \tilde{\mathcal{U}}}{\tilde{\mathcal{P}}^{1 / 2}}+\frac{1}{2 A^{3}} \frac{\tilde{\mathcal{R}}}{\tilde{\mathcal{P}}^{1 / 2}}
$$

where

$$
\begin{aligned}
\tilde{\mathcal{Q}}(t, \eta) & =A^{3} \mathcal{Q}\left(t, A^{2} \eta\right) \\
& =-\frac{1}{4} \int_{0}^{1} \operatorname{sign}(\eta-\theta) e^{-\frac{1}{A}|\tilde{\mathcal{Y}}(t, \eta)-\tilde{\mathcal{Y}}(t, \theta)|}\left(2\left(\tilde{\mathcal{U}}^{2}-\tilde{\mathcal{P}}\right) \tilde{\mathcal{Y}}_{\eta}(t, \theta)+A^{5}\right) d \theta \\
\tilde{\mathcal{S}}(t, \eta) & =A^{4} \mathcal{S}\left(t, A^{2} \eta\right) \\
& =\int_{0}^{1} e^{-\frac{1}{A}|\tilde{\mathcal{Y}}(t, \eta)-\tilde{\mathcal{Y}}(t, \theta)|}\left(\frac{2}{3} \tilde{\mathcal{U}}^{3} \tilde{\mathcal{Y}}_{\eta}-\tilde{\mathcal{U}}_{\eta} \tilde{\mathcal{Q}}-2 \tilde{\mathcal{P}} \tilde{\mathcal{Y}} \tilde{\mathcal{Y}}_{\eta}\right)(t, \theta) d \theta
\end{aligned}
$$




$$
\begin{aligned}
\tilde{\mathcal{R}}(t, \eta)= & A^{5} \mathcal{R}\left(t, A^{2} \eta\right) \\
= & \frac{1}{4} \int_{0}^{1} \operatorname{sign}(\eta-\theta) e^{-\frac{1}{A}|\tilde{\mathcal{Y}}(t, \eta)-\tilde{\mathcal{Y}}(t, \theta)|}\left(\frac{2}{3} A \tilde{\mathcal{U}}^{3} \tilde{\mathcal{Y}}_{\eta}+A^{6} \tilde{\mathcal{U}}\right)(t, \theta) \\
& -\frac{1}{2} \int_{0}^{1} e^{-\frac{1}{A}|\tilde{\mathcal{Y}}(t, \eta)-\tilde{\mathcal{Y}}(t, \theta)|} \tilde{\mathcal{U}} \tilde{\mathcal{Q}} \tilde{\mathcal{Y}}_{\eta}(t, \theta) d \theta .
\end{aligned}
$$

Now, we can define the metric between two general conservative solutions to (1.1). To simplify the notation we will from now on assume that all norms are on $L^{2}([0,1])$, unless otherwise indicated, and write

$$
\|\Psi\|=\|\Psi\|_{L^{2}([0,1])},
$$

for any function $\Psi$. We will not always explicitly indicate the time dependence in all quantities and write $\|\Psi\|$ rather than $\|\Psi(t)\|$.

Definition 4.2. Let $\left(u_{i}, \mu_{i}\right)$ for $i=1,2$ denote two conservative solutions of (1.1). We define the distance between them as

$$
\begin{aligned}
d\left(\left(u_{1}, \mu_{1}\right),\left(u_{2}, \mu_{2}\right)\right)= & \left\|\tilde{\mathcal{Y}}_{1}-\tilde{\mathcal{Y}}_{2}\right\|+\left\|\tilde{\mathcal{U}}_{1}-\tilde{\mathcal{U}}_{2}\right\| \\
& +\left\|\tilde{\mathcal{P}}_{1}^{1 / 2}-\tilde{\mathcal{P}}_{2}^{1 / 2}\right\|+\left|A_{1}-A_{2}\right|,
\end{aligned}
$$

where $A_{i}=\sqrt{2 C_{i}}$ and $C_{i}=\mu_{i}(\mathbb{R}), i=1,2$, and $\left(\tilde{\mathcal{Y}}_{i}, \tilde{\mathcal{U}}_{i}, \tilde{\mathcal{P}}_{i}^{1 / 2}\right)$ are given by (4.1).

REMARK 4.3. Notice that in the case of one of the two solutions being the trivial solution, the distance reduces to the $L^{2}$-norm of the solution in the right functional space, that is,

$$
d((u, \mu),(0,0))=\|\tilde{\mathcal{Y}}\|+\|\tilde{\mathcal{U}}\|+\left\|\tilde{\mathcal{P}}^{1 / 2}\right\|+\sqrt{2 C},
$$

where $C=\mu(\mathbb{R})$, that we already estimated in Section 3.6. It remains to show that the right-hand side of system (4.3) is Lipschitz continuous with respect to the new unknowns $\left(\tilde{\mathcal{Y}}, \tilde{\mathcal{U}}, \tilde{\mathcal{P}}^{1 / 2}\right)$.

The main result of this work can finally be stated. The proof consists in showing the corresponding Lipschitz estimates in each of the components of the distance in Definition 4.2. This is done in the next section in Lemmas 5.3-5.5. Collecting these results leads to our main theorem due to Gronwall's lemma.

THEOREM 4.4. Consider initial data $u_{i, 0} \in H^{1}(\mathbb{R}), \mu_{i, 0} \in \mathcal{M}_{+}(\mathbb{R})$ such that $d\left(\mu_{\mathrm{ac}}\right)_{i, 0}=\left(u_{i}^{2}+u_{i, x}^{2}\right) d x$ and $C_{i}=\mu_{i}(\mathbb{R})$, and let $\left(u_{i}, \mu_{i}\right)$ for $i=1,2$ denote 
the corresponding conservative solutions of the CH equation (1.1). Then we have that

$$
d\left(\left(u_{1}(t), \mu_{1}(t)\right),\left(u_{2}(t), \mu_{2}(t)\right)\right) \leqslant e^{\mathcal{O}(1) t} d\left(\left(u_{1,0}, \mu_{1,0}\right),\left(u_{2,0}, \mu_{2,0}\right)\right),
$$

where $\mathcal{O}(1)$ denotes a constant depending only on $\max _{j}\left(C_{j}\right)$ remaining bounded as $\max _{j}\left(C_{j}\right) \rightarrow 0$.

Now, let us start to do some preparatory work for the next section by collecting the main estimates we need in the new variables. Introduce

$$
\begin{aligned}
\tilde{\mathcal{D}}(t, \eta) & =\int_{0}^{\eta} e^{\frac{1}{A}(\tilde{\mathcal{Y}}(t, \theta)-\tilde{\mathcal{Y}}(t, \eta))}\left(\left(\tilde{\mathcal{U}}^{2}-\tilde{\mathcal{P}}\right) \tilde{\mathcal{Y}}_{\eta}(t, \theta)+\frac{1}{2} A^{5}\right) d \theta \\
& =\frac{1}{2} \int_{0}^{\eta} e^{\frac{1}{A}(\tilde{\mathcal{Y}}(t, \theta)-\tilde{\mathcal{Y}}(t, \eta))}\left(\tilde{\mathcal{U}}^{2} \tilde{\mathcal{Y}}_{\eta}+\tilde{\mathcal{H}}_{\eta}\right)(t, \theta) d \theta, \\
\tilde{\mathcal{E}}(t, \eta) & =\int_{\eta}^{1} e^{\frac{1}{A}(\tilde{\mathcal{Y}}(t, \eta)-\tilde{\mathcal{Y}}(t, \theta))}\left(\left(\tilde{\mathcal{U}}^{2}-\tilde{\mathcal{P}}\right) \tilde{\mathcal{Y}}_{\eta}(t, \theta)+\frac{1}{2} A^{5}\right) d \theta \\
& =\frac{1}{2} \int_{\eta}^{1} e^{\frac{1}{A}(\tilde{\mathcal{Y}}(t, \eta)-\tilde{\mathcal{Y}}(t, \theta))}\left(\tilde{\mathcal{U}}^{2} \tilde{\mathcal{Y}}_{\eta}+\tilde{\mathcal{H}}_{\eta}\right)(t, \theta) d \theta,
\end{aligned}
$$

so that we can write

$$
\tilde{\mathcal{P}}(t, \eta)=\frac{1}{2 A}(\tilde{\mathcal{D}}(t, \eta)+\tilde{\mathcal{E}}(t, \eta)) \quad \text { and } \quad \tilde{\mathcal{Q}}(t, \eta)=\frac{1}{2}(-\tilde{\mathcal{D}}(t, \eta)+\tilde{\mathcal{E}}(t, \eta))
$$

and

$$
\tilde{\mathcal{Q}}(t, \eta)=-\tilde{\mathcal{D}}(t, \eta)+A \tilde{\mathcal{P}}(t, \eta) .
$$

Note that both $\tilde{\mathcal{D}}(t, \eta)$ and $\tilde{\mathcal{E}}(t, \eta)$ have some very nice properties. Namely,

$$
0 \leqslant \tilde{\mathcal{D}}(t, \eta) \leqslant 2 A \tilde{\mathcal{P}}(t, \eta) \text { and } 0 \leqslant \tilde{\mathcal{E}}(t, \eta) \leqslant 2 A \tilde{\mathcal{P}}(t, \eta),
$$

and

$$
\begin{aligned}
\left|\frac{d}{d \eta} \tilde{\mathcal{D}}(t, \eta)\right| & =\left|\left(\tilde{\mathcal{U}}^{2}-\tilde{\mathcal{P}}\right) \tilde{\mathcal{Y}}_{\eta}(t, \eta)+\frac{1}{2} A^{5}-\frac{1}{A} \tilde{\mathcal{D}} \tilde{\mathcal{Y}}_{\eta}(t, \eta)\right| \leqslant \mathcal{O}(1) A^{5}, \\
\left|\frac{d}{d \eta} \tilde{\mathcal{E}}(t, \eta)\right| & =\left|-\left(\tilde{\mathcal{U}}^{2}-\tilde{\mathcal{P}}\right) \tilde{\mathcal{Y}}_{\eta}(t, \eta)-\frac{1}{2} A^{5}+\frac{1}{A} \tilde{\mathcal{E}} \tilde{\mathcal{Y}}_{\eta}(t, \eta)\right| \leqslant \mathcal{O}(1) A^{5} .
\end{aligned}
$$

The scaling leads to some changes in the standard estimates. Some of the problematic terms depending on $\frac{1}{A}$ in system (4.3) can be controlled since

$$
\begin{array}{ll}
2 \tilde{\mathcal{P}} \tilde{\mathcal{Y}}_{\eta}(t, \eta) \leqslant A^{5}, & 2\left|\tilde{\mathcal{U}} \tilde{\mathcal{U}}_{\eta}(t, \eta)\right| \leqslant A^{4}, \quad \tilde{\mathcal{U}}^{2} \tilde{\mathcal{Y}}_{\eta}(t, \eta) \leqslant A^{5}, \\
& \text { and } \tilde{\mathcal{H}}_{\eta}(t, \eta) \leqslant A^{5},
\end{array}
$$


from (3.33) and (4.1), as well as

$$
A^{2} \tilde{\mathcal{U}}_{\eta}^{2}(t, \eta) \leqslant \tilde{\mathcal{H}}_{\eta} \tilde{\mathcal{Y}}_{\eta}(t, \eta) \leqslant A^{5} \tilde{\mathcal{Y}}_{\eta}(t, \eta) .
$$

In addition, equation (3.32) becomes

$$
2 \tilde{\mathcal{P}} \tilde{\mathcal{Y}}_{\eta}(t, \eta)-\tilde{\mathcal{U}}^{2} \tilde{\mathcal{Y}}_{\eta}(t, \eta)+\tilde{\mathcal{H}}_{\eta}(t, \eta)=A^{5}
$$

Moreover, we can show that

$$
\begin{gathered}
4\|\tilde{\mathcal{P}}(t, \cdot)\|_{L^{\infty}}=4\left\|A^{2} \mathcal{P}(t, \cdot)\right\|_{L^{\infty}} \leqslant A^{4} \\
2\left\|\tilde{\mathcal{U}}^{2}(t, \cdot)\right\|_{L^{\infty}}=2\left\|A^{2} \mathcal{U}^{2}(t, \cdot)\right\|_{L^{\infty}} \leqslant A^{4} .
\end{gathered}
$$

We now collect all the estimates we will need on our solutions in the new variables with the explicit dependence on $A$. This will be repeatedly used in the next section.

$$
\begin{aligned}
& 0 \leqslant 4 \tilde{\mathcal{P}} \leqslant A^{4}, \quad \text { [from (4.14) and (3.13)], } \\
& \sqrt{2}|\tilde{\mathcal{U}}| \leqslant A^{2}, \quad \text { [from (4.14) and (3.10)], } \\
& |\tilde{\mathcal{Q}}| \leqslant A \tilde{\mathcal{P}}, \quad \text { [from common sense], } \\
& \tilde{\mathcal{U}}^{2} \leqslant 2 \tilde{\mathcal{P}}, \quad \text { [from (3.30)], } \\
& 2 \tilde{\mathcal{P}} \tilde{\mathcal{Y}}_{\eta} \leqslant A^{5}, \quad \text { [from (4.11)], } \\
& 2\left|\tilde{\mathcal{U}} \tilde{\mathcal{U}}_{\eta}\right| \leqslant A^{4}, \quad \text { [from (4.11)], } \\
& 0 \leqslant \tilde{\mathcal{U}}^{2} \tilde{\mathcal{Y}}_{\eta} \leqslant A^{5}, \quad \text { [from (4.11)], } \\
& \tilde{\mathcal{U}}_{\eta}^{2} \leqslant A^{3} \tilde{\mathcal{Y}}_{\eta}, \quad \text { [from (4.12) and (3.12a)], } \\
& \sqrt{2}|\tilde{\mathcal{U}}| \tilde{\mathcal{P}}^{1 / 2} \tilde{\mathcal{Y}}_{\eta} \leqslant A^{5}, \quad \text { [from (4.15e) and (4.15g)], } \\
& |\tilde{\mathcal{R}}| \leqslant \mathcal{O}(1) A^{3} \tilde{\mathcal{P}}, \quad \text { [from (3.37)], } \\
& 0 \leqslant \tilde{\mathcal{H}}_{\eta} \leqslant A^{5}, \quad \text { [from (4.11)], } \\
& 0 \leqslant \tilde{\mathcal{Y}}_{\eta}, \quad \text { [from the definition], } \\
& A^{2} \tilde{\mathcal{U}}_{\eta}^{2} \leqslant \tilde{\mathcal{H}}_{\eta} \tilde{\mathcal{Y}}_{\eta}, \quad \text { [from (4.12) and (3.12a)], } \\
& 0 \leqslant \tilde{\mathcal{D}} \leqslant 2 A \tilde{\mathcal{P}}, \quad \text { [from (4.8)] } \\
& 0 \leqslant \tilde{\mathcal{E}} \leqslant 2 A \tilde{\mathcal{P}}, \quad \text { [from (4.8)], } \\
& 2 \sqrt{2} \tilde{\mathcal{P}} \tilde{\mathcal{U}}_{\eta} \leqslant A^{6}, \quad \text { [from (4.15a), (4.15e), and (4.15h)], } \\
& 2 \tilde{\mathcal{P}} \tilde{\mathcal{U}}_{\eta}^{2} \leqslant A^{8}, \quad \text { [from (4.15e) and (4.15h)], } \\
& 4 \tilde{\mathcal{P}} \tilde{\mathcal{U}}_{\eta}^{2} \leqslant A^{7} \tilde{\mathcal{Y}}_{\eta}, \quad \text { [from (4.15a) and (4.15h)]. }
\end{aligned}
$$


In addition, we have several integrals that appear frequently:

$$
\begin{aligned}
& \int_{0}^{\eta} e^{-\frac{1}{A}(\tilde{\mathcal{Y}}(t, \eta)-\tilde{\mathcal{Y}}(t, \theta))} \tilde{\mathcal{U}}^{2}(t, \theta) d \theta \leqslant 6 \tilde{\mathcal{P}}(t, \eta), \quad[\text { from }(4.16 \mathrm{~g})], \\
& \int_{0}^{\eta} e^{-\frac{1}{A}(\tilde{\mathcal{Y}}(t, \eta)-\tilde{\mathcal{Y}}(t, \theta))} \tilde{\mathcal{P}}^{2} \tilde{\mathcal{Y}}_{\eta}(t, \theta) d \theta \leqslant \frac{3}{2} A^{5} \tilde{\mathcal{P}}(t, \eta), \\
& \text { [from (3.36) and (4.15a)], } \\
& \int_{0}^{\eta} e^{-\frac{1}{A}(\tilde{\mathcal{Y}}(t, \eta)-\tilde{\mathcal{Y}}(t, \theta)} \tilde{\mathcal{U}}^{2} \tilde{\mathcal{Y}}_{\eta}(t, \theta) d \theta \leqslant 4 A \tilde{\mathcal{P}}(t, \eta), \quad \text { [from (3.38)], } \\
& \int_{0}^{\eta} e^{-\frac{1}{A}(\tilde{\mathcal{Y}}(t, \eta)-\tilde{\mathcal{Y}}(t, \theta))} \tilde{\mathcal{H}}_{\eta}(t, \theta) d \theta \leqslant 4 A \tilde{\mathcal{P}}(t, \eta), \quad[\text { from (3.38)], } \\
& \int_{0}^{\eta} e^{-\frac{3}{2 A}(\tilde{\mathcal{Y}}(t, \eta)-\tilde{\mathcal{Y}}(t, \theta))} \tilde{\mathcal{P}} \tilde{\mathcal{Y}}_{\eta}(t, \theta) d \theta \leqslant 2 A \tilde{\mathcal{P}}(t, \eta), \quad \text { [from Lemma A.10], }
\end{aligned}
$$

$$
\int_{0}^{\eta} e^{-\frac{3}{2 A}(\tilde{\mathcal{Y}}(t, \eta)-\tilde{\mathcal{Y}}(t, \theta))} \tilde{\mathcal{H}}_{\eta}(t, \theta) d \theta \leqslant 4 A \tilde{\mathcal{P}}(t, \eta), \quad \text { [from Lemma A.10] }
$$

$$
\int_{0}^{\eta} e^{-\frac{1}{A}(\tilde{\mathcal{Y}}(t, \eta)-\tilde{\mathcal{Y}}(t, \theta))} \tilde{\mathcal{U}}^{2}(t, \theta) d \theta \leqslant 6 \tilde{\mathcal{P}}(t, \eta), \quad \text { [from Lemma A.10], }
$$

$$
\int_{0}^{\eta} e^{-\frac{5}{4 A}(\tilde{\mathcal{Y}}(t, \eta)-\tilde{\mathcal{Y}}(t, \theta))} \tilde{\mathcal{P}} \tilde{\mathcal{Y}}_{\eta}(t, \theta) d \theta \leqslant 4 A \tilde{\mathcal{P}}(t, \eta), \quad \text { [from Lemma A.10] }
$$

$$
\int_{0}^{\eta} e^{-\frac{3}{2 A}(\tilde{\mathcal{Y}}(t, \eta)-\tilde{\mathcal{Y}}(t, \theta))} \tilde{\mathcal{P}}(t, \theta) d \theta \leqslant 7 \tilde{\mathcal{P}}(t, \eta), \quad[\text { from Lemma A.10] }
$$

$$
\begin{gathered}
\int_{0}^{\eta} e^{-\frac{1}{2 A}(\tilde{\mathcal{Y}}(t, \eta)-\tilde{\mathcal{Y}}(t, \theta))} \tilde{\mathcal{P}}^{2} \tilde{\mathcal{Y}}_{\eta}(t, \theta) d \theta \leqslant A \mathcal{O}(1) \tilde{\mathcal{P}}^{1 / 2}(t, \eta), \quad \text { [from Lemma A.10] }, \\
\int_{0}^{\eta} e^{-\frac{1}{A}(\tilde{\mathcal{Y}}(t, \eta)-\tilde{\mathcal{Y}}(t, \theta))} \tilde{\mathcal{P}}^{1+\beta} \tilde{\mathcal{Y}}_{\eta}(t, \theta) d \theta \leqslant 3 \frac{1+\beta}{\beta} \frac{A^{1+4 \beta}}{4^{\beta}} \tilde{\mathcal{P}}(t, \eta), \quad \beta>0 \\
\text { [from Lemma A.10]. }
\end{gathered}
$$

As for derivatives of the quantity $\tilde{\mathcal{P}}^{1 / 2}$ we have

$$
\begin{aligned}
\left|\left(\tilde{\mathcal{P}}^{1 / 2}\right)_{\eta}\right| & \leqslant \frac{1}{2 A} \tilde{\mathcal{P}}^{1 / 2} \tilde{\mathcal{Y}}_{\eta}, \\
\left|\tilde{\mathcal{U}}\left(\tilde{\mathcal{P}}^{1 / 2}\right)_{\eta}\right| & \leqslant \frac{3}{8} A^{4},
\end{aligned}
$$




$$
\begin{aligned}
& \left(\left(\tilde{\mathcal{P}}^{1 / 2}\right)_{\eta}\right)^{2} \leqslant \frac{1}{8} A^{3} \tilde{\mathcal{Y}}_{\eta}, \\
& \left(\left(\tilde{\mathcal{P}}^{1 / 2}\right)_{\eta}\right)^{2} \leqslant \frac{1}{16} A^{2} \tilde{\mathcal{Y}}_{\eta}^{2} .
\end{aligned}
$$

\section{Lipschitz estimates in the new metric}

We will repeatedly use the following elementary identity and estimate.

Lemma 5.1. Let $a_{j}, b_{j} \in \mathbb{R}$ for $j=1,2$. Then we have

$$
a_{2} b_{2}-a_{1} b_{1}=\left(b_{2}-b_{1}\right)\left(a_{2} \mathbb{1}_{b_{1}<b_{2}}+a_{1} \mathbb{1}_{b_{1} \geqslant b_{2}}\right)+\min \left(b_{1}, b_{2}\right)\left(a_{2}-a_{1}\right) .
$$

Here

$$
\mathbb{1}_{\mathcal{K}}= \begin{cases}1, & \mathcal{K} \text { is true }, \\ 0, & \mathcal{K} \text { is false }\end{cases}
$$

We also use $\mathbb{1}_{\mathcal{K}}$ to denote the characteristic (indicator) function of a set $\mathcal{K}$. Furthermore, we have the estimate

$$
\left|\min \left(a_{1}, b_{1}\right)-\min \left(a_{2}, b_{2}\right)\right| \leqslant \max \left(\left|a_{1}-a_{2}\right|,\left|b_{1}-b_{2}\right|\right) .
$$

LEMMA 5.2. Let $f, g: \mathbb{R} \rightarrow \mathbb{R}$ be two Lipschitz continuous functions with Lipschitz constants $c_{f}$ and $c_{g}$, respectively. Then the function $h: \mathbb{R} \rightarrow \mathbb{R}$ defined by $h=\min (f, g)$ is a Lipschitz continuous function with Lipschitz constant bounded by $\max \left(c_{f}, c_{g}\right)$.

Proof. Use the previous lemma.

Introduce for any function $\Phi$ the functions

$$
\Phi^{-}=\min (0, \Phi), \quad \Phi^{+}=\max (0, \Phi) .
$$

We then have

$$
\Phi^{-} \leqslant 0 \leqslant \Phi^{+}, \quad \Phi^{-} \Phi^{+}=0, \quad \Phi=\Phi^{+}+\Phi^{-}, \quad|\Phi|=\Phi^{+}-\Phi^{-} .
$$

For two functions $\Phi, \Psi$ we have

$$
\left|\Phi^{ \pm}-\Psi^{ \pm}\right| \leqslant|\Phi-\Psi|
$$

Frequently, we will have to estimate quantities like

$$
\int_{0}^{1} e^{-\frac{1}{A}|\tilde{\mathcal{Y}}(t, \eta)-\tilde{\mathcal{Y}}(t, \theta)|}(\cdots)(t, \theta) d \theta .
$$


We will rewrite it as follows:

$$
\begin{aligned}
\int_{0}^{1} & e^{-\frac{1}{A}|\tilde{\mathcal{Y}}(t, \eta)-\tilde{\mathcal{Y}}(t, \theta)|}(\cdots)(t, \theta) d \theta \\
= & \left(\int_{0}^{\eta}+\int_{\eta}^{1}\right) e^{-\frac{1}{A}|\tilde{\mathcal{Y}}(t, \eta)-\tilde{\mathcal{Y}}(t, \theta)|}(\cdots)(t, \theta) d \theta \\
= & \int_{0}^{\eta} e^{-\frac{1}{A}(\tilde{\mathcal{Y}}(t, \eta)-\tilde{\mathcal{Y}}(t, \theta))}(\cdots)(t, \theta) d \theta \\
& +\int_{\eta}^{1} e^{\frac{1}{A}(\tilde{\mathcal{Y}}(t, \eta)-\tilde{\mathcal{Y}}(t, \theta))}(\cdots)(t, \theta) d \theta .
\end{aligned}
$$

Both integrals can be estimated in the same manner, using that the argument in the exponential is negative in both cases. Eliminating the absolute value will allow us to perform integration by parts.

5.1. Lipschitz estimates for $\tilde{\mathcal{Y}}$. From the system of differential equations, we have

$$
\tilde{\mathcal{Y}}_{i, t}+\left(\frac{2}{3} \frac{1}{A_{i}^{5}} \tilde{\mathcal{U}}_{i}^{3}+\frac{1}{A_{i}^{6}} \tilde{\mathcal{S}}_{i}\right) \tilde{\mathcal{Y}}_{i, \eta}=\tilde{\mathcal{U}}_{i}
$$

where

$$
\begin{aligned}
& \tilde{\mathcal{P}}_{i}(t, \eta)=\frac{1}{4 A_{i}} \int_{0}^{1} e^{-\frac{1}{A_{i}}\left|\tilde{\mathcal{Y}}_{i}(t, \eta)-\tilde{\mathcal{Y}}_{i}(t, \theta)\right|}\left(2\left(\tilde{\mathcal{U}}_{i}^{2}-\tilde{\mathcal{P}}_{i}\right) \tilde{\mathcal{Y}}_{i, \eta}(t, \theta)+A_{i}^{5}\right) d \theta \\
& \tilde{\mathcal{Q}}_{i}(t, \eta)=-\frac{1}{4} \int_{0}^{1} \operatorname{sign}(\eta-\theta) e^{-\frac{1}{A_{i}}\left|\tilde{\mathcal{Y}}_{i}(t, \eta)-\tilde{\mathcal{Y}}_{i}(t, \theta)\right|}\left(2\left(\tilde{\mathcal{U}}_{i}^{2}-\tilde{\mathcal{P}}_{i}\right) \tilde{\mathcal{Y}}_{i, \eta}(t, \theta)+A_{i}^{5}\right) d \theta \\
& \tilde{\mathcal{S}}_{i}(t, \eta)=\int_{0}^{1} e^{-\frac{1}{A_{i}}\left|\tilde{\mathcal{Y}}_{i}(t, \eta)-\tilde{\mathcal{Y}}_{i}(t, \theta)\right|}\left(\frac{2}{3} \tilde{\mathcal{U}}_{i}^{3} \tilde{\mathcal{Y}}_{i, \eta}-\tilde{\mathcal{Q}}_{i} \tilde{\mathcal{U}}_{i, \eta}-2 \tilde{\mathcal{P}}_{i} \tilde{\mathcal{U}}_{i} \tilde{\mathcal{Y}}_{i, \eta}\right)(t, \theta) d \theta
\end{aligned}
$$

Thus we have

$$
\begin{aligned}
\frac{d}{d t} & \int_{0}^{1}\left(\tilde{\mathcal{Y}}_{1}-\tilde{\mathcal{Y}}_{2}\right)^{2}(t, \eta) d \eta \\
= & 2 \int_{0}^{1}\left(\tilde{\mathcal{Y}}_{1}-\tilde{\mathcal{Y}}_{2}\right)\left(\tilde{\mathcal{Y}}_{1, t}-\tilde{\mathcal{Y}}_{2, t}\right)(t, \eta) d \eta \\
= & 2 \int_{0}^{1}\left(\tilde{\mathcal{Y}}_{1}-\tilde{\mathcal{Y}}_{2}\right)\left(\tilde{\mathcal{U}}_{1}-\tilde{\mathcal{U}}_{2}\right)(t, \eta) d \eta \\
& +\frac{4}{3} \int_{0}^{1}\left(\tilde{\mathcal{Y}}_{1}-\tilde{\mathcal{Y}}_{2}\right)\left(\frac{1}{A_{2}^{5}} \tilde{\mathcal{U}}_{2}^{3} \tilde{\mathcal{Y}}_{2, \eta}-\frac{1}{A_{1}^{5}} \tilde{\mathcal{U}}_{1}^{3} \tilde{\mathcal{Y}}_{1, \eta}\right)(t, \eta) d \eta
\end{aligned}
$$




$$
\begin{aligned}
& +2 \int_{0}^{1}\left(\tilde{\mathcal{Y}}_{1}-\tilde{\mathcal{Y}}_{2}\right)\left(\frac{1}{A_{2}^{6}} \tilde{\mathcal{S}}_{2} \tilde{\mathcal{Y}}_{2, \eta}-\frac{1}{A_{1}^{6}} \tilde{\mathcal{S}}_{1} \tilde{\mathcal{Y}}_{1, \eta}\right)(t, \eta) d \eta \\
= & I_{1}+I_{2}+I_{3} .
\end{aligned}
$$

The strategy is to use integration by parts for the last two integrals $I_{2}$ and $I_{3}$, while we want to use straightforward estimates for $I_{1}$, which will finally yield that

$$
\begin{aligned}
& \frac{d}{d t}\left\|\tilde{\mathcal{Y}}_{1}-\tilde{\mathcal{Y}}_{2}\right\|^{2} \\
& \quad \leqslant \mathcal{O}(1)\left(\left\|\tilde{\mathcal{Y}}_{1}-\tilde{\mathcal{Y}}_{2}\right\|^{2}+\left\|\tilde{\mathcal{U}}_{1}-\tilde{\mathcal{U}}_{2}\right\|^{2}+\left\|\tilde{\mathcal{P}}_{1}^{1 / 2}-\tilde{\mathcal{P}}_{2}^{1 / 2}\right\|^{2}+\left|A_{1}-A_{2}\right|^{2}\right)
\end{aligned}
$$

where $\mathcal{O}(1)$ denotes some constant which depends on $A=\max _{j}\left(A_{j}\right)$ and which remains bounded as $A \rightarrow 0$.

The first integral $I_{1}$ : Note that

$$
\begin{aligned}
\left|I_{1}\right| & =\left|2 \int_{0}^{1}\left(\tilde{\mathcal{Y}}_{1}-\tilde{\mathcal{Y}}_{2}\right)\left(\tilde{\mathcal{U}}_{1}-\tilde{\mathcal{U}}_{2}\right)(t, \eta) d \eta\right| \\
& \leqslant \int_{0}^{1}\left(\left(\tilde{\mathcal{Y}}_{1}-\tilde{\mathcal{Y}}_{2}\right)^{2}+\left(\tilde{\mathcal{U}}_{1}-\tilde{\mathcal{U}}_{2}\right)^{2}\right)(t, \eta) d \eta \\
& =\left\|\tilde{\mathcal{Y}}_{1}-\tilde{\mathcal{Y}}_{2}\right\|^{2}+\left\|\tilde{\mathcal{U}}_{1}-\tilde{\mathcal{U}}_{2}\right\|^{2} .
\end{aligned}
$$

The second integral $\mathrm{I}_{2}$ : Note that

$$
\begin{aligned}
& \frac{3}{4}\left|I_{2}\right|=\left|\int_{0}^{1}\left(\tilde{\mathcal{Y}}_{1}-\tilde{\mathcal{Y}}_{2}\right)\left(\frac{1}{A_{2}^{5}} \tilde{\mathcal{U}}_{2}^{3} \tilde{\mathcal{Y}}_{2, \eta}-\frac{1}{A_{1}^{5}} \tilde{\mathcal{U}}_{1}^{3} \tilde{\mathcal{Y}}_{1, \eta}\right)(t, \eta) d \eta\right| \\
& \leqslant \frac{1}{\left(\max _{j}\left(A_{j}\right)\right)^{5}}\left|\int_{0}^{1}\left(\tilde{\mathcal{Y}}_{1}-\tilde{\mathcal{Y}}_{2}\right)\left(\tilde{\mathcal{U}}_{2}^{3} \tilde{\mathcal{Y}}_{2, \eta}-\tilde{\mathcal{U}}_{1}^{3} \tilde{\mathcal{Y}}_{1, \eta}\right)(t, \eta) d \eta\right| \\
& +\frac{\left|A_{1}^{5}-A_{2}^{5}\right|}{A_{1}^{5} A_{2}^{5}}\left|\int_{0}^{1}\left(\tilde{\mathcal{Y}}_{1}-\tilde{\mathcal{Y}}_{2}\right)\left(\tilde{\mathcal{U}}_{1}^{3} \tilde{\mathcal{Y}}_{1, \eta} \mathbb{1}_{A_{1} \leqslant A_{2}}+\tilde{\mathcal{U}}_{2}^{3} \tilde{\mathcal{Y}}_{2, \eta} \mathbb{1}_{A_{2}<A_{1}}\right)(t, \eta) d \eta\right| \\
& \leqslant \frac{1}{A^{5}}\left|\int_{0}^{1}\left(\tilde{\mathcal{Y}}_{1}-\tilde{\mathcal{Y}}_{2}\right)\left(\tilde{\mathcal{U}}_{2}-\tilde{\mathcal{U}}_{1}\right) \tilde{\mathcal{U}}_{2}^{2} \tilde{\mathcal{Y}}_{2, \eta}(t, \eta) d \eta\right| \\
& +\frac{1}{A^{5}} \mid \int_{0}^{1}\left(\tilde{\mathcal{Y}}_{1}-\tilde{\mathcal{Y}}_{2}\right)\left[\tilde{\mathcal{U}}_{1} \tilde{\mathcal{Y}}_{1, \eta} \mathbb{1}_{\tilde{\mathcal{U}}_{2}^{2} \leqslant \tilde{\mathcal{U}}_{1}^{2}}+\tilde{\mathcal{U}}_{1} \tilde{\mathcal{Y}}_{2, \eta} \mathbb{1}_{\tilde{\mathcal{U}}_{1}^{2}<\tilde{\mathcal{U}}_{2}^{2}}\right] \\
& \times\left(\tilde{\mathcal{U}}_{2}+\tilde{\mathcal{U}}_{1}\right)\left(\tilde{\mathcal{U}}_{2}-\tilde{\mathcal{U}}_{1}\right)(t, \eta) d \eta \\
& +\frac{1}{A^{5}}\left|\int_{0}^{1}\left(\tilde{\mathcal{Y}}_{1}-\tilde{\mathcal{Y}}_{2}\right) \tilde{\mathcal{U}}_{1} \min _{j}\left(\tilde{\mathcal{U}}_{j}^{2}\right)\left(\tilde{\mathcal{Y}}_{2, \eta}-\tilde{\mathcal{Y}}_{1, \eta}\right)(t, \eta) d \eta\right|
\end{aligned}
$$




$$
\begin{aligned}
& +\frac{\left|A_{1}^{5}-A_{2}^{5}\right|}{A_{1}^{5} A_{2}^{5}}\left|\int_{0}^{1}\left(\tilde{\mathcal{Y}}_{1}-\tilde{\mathcal{Y}}_{2}\right)\left(\tilde{\mathcal{U}}_{1}^{3} \tilde{\mathcal{Y}}_{1, \eta} \mathbb{1}_{A_{1} \leqslant A_{2}}+\tilde{\mathcal{U}}_{2}^{3} \tilde{\mathcal{Y}}_{2, \eta} \mathbb{1}_{A_{2}<A_{1}}\right)(t, \eta) d \eta\right| \\
= & J_{1}+J_{2}+J_{3}+J_{4} .
\end{aligned}
$$

We will write

$$
A=\max _{j}\left(A_{j}\right), \quad a=\min _{j}\left(A_{j}\right)
$$

We commence with $J_{1}$ : Since $\tilde{\mathcal{U}}_{i}^{2} \tilde{\mathcal{Y}}_{i, \eta}(t, \eta) \leqslant A_{i}^{5} \leqslant A^{5}$ for all $t$ and $\eta$, we have

$$
\begin{aligned}
J_{1} & =\frac{1}{A^{5}}\left|\int_{0}^{1}\left(\tilde{\mathcal{Y}}_{1}-\tilde{\mathcal{Y}}_{2}\right)\left(\tilde{\mathcal{U}}_{2}-\tilde{\mathcal{U}}_{1}\right) \tilde{\mathcal{U}}_{2}^{2} \tilde{\mathcal{Y}}_{2, \eta}(t, \eta) d \eta\right| \\
& \leqslant\left\|\tilde{\mathcal{Y}}_{1}-\tilde{\mathcal{Y}}_{2}\right\|^{2}+\left\|\tilde{\mathcal{U}}_{1}-\tilde{\mathcal{U}}_{2}\right\|^{2} .
\end{aligned}
$$

Next term is $J_{2}$ : Using once more that $\tilde{\mathcal{U}}_{i}^{2} \tilde{\mathcal{Y}}_{i, \eta}(t, \eta) \leqslant A^{5}$ for all $t$ and $\eta$, we get

$$
\begin{aligned}
J_{2}= & \frac{1}{A^{5}} \mid \int_{0}^{1}\left(\tilde{\mathcal{Y}}_{1}-\tilde{\mathcal{Y}}_{2}\right)\left[\tilde{\mathcal{U}}_{1} \tilde{\mathcal{Y}}_{1, \eta} \mathbb{1}_{\tilde{\mathcal{U}}_{2}^{2} \leqslant \tilde{\mathcal{U}}_{1}^{2}}+\tilde{\mathcal{U}}_{1} \tilde{\mathcal{Y}}_{2, \eta} \mathbb{1}_{\tilde{\mathcal{U}}_{1}^{2}<\tilde{\mathcal{U}}_{2}^{2}}\right] \\
& \times\left(\tilde{\mathcal{U}}_{2}+\tilde{\mathcal{U}}_{1}\right)\left(\tilde{\mathcal{U}}_{2}-\tilde{\mathcal{U}}_{1}\right)(t, \eta) d \eta \mid \\
\leqslant & \frac{1}{A^{5}} \int_{0}^{1}\left|\tilde{\mathcal{Y}}_{1}-\tilde{\mathcal{Y}}_{2}\right|\left|\tilde{\mathcal{U}}_{1}-\tilde{\mathcal{U}}_{2}\right|\left(2 \tilde{\mathcal{U}}_{1}^{2} \tilde{\mathcal{Y}}_{1, \eta} \mathbb{1}_{\tilde{\mathcal{U}}_{2}^{2} \leqslant \tilde{\mathcal{U}}_{1}^{2}}+2 \tilde{\mathcal{U}}_{2}^{2} \tilde{\mathcal{Y}}_{2, \eta} \mathbb{1}_{\tilde{\mathcal{U}}_{1}^{2}<\tilde{\mathcal{U}}_{2}^{2}}\right)(t, \eta) d \eta \\
\leqslant & 2\left(\left\|\tilde{\mathcal{Y}}_{1}-\tilde{\mathcal{Y}}_{2}\right\|^{2}+\left\|\tilde{\mathcal{U}}_{1}-\tilde{\mathcal{U}}_{2}\right\|^{2}\right) .
\end{aligned}
$$

Next, it is $J_{3}$ : Here integration by parts plays an essential role. Thus we have to determine first whether or not the function $\eta \mapsto \tilde{\mathcal{U}}_{1} \min _{j}\left(\tilde{\mathcal{U}}_{j}^{2}\right)(t, \eta)$ is differentiable, and, if so, if its derivative is bounded. Recall from Lemma A.2 (ii) that the function $\eta \mapsto \min _{j}\left(\tilde{\mathcal{U}}_{j}^{2}\right)(t, \eta)$ is Lipschitz continuous with Lipschitz constant at most $A^{4}$. Thus

$$
\left|\frac{d}{d \eta}\left(\tilde{\mathcal{U}}_{1}(t, \eta) \min _{j}\left(\tilde{\mathcal{U}}_{j}^{2}(t, \eta)\right)\right)\right| \leqslant \frac{3}{2} A^{4}\left\|\tilde{\mathcal{U}}_{1}\right\|_{L^{\infty}} \leqslant \mathcal{O}(1) A^{6},
$$

and integration by parts together with (3.40) yields

$$
\begin{aligned}
J_{3} & =\frac{1}{A^{5}}\left|\int_{0}^{1}\left(\tilde{\mathcal{Y}}_{1}-\tilde{\mathcal{Y}}_{2}\right) \tilde{\mathcal{U}}_{1} \min _{j}\left(\tilde{\mathcal{U}}_{j}^{2}\right)\left(\tilde{\mathcal{Y}}_{2, \eta}-\tilde{\mathcal{Y}}_{1, \eta}\right)(t, \eta) d \eta\right| \\
& =\left|-\frac{1}{2 A^{5}}\left(\tilde{\mathcal{Y}}_{1}-\tilde{\mathcal{Y}}_{2}\right)^{2} \tilde{\mathcal{U}}_{1} \min _{j}\left(\tilde{\mathcal{U}}_{j}^{2}\right)(t, \eta)\right|_{\eta=0}^{1}
\end{aligned}
$$




$$
\begin{aligned}
& +\frac{1}{2 A^{5}} \int_{0}^{1}\left(\tilde{\mathcal{Y}}_{1}-\tilde{\mathcal{Y}}_{2}\right)^{2} \frac{d}{d \eta}\left(\tilde{\mathcal{U}}_{1} \min _{j}\left(\tilde{\mathcal{U}}_{j}^{2}\right)\right)(t, \eta) d \eta \mid \\
= & \frac{1}{2 A^{5}}\left|\int_{0}^{1}\left(\tilde{\mathcal{Y}}_{1}-\tilde{\mathcal{Y}}_{2}\right)^{2} \frac{d}{d \eta}\left(\tilde{\mathcal{U}}_{1} \min _{j}\left(\tilde{\mathcal{U}}_{j}^{2}\right)\right)(t, \eta) d \eta\right| \\
\leqslant & \frac{3}{4 A}\left\|\tilde{\mathcal{U}}_{1}(t, \cdot)\right\|_{L^{\infty}} \int_{0}^{1}\left(\tilde{\mathcal{Y}}_{1}-\tilde{\mathcal{Y}}_{2}\right)^{2}(t, \eta) d \eta \\
\leqslant & \mathcal{O}(1)\left\|\tilde{\mathcal{Y}}_{1}-\tilde{\mathcal{Y}}_{2}\right\|^{2} .
\end{aligned}
$$

Finally, we consider $J_{4}$ : Direct calculations yield

$$
\begin{aligned}
J_{4} \leqslant & \frac{\left|A_{1}^{5}-A_{2}^{5}\right|}{A_{1}^{5} A_{2}^{5}}\left(\mathbb{1}_{A_{1} \leqslant A_{2}}\left|\int_{0}^{1}\left(\tilde{\mathcal{Y}}_{1}-\tilde{\mathcal{Y}}_{2}\right) \tilde{\mathcal{U}}_{1}^{3} \tilde{\mathcal{Y}}_{1, \eta}(t, \eta) d \eta\right|\right. \\
& \left.+\mathbb{1}_{A_{2}<A_{1}}\left|\int_{0}^{1}\left(\tilde{\mathcal{Y}}_{1}-\tilde{\mathcal{Y}}_{2}\right) \tilde{\mathcal{U}}_{2}^{3} \tilde{\mathcal{Y}}_{2, \eta}(t, \eta) d \eta\right|\right) \\
\leqslant & \frac{\left|A_{1}^{5}-A_{2}^{5}\right|}{A_{1}^{5} A_{2}^{5}}\left(\mathbb{1}_{A_{1} \leqslant A_{2}} A_{1}^{7}+\mathbb{1}_{A_{2}<A_{1}} A_{2}^{7}\right) \int_{0}^{1}\left|\tilde{\mathcal{Y}}_{1}-\tilde{\mathcal{Y}}_{2}\right|(t, \eta) d \eta \\
\leqslant & \frac{\left|A_{1}^{5}-A_{2}^{5}\right|}{A^{3}}\left\|\tilde{\mathcal{Y}}_{1}-\tilde{\mathcal{Y}}_{2}\right\| \\
\leqslant & 5 A\left|A_{1}-A_{2}\right|\left\|\tilde{\mathcal{Y}}_{1}-\tilde{\mathcal{Y}}_{2}\right\| \\
\leqslant & \mathcal{O}(1)\left(\left\|\tilde{\mathcal{Y}}_{1}-\tilde{\mathcal{Y}}_{2}\right\|^{2}+\left|A_{1}-A_{2}\right|^{2}\right)
\end{aligned}
$$

where we again used that $\tilde{\mathcal{U}}_{i}^{2} \tilde{\mathcal{Y}}_{i, \eta} \leqslant A_{i}^{5}$ and $\left\|\tilde{\mathcal{U}}_{i}\right\|_{L^{\infty}} \leqslant A_{i}^{2}$.

The third integral $I_{3}$ : We will consider several smaller parts of $I_{3}$ by inserting the definition of $\tilde{\mathcal{S}}_{i}$, and combine them in the end. We write

$$
I_{3}=\frac{4}{3} I_{31}-4 I_{32}-2 I_{33},
$$

where

$$
\begin{aligned}
I_{31}= & \int_{0}^{1}\left(\tilde{\mathcal{Y}}_{1}-\tilde{\mathcal{Y}}_{2}\right)(t, \eta) \\
& \times\left(\frac{1}{A_{2}^{6}} \tilde{\mathcal{Y}}_{2, \eta}(t, \eta) \int_{0}^{1} e^{-\frac{1}{A_{2}}\left|\tilde{\mathcal{Y}}_{2}(t, \eta)-\tilde{\mathcal{Y}}_{2}(t, \theta)\right|} \tilde{\mathcal{U}}_{2}^{3} \tilde{\mathcal{Y}}_{2, \eta}(t, \theta) d \theta\right. \\
& \left.-\frac{1}{A_{1}^{6}} \tilde{\mathcal{Y}}_{1, \eta}(t, \eta) \int_{0}^{1} e^{-\frac{1}{A_{1}}\left|\tilde{\mathcal{Y}}_{1}(t, \eta)-\tilde{\mathcal{Y}}_{1}(t, \theta)\right|} \tilde{\mathcal{U}}_{1}^{3} \tilde{\mathcal{Y}}_{1, \eta}(t, \theta) d \theta\right) d \eta
\end{aligned}
$$




$$
\begin{aligned}
& I_{32}=\int_{0}^{1}\left(\tilde{\mathcal{Y}}_{1}-\tilde{\mathcal{Y}}_{2}\right)(t, \eta) \\
& \times\left(\frac{1}{A_{2}^{6}} \tilde{\mathcal{Y}}_{2, \eta}(t, \eta) \int_{0}^{1} e^{-\frac{1}{A_{2}}\left|\tilde{\mathcal{Y}}_{2}(t, \eta)-\tilde{\mathcal{Y}}_{2}(t, \theta)\right|} \tilde{\mathcal{P}}_{2} \tilde{\mathcal{U}}_{2} \tilde{\mathcal{Y}}_{2, \eta}(t, \theta) d \theta\right. \\
& \left.-\frac{1}{A_{1}^{6}} \tilde{\mathcal{Y}}_{1, \eta}(t, \eta) \int_{0}^{1} e^{-\frac{1}{A_{1}}\left|\tilde{\mathcal{Y}}_{1}(t, \eta)-\tilde{\mathcal{Y}}_{1}(t, \theta)\right|} \tilde{\mathcal{P}}_{1} \tilde{\mathcal{U}}_{1} \tilde{\mathcal{Y}}_{1, \eta}(t, \theta) d \theta\right) d \eta, \\
& I_{33}=\int_{0}^{1}\left(\tilde{\mathcal{Y}}_{1}-\tilde{\mathcal{Y}}_{2}\right)(t, \eta) \\
& \times\left(\frac{1}{A_{2}^{6}} \tilde{\mathcal{Y}}_{2, \eta}(t, \eta) \int_{0}^{1} e^{-\frac{1}{A_{2}}\left|\tilde{\mathcal{Y}}_{2}(t, \eta)-\tilde{\mathcal{Y}}_{2}(t, \theta)\right|} \tilde{\mathcal{Q}}_{2} \tilde{\mathcal{U}}_{2, \eta}(t, \theta) d \theta\right. \\
& \left.-\frac{1}{A_{1}^{6}} \tilde{\mathcal{Y}}_{1, \eta}(t, \eta) \int_{0}^{1} e^{-\frac{1}{A_{1}}\left|\tilde{\mathcal{Y}}_{1}(t, \eta)-\tilde{\mathcal{Y}}_{1}(t, \theta)\right|} \tilde{\mathcal{Q}}_{1} \tilde{\mathcal{U}}_{1, \eta}(t, \theta) d \theta\right) d \eta .
\end{aligned}
$$

Recall definitions (5.1). Then we have

$$
\begin{aligned}
& I_{31}=\int_{0}^{1}\left(\tilde{\mathcal{Y}}_{1}-\tilde{\mathcal{Y}}_{2}\right)(t, \eta) \\
& \times\left(\frac{1}{A_{2}^{6}} \tilde{\mathcal{Y}}_{2, \eta}(t, \eta) \int_{0}^{1} e^{-\frac{1}{A_{2}}\left|\tilde{\mathcal{Y}}_{2}(t, \eta)-\tilde{\mathcal{Y}}_{2}(t, \theta)\right|} \tilde{\mathcal{U}}_{2}^{3} \tilde{\mathcal{Y}}_{2, \eta}(t, \theta) d \theta\right. \\
& \left.-\frac{1}{A_{1}^{6}} \tilde{\mathcal{Y}}_{1, \eta}(t, \eta) \int_{0}^{1} e^{-\frac{1}{A_{1}}\left|\tilde{\mathcal{Y}}_{1}(t, \eta)-\tilde{\mathcal{Y}}_{1}(t, \theta)\right|} \tilde{\mathcal{U}}_{1}^{3} \tilde{\mathcal{Y}}_{1, \eta}(t, \theta) d \theta\right) d \eta \\
& =\int_{0}^{1}\left(\tilde{\mathcal{Y}}_{1}-\tilde{\mathcal{Y}}_{2}\right)(t, \eta) \\
& \times\left(\frac{1}{A_{2}^{6}} \tilde{\mathcal{Y}}_{2, \eta}(t, \eta) \int_{0}^{1} e^{-\frac{1}{A_{2}}\left|\tilde{\mathcal{Y}}_{2}(t, \eta)-\tilde{\mathcal{Y}}_{2}(t, \theta)\right|}\left(\tilde{\mathcal{U}}_{2}^{-}\right)^{3} \tilde{\mathcal{Y}}_{2, \eta}(t, \theta) d \theta\right. \\
& \left.-\frac{1}{A_{1}^{6}} \tilde{\mathcal{Y}}_{1, \eta}(t, \eta) \int_{0}^{1} e^{-\frac{1}{A_{1}}\left|\tilde{\mathcal{Y}}_{1}(t, \eta)-\tilde{\mathcal{Y}}_{1}(t, \theta)\right|}\left(\tilde{\mathcal{U}}_{1}^{-}\right)^{3} \tilde{\mathcal{Y}}_{1, \eta}(t, \theta) d \theta\right) d \eta \\
& +\int_{0}^{1}\left(\tilde{\mathcal{Y}}_{1}-\tilde{\mathcal{Y}}_{2}\right)(t, \eta) \\
& \times\left(\frac{1}{A_{2}^{6}} \tilde{\mathcal{Y}}_{2, \eta}(t, \eta) \int_{0}^{1} e^{-\frac{1}{A_{2}}\left|\tilde{\mathcal{Y}}_{2}(t, \eta)-\tilde{\mathcal{Y}}_{2}(t, \theta)\right|}\left(\tilde{\mathcal{U}}_{2}^{+}\right)^{3} \tilde{\mathcal{Y}}_{2, \eta}(t, \theta) d \theta\right. \\
& \left.-\frac{1}{A_{1}^{6}} \tilde{\mathcal{Y}}_{1, \eta}(t, \eta) \int_{0}^{1} e^{-\frac{1}{A_{1}}\left|\tilde{\mathcal{Y}}_{1}(t, \eta)-\tilde{\mathcal{Y}}_{1}(t, \theta)\right|}\left(\tilde{\mathcal{U}}_{1}^{+}\right)^{3} \tilde{\mathcal{Y}}_{1, \eta}(t, \theta) d \theta\right) d \eta \\
& =\int_{0}^{1}\left(\tilde{\mathcal{Y}}_{1}-\tilde{\mathcal{Y}}_{2}\right)\left(\tilde{K}^{-}+\tilde{K}^{+}\right)(t, \eta) d \eta \text {. }
\end{aligned}
$$


Since both integrals have the same structure, it suffices to consider the second integral. Furthermore, applying the device described in (5.4), it suffices to study the terms, denoted by $K^{ \pm}$, with the upper limit of the inner integral replaced by $\eta$. Therefore observe that we can write

$$
\begin{aligned}
& K^{+}(t, \eta) \\
& =\frac{1}{A_{2}^{6}} \tilde{\mathcal{Y}}_{2, \eta}(t, \eta) \int_{0}^{\eta} e^{-\frac{1}{A_{2}}\left(\tilde{\mathcal{Y}}_{2}(t, \eta)-\tilde{\mathcal{Y}}_{2}(t, \theta)\right)}\left(\tilde{\mathcal{U}}_{2}^{+}\right)^{3} \tilde{\mathcal{Y}}_{2, \eta}(t, \theta) d \theta \\
& -\frac{1}{A_{1}^{6}} \tilde{\mathcal{Y}}_{1, \eta}(t, \eta) \int_{0}^{\eta} e^{-\frac{1}{A_{1}}\left(\tilde{\mathcal{Y}}_{1}(t, \eta)-\tilde{\mathcal{Y}}_{1}(t, \theta)\right)}\left(\tilde{\mathcal{U}}_{1}^{+}\right)^{3} \tilde{\mathcal{Y}}_{1, \eta}(t, \theta) d \theta \\
& =\frac{1}{\left(\max _{j}\left(A_{j}\right)\right)^{6}}\left(\tilde{\mathcal{Y}}_{2, \eta}(t, \eta) \int_{0}^{\eta} e^{-\frac{1}{A_{2}}\left(\tilde{\mathcal{Y}}_{2}(t, \eta)-\tilde{\mathcal{Y}}_{2}(t, \theta)\right)}\left(\tilde{\mathcal{U}}_{2}^{+}\right)^{3} \tilde{\mathcal{Y}}_{2, \eta}(t, \theta) d \theta\right. \\
& \left.-\tilde{\mathcal{Y}}_{1, \eta}(t, \eta) \int_{0}^{\eta} e^{-\frac{1}{A_{1}}\left(\tilde{\mathcal{Y}}_{1}(t, \eta)-\tilde{\mathcal{Y}}_{1}(t, \theta)\right)}\left(\tilde{\mathcal{U}}_{1}^{+}\right)^{3} \tilde{\mathcal{Y}}_{1, \eta}(t, \theta) d \theta\right) \\
& +\frac{A_{1}^{6}-A_{2}^{6}}{A_{1}^{6} A_{2}^{6}} \mathbb{1}_{A_{1} \leqslant A_{2}} \tilde{\mathcal{Y}}_{1, \eta}(t, \eta) \int_{0}^{\eta} e^{-\frac{1}{A_{1}}\left(\tilde{\mathcal{Y}}_{1}(t, \eta)-\tilde{\mathcal{Y}}_{1}(t, \theta)\right)}\left(\tilde{\mathcal{U}}_{1}^{+}\right)^{3} \tilde{\mathcal{Y}}_{1, \eta}(t, \theta) d \theta \\
& +\frac{A_{1}^{6}-A_{2}^{6}}{A_{1}^{6} A_{2}^{6}} \mathbb{1}_{A_{2}<A_{1}} \tilde{\mathcal{Y}}_{2, \eta}(t, \eta) \int_{0}^{\eta} e^{-\frac{1}{A_{2}}\left(\tilde{\mathcal{Y}}_{2}(t, \eta)-\tilde{\mathcal{Y}}_{2}(t, \theta)\right)}\left(\tilde{\mathcal{U}}_{2}^{+}\right)^{3} \tilde{\mathcal{Y}}_{2, \eta}(t, \theta) d \theta \\
& =\frac{1}{A^{6}} \tilde{\mathcal{Y}}_{2, \eta}(t, \eta) \\
& \times\left(\int_{0}^{\eta} e^{-\frac{1}{A_{2}}\left(\tilde{\mathcal{Y}}_{2}(t, \eta)-\tilde{\mathcal{Y}}_{2}(t, \theta)\right)}\left(\left(\tilde{\mathcal{U}}_{2}^{+}\right)^{3}-\left(\tilde{\mathcal{U}}_{1}^{+}\right)^{3}\right) \tilde{\mathcal{Y}}_{2, \eta}(t, \theta) \mathbb{1}_{\tilde{\mathcal{U}}_{1}^{+} \leqslant \tilde{\mathcal{U}}_{2}^{+}}(t, \theta) d \theta\right) \\
& +\frac{1}{A^{6}} \tilde{\mathcal{Y}}_{1, \eta}(t, \eta) \\
& \times\left(\int_{0}^{\eta} e^{-\frac{1}{A_{1}}\left(\tilde{\mathcal{Y}}_{1}(t, \eta)-\tilde{\mathcal{Y}}_{1}(t, \theta)\right)}\left(\left(\tilde{\mathcal{U}}_{2}^{+}\right)^{3}-\left(\tilde{\mathcal{U}}_{1}^{+}\right)^{3}\right) \tilde{\mathcal{Y}}_{1, \eta}(t, \theta) \mathbb{1}_{\tilde{\mathcal{U}}_{2}^{+}<\tilde{\mathcal{U}}_{1}^{+}}(t, \theta) d \theta\right) \\
& +\frac{1}{A^{6}} \tilde{\mathcal{Y}}_{2, \eta}(t, \eta)\left(\int_{0}^{\eta} e^{-\frac{1}{A_{2}}\left(\tilde{\mathcal{Y}}_{2}(t, \eta)-\tilde{\mathcal{Y}}_{2}(t, \theta)\right)} \min _{j}\left(\tilde{\mathcal{U}}_{j}^{+}\right)^{3} \tilde{\mathcal{Y}}_{2, \eta}(t, \theta) d \theta\right) \\
& -\frac{1}{A^{6}} \tilde{\mathcal{Y}}_{1, \eta}(t, \eta)\left(\int_{0}^{\eta} e^{-\frac{1}{A_{1}}\left(\tilde{\mathcal{Y}}_{1}(t, \eta)-\tilde{\mathcal{Y}}_{1}(t, \theta)\right)} \min _{j}\left(\tilde{\mathcal{U}}_{j}^{+}\right)^{3} \tilde{\mathcal{Y}}_{1, \eta}(t, \theta) d \theta\right) \\
& +\frac{A_{1}^{6}-A_{2}^{6}}{A_{1}^{6} A_{2}^{6}} \mathbb{1}_{A_{1} \leqslant A_{2}} \tilde{\mathcal{Y}}_{1, \eta}(t, \eta) \int_{0}^{\eta} e^{-\frac{1}{A_{1}}\left(\tilde{\mathcal{Y}}_{1}(t, \eta)-\tilde{\mathcal{Y}}_{1}(t, \theta)\right)}\left(\tilde{\mathcal{U}}_{1}^{+}\right)^{3} \tilde{\mathcal{Y}}_{1, \eta}(t, \theta) d \theta \\
& +\frac{A_{1}^{6}-A_{2}^{6}}{A_{1}^{6} A_{2}^{6}} \mathbb{1}_{A_{2}<A_{1}} \tilde{\mathcal{Y}}_{2, \eta}(t, \eta) \int_{0}^{\eta} e^{-\frac{1}{A_{2}}\left(\tilde{\mathcal{Y}}_{2}(t, \eta)-\tilde{\mathcal{Y}}_{2}(t, \theta)\right)}\left(\tilde{\mathcal{U}}_{2}^{+}\right)^{3} \tilde{\mathcal{Y}}_{2, \eta}(t, \theta) d \theta \\
& =\frac{1}{A^{6}} \tilde{\mathcal{Y}}_{2, \eta}(t, \eta)\left(\int_{0}^{\eta} e^{-\frac{1}{A_{2}}\left(\tilde{\mathcal{Y}}_{2}(t, \eta)-\tilde{\mathcal{Y}}_{2}(t, \theta)\right)}\right.
\end{aligned}
$$




$$
\begin{aligned}
& \left.\times\left(\left(\tilde{\mathcal{U}}_{2}^{+}\right)^{3}-\left(\tilde{\mathcal{U}}_{1}^{+}\right)^{3}\right) \tilde{\mathcal{Y}}_{2, \eta}(t, \theta) \mathbb{1}_{\tilde{\mathcal{U}}_{1}^{+} \leqslant \tilde{\mathcal{U}}_{2}^{+}}(t, \theta) d \theta\right) \\
& +\frac{1}{A^{6}} \tilde{\mathcal{Y}}_{1, \eta}(t, \eta)\left(\int_{0}^{\eta} e^{-\frac{1}{A_{1}}\left(\tilde{\mathcal{Y}}_{1}(t, \eta)-\tilde{\mathcal{Y}}_{1}(t, \theta)\right)}\right. \\
& \left.\times\left(\left(\tilde{\mathcal{U}}_{2}^{+}\right)^{3}-\left(\tilde{\mathcal{U}}_{1}^{+}\right)^{3}\right) \tilde{\mathcal{Y}}_{1, \eta}(t, \theta) \mathbb{1}_{\tilde{\mathcal{U}}_{2}^{+}<\tilde{\mathcal{U}}_{1}^{+}}(t, \theta) d \theta\right) \\
& +\frac{1}{A^{6}} \mathbb{1}_{A_{1} \leqslant A_{2}} \tilde{\mathcal{Y}}_{2, \eta}(t, \eta) \\
& \times\left(\int_{0}^{\eta}\left(e^{-\frac{1}{A_{2}}\left(\tilde{\mathcal{Y}}_{2}(t, \eta)-\tilde{\mathcal{Y}}_{2}(t, \theta)\right)}-e^{-\frac{1}{A_{1}}\left(\tilde{\mathcal{Y}}_{2}(t, \eta)-\tilde{\mathcal{Y}}_{2}(t, \theta)\right)}\right) \min _{j}\left(\tilde{\mathcal{U}}_{j}^{+}\right)^{3} \tilde{\mathcal{Y}}_{2, \eta}(t, \theta) d \theta\right) \\
& +\frac{1}{A^{6}} \mathbb{1}_{A_{2}<A_{1}} \tilde{\mathcal{Y}}_{1, \eta}(t, \eta) \\
& \times\left(\int_{0}^{\eta}\left(e^{-\frac{1}{A_{2}}\left(\tilde{\mathcal{Y}}_{1}(t, \eta)-\tilde{\mathcal{Y}}_{1}(t, \theta)\right)}-e^{-\frac{1}{A_{1}}\left(\tilde{\mathcal{Y}}_{1}(t, \eta)-\tilde{\mathcal{Y}}_{1}(t, \theta)\right)}\right) \min _{j}\left(\tilde{\mathcal{U}}_{j}^{+}\right)^{3} \tilde{\mathcal{Y}}_{1, \eta}(t, \theta) d \theta\right) \\
& +\frac{1}{A^{6}} \tilde{\mathcal{Y}}_{2, \eta}(t, \eta)\left(\int_{0}^{\eta}\left(e^{-\frac{1}{a}\left(\tilde{\mathcal{Y}}_{2}(t, \eta)-\tilde{\mathcal{Y}}_{2}(t, \theta)\right)}-e^{-\frac{1}{a}\left(\tilde{\mathcal{Y}}_{1}(t, \eta)-\tilde{\mathcal{Y}}_{1}(t, \theta)\right)}\right)\right. \\
& \left.\times \min _{j}\left(\tilde{\mathcal{U}}_{j}^{+}\right)^{3} \tilde{\mathcal{Y}}_{2, \eta}(t, \theta) \mathbb{1}_{B(\eta)}(t, \theta) d \theta\right) \\
& -\frac{1}{A^{6}} \tilde{\mathcal{Y}}_{1, \eta}(t, \eta)\left(\int_{0}^{\eta}\left(e^{-\frac{1}{a}\left(\tilde{\mathcal{Y}}_{1}(t, \eta)-\tilde{\mathcal{Y}}_{1}(t, \theta)\right)}-e^{-\frac{1}{a}\left(\tilde{\mathcal{Y}}_{2}(t, \eta)-\tilde{\mathcal{Y}}_{2}(t, \theta)\right)}\right)\right. \\
& \left.\times \min _{j}\left(\tilde{\mathcal{U}}_{j}^{+}\right)^{3} \tilde{\mathcal{Y}}_{1, \eta}(t, \theta) \mathbb{1}_{B^{c}(\eta)}(t, \theta) d \theta\right) \\
& +\frac{1}{A^{6}} \tilde{\mathcal{Y}}_{2, \eta}(t, \eta)\left(\int_{0}^{\eta} \min _{j}\left(e^{-\frac{1}{a}\left(\tilde{\mathcal{Y}}_{j}(t, \eta)-\tilde{\mathcal{Y}}_{j}(t, \theta)\right)}\right)\right. \\
& \left.\times \min _{j}\left(\tilde{\mathcal{U}}_{j}^{+}\right)^{3} \tilde{\mathcal{Y}}_{2, \eta}(t, \theta) d \theta\right) \\
& -\frac{1}{A^{6}} \tilde{\mathcal{Y}}_{1, \eta}(t, \eta)\left(\int_{0}^{\eta} \min _{j}\left(e^{-\frac{1}{a}\left(\tilde{\mathcal{Y}}_{j}(t, \eta)-\tilde{\mathcal{Y}}_{j}(t, \theta)\right)}\right)\right. \\
& \left.\times \min _{j}\left(\tilde{\mathcal{U}}_{j}^{+}\right)^{3} \tilde{\mathcal{Y}}_{1, \eta}(t, \theta) d \theta\right) \\
& +\frac{A_{1}^{6}-A_{2}^{6}}{A_{1}^{6} A_{2}^{6}} \mathbb{1}_{A_{1} \leqslant A_{2}} \tilde{\mathcal{Y}}_{1, \eta}(t, \eta) \int_{0}^{\eta} e^{-\frac{1}{A_{1}}\left(\tilde{\mathcal{Y}}_{1}(t, \eta)-\tilde{\mathcal{Y}}_{1}(t, \theta)\right)}\left(\tilde{\mathcal{U}}_{1}^{+}\right)^{3} \tilde{\mathcal{Y}}_{1, \eta}(t, \theta) d \theta \\
& +\frac{A_{1}^{6}-A_{2}^{6}}{A_{1}^{6} A_{2}^{6}} \mathbb{1}_{A_{2}<A_{1}} \tilde{\mathcal{Y}}_{2, \eta}(t, \eta) \int_{0}^{\eta} e^{-\frac{1}{A_{2}}\left(\tilde{\mathcal{Y}}_{2}(t, \eta)-\tilde{\mathcal{Y}}_{2}(t, \theta)\right)}\left(\tilde{\mathcal{U}}_{2}^{+}\right)^{3} \tilde{\mathcal{Y}}_{2, \eta}(t, \theta) d \theta \\
& =\left(J_{1}+J_{2}+J_{3}+J_{4}+J_{5}+J_{6}+J_{7}+J_{8}+J_{9}+J_{10}\right)(t, \eta) \text {. }
\end{aligned}
$$


Here

$$
B(\eta)=\left\{(t, \theta) \mid e^{\tilde{\mathcal{Y}}_{1}(t, \theta)-\tilde{\mathcal{Y}}_{1}(t, \eta)} \leqslant e^{\tilde{\mathcal{Y}}_{2}(t, \theta)-\tilde{\mathcal{Y}}_{2}(t, \eta)}\right\}
$$

which means especially that $B(\eta)$ depends heavily on $\eta$ ! Observe that the set $B(\eta)$ is scale invariant in the sense that $\left(\tilde{\mathcal{Y}}_{1}, \tilde{\mathcal{Y}}_{2}\right)$ and $\left(c \tilde{\mathcal{Y}}_{1}, c \tilde{\mathcal{Y}}_{2}\right)$ define the same set $B(\eta)$ for any constant $c$.

As far as the first two terms $J_{1}$ and $J_{2}$ are concerned, they have the same structure, and hence we only consider the term $J_{1}$, that is,

$$
\begin{aligned}
\int_{0}^{1} & J_{1}\left(\tilde{\mathcal{Y}}_{1}-\tilde{\mathcal{Y}}_{2}\right)(t, \eta) d \eta \\
= & \frac{1}{A^{6}} \int_{0}^{1}\left(\tilde{\mathcal{Y}}_{1}-\tilde{\mathcal{Y}}_{2}\right) \tilde{\mathcal{Y}}_{2, \eta}(t, \eta) \\
& \times\left(\int_{0}^{\eta} e^{-\frac{1}{A_{2}}\left(\tilde{\mathcal{Y}}_{2}(t, \eta)-\tilde{\mathcal{Y}}_{2}(t, \theta)\right)}\left(\left(\tilde{\mathcal{U}}_{2}^{+}\right)^{3}-\left(\tilde{\mathcal{U}}_{1}^{+}\right)^{3}\right) \tilde{\mathcal{Y}}_{2, \eta}(t, \theta) \mathbb{1}_{\tilde{\mathcal{U}}_{1}^{+} \leqslant \tilde{\mathcal{U}}_{2}^{+}}(t, \theta) d \theta\right) d \eta .
\end{aligned}
$$

The main ingredients are the following observations:

$$
\left|\left(\tilde{\mathcal{U}}_{1}^{+}\right)^{3}(t, \eta)-\left(\tilde{\mathcal{U}}_{2}^{+}\right)^{3}(t, \eta)\right| \mathbb{1}_{\tilde{\mathcal{U}}_{1}^{+} \leqslant \tilde{\mathcal{U}}_{2}^{+}} \leqslant 3 \tilde{\mathcal{U}}_{2}^{2}(t, \eta)\left|\tilde{\mathcal{U}}_{1}(t, \eta)-\tilde{\mathcal{U}}_{2}(t, \eta)\right|,
$$

and (4.16c).

Thus we have

$$
\begin{aligned}
& \left|\int_{0}^{1} J_{1}\left(\tilde{\mathcal{Y}}_{1}-\tilde{\mathcal{Y}}_{2}\right)(t, \eta) d \eta\right| \\
& =\frac{1}{A^{6}} \mid \int_{0}^{1}\left(\tilde{\mathcal{Y}}_{1}-\tilde{\mathcal{Y}}_{2}\right) \tilde{\mathcal{Y}}_{2, \eta}(t, \eta) \\
& \times\left(\int_{0}^{\eta} e^{-\frac{1}{A_{2}}\left(\tilde{\mathcal{Y}}_{2}(t, \eta)-\tilde{\mathcal{Y}}_{2}(t, \theta)\right)}\left(\left(\tilde{\mathcal{U}}_{2}^{+}\right)^{3}-\left(\tilde{\mathcal{U}}_{1}^{+}\right)^{3}\right) \tilde{\mathcal{Y}}_{2, \eta}(t, \theta) \mathbb{1}_{\tilde{\mathcal{U}}_{1}^{+} \leqslant \tilde{\mathcal{U}}_{2}^{+}}(t, \theta) d \theta\right) d \eta \mid \\
& \leqslant\left\|\tilde{\mathcal{Y}}_{1}-\tilde{\mathcal{Y}}_{2}\right\|^{2}+\frac{1}{A^{12}} \int_{0}^{1} \tilde{\mathcal{Y}}_{2, \eta}^{2}(t, \eta) \\
& \times\left(\int_{0}^{\eta} e^{-\frac{1}{A_{2}}\left(\tilde{\mathcal{Y}}_{2}(t, \eta)-\tilde{\mathcal{Y}}_{2}(t, \theta)\right)}\left(\left(\tilde{\mathcal{U}}_{2}^{+}\right)^{3}-\left(\tilde{\mathcal{U}}_{1}^{+}\right)^{3}\right) \tilde{\mathcal{Y}}_{2, \eta}(t, \theta) \mathbb{1}_{\tilde{\mathcal{U}}_{1}^{+} \leqslant \tilde{\mathcal{U}}_{2}^{+}}(t, \theta) d \theta\right)^{2} d \eta \\
& \leqslant\left\|\tilde{\mathcal{Y}}_{1}-\tilde{\mathcal{Y}}_{2}\right\|^{2} \\
& +\frac{9}{A^{12}} \int_{0}^{1} \tilde{\mathcal{Y}}_{2, \eta}^{2}(t, \eta)\left(\int_{0}^{\eta} e^{-\frac{1}{A_{2}}\left(\tilde{\mathcal{Y}}_{2}(t, \eta)-\tilde{\mathcal{Y}}_{2}(t, \theta)\right)} \tilde{\mathcal{U}}_{2}^{2} \tilde{\mathcal{Y}}_{2, \eta}(t, \theta) d \theta\right) \\
& \times\left(\int_{0}^{\eta} e^{-\frac{1}{A_{2}}\left(\tilde{\mathcal{Y}}_{2}(t, \eta)-\tilde{\mathcal{Y}}_{2}(t, \theta)\right)} \tilde{\mathcal{U}}_{2}^{2} \tilde{\mathcal{Y}}_{2, \eta}\left(\tilde{\mathcal{U}}_{1}-\tilde{\mathcal{U}}_{2}\right)^{2}(t, \theta) d \theta\right) d \eta \\
& \leqslant\left\|\tilde{\mathcal{Y}}_{1}-\tilde{\mathcal{Y}}_{2}\right\|^{2}
\end{aligned}
$$




$$
\begin{aligned}
& +\frac{36}{A^{11}} \int_{0}^{1} \tilde{\mathcal{P}}_{2} \tilde{\mathcal{Y}}_{2, \eta}^{2}(t, \eta)\left(\int_{0}^{\eta} e^{-\frac{1}{A_{2}}\left(\tilde{\mathcal{Y}}_{2}(t, \eta)-\tilde{\mathcal{Y}}_{2}(t, \theta)\right)} \tilde{\mathcal{U}}_{2}^{2} \tilde{\mathcal{Y}}_{2, \eta}\left(\tilde{\mathcal{U}}_{1}-\tilde{\mathcal{U}}_{2}\right)^{2}(t, \theta) d \theta\right) d \eta \\
\leqslant & \left\|\tilde{\mathcal{Y}}_{1}-\tilde{\mathcal{Y}}_{2}\right\|^{2} \\
& +\frac{18}{A^{6}} \int_{0}^{1} \tilde{\mathcal{Y}}_{2, \eta}(t, \eta) e^{-\frac{1}{A_{2}} \tilde{\mathcal{Y}}_{2}(t, \eta)} \int_{0}^{\eta} e^{\frac{1}{A_{2}} \tilde{\mathcal{Y}}_{2}(t, \theta)} \tilde{\mathcal{U}}_{2}^{2} \tilde{\mathcal{Y}}_{2, \eta}\left(\tilde{\mathcal{U}}_{1}-\tilde{\mathcal{U}}_{2}\right)^{2}(t, \theta) d \theta d \eta \\
= & \left\|\tilde{\mathcal{Y}}_{1}-\tilde{\mathcal{Y}}_{2}\right\|^{2} \\
& -\left.\frac{18 A_{2}}{A^{6}} \int_{0}^{\eta} e^{-\frac{1}{A_{2}}\left(\tilde{\mathcal{Y}}_{2}(t, \eta)-\tilde{\mathcal{Y}}_{2}(t, \theta)\right)} \tilde{\mathcal{U}}_{2}^{2} \tilde{\mathcal{Y}}_{2, \eta}\left(\tilde{\mathcal{U}}_{1}-\tilde{\mathcal{U}}_{2}\right)^{2}(t, \theta) d \theta\right|_{\eta=0} ^{1} \\
& +\frac{18 A_{2}}{A^{6}} \int_{0}^{1} \tilde{\mathcal{U}}_{2}^{2} \tilde{\mathcal{Y}}_{2, \eta}\left(\tilde{\mathcal{U}}_{1}-\tilde{\mathcal{U}}_{2}\right)^{2}(t, \eta) d \eta \\
\leqslant & \left\|\tilde{\mathcal{Y}}_{1}-\tilde{\mathcal{Y}}_{2}\right\|^{2}+18\left\|\tilde{\mathcal{U}}_{1}-\tilde{\mathcal{U}}_{2}\right\|^{2},
\end{aligned}
$$

where in the last step we used (4.15b) and (4.16c), which imply that

$$
\int_{0}^{\eta} e^{-\frac{1}{A_{2}}\left(\tilde{\mathcal{Y}}_{2}(t, \eta)-\tilde{\mathcal{Y}}_{2}(t, \theta)\right)} \tilde{\mathcal{U}}_{2}^{2} \tilde{\mathcal{Y}}_{2, \eta}\left(\tilde{\mathcal{U}}_{1}-\tilde{\mathcal{U}}_{2}\right)^{2}(t, \theta) d \theta \leqslant 8 A^{5} \tilde{\mathcal{P}}_{2}(t, \eta),
$$

and hence the left-hand side of (5.12) tends to 0 as $\eta$ to 0 or 1 according to (3.40). Thus the second term above vanishes.

As far as the third and fourth terms $J_{3}$ and $J_{4}$ are concerned, they again have the same structure, and hence we only consider the integral corresponding to $J_{3}$, that is,

$$
\begin{aligned}
\int_{0}^{1} & J_{3}\left(\tilde{\mathcal{Y}}_{1}-\tilde{\mathcal{Y}}_{2}\right)(t, \eta) d \eta \\
= & \frac{1}{A^{6}} \mathbb{1}_{A_{1} \leqslant A_{2}} \int_{0}^{1}\left(\tilde{\mathcal{Y}}_{1}-\tilde{\mathcal{Y}}_{2}\right) \tilde{\mathcal{Y}}_{2, \eta}(t, \eta) \\
& \times\left(\int_{0}^{\eta}\left(e^{-\frac{1}{A_{2}}\left(\tilde{\mathcal{Y}}_{2}(t, \eta)-\tilde{\mathcal{Y}}_{2}(t, \theta)\right)}-e^{-\frac{1}{A_{1}}\left(\tilde{\mathcal{Y}}_{2}(t, \eta)-\tilde{\mathcal{Y}}_{2}(t, \theta)\right)}\right) \min _{j}\left(\tilde{\mathcal{U}}_{j}^{+}\right)^{3} \tilde{\mathcal{Y}}_{2, \eta}(t, \theta) d \theta\right) d \eta
\end{aligned}
$$

Recall Lemma A.1(ii). Direct computations yield

$$
\begin{aligned}
& \left|\int_{0}^{1} J_{3}\left(\tilde{\mathcal{Y}}_{1}-\tilde{\mathcal{Y}}_{2}\right)(t, \eta) d \eta\right| \\
& \quad=\frac{1}{A^{6}} \mathbb{1}_{A_{1} \leqslant A_{2}} \mid \int_{0}^{1}\left(\tilde{\mathcal{Y}}_{1}-\tilde{\mathcal{Y}}_{2}\right) \tilde{\mathcal{Y}}_{2, \eta}(t, \eta) \\
& \quad \times\left(\int_{0}^{\eta}\left(e^{-\frac{1}{A_{2}}\left(\tilde{\mathcal{Y}}_{2}(t, \eta)-\tilde{\mathcal{Y}}_{2}(t, \theta)\right)}-e^{-\frac{1}{A_{1}}\left(\tilde{\mathcal{Y}}_{2}(t, \eta)-\tilde{\mathcal{Y}}_{2}(t, \theta)\right)}\right) \min _{j}\left(\tilde{\mathcal{U}}_{j}^{+}\right)^{3} \tilde{\mathcal{Y}}_{2, \eta}(t, \theta) d \theta\right) d \eta \mid \\
& \quad \leqslant \frac{4\left|A_{1}-A_{2}\right|}{a A^{6} e} \mathbb{1}_{A_{1} \leqslant A_{2}} \int_{0}^{1}\left|\tilde{\mathcal{Y}}_{1}-\tilde{\mathcal{Y}}_{2}\right| \tilde{\mathcal{Y}}_{2, \eta}(t, \eta)
\end{aligned}
$$




$$
\begin{aligned}
& \times\left(\int_{0}^{\eta} e^{-\frac{3}{4 A_{2}}\left(\tilde{\mathcal{Y}}_{2}(t, \eta)-\tilde{\mathcal{Y}}_{2}(t, \theta)\right)} \min _{j}\left(\tilde{\mathcal{U}}_{j}^{+}\right)^{3} \tilde{\mathcal{Y}}_{2, \eta}(t, \theta) d \theta\right) d \eta \\
& \leqslant\left\|\tilde{\mathcal{Y}}_{1}-\tilde{\mathcal{Y}}_{2}\right\|^{2}+\frac{16\left|A_{1}-A_{2}\right|^{2}}{a^{2} A^{12} e^{2}} \mathbb{1}_{A_{1} \leqslant A_{2}} \\
& \times \int_{0}^{1} \tilde{\mathcal{Y}}_{2, \eta}^{2}(t, \eta)\left(\int_{0}^{\eta} e^{-\frac{3}{4 A_{2}}\left(\tilde{\mathcal{Y}}_{2}(t, \eta)-\tilde{\mathcal{Y}}_{2}(t, \theta)\right)}\left|\tilde{\mathcal{U}}_{1}\right| \tilde{\mathcal{U}}_{2}^{2} \tilde{\mathcal{Y}}_{2, \eta}(t, \theta) d \theta\right)^{2} d \eta \\
& \leqslant\left\|\tilde{\mathcal{Y}}_{1}-\tilde{\mathcal{Y}}_{2}\right\|^{2} \\
& +\frac{16 a^{4}\left|A_{1}-A_{2}\right|^{2}}{a^{2} A^{12} e^{2}} \int_{0}^{1} \tilde{\mathcal{Y}}_{2, \eta}^{2}(t, \eta)\left(\int_{0}^{\eta} e^{-\frac{1}{A_{2}}\left(\tilde{\mathcal{Y}}_{2}(t, \eta)-\tilde{\mathcal{Y}}_{2}(t, \theta)\right)} \tilde{\mathcal{U}}_{2}^{2} \tilde{\mathcal{Y}}_{2, \eta}(t, \theta) d \theta\right) \\
& \times\left(\int_{0}^{\eta} e^{-\frac{1}{2 A_{2}}\left(\tilde{\mathcal{Y}}_{2}(t, \eta)-\tilde{\mathcal{Y}}_{2}(t, \theta)\right)} \tilde{\mathcal{U}}_{2}^{2} \tilde{\mathcal{Y}}_{2, \eta}(t, \theta) d \theta\right) d \eta \\
& \leqslant\left\|\tilde{\mathcal{Y}}_{1}-\tilde{\mathcal{Y}}_{2}\right\|^{2} \\
& +\frac{64\left|A_{1}-A_{2}\right|^{2}}{A^{9} e^{2}} \int_{0}^{1} \tilde{\mathcal{P}}_{2} \tilde{\mathcal{Y}}_{2, \eta}^{2}(t, \eta)\left(\int_{0}^{\eta} e^{-\frac{1}{2 A_{2}}\left(\tilde{\mathcal{Y}}_{2}(t, \eta)-\tilde{\mathcal{Y}}_{2}(t, \theta)\right)} \tilde{\mathcal{U}}_{2}^{2} \tilde{\mathcal{Y}}_{2, \eta}(t, \theta) d \theta\right) d \eta \\
& \leqslant\left\|\tilde{\mathcal{Y}}_{1}-\tilde{\mathcal{Y}}_{2}\right\|^{2}
\end{aligned}
$$$$
+\frac{32\left|A_{1}-A_{2}\right|^{2}}{A^{4} e^{2}} \int_{0}^{1} \tilde{\mathcal{Y}}_{2, \eta}(t, \eta) e^{-\frac{1}{2 A_{2}} \tilde{\mathcal{Y}}_{2}(t, \eta)} \int_{0}^{\eta} e^{\frac{1}{2 A_{2}} \tilde{\mathcal{Y}}_{2}(t, \theta)} \tilde{\mathcal{U}}_{2}^{2} \tilde{\mathcal{Y}}_{2, \eta}(t, \theta) d \theta d \eta
$$$$
\leqslant\left\|\tilde{\mathcal{Y}}_{1}-\tilde{\mathcal{Y}}_{2}\right\|^{2}+\frac{64 A_{2}\left|A_{1}-A_{2}\right|^{2}}{A^{4} e^{2}}
$$$$
\times\left(-\left.\int_{0}^{\eta} e^{-\frac{1}{2 A_{2}}\left(\tilde{\mathcal{Y}}_{2}(t, \eta)-\tilde{\mathcal{Y}}_{2}(t, \theta)\right)} \tilde{\mathcal{U}}_{2}^{2} \tilde{\mathcal{Y}}_{2, \eta}(t, \theta) d \theta\right|_{\eta=0} ^{1}+\int_{0}^{1} \tilde{\mathcal{U}}_{2}^{2} \tilde{\mathcal{Y}}_{2, \eta}(t, \eta) d \eta\right)
$$$$
\leqslant\left\|\tilde{\mathcal{Y}}_{1}-\tilde{\mathcal{Y}}_{2}\right\|^{2}+\frac{128 A^{2}}{e^{2}}\left|A_{1}-A_{2}\right|^{2}
$$$$
\leqslant \mathcal{O}(1)\left(\left\|\tilde{\mathcal{Y}}_{1}-\tilde{\mathcal{Y}}_{2}\right\|^{2}+\left|A_{1}-A_{2}\right|^{2}\right) \text {. }
$$

As far as the terms $J_{5}$ and $J_{6}$ are concerned, they again have the same structure, and hence we only consider the integral corresponding to $J_{5}$, that is,

$$
\begin{aligned}
\int_{0}^{1} J_{5}\left(\tilde{\mathcal{Y}}_{1}-\tilde{\mathcal{Y}}_{2}\right)(t, \eta) d \eta= & \frac{1}{A^{6}} \int_{0}^{1}\left(\tilde{\mathcal{Y}}_{1}-\tilde{\mathcal{Y}}_{2}\right) \tilde{\mathcal{Y}}_{2, \eta}(t, \eta) \\
& \times\left(\int_{0}^{\eta}\left(e^{-\frac{1}{a}\left(\tilde{\mathcal{Y}}_{2}(t, \eta)-\tilde{\mathcal{Y}}_{2}(t, \theta)\right)}-e^{-\frac{1}{a}\left(\tilde{\mathcal{Y}}_{1}(t, \eta)-\tilde{\mathcal{Y}}_{1}(t, \theta)\right)}\right)\right. \\
& \left.\times \min _{j}\left(\tilde{\mathcal{U}}_{j}^{+}\right)^{3} \tilde{\mathcal{Y}}_{2, \eta}(t, \theta) \mathbb{1}_{B(\eta)}(t, \theta) d \theta\right) d \eta
\end{aligned}
$$


The main ingredient is the estimate

$$
\begin{aligned}
& \left|e^{-\frac{1}{a}\left(\tilde{\mathcal{Y}}_{2}(t, \eta)-\tilde{\mathcal{Y}}_{2}(t, \theta)\right)}-e^{-\frac{1}{a}\left(\tilde{\mathcal{Y}}_{1}(t, \eta)-\tilde{\mathcal{Y}}_{1}(t, \theta)\right)}\right| \\
& \leqslant \frac{1}{a} e^{-\frac{1}{a}\left(\tilde{\mathcal{Y}}_{2}(t, \eta)-\tilde{\mathcal{Y}}_{2}(t, \theta)\right)}\left(\left|\tilde{\mathcal{Y}}_{2}(t, \theta)-\tilde{\mathcal{Y}}_{1}(t, \theta)\right|+\left|\tilde{\mathcal{Y}}_{2}(t, \eta)-\tilde{\mathcal{Y}}_{1}(t, \eta)\right|\right) \\
& \quad \text { for all }(t, \theta) \in B(\eta),
\end{aligned}
$$

which follows from Lemma A.1 (i).

Direct computations yield

$$
\begin{aligned}
& \left|\int_{0}^{1} J_{5}\left(\tilde{\mathcal{Y}}_{1}-\tilde{\mathcal{Y}}_{2}\right)(t, \eta) d \eta\right| \\
& =\frac{1}{A^{6}} \mid \int_{0}^{1}\left(\tilde{\mathcal{Y}}_{1}-\tilde{\mathcal{Y}}_{2}\right) \tilde{\mathcal{Y}}_{2, \eta}(t, \eta)\left(\int_{0}^{\eta}\left(e^{-\frac{1}{a}\left(\tilde{\mathcal{Y}}_{2}(t, \eta)-\tilde{\mathcal{Y}}_{2}(t, \theta)\right)}-e^{-\frac{1}{a}\left(\tilde{\mathcal{Y}}_{1}(t, \eta)-\tilde{\mathcal{Y}}_{1}(t, \theta)\right)}\right)\right. \\
& \left.\times \min _{j}\left(\tilde{\mathcal{U}}_{j}^{+}\right)^{3} \tilde{\mathcal{Y}}_{2, \eta}(t, \theta) \mathbb{1}_{B(\eta)}(t, \theta) d \theta\right) d \eta \mid \\
& \leqslant \frac{1}{a A^{6}} \int_{0}^{1}\left|\tilde{\mathcal{Y}}_{1}-\tilde{\mathcal{Y}}_{2}\right| \tilde{\mathcal{Y}}_{2, \eta}(t, \eta) \\
& \times\left(\int_{0}^{\eta}\left(\left|\tilde{\mathcal{Y}}_{2}(t, \eta)-\tilde{\mathcal{Y}}_{1}(t, \eta)\right|+\left|\tilde{\mathcal{Y}}_{2}(t, \theta)-\tilde{\mathcal{Y}}_{1}(t, \theta)\right|\right)\right. \\
& \left.\times e^{-\frac{1}{a}\left(\tilde{\mathcal{Y}}_{2}(t, \eta)-\tilde{\mathcal{Y}}_{2}(t, \theta)\right)} \min _{j}\left(\tilde{\mathcal{U}}_{j}^{+}\right)^{3} \tilde{\mathcal{Y}}_{2, \eta}(t, \theta) d \theta\right) d \eta \\
& \leqslant \frac{1}{a A^{6}} \int_{0}^{1}\left(\tilde{\mathcal{Y}}_{1}-\tilde{\mathcal{Y}}_{2}\right)^{2} \tilde{\mathcal{Y}}_{2, \eta}(t, \eta) \\
& \times\left(\int_{0}^{\eta} e^{-\frac{1}{a}\left(\tilde{\mathcal{Y}}_{2}(t, \eta)-\tilde{\mathcal{Y}}_{2}(t, \theta)\right)} \min _{j}\left(\tilde{\mathcal{U}}_{j}^{+}\right)^{3} \tilde{\mathcal{Y}}_{2, \eta}(t, \theta) d \theta\right) d \eta \\
& +\left\|\tilde{\mathcal{Y}}_{1}-\tilde{\mathcal{Y}}_{2}\right\|^{2}+\frac{1}{a^{2} A^{12}} \int_{0}^{1} \tilde{\mathcal{Y}}_{2, \eta}^{2}(t, \eta) \\
& \times\left(\int_{0}^{\eta}\left|\tilde{\mathcal{Y}}_{1}(t, \theta)-\tilde{\mathcal{Y}}_{2}(t, \theta)\right| e^{-\frac{1}{a}\left(\tilde{\mathcal{Y}}_{2}(t, \eta)-\tilde{\mathcal{Y}}_{2}(t, \theta)\right)} \min _{j}\left(\tilde{\mathcal{U}}_{j}^{+}\right)^{3} \tilde{\mathcal{Y}}_{2, \eta}(t, \theta) d \theta\right)^{2} d \eta \\
& \leqslant \frac{4}{A^{4}} \int_{0}^{1}\left(\tilde{\mathcal{Y}}_{1}-\tilde{\mathcal{Y}}_{2}\right)^{2} \tilde{\mathcal{P}}_{2} \tilde{\mathcal{Y}}_{2, \eta}(t, \eta) d \eta+\left\|\tilde{\mathcal{Y}}_{1}-\tilde{\mathcal{Y}}_{2}\right\|^{2} \\
& +\frac{1}{a^{2} A^{12}} \int_{0}^{1} \tilde{\mathcal{Y}}_{2, \eta}^{2}(t, \eta)\left(\int_{0}^{\eta} e^{-\frac{1}{a}\left(\tilde{\mathcal{Y}}_{2}(t, \eta)-\tilde{\mathcal{Y}}_{2}(t, \theta)\right)} \tilde{\mathcal{U}}_{2}^{2} \tilde{\mathcal{Y}}_{2, \eta}(t, \theta) d \theta\right) \\
& \times\left(\int_{0}^{\eta}\left(\tilde{\mathcal{Y}}_{1}-\tilde{\mathcal{Y}}_{2}\right)^{2}(t, \theta) e^{-\frac{1}{a}\left(\tilde{\mathcal{Y}}_{2}(t, \eta)-\tilde{\mathcal{Y}}_{2}(t, \theta)\right)} \min _{j}\left(\tilde{\mathcal{U}}_{j}^{4}\right) \tilde{\mathcal{Y}}_{2, \eta}(t, \theta) d \theta\right) d \eta
\end{aligned}
$$




$$
\begin{aligned}
& \leqslant(2 A+1)\left\|\tilde{\mathcal{Y}}_{1}-\tilde{\mathcal{Y}}_{2}\right\|^{2}+\frac{4}{a^{2} A^{11}} \int_{0}^{1} \tilde{\mathcal{P}}_{2} \tilde{\mathcal{Y}}_{2, \eta}^{2}(t, \eta) \\
& \times\left(\int_{0}^{\eta}\left(\tilde{\mathcal{Y}}_{1}-\tilde{\mathcal{Y}}_{2}\right)^{2}(t, \theta) e^{-\frac{1}{a}\left(\tilde{\mathcal{Y}}_{2}(t, \eta)-\tilde{\mathcal{Y}}_{2}(t, \theta)\right)} \min _{j}\left(\tilde{\mathcal{U}}_{j}^{4}\right) \tilde{\mathcal{Y}}_{2, \eta}(t, \theta) d \theta\right) d \eta \\
& \leqslant \mathcal{O}(1)\left\|\tilde{\mathcal{Y}}_{1}-\tilde{\mathcal{Y}}_{2}\right\|^{2}+\frac{2}{a^{2} A^{6}} \int_{0}^{1} \tilde{\mathcal{Y}}_{2, \eta}(t, \eta) \\
& \times\left(\int_{0}^{\eta}\left(\tilde{\mathcal{Y}}_{1}-\tilde{\mathcal{Y}}_{2}\right)^{2}(t, \theta) e^{-\frac{1}{a}\left(\tilde{\mathcal{Y}}_{2}(t, \eta)-\tilde{\mathcal{Y}}_{2}(t, \theta)\right)} \min _{j}\left(\tilde{\mathcal{U}}_{j}^{4}\right) \tilde{\mathcal{Y}}_{2, \eta}(t, \theta) d \theta\right) d \eta \\
& =\mathcal{O}(1)\left\|\tilde{\mathcal{Y}}_{1}-\tilde{\mathcal{Y}}_{2}\right\|^{2}+\frac{2}{a^{2} A^{6}} \int_{0}^{1} \tilde{\mathcal{Y}}_{2, \eta}(t, \eta) e^{-\frac{1}{a} \tilde{\mathcal{Y}}_{2}(t, \eta)} \\
& \times \int_{0}^{\eta}\left(\tilde{\mathcal{Y}}_{1}-\tilde{\mathcal{Y}}_{2}\right)^{2}(t, \theta) e^{\frac{1}{a} \tilde{\mathcal{Y}}_{2}(t, \theta)} \min _{j}\left(\tilde{\mathcal{U}}_{j}^{4}\right) \tilde{\mathcal{Y}}_{2, \eta}(t, \theta) d \theta d \eta \\
& =\mathcal{O}(1)\left\|\tilde{\mathcal{Y}}_{1}-\tilde{\mathcal{Y}}_{2}\right\|^{2} \\
& -\left.\frac{2}{a A^{6}} \int_{0}^{\eta} e^{-\frac{1}{a}\left(\tilde{\mathcal{Y}}_{2}(t, \eta)-\tilde{\mathcal{Y}}_{2}(t, \theta)\right)}\left(\tilde{\mathcal{Y}}_{1}-\tilde{\mathcal{Y}}_{2}\right)^{2} \min _{j}\left(\tilde{\mathcal{U}}_{j}^{4}\right) \tilde{\mathcal{Y}}_{2, \eta}(t, \theta) d \theta\right|_{\eta=0} ^{1} \\
& +\frac{2}{a A^{6}} \int_{0}^{1}\left(\tilde{\mathcal{Y}}_{1}-\tilde{\mathcal{Y}}_{2}\right)^{2} \min _{j}\left(\tilde{\mathcal{U}}_{j}^{4}\right) \tilde{\mathcal{Y}}_{2, \eta}(t, \eta) d \eta \\
& \leqslant \mathcal{O}(1)\left\|\tilde{\mathcal{Y}}_{1}-\tilde{\mathcal{Y}}_{2}\right\|^{2} \text {, }
\end{aligned}
$$

where $\mathcal{O}(1)$ denotes some constant which only depends on $A$ and which remains bounded as $A \rightarrow 0$. We used (cf. (4.16c)) that

$$
\begin{aligned}
\int_{0}^{\eta} e^{-\frac{1}{a}\left(\tilde{\mathcal{Y}}_{2}(t, \eta)-\tilde{\mathcal{Y}}_{2}(t, \theta)\right)} \tilde{\mathcal{U}}_{2}^{2} \tilde{\mathcal{Y}}_{2, \eta}(t, \theta) d \theta & \leqslant \int_{0}^{\eta} e^{-\frac{1}{A_{2}}\left(\tilde{\mathcal{Y}}_{2}(t, \eta)-\tilde{\mathcal{Y}}_{2}(t, \theta)\right)} \tilde{\mathcal{U}}_{2}^{2} \tilde{\mathcal{Y}}_{2, \eta}(t, \theta) d \theta \\
& \leqslant 4 A_{2} \tilde{\mathcal{P}}_{2}(t, \eta)
\end{aligned}
$$

In the last step we used (4.15b), (4.15g), and finally that

$$
\begin{aligned}
& \left|\int_{0}^{\eta} e^{-\frac{1}{a}\left(\tilde{\mathcal{Y}}_{2}(t, \eta)-\tilde{\mathcal{Y}}_{2}(t, \theta)\right)}\left(\tilde{\mathcal{Y}}_{1}-\tilde{\mathcal{Y}}_{2}\right)^{2} \min _{j}\left(\tilde{\mathcal{U}}_{j}^{4}\right) \tilde{\mathcal{Y}}_{2, \eta}(t, \theta) d \theta\right| \\
& \quad \leqslant\left(\left\|\tilde{\mathcal{Y}}_{1} \tilde{\mathcal{U}}_{1}\right\|_{L^{\infty}}^{2}+\left\|\tilde{\mathcal{Y}}_{2} \tilde{\mathcal{U}}_{2}\right\|_{L^{\infty}}^{2}\right) 8 A \tilde{\mathcal{P}}_{2}(t, \eta) .
\end{aligned}
$$

Since $\tilde{\mathcal{P}}_{i}(t, \eta)$ tends to 0 as $\eta$ tends to 0 and 1 , the term on the left-hand side tends to zero as $\eta$ tends to 0 and 1 , respectively (cf. (3.40)). 
Consider next the terms $J_{7}$ and $J_{8}$, that is,

$$
\begin{aligned}
\left(J_{7}+J_{8}\right)(t, \eta)= & \frac{1}{A^{6}} \tilde{\mathcal{Y}}_{2, \eta}(t, \eta) \\
& \times\left(\int_{0}^{\eta} \min _{j}\left(e^{-\frac{1}{a}\left(\tilde{\mathcal{Y}}_{j}(t, \eta)-\tilde{\mathcal{Y}}_{j}(t, \theta)\right)}\right) \min _{j}\left(\tilde{\mathcal{U}}_{j}^{+}\right)^{3} \tilde{\mathcal{Y}}_{2, \eta}(t, \theta) d \theta\right) \\
& -\frac{1}{A^{6}} \tilde{\mathcal{Y}}_{1, \eta}(t, \eta) \\
& \times\left(\int_{0}^{\eta} \min _{j}\left(e^{-\frac{1}{a}\left(\tilde{\mathcal{Y}}_{j}(t, \eta)-\tilde{\mathcal{Y}}_{j}(t, \theta)\right)}\right) \min _{j}\left(\tilde{\mathcal{U}}_{j}^{+}\right)^{3} \tilde{\mathcal{Y}}_{1, \eta}(t, \theta) d \theta\right) .
\end{aligned}
$$

Here we have to be a bit more careful. Introducing

$$
E=\left\{(t, \eta) \mid \int_{0}^{\eta} \Omega(t, \eta, \theta) \tilde{\mathcal{Y}}_{2, \eta}(t, \theta) d \theta \leqslant \int_{0}^{\eta} \Omega(t, \eta, \theta) \tilde{\mathcal{Y}}_{1, \eta}(t, \theta) d \theta\right\},
$$

with

$$
\Omega(t, \eta, \theta)=\min _{j}\left(e^{-\frac{1}{a}\left(\tilde{\mathcal{Y}}_{j}(t, \eta)-\tilde{\mathcal{Y}}_{j}(t, \theta)\right)}\right) \min _{j}\left(\tilde{\mathcal{U}}_{j}^{+}\right)^{3}(t, \theta),
$$

we can write

$$
\begin{aligned}
\left(J_{7}+\right. & \left.J_{8}\right)(t, \eta) \\
= & \frac{1}{A^{6}} \tilde{\mathcal{Y}}_{2, \eta}(t, \eta) \\
& \times\left(\int_{0}^{\eta} \min _{j}\left(e^{-\frac{1}{a}\left(\tilde{\mathcal{Y}}_{j}(t, \eta)-\tilde{\mathcal{Y}}_{j}(t, \theta)\right)}\right) \min _{j}\left(\tilde{\mathcal{U}}_{j}^{+}\right)^{3} \tilde{\mathcal{Y}}_{2, \eta}(t, \theta) d \theta\right) \\
& -\frac{1}{A^{6}} \tilde{\mathcal{Y}}_{1, \eta}(t, \eta) \\
& \times\left(\int_{0}^{\eta} \min _{j}\left(e^{-\frac{1}{a}\left(\tilde{\mathcal{Y}}_{j}(t, \eta)-\tilde{\mathcal{Y}}_{j}(t, \theta)\right)}\right) \min _{j}\left(\tilde{\mathcal{U}}_{j}^{+}\right)^{3} \tilde{\mathcal{Y}}_{1, \eta}(t, \theta) d \theta\right) \\
= & \frac{1}{A^{6}}\left(\tilde{\mathcal{Y}}_{2, \eta}-\tilde{\mathcal{Y}}_{1, \eta}\right)(t, \eta) \\
& \times \min _{k}\left[\int_{0}^{\eta} \min _{j}\left(e^{-\frac{1}{a}\left(\tilde{\mathcal{Y}}_{j}(t, \eta)-\tilde{\mathcal{Y}}_{j}(t, \theta)\right)}\right) \min _{j}\left(\tilde{\mathcal{U}}_{j}^{+}\right)^{3} \tilde{\mathcal{Y}}_{k, \eta}(t, \theta) d \theta\right] \\
& +\frac{1}{A^{6}} \tilde{\mathcal{Y}}_{1, \eta}(t, \eta) \int_{0}^{\eta} \min _{j}\left(e^{-\frac{1}{a}\left(\tilde{\mathcal{Y}}_{j}(t, \eta)-\tilde{\mathcal{Y}}_{j}(t, \theta)\right)}\right) \\
& \times \min _{j}\left(\tilde{\mathcal{U}}_{j}^{+}\right)^{3}\left(\tilde{\mathcal{Y}}_{2, \eta}-\tilde{\mathcal{Y}}_{1, \eta}\right)(t, \theta) d \theta \mathbb{1}_{E}(t, \eta) \\
& +\frac{1}{A^{6}} \tilde{\mathcal{Y}}_{2, \eta}(t, \eta) \int_{0}^{\eta} \min _{j}\left(e^{-\frac{1}{a}\left(\tilde{\mathcal{Y}}_{j}(t, \eta)-\tilde{\mathcal{Y}}_{j}(t, \theta)\right)}\right)
\end{aligned}
$$




$$
\begin{aligned}
& \times \min _{j}\left(\tilde{\mathcal{U}}_{j}^{+}\right)^{3}\left(\tilde{\mathcal{Y}}_{2, \eta}-\tilde{\mathcal{Y}}_{1, \eta}\right)(t, \theta) d \theta \mathbb{1}_{E^{c}}(t, \eta) \\
= & \left(L_{1}+L_{2}+L_{3}\right)(t, \eta) .
\end{aligned}
$$

As far as the first term $L_{1}$ is concerned, the corresponding integral can be estimated as follows (we use (3.40)):

$$
\begin{aligned}
\mid \int_{0}^{1} & L_{1}\left(\tilde{\mathcal{Y}}_{1}-\tilde{\mathcal{Y}}_{2}\right)(t, \eta) d \eta \mid \\
= & \frac{1}{A^{6}} \mid \int_{0}^{1}\left(\tilde{\mathcal{Y}}_{1}-\tilde{\mathcal{Y}}_{2}\right)\left(\tilde{\mathcal{Y}}_{2, \eta}-\tilde{\mathcal{Y}}_{1, \eta}\right)(t, \eta) \\
& \times \min _{k}\left[\int_{0}^{\eta} \min _{j}\left(e^{-\frac{1}{a}\left(\tilde{\mathcal{Y}}_{j}(t, \eta)-\tilde{\mathcal{Y}}_{j}(t, \theta)\right)}\right) \min _{j}\left(\tilde{\mathcal{U}}_{j}^{+}\right)^{3} \tilde{\mathcal{Y}}_{k, \eta}(t, \theta) d \theta\right] d \eta \mid \\
= & \mid-\frac{1}{2 A^{6}}\left(\tilde{\mathcal{Y}}_{1}-\tilde{\mathcal{Y}}_{2}\right)^{2}(t, \eta) \\
& \times\left.\min _{k}\left[\int_{0}^{\eta} \min _{j}\left(e^{-\frac{1}{a}\left(\tilde{\mathcal{Y}}_{j}(t, \eta)-\tilde{\mathcal{Y}}_{j}(t, \theta)\right)}\right) \min _{j}\left(\tilde{\mathcal{U}}_{j}^{+}\right)^{3} \tilde{\mathcal{Y}}_{k, \eta}(t, \theta) d \theta\right]\right|_{\eta=0} \\
& +\frac{1}{2 A^{6}} \int_{0}^{1}\left(\tilde{\mathcal{Y}}_{1}-\tilde{\mathcal{Y}}_{2}\right)^{2}(t, \eta) \\
& \times \frac{d}{d \eta} \min _{k}\left[\int_{0}^{\eta} \min _{j}\left(e^{-\frac{1}{a}\left(\tilde{\mathcal{Y}}_{j}(t, \eta)-\tilde{\mathcal{Y}}_{j}(t, \theta)\right)}\right) \min _{j}\left(\tilde{\mathcal{U}}_{j}^{+}\right)^{3} \tilde{\mathcal{Y}}_{k, \eta}(t, \theta) d \theta\right] d \eta \mid \\
\leqslant & \mathcal{O}(1)\left\|\tilde{\mathcal{Y}}_{1}-\tilde{\mathcal{Y}}_{2}\right\|^{2},
\end{aligned}
$$

where $\mathcal{O}(1)$ denotes some constant only depending on $A$, which remains bounded as $A \rightarrow 0$, since the derivative

$$
\frac{d}{d \eta} \min _{k}\left[\int_{0}^{\eta} \min _{j}\left(e^{-\frac{1}{a}\left(\tilde{\mathcal{Y}}_{j}(t, \eta)-\tilde{\mathcal{Y}}_{j}(t, \theta)\right)}\right) \min _{j}\left(\tilde{\mathcal{U}}_{j}^{+}\right)^{3} \tilde{\mathcal{Y}}_{k, \eta}(t, \theta) d \theta\right]
$$

exists and is uniformly bounded; see Lemma A.8.

As far as the last term $L_{3}$ (a similar argument works for $L_{2}$ ) is concerned, the corresponding integral can be estimated as follows:

$$
\begin{aligned}
\int_{0}^{1} & L_{3}\left(\tilde{\mathcal{Y}}_{1}-\tilde{\mathcal{Y}}_{2}\right)(t, \eta) d \eta \\
= & \frac{1}{A^{6}} \int_{0}^{1}\left(\tilde{\mathcal{Y}}_{1}-\tilde{\mathcal{Y}}_{2}\right) \tilde{\mathcal{Y}}_{2, \eta}(t, \eta) \int_{0}^{\eta} \min _{j}\left(e^{-\frac{1}{a}\left(\tilde{\mathcal{Y}}_{j}(t, \eta)-\tilde{\mathcal{Y}}_{j}(t, \theta)\right)}\right) \\
& \times \min _{j}\left(\tilde{\mathcal{U}}_{j}^{+}\right)^{3}\left(\tilde{\mathcal{Y}}_{2, \eta}-\tilde{\mathcal{Y}}_{1, \eta}\right)(t, \theta) d \theta \mathbb{1}_{E^{c}}(t, \eta) d \eta
\end{aligned}
$$




$$
\begin{aligned}
& =\frac{1}{A^{6}} \int_{0}^{1}\left(\tilde{\mathcal{Y}}_{1}-\tilde{\mathcal{Y}}_{2}\right) \tilde{\mathcal{Y}}_{2, \eta}(t, \eta) \mathbb{1}_{E^{c}}(t, \eta) \\
& \times\left[\left.\left(\tilde{\mathcal{Y}}_{2}-\tilde{\mathcal{Y}}_{1}\right)(t, \theta) \min _{j}\left(e^{-\frac{1}{a}\left(\tilde{\mathcal{Y}}_{j}(t, \eta)-\tilde{\mathcal{Y}}_{j}(t, \theta)\right)}\right) \min _{j}\left(\tilde{\mathcal{U}}_{j}^{+}\right)^{3}(t, \theta)\right|_{\theta=0} ^{\eta}\right. \\
& -\int_{0}^{\eta}\left(\tilde{\mathcal{Y}}_{2}-\tilde{\mathcal{Y}}_{1}\right)(t, \theta) \\
& \times\left[\left(\frac{d}{d \theta} \min _{j}\left(e^{-\frac{1}{a}\left(\tilde{\mathcal{Y}}_{j}(t, \eta)-\tilde{\mathcal{Y}}_{j}(t, \theta)\right)}\right)\right) \min _{j}\left(\tilde{\mathcal{U}}_{j}^{+}\right)^{3}(t, \theta)\right. \\
& \left.\left.+\min _{j}\left(e^{-\frac{1}{a}\left(\tilde{\mathcal{Y}}_{j}(t, \eta)-\tilde{\mathcal{Y}}_{j}(t, \theta)\right)}\right)\left(\frac{d}{d \theta} \min _{j}\left(\tilde{\mathcal{U}}_{j}^{+}\right)^{3}\right)(t, \theta)\right] d \theta\right] d \eta \\
& =-\frac{1}{A^{6}} \int_{0}^{1}\left(\tilde{\mathcal{Y}}_{1}-\tilde{\mathcal{Y}}_{2}\right)^{2} \min \left(\tilde{\mathcal{U}}_{j}^{+}\right)^{3} \tilde{\mathcal{Y}}_{2, \eta}(t, \eta) \mathbb{1}_{E^{c}}(t, \eta) d \eta \\
& +\frac{1}{A^{6}} \int_{0}^{1}\left(\tilde{\mathcal{Y}}_{1}-\tilde{\mathcal{Y}}_{2}\right) \tilde{\mathcal{Y}}_{2, \eta}(t, \eta) \mathbb{1}_{E^{c}}(t, \eta) \\
& \times \int_{0}^{\eta}\left(\tilde{\mathcal{Y}}_{1}-\tilde{\mathcal{Y}}_{2}\right)(t, \theta) \\
& \times\left[\left(\frac{d}{d \theta} \min _{j}\left(e^{-\frac{1}{a}\left(\tilde{\mathcal{Y}}_{j}(t, \eta)-\tilde{\mathcal{Y}}_{j}(t, \theta)\right)}\right)\right) \min _{j}\left(\tilde{\mathcal{U}}_{j}^{+}\right)^{3}(t, \theta)\right. \\
& \left.+\min _{j}\left(e^{-\frac{1}{a}\left(\tilde{\mathcal{Y}}_{j}(t, \eta)-\tilde{\mathcal{Y}}_{j}(t, \theta)\right)}\right)\left(\frac{d}{d \theta} \min _{j}\left(\tilde{\mathcal{U}}_{j}^{+}\right)^{3}\right)(t, \theta)\right] d \theta d \eta \\
& =L_{31}+L_{32} \text {. }
\end{aligned}
$$

As far as the first term $L_{31}$ is concerned, we have, since

$$
\min _{j}\left(\tilde{\mathcal{U}}_{j}^{+}\right)^{3} \tilde{\mathcal{Y}}_{i, \eta}(t, \eta) \leqslant A^{5} \min _{j}\left(\tilde{\mathcal{U}}_{j}^{+}\right) \leqslant \frac{1}{\sqrt{2}} A^{7}
$$

that

$$
\begin{aligned}
\left|L_{31}\right| & \leqslant\left|\frac{1}{A^{6}} \int_{0}^{1}\left(\tilde{\mathcal{Y}}_{1}-\tilde{\mathcal{Y}}_{2}\right)^{2} \min _{j}\left(\tilde{\mathcal{U}}_{j}^{+}\right)^{3} \tilde{\mathcal{Y}}_{2, \eta}(t, \eta) \mathbb{1}_{E^{c}}(t, \eta) d \eta\right| \\
& \leqslant \frac{A}{\sqrt{2}}\left\|\tilde{\mathcal{Y}}_{1}-\tilde{\mathcal{Y}}_{2}\right\|^{2}=\mathcal{O}(1)\left\|\tilde{\mathcal{Y}}_{1}-\tilde{\mathcal{Y}}_{2}\right\|^{2},
\end{aligned}
$$

where $\mathcal{O}(1)$ denotes a constant, which only depends on $A$ and which remains bounded as $A \rightarrow 0$.

The second term $L_{32}$, on the other hand, is a bit more demanding. We start by considering the first part of $L_{32}$. From Lemma A.2 we have that

$$
\left|\frac{d}{d \theta} \min _{j}\left(e^{-\frac{1}{a}\left(\tilde{\mathcal{Y}}_{j}(t, \eta)-\tilde{\mathcal{Y}}_{j}(t, \theta)\right)}\right)\right| \leqslant \frac{1}{a} \min _{j}\left(e^{-\frac{1}{a}\left(\tilde{\mathcal{Y}}_{j}(t, \eta)-\tilde{\mathcal{Y}}_{j}(t, \theta)\right)}\right) \max _{j}\left(\tilde{\mathcal{Y}}_{j, \eta}(t, \theta)\right) .
$$


This implies that

$$
\begin{aligned}
& \frac{1}{A^{6}} \mid \int_{0}^{1}\left(\tilde{\mathcal{Y}}_{1}-\tilde{\mathcal{Y}}_{2}\right) \tilde{\mathcal{Y}}_{2, \eta}(t, \eta) \mathbb{1}_{E^{c}}(t, \eta) \int_{0}^{\eta}\left(\tilde{\mathcal{Y}}_{1}-\tilde{\mathcal{Y}}_{2}\right)(t, \theta) \\
& \times\left(\frac{d}{d \theta} \min _{j}\left(e^{-\frac{1}{a}\left(\tilde{\mathcal{Y}}_{j}(t, \eta)-\tilde{\mathcal{Y}}_{j}(t, \theta)\right)}\right)\right) \min _{j}\left(\tilde{\mathcal{U}}_{j}^{+}\right)^{3}(t, \theta) d \theta d \eta \mid \\
& \leqslant\left\|\tilde{\mathcal{Y}}_{1}-\tilde{\mathcal{Y}}_{2}\right\|^{2}+\frac{1}{A^{12}} \int_{0}^{1} \tilde{\mathcal{Y}}_{2, \eta}^{2}(t, \eta)\left(\int_{0}^{\eta}\left(\tilde{\mathcal{Y}}_{1}-\tilde{\mathcal{Y}}_{2}\right)(t, \theta)\right. \\
& \left.\times\left(\frac{d}{d \theta} \min _{j}\left(e^{-\frac{1}{a}\left(\tilde{\mathcal{Y}}_{j}(t, \eta)-\tilde{\mathcal{Y}}_{j}(t, \theta)\right)}\right)\right) \min _{j}\left(\tilde{\mathcal{U}}_{j}^{+}\right)^{3}(t, \theta) d \theta\right)^{2} d \eta \\
& \leqslant\left\|\tilde{\mathcal{Y}}_{1}-\tilde{\mathcal{Y}}_{2}\right\|^{2}+\frac{1}{a^{2} A^{12}} \int_{0}^{1} \tilde{\mathcal{Y}}_{2, \eta}^{2}(t, \eta) \\
& \times\left(\int_{0}^{\eta}\left(\tilde{\mathcal{Y}}_{1}-\tilde{\mathcal{Y}}_{2}\right)(t, \theta) \min _{j}\left(e^{-\frac{1}{a}\left(\tilde{\mathcal{Y}}_{j}(t, \eta)-\tilde{\mathcal{Y}}_{j}(t, \theta)\right)}\right)\right. \\
& \left.\times \min _{j}\left(\tilde{\mathcal{U}}_{j}^{+}\right)^{3} \max _{j}\left(\tilde{\mathcal{Y}}_{j, \eta}\right)(t, \theta) d \theta\right)^{2} d \eta \\
& \leqslant\left\|\tilde{\mathcal{Y}}_{1}-\tilde{\mathcal{Y}}_{2}\right\|^{2}+\frac{1}{a^{2} A^{2}} \int_{0}^{1} \tilde{\mathcal{Y}}_{2, \eta}^{2}(t, \eta) \\
& \times\left(\int_{0}^{\eta}\left(\tilde{\mathcal{Y}}_{1}-\tilde{\mathcal{Y}}_{2}\right)(t, \theta) \min _{j}\left(e^{-\frac{1}{a}\left(\tilde{\mathcal{Y}}_{j}(t, \eta)-\tilde{\mathcal{Y}}_{j}(t, \theta)\right)}\right) \min _{j}\left(\tilde{\mathcal{U}}_{j}^{+}\right)(t, \theta) d \theta\right)^{2} d \eta \\
& \leqslant\left\|\tilde{\mathcal{Y}}_{1}-\tilde{\mathcal{Y}}_{2}\right\|^{2}+\frac{a^{2}}{2 A^{2}} \int_{0}^{1} \tilde{\mathcal{Y}}_{2, \eta}^{2}(t, \eta)\left(\int_{0}^{\eta}\left(\tilde{\mathcal{Y}}_{1}-\tilde{\mathcal{Y}}_{2}\right)(t, \theta)\right. \\
& \left.\times \min _{j}\left(e^{-\frac{1}{a}\left(\tilde{\mathcal{Y}}_{j}(t, \eta)-\tilde{\mathcal{Y}}_{j}(t, \theta)\right)}\right) d \theta\right)^{2} d \eta \\
& \leqslant\left\|\tilde{\mathcal{Y}}_{1}-\tilde{\mathcal{Y}}_{2}\right\|^{2} \\
& +\frac{1}{2} \int_{0}^{1} \tilde{\mathcal{Y}}_{2, \eta}^{2}(t, \eta)\left(\int_{0}^{\eta} e^{-\frac{1}{2 A_{2}}\left(\tilde{\mathcal{Y}}_{2}(t, \eta)-\tilde{\mathcal{Y}}_{2}(t, \theta)\right)}\left(\tilde{\mathcal{Y}}_{1}-\tilde{\mathcal{Y}}_{2}\right)^{2}(t, \theta) d \theta\right) \\
& \times\left(\int_{0}^{\eta} e^{-\frac{3}{2 A_{2}}\left(\tilde{\mathcal{Y}}_{2}(t, \eta)-\tilde{\mathcal{Y}}_{2}(t, \theta)\right)} d \theta\right) d \eta \\
& \leqslant\left\|\tilde{\mathcal{Y}}_{1}-\tilde{\mathcal{Y}}_{2}\right\|^{2} \\
& +\frac{1}{2 A^{5}} \int_{0}^{1} \tilde{\mathcal{Y}}_{2, \eta}^{2}(t, \eta)\left(\int_{0}^{\eta} e^{-\frac{1}{2 A_{2}}\left(\tilde{\mathcal{Y}}_{2}(t, \eta)-\tilde{\mathcal{Y}}_{2}(t, \theta)\right)}\left(\tilde{\mathcal{Y}}_{1}-\tilde{\mathcal{Y}}_{2}\right)^{2}(t, \theta) d \theta\right) \\
& \times\left(\int_{0}^{\eta} e^{-\frac{3}{2 A_{2}}\left(\tilde{\mathcal{Y}}_{2}(t, \eta)-\tilde{\mathcal{Y}}_{2}(t, \theta)\right)}\left(2 \tilde{\mathcal{P}}_{2} \tilde{\mathcal{Y}}_{2, \eta}+\tilde{\mathcal{H}}_{2, \eta}\right)(t, \theta) d \theta\right) d \eta
\end{aligned}
$$




$$
\begin{aligned}
\leqslant & \left\|\tilde{\mathcal{Y}}_{1}-\tilde{\mathcal{Y}}_{2}\right\|^{2} \\
& +\frac{1}{2 A^{5}} \int_{0}^{1} 8 A \tilde{\mathcal{P}}_{2} \tilde{\mathcal{Y}}_{2, \eta}^{2}(t, \eta)\left(\int_{0}^{\eta} e^{-\frac{1}{2 A_{2}}\left(\tilde{\mathcal{Y}}_{2}(t, \eta)-\tilde{\mathcal{Y}}_{2}(t, \theta)\right)}\left(\tilde{\mathcal{Y}}_{1}-\tilde{\mathcal{Y}}_{2}\right)^{2}(t, \theta) d \theta\right) d \eta \\
\leqslant & \left\|\tilde{\mathcal{Y}}_{1}-\tilde{\mathcal{Y}}_{2}\right\|^{2} \\
& +2 A \int_{0}^{1} \tilde{\mathcal{Y}}_{2, \eta}(t, \eta) e^{-\frac{1}{2 A_{2}} \tilde{\mathcal{Y}}_{2}(t, \eta)} \int_{0}^{\eta} e^{\frac{1}{2 A_{2}} \tilde{\mathcal{Y}}_{2}(t, \theta)}\left(\tilde{\mathcal{Y}}_{1}-\tilde{\mathcal{Y}}_{2}\right)^{2}(t, \theta) d \theta d \eta \\
\leqslant & \left\|\tilde{\mathcal{Y}}_{1}-\tilde{\mathcal{Y}}_{2}\right\|^{2}+4 A A_{2}\left(-\left.\int_{0}^{\eta} e^{-\frac{1}{2 A_{2}}\left(\tilde{\mathcal{Y}}_{2}(t, \eta)-\tilde{\mathcal{Y}}_{2}(t, \theta)\right)}\left(\tilde{\mathcal{Y}}_{1}-\tilde{\mathcal{Y}}_{2}\right)^{2}(t, \theta) d \theta\right|_{\eta=0} ^{1}\right. \\
& \left.+\int_{0}^{1}\left(\tilde{\mathcal{Y}}_{1}-\tilde{\mathcal{Y}}_{2}\right)^{2}(t, \eta) d \eta\right) \\
\leqslant & \mathcal{O}(1)\left\|\tilde{\mathcal{Y}}_{1}-\tilde{\mathcal{Y}}_{2}\right\|^{2} .
\end{aligned}
$$

Here we used (4.13) and (4.16e). Furthermore, we use (4.16f).

Next, we turn to the second half of $L_{32}$. Recall first (4.16g).

From Lemma A.2 we have that

$$
\left|\frac{d}{d \theta} \min _{j}\left(\tilde{\mathcal{U}}_{j}^{+}\right)^{3}(t, \theta)\right| \leqslant 2 A^{4} \min _{j}\left(\tilde{\mathcal{U}}_{j}^{+}\right)(t, \theta) .
$$

Thus we can conclude as before

$$
\begin{aligned}
& \frac{1}{A^{6}} \mid \int_{0}^{1}\left(\tilde{\mathcal{Y}}_{1}-\tilde{\mathcal{Y}}_{2}\right) \tilde{\mathcal{Y}}_{2, \eta}(t, \eta) \mathbb{1}_{E^{c}}(t, \eta) \int_{0}^{\eta}\left(\tilde{\mathcal{Y}}_{1}-\tilde{\mathcal{Y}}_{2}\right)(t, \theta) \min _{j}\left(e^{-\frac{1}{a}\left(\tilde{\mathcal{Y}}_{j}(t, \eta)-\tilde{\mathcal{Y}}_{j}(t, \theta)\right)}\right) \\
& \times\left(\frac{d}{d \theta} \min _{j}\left(\tilde{\mathcal{U}}_{j}^{+}\right)^{3}\right)(t, \theta) d \theta d \eta \\
& \leqslant\left\|\tilde{\mathcal{Y}}_{1}-\tilde{\mathcal{Y}}_{2}\right\|^{2}+\frac{1}{A^{12}} \int_{0}^{1} \tilde{\mathcal{Y}}_{2, \eta}^{2}(t, \eta)\left(\int_{0}^{\eta}\left(\tilde{\mathcal{Y}}_{1}-\tilde{\mathcal{Y}}_{2}\right)(t, \theta) \min _{j}\left(e^{-\frac{1}{a}\left(\tilde{\mathcal{Y}}_{j}(t, \eta)-\tilde{\mathcal{Y}}_{j}(t, \theta)\right)}\right)\right. \\
& \left.\times\left(\frac{d}{d \theta} \min _{j}\left(\tilde{\mathcal{U}}_{j}^{+}\right)^{3}\right)(t, \theta) d \theta\right)^{2} d \eta \\
& \leqslant\left\|\tilde{\mathcal{Y}}_{1}-\tilde{\mathcal{Y}}_{2}\right\|^{2}+\frac{4}{A^{4}} \int_{0}^{1} \tilde{\mathcal{Y}}_{2, \eta}^{2}(t, \eta)\left(\int_{0}^{\eta}\left|\tilde{\mathcal{Y}}_{1}-\tilde{\mathcal{Y}}_{2}\right|(t, \theta) e^{-\frac{1}{a}\left(\tilde{\mathcal{Y}}_{2}(t, \eta)-\tilde{\mathcal{Y}}_{2}(t, \theta)\right)}\right. \\
& \left.\times \min _{j}\left(\tilde{\mathcal{U}}_{j}^{+}\right)(t, \theta) d \theta\right)^{2} d \eta \\
& \leqslant\left\|\tilde{\mathcal{Y}}_{1}-\tilde{\mathcal{Y}}_{2}\right\|^{2} \\
& +\frac{4}{A^{4}} \int_{0}^{1} \tilde{\mathcal{Y}}_{2, \eta}^{2}(t, \eta)\left(\int_{0}^{\eta}\left(\tilde{\mathcal{Y}}_{1}-\tilde{\mathcal{Y}}_{2}\right)^{2}(t, \theta) e^{-\frac{1}{a}\left(\tilde{\mathcal{Y}}_{2}(t, \eta)-\tilde{\mathcal{Y}}_{2}(t, \theta)\right)} d \theta\right) \\
& \times\left(\int_{0}^{\eta} e^{-\frac{1}{A_{2}}\left(\tilde{\mathcal{Y}}_{2}(t, \eta)-\tilde{\mathcal{Y}}_{2}(t, \theta)\right)} \tilde{\mathcal{U}}_{2}^{2}(t, \theta) d \theta\right) d \eta
\end{aligned}
$$




$$
\begin{aligned}
& \leqslant\left\|\tilde{\mathcal{Y}}_{1}-\tilde{\mathcal{Y}}_{2}\right\|^{2} \\
& +\frac{24}{A^{4}} \int_{0}^{1} \tilde{\mathcal{P}}_{2} \tilde{\mathcal{Y}}_{2, \eta}^{2}(t, \eta)\left(\int_{0}^{\eta}\left(\tilde{\mathcal{Y}}_{1}-\tilde{\mathcal{Y}}_{2}\right)^{2}(t, \theta) e^{-\frac{1}{a}\left(\tilde{\mathcal{Y}}_{2}(t, \eta)-\tilde{\mathcal{Y}}_{2}(t, \theta)\right)} d \theta\right) d \eta \\
& \leqslant\left\|\tilde{\mathcal{Y}}_{1}-\tilde{\mathcal{Y}}_{2}\right\|^{2} \\
& +12 A \int_{0}^{1} \tilde{\mathcal{Y}}_{2, \eta}(t, \eta) e^{-\frac{1}{a} \tilde{\mathcal{Y}}_{2}(t, \eta)}\left(\int_{0}^{\eta}\left(\tilde{\mathcal{Y}}_{1}-\tilde{\mathcal{Y}}_{2}\right)^{2}(t, \theta) e^{\frac{1}{a} \tilde{\mathcal{Y}}_{2}(t, \theta)} d \theta\right) d \eta \\
& =\left\|\tilde{\mathcal{Y}}_{1}-\tilde{\mathcal{Y}}_{2}\right\|^{2}+12 a A\left(-\left.\int_{0}^{\eta} e^{-\frac{1}{a}\left(\tilde{\mathcal{Y}}_{2}(t, \eta)-\tilde{\mathcal{Y}}_{2}(t, \theta)\right)}\left(\tilde{\mathcal{Y}}_{1}-\tilde{\mathcal{Y}}_{2}\right)^{2}(t, \theta) d \theta\right|_{\eta=0} ^{1}\right. \\
& \left.+\int_{0}^{1}\left(\tilde{\mathcal{Y}}_{1}-\tilde{\mathcal{Y}}_{2}\right)^{2}(t, \eta) d \eta\right) \\
& \leqslant \mathcal{O}(1)\left\|\tilde{\mathcal{Y}}_{1}-\tilde{\mathcal{Y}}_{2}\right\|^{2} \text {. }
\end{aligned}
$$

We conclude that

$$
\left|L_{32}\right| \leqslant \mathcal{O}(1)\left\|\tilde{\mathcal{Y}}_{1}-\tilde{\mathcal{Y}}_{2}\right\|^{2}
$$

Finally, we have a look at $J_{9}$ (the argument for $J_{10}$ follows the same lines). We have

$$
\begin{aligned}
& \left|\int_{0}^{1} J_{9}\left(\tilde{\mathcal{Y}}_{1}-\tilde{\mathcal{Y}}_{2}\right)(t, \eta) d \eta\right| \\
& =\mid \frac{A_{1}^{6}-A_{2}^{6}}{A_{1}^{6} A_{2}^{6}} \mathbb{1}_{A_{1} \leqslant A_{2}} \int_{0}^{1}\left(\tilde{\mathcal{Y}}_{1}-\tilde{\mathcal{Y}}_{2}\right) \tilde{\mathcal{Y}}_{1, \eta}(t, \eta) \\
& \times\left(\int_{0}^{\eta} e^{-\frac{1}{A_{1}}\left(\tilde{\mathcal{Y}}_{1}(t, \eta)-\tilde{\mathcal{Y}}_{1}(t, \theta)\right)}\left(\tilde{\mathcal{U}}_{1}^{+}\right)^{3} \tilde{\mathcal{Y}}_{1, \eta}(t, \theta) d \theta\right) d \eta \\
& \leqslant \frac{A_{2}^{6}-A_{1}^{6}}{\sqrt{2} A_{1}^{6} A_{2}^{6}} A_{1}^{2} \mathbb{1}_{A_{1} \leqslant A_{2}} \\
& \times \int_{0}^{1}\left|\tilde{\mathcal{Y}}_{1}-\tilde{\mathcal{Y}}_{2}\right| \tilde{\mathcal{Y}}_{1, \eta}(t, \eta)\left(\int_{0}^{\eta} e^{-\frac{1}{A_{1}}\left(\tilde{\mathcal{Y}}_{1}(t, \eta)-\tilde{\mathcal{Y}}_{1}(t, \theta)\right)} \tilde{\mathcal{U}}_{1}^{2} \tilde{\mathcal{Y}}_{1, \eta}(t, \theta) d \theta\right) d \eta \\
& \leqslant 4 \frac{A_{2}^{6}-A_{1}^{6}}{\sqrt{2} A_{1}^{6} A_{2}^{6}} A_{1}^{3} \mathbb{1}_{A_{1} \leqslant A_{2}} \int_{0}^{1}\left|\tilde{\mathcal{Y}}_{1}-\tilde{\mathcal{Y}}_{2}\right| \tilde{\mathcal{P}}_{1} \tilde{\mathcal{Y}}_{1, \eta}(t, \eta) d \eta \\
& \leqslant 2 \frac{A_{2}^{6}-A_{1}^{6}}{\sqrt{2} A_{1}^{6} A_{2}^{6}} A_{1}^{8} \mathbb{1}_{A_{1} \leqslant A_{2}} \int_{0}^{1}\left|\tilde{\mathcal{Y}}_{1}-\tilde{\mathcal{Y}}_{2}\right|(t, \eta) d \eta \\
& \leqslant 6 \sqrt{2} A\left(A_{2}-A_{1}\right)\left\|\tilde{\mathcal{Y}}_{1}-\tilde{\mathcal{Y}}_{2}\right\| \\
& \leqslant 6 A\left(\left\|\tilde{\mathcal{Y}}_{1}-\tilde{\mathcal{Y}}_{2}\right\|^{2}+\left|A_{1}-A_{2}\right|^{2}\right) \text {. }
\end{aligned}
$$


We now turn our attention to $I_{32}$, that is,

$$
\begin{aligned}
I_{32}= & \int_{0}^{1}\left(\tilde{\mathcal{Y}}_{1}-\tilde{\mathcal{Y}}_{2}\right)(t, \eta)\left(\frac{1}{A_{2}^{6}} \tilde{\mathcal{Y}}_{2, \eta}(t, \eta) \int_{0}^{1} e^{-\frac{1}{A_{2}}\left|\tilde{\mathcal{Y}}_{2}(t, \eta)-\tilde{\mathcal{Y}}_{2}(t, \theta)\right|} \tilde{\mathcal{P}}_{2} \tilde{\mathcal{U}}_{2} \tilde{\mathcal{Y}}_{2, \eta}(t, \theta) d \theta\right. \\
& \left.-\frac{1}{A_{1}^{6}} \tilde{\mathcal{Y}}_{1, \eta}(t, \eta) \int_{0}^{1} e^{-\frac{1}{A_{1}}\left|\tilde{\mathcal{Y}}_{1}(t, \eta)-\tilde{\mathcal{Y}}_{1}(t, \theta)\right|} \tilde{\mathcal{P}}_{1} \tilde{\mathcal{U}}_{1} \tilde{\mathcal{Y}}_{1, \eta}(t, \theta) d \theta\right) d \eta
\end{aligned}
$$

As before, we are only going to establish the estimates for one part of it, since the other parts can be treated similarly. Let

$$
\begin{aligned}
& \tilde{I}_{32}=\int_{0}^{1}\left(\tilde{\mathcal{Y}}_{1}-\tilde{\mathcal{Y}}_{2}\right)(t, \eta)\left(\frac{1}{A_{2}^{6}} \tilde{\mathcal{Y}}_{2, \eta}(t, \eta) \int_{0}^{\eta} e^{-\frac{1}{A_{2}}\left(\tilde{\mathcal{Y}}_{2}(t, \eta)-\tilde{\mathcal{Y}}_{2}(t, \theta)\right)} \tilde{\mathcal{P}}_{2} \tilde{\mathcal{U}}_{2}^{+} \tilde{\mathcal{Y}}_{2, \eta}(t, \theta) d \theta\right. \\
& \left.-\frac{1}{A_{1}^{6}} \tilde{\mathcal{Y}}_{1, \eta}(t, \eta) \int_{0}^{\eta} e^{-\frac{1}{A_{1}}\left(\tilde{\mathcal{Y}}_{1}(t, \eta)-\tilde{\mathcal{Y}}_{1}(t, \theta)\right)} \tilde{\mathcal{P}}_{1} \tilde{\mathcal{U}}_{1}^{+} \tilde{\mathcal{Y}}_{1, \eta}(t, \theta) d \theta\right) d \eta \\
& =\frac{1}{A^{6}} \int_{0}^{1}\left(\tilde{\mathcal{Y}}_{1}-\tilde{\mathcal{Y}}_{2}\right)(t, \eta)\left(\tilde{\mathcal{Y}}_{2, \eta}(t, \eta) \int_{0}^{\eta} e^{-\frac{1}{A_{2}}\left(\tilde{\mathcal{Y}}_{2}(t, \eta)-\tilde{\mathcal{Y}}_{2}(t, \theta)\right)} \tilde{\mathcal{P}}_{2} \tilde{\mathcal{U}}_{2}^{+} \tilde{\mathcal{Y}}_{2, \eta}(t, \theta) d \theta\right. \\
& \left.-\tilde{\mathcal{Y}}_{1, \eta}(t, \eta) \int_{0}^{\eta} e^{-\frac{1}{A_{1}}\left(\tilde{\mathcal{Y}}_{1}(t, \eta)-\tilde{\mathcal{Y}}_{1}(t, \theta)\right)} \tilde{\mathcal{P}}_{1} \tilde{\mathcal{U}}_{1}^{+} \tilde{\mathcal{Y}}_{1, \eta}(t, \theta) d \theta\right) d \eta \\
& +\left(\frac{1}{A_{2}^{6}}-\frac{1}{A_{1}^{6}}\right) \mathbb{1}_{A_{1} \leqslant A_{2}} \int_{0}^{1}\left(\tilde{\mathcal{Y}}_{1}-\tilde{\mathcal{Y}}_{2}\right) \tilde{\mathcal{Y}}_{1, \eta}(t, \eta) \\
& \times\left(\int_{0}^{\eta} e^{-\frac{1}{A_{1}}\left(\tilde{\mathcal{Y}}_{1}(t, \eta)-\tilde{\mathcal{Y}}_{1}(t, \theta)\right)} \tilde{\mathcal{P}}_{1} \tilde{\mathcal{U}}_{1}^{+} \tilde{\mathcal{Y}}_{1, \eta}(t, \theta) d \theta\right) d \eta \\
& +\left(\frac{1}{A_{2}^{6}}-\frac{1}{A_{1}^{6}}\right) \mathbb{1}_{A_{2}<A_{1}} \int_{0}^{1}\left(\tilde{\mathcal{Y}}_{1}-\tilde{\mathcal{Y}}_{2}\right) \tilde{\mathcal{Y}}_{2, \eta}(t, \eta) \\
& \times\left(\int_{0}^{\eta} e^{-\frac{1}{A_{2}}\left(\tilde{\mathcal{Y}}_{2}(t, \eta)-\tilde{\mathcal{Y}}_{2}(t, \theta)\right)} \tilde{\mathcal{P}}_{2} \tilde{\mathcal{U}}_{2}^{+} \tilde{\mathcal{Y}}_{2, \eta}(t, \theta) d \theta\right) d \eta \\
& =\tilde{K}_{1}+\tilde{K}_{2}+\tilde{K}_{3} \text {. }
\end{aligned}
$$

Direct calculations yield for $\tilde{K}_{2}$ (and similarly for $\tilde{K}_{3}$ ) that

$$
\begin{aligned}
\left|\tilde{K}_{2}\right| \leqslant & \frac{A_{2}^{6}-A_{1}^{6}}{A_{1}^{6} A_{2}^{6}} \mathbb{1}_{A_{1} \leqslant A_{2}} \\
& \times \int_{0}^{1}\left|\tilde{\mathcal{Y}}_{1}-\tilde{\mathcal{Y}}_{2}\right| \tilde{\mathcal{Y}}_{1, \eta}(t, \eta)\left(\int_{0}^{\eta} e^{-\frac{1}{A_{1}}\left(\tilde{\mathcal{Y}}_{1}(t, \eta)-\tilde{\mathcal{Y}}_{1}(t, \theta)\right)} \tilde{\mathcal{P}}_{1} \tilde{\mathcal{U}}_{1}^{+} \tilde{\mathcal{Y}}_{1, \eta}(t, \theta) d \theta\right) d \eta \\
\leqslant & \frac{A_{2}^{6}-A_{1}^{6}\left\|\tilde{\mathcal{Y}}_{1}-\tilde{\mathcal{Y}}_{2}\right\|}{A_{1}^{6} A_{2}^{6}} \\
& \times\left(\int_{0}^{1} \tilde{\mathcal{Y}}_{1, \eta}^{2}(t, \eta)\left(\int_{0}^{\eta} e^{-\frac{1}{A_{1}}\left(\tilde{\mathcal{Y}}_{1}(t, \eta)-\tilde{\mathcal{Y}}_{1}(t, \theta)\right)} \tilde{\mathcal{P}}_{1} \tilde{\mathcal{U}}_{1}^{+} \tilde{\mathcal{Y}}_{1, \eta}(t, \theta) d \theta\right)^{2} d \eta\right)^{1 / 2}
\end{aligned}
$$




$$
\begin{aligned}
& \leqslant \frac{A_{2}^{6}-A_{1}^{6}}{A_{1}^{6} A_{2}^{6}}\left\|\tilde{\mathcal{Y}}_{1}-\tilde{\mathcal{Y}}_{2}\right\|\left(\int_{0}^{1} \tilde{\mathcal{Y}}_{1, \eta}^{2}(t, \eta) \int_{0}^{\eta} e^{-\frac{1}{A_{1}}\left(\tilde{\mathcal{Y}}_{1}(t, \eta)-\tilde{\mathcal{Y}}_{1}(t, \theta)\right)} \tilde{\mathcal{P}}_{1}^{2} \tilde{\mathcal{Y}}_{1, \eta}(t, \theta) d \theta\right. \\
& \left.\times \int_{0}^{\eta} e^{-\frac{1}{A_{1}}\left(\tilde{\mathcal{Y}}_{1}(t, \eta)-\tilde{\mathcal{Y}}_{1}(t, \theta)\right)} \tilde{\mathcal{U}}_{1}^{2} \tilde{\mathcal{Y}}_{1, \eta}(t, \theta) d \theta d \eta\right)^{1 / 2} \\
& \leqslant \sqrt{6} \frac{A_{2}^{6}-A_{1}^{6}}{A_{1}^{3} A_{2}^{6}}\left\|\tilde{\mathcal{Y}}_{1}-\tilde{\mathcal{Y}}_{2}\right\|\left(\int_{0}^{1} \tilde{\mathcal{P}}_{1}^{2} \tilde{\mathcal{Y}}_{1, \eta}^{2}(t, \eta) d \eta\right)^{1 / 2} \\
& \leqslant \frac{\sqrt{6}}{2} \frac{A_{2}^{6}-A_{1}^{6}}{A^{4}}\left\|\tilde{\mathcal{Y}}_{1}-\tilde{\mathcal{Y}}_{2}\right\| \\
& \leqslant \mathcal{O}(1)\left(\left\|\tilde{\mathcal{Y}}_{1}-\tilde{\mathcal{Y}}_{2}\right\|^{2}+\left|A_{2}-A_{1}\right|^{2}\right) \text {, }
\end{aligned}
$$

where we used (4.16b), (4.16c), and $A_{1} \leqslant A_{2}$.

On the other hand, the term $\tilde{K}_{1}$ needs to be rewritten a bit more. Namely,

$$
\begin{aligned}
& \tilde{K}_{1}=\frac{1}{A^{6}} \int_{0}^{1}\left(\tilde{\mathcal{Y}}_{1}-\tilde{\mathcal{Y}}_{2}\right)(t, \eta)\left(\tilde{\mathcal{Y}}_{2, \eta}(t, \eta) \int_{0}^{\eta} e^{-\frac{1}{A_{2}}\left(\tilde{\mathcal{Y}}_{2}(t, \eta)-\tilde{\mathcal{Y}}_{2}(t, \theta)\right)} \tilde{\mathcal{P}}_{2} \tilde{\mathcal{U}}_{2}^{+} \tilde{\mathcal{Y}}_{2, \eta}(t, \theta) d \theta\right. \\
& \left.-\tilde{\mathcal{Y}}_{1, \eta}(t, \eta) \int_{0}^{\eta} e^{-\frac{1}{A_{1}}\left(\tilde{\mathcal{Y}}_{1}(t, \eta)-\tilde{\mathcal{Y}}_{1}(t, \theta)\right)} \tilde{\mathcal{P}}_{1} \tilde{\mathcal{U}}_{1}^{+} \tilde{\mathcal{Y}}_{1, \eta}(t, \theta) d \theta\right) d \eta \\
& =\frac{1}{A^{6}} \int_{0}^{1}\left(\tilde{\mathcal{Y}}_{1}-\tilde{\mathcal{Y}}_{2}\right) \tilde{\mathcal{Y}}_{2, \eta}(t, \eta) \\
& \times\left(\int_{0}^{\eta} e^{-\frac{1}{A_{2}}\left(\tilde{\mathcal{Y}}_{2}(t, \eta)-\tilde{\mathcal{Y}}_{2}(t, \theta)\right)}\left(\tilde{\mathcal{P}}_{2}-\tilde{\mathcal{P}}_{1}\right) \tilde{\mathcal{U}}_{2}^{+} \tilde{\mathcal{Y}}_{2, \eta}(t, \theta) \mathbb{1}_{\tilde{\mathcal{P}}_{1} \leqslant \tilde{\mathcal{P}}_{2}}(t, \theta) d \theta\right) d \eta \\
& +\frac{1}{A^{6}} \int_{0}^{1}\left(\tilde{\mathcal{Y}}_{1}-\tilde{\mathcal{Y}}_{2}\right) \tilde{\mathcal{Y}}_{1, \eta}(t, \eta) \\
& \times\left(\int_{0}^{\eta} e^{-\frac{1}{A_{1}}\left(\tilde{\mathcal{Y}}_{1}(t, \eta)-\tilde{\mathcal{Y}}_{1}(t, \theta)\right)}\left(\tilde{\mathcal{P}}_{2}-\tilde{\mathcal{P}}_{1}\right) \tilde{\mathcal{U}}_{1}^{+} \tilde{\mathcal{Y}}_{1, \eta}(t, \theta) \mathbb{1}_{\tilde{\mathcal{P}}_{2}<\tilde{\mathcal{P}}_{1}}(t, \theta) d \theta\right) d \eta \\
& +\frac{1}{A^{6}} \int_{0}^{1}\left(\tilde{\mathcal{Y}}_{1}-\tilde{\mathcal{Y}}_{2}\right) \tilde{\mathcal{Y}}_{2, \eta}(t, \eta) \\
& \times\left(\int_{0}^{\eta} e^{-\frac{1}{A_{2}}\left(\tilde{\mathcal{Y}}_{2}(t, \eta)-\tilde{\mathcal{Y}}_{2}(t, \theta)\right)} \min _{j}\left(\tilde{\mathcal{P}}_{j}\right)\left(\tilde{\mathcal{U}}_{2}^{+}-\tilde{\mathcal{U}}_{1}^{+}\right) \tilde{\mathcal{Y}}_{2, \eta}(t, \theta) \mathbb{1}_{\tilde{\mathcal{U}}_{1}^{+} \leqslant \tilde{\mathcal{U}}_{2}^{+}}(t, \theta) d \theta\right) d \eta \\
& +\frac{1}{A^{6}} \int_{0}^{1}\left(\tilde{\mathcal{Y}}_{1}-\tilde{\mathcal{Y}}_{2}\right) \tilde{\mathcal{Y}}_{1, \eta}(t, \eta) \\
& \times\left(\int_{0}^{\eta} e^{-\frac{1}{A_{1}}\left(\tilde{\mathcal{Y}}_{1}(t, \eta)-\tilde{\mathcal{Y}}_{1}(t, \theta)\right)} \min _{j}\left(\tilde{\mathcal{P}}_{j}\right)\left(\tilde{\mathcal{U}}_{2}^{+}-\tilde{\mathcal{U}}_{1}^{+}\right) \tilde{\mathcal{Y}}_{1, \eta}(t, \theta) \mathbb{1}_{\tilde{\mathcal{U}}_{2}^{+}<\tilde{\mathcal{U}}_{1}^{+}}(t, \theta) d \theta\right) d \eta \\
& +\frac{1}{A^{6}} \int_{0}^{1}\left(\tilde{\mathcal{Y}}_{1}-\tilde{\mathcal{Y}}_{2}\right)(t, \eta) \\
& \times\left(\tilde{\mathcal{Y}}_{2, \eta}(t, \eta) \int_{0}^{\eta} e^{-\frac{1}{A_{2}}\left(\tilde{\mathcal{Y}}_{2}(t, \eta)-\tilde{\mathcal{Y}}_{2}(t, \theta)\right)} \min _{j}\left(\tilde{\mathcal{P}}_{j}\right) \min _{j}\left(\tilde{\mathcal{U}}_{j}^{+}\right) \tilde{\mathcal{Y}}_{2, \eta}(t, \theta) d \theta\right.
\end{aligned}
$$




$$
\begin{aligned}
& \left.-\tilde{\mathcal{Y}}_{1, \eta}(t, \eta) \int_{0}^{\eta} e^{-\frac{1}{A_{1}}\left(\tilde{\mathcal{Y}}_{1}(t, \eta)-\tilde{\mathcal{Y}}_{1}(t, \theta)\right)} \min _{j}\left(\tilde{\mathcal{P}}_{j}\right) \min _{j}\left(\tilde{\mathcal{U}}_{j}^{+}\right) \tilde{\mathcal{Y}}_{1, \eta}(t, \theta) d \theta\right) d \eta \\
= & \tilde{J}_{1}+\tilde{J}_{2}+\tilde{J}_{3}+\tilde{J}_{4}+\tilde{J}_{5} .
\end{aligned}
$$

We start by having a close look at $\tilde{J}_{1}$ ( $\tilde{J}_{2}$ can be handled similarly). One has

$$
\begin{aligned}
& \left|\tilde{J}_{1}\right| \leqslant \frac{1}{A^{6}} \mid \int_{0}^{1}\left(\tilde{\mathcal{Y}}_{1}-\tilde{\mathcal{Y}}_{2}\right) \tilde{\mathcal{Y}}_{2, \eta}(t, \eta) \\
& \times\left(\int_{0}^{\eta} e^{-\frac{1}{A_{2}}\left(\tilde{\mathcal{Y}}_{2}(t, \eta)-\tilde{\mathcal{Y}}_{2}(t, \theta)\right)}\left(\tilde{\mathcal{P}}_{2}-\tilde{\mathcal{P}}_{1}\right) \tilde{\mathcal{U}}_{2}^{+} \tilde{\mathcal{Y}}_{2, \eta}(t, \theta) \mathbb{1}_{\tilde{\mathcal{P}}_{1} \leqslant \tilde{\mathcal{P}}_{2}}(t, \theta) d \theta\right) d \eta \mid \\
& \leqslant\left\|\tilde{\mathcal{Y}}_{1}-\tilde{\mathcal{Y}}_{2}\right\|^{2}+\frac{1}{A^{12}} \int_{0}^{1} \tilde{\mathcal{Y}}_{2, \eta}^{2}(t, \eta)\left(\int_{0}^{\eta} e^{-\frac{1}{A_{2}}\left(\tilde{\mathcal{Y}}_{2}(t, \eta)-\tilde{\mathcal{Y}}_{2}(t, \theta)\right)}\right. \\
& \left.\times\left(\tilde{\mathcal{P}}_{2}^{1 / 2}-\tilde{\mathcal{P}}_{1}^{1 / 2}\right)\left(\tilde{\mathcal{P}}_{2}^{1 / 2}+\tilde{\mathcal{P}}_{1}^{1 / 2}\right) \tilde{\mathcal{U}}_{2}^{+} \tilde{\mathcal{Y}}_{2, \eta}(t, \theta) \mathbb{1}_{\tilde{\mathcal{P}}_{1} \leqslant \tilde{\mathcal{P}}_{2}}(t, \theta) d \theta\right)^{2} d \eta \\
& \leqslant\left\|\tilde{\mathcal{Y}}_{1}-\tilde{\mathcal{Y}}_{2}\right\|^{2}+\frac{1}{A^{12}} \int_{0}^{1} \tilde{\mathcal{Y}}_{2, \eta}^{2}(t, \eta) \\
& \times\left(2 \int_{0}^{\eta} e^{-\frac{1}{A_{2}}\left(\tilde{\mathcal{Y}}_{2}(t, \eta)-\tilde{\mathcal{Y}}_{2}(t, \theta)\right)}\left(\tilde{\mathcal{P}}_{2}^{1 / 2}-\tilde{\mathcal{P}}_{1}^{1 / 2}\right) \tilde{\mathcal{P}}_{2}^{1 / 2} \tilde{\mathcal{U}}_{2}^{+} \tilde{\mathcal{Y}}_{2, \eta}(t, \theta) d \theta\right)^{2} d \eta \\
& \leqslant\left\|\tilde{\mathcal{Y}}_{1}-\tilde{\mathcal{Y}}_{2}\right\|^{2}+\frac{4}{A^{12}} \int_{0}^{1} \tilde{\mathcal{Y}}_{2, \eta}^{2}(t, \eta)\left(\int_{0}^{\eta} e^{-\frac{1}{A_{2}}\left(\tilde{\mathcal{Y}}_{2}(t, \eta)-\tilde{\mathcal{Y}}_{2}(t, \theta)\right)} \tilde{\mathcal{U}}_{2}^{2} \tilde{\mathcal{Y}}_{2, \eta}(t, \theta) d \theta\right) \\
& \times\left(\int_{0}^{\eta} e^{-\frac{1}{A_{2}}\left(\tilde{\mathcal{Y}}_{2}(t, \eta)-\tilde{\mathcal{Y}}_{2}(t, \theta)\right)}\left(\tilde{\mathcal{P}}_{2}^{1 / 2}-\tilde{\mathcal{P}}_{1}^{1 / 2}\right)^{2} \tilde{\mathcal{P}}_{2} \tilde{\mathcal{Y}}_{2, \eta}(t, \theta) d \theta\right) d \eta \\
& \leqslant\left\|\tilde{\mathcal{Y}}_{1}-\tilde{\mathcal{Y}}_{2}\right\|^{2}+\frac{16}{A^{11}} \int_{0}^{1} \tilde{\mathcal{P}}_{2} \tilde{\mathcal{Y}}_{2, \eta}^{2}(t, \eta) \\
& \times\left(\int_{0}^{\eta} e^{-\frac{1}{A_{2}}\left(\tilde{\mathcal{Y}}_{2}(t, \eta)-\tilde{\mathcal{Y}}_{2}(t, \theta)\right)}\left(\tilde{\mathcal{P}}_{2}^{1 / 2}-\tilde{\mathcal{P}}_{1}^{1 / 2}\right)^{2} \tilde{\mathcal{P}}_{2} \tilde{\mathcal{Y}}_{2, \eta}(t, \theta) d \theta\right) d \eta \\
& \leqslant\left\|\tilde{\mathcal{Y}}_{1}-\tilde{\mathcal{Y}}_{2}\right\|^{2}+\frac{8}{A^{6}} \int_{0}^{1} \tilde{\mathcal{Y}}_{2, \eta}(t, \eta) \\
& \times\left(\int_{0}^{\eta} e^{-\frac{1}{A_{2}}\left(\tilde{\mathcal{Y}}_{2}(t, \eta)-\tilde{\mathcal{Y}}_{2}(t, \theta)\right)}\left(\tilde{\mathcal{P}}_{2}^{1 / 2}-\tilde{\mathcal{P}}_{1}^{1 / 2}\right)^{2} \tilde{\mathcal{P}}_{2} \tilde{\mathcal{Y}}_{2, \eta}(t, \theta) d \theta\right) d \eta \\
& \leqslant\left\|\tilde{\mathcal{Y}}_{1}-\tilde{\mathcal{Y}}_{2}\right\|^{2}+\frac{8}{A^{6}} \int_{0}^{1} \tilde{\mathcal{Y}}_{2, \eta}(t, \eta) e^{-\frac{1}{A_{2}} \tilde{\mathcal{Y}}_{2}(t, \eta)} \\
& \times\left(\int_{0}^{\eta} e^{\frac{1}{A_{2}} \tilde{\mathcal{Y}}_{2}(t, \theta)}\left(\tilde{\mathcal{P}}_{2}^{1 / 2}-\tilde{\mathcal{P}}_{1}^{1 / 2}\right)^{2} \tilde{\mathcal{P}}_{2} \tilde{\mathcal{Y}}_{2, \eta}(t, \theta) d \theta\right) d \eta \\
& \leqslant\left\|\tilde{\mathcal{Y}}_{1}-\tilde{\mathcal{Y}}_{2}\right\|^{2}
\end{aligned}
$$




$$
\begin{aligned}
& +\frac{8 A_{2}}{A^{6}}\left(-\left.\int_{0}^{\eta} e^{-\frac{1}{A_{2}}\left(\tilde{\mathcal{Y}}_{2}(t, \eta)-\tilde{\mathcal{Y}}_{2}(t, \theta)\right)}\left(\tilde{\mathcal{P}}_{2}^{1 / 2}-\tilde{\mathcal{P}}_{1}^{1 / 2}\right)^{2} \tilde{\mathcal{P}}_{2} \tilde{\mathcal{Y}}_{2, \eta}(t, \theta) d \theta\right|_{\eta=0} ^{1}\right. \\
& \left.+\int_{0}^{1}\left(\tilde{\mathcal{P}}_{2}^{1 / 2}-\tilde{\mathcal{P}}_{1}^{1 / 2}\right)^{2} \tilde{\mathcal{P}}_{2} \tilde{\mathcal{Y}}_{2, \eta}(t, \eta) d \eta\right) \\
& \leqslant \mathcal{O}(1)\left(\left\|\tilde{\mathcal{Y}}_{1}-\tilde{\mathcal{Y}}_{2}\right\|^{2}+\left\|\tilde{\mathcal{P}}_{1}^{1 / 2}-\tilde{\mathcal{P}}_{2}^{1 / 2}\right\|^{2}\right),
\end{aligned}
$$

where we used that

$$
\begin{aligned}
0 \leqslant & \int_{0}^{\eta} e^{-\frac{1}{A_{2}}\left(\tilde{\mathcal{Y}}_{2}(t, \eta)-\tilde{\mathcal{Y}}_{2}(t, \theta)\right)}\left(\tilde{\mathcal{P}}_{2}^{1 / 2}-\tilde{\mathcal{P}}_{1}^{1 / 2}\right)^{2} \tilde{\mathcal{P}}_{2} \tilde{\mathcal{Y}}_{2, \eta}(t, \eta) d \eta \\
\leqslant & A^{4} \int_{0}^{\eta} e^{-\frac{1}{A_{2}}\left(\tilde{\mathcal{Y}}_{2}(t, \eta)-\tilde{\mathcal{Y}}_{2}(t, \theta)\right)} \tilde{\mathcal{P}}_{2} \tilde{\mathcal{Y}}_{2, \eta}(t, \theta) d \theta \\
\leqslant & A^{4}\left(\int_{0}^{\eta} e^{-\frac{1}{A_{2}}\left(\tilde{\mathcal{Y}}_{2}(t, \eta)-\tilde{\mathcal{Y}}_{2}(t, \theta)\right)} \tilde{\mathcal{Y}}_{2, \eta}(t, \theta) d \theta\right)^{1 / 2} \\
& \times\left(\int_{0}^{\eta} e^{-\frac{1}{A_{2}}\left(\tilde{\mathcal{Y}}_{2}(t, \eta)-\tilde{\mathcal{Y}}_{2}(t, \theta)\right)} \tilde{\mathcal{P}}_{2}^{2} \tilde{\mathcal{Y}}_{2, \eta}(t, \theta) d \theta\right)^{1 / 2} \\
\leqslant & A^{5} \mathcal{O}(1) \tilde{\mathcal{P}}_{2}^{1 / 2}(t, \eta),
\end{aligned}
$$

where the very last term tends to 0 as $\eta \rightarrow 0,1$. In the last step we used (4.16b).

Next, we investigate $\tilde{J}_{3}\left(\tilde{J}_{4}\right.$ can be handled in a similar way). The argument is a bit more involved and hence we start with some preliminary estimates. One has

$$
\begin{aligned}
\int_{0}^{\eta} & e^{-\frac{1}{2 A_{2}}\left(\tilde{\mathcal{Y}}_{2}(t, \eta)-\tilde{\mathcal{Y}}_{2}(t, \theta)\right)}\left(\tilde{\mathcal{U}}_{2}^{+}-\tilde{\mathcal{U}}_{1}^{+}\right)^{2} \tilde{\mathcal{P}}_{2} \tilde{\mathcal{Y}}_{2, \eta}(t, \theta) d \theta \\
\leqslant & 2 A^{4} \int_{0}^{\eta} e^{-\frac{1}{2 A_{2}}\left(\tilde{\mathcal{Y}}_{2}(t, \eta)-\tilde{\mathcal{Y}}_{2}(t, \theta)\right)} \tilde{\mathcal{P}}_{2} \tilde{\mathcal{Y}}_{2, \eta}(t, \theta) d \theta \\
\leqslant & 2 A^{4}\left(\int_{0}^{\eta} e^{-\frac{1}{2 A_{2}}\left(\tilde{\mathcal{Y}}_{2}(t, \eta)-\tilde{\mathcal{Y}}_{2}(t, \theta)\right)} \tilde{\mathcal{Y}}_{2, \eta}(t, \theta) d \theta\right)^{1 / 2} \\
& \times\left(\int_{0}^{\eta} e^{-\frac{1}{2 A_{2}}\left(\tilde{\mathcal{Y}}_{2}(t, \eta)-\tilde{\mathcal{Y}}_{2}(t, \theta)\right)} \tilde{\mathcal{P}}_{2}^{2} \tilde{\mathcal{Y}}_{2, \eta}(t, \theta) d \theta\right)^{1 / 2} \\
\leqslant & A^{5} \mathcal{O}(1) \tilde{\mathcal{P}}_{2}^{1 / 4}(t, \eta),
\end{aligned}
$$

where the term on the right-hand side tends to 0 for $\eta \rightarrow 0,1$. In the last step we used (4.16j); cf. (3.35) (and the derivative $\tilde{\mathcal{Q}}_{2, \eta}$ can be found from (4.7) and (4.9)). 
With these estimates in mind, we end up with, recalling (4.16e),

$$
\begin{aligned}
& \left|\tilde{J}_{3}\right|=\frac{1}{A^{6}} \mid \int_{0}^{1}\left(\tilde{\mathcal{Y}}_{1}-\tilde{\mathcal{Y}}_{2}\right) \tilde{\mathcal{Y}}_{2, \eta}(t, \eta) \\
& \times\left(\int_{0}^{\eta} e^{-\frac{1}{A_{2}}\left(\tilde{\mathcal{Y}}_{2}(t, \eta)-\tilde{\mathcal{Y}}_{2}(t, \theta)\right)} \min _{j}\left(\tilde{\mathcal{P}}_{j}\right)\left(\tilde{\mathcal{U}}_{2}^{+}-\tilde{\mathcal{U}}_{1}^{+}\right) \tilde{\mathcal{Y}}_{2, \eta}(t, \theta) \mathbb{1}_{\tilde{\mathcal{U}}_{1}^{+} \leqslant \tilde{\mathcal{U}}_{2}^{+}}(t, \theta) d \theta\right) d \eta \mid \\
& \leqslant\left\|\tilde{\mathcal{Y}}_{1}-\tilde{\mathcal{Y}}_{2}\right\|^{2} \\
& +\frac{1}{A^{12}} \int_{0}^{1} \tilde{\mathcal{Y}}_{2, \eta}^{2}(t, \eta) \\
& \times\left(\int_{0}^{\eta} e^{-\frac{1}{A_{2}}\left(\tilde{\mathcal{Y}}_{2}(t, \eta)-\tilde{\mathcal{Y}}_{2}(t, \theta)\right)} \min _{j}\left(\tilde{\mathcal{P}}_{j}\right)\left(\tilde{\mathcal{U}}_{2}^{+}-\tilde{\mathcal{U}}_{1}^{+}\right) \tilde{\mathcal{Y}}_{2, \eta}(t, \theta) \mathbb{1}_{\tilde{\mathcal{U}}_{1}^{+} \leqslant \tilde{\mathcal{U}}_{2}^{+}}(t, \theta) d \theta\right)^{2} d \eta \\
& \leqslant\left\|\tilde{\mathcal{Y}}_{1}-\tilde{\mathcal{Y}}_{2}\right\|^{2} \\
& +\frac{1}{A^{12}} \int_{0}^{1} \tilde{\mathcal{Y}}_{2, \eta}^{2}(t, \eta)\left(\int_{0}^{\eta} e^{-\frac{3}{2 A_{2}}\left(\tilde{\mathcal{Y}}_{2}(t, \eta)-\tilde{\mathcal{Y}}_{2}(t, \theta)\right)} \tilde{\mathcal{P}}_{2} \tilde{\mathcal{Y}}_{2, \eta}(t, \theta) d \theta\right) \\
& \times\left(\int_{0}^{\eta} e^{-\frac{1}{2 A_{2}}\left(\tilde{\mathcal{Y}}_{2}(t, \eta)-\tilde{\mathcal{Y}}_{2}(t, \theta)\right)}\left(\tilde{\mathcal{U}}_{2}^{+}-\tilde{\mathcal{U}}_{1}^{+}\right)^{2} \tilde{\mathcal{P}}_{2} \tilde{\mathcal{Y}}_{2, \eta}(t, \theta) d \theta\right) d \eta \\
& \leqslant\left\|\tilde{\mathcal{Y}}_{1}-\tilde{\mathcal{Y}}_{2}\right\|^{2}+\frac{2}{A^{11}} \int_{0}^{1} \tilde{\mathcal{P}}_{2} \tilde{\mathcal{Y}}_{2, \eta}^{2}(t, \eta) \\
& \times\left(\int_{0}^{\eta} e^{-\frac{1}{2 A_{2}}\left(\tilde{\mathcal{Y}}_{2}(t, \eta)-\tilde{\mathcal{Y}}_{2}(t, \theta)\right)}\left(\tilde{\mathcal{U}}_{2}^{+}-\tilde{\mathcal{U}}_{1}^{+}\right)^{2} \tilde{\mathcal{P}}_{2} \tilde{\mathcal{Y}}_{2, \eta}(t, \theta) d \theta\right) d \eta \\
& \leqslant\left\|\tilde{\mathcal{Y}}_{1}-\tilde{\mathcal{Y}}_{2}\right\|^{2} \\
& +\frac{1}{A^{6}} \int_{0}^{1} \tilde{\mathcal{Y}}_{2, \eta}(t, \eta) e^{-\frac{1}{2 A_{2}} \tilde{\mathcal{Y}}_{2}(t, \eta)}\left(\int_{0}^{\eta} e^{\frac{1}{2 A_{2}} \tilde{\mathcal{Y}}_{2}(t, \theta)}\left(\tilde{\mathcal{U}}_{2}^{+}-\tilde{\mathcal{U}}_{1}^{+}\right)^{2} \tilde{\mathcal{P}}_{2} \tilde{\mathcal{Y}}_{2, \eta}(t, \theta) d \theta\right) d \eta \\
& =\left\|\tilde{\mathcal{Y}}_{1}-\tilde{\mathcal{Y}}_{2}\right\|^{2} \\
& +\frac{1}{A^{6}}\left(-\left.2 A_{2} \int_{0}^{\eta} e^{-\frac{1}{2 A_{2}}\left(\tilde{\mathcal{Y}}_{2}(t, \eta)-\tilde{\mathcal{Y}}_{2}(t, \theta)\right)}\left(\tilde{\mathcal{U}}_{2}^{+}-\tilde{\mathcal{U}}_{1}^{+}\right)^{2} \tilde{\mathcal{P}}_{2} \tilde{\mathcal{Y}}_{2, \eta}(t, \theta) d \theta\right|_{\eta=0} ^{1}\right. \\
& \left.+2 A_{2} \int_{0}^{1}\left(\tilde{\mathcal{U}}_{2}^{+}-\tilde{\mathcal{U}}_{1}^{+}\right)^{2} \tilde{\mathcal{P}}_{2} \tilde{\mathcal{Y}}_{2, \eta}(t, \eta) d \eta\right) \\
& \leqslant \mathcal{O}(1)\left(\left\|\tilde{\mathcal{Y}}_{1}-\tilde{\mathcal{Y}}_{2}\right\|^{2}+\left\|\tilde{\mathcal{U}}_{2}-\tilde{\mathcal{U}}_{1}\right\|^{2}\right) \text {. }
\end{aligned}
$$

As far as $\tilde{J}_{5}$ is concerned, we have to rewrite it a bit more. Namely,

$$
\begin{aligned}
\tilde{J}_{5}= & \frac{1}{A^{6}} \int_{0}^{1}\left(\tilde{\mathcal{Y}}_{1}-\tilde{\mathcal{Y}}_{2}\right)(t, \eta) \\
& \times\left(\tilde{\mathcal{Y}}_{2, \eta}(t, \eta) \int_{0}^{\eta} e^{-\frac{1}{A_{2}}\left(\tilde{\mathcal{Y}}_{2}(t, \eta)-\tilde{\mathcal{Y}}_{2}(t, \theta)\right)} \min _{j}\left(\tilde{\mathcal{P}}_{j}\right) \min _{j}\left(\tilde{\mathcal{U}}_{j}^{+}\right) \tilde{\mathcal{Y}}_{2, \eta}(t, \theta) d \theta\right.
\end{aligned}
$$




$$
\begin{aligned}
& \left.-\tilde{\mathcal{Y}}_{1, \eta}(t, \eta) \int_{0}^{\eta} e^{-\frac{1}{A_{1}}\left(\tilde{\mathcal{Y}}_{1}(t, \eta)-\tilde{\mathcal{Y}}_{1}(t, \theta)\right)} \min _{j}\left(\tilde{\mathcal{P}}_{j}\right) \min _{j}\left(\tilde{\mathcal{U}}_{j}^{+}\right) \tilde{\mathcal{Y}}_{1, \eta}(t, \theta) d \theta\right) d \eta \\
& =\frac{1}{A^{6}} \mathbb{1}_{A_{1} \leqslant A_{2}} \int_{0}^{1}\left(\tilde{\mathcal{Y}}_{1}-\tilde{\mathcal{Y}}_{2}\right) \tilde{\mathcal{Y}}_{2, \eta}(t, \eta) \\
& \times\left(\int_{0}^{\eta}\left(e^{-\frac{1}{A_{2}}\left(\tilde{\mathcal{Y}}_{2}(t, \eta)-\tilde{\mathcal{Y}}_{2}(t, \theta)\right)}-e^{-\frac{1}{A_{1}}\left(\tilde{\mathcal{Y}}_{2}(t, \eta)-\tilde{\mathcal{Y}}_{2}(t, \theta)\right)}\right)\right. \\
& \left.\times \min _{j}\left(\tilde{\mathcal{P}}_{j}\right) \min _{j}\left(\tilde{\mathcal{U}}_{j}^{+}\right) \tilde{\mathcal{Y}}_{2, \eta}(t, \theta) d \theta\right) d \eta \\
& +\frac{1}{A^{6}} \mathbb{1}_{A_{2}<A_{1}} \int_{0}^{1}\left(\tilde{\mathcal{Y}}_{1}-\tilde{\mathcal{Y}}_{2}\right) \tilde{\mathcal{Y}}_{1, \eta}(t, \eta) \\
& \times\left(\int_{0}^{\eta}\left(e^{-\frac{1}{A_{2}}\left(\tilde{\mathcal{Y}}_{1}(t, \eta)-\tilde{\mathcal{Y}}_{1}(t, \theta)\right)}-e^{-\frac{1}{A_{1}}\left(\tilde{\mathcal{Y}}_{1}(t, \eta)-\tilde{\mathcal{Y}}_{1}(t, \theta)\right)}\right)\right. \\
& \left.\times \min _{j}\left(\tilde{\mathcal{P}}_{j}\right) \min _{j}\left(\tilde{\mathcal{U}}_{j}^{+}\right) \tilde{\mathcal{Y}}_{1, \eta}(t, \theta) d \theta\right) d \eta \\
& +\frac{1}{A^{6}} \int_{0}^{1}\left(\tilde{\mathcal{Y}}_{1}-\tilde{\mathcal{Y}}_{2}\right) \tilde{\mathcal{Y}}_{2, \eta}(t, \eta) \\
& \times\left(\int_{0}^{\eta}\left(e^{-\frac{1}{a}\left(\tilde{\mathcal{Y}}_{2}(t, \eta)-\tilde{\mathcal{Y}}_{2}(t, \theta)\right)}-e^{-\frac{1}{a}\left(\tilde{\mathcal{Y}}_{1}(t, \eta)-\tilde{\mathcal{Y}}_{1}(t, \theta)\right)}\right) \min _{j}\left(\tilde{\mathcal{P}}_{j}\right)\right. \\
& \left.\times \min _{j}\left(\tilde{\mathcal{U}}_{j}^{+}\right) \tilde{\mathcal{Y}}_{2, \eta}(t, \theta) \mathbb{1}_{B(\eta)}(t, \theta) d \theta\right) d \eta \\
& +\frac{1}{A^{6}} \int_{0}^{1}\left(\tilde{\mathcal{Y}}_{1}-\tilde{\mathcal{Y}}_{2}\right) \tilde{\mathcal{Y}}_{1, \eta}(t, \eta) \\
& \times\left(\int_{0}^{\eta}\left(e^{-\frac{1}{a}\left(\tilde{\mathcal{Y}}_{2}(t, \eta)-\tilde{\mathcal{Y}}_{2}(t, \theta)\right)}-e^{-\frac{1}{a}\left(\tilde{\mathcal{Y}}_{1}(t, \eta)-\tilde{\mathcal{Y}}_{1}(t, \theta)\right)}\right) \min _{j}\left(\tilde{\mathcal{P}}_{j}\right)\right. \\
& \left.\times \min _{j}\left(\tilde{\mathcal{U}}_{j}^{+}\right) \tilde{\mathcal{Y}}_{1, \eta}(t, \theta) \mathbb{1}_{B^{c}(\eta)}(t, \theta) d \theta\right) d \eta \\
& +\frac{1}{A^{6}} \int_{0}^{1}\left(\tilde{\mathcal{Y}}_{1}-\tilde{\mathcal{Y}}_{2}\right)(t, \eta) \\
& \times\left(\tilde{\mathcal{Y}}_{2, \eta}(t, \eta) \int_{0}^{\eta} \min _{j}\left(e^{-\frac{1}{a}\left(\tilde{\mathcal{Y}}_{j}(t, \eta)-\tilde{\mathcal{Y}}_{j}(t, \theta)\right)}\right) \min _{j}\left(\tilde{\mathcal{P}}_{j}\right)\right. \\
& \times \min _{j}\left(\tilde{\mathcal{U}}_{j}^{+}\right) \tilde{\mathcal{Y}}_{2, \eta}(t, \theta) d \theta \\
& -\tilde{\mathcal{Y}}_{1, \eta}(t, \eta) \int_{0}^{\eta} \min _{j}\left(e^{-\frac{1}{a}\left(\tilde{\mathcal{Y}}_{j}(t, \eta)-\tilde{\mathcal{Y}}_{j}(t, \theta)\right)}\right) \min _{j}\left(\tilde{\mathcal{P}}_{j}\right)
\end{aligned}
$$




$$
\begin{aligned}
& \left.\times \min _{j}\left(\tilde{\mathcal{U}}_{j}^{+}\right) \tilde{\mathcal{Y}}_{1, \eta}(t, \theta) d \theta\right) d \eta \\
= & \tilde{L}_{1}+\tilde{L}_{2}+\tilde{L}_{3}+\tilde{L}_{4}+\tilde{L}_{5},
\end{aligned}
$$

where $B(\eta)$ is given by $(5.11)$.

Both $\tilde{L}_{1}$ and $\tilde{L}_{2}$ can be handled in much the same way, and therefore we only consider $\tilde{L}_{1}$. One has

$$
\begin{aligned}
& \left|\tilde{L}_{1}\right|=\frac{1}{A^{6}} \mathbb{1}_{A_{1} \leqslant A_{2}} \mid \int_{0}^{1}\left(\tilde{\mathcal{Y}}_{1}-\tilde{\mathcal{Y}}_{2}\right) \tilde{\mathcal{Y}}_{2, \eta}(t, \eta) \\
& \times\left(\int_{0}^{\eta}\left(e^{-\frac{1}{A_{2}}\left(\tilde{\mathcal{Y}}_{2}(t, \eta)-\tilde{\mathcal{Y}}_{2}(t, \theta)\right)}-e^{-\frac{1}{A_{1}}\left(\tilde{\mathcal{Y}}_{2}(t, \eta)-\tilde{\mathcal{Y}}_{2}(t, \theta)\right)}\right)\right. \\
& \left.\times \min _{j}\left(\tilde{\mathcal{P}}_{j}\right) \min _{j}\left(\tilde{\mathcal{U}}_{j}^{+}\right) \tilde{\mathcal{Y}}_{2, \eta}(t, \theta) d \theta\right) d \eta \mid \\
& \leqslant \frac{4}{a A^{6} e} \mathbb{1}_{A_{1} \leqslant A_{2}} \int_{0}^{1}\left|\tilde{\mathcal{Y}}_{1}-\tilde{\mathcal{Y}}_{2}\right| \tilde{\mathcal{Y}}_{2, \eta}(t, \eta) \\
& \times\left(\int_{0}^{\eta} e^{-\frac{3}{4 A_{2}}\left(\tilde{\mathcal{Y}}_{2}(t, \eta)-\tilde{\mathcal{Y}}_{2}(t, \theta)\right)} \min _{j}\left(\tilde{\mathcal{P}}_{j}\right) \min \left(\tilde{\mathcal{U}}_{j}^{+}\right) \tilde{\mathcal{Y}}_{2, \eta}(t, \theta) d \theta\right) d \eta\left|A_{1}-A_{2}\right| \\
& \leqslant\left\|\tilde{\mathcal{Y}}_{1}-\tilde{\mathcal{Y}}_{2}\right\|^{2}+\frac{16}{a^{2} A^{12} e^{2}} \mathbb{1}_{A_{1} \leqslant A_{2}} \int_{0}^{1} \tilde{\mathcal{Y}}_{2, \eta}^{2}(t, \eta) \\
& \times\left(\int_{0}^{\eta} e^{-\frac{3}{4 A_{2}}\left(\tilde{\mathcal{Y}}_{2}(t, \eta)-\tilde{\mathcal{Y}}_{2}(t, \theta)\right)}\left|\tilde{\mathcal{U}}_{1}\right| \tilde{\mathcal{P}}_{2} \tilde{\mathcal{Y}}_{2, \eta}(t, \theta) d \theta\right)^{2} d \eta\left|A_{1}-A_{2}\right|^{2} \\
& \leqslant\left\|\tilde{\mathcal{Y}}_{1}-\tilde{\mathcal{Y}}_{2}\right\|^{2}+\frac{8}{A^{10} e^{2}} \int_{0}^{1} \tilde{\mathcal{Y}}_{2, \eta}^{2}(t, \eta) \\
& \times\left(\int_{0}^{\eta} e^{-\frac{3}{4 A_{2}}\left(\tilde{\mathcal{Y}}_{2}(t, \eta)-\tilde{\mathcal{Y}}_{2}(t, \theta)\right)} \tilde{\mathcal{P}}_{2} \tilde{\mathcal{Y}}_{2, \eta}(t, \theta) d \theta\right)^{2} d \eta\left|A_{1}-A_{2}\right|^{2} \\
& \leqslant\left\|\tilde{\mathcal{Y}}_{1}-\tilde{\mathcal{Y}}_{2}\right\|^{2}+\frac{8}{A^{10} e^{2}} \int_{0}^{1} \tilde{\mathcal{Y}}_{2, \eta}^{2}(t, \eta)\left(\int_{0}^{\eta} e^{-\frac{5}{4 A_{2}}\left(\tilde{\mathcal{Y}}_{2}(t, \eta)-\tilde{\mathcal{Y}}_{2}(t, \theta)\right)} \tilde{\mathcal{P}}_{2} \tilde{\mathcal{Y}}_{2, \eta}(t, \theta) d \theta\right) \\
& \times\left(\int_{0}^{\eta} e^{-\frac{1}{4 A_{2}}\left(\tilde{\mathcal{Y}}_{2}(t, \eta)-\tilde{\mathcal{Y}}_{2}(t, \theta)\right)} \tilde{\mathcal{P}}_{2} \tilde{\mathcal{Y}}_{2, \eta}(t, \theta) d \theta\right) d \eta\left|A_{1}-A_{2}\right|^{2} \\
& \leqslant\left\|\tilde{\mathcal{Y}}_{1}-\tilde{\mathcal{Y}}_{2}\right\|^{2}+\frac{32}{A^{9} e^{2}} \int_{0}^{1} \tilde{\mathcal{P}}_{2} \tilde{\mathcal{Y}}_{2, \eta}^{2}(t, \eta) \\
& \times\left(\int_{0}^{\eta} e^{-\frac{1}{4 A_{2}}\left(\tilde{\mathcal{Y}}_{2}(t, \eta)-\tilde{\mathcal{Y}}_{2}(t, \theta)\right)} \tilde{\mathcal{P}}_{2} \tilde{\mathcal{Y}}_{2, \eta}(t, \theta) d \theta\right) d \eta\left|A_{1}-A_{2}\right|^{2} \\
& \leqslant\left\|\tilde{\mathcal{Y}}_{1}-\tilde{\mathcal{Y}}_{2}\right\|^{2}+\frac{16}{A^{4} e^{2}} \int_{0}^{1} \tilde{\mathcal{Y}}_{2, \eta}(t, \eta) e^{-\frac{1}{4 A_{2}} \tilde{\mathcal{Y}}_{2}(t, \eta)} \\
& \times \int_{0}^{\eta} e^{\frac{1}{4 A_{2}} \tilde{\mathcal{Y}}_{2}(t, \theta)} \tilde{\mathcal{P}}_{2} \tilde{\mathcal{Y}}_{2, \eta}(t, \theta) d \theta d \eta\left|A_{1}-A_{2}\right|^{2}
\end{aligned}
$$




$$
\begin{aligned}
\leqslant & \left\|\tilde{\mathcal{Y}}_{1}-\tilde{\mathcal{Y}}_{2}\right\|^{2}+\frac{64}{A^{3} e^{2}}\left(-\left.\int_{0}^{\eta} e^{-\frac{1}{4 A_{2}}\left(\tilde{\mathcal{Y}}_{2}(t, \eta)-\tilde{\mathcal{Y}}_{2}(t, \theta)\right)} \tilde{\mathcal{P}}_{2} \tilde{\mathcal{Y}}_{2, \eta}(t, \theta) d \theta\right|_{\eta=0} ^{1}\right. \\
& \left.+\int_{0}^{1} \tilde{\mathcal{P}}_{2} \tilde{\mathcal{Y}}_{2, \eta}(t, \eta) d \eta\right)\left|A_{1}-A_{2}\right|^{2} \\
\leqslant & \mathcal{O}(1)\left(\left\|\tilde{\mathcal{Y}}_{1}-\tilde{\mathcal{Y}}_{2}\right\|^{2}+\left|A_{1}-A_{2}\right|^{2}\right),
\end{aligned}
$$

where we used (4.16h).

Next we turn our attention to $\tilde{L}_{3}$ and $\tilde{L}_{4}$, which can be handled in much the same way, and therefore we only consider $\tilde{L}_{3}$. One has

$$
\begin{aligned}
& \left|\tilde{L}_{3}\right|=\frac{1}{A^{6}} \mid \int_{0}^{1}\left(\tilde{\mathcal{Y}}_{1}-\tilde{\mathcal{Y}}_{2}\right) \tilde{\mathcal{Y}}_{2, \eta}(t, \eta) \\
& \times\left(\int_{0}^{\eta}\left(e^{-\frac{1}{a}\left(\tilde{\mathcal{Y}}_{2}(t, \eta)-\tilde{\mathcal{Y}}_{2}(t, \theta)\right)}-e^{-\frac{1}{a}\left(\tilde{\mathcal{Y}}_{1}(t, \eta)-\tilde{\mathcal{Y}}_{1}(t, \theta)\right)}\right) \min _{j}\left(\tilde{\mathcal{P}}_{j}\right)\right. \\
& \left.\times \min _{j}\left(\tilde{\mathcal{U}}_{j}^{+}\right) \tilde{\mathcal{Y}}_{2, \eta}(t, \theta) \mathbb{1}_{B(\eta)}(t, \theta) d \theta\right) d \eta \mid \\
& \leqslant \frac{1}{a A^{6}} \int_{0}^{1}\left|\tilde{\mathcal{Y}}_{1}-\tilde{\mathcal{Y}}_{2}\right| \tilde{\mathcal{Y}}_{2, \eta}(t, \eta) \\
& \times\left(\int_{0}^{\eta}\left(\left|\tilde{\mathcal{Y}}_{1}(t, \eta)-\tilde{\mathcal{Y}}_{2}(t, \eta)\right|+\left|\tilde{\mathcal{Y}}_{1}(t, \theta)-\tilde{\mathcal{Y}}_{2}(t, \theta)\right|\right)\right. \\
& \left.\times e^{-\frac{1}{a}\left(\tilde{\mathcal{Y}}_{2}(t, \eta)-\tilde{\mathcal{Y}}_{2}(t, \theta)\right)} \min _{j}\left(\tilde{\mathcal{P}}_{j}\right) \min _{j}\left(\tilde{\mathcal{U}}_{j}^{+}\right) \tilde{\mathcal{Y}}_{2, \eta}(t, \theta) d \theta\right) d \eta \\
& =\frac{1}{a A^{6}} \int_{0}^{1}\left(\tilde{\mathcal{Y}}_{1}-\tilde{\mathcal{Y}}_{2}\right)^{2} \tilde{\mathcal{Y}}_{2, \eta}(t, \eta) \\
& \times\left(\int_{0}^{\eta} e^{-\frac{1}{a}\left(\tilde{\mathcal{Y}}_{2}(t, \eta)-\tilde{\mathcal{Y}}_{2}(t, \theta)\right)} \min _{j}\left(\tilde{\mathcal{P}}_{j}\right) \min _{j}\left(\tilde{\mathcal{U}}_{j}^{+}\right) \tilde{\mathcal{Y}}_{2, \eta}(t, \theta) d \theta\right) d \eta \\
& +\frac{1}{a A^{6}} \int_{0}^{1}\left|\tilde{\mathcal{Y}}_{1}-\tilde{\mathcal{Y}}_{2}\right| \tilde{\mathcal{Y}}_{2, \eta}(t, \eta)\left(\int_{0}^{\eta}\left|\tilde{\mathcal{Y}}_{1}(t, \theta)-\tilde{\mathcal{Y}}_{2}(t, \theta)\right|\right. \\
& \left.\times e^{-\frac{1}{a}\left(\tilde{\mathcal{Y}}_{2}(t, \eta)-\tilde{\mathcal{Y}}_{2}(t, \theta)\right)} \min _{j}\left(\tilde{\mathcal{P}}_{j}\right) \min _{j}\left(\tilde{\mathcal{U}}_{j}^{+}\right) \tilde{\mathcal{Y}}_{2, \eta}(t, \theta) d \theta\right) d \eta \\
& \leqslant \frac{1}{\sqrt{2} A^{6}} \int_{0}^{1}\left(\tilde{\mathcal{Y}}_{1}-\tilde{\mathcal{Y}}_{2}\right)^{2} \tilde{\mathcal{Y}}_{2, \eta}(t, \eta) \\
& \times\left(\int_{0}^{\eta} e^{-\frac{1}{A_{2}}\left(\tilde{\mathcal{Y}}_{2}(t, \eta)-\tilde{\mathcal{Y}}_{2}(t, \theta)\right)} \tilde{\mathcal{P}}_{2}^{3 / 4} \tilde{\mathcal{U}}_{2}^{+} \tilde{\mathcal{Y}}_{2, \eta}(t, \theta) d \theta\right) d \eta \\
& +\frac{1}{\sqrt{2} A^{6}} \int_{0}^{1}\left|\tilde{\mathcal{Y}}_{1}-\tilde{\mathcal{Y}}_{2}\right| \tilde{\mathcal{Y}}_{2, \eta}(t, \eta) \\
& \times\left(\int_{0}^{\eta}\left|\tilde{\mathcal{Y}}_{1}(t, \theta)-\tilde{\mathcal{Y}}_{2}(t, \theta)\right| e^{-\frac{1}{A_{2}}\left(\tilde{\mathcal{Y}}_{2}(t, \eta)-\tilde{\mathcal{Y}}_{2}(t, \theta)\right)} \tilde{\mathcal{P}}_{2}^{3 / 4} \tilde{\mathcal{U}}_{2}^{+} \tilde{\mathcal{Y}}_{2, \eta}(t, \theta) d \theta\right) d \eta
\end{aligned}
$$




$$
\begin{aligned}
& \leqslant \frac{1}{\sqrt{2} A^{6}} \int_{0}^{1}\left(\tilde{\mathcal{Y}}_{1}-\tilde{\mathcal{Y}}_{2}\right)^{2} \tilde{\mathcal{Y}}_{2, \eta}(t, \eta)\left(\int_{0}^{\eta} e^{-\frac{1}{A_{2}}\left(\tilde{\mathcal{Y}}_{2}(t, \eta)-\tilde{\mathcal{Y}}_{2}(t, \theta)\right)} \tilde{\mathcal{P}}_{2}^{3 / 2} \tilde{\mathcal{Y}}_{2, \eta}(t, \theta) d \theta\right)^{1 / 2} \\
& \times\left(\int_{0}^{\eta} e^{-\frac{1}{A_{2}}\left(\tilde{\mathcal{Y}}_{2}(t, \eta)-\tilde{\mathcal{Y}}_{2}(t, \theta)\right)} \tilde{\mathcal{U}}_{2}^{2} \tilde{\mathcal{Y}}_{2, \eta}(t, \theta) d \theta\right)^{1 / 2} d \eta \\
& +\left\|\tilde{\mathcal{Y}}_{1}-\tilde{\mathcal{Y}}_{2}\right\|^{2}+\frac{1}{2 A^{12}} \int_{0}^{1} \tilde{\mathcal{Y}}_{2, \eta}^{2}(t, \eta) \\
& \times\left(\int_{0}^{\eta} e^{-\frac{1}{A_{2}}\left(\tilde{\mathcal{Y}}_{2}(t, \eta)-\tilde{\mathcal{Y}}_{2}(t, \theta)\right)}\left|\tilde{\mathcal{Y}}_{1}-\tilde{\mathcal{Y}}_{2}\right| \tilde{\mathcal{P}}_{2}^{3 / 4} \tilde{\mathcal{U}}_{2}^{+} \tilde{\mathcal{Y}}_{2, \eta}(t, \theta) d \theta\right)^{2} d \eta \\
& \leqslant \frac{3}{A^{4}} \int_{0}^{1}\left(\tilde{\mathcal{Y}}_{1}-\tilde{\mathcal{Y}}_{2}\right)^{2} \tilde{\mathcal{P}}_{2} \tilde{\mathcal{Y}}_{2, \eta}(t, \eta) d \eta+\left\|\tilde{\mathcal{Y}}_{1}-\tilde{\mathcal{Y}}_{2}\right\|^{2} \\
& +\frac{1}{2 A^{12}} \int_{0}^{1} \tilde{\mathcal{Y}}_{2, \eta}^{2}(t, \eta)\left(\int_{0}^{\eta} e^{-\frac{1}{A_{2}}\left(\tilde{\mathcal{Y}}_{2}(t, \eta)-\tilde{\mathcal{Y}}_{2}(t, \theta)\right)} \tilde{\mathcal{U}}_{2}^{2} \tilde{\mathcal{Y}}_{2, \eta}(t, \theta) d \theta\right) \\
& \times\left(\int_{0}^{\eta} e^{-\frac{1}{A_{2}}\left(\tilde{\mathcal{Y}}_{2}(t, \eta)-\tilde{\mathcal{Y}}_{2}(t, \theta)\right)}\left(\tilde{\mathcal{Y}}_{1}-\tilde{\mathcal{Y}}_{2}\right)^{2} \tilde{\mathcal{P}}_{2}^{3 / 2} \tilde{\mathcal{Y}}_{2, \eta}(t, \theta) d \theta\right) d \eta \\
& \leqslant \mathcal{O}(1)\left\|\tilde{\mathcal{Y}}_{1}-\tilde{\mathcal{Y}}_{2}\right\|^{2} \\
& +\frac{1}{A^{6}} \int_{0}^{1} \tilde{\mathcal{Y}}_{2, \eta}(t, \eta) e^{-\frac{1}{A_{2}} \tilde{\mathcal{Y}}_{2}(t, \eta)}\left(\int_{0}^{\eta} e^{\frac{1}{A_{2}} \tilde{\mathcal{Y}}_{2}(t, \theta)}\left(\tilde{\mathcal{Y}}_{1}-\tilde{\mathcal{Y}}_{2}\right)^{2} \tilde{\mathcal{P}}_{2}^{3 / 2} \tilde{\mathcal{Y}}_{2, \eta}(t, \theta) d \theta\right) d \eta \\
& \leqslant \mathcal{O}(1)\left\|\tilde{\mathcal{Y}}_{1}-\tilde{\mathcal{Y}}_{2}\right\|^{2} \\
& +\frac{1}{A^{5}}\left(-\left.\int_{0}^{\eta} e^{-\frac{1}{A_{2}}\left(\tilde{\mathcal{Y}}_{2}(t, \eta)-\tilde{\mathcal{Y}}_{2}(t, \theta)\right)}\left(\tilde{\mathcal{Y}}_{1}-\tilde{\mathcal{Y}}_{2}\right)^{2} \tilde{\mathcal{P}}_{2}^{3 / 2} \tilde{\mathcal{Y}}_{2, \eta}(t, \theta) d \theta\right|_{\eta=0} ^{1}\right. \\
& \left.+\int_{0}^{1}\left(\tilde{\mathcal{Y}}_{1}-\tilde{\mathcal{Y}}_{2}\right)^{2} \tilde{\mathcal{P}}_{2}^{3 / 2} \tilde{\mathcal{Y}}_{2, \eta}(t, \eta) d \eta\right) \\
& \leqslant \mathcal{O}(1)\left\|\tilde{\mathcal{Y}}_{1}-\tilde{\mathcal{Y}}_{2}\right\|^{2} \text {, }
\end{aligned}
$$

where we used (4.16k) for $\beta=\frac{1}{2}$.

Finally, we can turn our attention to $\tilde{L}_{5}$, which we need to split into several parts. We write

$$
\begin{aligned}
\tilde{L}_{5}= & \frac{1}{A^{6}} \int_{0}^{1}\left(\tilde{\mathcal{Y}}_{1}-\tilde{\mathcal{Y}}_{2}\right)(t, \eta) \\
& \times\left(\tilde{\mathcal{Y}}_{2, \eta}(t, \eta) \int_{0}^{\eta} \min _{j}\left(e^{-\frac{1}{a}\left(\tilde{\mathcal{Y}}_{j}(t, \eta)-\tilde{\mathcal{Y}}_{j}(t, \theta)\right)}\right) \min _{j}\left(\tilde{\mathcal{P}}_{j}\right)\right. \\
& \times \min _{j}\left(\tilde{\mathcal{U}}_{j}^{+}\right) \tilde{\mathcal{Y}}_{2, \eta}(t, \theta) d \theta \\
& -\tilde{\mathcal{Y}}_{1, \eta}(t, \eta) \int_{0}^{\eta} \min _{j}\left(e^{-\frac{1}{a}\left(\tilde{\mathcal{Y}}_{j}(t, \eta)-\tilde{\mathcal{Y}}_{j}(t, \theta)\right)}\right) \min _{j}\left(\tilde{\mathcal{P}}_{j}\right)
\end{aligned}
$$




$$
\begin{aligned}
& \left.\times \min _{j}\left(\tilde{\mathcal{U}}_{j}^{+}\right) \tilde{\mathcal{Y}}_{1, \eta}(t, \theta) d \theta\right) d \eta \\
& =\frac{1}{A^{6}} \int_{0}^{1}\left(\tilde{\mathcal{Y}}_{1}-\tilde{\mathcal{Y}}_{2}\right)\left(\tilde{\mathcal{Y}}_{2, \eta}-\tilde{\mathcal{Y}}_{1, \eta}\right)(t, \eta) \\
& \times \min _{k}\left(\int_{0}^{\eta} \min _{j}\left(e^{-\frac{1}{a}\left(\tilde{\mathcal{Y}}_{j}(t, \eta)-\tilde{\mathcal{Y}}_{j}(t, \theta)\right)}\right) \min _{j}\left(\tilde{\mathcal{P}}_{j}\right)\right. \\
& \left.\times \min _{j}\left(\tilde{\mathcal{U}}_{j}^{+}\right) \tilde{\mathcal{Y}}_{k, \eta}(t, \theta) d \theta\right) d \eta \\
& +\frac{1}{A^{6}} \int_{0}^{1}\left(\tilde{\mathcal{Y}}_{1}-\tilde{\mathcal{Y}}_{2}\right) \tilde{\mathcal{Y}}_{2, \eta}(t, \eta) \mathbb{1}_{\tilde{E}^{c}}(t, \eta) \\
& \times\left(\int_{0}^{\eta} \min _{j}\left(e^{-\frac{1}{a}\left(\tilde{\mathcal{Y}}_{j}(t, \eta)-\tilde{\mathcal{Y}}_{j}(t, \theta)\right)}\right) \min _{j}\left(\tilde{\mathcal{P}}_{j}\right)\right. \\
& \left.\times \min _{j}\left(\tilde{\mathcal{U}}_{j}^{+}\right)\left(\tilde{\mathcal{Y}}_{2, \eta}-\tilde{\mathcal{Y}}_{1, \eta}\right)(t, \theta) d \theta\right) d \eta \\
& +\frac{1}{A^{6}} \int_{0}^{1}\left(\tilde{\mathcal{Y}}_{1}-\tilde{\mathcal{Y}}_{2}\right) \tilde{\mathcal{Y}}_{1, \eta}(t, \eta) \mathbb{1}_{\tilde{E}}(t, \eta) \\
& \times\left(\int_{0}^{\eta} \min _{j}\left(e^{-\frac{1}{a}\left(\tilde{\mathcal{Y}}_{j}(t, \eta)-\tilde{\mathcal{Y}}_{j}(t, \theta)\right)}\right) \min _{j}\left(\tilde{\mathcal{P}}_{j}\right)\right. \\
& \left.\times \min _{j}\left(\tilde{\mathcal{U}}_{j}^{+}\right)\left(\tilde{\mathcal{Y}}_{2, \eta}-\tilde{\mathcal{Y}}_{1, \eta}\right)(t, \theta) d \theta\right) d \eta \\
& =\tilde{L}_{51}+\tilde{L}_{52}+\tilde{L}_{53} \text {, }
\end{aligned}
$$

where $\tilde{E}$ is given by

$$
\begin{aligned}
\tilde{E}= & \left\{(t, \eta) \mid \int_{0}^{\eta} \min \left(e^{-\frac{1}{a}\left(\tilde{\mathcal{Y}}_{j}(t, \eta)-\tilde{\mathcal{Y}}_{j}(t, \theta)\right)}\right) \min _{j}\left(\tilde{\mathcal{P}}_{j}\right) \min _{j}\left(\tilde{\mathcal{U}}_{j}^{+}\right) \tilde{\mathcal{Y}}_{2, \eta}(t, \theta) d \theta\right. \\
& \left.\leqslant \int_{0}^{\eta} \min _{j}\left(e^{-\frac{1}{a}\left(\tilde{\mathcal{Y}}_{j}(t, \eta)-\tilde{\mathcal{Y}}_{j}(t, \theta)\right)}\right) \min _{j}\left(\tilde{\mathcal{P}}_{j}\right) \min _{j}\left(\tilde{\mathcal{U}}_{j}^{+}\right) \tilde{\mathcal{Y}}_{1, \eta}(t, \theta) d \theta\right\} .
\end{aligned}
$$

As far as the first term $\tilde{L}_{51}$ is concerned, it can be estimated as follows (we use $(3.40))$ :

$$
\begin{aligned}
\left|\tilde{L}_{51}\right|= & \frac{1}{A^{6}} \mid \int_{0}^{1}\left(\tilde{\mathcal{Y}}_{1}-\tilde{\mathcal{Y}}_{2}\right)\left(\tilde{\mathcal{Y}}_{2, \eta}-\tilde{\mathcal{Y}}_{1, \eta}\right)(t, \eta) \\
& \times \min _{k}\left(\int_{0}^{\eta} \min _{j}\left(e^{-\frac{1}{a}\left(\tilde{\mathcal{Y}}_{j}(t, \eta)-\tilde{\mathcal{Y}}_{j}(t, \theta)\right)}\right) \min _{j}\left(\tilde{\mathcal{P}}_{j}\right)\right. \\
& \left.\times \min _{j}\left(\tilde{\mathcal{U}}_{j}^{+}\right) \tilde{\mathcal{Y}}_{k, \eta}(t, \theta) d \theta\right) d \eta \mid
\end{aligned}
$$




$$
\begin{aligned}
& =\frac{1}{2 A^{6}} \mid-\left(\tilde{\mathcal{Y}}_{1}-\tilde{\mathcal{Y}}_{2}\right)^{2}(t, \eta) \\
& \quad \times \min _{k}\left(\int_{0}^{\eta} \min _{j}\left(e^{-\frac{1}{a}\left(\tilde{\mathcal{Y}}_{j}(t, \eta)-\tilde{\mathcal{Y}}_{j}(t, \theta)\right)}\right) \min _{j}\left(\tilde{\mathcal{P}}_{j}\right)\right. \\
& \left.\quad \times \min _{j}\left(\tilde{\mathcal{U}}_{j}^{+}\right) \tilde{\mathcal{Y}}_{k, \eta}(t, \theta) d \theta\right)\left.\right|_{\eta=0} ^{1} \\
& \quad+\int_{0}^{1}\left(\tilde{\mathcal{Y}}_{1}-\tilde{\mathcal{Y}}_{2}\right)^{2}(t, \eta) \\
& \quad \times \frac{d}{d \eta} \min _{k}\left(\int_{0}^{\eta} \min _{j}\left(e^{-\frac{1}{a}\left(\tilde{\mathcal{Y}}_{j}(t, \eta)-\tilde{\mathcal{Y}}_{j}(t, \theta)\right)}\right) \min _{j}\left(\tilde{\mathcal{P}}_{j}\right)\right. \\
& \left.\quad \times \min _{j}\left(\tilde{\mathcal{U}}_{j}^{+}\right) \tilde{\mathcal{Y}}_{k, \eta}(t, \theta) d \theta\right) d \eta \mid \\
& \quad \leqslant \mathcal{O}_{(1)}\left\|\tilde{\mathcal{Y}}_{1}-\tilde{\mathcal{Y}}_{2}\right\|^{2},
\end{aligned}
$$

where $\mathcal{O}(1)$ denotes some constant only depending on $A$, which remains bounded as $A \rightarrow 0$, provided we can show that the derivative in the latter integral exists and is uniformly bounded. In fact, from Lemma A.6 we have that

$$
\frac{d}{d \eta} \min _{k}\left(\int_{0}^{\eta} \min _{j}\left(e^{-\frac{1}{a}\left(\tilde{\mathcal{Y}}_{j}(t, \eta)-\tilde{\mathcal{Y}}_{j}(t, \theta)\right)}\right) \min _{j}\left(\tilde{\mathcal{P}}_{j}\right) \min _{j}\left(\tilde{\mathcal{U}}_{j}^{+}\right) \tilde{\mathcal{Y}}_{k, \eta}(t, \theta) d \theta\right)
$$

exists and is uniformly bounded.

As far as the term $\tilde{L}_{52}$ (a similar argument works for $\tilde{L}_{53}$ ) is concerned, the integral can be estimated as follows:

$$
\begin{aligned}
\tilde{L}_{52}= & \frac{1}{A^{6}} \int_{0}^{1}\left(\tilde{\mathcal{Y}}_{1}-\tilde{\mathcal{Y}}_{2}\right) \tilde{\mathcal{Y}}_{2, \eta}(t, \eta) \mathbb{1}_{\tilde{E}^{c}}(t, \eta) \\
& \times\left(\int_{0}^{\eta} \min _{j}\left(e^{-\frac{1}{a}\left(\tilde{\mathcal{Y}}_{j}(t, \eta)-\tilde{\mathcal{Y}}_{j}(t, \theta)\right)}\right) \min _{j}\left(\tilde{\mathcal{P}}_{j}\right)\right. \\
& \left.\times \min _{j}\left(\tilde{\mathcal{U}}_{j}^{+}\right)\left(\tilde{\mathcal{Y}}_{2, \eta}-\tilde{\mathcal{Y}}_{1, \eta}\right)(t, \theta) d \theta\right) d \eta \\
= & \frac{1}{A^{6}} \int_{0}^{1}\left(\tilde{\mathcal{Y}}_{1}-\tilde{\mathcal{Y}}_{2}\right) \tilde{\mathcal{Y}}_{2, \eta}(t, \eta) \mathbb{1}_{\tilde{E}^{c}}(t, \eta) \\
& \times\left[\left(\tilde{\mathcal{Y}}_{2}-\tilde{\mathcal{Y}}_{1}\right)(t, \theta) \min _{j}\left(e^{-\frac{1}{a}\left(\tilde{\mathcal{Y}}_{j}(t, \eta)-\tilde{\mathcal{Y}}_{j}(t, \theta)\right)}\right) \min _{j}\left(\tilde{\mathcal{P}}_{j}\right)\right. \\
& \times\left.\min _{j}\left(\tilde{\mathcal{U}}_{j}^{+}\right)(t, \theta)\right|_{\theta=0} ^{\eta}
\end{aligned}
$$




$$
\begin{aligned}
& -\int_{0}^{\eta}\left(\tilde{\mathcal{Y}}_{2}-\tilde{\mathcal{Y}}_{1}\right)(t, \theta) \\
\times & {\left[\left(\frac{d}{d \theta} \min _{j}\left(e^{-\frac{1}{a}\left(\tilde{\mathcal{Y}}_{j}(t, \eta)-\tilde{\mathcal{Y}}_{j}(t, \theta)\right)}\right)\right) \min _{j}\left(\tilde{\mathcal{P}}_{j}\right) \min _{j}\left(\tilde{\mathcal{U}}_{j}^{+}\right)(t, \theta)\right.} \\
+ & \left.\left.\min _{j}\left(e^{-\frac{1}{a}\left(\tilde{\mathcal{Y}}_{j}(t, \eta)-\tilde{\mathcal{Y}}_{j}(t, \theta)\right)}\right)\left(\frac{d}{d \theta} \min _{j}\left(\tilde{\mathcal{P}}_{j}\right) \min _{j}\left(\tilde{\mathcal{U}}_{j}^{+}\right)\right)(t, \theta) d \theta\right] d \eta\right] \\
= & \frac{1}{A^{6}} \int_{0}^{1}\left(\tilde{\mathcal{Y}}_{1}-\tilde{\mathcal{Y}}_{2}\right)^{2} \min _{j}\left(\tilde{\mathcal{P}}_{j}\right) \min _{j}\left(\tilde{\mathcal{U}}_{j}^{+}\right) \tilde{\mathcal{Y}}_{2, \eta}(t, \eta) \mathbb{1}_{\tilde{E}^{c}}(t, \eta) d \eta \\
+ & \frac{1}{A^{6}} \int_{0}^{1}\left(\tilde{\mathcal{Y}}_{1}-\tilde{\mathcal{Y}}_{2}\right) \tilde{\mathcal{Y}}_{2, \eta}(t, \eta) \mathbb{1}_{\tilde{E}^{c}}(t, \eta) \\
\times & \int_{0}^{\eta}\left(\tilde{\mathcal{Y}}_{1}-\tilde{\mathcal{Y}}_{2}\right)(t, \theta) \\
\times & {\left[\left(\frac{d}{d \theta} \min _{j}\left(e^{-\frac{1}{a}\left(\tilde{\mathcal{Y}}_{j}(t, \eta)-\tilde{\mathcal{Y}}_{j}(t, \theta)\right)}\right)\right) \min _{j}\left(\tilde{\mathcal{P}}_{j}\right) \min _{j}\left(\tilde{\mathcal{U}}_{j}^{+}\right)(t, \theta)\right.} \\
+ & \left.\min _{j}\left(e^{-\frac{1}{a}\left(\tilde{\mathcal{Y}}_{j}(t, \eta)-\tilde{\mathcal{Y}}_{j}(t, \theta)\right)}\right)\left(\frac{d}{d \theta} \min _{j}\left(\tilde{\mathcal{P}}_{j}\right) \min _{j}\left(\tilde{\mathcal{U}}_{j}^{+}\right)\right)(t, \theta) d \theta\right] d \eta \\
= & M_{1}+M_{2} .
\end{aligned}
$$

As far as the first term $M_{1}$ is concerned, we have since

$$
2 \min _{j}\left(\tilde{\mathcal{P}}_{j}\right) \min _{j}\left(\tilde{\mathcal{U}}_{j}^{+}\right) \tilde{\mathcal{Y}}_{i, \eta}(t, \eta) \leqslant A^{5} \min _{j}\left(\tilde{\mathcal{U}}_{j}^{+}\right)(t, \eta) \leqslant \frac{A^{7}}{\sqrt{2}}
$$

that

$$
\begin{aligned}
\left|M_{1}\right| & \leqslant \frac{1}{A^{6}} \int_{0}^{1}\left(\tilde{\mathcal{Y}}_{1}-\tilde{\mathcal{Y}}_{2}\right)^{2} \min _{j}\left(\tilde{\mathcal{P}}_{j}\right) \min _{j}\left(\tilde{\mathcal{U}}_{j}^{+}\right) \tilde{\mathcal{Y}}_{2, \eta}(t, \eta) d \eta \\
& \leqslant \frac{A}{2 \sqrt{2}}\left\|\tilde{\mathcal{Y}}_{1}-\tilde{\mathcal{Y}}_{2}\right\|^{2}=\mathcal{O}(1)\left\|\tilde{\mathcal{Y}}_{1}-\tilde{\mathcal{Y}}_{2}\right\|^{2}
\end{aligned}
$$

where $\mathcal{O}(1)$ denotes some constant, which only depends on $A$ and which remains bounded as $A \rightarrow 0$.

The second term $M_{2}$, on the other hand, is a bit more demanding. (i): First of all, recall (A.4), that is,

$$
\left|\frac{d}{d \theta} \min _{j}\left(e^{-\frac{1}{a}\left(\tilde{\mathcal{Y}}_{j}(t, \eta)-\tilde{\mathcal{Y}}_{j}(t, \theta)\right)}\right)\right| \leqslant \frac{1}{a} \min _{j}\left(e^{-\frac{1}{a}\left(\tilde{\mathcal{Y}}_{j}(t, \eta)-\tilde{\mathcal{Y}}_{j}(t, \theta)\right)}\right) \max _{j}\left(\tilde{\mathcal{Y}}_{j, \eta}\right)(t, \theta),
$$


which implies that

$$
\begin{aligned}
& \frac{1}{A^{6}} \mid \int_{0}^{1}\left(\tilde{\mathcal{Y}}_{1}-\tilde{\mathcal{Y}}_{2}\right) \tilde{\mathcal{Y}}_{2, \eta}(t, \eta) \mathbb{1}_{\tilde{E}^{c}}(t, \eta) \int_{0}^{\eta}\left(\tilde{\mathcal{Y}}_{1}-\tilde{\mathcal{Y}}_{2}\right)(t, \theta) \\
& \times\left(\frac{d}{d \theta} \min _{j}\left(e^{-\frac{1}{a}\left(\tilde{\mathcal{Y}}_{j}(t, \eta)-\tilde{\mathcal{Y}}_{j}(t, \theta)\right)}\right)\right) \min _{j}\left(\tilde{\mathcal{P}}_{j}\right) \min _{j}\left(\tilde{\mathcal{U}}_{j}^{+}\right)(t, \theta) d \theta d \eta \\
& \leqslant\left\|\tilde{\mathcal{Y}}_{1}-\tilde{\mathcal{Y}}_{2}\right\|^{2}+\frac{1}{A^{12}} \int_{0}^{1} \tilde{\mathcal{Y}}_{2, \eta}^{2}(t, \eta) \mathbb{1}_{\tilde{E}^{c}}(t, \eta)\left(\int_{0}^{\eta}\left(\tilde{\mathcal{Y}}_{1}-\tilde{\mathcal{Y}}_{2}\right)(t, \theta)\right. \\
& \left.\times\left(\frac{d}{d \theta} \min _{j}\left(e^{-\frac{1}{a}\left(\tilde{\mathcal{Y}}_{j}(t, \eta)-\tilde{\mathcal{Y}}_{j}(t, \theta)\right)}\right)\right) \min _{j}\left(\tilde{\mathcal{P}}_{j}\right) \min _{j}\left(\tilde{\mathcal{U}}_{j}^{+}\right)(t, \theta) d \theta\right)^{2} d \eta \\
& \leqslant\left\|\tilde{\mathcal{Y}}_{1}-\tilde{\mathcal{Y}}_{2}\right\|^{2}+\frac{1}{4 a^{2} A^{2}} \int_{0}^{1} \tilde{\mathcal{Y}}_{2, \eta}^{2}(t, \eta) \\
& \times\left(\int_{0}^{\eta}\left|\tilde{\mathcal{Y}}_{1}-\tilde{\mathcal{Y}}_{2}\right|(t, \theta) e^{-\frac{1}{a}\left(\tilde{\mathcal{Y}}_{2}(t, \eta)-\tilde{\mathcal{Y}}_{2}(t, \theta)\right)} \min _{j}\left(\tilde{\mathcal{U}}_{j}^{+}\right)(t, \theta) d \theta\right)^{2} d \eta \\
& \leqslant\left\|\tilde{\mathcal{Y}}_{1}-\tilde{\mathcal{Y}}_{2}\right\|^{2}+\frac{a^{2}}{8 A^{2} A_{2}^{5}} \int_{0}^{1} \tilde{\mathcal{Y}}_{2, \eta}^{2}(t, \eta) \\
& \times\left(\int_{0}^{\eta}\left|\tilde{\mathcal{Y}}_{1}-\tilde{\mathcal{Y}}_{2}\right|(t, \theta) e^{-\frac{1}{A_{2}}\left(\tilde{\mathcal{Y}}_{2}(t, \eta)-\tilde{\mathcal{Y}}_{2}(t, \theta)\right)}\left(2 \tilde{\mathcal{P}}_{2} \tilde{\mathcal{Y}}_{2, \eta}+\tilde{\mathcal{H}}_{2, \eta}\right)^{1 / 2}(t, \theta) d \theta\right)^{2} d \eta \\
& \leqslant\left\|\tilde{\mathcal{Y}}_{1}-\tilde{\mathcal{Y}}_{2}\right\|^{2} \\
& +\frac{1}{8 A_{2}^{5}} \int_{0}^{1} \tilde{\mathcal{Y}}_{2, \eta}^{2}(t, \eta)\left(\int_{0}^{\eta}\left(\tilde{\mathcal{Y}}_{1}-\tilde{\mathcal{Y}}_{2}\right)^{2}(t, \theta) e^{-\frac{1}{2 A_{2}}\left(\tilde{\mathcal{Y}}_{2}(t, \eta)-\tilde{\mathcal{Y}}_{2}(t, \theta)\right)} d \theta\right) \\
& \times\left(\int_{0}^{\eta} e^{-\frac{3}{2 A_{2}}\left(\tilde{\mathcal{Y}}_{2}(t, \eta)-\tilde{\mathcal{Y}}_{2}(t, \theta)\right)}\left(2 \tilde{\mathcal{P}}_{2} \tilde{\mathcal{Y}}_{2, \eta}+\tilde{\mathcal{H}}_{2, \eta}\right)(t, \theta) d \theta\right) d \eta \\
& \leqslant\left\|\tilde{\mathcal{Y}}_{1}-\tilde{\mathcal{Y}}_{2}\right\|^{2} \\
& +\frac{1}{A_{2}^{4}} \int_{0}^{1} \tilde{\mathcal{P}}_{2} \tilde{\mathcal{Y}}_{2, \eta}^{2}(t, \eta)\left(\int_{0}^{\eta}\left(\tilde{\mathcal{Y}}_{1}-\tilde{\mathcal{Y}}_{2}\right)^{2}(t, \theta) e^{-\frac{1}{2 A_{2}}\left(\tilde{\mathcal{Y}}_{2}(t, \eta)-\tilde{\mathcal{Y}}_{2}(t, \theta)\right)} d \theta\right) d \eta \\
& \leqslant\left\|\tilde{\mathcal{Y}}_{1}-\tilde{\mathcal{Y}}_{2}\right\|^{2} \\
& +\frac{A}{2} \int_{0}^{1} \tilde{\mathcal{Y}}_{2, \eta}(t, \eta) e^{-\frac{1}{2 A_{2}} \tilde{\mathcal{Y}}_{2}(t, \eta)}\left(\int_{0}^{\eta}\left(\tilde{\mathcal{Y}}_{1}-\tilde{\mathcal{Y}}_{2}\right)^{2}(t, \theta) e^{\frac{1}{2 A_{2}} \tilde{\mathcal{Y}}_{2}(t, \theta)} d \theta\right) d \eta \\
& =\left\|\tilde{\mathcal{Y}}_{1}-\tilde{\mathcal{Y}}_{2}\right\|^{2}-\left.A A_{2} \int_{0}^{\eta} e^{-\frac{1}{2 A_{2}}\left(\tilde{\mathcal{Y}}_{2}(t, \eta)-\tilde{\mathcal{Y}}_{2}(t, \theta)\right)}\left(\tilde{\mathcal{Y}}_{1}-\tilde{\mathcal{Y}}_{2}\right)^{2}(t, \theta) d \theta\right|_{\eta=0} ^{1} \\
& +A A_{2} \int_{0}^{1}\left(\tilde{\mathcal{Y}}_{1}-\tilde{\mathcal{Y}}_{2}\right)^{2}(t, \eta) d \eta \\
& \leqslant \mathcal{O}(1)\left\|\tilde{\mathcal{Y}}_{1}-\tilde{\mathcal{Y}}_{2}\right\|^{2}
\end{aligned}
$$

where we used (4.16e) and (4.16f). 
(ii): First of all, we have to establish that $\min _{j}\left(\tilde{\mathcal{P}}_{j}\right) \min _{j}\left(\tilde{\mathcal{U}}_{j}^{+}\right)(t, \theta)$ is Lipschitz continuous with a uniformly bounded Lipschitz constant. More precisely, in Lemma A. 3 we show that

$$
\left|\frac{d}{d \theta}\left(\min _{j}\left(\tilde{\mathcal{P}}_{j}\right) \min _{j}\left(\tilde{\mathcal{U}}_{j}^{+}\right)\right)(t, \theta)\right| \leqslant 2 A^{4}\left(\min _{j}\left(\tilde{\mathcal{P}}_{j}\right)^{1 / 2}+\left|\tilde{\mathcal{U}}_{2}\right|\right)(t, \theta) .
$$

We are now ready to establish a Lipschitz estimate for the second part of $M_{2}$. Indeed,

$$
\begin{aligned}
& \frac{1}{A^{6}} \mid \int_{0}^{1}\left(\tilde{\mathcal{Y}}_{1}-\tilde{\mathcal{Y}}_{2}\right) \tilde{\mathcal{Y}}_{2, \eta}(t, \eta) \mathbb{1}_{\tilde{E}^{c}}(t, \eta) \\
& \times \int_{0}^{\eta}\left(\tilde{\mathcal{Y}}_{1}-\tilde{\mathcal{Y}}_{2}\right)(t, \theta) \min _{j}\left(e^{-\frac{1}{a}\left(\tilde{\mathcal{Y}}_{j}(t, \eta)-\tilde{\mathcal{Y}}_{j}(t, \theta)\right)}\right) \\
& \times\left(\frac{d}{d \theta} \min _{j}\left(\tilde{\mathcal{P}}_{j}\right) \min _{j}\left(\tilde{\mathcal{U}}_{j}^{+}\right)\right)(t, \theta) d \theta d \eta \mid \\
& \leqslant\left\|\tilde{\mathcal{Y}}_{1}-\tilde{\mathcal{Y}}_{2}\right\|^{2}+\frac{1}{A^{12}} \int_{0}^{1} \tilde{\mathcal{Y}}_{2, \eta}^{2}(t, \eta) \mathbb{1}_{\tilde{E}^{c}}(t, \eta) \\
& \times\left(\int_{0}^{\eta}\left(\tilde{\mathcal{Y}}_{1}-\tilde{\mathcal{Y}}_{2}\right)(t, \theta) \min _{j}\left(e^{-\frac{1}{a}\left(\tilde{\mathcal{Y}}_{j}(t, \eta)-\tilde{\mathcal{Y}}_{j}(t, \theta)\right)}\right)\right. \\
& \left.\times\left(\frac{d}{d \theta} \min _{j}\left(\tilde{\mathcal{P}}_{j}\right) \min _{j}\left(\tilde{\mathcal{U}}_{j}^{+}\right)\right)(t, \theta) d \theta\right)^{2} d \eta \\
& \leqslant\left\|\tilde{\mathcal{Y}}_{1}-\tilde{\mathcal{Y}}_{2}\right\|^{2}+\frac{4}{A^{4}} \int_{0}^{1} \tilde{\mathcal{Y}}_{2, \eta}^{2}(t, \eta) \\
& \times\left(\int_{0}^{\eta} \min _{j}\left(e^{-\frac{1}{a}\left(\tilde{\mathcal{Y}}_{j}(t, \eta)-\tilde{\mathcal{Y}}_{j}(t, \theta)\right)}\right)\right. \\
& \left.\times\left|\tilde{\mathcal{Y}}_{1}-\tilde{\mathcal{Y}}_{2}\right|\left(\min _{j}\left(\tilde{\mathcal{P}}_{j}\right)^{1 / 2}+\left|\tilde{\mathcal{U}}_{2}\right|\right)(t, \theta) d \theta\right)^{2} d \eta \\
& \leqslant\left\|\tilde{\mathcal{Y}}_{1}-\tilde{\mathcal{Y}}_{2}\right\|^{2}+\frac{4(1+\sqrt{2})^{2}}{A^{4}} \int_{0}^{1} \tilde{\mathcal{Y}}_{2, \eta}^{2}(t, \eta) \\
& \times\left(\int_{0}^{\eta} e^{-\frac{1}{A_{2}}\left(\tilde{\mathcal{Y}}_{2}(t, \eta)-\tilde{\mathcal{Y}}_{2}(t, \theta)\right)}\left|\tilde{\mathcal{Y}}_{1}-\tilde{\mathcal{Y}}_{2}\right| \tilde{\mathcal{P}}_{2}^{1 / 2}(t, \theta) d \theta\right)^{2} d \eta \\
& \leqslant\left\|\tilde{\mathcal{Y}}_{1}-\tilde{\mathcal{Y}}_{2}\right\|^{2} \\
& +\frac{36}{A^{4}} \int_{0}^{1} \tilde{\mathcal{Y}}_{2, \eta}^{2}(t, \eta)\left(\int_{0}^{\eta} e^{-\frac{3}{2 A_{2}}\left(\tilde{\mathcal{Y}}_{2}(t, \eta)-\tilde{\mathcal{Y}}_{2}(t, \theta)\right)} \tilde{\mathcal{P}}_{2}(t, \theta) d \theta\right) \\
& \times\left(\int_{0}^{\eta} e^{-\frac{1}{2 A_{2}}\left(\tilde{\mathcal{Y}}_{2}(t, \eta)-\tilde{\mathcal{Y}}_{2}(t, \theta)\right)}\left(\tilde{\mathcal{Y}}_{1}-\tilde{\mathcal{Y}}_{2}\right)^{2}(t, \theta) d \theta\right) d \eta
\end{aligned}
$$




$$
\begin{aligned}
\leqslant & \left\|\tilde{\mathcal{Y}}_{1}-\tilde{\mathcal{Y}}_{2}\right\|^{2} \\
& +\frac{252}{A^{4}} \int_{0}^{1} \tilde{\mathcal{P}}_{2} \tilde{\mathcal{Y}}_{2, \eta}^{2}(t, \eta)\left(\int_{0}^{\eta} e^{-\frac{1}{2 A_{2}}\left(\tilde{\mathcal{Y}}_{2}(t, \eta)-\tilde{\mathcal{Y}}_{2}(t, \theta)\right)}\left(\tilde{\mathcal{Y}}_{1}-\tilde{\mathcal{Y}}_{2}\right)^{2}(t, \theta) d \theta\right) d \eta \\
\leqslant & \left\|\tilde{\mathcal{Y}}_{1}-\tilde{\mathcal{Y}}_{2}\right\|^{2} \\
& +126 A \int_{0}^{1} \tilde{\mathcal{Y}}_{2, \eta}(t, \eta) e^{-\frac{1}{2 A_{2}} \tilde{\mathcal{Y}}_{2}(t, \eta)}\left(\int_{0}^{\eta} e^{\frac{1}{2 A_{2}} \tilde{\mathcal{Y}}_{2}(t, \theta)}\left(\tilde{\mathcal{Y}}_{1}-\tilde{\mathcal{Y}}_{2}\right)^{2}(t, \theta) d \theta\right) d \eta \\
\leqslant & \left\|\tilde{\mathcal{Y}}_{1}-\tilde{\mathcal{Y}}_{2}\right\|^{2} \\
& +126 A\left(-\left.2 A_{2} \int_{0}^{\eta} e^{-\frac{1}{2 A_{2}}\left(\tilde{\mathcal{Y}}_{2}(t, \eta)-\tilde{\mathcal{Y}}_{2}(t, \theta)\right)}\left(\tilde{\mathcal{Y}}_{1}-\tilde{\mathcal{Y}}_{2}\right)^{2}(t, \theta) d \theta\right|_{\eta=0} ^{1}\right. \\
& \left.+\int_{0}^{1} 2 A_{2}\left(\tilde{\mathcal{Y}}_{1}-\tilde{\mathcal{Y}}_{2}\right)^{2}(t, \eta) d \eta\right) \\
\leqslant & \mathcal{O}(1)\left\|\tilde{\mathcal{Y}}_{1}-\tilde{\mathcal{Y}}_{2}\right\|^{2},
\end{aligned}
$$

where we used (4.16i). Thus we find that

$$
\left|\tilde{L}_{52}\right|+\left|\tilde{L}_{53}\right| \leqslant \mathcal{O}(1)\left\|\tilde{\mathcal{Y}}_{1}-\tilde{\mathcal{Y}}_{2}\right\|^{2}
$$

We now turn our attention to $I_{33}$, that is,

$$
\begin{aligned}
I_{33}= & \int_{0}^{1}\left(\tilde{\mathcal{Y}}_{1}-\tilde{\mathcal{Y}}_{2}\right)(t, \eta)\left(\frac{1}{A_{2}^{6}} \tilde{\mathcal{Y}}_{2, \eta}(t, \eta) \int_{0}^{1} e^{-\frac{1}{A_{2}}\left|\tilde{\mathcal{Y}}_{2}(t, \eta)-\tilde{\mathcal{Y}}_{2}(t, \theta)\right|} \tilde{\mathcal{Q}}_{2} \tilde{\mathcal{U}}_{2, \eta}(t, \theta) d \theta\right. \\
& \left.-\frac{1}{A_{1}^{6}} \tilde{\mathcal{Y}}_{1, \eta}(t, \eta) \int_{0}^{1} e^{-\frac{1}{A_{1}}\left|\tilde{\mathcal{Y}}_{1}(t, \eta)-\tilde{\mathcal{Y}}_{1}(t, \theta)\right|} \tilde{\mathcal{Q}}_{1} \tilde{\mathcal{U}}_{1, \eta}(t, \theta) d \theta\right) d \eta .
\end{aligned}
$$

As before, we apply (5.4). Thus it suffices to study the following term.

$$
\begin{aligned}
& \bar{I}_{33}=\int_{0}^{1}\left(\tilde{\mathcal{Y}}_{1}-\tilde{\mathcal{Y}}_{2}\right)(t, \eta)\left(\frac{1}{A_{2}^{6}} \tilde{\mathcal{Y}}_{2, \eta}(t, \eta) \int_{0}^{\eta} e^{-\frac{1}{A_{2}}\left(\tilde{\mathcal{Y}}_{2}(t, \eta)-\tilde{\mathcal{Y}}_{2}(t, \theta)\right)} \tilde{\mathcal{Q}}_{2} \tilde{\mathcal{U}}_{2, \eta}(t, \theta) d \theta\right. \\
& \left.-\frac{1}{A_{1}^{6}} \tilde{\mathcal{Y}}_{1, \eta}(t, \eta) \int_{0}^{\eta} e^{-\frac{1}{A_{1}}\left(\tilde{\mathcal{Y}}_{1}(t, \eta)-\tilde{\mathcal{Y}}_{1}(t, \theta)\right)} \tilde{\mathcal{Q}}_{1} \tilde{\mathcal{U}}_{1, \eta}(t, \theta) d \theta\right) d \eta \\
& =\frac{1}{A^{6}} \int_{0}^{1}\left(\tilde{\mathcal{Y}}_{1}-\tilde{\mathcal{Y}}_{2}\right)(t, \eta)\left(\tilde{\mathcal{Y}}_{2, \eta}(t, \eta) \int_{0}^{\eta} e^{-\frac{1}{A_{2}}\left(\tilde{\mathcal{Y}}_{2}(t, \eta)-\tilde{\mathcal{Y}}_{2}(t, \theta)\right)} \tilde{\mathcal{Q}}_{2} \tilde{\mathcal{U}}_{2, \eta}(t, \theta) d \theta\right. \\
& \left.-\tilde{\mathcal{Y}}_{1, \eta}(t, \eta) \int_{0}^{\eta} e^{-\frac{1}{A_{1}}\left(\tilde{\mathcal{Y}}_{1}(t, \eta)-\tilde{\mathcal{Y}}_{1}(t, \theta)\right)} \tilde{\mathcal{Q}}_{1} \tilde{\mathcal{U}}_{1, \eta}(t, \theta) d \theta\right) d \eta \\
& +\left(\frac{1}{A_{2}^{6}}-\frac{1}{A_{1}^{6}}\right) \mathbb{1}_{A_{1} \leqslant A_{2}} \int_{0}^{1}\left(\tilde{\mathcal{Y}}_{1}-\tilde{\mathcal{Y}}_{2}\right) \tilde{\mathcal{Y}}_{1, \eta}(t, \eta) \\
& \times\left(\int_{0}^{\eta} e^{-\frac{1}{A_{1}}\left(\tilde{\mathcal{Y}}_{1}(t, \eta)-\tilde{\mathcal{Y}}_{1}(t, \theta)\right)} \tilde{\mathcal{Q}}_{1} \tilde{\mathcal{U}}_{1, \eta}(t, \theta) d \theta\right) d \eta
\end{aligned}
$$




$$
\begin{aligned}
& +\left(\frac{1}{A_{2}^{6}}-\frac{1}{A_{1}^{6}}\right) \mathbb{1}_{A_{2}<A_{1}} \int_{0}^{1}\left(\tilde{\mathcal{Y}}_{1}-\tilde{\mathcal{Y}}_{2}\right) \tilde{\mathcal{Y}}_{2, \eta}(t, \eta) \\
& \times\left(\int_{0}^{\eta} e^{-\frac{1}{A_{2}}\left(\tilde{\mathcal{Y}}_{2}(t, \eta)-\tilde{\mathcal{Y}}_{2}(t, \theta)\right)} \tilde{\mathcal{Q}}_{2} \tilde{\mathcal{U}}_{2, \eta}(t, \theta) d \theta\right) d \eta \\
& =\bar{K}_{1}+\bar{K}_{2}+\bar{K}_{3} .
\end{aligned}
$$

Direct calculations yield for $\bar{K}_{2}$ (and similarly for $\bar{K}_{3}$ ) that

$$
\begin{aligned}
\left|\bar{K}_{2}\right| \leqslant & \frac{A_{2}^{6}-A_{1}^{6}}{A_{1}^{5} A_{2}^{6}} \int_{0}^{1}\left|\tilde{\mathcal{Y}}_{1}-\tilde{\mathcal{Y}}_{2}\right| \tilde{\mathcal{Y}}_{1, \eta}(t, \eta)\left(\int_{0}^{\eta} e^{-\frac{1}{A_{1}}\left(\tilde{\mathcal{Y}}_{1}(t, \eta)-\tilde{\mathcal{Y}}_{1}(t, \theta)\right)} \tilde{\mathcal{P}}_{1}\left|\tilde{\mathcal{U}}_{1, \eta}\right|(t, \theta) d \theta\right) d \eta \\
\leqslant & \frac{A_{2}^{6}-A_{1}^{6}}{A_{1}^{5} A_{2}^{6}}\left\|\tilde{\mathcal{Y}}_{1}-\tilde{\mathcal{Y}}_{2}\right\| \\
& \times\left(\int_{0}^{1} \tilde{\mathcal{Y}}_{1, \eta}^{2}(t, \eta)\left(\int_{0}^{\eta} e^{-\frac{1}{A_{1}}\left(\tilde{\mathcal{Y}}_{1}(t, \eta)-\tilde{\mathcal{Y}}_{1}(t, \theta)\right)} \tilde{\mathcal{P}}_{1}\left|\tilde{\mathcal{U}}_{1, \eta}\right|(t, \theta) d \theta\right)^{2} d \eta\right)^{1 / 2} \\
\leqslant & \frac{A_{2}^{6}-A_{1}^{6}}{A_{1}^{6} A_{2}^{6}}\left\|\tilde{\mathcal{Y}}_{1}-\tilde{\mathcal{Y}}_{2}\right\|\left(\int_{0}^{1} \tilde{\mathcal{Y}}_{1, \eta}^{2}(t, \eta)\left(\int_{0}^{\eta} e^{-\frac{1}{A_{1}}\left(\tilde{\mathcal{Y}}_{1}(t, \eta)-\tilde{\mathcal{Y}}_{1}(t, \theta)\right)} \tilde{\mathcal{P}}_{1}^{2} \tilde{\mathcal{Y}}_{1, \eta}(t, \theta) d \theta\right)\right. \\
& \left.\times\left(\int_{0}^{\eta} e^{-\frac{1}{A_{1}}\left(\tilde{\mathcal{Y}}_{1}(t, \eta)-\tilde{\mathcal{Y}}_{1}(t, \theta)\right)} \tilde{\mathcal{H}}_{1, \eta}(t, \theta) d \theta\right) d \eta\right)^{1 / 2} \\
\leqslant & \sqrt{6} \frac{A_{2}^{6}-A_{1}^{6}}{A_{1}^{3} A_{2}^{6}}\left\|\tilde{\mathcal{Y}}_{1}-\tilde{\mathcal{Y}}_{2}\right\|\left(\int_{0}^{1} \tilde{\mathcal{P}}_{1}^{2} \tilde{\mathcal{Y}}_{1, \eta}^{2}(t, \eta) d \eta\right)^{1 / 2} \\
\leqslant & \frac{\sqrt{6}}{2} \frac{A_{2}^{6}-A_{1}^{6}}{A^{4}}\left\|\tilde{\mathcal{Y}}_{1}-\tilde{\mathcal{Y}}_{2}\right\| \\
\leqslant & \mathcal{O}(1)\left(\left\|\tilde{\mathcal{Y}}_{1}-\tilde{\mathcal{Y}}_{2}\right\|^{2}+\left|A_{1}-A_{2}\right|^{2}\right) .
\end{aligned}
$$

Here we used (4.15m), (4.16b), and (4.16d).

$\bar{K}_{1}$, on the other hand, needs to be rewritten a bit more. Recall (4.7). Then we can write

$$
\begin{aligned}
\bar{K}_{1}= & \frac{1}{A^{6}} \int_{0}^{1}\left(\tilde{\mathcal{Y}}_{1}-\tilde{\mathcal{Y}}_{2}\right)(t, \eta)\left(\tilde{\mathcal{Y}}_{2, \eta}(t, \eta) \int_{0}^{\eta} e^{-\frac{1}{A_{2}}\left(\tilde{\mathcal{Y}}_{2}(t, \eta)-\tilde{\mathcal{Y}}_{2}(t, \theta)\right)} \tilde{\mathcal{Q}}_{2} \tilde{\mathcal{U}}_{2, \eta}(t, \theta) d \theta\right. \\
& \left.-\tilde{\mathcal{Y}}_{1, \eta}(t, \eta) \int_{0}^{\eta} e^{-\frac{1}{A_{1}}\left(\tilde{\mathcal{Y}}_{1}(t, \eta)-\tilde{\mathcal{Y}}_{1}(t, \theta)\right)} \tilde{\mathcal{Q}}_{1} \tilde{\mathcal{U}}_{1, \eta}(t, \theta) d \theta\right) d \eta \\
= & \frac{1}{A^{6}} \int_{0}^{1}\left(\tilde{\mathcal{Y}}_{1}-\tilde{\mathcal{Y}}_{2}\right)(t, \eta)\left(A_{2} \tilde{\mathcal{Y}}_{2, \eta}(t, \eta) \int_{0}^{\eta} e^{-\frac{1}{A_{2}}\left(\tilde{\mathcal{Y}}_{2}(t, \eta)-\tilde{\mathcal{Y}}_{2}(t, \theta)\right)} \tilde{\mathcal{P}}_{2} \tilde{\mathcal{U}}_{2, \eta}(t, \theta) d \theta\right. \\
& \left.-A_{1} \tilde{\mathcal{Y}}_{1, \eta}(t, \eta) \int_{0}^{\eta} e^{-\frac{1}{A_{1}}\left(\tilde{\mathcal{Y}}_{1}(t, \eta)-\tilde{\mathcal{Y}}_{1}(t, \theta)\right)} \tilde{\mathcal{P}}_{1} \tilde{\mathcal{U}}_{1, \eta}(t, \theta) d \theta\right) d \eta
\end{aligned}
$$




$$
\begin{aligned}
& +\frac{1}{A^{6}} \int_{0}^{1}\left(\tilde{\mathcal{Y}}_{1}-\tilde{\mathcal{Y}}_{2}\right)(t, \eta)\left(\tilde{\mathcal{Y}}_{1, \eta}(t, \eta) \int_{0}^{\eta} e^{-\frac{1}{A_{1}}\left(\tilde{\mathcal{Y}}_{1}(t, \eta)-\tilde{\mathcal{Y}}_{1}(t, \theta)\right)} \tilde{\mathcal{D}}_{1} \tilde{\mathcal{U}}_{1, \eta}(t, \theta) d \theta\right. \\
& \left.-\tilde{\mathcal{Y}}_{2, \eta}(t, \eta) \int_{0}^{\eta} e^{-\frac{1}{A_{2}}\left(\tilde{\mathcal{Y}}_{2}(t, \eta)-\tilde{\mathcal{Y}}_{2}(t, \theta)\right)} \tilde{\mathcal{D}}_{2} \tilde{\mathcal{U}}_{2, \eta}(t, \theta) d \theta\right) d \eta .
\end{aligned}
$$

In the next step we are applying integration by parts, so that we get rid of $\tilde{\mathcal{U}}_{j, \eta}(t$, $\theta$ ) in the integrands and hence can therefore have a splitting into positive and negative parts. Therefore note that for $i=1,2$, we have

$$
\begin{aligned}
\int_{0}^{\eta} & e^{-\frac{1}{A_{i}}\left(\tilde{\mathcal{Y}}_{i}(t, \eta)-\tilde{\mathcal{Y}}_{i}(t, \theta)\right)} \tilde{\mathcal{P}}_{i} \tilde{\mathcal{U}}_{i, \eta}(t, \theta) d \theta \\
= & \left.e^{-\frac{1}{A_{i}}\left(\tilde{\mathcal{Y}}_{i}(t, \eta)-\tilde{\mathcal{Y}}_{i}(t, \theta)\right)} \tilde{\mathcal{P}}_{i} \tilde{\mathcal{U}}_{i}(t, \theta)\right|_{\theta=0} ^{\eta} \\
& -\int_{0}^{\eta} e^{-\frac{1}{A_{i}}\left(\tilde{\mathcal{Y}}_{i}(t, \eta)-\tilde{\mathcal{Y}}_{i}(t, \theta)\right)}\left(\frac{1}{A_{i}} \tilde{\mathcal{P}}_{i}+\frac{1}{A_{i}^{2}} \tilde{\mathcal{Q}}_{i}\right) \tilde{\mathcal{U}}_{i} \tilde{\mathcal{Y}}_{i, \eta}(t, \theta) d \theta \\
= & \tilde{\mathcal{P}}_{i} \tilde{\mathcal{U}}_{i}(t, \eta) \\
& -\int_{0}^{\eta} e^{-\frac{1}{A_{i}}\left(\tilde{\mathcal{Y}}_{i}(t, \eta)-\tilde{\mathcal{Y}}_{i}(t, \theta)\right)}\left(\frac{2}{A_{i}} \tilde{\mathcal{P}}_{i}-\frac{1}{A_{i}^{2}} \tilde{\mathcal{D}}_{i}\right) \tilde{\mathcal{U}}_{i} \tilde{\mathcal{Y}}_{i, \eta}(t, \theta) d \theta \\
= & \tilde{\mathcal{P}}_{i} \tilde{\mathcal{U}}_{i}(t, \eta) \\
& -\frac{2}{A_{i}} \int_{0}^{\eta} e^{-\frac{1}{A_{i}}\left(\tilde{\mathcal{Y}}_{i}(t, \eta)-\tilde{\mathcal{Y}}_{i}(t, \theta)\right)} \tilde{\mathcal{P}}_{i} \tilde{\mathcal{U}}_{i} \tilde{\mathcal{Y}}_{i, \eta}(t, \theta) d \theta \\
& +\frac{1}{A_{i}^{2}} \int_{0}^{\eta} e^{-\frac{1}{A_{i}}\left(\tilde{\mathcal{Y}}_{i}(t, \eta)-\tilde{\mathcal{Y}}_{i}(t, \theta)\right)} \tilde{\mathcal{D}}_{i} \tilde{\mathcal{U}}_{i} \tilde{\mathcal{Y}}_{i, \eta}(t, \theta) d \theta
\end{aligned}
$$

and

$$
\begin{aligned}
\int_{0}^{\eta} & e^{-\frac{1}{A_{i}}\left(\tilde{\mathcal{Y}}_{i}(t, \eta)-\tilde{\mathcal{Y}}_{i}(t, \theta)\right)} \tilde{\mathcal{D}}_{i} \tilde{\mathcal{U}}_{i, \eta}(t, \theta) d \theta \\
= & \int_{0}^{\eta} e^{-\frac{1}{A_{i}}\left(\tilde{\mathcal{Y}}_{i}(t, \eta)-\tilde{\mathcal{Y}}_{i}(t, \theta)\right)} \\
& \times\left(\int_{0}^{\theta} e^{-\frac{1}{A_{i}}\left(\tilde{\mathcal{Y}}_{i}(t, \theta)-\tilde{\mathcal{Y}}_{i}(t, l)\right)}\left(\left(\tilde{\mathcal{U}}_{i}^{2}-\tilde{\mathcal{P}}_{i}\right) \tilde{\mathcal{Y}}_{i, \eta}(t, l)+\frac{1}{2} A_{i}^{5}\right) d l\right) \tilde{\mathcal{U}}_{i, \eta}(t, \theta) d \theta \\
= & \int_{0}^{\eta}\left(\int_{0}^{\theta} e^{-\frac{1}{A_{i}}\left(\tilde{\mathcal{Y}}_{i}(t, \eta)-\tilde{\mathcal{Y}}_{i}(t, l)\right)}\left(\left(\tilde{\mathcal{U}}_{i}^{2}-\tilde{\mathcal{P}}_{i}\right) \tilde{\mathcal{Y}}_{i, \eta}(t, l)+\frac{1}{2} A_{i}^{5}\right) d l\right) \tilde{\mathcal{U}}_{i, \eta}(t, \theta) d \theta \\
= & \left.\left(\int_{0}^{\theta} e^{-\frac{1}{A_{i}}\left(\tilde{\mathcal{Y}}_{i}(t, \eta)-\tilde{\mathcal{Y}}_{i}(t, l)\right)}\left(\left(\tilde{\mathcal{U}}_{i}^{2}-\tilde{\mathcal{P}}_{i}\right) \tilde{\mathcal{Y}}_{i, \eta}(t, l)+\frac{1}{2} A_{i}^{5}\right) d l\right) \tilde{\mathcal{U}}_{i}(t, \theta)\right|_{\theta=0} ^{\eta} \\
& -\int_{0}^{\eta} e^{-\frac{1}{A_{i}}\left(\tilde{\mathcal{Y}}_{i}(t, \eta)-\tilde{\mathcal{Y}}_{i}(t, \theta)\right)}\left(\left(\tilde{\mathcal{U}}_{i}^{2}-\tilde{\mathcal{P}}_{i}\right) \tilde{\mathcal{Y}}_{i, \eta}(t, \theta)+\frac{1}{2} A_{i}^{5}\right) \tilde{\mathcal{U}}_{i}(t, \theta) d \theta
\end{aligned}
$$




$$
\begin{aligned}
= & \tilde{\mathcal{D}}_{i} \tilde{\mathcal{U}}_{i}(t, \eta) \\
& -\int_{0}^{\eta} e^{-\frac{1}{A_{i}}\left(\tilde{\mathcal{Y}}_{i}(t, \eta)-\tilde{\mathcal{Y}}_{i}(t, \theta)\right)}\left(\left(\tilde{\mathcal{U}}_{i}^{2}-\tilde{\mathcal{P}}_{i}\right) \tilde{\mathcal{Y}}_{i, \eta}(t, \theta)+\frac{1}{2} A_{i}^{5}\right) \tilde{\mathcal{U}}_{i}(t, \theta) d \theta .
\end{aligned}
$$

Here we used that for $\theta \leqslant \eta$,

$$
\begin{aligned}
0 & \leqslant \int_{0}^{\theta} e^{-\frac{1}{A_{i}}\left(\tilde{\mathcal{Y}}_{i}(t, \eta)-\tilde{\mathcal{Y}}_{i}(t, l)\right)}\left(\left(\tilde{\mathcal{U}}_{i}^{2}-\tilde{\mathcal{P}}_{i}\right) \tilde{\mathcal{Y}}_{i, \eta}(t, l)+\frac{1}{2} A_{i}^{5}\right) d l \\
& \leqslant \int_{0}^{\eta} e^{-\frac{1}{A_{i}}\left(\tilde{\mathcal{Y}}_{i}(t, \eta)-\tilde{\mathcal{Y}}_{i}(t, l)\right)}\left(\left(\tilde{\mathcal{U}}_{i}^{2}-\tilde{\mathcal{P}}_{i}\right) \tilde{\mathcal{Y}}_{i, \eta}(t, l)+\frac{1}{2} A_{i}^{5}\right) d l \\
& \leqslant \tilde{\mathcal{D}}_{i}(t, \eta) \leqslant 2 A_{i} \tilde{\mathcal{P}}_{i}(t, \eta) .
\end{aligned}
$$

We finally end up with

$$
\begin{aligned}
& \bar{K}_{1}=\frac{1}{A^{6}} \int_{0}^{1}\left(\tilde{\mathcal{Y}}_{1}-\tilde{\mathcal{Y}}_{2}\right)\left(A_{2} \tilde{\mathcal{P}}_{2} \tilde{\mathcal{U}}_{2} \tilde{\mathcal{Y}}_{2, \eta}-A_{1} \tilde{\mathcal{P}}_{1} \tilde{\mathcal{U}}_{1} \tilde{\mathcal{Y}}_{1, \eta}\right)(t, \eta) d \eta \\
& +\frac{1}{A^{6}} \int_{0}^{1}\left(\tilde{\mathcal{Y}}_{1}-\tilde{\mathcal{Y}}_{2}\right)\left(\tilde{\mathcal{D}}_{1} \tilde{\mathcal{U}}_{1} \tilde{\mathcal{Y}}_{1, \eta}-\tilde{\mathcal{D}}_{2} \tilde{\mathcal{U}}_{2} \tilde{\mathcal{Y}}_{2, \eta}\right)(t, \eta) d \eta \\
& +\frac{1}{A^{6}} \int_{0}^{1}\left(\tilde{\mathcal{Y}}_{1}-\tilde{\mathcal{Y}}_{2}\right)(t, \eta) \\
& \times\left(\tilde{\mathcal{Y}}_{2, \eta}(t, \eta) \int_{0}^{\eta} e^{-\frac{1}{A_{2}}\left(\tilde{\mathcal{Y}}_{2}(t, \eta)-\tilde{\mathcal{Y}}_{2}(t, \theta)\right)} \frac{1}{A_{2}} \tilde{\mathcal{D}}_{2} \tilde{\mathcal{U}}_{2} \tilde{\mathcal{Y}}_{2, \eta}(t, \theta) d \theta\right. \\
& \left.-\tilde{\mathcal{Y}}_{1, \eta}(t, \eta) \int_{0}^{\eta} e^{-\frac{1}{A_{1}}\left(\tilde{\mathcal{Y}}_{1}(t, \eta)-\tilde{\mathcal{Y}}_{1}(t, \theta)\right)} \frac{1}{A_{1}} \tilde{\mathcal{D}}_{1} \tilde{\mathcal{U}}_{1} \tilde{\mathcal{Y}}_{1, \eta}(t, \theta) d \theta\right) d \eta \\
& +\frac{3}{A^{6}} \int_{0}^{1}\left(\tilde{\mathcal{Y}}_{1}-\tilde{\mathcal{Y}}_{2}\right)(t, \eta)\left(\tilde{\mathcal{Y}}_{1, \eta}(t, \eta) \int_{0}^{\eta} e^{-\frac{1}{A_{1}}\left(\tilde{\mathcal{Y}}_{1}(t, \eta)-\tilde{\mathcal{Y}}_{1}(t, \theta)\right)} \tilde{\mathcal{P}}_{1} \tilde{\mathcal{U}}_{1} \tilde{\mathcal{Y}}_{1, \eta}(t, \theta) d \theta\right. \\
& \left.-\tilde{\mathcal{Y}}_{2, \eta}(t, \eta) \int_{0}^{\eta} e^{-\frac{1}{A_{2}}\left(\tilde{\mathcal{Y}}_{2}(t, \eta)-\tilde{\mathcal{Y}}_{2}(t, \theta)\right)} \tilde{\mathcal{P}}_{2} \tilde{\mathcal{U}}_{2} \tilde{\mathcal{Y}}_{2, \eta}(t, \theta) d \theta\right) d \eta \\
& +\frac{1}{A^{6}} \int_{0}^{1}\left(\tilde{\mathcal{Y}}_{1}-\tilde{\mathcal{Y}}_{2}\right)(t, \eta)\left(\tilde{\mathcal{Y}}_{2, \eta}(t, \eta) \int_{0}^{\eta} e^{-\frac{1}{A_{2}}\left(\tilde{\mathcal{Y}}_{2}(t, \eta)-\tilde{\mathcal{Y}}_{2}(t, \theta)\right)} \tilde{\mathcal{U}}_{2}^{3} \tilde{\mathcal{Y}}_{2, \eta}(t, \theta) d \theta\right. \\
& \left.-\tilde{\mathcal{Y}}_{1, \eta}(t, \eta) \int_{0}^{\eta} e^{-\frac{1}{A_{1}}\left(\tilde{\mathcal{Y}}_{1}(t, \eta)-\tilde{\mathcal{Y}}_{1}(t, \theta)\right)} \tilde{\mathcal{U}}_{1}^{3} \tilde{\mathcal{Y}}_{1, \eta}(t, \theta) d \theta\right) d \eta \\
& +\frac{1}{2 A^{6}} \int_{0}^{1}\left(\tilde{\mathcal{Y}}_{1}-\tilde{\mathcal{Y}}_{2}\right)(t, \eta)\left(\tilde{\mathcal{Y}}_{2, \eta}(t, \eta) \int_{0}^{\eta} e^{-\frac{1}{A_{2}}\left(\tilde{\mathcal{Y}}_{2}(t, \eta)-\tilde{\mathcal{Y}}_{2}(t, \theta)\right)} A_{2}^{5} \tilde{\mathcal{U}}_{2}(t, \theta) d \theta\right. \\
& \left.-\tilde{\mathcal{Y}}_{1, \eta}(t, \eta) \int_{0}^{\eta} e^{-\frac{1}{A_{1}}\left(\tilde{\mathcal{Y}}_{1}(t, \eta)-\tilde{\mathcal{Y}}_{1}(t, \theta)\right)} A_{1}^{5} \tilde{\mathcal{U}}_{1}(t, \theta) d \theta\right) d \eta \\
& =\bar{K}_{11}+\bar{K}_{12}+\bar{K}_{13}+\bar{K}_{14}+\bar{K}_{15}+\bar{K}_{16} \text {. }
\end{aligned}
$$


We start by considering $\bar{K}_{11}$, which can be further split into

$$
\begin{aligned}
\bar{K}_{11}= & \frac{1}{A^{6}} \int_{0}^{1}\left(\tilde{\mathcal{Y}}_{1}-\tilde{\mathcal{Y}}_{2}\right)\left(A_{2} \tilde{\mathcal{P}}_{2} \tilde{\mathcal{U}}_{2} \tilde{\mathcal{Y}}_{2, \eta}-A_{1} \tilde{\mathcal{P}}_{1} \tilde{\mathcal{U}}_{1} \tilde{\mathcal{Y}}_{1, \eta}\right)(t, \eta) d \eta \\
= & \frac{1}{A^{6}} \mathbb{1}_{A_{1} \leqslant A_{2}}\left(A_{2}-A_{1}\right) \int_{0}^{1}\left(\tilde{\mathcal{Y}}_{1}-\tilde{\mathcal{Y}}_{2}\right) \tilde{\mathcal{P}}_{2} \tilde{\mathcal{U}}_{2} \tilde{\mathcal{Y}}_{2, \eta}(t, \eta) d \eta \\
& +\frac{1}{A^{6}} \mathbb{1}_{A_{2}<A_{1}}\left(A_{2}-A_{1}\right) \int_{0}^{1}\left(\tilde{\mathcal{Y}}_{1}-\tilde{\mathcal{Y}}_{2}\right) \tilde{\mathcal{P}}_{1} \tilde{\mathcal{U}}_{1} \tilde{\mathcal{Y}}_{1, \eta}(t, \eta) d \eta \\
& +\frac{a}{A^{6}} \int_{0}^{1}\left(\tilde{\mathcal{Y}}_{1}-\tilde{\mathcal{Y}}_{2}\right)\left(\tilde{\mathcal{P}}_{2}-\tilde{\mathcal{P}}_{1}\right) \tilde{\mathcal{U}}_{2} \tilde{\mathcal{Y}}_{2, \eta} \mathbb{1}_{\tilde{\mathcal{P}}_{1} \leqslant \tilde{\mathcal{P}}_{2}}(t, \eta) d \eta \\
& +\frac{a}{A^{6}} \int_{0}^{1}\left(\tilde{\mathcal{Y}}_{1}-\tilde{\mathcal{Y}}_{2}\right)\left(\tilde{\mathcal{P}}_{2}-\tilde{\mathcal{P}}_{1}\right) \tilde{\mathcal{U}}_{1} \tilde{\mathcal{Y}}_{1, \eta} \mathbb{1}_{\tilde{\mathcal{P}}_{2}<\tilde{\mathcal{P}}_{1}}(t, \eta) d \eta \\
& +\frac{a}{A^{6}} \int_{0}^{1}\left(\tilde{\mathcal{Y}}_{1}-\tilde{\mathcal{Y}}_{2}\right)\left(\tilde{\mathcal{Y}}_{2, \eta}-\tilde{\mathcal{Y}}_{1, \eta}\right) \min \left(\tilde{\mathcal{P}}_{j}\right) \tilde{\mathcal{U}}_{2}(t, \eta) d \eta \\
& +\frac{a}{A^{6}} \int_{0}^{1}\left(\tilde{\mathcal{Y}}_{1}-\tilde{\mathcal{Y}}_{2}\right)\left(\tilde{\mathcal{U}}_{2}-\tilde{\mathcal{U}}_{1}\right) \min _{j}\left(\tilde{\mathcal{P}}_{j}\right) \tilde{\mathcal{Y}}_{1, \eta}(t, \eta) d \eta \\
= & \bar{B}_{11}+\bar{B}_{12}+\bar{B}_{13}+\bar{B}_{14}+\bar{B}_{15}+\bar{B}_{16} .
\end{aligned}
$$

For $\bar{B}_{11}$ we have (and similarly for $B_{12}$ ) that

$$
\begin{aligned}
\left|\bar{B}_{11}\right| & =\frac{1}{A^{6}} \mathbb{1}_{A_{1} \leqslant A_{2}}\left(A_{2}-A_{1}\right)\left|\int_{0}^{1}\left(\tilde{\mathcal{Y}}_{1}-\tilde{\mathcal{Y}}_{2}\right) \tilde{\mathcal{P}}_{2} \tilde{\mathcal{U}}_{2} \tilde{\mathcal{Y}}_{2, \eta}(t, \eta) d \eta\right| \\
& \leqslant \frac{A}{2 \sqrt{2}}\left|A_{1}-A_{2}\right| \int_{0}^{1}\left|\tilde{\mathcal{Y}}_{1}-\tilde{\mathcal{Y}}_{2}\right|(t, \eta) d \eta \\
& \leqslant \mathcal{O}(1)\left(\left\|\tilde{\mathcal{Y}}_{1}-\tilde{\mathcal{Y}}_{2}\right\|^{2}+\left|A_{1}-A_{2}\right|\right) .
\end{aligned}
$$

Recalling (4.15i), we have for $\bar{B}_{13}$ (and similarly for $\bar{B}_{14}$ ) that

$$
\begin{aligned}
\left|\bar{B}_{13}\right| & =\frac{a}{A^{6}}\left|\int_{0}^{1}\left(\tilde{\mathcal{Y}}_{1}-\tilde{\mathcal{Y}}_{2}\right)\left(\tilde{\mathcal{P}}_{2}-\tilde{\mathcal{P}}_{1}\right) \tilde{\mathcal{U}}_{2} \tilde{\mathcal{Y}}_{2, \eta} \mathbb{1}_{\tilde{\mathcal{P}}_{1} \leqslant \tilde{\mathcal{P}}_{2}}(t, \eta) d \eta\right| \\
& \leqslant \frac{2}{A^{5}} \int_{0}^{1}\left|\tilde{\mathcal{Y}}_{1}-\tilde{\mathcal{Y}}_{2}\right|\left|\tilde{\mathcal{P}}_{2}^{1 / 2}-\tilde{\mathcal{P}}_{1}^{1 / 2}\right| \tilde{\mathcal{P}}_{2}^{1 / 2}\left|\tilde{\mathcal{U}}_{2}\right| \tilde{\mathcal{Y}}_{2, \eta}(t, \eta) d \eta \\
& \leqslant \sqrt{2} \int_{0}^{1}\left|\tilde{\mathcal{Y}}_{1}-\tilde{\mathcal{Y}}_{2}\right|\left|\tilde{\mathcal{P}}_{2}^{1 / 2}-\tilde{\mathcal{P}}_{1}^{1 / 2}\right|(t, \eta) d \eta \\
& \leqslant \mathcal{O}(1)\left(\left\|\tilde{\mathcal{Y}}_{1}-\tilde{\mathcal{Y}}_{2}\right\|^{2}+\left\|\tilde{\mathcal{P}}_{2}^{1 / 2}-\tilde{\mathcal{P}}_{1}^{1 / 2}\right\|^{2}\right)
\end{aligned}
$$


As far as $\bar{B}_{15}$ is concerned, we want to use integration by parts. Therefore it is important to recall (3.40) and Lemma A.3 (ii), which imply that

$$
\begin{aligned}
\left|\bar{B}_{15}\right|= & \frac{a}{A^{6}}\left|\int_{0}^{1}\left(\tilde{\mathcal{Y}}_{1}-\tilde{\mathcal{Y}}_{2}\right)\left(\tilde{\mathcal{Y}}_{1, \eta}-\tilde{\mathcal{Y}}_{2, \eta}\right) \min _{j}\left(\tilde{\mathcal{P}}_{j}\right) \tilde{\mathcal{U}}_{2}(t, \eta) d \eta\right| \\
= & \frac{a}{2 A^{6}}\left|\left(\tilde{\mathcal{Y}}_{1}-\tilde{\mathcal{Y}}_{2}\right)^{2} \min _{j}\left(\tilde{\mathcal{P}}_{j}\right) \tilde{\mathcal{U}}_{2}(t, \eta)\right|_{\eta=0}^{1} \\
& -\int_{0}^{1}\left(\tilde{\mathcal{Y}}_{1}-\tilde{\mathcal{Y}}_{2}\right)^{2} \frac{d}{d \eta}\left(\min _{j}\left(\tilde{\mathcal{P}}_{j}\right) \tilde{\mathcal{U}}_{2}\right)(t, \eta) d \eta \mid \\
= & \frac{a}{2 A^{6}}\left|\int_{0}^{1}\left(\tilde{\mathcal{Y}}_{1}-\tilde{\mathcal{Y}}_{2}\right)^{2} \frac{d}{d \eta}\left(\min _{j}\left(\tilde{\mathcal{P}}_{j}\right) \tilde{\mathcal{U}}_{2}\right)(t, \eta) d \eta\right| \\
\leqslant & \frac{A}{2 \sqrt{2}}\left\|\tilde{\mathcal{Y}}_{1}-\tilde{\mathcal{Y}}_{2}\right\|^{2} .
\end{aligned}
$$

$\bar{B}_{16}$ is straightforward. Indeed, one has using (4.15e) that

$$
\begin{aligned}
\left|\bar{B}_{16}\right| & =\frac{a}{A^{6}}\left|\int_{0}^{1}\left(\tilde{\mathcal{Y}}_{1}-\tilde{\mathcal{Y}}_{2}\right)\left(\tilde{\mathcal{U}}_{1}-\tilde{\mathcal{U}}_{2}\right) \min _{j}\left(\tilde{\mathcal{P}}_{j}\right) \tilde{\mathcal{Y}}_{1, \eta}(t, \eta) d \eta\right| \\
& \leqslant \frac{1}{2} \int_{0}^{1}\left|\tilde{\mathcal{Y}}_{1}-\tilde{\mathcal{Y}}_{2}\right|\left|\tilde{\mathcal{U}}_{1}-\tilde{\mathcal{U}}_{2}\right|(t, \eta) d \eta \\
& \leqslant\left\|\tilde{\mathcal{Y}}_{1}-\tilde{\mathcal{Y}}_{2}\right\|^{2}+\left\|\tilde{\mathcal{U}}_{1}-\tilde{\mathcal{U}}_{2}\right\| .
\end{aligned}
$$

We continue with $\bar{K}_{12}$. We can split $\bar{K}_{12}$ as follows:

$$
\begin{aligned}
\bar{K}_{12}= & \frac{1}{A^{6}} \int_{0}^{1}\left(\tilde{\mathcal{Y}}_{1}-\tilde{\mathcal{Y}}_{2}\right)\left(\tilde{\mathcal{D}}_{1} \tilde{\mathcal{U}}_{1} \tilde{\mathcal{Y}}_{1, \eta}-\tilde{\mathcal{D}}_{2} \tilde{\mathcal{U}}_{2} \tilde{\mathcal{Y}}_{2, \eta}\right)(t, \eta) d \eta \\
= & \frac{1}{A^{6}} \int_{0}^{1}\left(\tilde{\mathcal{Y}}_{1}-\tilde{\mathcal{Y}}_{2}\right)\left(\tilde{\mathcal{D}}_{1}-\tilde{\mathcal{D}}_{2}\right) \tilde{\mathcal{U}}_{1} \tilde{\mathcal{Y}}_{1, \eta} \mathbb{1}_{\tilde{\mathcal{D}}_{2} \leqslant \tilde{\mathcal{D}}_{1}}(t, \eta) d \eta \\
& +\frac{1}{A^{6}} \int_{0}^{1}\left(\tilde{\mathcal{Y}}_{1}-\tilde{\mathcal{Y}}_{2}\right)\left(\tilde{\mathcal{D}}_{1}-\tilde{\mathcal{D}}_{2}\right) \tilde{\mathcal{U}}_{2} \tilde{\mathcal{Y}}_{2, \eta} \mathbb{1}_{\tilde{\mathcal{D}}_{1}<\tilde{\mathcal{D}}_{2}}(t, \eta) d \eta \\
& +\frac{1}{A^{6}} \int_{0}^{1}\left(\tilde{\mathcal{Y}}_{1}-\tilde{\mathcal{Y}}_{2}\right)\left(\tilde{\mathcal{U}}_{1}-\tilde{\mathcal{U}}_{2}\right) \min _{j}\left(\tilde{\mathcal{D}}_{j}\right) \tilde{\mathcal{Y}}_{1, \eta}(t, \eta) d \eta \\
& +\frac{1}{A^{6}} \int_{0}^{1}\left(\tilde{\mathcal{Y}}_{1}-\tilde{\mathcal{Y}}_{2}\right)\left(\tilde{\mathcal{Y}}_{1, \eta}-\tilde{\mathcal{Y}}_{2, \eta}\right) \min _{j}\left(\tilde{\mathcal{D}}_{j}\right) \tilde{\mathcal{U}}_{2}(t, \eta) d \eta \\
= & \bar{B}_{21}+\bar{B}_{22}+\bar{B}_{23}+\bar{B}_{24} .
\end{aligned}
$$


As the first step we need to establish an estimate for $\left(\tilde{\mathcal{D}}_{1}-\tilde{\mathcal{D}}_{2}\right)(t, \eta)$. Note that $\left|\tilde{\mathcal{U}}_{i} \tilde{\mathcal{Y}}_{i, \eta}(t, \eta)\right| \leqslant A^{5 / 2} \sqrt{\tilde{\mathcal{Y}}_{i, \eta}}(t, \eta)$, and hence it is in general unbounded. Thus our estimate for $\left(\tilde{\mathcal{D}}_{1}-\tilde{\mathcal{D}}_{2}\right)(t, \eta)$ must take care of this problem.

Applying Lemma A.9 finally yields

$$
\begin{aligned}
& \left|\bar{B}_{21}\right| \leqslant \frac{1}{A^{6}} \int_{0}^{1}\left|\tilde{\mathcal{Y}}_{1}-\tilde{\mathcal{Y}}_{2}\right|\left|\tilde{\mathcal{D}}_{1}-\tilde{\mathcal{D}}_{2}\right|\left|\tilde{\mathcal{U}}_{1}\right| \tilde{\mathcal{Y}}_{1, \eta}(t, \eta) \mathbb{1}_{\tilde{\mathcal{D}}_{2} \leqslant \tilde{\mathcal{D}}_{1}}(t, \eta) d \eta \\
& \leqslant \frac{2}{A^{9 / 2}} \int_{0}^{1}\left(\tilde{\mathcal{Y}}_{1}-\tilde{\mathcal{Y}}_{2}\right)^{2} \tilde{\mathcal{D}}_{1}^{1 / 2}\left|\tilde{\mathcal{U}}_{1}\right| \tilde{\mathcal{Y}}_{1, \eta}(t, \eta) d \eta \\
& +\frac{1}{A^{6}} \int_{0}^{1}\left(\tilde{\mathcal{Y}}_{1}-\tilde{\mathcal{Y}}_{2}\right)^{2}\left(\tilde{\mathcal{U}}_{1}^{2}+\tilde{\mathcal{P}}_{1}\right)\left|\tilde{\mathcal{U}}_{1}\right| \tilde{\mathcal{Y}}_{1, \eta}(t, \eta) d \eta \\
& +\frac{2 \sqrt{2}}{A^{9 / 2}}\left\|\tilde{\mathcal{Y}}_{1}-\tilde{\mathcal{Y}}_{2}\right\| \int_{0}^{1}\left|\tilde{\mathcal{Y}}_{1}-\tilde{\mathcal{Y}}_{2}\right| \tilde{\mathcal{D}}_{1}^{1 / 2}\left|\tilde{\mathcal{U}}_{1}\right| \tilde{\mathcal{Y}}_{1, \eta}(t, \eta) d \eta \\
& +\frac{4}{A^{3}} \int_{0}^{1}\left|\tilde{\mathcal{Y}}_{1}-\tilde{\mathcal{Y}}_{2}\right|(t, \eta) \\
& \times\left(\int_{0}^{\eta} e^{-\frac{1}{A}\left(\tilde{\mathcal{Y}}_{1}(t, \eta)-\tilde{\mathcal{Y}}_{1}(t, \theta)\right)}\left(\tilde{\mathcal{U}}_{1}-\tilde{\mathcal{U}}_{2}\right)^{2}(t, \theta) d \theta\right)^{1 / 2}\left|\tilde{\mathcal{U}}_{1}\right| \tilde{\mathcal{Y}}_{1, \eta}(t, \eta) d \eta \\
& +\frac{2 \sqrt{2}}{A^{3}} \int_{0}^{1}\left|\tilde{\mathcal{Y}}_{1}-\tilde{\mathcal{Y}}_{2}\right|(t, \eta) \\
& \times\left(\int_{0}^{\eta} e^{-\frac{1}{A}\left(\tilde{\mathcal{Y}}_{1}(t, \eta)-\tilde{\mathcal{Y}}_{1}(t, \theta)\right)}\left(\tilde{\mathcal{P}}_{1}^{1 / 2}-\tilde{\mathcal{P}}_{2}^{1 / 2}\right)^{2}(t, \theta) d \theta\right)^{1 / 2}\left|\tilde{\mathcal{U}}_{1}\right| \tilde{\mathcal{Y}}_{1, \eta}(t, \eta) d \eta \\
& +\frac{3}{\sqrt{2} A^{2}} \int_{0}^{1}\left|\tilde{\mathcal{Y}}_{1}-\tilde{\mathcal{Y}}_{2}\right|(t, \eta) \\
& \times\left(\int_{0}^{\eta} e^{-\frac{1}{a}\left(\tilde{\mathcal{Y}}_{1}(t, \eta)-\tilde{\mathcal{Y}}_{1}(t, \theta)\right)}\left(\tilde{\mathcal{Y}}_{1}-\tilde{\mathcal{Y}}_{2}\right)^{2}(t, \theta) d \theta\right)^{1 / 2}\left|\tilde{\mathcal{U}}_{1}\right| \tilde{\mathcal{Y}}_{1, \eta}(t, \eta) d \eta \\
& +\frac{12 \sqrt{2}}{\sqrt{3} e A^{2}}\left|A_{1}-A_{2}\right| \int_{0}^{1}\left|\tilde{\mathcal{Y}}_{1}-\tilde{\mathcal{Y}}_{2}\right|(t, \eta) \\
& \times\left(\int_{0}^{\eta} e^{-\frac{3}{4 A}\left(\tilde{\mathcal{Y}}_{1}(t, \eta)-\tilde{\mathcal{Y}}_{1}(t, \theta)\right)} d \theta\right)^{1 / 2}\left|\tilde{\mathcal{U}}_{1}\right| \tilde{\mathcal{Y}}_{1, \eta}(t, \eta) d \eta \\
& +\frac{3}{2 A^{2}} \int_{0}^{1}\left|\tilde{\mathcal{Y}}_{1}-\tilde{\mathcal{Y}}_{2}\right|(t, \eta) \\
& \times\left(\int_{0}^{\eta} e^{-\frac{1}{a}\left(\tilde{\mathcal{Y}}_{1}(t, \eta)-\tilde{\mathcal{Y}}_{1}(t, \theta)\right)}\left|\tilde{\mathcal{Y}}_{1}-\tilde{\mathcal{Y}}_{2}\right|(t, \theta) d \theta\right)\left|\tilde{\mathcal{U}}_{1}\right| \tilde{\mathcal{Y}}_{1, \eta}(t, \eta) d \eta \\
& +\frac{6}{A^{2}}\left|A_{1}-A_{2}\right| \int_{0}^{1}\left|\tilde{\mathcal{Y}}_{1}-\tilde{\mathcal{Y}}_{2}\right|(t, \eta)
\end{aligned}
$$




$$
\begin{aligned}
& \times\left(\int_{0}^{\eta} e^{-\frac{3}{4 A}\left(\tilde{\mathcal{Y}}_{1}(t, \eta)-\tilde{\mathcal{Y}}_{1}(t, \theta)\right)} d \theta\right)\left|\tilde{\mathcal{U}}_{1}\right| \tilde{\mathcal{Y}}_{1, \eta}(t, \eta) d \eta \\
& \leqslant \mathcal{O}(1)\left\|\tilde{\mathcal{Y}}_{1}-\tilde{\mathcal{Y}}_{2}\right\|^{2}+\frac{16}{A} \int_{0}^{1} \tilde{\mathcal{Y}}_{1, \eta}(t, \eta) e^{-\frac{1}{A} \tilde{\mathcal{Y}}_{1}(t, \eta)} \\
& \times\left(\int_{0}^{\eta} e^{\frac{1}{A} \tilde{\mathcal{Y}}_{1}(t, \theta)}\left(\left(\tilde{\mathcal{U}}_{1}-\tilde{\mathcal{U}}_{2}\right)^{2}+\left(\tilde{\mathcal{P}}_{1}^{1 / 2}-\tilde{\mathcal{P}}_{2}^{1 / 2}\right)^{2}+A\left(\tilde{\mathcal{Y}}_{1}-\tilde{\mathcal{Y}}_{2}\right)^{2}\right)(t, \theta) d \theta\right) d \eta \\
& +12 A^{1 / 2}\left|A_{1}-A_{2}\right|\left\|\tilde{\mathcal{Y}}_{1}-\tilde{\mathcal{Y}}_{2}\right\| \\
& \times\left(\int_{0}^{1} \tilde{\mathcal{Y}}_{1, \eta}(t, \eta) e^{-\frac{3}{4 A} \tilde{\mathcal{Y}}_{1}(t, \eta)}\left(\int_{0}^{\eta} e^{\frac{3}{4 A} \tilde{\mathcal{Y}}_{1}(t, \theta)} d \theta\right) d \eta\right)^{1 / 2} \\
& +\frac{3 A^{1 / 2}}{2}\left(\int_{0}^{1} \tilde{\mathcal{Y}}_{1, \eta}(t, \eta) e^{-\frac{2}{a} \tilde{\mathcal{Y}}_{1}(t, \eta)}\left(\int_{0}^{\eta} e^{\frac{2}{a} \tilde{\mathcal{Y}}_{1}(t, \theta)} d \theta\right) d \eta\right)^{1 / 2}\left\|\tilde{\mathcal{Y}}_{1}-\tilde{\mathcal{Y}}_{2}\right\|^{2} \\
& +6 A^{1 / 2}\left|A_{1}-A_{2}\right|\left\|\tilde{\mathcal{Y}}_{1}-\tilde{\mathcal{Y}}_{2}\right\| \\
& \times\left(\int_{0}^{1} \tilde{\mathcal{Y}}_{1, \eta}(t, \eta) e^{-\frac{3}{4 A} \tilde{\mathcal{Y}}_{1}(t, \eta)}\left(\int_{0}^{\eta} e^{\frac{3}{4 A} \tilde{\mathcal{Y}}_{1}(t, \theta)} d \theta\right) d \eta\right)^{1 / 2} \\
& \leqslant \mathcal{O}(1)\left(\left\|\tilde{\mathcal{Y}}_{1}-\tilde{\mathcal{Y}}_{2}\right\|^{2}+\left\|\tilde{\mathcal{U}}_{1}-\tilde{\mathcal{U}}_{2}\right\|^{2}+\left\|\tilde{\mathcal{P}}_{1}^{1 / 2}-\tilde{\mathcal{P}}_{2}^{1 / 2}\right\|^{2}+\left|A_{1}-A_{2}\right|^{2}\right) .
\end{aligned}
$$

Following the same lines, one obtains

$$
\left|\bar{B}_{22}\right| \leqslant \mathcal{O}(1)\left(\left\|\tilde{\mathcal{Y}}_{1}-\tilde{\mathcal{Y}}_{2}\right\|^{2}+\left\|\tilde{\mathcal{U}}_{1}-\tilde{\mathcal{U}}_{2}\right\|^{2}+\left\|\tilde{\mathcal{P}}_{1}^{1 / 2}-\tilde{\mathcal{P}}_{2}^{1 / 2}\right\|^{2}+\left|A_{1}-A_{2}\right|^{2}\right) .
$$

The estimate for $\bar{B}_{23}$ is straightforward. Namely,

$$
\begin{aligned}
\left|\bar{B}_{23}\right| & \leqslant \frac{2}{A^{5}} \int_{0}^{1}\left|\tilde{\mathcal{Y}}_{1}-\tilde{\mathcal{Y}}_{2}\right|\left|\tilde{\mathcal{U}}_{1}-\tilde{\mathcal{U}}_{2}\right| \tilde{\mathcal{P}}_{1} \tilde{\mathcal{Y}}_{1, \eta}(t, \eta) d \eta \\
& \leqslant\left\|\tilde{\mathcal{Y}}_{1}-\tilde{\mathcal{Y}}_{2}\right\|^{2}+\left\|\tilde{\mathcal{U}}_{1}-\tilde{\mathcal{U}}_{2}\right\|^{2} .
\end{aligned}
$$

As far as $\bar{B}_{24}$ is concerned, recall from Lemma A.4 (ii) that

$$
\left|\frac{d}{d \eta}\left(\min _{j}\left(\tilde{\mathcal{D}}_{j}\right) \tilde{\mathcal{U}}_{2}(t, \eta)\right)\right| \leqslant \mathcal{O}(1) A^{7},
$$

which yields, together with (3.40), that

$$
\begin{aligned}
\left|\bar{B}_{24}\right|= & \frac{1}{A^{6}}\left|\int_{0}^{1}\left(\tilde{\mathcal{Y}}_{1}-\tilde{\mathcal{Y}}_{2}\right)\left(\tilde{\mathcal{Y}}_{1, \eta}-\tilde{\mathcal{Y}}_{2, \eta}\right) \min _{j}\left(\tilde{\mathcal{D}}_{j}\right) \tilde{\mathcal{U}}_{2}(t, \eta) d \eta\right| \\
= & \frac{1}{2 A^{6}}\left|\left(\tilde{\mathcal{Y}}_{1}-\tilde{\mathcal{Y}}_{2}\right)^{2} \min _{j}\left(\tilde{\mathcal{D}}_{j}\right) \tilde{\mathcal{U}}_{2}(t, \eta)\right|_{\eta=0}^{1} \\
& -\int_{0}^{1}\left(\tilde{\mathcal{Y}}_{1}-\tilde{\mathcal{Y}}_{2}\right)^{2}(t, \eta) \frac{d}{d \eta}\left(\min _{j}\left(\tilde{\mathcal{D}}_{j}\right) \tilde{\mathcal{U}}_{2}\right)(t, \eta) d \eta \mid
\end{aligned}
$$




$$
\begin{aligned}
& =\frac{1}{2 A^{6}}\left|\int_{0}^{1}\left(\tilde{\mathcal{Y}}_{1}-\tilde{\mathcal{Y}}_{2}\right)^{2}(t, \eta) \frac{d}{d \eta}\left(\min _{j}\left(\tilde{\mathcal{D}}_{j}\right) \tilde{\mathcal{U}}_{2}\right)(t, \eta) d \eta\right| \\
& \leqslant \mathcal{O}(1)\left\|\tilde{\mathcal{Y}}_{1}-\tilde{\mathcal{Y}}_{2}\right\|^{2} .
\end{aligned}
$$

Next, we have a look at $\bar{K}_{13}$, which can be rewritten as follows:

$$
\begin{aligned}
& \bar{K}_{13}=\frac{1}{A^{6}} \int_{0}^{1}\left(\tilde{\mathcal{Y}}_{1}-\tilde{\mathcal{Y}}_{2}\right)(t, \eta) \\
& \times\left(\tilde{\mathcal{Y}}_{2, \eta}(t, \eta) \int_{0}^{\eta} e^{-\frac{1}{A_{2}}\left(\tilde{\mathcal{Y}}_{2}(t, \eta)-\tilde{\mathcal{Y}}_{2}(t, \theta)\right)} \frac{1}{A_{2}} \tilde{\mathcal{D}}_{2} \tilde{\mathcal{U}}_{2} \tilde{\mathcal{Y}}_{2, \eta}(t, \theta) d \theta\right. \\
& \left.-\tilde{\mathcal{Y}}_{1, \eta}(t, \eta) \int_{0}^{\eta} e^{-\frac{1}{A_{1}}\left(\tilde{\mathcal{Y}}_{1}(t, \eta)-\tilde{\mathcal{Y}}_{1}(t, \theta)\right)} \frac{1}{A_{1}} \tilde{\mathcal{D}}_{1} \tilde{\mathcal{U}}_{1} \tilde{\mathcal{Y}}_{1, \eta}(t, \theta) d \theta\right) d \eta \\
& =\frac{1}{A^{6}} \int_{0}^{1}\left(\tilde{\mathcal{Y}}_{1}-\tilde{\mathcal{Y}}_{2}\right)(t, \eta) \\
& \times\left(\tilde{\mathcal{Y}}_{2, \eta}(t, \eta) \int_{0}^{\eta} e^{-\frac{1}{A_{2}}\left(\tilde{\mathcal{Y}}_{2}(t, \eta)-\tilde{\mathcal{Y}}_{2}(t, \theta)\right)} \frac{1}{A_{2}} \tilde{\mathcal{D}}_{2} \tilde{\mathcal{U}}_{2}^{+} \tilde{\mathcal{Y}}_{2, \eta}(t, \theta) d \theta\right. \\
& \left.-\tilde{\mathcal{Y}}_{1, \eta}(t, \eta) \int_{0}^{\eta} e^{-\frac{1}{A_{1}}\left(\tilde{\mathcal{Y}}_{1}(t, \eta)-\tilde{\mathcal{Y}}_{1}(t, \theta)\right)} \frac{1}{A_{1}} \tilde{\mathcal{D}}_{1} \tilde{\mathcal{U}}_{1}^{+} \tilde{\mathcal{Y}}_{1, \eta}(t, \theta) d \theta\right) d \eta \\
& +\frac{1}{A^{6}} \int_{0}^{1}\left(\tilde{\mathcal{Y}}_{1}-\tilde{\mathcal{Y}}_{2}\right)(t, \eta) \\
& \times\left(\tilde{\mathcal{Y}}_{2, \eta}(t, \eta) \int_{0}^{\eta} e^{-\frac{1}{A_{2}}\left(\tilde{\mathcal{Y}}_{2}(t, \eta)-\tilde{\mathcal{Y}}_{2}(t, \theta)\right)} \frac{1}{A_{2}} \tilde{\mathcal{D}}_{2} \tilde{\mathcal{U}}_{2}^{-} \tilde{\mathcal{Y}}_{2, \eta}(t, \theta) d \theta\right. \\
& \left.-\tilde{\mathcal{Y}}_{1, \eta}(t, \eta) \int_{0}^{\eta} e^{-\frac{1}{A_{1}}\left(\tilde{\mathcal{Y}}_{1}(t, \eta)-\tilde{\mathcal{Y}}_{1}(t, \theta)\right)} \frac{1}{A_{1}} \tilde{\mathcal{D}}_{1} \tilde{\mathcal{U}}_{1}^{-} \tilde{\mathcal{Y}}_{1, \eta}(t, \theta) d \theta\right) d \eta \\
& =\bar{K}_{13}^{+}+\bar{K}_{13}^{-} \text {. }
\end{aligned}
$$

Note that both $\bar{K}_{13}^{+}$and $\bar{K}_{13}^{-}$have the same structure and hence we are only going to present the details for $K_{13}^{+}$, which needs to be rewritten a bit more. Namely,

$$
\begin{aligned}
\bar{K}_{13}^{+}= & \frac{1}{A^{6}} \int_{0}^{1}\left(\tilde{\mathcal{Y}}_{1}-\tilde{\mathcal{Y}}_{2}\right)(t, \eta) \\
& \times\left(\tilde{\mathcal{Y}}_{2, \eta}(t, \eta) \int_{0}^{\eta} e^{-\frac{1}{A_{2}}\left(\tilde{\mathcal{Y}}_{2}(t, \eta)-\tilde{\mathcal{Y}}_{2}(t, \theta)\right)} \frac{1}{A_{2}} \tilde{\mathcal{D}}_{2} \tilde{\mathcal{U}}_{2}^{+} \tilde{\mathcal{Y}}_{2, \eta}(t, \theta) d \theta\right. \\
& \left.-\tilde{\mathcal{Y}}_{1, \eta}(t, \eta) \int_{0}^{\eta} e^{-\frac{1}{A_{1}}\left(\tilde{\mathcal{Y}}_{1}(t, \eta)-\tilde{\mathcal{Y}}_{1}(t, \theta)\right)} \frac{1}{A_{1}} \tilde{\mathcal{D}}_{1} \tilde{\mathcal{U}}_{1}^{+} \tilde{\mathcal{Y}}_{1, \eta}(t, \theta) d \theta\right) d \eta \\
= & \mathbb{1}_{A_{1} \leqslant A_{2}} \frac{1}{A^{6}}\left(\frac{1}{A_{2}}-\frac{1}{A_{1}}\right) \int_{0}^{1}\left(\tilde{\mathcal{Y}}_{1}-\tilde{\mathcal{Y}}_{2}\right) \tilde{\mathcal{Y}}_{1, \eta}(t, \eta)
\end{aligned}
$$




$$
\begin{aligned}
& \times\left(\int_{0}^{\eta} e^{-\frac{1}{A_{1}}\left(\tilde{\mathcal{Y}}_{1}(t, \eta)-\tilde{\mathcal{Y}}_{1}(t, \theta)\right)} \tilde{\mathcal{D}}_{1} \tilde{\mathcal{U}}_{1}^{+} \tilde{\mathcal{Y}}_{1, \eta}(t, \theta) d \theta\right) d \eta \\
& +\mathbb{1}_{A_{2}<A_{1}} \frac{1}{A^{6}}\left(\frac{1}{A_{2}}-\frac{1}{A_{1}}\right) \int_{0}^{1}\left(\tilde{\mathcal{Y}}_{1}-\tilde{\mathcal{Y}}_{2}\right) \tilde{\mathcal{Y}}_{2, \eta}(t, \eta) \\
& \times\left(\int_{0}^{\eta} e^{-\frac{1}{A_{2}}\left(\tilde{\mathcal{Y}}_{2}(t, \eta)-\tilde{\mathcal{Y}}_{2}(t, \theta)\right)} \tilde{\mathcal{D}}_{2} \tilde{\mathcal{U}}_{2}^{+} \tilde{\mathcal{Y}}_{2, \eta}(t, \theta) d \theta\right) d \eta \\
& +\frac{1}{A^{7}} \int_{0}^{1}\left(\tilde{\mathcal{Y}}_{1}-\tilde{\mathcal{Y}}_{2}\right) \tilde{\mathcal{Y}}_{2, \eta}(t, \eta) \\
& \times\left(\int_{0}^{\eta} e^{-\frac{1}{A_{2}}\left(\tilde{\mathcal{Y}}_{2}(t, \eta)-\tilde{\mathcal{Y}}_{2}(t, \theta)\right)}\left(\tilde{\mathcal{D}}_{2}-\tilde{\mathcal{D}}_{1}\right) \tilde{\mathcal{U}}_{2}^{+} \tilde{\mathcal{Y}}_{2, \eta} \mathbb{1}_{\tilde{\mathcal{D}}_{1} \leqslant \tilde{\mathcal{D}}_{2}}(t, \theta) d \theta\right) d \eta \\
& +\frac{1}{A^{7}} \int_{0}^{1}\left(\tilde{\mathcal{Y}}_{1}-\tilde{\mathcal{Y}}_{2}\right) \tilde{\mathcal{Y}}_{1, \eta}(t, \eta) \\
& \times\left(\int_{0}^{\eta} e^{-\frac{1}{A_{1}}\left(\tilde{\mathcal{Y}}_{1}(t, \eta)-\tilde{\mathcal{Y}}_{1}(t, \theta)\right)}\left(\tilde{\mathcal{D}}_{2}-\tilde{\mathcal{D}}_{1}\right) \tilde{\mathcal{U}}_{1}^{+} \tilde{\mathcal{Y}}_{1, \eta} \mathbb{1}_{\tilde{\mathcal{D}}_{2}<\tilde{\mathcal{D}}_{1}}(t, \theta) d \theta\right) d \eta \\
& +\frac{1}{A^{7}} \int_{0}^{1}\left(\tilde{\mathcal{Y}}_{1}-\tilde{\mathcal{Y}}_{2}\right) \tilde{\mathcal{Y}}_{2, \eta}(t, \eta) \\
& \times\left(\int_{0}^{\eta} e^{-\frac{1}{A_{2}}\left(\tilde{\mathcal{Y}}_{2}(t, \eta)-\tilde{\mathcal{Y}}_{2}(t, \theta)\right)} \min _{j}\left(\tilde{\mathcal{D}}_{j}\right)\left(\tilde{\mathcal{U}}_{2}^{+}-\tilde{\mathcal{U}}_{1}^{+}\right) \tilde{\mathcal{Y}}_{2, \eta} \mathbb{1}_{\tilde{\mathcal{U}}_{1}^{+} \leqslant \tilde{\mathcal{U}}_{2}^{+}}(t, \theta) d \theta\right) d \eta \\
& +\frac{1}{A^{7}} \int_{0}^{1}\left(\tilde{\mathcal{Y}}_{1}-\tilde{\mathcal{Y}}_{2}\right) \tilde{\mathcal{Y}}_{1, \eta}(t, \eta) \\
& \times\left(\int_{0}^{\eta} e^{-\frac{1}{A_{1}}\left(\tilde{\mathcal{Y}}_{1}(t, \eta)-\tilde{\mathcal{Y}}_{1}(t, \theta)\right)} \min _{j}\left(\tilde{\mathcal{D}}_{j}\right)\left(\tilde{\mathcal{U}}_{2}^{+}-\tilde{\mathcal{U}}_{1}^{+}\right) \tilde{\mathcal{Y}}_{1, \eta} \mathbb{1}_{\tilde{\mathcal{U}}_{2}^{+}<\tilde{\mathcal{U}}_{1}^{+}}(t, \theta) d \theta\right) d \eta \\
& +\frac{1}{A^{7}} \int_{0}^{1}\left(\tilde{\mathcal{Y}}_{1}-\tilde{\mathcal{Y}}_{2}\right) \tilde{\mathcal{Y}}_{2, \eta}(t, \eta) \\
& \times\left(\int_{0}^{\eta}\left(e^{-\frac{1}{A_{2}}\left(\tilde{\mathcal{Y}}_{2}(t, \eta)-\tilde{\mathcal{Y}}_{2}(t, \theta)\right)}-e^{-\frac{1}{A_{2}}\left(\tilde{\mathcal{Y}}_{1}(t, \eta)-\tilde{\mathcal{Y}}_{1}(t, \theta)\right)}\right)\right. \\
& \left.\times \min _{j}\left(\tilde{\mathcal{D}}_{j}\right) \min _{j}\left(\tilde{\mathcal{U}}_{j}^{+}\right) \tilde{\mathcal{Y}}_{2, \eta} \mathbb{1}_{B(\eta)}(t, \theta) d \theta\right) d \eta \\
& +\frac{1}{A^{7}} \int_{0}^{1}\left(\tilde{\mathcal{Y}}_{1}-\tilde{\mathcal{Y}}_{2}\right) \tilde{\mathcal{Y}}_{1, \eta}(t, \eta) \\
& \times\left(\int_{0}^{\eta}\left(e^{-\frac{1}{A_{1}}\left(\tilde{\mathcal{Y}}_{2}(t, \eta)-\tilde{\mathcal{Y}}_{2}(t, \theta)\right)}-e^{-\frac{1}{A_{1}}\left(\tilde{\mathcal{Y}}_{1}(t, \eta)-\tilde{\mathcal{Y}}_{1}(t, \theta)\right)}\right)\right. \\
& \left.\times \min _{j}\left(\tilde{\mathcal{D}}_{j}\right) \min _{j}\left(\tilde{\mathcal{U}}_{j}^{+}\right) \tilde{\mathcal{Y}}_{1, \eta} \mathbb{1}_{B(\eta)^{c}}(t, \theta) d \theta\right) d \eta
\end{aligned}
$$




$$
\begin{aligned}
& +\mathbb{1}_{A_{1} \leqslant A_{2}} \frac{1}{A^{7}} \int_{0}^{1}\left(\tilde{\mathcal{Y}}_{1}-\tilde{\mathcal{Y}}_{2}\right) \tilde{\mathcal{Y}}_{2, \eta}(t, \eta) \\
& \times\left(\int_{0}^{\eta}\left(\min _{j}\left(e^{-\frac{1}{A_{2}}\left(\tilde{\mathcal{Y}}_{j}(t, \eta)-\tilde{\mathcal{Y}}_{j}(t, \theta)\right)}\right)-\min _{j}\left(e^{-\frac{1}{A_{1}}\left(\tilde{\mathcal{Y}}_{j}(t, \eta)-\tilde{\mathcal{Y}}_{j}(t, \theta)\right)}\right)\right)\right. \\
& \left.\times \min _{j}\left(\tilde{\mathcal{D}}_{j}\right) \min _{j}\left(\tilde{\mathcal{U}}_{j}^{+}\right) \tilde{\mathcal{Y}}_{2, \eta}(t, \theta) d \theta\right) d \eta \\
& +\mathbb{1}_{A_{2}<A_{1}} \frac{1}{A^{7}} \int_{0}^{1}\left(\tilde{\mathcal{Y}}_{1}-\tilde{\mathcal{Y}}_{2}\right) \tilde{\mathcal{Y}}_{1, \eta}(t, \eta) \\
& \times\left(\int_{0}^{\eta}\left(\min _{j}\left(e^{-\frac{1}{A_{2}}\left(\tilde{\mathcal{Y}}_{j}(t, \eta)-\tilde{\mathcal{Y}}_{j}(t, \theta)\right)}\right)-\min _{j}\left(e^{-\frac{1}{A_{1}}\left(\tilde{\mathcal{Y}}_{j}(t, \eta)-\tilde{\mathcal{Y}}_{j}(t, \theta)\right)}\right)\right)\right. \\
& \left.\times \min _{j}\left(\tilde{\mathcal{D}}_{j}\right) \min _{j}\left(\tilde{\mathcal{U}}_{j}^{+}\right) \tilde{\mathcal{Y}}_{1, \eta}(t, \theta) d \theta\right) d \eta \\
& +\frac{1}{A^{7}} \int_{0}^{1}\left(\tilde{\mathcal{Y}}_{1}-\tilde{\mathcal{Y}}_{2}\right)\left(\tilde{\mathcal{Y}}_{2, \eta}-\tilde{\mathcal{Y}}_{1, \eta}\right)(t, \eta) \\
& \times \min _{k}\left(\int_{0}^{\eta} \min \left(e^{-\frac{1}{a}\left(\tilde{\mathcal{Y}}_{j}(t, \eta)-\tilde{\mathcal{Y}}_{j}(t, \theta)\right)}\right) \min _{j}\left(\tilde{\mathcal{D}}_{j}\right) \min _{j}\left(\tilde{\mathcal{U}}_{j}^{+}\right) \tilde{\mathcal{Y}}_{k, \eta}(t, \theta) d \theta\right) d \eta \\
& +\frac{1}{A^{7}} \int_{0}^{1}\left(\tilde{\mathcal{Y}}_{1}-\tilde{\mathcal{Y}}_{2}\right) \tilde{\mathcal{Y}}_{2, \eta} \mathbb{1}_{D^{c}}(t, \eta) \\
& \times\left(\int_{0}^{\eta} \min _{j}\left(e^{-\frac{1}{a}\left(\tilde{\mathcal{Y}}_{j}(t, \eta)-\tilde{\mathcal{Y}}_{j}(t, \theta)\right)}\right)\right. \\
& \left.\times \min _{j}\left(\tilde{\mathcal{D}}_{j}\right) \min _{j}\left(\tilde{\mathcal{U}}_{j}^{+}\right)\left(\tilde{\mathcal{Y}}_{2, \eta}-\tilde{\mathcal{Y}}_{1, \eta}\right)(t, \theta) d \theta\right) d \eta \\
& +\frac{1}{A^{7}} \int_{0}^{1}\left(\tilde{\mathcal{Y}}_{1}-\tilde{\mathcal{Y}}_{2}\right) \tilde{\mathcal{Y}}_{1, \eta} \mathbb{1}_{D}(t, \eta) \\
& \times\left(\int_{0}^{\eta} \min _{j}\left(e^{-\frac{1}{a}\left(\tilde{\mathcal{Y}}_{j}(t, \eta)-\tilde{\mathcal{Y}}_{j}(t, \theta)\right)}\right)\right. \\
& \left.\times \min _{j}\left(\tilde{\mathcal{D}}_{j}\right) \min _{j}\left(\tilde{\mathcal{U}}_{j}^{+}\right)\left(\tilde{\mathcal{Y}}_{2, \eta}-\tilde{\mathcal{Y}}_{1, \eta}\right)(t, \theta) d \theta\right) d \eta \\
& =\hat{B}_{31}+\hat{B}_{32}+\bar{B}_{31}+\bar{B}_{32}+\bar{B}_{33}+\bar{B}_{34} \\
& +\bar{B}_{35}+\bar{B}_{36}+\hat{B}_{35}+\hat{B}_{36}+\bar{B}_{37}+\bar{B}_{38}+\bar{B}_{39} \text {, }
\end{aligned}
$$

where $B(\eta)$ by $(5.11)$ and

$$
D=\left\{(t, \eta) \mid \int_{0}^{\eta} \Upsilon(t, \eta, \theta) \tilde{\mathcal{Y}}_{2, \eta}(t, \theta) d \theta \leqslant \int_{0}^{\eta} \Upsilon(t, \eta, \theta) \tilde{\mathcal{Y}}_{1, \eta}(t, \theta) d \theta\right\},
$$


where

$$
\Upsilon(t, \eta, \theta)=\min _{j}\left(e^{-\frac{1}{a}\left(\tilde{\mathcal{Y}}_{j}(t, \eta)-\tilde{\mathcal{Y}}_{j}(t, \theta)\right)}\right) \min _{j}\left(\tilde{\mathcal{D}}_{j}\right) \min _{j}\left(\tilde{\mathcal{U}}_{j}^{+}\right)
$$

We start by estimating $\hat{B}_{31}$ (a similar argument works for $\hat{B}_{32}$ ). Direct calculations yield

$$
\begin{aligned}
& \left|\hat{B}_{31}\right|=\mathbb{1}_{A_{1} \leqslant A_{2}} \frac{1}{a A^{7}}\left|A_{2}-A_{1}\right| \int_{0}^{1}\left|\tilde{\mathcal{Y}}_{1}-\tilde{\mathcal{Y}}_{2}\right| \tilde{\mathcal{Y}}_{1, \eta}(t, \eta) \\
& \times\left(\int_{0}^{\eta} e^{-\frac{1}{A_{1}}\left(\tilde{\mathcal{Y}}_{1}(t, \eta)-\tilde{\mathcal{Y}}_{1}(t, \theta)\right)} \tilde{\mathcal{D}}_{1} \tilde{\mathcal{U}}_{1}^{+} \tilde{\mathcal{Y}}_{1, \eta}(t, \theta) d \theta\right) d \eta \\
& \leqslant \frac{2}{A^{7}}\left|A_{1}-A_{2}\right| \int_{0}^{1}\left|\tilde{\mathcal{Y}}_{1}-\tilde{\mathcal{Y}}_{2}\right| \tilde{\mathcal{Y}}_{1, \eta}(t, \eta) \\
& \times\left(\int_{0}^{\eta} e^{-\frac{1}{A_{1}}\left(\tilde{\mathcal{Y}}_{1}(t, \eta)-\tilde{\mathcal{Y}}_{1}(t, \theta)\right)} \tilde{\mathcal{P}}_{1} \tilde{\mathcal{U}}_{1}^{+} \tilde{\mathcal{Y}}_{1, \eta}(t, \theta) d \theta\right) d \eta \\
& \leqslant \frac{2}{A^{7}}\left|A_{1}-A_{2}\right| \int_{0}^{1}\left|\tilde{\mathcal{Y}}_{1}-\tilde{\mathcal{Y}}_{2}\right| \tilde{\mathcal{Y}}_{1, \eta}(t, \eta) \\
& \times\left(\int_{0}^{\eta} e^{-\frac{1}{A_{1}}\left(\tilde{\mathcal{Y}}_{1}(t, \eta)-\tilde{\mathcal{Y}}_{1}(t, \theta)\right)} \tilde{\mathcal{P}}_{1}^{2} \tilde{\mathcal{Y}}_{1, \eta}(t, \theta) d \theta\right)^{1 / 2} \\
& \times\left(\int_{0}^{\eta} e^{-\frac{1}{A_{1}}\left(\tilde{\mathcal{Y}}_{1}(t, \eta)-\tilde{\mathcal{Y}}_{1}(t, \theta)\right)} \tilde{\mathcal{U}}_{1}^{2} \tilde{\mathcal{Y}}_{1, \eta}(t, \theta) d \theta\right)^{1 / 2} d \eta \\
& \leqslant \frac{2 \sqrt{6}}{A^{4}}\left|A_{1}-A_{2}\right| \int_{0}^{1}\left|\tilde{\mathcal{Y}}_{1}-\tilde{\mathcal{Y}}_{2}\right| \tilde{\mathcal{P}}_{1} \tilde{\mathcal{Y}}_{1, \eta}(t, \eta) d \eta \\
& \leqslant \sqrt{6} A\left|A_{1}-A_{2}\right| \int_{0}^{1}\left|\tilde{\mathcal{Y}}_{1}-\tilde{\mathcal{Y}}_{2}\right|(t, \eta) d \eta \\
& \leqslant \mathcal{O}(1)\left(\left\|\tilde{\mathcal{Y}}_{1}-\tilde{\mathcal{Y}}_{2}\right\|^{2}+\left|A_{1}-A_{2}\right|^{2}\right) \text {. }
\end{aligned}
$$

For $\bar{B}_{31}$ (a similar argument works for $\bar{B}_{32}$ ) we would like to apply some of the estimates established when investigating $\bar{K}_{1}$. Thus we split $\bar{B}_{31}$ into even smaller parts, that is,

$$
\begin{aligned}
\left|\bar{B}_{31}\right| \leqslant & \frac{1}{A^{7}} \int_{0}^{1}\left|\tilde{\mathcal{Y}}_{1}-\tilde{\mathcal{Y}}_{2}\right| \tilde{\mathcal{Y}}_{2, \eta}(t, \eta) \\
& \times\left(\int_{0}^{\eta} e^{-\frac{1}{A_{2}}\left(\tilde{\mathcal{Y}}_{2}(t, \eta)-\tilde{\mathcal{Y}}_{2}(t, \theta)\right)}\left(\bar{d}_{11}+\bar{d}_{12}\right) \tilde{\mathcal{U}}_{2}^{+} \tilde{\mathcal{Y}}_{2, \eta} \mathbb{1}_{\tilde{\mathcal{D}}_{1} \leqslant \tilde{\mathcal{D}}_{2}}(t, \theta) d \theta\right) d \eta \\
& +\frac{1}{A^{7}} \int_{0}^{1}\left|\tilde{\mathcal{Y}}_{1}-\tilde{\mathcal{Y}}_{2}\right| \tilde{\mathcal{Y}}_{2, \eta}(t, \eta)
\end{aligned}
$$




$$
\begin{aligned}
& \times\left(\int_{0}^{\eta} e^{-\frac{1}{A_{2}}\left(\tilde{\mathcal{Y}}_{2}(t, \eta)-\tilde{\mathcal{Y}}_{2}(t, \theta)\right)} \bar{T}_{1} \tilde{\mathcal{U}}_{2}^{+} \tilde{\mathcal{Y}}_{2, \eta} \mathbb{1}_{\tilde{\mathcal{D}}_{1} \leqslant \tilde{\mathcal{D}}_{2}}(t, \theta) d \theta\right) d \eta \\
& +\frac{1}{A^{7}} \int_{0}^{1}\left|\tilde{\mathcal{Y}}_{1}-\tilde{\mathcal{Y}}_{2}\right| \tilde{\mathcal{Y}}_{2, \eta}(t, \eta) \\
& \times\left(\int_{0}^{\eta} e^{-\frac{1}{A_{2}}\left(\tilde{\mathcal{Y}}_{2}(t, \eta)-\tilde{\mathcal{Y}}_{2}(t, \theta)\right)} \bar{T}_{2} \tilde{\mathcal{U}}_{2}^{+} \tilde{\mathcal{Y}}_{2, \eta} \mathbb{1}_{\tilde{\mathcal{D}}_{1} \leqslant \tilde{\mathcal{D}}_{2}}(t, \theta) d \theta\right) d \eta \\
& +\frac{1}{A^{7}} \int_{0}^{1}\left|\tilde{\mathcal{Y}}_{1}-\tilde{\mathcal{Y}}_{2}\right| \tilde{\mathcal{Y}}_{2, \eta}(t, \eta) \\
& \times\left(\int_{0}^{\eta} e^{-\frac{1}{A_{2}}\left(\tilde{\mathcal{Y}}_{2}(t, \eta)-\tilde{\mathcal{Y}}_{2}(t, \theta)\right)} \bar{T}_{3} \tilde{\mathcal{U}}_{2}^{+} \tilde{\mathcal{Y}}_{2, \eta} \mathbb{1}_{\tilde{\mathcal{D}}_{1} \leqslant \tilde{\mathcal{D}}_{2}}(t, \theta) d \theta\right) d \eta \\
& =B_{311}+B_{312}+B_{313}+B_{314} \cdot
\end{aligned}
$$

By (A.12) and (A.13), we have that

$$
\left(\bar{d}_{11}+\bar{d}_{12}\right)(t, \theta) \leqslant 2 A^{3 / 2} \tilde{\mathcal{D}}_{2}^{1 / 2}\left|\tilde{\mathcal{Y}}_{1}-\tilde{\mathcal{Y}}_{2}\right|(t, \theta)+2 \sqrt{2} A^{3 / 2} \tilde{\mathcal{D}}_{2}^{1 / 2}(t, \theta)\left\|\tilde{\mathcal{Y}}_{1}-\tilde{\mathcal{Y}}_{2}\right\|
$$

and hence

$$
\begin{aligned}
& B_{311} \leqslant \frac{2}{A^{11 / 2}} \int_{0}^{1}\left|\tilde{\mathcal{Y}}_{1}-\tilde{\mathcal{Y}}_{2}\right| \tilde{\mathcal{Y}}_{2, \eta}(t, \eta) \\
& \times\left(\int_{0}^{\eta} e^{-\frac{1}{A_{2}}\left(\tilde{\mathcal{Y}}_{2}(t, \eta)-\tilde{\mathcal{Y}}_{2}(t, \theta)\right)}\left|\tilde{\mathcal{Y}}_{1}-\tilde{\mathcal{Y}}_{2}\right| \tilde{\mathcal{D}}_{2}^{1 / 2} \tilde{\mathcal{U}}_{2}^{+} \tilde{\mathcal{Y}}_{2, \eta}(t, \theta) d \theta\right) d \eta \\
& +\frac{2 \sqrt{2}}{A^{11 / 2}} \int_{0}^{1}\left|\tilde{\mathcal{Y}}_{1}-\tilde{\mathcal{Y}}_{2}\right| \tilde{\mathcal{Y}}_{2, \eta}(t, \eta) \\
& \times\left(\int_{0}^{\eta} e^{-\frac{1}{A_{2}}\left(\tilde{\mathcal{Y}}_{2}(t, \eta)-\tilde{\mathcal{Y}}_{2}(t, \theta)\right)} \tilde{\mathcal{D}}_{2}^{1 / 2} \tilde{\mathcal{U}}_{2}^{+} \tilde{\mathcal{Y}}_{2, \eta}(t, \theta) d \theta\right) d \eta\left\|\tilde{\mathcal{Y}}_{1}-\tilde{\mathcal{Y}}_{2}\right\| \\
& \leqslant\left\|\tilde{\mathcal{Y}}_{1}-\tilde{\mathcal{Y}}_{2}\right\|^{2} \\
& +\frac{4}{A^{11}} \int_{0}^{1} \tilde{\mathcal{Y}}_{2, \eta}^{2}(t, \eta) \\
& \times\left(\int_{0}^{\eta} e^{-\frac{1}{A_{2}}\left(\tilde{\mathcal{Y}}_{2}(t, \eta)-\tilde{\mathcal{Y}}_{2}(t, \theta)\right)}\left|\tilde{\mathcal{Y}}_{1}-\tilde{\mathcal{Y}}_{2}\right| \tilde{\mathcal{D}}_{2}^{1 / 2} \tilde{\mathcal{U}}_{2}^{+} \tilde{\mathcal{Y}}_{2, \eta}(t, \theta) d \theta\right)^{2} d \eta \\
& +\frac{2 \sqrt{2}}{A^{11 / 2}}\left\|\tilde{\mathcal{Y}}_{1}-\tilde{\mathcal{Y}}_{2}\right\|^{2} \\
& \times\left(\int_{0}^{1} \tilde{\mathcal{Y}}_{2, \eta}^{2}(t, \eta)\left(\int_{0}^{\eta} e^{-\frac{1}{A_{2}}\left(\tilde{\mathcal{Y}}_{2}(t, \eta)-\tilde{\mathcal{Y}}_{2}(t, \theta)\right)} \tilde{\mathcal{D}}_{2}^{1 / 2} \tilde{\mathcal{U}}_{2}^{+} \tilde{\mathcal{Y}}_{2, \eta}(t, \theta) d \theta\right)^{2} d \eta\right)^{1 / 2} \\
& \leqslant\left\|\tilde{\mathcal{Y}}_{1}-\tilde{\mathcal{Y}}_{2}\right\|^{2} \\
& +\frac{4}{A^{11}} \int_{0}^{1} \tilde{\mathcal{Y}}_{2, \eta}^{2}(t, \eta)\left(\int_{0}^{\eta} e^{-\frac{1}{A_{2}}\left(\tilde{\mathcal{Y}}_{2}(t, \eta)-\tilde{\mathcal{Y}}_{2}(t, \theta)\right)} \tilde{\mathcal{U}}_{2}^{2} \tilde{\mathcal{Y}}_{2, \eta}(t, \theta) d \theta\right)
\end{aligned}
$$




$$
\begin{aligned}
& \times\left(\int_{0}^{\eta} e^{-\frac{1}{A_{2}}\left(\tilde{\mathcal{Y}}_{2}(t, \eta)-\tilde{\mathcal{Y}}_{2}(t, \theta)\right)}\left(\tilde{\mathcal{Y}}_{1}-\tilde{\mathcal{Y}}_{2}\right)^{2} \tilde{\mathcal{D}}_{2} \tilde{\mathcal{Y}}_{2, \eta}(t, \theta) d \theta\right) d \eta \\
& +\frac{2 \sqrt{2}}{A^{11 / 2}}\left\|\tilde{\mathcal{Y}}_{1}-\tilde{\mathcal{Y}}_{2}\right\|^{2}\left(\int_{0}^{1} \tilde{\mathcal{Y}}_{2, \eta}^{2}(t, \eta)\left(\int_{0}^{\eta} e^{-\frac{1}{A_{2}}\left(\tilde{\mathcal{Y}}_{2}(t, \eta)-\tilde{\mathcal{Y}}_{2}(t, \theta)\right)} \tilde{\mathcal{U}}_{2}^{2} \tilde{\mathcal{Y}}_{2, \eta}(t, \theta) d \theta\right)\right. \\
& \left.\times\left(\int_{0}^{\eta} e^{-\frac{1}{A_{2}}\left(\tilde{\mathcal{Y}}_{2}(t, \eta)-\tilde{\mathcal{Y}}_{2}(t, \theta)\right)} \tilde{\mathcal{D}}_{2} \tilde{\mathcal{Y}}_{2, \eta}(t, \theta) d \theta\right) d \eta\right)^{1 / 2} \\
& \leqslant\left\|\tilde{\mathcal{Y}}_{1}-\tilde{\mathcal{Y}}_{2}\right\|^{2} \\
& +\frac{8}{A^{5}} \int_{0}^{1} \tilde{\mathcal{Y}}_{2, \eta}(t, \eta) e^{-\frac{1}{A_{2}} \tilde{\mathcal{Y}}_{2}(t, \eta)}\left(\int_{0}^{\eta} e^{\frac{1}{A_{2}} \tilde{\mathcal{Y}}_{2}(t, \theta)}\left(\tilde{\mathcal{Y}}_{1}-\tilde{\mathcal{Y}}_{2}\right)^{2} \tilde{\mathcal{D}}_{2} \tilde{\mathcal{Y}}_{2, \eta}(t, \theta) d \theta\right) d \eta \\
& +\frac{4}{A^{5 / 2}}\left\|\tilde{\mathcal{Y}}_{1}-\tilde{\mathcal{Y}}_{2}\right\|^{2} \\
& \times\left(\int_{0}^{1} \tilde{\mathcal{Y}}_{2, \eta}(t, \eta)\left(\int_{0}^{\eta} e^{-\frac{1}{A_{2}}\left(\tilde{\mathcal{Y}}_{2}(t, \eta)-\tilde{\mathcal{Y}}_{2}(t, \theta)\right)} \tilde{\mathcal{D}}_{2} \tilde{\mathcal{Y}}_{2, \eta}(t, \theta) d \theta\right) d \eta\right)^{1 / 2} \\
& \leqslant\left\|\tilde{\mathcal{Y}}_{1}-\tilde{\mathcal{Y}}_{2}\right\|^{2} \\
& +\frac{8 A_{2}}{A^{5}}\left[-\left.\int_{0}^{\eta} e^{-\frac{1}{A_{2}}\left(\tilde{\mathcal{Y}}_{2}(t, \eta)-\tilde{\mathcal{Y}}_{2}(t, \theta)\right)}\left(\tilde{\mathcal{Y}}_{1}-\tilde{\mathcal{Y}}_{2}\right)^{2} \tilde{\mathcal{D}}_{2} \tilde{\mathcal{Y}}_{2, \eta}(t, \theta) d \theta\right|_{\eta=0} ^{1}\right. \\
& \left.+\int_{0}^{1}\left(\tilde{\mathcal{Y}}_{1}-\tilde{\mathcal{Y}}_{2}\right)^{2} \tilde{\mathcal{D}}_{2} \tilde{\mathcal{Y}}_{2, \eta}(t, \eta) d \eta\right] \\
& +4 A^{1 / 2}\left\|\tilde{\mathcal{Y}}_{1}-\tilde{\mathcal{Y}}_{2}\right\|^{2}\left(\int_{0}^{1} \tilde{\mathcal{Y}}_{2, \eta}(t, \eta) e^{-\frac{1}{A_{2}} \tilde{\mathcal{Y}}_{2}(t, \eta)}\left(\int_{0}^{\eta} e^{\frac{1}{A_{2}} \tilde{\mathcal{Y}}_{2}(t, \theta)} d \theta\right) d \eta\right)^{1 / 2} \\
& \leqslant \mathcal{O}(1)\left\|\tilde{\mathcal{Y}}_{1}-\tilde{\mathcal{Y}}_{2}\right\|^{2}
\end{aligned}
$$

Recalling the estimate for $\bar{T}_{1}$, we have that

$$
\begin{aligned}
B_{312} \leqslant & \frac{1}{A^{7}} \int_{0}^{1}\left|\tilde{\mathcal{Y}}_{1}-\tilde{\mathcal{Y}}_{2}\right| \tilde{\mathcal{Y}}_{2, \eta}(t, \eta) \\
& \times\left(\int_{0}^{\eta} e^{-\frac{1}{A_{2}}\left(\tilde{\mathcal{Y}}_{2}(t, \eta)-\tilde{\mathcal{Y}}_{2}(t, \theta)\right)}\left|\tilde{\mathcal{Y}}_{1}-\tilde{\mathcal{Y}}_{2}\right|\left|\tilde{\mathcal{U}}_{2}\right|^{3} \tilde{\mathcal{Y}}_{2, \eta}(t, \theta) d \theta\right) d \eta \\
& +\frac{4}{A^{4}} \int_{0}^{1}\left|\tilde{\mathcal{Y}}_{1}-\tilde{\mathcal{Y}}_{2}\right| \tilde{\mathcal{Y}}_{2, \eta}(t, \eta)\left(\int_{0}^{\eta} e^{-\frac{1}{A_{2}}\left(\tilde{\mathcal{Y}}_{2}(t, \eta)-\tilde{\mathcal{Y}}_{2}(t, \theta)\right)}\right. \\
& \left.\times\left(\int_{0}^{\theta} e^{-\frac{1}{A}\left(\tilde{\mathcal{Y}}_{2}(t, \theta)-\tilde{\mathcal{Y}}_{2}(t, l)\right)}\left(\tilde{\mathcal{U}}_{1}-\tilde{\mathcal{U}}_{2}\right)^{2}(t, l) d l\right)^{1 / 2} \tilde{\mathcal{U}}_{2}^{+} \tilde{\mathcal{Y}}_{2, \eta}(t, \theta) d \theta\right) d \eta \\
& +\frac{\sqrt{2}}{A^{3}} \int_{0}^{1}\left|\tilde{\mathcal{Y}}_{1}-\tilde{\mathcal{Y}}_{2}\right| \tilde{\mathcal{Y}}_{2, \eta}(t, \eta)\left(\int_{0}^{\eta} e^{-\frac{1}{A_{2}}\left(\tilde{\mathcal{Y}}_{2}(t, \eta)-\tilde{\mathcal{Y}}_{2}(t, \theta)\right)}\right.
\end{aligned}
$$




$$
\begin{aligned}
& \left.\times\left(\int_{0}^{\theta} e^{-\frac{1}{a}\left(\tilde{\mathcal{Y}}_{2}(t, \theta)-\tilde{\mathcal{Y}}_{2}(t, l)\right)}\left(\tilde{\mathcal{Y}}_{1}-\tilde{\mathcal{Y}}_{2}\right)^{2}(t, l) d l\right)^{1 / 2} \tilde{\mathcal{U}}_{2}^{+} \tilde{\mathcal{Y}}_{2, \eta}(t, \theta) d \theta\right) d \eta \\
& +\frac{1}{A^{3}} \int_{0}^{1}\left|\tilde{\mathcal{Y}}_{1}-\tilde{\mathcal{Y}}_{2}\right| \tilde{\mathcal{Y}}_{2, \eta}(t, \eta)\left(\int_{0}^{\eta} e^{-\frac{1}{A_{2}}\left(\tilde{\mathcal{Y}}_{2}(t, \eta)-\tilde{\mathcal{Y}}_{2}(t, \theta)\right)}\right. \\
& \left.\times\left(\int_{0}^{\theta} e^{-\frac{1}{a}\left(\tilde{\mathcal{Y}}_{2}(t, \theta)-\tilde{\mathcal{Y}}_{2}(t, l)\right)}\left|\tilde{\mathcal{Y}}_{1}-\tilde{\mathcal{Y}}_{2}\right|(t, l) d l\right) \tilde{\mathcal{U}}_{2}^{+} \tilde{\mathcal{Y}}_{2, \eta}(t, \theta) d \theta\right) d \eta \\
& +\frac{8 \sqrt{2}}{\sqrt{3} e A^{3}} \int_{0}^{1}\left|\tilde{\mathcal{Y}}_{1}-\tilde{\mathcal{Y}}_{2}\right| \tilde{\mathcal{Y}}_{2, \eta}(t, \eta)\left(\int_{0}^{\eta} e^{-\frac{1}{A_{2}}\left(\tilde{\mathcal{Y}}_{2}(t, \eta)-\tilde{\mathcal{Y}}_{2}(t, \theta)\right)}\right. \\
& \left.\times\left(\int_{0}^{\theta} e^{-\frac{3}{4 A}\left(\tilde{\mathcal{Y}}_{2}(t, \theta)-\tilde{\mathcal{Y}}_{2}(t, l)\right)} d l\right)^{1 / 2} \tilde{\mathcal{U}}_{2}^{+} \tilde{\mathcal{Y}}_{2, \eta}(t, \theta) d \theta\right) d \eta\left|A_{1}-A_{2}\right| \\
& \leqslant 5\left\|\tilde{\mathcal{Y}}_{1}-\tilde{\mathcal{Y}}_{2}\right\|^{2}+\frac{1}{A^{14}} \int_{0}^{1} \tilde{\mathcal{Y}}_{2, \eta}^{2}(t, \eta) \\
& \times\left(\int_{0}^{\eta} e^{-\frac{1}{A_{2}}\left(\tilde{\mathcal{Y}}_{2}(t, \eta)-\tilde{\mathcal{Y}}_{2}(t, \theta)\right)}\left|\tilde{\mathcal{Y}}_{1}-\tilde{\mathcal{Y}}_{2}\right|\left|\tilde{\mathcal{U}}_{2}\right|^{3} \tilde{\mathcal{Y}}_{2, \eta}(t, \theta) d \theta\right)^{2} d \eta \\
& +\frac{16}{A^{8}} \int_{0}^{1} \tilde{\mathcal{Y}}_{2, \eta}^{2}(t, \eta)\left(\int_{0}^{\eta} e^{-\frac{1}{A_{2}}\left(\tilde{\mathcal{Y}}_{2}(t, \eta)-\tilde{\mathcal{Y}}_{2}(t, \theta)\right)}\right. \\
& \left.\times\left(\int_{0}^{\theta} e^{-\frac{1}{A}\left(\tilde{\mathcal{Y}}_{2}(t, \theta)-\tilde{\mathcal{Y}}_{2}(t, l)\right)}\left(\tilde{\mathcal{U}}_{1}-\tilde{\mathcal{U}}_{2}\right)^{2}(t, l) d l\right)^{1 / 2} \tilde{\mathcal{U}}_{2}^{+} \tilde{\mathcal{Y}}_{2, \eta}(t, \theta) d \theta\right)^{2} d \eta \\
& +\frac{2}{A^{6}} \int_{0}^{1} \tilde{\mathcal{Y}}_{2, \eta}^{2}(t, \eta)\left(\int_{0}^{\eta} e^{-\frac{1}{A_{2}}\left(\tilde{\mathcal{Y}}_{2}(t, \eta)-\tilde{\mathcal{Y}}_{2}(t, \theta)\right)}\right. \\
& \left.\times\left(\int_{0}^{\theta} e^{-\frac{1}{a}\left(\tilde{\mathcal{Y}}_{2}(t, \theta)-\tilde{\mathcal{Y}}_{2}(t, l)\right)}\left(\tilde{\mathcal{Y}}_{1}-\tilde{\mathcal{Y}}_{2}\right)^{2}(t, l) d l\right)^{1 / 2} \tilde{\mathcal{U}}_{2}^{+} \tilde{\mathcal{Y}}_{2, \eta}(t, \theta) d \theta\right)^{2} d \eta \\
& +\frac{1}{A^{6}} \int_{0}^{1} \tilde{\mathcal{Y}}_{2, \eta}^{2}(t, \eta)\left(\int_{0}^{\eta} e^{-\frac{1}{A_{2}}\left(\tilde{\mathcal{Y}}_{2}(t, \eta)-\tilde{\mathcal{Y}}_{2}(t, \theta)\right)}\right. \\
& \left.\times\left(\int_{0}^{\theta} e^{-\frac{1}{a}\left(\tilde{\mathcal{Y}}_{2}(t, \theta)-\tilde{\mathcal{Y}}_{2}(t, l)\right)}\left|\tilde{\mathcal{Y}}_{1}-\tilde{\mathcal{Y}}_{2}\right|(t, l) d l\right) \tilde{\mathcal{U}}_{2}^{+} \tilde{\mathcal{Y}}_{2, \eta}(t, \theta) d \theta\right)^{2} d \eta \\
& +\frac{128}{3 e^{2} A^{6}} \int_{0}^{1} \tilde{\mathcal{Y}}_{2, \eta}^{2}(t, \eta)\left(\int_{0}^{\eta} e^{-\frac{1}{A_{2}}\left(\tilde{\mathcal{Y}}_{2}(t, \eta)-\tilde{\mathcal{Y}}_{2}(t, \theta)\right)}\right. \\
& \left.\times\left(\int_{0}^{\theta} e^{-\frac{3}{4 A}\left(\tilde{\mathcal{Y}}_{2}(t, \theta)-\tilde{\mathcal{Y}}_{2}(t, l)\right)} d l\right)^{1 / 2} \tilde{\mathcal{U}}_{2}^{+} \tilde{\mathcal{Y}}_{2, \eta}(t, \theta) d \theta\right)^{2} d \eta\left|A_{1}-A_{2}\right|^{2} \\
& \leqslant 5\left\|\tilde{\mathcal{Y}}_{1}-\tilde{\mathcal{Y}}_{2}\right\|^{2}+\frac{1}{A^{14}} \int_{0}^{1} \tilde{\mathcal{Y}}_{2, \eta}^{2}(t, \eta)\left(\int_{0}^{\eta} e^{-\frac{1}{A_{2}}\left(\tilde{\mathcal{Y}}_{2}(t, \eta)-\tilde{\mathcal{Y}}_{2}(t, \theta)\right)} \tilde{\mathcal{U}}_{2}^{4} \tilde{\mathcal{Y}}_{2, \eta}(t, \theta) d \theta\right)
\end{aligned}
$$




$$
\begin{aligned}
& \times\left(\int_{0}^{\eta} e^{-\frac{1}{A_{2}}\left(\tilde{\mathcal{Y}}_{2}(t, \eta)-\tilde{\mathcal{Y}}_{2}(t, \theta)\right)}\left(\tilde{\mathcal{Y}}_{1}-\tilde{\mathcal{Y}}_{2}\right)^{2} \tilde{\mathcal{U}}_{2}^{2} \tilde{\mathcal{Y}}_{2, \eta}(t, \theta) d \theta\right) d \eta \\
& +\frac{16}{A^{8}} \int_{0}^{1} \tilde{\mathcal{Y}}_{2, \eta}^{2}(t, \eta)\left(\int_{0}^{\eta} e^{-\frac{1}{A_{2}}\left(\tilde{\mathcal{Y}}_{2}(t, \eta)-\tilde{\mathcal{Y}}_{2}(t, \theta)\right)} \tilde{\mathcal{U}}_{2}^{2} \tilde{\mathcal{Y}}_{2, \eta}(t, \theta) d \theta\right) \\
& \times\left(\int_{0}^{\eta} e^{-\frac{1}{A_{2}}\left(\tilde{\mathcal{Y}}_{2}(t, \eta)-\tilde{\mathcal{Y}}_{2}(t, \theta)\right)}\right. \\
& \left.\times\left(\int_{0}^{\theta} e^{-\frac{1}{A}\left(\tilde{\mathcal{Y}}_{2}(t, \theta)-\tilde{\mathcal{Y}}_{2}(t, l)\right)}\left(\tilde{\mathcal{U}}_{1}-\tilde{\mathcal{U}}_{2}\right)^{2}(t, l) d l\right) \tilde{\mathcal{Y}}_{2, \eta}(t, \theta) d \theta\right) d \eta \\
& +\frac{2}{A^{6}} \int_{0}^{1} \tilde{\mathcal{Y}}_{2, \eta}^{2}(t, \eta)\left(\int_{0}^{\eta} e^{-\frac{1}{A_{2}}\left(\tilde{\mathcal{Y}}_{2}(t, \eta)-\tilde{\mathcal{Y}}_{2}(t, \theta)\right)} \tilde{\mathcal{U}}_{2}^{2} \tilde{\mathcal{Y}}_{2, \eta}(t, \theta) d \theta\right) \\
& \times\left(\int_{0}^{\eta} e^{-\frac{1}{A_{2}}\left(\tilde{\mathcal{Y}}_{2}(t, \eta)-\tilde{\mathcal{Y}}_{2}(t, \theta)\right)}\right. \\
& \left.\times\left(\int_{0}^{\theta} e^{-\frac{1}{a}\left(\tilde{\mathcal{Y}}_{2}(t, \theta)-\tilde{\mathcal{Y}}_{2}(t, l)\right)}\left(\tilde{\mathcal{Y}}_{1}-\tilde{\mathcal{Y}}_{2}\right)^{2}(t, l) d l\right) \tilde{\mathcal{Y}}_{2, \eta}(t, \theta) d \theta\right) d \eta \\
& +\frac{1}{A^{6}} \int_{0}^{1} \tilde{\mathcal{Y}}_{2, \eta}^{2}(t, \eta)\left(\int_{0}^{\eta} e^{-\frac{1}{A_{2}}\left(\tilde{\mathcal{Y}}_{2}(t, \eta)-\tilde{\mathcal{Y}}_{2}(t, \theta)\right)} \tilde{\mathcal{U}}_{2}^{2} \tilde{\mathcal{Y}}_{2, \eta}(t, \theta) d \theta\right) \\
& \times\left(\int_{0}^{\eta} e^{-\frac{1}{A_{2}}\left(\tilde{\mathcal{Y}}_{2}(t, \eta)-\tilde{\mathcal{Y}}_{2}(t, \theta)\right)}\right. \\
& \left.\times\left(\int_{0}^{\theta} e^{-\frac{1}{a}\left(\tilde{\mathcal{Y}}_{2}(t, \theta)-\tilde{\mathcal{Y}}_{2}(t, l)\right)}\left|\tilde{\mathcal{Y}}_{1}-\tilde{\mathcal{Y}}_{2}\right|(t, l) d l\right)^{2} \tilde{\mathcal{Y}}_{2, \eta}(t, \theta) d \theta\right) d \eta \\
& +\frac{128}{3 e^{2} A^{6}} \int_{0}^{1} \tilde{\mathcal{Y}}_{2, \eta}^{2}(t, \eta)\left(\int_{0}^{\eta} e^{-\frac{1}{A_{2}}\left(\tilde{\mathcal{Y}}_{2}(t, \eta)-\tilde{\mathcal{Y}}_{2}(t, \theta)\right)} \tilde{\mathcal{U}}_{2}^{2} \tilde{\mathcal{Y}}_{2, \eta}(t, \theta) d \theta\right) \\
& \times\left(\int_{0}^{\eta} e^{-\frac{1}{A_{2}}\left(\tilde{\mathcal{Y}}_{2}(t, \eta)-\tilde{\mathcal{Y}}_{2}(t, \theta)\right)}\right. \\
& \left.\times\left(\int_{0}^{\theta} e^{-\frac{3}{4 A}\left(\tilde{\mathcal{Y}}_{2}(t, \theta)-\tilde{\mathcal{Y}}_{2}(t, l)\right.} d l\right) \tilde{\mathcal{Y}}_{2, \eta}(t, \theta) d \theta\right) d \eta\left|A_{1}-A_{2}\right|^{2} \\
& \leqslant 5\left\|\tilde{\mathcal{Y}}_{1}-\tilde{\mathcal{Y}}_{2}\right\|^{2} \\
& +\frac{2}{A^{4}} \int_{0}^{1} \tilde{\mathcal{P}}_{2} \tilde{\mathcal{Y}}_{2, \eta}^{2}(t, \eta)\left(\int_{0}^{\eta} e^{-\frac{1}{A_{2}}\left(\tilde{\mathcal{Y}}_{2}(t, \eta)-\tilde{\mathcal{Y}}_{2}(t, \theta)\right)}\left(\tilde{\mathcal{Y}}_{1}-\tilde{\mathcal{Y}}_{2}\right)^{2}(t, \theta) d \theta\right) d \eta \\
& +\frac{64}{A^{7}} \int_{0}^{1} \tilde{\mathcal{P}}_{2} \tilde{\mathcal{Y}}_{2, \eta}^{2}(t, \eta)\left(\int_{0}^{\eta} e^{-\frac{1}{2 A}\left(\tilde{\mathcal{Y}}_{2}(t, \eta)-\tilde{\mathcal{Y}}_{2}(t, \theta)\right)}\right.
\end{aligned}
$$




$$
\begin{aligned}
& \left.\times\left(\int_{0}^{\theta} e^{-\frac{1}{A}\left(\tilde{\mathcal{Y}}_{2}(t, \theta)-\tilde{\mathcal{Y}}_{2}(t, l)\right)}\left(\tilde{\mathcal{U}}_{1}-\tilde{\mathcal{U}}_{2}\right)^{2}(t, l) d l\right) \tilde{\mathcal{Y}}_{2, \eta}(t, \theta) d \theta\right) d \eta \\
& +\frac{8}{A^{5}} \int_{0}^{1} \tilde{\mathcal{P}}_{2} \tilde{\mathcal{Y}}_{2, \eta}^{2}(t, \eta)\left(\int_{0}^{\eta} e^{-\frac{1}{2 A_{2}}\left(\tilde{\mathcal{Y}}_{2}(t, \eta)-\tilde{\mathcal{Y}}_{2}(t, \theta)\right)}\right. \\
& \left.\times\left(\int_{0}^{\theta} e^{-\frac{1}{A_{2}}\left(\tilde{\mathcal{Y}}_{2}(t, \theta)-\tilde{\mathcal{Y}}_{2}(t, l)\right)}\left(\tilde{\mathcal{Y}}_{1}-\tilde{\mathcal{Y}}_{2}\right)^{2}(t, l) d l\right) \tilde{\mathcal{Y}}_{2, \eta}(t, \theta) d \theta\right) d \eta \\
& +\frac{4}{A^{5}} \int_{0}^{1} \tilde{\mathcal{P}}_{2} \tilde{\mathcal{Y}}_{2, \eta}^{2}(t, \eta)\left(\int_{0}^{\eta} e^{-\frac{1}{2 A_{2}}\left(\tilde{\mathcal{Y}}_{2}(t, \eta)-\tilde{\mathcal{Y}}_{2}(t, \theta)\right)}\left(\int_{0}^{\theta} e^{-\frac{1}{A_{2}}\left(\tilde{\mathcal{Y}}_{2}(t, \theta)-\tilde{\mathcal{Y}}_{2}(t, l)\right)} d l\right)\right. \\
& \left.\times\left(\int_{0}^{\theta} e^{-\frac{1}{A_{2}}\left(\tilde{\mathcal{Y}}_{2}(t, \theta)-\tilde{\mathcal{Y}}_{2}(t, l)\right)}\left(\tilde{\mathcal{Y}}_{1}-\tilde{\mathcal{Y}}_{2}\right)^{2}(t, l) d l\right) \tilde{\mathcal{Y}}_{2, \eta}(t, \theta) d \theta\right) d \eta \\
& +\frac{512}{3 e^{2} A^{5}} \int_{0}^{1} \tilde{\mathcal{P}}_{2} \tilde{\mathcal{Y}}_{2, \eta}^{2}(t, \eta)\left(\int_{0}^{\eta} e^{-\frac{1}{2 A}\left(\tilde{\mathcal{Y}}_{2}(t, \eta)-\tilde{\mathcal{Y}}_{2}(t, \theta)\right)}\right. \\
& \left.\times\left(\int_{0}^{\theta} e^{-\frac{3}{4 A}\left(\tilde{\mathcal{Y}}_{2}(t, \theta)-\tilde{\mathcal{Y}}_{2}(t, l)\right)} d l\right) \tilde{\mathcal{Y}}_{2, \eta}(t, \theta) d \theta\right) d \eta\left|A_{1}-A_{2}\right|^{2} \\
& \leqslant 5\left\|\tilde{\mathcal{Y}}_{1}-\tilde{\mathcal{Y}}_{2}\right\|^{2} \\
& +A \int_{0}^{1} \tilde{\mathcal{Y}}_{2, \eta}(t, \eta) e^{-\frac{1}{A_{2}} \tilde{\mathcal{Y}}_{2}(t, \eta)}\left(\int_{0}^{\eta} e^{\frac{1}{A_{2}} \tilde{\mathcal{Y}}_{2}(t, \theta)}\left(\tilde{\mathcal{Y}}_{1}-\tilde{\mathcal{Y}}_{2}\right)^{2}(t, \theta) d \theta\right) d \eta \\
& +\frac{32}{A^{2}} \int_{0}^{1} \tilde{\mathcal{Y}}_{2, \eta}(t, \eta)\left(\int_{0}^{\eta} \tilde{\mathcal{Y}}_{2, \eta}(t, \theta) e^{-\frac{1}{2 A} \tilde{\mathcal{Y}}_{2}(t, \theta)}\right. \\
& \left.\times\left(\int_{0}^{\theta} e^{-\left(\frac{1}{2 A} \tilde{\mathcal{Y}}_{2}(t, \eta)-\frac{1}{A} \tilde{\mathcal{Y}}_{2}(t, l)\right)}\left(\tilde{\mathcal{U}}_{1}-\tilde{\mathcal{U}}_{2}\right)^{2}(t, l) d l\right) d \theta\right) d \eta \\
& +4 \int_{0}^{1} \tilde{\mathcal{Y}}_{2, \eta}(t, \eta)\left(\int_{0}^{\eta} \tilde{\mathcal{Y}}_{2, \eta}(t, \theta) e^{-\frac{1}{2 A_{2}} \tilde{\mathcal{Y}}_{2}(t, \theta)}\right. \\
& \left.\times\left(\int_{0}^{\theta} e^{-\left(\frac{1}{2 A_{2}} \tilde{\mathcal{Y}}_{2}(t, \eta)-\frac{1}{A_{2}} \tilde{\mathcal{Y}}_{2}(t, l)\right)}\left(\tilde{\mathcal{Y}}_{1}-\tilde{\mathcal{Y}}_{2}\right)^{2}(t, l) d l\right) d \theta\right) d \eta \\
& +2 \int_{0}^{1} \tilde{\mathcal{Y}}_{2, \eta}(t, \eta)\left(\int_{0}^{\eta} \tilde{\mathcal{Y}}_{2, \eta}(t, \theta) e^{-\frac{1}{2 A_{2}} \tilde{\mathcal{Y}}_{2}(t, \theta)}\right. \\
& \left.\times\left(\int_{0}^{\theta} e^{-\left(\frac{1}{2 A_{2}} \tilde{\mathcal{Y}}_{2}(t, \eta)-\frac{1}{A_{2}} \tilde{\mathcal{Y}}_{2}(t, l)\right)} d l\right) d \theta\right) d \eta\left\|\tilde{\mathcal{Y}}_{1}-\tilde{\mathcal{Y}}_{2}\right\|^{2} \\
& +\frac{256}{3 e^{2}} \int_{0}^{1} \tilde{\mathcal{Y}}_{2, \eta}(t, \eta)\left(\int_{0}^{\eta} \tilde{\mathcal{Y}}_{2, \eta}(t, \theta) e^{-\frac{1}{4 A} \tilde{\mathcal{Y}}_{2}(t, \theta)}\right.
\end{aligned}
$$




$$
\begin{aligned}
& \left.\times\left(\int_{0}^{\theta} e^{-\left(\frac{1}{2 A} \tilde{\mathcal{Y}}_{2}(t, \eta)-\frac{3}{4 A} \tilde{\mathcal{Y}}_{2}(t, l)\right)} d l\right) d \theta\right) d \eta\left|A_{1}-A_{2}\right|^{2} \\
& \leqslant 5\left\|\tilde{\mathcal{Y}}_{1}-\tilde{\mathcal{Y}}_{2}\right\|^{2}+A\left[-\left.A_{2} \int_{0}^{\eta} e^{-\frac{1}{A_{2}}\left(\tilde{\mathcal{Y}}_{2}(t, \eta)-\tilde{\mathcal{Y}}_{2}(t, \theta)\right)}\left(\tilde{\mathcal{Y}}_{1}-\tilde{\mathcal{Y}}_{2}\right)^{2}(t, \theta) d \theta\right|_{\eta=0} ^{1}\right. \\
& \left.+\int_{0}^{1}\left(\tilde{\mathcal{Y}}_{1}-\tilde{\mathcal{Y}}_{2}\right)^{2}(t, \eta) d \eta\right] \\
& +\frac{32}{A} \int_{0}^{1} \tilde{\mathcal{Y}}_{2, \eta}(t, \eta)\left[-\left.2 \int_{0}^{\theta} e^{\frac{1}{A} \tilde{\mathcal{Y}}_{2}(t, l)-\frac{1}{2 A}\left(\tilde{\mathcal{Y}}_{2}(t, \eta)+\tilde{\mathcal{Y}}_{2}(t, \theta)\right)}\left(\tilde{\mathcal{U}}_{1}-\tilde{\mathcal{U}}_{2}\right)^{2}(t, l) d l\right|_{\theta=0} ^{\eta}\right. \\
& \left.+2 \int_{0}^{\eta} e^{\frac{1}{2 A}\left(\tilde{\mathcal{Y}}_{2}(t, \theta)-\tilde{\mathcal{Y}}_{2}(t, \eta)\right)}\left(\tilde{\mathcal{U}}_{1}-\tilde{\mathcal{U}}_{2}\right)^{2}(t, \theta) d \theta\right] d \eta \\
& +4 A_{2} \int_{0}^{1} \tilde{\mathcal{Y}}_{2, \eta}(t, \eta) \\
& \times\left[-\left.2 \int_{0}^{\theta} e^{\frac{1}{A_{2}} \tilde{\mathcal{Y}}_{2}(t, l)-\frac{1}{2 A_{2}}\left(\tilde{\mathcal{Y}}_{2}(t, \eta)+\tilde{\mathcal{Y}}_{2}(t, \theta)\right)}\left(\tilde{\mathcal{Y}}_{1}-\tilde{\mathcal{Y}}_{2}\right)^{2}(t, l) d l\right|_{\theta=0} ^{\eta}\right. \\
& \left.+2 \int_{0}^{\eta} e^{\frac{1}{2 A_{2}}\left(\tilde{\mathcal{Y}}_{2}(t, \theta)-\tilde{\mathcal{Y}}_{2}(t, \eta)\right)}\left(\tilde{\mathcal{Y}}_{1}-\tilde{\mathcal{Y}}_{2}\right)^{2}(t, \theta) d \theta\right] d \eta \\
& +2 A_{2} \int_{0}^{1} \tilde{\mathcal{Y}}_{2, \eta}(t, \eta)\left[-\left.2 \int_{0}^{\theta} e^{\frac{1}{A_{2}} \tilde{\mathcal{Y}}_{2}(t, l)-\frac{1}{2 A_{2}}\left(\tilde{\mathcal{Y}}_{2}(t, \theta)+\tilde{\mathcal{Y}}_{2}(t, \eta)\right)} d l\right|_{\theta=0} ^{\eta}\right. \\
& \left.+2 \int_{0}^{\eta} e^{\frac{1}{2 A_{2}}\left(\tilde{\mathcal{Y}}_{2}(t, \theta)-\tilde{\mathcal{Y}}_{2}(t, \eta)\right)} d \theta\right] d \eta\left\|\tilde{\mathcal{Y}}_{1}-\tilde{\mathcal{Y}}_{2}\right\|^{2} \\
& +\frac{256 A}{3 e^{2}} \int_{0}^{1} \tilde{\mathcal{Y}}_{2, \eta}(t, \eta)\left[-\left.4 \int_{0}^{\theta} e^{\frac{3}{4 A} \tilde{\mathcal{Y}}_{2}(t, l)-\left(\frac{1}{4 A} \tilde{\mathcal{Y}}_{2}(t, \theta)+\frac{1}{2 A} \tilde{\mathcal{Y}}_{2}(t, \eta)\right)}\right|_{\theta=0} ^{\eta}\right. \\
& \left.+4 \int_{0}^{\eta} e^{\frac{1}{2 A}\left(\tilde{\mathcal{Y}}_{2}(t, \theta)-\tilde{\mathcal{Y}}_{2}(t, \eta)\right)} d \theta\right] d \eta\left|A_{1}-A_{2}\right|^{2} \\
& \leqslant \mathcal{O}(1)\left\|\tilde{\mathcal{Y}}_{1}-\tilde{\mathcal{Y}}_{2}\right\|^{2} \\
& +\frac{64}{A} \int_{0}^{1} \tilde{\mathcal{Y}}_{2, \eta}(t, \eta) e^{-\frac{1}{2 A} \tilde{\mathcal{Y}}_{2}(t, \eta)}\left(\int_{0}^{\eta} e^{\frac{1}{2 A} \tilde{\mathcal{Y}}_{2}(t, \theta)}\left(\tilde{\mathcal{U}}_{1}-\tilde{\mathcal{U}}_{2}\right)^{2}(t, \theta) d \theta\right) d \eta \\
& +8 A \int_{0}^{1} \tilde{\mathcal{Y}}_{2, \eta}(t, \eta) e^{-\frac{1}{2 A_{2}} \tilde{\mathcal{Y}}_{2}(t, \eta)}\left(\int_{0}^{\eta} e^{\frac{1}{2 A_{2}} \tilde{\mathcal{Y}}_{2}(t, \theta)}\left(\tilde{\mathcal{Y}}_{1}-\tilde{\mathcal{Y}}_{2}\right)^{2}(t, \theta) d \theta\right) d \eta \\
& +4 A \int_{0}^{1} \tilde{\mathcal{Y}}_{2, \eta}(t, \eta) e^{-\frac{1}{2 A_{2}} \tilde{Y}_{2}(t, \eta)}\left(\int_{0}^{\eta} e^{\frac{1}{2 \lambda_{2}} \tilde{\mathcal{Y}}_{2}(t, \theta)} d \theta\right) d \eta\left\|\tilde{\mathcal{Y}}_{1}-\tilde{\mathcal{Y}}_{2}\right\|^{2}
\end{aligned}
$$




$$
+\frac{1024 A}{3 e^{2}} \int_{0}^{1} \tilde{\mathcal{Y}}_{2, \eta}(t, \eta) e^{-\frac{1}{2 A} \tilde{\mathcal{Y}}_{2}(t, \eta)}\left(\int_{0}^{\eta} e^{\frac{1}{2 A} \tilde{\mathcal{Y}}_{2}(t, \theta)} d \theta\right) d \eta\left|A_{1}-A_{2}\right|^{2}
$$

$\leqslant \mathcal{O}(1)\left\|\tilde{\mathcal{Y}}_{1}-\tilde{\mathcal{Y}}_{2}\right\|^{2}$

$$
\begin{aligned}
& +64\left[-\left.2 \int_{0}^{\eta} e^{\frac{1}{2 A}\left(\tilde{\mathcal{Y}}_{2}(t, \theta)-\tilde{\mathcal{Y}}_{2}(t, \eta)\right)}\left(\tilde{\mathcal{U}}_{1}-\tilde{\mathcal{U}}_{2}\right)^{2}(t, \theta) d \theta\right|_{\eta=0} ^{1}\right. \\
& \left.+2 \int_{0}^{1}\left(\tilde{\mathcal{U}}_{1}-\tilde{\mathcal{U}}_{2}\right)^{2}(t, \eta) d \eta\right] \\
& +8 A A_{2}\left[-\left.2 \int_{0}^{\eta} e^{\frac{1}{2 A_{2}}\left(\tilde{\mathcal{Y}}_{2}(t, \theta)-\tilde{\mathcal{Y}}_{2}(t, \eta)\right)}\left(\tilde{\mathcal{Y}}_{1}-\tilde{\mathcal{Y}}_{2}\right)^{2}(t, \theta) d \theta\right|_{\eta=0} ^{1}\right. \\
& \left.+2 \int_{0}^{1}\left(\tilde{\mathcal{Y}}_{1}-\tilde{\mathcal{Y}}_{2}\right)^{2}(t, \eta) d \eta\right] \\
& +4 A A_{2}\left[-\left.2 \int_{0}^{\eta} e^{\frac{1}{2 A_{2}}\left(\tilde{\mathcal{Y}}_{2}(t, \theta)-\tilde{\mathcal{Y}}_{2}(t, \eta)\right)} d \theta\right|_{\eta=0} ^{1}+2 \int_{0}^{1} d \eta\right]\left\|\tilde{\mathcal{Y}}_{1}-\tilde{\mathcal{Y}}_{2}\right\|^{2} \\
& +\frac{1024 A^{2}}{3 e^{2}}\left[-\left.2 \int_{0}^{\eta} e^{\frac{1}{2 A}\left(\tilde{\mathcal{Y}}_{2}(t, \theta)-\tilde{\mathcal{Y}}_{2}(t, \eta)\right)} d \theta\right|_{\eta=0} ^{1}+2 \int_{0}^{1} d \eta\right]\left|A_{1}-A_{2}\right|^{2} \\
& \leqslant \\
& \mathcal{O}(1)\left(\left\|\tilde{\mathcal{Y}}_{1}-\tilde{\mathcal{Y}}_{2}\right\|^{2}+\left\|\tilde{\mathcal{U}}_{1}-\tilde{\mathcal{U}}_{2}\right\|^{2}+\left|A_{1}-A_{2}\right|^{2}\right) .
\end{aligned}
$$

Similar calculations yield for $B_{313}$,

$$
\begin{aligned}
& B_{313} \leqslant \frac{1}{A^{7}} \int_{0}^{1}\left|\tilde{\mathcal{Y}}_{1}-\tilde{\mathcal{Y}}_{2}\right| \tilde{\mathcal{Y}}_{2, \eta}(t, \eta) \\
& \times\left(\int_{0}^{\eta} e^{-\frac{1}{A_{2}}\left(\tilde{\mathcal{Y}}_{2}(t, \eta)-\tilde{\mathcal{Y}}_{2}(t, \theta)\right)}\left|\tilde{\mathcal{Y}}_{1}-\tilde{\mathcal{Y}}_{2}\right| \tilde{\mathcal{P}}_{2}\left|\tilde{\mathcal{U}}_{2}\right| \tilde{\mathcal{Y}}_{2, \eta}(t, \theta) d \theta\right) d \eta \\
& +\frac{2 \sqrt{2}}{A^{4}} \int_{0}^{1}\left|\tilde{\mathcal{Y}}_{1}-\tilde{\mathcal{Y}}_{2}\right| \tilde{\mathcal{Y}}_{2, \eta}(t, \eta)\left(\int_{0}^{\eta} e^{-\frac{1}{A_{2}}\left(\tilde{\mathcal{Y}}_{2}(t, \eta)-\tilde{\mathcal{Y}}_{2}(t, \theta)\right)}\right. \\
& \left.\times\left(\int_{0}^{\theta} e^{-\frac{1}{A}\left(\tilde{\mathcal{Y}}_{2}(t, \theta)-\tilde{\mathcal{Y}}_{2}(t, l)\right)}\left(\tilde{\mathcal{P}}_{1}^{1 / 2}-\tilde{\mathcal{P}}_{2}^{1 / 2}\right)^{2}(t, l) d l\right)^{1 / 2} \tilde{\mathcal{U}}_{2}^{+} \tilde{\mathcal{Y}}_{2, \eta}(t, \theta) d \theta\right) d \eta \\
& +\frac{1}{\sqrt{2} A^{3}} \int_{0}^{1}\left|\tilde{\mathcal{Y}}_{1}-\tilde{\mathcal{Y}}_{2}\right| \tilde{\mathcal{Y}}_{2, \eta}(t, \eta)\left(\int_{0}^{\eta} e^{-\frac{1}{A_{2}}\left(\tilde{\mathcal{Y}}_{2}(t, \eta)-\tilde{\mathcal{Y}}_{2}(t, \theta)\right)}\right. \\
& \left.\times\left(\int_{0}^{\theta} e^{-\frac{1}{a}\left(\tilde{\mathcal{Y}}_{2}(t, \theta)-\tilde{\mathcal{Y}}_{2}(t, l)\right)}\left(\tilde{\mathcal{Y}}_{1}-\tilde{\mathcal{Y}}_{2}\right)^{2}(t, l) d l\right)^{1 / 2} \tilde{\mathcal{U}}_{2}^{+} \tilde{\mathcal{Y}}_{2, \eta}(t, \theta) d \theta\right) d \eta \\
& +\frac{1}{2 A^{3}} \int_{0}^{1}\left|\tilde{\mathcal{Y}}_{1}-\tilde{\mathcal{Y}}_{2}\right| \tilde{\mathcal{Y}}_{2, \eta}(t, \eta)\left(\int_{0}^{\eta} e^{-\frac{1}{A_{2}}\left(\tilde{\mathcal{Y}}_{2}(t, \eta)-\tilde{\mathcal{Y}}_{2}(t, \theta)\right)}\right.
\end{aligned}
$$




$$
\begin{aligned}
& \left.\times\left(\int_{0}^{\theta} e^{-\frac{1}{a}\left(\tilde{\mathcal{Y}}_{2}(t, \theta)-\tilde{\mathcal{Y}}_{2}(t, l)\right)}\left|\tilde{\mathcal{Y}}_{1}-\tilde{\mathcal{Y}}_{2}\right|(t, l) d l\right) \tilde{\mathcal{U}}_{2}^{+} \tilde{\mathcal{Y}}_{2, \eta}(t, \theta) d \theta\right) d \eta \\
& +\frac{4 \sqrt{2}}{\sqrt{3} e A^{3}} \int_{0}^{1}\left|\tilde{\mathcal{Y}}_{1}-\tilde{\mathcal{Y}}_{2}\right| \tilde{\mathcal{Y}}_{2, \eta}(t, \eta)\left(\int_{0}^{\eta} e^{-\frac{1}{A_{2}}\left(\tilde{\mathcal{Y}}_{2}(t, \eta)-\tilde{\mathcal{Y}}_{2}(t, \theta)\right)}\right. \\
& \left.\times\left(\int_{0}^{\theta} e^{-\frac{3}{4 A}\left(\tilde{\mathcal{Y}}_{2}(t, \theta)-\tilde{\mathcal{Y}}_{2}(t, l)\right)} d l\right)^{1 / 2} \tilde{\mathcal{U}}_{2}^{+} \tilde{\mathcal{Y}}_{2, \eta}(t, \theta) d \theta\right) d \eta\left|A_{1}-A_{2}\right| \\
& \leqslant 5\left\|\tilde{\mathcal{Y}}_{1}-\tilde{\mathcal{Y}}_{2}\right\|^{2}+\frac{1}{A^{14}} \int_{0}^{1} \tilde{\mathcal{Y}}_{2, \eta}^{2}(t, \eta) \\
& \times\left(\int_{0}^{\eta} e^{-\frac{1}{A_{2}}\left(\tilde{\mathcal{Y}}_{2}(t, \eta)-\tilde{\mathcal{Y}}_{2}(t, \theta)\right)}\left|\tilde{\mathcal{Y}}_{1}-\tilde{\mathcal{Y}}_{2}\right| \tilde{\mathcal{P}}_{2}\left|\tilde{\mathcal{U}}_{2}\right| \tilde{\mathcal{Y}}_{2, \eta}(t, \theta) d \theta\right)^{2} d \eta \\
& +\frac{8}{A^{8}} \int_{0}^{1} \tilde{\mathcal{Y}}_{2, \eta}^{2}(t, \eta)\left(\int_{0}^{\eta} e^{-\frac{1}{A_{2}}\left(\tilde{\mathcal{Y}}_{2}(t, \eta)-\tilde{\mathcal{Y}}_{2}(t, \theta)\right)}\right. \\
& \left.\times\left(\int_{0}^{\theta} e^{-\frac{1}{A}\left(\tilde{\mathcal{Y}}_{2}(t, \theta)-\tilde{\mathcal{Y}}_{2}(t, l)\right)}\left(\tilde{\mathcal{P}}_{1}^{1 / 2}-\tilde{\mathcal{P}}_{2}^{1 / 2}\right)^{2}(t, l) d l\right)^{1 / 2} \tilde{\mathcal{U}}_{2}^{+} \tilde{\mathcal{Y}}_{2, \eta}(t, \theta) d \theta\right)^{2} d \eta \\
& +\frac{1}{2 A^{6}} \int_{0}^{1} \tilde{\mathcal{Y}}_{2, \eta}^{2}(t, \eta)\left(\int_{0}^{\eta} e^{-\frac{1}{A_{2}}\left(\tilde{\mathcal{Y}}_{2}(t, \eta)-\tilde{\mathcal{Y}}_{2}(t, \theta)\right)}\right. \\
& \left.\times\left(\int_{0}^{\theta} e^{-\frac{1}{a}\left(\tilde{\mathcal{Y}}_{2}(t, \theta)-\tilde{\mathcal{Y}}_{2}(t, l)\right)}\left(\tilde{\mathcal{Y}}_{1}-\tilde{\mathcal{Y}}_{2}\right)^{2}(t, l) d l\right)^{1 / 2} \tilde{\mathcal{U}}_{2}^{+} \tilde{\mathcal{Y}}_{2, \eta}(t, \theta) d \theta\right)^{2} d \eta \\
& +\frac{1}{4 A^{6}} \int_{0}^{1} \tilde{\mathcal{Y}}_{2, \eta}^{2}(t, \eta)\left(\int_{0}^{\eta} e^{-\frac{1}{A_{2}}\left(\tilde{\mathcal{Y}}_{2}(t, \eta)-\tilde{\mathcal{Y}}_{2}(t, \theta)\right)}\right. \\
& \left.\times\left(\int_{0}^{\theta} e^{-\frac{1}{a}\left(\tilde{\mathcal{Y}}_{2}(t, \theta)-\tilde{\mathcal{Y}}_{2}(t, l)\right)}\left|\tilde{\mathcal{Y}}_{1}-\tilde{\mathcal{Y}}_{2}\right|(t, l) d l\right) \tilde{\mathcal{U}}_{2}^{+} \tilde{\mathcal{Y}}_{2, \eta}(t, \theta) d \theta\right)^{2} d \eta \\
& +\frac{32}{3 e^{2} A^{6}} \int_{0}^{1} \tilde{\mathcal{Y}}_{2, \eta}^{2}(t, \eta)\left(\int_{0}^{\eta} e^{-\frac{1}{A_{2}}\left(\tilde{\mathcal{Y}}_{2}(t, \eta)-\tilde{\mathcal{Y}}_{2}(t, \theta)\right)}\right. \\
& \left.\times\left(\int_{0}^{\theta} e^{-\frac{3}{4 A}\left(\tilde{\mathcal{Y}}_{2}(t, \theta)-\tilde{\mathcal{Y}}_{2}(t, l)\right)} d l\right)^{1 / 2} \tilde{\mathcal{U}}_{2}^{+} \tilde{\mathcal{Y}}_{2, \eta}(t, \theta) d \theta\right)^{2} d \eta\left|A_{1}-A_{2}\right|^{2} \\
& \leqslant 5\left\|\tilde{\mathcal{Y}}_{1}-\tilde{\mathcal{Y}}_{2}\right\|^{2} \\
& +\frac{1}{A^{14}} \int_{0}^{1} \tilde{\mathcal{Y}}_{2, \eta}^{2}(t, \eta)\left(\int_{0}^{\eta} e^{-\frac{1}{A_{2}}\left(\tilde{\mathcal{Y}}_{2}(t, \eta)-\tilde{\mathcal{Y}}_{2}(t, \theta)\right)} \tilde{\mathcal{U}}_{2}^{2} \tilde{\mathcal{Y}}_{2, \eta}(t, \theta) d \theta\right) \\
& \times\left(\int_{0}^{\eta} e^{-\frac{1}{A_{2}}\left(\tilde{\mathcal{Y}}_{2}(t, \eta)-\tilde{\mathcal{Y}}_{2}(t, \theta)\right)}\left(\tilde{\mathcal{Y}}_{1}-\tilde{\mathcal{Y}}_{2}\right)^{2} \tilde{\mathcal{P}}_{2}^{2} \tilde{\mathcal{Y}}_{2, \eta}(t, \theta) d \theta\right) d \eta \\
& +\frac{8}{A^{8}} \int_{0}^{1} \tilde{\mathcal{Y}}_{2, \eta}^{2}(t, \eta)\left(\int_{0}^{\eta} e^{-\frac{1}{A_{2}}\left(\tilde{\mathcal{Y}}_{2}(t, \eta)-\tilde{\mathcal{Y}}_{2}(t, \theta)\right)} \tilde{\mathcal{U}}_{2}^{2} \tilde{\mathcal{Y}}_{2, \eta}(t, \theta) d \theta\right)
\end{aligned}
$$




$$
\begin{aligned}
& \times\left(\int_{0}^{\eta} e^{-\frac{1}{A_{2}}\left(\tilde{\mathcal{Y}}_{2}(t, \eta)-\tilde{\mathcal{Y}}_{2}(t, \theta)\right)}\right. \\
& \left.\times\left(\int_{0}^{\theta} e^{-\frac{1}{A}\left(\tilde{\mathcal{Y}}_{2}(t, \theta)-\tilde{\mathcal{Y}}_{2}(t, l)\right)}\left(\tilde{\mathcal{P}}_{1}^{1 / 2}-\tilde{\mathcal{P}}_{2}^{1 / 2}\right)^{2}(t, l) d l\right) \tilde{\mathcal{Y}}_{2, \eta}(t, \theta) d \theta\right) d \eta \\
& +\frac{1}{2 A^{6}} \int_{0}^{1} \tilde{\mathcal{Y}}_{2, \eta}^{2}(t, \eta)\left(\int_{0}^{\eta} e^{-\frac{1}{A_{2}}\left(\tilde{\mathcal{Y}}_{2}(t, \eta)-\tilde{\mathcal{Y}}_{2}(t, \theta)\right)} \tilde{\mathcal{U}}_{2}^{2} \tilde{\mathcal{Y}}_{2, \eta}(t, \theta) d \theta\right) \\
& \times\left(\int_{0}^{\eta} e^{-\frac{1}{A_{2}}\left(\tilde{\mathcal{Y}}_{2}(t, \eta)-\tilde{\mathcal{Y}}_{2}(t, \theta)\right)}\right. \\
& \left.\times\left(\int_{0}^{\theta} e^{-\frac{1}{a}\left(\tilde{\mathcal{Y}}_{2}(t, \theta)-\tilde{\mathcal{Y}}_{2}(t, l)\right)}\left(\tilde{\mathcal{Y}}_{1}-\tilde{\mathcal{Y}}_{2}\right)^{2}(t, l) d l\right) \tilde{\mathcal{Y}}_{2, \eta}(t, \theta) d \theta\right) d \eta \\
& +\frac{1}{4 A^{6}} \int_{0}^{1} \tilde{\mathcal{Y}}_{2, \eta}^{2}(t, \eta)\left(\int_{0}^{\eta} e^{-\frac{1}{A_{2}}\left(\tilde{\mathcal{Y}}_{2}(t, \eta)-\tilde{\mathcal{Y}}_{2}(t, \theta)\right)} \tilde{\mathcal{U}}_{2}^{2} \tilde{\mathcal{Y}}_{2, \eta}(t, \theta) d \theta\right) \\
& \times\left(\int_{0}^{\eta} e^{-\frac{1}{A_{2}}\left(\tilde{\mathcal{Y}}_{2}(t, \eta)-\tilde{\mathcal{Y}}_{2}(t, \theta)\right)}\right. \\
& \left.\times\left(\int_{0}^{\theta} e^{-\frac{1}{a}\left(\tilde{\mathcal{Y}}_{2}(t, \theta)-\tilde{\mathcal{Y}}_{2}(t, l)\right)}\left|\tilde{\mathcal{Y}}_{1}-\tilde{\mathcal{Y}}_{2}\right|(t, l) d l\right)^{2} \tilde{\mathcal{Y}}_{2, \eta}(t, \theta) d \theta\right) d \eta \\
& +\frac{32}{3 e^{2} A^{6}} \int_{0}^{1} \tilde{\mathcal{Y}}_{2, \eta}^{2}(t, \eta)\left(\int_{0}^{\eta} e^{-\frac{1}{A_{2}}\left(\tilde{\mathcal{Y}}_{2}(t, \eta)-\tilde{\mathcal{Y}}_{2}(t, \theta)\right)} \tilde{\mathcal{U}}_{2}^{2} \tilde{\mathcal{Y}}_{2, \eta}(t, \theta) d \theta\right) \\
& \times\left(\int_{0}^{\eta} e^{-\frac{1}{A_{2}}\left(\tilde{\mathcal{Y}}_{2}(t, \eta)-\tilde{\mathcal{Y}}_{2}(t, \theta)\right)}\right. \\
& \left.\times\left(\int_{0}^{\theta} e^{-\frac{3}{4 A}\left(\tilde{\mathcal{Y}}_{2}(t, \theta)-\tilde{\mathcal{Y}}_{2}(t, l)\right)} d l\right) \tilde{\mathcal{Y}}_{2, \eta}(t, \theta) d \theta\right) d \eta\left|A_{1}-A_{2}\right|^{2} \\
& \leqslant 5\left\|\tilde{\mathcal{Y}}_{1}-\tilde{\mathcal{Y}}_{2}\right\|^{2} \\
& +\frac{1}{2 A^{4}} \int_{0}^{1} \tilde{\mathcal{P}}_{2} \tilde{\mathcal{Y}}_{2, \eta}^{2}(t, \eta)\left(\int_{0}^{\eta} e^{-\frac{1}{A_{2}}\left(\tilde{\mathcal{Y}}_{2}(t, \eta)-\tilde{\mathcal{Y}}_{2}(t, \theta)\right)}\left(\tilde{\mathcal{Y}}_{1}-\tilde{\mathcal{Y}}_{2}\right)^{2}(t, \theta) d \theta\right) d \eta \\
& +\frac{32}{A^{7}} \int_{0}^{1} \tilde{\mathcal{P}}_{2} \tilde{\mathcal{Y}}_{2, \eta}^{2}(t, \eta)\left(\int_{0}^{\eta} e^{-\frac{1}{2 A}\left(\tilde{\mathcal{Y}}_{2}(t, \eta)-\tilde{\mathcal{Y}}_{2}(t, \theta)\right)}\right. \\
& \left.\times\left(\int_{0}^{\theta} e^{-\frac{1}{A}\left(\tilde{\mathcal{Y}}_{2}(t, \theta)-\tilde{\mathcal{Y}}_{2}(t, l)\right)}\left(\tilde{\mathcal{P}}_{1}^{1 / 2}-\tilde{\mathcal{P}}_{2}^{1 / 2}\right)^{2}(t, l) d l\right) \tilde{\mathcal{Y}}_{2, \eta}(t, \theta) d \theta\right) d \eta \\
& +\frac{2}{A^{5}} \int_{0}^{1} \tilde{\mathcal{P}}_{2} \tilde{\mathcal{Y}}_{2, \eta}^{2}(t, \eta)\left(\int_{0}^{\eta} e^{-\frac{1}{2 A_{2}}\left(\tilde{\mathcal{Y}}_{2}(t, \eta)-\tilde{\mathcal{Y}}_{2}(t, \theta)\right)}\right. \\
& \left.\times\left(\int_{0}^{\theta} e^{-\frac{1}{A_{2}}\left(\tilde{\mathcal{Y}}_{2}(t, \theta)-\tilde{\mathcal{Y}}_{2}(t, l)\right)}\left(\tilde{\mathcal{Y}}_{1}-\tilde{\mathcal{Y}}_{2}\right)^{2}(t, l) d l\right) \tilde{\mathcal{Y}}_{2, \eta}(t, \theta) d \theta\right) d \eta
\end{aligned}
$$




$$
\begin{aligned}
& +\frac{1}{A^{5}} \int_{0}^{1} \tilde{\mathcal{P}}_{2} \tilde{\mathcal{Y}}_{2, \eta}^{2}(t, \eta)\left(\int_{0}^{\eta} e^{-\frac{1}{2 A_{2}}\left(\tilde{\mathcal{Y}}_{2}(t, \eta)-\tilde{\mathcal{Y}}_{2}(t, \theta)\right)}\left(\int_{0}^{\theta} e^{-\frac{1}{A_{2}}\left(\tilde{\mathcal{Y}}_{2}(t, \theta)-\tilde{\mathcal{Y}}_{2}(t, l)\right)} d l\right)\right. \\
& \left.\times\left(\int_{0}^{\theta} e^{-\frac{1}{A_{2}}\left(\tilde{\mathcal{Y}}_{2}(t, \theta)-\tilde{\mathcal{Y}}_{2}(t, l)\right)}\left(\tilde{\mathcal{Y}}_{1}-\tilde{\mathcal{Y}}_{2}\right)^{2}(t, l) d l\right) \tilde{\mathcal{Y}}_{2, \eta}(t, \theta) d \theta\right) d \eta \\
& +\frac{128}{3 e^{2} A^{5}} \int_{0}^{1} \tilde{\mathcal{P}}_{2} \tilde{\mathcal{Y}}_{2, \eta}^{2}(t, \eta)\left(\int_{0}^{\eta} e^{-\frac{1}{2 A}\left(\tilde{\mathcal{Y}}_{2}(t, \eta)-\tilde{\mathcal{Y}}_{2}(t, \theta)\right)}\right. \\
& \left.\times\left(\int_{0}^{\theta} e^{-\frac{3}{4 A}\left(\tilde{\mathcal{Y}}_{2}(t, \theta)-\tilde{\mathcal{Y}}_{2}(t, l)\right)} d l\right) \tilde{\mathcal{Y}}_{2, \eta}(t, \theta) d \theta\right) d \eta\left|A_{1}-A_{2}\right|^{2} \\
& \leqslant 5\left\|\tilde{\mathcal{Y}}_{1}-\tilde{\mathcal{Y}}_{2}\right\|^{2} \\
& +\frac{A}{4} \int_{0}^{1} \tilde{\mathcal{Y}}_{2, \eta}(t, \eta) e^{-\frac{1}{A_{2}} \tilde{\mathcal{Y}}_{2}(t, \eta)}\left(\int_{0}^{\eta} e^{\frac{1}{A_{2}} \tilde{\mathcal{Y}}_{2}(t, \theta)}\left(\tilde{\mathcal{Y}}_{1}-\tilde{\mathcal{Y}}_{2}\right)^{2}(t, \theta) d \theta\right) d \eta \\
& +\frac{16}{A^{2}} \int_{0}^{1} \tilde{\mathcal{Y}}_{2, \eta}(t, \eta)\left(\int_{0}^{\eta} \tilde{\mathcal{Y}}_{2, \eta}(t, \theta) e^{-\frac{1}{2 A} \tilde{\mathcal{Y}}_{2}(t, \theta)}\right. \\
& \left.\times\left(\int_{0}^{\theta} e^{-\left(\frac{1}{2 A} \tilde{\mathcal{Y}}_{2}(t, \eta)-\frac{1}{A} \tilde{\mathcal{Y}}_{2}(t, l)\right)}\left(\tilde{\mathcal{P}}_{1}^{1 / 2}-\tilde{\mathcal{P}}_{2}^{1 / 2}\right)^{2}(t, l) d l\right) d \theta\right) d \eta \\
& +\int_{0}^{1} \tilde{\mathcal{Y}}_{2, \eta}(t, \eta)\left(\int_{0}^{\eta} \tilde{\mathcal{Y}}_{2, \eta}(t, \theta) e^{-\frac{1}{2 A_{2}} \tilde{\mathcal{Y}}_{2}(t, \theta)}\right. \\
& \left.\times\left(\int_{0}^{\theta} e^{-\left(\frac{1}{2 A_{2}} \tilde{\mathcal{Y}}_{2}(t, \eta)-\frac{1}{A_{2}} \tilde{\mathcal{Y}}_{2}(t, l)\right)}\left(\tilde{\mathcal{Y}}_{1}-\tilde{\mathcal{Y}}_{2}\right)^{2}(t, l) d l\right) d \theta\right) d \eta \\
& +\frac{1}{2} \int_{0}^{1} \tilde{\mathcal{Y}}_{2, \eta}(t, \eta)\left(\int_{0}^{\eta} \tilde{\mathcal{Y}}_{2, \eta}(t, \theta) e^{-\frac{1}{2 A_{2}} \tilde{\mathcal{Y}}_{2}(t, \theta)}\right. \\
& \left.\times\left(\int_{0}^{\theta} e^{-\left(\frac{1}{2 A_{2}} \tilde{\mathcal{Y}}_{2}(t, \eta)-\frac{1}{A_{2}} \tilde{\mathcal{Y}}_{2}(t, l)\right)} d l\right) d \theta\right) d \eta\left\|\tilde{\mathcal{Y}}_{1}-\tilde{\mathcal{Y}}_{2}\right\|^{2} \\
& +\frac{64}{3 e^{2}} \int_{0}^{1} \tilde{\mathcal{Y}}_{2, \eta}(t, \eta)\left(\int_{0}^{\eta} \tilde{\mathcal{Y}}_{2, \eta}(t, \theta) e^{-\frac{1}{4 A} \tilde{\mathcal{Y}}_{2}(t, \theta)}\right. \\
& \left.\times\left(\int_{0}^{\theta} e^{-\left(\frac{1}{2 A} \tilde{\mathcal{Y}}_{2}(t, \eta)-\frac{3}{4 A} \tilde{\mathcal{Y}}_{2}(t, l)\right)} d l\right) d \theta\right) d \eta\left|A_{1}-A_{2}\right|^{2} \\
& \leqslant 5\left\|\tilde{\mathcal{Y}}_{1}-\tilde{\mathcal{Y}}_{2}\right\|^{2}+\frac{A}{4}\left[-\left.A_{2} \int_{0}^{\eta} e^{-\frac{1}{A_{2}}\left(\tilde{\mathcal{Y}}_{2}(t, \eta)-\tilde{\mathcal{Y}}_{2}(t, \theta)\right)}\left(\tilde{\mathcal{Y}}_{1}-\tilde{\mathcal{Y}}_{2}\right)^{2}(t, \theta) d \theta\right|_{\eta=0} ^{1}\right. \\
& \left.+\int_{0}^{1}\left(\tilde{\mathcal{Y}}_{1}-\tilde{\mathcal{Y}}_{2}\right)^{2}(t, \eta) d \eta\right] \\
& +\frac{16}{A} \int_{0}^{1} \tilde{\mathcal{Y}}_{2, \eta}(t, \eta)
\end{aligned}
$$




$$
\begin{aligned}
& \times\left[-\left.2 \int_{0}^{\theta} e^{\frac{1}{A} \tilde{\mathcal{Y}}_{2}(t, l)-\frac{1}{2 A}\left(\tilde{\mathcal{Y}}_{2}(t, \eta)+\tilde{\mathcal{Y}}_{2}(t, \theta)\right)}\left(\tilde{\mathcal{P}}_{1}^{1 / 2}-\tilde{\mathcal{P}}_{2}^{1 / 2}\right)^{2}(t, l) d l\right|_{\theta=0} ^{\eta}\right. \\
& \left.+2 \int_{0}^{\eta} e^{\frac{1}{2 A}\left(\tilde{\mathcal{Y}}_{2}(t, \theta)-\tilde{\mathcal{Y}}_{2}(t, \eta)\right)}\left(\tilde{\mathcal{P}}_{1}^{1 / 2}-\tilde{\mathcal{P}}_{2}^{1 / 2}\right)^{2}(t, \theta) d \theta\right] d \eta \\
& +A_{2} \int_{0}^{1} \tilde{\mathcal{Y}}_{2, \eta}(t, \eta) \\
& \times\left[-\left.2 \int_{0}^{\theta} e^{\frac{1}{A_{2}} \tilde{\mathcal{Y}}_{2}(t, l)-\frac{1}{2 A_{2}}\left(\tilde{\mathcal{Y}}_{2}(t, \eta)+\tilde{\mathcal{Y}}_{2}(t, \theta)\right)}\left(\tilde{\mathcal{Y}}_{1}-\tilde{\mathcal{Y}}_{2}\right)^{2}(t, l) d l\right|_{\theta=0} ^{\eta}\right. \\
& \left.+2 \int_{0}^{\eta} e^{\frac{1}{2 A_{2}}\left(\tilde{\mathcal{Y}}_{2}(t, \theta)-\tilde{\mathcal{Y}}_{2}(t, \eta)\right)}\left(\tilde{\mathcal{Y}}_{1}-\tilde{\mathcal{Y}}_{2}\right)^{2}(t, \theta) d \theta\right] d \eta \\
& +\frac{A_{2}}{2} \int_{0}^{1} \tilde{\mathcal{Y}}_{2, \eta}(t, \eta)\left[-\left.2 \int_{0}^{\theta} e^{\frac{1}{A_{2}} \tilde{\mathcal{Y}}_{2}(t, l)-\frac{1}{2 A_{2}}\left(\tilde{\mathcal{Y}}_{2}(t, \theta)+\tilde{\mathcal{Y}}_{2}(t, \eta)\right)} d l\right|_{\theta=0} ^{\eta}\right. \\
& \left.+2 \int_{0}^{\eta} e^{\frac{1}{2 A_{2}}\left(\tilde{\mathcal{Y}}_{2}(t, \theta)-\tilde{\mathcal{Y}}_{2}(t, \eta)\right)} d \theta\right] d \eta\left\|\tilde{\mathcal{Y}}_{1}-\tilde{\mathcal{Y}}_{2}\right\|^{2} \\
& +\frac{64 A}{3 e^{2}} \int_{0}^{1} \tilde{\mathcal{Y}}_{2, \eta}(t, \eta)\left[-4 \int_{0}^{\theta} e^{\frac{3}{4 A}} \tilde{\mathcal{Y}}_{2}(t, l)-\left.\left(\frac{1}{4 A} \tilde{\mathcal{Y}}_{2}(t, \theta)+\frac{1}{2 A} \tilde{\mathcal{Y}}_{2}(t, \eta)\right)\right|_{\theta=0} ^{\eta}\right. \\
& \left.+4 \int_{0}^{\eta} e^{\frac{1}{2 A}\left(\tilde{\mathcal{Y}}_{2}(t, \theta)-\tilde{\mathcal{Y}}_{2}(t, \eta)\right)} d \theta\right] d \eta\left|A_{1}-A_{2}\right|^{2} \\
& \leqslant \mathcal{O}(1)\left\|\tilde{\mathcal{Y}}_{1}-\tilde{\mathcal{Y}}_{2}\right\|^{2} \\
& +\frac{32}{A} \int_{0}^{1} \tilde{\mathcal{Y}}_{2, \eta}(t, \eta) e^{-\frac{1}{2 A} \tilde{\mathcal{Y}}_{2}(t, \eta)}\left(\int_{0}^{\eta} e^{\frac{1}{2 A} \tilde{\mathcal{Y}}_{2}(t, \theta)}\left(\tilde{\mathcal{P}}_{1}^{1 / 2}-\tilde{\mathcal{P}}_{2}^{1 / 2}\right)^{2}(t, \theta) d \theta\right) d \eta \\
& +2 A \int_{0}^{1} \tilde{\mathcal{Y}}_{2, \eta}(t, \eta) e^{-\frac{1}{2 A_{2}} \tilde{\mathcal{Y}}_{2}(t, \eta)}\left(\int_{0}^{\eta} e^{\frac{1}{2 A_{2}} \tilde{\mathcal{Y}}_{2}(t, \theta)}\left(\tilde{\mathcal{Y}}_{1}-\tilde{\mathcal{Y}}_{2}\right)^{2}(t, \theta) d \theta\right) d \eta \\
& +A \int_{0}^{1} \tilde{\mathcal{Y}}_{2, \eta}(t, \eta) e^{-\frac{1}{2 A_{2}} \tilde{\mathcal{Y}}_{2}(t, \eta)}\left(\int_{0}^{\eta} e^{\frac{1}{2 A_{2}} \tilde{\mathcal{Y}}_{2}(t, \theta)} d \theta\right) d \eta\left\|\tilde{\mathcal{Y}}_{1}-\tilde{\mathcal{Y}}_{2}\right\|^{2} \\
& +\frac{256 A}{3 e^{2}} \int_{0}^{1} \tilde{\mathcal{Y}}_{2, \eta}(t, \eta) e^{-\frac{1}{2 A} \tilde{\mathcal{Y}}_{2}(t, \eta)}\left(\int_{0}^{\eta} e^{\frac{1}{2 A} \tilde{\mathcal{Y}}_{2}(t, \theta)} d \theta\right) d \eta\left|A_{1}-A_{2}\right|^{2} \\
& \leqslant \mathcal{O}(1)\left\|\tilde{\mathcal{Y}}_{1}-\tilde{\mathcal{Y}}_{2}\right\|^{2} \\
& +32\left[-\left.2 \int_{0}^{\eta} e^{\frac{1}{2 A}\left(\tilde{\mathcal{Y}}_{2}(t, \theta)-\tilde{\mathcal{Y}}_{2}(t, \eta)\right)}\left(\tilde{\mathcal{P}}_{1}^{1 / 2}-\tilde{\mathcal{P}}_{2}^{1 / 2}\right)^{2}(t, \theta) d \theta\right|_{\eta=0} ^{1}\right. \\
& \left.+2 \int_{0}^{1}\left(\tilde{\mathcal{P}}_{1}^{1 / 2}-\tilde{\mathcal{P}}_{2}^{1 / 2}\right)^{2}(t, \eta) d \eta\right]
\end{aligned}
$$




$$
\begin{aligned}
& +2 A A_{2}\left[-\left.2 \int_{0}^{\eta} e^{\frac{1}{2 A_{2}}\left(\tilde{\mathcal{Y}}_{2}(t, \theta)-\tilde{\mathcal{Y}}_{2}(t, \eta)\right)}\left(\tilde{\mathcal{Y}}_{1}-\tilde{\mathcal{Y}}_{2}\right)^{2}(t, \theta) d \theta\right|_{\eta=0} ^{1}\right. \\
& \left.+2 \int_{0}^{1}\left(\tilde{\mathcal{Y}}_{1}-\tilde{\mathcal{Y}}_{2}\right)^{2}(t, \eta) d \eta\right] \\
& +A A_{2}\left[-\left.2 \int_{0}^{\eta} e^{\frac{1}{2 A_{2}}\left(\tilde{\mathcal{Y}}_{2}(t, \theta)-\tilde{\mathcal{Y}}_{2}(t, \eta)\right)} d \theta\right|_{\eta=0} ^{1}+2 \int_{0}^{1} d \eta\right]\left\|\tilde{\mathcal{Y}}_{1}-\tilde{\mathcal{Y}}_{2}\right\|^{2} \\
& +\frac{256 A^{2}}{3 e^{2}}\left[-\left.2 \int_{0}^{\eta} e^{\frac{1}{2 A}\left(\tilde{\mathcal{Y}}_{2}(t, \theta)-\tilde{\mathcal{Y}}_{2}(t, \eta)\right)} d \theta\right|_{\eta=0} ^{1}+2 \int_{0}^{1} d \eta\right]\left|A_{1}-A_{2}\right|^{2} \\
& \leqslant \mathcal{O}(1)\left(\left\|\tilde{\mathcal{Y}}_{1}-\tilde{\mathcal{Y}}_{2}\right\|^{2}+\left\|\tilde{\mathcal{P}}_{1}^{1 / 2}-\tilde{\mathcal{P}}_{2}^{1 / 2}\right\|^{2}+\left|A_{1}-A_{2}\right|^{2}\right) .
\end{aligned}
$$

Last but not least, direct calculations for $B_{314}$ yield

$$
\begin{aligned}
& B_{314} \leqslant \frac{6}{A^{3}}\left|A_{1}-A_{2}\right| \int_{0}^{1}\left|\tilde{\mathcal{Y}}_{1}-\tilde{\mathcal{Y}}_{2}\right| \tilde{\mathcal{Y}}_{2, \eta}(t, \eta) \\
& \times\left(\int_{0}^{\eta} e^{-\frac{1}{A_{2}}\left(\tilde{\mathcal{Y}}_{2}(t, \eta)-\tilde{\mathcal{Y}}_{2}(t, \theta)\right)}\left(\int_{0}^{\theta} e^{-\frac{3}{4 A}\left(\tilde{\mathcal{Y}}_{2}(t, \theta)-\tilde{\mathcal{Y}}_{2}(t, l)\right)} d l\right) \tilde{\mathcal{U}}_{2}^{+} \tilde{\mathcal{Y}}_{2, \eta}(t, \theta) d \theta\right) d \eta \\
& \leqslant \frac{6}{A^{3}}\left|A_{1}-A_{2}\right| \| \tilde{\mathcal{Y}}_{1}-\tilde{\mathcal{Y}}_{2}|| \\
& \times\left(\int _ { 0 } ^ { 1 } \tilde { \mathcal { Y } } _ { 2 , \eta } ^ { 2 } ( t , \eta ) \left(\int_{0}^{\eta} e^{-\frac{1}{A_{2}}\left(\tilde{\mathcal{Y}}_{2}(t, \eta)-\tilde{\mathcal{Y}}_{2}(t, \theta)\right)}\right.\right. \\
& \left.\left.\times\left(\int_{0}^{\theta} e^{-\frac{3}{4 A}\left(\tilde{\mathcal{Y}}_{2}(t, \theta)-\tilde{\mathcal{Y}}_{2}(t, l)\right)} d l\right) \tilde{\mathcal{U}}_{2}^{+} \tilde{\mathcal{Y}}_{2, \eta}(t, \theta) d \theta\right)^{2} d \eta\right)^{1 / 2} \\
& \leqslant \frac{6}{A^{3}}\left|A_{1}-A_{2}\right|\left\|\tilde{\mathcal{Y}}_{1}-\tilde{\mathcal{Y}}_{2}\right\| \\
& \times\left(\int_{0}^{1} \tilde{\mathcal{Y}}_{2, \eta}^{2}(t, \eta)\left(\int_{0}^{\eta} e^{-\frac{1}{A_{2}}\left(\tilde{\mathcal{Y}}_{2}(t, \eta)-\tilde{\mathcal{Y}}_{2}(t, \theta)\right)} \tilde{\mathcal{U}}_{2}^{2} \tilde{\mathcal{Y}}_{2, \eta}(t, \theta) d \theta\right)\right. \\
& \left.\times\left(\int_{0}^{\eta} e^{-\frac{1}{A_{2}}\left(\tilde{\mathcal{Y}}_{2}(t, \eta)-\tilde{\mathcal{Y}}_{2}(t, \theta)\right)}\left(\int_{0}^{\theta} e^{-\frac{3}{4 A}\left(\tilde{\mathcal{Y}}_{2}(t, \theta)-\tilde{\mathcal{Y}}_{2}(t, l)\right)} d l\right)^{2} \tilde{\mathcal{Y}}_{2, \eta}(t, \theta) d \theta\right) d \eta\right)^{1 / 2} \\
& \leqslant \frac{12}{A^{5 / 2}}\left|A_{1}-A_{2}\right|\left\|\tilde{\mathcal{Y}}_{1}-\tilde{\mathcal{Y}}_{2}\right\| \\
& \times\left(\int _ { 0 } ^ { 1 } \tilde { \mathcal { P } } _ { 2 } \tilde { \mathcal { Y } } _ { 2 , \eta } ^ { 2 } ( t , \eta ) \left(\int_{0}^{\eta} e^{-\frac{1}{2 A}\left(\tilde{\mathcal{Y}}_{2}(t, \eta)-\tilde{\mathcal{Y}}_{2}(t, \theta)\right)}\right.\right. \\
& \left.\left.\times\left(\int_{0}^{\theta} e^{-\frac{3}{4 A}\left(\tilde{\mathcal{Y}}_{2}(t, \theta)-\tilde{\mathcal{Y}}_{2}(t, l)\right)} d l\right) \tilde{\mathcal{Y}}_{2, \eta}(t, \theta) d \theta\right) d \eta\right)^{1 / 2} \\
& \leqslant 6 \sqrt{2}\left|A_{1}-A_{2}\right|\left\|\tilde{\mathcal{Y}}_{1}-\tilde{\mathcal{Y}}_{2}\right\|
\end{aligned}
$$




$$
\begin{aligned}
& \times\left(\int _ { 0 } ^ { 1 } \tilde { \mathcal { Y } } _ { 2 , \eta } ( t , \eta ) \left(\int_{0}^{\eta} \tilde{\mathcal{Y}}_{2, \eta}(t, \theta) e^{-\frac{1}{4 A}} \tilde{\mathcal{Y}}_{2}(t, \theta)\right.\right. \\
& \left.\left.\times\left(\int_{0}^{\theta} e^{\frac{3}{4 A} \tilde{\mathcal{Y}}_{2}(t, l)-\frac{1}{2 A} \tilde{\mathcal{Y}}_{2}(t, \eta)} d l\right) d \theta\right) d \eta\right)^{1 / 2} \\
\leqslant & \mathcal{O}(1)\left|A_{1}-A_{2}\right|\left\|\tilde{\mathcal{Y}}_{1}-\tilde{\mathcal{Y}}_{2}\right\| .
\end{aligned}
$$

Collecting the estimates we have obtained, we conclude

$$
\left|\bar{B}_{31}\right| \leqslant \mathcal{O}(1)\left(\left\|\tilde{\mathcal{U}}_{1}-\tilde{\mathcal{U}}_{2}\right\|^{2}+\left\|\tilde{\mathcal{Y}}_{1}-\tilde{\mathcal{Y}}_{2}\right\|^{2}+\left\|\tilde{\mathcal{P}}_{1}^{1 / 2}-\tilde{\mathcal{P}}_{2}^{1 / 2}\right\|^{2}+\left|A_{1}-A_{2}\right|^{2}\right) .
$$

Recalling (4.16e), a direct computation yields for $\bar{B}_{33}$ (and in much the same way for $\left.\bar{B}_{34}\right)$ that

$$
\begin{aligned}
& \left|\bar{B}_{33}\right| \leqslant \frac{1}{A^{7}} \int_{0}^{1}\left|\tilde{\mathcal{Y}}_{1}-\tilde{\mathcal{Y}}_{2}\right| \tilde{\mathcal{Y}}_{2, \eta}(t, \eta) \\
& \times\left(\int_{0}^{\eta} e^{-\frac{1}{A_{2}}\left(\tilde{\mathcal{Y}}_{2}(t, \eta)-\tilde{\mathcal{Y}}_{2}(t, \theta)\right)} \min _{j}\left(\tilde{\mathcal{D}}_{j}\right)\left|\tilde{\mathcal{U}}_{1}-\tilde{\mathcal{U}}_{2}\right| \tilde{\mathcal{Y}}_{2, \eta}(t, \theta) d \theta\right) d \eta \\
& \leqslant\left\|\tilde{\mathcal{Y}}_{1}-\tilde{\mathcal{Y}}_{2}\right\|^{2}+\frac{1}{A^{14}} \int_{0}^{1} \tilde{\mathcal{Y}}_{2, \eta}^{2}(t, \eta) \\
& \times\left(\int_{0}^{\eta} e^{-\frac{1}{A_{2}}\left(\tilde{\mathcal{Y}}_{2}(t, \eta)-\tilde{\mathcal{Y}}_{2}(t, \theta)\right)} \min _{j}\left(\tilde{\mathcal{D}}_{j}\right) \tilde{\mathcal{Y}}_{2, \eta}\left|\tilde{\mathcal{U}}_{1}-\tilde{\mathcal{U}}_{2}\right|(t, \theta) d \theta\right)^{2} d \eta \\
& \leqslant\left\|\tilde{\mathcal{Y}}_{1}-\tilde{\mathcal{Y}}_{2}\right\|^{2} \\
& +\frac{4}{A^{12}} \int_{0}^{1} \tilde{\mathcal{Y}}_{2, \eta}^{2}(t, \eta)\left(\int_{0}^{\eta} e^{-\frac{3}{2 A_{2}}\left(\tilde{\mathcal{Y}}_{2}(t, \eta)-\tilde{\mathcal{Y}}_{2}(t, \theta)\right)} \tilde{\mathcal{P}}_{2} \tilde{\mathcal{Y}}_{2, \eta}(t, \theta) d \theta\right) \\
& \times\left(\int_{0}^{\eta} e^{-\frac{1}{2 A_{2}}\left(\tilde{\mathcal{Y}}_{2}(t, \eta)-\tilde{\mathcal{Y}}_{2}(t, \theta)\right)} \tilde{\mathcal{P}}_{2} \tilde{\mathcal{Y}}_{2, \eta}\left(\tilde{\mathcal{U}}_{1}-\tilde{\mathcal{U}}_{2}\right)^{2}(t, \theta) d \theta\right) d \eta \\
& \leqslant\left\|\tilde{\mathcal{Y}}_{1}-\tilde{\mathcal{Y}}_{2}\right\|^{2} \\
& +\frac{4}{A^{6}} \int_{0}^{1} \tilde{\mathcal{P}}_{2} \tilde{\mathcal{Y}}_{2, \eta}^{2}(t, \eta)\left(\int_{0}^{\eta} e^{-\frac{1}{2 A_{2}}\left(\tilde{\mathcal{Y}}_{2}(t, \eta)-\tilde{\mathcal{Y}}_{2}(t, \theta)\right)}\left(\tilde{\mathcal{U}}_{1}-\tilde{\mathcal{U}}_{2}\right)^{2}(t, \theta) d \theta\right) d \eta \\
& \leqslant\left\|\tilde{\mathcal{Y}}_{1}-\tilde{\mathcal{Y}}_{2}\right\|^{2} \\
& +\frac{2}{A} \int_{0}^{1} \tilde{\mathcal{Y}}_{2, \eta}(t, \eta) e^{-\frac{1}{2 A_{2}} \tilde{\mathcal{Y}}_{2}(t, \eta)}\left(\int_{0}^{\eta} e^{\frac{1}{2 A_{2}} \tilde{\mathcal{Y}}_{2}(t, \theta)}\left(\tilde{\mathcal{U}}_{1}-\tilde{\mathcal{U}}_{2}\right)^{2}(t, \theta) d \theta\right) d \eta \\
& \leqslant\left\|\tilde{\mathcal{Y}}_{1}-\tilde{\mathcal{Y}}_{2}\right\|^{2} \\
& +\frac{2 A_{2}}{A}\left[-\left.2 \int_{0}^{\eta} e^{-\frac{1}{2 A_{2}}\left(\tilde{\mathcal{Y}}_{2}(t, \eta)-\tilde{\mathcal{Y}}_{2}(t, \theta)\right)}\left(\tilde{\mathcal{U}}_{1}-\tilde{\mathcal{U}}_{2}\right)^{2}(t, \theta) d \theta\right|_{\eta=0} ^{1}\right.
\end{aligned}
$$




$$
\begin{aligned}
& \left.+2 \int_{0}^{1}\left(\tilde{\mathcal{U}}_{1}-\tilde{\mathcal{U}}_{2}\right)^{2}(t, \eta) d \eta\right] \\
\leqslant & \mathcal{O}(1)\left(\left\|\tilde{\mathcal{Y}}_{1}-\tilde{\mathcal{Y}}_{2}\right\|^{2}+\left\|\tilde{\mathcal{U}}_{1}-\tilde{\mathcal{U}}_{2}\right\|^{2}\right) .
\end{aligned}
$$

Direct calculations yield for $\bar{B}_{35}$ (and in much the same way for $\bar{B}_{36}$ ) that

$$
\begin{aligned}
& \left|\bar{B}_{35}\right| \leqslant \frac{1}{A^{7}} \int_{0}^{1}\left|\tilde{\mathcal{Y}}_{1}-\tilde{\mathcal{Y}}_{2}\right| \tilde{\mathcal{Y}}_{2, \eta}(t, \eta) \\
& \times\left(\int_{0}^{\eta}\left(e^{-\frac{1}{A_{2}}\left(\tilde{\mathcal{Y}}_{2}(t, \eta)-\tilde{\mathcal{Y}}_{2}(t, \theta)\right)}-e^{-\frac{1}{A_{2}}\left(\tilde{\mathcal{Y}}_{1}(t, \eta)-\tilde{\mathcal{Y}}_{1}(t, \theta)\right)}\right)\right. \\
& \left.\times \min _{j}\left(\tilde{\mathcal{D}}_{j}\right) \min _{j}\left(\tilde{\mathcal{U}}_{j}^{+}\right) \tilde{\mathcal{Y}}_{2, \eta} \mathbb{1}_{B(\eta)}(t, \theta) d \theta\right) d \eta \\
& \leqslant \frac{1}{A^{7} A_{2}} \int_{0}^{1}\left|\tilde{\mathcal{Y}}_{1}-\tilde{\mathcal{Y}}_{2}\right| \tilde{\mathcal{Y}}_{2, \eta}(t, \eta)\left(\int_{0}^{\eta}\left(\left|\tilde{\mathcal{Y}}_{1}-\tilde{\mathcal{Y}}_{2}\right|(t, \eta)+\left|\tilde{\mathcal{Y}}_{1}-\tilde{\mathcal{Y}}_{2}\right|(t, \theta)\right)\right. \\
& \left.\times e^{-\frac{1}{A_{2}}\left(\tilde{\mathcal{Y}}_{2}(t, \eta)-\tilde{\mathcal{Y}}_{2}(t, \theta)\right)} \tilde{\mathcal{D}}_{2} \tilde{\mathcal{U}}_{2}^{+} \tilde{\mathcal{Y}}_{2, \eta}(t, \theta) d \theta\right) d \eta \\
& \leqslant \frac{1}{A^{7} A_{2}} \int_{0}^{1}\left(\tilde{\mathcal{Y}}_{1}-\tilde{\mathcal{Y}}_{2}\right)^{2} \tilde{\mathcal{Y}}_{2, \eta}(t, \eta) \\
& \times\left(\int_{0}^{\eta} e^{-\frac{1}{A_{2}}\left(\tilde{\mathcal{Y}}_{2}(t, \eta)-\tilde{\mathcal{Y}}_{2}(t, \theta)\right)} \tilde{\mathcal{D}}_{2} \tilde{\mathcal{U}}_{2}^{+} \tilde{\mathcal{Y}}_{2, \eta}(t, \theta) d \theta\right) d \eta \\
& +\frac{1}{A^{7} A_{2}} \int_{0}^{1}\left|\tilde{\mathcal{Y}}_{1}-\tilde{\mathcal{Y}}_{2}\right| \tilde{\mathcal{Y}}_{2, \eta}(t, \eta) \\
& \times\left(\int_{0}^{\eta} e^{-\frac{1}{A_{2}}\left(\tilde{\mathcal{Y}}_{2}(t, \eta)-\tilde{\mathcal{Y}}_{2}(t, \theta)\right)} \tilde{\mathcal{D}}_{2} \tilde{\mathcal{U}}_{2}^{+} \tilde{\mathcal{Y}}_{2, \eta}\left|\tilde{\mathcal{Y}}_{1}-\tilde{\mathcal{Y}}_{2}\right|(t, \theta) d \theta\right) d \eta \\
& \leqslant \frac{2}{A^{7}} \int_{0}^{1}\left(\tilde{\mathcal{Y}}_{1}-\tilde{\mathcal{Y}}_{2}\right)^{2} \tilde{\mathcal{Y}}_{2, \eta}(t, \eta)\left(\int_{0}^{\eta} e^{-\frac{1}{A_{2}}\left(\tilde{\mathcal{Y}}_{2}(t, \eta)-\tilde{\mathcal{Y}}_{2}(t, \theta)\right)} \tilde{\mathcal{P}}_{2}^{2} \tilde{\mathcal{Y}}_{2, \eta}(t, \theta) d \theta\right)^{1 / 2} \\
& \times\left(\int_{0}^{\eta} e^{-\frac{1}{A_{2}}\left(\tilde{\mathcal{Y}}_{2}(t, \eta)-\tilde{\mathcal{Y}}_{2}(t, \theta)\right)} \tilde{\mathcal{U}}_{2}^{2} \tilde{\mathcal{Y}}_{2, \eta}(t, \theta) d \theta\right)^{1 / 2} d \eta \\
& +\left\|\tilde{\mathcal{Y}}_{1}-\tilde{\mathcal{Y}}_{2}\right\|^{2} \\
& +\frac{4}{A^{14}} \int_{0}^{1} \tilde{\mathcal{Y}}_{2, \eta}^{2}(t, \eta)\left(\int_{0}^{\eta} e^{-\frac{1}{A_{2}}\left(\tilde{\mathcal{Y}}_{2}(t, \eta)-\tilde{\mathcal{Y}}_{2}(t, \theta)\right)} \tilde{\mathcal{U}}_{2}^{2} \tilde{\mathcal{Y}}_{2, \eta}(t, \theta) d \theta\right) \\
& \times\left(\int_{0}^{\eta} e^{-\frac{1}{A_{2}}\left(\tilde{\mathcal{Y}}_{2}(t, \eta)-\tilde{\mathcal{Y}}_{2}(t, \theta)\right)} \tilde{\mathcal{P}}_{2}^{2} \tilde{\mathcal{Y}}_{2, \eta}\left(\tilde{\mathcal{Y}}_{1}-\tilde{\mathcal{Y}}_{2}\right)^{2}(t, \theta) d \theta\right) d \eta
\end{aligned}
$$




$$
\begin{aligned}
\leqslant & \left\|\tilde{\mathcal{Y}}_{1}-\tilde{\mathcal{Y}}_{2}\right\|^{2}+\frac{2 \sqrt{6}}{A^{4}} \int_{0}^{1}\left(\tilde{\mathcal{Y}}_{1}-\tilde{\mathcal{Y}}_{2}\right)^{2} \tilde{\mathcal{P}}_{2} \tilde{\mathcal{Y}}_{2, \eta}(t, \eta) d \eta \\
& +\frac{16}{A^{13}} \int_{0}^{1} \tilde{\mathcal{P}}_{2} \tilde{\mathcal{Y}}_{2, \eta}^{2}(t, \eta) \\
& \times\left(\int_{0}^{\eta} e^{-\frac{1}{A_{2}}\left(\tilde{\mathcal{Y}}_{2}(t, \eta)-\tilde{\mathcal{Y}}_{2}(t, \theta)\right)} \tilde{\mathcal{P}}_{2}^{2} \tilde{\mathcal{Y}}_{2, \eta}\left(\tilde{\mathcal{Y}}_{1}-\tilde{\mathcal{Y}}_{2}\right)^{2}(t, \theta) d \theta\right) d \eta \\
\leqslant & (1+\sqrt{6} A)\left\|\tilde{\mathcal{Y}}_{1}-\tilde{\mathcal{Y}}_{2}\right\|^{2} \\
& +A \int_{0}^{1} \tilde{\mathcal{Y}}_{2, \eta}(t, \eta) e^{-\frac{1}{A_{2}} \tilde{\mathcal{Y}}_{2}(t, \eta)}\left(\int_{0}^{\eta} e^{\frac{1}{A_{2}} \tilde{\mathcal{Y}}_{2}(t, \theta)}\left(\tilde{\mathcal{Y}}_{1}-\tilde{\mathcal{Y}}_{2}\right)^{2}(t, \theta) d \theta\right) d \eta \\
= & (1+\sqrt{6} A)\left\|\tilde{\mathcal{Y}}_{1}-\tilde{\mathcal{Y}}_{2}\right\|^{2} \\
& +A A_{2}\left[-\left.\int_{0}^{\eta} e^{-\frac{1}{A_{2}}\left(\tilde{\mathcal{Y}}_{2}(t, \eta)-\tilde{\mathcal{Y}}_{2}(t, \theta)\right)}\left(\tilde{\mathcal{Y}}_{1}-\tilde{\mathcal{Y}}_{2}\right)^{2}(t, \theta) d \theta\right|_{\eta=0} ^{1}\right. \\
& \left.+\int_{0}^{1}\left(\tilde{\mathcal{Y}}_{1}-\tilde{\mathcal{Y}}_{2}\right)^{2}(t, \eta) d \eta\right] \\
\leqslant & \mathcal{O}(1)\left\|\tilde{\mathcal{Y}}_{1}-\tilde{\mathcal{Y}}_{2}\right\|^{2} .
\end{aligned}
$$

Direct calculations yield for $\hat{B}_{35}$ (and in much the same way for $\hat{B}_{36}$ ) that

$$
\begin{aligned}
\left|\hat{B}_{35}\right| \leqslant & \mathbb{1}_{A_{1} \leqslant A_{2}} \frac{4\left|A_{1}-A_{2}\right|}{a A^{7} e} \int_{0}^{1}\left|\tilde{\mathcal{Y}}_{1}-\tilde{\mathcal{Y}}_{2}\right| \tilde{\mathcal{Y}}_{2, \eta}(t, \eta) \\
& \times\left(\int_{0}^{\eta} \min \left(e^{-\frac{3}{4 A}\left(\tilde{\mathcal{Y}}_{j}(t, \eta)-\tilde{\mathcal{Y}}_{j}(t, \theta)\right)}\right) \min _{j}\left(\tilde{\mathcal{D}}_{j}\right) \min _{j}\left(\tilde{\mathcal{U}}_{j}^{+}\right) \tilde{\mathcal{Y}}_{2, \eta}(t, \theta) d \theta\right) d \eta \\
\leqslant & \frac{8}{a A^{6} e} \int_{0}^{1}\left|\tilde{\mathcal{Y}}_{1}-\tilde{\mathcal{Y}}_{2}\right| \tilde{\mathcal{Y}}_{2, \eta}(t, \eta) \\
& \times\left(\int_{0}^{\eta} e^{-\frac{1}{A_{2}}\left(\tilde{\mathcal{Y}}_{2}(t, \eta)-\tilde{\mathcal{Y}}_{2}(t, \theta)\right)} \tilde{\mathcal{U}}_{2}^{2} \tilde{\mathcal{Y}}_{2, \eta}(t, \theta) d \theta\right)^{1 / 2} \\
& \times\left(\int_{0}^{\eta} e^{-\frac{1}{2 A_{2}}\left(\tilde{\mathcal{Y}}_{2}(t, \eta)-\tilde{\mathcal{Y}}_{2}(t, \theta)\right)} \tilde{\mathcal{P}}_{1} \tilde{\mathcal{P}}_{2} \tilde{\mathcal{Y}}_{2, \eta}(t, \theta) d \theta\right)^{1 / 2} d \eta\left|A_{1}-A_{2}\right| \\
\leqslant & \frac{16}{a A^{11 / 2} e} \int_{0}^{1}\left|\tilde{\mathcal{Y}}_{1}-\tilde{\mathcal{Y}}_{2}\right| \tilde{\mathcal{P}}_{2}^{1 / 2} \tilde{\mathcal{Y}}_{2, \eta}(t, \eta) \\
& \times\left(\int_{0}^{\eta} e^{-\frac{1}{2 A_{2}}\left(\tilde{\mathcal{Y}}_{2}(t, \eta)-\tilde{\mathcal{Y}}_{2}(t, \theta)\right)} \tilde{\mathcal{P}}_{1} \tilde{\mathcal{P}}_{2} \tilde{\mathcal{Y}}_{2, \eta}(t, \theta) d \theta\right)^{1 / 2} d \eta\left|A_{1}-A_{2}\right| \\
\leqslant & \left\|\tilde{\mathcal{Y}}_{1}-\tilde{\mathcal{Y}}_{2}\right\|^{2}
\end{aligned}
$$




$$
\begin{aligned}
& +\frac{256}{a^{2} A^{11} e^{2}} \int_{0}^{1} \tilde{\mathcal{P}}_{2} \tilde{\mathcal{Y}}_{2, \eta}^{2}(t, \eta) \\
& \times\left(\int_{0}^{\eta} e^{-\frac{1}{2 A_{2}}\left(\tilde{\mathcal{Y}}_{2}(t, \eta)-\tilde{\mathcal{Y}}_{2}(t, \theta)\right)} \tilde{\mathcal{P}}_{1} \tilde{\mathcal{P}}_{2} \tilde{\mathcal{Y}}_{2, \eta}(t, \theta) d \theta\right) d \eta\left|A_{1}-A_{2}\right|^{2} \\
& \leqslant\left\|\tilde{\mathcal{Y}}_{1}-\tilde{\mathcal{Y}}_{2}\right\|^{2} \\
& +\frac{16 A}{e^{2}} \int_{0}^{1} \tilde{\mathcal{Y}}_{2, \eta}(t, \eta) e^{-\frac{1}{2 A_{2}} \tilde{\mathcal{Y}}_{2}(t, \eta)}\left(\int_{0}^{\eta} e^{\frac{1}{2 A_{2}} \tilde{\mathcal{Y}}_{2}(t, \theta)} d \theta\right) d \eta\left|A_{1}-A_{2}\right|^{2} \\
& \leqslant \mathcal{O}(1)\left(\left\|\tilde{\mathcal{Y}}_{1}-\tilde{\mathcal{Y}}_{2}\right\|^{2}+\left|A_{1}-A_{2}\right|^{2}\right) \text {. }
\end{aligned}
$$

As far as the term $\bar{B}_{37}$ is concerned, it can be estimated as follows (we use $(3.40))$ :

$$
\begin{aligned}
& \left|\bar{B}_{37}\right|=\frac{1}{A^{7}} \mid \int_{0}^{1}\left(\tilde{\mathcal{Y}}_{1}-\tilde{\mathcal{Y}}_{2}\right)\left(\tilde{\mathcal{Y}}_{1, \eta}-\tilde{\mathcal{Y}}_{2, \eta}\right)(t, \eta) \\
& \times \min _{k}\left(\int_{0}^{\eta} \min _{j}\left(e^{-\frac{1}{a}\left(\tilde{\mathcal{Y}}_{j}(t, \eta)-\tilde{\mathcal{Y}}_{j}(t, \theta)\right)}\right)\right. \\
& \left.\times \min _{j}\left(\tilde{\mathcal{D}}_{j}\right) \min _{j}\left(\tilde{\mathcal{U}}_{j}^{+}\right) \tilde{\mathcal{Y}}_{k, \eta}(t, \theta) d \theta\right) d \eta \\
& =\frac{1}{2 A^{7}} \mid\left(\tilde{\mathcal{Y}}_{1}-\tilde{\mathcal{Y}}_{2}\right)^{2}(t, \eta) \min _{k}\left(\int_{0}^{\eta} \min \left(e^{-\frac{1}{a}\left(\tilde{\mathcal{Y}}_{j}(t, \eta)-\tilde{\mathcal{Y}}_{j}(t, \theta)\right)}\right)\right. \\
& \left.\times \min _{j}\left(\tilde{\mathcal{D}}_{j}\right) \min _{j}\left(\tilde{\mathcal{U}}_{j}^{+}\right) \tilde{\mathcal{Y}}_{k, \eta}(t, \theta) d \theta\right)\left.\right|_{\eta=0} ^{1} \\
& -\int_{0}^{1}\left(\tilde{\mathcal{Y}}_{1}-\tilde{\mathcal{Y}}_{2}\right)^{2}(t, \eta) \frac{d}{d \eta} \min _{k}\left(\int_{0}^{\eta} \min _{j}\left(e^{-\frac{1}{a}\left(\tilde{\mathcal{Y}}_{j}(t, \eta)-\tilde{\mathcal{Y}}_{j}(t, \theta)\right)}\right)\right. \\
& \left.\times \min _{j}\left(\tilde{\mathcal{D}}_{j}\right) \min _{j}\left(\tilde{\mathcal{U}}_{j}^{+}\right) \tilde{\mathcal{Y}}_{k, \eta}(t, \theta) d \theta\right) d \eta \mid \\
& =\frac{1}{2 A^{7}} \mid \int_{0}^{1}\left(\tilde{\mathcal{Y}}_{1}-\tilde{\mathcal{Y}}_{2}\right)^{2}(t, \eta) \frac{d}{d \eta} \min _{k}\left(\int_{0}^{\eta} \min \left(e^{-\frac{1}{a}\left(\tilde{\mathcal{Y}}_{j}(t, \eta)-\tilde{\mathcal{Y}}_{j}(t, \theta)\right)}\right)\right. \\
& \left.\times \min _{j}\left(\tilde{\mathcal{D}}_{j}\right) \min _{j}\left(\tilde{\mathcal{U}}_{j}^{+}\right) \tilde{\mathcal{Y}}_{k, \eta}(t, \theta) d \theta\right) d \eta \mid \\
& \leqslant \mathcal{O}(1)\left\|\tilde{\mathcal{Y}}_{1}-\tilde{\mathcal{Y}}_{2}\right\|^{2} \text {, }
\end{aligned}
$$

where $\mathcal{O}(1)$ denotes some constant only depending on $A$, which remains bounded as $A \rightarrow 0$, provided we can show that the derivative in the latter integral exists and is uniformly bounded; see Lemma A.7. 
As far as the term $\bar{B}_{38}$ (a similar argument works for $\bar{B}_{39}$ ) is concerned, the integral can be estimated as follows:

$$
\begin{aligned}
& \bar{B}_{38}=\frac{1}{A^{7}} \int_{0}^{1}\left(\tilde{\mathcal{Y}}_{1}-\tilde{\mathcal{Y}}_{2}\right) \tilde{\mathcal{Y}}_{2, \eta} \mathbb{1}_{D^{c}}(t, \eta)\left(\int_{0}^{\eta} \min _{j}\left(e^{-\frac{1}{a}\left(\tilde{\mathcal{Y}}_{j}(t, \eta)-\tilde{\mathcal{Y}}_{j}(t, \theta)\right)}\right)\right. \\
& \left.\times \min _{j}\left(\tilde{\mathcal{D}}_{j}\right) \min _{j}\left(\tilde{\mathcal{U}}_{j}^{+}\right)\left(\tilde{\mathcal{Y}}_{2, \eta}-\tilde{\mathcal{Y}}_{1, \eta}\right)(t, \theta) d \theta\right) d \eta \\
& =\frac{1}{A^{7}} \int_{0}^{1}\left(\tilde{\mathcal{Y}}_{1}-\tilde{\mathcal{Y}}_{2}\right) \tilde{\mathcal{Y}}_{2, \eta} \mathbb{1}_{D^{c}}(t, \eta)\left[\left(\tilde{\mathcal{Y}}_{2}-\tilde{\mathcal{Y}}_{1}\right)(t, \theta) \min _{j}\left(e^{-\frac{1}{a}\left(\tilde{\mathcal{Y}}_{j}(t, \eta)-\tilde{\mathcal{Y}}_{j}(t, \theta)\right)}\right)\right. \\
& \times\left.\min _{j}\left(\tilde{\mathcal{D}}_{j}\right) \min _{j}\left(\tilde{\mathcal{U}}_{j}^{+}\right)(t, \theta)\right|_{\theta=0} ^{\eta} \\
& -\int_{0}^{\eta}\left(\tilde{\mathcal{Y}}_{2}-\tilde{\mathcal{Y}}_{1}\right)(t, \theta)\left[\left(\frac{d}{d \theta} \min _{j}\left(e^{-\frac{1}{a}\left(\tilde{\mathcal{Y}}_{j}(t, \eta)-\tilde{\mathcal{Y}}_{j}(t, \theta)\right)}\right)\right)\right. \\
& \times \min _{j}\left(\tilde{\mathcal{D}}_{j}\right) \min _{j}\left(\tilde{\mathcal{U}}_{j}^{+}\right)(t, \theta) \\
& \left.\left.+\min _{j}\left(e^{-\frac{1}{a}\left(\tilde{\mathcal{Y}}_{j}(t, \eta)-\tilde{\mathcal{Y}}_{j}(t, \theta)\right)}\right)\left(\frac{d}{d \theta} \min _{j}\left(\tilde{\mathcal{D}}_{j}\right) \min _{j}\left(\tilde{\mathcal{U}}_{j}^{+}\right)\right)(t, \theta) d \theta\right] d \eta\right] \\
& =-\frac{1}{A^{7}} \int_{0}^{1}\left(\tilde{\mathcal{Y}}_{1}-\tilde{\mathcal{Y}}_{2}\right)^{2} \min _{j}\left(\tilde{\mathcal{D}}_{j}\right) \min _{j}\left(\tilde{\mathcal{U}}_{j}^{+}\right) \tilde{\mathcal{Y}}_{2, \eta} \mathbb{1}_{D^{c}}(t, \eta) d \eta \\
& +\frac{1}{A^{7}} \int_{0}^{1}\left(\tilde{\mathcal{Y}}_{1}-\tilde{\mathcal{Y}}_{2}\right) \tilde{\mathcal{Y}}_{2, \eta}(t, \eta) \mathbb{1}_{D^{c}}(t, \eta) \\
& \times \int_{0}^{\eta}\left(\tilde{\mathcal{Y}}_{1}-\tilde{\mathcal{Y}}_{2}\right)(t, \theta)\left[\left(\frac{d}{d \theta} \min _{j}\left(e^{-\frac{1}{a}\left(\tilde{\mathcal{Y}}_{j}(t, \eta)-\tilde{\mathcal{Y}}_{j}(t, \theta)\right)}\right)\right)\right. \\
& \times \min _{j}\left(\tilde{\mathcal{D}}_{j}\right) \min _{j}\left(\tilde{\mathcal{U}}_{j}^{+}\right)(t, \theta) \\
& \left.+\min _{j}\left(e^{-\frac{1}{a}\left(\tilde{\mathcal{Y}}_{j}(t, \eta)-\tilde{\mathcal{Y}}_{j}(t, \theta)\right)}\right)\left(\frac{d}{d \theta} \min _{j}\left(\tilde{\mathcal{D}}_{j}\right) \min _{j}\left(\tilde{\mathcal{U}}_{j}^{+}\right)\right)(t, \theta) d \theta\right] d \eta \\
& =\bar{M}_{1}+\bar{M}_{2} \text {. }
\end{aligned}
$$

As far as the first term $\bar{M}_{1}$ is concerned, we have since

$$
\begin{aligned}
\min _{j}\left(\tilde{\mathcal{D}}_{j}\right) \min _{j}\left(\tilde{\mathcal{U}}_{j}^{+}\right) \tilde{\mathcal{Y}}_{i, \eta}(t, \eta) & \leqslant 2 \min _{j}\left(A_{j} \tilde{\mathcal{P}}_{j}\right) \min _{j}\left(\tilde{\mathcal{U}}_{j}^{+}\right) \tilde{\mathcal{Y}}_{i, \eta}(t, \eta) \\
& \leqslant A^{6} \min _{j}\left(\tilde{\mathcal{U}}_{j}^{+}\right)(t, \eta) \leqslant \frac{A^{8}}{\sqrt{2}}
\end{aligned}
$$


that

$$
\begin{aligned}
\left|\bar{M}_{1}\right| & \leqslant \frac{1}{A^{7}} \int_{0}^{1}\left(\tilde{\mathcal{Y}}_{1}-\tilde{\mathcal{Y}}_{2}\right)^{2} \min _{j}\left(\tilde{\mathcal{D}}_{j}\right) \min _{j}\left(\tilde{\mathcal{U}}_{j}^{+}\right) \tilde{\mathcal{Y}}_{2, \eta}(t, \eta) d \eta \\
& \leqslant \frac{A}{\sqrt{2}}\left\|\tilde{\mathcal{Y}}_{1}-\tilde{\mathcal{Y}}_{2}\right\|^{2}=\mathcal{O}(1)\left\|\tilde{\mathcal{Y}}_{1}-\tilde{\mathcal{Y}}_{2}\right\|^{2},
\end{aligned}
$$

where $\mathcal{O}(1)$ denotes some constant, which only depends on $A$ and which remains bounded as $A \rightarrow 0$.

The second term $\bar{M}_{2}$, on the other hand, is a bit more demanding.

(i): First of all, recall (A.4), that is,

$$
\left|\frac{d}{d \theta} \min _{j}\left(e^{-\frac{1}{a}\left(\tilde{\mathcal{Y}}_{j}(t, \eta)-\tilde{\mathcal{Y}}_{j}(t, \theta)\right)}\right)\right| \leqslant \frac{1}{a} \min _{j}\left(e^{-\frac{1}{a}\left(\tilde{\mathcal{Y}}_{j}(t, \eta)-\tilde{\mathcal{Y}}_{j}(t, \theta)\right)}\right) \max _{j}\left(\tilde{\mathcal{Y}}_{j, \eta}\right)(t, \theta),
$$

which implies that

$$
\begin{aligned}
& \frac{1}{A^{7}} \mid \int_{0}^{1}\left(\tilde{\mathcal{Y}}_{1}-\tilde{\mathcal{Y}}_{2}\right) \tilde{\mathcal{Y}}_{2, \eta}(t, \eta) \mathbb{1}_{D^{c}}(t, \eta) \int_{0}^{\eta}\left(\tilde{\mathcal{Y}}_{1}-\tilde{\mathcal{Y}}_{2}\right)(t, \theta) \\
& \times\left(\frac{d}{d \theta} \min _{j}\left(e^{-\frac{1}{a}\left(\tilde{\mathcal{Y}}_{j}(t, \eta)-\tilde{\mathcal{Y}}_{j}(t, \theta)\right)}\right)\right) \min _{j}\left(\tilde{\mathcal{D}}_{j}\right) \min _{j}\left(\tilde{\mathcal{U}}_{j}^{+}\right)(t, \theta) d \theta d \eta \mid \\
& \leqslant\left\|\tilde{\mathcal{Y}}_{1}-\tilde{\mathcal{Y}}_{2}\right\|^{2}+\frac{1}{A^{14}} \int_{0}^{1} \tilde{\mathcal{Y}}_{2, \eta}^{2}(t, \eta) \mathbb{1}_{D^{c}}(t, \eta) \\
& \times\left(\int_{0}^{\eta}\left(\tilde{\mathcal{Y}}_{1}-\tilde{\mathcal{Y}}_{2}\right)(t, \theta)\left(\frac{d}{d \theta} \min _{j}\left(e^{-\frac{1}{a}\left(\tilde{\mathcal{Y}}_{j}(t, \eta)-\tilde{\mathcal{Y}}_{j}(t, \theta)\right)}\right)\right)\right. \\
& \left.\times \min _{j}\left(\tilde{\mathcal{D}}_{j}\right) \min _{j}\left(\tilde{\mathcal{U}}_{j}^{+}\right)(t, \theta) d \theta\right)^{2} d \eta \\
& \leqslant\left\|\tilde{\mathcal{Y}}_{1}-\tilde{\mathcal{Y}}_{2}\right\|^{2}+\frac{1}{a^{2} A^{2}} \int_{0}^{1} \tilde{\mathcal{Y}}_{2, \eta}^{2}(t, \eta) \\
& \times\left(\int_{0}^{\eta}\left|\tilde{\mathcal{Y}}_{1}-\tilde{\mathcal{Y}}_{2}\right|(t, \theta) e^{-\frac{1}{A_{2}}\left(\tilde{\mathcal{Y}}_{2}(t, \eta)-\tilde{\mathcal{Y}}_{2}(t, \theta)\right)} \min _{j}\left(\tilde{\mathcal{U}}_{j}^{+}\right)(t, \theta) d \theta\right)^{2} d \eta \\
& \leqslant\left\|\tilde{\mathcal{Y}}_{1}-\tilde{\mathcal{Y}}_{2}\right\|^{2} \\
& +\frac{a^{2}}{2 A^{2}} \int_{0}^{1} \tilde{\mathcal{Y}}_{2, \eta}^{2}(t, \eta)\left(\int_{0}^{\eta}\left|\tilde{\mathcal{Y}}_{1}-\tilde{\mathcal{Y}}_{2}\right|(t, \theta) e^{-\frac{1}{A_{2}}\left(\tilde{\mathcal{Y}}_{2}(t, \eta)-\tilde{\mathcal{Y}}_{2}(t, \theta)\right)} d \theta\right)^{2} d \eta \\
& \leqslant\left\|\tilde{\mathcal{Y}}_{1}-\tilde{\mathcal{Y}}_{2}\right\|^{2} \\
& +\frac{1}{2} \int_{0}^{1} \tilde{\mathcal{Y}}_{2, \eta}^{2}(t, \eta)\left(\int_{0}^{\eta}\left(\tilde{\mathcal{Y}}_{1}-\tilde{\mathcal{Y}}_{2}\right)^{2}(t, \theta) e^{-\frac{1}{2 A_{2}}\left(\tilde{\mathcal{Y}}_{2}(t, \eta)-\tilde{\mathcal{Y}}_{2}(t, \theta)\right)} d \theta\right)
\end{aligned}
$$




$$
\begin{aligned}
& \times\left(\int_{0}^{\eta} e^{-\frac{3}{2 A_{2}}\left(\tilde{\mathcal{Y}}_{2}(t, \eta)-\tilde{\mathcal{Y}}_{2}(t, \theta)\right)} d \theta\right) d \eta \\
& \leqslant\left\|\tilde{\mathcal{Y}}_{1}-\tilde{\mathcal{Y}}_{2}\right\|^{2} \\
& +\frac{1}{A_{2}^{5}} \int_{0}^{1} \tilde{\mathcal{Y}}_{2, \eta}^{2}(t, \eta)\left(\int_{0}^{\eta}\left(\tilde{\mathcal{Y}}_{1}-\tilde{\mathcal{Y}}_{2}\right)^{2}(t, \theta) e^{-\frac{1}{2 A_{2}}\left(\tilde{\mathcal{Y}}_{2}(t, \eta)-\tilde{\mathcal{Y}}_{2}(t, \theta)\right)} d \theta\right) \\
& \times\left(\int_{0}^{\eta} e^{-\frac{3}{2 A_{2}}\left(\tilde{\mathcal{Y}}_{2}(t, \eta)-\tilde{\mathcal{Y}}_{2}(t, \theta)\right)}\left(\tilde{\mathcal{P}}_{2} \tilde{\mathcal{Y}}_{2, \eta}+\tilde{\mathcal{H}}_{2, \eta}\right)(t, \theta) d \theta\right) d \eta \\
& \leqslant\left\|\tilde{\mathcal{Y}}_{1}-\tilde{\mathcal{Y}}_{2}\right\|^{2} \\
& +\frac{6}{A_{2}^{4}} \int_{0}^{1} \tilde{\mathcal{P}}_{2} \tilde{\mathcal{Y}}_{2, \eta}^{2}(t, \eta)\left(\int_{0}^{\eta}\left(\tilde{\mathcal{Y}}_{1}-\tilde{\mathcal{Y}}_{2}\right)^{2}(t, \theta) e^{-\frac{1}{2 A_{2}}\left(\tilde{\mathcal{Y}}_{2}(t, \eta)-\tilde{\mathcal{Y}}_{2}(t, \theta)\right)} d \theta\right) d \eta \\
& \leqslant\left\|\tilde{\mathcal{Y}}_{1}-\tilde{\mathcal{Y}}_{2}\right\|^{2} \\
& +3 A \int_{0}^{1} \tilde{\mathcal{Y}}_{2, \eta}(t, \eta) e^{-\frac{1}{2 A_{2}} \tilde{\mathcal{Y}}_{2}(t, \eta)}\left(\int_{0}^{\eta}\left(\tilde{\mathcal{Y}}_{1}-\tilde{\mathcal{Y}}_{2}\right)^{2}(t, \theta) e^{\frac{1}{2 A_{2}} \tilde{\mathcal{Y}}_{2}(t, \theta)} d \theta\right) d \eta \\
& =\left\|\tilde{\mathcal{Y}}_{1}-\tilde{\mathcal{Y}}_{2}\right\|^{2}-\left.6 A A_{2} \int_{0}^{\eta} e^{-\frac{1}{2 A_{2}}\left(\tilde{\mathcal{Y}}_{2}(t, \eta)-\tilde{\mathcal{Y}}_{2}(t, \theta)\right)}\left(\tilde{\mathcal{Y}}_{1}-\tilde{\mathcal{Y}}_{2}\right)^{2}(t, \theta) d \theta\right|_{\eta=0} ^{1} \\
& +6 A A_{2} \int_{0}^{1}\left(\tilde{\mathcal{Y}}_{1}-\tilde{\mathcal{Y}}_{2}\right)^{2}(t, \eta) d \eta \\
& \leqslant \mathcal{O}(1)\left\|\tilde{\mathcal{Y}}_{1}-\tilde{\mathcal{Y}}_{2}\right\|^{2},
\end{aligned}
$$

where we used (4.13), (4.16e), and (4.16f). Again, $\mathcal{O}(1)$ denotes some constant only depending on $A$, which remains bounded as $A \rightarrow 0$.

(ii): First of all, we have to establish that $\min _{j}\left(\tilde{\mathcal{D}}_{j}\right) \min _{j}\left(\tilde{\mathcal{U}}_{j}^{+}\right)(t, \theta)$ is Lipschitz continuous with a uniformly bounded Lipschitz constant. More precisely, in Lemma A.4 we show that

$$
\left|\frac{d}{d \theta}\left(\min _{j}\left(\tilde{\mathcal{D}}_{j}\right) \min _{j}\left(\tilde{\mathcal{U}}_{j}^{+}\right)\right)(t, \theta)\right| \leqslant \mathcal{O}(1) \sqrt{A} A^{4}\left(\min _{j}\left(\tilde{\mathcal{D}}_{j}\right)^{1 / 2}+\left|\tilde{\mathcal{U}}_{2}\right|\right)(t, \theta) .
$$

We are now ready to establish a Lipschitz estimate for the second part of $\bar{M}_{2}$. Indeed,

$$
\begin{aligned}
& \frac{1}{A^{7}} \mid \int_{0}^{1}\left(\tilde{\mathcal{Y}}_{1}-\tilde{\mathcal{Y}}_{2}\right) \tilde{\mathcal{Y}}_{2, \eta} \mathbb{1}_{D^{c}}(t, \eta) \int_{0}^{\eta}\left(\tilde{\mathcal{Y}}_{1}-\tilde{\mathcal{Y}}_{2}\right)(t, \theta) \min _{j}\left(e^{-\frac{1}{a}\left(\tilde{\mathcal{Y}}_{j}(t, \eta)-\tilde{\mathcal{Y}}_{j}(t, \theta)\right)}\right) \\
& \quad \times\left(\frac{d}{d \theta} \min _{j}\left(\tilde{\mathcal{D}}_{j}\right) \min _{j}\left(\tilde{\mathcal{U}}_{j}^{+}\right)\right)(t, \theta) d \theta d \eta \mid
\end{aligned}
$$




$$
\begin{aligned}
& \leqslant\left\|\tilde{\mathcal{Y}}_{1}-\tilde{\mathcal{Y}}_{2}\right\|^{2}+\frac{1}{A^{14}} \int_{0}^{1} \tilde{\mathcal{Y}}_{2, \eta}^{2} \mathbb{1}_{D^{c}}(t, \eta) \\
& \times\left(\int_{0}^{\eta}\left(\tilde{\mathcal{Y}}_{1}-\tilde{\mathcal{Y}}_{2}\right)(t, \theta) \min _{j}\left(e^{-\frac{1}{a}\left(\tilde{\mathcal{Y}}_{j}(t, \eta)-\tilde{\mathcal{Y}}_{j}(t, \theta)\right)}\right)\right. \\
& \left.\times\left(\frac{d}{d \theta} \min _{j}\left(\tilde{\mathcal{D}}_{j}\right) \min _{j}\left(\tilde{\mathcal{U}}_{j}^{+}\right)\right)(t, \theta) d \theta\right)^{2} d \eta \\
& \leqslant\left\|\tilde{\mathcal{Y}}_{1}-\tilde{\mathcal{Y}}_{2}\right\|^{2} \\
& +\mathcal{O}(1) \frac{1}{A^{5}} \int_{0}^{1} \tilde{\mathcal{Y}}_{2, \eta}^{2}(t, \eta)\left(\int_{0}^{\eta} \min _{j}\left(e^{-\frac{1}{a}\left(\tilde{\mathcal{Y}}_{j}(t, \eta)-\tilde{\mathcal{Y}}_{j}(t, \theta)\right)}\right)\left|\tilde{\mathcal{Y}}_{1}-\tilde{\mathcal{Y}}_{2}\right|\right. \\
& \left.\times\left(\min _{j}\left(\tilde{\mathcal{D}}_{j}\right)^{1 / 2}+\left|\tilde{\mathcal{U}}_{2}\right|\right)(t, \theta) d \theta\right)^{2} d \eta \\
& \leqslant\left\|\tilde{\mathcal{Y}}_{1}-\tilde{\mathcal{Y}}_{2}\right\|^{2} \\
& +\mathcal{O}(1) \frac{1}{A^{5}} \int_{0}^{1} \tilde{\mathcal{Y}}_{2, \eta}^{2}(t, \eta)\left(\int_{0}^{\eta} e^{-\frac{1}{A_{2}}\left(\tilde{\mathcal{Y}}_{2}(t, \eta)-\tilde{\mathcal{Y}}_{2}(t, \theta)\right)}\left|\tilde{\mathcal{Y}}_{1}-\tilde{\mathcal{Y}}_{2}\right| \tilde{\mathcal{P}}_{2}^{1 / 2}(t, \theta) d \theta\right)^{2} d \eta \\
& \leqslant\left\|\tilde{\mathcal{Y}}_{1}-\tilde{\mathcal{Y}}_{2}\right\|^{2}
\end{aligned}
$$$$
+\mathcal{O}(1) \frac{1}{A^{5}} \int_{0}^{1} \tilde{\mathcal{Y}}_{2, \eta}^{2}(t, \eta)\left(\int_{0}^{\eta} e^{-\frac{3}{2 A_{2}}\left(\tilde{\mathcal{Y}}_{2}(t, \eta)-\tilde{\mathcal{Y}}_{2}(t, \theta)\right)} \tilde{\mathcal{P}}_{2}(t, \theta) d \theta\right)
$$$$
\times\left(\int_{0}^{\eta} e^{-\frac{1}{2 A_{2}}\left(\tilde{\mathcal{Y}}_{2}(t, \eta)-\tilde{\mathcal{Y}}_{2}(t, \theta)\right)}\left(\tilde{\mathcal{Y}}_{1}-\tilde{\mathcal{Y}}_{2}\right)^{2}(t, \theta) d \theta\right) d \eta
$$$$
\leqslant\left\|\tilde{\mathcal{Y}}_{1}-\tilde{\mathcal{Y}}_{2}\right\|^{2}
$$$$
+\mathcal{O}(1) \frac{1}{A^{5}} \int_{0}^{1} \tilde{\mathcal{P}}_{2} \tilde{\mathcal{Y}}_{2, \eta}^{2}(t, \eta)\left(\int_{0}^{\eta} e^{-\frac{1}{2 A_{2}}\left(\tilde{\mathcal{Y}}_{2}(t, \eta)-\tilde{\mathcal{Y}}_{2}(t, \theta)\right)}\left(\tilde{\mathcal{Y}}_{1}-\tilde{\mathcal{Y}}_{2}\right)^{2}(t, \theta) d \theta\right) d \eta
$$$$
\leqslant\left\|\tilde{\mathcal{Y}}_{1}-\tilde{\mathcal{Y}}_{2}\right\|^{2}
$$$$
+\mathcal{O}(1) \int_{0}^{1} \tilde{\mathcal{Y}}_{2, \eta}(t, \eta) e^{-\frac{1}{2 A_{2}} \tilde{\mathcal{Y}}_{2}(t, \eta)}\left(\int_{0}^{\eta} e^{\frac{1}{2 A_{2}} \tilde{\mathcal{Y}}_{2}(t, \theta)}\left(\tilde{\mathcal{Y}}_{1}-\tilde{\mathcal{Y}}_{2}\right)^{2}(t, \theta) d \theta\right) d \eta
$$

$\leqslant\left\|\tilde{\mathcal{Y}}_{1}-\tilde{\mathcal{Y}}_{2}\right\|^{2}$

$$
\begin{aligned}
& +\mathcal{O}(1) A_{2}\left(-\left.2 \int_{0}^{\eta} e^{-\frac{1}{2 A_{2}}\left(\tilde{\mathcal{Y}}_{2}(t, \eta)-\tilde{\mathcal{Y}}_{2}(t, \theta)\right)}\left(\tilde{\mathcal{Y}}_{1}-\tilde{\mathcal{Y}}_{2}\right)^{2}(t, \theta) d \theta\right|_{\eta=0} ^{1}\right. \\
& \left.+\int_{0}^{1} 2\left(\tilde{\mathcal{Y}}_{1}-\tilde{\mathcal{Y}}_{2}\right)^{2}(t, \eta) d \eta\right) \\
& \leqslant \mathcal{O}(1)\left\|\tilde{\mathcal{Y}}_{1}-\tilde{\mathcal{Y}}_{2}\right\|^{2}
\end{aligned}
$$


where we used (4.16i). Thus we conclude that

$$
\left|\bar{B}_{38}\right|+\left|\bar{B}_{39}\right| \leqslant \mathcal{O}(1)\left\|\tilde{\mathcal{Y}}_{1}-\tilde{\mathcal{Y}}_{2}\right\|^{2} .
$$

Next we have a look at $\bar{K}_{14}$, which can be rewritten as follows:

$$
\begin{aligned}
\bar{K}_{14}= & \frac{3}{A^{6}} \int_{0}^{1}\left(\tilde{\mathcal{Y}}_{1}-\tilde{\mathcal{Y}}_{2}\right)(t, \eta)\left(\tilde{\mathcal{Y}}_{1, \eta}(t, \eta) \int_{0}^{\eta} e^{-\frac{1}{A_{1}}\left(\tilde{\mathcal{Y}}_{1}(t, \eta)-\tilde{\mathcal{Y}}_{1}(t, \theta)\right)} \tilde{\mathcal{P}}_{1} \tilde{\mathcal{U}}_{1} \tilde{\mathcal{Y}}_{1, \eta}(t, \theta) d \theta\right. \\
& \left.-\tilde{\mathcal{Y}}_{2, \eta}(t, \eta) \int_{0}^{\eta} e^{-\frac{1}{A_{2}}\left(\tilde{\mathcal{Y}}_{2}(t, \eta)-\tilde{\mathcal{Y}}_{2}(t, \theta)\right)} \tilde{\mathcal{P}}_{2} \tilde{\mathcal{U}}_{2} \tilde{\mathcal{Y}}_{2, \eta}(t, \theta) d \theta\right) d \eta \\
= & \frac{3}{A^{6}} \int_{0}^{1}\left(\tilde{\mathcal{Y}}_{1}-\tilde{\mathcal{Y}}_{2}\right)(t, \eta)\left(\tilde{\mathcal{Y}}_{1, \eta}(t, \eta) \int_{0}^{\eta} e^{-\frac{1}{A_{1}}\left(\tilde{\mathcal{Y}}_{1}(t, \eta)-\tilde{\mathcal{Y}}_{1}(t, \theta)\right)} \tilde{\mathcal{P}}_{1} \tilde{\mathcal{U}}_{1}^{+} \tilde{\mathcal{Y}}_{1, \eta}(t, \theta) d \theta\right. \\
& \left.-\tilde{\mathcal{Y}}_{2, \eta}(t, \eta) \int_{0}^{\eta} e^{-\frac{1}{A_{2}}\left(\tilde{\mathcal{Y}}_{2}(t, \eta)-\tilde{\mathcal{Y}}_{2}(t, \theta)\right)} \tilde{\mathcal{P}}_{2} \tilde{\mathcal{U}}_{2}^{+} \tilde{\mathcal{Y}}_{2, \eta}(t, \theta) d \theta\right) d \eta \\
& +\frac{3}{A^{6}} \int_{0}^{1}\left(\tilde{\mathcal{Y}}_{1}-\tilde{\mathcal{Y}}_{2}\right)(t, \eta)\left(\tilde{\mathcal{Y}}_{1, \eta}(t, \eta) \int_{0}^{\eta} e^{-\frac{1}{A_{1}}\left(\tilde{\mathcal{Y}}_{1}(t, \eta)-\tilde{\mathcal{Y}}_{1}(t, \theta)\right)} \tilde{\mathcal{P}}_{1} \tilde{\mathcal{U}}_{1}^{-} \tilde{\mathcal{Y}}_{1, \eta}(t, \theta) d \theta\right. \\
& \left.-\tilde{\mathcal{Y}}_{2, \eta}(t, \eta) \int_{0}^{\eta} e^{-\frac{1}{A_{2}}\left(\tilde{\mathcal{Y}}_{2}(t, \eta)-\tilde{\mathcal{Y}}_{2}(t, \theta)\right)} \tilde{\mathcal{P}}_{2} \tilde{\mathcal{U}}_{2}^{-} \tilde{\mathcal{Y}}_{2, \eta}(t, \theta) d \theta\right) d \eta \\
= & \bar{K}_{14}^{+}+\bar{K}_{14}^{-} .
\end{aligned}
$$

Note that both $\bar{K}_{14}^{+}$and $\bar{K}_{14}^{-}$have the same structure. Moreover, having a close look at $\bar{K}_{14}^{+}$one has

$$
\bar{K}_{14}^{+}=-3 \tilde{K}_{1},
$$

where $\tilde{K}_{1}$ is defined in (5.15). Thus we can immediately conclude that

$$
\left|\bar{K}_{14}^{+}\right| \leqslant \mathcal{O}(1)\left(\left\|\tilde{\mathcal{Y}}_{1}-\tilde{\mathcal{Y}}_{2}\right\|^{2}+\left\|\tilde{\mathcal{U}}_{1}-\tilde{\mathcal{U}}_{2}\right\|^{2}+\left\|\tilde{\mathcal{P}}_{1}^{1 / 2}-\tilde{\mathcal{P}}_{2}^{1 / 2}\right\|^{2}+\left|A_{1}-A_{2}\right|\right) .
$$

Next, we have a look at $\bar{K}_{15}$, which can be rewritten as follows:

$$
\begin{aligned}
\bar{K}_{15}= & \frac{1}{A^{6}} \int_{0}^{1}\left(\tilde{\mathcal{Y}}_{1}-\tilde{\mathcal{Y}}_{2}\right)(t, \eta)\left(\tilde{\mathcal{Y}}_{2, \eta}(t, \eta) \int_{0}^{\eta} e^{-\frac{1}{A_{2}}\left(\tilde{\mathcal{Y}}_{2}(t, \eta)-\tilde{\mathcal{Y}}_{2}(t, \theta)\right)} \tilde{\mathcal{U}}_{2}^{3} \tilde{\mathcal{Y}}_{2, \eta}(t, \theta) d \theta\right. \\
& \left.-\tilde{\mathcal{Y}}_{1, \eta}(t, \eta) \int_{0}^{\eta} e^{-\frac{1}{A_{1}}\left(\tilde{\mathcal{Y}}_{1}(t, \eta)-\tilde{\mathcal{Y}}_{1}(t, \theta)\right)} \tilde{\mathcal{U}}_{1}^{3} \tilde{\mathcal{Y}}_{1, \eta}(t, \theta) d \theta\right) d \eta \\
= & \frac{1}{A^{6}} \int_{0}^{1}\left(\tilde{\mathcal{Y}}_{1}-\tilde{\mathcal{Y}}_{2}\right)(t, \eta)\left(\tilde{\mathcal{Y}}_{2, \eta}(t, \eta) \int_{0}^{\eta} e^{-\frac{1}{A_{2}}\left(\tilde{\mathcal{Y}}_{2}(t, \eta)-\tilde{\mathcal{Y}}_{2}(t, \theta)\right)}\left(\tilde{\mathcal{U}}_{2}^{+}\right)^{3} \tilde{\mathcal{Y}}_{2, \eta}(t, \theta) d \theta\right. \\
& \left.-\tilde{\mathcal{Y}}_{1, \eta}(t, \eta) \int_{0}^{\eta} e^{-\frac{1}{A_{1}}\left(\tilde{\mathcal{Y}}_{1}(t, \eta)-\tilde{\mathcal{Y}}_{1}(t, \theta)\right)}\left(\tilde{\mathcal{U}}_{1}^{+}\right)^{3} \tilde{\mathcal{Y}}_{1, \eta}(t, \theta) d \theta\right) d \eta \\
& +\frac{1}{A^{6}} \int_{0}^{1}\left(\tilde{\mathcal{Y}}_{1}-\tilde{\mathcal{Y}}_{2}\right)(t, \eta)\left(\tilde{\mathcal{Y}}_{2, \eta}(t, \eta) \int_{0}^{\eta} e^{-\frac{1}{A_{2}}\left(\tilde{\mathcal{Y}}_{2}(t, \eta)-\tilde{\mathcal{Y}}_{2}(t, \theta)\right)}\left(\tilde{\mathcal{U}}_{2}^{-}\right)^{3} \tilde{\mathcal{Y}}_{2, \eta}(t, \theta) d \theta\right.
\end{aligned}
$$




$$
\begin{aligned}
& \left.-\tilde{\mathcal{Y}}_{1, \eta}(t, \eta) \int_{0}^{\eta} e^{-\frac{1}{A_{1}}\left(\tilde{\mathcal{Y}}_{1}(t, \eta)-\tilde{\mathcal{Y}}_{1}(t, \theta)\right)}\left(\tilde{\mathcal{U}}_{1}^{-}\right)^{3} \tilde{\mathcal{Y}}_{1, \eta}(t, \theta) d \theta\right) d \eta \\
= & \bar{K}_{15}^{+}+\bar{K}_{15}^{-} .
\end{aligned}
$$

Note that both $\bar{K}_{15}^{+}$and $\bar{K}_{15}^{-}$have the same structure. Having a close look at $\bar{K}_{15}^{+}$ one has

$$
\bar{K}_{15}^{+}=\int_{0}^{1}\left(\tilde{\mathcal{Y}}_{1}-\tilde{\mathcal{Y}}_{2}\right)\left(J_{1}+J_{2}+J_{3}+J_{4}+J_{5}+J_{6}+J_{7}+J_{8}\right)(t, \eta) d \eta,
$$

where $J_{1}, \ldots, J_{8}$ are defined in (5.10). Thus we can conclude immediately that

$$
\begin{aligned}
\left|\bar{K}_{15}^{+}\right| \leqslant & \mathcal{O}(1)\left(\left\|\tilde{\mathcal{Y}}_{1}-\tilde{\mathcal{Y}}_{2}\right\|^{2}+\left\|\tilde{\mathcal{U}}_{1}-\tilde{\mathcal{U}}_{2}\right\|^{2}\right. \\
& \left.+\left\|\tilde{\mathcal{P}}_{1}^{1 / 2}-\tilde{\mathcal{P}}_{2}^{1 / 2}\right\|^{2}+\left|A_{1}-A_{2}\right|^{2}\right)
\end{aligned}
$$

Finally, we have a look at $\bar{K}_{16}$, which can be rewritten as follows:

$$
\begin{aligned}
& \bar{K}_{16}=\frac{1}{2 A^{6}} \int_{0}^{1}\left(\tilde{\mathcal{Y}}_{1}-\tilde{\mathcal{Y}}_{2}\right)(t, \eta)\left(\tilde{\mathcal{Y}}_{2, \eta}(t, \eta) \int_{0}^{\eta} e^{-\frac{1}{A_{2}}\left(\tilde{\mathcal{Y}}_{2}(t, \eta)-\tilde{\mathcal{Y}}_{2}(t, \theta)\right)} A_{2}^{5} \tilde{\mathcal{U}}_{2}(t, \theta) d \theta\right. \\
& \left.-\tilde{\mathcal{Y}}_{1, \eta}(t, \eta) \int_{0}^{\eta} e^{-\frac{1}{A_{1}}\left(\tilde{\mathcal{Y}}_{1}(t, \eta)-\tilde{\mathcal{Y}}_{1}(t, \theta)\right)} A_{1}^{5} \tilde{\mathcal{U}}_{1}(t, \theta) d \theta\right) d \eta \\
& =\frac{A_{2}^{5}-A_{1}^{5}}{2 A^{6}} \mathbb{1}_{A_{1} \leqslant A_{2}} \int_{0}^{1}\left(\tilde{\mathcal{Y}}_{1}-\tilde{\mathcal{Y}}_{2}\right) \tilde{\mathcal{Y}}_{2, \eta}(t, \eta) \\
& \times\left(\int_{0}^{\eta} e^{-\frac{1}{A_{2}}\left(\tilde{\mathcal{Y}}_{2}(t, \eta)-\tilde{\mathcal{Y}}_{2}(t, \theta)\right)} \tilde{\mathcal{U}}_{2}(t, \theta) d \theta\right) d \eta \\
& +\frac{A_{2}^{5}-A_{1}^{5}}{2 A^{6}} \mathbb{1}_{A_{2}<A_{1}} \int_{0}^{1}\left(\tilde{\mathcal{Y}}_{1}-\tilde{\mathcal{Y}}_{2}\right) \tilde{\mathcal{Y}}_{1, \eta}(t, \eta) \\
& \times\left(\int_{0}^{\eta} e^{-\frac{1}{A_{1}}\left(\tilde{\mathcal{Y}}_{1}(t, \eta)-\tilde{\mathcal{Y}}_{1}(t, \theta)\right)} \tilde{\mathcal{U}}_{1}(t, \theta) d \theta\right) d \eta \\
& +\frac{a^{5}}{2 A^{6}} \int_{0}^{1}\left(\tilde{\mathcal{Y}}_{1}-\tilde{\mathcal{Y}}_{2}\right) \tilde{\mathcal{Y}}_{2, \eta}(t, \eta) \\
& \times\left(\int_{0}^{\eta}\left(e^{-\frac{1}{A_{2}}\left(\tilde{\mathcal{Y}}_{2}(t, \eta)-\tilde{\mathcal{Y}}_{2}(t, \theta)\right)}-e^{-\frac{1}{A_{2}}\left(\tilde{\mathcal{Y}}_{1}(t, \eta)-\tilde{\mathcal{Y}}_{1}(t, \theta)\right)}\right) \tilde{\mathcal{U}}_{2} \mathbb{1}_{B(\eta)}(t, \theta) d \theta\right) d \eta \\
& +\frac{a^{5}}{2 A^{6}} \int_{0}^{1}\left(\tilde{\mathcal{Y}}_{1}-\tilde{\mathcal{Y}}_{2}\right) \tilde{\mathcal{Y}}_{1, \eta}(t, \eta) \\
& \times\left(\int_{0}^{\eta}\left(e^{-\frac{1}{A_{1}}\left(\tilde{\mathcal{Y}}_{2}(t, \eta)-\tilde{\mathcal{Y}}_{2}(t, \theta)\right)}-e^{-\frac{1}{A_{1}}\left(\tilde{\mathcal{Y}}_{1}(t, \eta)-\tilde{\mathcal{Y}}_{1}(t, \theta)\right)}\right) \tilde{\mathcal{U}}_{1} \mathbb{1}_{B(\eta) c}(t, \theta) d \theta\right) d \eta
\end{aligned}
$$




$$
\begin{aligned}
& +\mathbb{1}_{A_{1} \leqslant A_{2}} \frac{a^{5}}{2 A^{6}} \int_{0}^{1}\left(\tilde{\mathcal{Y}}_{1}-\tilde{\mathcal{Y}}_{2}\right) \tilde{\mathcal{Y}}_{2, \eta}(t, \eta)\left(\int _ { 0 } ^ { \eta } \left(\min _{j}\left(e^{-\frac{1}{A_{2}}\left(\tilde{\mathcal{Y}}_{j}(t, \eta)-\tilde{\mathcal{Y}}_{j}(t, \theta)\right)}\right)\right.\right. \\
& \left.\left.-\min _{j}\left(e^{-\frac{1}{A_{1}}\left(\tilde{\mathcal{Y}}_{j}(t, \eta)-\tilde{\mathcal{Y}}_{j}(t, \theta)\right)}\right)\right) \tilde{\mathcal{U}}_{2}(t, \theta) d \theta\right) d \eta \\
& +\mathbb{1}_{A_{2}<A_{1}} \frac{a^{5}}{2 A^{6}} \int_{0}^{1}\left(\tilde{\mathcal{Y}}_{1}-\tilde{\mathcal{Y}}_{2}\right) \tilde{\mathcal{Y}}_{1, \eta}(t, \eta)\left(\int _ { 0 } ^ { \eta } \left(\min _{j}\left(e^{-\frac{1}{A_{2}}\left(\tilde{\mathcal{Y}}_{j}(t, \eta)-\tilde{\mathcal{Y}}_{j}(t, \theta)\right)}\right)\right.\right. \\
& \left.\left.-\min _{j}\left(e^{-\frac{1}{A_{1}}\left(\tilde{\mathcal{Y}}_{j}(t, \eta)-\tilde{\mathcal{Y}}_{j}(t, \theta)\right)}\right)\right) \tilde{\mathcal{U}}_{1}(t, \theta) d \theta\right) d \eta \\
& +\frac{a^{5}}{2 A^{6}} \int_{0}^{1}\left(\tilde{\mathcal{Y}}_{1}-\tilde{\mathcal{Y}}_{2}\right) \tilde{\mathcal{Y}}_{2, \eta}(t, \eta) \\
& \times\left(\int_{0}^{\eta} \min _{j}\left(e^{-\frac{1}{a}\left(\tilde{\mathcal{Y}}_{j}(t, \eta)-\tilde{\mathcal{Y}}_{j}(t, \theta)\right)}\right)\left(\tilde{\mathcal{U}}_{2}^{+}-\tilde{\mathcal{U}}_{1}^{+}\right) \mathbb{1}_{\tilde{\mathcal{U}}_{1}^{+} \leqslant \tilde{\mathcal{U}}_{2}^{+}}(t, \theta) d \theta\right) d \eta \\
& +\frac{a^{5}}{2 A^{6}} \int_{0}^{1}\left(\tilde{\mathcal{Y}}_{1}-\tilde{\mathcal{Y}}_{2}\right) \tilde{\mathcal{Y}}_{2, \eta}(t, \eta) \\
& \times\left(\int_{0}^{\eta} \min _{j}\left(e^{-\frac{1}{a}\left(\tilde{\mathcal{Y}}_{j}(t, \eta)-\tilde{\mathcal{Y}}_{j}(t, \theta)\right)}\right)\left(\tilde{\mathcal{U}}_{2}^{-}-\tilde{\mathcal{U}}_{1}^{-}\right) \mathbb{1}_{\tilde{\mathcal{U}}_{2}^{-} \leqslant \tilde{\mathcal{U}}_{1}^{-}}(t, \theta) d \theta\right) d \eta \\
& +\frac{a^{5}}{2 A^{6}} \int_{0}^{1}\left(\tilde{\mathcal{Y}}_{1}-\tilde{\mathcal{Y}}_{2}\right) \tilde{\mathcal{Y}}_{1, \eta}(t, \eta) \\
& \times\left(\int_{0}^{\eta} \min _{j}\left(e^{-\frac{1}{a}\left(\tilde{\mathcal{Y}}_{j}(t, \eta)-\tilde{\mathcal{Y}}_{j}(t, \theta)\right)}\right)\left(\tilde{\mathcal{U}}_{2}^{+}-\tilde{\mathcal{U}}_{1}^{+}\right) \mathbb{1}_{\tilde{\mathcal{U}}_{2}^{+} \leqslant \tilde{\mathcal{U}}_{1}^{+}}(t, \theta) d \theta\right) d \eta \\
& +\frac{a^{5}}{2 A^{6}} \int_{0}^{1}\left(\tilde{\mathcal{Y}}_{1}-\tilde{\mathcal{Y}}_{2}\right) \tilde{\mathcal{Y}}_{1, \eta}(t, \eta) \\
& \times\left(\int_{0}^{\eta} \min _{j}\left(e^{-\frac{1}{a}\left(\tilde{\mathcal{Y}}_{j}(t, \eta)-\tilde{\mathcal{Y}}_{j}(t, \theta)\right)}\right)\left(\tilde{\mathcal{U}}_{2}^{-}-\tilde{\mathcal{U}}_{1}^{-}\right) \mathbb{1}_{\tilde{\mathcal{U}}_{1}^{-} \leqslant \tilde{\mathcal{U}}_{2}^{-}}(t, \theta) d \theta\right) d \eta \\
& +\frac{a^{5}}{2 A^{6}} \int_{0}^{1}\left(\tilde{\mathcal{Y}}_{1}-\tilde{\mathcal{Y}}_{2}\right)\left(\tilde{\mathcal{Y}}_{2, \eta}-\tilde{\mathcal{Y}}_{1, \eta}\right)(t, \eta) \\
& \times\left(\int_{0}^{\eta} \min _{j}\left(e^{-\frac{1}{a}\left(\tilde{\mathcal{Y}}_{j}(t, \eta)-\tilde{\mathcal{Y}}_{j}(t, \theta)\right)}\right) \min _{j}\left(\tilde{\mathcal{U}}_{j}^{+}\right)(t, \theta) d \theta\right) d \eta \\
& +\frac{a^{5}}{2 A^{6}} \int_{0}^{1}\left(\tilde{\mathcal{Y}}_{1}-\tilde{\mathcal{Y}}_{2}\right)\left(\tilde{\mathcal{Y}}_{2, \eta}-\tilde{\mathcal{Y}}_{1, \eta}\right)(t, \eta) \\
& \times\left(\int_{0}^{\eta} \min _{j}\left(e^{-\frac{1}{a}\left(\tilde{\mathcal{Y}}_{j}(t, \eta)-\tilde{\mathcal{Y}}_{j}(t, \theta)\right)}\right) \max _{j}\left(\tilde{\mathcal{U}}_{j}^{-}\right)(t, \theta) d \theta\right) d \eta \\
& =\bar{B}_{61}+\bar{B}_{62}+\bar{B}_{63}+\bar{B}_{64}+\bar{B}_{65}+\bar{B}_{66} \\
& +\bar{B}_{67}^{+}+\bar{B}_{67}^{-}+\bar{B}_{68}^{+}+\bar{B}_{68}^{-}+\bar{B}_{69}^{+}+\bar{B}_{69}^{-} \text {. }
\end{aligned}
$$


The key observation, which rescues the whole paper, is again

$$
A_{i}^{5} \leqslant 2\left(\tilde{\mathcal{P}}_{i} \tilde{\mathcal{Y}}_{i, \eta}+\tilde{\mathcal{H}}_{i, \eta}\right)(t, \eta)
$$

which yields for $\bar{B}_{61}$ (and similarly for $\bar{B}_{62}$ ) that

$$
\begin{aligned}
& \left|\bar{B}_{61}\right| \leqslant \frac{A_{2}^{5}-A_{1}^{5}}{2 A^{11}} \int_{0}^{1}\left|\tilde{\mathcal{Y}}_{1}-\tilde{\mathcal{Y}}_{2}\right| \tilde{\mathcal{Y}}_{2, \eta}(t, \eta) \\
& \times\left(\int_{0}^{\eta} e^{-\frac{1}{A_{2}}\left(\tilde{\mathcal{Y}}_{2}(t, \eta)-\tilde{\mathcal{Y}}_{2}(t, \theta)\right)} A_{2}^{5}\left|\tilde{\mathcal{U}}_{2}\right|(t, \theta) d \theta\right) d \eta \\
& \leqslant \frac{5}{2 A^{7}}\left|A_{2}-A_{1}\right|\left\|\tilde{\mathcal{Y}}_{1}-\tilde{\mathcal{Y}}_{2}\right\| \\
& \times\left(\int_{0}^{1} \tilde{\mathcal{Y}}_{2, \eta}^{2}(t, \eta)\left(\int_{0}^{\eta} e^{-\frac{1}{A_{2}}\left(\tilde{\mathcal{Y}}_{2}(t, \eta)-\tilde{\mathcal{Y}}_{2}(t, \theta)\right)} A_{2}^{5}\left|\tilde{\mathcal{U}}_{2}\right|(t, \theta)\right)^{2} d \eta\right)^{1 / 2} \\
& \leqslant \frac{10}{A^{7}}\left|A_{2}-A_{1}\right|\left\|\tilde{\mathcal{Y}}_{1}-\tilde{\mathcal{Y}}_{2}\right\| \\
& \times\left(\int _ { 0 } ^ { 1 } \tilde { \mathcal { Y } } _ { 2 , \eta } ^ { 2 } ( t , \eta ) \left[\left(\int_{0}^{\eta} e^{-\frac{1}{A_{2}}\left(\tilde{\mathcal{Y}}_{2}(t, \eta)-\tilde{\mathcal{Y}}_{2}(t, \theta)\right)} \tilde{\mathcal{P}}_{2}\left|\tilde{\mathcal{U}}_{2}\right| \tilde{\mathcal{Y}}_{2, \eta}(t, \theta) d \theta\right)^{2}\right.\right. \\
& \left.\left.+\left(\int_{0}^{\eta} e^{-\frac{1}{A_{2}}\left(\tilde{\mathcal{Y}}_{2}(t, \eta)-\tilde{\mathcal{Y}}_{2}(t, \theta)\right)}\left|\tilde{\mathcal{U}}_{2}\right| \tilde{\mathcal{H}}_{2, \eta}(t, \theta) d \theta\right)^{2}\right] d \eta\right)^{1 / 2} \\
& \leqslant \frac{10}{A^{7}}\left|A_{2}-A_{1}\right|\left\|\tilde{\mathcal{Y}}_{1}-\tilde{\mathcal{Y}}_{2}\right\|\left(\int_{0}^{1} \tilde{\mathcal{Y}}_{2, \eta}^{2}(t, \eta)\right. \\
& \times\left[\left(\int_{0}^{\eta} e^{-\frac{1}{A_{2}}\left(\tilde{\mathcal{Y}}_{2}(t, \eta)-\tilde{\mathcal{Y}}_{2}(t, \theta)\right)} \tilde{\mathcal{U}}_{2}^{2} \tilde{\mathcal{Y}}_{2, \eta}(t, \theta) d \theta\right)\right. \\
& \times\left(\int_{0}^{\eta} e^{-\frac{1}{A_{2}}\left(\tilde{\mathcal{Y}}_{2}(t, \eta)-\tilde{\mathcal{Y}}_{2}(t, \theta)\right)} \tilde{\mathcal{P}}_{2}^{2} \tilde{\mathcal{Y}}_{2, \eta}(t, \theta) d \theta\right) \\
& \left.\left.+\left\|\tilde{\mathcal{U}}_{2}(t, \cdot)\right\|_{L^{\infty}}^{2}\left(\int_{0}^{\eta} e^{-\frac{1}{A_{2}}\left(\tilde{\mathcal{Y}}_{2}(t, \eta)-\tilde{\mathcal{Y}}_{2}(t, \theta)\right)} \tilde{\mathcal{H}}_{2, \eta}(t, \theta) d \theta\right)^{2}\right] d \eta\right)^{1 / 2} \\
& \leqslant \frac{10}{A^{7}}\left|A_{2}-A_{1}\right|\left\|\tilde{\mathcal{Y}}_{1}-\tilde{\mathcal{Y}}_{2}\right\| \\
& \times\left(\int_{0}^{1}\left(6 A^{6}+16 A^{2}\left\|\tilde{\mathcal{U}}_{2}(t, \cdot)\right\|_{L^{\infty}}^{2}\right) \tilde{\mathcal{P}}_{2}^{2} \tilde{\mathcal{Y}}_{2, \eta}^{2}(t, \eta) d \eta\right)^{1 / 2} \\
& \leqslant \mathcal{O}(1)\left(\left\|\tilde{\mathcal{Y}}_{1}-\tilde{\mathcal{Y}}_{2}\right\|^{2}+\left|A_{2}-A_{1}\right|^{2}\right) \text {. }
\end{aligned}
$$


Next, we have a close look at $\bar{B}_{63}$ (and similarly for $\bar{B}_{64}$ ). Recalling the definition of $B(\eta)$ (5.11), we have

$$
\begin{aligned}
& \left|\bar{B}_{63}\right| \leqslant \frac{a^{5}}{2 A^{6} A_{2}} \int_{0}^{1}\left|\tilde{\mathcal{Y}}_{1}-\tilde{\mathcal{Y}}_{2}\right| \tilde{\mathcal{Y}}_{2, \eta}(t, \eta)\left(\int_{0}^{\eta}\left(\left|\tilde{\mathcal{Y}}_{1}-\tilde{\mathcal{Y}}_{2}\right|(t, \eta)+\left|\tilde{\mathcal{Y}}_{1}-\tilde{\mathcal{Y}}_{2}\right|(t, \theta)\right)\right. \\
& \left.\times e^{-\frac{1}{A_{2}}\left(\tilde{\mathcal{Y}}_{2}(t, \eta)-\tilde{\mathcal{Y}}_{2}(t, \theta)\right)}\left|\tilde{\mathcal{U}}_{2}\right|(t, \theta) d \theta\right) d \eta \\
& \leqslant \frac{a^{5}}{2 A^{6} A_{2}} \int_{0}^{1}\left(\tilde{\mathcal{Y}}_{1}-\tilde{\mathcal{Y}}_{2}\right)^{2} \tilde{\mathcal{Y}}_{2, \eta}(t, \eta)\left(\int_{0}^{\eta} e^{-\frac{1}{A_{2}}\left(\tilde{\mathcal{Y}}_{2}(t, \eta)-\tilde{\mathcal{Y}}_{2}(t, \theta)\right)}\left|\tilde{\mathcal{U}}_{2}\right|(t, \theta) d \theta\right) d \eta \\
& +\frac{a^{5}}{2 A^{6} A_{2}} \int_{0}^{1}\left|\tilde{\mathcal{Y}}_{1}-\tilde{\mathcal{Y}}_{2}\right| \tilde{\mathcal{Y}}_{2, \eta}(t, \eta) \\
& \times\left(\int_{0}^{\eta}\left|\tilde{\mathcal{Y}}_{1}-\tilde{\mathcal{Y}}_{2}\right|(t, \theta) e^{-\frac{1}{A_{2}}\left(\tilde{\mathcal{Y}}_{2}(t, \eta)-\tilde{\mathcal{Y}}_{2}(t, \theta)\right)}\left|\tilde{\mathcal{U}}_{2}\right|(t, \theta) d \theta\right) d \eta \\
& \leqslant \frac{a^{5}}{A^{6} A_{2}^{6}} \int_{0}^{1}\left(\tilde{\mathcal{Y}}_{1}-\tilde{\mathcal{Y}}_{2}\right)^{2} \tilde{\mathcal{Y}}_{2, \eta}(t, \eta) \\
& \times\left(\int_{0}^{\eta} e^{-\frac{1}{A_{2}}\left(\tilde{\mathcal{Y}}_{2}(t, \eta)-\tilde{\mathcal{Y}}_{2}(t, \theta)\right)}\left(\tilde{\mathcal{P}}_{2} \tilde{\mathcal{Y}}_{2, \eta}+\tilde{\mathcal{H}}_{2, \eta}\right)\left|\tilde{\mathcal{U}}_{2}\right|(t, \theta) d \theta\right) d \eta \\
& +\frac{a^{5}}{A^{6} A_{2}^{6}} \int_{0}^{1}\left|\tilde{\mathcal{Y}}_{1}-\tilde{\mathcal{Y}}_{2}\right| \tilde{\mathcal{Y}}_{2, \eta}(t, \eta) \\
& \times\left(\int_{0}^{\eta}\left|\tilde{\mathcal{Y}}_{1}-\tilde{\mathcal{Y}}_{2}\right|(t, \theta) e^{-\frac{1}{A_{2}}\left(\tilde{\mathcal{Y}}_{2}(t, \eta)-\tilde{\mathcal{Y}}_{2}(t, \theta)\right)}\left(\tilde{\mathcal{P}}_{2} \tilde{\mathcal{Y}}_{2, \eta}+\tilde{\mathcal{H}}_{2, \eta}\right)\left|\tilde{\mathcal{U}}_{2}\right|(t, \theta) d \theta\right) d \eta \\
& \leqslant \frac{1}{A^{6} A_{2}} \int_{0}^{1}\left(\tilde{\mathcal{Y}}_{1}-\tilde{\mathcal{Y}}_{2}\right)^{2} \tilde{\mathcal{Y}}_{2, \eta}(t, \eta)\left(\int_{0}^{\eta} e^{-\frac{1}{A_{2}}\left(\tilde{\mathcal{Y}}_{2}(t, \eta)-\tilde{\mathcal{Y}}_{2}(t, \theta)\right)}\right. \\
& \left.\times\left(\frac{1}{A_{2}} \tilde{\mathcal{P}}_{2}^{2} \tilde{\mathcal{Y}}_{2, \eta}+A_{2} \tilde{\mathcal{U}}_{2}^{2} \tilde{\mathcal{Y}}_{2, \eta}+\left\|\tilde{\mathcal{U}}_{2}(t, \cdot)\right\|_{L^{\infty}} \tilde{\mathcal{H}}_{2, \eta}\right)(t, \theta) d \theta\right) d \eta \\
& +\left\|\tilde{\mathcal{Y}}_{1}-\tilde{\mathcal{Y}}_{2}\right\|^{2} \\
& +\frac{1}{A^{12} A_{2}^{2}} \int_{0}^{1} \tilde{\mathcal{Y}}_{2, \eta}^{2}(t, \eta)\left(\int_{0}^{\eta}\left|\tilde{\mathcal{Y}}_{1}-\tilde{\mathcal{Y}}_{2}\right|(t, \theta) e^{-\frac{1}{A_{2}}\left(\tilde{\mathcal{Y}}_{2}(t, \eta)-\tilde{\mathcal{Y}}_{2}(t, \theta)\right)}\right. \\
& \left.\times\left(\tilde{\mathcal{P}}_{2} \tilde{\mathcal{Y}}_{2, \eta}+\tilde{\mathcal{H}}_{2, \eta}\right)\left|\tilde{\mathcal{U}}_{2}\right|(t, \theta) d \theta\right)^{2} d \eta \\
& \leqslant\left\|\tilde{\mathcal{Y}}_{1}-\tilde{\mathcal{Y}}_{2}\right\|^{2}+\mathcal{O}(1) \frac{1}{A^{5}} \int_{0}^{1}\left(\tilde{\mathcal{Y}}_{1}-\tilde{\mathcal{Y}}_{2}\right)^{2} \tilde{\mathcal{P}}_{2} \tilde{\mathcal{Y}}_{2, \eta}(t, \eta) d \eta \\
& +\frac{2}{A^{12} A_{2}^{2}} \int_{0}^{1} \tilde{\mathcal{Y}}_{2, \eta}^{2}(t, \eta) \\
& \times\left[\left(\int_{0}^{\eta}\left|\tilde{\mathcal{Y}}_{1}-\tilde{\mathcal{Y}}_{2}\right|(t, \theta) e^{-\frac{1}{A_{2}}\left(\tilde{\mathcal{Y}}_{2}(t, \eta)-\tilde{\mathcal{Y}}_{2}(t, \theta)\right)} \tilde{\mathcal{P}}_{2}\left|\tilde{\mathcal{U}}_{2}\right| \tilde{\mathcal{Y}}_{2, \eta}(t, \theta) d \theta\right)^{2}\right.
\end{aligned}
$$




$$
\begin{aligned}
& \left.+\left(\int_{0}^{\eta}\left|\tilde{\mathcal{Y}}_{1}-\tilde{\mathcal{Y}}_{2}\right|(t, \theta) e^{-\frac{1}{A_{2}}\left(\tilde{\mathcal{Y}}_{2}(t, \eta)-\tilde{\mathcal{Y}}_{2}(t, \theta)\right)}\left|\tilde{\mathcal{U}}_{2}\right| \tilde{\mathcal{H}}_{2, \eta}(t, \theta) d \theta\right)^{2}\right] d \eta \\
& \leqslant \mathcal{O}(1)\left\|\tilde{\mathcal{Y}}_{1}-\tilde{\mathcal{Y}}_{2}\right\|^{2}+\frac{2}{A^{12} A_{2}^{2}} \int_{0}^{1} \tilde{\mathcal{Y}}_{2, \eta}^{2}(t, \eta) \\
& \times\left[\left(\int_{0}^{\eta}\left(\tilde{\mathcal{Y}}_{1}-\tilde{\mathcal{Y}}_{2}\right)^{2}(t, \theta) e^{-\frac{1}{A_{2}}\left(\tilde{\mathcal{Y}}_{2}(t, \eta)-\tilde{\mathcal{Y}}_{2}(t, \theta)\right)} \tilde{\mathcal{P}}_{2}^{2} \tilde{\mathcal{Y}}_{2, \eta}(t, \theta) d \theta\right)\right. \\
& \times\left(\int_{0}^{\eta} e^{-\frac{1}{A_{2}}\left(\tilde{\mathcal{Y}}_{2}(t, \eta)-\tilde{\mathcal{Y}}_{2}(t, \theta)\right)} \tilde{\mathcal{U}}_{2}^{2} \tilde{\mathcal{Y}}_{2, \eta}(t, \theta) d \theta\right) \\
& +\left(\int_{0}^{\eta}\left(\tilde{\mathcal{Y}}_{1}-\tilde{\mathcal{Y}}_{2}\right)^{2}(t, \theta) e^{-\frac{1}{A_{2}}\left(\tilde{\mathcal{Y}}_{2}(t, \eta)-\tilde{\mathcal{Y}}_{2}(t, \theta)\right)} \tilde{\mathcal{U}}_{2}^{2} \tilde{\mathcal{H}}_{2, \eta}(t, \theta) d \theta\right) \\
& \left.\times\left(\int_{0}^{\eta} e^{-\frac{1}{A_{2}}\left(\tilde{\mathcal{Y}}_{2}(t, \eta)-\tilde{\mathcal{Y}}_{2}(t, \theta)\right)} \tilde{\mathcal{H}}_{2, \eta}(t, \theta) d \theta\right)\right] d \eta \\
& \leqslant \mathcal{O}(1)\left\|\tilde{\mathcal{Y}}_{1}-\tilde{\mathcal{Y}}_{2}\right\|^{2} \\
& +\frac{8}{A^{12} A_{2}} \int_{0}^{1} \tilde{\mathcal{P}}_{2} \tilde{\mathcal{Y}}_{2, \eta}^{2}(t, \eta) \\
& \times\left(\int_{0}^{\eta}\left(\tilde{\mathcal{Y}}_{1}-\tilde{\mathcal{Y}}_{2}\right)^{2}(t, \theta) e^{-\frac{1}{A_{2}}\left(\tilde{\mathcal{Y}}_{2}(t, \eta)-\tilde{\mathcal{Y}}_{2}(t, \theta)\right)}\left(\tilde{\mathcal{P}}_{2}^{2} \tilde{\mathcal{Y}}_{2, \eta}+\tilde{\mathcal{U}}_{2}^{2} \tilde{\mathcal{H}}_{2, \eta}\right)(t, \theta) d \theta\right) d \eta \\
& \leqslant \mathcal{O}(1)\left\|\tilde{\mathcal{Y}}_{1}-\tilde{\mathcal{Y}}_{2}\right\|^{2}+\frac{4}{A^{8}} \int_{0}^{1} \tilde{\mathcal{Y}}_{2, \eta}(t, \eta) e^{-\frac{1}{A_{2}} \tilde{\mathcal{Y}}_{2}(t, \eta)} \\
& \times\left(\int_{0}^{\eta}\left(\tilde{\mathcal{Y}}_{1}-\tilde{\mathcal{Y}}_{2}\right)^{2} e^{\frac{1}{A_{2}} \tilde{\mathcal{Y}}_{2}(t, \theta)}\left(\tilde{\mathcal{P}}_{2}^{2} \tilde{\mathcal{Y}}_{2, \eta}+\tilde{\mathcal{U}}_{2}^{2} \tilde{\mathcal{H}}_{2, \eta}\right)(t, \theta) d \theta\right) d \eta \\
& =\mathcal{O}(1)\left\|\tilde{\mathcal{Y}}_{1}-\tilde{\mathcal{Y}}_{2}\right\|^{2}+\frac{4 A_{2}}{A^{8}}\left(-\int_{0}^{\eta}\left(\tilde{\mathcal{Y}}_{1}-\tilde{\mathcal{Y}}_{2}\right)^{2}(t, \theta) e^{-\frac{1}{A_{2}}\left(\tilde{\mathcal{Y}}_{2}(t, \eta)-\tilde{\mathcal{Y}}_{2}(t, \theta)\right)}\right. \\
& \times\left.\left(\tilde{\mathcal{P}}_{2}^{2} \tilde{\mathcal{Y}}_{2, \eta}+\tilde{\mathcal{U}}_{2}^{2} \tilde{\mathcal{H}}_{2, \eta}\right)(t, \theta) d \theta\right|_{\eta=0} ^{1} \\
& \left.+\int_{0}^{1}\left(\tilde{\mathcal{Y}}_{1}-\tilde{\mathcal{Y}}_{2}\right)^{2}\left(\tilde{\mathcal{P}}_{2}^{2} \tilde{\mathcal{Y}}_{2, \eta}+\tilde{\mathcal{U}}_{2}^{2} \tilde{\mathcal{H}}_{2, \eta}\right)(t, \eta) d \eta\right) \\
& \leqslant \mathcal{O}(1)\left\|\tilde{\mathcal{Y}}_{1}-\tilde{\mathcal{Y}}_{2}\right\|^{2} \text {. }
\end{aligned}
$$

Next, we have a look at $\bar{B}_{65}$ (a similar argument works for $\bar{B}_{66}$ ). Direct calculations yield

$$
\begin{aligned}
\left|\bar{B}_{65}\right| \leqslant & \frac{a^{5}}{2 A^{6}} \int_{0}^{1}\left|\tilde{\mathcal{Y}}_{1}-\tilde{\mathcal{Y}}_{2}\right| \tilde{\mathcal{Y}}_{2, \eta}(t, \eta) \\
& \times\left(\frac{4}{a e} \int_{0}^{\eta} \min _{j}\left(e^{-\frac{3}{4 A_{2}}\left(\tilde{\mathcal{Y}}_{j}(t, \eta)-\tilde{\mathcal{Y}}_{j}(t, \theta)\right)}\right)\left|\tilde{\mathcal{U}}_{2}\right|(t, \theta) d \theta\right) d \eta\left|A_{1}-A_{2}\right|
\end{aligned}
$$




$$
\begin{aligned}
& \leqslant \frac{2}{A^{2} e} \int_{0}^{1}\left|\tilde{\mathcal{Y}}_{1}-\tilde{\mathcal{Y}}_{2}\right| \tilde{\mathcal{Y}}_{2, \eta}(t, \eta) \\
& \times\left(\int_{0}^{\eta} e^{-\frac{3}{4 A_{2}}\left(\tilde{\mathcal{Y}}_{2}(t, \eta)-\tilde{\mathcal{Y}}_{2}(t, \theta)\right)}\left|\tilde{\mathcal{U}}_{2}\right|(t, \theta) d \theta\right) d \eta\left|A_{1}-A_{2}\right| \\
& \leqslant \frac{4}{A^{2} A_{2}^{5} e} \int_{0}^{1}\left|\tilde{\mathcal{Y}}_{1}-\tilde{\mathcal{Y}}_{2}\right| \tilde{\mathcal{Y}}_{2, \eta}(t, \eta) \\
& \times\left(\int_{0}^{\eta} e^{-\frac{3}{4 A_{2}}\left(\tilde{\mathcal{Y}}_{2}(t, \eta)-\tilde{\mathcal{Y}}_{2}(t, \theta)\right)}\left(\tilde{\mathcal{P}}_{2} \tilde{\mathcal{Y}}_{2, \eta}+\tilde{\mathcal{H}}_{2, \eta}\right)\left|\tilde{\mathcal{U}}_{2}\right|(t, \theta) d \theta\right) d \eta\left|A_{1}-A_{2}\right| \\
& \leqslant\left|A_{1}-A_{2}\right|^{2}+\frac{32}{A^{4} A_{2}^{10} e^{2}}\left\|\tilde{\mathcal{Y}}_{1}-\tilde{\mathcal{Y}}_{2}\right\|^{2} \int_{0}^{1} \tilde{\mathcal{Y}}_{2, \eta}^{2}(t, \eta) \\
& \times\left[\left(\int_{0}^{\eta} e^{-\frac{1}{2 A_{2}}\left(\tilde{\mathcal{Y}}_{2}(t, \eta)-\tilde{\mathcal{Y}}_{2}(t, \theta)\right)} \tilde{\mathcal{P}}_{2}^{2} \tilde{\mathcal{Y}}_{2, \eta}(t, \theta) d \theta\right)\right. \\
& \times\left(\int_{0}^{\eta} e^{-\frac{1}{A_{2}}\left(\tilde{\mathcal{Y}}_{2}(t, \eta)-\tilde{\mathcal{Y}}_{2}(t, \theta)\right)} \tilde{\mathcal{U}}_{2}^{2} \tilde{\mathcal{Y}}_{2, \eta}(t, \theta) d \theta\right) \\
& +\left(\int_{0}^{\eta} e^{-\frac{1}{2 A_{2}}\left(\tilde{\mathcal{Y}}_{2}(t, \eta)-\tilde{\mathcal{Y}}_{2}(t, \theta)\right)} \tilde{\mathcal{U}}_{2}^{2} \tilde{\mathcal{H}}_{2, \eta}(t, \theta) d \theta\right) \\
& \left.\times\left(\int_{0}^{\eta} e^{-\frac{1}{A_{2}}\left(\tilde{\mathcal{Y}}_{2}(t, \eta)-\tilde{\mathcal{Y}}_{2}(t, \theta)\right)} \tilde{\mathcal{H}}_{2, \eta}(t, \theta) d \theta\right)\right] d \eta \\
& \leqslant\left|A_{1}-A_{2}\right|^{2}+\frac{128}{A^{4} A_{2}^{9} e^{2}}\left\|\tilde{\mathcal{Y}}_{1}-\tilde{\mathcal{Y}}_{2}\right\|^{2} \int_{0}^{1} \tilde{\mathcal{P}}_{2} \tilde{\mathcal{Y}}_{2, \eta}^{2}(t, \eta) \\
& \times\left(\int_{0}^{\eta} e^{-\frac{1}{2 A_{2}}\left(\tilde{\mathcal{Y}}_{2}(t, \eta)-\tilde{\mathcal{Y}}_{2}(t, \theta)\right)}\left(\tilde{\mathcal{P}}_{2}^{2} \tilde{\mathcal{Y}}_{2, \eta}+\tilde{\mathcal{U}}_{2}^{2} \tilde{\mathcal{H}}_{2, \eta}\right)(t, \theta) d \theta\right) d \eta \\
& \leqslant\left|A_{1}-A_{2}\right|^{2}+\frac{64}{A^{4} A_{2}^{4} e^{2}}\left\|\tilde{\mathcal{Y}}_{1}-\tilde{\mathcal{Y}}_{2}\right\|^{2} \\
& \times \int_{0}^{1} \tilde{\mathcal{Y}}_{2, \eta}(t, \eta) e^{-\frac{1}{2 A_{2}} \tilde{\mathcal{Y}}_{2}(t, \eta)}\left(\int_{0}^{\eta} e^{\frac{1}{2 A_{2}} \tilde{\mathcal{Y}}_{2}(t, \theta)}\left(\tilde{\mathcal{P}}_{2}^{2} \tilde{\mathcal{Y}}_{2, \eta}+\tilde{\mathcal{U}}_{2}^{2} \tilde{\mathcal{H}}_{2, \eta}\right)(t, \theta) d \theta\right) d \eta \\
& \leqslant\left|A_{1}-A_{2}\right|^{2}+\frac{128}{A^{4} A_{2}^{3} e^{2}}\left\|\tilde{\mathcal{Y}}_{1}-\tilde{\mathcal{Y}}_{2}\right\|^{2} \\
& \times\left[-\left.\int_{0}^{\eta} e^{-\frac{1}{2 A_{2}}\left(\tilde{\mathcal{Y}}_{2}(t, \eta)-\tilde{\mathcal{Y}}_{2}(t, \theta)\right)}\left(\tilde{\mathcal{P}}_{2}^{2} \tilde{\mathcal{Y}}_{2, \eta}+\tilde{\mathcal{U}}_{2}^{2} \tilde{\mathcal{H}}_{2, \eta}\right)(t, \theta) d \theta\right|_{\eta=0} ^{1}\right. \\
& \left.+\int_{0}^{1}\left(\tilde{\mathcal{P}}_{2}^{2} \tilde{\mathcal{Y}}_{2, \eta}+\tilde{\mathcal{U}}_{2}^{2} \tilde{\mathcal{H}}_{2, \eta}\right)(t, \eta) d \eta\right] \\
& \leqslant \mathcal{O}(1)\left(\left\|\tilde{\mathcal{Y}}_{1}-\tilde{\mathcal{Y}}_{2}\right\|^{2}+\left|A_{1}-A_{2}\right|^{2}\right) \text {. }
\end{aligned}
$$


Next, we have a look at $\bar{B}_{67}^{+}$(a similar argument works for $\bar{B}_{67}^{-}$and $\bar{B}_{68}^{ \pm}$). Direct calculations yield

$$
\begin{aligned}
& \left|\bar{B}_{67}^{+}\right| \leqslant \frac{a^{5}}{2 A^{6}} \int_{0}^{1}\left|\tilde{\mathcal{Y}}_{1}-\tilde{\mathcal{Y}}_{2}\right| \tilde{\mathcal{Y}}_{2, \eta}(t, \eta)\left(\int_{0}^{\eta} e^{-\frac{1}{A_{2}}\left(\tilde{\mathcal{Y}}_{2}(t, \eta)-\tilde{\mathcal{Y}}_{2}(t, \theta)\right)}\left|\tilde{\mathcal{U}}_{2}^{+}-\tilde{\mathcal{U}}_{1}^{+}\right|(t, \theta) d \theta\right) d \eta \\
& \leqslant\left\|\tilde{\mathcal{Y}}_{1}-\tilde{\mathcal{Y}}_{2}\right\|^{2} \\
& +\frac{A_{2}^{5}}{4 A^{7}} \int_{0}^{1} \tilde{\mathcal{Y}}_{2, \eta}^{2}(t, \eta)\left(\int_{0}^{\eta} e^{-\frac{1}{A_{2}}\left(\tilde{\mathcal{Y}}_{2}(t, \eta)-\tilde{\mathcal{Y}}_{2}(t, \theta)\right)}\left|\tilde{\mathcal{U}}_{2}-\tilde{\mathcal{U}}_{1}\right|(t, \theta) d \theta\right)^{2} d \eta \\
& \leqslant\left\|\tilde{\mathcal{Y}}_{1}-\tilde{\mathcal{Y}}_{2}\right\|^{2}+\frac{1}{2 A^{7}} \int_{0}^{1} \tilde{\mathcal{Y}}_{2, \eta}^{2}(t, \eta) \\
& \times\left(\int_{0}^{\eta} e^{-\frac{1}{A_{2}}\left(\tilde{\mathcal{Y}}_{2}(t, \eta)-\tilde{\mathcal{Y}}_{2}(t, \theta)\right)}\left(\tilde{\mathcal{P}}_{2} \tilde{\mathcal{Y}}_{2, \eta}+\tilde{\mathcal{H}}_{2, \eta}\right)\left|\tilde{\mathcal{U}}_{2}-\tilde{\mathcal{U}}_{1}\right|(t, \theta) d \theta\right)^{2} d \eta \\
& \leqslant\left\|\tilde{\mathcal{Y}}_{1}-\tilde{\mathcal{Y}}_{2}\right\|^{2} \\
& +\frac{1}{A^{7}} \int_{0}^{1} \tilde{\mathcal{Y}}_{2, \eta}^{2}(t, \eta)\left[\left(\int_{0}^{\eta} e^{-\frac{1}{A_{2}}\left(\tilde{\mathcal{Y}}_{2}(t, \eta)-\tilde{\mathcal{Y}}_{2}(t, \theta)\right)} \tilde{\mathcal{P}}_{2} \tilde{\mathcal{Y}}_{2, \eta}\left|\tilde{\mathcal{U}}_{2}-\tilde{\mathcal{U}}_{1}\right|(t, \theta) d \theta\right)^{2}\right. \\
& \left.+\left(\int_{0}^{\eta} e^{-\frac{1}{A_{2}}\left(\tilde{\mathcal{Y}}_{2}(t, \eta)-\tilde{\mathcal{Y}}_{2}(t, \theta)\right)} \tilde{\mathcal{H}}_{2, \eta}\left|\tilde{\mathcal{U}}_{2}-\tilde{\mathcal{U}}_{1}\right|(t, \theta) d \theta\right)^{2}\right] d \eta \\
& \leqslant\left\|\tilde{\mathcal{Y}}_{1}-\tilde{\mathcal{Y}}_{2}\right\|^{2}+\frac{1}{A^{7}} \int_{0}^{1} \tilde{\mathcal{Y}}_{2, \eta}^{2}(t, \eta)\left(\int_{0}^{\eta} e^{-\frac{3}{2 A_{2}}\left(\tilde{\mathcal{Y}}_{2}(t, \eta)-\tilde{\mathcal{Y}}_{2}(t, \theta)\right)} \tilde{\mathcal{P}}_{2} \tilde{\mathcal{Y}}_{2, \eta}(t, \theta) d \theta\right) \\
& \times\left(\int_{0}^{\eta} e^{-\frac{1}{2 A_{2}}\left(\tilde{\mathcal{Y}}_{2}(t, \eta)-\tilde{\mathcal{Y}}_{2}(t, \theta)\right)}\left(\tilde{\mathcal{U}}_{2}-\tilde{\mathcal{U}}_{1}\right)^{2} \tilde{\mathcal{P}}_{2} \tilde{\mathcal{Y}}_{2, \eta}(t, \theta) d \theta\right) d \eta \\
& +\frac{1}{A^{7}} \int_{0}^{1} \tilde{\mathcal{Y}}_{2, \eta}^{2}(t, \eta)\left(\int_{0}^{\eta} e^{-\frac{1}{A_{2}}\left(\tilde{\mathcal{Y}}_{2}(t, \eta)-\tilde{\mathcal{Y}}_{2}(t, \theta)\right)} \tilde{\mathcal{H}}_{2, \eta}(t, \theta) d \theta\right) \\
& \times\left(\int_{0}^{\eta} e^{-\frac{1}{A_{2}}\left(\tilde{\mathcal{Y}}_{2}(t, \eta)-\tilde{\mathcal{Y}}_{2}(t, \theta)\right)}\left(\tilde{\mathcal{U}}_{1}-\tilde{\mathcal{U}}_{2}\right)^{2} \tilde{\mathcal{H}}_{2, \eta}(t, \theta) d \theta\right) d \eta \\
& \leqslant\left\|\tilde{\mathcal{Y}}_{1}-\tilde{\mathcal{Y}}_{2}\right\|^{2} \\
& +\frac{2}{A^{6}} \int_{0}^{1} \tilde{\mathcal{Y}}_{2, \eta}(t, \eta) e^{-\frac{1}{2 A_{2}} \tilde{\mathcal{Y}}_{2}(t, \eta)}\left(\int_{0}^{\eta} e^{\frac{1}{2 A_{2}} \tilde{\mathcal{Y}}_{2}(t, \theta)}\left(\tilde{\mathcal{U}}_{1}-\tilde{\mathcal{U}}_{2}\right)^{2} \tilde{\mathcal{P}}_{2} \tilde{\mathcal{Y}}_{2 \eta}(t, \theta) d \theta\right) d \eta \\
& +\frac{4}{A^{6}} \int_{0}^{1} \tilde{\mathcal{Y}}_{2, \eta}(t, \eta) e^{-\frac{1}{A_{2}} \tilde{\mathcal{Y}}_{2}(t, \eta)}\left(\int_{0}^{\eta} e^{\frac{1}{A_{2}} \tilde{\mathcal{Y}}_{2}(t, \theta)}\left(\tilde{\mathcal{U}}_{1}-\tilde{\mathcal{U}}_{2}\right)^{2} \tilde{\mathcal{H}}_{2, \eta}(t, \theta) d \theta\right) d \eta \\
& \leqslant\left\|\tilde{\mathcal{Y}}_{1}-\tilde{\mathcal{Y}}_{2}\right\|^{2} \\
& +\frac{2 A_{2}}{A^{6}}\left(-\left.2 \int_{0}^{\eta} e^{-\frac{1}{2 A_{2}}\left(\tilde{\mathcal{Y}}_{2}(t, \eta)-\tilde{\mathcal{Y}}_{2}(t, \theta)\right)}\left(\tilde{\mathcal{U}}_{1}-\tilde{\mathcal{U}}_{2}\right)^{2} \tilde{\mathcal{P}}_{2} \tilde{\mathcal{Y}}_{2, \eta}(t, \theta) d \theta\right|_{\eta=0} ^{1}\right. \\
& \left.+2 \int_{0}^{1}\left(\tilde{\mathcal{U}}_{1}-\tilde{\mathcal{U}}_{2}\right)^{2} \tilde{\mathcal{P}}_{2} \tilde{\mathcal{Y}}_{2, \eta}(t, \eta) d \eta\right)
\end{aligned}
$$




$$
\begin{aligned}
& +\frac{4 A_{2}}{A^{6}}\left(-\left.\int_{0}^{\eta} e^{-\frac{1}{A_{2}}\left(\tilde{\mathcal{Y}}_{2}(t, \eta)-\tilde{\mathcal{Y}}_{2}(t, \theta)\right)}\left(\tilde{\mathcal{U}}_{1}-\tilde{\mathcal{U}}_{2}\right)^{2} \tilde{\mathcal{H}}_{2, \eta}(t, \theta) d \theta\right|_{\eta=0} ^{1}\right. \\
& \left.+\int_{0}^{1}\left(\tilde{\mathcal{U}}_{1}-\tilde{\mathcal{U}}_{2}\right)^{2} \tilde{\mathcal{H}}_{2, \eta}(t, \eta) d \eta\right) \\
& \leqslant \mathcal{O}(1)\left(\left\|\tilde{\mathcal{Y}}_{1}-\tilde{\mathcal{Y}}_{2}\right\|^{2}+\left\|\tilde{\mathcal{U}}_{1}-\tilde{\mathcal{U}}_{2}\right\|^{2}\right) .
\end{aligned}
$$

Finally, we consider $\bar{B}_{69}^{+}$(a similar argument works for $\bar{B}_{69}^{-}$). Here integration by parts will play the main role. Indeed, we have

$$
\begin{aligned}
& \left|\bar{B}_{69}^{+}\right|=\frac{a^{5}}{2 A^{6}} \mid \int_{0}^{1}\left(\tilde{\mathcal{Y}}_{1}-\tilde{\mathcal{Y}}_{2}\right)\left(\tilde{\mathcal{Y}}_{1, \eta}-\tilde{\mathcal{Y}}_{2, \eta}\right)(t, \eta) \\
& \times\left(\int_{0}^{\eta} \min _{j}\left(e^{-\frac{1}{a}\left(\tilde{\mathcal{Y}}_{j}(t, \eta)-\tilde{\mathcal{Y}}_{j}(t, \theta)\right)}\right) \min _{j}\left(\tilde{\mathcal{U}}_{j}^{+}\right)(t, \theta) d \theta\right) d \eta \mid \\
& =\frac{a^{5}}{4 A^{6}} \mid\left(\tilde{\mathcal{Y}}_{1}-\tilde{\mathcal{Y}}_{2}\right)^{2}(t, \eta) \\
& \times\left.\left(\int_{0}^{\eta} \min _{j}\left(e^{-\frac{1}{a}\left(\tilde{\mathcal{Y}}_{j}(t, \eta)-\tilde{\mathcal{Y}}_{j}(t, \theta)\right)}\right) \min _{j}\left(\tilde{\mathcal{U}}_{j}^{+}\right)(t, \theta) d \theta\right)\right|_{\eta=0} ^{1} \\
& -\int_{0}^{1}\left(\tilde{\mathcal{Y}}_{1}-\tilde{\mathcal{Y}}_{2}\right)^{2}(t, \eta) \\
& \times \frac{d}{d \eta}\left(\int_{0}^{\eta} \min _{j}\left(e^{-\frac{1}{a}\left(\tilde{\mathcal{Y}}_{j}(t, \eta)-\tilde{\mathcal{Y}}_{j}(t, \theta)\right)}\right) \min _{j}\left(\tilde{\mathcal{U}}_{j}^{+}\right)(t, \theta) d \theta\right) d \eta \mid \\
& \leqslant \frac{a^{5}}{4 A^{6}} \mid \int_{0}^{1}\left(\tilde{\mathcal{Y}}_{1}-\tilde{\mathcal{Y}}_{2}\right)^{2}(t, \eta) \\
& \times \frac{d}{d \eta}\left(\int_{0}^{\eta} \min _{j}\left(e^{-\frac{1}{a}\left(\tilde{\mathcal{Y}}_{j}(t, \eta)-\tilde{\mathcal{Y}}_{j}(t, \theta)\right)}\right) \min _{j}\left(\tilde{\mathcal{U}}_{j}^{+}\right)(t, \theta) d \theta\right) d \eta \mid \\
& \leqslant \mathcal{O}(1)\left\|\tilde{\mathcal{Y}}_{1}-\tilde{\mathcal{Y}}_{2}\right\|
\end{aligned}
$$

where $\mathcal{O}(1)$ denotes some constant depending on $A$, which remains bounded as $A \rightarrow 0$, provided that we can show that both

$$
a^{5} \int_{0}^{\eta} \min _{j}\left(e^{-\frac{1}{a}\left(\tilde{\mathcal{Y}}_{j}(t, \eta)-\tilde{\mathcal{Y}}_{j}(t, \theta)\right)}\right) \min _{j}\left(\tilde{\mathcal{U}}_{j}^{+}\right)(t, \theta) d \theta \leqslant \mathcal{O}(1) A^{2} \min _{j}\left(\tilde{\mathcal{P}}_{j}\right)(t, \eta)
$$

and the derivative

$$
a \frac{d}{d \eta}\left(\int_{0}^{\eta} \min _{j}\left(e^{-\frac{1}{a}\left(\tilde{\mathcal{Y}}_{j}(t, \eta)-\tilde{\mathcal{Y}}_{j}(t, \theta)\right)}\right) \min _{j}\left(\tilde{\mathcal{U}}_{j}^{+}\right)(t, \theta) d \theta\right)
$$

exist and are uniformly bounded. 
Direct computations yield

$$
\begin{aligned}
& a^{5} \int_{0}^{\eta} \min _{j}\left(e^{-\frac{1}{a}\left(\tilde{\mathcal{Y}}_{j}(t, \eta)-\tilde{\mathcal{Y}}_{j}(t, \theta)\right)}\right) \min _{j}\left(\tilde{\mathcal{U}}_{j}^{+}\right)(t, \theta) d \theta \\
& \quad \leqslant \int_{0}^{\eta} e^{-\frac{1}{A_{i}}\left(\tilde{\mathcal{Y}}_{i}(t, \eta)-\tilde{\mathcal{Y}}_{i}(t, \theta)\right)} A_{i}^{5}\left|\tilde{\mathcal{U}}_{i}\right|(t, \theta) d \theta \\
& \quad \leqslant 2 \int_{0}^{\eta} e^{-\frac{1}{A_{i}}\left(\tilde{\mathcal{Y}}_{i}(t, \eta)-\tilde{\mathcal{Y}}_{i}(t, \theta)\right)}\left(\tilde{\mathcal{P}}_{i} \tilde{\mathcal{Y}}_{i, \eta}+\tilde{\mathcal{H}}_{i, \eta}\right)\left|\tilde{\mathcal{U}}_{i}\right|(t, \theta) d \theta \\
& \quad \leqslant 2 \int_{0}^{\eta} e^{-\frac{1}{A_{i}}\left(\tilde{\mathcal{Y}}_{i}(t, \eta)-\tilde{\mathcal{Y}}_{i}(t, \theta)\right)}\left(\frac{1}{A_{i}} \tilde{\mathcal{P}}_{i}^{2} \tilde{\mathcal{Y}}_{i, \eta}+A_{i} \tilde{\mathcal{U}}_{i}^{2} \tilde{\mathcal{Y}}_{i, \eta}+\left\|\tilde{\mathcal{U}}_{i}(t, \cdot)\right\|_{L^{\infty}} \tilde{\mathcal{H}}_{i, \eta}\right)(t, \theta) d \theta \\
& \quad \leqslant \mathcal{O}(1) A_{i}^{2} \tilde{\mathcal{P}}_{i}(t, \eta) .
\end{aligned}
$$

The result for (5.26) is contained in Lemma A.5.

LEMMA 5.3. Let $\tilde{\mathcal{Y}}_{i}$ denote two solutions of (5.5). Then we have

$$
\begin{aligned}
\frac{d}{d t}\left\|\tilde{\mathcal{Y}}_{1}-\tilde{\mathcal{Y}}_{2}\right\|^{2} \leqslant & \mathcal{O}(1)\left(\left\|\tilde{\mathcal{Y}}_{1}-\tilde{\mathcal{Y}}_{2}\right\|^{2}+\left\|\tilde{\mathcal{U}}_{1}-\tilde{\mathcal{U}}_{2}\right\|^{2}\right. \\
& \left.+\left\|\tilde{\mathcal{P}}_{1}^{1 / 2}-\tilde{\mathcal{P}}_{2}^{1 / 2}\right\|^{2}+\left|A_{1}-A_{2}\right|^{2}\right)
\end{aligned}
$$

where $\mathcal{O}(1)$ denotes some constant which depends on $A=\max _{j}\left(A_{j}\right)$, which remains bounded as $A \rightarrow 0$.

5.2. Lipschitz estimates for $\tilde{\mathcal{U}}$. From the system of differential equations, we have

$$
\tilde{\mathcal{U}}_{i, t}+\left(\frac{2}{3} \frac{1}{A_{i}^{5}} \tilde{\mathcal{U}}_{i}^{3}+\frac{1}{A_{i}^{6}} \tilde{\mathcal{S}}_{i}\right) \tilde{\mathcal{U}}_{i, \eta}=-\frac{1}{A_{i}^{2}} \tilde{\mathcal{Q}}_{i},
$$

where

$$
\begin{aligned}
& \tilde{\mathcal{Q}}_{i}(t, \eta)=-\frac{1}{4} \int_{0}^{1} \operatorname{sign}(\eta-\theta) e^{-\frac{1}{A_{i}}\left|\tilde{\mathcal{Y}}_{i}(t, \eta)-\tilde{\mathcal{Y}}_{i}(t, \theta)\right|}\left(2\left(\tilde{\mathcal{U}}_{i}^{2}-\tilde{\mathcal{P}}_{i}\right) \tilde{\mathcal{Y}}_{i, \eta}(t, \theta)+A_{i}^{5}\right) d \theta \\
& \tilde{\mathcal{S}}_{i}(t, \eta)=\int_{0}^{1} e^{-\frac{1}{A_{i}}\left|\tilde{\mathcal{Y}}_{i}(t, \eta)-\tilde{\mathcal{Y}}_{i}(t, \theta)\right|}\left(\frac{2}{3} \tilde{\mathcal{U}}_{i}^{3} \tilde{\mathcal{Y}}_{i, \eta}-\tilde{\mathcal{Q}}_{i} \tilde{\mathcal{U}}_{i, \eta}-2 \tilde{\mathcal{P}}_{i} \tilde{\mathcal{U}}_{i} \tilde{\mathcal{Y}}_{i, \eta}\right)(t, \theta) d \theta
\end{aligned}
$$

Thus we have

$$
\begin{aligned}
& \frac{d}{d t} \int_{0}^{1}\left(\tilde{\mathcal{U}}_{1}-\tilde{\mathcal{U}}_{2}\right)^{2}(t, \eta) d \eta \\
& \quad=2 \int_{0}^{1}\left(\tilde{\mathcal{U}}_{1}-\tilde{\mathcal{U}}_{2}\right)\left(\tilde{\mathcal{U}}_{1, t}-\tilde{\mathcal{U}}_{2, t}\right)(t, \eta) d \eta
\end{aligned}
$$




$$
\begin{aligned}
= & 2 \int_{0}^{1}\left(\tilde{\mathcal{U}}_{1}-\tilde{\mathcal{U}}_{2}\right)\left(\frac{1}{A_{2}^{2}} \tilde{\mathcal{Q}}_{2}-\frac{1}{A_{1}^{2}} \tilde{\mathcal{Q}}_{1}\right)(t, \eta) d \eta \\
& +\frac{4}{3} \int_{0}^{1}\left(\tilde{\mathcal{U}}_{1}-\tilde{\mathcal{U}}_{2}\right)\left(\frac{1}{A_{2}^{5}} \tilde{\mathcal{U}}_{2}^{3} \tilde{\mathcal{U}}_{2, \eta}-\frac{1}{A_{1}^{5}} \tilde{\mathcal{U}}_{1}^{3} \tilde{\mathcal{U}}_{1, \eta}\right)(t, \eta) d \eta \\
& +2 \int_{0}^{1}\left(\tilde{\mathcal{U}}_{1}-\tilde{\mathcal{U}}_{2}\right)\left(\frac{1}{A_{2}^{6}} \tilde{\mathcal{S}}_{2} \tilde{\mathcal{U}}_{2, \eta}-\frac{1}{A_{1}^{6}} \tilde{\mathcal{S}}_{1} \tilde{\mathcal{U}}_{1, \eta}\right)(t, \eta) d \eta \\
= & I_{1}+I_{2}+I_{3} .
\end{aligned}
$$

The strategy is to use integration by parts for the last two integrals $I_{2}$ and $I_{3}$, while we want to use straightforward estimates for $I_{1}$, which will finally yield that

$$
\begin{aligned}
& \frac{d}{d t}\left\|\tilde{\mathcal{U}}_{1}-\tilde{\mathcal{U}}_{2}\right\|^{2} \\
& \quad \leqslant \mathcal{O}(1)\left(\left\|\tilde{\mathcal{Y}}_{1}-\tilde{\mathcal{Y}}_{2}\right\|^{2}+\left\|\tilde{\mathcal{U}}_{1}-\tilde{\mathcal{U}}_{2}\right\|^{2}+\left\|\tilde{\mathcal{P}}_{1}^{1 / 2}-\tilde{\mathcal{P}}_{2}^{1 / 2}\right\|^{2}+\left|A_{1}-A_{2}\right|^{2}\right),
\end{aligned}
$$

where $\mathcal{O}(1)$ denotes some constant which only depends on $A=\max _{j}\left(A_{j}\right)$ and which remains bounded as $A \rightarrow 0$.

The first integral $I_{1}$ : Note that we can split $I_{1}$ as follows:

$$
\begin{aligned}
I_{1}= & \mathbb{1}_{A_{1} \leqslant A_{2}} 2 \frac{A_{1}^{2}-A_{2}^{2}}{A_{1}^{2} A_{2}^{2}} \int_{0}^{1}\left(\tilde{\mathcal{U}}_{1}-\tilde{\mathcal{U}}_{2}\right) \tilde{\mathcal{Q}}_{1}(t, \eta) d \eta \\
& +\mathbb{1}_{A_{2}<A_{1}} 2 \frac{A_{1}^{2}-A_{2}^{2}}{A_{1}^{2} A_{2}^{2}} \int_{0}^{1}\left(\tilde{\mathcal{U}}_{1}-\tilde{\mathcal{U}}_{2}\right) \tilde{\mathcal{Q}}_{2}(t, \eta) d \eta \\
& +2 \frac{1}{A^{2}} \int_{0}^{1}\left(\tilde{\mathcal{U}}_{1}-\tilde{\mathcal{U}}_{2}\right)\left(\tilde{\mathcal{Q}}_{2}-\tilde{\mathcal{Q}}_{1}\right)(t, \eta) d \eta \\
= & I_{11}+I_{12}+I_{13} .
\end{aligned}
$$

As far as $I_{11}$ is concerned (a similar argument works for $I_{12}$ ), we have

$$
\begin{aligned}
\left|I_{11}\right| & \leqslant \mathbb{1}_{A_{1} \leqslant A_{2}} 4 \frac{\left|A_{1}-A_{2}\right|}{A_{1} A_{2}} \int_{0}^{1}\left|\tilde{\mathcal{U}}_{1}-\tilde{\mathcal{U}}_{2}\right| \tilde{\mathcal{P}}_{1}(t, \eta) d \eta \\
& \leqslant A^{2}\left|A_{1}-A_{2}\right| \int_{0}^{1}\left|\tilde{\mathcal{U}}_{1}-\tilde{\mathcal{U}}_{2}\right|(t, \eta) d \eta \\
& \leqslant \mathcal{O}(1)\left(\left\|\tilde{\mathcal{U}}_{1}-\tilde{\mathcal{U}}_{2}\right\|^{2}+\left|A_{1}-A_{2}\right|^{2}\right) .
\end{aligned}
$$


Note that

$$
\begin{aligned}
\left|I_{13}\right| & =\left|2 \frac{1}{A^{2}} \int_{0}^{1}\left(\tilde{\mathcal{U}}_{1}-\tilde{\mathcal{U}}_{2}\right)\left(\tilde{\mathcal{Q}}_{1}-\tilde{\mathcal{Q}}_{2}\right)(t, \eta) d \eta\right| \\
& \leqslant \int_{0}^{1}\left(\left(\tilde{\mathcal{U}}_{1}-\tilde{\mathcal{U}}_{2}\right)^{2}+\frac{1}{A^{4}}\left(\tilde{\mathcal{Q}}_{1}-\tilde{\mathcal{Q}}_{2}\right)^{2}\right)(t, \eta) d \eta,
\end{aligned}
$$

and hence it suffices to show that

$$
\begin{aligned}
\left\|\tilde{\mathcal{Q}}_{1}-\tilde{\mathcal{Q}}_{2}\right\| \leqslant & \mathcal{O}(1) A^{2}\left(\left\|\tilde{\mathcal{Y}}_{1}-\tilde{\mathcal{Y}}_{2}\right\|+\left\|\tilde{\mathcal{U}}_{1}-\tilde{\mathcal{U}}_{2}\right\|\right. \\
& \left.+\left\|\tilde{\mathcal{P}}_{1}^{1 / 2}-\tilde{\mathcal{P}}_{2}^{1 / 2}\right\|+\left|A_{1}-A_{2}\right|\right),
\end{aligned}
$$

which is equivalent to

$$
\begin{aligned}
\left\|\tilde{\mathcal{Q}}_{1}-\tilde{\mathcal{Q}}_{2}\right\|^{2} \leqslant & \mathcal{O}(1) A^{4}\left(\left\|\tilde{\mathcal{Y}}_{1}-\tilde{\mathcal{Y}}_{2}\right\|^{2}+\left\|\tilde{\mathcal{U}}_{1}-\tilde{\mathcal{U}}_{2}\right\|^{2}\right. \\
& \left.+\left\|\tilde{\mathcal{P}}_{1}^{1 / 2}-\tilde{\mathcal{P}}_{2}^{1 / 2}\right\|^{2}+\left|A_{1}-A_{2}\right|^{2}\right) .
\end{aligned}
$$

To begin with, we observe that we can write

$$
\begin{aligned}
\left(\tilde{\mathcal{Q}}_{1}-\tilde{\mathcal{Q}}_{2}\right)(t, \eta)= & \left(A_{1} \tilde{\mathcal{P}}_{1}-A_{2} \tilde{\mathcal{P}}_{2}\right)(t, \eta) \\
& +\left(\tilde{\mathcal{D}}_{2}-\tilde{\mathcal{D}}_{1}\right)(t, \eta) \\
= & \left(A_{1}-A_{2}\right) \tilde{\mathcal{P}}_{1} \\
& +A_{2}\left(\tilde{\mathcal{P}}_{1}^{1 / 2}+\tilde{\mathcal{P}}_{2}^{1 / 2}\right)\left(\tilde{\mathcal{P}}_{1}^{1 / 2}-\tilde{\mathcal{P}}_{2}^{1 / 2}\right)(t, \eta) \\
& +\left(\tilde{\mathcal{D}}_{2}-\tilde{\mathcal{D}}_{1}\right)(t, \eta) \\
= & K_{1}(t, \eta)+K_{2}(t, \eta)+K_{3}(t, \eta) .
\end{aligned}
$$

As far as $K_{1}(t, \eta)$ is concerned, we have

$$
\left|A_{1}-A_{2}\right|\left\|\tilde{\mathcal{P}}_{1}\right\|_{L^{\infty}} \leqslant \frac{A^{4}}{4}\left|A_{1}-A_{2}\right| .
$$

As far as $K_{2}(t, \eta)$ is concerned, we have

$$
\left\|\tilde{\mathcal{P}}_{1}-\tilde{\mathcal{P}}_{2}\right\| \leqslant A^{2}\left\|\tilde{\mathcal{P}}_{1}^{1 / 2}-\tilde{\mathcal{P}}_{2}^{1 / 2}\right\|,
$$

since $\left\|\tilde{\mathcal{P}}_{i}^{1 / 2}\right\|_{L^{\infty}}$ can be bounded by a constant, which only depends on $A$.

As far as $K_{3}(t, \eta)$ is concerned, Lemma A.9 implies immediately that

$$
\begin{aligned}
\left\|\tilde{\mathcal{D}}_{1}-\tilde{\mathcal{D}}_{2}\right\| \leqslant & \mathcal{O}(1) A^{2}\left(\left\|\tilde{\mathcal{Y}}_{1}-\tilde{\mathcal{Y}}_{2}\right\|+\left\|\tilde{\mathcal{U}}_{1}-\tilde{\mathcal{U}}_{2}\right\|\right. \\
& \left.+\left\|\tilde{\mathcal{P}}_{1}^{1 / 2}-\tilde{\mathcal{P}}_{2}^{1 / 2}\right\|+\left|A_{1}-A_{2}\right|\right) .
\end{aligned}
$$

This finishes the proof of (5.29). 
The second integral $I_{2}$ : Note that we can write

$$
\begin{aligned}
& \frac{3}{4} I_{2}=\int_{0}^{1}\left(\tilde{\mathcal{U}}_{1}-\tilde{\mathcal{U}}_{2}\right)\left(\frac{1}{A_{2}^{5}} \tilde{\mathcal{U}}_{2}^{3} \tilde{\mathcal{U}}_{2, \eta}-\frac{1}{A_{1}^{5}} \tilde{\mathcal{U}}_{1}^{3} \tilde{\mathcal{U}}_{1, \eta}\right)(t, \eta) d \eta \\
& =\frac{1}{A^{5}} \int_{0}^{1}\left(\tilde{\mathcal{U}}_{1}-\tilde{\mathcal{U}}_{2}\right)\left(\tilde{\mathcal{U}}_{2}^{3} \tilde{\mathcal{U}}_{2, \eta}-\tilde{\mathcal{U}}_{1}^{3} \tilde{\mathcal{U}}_{1, \eta}\right)(t, \eta) d \eta \\
& +\frac{A_{1}^{5}-A_{2}^{5}}{A_{1}^{5} A_{2}^{5}} \int_{0}^{1}\left(\tilde{\mathcal{U}}_{1}-\tilde{\mathcal{U}}_{2}\right)\left(\tilde{\mathcal{U}}_{1}^{3} \tilde{\mathcal{U}}_{1, \eta} \mathbb{1}_{A_{1} \leqslant A_{2}}+\tilde{\mathcal{U}}_{2}^{3} \tilde{\mathcal{U}}_{2, \eta} \mathbb{1}_{A_{2}<A_{1}}\right)(t, \eta) d \eta \\
& =\frac{1}{A^{5}} \int_{0}^{1}\left(\tilde{\mathcal{U}}_{1}-\tilde{\mathcal{U}}_{2}\right)\left(\tilde{\mathcal{U}}_{2}-\tilde{\mathcal{U}}_{1}\right) \tilde{\mathcal{U}}_{2}^{2} \tilde{\mathcal{U}}_{2, \eta}(t, \eta) d \eta \\
& +\frac{1}{A^{5}} \int_{0}^{1}\left(\tilde{\mathcal{U}}_{1}-\tilde{\mathcal{U}}_{2}\right)\left(\tilde{\mathcal{U}}_{2}^{2} \tilde{\mathcal{U}}_{2, \eta}-\tilde{\mathcal{U}}_{1}^{2} \tilde{\mathcal{U}}_{1, \eta}\right) \tilde{\mathcal{U}}_{1}(t, \eta) d \eta \\
& +\frac{A_{1}^{5}-A_{2}^{5}}{A_{1}^{5} A_{2}^{5}} \int_{0}^{1}\left(\tilde{\mathcal{U}}_{1}-\tilde{\mathcal{U}}_{2}\right)\left(\tilde{\mathcal{U}}_{1}^{3} \tilde{\mathcal{U}}_{1, \eta} \mathbb{1}_{A_{1} \leqslant A_{2}}+\tilde{\mathcal{U}}_{2}^{3} \tilde{\mathcal{U}}_{2, \eta} \mathbb{1}_{A_{2}<A_{1}}\right)(t, \eta) d \eta \\
& =\frac{1}{A^{5}} \int_{0}^{1}\left(\tilde{\mathcal{U}}_{1}-\tilde{\mathcal{U}}_{2}\right)\left(\tilde{\mathcal{U}}_{2}-\tilde{\mathcal{U}}_{1}\right) \tilde{\mathcal{U}}_{2}^{2} \tilde{\mathcal{U}}_{2, \eta}(t, \eta) d \eta \\
& +\frac{1}{A^{5}} \int_{0}^{1}\left(\tilde{\mathcal{U}}_{1}-\tilde{\mathcal{U}}_{2}\right)\left(\tilde{\mathcal{U}}_{2}^{2}-\tilde{\mathcal{U}}_{1}^{2}\right) \tilde{\mathcal{U}}_{2, \eta} \tilde{\mathcal{U}}_{1}(t, \eta) \mathbb{1}_{\tilde{\mathcal{U}}_{1}^{2} \leqslant \tilde{\mathcal{U}}_{2}^{2}}(t, \eta) d \eta \\
& +\frac{1}{A^{5}} \int_{0}^{1}\left(\tilde{\mathcal{U}}_{1}-\tilde{\mathcal{U}}_{2}\right)\left(\tilde{\mathcal{U}}_{2}^{2}-\tilde{\mathcal{U}}_{1}^{2}\right) \tilde{\mathcal{U}}_{1} \tilde{\mathcal{U}}_{1, \eta}(t, \eta) \mathbb{1}_{\tilde{\mathcal{U}}_{2}^{2}<\tilde{\mathcal{U}}_{1}^{2}}(t, \eta) d \eta \\
& +\frac{1}{A^{5}} \int_{0}^{1}\left(\tilde{\mathcal{U}}_{1}-\tilde{\mathcal{U}}_{2}\right)\left(\tilde{\mathcal{U}}_{2, \eta}-\tilde{\mathcal{U}}_{1, \eta}\right) \tilde{\mathcal{U}}_{1} \min _{j}\left(\tilde{\mathcal{U}}_{j}^{2}\right)(t, \eta) d \eta \\
& +\frac{A_{1}^{5}-A_{2}^{5}}{A_{1}^{5} A_{2}^{5}} \int_{0}^{1}\left(\tilde{\mathcal{U}}_{1}-\tilde{\mathcal{U}}_{2}\right)\left(\tilde{\mathcal{U}}_{1}^{3} \tilde{\mathcal{U}}_{1, \eta} \mathbb{1}_{A_{1} \leqslant A_{2}}+\tilde{\mathcal{U}}_{2}^{3} \tilde{\mathcal{U}}_{2, \eta} \mathbb{1}_{A_{2}<A_{1}}\right)(t, \eta) d \eta \\
& =\frac{1}{A^{5}} \int_{0}^{1}\left(\tilde{\mathcal{U}}_{1}-\tilde{\mathcal{U}}_{2}\right)\left(\tilde{\mathcal{U}}_{2}-\tilde{\mathcal{U}}_{1}\right) \tilde{\mathcal{U}}_{2}^{2} \tilde{\mathcal{U}}_{2, \eta}(t, \eta) d \eta \\
& +\frac{1}{A^{5}} \int_{0}^{1}\left(\tilde{\mathcal{U}}_{1}-\tilde{\mathcal{U}}_{2}\right)\left(\tilde{\mathcal{U}}_{2}^{2}-\tilde{\mathcal{U}}_{1}^{2}\right) \tilde{\mathcal{U}}_{2, \eta} \tilde{\mathcal{U}}_{1}(t, \eta) \mathbb{1}_{\tilde{\mathcal{U}}_{1}^{2} \leqslant \tilde{\mathcal{U}}_{2}^{2}}(t, \eta) d \eta \\
& +\frac{1}{A^{5}} \int_{0}^{1}\left(\tilde{\mathcal{U}}_{1}-\tilde{\mathcal{U}}_{2}\right)\left(\tilde{\mathcal{U}}_{2}^{2}-\tilde{\mathcal{U}}_{1}^{2}\right) \tilde{\mathcal{U}}_{1} \tilde{\mathcal{U}}_{1, \eta}(t, \eta) \mathbb{1}_{\tilde{\mathcal{U}}_{2}^{2}<\tilde{\mathcal{U}}_{1}^{2}}(t, \eta) d \eta \\
& +\frac{1}{2 A^{5}} \int_{0}^{1}\left(\tilde{\mathcal{U}}_{1}-\tilde{\mathcal{U}}_{2}\right)^{2} \frac{d}{d \eta}\left(\tilde{\mathcal{U}}_{1} \min _{j}\left(\tilde{\mathcal{U}}_{j}^{2}\right)\right)(t, \eta) d \eta \\
& +\frac{A_{1}^{5}-A_{2}^{5}}{A_{1}^{5} A_{2}^{5}} \int_{0}^{1}\left(\tilde{\mathcal{U}}_{1}-\tilde{\mathcal{U}}_{2}\right)\left(\tilde{\mathcal{U}}_{1}^{3} \tilde{\mathcal{U}}_{1, \eta} \mathbb{1}_{A_{1} \leqslant A_{2}}+\tilde{\mathcal{U}}_{2}^{3} \tilde{\mathcal{U}}_{2, \eta} \mathbb{1}_{A_{2}<A_{1}}\right)(t, \eta) d \eta,
\end{aligned}
$$


where we used integration by parts in the last step together with $\tilde{\mathcal{U}}_{i}(t, \eta) \rightarrow 0$ as $\eta \rightarrow 0,1$. As far as the derivative in the last integral is concerned, observe that

$$
\left|\tilde{\mathcal{U}}_{1, \eta} \min _{j}\left(\tilde{\mathcal{U}}_{j}^{2}\right)(t, \eta)\right| \leqslant\left|\tilde{\mathcal{U}}_{1}^{2} \tilde{\mathcal{U}}_{1, \eta}(t, \eta)\right| \leqslant \frac{A^{4}}{2}\left\|\tilde{\mathcal{U}}_{1}\right\|_{L^{\infty}} \leqslant \frac{A^{6}}{2 \sqrt{2}},
$$

since $2\left|\tilde{\mathcal{U}}_{i} \tilde{\mathcal{U}}_{i, \eta}(t, \eta)\right| \leqslant A_{i}^{4} \leqslant A^{4}$ for all $t$ and $\eta$. Furthermore, we established before that the function $\theta \mapsto \min _{j}\left(\tilde{\mathcal{U}}_{j}^{2}(t, \eta)\right)$ is Lipschitz continuous with Lipschitz constant at most $A^{4}$; see (5.8). Thus

$$
\left|\frac{d}{d \eta} \tilde{\mathcal{U}}_{1} \min _{j}\left(\tilde{\mathcal{U}}_{j}^{2}\right)(t, \eta)\right| \leqslant \frac{3}{2} A^{4}\left\|\tilde{\mathcal{U}}_{1}\right\|_{L^{\infty}} \leqslant \frac{3}{2 \sqrt{2}} A^{6}
$$

and

$$
\left|\frac{1}{A_{1}^{5} A_{2}^{5}} \tilde{\mathcal{U}}_{i}^{3} \tilde{\mathcal{U}}_{i, n} \mathbb{1}_{A_{i}=a}\right| \leqslant \frac{1}{A_{1}^{5} A_{2}^{5}} \frac{1}{4} A_{i}^{8} \mathbb{1}_{A_{i}=a} \leqslant \frac{1}{A^{2}} .
$$

Finally, we get that

$$
\begin{aligned}
\frac{3}{4}\left|I_{2}\right| & =\left|\int_{0}^{1}\left(\tilde{\mathcal{U}}_{1}-\tilde{\mathcal{U}}_{2}\right)\left(\frac{1}{A_{2}^{5}} \tilde{\mathcal{U}}_{2}^{3} \tilde{\mathcal{U}}_{2, \eta}-\frac{1}{A_{1}^{5}} \tilde{\mathcal{U}}_{1}^{3} \tilde{\mathcal{U}}_{1, \eta}\right)(t, \eta) d \eta\right| \\
& \leqslant \mathcal{O}(1)\left\|\tilde{\mathcal{U}}_{1}-\tilde{\mathcal{U}}_{2}\right\|^{2}+\frac{2}{A^{2}}\left|A_{1}^{5}-A_{2}^{5}\right| \int_{0}^{1}\left|\tilde{\mathcal{U}}_{1}-\tilde{\mathcal{U}}_{2}\right|(t, \eta) d \eta \\
& \leqslant \mathcal{O}(1)\left(\left\|\tilde{\mathcal{U}}_{1}-\tilde{\mathcal{U}}_{2}\right\|^{2}+\left|A_{1}-A_{2}\right|^{2}\right) .
\end{aligned}
$$

The third integral $I_{3}$ : We will split $I_{3}$ into several terms that we treat separately, and combine them in the end.

Recall that we introduced the functions $\tilde{\mathcal{U}}_{i}^{-}=\min \left(0, \tilde{\mathcal{U}}_{i}\right)$ and $\tilde{\mathcal{U}}_{i}^{+}=\max \left(0, \tilde{\mathcal{U}}_{i}\right)$ with properties (5.2) and (5.3). We write

$$
I_{3}=\frac{4}{3} I_{31}-4 I_{32}-2 I_{33},
$$

where

$$
\begin{aligned}
I_{31}= & \int_{0}^{1}\left(\tilde{\mathcal{U}}_{1}-\tilde{\mathcal{U}}_{2}\right)(t, \eta)\left(\frac{1}{A_{2}^{6}} \tilde{\mathcal{U}}_{2, \eta}(t, \eta) \int_{0}^{1} e^{-\frac{1}{A_{2}}\left|\tilde{\mathcal{Y}}_{2}(t, \eta)-\tilde{\mathcal{Y}}_{2}(t, \theta)\right|} \tilde{\mathcal{U}}_{2}^{3} \tilde{\mathcal{Y}}_{2, \eta}(t, \theta) d \theta\right. \\
& \left.-\frac{1}{A_{1}^{6}} \tilde{\mathcal{U}}_{1, \eta}(t, \eta) \int_{0}^{1} e^{-\frac{1}{A_{1}}\left|\tilde{\mathcal{Y}}_{1}(t, \eta)-\tilde{\mathcal{Y}}_{1}(t, \theta)\right|} \tilde{\mathcal{U}}_{1}^{3} \tilde{\mathcal{Y}}_{1, \eta}(t, \theta) d \theta\right) d \eta \\
I_{32}= & \int_{0}^{1}\left(\tilde{\mathcal{U}}_{1}-\tilde{\mathcal{U}}_{2}\right)(t, \eta)\left(\frac{1}{A_{2}^{6}} \tilde{\mathcal{U}}_{2, \eta}(t, \eta) \int_{0}^{1} e^{-\frac{1}{A_{2}}\left|\tilde{\mathcal{Y}}_{2}(t, \eta)-\tilde{\mathcal{Y}}_{2}(t, \theta)\right|} \tilde{\mathcal{P}}_{2} \tilde{\mathcal{U}}_{2} \tilde{\mathcal{Y}}_{2, \eta}(t, \theta) d \theta\right.
\end{aligned}
$$




$$
\begin{aligned}
& \left.-\frac{1}{A_{1}^{6}} \tilde{\mathcal{U}}_{1, \eta}(t, \eta) \int_{0}^{1} e^{-\frac{1}{A_{1}}\left|\tilde{\mathcal{Y}}_{1}(t, \eta)-\tilde{\mathcal{Y}}_{1}(t, \theta)\right|} \tilde{\mathcal{P}}_{1} \tilde{\mathcal{U}}_{1} \tilde{\mathcal{Y}}_{1, \eta}(t, \theta) d \theta\right) d \eta \\
I_{33}= & \int_{0}^{1}\left(\tilde{\mathcal{U}}_{1}-\tilde{\mathcal{U}}_{2}\right)(t, \eta)\left(\frac{1}{A_{2}^{6}} \tilde{\mathcal{U}}_{2, \eta}(t, \eta) \int_{0}^{1} e^{-\frac{1}{A_{2}}\left|\tilde{\mathcal{Y}}_{2}(t, \eta)-\tilde{\mathcal{Y}}_{2}(t, \theta)\right|} \tilde{\mathcal{Q}}_{2} \tilde{\mathcal{U}}_{2, \eta}(t, \theta) d \theta\right. \\
& \left.-\frac{1}{A_{1}^{6}} \tilde{\mathcal{U}}_{1, \eta}(t, \eta) \int_{0}^{1} e^{-\frac{1}{A_{1}}\left|\tilde{\mathcal{Y}}_{1}(t, \eta)-\tilde{\mathcal{Y}}_{1}(t, \theta)\right|} \tilde{\mathcal{Q}}_{1} \tilde{\mathcal{U}}_{1, \eta}(t, \theta) d \theta\right) d \eta
\end{aligned}
$$

Thus we have

$$
\begin{aligned}
& I_{31}=\int_{0}^{1}\left(\tilde{\mathcal{U}}_{1}-\tilde{\mathcal{U}}_{2}\right)(t, \eta) \\
& \times\left(\frac{1}{A_{2}^{6}} \tilde{\mathcal{U}}_{2, \eta}(t, \eta) \int_{0}^{1} e^{-\frac{1}{A_{2}}\left|\tilde{\mathcal{Y}}_{2}(t, \eta)-\tilde{\mathcal{Y}}_{2}(t, \theta)\right|} \tilde{\mathcal{U}}_{2}^{3} \tilde{\mathcal{Y}}_{2, \eta}(t, \theta) d \theta\right. \\
& \left.-\frac{1}{A_{1}^{6}} \tilde{\mathcal{U}}_{1, \eta}(t, \eta) \int_{0}^{1} e^{-\frac{1}{A_{1}}\left|\tilde{\mathcal{Y}}_{1}(t, \eta)-\tilde{\mathcal{Y}}_{1}(t, \theta)\right|} \tilde{\mathcal{U}}_{1}^{3} \tilde{\mathcal{Y}}_{1, \eta}(t, \theta) d \theta\right) d \eta \\
& =\int_{0}^{1}\left(\tilde{\mathcal{U}}_{1}-\tilde{\mathcal{U}}_{2}\right)(t, \eta) \\
& \times\left(\frac{1}{A_{2}^{6}} \tilde{\mathcal{U}}_{2, \eta}(t, \eta) \int_{0}^{1} e^{-\frac{1}{A_{2}}\left|\tilde{\mathcal{Y}}_{2}(t, \eta)-\tilde{\mathcal{Y}}_{2}(t, \theta)\right|}\left(\tilde{\mathcal{U}}_{2}^{-}\right)^{3} \tilde{\mathcal{Y}}_{2, \eta}(t, \theta) d \theta\right. \\
& \left.-\frac{1}{A_{1}^{6}} \tilde{\mathcal{U}}_{1, \eta}(t, \eta) \int_{0}^{1} e^{-\frac{1}{A_{1}}\left|\tilde{\mathcal{Y}}_{1}(t, \eta)-\tilde{\mathcal{Y}}_{1}(t, \theta)\right|}\left(\tilde{\mathcal{U}}_{1}^{-}\right)^{3} \tilde{\mathcal{Y}}_{1, \eta}(t, \theta) d \theta\right) d \eta \\
& +\int_{0}^{1}\left(\tilde{\mathcal{U}}_{1}-\tilde{\mathcal{U}}_{2}\right)(t, \eta) \\
& \times\left(\frac{1}{A_{2}^{6}} \tilde{\mathcal{U}}_{2, \eta}(t, \eta) \int_{0}^{1} e^{-\frac{1}{A_{2}}\left|\tilde{\mathcal{Y}}_{2}(t, \eta)-\tilde{\mathcal{Y}}_{2}(t, \theta)\right|}\left(\tilde{\mathcal{U}}_{2}^{+}\right)^{3} \tilde{\mathcal{Y}}_{2, \eta}(t, \theta) d \theta\right. \\
& \left.-\frac{1}{A_{1}^{6}} \tilde{\mathcal{U}}_{1, \eta}(t, \eta) \int_{0}^{1} e^{-\frac{1}{A_{1}}\left|\tilde{\mathcal{Y}}_{1}(t, \eta)-\tilde{\mathcal{Y}}_{1}(t, \theta)\right|}\left(\tilde{\mathcal{U}}_{1}^{+}\right)^{3} \tilde{\mathcal{Y}}_{1, \eta}(t, \theta) d \theta\right) d \eta \text {. }
\end{aligned}
$$

Since both inner integrals have the same structure, it suffices to consider the second integral. Recall rewrite (5.4). Thus we need to estimate the following term:

$$
\begin{aligned}
& \frac{1}{A_{2}^{6}} \tilde{\mathcal{U}}_{2, \eta}(t, \eta) \int_{0}^{\eta} e^{-\frac{1}{A_{2}}\left(\tilde{\mathcal{Y}}_{2}(t, \eta)-\tilde{\mathcal{Y}}_{2}(t, \theta)\right)}\left(\tilde{\mathcal{U}}_{2}^{+}\right)^{3} \tilde{\mathcal{Y}}_{2, \eta}(t, \theta) d \theta \\
& \quad-\frac{1}{A_{1}^{6}} \tilde{\mathcal{U}}_{1, \eta}(t, \eta) \int_{0}^{\eta} e^{-\frac{1}{A_{1}}\left(\tilde{\mathcal{Y}}_{1}(t, \eta)-\tilde{\mathcal{Y}}_{1}(t, \theta)\right)}\left(\tilde{\mathcal{U}}_{1}^{+}\right)^{3} \tilde{\mathcal{Y}}_{1, \eta}(t, \theta) d \theta \\
& =\frac{1}{A^{6}}\left(\tilde{\mathcal{U}}_{2, \eta}(t, \eta) \int_{0}^{\eta} e^{-\frac{1}{A_{2}}\left(\tilde{\mathcal{Y}}_{2}(t, \eta)-\tilde{\mathcal{Y}}_{2}(t, \theta)\right)}\left(\tilde{\mathcal{U}}_{2}^{+}\right)^{3} \tilde{\mathcal{Y}}_{2, \eta}(t, \theta) d \theta\right.
\end{aligned}
$$




$$
\begin{aligned}
& \left.-\tilde{\mathcal{U}}_{1, \eta}(t, \eta) \int_{0}^{\eta} e^{-\frac{1}{A_{1}}\left(\tilde{\mathcal{Y}}_{1}(t, \eta)-\tilde{\mathcal{Y}}_{1}(t, \theta)\right)}\left(\tilde{\mathcal{U}}_{1}^{+}\right)^{3} \tilde{\mathcal{Y}}_{1, \eta}(t, \theta) d \theta\right) \\
& +\mathbb{1}_{A_{1} \leqslant A_{2}}\left(\frac{1}{A_{2}^{6}}-\frac{1}{A_{1}^{6}}\right)\left(\tilde{\mathcal{U}}_{1, \eta}(t, \eta) \int_{0}^{\eta} e^{-\frac{1}{A_{1}}\left(\tilde{\mathcal{Y}}_{1}(t, \eta)-\tilde{\mathcal{Y}}_{1}(t, \theta)\right)}\left(\tilde{\mathcal{U}}_{1}^{+}\right)^{3} \tilde{\mathcal{Y}}_{1, \eta}(t, \theta) d \theta\right) \\
& +\mathbb{1}_{A_{2}<A_{1}}\left(\frac{1}{A_{2}^{6}}-\frac{1}{A_{1}^{6}}\right)\left(\tilde{\mathcal{U}}_{2, \eta}(t, \eta) \int_{0}^{\eta} e^{-\frac{1}{A_{2}}\left(\tilde{\mathcal{Y}}_{2}(t, \eta)-\tilde{\mathcal{Y}}_{2}(t, \theta)\right)}\left(\tilde{\mathcal{U}}_{2}^{+}\right)^{3} \tilde{\mathcal{Y}}_{2, \eta}(t, \theta) d \theta\right) \\
& =\frac{1}{A^{6}} \tilde{\mathcal{U}}_{2, \eta}(t, \eta) \int_{0}^{\eta} e^{-\frac{1}{A_{2}}\left(\tilde{\mathcal{Y}}_{2}(t, \eta)-\tilde{\mathcal{Y}}_{2}(t, \theta)\right)}\left(\left(\tilde{\mathcal{U}}_{2}^{+}\right)^{3}-\left(\tilde{\mathcal{U}}_{1}^{+}\right)^{3}\right) \tilde{\mathcal{Y}}_{2, \eta}(t, \theta) \mathbb{1}_{\tilde{\mathcal{U}}_{1}^{+} \leqslant \tilde{\mathcal{U}}_{2}^{+}}(t, \theta) d \theta \\
& +\frac{1}{A^{6}} \tilde{\mathcal{U}}_{1, \eta}(t, \eta) \int_{0}^{\eta} e^{-\frac{1}{A_{1}}\left(\tilde{\mathcal{Y}}_{1}(t, \eta)-\tilde{\mathcal{Y}}_{1}(t, \theta)\right)}\left(\left(\tilde{\mathcal{U}}_{2}^{+}\right)^{3}-\left(\tilde{\mathcal{U}}_{1}^{+}\right)^{3}\right) \tilde{\mathcal{Y}}_{1, \eta}(t, \theta) \mathbb{1}_{\tilde{\mathcal{U}}_{2}^{+}<\tilde{\mathcal{U}}_{1}^{+}}(t, \theta) d \theta \\
& +\frac{1}{A^{6}} \tilde{\mathcal{U}}_{2, \eta}(t, \eta) \int_{0}^{\eta} e^{-\frac{1}{A_{2}}\left(\tilde{\mathcal{Y}}_{2}(t, \eta)-\tilde{\mathcal{Y}}_{2}(t, \theta)\right)} \min _{j}\left(\tilde{\mathcal{U}}_{j}^{+}\right)^{3} \tilde{\mathcal{Y}}_{2, \eta}(t, \theta) d \theta \\
& -\frac{1}{A^{6}} \tilde{\mathcal{U}}_{1, \eta}(t, \eta) \int_{0}^{\eta} e^{-\frac{1}{A_{1}}\left(\tilde{\mathcal{Y}}_{1}(t, \eta)-\tilde{\mathcal{Y}}_{1}(t, \theta)\right)} \min _{j}\left(\tilde{\mathcal{U}}_{j}^{+}\right)^{3} \tilde{\mathcal{Y}}_{1, \eta}(t, \theta) d \theta \\
& +\mathbb{1}_{A_{1} \leqslant A_{2}}\left(\frac{1}{A_{2}^{6}}-\frac{1}{A_{1}^{6}}\right)\left(\tilde{\mathcal{U}}_{1, \eta}(t, \eta) \int_{0}^{\eta} e^{-\frac{1}{A_{1}}\left(\tilde{\mathcal{Y}}_{1}(t, \eta)-\tilde{\mathcal{Y}}_{1}(t, \theta)\right)}\left(\tilde{\mathcal{U}}_{1}^{+}\right)^{3} \tilde{\mathcal{Y}}_{1, \eta}(t, \theta) d \theta\right) \\
& +\mathbb{1}_{A_{2}<A_{1}}\left(\frac{1}{A_{2}^{6}}-\frac{1}{A_{1}^{6}}\right)\left(\tilde{\mathcal{U}}_{2, \eta}(t, \eta) \int_{0}^{\eta} e^{-\frac{1}{A_{2}}\left(\tilde{\mathcal{Y}}_{2}(t, \eta)-\tilde{\mathcal{Y}}_{2}(t, \theta)\right)}\left(\tilde{\mathcal{U}}_{2}^{+}\right)^{3} \tilde{\mathcal{Y}}_{2, \eta}(t, \theta) d \theta\right) \\
& =\frac{1}{A^{6}} \tilde{\mathcal{U}}_{2, \eta}(t, \eta) \int_{0}^{\eta} e^{-\frac{1}{A_{2}}\left(\tilde{\mathcal{Y}}_{2}(t, \eta)-\tilde{\mathcal{Y}}_{2}(t, \theta)\right)}\left(\left(\tilde{\mathcal{U}}_{2}^{+}\right)^{3}-\left(\tilde{\mathcal{U}}_{1}^{+}\right)^{3}\right) \tilde{\mathcal{Y}}_{2, \eta}(t, \theta) \mathbb{1}_{\tilde{\mathcal{U}}_{1}^{+} \leqslant \tilde{\mathcal{U}}_{2}^{+}}(t, \theta) d \theta \\
& +\frac{1}{A^{6}} \tilde{\mathcal{U}}_{1, \eta}(t, \eta) \int_{0}^{\eta} e^{-\frac{1}{A_{1}}\left(\tilde{\mathcal{Y}}_{1}(t, \eta)-\tilde{\mathcal{Y}}_{1}(t, \theta)\right)}\left(\left(\tilde{\mathcal{U}}_{2}^{+}\right)^{3}-\left(\tilde{\mathcal{U}}_{1}^{+}\right)^{3}\right) \tilde{\mathcal{Y}}_{1, \eta}(t, \theta) \mathbb{1}_{\tilde{\mathcal{U}}_{2}^{+}<\tilde{\mathcal{U}}_{1}^{+}}(t, \theta) d \theta \\
& +\frac{1}{A^{6}} \mathbb{1}_{A_{1} \leqslant A_{2}} \tilde{\mathcal{U}}_{2, \eta}(t, \eta) \int_{0}^{\eta}\left(e^{-\frac{1}{A_{2}}\left(\tilde{\mathcal{Y}}_{2}(t, \eta)-\tilde{\mathcal{Y}}_{2}(t, \theta)\right)}-e^{-\frac{1}{A_{1}}\left(\tilde{\mathcal{Y}}_{2}(t, \eta)-\tilde{\mathcal{Y}}_{2}(t, \theta)\right)}\right) \\
& \times \min _{j}\left(\tilde{\mathcal{U}}_{j}^{+}\right)^{3} \tilde{\mathcal{Y}}_{2, \eta}(t, \theta) d \theta \\
& +\frac{1}{A^{6}} \mathbb{1}_{A_{2}<A_{1}} \tilde{\mathcal{U}}_{1, \eta}(t, \eta) \int_{0}^{\eta}\left(e^{-\frac{1}{A_{2}}\left(\tilde{\mathcal{Y}}_{1}(t, \eta)-\tilde{\mathcal{Y}}_{1}(t, \theta)\right)}-e^{-\frac{1}{A_{1}}\left(\tilde{\mathcal{Y}}_{1}(t, \eta)-\tilde{\mathcal{Y}}_{1}(t, \theta)\right)}\right) \\
& \times \min _{j}\left(\tilde{\mathcal{U}}_{j}^{+}\right) \tilde{\mathcal{Y}}_{1, \eta}(t, \theta) d \theta \\
& +\frac{1}{A^{6}} \tilde{\mathcal{U}}_{2, \eta}(t, \eta) \int_{0}^{\eta}\left(e^{-\frac{1}{a}\left(\tilde{\mathcal{Y}}_{2}(t, \eta)-\tilde{\mathcal{Y}}_{2}(t, \theta)\right)}-e^{-\frac{1}{a}\left(\tilde{\mathcal{Y}}_{1}(t, \eta)-\tilde{\mathcal{Y}}_{1}(t, \theta)\right)}\right) \\
& \times \min _{j}\left(\tilde{\mathcal{U}}_{j}^{+}\right)^{3} \tilde{\mathcal{Y}}_{2, \eta}(t, \theta) \mathbb{1}_{B(\eta)}(t, \theta) d \theta \\
& -\frac{1}{A^{6}} \tilde{\mathcal{U}}_{1, \eta}(t, \eta) \int_{0}^{\eta}\left(e^{-\frac{1}{a}\left(\tilde{\mathcal{Y}}_{1}(t, \eta)-\tilde{\mathcal{Y}}_{1}(t, \theta)\right)}-e^{-\frac{1}{a}\left(\tilde{\mathcal{Y}}_{2}(t, \eta)-\tilde{\mathcal{Y}}_{2}(t, \theta)\right)}\right) \\
& \left.\times \min _{j}\left(\tilde{\mathcal{U}}_{j}^{+}\right)^{3} \tilde{\mathcal{Y}}_{1, \eta}(t, \theta) \mathbb{1}_{B^{c}(\eta)}(t, \theta) d \theta\right)
\end{aligned}
$$




$$
\begin{aligned}
& +\frac{1}{A^{6}} \tilde{\mathcal{U}}_{2, \eta}(t, \eta) \int_{0}^{\eta} \min _{j}\left(e^{-\frac{1}{a}\left(\tilde{\mathcal{Y}}_{j}(t, \eta)-\tilde{\mathcal{Y}}_{j}(t, \theta)\right)}\right) \min _{j}\left(\tilde{\mathcal{U}}_{j}^{+}\right)^{3} \tilde{\mathcal{Y}}_{2, \eta}(t, \theta) d \theta \\
& -\frac{1}{A^{6}} \tilde{\mathcal{U}}_{1, \eta}(t, \eta) \int_{0}^{\eta} \min _{j}\left(e^{-\frac{1}{a}\left(\tilde{\mathcal{Y}}_{j}(t, \eta)-\tilde{\mathcal{Y}}_{j}(t, \theta)\right)}\right) \min _{j}\left(\tilde{\mathcal{U}}_{j}^{+}\right)^{3} \tilde{\mathcal{Y}}_{1, \eta}(t, \theta) d \theta \\
& +\mathbb{1}_{A_{1} \leqslant A_{2}}\left(\frac{1}{A_{2}^{6}}-\frac{1}{A_{1}^{6}}\right)\left(\tilde{\mathcal{U}}_{1, \eta}(t, \eta) \int_{0}^{\eta} e^{-\frac{1}{A_{1}}\left(\tilde{\mathcal{Y}}_{1}(t, \eta)-\tilde{\mathcal{Y}}_{1}(t, \theta)\right)}\left(\tilde{\mathcal{U}}_{1}^{+}\right)^{3} \tilde{\mathcal{Y}}_{1, \eta}(t, \theta) d \theta\right) \\
& +\mathbb{1}_{A_{2}<A_{1}}\left(\frac{1}{A_{2}^{6}}-\frac{1}{A_{1}^{6}}\right)\left(\tilde{\mathcal{U}}_{2, \eta}(t, \eta) \int_{0}^{\eta} e^{-\frac{1}{A_{2}}\left(\tilde{\mathcal{Y}}_{2}(t, \eta)-\tilde{\mathcal{Y}}_{2}(t, \theta)\right)}\left(\tilde{\mathcal{U}}_{2}^{+}\right)^{3} \hat{\mathcal{Y}}_{2, \eta}(t, \theta) d \theta\right) \\
& =\left(J_{1}+J_{2}+J_{3}+J_{4}+J_{5}+J_{6}+J_{7}+J_{8}+J_{9}+J_{10}\right)(t, \eta),
\end{aligned}
$$

where $B(\eta)$ is given by (5.11).

As far as the integral that contains $J_{1}$ (a similar argument works for $J_{2}$ ) is concerned, we have

$$
\begin{aligned}
\mid \int_{0}^{1} & J_{1}\left(\tilde{\mathcal{U}}_{1}-\tilde{\mathcal{U}}_{2}\right)(t, \eta) d \eta \mid \\
\leqslant & \left\|\tilde{\mathcal{U}}_{1}-\tilde{\mathcal{U}}_{2}\right\|^{2}+\int_{0}^{1} J_{1}^{2}(t, \eta) d \eta \\
\leqslant & \left\|\tilde{\mathcal{U}}_{1}-\tilde{\mathcal{U}}_{2}\right\|_{L^{2}}^{2}+\frac{1}{A^{12}} \int_{0}^{1} \tilde{\mathcal{U}}_{2, \eta}^{2}(t, \eta) \\
& \times\left(\int_{0}^{\eta} e^{-\frac{1}{A_{2}}\left(\tilde{\mathcal{Y}}_{2}(t, \eta)-\tilde{\mathcal{Y}}_{2}(t, \theta)\right)}\left(\left(\tilde{\mathcal{U}}_{2}^{+}\right)^{3}-\left(\tilde{\mathcal{U}}_{1}^{+}\right)^{3}\right) \tilde{\mathcal{Y}}_{2, \eta}(t, \theta) \tilde{\mathbb{U}}_{1}^{+} \leqslant \tilde{\mathcal{U}}_{2}^{+}(t, \theta) d \theta\right)^{2} d \eta \\
\leqslant & \left\|\tilde{\mathcal{U}}_{1}-\tilde{\mathcal{U}}_{2}\right\|^{2} \\
& +\frac{1}{A^{9}} \int_{0}^{1} \tilde{\mathcal{Y}}_{2, \eta}(t, \eta)\left(\int_{0}^{\eta} e^{-\frac{1}{A_{2}}\left(\tilde{\mathcal{Y}}_{2}(t, \eta)-\tilde{\mathcal{Y}}_{2}(t, \theta)\right)} 3\left(\tilde{\mathcal{U}}_{2}^{+}\right)^{2}\left|\tilde{\mathcal{U}}_{2}^{+}-\tilde{\mathcal{U}}_{1}^{+}\right| \tilde{\mathcal{Y}}_{2, \eta}(t, \theta) d \theta\right)^{2} d \eta \\
\leqslant & \left\|\tilde{\mathcal{U}}_{1}-\tilde{\mathcal{U}}_{2}\right\|^{2} \\
& +\frac{9}{A^{4}} \int_{0}^{1} \tilde{\mathcal{Y}}_{2, \eta}(t, \eta)\left(\int_{0}^{\eta} e^{-\frac{1}{A_{2}}\left(\tilde{\mathcal{Y}}_{2}(t, \eta)-\tilde{\mathcal{Y}}_{2}(t, \theta)\right)} \tilde{\mathcal{U}}_{2}^{2} \tilde{\mathcal{Y}}_{2, \eta}(t, \theta) d \theta\right) d \eta\left\|\tilde{\mathcal{U}}_{1}-\tilde{\mathcal{U}}_{2}\right\|^{2} \\
\leqslant & \left(1+\frac{36}{A^{3}} \int_{0}^{1} \tilde{\mathcal{P}}_{2} \tilde{\mathcal{Y}}_{2, \eta}(t, \eta) d \eta\right)\left\|\tilde{\mathcal{U}}_{1}-\tilde{\mathcal{U}}_{2}\right\|^{2} \\
\leqslant & \mathcal{O}(1)\left\|\tilde{\mathcal{U}}_{1}-\tilde{\mathcal{U}}_{2}\right\|^{2},
\end{aligned}
$$

where we used (4.15e), (4.15g), and (4.15h).

As far as the third and fourth terms $J_{3}$ and $J_{4}$ are concerned, they again have the same structure, and hence we only consider the integral corresponding to $J_{3}$.

$$
\begin{aligned}
& \left|\int_{0}^{1} J_{3}\left(\tilde{\mathcal{U}}_{1}-\tilde{\mathcal{U}}_{2}\right)(t, \eta) d \eta\right| \\
& \quad=\mathbb{1}_{A_{1} \leqslant A_{2}} \frac{1}{A^{6}} \mid \int_{0}^{1}\left(\tilde{\mathcal{U}}_{1}-\tilde{\mathcal{U}}_{2}\right) \tilde{\mathcal{U}}_{2, \eta}(t, \eta)
\end{aligned}
$$




$$
\begin{aligned}
& \times \int_{0}^{\eta}\left(e^{-\frac{1}{A_{2}}\left(\tilde{\mathcal{Y}}_{2}(t, \eta)-\tilde{\mathcal{Y}}_{2}(t, \theta)\right)}-e^{-\frac{1}{A_{1}}\left(\tilde{\mathcal{Y}}_{2}(t, \eta)-\tilde{\mathcal{Y}}_{2}(t, \theta)\right)}\right) \min _{j}\left(\tilde{\mathcal{U}}_{j}^{+}\right)^{3} \tilde{\mathcal{Y}}_{2, \eta}(t, \theta) d \theta d \eta \mid \\
\leqslant & \frac{2 \sqrt{2} a}{A^{6} e} \int_{0}^{1}\left|\tilde{\mathcal{U}}_{1}-\tilde{\mathcal{U}}_{2}\right|\left|\tilde{\mathcal{U}}_{2, \eta}\right|(t, \eta) \\
& \times \int_{0}^{\eta} e^{-\frac{3}{4 A_{2}}\left(\tilde{\mathcal{Y}}_{2}(t, \eta)-\tilde{\mathcal{Y}}_{2}(t, \theta)\right)} \tilde{\mathcal{U}}_{2}^{2} \tilde{\mathcal{Y}}_{2, \eta}(t, \theta) d \theta d \eta\left|A_{1}-A_{2}\right| \\
\leqslant & \left\|\tilde{\mathcal{U}}_{1}-\tilde{\mathcal{U}}_{2}\right\|^{2}+\frac{8}{A^{10} e^{2}} \int_{0}^{1} \tilde{\mathcal{U}}_{2, \eta}^{2}(t, \eta)\left(\int_{0}^{\eta} e^{-\frac{1}{A_{2}}\left(\tilde{\mathcal{Y}}_{2}(t, \eta)-\tilde{\mathcal{Y}}_{2}(t, \theta)\right)} \tilde{\mathcal{U}}_{2}^{2} \tilde{\mathcal{Y}}_{2, \eta}(t, \theta) d \theta\right) \\
& \times\left(\int_{0}^{\eta} e^{-\frac{1}{2 A_{2}}\left(\tilde{\mathcal{Y}}_{2}(t, \eta)-\tilde{\mathcal{Y}}_{2}(t, \theta)\right)} \tilde{\mathcal{U}}_{2}^{2} \tilde{\mathcal{Y}}_{2, \eta}(t, \theta) d \theta\right) d \eta\left|A_{1}-A_{2}\right|^{2} \\
\leqslant & \left\|\tilde{\mathcal{U}}_{1}-\tilde{\mathcal{U}}_{2}\right\|^{2}+\frac{32}{A e^{2}} \int_{0}^{1} \tilde{\mathcal{P}}_{2} \tilde{\mathcal{Y}}_{2, \eta}(t, \eta) d \eta\left|A_{1}-A_{2}\right|^{2} \\
\leqslant & \mathcal{O}(1)\left(\left\|\tilde{\mathcal{U}}_{1}-\tilde{\mathcal{U}}_{2}\right\|^{2}+\left|A_{1}-A_{2}\right|^{2}\right) .
\end{aligned}
$$

As far as the third and fourth terms $J_{5}$ and $J_{6}$ are concerned, they again have the same structure, and hence we only consider the integral corresponding to $J_{5}$. Thus, using (5.13),

$$
\begin{aligned}
\mid \int_{0}^{1} & J_{5}\left(\tilde{\mathcal{U}}_{1}-\tilde{\mathcal{U}}_{2}\right)(t, \eta) d \eta \mid \\
= & \frac{1}{A^{6}} \mid \int_{0}^{1}\left(\tilde{\mathcal{U}}_{1}-\tilde{\mathcal{U}}_{2}\right) \tilde{\mathcal{U}}_{2, \eta}(t, \eta)\left(\int_{0}^{\eta}\left(e^{-\frac{1}{a}\left(\tilde{\mathcal{Y}}_{2}(t, \eta)-\tilde{\mathcal{Y}}_{2}(t, \theta)\right)}-e^{-\frac{1}{a}\left(\tilde{\mathcal{Y}}_{1}(t, \eta)-\tilde{\mathcal{Y}}_{1}(t, \theta)\right)}\right)\right. \\
& \left.\times \min _{j}\left(\tilde{\mathcal{U}}_{j}^{+}\right)^{3} \tilde{\mathcal{Y}}_{2, \eta}(t, \theta) \mathbb{1}_{B(\eta)}(t, \theta) d \theta\right) d \eta \mid \\
\leqslant & \frac{1}{a A^{6}} \int_{0}^{1}\left|\tilde{\mathcal{U}}_{1}-\tilde{\mathcal{U}}_{2}\right|\left|\tilde{\mathcal{U}}_{2, \eta}\right|(t, \eta) \\
& \times\left(\int_{0}^{\eta}\left(\left|\tilde{\mathcal{Y}}_{2}(t, \eta)-\tilde{\mathcal{Y}}_{1}(t, \eta)\right|+\left|\tilde{\mathcal{Y}}_{2}(t, \theta)-\tilde{\mathcal{Y}}_{1}(t, \theta)\right|\right)\right. \\
& \left.\times e^{-\frac{1}{a}\left(\tilde{\mathcal{Y}}_{2}(t, \eta)-\tilde{\mathcal{Y}}_{2}(t, \theta)\right)} \min _{j}\left(\tilde{\mathcal{U}}_{j}^{+}\right)^{3} \tilde{\mathcal{Y}}_{2, \eta}(t, \theta) d \theta\right) d \eta \\
\leqslant & \frac{1}{a A^{6}} \int_{0}^{1}\left|\tilde{\mathcal{U}}_{1}-\tilde{\mathcal{U}}_{2}\right|\left|\tilde{\mathcal{Y}}_{1}-\tilde{\mathcal{Y}}_{2}\right|\left|\tilde{\mathcal{U}}_{2, \eta}\right|(t, \eta) \\
& \times \int_{0}^{\eta} e^{-\frac{1}{a}\left(\tilde{\mathcal{Y}}_{2}(t, \eta)-\tilde{\mathcal{Y}}_{2}(t, \theta)\right)} \min _{j}\left(\tilde{\mathcal{U}}_{j}^{+}\right)^{3} \tilde{\mathcal{Y}}_{2, \eta}(t, \theta) d \theta d \eta \\
& +\left\|\tilde{\mathcal{U}}_{1}-\tilde{\mathcal{U}}_{2}\right\|^{2}+\frac{1}{a^{2} A^{12}} \int_{0}^{1} \tilde{\mathcal{U}}_{2, \eta}^{2}(t, \eta)
\end{aligned}
$$




$$
\begin{aligned}
& \times\left(\int_{0}^{\eta}\left|\tilde{\mathcal{Y}}_{1}(t, \theta)-\tilde{\mathcal{Y}}_{2}(t, \theta)\right| e^{-\frac{1}{a}\left(\tilde{\mathcal{Y}}_{2}(t, \eta)-\tilde{\mathcal{Y}}_{2}(t, \theta)\right)} \min _{j}\left(\tilde{\mathcal{U}}_{j}^{+}\right)^{3} \tilde{\mathcal{Y}}_{2, \eta}(t, \theta) d \theta\right)^{2} d \eta \\
\leqslant & \frac{4 a}{A^{5}} \int_{0}^{1}\left|\tilde{\mathcal{U}}_{1}-\tilde{\mathcal{U}}_{2}\right|\left|\tilde{\mathcal{Y}}_{1}-\tilde{\mathcal{Y}}_{2}\right| \tilde{\mathcal{P}}_{2}\left|\tilde{\mathcal{U}}_{2, \eta}\right|(t, \eta) d \eta+\left\|\tilde{\mathcal{U}}_{1}-\tilde{\mathcal{U}}_{2}\right\|^{2} \\
& +\frac{1}{a^{2} A^{9}} \int_{0}^{1} \tilde{\mathcal{Y}}_{2, \eta}(t, \eta)\left(\int_{0}^{\eta} e^{-\frac{1}{A_{2}}\left(\tilde{\mathcal{Y}}_{2}(t, \eta)-\tilde{\mathcal{Y}}_{2}(t, \theta)\right)} \tilde{\mathcal{U}}_{2}^{2} \tilde{\mathcal{Y}}_{2, \eta}(t, \theta) d \theta\right) \\
& \times\left(\int_{0}^{\eta}\left(\tilde{\mathcal{Y}}_{1}-\tilde{\mathcal{Y}}_{2}\right)^{2}(t, \theta) e^{-\frac{1}{a}\left(\tilde{\mathcal{Y}}_{2}(t, \eta)-\tilde{\mathcal{Y}}_{2}(t, \theta)\right)} \min _{j}\left(\tilde{\mathcal{U}}_{j}^{4}\right) \tilde{\mathcal{Y}}_{2, \eta}(t, \theta) d \theta\right) d \eta \\
\leqslant & \left(\sqrt{2} A^{2}+1\right)\left(\left\|\tilde{\mathcal{Y}}_{1}-\tilde{\mathcal{Y}}_{2}\right\|^{2}+\left\|\tilde{\mathcal{U}}_{1}-\tilde{\mathcal{U}}_{2}\right\|^{2}\right)+\frac{2}{A^{6}} \int_{0}^{1} \tilde{\mathcal{P}}_{2} \tilde{\mathcal{Y}}_{2, \eta}(t, \eta) \\
\times & \left(\int_{0}^{\eta}\left(\tilde{\mathcal{Y}}_{1}-\tilde{\mathcal{Y}}_{2}\right)^{2}(t, \theta) e^{-\frac{1}{a}\left(\tilde{\mathcal{Y}}_{2}(t, \eta)-\tilde{\mathcal{Y}}_{2}(t, \theta)\right)} \tilde{\mathcal{U}}_{2}^{2} \tilde{\mathcal{Y}}_{2, \eta}(t, \theta) d \theta\right) d \eta \\
\leqslant & \mathcal{O}(1)\left(\left\|\tilde{\mathcal{Y}}_{1}-\tilde{\mathcal{Y}}_{2}\right\|^{2}+\left\|\tilde{\mathcal{U}}_{1}-\tilde{\mathcal{U}}_{2}\right\|^{2}\right) .
\end{aligned}
$$

Next are the terms $J_{7}$ and $J_{8}$. Therefore recall (5.14), which implies

$$
\begin{aligned}
J_{7}+J_{8}= & \frac{1}{A^{6}} \tilde{\mathcal{U}}_{2, \eta}(t, \eta) \\
& \times\left(\int_{0}^{\eta} \min _{j}\left(e^{-\frac{1}{a}\left(\tilde{\mathcal{Y}}_{j}(t, \eta)-\tilde{\mathcal{Y}}_{j}(t, \theta)\right)}\right) \min _{j}\left(\tilde{\mathcal{U}}_{j}^{+}\right)^{3} \tilde{\mathcal{Y}}_{2, \eta}(t, \theta) d \theta\right) \\
& -\frac{1}{A^{6}} \tilde{\mathcal{U}}_{1, \eta}(t, \eta) \\
& \times\left(\int_{0}^{\eta} \min _{j}\left(e^{-\frac{1}{a}\left(\tilde{\mathcal{Y}}_{j}(t, \eta)-\tilde{\mathcal{Y}}_{j}(t, \theta)\right)}\right) \min _{j}\left(\tilde{\mathcal{U}}_{j}^{+}\right)^{3} \tilde{\mathcal{Y}}_{1, \eta}(t, \theta) d \theta\right) \\
= & \frac{1}{A^{6}}\left(\tilde{\mathcal{U}}_{2, \eta}-\tilde{\mathcal{U}}_{1, \eta}\right)(t, \eta) \\
& \times \min _{k}\left[\int_{0}^{\eta} \min _{j}\left(e^{-\frac{1}{a}\left(\tilde{\mathcal{Y}}_{j}(t, \eta)-\tilde{\mathcal{Y}}_{j}(t, \theta)\right)}\right) \min _{j}\left(\tilde{\mathcal{U}}_{j}^{+}\right)^{3} \tilde{\mathcal{Y}}_{k, \eta}(t, \theta) d \theta\right] \\
& +\frac{1}{A^{6}} \tilde{\mathcal{U}}_{1, \eta}(t, \eta) \int_{0}^{\eta} \min _{j}\left(e^{-\frac{1}{a}\left(\tilde{\mathcal{Y}}_{j}(t, \eta)-\tilde{\mathcal{Y}}_{j}(t, \theta)\right)}\right) \\
& \times \min _{j}\left(\tilde{\mathcal{U}}_{j}^{+}\right)^{3}\left(\tilde{\mathcal{Y}}_{2, \eta}-\tilde{\mathcal{Y}}_{1, \eta}\right)(t, \theta) d \theta \mathbb{1}_{E}(t, \eta) \\
& +\frac{1}{A^{6}} \tilde{\mathcal{U}}_{2, \eta}(t, \eta) \int_{0}^{\eta} \min _{j}\left(e^{-\frac{1}{a}\left(\tilde{\mathcal{Y}}_{j}(t, \eta)-\tilde{\mathcal{Y}}_{j}(t, \theta)\right)}\right) \\
& \times \min _{j}\left(\tilde{\mathcal{U}}_{j}^{+}\right)^{3}\left(\tilde{\mathcal{Y}}_{2, \eta}-\tilde{\mathcal{Y}}_{1, \eta}\right)(t, \theta) d \theta \mathbb{1}_{E^{c}}(t, \eta) \\
= & L_{1}+L_{2}+L_{3} .
\end{aligned}
$$


As far as the first term $L_{1}$ is concerned, the corresponding integral can be estimated as follows, using (A.11),

$$
\begin{aligned}
& \left|\int_{0}^{1} L_{1}\left(\tilde{\mathcal{U}}_{1}-\tilde{\mathcal{U}}_{2}\right)(t, \eta) d \eta\right| \\
& =\frac{1}{A^{6}} \mid \int_{0}^{1}\left(\tilde{\mathcal{U}}_{1}-\tilde{\mathcal{U}}_{2}\right)\left(\tilde{\mathcal{U}}_{2, \eta}-\tilde{\mathcal{U}}_{1, \eta}\right)(t, \eta) \\
& \times \min _{k}\left[\int_{0}^{\eta} \min _{j}\left(e^{-\frac{1}{a}\left(\tilde{\mathcal{Y}}_{j}(t, \eta)-\tilde{\mathcal{Y}}_{j}(t, \theta)\right)}\right) \min _{j}\left(\tilde{\mathcal{U}}_{j}^{+}\right)^{3} \tilde{\mathcal{Y}}_{k, \eta}(t, \theta) d \theta\right] d \eta \mid \\
& =\mid-\frac{1}{2 A^{6}}\left(\tilde{\mathcal{U}}_{1}-\tilde{\mathcal{U}}_{2}\right)^{2}(t, \eta) \\
& \times\left.\min _{k}\left[\int_{0}^{\eta} \min _{j}\left(e^{-\frac{1}{a}\left(\tilde{\mathcal{Y}}_{j}(t, \eta)-\tilde{\mathcal{Y}}_{j}(t, \theta)\right)}\right) \min _{j}\left(\tilde{\mathcal{U}}_{j}^{+}\right)^{3} \tilde{\mathcal{Y}}_{k, \eta}(t, \theta) d \theta\right]\right|_{\eta=0} ^{1} \\
& +\frac{1}{2 A^{6}} \int_{0}^{1}\left(\tilde{\mathcal{U}}_{1}-\tilde{\mathcal{U}}_{2}\right)^{2}(t, \eta) \\
& \times \frac{d}{d \eta} \min _{k}\left[\int_{0}^{\eta} \min _{j}\left(e^{-\frac{1}{a}\left(\tilde{\mathcal{Y}}_{j}(t, \eta)-\tilde{\mathcal{Y}}_{j}(t, \theta)\right)}\right) \min _{j}\left(\tilde{\mathcal{U}}_{j}^{+}\right)^{3} \tilde{\mathcal{Y}}_{k, \eta}(t, \theta) d \theta\right] d \eta \\
& \leqslant \mathcal{O}(1)\left\|\tilde{\mathcal{U}}_{1}-\tilde{\mathcal{U}}_{2}\right\|^{2},
\end{aligned}
$$

where $\mathcal{O}(1)$ denotes some constant only depending on $A$, which remains bounded as $A \rightarrow 0$.

As far as the last term $L_{3}$ (a similar argument works for $L_{2}$ ) is concerned, the corresponding integral can be estimated as follows:

$$
\begin{aligned}
& \left|\int_{0}^{1} L_{3}\left(\tilde{\mathcal{U}}_{1}-\tilde{\mathcal{U}}_{2}\right)(t, \eta) d \eta\right| \\
& =\frac{1}{A^{6}} \mid \int_{0}^{1}\left(\tilde{\mathcal{U}}_{1}-\tilde{\mathcal{U}}_{2}\right) \tilde{\mathcal{U}}_{2, \eta}(t, \eta) \int_{0}^{\eta} \min \left(e^{-\frac{1}{a}\left(\tilde{\mathcal{Y}}_{j}(t, \eta)-\tilde{\mathcal{Y}}_{j}(t, \theta)\right)}\right) \\
& \times \min _{j}\left(\tilde{\mathcal{U}}_{j}^{+}\right)^{3}\left(\tilde{\mathcal{Y}}_{2, \eta}-\tilde{\mathcal{Y}}_{1, \eta}\right)(t, \theta) d \theta \mathbb{1}_{E^{c}}(t, \eta) d \eta \mid \\
& =\frac{1}{A^{6}} \mid \int_{0}^{1}\left(\tilde{\mathcal{U}}_{1}-\tilde{\mathcal{U}}_{2}\right) \tilde{\mathcal{U}}_{2, \eta}(t, \eta) \mathbb{1}_{E^{c}}(t, \eta) \\
& \times\left[\left.\left(\tilde{\mathcal{Y}}_{2}-\tilde{\mathcal{Y}}_{1}\right)(t, \theta) \min _{j}\left(e^{-\frac{1}{a}\left(\tilde{\mathcal{Y}}_{j}(t, \eta)-\tilde{\mathcal{Y}}_{j}(t, \theta)\right)}\right) \min _{j}\left(\tilde{\mathcal{U}}_{j}^{+}\right)^{3}(t, \theta)\right|_{\theta=0} ^{\eta}\right. \\
& -\int_{0}^{\eta}\left(\tilde{\mathcal{Y}}_{2}-\tilde{\mathcal{Y}}_{1}\right)(t, \theta)\left[\left(\frac{d}{d \theta} \min _{j}\left(e^{-\frac{1}{a}\left(\tilde{\mathcal{Y}}_{j}(t, \eta)-\tilde{\mathcal{Y}}_{j}(t, \theta)\right)}\right)\right) \min _{j}\left(\tilde{\mathcal{U}}_{j}^{+}\right)^{3}(t, \theta)\right. \\
& \left.\left.+\min _{j}\left(e^{-\frac{1}{a}\left(\tilde{\mathcal{Y}}_{j}(t, \eta)-\tilde{\mathcal{Y}}_{j}(t, \theta)\right)}\right)\left(\frac{d}{d \theta} \min _{j}\left(\tilde{\mathcal{U}}_{j}^{+}\right)^{3}\right)(t, \theta)\right] d \theta\right] \mathbb{1}_{E^{c}}(t, \eta) d \eta \mid
\end{aligned}
$$




$$
\begin{aligned}
& =\frac{1}{A^{6}} \mid-\int_{0}^{1}\left(\tilde{\mathcal{U}}_{1}-\tilde{\mathcal{U}}_{2}\right)\left(\tilde{\mathcal{Y}}_{1}-\tilde{\mathcal{Y}}_{2}\right) \min _{j}\left(\tilde{\mathcal{U}}_{j}^{+}\right)^{3} \tilde{\mathcal{U}}_{2, \eta}(t, \eta) \mathbb{1}_{E^{c}}(t, \eta) d \eta \\
& +\int_{0}^{1}\left(\tilde{\mathcal{U}}_{1}-\tilde{\mathcal{U}}_{2}\right) \tilde{\mathcal{U}}_{2, \eta}(t, \eta) \mathbb{1}_{E^{c}}(t, \eta) \\
& \times \int_{0}^{\eta}\left(\tilde{\mathcal{Y}}_{1}-\tilde{\mathcal{Y}}_{2}\right)(t, \theta)\left[\left(\frac{d}{d \theta} \min _{j}\left(e^{-\frac{1}{a}\left(\tilde{\mathcal{Y}}_{j}(t, \eta)-\tilde{\mathcal{Y}}_{j}(t, \theta)\right)}\right)\right) \min _{j}\left(\tilde{\mathcal{U}}_{j}^{+}\right)^{3}(t, \theta)\right. \\
& \left.+\min _{j}\left(e^{-\frac{1}{a}\left(\tilde{\mathcal{Y}}_{j}(t, \eta)-\tilde{\mathcal{Y}}_{j}(t, \theta)\right)}\right)\left(\frac{d}{d \theta} \min _{j}\left(\tilde{\mathcal{U}}_{j}^{+}\right)^{3}\right)(t, \theta)\right] d \theta d \eta \mid \\
& \leqslant \mathcal{O}(1)\left(\left\|\tilde{\mathcal{Y}}_{1}-\tilde{\mathcal{Y}}_{2}\right\|^{2}+\left\|\tilde{\mathcal{U}}_{1}-\tilde{\mathcal{U}}_{2}\right\|^{2}\right)+\frac{1}{A^{12}} \int_{0}^{1} \tilde{\mathcal{U}}_{2, \eta}^{2}(t, \eta)\left(\int_{0}^{\eta}\left|\tilde{\mathcal{Y}}_{1}-\tilde{\mathcal{Y}}_{2}\right|(t, \theta)\right. \\
& \times\left[\frac{1}{a} \min _{j}\left(e^{-\frac{1}{a}\left(\tilde{\mathcal{Y}}_{j}(t, \eta)-\tilde{\mathcal{Y}}_{j}(t, \theta)\right)}\right) \min _{j}\left(\tilde{\mathcal{U}}_{j}^{+}\right)^{3} \max _{j}\left(\tilde{\mathcal{Y}}_{j, \eta}\right)(t, \theta)\right. \\
& \left.\left.+2 A^{4} \min _{j}\left(e^{-\frac{1}{a}\left(\tilde{\mathcal{Y}}_{j}(t, \eta)-\tilde{\mathcal{Y}}_{j}(t, \theta)\right)}\right) \min _{j}\left(\tilde{\mathcal{U}}_{j}^{+}\right)(t, \theta)\right] d \theta\right)^{2} d \eta \\
& \leqslant \mathcal{O}(1)\left(\left\|\tilde{\mathcal{Y}}_{1}-\tilde{\mathcal{Y}}_{2}\right\|^{2}+\left\|\tilde{\mathcal{U}}_{1}-\tilde{\mathcal{U}}_{2}\right\|^{2}\right)+\frac{54}{A} \int_{0}^{1} \tilde{\mathcal{P}}_{2} \tilde{\mathcal{Y}}_{2, \eta}(t, \eta) d \eta\left\|\tilde{\mathcal{Y}}_{1}-\tilde{\mathcal{Y}}_{2}\right\|^{2} \\
& \leqslant \mathcal{O}(1)\left(\left\|\tilde{\mathcal{Y}}_{1}-\tilde{\mathcal{Y}}_{2}\right\|^{2}+\left\|\tilde{\mathcal{U}}_{1}-\tilde{\mathcal{U}}_{2}\right\|^{2}\right),
\end{aligned}
$$

where we used (A.4), (4.16a), and (A.6).

Finally, we have a look at the integral, which contains $J_{9}$ (a similar argument works for $J_{10}$ ), where we can assume $A_{1} \leqslant A_{2}$. Thus

$$
\begin{aligned}
&\left|\int_{0}^{1} J_{9}\left(\tilde{\mathcal{U}}_{1}-\tilde{\mathcal{U}}_{2}\right)(t, \eta) d \eta\right| \\
&=\left(\frac{1}{A_{1}^{6}}-\frac{1}{A_{2}^{6}}\right) \mid \int_{0}^{1}\left(\tilde{\mathcal{U}}_{1}-\tilde{\mathcal{U}}_{2}\right) \tilde{\mathcal{U}}_{1, \eta}(t, \eta) \\
& \quad \times\left(\int_{0}^{\eta} e^{-\frac{1}{A_{1}}\left(\tilde{\mathcal{Y}}_{1}(t, \eta)-\tilde{\mathcal{Y}}_{1}(t, \theta)\right)}\left(\tilde{\mathcal{U}}_{1}^{+}\right)^{3} \tilde{\mathcal{Y}}_{1, \eta}(t, \theta) d \theta\right) d \eta \mid \\
&=\frac{A_{2}^{6}-A_{1}^{6} \mid \int_{0}^{1}\left(\tilde{\mathcal{U}}_{1}-\tilde{\mathcal{U}}_{2}\right) \tilde{\mathcal{U}}_{1, \eta}(t, \eta)}{} \\
& \quad \times\left(\int_{0}^{\eta} e^{-\frac{1}{A_{1}}\left(\tilde{\mathcal{Y}}_{1}(t, \eta)-\tilde{\mathcal{Y}}_{1}(t, \theta)\right)}\left(\tilde{\mathcal{U}}_{1}^{+}\right)^{3} \tilde{\mathcal{Y}}_{1, \eta}(t, \theta) d \theta\right) d \eta \mid \\
& \leqslant 3 \frac{A_{2}-A_{1}}{A_{1}^{2} A_{2}} \mid \int_{0}^{1}\left(\tilde{\mathcal{U}}_{1}-\tilde{\mathcal{U}}_{2}\right) \tilde{\mathcal{U}}_{1, \eta}(t, \eta) \\
& \times\left(\int_{0}^{\eta} e^{-\frac{1}{A_{1}}\left(\tilde{\mathcal{Y}}_{1}(t, \eta)-\tilde{\mathcal{Y}}_{1}(t, \theta)\right)} \tilde{\mathcal{U}}_{1}^{+} \tilde{\mathcal{Y}}_{1, \eta}(t, \theta) d \theta\right) d \eta \mid
\end{aligned}
$$




$$
\begin{aligned}
\leqslant & \left|A_{1}-A_{2}\right|^{2}+\frac{9}{A_{1}^{4} A_{2}^{2}} \int_{0}^{1}\left(\tilde{\mathcal{U}}_{2}-\tilde{\mathcal{U}}_{1}\right)^{2} \tilde{\mathcal{U}}_{1, \eta}^{2}(t, \eta) \\
& \times\left(\int_{0}^{\eta} e^{-\frac{1}{A_{1}}\left(\tilde{\mathcal{Y}}_{1}(t, \eta)-\tilde{\mathcal{Y}}_{1}(t, \theta)\right)} \tilde{\mathcal{U}}_{1}^{+} \tilde{\mathcal{Y}}_{1, \eta}(t, \theta) d \theta\right)^{2} d \eta \\
\leqslant & \left|A_{1}-A_{2}\right|^{2}+\frac{9}{A_{1} A_{2}^{2}} \int_{0}^{1}\left(\tilde{\mathcal{U}}_{1}-\tilde{\mathcal{U}}_{2}\right)^{2} \tilde{\mathcal{Y}}_{1, \eta}(t, \eta) \\
& \times\left(\int_{0}^{\eta} e^{-\frac{1}{A_{1}}\left(\tilde{\mathcal{Y}}_{1}(t, \eta)-\tilde{\mathcal{Y}}_{1}(t, \theta)\right)} \tilde{\mathcal{Y}}_{1, \eta}(t, \theta) d \theta\right) \\
& \times\left(\int_{0}^{\eta} e^{-\frac{1}{A_{1}}\left(\tilde{\mathcal{Y}}_{1}(t, \eta)-\tilde{\mathcal{Y}}_{1}(t, \theta)\right)}\left(\tilde{\mathcal{U}}_{1}^{+}\right)^{2} \tilde{\mathcal{Y}}_{1, \eta}(t, \theta) d \theta\right) d \eta \\
\leqslant & \left|A_{1}-A_{2}\right|^{2}+\frac{9}{A_{2}^{2}} \int_{0}^{1}\left(\tilde{\mathcal{U}}_{1}-\tilde{\mathcal{U}}_{2}\right)^{2} \tilde{\mathcal{Y}}_{1, \eta}(t, \eta) \\
& \times\left(\int_{0}^{\eta} e^{-\frac{1}{A_{1}}\left(\tilde{\mathcal{Y}}_{1}(t, \eta)-\tilde{\mathcal{Y}}_{1}(t, \theta)\right)} \tilde{\mathcal{U}}_{1}^{2} \tilde{\mathcal{Y}}_{1, \eta}(t, \theta) d \theta\right) d \eta \\
\leqslant & \left|A_{1}-A_{1}\right|^{2}+\frac{36}{A} \int_{0}^{1}\left(\tilde{\mathcal{U}}_{1}-\tilde{\mathcal{U}}_{2}\right)^{2} \tilde{\mathcal{P}}_{1} \tilde{\mathcal{Y}}_{1, \eta}(t, \eta) d \eta \\
& \mid \tilde{\mathcal{U}}_{1}-\tilde{\mathcal{U}}_{2} \|^{2} .
\end{aligned}
$$

We now turn to the integral $I_{32}$. Recall equation $(5.32 \mathrm{~b})$, namely,

$$
\begin{aligned}
I_{32}= & \int_{0}^{1}\left(\tilde{\mathcal{U}}_{1}-\tilde{\mathcal{U}}_{2}\right)(t, \eta)\left(\frac{1}{A_{2}^{6}} \tilde{\mathcal{U}}_{2, \eta}(t, \eta) \int_{0}^{1} e^{-\frac{1}{A_{2}}\left|\tilde{\mathcal{Y}}_{2}(t, \eta)-\tilde{\mathcal{Y}}_{2}(t, \theta)\right|} \tilde{\mathcal{P}}_{2} \tilde{\mathcal{U}}_{2} \tilde{\mathcal{Y}}_{2, \eta}(t, \theta) d \theta\right. \\
& \left.-\frac{1}{A_{1}^{6}} \tilde{\mathcal{U}}_{1, \eta}(t, \eta) \int_{0}^{1} e^{-\frac{1}{A_{1}}\left|\tilde{\mathcal{Y}}_{1}(t, \eta)-\tilde{\mathcal{Y}}_{1}(t, \theta)\right|} \tilde{\mathcal{P}}_{1} \tilde{\mathcal{U}}_{1} \tilde{\mathcal{Y}}_{1, \eta}(t, \theta) d \theta\right) d \eta .
\end{aligned}
$$

By first using the decomposition $\tilde{\mathcal{U}}_{j}=\tilde{\mathcal{U}}_{j}^{+}+\tilde{\mathcal{U}}_{j}^{-}$, and then involving (5.4), we need to estimate terms of the type

$$
\begin{aligned}
\tilde{I}_{32}= & \int_{0}^{1}\left(\tilde{\mathcal{U}}_{1}-\tilde{\mathcal{U}}_{2}\right)(t, \eta)\left(\frac{1}{A_{2}^{6}} \tilde{\mathcal{U}}_{2, \eta}(t, \eta) \int_{0}^{\eta} e^{-\frac{1}{A_{2}}\left(\tilde{\mathcal{Y}}_{2}(t, \eta)-\tilde{\mathcal{Y}}_{2}(t, \theta)\right)} \tilde{\mathcal{P}}_{2} \tilde{\mathcal{U}}_{2}^{+} \tilde{\mathcal{Y}}_{2, \eta}(t, \theta) d \theta\right. \\
& \left.-\frac{1}{A_{1}^{6}} \tilde{\mathcal{U}}_{1, \eta}(t, \eta) \int_{0}^{\eta} e^{-\frac{1}{A_{1}}\left(\tilde{\mathcal{Y}}_{1}(t, \eta)-\tilde{\mathcal{Y}}_{1}(t, \theta)\right)} \tilde{\mathcal{P}}_{1} \tilde{\mathcal{U}}_{1}^{+} \tilde{\mathcal{Y}}_{1, \eta}(t, \theta) d \theta\right) d \eta .
\end{aligned}
$$

We invoke Lemma 5.1 and find

$$
\begin{aligned}
\tilde{I}_{32}= & \frac{1}{A^{6}} \int_{0}^{1}\left(\tilde{\mathcal{U}}_{1}-\tilde{\mathcal{U}}_{2}\right)(t, \eta)\left(\tilde{\mathcal{U}}_{2, \eta}(t, \eta) \int_{0}^{\eta} e^{-\frac{1}{A_{2}}\left(\tilde{\mathcal{Y}}_{2}(t, \eta)-\tilde{\mathcal{Y}}_{2}(t, \theta)\right)} \tilde{\mathcal{P}}_{2} \tilde{\mathcal{U}}_{2}^{+} \tilde{\mathcal{Y}}_{2, \eta}(t, \theta) d \theta\right. \\
& \left.-\tilde{\mathcal{U}}_{1, \eta}(t, \eta) \int_{0}^{\eta} e^{-\frac{1}{A_{1}}\left(\tilde{\mathcal{Y}}_{1}(t, \eta)-\tilde{\mathcal{Y}}_{1}(t, \theta)\right)} \tilde{\mathcal{P}}_{1} \tilde{\mathcal{U}}_{1}^{+} \tilde{\mathcal{Y}}_{1, \eta}(t, \theta) d \theta\right) d \eta
\end{aligned}
$$




$$
\begin{aligned}
& +\frac{A_{1}^{6}-A_{2}^{6}}{A_{1}^{6} A_{2}^{6}} \mathbb{1}_{A_{2} \leqslant A_{1}} \int_{0}^{1}\left(\tilde{\mathcal{U}}_{1}-\tilde{\mathcal{U}}_{2}\right) \tilde{\mathcal{U}}_{2, \eta}(t, \eta) \\
& \times\left(\int_{0}^{\eta} e^{-\frac{1}{A_{2}}\left(\tilde{\mathcal{Y}}_{2}(t, \eta)-\tilde{\mathcal{Y}}_{2}(t, \theta)\right)} \tilde{\mathcal{P}}_{2} \tilde{\mathcal{U}}_{2}^{+} \tilde{\mathcal{Y}}_{2, \eta}(t, \theta) d \theta\right) d \eta \\
& +\frac{A_{1}^{6}-A_{2}^{6}}{A_{1}^{6} A_{2}^{6}} \mathbb{1}_{A_{1}<A_{2}} \int_{0}^{1}\left(\tilde{\mathcal{U}}_{1}-\tilde{\mathcal{U}}_{2}\right) \tilde{\mathcal{U}}_{1, \eta}(t, \eta) \\
& \times\left(\int_{0}^{\eta} e^{-\frac{1}{A_{1}}\left(\tilde{\mathcal{Y}}_{1}(t, \eta)-\tilde{\mathcal{Y}}_{1}(t, \theta)\right)} \tilde{\mathcal{P}}_{1} \tilde{\mathcal{U}}_{1}^{+} \tilde{\mathcal{Y}}_{1, \eta}(t, \theta) d \theta\right) d \eta \\
& =N_{1}+N_{2}+N_{3} .
\end{aligned}
$$

We consider first $N_{1}$, where we get

$$
\begin{aligned}
& N_{1}=\frac{1}{A^{6}} \int_{0}^{1}\left(\tilde{\mathcal{U}}_{1}-\tilde{\mathcal{U}}_{2}\right)(t, \eta)\left[\tilde{\mathcal{U}}_{2, \eta}(t, \eta) \int_{0}^{\eta} e^{-\frac{1}{A_{2}}\left(\tilde{\mathcal{Y}}_{2}(t, \eta)-\tilde{\mathcal{Y}}_{2}(t, \theta)\right)} \tilde{\mathcal{P}}_{2} \tilde{\mathcal{U}}_{2}^{+} \tilde{\mathcal{Y}}_{2, \eta}(t, \theta) d \theta\right. \\
& \left.-\tilde{\mathcal{U}}_{1, \eta}(t, \eta) \int_{0}^{\eta} e^{-\frac{1}{A_{1}}\left(\tilde{\mathcal{Y}}_{1}(t, \eta)-\tilde{\mathcal{Y}}_{1}(t, \theta)\right)} \tilde{\mathcal{P}}_{1} \tilde{\mathcal{U}}_{1}^{+} \tilde{\mathcal{Y}}_{1, \eta}(t, \theta) d \theta\right] d \eta \\
& =\frac{1}{A^{6}} \int_{0}^{1}\left(\tilde{\mathcal{U}}_{1}-\tilde{\mathcal{U}}_{2}\right)(t, \eta) \\
& \times\left[\tilde{\mathcal{U}}_{2, \eta}(t, \eta) \int_{0}^{\eta} e^{-\frac{1}{A_{2}}\left(\tilde{\mathcal{Y}}_{2}(t, \eta)-\tilde{\mathcal{Y}}_{2}(t, \theta)\right)}\left(\tilde{\mathcal{P}}_{2}-\tilde{\mathcal{P}}_{1}\right) \tilde{\mathcal{U}}_{2}^{+} \tilde{\mathcal{Y}}_{2, \eta} \mathbb{1}_{\tilde{\mathcal{P}}_{1} \leqslant \tilde{\mathcal{P}}_{2}}(t, \theta) d \theta\right. \\
& +\tilde{\mathcal{U}}_{1, \eta}(t, \eta) \int_{0}^{\eta} e^{-\frac{1}{A_{1}}\left(\tilde{\mathcal{Y}}_{1}(t, \eta)-\tilde{\mathcal{Y}}_{1}(t, \theta)\right)}\left(\tilde{\mathcal{P}}_{2}-\tilde{\mathcal{P}}_{1}\right) \tilde{\mathcal{U}}_{1}^{+} \tilde{\mathcal{Y}}_{1, \eta} \mathbb{1}_{\tilde{\mathcal{P}}_{2}<\tilde{\mathcal{P}}_{1}}(t, \theta) d \theta \\
& +\tilde{\mathcal{U}}_{2, \eta}(t, \eta) \int_{0}^{\eta} e^{-\frac{1}{A_{2}}\left(\tilde{\mathcal{Y}}_{2}(t, \eta)-\tilde{\mathcal{Y}}_{2}(t, \theta)\right)} \min _{j}\left(\tilde{\mathcal{P}}_{j}\right)\left(\tilde{\mathcal{U}}_{2}^{+}-\tilde{\mathcal{U}}_{1}^{+}\right) \tilde{\mathcal{Y}}_{2, \eta} \mathbb{1}_{\tilde{\mathcal{U}}_{1}^{+} \leqslant \tilde{\mathcal{U}}_{2}^{+}}(t, \theta) d \theta \\
& +\tilde{\mathcal{U}}_{1, \eta}(t, \eta) \int_{0}^{\eta} e^{-\frac{1}{A_{1}}\left(\tilde{\mathcal{Y}}_{1}(t, \eta)-\tilde{\mathcal{Y}}_{1}(t, \theta)\right)} \min _{j}\left(\tilde{\mathcal{P}}_{j}\right)\left(\tilde{\mathcal{U}}_{2}^{+}-\tilde{\mathcal{U}}_{1}^{+}\right) \tilde{\mathcal{Y}}_{1, \eta} \mathbb{1}_{\tilde{\mathcal{U}}_{2}^{+}<\tilde{\mathcal{U}}_{1}^{+}}(t, \theta) d \theta \\
& +\tilde{\mathcal{U}}_{2, \eta}(t, \eta) \int_{0}^{\eta} e^{-\frac{1}{A_{2}}\left(\tilde{\mathcal{Y}}_{2}(t, \eta)-\tilde{\mathcal{Y}}_{2}(t, \theta)\right)} \min _{j}\left(\tilde{\mathcal{P}}_{j}\right) \min _{j}\left(\tilde{\mathcal{U}}_{j}^{+}\right) \tilde{\mathcal{Y}}_{2, \eta}(t, \theta) d \theta \\
& \left.-\tilde{\mathcal{U}}_{1, \eta}(t, \eta) \int_{0}^{\eta} e^{-\frac{1}{A_{1}}\left(\tilde{\mathcal{Y}}_{1}(t, \eta)-\tilde{\mathcal{Y}}_{1}(t, \theta)\right)} \min _{j}\left(\tilde{\mathcal{P}}_{j}\right) \min _{j}\left(\tilde{\mathcal{U}}_{j}^{+}\right) \tilde{\mathcal{Y}}_{1, \eta}(t, \theta) d \theta\right] d \eta \\
& =N_{11}+N_{12}+N_{13}+N_{14}+N_{15}+N_{16} \text {. }
\end{aligned}
$$

The terms $N_{11}$ and $N_{12}$ can be treated similarly. To that end, we find

$$
\begin{aligned}
\left|N_{11}\right|= & \frac{1}{A^{6}} \mid \int_{0}^{1}\left(\tilde{\mathcal{U}}_{1}-\tilde{\mathcal{U}}_{2}\right)(t, \eta) \\
& \left.\times\left[\tilde{\mathcal{U}}_{2, \eta}(t, \eta) \int_{0}^{\eta} e^{-\frac{1}{A_{2}}\left(\tilde{\mathcal{Y}}_{2}(t, \eta)-\tilde{\mathcal{Y}}_{2}(t, \theta)\right)}\left(\tilde{\mathcal{P}}_{2}-\tilde{\mathcal{P}}_{1}\right) \tilde{\mathcal{U}}_{2}^{+} \tilde{\mathcal{Y}}_{2, \eta} \mathbb{1}_{\tilde{\mathcal{P}}_{1} \leqslant \tilde{\mathcal{P}}_{2}}(t, \theta) d \theta\right] d \eta\right]
\end{aligned}
$$




$$
\begin{aligned}
\leqslant & \left\|\tilde{\mathcal{U}}_{1}-\tilde{\mathcal{U}}_{2}\right\|^{2}+\frac{1}{A^{12}} \int_{0}^{1} \tilde{\mathcal{U}}_{2, \eta}^{2}(t, \eta) \\
& \times\left(\int_{0}^{\eta} e^{-\frac{1}{A_{2}}\left(\tilde{\mathcal{Y}}_{2}(t, \eta)-\tilde{\mathcal{Y}}_{2}(t, \theta)\right)}\left(\tilde{\mathcal{P}}_{2}-\tilde{\mathcal{P}}_{1}\right) \tilde{\mathcal{U}}_{2}^{+} \tilde{\mathcal{Y}}_{2, \eta} \mathbb{1}_{\tilde{\mathcal{P}}_{1} \leqslant \tilde{\mathcal{P}}_{2}}(t, \theta) d \theta\right)^{2} d \eta \\
\leqslant & \left\|\tilde{\mathcal{U}}_{1}-\tilde{\mathcal{U}}_{2}\right\|^{2} \\
& +\frac{4}{A^{9}} \int_{0}^{1} \tilde{\mathcal{Y}}_{2, \eta}(t, \eta)\left(\int_{0}^{\eta} e^{-\frac{1}{A_{2}}\left(\tilde{\mathcal{Y}}_{2}(t, \eta)-\tilde{\mathcal{Y}}_{2}(t, \theta)\right)}\left(\tilde{\mathcal{U}}_{2}^{+}\right)^{2} \tilde{\mathcal{Y}}_{2, \eta}(t, \theta) d \theta\right) \\
& \times\left(\int_{0}^{\eta} e^{-\frac{1}{A_{2}}\left(\tilde{\mathcal{Y}}_{2}(t, \eta)-\tilde{\mathcal{Y}}_{2}(t, \theta)\right)}\left|\tilde{\mathcal{P}}_{2}^{1 / 2}-\tilde{\mathcal{P}}_{1}^{1 / 2}\right|^{2} \tilde{\mathcal{P}}_{2} \tilde{\mathcal{Y}}_{2, \eta}(t, \theta) d \theta\right) d \eta \\
\leqslant & \left\|\tilde{\mathcal{U}}_{1}-\tilde{\mathcal{U}}_{2}\right\|^{2}+\frac{16}{A^{8}} \int_{0}^{1} \tilde{\mathcal{P}}_{2} \tilde{\mathcal{Y}}_{2, \eta}(t, \eta) \\
& \times\left(\int_{0}^{\eta} e^{-\frac{1}{A_{2}}\left(\tilde{\mathcal{Y}}_{2}(t, \eta)-\tilde{\mathcal{Y}}_{2}(t, \theta)\right)}\left|\tilde{\mathcal{P}}_{2}^{1 / 2}-\tilde{\mathcal{P}}_{1}^{1 / 2}\right|^{2} \tilde{\mathcal{P}}_{2} \tilde{\mathcal{Y}}_{2, \eta}(t, \theta) d \theta\right) d \eta \\
\leqslant & \mathcal{O}(1)\left(\left\|\tilde{\mathcal{U}}_{1}-\tilde{\mathcal{U}}_{2}\right\|^{2}+\left\|\tilde{\mathcal{P}}_{1}^{1 / 2}-\tilde{\mathcal{P}}_{2}^{1 / 2}\right\|^{2}\right),
\end{aligned}
$$

where we have used (4.15e), (4.15h), and (4.16c).

The terms $N_{13}$ and $N_{14}$ follow the same estimates. More precisely,

$$
\begin{aligned}
\left|N_{13}\right|= & \frac{1}{A^{6}} \mid \int_{0}^{1}\left(\tilde{\mathcal{U}}_{1}-\tilde{\mathcal{U}}_{2}\right) \tilde{\mathcal{U}}_{2, \eta}(t, \eta) \\
& \times \int_{0}^{\eta} e^{-\frac{1}{A_{2}}\left(\tilde{\mathcal{Y}}_{2}(t, \eta)-\tilde{\mathcal{Y}}_{2}(t, \theta)\right)} \min _{j}\left(\tilde{\mathcal{P}}_{j}\right)\left(\tilde{\mathcal{U}}_{2}^{+}-\tilde{\mathcal{U}}_{1}^{+}\right) \tilde{\mathcal{Y}}_{2, \eta} \mathbb{1}_{\tilde{\mathcal{U}}_{1}^{+} \leqslant \tilde{\mathcal{U}}_{2}^{+}}(t, \theta) d \theta d \eta \mid \\
\leqslant & \frac{1}{A^{6}} \int_{0}^{1}\left|\tilde{\mathcal{U}}_{1}-\tilde{\mathcal{U}}_{2}\right|\left|\tilde{\mathcal{U}}_{2, \eta}\right|(t, \eta) \\
& \times \int_{0}^{\eta} e^{-\frac{1}{A_{2}}\left(\tilde{\mathcal{Y}}_{2}(t, \eta)-\tilde{\mathcal{Y}}_{2}(t, \theta)\right)} \min \left(\tilde{\mathcal{P}}_{j}\right)\left|\tilde{\mathcal{U}}_{2}^{+}-\tilde{\mathcal{U}}_{1}^{+}\right| \tilde{\mathcal{Y}}_{2, \eta} \mathbb{1}_{\tilde{\mathcal{U}}_{1}^{+} \leqslant \tilde{\mathcal{U}}_{2}^{+}}(t, \theta) d \theta d \eta \\
\leqslant & \left\|\tilde{\mathcal{U}}_{1}-\tilde{\mathcal{U}}_{2}\right\|^{2}+\frac{1}{A^{12}} \int_{0}^{1} \tilde{\mathcal{U}}_{2, \eta}^{2}(t, \eta) \\
& \times\left(\int_{0}^{\eta} e^{-\frac{1}{A_{2}}\left(\tilde{\mathcal{Y}}_{2}(t, \eta)-\tilde{\mathcal{Y}}_{2}(t, \theta)\right)} \min _{j}\left(\tilde{\mathcal{P}}_{j}\right)\left|\tilde{\mathcal{U}}_{2}^{+}-\tilde{\mathcal{U}}_{1}^{+}\right| \tilde{\mathcal{Y}}_{2, \eta} \mathbb{1}_{\tilde{\mathcal{U}}_{1}^{+} \leqslant \tilde{\mathcal{U}}_{2}^{+}}(t, \theta) d \theta\right)^{2} d \eta \\
\leqslant & \left\|\tilde{\mathcal{U}}_{1}-\tilde{\mathcal{U}}_{2}\right\|^{2} \\
& +\frac{1}{A^{12}} \int_{0}^{1} \tilde{\mathcal{U}}_{2, \eta}^{2}(t, \eta)\left(\int_{0}^{\eta} e^{-\frac{3}{2 A_{2}}\left(\tilde{\mathcal{Y}}_{2}(t, \eta)-\tilde{\mathcal{Y}}_{2}(t, \theta)\right)} \tilde{\mathcal{P}}_{2} \tilde{\mathcal{Y}}_{2, \eta}(t, \theta) d \theta\right) \\
& \times\left(\int_{0}^{\eta} e^{-\frac{1}{2 A_{2}}\left(\tilde{\mathcal{Y}}_{2}(t, \eta)-\tilde{\mathcal{Y}}_{2}(t, \theta)\right)}\left(\tilde{\mathcal{U}}_{2}^{+}-\tilde{\mathcal{U}}_{1}^{+}\right)^{2} \tilde{\mathcal{P}}_{2} \tilde{\mathcal{Y}}_{2, \eta}(t, \theta) d \theta\right) d \eta
\end{aligned}
$$




$$
\begin{aligned}
\leqslant & \left\|\tilde{\mathcal{U}}_{1}-\tilde{\mathcal{U}}_{2}\right\|^{2}+\frac{2}{A^{11}} \int_{0}^{1} \tilde{\mathcal{P}}_{2} \tilde{\mathcal{U}}_{2, \eta}^{2}(t, \eta) \\
& \times\left(\int_{0}^{\eta} e^{-\frac{1}{2 A_{2}}\left(\tilde{\mathcal{Y}}_{2}(t, \eta)-\tilde{\mathcal{Y}}_{2}(t, \theta)\right)}\left(\tilde{\mathcal{U}}_{2}-\tilde{\mathcal{U}}_{1}\right)^{2} \tilde{\mathcal{P}}_{2} \tilde{\mathcal{Y}}_{2, \eta}(t, \theta) d \theta\right) d \eta \\
\leqslant & \mathcal{O}(1)\left\|\tilde{\mathcal{U}}_{2}-\tilde{\mathcal{U}}_{1}\right\|^{2},
\end{aligned}
$$

by applying (4.15e), (4.15q), and (4.16e).

The term $N_{15}+N_{16}$ can be estimated as follows:

$$
\begin{aligned}
& N_{15}+N_{16}=\frac{1}{A^{6}} \int_{0}^{1}\left(\tilde{\mathcal{U}}_{1}-\tilde{\mathcal{U}}_{2}\right)(t, \eta) \\
& \times\left[\tilde{\mathcal{U}}_{2, \eta}(t, \eta) \int_{0}^{\eta} e^{-\frac{1}{A_{2}}\left(\tilde{\mathcal{Y}}_{2}(t, \eta)-\tilde{\mathcal{Y}}_{2}(t, \theta)\right)} \min _{j}\left(\tilde{\mathcal{P}}_{j}\right) \min _{j}\left(\tilde{\mathcal{U}}_{j}^{+}\right) \tilde{\mathcal{Y}}_{2, \eta}(t, \theta) d \theta\right. \\
& \left.-\tilde{\mathcal{U}}_{1, \eta}(t, \eta) \int_{0}^{\eta} e^{-\frac{1}{A_{1}}\left(\tilde{\mathcal{Y}}_{1}(t, \eta)-\tilde{\mathcal{Y}}_{1}(t, \theta)\right)} \min _{j}\left(\tilde{\mathcal{P}}_{j}\right) \min _{j}\left(\tilde{\mathcal{U}}_{j}^{+}\right) \tilde{\mathcal{Y}}_{1, \eta}(t, \theta) d \theta\right] d \eta \\
& =\frac{1}{A^{6}} \mathbb{1}_{A_{1} \leqslant A_{2}} \int_{0}^{1}\left(\tilde{\mathcal{U}}_{1}-\tilde{\mathcal{U}}_{2}\right) \tilde{\mathcal{U}}_{2, \eta}(t, \eta) \\
& \times\left(\int_{0}^{\eta}\left(e^{-\frac{1}{A_{2}}\left(\tilde{\mathcal{Y}}_{2}(t, \eta)-\tilde{\mathcal{Y}}_{2}(t, \theta)\right)}-e^{-\frac{1}{A_{1}}\left(\tilde{\mathcal{Y}}_{2}(t, \eta)-\tilde{\mathcal{Y}}_{2}(t, \theta)\right)}\right)\right. \\
& \left.\times \min _{j}\left(\tilde{\mathcal{P}}_{j}\right) \min _{j}\left(\tilde{\mathcal{U}}_{j}^{+}\right) \tilde{\mathcal{Y}}_{2, \eta}(t, \theta) d \theta\right) d \eta \\
& +\frac{1}{A^{6}} \mathbb{1}_{A_{2}<A_{1}} \int_{0}^{1}\left(\tilde{\mathcal{U}}_{1}-\tilde{\mathcal{U}}_{2}\right) \tilde{\mathcal{U}}_{1, \eta}(t, \eta) \\
& \times\left(\int_{0}^{\eta}\left(e^{-\frac{1}{A_{2}}\left(\tilde{\mathcal{Y}}_{1}(t, \eta)-\tilde{\mathcal{Y}}_{1}(t, \theta)\right)}-e^{-\frac{1}{A_{1}}\left(\tilde{\mathcal{Y}}_{1}(t, \eta)-\tilde{\mathcal{Y}}_{1}(t, \theta)\right)}\right)\right. \\
& \left.\times \min _{j}\left(\tilde{\mathcal{P}}_{j}\right) \min _{j}\left(\tilde{\mathcal{U}}_{j}^{+}\right) \tilde{\mathcal{Y}}_{1, \eta}(t, \theta) d \theta\right) d \eta \\
& +\frac{1}{A^{6}} \int_{0}^{1}\left(\tilde{\mathcal{U}}_{1}-\tilde{\mathcal{U}}_{2}\right) \tilde{\mathcal{U}}_{2, \eta}(t, \eta) \\
& \times\left(\int_{0}^{\eta}\left(e^{-\frac{1}{a}\left(\tilde{\mathcal{Y}}_{2}(t, \eta)-\tilde{\mathcal{Y}}_{2}(t, \theta)\right)}-e^{-\frac{1}{a}\left(\tilde{\mathcal{Y}}_{1}(t, \eta)-\tilde{\mathcal{Y}}_{1}(t, \theta)\right)}\right)\right. \\
& \left.\times \min _{j}\left(\tilde{\mathcal{P}}_{j}\right) \min _{j}\left(\tilde{\mathcal{U}}_{j}^{+}\right) \tilde{\mathcal{Y}}_{2, \eta}(t, \theta) \mathbb{1}_{B(\eta)}(t, \theta) d \theta\right) d \eta \\
& +\frac{1}{A^{6}} \int_{0}^{1}\left(\tilde{\mathcal{U}}_{1}-\tilde{\mathcal{U}}_{2}\right) \tilde{\mathcal{U}}_{1, \eta}(t, \eta) \\
& \times\left(\int_{0}^{\eta}\left(e^{-\frac{1}{a}\left(\tilde{\mathcal{Y}}_{2}(t, \eta)-\tilde{\mathcal{Y}}_{2}(t, \theta)\right)}-e^{-\frac{1}{a}\left(\tilde{\mathcal{Y}}_{1}(t, \eta)-\tilde{\mathcal{Y}}_{1}(t, \theta)\right)}\right)\right.
\end{aligned}
$$




$$
\begin{aligned}
& \left.\times \min _{j}\left(\tilde{\mathcal{P}}_{j}\right) \min _{j}\left(\tilde{\mathcal{U}}_{j}^{+}\right) \tilde{\mathcal{Y}}_{1, \eta}(t, \theta) \mathbb{1}_{B^{c}(\eta)}(t, \theta) d \theta\right) d \eta \\
& +\frac{1}{A^{6}} \int_{0}^{1}\left(\tilde{\mathcal{U}}_{1}-\tilde{\mathcal{U}}_{2}\right)(t, \eta)\left[\tilde{\mathcal{U}}_{2, \eta}(t, \eta) \int_{0}^{\eta} \min _{j}\left(e^{-\frac{1}{a}\left(\tilde{\mathcal{Y}}_{j}(t, \eta)-\tilde{\mathcal{Y}}_{j}(t, \theta)\right)}\right)\right. \\
& \times \min _{j}\left(\tilde{\mathcal{P}}_{j}\right) \min _{j}\left(\tilde{\mathcal{U}}_{j}^{+}\right) \tilde{\mathcal{Y}}_{2, \eta}(t, \theta) d \theta \\
& -\tilde{\mathcal{U}}_{1, \eta}(t, \eta) \int_{0}^{\eta} \min \left(e^{-\frac{1}{a}\left(\tilde{\mathcal{Y}}_{j}(t, \eta)-\tilde{\mathcal{Y}}_{j}(t, \theta)\right)}\right) \\
& \left.\times \min _{j}\left(\tilde{\mathcal{P}}_{j}\right) \min _{j}\left(\tilde{\mathcal{U}}_{j}^{+}\right) \tilde{\mathcal{Y}}_{1, \eta}(t, \theta) d \theta\right] d \eta \\
& =N_{151}+N_{152}+N_{153}+N_{154}+N_{155} .
\end{aligned}
$$

Unfortunately, each of these terms needs a special treatment.

The terms $N_{151}$ and $N_{152}$ can be handled as follows:

$$
\begin{aligned}
& \left|N_{151}\right|=\frac{1}{A^{6}} \mathbb{1}_{A_{1} \leqslant A_{2}} \mid \int_{0}^{1}\left(\tilde{\mathcal{U}}_{1}-\tilde{\mathcal{U}}_{2}\right) \tilde{\mathcal{U}}_{2, \eta}(t, \eta) \\
& \times\left(\int_{0}^{\eta}\left(e^{-\frac{1}{A_{2}}\left(\tilde{\mathcal{Y}}_{2}(t, \eta)-\tilde{\mathcal{Y}}_{2}(t, \theta)\right)}-e^{-\frac{1}{A_{1}}\left(\tilde{\mathcal{Y}}_{2}(t, \eta)-\tilde{\mathcal{Y}}_{2}(t, \theta)\right)}\right)\right. \\
& \left.\times \min _{j}\left(\tilde{\mathcal{P}}_{j}\right) \min _{j}\left(\tilde{\mathcal{U}}_{j}^{+}\right) \tilde{\mathcal{Y}}_{2, \eta}(t, \theta) d \theta\right) d \eta \mid \\
& \leqslant \frac{4}{A^{6} A_{1} e} \int_{0}^{1}\left|\tilde{\mathcal{U}}_{1}-\tilde{\mathcal{U}}_{2}\right|\left|\tilde{\mathcal{U}}_{2, \eta}\right|(t, \eta) \\
& \times\left(\int_{0}^{\eta} e^{-\frac{3}{4 A_{2}}\left(\tilde{\mathcal{Y}}_{2}(t, \eta)-\tilde{\mathcal{Y}}_{2}(t, \theta)\right)} \min _{j}\left(\tilde{\mathcal{P}}_{j}\right) \min _{j}\left(\tilde{\mathcal{U}}_{j}^{+}\right) \tilde{\mathcal{Y}}_{2, \eta}(t, \theta) d \theta\right) d \eta\left|A_{1}-A_{2}\right| \\
& \leqslant\left\|\tilde{\mathcal{U}}_{1}-\tilde{\mathcal{U}}_{2}\right\|^{2}+\frac{16}{A^{12} A_{1}^{2} e^{2}} \int_{0}^{1} \tilde{\mathcal{U}}_{2, \eta}^{2}(t, \eta) \\
& \times\left(\int_{0}^{\eta} e^{-\frac{3}{4 A_{2}}\left(\tilde{\mathcal{Y}}_{2}(t, \eta)-\tilde{\mathcal{Y}}_{2}(t, \theta)\right)}\left|\tilde{\mathcal{U}}_{1}\right| \tilde{\mathcal{P}}_{2} \tilde{\mathcal{Y}}_{2, \eta}(t, \theta) d \theta\right)^{2} d \eta\left|A_{1}-A_{2}\right|^{2} \\
& \leqslant\left\|\tilde{\mathcal{U}}_{1}-\tilde{\mathcal{U}}_{2}\right\|^{2}+\frac{8}{A^{4} e^{2}} \int_{0}^{1} \tilde{\mathcal{P}}_{2} \tilde{\mathcal{U}}_{2, \eta}^{2}(t, \eta) d \eta\left|A_{1}-A_{2}\right|^{2} \\
& \leqslant \mathcal{O}(1)\left(\left\|\tilde{\mathcal{U}}_{1}-\tilde{\mathcal{U}}_{2}\right\|^{2}+\left|A_{1}-A_{2}\right|^{2}\right),
\end{aligned}
$$

where we used (4.16e).

The terms $N_{153}$ and $N_{154}$ can be handled as follows:

$$
\begin{aligned}
\left|N_{153}\right|= & \frac{1}{A^{6}} \mid \int_{0}^{1}\left(\tilde{\mathcal{U}}_{1}-\tilde{\mathcal{U}}_{2}\right) \tilde{\mathcal{U}}_{2, \eta}(t, \eta) \\
& \times \int_{0}^{\eta}\left(e^{-\frac{1}{a}\left(\tilde{\mathcal{Y}}_{2}(t, \eta)-\tilde{\mathcal{Y}}_{2}(t, \theta)\right)}-e^{-\frac{1}{a}\left(\tilde{\mathcal{Y}}_{1}(t, \eta)-\tilde{\mathcal{Y}}_{1}(t, \theta)\right)}\right)
\end{aligned}
$$




$$
\begin{aligned}
& \times \min _{j}\left(\tilde{\mathcal{P}}_{j}\right) \min _{j}\left(\tilde{\mathcal{U}}_{j}^{+}\right) \tilde{\mathcal{Y}}_{2, \eta}(t, \theta) \mathbb{1}_{B(\eta)}(t, \theta) d \theta d \eta \\
& \leqslant \frac{1}{A^{6} a} \int_{0}^{1}\left|\tilde{\mathcal{U}}_{1}-\tilde{\mathcal{U}}_{2}\right|\left|\tilde{\mathcal{U}}_{2, \eta}\right|(t, \eta) \\
& \times \int_{0}^{\eta}\left(\left|\tilde{\mathcal{Y}}_{2}(t, \eta)-\tilde{\mathcal{Y}}_{1}(t, \eta)\right|+\left|\tilde{\mathcal{Y}}_{2}(t, \theta)-\tilde{\mathcal{Y}}_{1}(t, \theta)\right|\right) e^{-\frac{1}{a}\left(\tilde{\mathcal{Y}}_{2}(t, \eta)-\tilde{\mathcal{Y}}_{2}(t, \theta)\right)} \\
& \times \min _{j}\left(\tilde{\mathcal{P}}_{j}\right) \min _{j}\left(\tilde{\mathcal{U}}_{j}^{+}\right) \tilde{\mathcal{Y}}_{2, \eta}(t, \theta) d \theta d \eta \\
& =\frac{1}{A^{6} a} \int_{0}^{1}\left|\tilde{\mathcal{U}}_{1}-\tilde{\mathcal{U}}_{2}\right|\left|\tilde{\mathcal{Y}}_{2}-\tilde{\mathcal{Y}}_{1}\right|\left|\tilde{\mathcal{U}}_{2, \eta}\right|(t, \eta) \\
& \times \int_{0}^{\eta} e^{-\frac{1}{a}\left(\tilde{\mathcal{Y}}_{2}(t, \eta)-\tilde{\mathcal{Y}}_{2}(t, \theta)\right)} \min _{j}\left(\tilde{\mathcal{P}}_{j}\right) \min _{j}\left(\tilde{\mathcal{U}}_{j}^{+}\right) \tilde{\mathcal{Y}}_{2, \eta}(t, \theta) d \theta d \eta \\
& +\frac{1}{A^{6} a} \int_{0}^{1}\left|\tilde{\mathcal{U}}_{1}-\tilde{\mathcal{U}}_{2}\right|\left|\tilde{\mathcal{U}}_{2, \eta}\right|(t, \eta) \\
& \times \int_{0}^{\eta} e^{-\frac{1}{a}\left(\tilde{\mathcal{Y}}_{2}(t, \eta)-\tilde{\mathcal{Y}}_{2}(t, \theta)\right)}\left|\tilde{\mathcal{Y}}_{2}-\tilde{\mathcal{Y}}_{1}\right| \min _{j}\left(\tilde{\mathcal{P}}_{j}\right) \min _{j}\left(\tilde{\mathcal{U}}_{j}^{+}\right) \tilde{\mathcal{Y}}_{2, \eta}(t, \theta) d \theta d \eta \\
& \leqslant \frac{1}{A^{6} a} \int_{0}^{1}\left|\tilde{\mathcal{U}}_{1}-\tilde{\mathcal{U}}_{2}\right|\left|\tilde{\mathcal{Y}}_{2}-\tilde{\mathcal{Y}}_{1}\right|\left|\tilde{\mathcal{U}}_{2, \eta}\right|(t, \eta) \\
& \times\left(\int_{0}^{\eta} e^{-\frac{1}{A_{2}}\left(\tilde{\mathcal{Y}}_{2}(t, \eta)-\tilde{\mathcal{Y}}_{2}(t, \theta)\right)} \tilde{\mathcal{P}}_{2}^{2} \tilde{\mathcal{Y}}_{2, \eta}(t, \theta) d \theta\right)^{1 / 2} \\
& \times\left(\int_{0}^{\eta} e^{-\frac{1}{a}\left(\tilde{\mathcal{Y}}_{2}(t, \eta)-\tilde{\mathcal{Y}}_{2}(t, \theta)\right)} \min _{j}\left(\tilde{\mathcal{U}}_{j}^{+}\right)^{2} \tilde{\mathcal{Y}}_{2, \eta}(t, \theta) d \theta\right)^{1 / 2} d \eta \\
& +\left\|\tilde{\mathcal{U}}_{1}-\tilde{\mathcal{U}}_{2}\right\|^{2}+\frac{1}{A^{12} a^{2}} \int_{0}^{1} \tilde{\mathcal{U}}_{2, \eta}^{2}(t, \eta) \\
& \times\left(\int_{0}^{\eta} e^{-\frac{1}{A_{2}}\left(\tilde{\mathcal{Y}}_{2}(t, \eta)-\tilde{\mathcal{Y}}_{2}(t, \theta)\right)}\left|\tilde{\mathcal{Y}}_{2}-\tilde{\mathcal{Y}}_{1}\right| \tilde{\mathcal{P}}_{2} \min _{j}\left(\tilde{\mathcal{U}}_{j}^{+}\right) \tilde{\mathcal{Y}}_{2, \eta}(t, \theta) d \theta\right)^{2} d \eta \\
& \leqslant \frac{\sqrt{3}}{2 A^{2}} \int_{0}^{1}\left|\tilde{\mathcal{U}}_{1}-\tilde{\mathcal{U}}_{2}\right|\left|\tilde{\mathcal{Y}}_{2}-\tilde{\mathcal{Y}}_{1}\right| \tilde{\mathcal{P}}_{2}^{1 / 2}\left|\tilde{\mathcal{U}}_{2, \eta}\right|(t, \eta) d \eta+\left\|\tilde{\mathcal{U}}_{1}-\tilde{\mathcal{U}}_{2}\right\|^{2} \\
& +\frac{9}{4 A^{4}} \int_{0}^{1} \tilde{\mathcal{P}}_{2} \tilde{\mathcal{U}}_{2, \eta}^{2}(t, \eta) d \eta\left\|\tilde{\mathcal{Y}}_{1}-\tilde{\mathcal{Y}}_{2}\right\|^{2} \\
& \leqslant \mathcal{O}(1)\left(\left\|\tilde{\mathcal{U}}_{1}-\tilde{\mathcal{U}}_{2}\right\|^{2}+\left\|\tilde{\mathcal{Y}}_{1}-\tilde{\mathcal{Y}}_{2}\right\|^{2}\right),
\end{aligned}
$$


where we used (4.16k). We consider now $N_{155}$ :

$$
\begin{aligned}
& N_{155}=\frac{1}{A^{6}} \int_{0}^{1}\left(\tilde{\mathcal{U}}_{1}-\tilde{\mathcal{U}}_{2}\right)(t, \eta) \\
& \times\left[\tilde{\mathcal{U}}_{2, \eta}(t, \eta) \int_{0}^{\eta} \min _{j}\left(e^{-\frac{1}{a}\left(\tilde{\mathcal{Y}}_{j}(t, \eta)-\tilde{\mathcal{Y}}_{j}(t, \theta)\right)}\right) \min _{j}\left(\tilde{\mathcal{P}}_{j}\right) \min _{j}\left(\tilde{\mathcal{U}}_{j}^{+}\right) \tilde{\mathcal{Y}}_{2, \eta}(t, \theta) d \theta\right. \\
& \left.-\tilde{\mathcal{U}}_{1, \eta}(t, \eta) \int_{0}^{\eta} \min _{j}\left(e^{-\frac{1}{a}\left(\tilde{\mathcal{Y}}_{j}(t, \eta)-\tilde{\mathcal{Y}}_{j}(t, \theta)\right)}\right) \min _{j}\left(\tilde{\mathcal{P}}_{j}\right) \min _{j}\left(\tilde{\mathcal{U}}_{j}^{+}\right) \tilde{\mathcal{Y}}_{1, \eta}(t, \theta) d \theta\right] d \eta \\
& =\frac{1}{A^{6}} \int_{0}^{1}\left(\tilde{\mathcal{U}}_{1}-\tilde{\mathcal{U}}_{2}\right)\left(\tilde{\mathcal{U}}_{2}-\tilde{\mathcal{U}}_{1}\right)_{\eta}(t, \eta) \\
& \times \min _{k}\left[\int_{0}^{\eta} \min _{j}\left(e^{-\frac{1}{a}\left(\tilde{\mathcal{Y}}_{j}(t, \eta)-\tilde{\mathcal{Y}}_{j}(t, \theta)\right)}\right) \min _{j}\left(\tilde{\mathcal{P}}_{j}\right) \min _{j}\left(\tilde{\mathcal{U}}_{j}^{+}\right) \tilde{\mathcal{Y}}_{k, \eta}(t, \theta) d \theta\right] d \eta \\
& +\frac{1}{A^{6}} \int_{0}^{1}\left(\tilde{\mathcal{U}}_{1}-\tilde{\mathcal{U}}_{2}\right) \tilde{\mathcal{U}}_{2, \eta}(t, \eta) \mathbb{1}_{\tilde{E}^{c}}(t, \eta) \\
& \times\left(\int_{0}^{\eta} \min _{j}\left(e^{-\frac{1}{a}\left(\tilde{\mathcal{Y}}_{j}(t, \eta)-\tilde{\mathcal{Y}}_{j}(t, \theta)\right)}\right) \min _{j}\left(\tilde{\mathcal{P}}_{j}\right) \min _{j}\left(\tilde{\mathcal{U}}_{j}^{+}\right)\left(\tilde{\mathcal{Y}}_{2}-\tilde{\mathcal{Y}}_{1}\right)_{\eta} d \theta\right) d \eta \\
& +\frac{1}{A^{6}} \int_{0}^{1}\left(\tilde{\mathcal{U}}_{1}-\tilde{\mathcal{U}}_{2}\right) \tilde{\mathcal{U}}_{1, \eta}(t, \eta) \mathbb{1}_{\tilde{E}}(t, \eta) \\
& \times\left(\int_{0}^{\eta} \min _{j}\left(e^{-\frac{1}{a}\left(\tilde{\mathcal{Y}}_{j}(t, \eta)-\tilde{\mathcal{Y}}_{j}(t, \theta)\right)}\right) \min _{j}\left(\tilde{\mathcal{P}}_{j}\right) \min _{j}\left(\tilde{\mathcal{U}}_{j}^{+}\right)\left(\tilde{\mathcal{Y}}_{2}-\tilde{\mathcal{Y}}_{1}\right)_{\eta} d \theta\right) d \eta \\
& =N_{1551}+N_{1552}+N_{1553} \text {, }
\end{aligned}
$$

which, yet again, requires separate treatment. Here $\tilde{E}$ is defined in (5.16).

The term $N_{1551}$ can be handled as the term $\tilde{L}_{31}$ (cf. (5.17)),

$$
\left|N_{1551}\right| \leqslant \mathcal{O}(1)\left\|\tilde{\mathcal{U}}_{1}-\tilde{\mathcal{U}}_{2}\right\|^{2} .
$$

The terms $N_{1552}$ and $N_{1553}$ can be treated in the same manner:

$$
\begin{aligned}
\left|N_{1552}\right| \leqslant & \frac{1}{A^{6}} \mid \int_{0}^{1}\left(\tilde{\mathcal{U}}_{1}-\tilde{\mathcal{U}}_{2}\right) \tilde{\mathcal{U}}_{2, \eta}(t, \eta) \mathbb{1}_{\tilde{E}^{c}}(t, \eta) \\
& \times\left(\int_{0}^{\eta} \min _{j}\left(e^{-\frac{1}{a}\left(\tilde{\mathcal{Y}}_{j}(t, \eta)-\tilde{\mathcal{Y}}_{j}(t, \theta)\right)}\right) \min _{j}\left(\tilde{\mathcal{P}}_{j}\right) \min _{j}\left(\tilde{\mathcal{U}}_{j}^{+}\right)\left(\tilde{\mathcal{Y}}_{2}-\tilde{\mathcal{Y}}_{1}\right)_{\eta}(t, \theta) d \theta\right) d \eta \mid \\
\leqslant & \frac{1}{A^{6}} \int_{0}^{1}\left|\tilde{\mathcal{U}}_{1}-\tilde{\mathcal{U}}_{2}\right|\left|\tilde{\mathcal{Y}}_{1}-\tilde{\mathcal{Y}}_{2}\right| \min _{j}\left(\tilde{\mathcal{P}}_{j}\right) \min _{j}\left(\tilde{\mathcal{U}}_{j}^{+}\right)\left|\tilde{\mathcal{U}}_{2, \eta}\right| \mathbb{1}_{\tilde{E}^{c}}(t, \eta) d \eta \\
& +\frac{1}{A^{6}} \mid \int_{0}^{1}\left(\tilde{\mathcal{U}}_{1}-\tilde{\mathcal{U}}_{2}\right) \tilde{\mathcal{U}}_{2, \eta}(t, \eta) \mathbb{1}_{\tilde{E}^{c}}(t, \eta) \\
& \times \int_{0}^{\eta}\left(\tilde{\mathcal{Y}}_{1}-\tilde{\mathcal{Y}}_{2}\right)\left[\frac{d}{d \theta}\left(\min _{j}\left(e^{-\frac{1}{a}\left(\tilde{\mathcal{Y}}_{j}(t, \eta)-\tilde{\mathcal{Y}}_{j}(t, \theta)\right)}\right)\right) \min _{j}\left(\tilde{\mathcal{P}}_{j}\right) \min _{j}\left(\tilde{\mathcal{U}}_{j}^{+}\right)\right.
\end{aligned}
$$




$$
\begin{aligned}
& \left.+\min _{j}\left(e^{-\frac{1}{a}\left(\tilde{\mathcal{Y}}_{j}(t, \eta)-\tilde{\mathcal{Y}}_{j}(t, \theta)\right)}\right) \frac{d}{d \theta}\left(\min _{j}\left(\tilde{\mathcal{P}}_{j}\right) \min _{j}\left(\tilde{\mathcal{U}}_{j}^{+}\right)\right)\right](t, \theta) d \theta d \eta \mid \\
\leqslant & \mathcal{O}(1)\left(\left\|\tilde{\mathcal{U}}_{1}-\tilde{\mathcal{U}}_{2}\right\|^{2}+\left\|\tilde{\mathcal{Y}}_{1}-\tilde{\mathcal{Y}}_{2}\right\|^{2}\right) .
\end{aligned}
$$

The term $N_{2}$ (and also $N_{3}$ ) can be treated as follows, keeping in mind that $A_{2} \leqslant A_{1}$.

$$
\begin{aligned}
\left|N_{2}\right| \leqslant & \frac{A_{1}^{6}-A_{2}^{6}}{A_{1}^{6} A_{2}^{6}} \int_{0}^{1}\left|\tilde{\mathcal{U}}_{1}-\tilde{\mathcal{U}}_{2}\right|\left|\tilde{\mathcal{U}}_{2, \eta}\right|(t, \eta)\left(\int_{0}^{\eta} e^{-\frac{1}{A_{2}}\left(\tilde{\mathcal{Y}}_{2}(t, \eta)-\tilde{\mathcal{Y}}_{2}(t, \theta)\right)} \tilde{\mathcal{P}}_{2}^{2} \tilde{\mathcal{Y}}_{2, \eta}(t, \theta) d \theta\right)^{1 / 2} \\
& \times\left(\int_{0}^{\eta} e^{-\frac{1}{A_{2}}\left(\tilde{\mathcal{Y}}_{2}(t, \eta)-\tilde{\mathcal{Y}}_{2}(t, \theta)\right)} \tilde{\mathcal{U}}_{2}^{2} \tilde{\mathcal{Y}}_{2, \eta}(t, \theta) d \theta\right)^{1 / 2} d \eta \\
\leqslant & \sqrt{6} \frac{A_{1}^{6}-A_{2}^{6}}{A^{6} A_{2}^{3}} \int_{0}^{1}\left|\tilde{\mathcal{U}}_{1}-\tilde{\mathcal{U}}_{2}\right| \tilde{\mathcal{P}}_{2}\left|\tilde{\mathcal{U}}_{2, \eta}\right|(t, \eta) d \eta \\
\leqslant & 3 \sqrt{3} A^{2}\left|A_{1}-A_{2}\right| \int_{0}^{1}\left|\tilde{\mathcal{U}}_{2}-\tilde{\mathcal{U}}_{1}\right|(t, \eta) d \eta \\
\leqslant & \mathcal{O}(1)\left(\left\|\tilde{\mathcal{U}}_{1}-\tilde{\mathcal{U}}_{2}\right\|^{2}+\left|A_{1}-A_{2}\right|^{2}\right) .
\end{aligned}
$$

Finally, we now turn to the integral $I_{33}$. Recall equation (5.32c), namely,

$$
\begin{aligned}
I_{33}= & \int_{0}^{1}\left(\tilde{\mathcal{U}}_{1}-\tilde{\mathcal{U}}_{2}\right)(t, \eta)\left(\frac{1}{A_{2}^{6}} \tilde{\mathcal{U}}_{2, \eta}(t, \eta) \int_{0}^{1} e^{-\frac{1}{A_{2}}\left|\tilde{\mathcal{Y}}_{2}(t, \eta)-\tilde{\mathcal{Y}}_{2}(t, \theta)\right|} \tilde{\mathcal{Q}}_{2} \tilde{\mathcal{U}}_{2, \eta}(t, \theta) d \theta\right. \\
& \left.-\frac{1}{A_{1}^{6}} \tilde{\mathcal{U}}_{1, \eta}(t, \eta) \int_{0}^{1} e^{-\frac{1}{A_{1}}\left|\tilde{\mathcal{Y}}_{1}(t, \eta)-\tilde{\mathcal{Y}}_{1}(t, \theta)\right|} \tilde{\mathcal{Q}}_{1} \tilde{\mathcal{U}}_{1, \eta}(t, \theta) d \theta\right) d \eta
\end{aligned}
$$

By first involving (5.4) and then Lemma 5.1, we see that it suffices to estimate

$$
\begin{aligned}
\tilde{I}_{33}= & \int_{0}^{1}\left(\tilde{\mathcal{U}}_{1}-\tilde{\mathcal{U}}_{2}\right)(t, \eta)\left(\frac{1}{A_{2}^{6}} \tilde{\mathcal{U}}_{2, \eta}(t, \eta) \int_{0}^{\eta} e^{-\frac{1}{A_{2}}\left(\tilde{\mathcal{Y}}_{2}(t, \eta)-\tilde{\mathcal{Y}}_{2}(t, \theta)\right)} \tilde{\mathcal{Q}}_{2} \tilde{\mathcal{U}}_{2, \eta}(t, \theta) d \theta\right. \\
& \left.-\frac{1}{A_{1}^{6}} \tilde{\mathcal{U}}_{1, \eta}(t, \eta) \int_{0}^{\eta} e^{-\frac{1}{A_{1}}\left(\tilde{\mathcal{Y}}_{1}(t, \eta)-\tilde{\mathcal{Y}}_{1}(t, \theta)\right)} \tilde{\mathcal{Q}}_{1} \tilde{\mathcal{U}}_{1, \eta}(t, \theta) d \theta\right) d \eta \\
= & \frac{1}{A^{6}} \int_{0}^{1}\left(\tilde{\mathcal{U}}_{1}-\tilde{\mathcal{U}}_{2}\right)(t, \eta)\left(\tilde{\mathcal{U}}_{2, \eta}(t, \eta) \int_{0}^{\eta} e^{-\frac{1}{A_{2}}\left(\tilde{\mathcal{Y}}_{2}(t, \eta)-\tilde{\mathcal{Y}}_{2}(t, \theta)\right)} \tilde{\mathcal{Q}}_{2} \tilde{\mathcal{U}}_{2, \eta}(t, \theta) d \theta\right. \\
& \left.-\tilde{\mathcal{U}}_{1, \eta}(t, \eta) \int_{0}^{\eta} e^{-\frac{1}{A_{1}}\left(\tilde{\mathcal{Y}}_{1}(t, \eta)-\tilde{\mathcal{Y}}_{1}(t, \theta)\right)} \tilde{\mathcal{Q}}_{1} \tilde{\mathcal{U}}_{1, \eta}(t, \theta) d \theta\right) d \eta \\
& +\mathbb{1}_{A_{2} \leqslant A_{1}}\left(\frac{1}{A_{2}^{6}}-\frac{1}{A_{1}^{6}}\right) \int_{0}^{1}\left(\tilde{\mathcal{U}}_{1}-\tilde{\mathcal{U}}_{2}\right) \tilde{\mathcal{U}}_{2, \eta}(t, \eta) \\
& \times \int_{0}^{\eta} e^{-\frac{1}{A_{2}}\left(\tilde{\mathcal{Y}}_{2}(t, \eta)-\tilde{\mathcal{Y}}_{2}(t, \theta)\right)} \tilde{\mathcal{Q}}_{2} \tilde{\mathcal{U}}_{2, \eta}(t, \theta) d \theta d \eta
\end{aligned}
$$




$$
\begin{aligned}
& +\mathbb{1}_{A_{1}<A_{2}}\left(\frac{1}{A_{2}^{6}}-\frac{1}{A_{1}^{6}}\right) \int_{0}^{1}\left(\tilde{\mathcal{U}}_{1}-\tilde{\mathcal{U}}_{2}\right) \tilde{\mathcal{U}}_{1, \eta}(t, \eta) \\
& \times \int_{0}^{\eta} e^{-\frac{1}{A_{1}}\left(\tilde{\mathcal{Y}}_{1}(t, \eta)-\tilde{\mathcal{Y}}_{1}(t, \theta)\right)} \tilde{\mathcal{Q}}_{1} \tilde{\mathcal{U}}_{1, \eta}(t, \theta) d \theta d \eta \\
& =M_{1}+M_{2}+M_{3} .
\end{aligned}
$$

To our dismay, the estimate for $M_{1}$ is rather involved. We estimate, using first that $\tilde{\mathcal{Q}}_{i}=A_{i} \tilde{\mathcal{P}}_{i}-\tilde{\mathcal{D}}_{i}$ (cf. (4.7)):

$$
\begin{aligned}
& M_{1}=\frac{1}{A^{6}} \int_{0}^{1}\left(\tilde{\mathcal{U}}_{1}-\tilde{\mathcal{U}}_{2}\right)(t, \eta)\left(\tilde{\mathcal{U}}_{2, \eta}(t, \eta) \int_{0}^{\eta} e^{-\frac{1}{A_{2}}\left(\tilde{\mathcal{Y}}_{2}(t, \eta)-\tilde{\mathcal{Y}}_{2}(t, \theta)\right)} \tilde{\mathcal{Q}}_{2} \tilde{\mathcal{U}}_{2, \eta}(t, \theta) d \theta\right. \\
& \left.-\tilde{\mathcal{U}}_{1, \eta}(t, \eta) \int_{0}^{\eta} e^{-\frac{1}{A_{1}}\left(\tilde{\mathcal{Y}}_{1}(t, \eta)-\tilde{\mathcal{Y}}_{1}(t, \theta)\right)} \tilde{\mathcal{Q}}_{1} \tilde{\mathcal{U}}_{1, \eta}(t, \theta) d \theta\right) d \eta \\
& =\frac{1}{A^{6}} \int_{0}^{1}\left(\tilde{\mathcal{U}}_{1}-\tilde{\mathcal{U}}_{2}\right)(t, \eta)\left(\tilde{\mathcal{U}}_{2, \eta}(t, \eta) \int_{0}^{\eta} e^{-\frac{1}{A_{2}}\left(\tilde{\mathcal{Y}}_{2}(t, \eta)-\tilde{\mathcal{Y}}_{2}(t, \theta)\right)} A_{2} \tilde{\mathcal{P}}_{2} \tilde{\mathcal{U}}_{2, \eta}(t, \theta) d \theta\right. \\
& \left.-\tilde{\mathcal{U}}_{1, \eta}(t, \eta) \int_{0}^{\eta} e^{-\frac{1}{A_{1}}\left(\tilde{\mathcal{Y}}_{1}(t, \eta)-\tilde{\mathcal{Y}}_{1}(t, \theta)\right)} A_{1} \tilde{\mathcal{P}}_{1} \tilde{\mathcal{U}}_{1, \eta}(t, \theta) d \theta\right) d \eta \\
& -\frac{1}{A^{6}} \int_{0}^{1}\left(\tilde{\mathcal{U}}_{1}-\tilde{\mathcal{U}}_{2}\right)(t, \eta)\left(\tilde{\mathcal{U}}_{2, \eta}(t, \eta) \int_{0}^{\eta} e^{-\frac{1}{A_{2}}\left(\tilde{\mathcal{Y}}_{2}(t, \eta)-\tilde{\mathcal{Y}}_{2}(t, \theta)\right)} \tilde{\mathcal{D}}_{2} \tilde{\mathcal{U}}_{2, \eta}(t, \theta) d \theta\right. \\
& \left.-\tilde{\mathcal{U}}_{1, \eta}(t, \eta) \int_{0}^{\eta} e^{-\frac{1}{A_{1}}\left(\tilde{\mathcal{Y}}_{1}(t, \eta)-\tilde{\mathcal{Y}}_{1}(t, \theta)\right)} \tilde{\mathcal{D}}_{1} \tilde{\mathcal{U}}_{1, \eta}(t, \theta) d \theta\right) d \eta \\
& =\frac{1}{A^{6}} \int_{0}^{1}\left(\tilde{\mathcal{U}}_{1}-\tilde{\mathcal{U}}_{2}\right)\left(A_{2} \tilde{\mathcal{P}}_{2} \tilde{\mathcal{U}}_{2} \tilde{\mathcal{U}}_{2, \eta}-A_{1} \tilde{\mathcal{P}}_{1} \tilde{\mathcal{U}}_{1} \tilde{\mathcal{U}}_{1, \eta}\right)(t, \eta) d \eta \\
& -\frac{1}{A^{6}} \int_{0}^{1}\left(\tilde{\mathcal{U}}_{1}-\tilde{\mathcal{U}}_{2}\right)\left(\tilde{\mathcal{D}}_{2} \tilde{\mathcal{U}}_{2} \tilde{\mathcal{U}}_{2, \eta}-\tilde{\mathcal{D}}_{1} \tilde{\mathcal{U}}_{1} \tilde{\mathcal{U}}_{1, \eta}\right)(t, \eta) d \eta \\
& +\frac{1}{A^{6}} \int_{0}^{1}\left(\tilde{\mathcal{U}}_{1}-\tilde{\mathcal{U}}_{2}\right)(t, \eta) \\
& \times\left(\tilde{\mathcal{U}}_{2, \eta}(t, \eta) \int_{0}^{\eta} e^{-\frac{1}{A_{2}}\left(\tilde{\mathcal{Y}}_{2}(t, \eta)-\tilde{\mathcal{Y}}_{2}(t, \theta)\right)} \frac{1}{A_{2}} \tilde{\mathcal{D}}_{2} \tilde{\mathcal{U}}_{2} \tilde{\mathcal{Y}}_{2, \eta}(t, \theta) d \theta\right. \\
& \left.-\tilde{\mathcal{U}}_{1, \eta}(t, \eta) \int_{0}^{\eta} e^{-\frac{1}{A_{1}}\left(\tilde{\mathcal{Y}}_{1}(t, \eta)-\tilde{\mathcal{Y}}_{1}(t, \theta)\right)} \frac{1}{A_{1}} \tilde{\mathcal{D}}_{1} \tilde{\mathcal{U}}_{1} \tilde{\mathcal{Y}}_{1, \eta}(t, \theta) d \theta\right) d \eta \\
& -\frac{3}{A^{6}} \int_{0}^{1}\left(\tilde{\mathcal{U}}_{1}-\tilde{\mathcal{U}}_{2}\right)(t, \eta) \\
& \times\left(\tilde{\mathcal{U}}_{2, \eta}(t, \eta) \int_{0}^{\eta} e^{-\frac{1}{A_{2}}\left(\tilde{\mathcal{Y}}_{2}(t, \eta)-\tilde{\mathcal{Y}}_{2}(t, \theta)\right)} \tilde{\mathcal{P}}_{2} \tilde{\mathcal{U}}_{2} \tilde{\mathcal{Y}}_{2, \eta}(t, \theta) d \theta\right.
\end{aligned}
$$




$$
\begin{aligned}
& \left.-\tilde{\mathcal{U}}_{1, \eta}(t, \eta) \int_{0}^{\eta} e^{-\frac{1}{A_{1}}\left(\tilde{\mathcal{Y}}_{1}(t, \eta)-\tilde{\mathcal{Y}}_{1}(t, \theta)\right)} \tilde{\mathcal{P}}_{1} \tilde{\mathcal{U}}_{1} \tilde{\mathcal{Y}}_{1, \eta}(t, \theta) d \theta\right) d \eta \\
& +\frac{1}{A^{6}} \int_{0}^{1}\left(\tilde{\mathcal{U}}_{1}-\tilde{\mathcal{U}}_{2}\right)(t, \eta)\left(\tilde{\mathcal{U}}_{2, \eta}(t, \eta) \int_{0}^{\eta} e^{-\frac{1}{A_{2}}\left(\tilde{\mathcal{Y}}_{2}(t, \eta)-\tilde{\mathcal{Y}}_{2}(t, \theta)\right)} \tilde{\mathcal{U}}_{2}^{3} \tilde{\mathcal{Y}}_{2, \eta}(t, \theta) d \theta\right. \\
& \left.-\tilde{\mathcal{U}}_{1, \eta}(t, \eta) \int_{0}^{\eta} e^{-\frac{1}{A_{1}}\left(\tilde{\mathcal{Y}}_{1}(t, \eta)-\tilde{\mathcal{Y}}_{1}(t, \theta)\right)} \tilde{\mathcal{U}}_{1}^{3} \tilde{\mathcal{Y}}_{1, \eta}(t, \theta) d \theta\right) d \eta \\
& +\frac{1}{2 A^{6}} \int_{0}^{1}\left(\tilde{\mathcal{U}}_{1}-\tilde{\mathcal{U}}_{2}\right)(t, \eta)\left(\tilde{\mathcal{U}}_{2, \eta}(t, \eta) \int_{0}^{\eta} e^{-\frac{1}{A_{2}}\left(\tilde{\mathcal{Y}}_{2}(t, \eta)-\tilde{\mathcal{Y}}_{2}(t, \theta)\right)} A_{2}^{5} \tilde{\mathcal{U}}_{2}(t, \theta) d \theta\right. \\
& \left.-\tilde{\mathcal{U}}_{1, \eta}(t, \eta) \int_{0}^{\eta} e^{-\frac{1}{A_{1}}\left(\tilde{\mathcal{Y}}_{1}(t, \eta)-\tilde{\mathcal{Y}}_{1}(t, \theta)\right)} A_{1}^{5} \tilde{\mathcal{U}}_{1}(t, \theta) d \theta\right) d \eta \\
& =W_{1}+W_{2}+W_{3}+W_{4}+W_{5}+W_{6} .
\end{aligned}
$$

Here we have used the rewrite employed when manipulating the term $\bar{K}_{1}$ from the expression (5.18) to (5.19). We start by considering the term $W_{1}$ :

$$
\begin{aligned}
W_{1}= & \frac{1}{A^{6}} \int_{0}^{1}\left(\tilde{\mathcal{U}}_{1}-\tilde{\mathcal{U}}_{2}\right)\left(A_{2} \tilde{\mathcal{P}}_{2} \tilde{\mathcal{U}}_{2} \tilde{\mathcal{U}}_{2, \eta}-A_{1} \tilde{\mathcal{P}}_{1} \tilde{\mathcal{U}}_{1} \tilde{\mathcal{U}}_{1, \eta}\right)(t, \eta) d \eta \\
= & \frac{1}{A^{6}} \mathbb{1}_{A_{1} \leqslant A_{2}}\left(A_{2}-A_{1}\right) \int_{0}^{1}\left(\tilde{\mathcal{U}}_{1}-\tilde{\mathcal{U}}_{2}\right) \tilde{\mathcal{P}}_{2} \tilde{\mathcal{U}}_{2} \tilde{\mathcal{U}}_{2, \eta}(t, \eta) d \eta \\
& +\frac{1}{A^{6}} \mathbb{1}_{A_{2}<A_{1}}\left(A_{2}-A_{1}\right) \int_{0}^{1}\left(\tilde{\mathcal{U}}_{1}-\tilde{\mathcal{U}}_{2}\right) \tilde{\mathcal{P}}_{1} \tilde{\mathcal{U}}_{1} \tilde{\mathcal{U}}_{1, \eta}(t, \eta) d \eta \\
& +\frac{a}{A^{6}} \int_{0}^{1}\left(\tilde{\mathcal{U}}_{1}-\tilde{\mathcal{U}}_{2}\right)\left(\tilde{\mathcal{P}}_{2}-\tilde{\mathcal{P}}_{1}\right) \tilde{\mathcal{U}}_{2} \tilde{\mathcal{U}}_{2, \eta}(t, \eta) \mathbb{1}_{\tilde{\mathcal{P}}_{1} \leqslant \tilde{\mathcal{P}}_{2}}(t, \eta) d \eta \\
& +\frac{a}{A^{6}} \int_{0}^{1}\left(\tilde{\mathcal{U}}_{1}-\tilde{\mathcal{U}}_{2}\right)\left(\tilde{\mathcal{P}}_{2}-\tilde{\mathcal{P}}_{1}\right) \tilde{\mathcal{U}}_{1} \tilde{\mathcal{U}}_{1, \eta}(t, \eta) \mathbb{1}_{\tilde{\mathcal{P}}_{2}<\tilde{\mathcal{P}}_{1}}(t, \eta) d \eta \\
& +\frac{a}{A^{6}} \int_{0}^{1}\left(\tilde{\mathcal{U}}_{1}-\tilde{\mathcal{U}}_{2}\right)\left(\tilde{\mathcal{U}}_{2, \eta}-\tilde{\mathcal{U}}_{1, \eta}\right) \min _{j}\left(\tilde{\mathcal{P}}_{j}\right) \tilde{\mathcal{U}}_{2}(t, \eta) d \eta \\
& -\frac{a}{A^{6}} \int_{0}^{1}\left(\tilde{\mathcal{U}}_{1}-\tilde{\mathcal{U}}_{2}\right)^{2} \min _{j}\left(\tilde{\mathcal{P}}_{j}\right) \tilde{\mathcal{U}}_{1, \eta}(t, \eta) d \eta \\
= & W_{11}+W_{12}+W_{13}+W_{14}+W_{15}+W_{16} .
\end{aligned}
$$

For $W_{11}$ we have (and similarly for $W_{12}$ ) that

$$
\begin{aligned}
\left|W_{11}\right| & \leqslant \frac{A^{2}}{8}\left|A_{1}-A_{2}\right| \int_{0}^{1}\left|\tilde{\mathcal{U}}_{1}-\tilde{\mathcal{U}}_{2}\right|(t, \eta) d \eta \\
& \leqslant \mathcal{O}(1)\left(\left\|\tilde{\mathcal{U}}_{1}-\tilde{\mathcal{U}}_{2}\right\|^{2}+\left|A_{1}-A_{2}\right|^{2}\right) .
\end{aligned}
$$


The terms $W_{13}$ and $W_{14}$ are similar:

$$
\begin{aligned}
\left|W_{11}\right| & =\frac{a}{A^{6}}\left|\int_{0}^{1}\left(\tilde{\mathcal{U}}_{1}-\tilde{\mathcal{U}}_{2}\right)\left(\tilde{\mathcal{P}}_{2}-\tilde{\mathcal{P}}_{1}\right) \tilde{\mathcal{U}}_{2} \tilde{\mathcal{U}}_{2, \eta}(t, \eta) \mathbb{1}_{\tilde{\mathcal{P}}_{1} \leqslant \tilde{\mathcal{P}}_{2}}(t, \eta) d \eta\right| \\
& \leqslant \frac{2 a}{A^{6}} \int_{0}^{1}\left|\tilde{\mathcal{U}}_{1}-\tilde{\mathcal{U}}_{2}\right|\left|\tilde{\mathcal{P}}_{2}^{1 / 2}-\tilde{\mathcal{P}}_{1}^{1 / 2}\right| \tilde{\mathcal{P}}_{2}^{1 / 2}\left|\tilde{\mathcal{U}}_{2} \tilde{\mathcal{U}}_{2, \eta}\right|(t, \eta) d \eta \\
& \leqslant \frac{A}{2} \int_{0}^{1}\left|\tilde{\mathcal{U}}_{1}-\tilde{\mathcal{U}}_{2}\right|\left|\tilde{\mathcal{P}}_{2}^{1 / 2}-\tilde{\mathcal{P}}_{1}^{1 / 2}\right| d \eta \\
& \leqslant \mathcal{O}(1)\left(\left\|\tilde{\mathcal{U}}_{1}-\tilde{\mathcal{U}}_{2}\right\|^{2}+\left\|\tilde{\mathcal{P}}_{2}^{1 / 2}-\tilde{\mathcal{P}}_{1}^{1 / 2}\right\|^{2}\right),
\end{aligned}
$$

using (4.15a) and (4.15f).

The term $W_{15}$ goes as follows:

$$
\begin{aligned}
\left|W_{15}\right|= & \frac{a}{A^{6}}\left|\int_{0}^{1}\left(\tilde{\mathcal{U}}_{1}-\tilde{\mathcal{U}}_{2}\right)\left(\tilde{\mathcal{U}}_{2, \eta}-\tilde{\mathcal{U}}_{1, \eta}\right) \min _{j}\left(\tilde{\mathcal{P}}_{j}\right) \tilde{\mathcal{U}}_{2}(t, \eta) d \eta\right| \\
= & \left|\frac{a}{2 A^{6}}\left(\tilde{\mathcal{U}}_{1}-\tilde{\mathcal{U}}_{2}\right)^{2} \min _{j}\left(\tilde{\mathcal{P}}_{j}\right) \tilde{\mathcal{U}}_{2}(t, \eta)\right|_{\eta=0}^{1} \\
& -\frac{a}{2 A^{6}} \int_{0}^{1}\left(\tilde{\mathcal{U}}_{1}-\tilde{\mathcal{U}}_{2}\right)^{2} \frac{d}{d \eta}\left(\min _{j}\left(\tilde{\mathcal{P}}_{j}\right) \tilde{\mathcal{U}}_{2}\right)(t, \eta) d \eta \mid \\
\leqslant & \frac{a}{2 A^{6}} \int_{0}^{1}\left(\tilde{\mathcal{U}}_{1}-\tilde{\mathcal{U}}_{2}\right)^{2}\left|\frac{d}{d \eta}\left(\min _{j}\left(\tilde{\mathcal{P}}_{j}\right) \tilde{\mathcal{U}}_{2}\right)\right|(t, \eta) d \eta \\
\leqslant & \mathcal{O}(1)\left\|\tilde{\mathcal{U}}_{1}-\tilde{\mathcal{U}}_{2}\right\|^{2} ;
\end{aligned}
$$

see the estimates for $\bar{B}_{15}$ (cf. (5.20)). As for the term $W_{16}$, we get

$$
\begin{aligned}
\left|W_{16}\right| & =\frac{a}{A^{6}} \int_{0}^{1}\left(\tilde{\mathcal{U}}_{1}-\tilde{\mathcal{U}}_{2}\right)^{2} \min _{j}\left(\tilde{\mathcal{P}}_{j}\right)\left|\tilde{\mathcal{U}}_{1, \eta}\right|(t, \eta) d \eta \\
& \leqslant \mathcal{O}(1)\left\|\tilde{\mathcal{U}}_{1}-\tilde{\mathcal{U}}_{2}\right\|^{2},
\end{aligned}
$$

using (4.15p).

As for the term $W_{2}$, we find

$$
\begin{aligned}
-W_{2}= & \frac{1}{A^{6}} \int_{0}^{1}\left(\tilde{\mathcal{U}}_{1}-\tilde{\mathcal{U}}_{2}\right)\left(\tilde{\mathcal{D}}_{2} \tilde{\mathcal{U}}_{2} \tilde{\mathcal{U}}_{2, \eta}-\tilde{\mathcal{D}}_{1} \tilde{\mathcal{U}}_{1} \tilde{\mathcal{U}}_{1, \eta}\right)(t, \eta) d \eta \\
= & \frac{1}{A^{6}} \int_{0}^{1}\left(\tilde{\mathcal{U}}_{1}-\tilde{\mathcal{U}}_{2}\right)\left(\tilde{\mathcal{D}}_{2}-\tilde{\mathcal{D}}_{1}\right) \tilde{\mathcal{U}}_{1} \tilde{\mathcal{U}}_{1, \eta}(t, \eta) \mathbb{1}_{\tilde{\mathcal{D}}_{2} \leqslant \tilde{\mathcal{D}}_{1}}(t, \eta) d \eta \\
& +\frac{1}{A^{6}} \int_{0}^{1}\left(\tilde{\mathcal{U}}_{1}-\tilde{\mathcal{U}}_{2}\right)\left(\tilde{\mathcal{D}}_{2}-\tilde{\mathcal{D}}_{1}\right) \tilde{\mathcal{U}}_{2} \tilde{\mathcal{U}}_{2, \eta}(t, \eta) \mathbb{1}_{\tilde{\mathcal{D}}_{1}<\tilde{\mathcal{D}}_{2}}(t, \eta) d \eta
\end{aligned}
$$




$$
\begin{aligned}
& +\frac{1}{A^{6}} \int_{0}^{1}\left(\tilde{\mathcal{U}}_{1}-\tilde{\mathcal{U}}_{2}\right)\left(\tilde{\mathcal{U}}_{2, \eta}-\tilde{\mathcal{U}}_{1, \eta}\right) \min _{j}\left(\tilde{\mathcal{D}}_{j}\right) \tilde{\mathcal{U}}_{2}(t, \eta) d \eta \\
& -\frac{1}{A^{6}} \int_{0}^{1}\left(\tilde{\mathcal{U}}_{1}-\tilde{\mathcal{U}}_{2}\right)^{2} \min _{j}\left(\tilde{\mathcal{D}}_{j}\right) \tilde{\mathcal{U}}_{1, \eta}(t, \eta) d \eta \\
& =W_{21}+W_{22}+W_{23}+W_{24} .
\end{aligned}
$$

The terms $W_{21}$ and $W_{22}$ can be treated similarly. We need to estimate $\tilde{\mathcal{D}}_{2}-\tilde{\mathcal{D}}_{1}$. Applying Lemma A.9, we have

$$
\begin{aligned}
& \left|W_{21}\right|=\frac{1}{A^{6}}\left|\int_{0}^{1}\left(\tilde{\mathcal{U}}_{1}-\tilde{\mathcal{U}}_{2}\right)\left(\tilde{\mathcal{D}}_{2}-\tilde{\mathcal{D}}_{1}\right) \tilde{\mathcal{U}}_{1} \tilde{\mathcal{U}}_{1, \eta}(t, \eta) \mathbb{1}_{\tilde{\mathcal{D}}_{2} \leqslant \tilde{\mathcal{D}}_{1}}(t, \eta) d \eta\right| \\
& \leqslant \frac{2}{A^{9 / 2}} \int_{0}^{1}\left|\tilde{\mathcal{U}}_{1}-\tilde{\mathcal{U}}_{2}\right|\left|\tilde{\mathcal{Y}}_{1}-\tilde{\mathcal{Y}}_{2}\right| \tilde{\mathcal{D}}_{1}^{1 / 2}\left|\tilde{\mathcal{U}}_{1} \tilde{\mathcal{U}}_{1, \eta}\right|(t, \eta) d \eta \\
& +\frac{1}{A^{6}} \int_{0}^{1}\left|\tilde{\mathcal{U}}_{1}-\tilde{\mathcal{U}}_{2}\right|\left|\tilde{\mathcal{Y}}_{1}-\tilde{\mathcal{Y}}_{2}\right|\left(\tilde{\mathcal{U}}_{1}^{2}+\tilde{\mathcal{P}}_{1}\right)\left|\tilde{\mathcal{U}}_{1} \tilde{\mathcal{U}}_{1, \eta}\right|(t, \eta) d \eta \\
& +\frac{2 \sqrt{2}}{A^{9 / 2}}\left\|\tilde{\mathcal{Y}}_{1}-\tilde{\mathcal{Y}}_{2}\right\| \int_{0}^{1}\left|\tilde{\mathcal{U}}_{1}-\tilde{\mathcal{U}}_{2}\right| \tilde{\mathcal{D}}_{1}^{1 / 2}\left|\tilde{\mathcal{U}}_{1} \tilde{\mathcal{U}}_{1, \eta}\right|(t, \eta) d \eta \\
& +\frac{4}{A^{3}} \int_{0}^{1}\left|\tilde{\mathcal{U}}_{1}-\tilde{\mathcal{U}}_{2}\right| \\
& \times\left(\int_{0}^{\eta} e^{-\frac{1}{A}\left(\tilde{\mathcal{Y}}_{1}(t, \eta)-\tilde{\mathcal{Y}}_{1}(t, \theta)\right)}\left(\tilde{\mathcal{U}}_{1}-\tilde{\mathcal{U}}_{2}\right)^{2}(t, \theta) d \theta\right)^{1 / 2}\left|\tilde{\mathcal{U}}_{1} \tilde{\mathcal{U}}_{1, \eta}\right|(t, \eta) d \eta \\
& +\frac{2 \sqrt{2}}{A^{3}} \int_{0}^{1}\left|\tilde{\mathcal{U}}_{1}-\tilde{\mathcal{U}}_{2}\right| \\
& \times\left(\int_{0}^{\eta} e^{-\frac{1}{A}\left(\tilde{\mathcal{Y}}_{1}(t, \eta)-\tilde{\mathcal{Y}}_{1}(t, \theta)\right)}\left(\tilde{\mathcal{P}}_{1}^{1 / 2}-\tilde{\mathcal{P}}_{2}^{1 / 2}\right)^{2}(t, \theta) d \theta\right)^{1 / 2}\left|\tilde{\mathcal{U}}_{1} \tilde{\mathcal{U}}_{1, \eta}\right|(t, \eta) d \eta \\
& +\frac{3}{\sqrt{2} A^{2}} \int_{0}^{1}\left|\tilde{\mathcal{U}}_{1}-\tilde{\mathcal{U}}_{2}\right| \\
& \times\left(\int_{0}^{\eta} e^{-\frac{1}{a}\left(\tilde{\mathcal{Y}}_{1}(t, \eta)-\tilde{\mathcal{Y}}_{1}(t, \theta)\right)}\left(\tilde{\mathcal{Y}}_{1}-\tilde{\mathcal{Y}}_{2}\right)^{2}(t, \theta) d \theta\right)^{1 / 2}\left|\tilde{\mathcal{U}}_{1} \tilde{\mathcal{U}}_{1, \eta}\right|(t, \eta) d \eta \\
& +\frac{12 \sqrt{2}}{\sqrt{3} e A^{2}} \int_{0}^{1}\left|\tilde{\mathcal{U}}_{1}-\tilde{\mathcal{U}}_{2}\right| \\
& \times\left(\int_{0}^{\eta} e^{-\frac{3}{4 A}\left(\tilde{\mathcal{Y}}_{1}(t, \eta)-\tilde{\mathcal{Y}}_{1}(t, \theta)\right)} d \theta\right)^{1 / 2}\left|\tilde{\mathcal{U}}_{1} \tilde{\mathcal{U}}_{1, \eta}\right|(t, \eta) d \eta\left|A_{1}-A_{2}\right| \\
& +\frac{3}{2 A^{2}} \int_{0}^{1}\left|\tilde{\mathcal{U}}_{1}-\tilde{\mathcal{U}}_{2}\right| \\
& \times\left(\int_{0}^{\eta} e^{-\frac{1}{a}\left(\tilde{\mathcal{Y}}_{1}(t, \eta)-\tilde{\mathcal{Y}}_{1}(t, \theta)\right)}\left|\tilde{\mathcal{Y}}_{1}-\tilde{\mathcal{Y}}_{2}\right|(t, \theta) d \theta\right)\left|\tilde{\mathcal{U}}_{1} \tilde{\mathcal{U}}_{1, \eta}\right|(t, \eta) d \eta
\end{aligned}
$$




$$
\begin{aligned}
& +\frac{6}{A^{2}}\left|A_{1}-A_{2}\right| \int_{0}^{1}\left|\tilde{\mathcal{U}}_{1}-\tilde{\mathcal{U}}_{2}\right|\left(\int_{0}^{\eta} e^{-\frac{3}{4 A}\left(\tilde{\mathcal{Y}}_{1}(t, \eta)-\tilde{\mathcal{Y}}_{1}(t, \theta)\right)} d \theta\right)\left|\tilde{\mathcal{U}}_{1} \tilde{\mathcal{U}}_{1, \eta}\right|(t, \eta) d \eta \\
& \leqslant \frac{2}{A^{9 / 2}} \int_{0}^{1}\left|\tilde{\mathcal{U}}_{1}-\tilde{\mathcal{U}}_{2}\right|\left|\tilde{\mathcal{Y}}_{1}-\tilde{\mathcal{Y}}_{2}\right|(t, \eta) \frac{A^{13 / 2}}{2 \sqrt{2}} d \eta \\
& +\frac{1}{A^{6}} \int_{0}^{1}\left|\tilde{\mathcal{U}}_{1}-\tilde{\mathcal{U}}_{2}\right|\left|\tilde{\mathcal{Y}}_{1}-\tilde{\mathcal{Y}}_{2}\right|(t, \eta) \frac{3 A^{8}}{8} d \eta \\
& +\frac{2 \sqrt{2}}{A^{9 / 2}}\left\|\tilde{\mathcal{Y}}_{1}-\tilde{\mathcal{Y}}_{2}\right\| \int_{0}^{1}\left|\tilde{\mathcal{U}}_{1}-\tilde{\mathcal{U}}_{2}\right|(t, \eta) \frac{A^{13 / 2}}{2 \sqrt{2}} d \eta \\
& +\frac{4}{A^{3}} \int_{0}^{1}\left|\tilde{\mathcal{U}}_{1}-\tilde{\mathcal{U}}_{2}\right|(t, \eta)\left(\int_{0}^{\eta}\left(\tilde{\mathcal{U}}_{1}-\tilde{\mathcal{U}}_{2}\right)^{2}(t, \theta) d \theta\right)^{1 / 2} \frac{A^{4}}{2} d \eta \\
& +\frac{2 \sqrt{2}}{A^{3}} \int_{0}^{1}\left|\tilde{\mathcal{U}}_{1}-\tilde{\mathcal{U}}_{2}\right|(t, \eta)\left(\int_{0}^{\eta}\left(\tilde{\mathcal{P}}_{1}^{1 / 2}-\tilde{\mathcal{P}}_{2}^{1 / 2}\right)^{2}(t, \theta) d \theta\right)^{1 / 2} \frac{A^{4}}{2} d \eta \\
& +\frac{3}{\sqrt{2} A^{2}} \int_{0}^{1}\left|\tilde{\mathcal{U}}_{1}-\tilde{\mathcal{U}}_{2}\right|(t, \eta)\left(\int_{0}^{\eta}\left(\tilde{\mathcal{Y}}_{1}-\tilde{\mathcal{Y}}_{2}\right)^{2}(t, \theta) d \theta\right)^{1 / 2} \frac{A^{4}}{2} d \eta \\
& +\frac{12 \sqrt{2}}{\sqrt{3} e A^{2}} \int_{0}^{1}\left|\tilde{\mathcal{U}}_{1}-\tilde{\mathcal{U}}_{2}\right| \frac{A^{4}}{2} d \eta\left|A_{1}-A_{2}\right| \\
& +\frac{3}{2 A^{2}} \int_{0}^{1}\left|\tilde{\mathcal{U}}_{1}-\tilde{\mathcal{U}}_{2}\right|(t, \eta)\left(\int_{0}^{\eta}\left|\tilde{\mathcal{Y}}_{1}-\tilde{\mathcal{Y}}_{2}\right|(t, \theta) d \theta\right) \frac{A^{4}}{2} d \eta \\
& +\frac{6}{A^{2}}\left|A_{1}-A_{2}\right| \int_{0}^{1}\left|\tilde{\mathcal{U}}_{1}-\tilde{\mathcal{U}}_{2}\right|(t, \eta) \frac{A^{4}}{2} d \eta \\
& \leqslant \mathcal{O}(1)\left(\left\|\tilde{\mathcal{U}}_{1}-\tilde{\mathcal{U}}_{2}\right\|^{2}+\left\|\tilde{\mathcal{P}}_{1}^{1 / 2}-\tilde{\mathcal{P}}_{2}^{1 / 2}\right\|^{2}+\left\|\tilde{\mathcal{Y}}_{1}-\tilde{\mathcal{Y}}_{2}\right\|^{2}+\left|A_{1}-A_{2}\right|^{2}\right),
\end{aligned}
$$

where we have used estimates (4.15a), (4.15b), (4.15f), and (4.15n). The term $W_{23}$ goes as follows:

$$
\begin{aligned}
\left|W_{23}\right|= & \frac{1}{A^{6}}\left|\int_{0}^{1}\left(\tilde{\mathcal{U}}_{1}-\tilde{\mathcal{U}}_{2}\right)\left(\tilde{\mathcal{U}}_{2, \eta}-\tilde{\mathcal{U}}_{1, \eta}\right) \min _{j}\left(\tilde{\mathcal{D}}_{j}\right) \tilde{\mathcal{U}}_{2}(t, \eta) d \eta\right| \\
\leqslant & \left|\frac{1}{2 A^{6}}\left(\tilde{\mathcal{U}}_{1}-\tilde{\mathcal{U}}_{2}\right)^{2} \min _{j}\left(\tilde{\mathcal{D}}_{j}\right) \tilde{\mathcal{U}}_{2}(t, \eta)\right|_{\eta=0}^{1} \\
& -\frac{1}{2 A^{6}} \int_{0}^{1}\left(\tilde{\mathcal{U}}_{1}-\tilde{\mathcal{U}}_{2}\right)^{2} \frac{d}{d \theta}\left(\min _{j}\left(\tilde{\mathcal{D}}_{j}\right) \tilde{\mathcal{U}}_{2}\right)(t, \eta) d \eta \mid \\
\leqslant & \frac{1}{2 A^{6}} \int_{0}^{1}\left(\tilde{\mathcal{U}}_{1}-\tilde{\mathcal{U}}_{2}\right)^{2}\left|\frac{d}{d \theta}\left(\min _{j}\left(\tilde{\mathcal{D}}_{j}\right) \tilde{\mathcal{U}}_{2}\right)\right|(t, \eta) d \eta \\
\leqslant & \mathcal{O}(1)\left\|\tilde{\mathcal{U}}_{1}-\tilde{\mathcal{U}}_{2}\right\|^{2},
\end{aligned}
$$

by applying Lemma A.4 (ii). 
The term $W_{24}$ goes as follows:

$$
\begin{aligned}
\left|W_{24}\right| & \leqslant \frac{1}{A^{6}} \int_{0}^{1}\left(\tilde{\mathcal{U}}_{1}-\tilde{\mathcal{U}}_{2}\right)^{2} \min _{j}\left(\tilde{\mathcal{D}}_{j}\right)\left|\tilde{\mathcal{U}}_{1, \eta}\right|(t, \eta) d \eta \\
& \leqslant \mathcal{O}(1)\left\|\tilde{\mathcal{U}}_{1}-\tilde{\mathcal{U}}_{2}\right\|^{2},
\end{aligned}
$$

using

$$
\min _{j}\left(\tilde{\mathcal{D}}_{j}\right)\left|\tilde{\mathcal{U}}_{1, \eta}\right| \leqslant 2 A_{1} \tilde{\mathcal{P}}_{1}\left|\tilde{\mathcal{U}}_{1, \eta}\right| \leqslant \frac{A^{7}}{\sqrt{2}},
$$

from $(4.15 n)$ and (4.15p).

Next, we turn to the term $W_{3}$ :

$$
\begin{aligned}
W_{3}= & \frac{1}{A^{6}} \int_{0}^{1}\left(\tilde{\mathcal{U}}_{1}-\tilde{\mathcal{U}}_{2}\right)(t, \eta) \\
& \times\left(\tilde{\mathcal{U}}_{2, \eta}(t, \eta) \int_{0}^{\eta} e^{-\frac{1}{A_{2}}\left(\tilde{\mathcal{Y}}_{2}(t, \eta)-\tilde{\mathcal{Y}}_{2}(t, \theta)\right)} \frac{1}{A_{2}} \tilde{\mathcal{D}}_{2} \tilde{\mathcal{U}}_{2} \tilde{\mathcal{Y}}_{2, \eta}(t, \theta) d \theta\right. \\
& \left.-\tilde{\mathcal{U}}_{1, \eta}(t, \eta) \int_{0}^{\eta} e^{-\frac{1}{A_{1}}\left(\tilde{\mathcal{Y}}_{1}(t, \eta)-\tilde{\mathcal{Y}}_{1}(t, \theta)\right)} \frac{1}{A_{1}} \tilde{\mathcal{D}}_{1} \tilde{\mathcal{U}}_{1} \tilde{\mathcal{Y}}_{1, \eta}(t, \theta) d \theta\right) d \eta \\
= & \frac{1}{A^{6}} \int_{0}^{1}\left(\tilde{\mathcal{U}}_{1}-\tilde{\mathcal{U}}_{2}\right)(t, \eta) \\
& \times\left(\tilde{\mathcal{U}}_{2, \eta}(t, \eta) \int_{0}^{\eta} e^{-\frac{1}{A_{2}}\left(\tilde{\mathcal{Y}}_{2}(t, \eta)-\tilde{\mathcal{Y}}_{2}(t, \theta)\right)} \frac{1}{A_{2}} \tilde{\mathcal{D}}_{2} \tilde{\mathcal{U}}_{2}^{+} \tilde{\mathcal{Y}}_{2, \eta}(t, \theta) d \theta\right. \\
& \left.-\tilde{\mathcal{U}}_{1, \eta}(t, \eta) \int_{0}^{\eta} e^{-\frac{1}{A_{1}}\left(\tilde{\mathcal{Y}}_{1}(t, \eta)-\tilde{\mathcal{Y}}_{1}(t, \theta)\right)} \frac{1}{A_{1}} \tilde{\mathcal{D}}_{1} \tilde{\mathcal{U}}_{1}^{+} \tilde{\mathcal{Y}}_{1, \eta}(t, \theta) d \theta\right) d \eta \\
& +\frac{1}{A^{6}} \int_{0}^{1}\left(\tilde{\mathcal{U}}_{1}-\tilde{\mathcal{U}}_{2}\right)(t, \eta) \\
& \times\left(\tilde{\mathcal{U}}_{2, \eta}(t, \eta) \int_{0}^{\eta} e^{-\frac{1}{A_{2}}\left(\tilde{\mathcal{Y}}_{2}(t, \eta)-\tilde{\mathcal{Y}}_{2}(t, \theta)\right)} \frac{1}{A_{2}} \tilde{\mathcal{D}}_{2} \tilde{\mathcal{U}}_{2}^{-} \tilde{\mathcal{Y}}_{2, \eta}(t, \theta) d \theta\right. \\
& \left.-\tilde{\mathcal{U}}_{1, \eta}(t, \eta) \int_{0}^{\eta} e^{-\frac{1}{A_{1}}\left(\tilde{\mathcal{Y}}_{1}(t, \eta)-\tilde{\mathcal{Y}}_{1}(t, \theta)\right)} \frac{1}{A_{1}} \tilde{\mathcal{D}}_{1} \tilde{\mathcal{U}}_{1}^{-} \tilde{\mathcal{Y}}_{1, \eta}(t, \theta) d \theta\right) d \eta \\
= & W_{31}+W_{32} .
\end{aligned}
$$

These terms can be treated similarly:

$$
\begin{aligned}
W_{31}= & \frac{1}{A^{6}} \int_{0}^{1}\left(\tilde{\mathcal{U}}_{1}-\tilde{\mathcal{U}}_{2}\right)(t, \eta) \\
& \times\left(\tilde{\mathcal{U}}_{2, \eta}(t, \eta) \int_{0}^{\eta} e^{-\frac{1}{A_{2}}\left(\tilde{\mathcal{Y}}_{2}(t, \eta)-\tilde{\mathcal{Y}}_{2}(t, \theta)\right)} \frac{1}{A_{2}} \tilde{\mathcal{D}}_{2} \tilde{\mathcal{U}}_{2}^{+} \tilde{\mathcal{Y}}_{2, \eta}(t, \theta) d \theta\right.
\end{aligned}
$$




$$
\begin{aligned}
& \left.-\tilde{\mathcal{U}}_{1, \eta}(t, \eta) \int_{0}^{\eta} e^{-\frac{1}{A_{1}}\left(\tilde{\mathcal{Y}}_{1}(t, \eta)-\tilde{\mathcal{Y}}_{1}(t, \theta)\right)} \frac{1}{A_{1}} \tilde{\mathcal{D}}_{1} \tilde{\mathcal{U}}_{1}^{+} \tilde{\mathcal{Y}}_{1, \eta}(t, \theta) d \theta\right) d \eta \\
& =\mathbb{1}_{A_{1} \leqslant A_{2}} \frac{1}{A^{6}}\left(\frac{1}{A_{2}}-\frac{1}{A_{1}}\right) \int_{0}^{1}\left(\tilde{\mathcal{U}}_{1}-\tilde{\mathcal{U}}_{2}\right) \tilde{\mathcal{U}}_{1, \eta}(t, \eta) \\
& \times\left(\int_{0}^{\eta} e^{-\frac{1}{A_{1}}\left(\tilde{\mathcal{Y}}_{1}(t, \eta)-\tilde{\mathcal{Y}}_{1}(t, \theta)\right)} \tilde{\mathcal{D}}_{1} \tilde{\mathcal{U}}_{1}^{+} \tilde{\mathcal{Y}}_{1, \eta}(t, \theta) d \theta\right) d \eta \\
& +\mathbb{1}_{A_{2}<A_{1}} \frac{1}{A^{6}}\left(\frac{1}{A_{2}}-\frac{1}{A_{1}}\right) \int_{0}^{1}\left(\tilde{\mathcal{U}}_{1}-\tilde{\mathcal{U}}_{2}\right) \tilde{\mathcal{U}}_{2, \eta}(t, \eta) \\
& \times\left(\int_{0}^{\eta} e^{-\frac{1}{A_{2}}\left(\tilde{\mathcal{Y}}_{2}(t, \eta)-\tilde{\mathcal{Y}}_{2}(t, \theta)\right)} \tilde{\mathcal{D}}_{2} \tilde{\mathcal{U}}_{2}^{+} \tilde{\mathcal{Y}}_{2, \eta}(t, \theta) d \theta\right) d \eta \\
& +\frac{1}{A^{7}} \int_{0}^{1}\left(\tilde{\mathcal{U}}_{1}-\tilde{\mathcal{U}}_{2}\right) \tilde{\mathcal{U}}_{2, \eta}(t, \eta) \\
& \times\left(\int_{0}^{\eta} e^{-\frac{1}{A_{2}}\left(\tilde{\mathcal{Y}}_{2}(t, \eta)-\tilde{\mathcal{Y}}_{2}(t, \theta)\right)}\left(\tilde{\mathcal{D}}_{2}-\tilde{\mathcal{D}}_{1}\right) \tilde{\mathcal{U}}_{2}^{+} \tilde{\mathcal{Y}}_{2, \eta} \mathbb{1}_{\tilde{\mathcal{D}}_{1} \leqslant \tilde{\mathcal{D}}_{2}}(t, \theta) d \theta\right) d \eta \\
& +\frac{1}{A^{7}} \int_{0}^{1}\left(\tilde{\mathcal{U}}_{1}-\tilde{\mathcal{U}}_{2}\right) \tilde{\mathcal{U}}_{1, \eta}(t, \eta) \\
& \times\left(\int_{0}^{\eta} e^{-\frac{1}{A_{1}}\left(\tilde{\mathcal{Y}}_{1}(t, \eta)-\tilde{\mathcal{Y}}_{1}(t, \theta)\right)}\left(\tilde{\mathcal{D}}_{2}-\tilde{\mathcal{D}}_{1}\right) \tilde{\mathcal{U}}_{1}^{+} \tilde{\mathcal{Y}}_{1, \eta} \mathbb{1}_{\tilde{\mathcal{D}}_{2}<\tilde{\mathcal{D}}_{1}}(t, \theta) d \theta\right) d \eta \\
& +\frac{1}{A^{7}} \int_{0}^{1}\left(\tilde{\mathcal{U}}_{1}-\tilde{\mathcal{U}}_{2}\right) \tilde{\mathcal{U}}_{2, \eta}(t, \eta) \\
& \times\left(\int_{0}^{\eta} e^{-\frac{1}{A_{2}}\left(\tilde{\mathcal{Y}}_{2}(t, \eta)-\tilde{\mathcal{Y}}_{2}(t, \theta)\right)}\left(\tilde{\mathcal{U}}_{2}^{+}-\tilde{\mathcal{U}}_{1}^{+}\right) \min _{j}\left(\tilde{\mathcal{D}}_{j}\right) \tilde{\mathcal{Y}}_{2, \eta} \mathbb{1}_{\tilde{\mathcal{U}}_{1}^{+} \leqslant \tilde{\mathcal{U}}_{2}^{+}}(t, \theta) d \theta\right) d \eta \\
& +\frac{1}{A^{7}} \int_{0}^{1}\left(\tilde{\mathcal{U}}_{1}-\tilde{\mathcal{U}}_{2}\right) \tilde{\mathcal{U}}_{1, \eta}(t, \eta) \\
& \times\left(\int_{0}^{\eta} e^{-\frac{1}{A_{1}}\left(\tilde{\mathcal{Y}}_{1}(t, \eta)-\tilde{\mathcal{Y}}_{1}(t, \theta)\right)}\left(\tilde{\mathcal{U}}_{2}^{+}-\tilde{\mathcal{U}}_{1}^{+}\right) \min _{j}\left(\tilde{\mathcal{D}}_{j}\right) \tilde{\mathcal{Y}}_{1, \eta} \mathbb{1}_{\tilde{\mathcal{U}}_{2}^{+}<\tilde{\mathcal{U}}_{1}^{+}}(t, \theta) d \theta\right) d \eta \\
& +\frac{1}{A^{7}} \mathbb{1}_{A_{1} \leqslant A_{2}} \int_{0}^{1}\left(\tilde{\mathcal{U}}_{1}-\tilde{\mathcal{U}}_{2}\right) \tilde{\mathcal{U}}_{2, \eta}(t, \eta) \\
& \times\left(\int_{0}^{\eta}\left(e^{-\frac{1}{A_{2}}\left(\tilde{\mathcal{Y}}_{2}(t, \eta)-\tilde{\mathcal{Y}}_{2}(t, \theta)\right)}-e^{-\frac{1}{A_{1}}\left(\tilde{\mathcal{Y}}_{2}(t, \eta)-\tilde{\mathcal{Y}}_{2}(t, \theta)\right)}\right)\right. \\
& \left.\times \min _{j}\left(\tilde{\mathcal{D}}_{j}\right) \min _{j}\left(\tilde{\mathcal{U}}_{j}^{+}\right) \tilde{\mathcal{Y}}_{2, \eta}(t, \theta) d \theta\right) d \eta \\
& +\frac{1}{A^{7}} \mathbb{1}_{A_{2}<A_{1}} \int_{0}^{1}\left(\tilde{\mathcal{U}}_{1}-\tilde{\mathcal{U}}_{2}\right) \tilde{\mathcal{U}}_{1, \eta}(t, \eta) \\
& \times\left(\int_{0}^{\eta}\left(e^{-\frac{1}{A_{2}}\left(\tilde{\mathcal{Y}}_{1}(t, \eta)-\tilde{\mathcal{Y}}_{1}(t, \theta)\right)}-e^{-\frac{1}{A_{1}}\left(\tilde{\mathcal{Y}}_{1}(t, \eta)-\tilde{\mathcal{Y}}_{1}(t, \theta)\right)}\right)\right.
\end{aligned}
$$




$$
\begin{aligned}
& \left.\times \min _{j}\left(\tilde{\mathcal{D}}_{j}\right) \min _{j}\left(\tilde{\mathcal{U}}_{j}^{+}\right) \tilde{\mathcal{Y}}_{1, \eta}(t, \theta) d \theta\right) d \eta \\
& +\frac{1}{A^{7}} \int_{0}^{1}\left(\tilde{\mathcal{U}}_{1}-\tilde{\mathcal{U}}_{2}\right) \tilde{\mathcal{U}}_{2, \eta}(t, \eta) \\
& \times\left(\int_{0}^{\eta}\left(e^{-\frac{1}{a}\left(\tilde{\mathcal{Y}}_{2}(t, \eta)-\tilde{\mathcal{Y}}_{2}(t, \theta)\right)}-e^{-\frac{1}{a}\left(\tilde{\mathcal{Y}}_{1}(t, \eta)-\tilde{\mathcal{Y}}_{1}(t, \theta)\right)}\right)\right. \\
& \left.\times \min _{j}\left(\tilde{\mathcal{D}}_{j}\right) \min _{j}\left(\tilde{\mathcal{U}}_{j}^{+}\right) \tilde{\mathcal{Y}}_{2, \eta} \mathbb{1}_{B(\eta)}(t, \theta) d \theta\right) d \eta \\
& +\frac{1}{A^{7}} \int_{0}^{1}\left(\tilde{\mathcal{U}}_{1}-\tilde{\mathcal{U}}_{2}\right) \tilde{\mathcal{U}}_{1, \eta}(t, \eta) \\
& \times\left(\int_{0}^{\eta}\left(e^{-\frac{1}{a}\left(\tilde{\mathcal{Y}}_{2}(t, \eta)-\tilde{\mathcal{Y}}_{2}(t, \theta)\right)}-e^{-\frac{1}{a}\left(\tilde{\mathcal{Y}}_{1}(t, \eta)-\tilde{\mathcal{Y}}_{1}(t, \theta)\right)}\right)\right. \\
& \left.\times \min _{j}\left(\tilde{\mathcal{D}}_{j}\right) \min _{j}\left(\tilde{\mathcal{U}}_{j}^{+}\right) \tilde{\mathcal{Y}}_{1, \eta} \mathbb{1}_{B^{c}(\eta)}(t, \theta) d \theta\right) d \eta \\
& -\frac{1}{A^{7}} \int_{0}^{1}\left(\tilde{\mathcal{U}}_{1}-\tilde{\mathcal{U}}_{2}\right)\left(\tilde{\mathcal{U}}_{1}-\tilde{\mathcal{U}}_{2}\right)_{\eta}(t, \eta) \\
& \times \min _{k}\left(\int_{0}^{\eta} \min _{j}\left(e^{-\frac{1}{a}\left(\tilde{\mathcal{Y}}_{j}(t, \eta)-\tilde{\mathcal{Y}}_{j}(t, \theta)\right)}\right) \min _{j}\left(\tilde{\mathcal{D}}_{j}\right) \min _{j}\left(\tilde{\mathcal{U}}_{j}^{+}\right) \tilde{\mathcal{Y}}_{k, \eta}(t, \theta) d \theta\right) d \eta \\
& +\frac{1}{A^{7}} \int_{0}^{1}\left(\tilde{\mathcal{U}}_{1}-\tilde{\mathcal{U}}_{2}\right) \tilde{\mathcal{U}}_{2, \eta} \mathbb{1}_{D^{c}}(t, \eta) \\
& \times\left(\int_{0}^{\eta} \min _{j}\left(e^{-\frac{1}{a}\left(\tilde{\mathcal{Y}}_{j}(t, \eta)-\tilde{\mathcal{Y}}_{j}(t, \theta)\right)}\right) \min _{j}\left(\tilde{\mathcal{D}}_{j}\right) \min _{j}\left(\tilde{\mathcal{U}}_{j}^{+}\right)\left(\tilde{\mathcal{Y}}_{2, \eta}-\tilde{\mathcal{Y}}_{1, \eta}\right)(t, \theta) d \theta\right) d \eta \\
& +\frac{1}{A^{7}} \int_{0}^{1}\left(\tilde{\mathcal{U}}_{1}-\tilde{\mathcal{U}}_{2}\right) \tilde{\mathcal{U}}_{1, \eta} \mathbb{1}_{D}(t, \eta) \\
& \times\left(\int_{0}^{\eta} \min _{j}\left(e^{-\frac{1}{a}\left(\tilde{\mathcal{Y}}_{j}(t, \eta)-\tilde{\mathcal{Y}}_{j}(t, \theta)\right)}\right) \min _{j}\left(\tilde{\mathcal{D}}_{j}\right) \min _{j}\left(\tilde{\mathcal{U}}_{j}^{+}\right)\left(\tilde{\mathcal{Y}}_{2, \eta}-\tilde{\mathcal{Y}}_{1, \eta}\right)(t, \theta) d \theta\right) d \eta \\
& =Z_{1}+Z_{2}+Z_{3}+Z_{4}+Z_{5}+Z_{6}+Z_{7}+Z_{8}+Z_{9}+Z_{10}+Z_{11}+Z_{12}+Z_{13} \text {, }
\end{aligned}
$$

where the set $D$ is defined by (5.21). At this point, it cannot come as a surprise that we have to treat these terms separately.

Let us start with $Z_{1}\left(Z_{2}\right.$ is similar):

$$
\begin{aligned}
\left|Z_{1}\right| \leqslant & \frac{2}{A^{7}}\left|A_{1}-A_{2}\right| \int_{0}^{1}\left|\tilde{\mathcal{U}}_{1}-\tilde{\mathcal{U}}_{2}\right|\left|\tilde{\mathcal{U}}_{1, \eta}\right|(t, \eta) \\
& \times\left(\int_{0}^{\eta} e^{-\frac{1}{A_{1}}\left(\tilde{\mathcal{Y}}_{1}(t, \eta)-\tilde{\mathcal{Y}}_{1}(t, \theta)\right)} \tilde{\mathcal{U}}_{1}^{2} \tilde{\mathcal{Y}}_{1, \eta}(t, \theta) d \theta\right)^{1 / 2} \\
& \times\left(\int_{0}^{\eta} e^{-\frac{1}{A_{1}}\left(\tilde{\mathcal{Y}}_{1}(t, \eta)-\tilde{\mathcal{Y}}_{1}(t, \theta)\right)} \tilde{\mathcal{P}}_{1}^{2} \tilde{\mathcal{Y}}_{1, \eta}(t, \theta) d \theta\right)^{1 / 2} d \eta
\end{aligned}
$$




$$
\begin{aligned}
& \leqslant \frac{2 \sqrt{6}}{A^{4}}\left|A_{1}-A_{2}\right| \int_{0}^{1}\left|\tilde{\mathcal{U}}_{1}-\tilde{\mathcal{U}}_{2}\right| \tilde{\mathcal{P}}_{1}\left|\tilde{\mathcal{U}}_{1, \eta}\right|(t, \eta) d \eta \\
& \leqslant \mathcal{O}(1)\left(\left\|\tilde{\mathcal{U}}_{1}-\tilde{\mathcal{U}}_{2}\right\|^{2}+\left|A_{1}-A_{2}\right|^{2}\right) .
\end{aligned}
$$

The terms $Z_{3}$ and $Z_{4}$ go as follows:

$$
\begin{aligned}
\left|Z_{3}\right|= & \frac{1}{A^{7}} \mid \int_{0}^{1}\left(\tilde{\mathcal{U}}_{1}-\tilde{\mathcal{U}}_{2}\right) \tilde{\mathcal{U}}_{2, \eta}(t, \eta) \\
& \times\left(\int_{0}^{\eta} e^{-\frac{1}{A_{2}}\left(\tilde{\mathcal{Y}}_{2}(t, \eta)-\tilde{\mathcal{Y}}_{2}(t, \theta)\right)}\left(\tilde{\mathcal{D}}_{2}-\tilde{\mathcal{D}}_{1}\right) \tilde{\mathcal{U}}_{2}^{+} \tilde{\mathcal{Y}}_{2, \eta} \mathbb{1}_{\tilde{\mathcal{D}}_{1} \leqslant \tilde{\mathcal{D}}_{2}}(t, \theta) d \theta\right) d \eta \mid \\
\leqslant & \mathcal{O}(1)\left(\left\|\tilde{\mathcal{U}}_{1}-\tilde{\mathcal{U}}_{2}\right\|^{2}+\left\|\tilde{\mathcal{Y}}_{1}-\tilde{\mathcal{Y}}_{2}\right\|^{2}+\left\|\tilde{\mathcal{P}}_{1}^{1 / 2}-\tilde{\mathcal{P}}_{2}^{1 / 2}\right\|^{2}+\left|A_{1}-A_{2}\right|^{2}\right),
\end{aligned}
$$

by Lemma A.9. The terms $Z_{5}$ and $Z_{6}$ can be treated similarly:

$$
\begin{aligned}
\left|Z_{5}\right|= & \frac{1}{A^{7}} \mid \int_{0}^{1}\left(\tilde{\mathcal{U}}_{1}-\tilde{\mathcal{U}}_{2}\right) \tilde{\mathcal{U}}_{2, \eta}(t, \eta) \\
& \times\left(\int_{0}^{\eta} e^{-\frac{1}{A_{2}}\left(\tilde{\mathcal{Y}}_{2}(t, \eta)-\tilde{\mathcal{Y}}_{2}(t, \theta)\right)}\left(\tilde{\mathcal{U}}_{2}^{+}-\tilde{\mathcal{U}}_{1}^{+}\right) \min _{j}\left(\tilde{\mathcal{D}}_{j}\right) \tilde{\mathcal{Y}}_{2, \eta} \mathbb{1}_{\tilde{\mathcal{U}}_{1}^{+} \leqslant \tilde{\mathcal{U}}_{2}^{+}}(t, \theta) d \theta\right) d \eta \mid \\
\leqslant & \left\|\tilde{\mathcal{U}}_{1}-\tilde{\mathcal{U}}_{2}\right\|^{2}+\frac{1}{A^{14}} \int_{0}^{1} \tilde{\mathcal{U}}_{2, \eta}^{2}(t, \eta) \\
& \times\left(\int_{0}^{\eta} e^{-\frac{1}{A_{2}}\left(\tilde{\mathcal{Y}}_{2}(t, \eta)-\tilde{\mathcal{Y}}_{2}(t, \theta)\right)}\left(\tilde{\mathcal{U}}_{2}^{+}-\tilde{\mathcal{U}}_{1}^{+}\right) \min _{j}\left(\tilde{\mathcal{D}}_{j}\right) \tilde{\mathcal{Y}}_{2, \eta} \mathbb{1}_{\tilde{\mathcal{U}}_{1}^{+} \leqslant \tilde{\mathcal{U}}_{2}^{+}}(t, \theta) d \theta\right)^{2} d \eta \\
\leqslant & \mathcal{O}(1)\left\|\tilde{\mathcal{U}}_{1}-\tilde{\mathcal{U}}_{2}\right\|^{2},
\end{aligned}
$$

by applying (4.16e), estimating $\tilde{\mathcal{D}}_{2} \leqslant 2 A_{2} \tilde{\mathcal{P}}_{2}$ (cf. (4.15n)), and subsequently $2 \sqrt{2} \tilde{\mathcal{P}}_{2} \tilde{\mathcal{U}}_{2, \eta}^{2} \leqslant A_{2}^{6}$ (cf. (4.15q)). The terms $Z_{7}$ and $Z_{8}$ follow this pattern:

$$
\begin{aligned}
\left|Z_{8}\right| \leqslant & \frac{4}{A^{7} a e} \int_{0}^{1}\left|\tilde{\mathcal{U}}_{1}-\tilde{\mathcal{U}}_{2}\right|\left|\tilde{\mathcal{U}}_{1, \eta}\right|(t, \eta) \\
& \times\left(\int_{0}^{\eta} e^{-\frac{3}{4 A_{1}}\left(\tilde{\mathcal{Y}}_{1}(t, \eta)-\tilde{\mathcal{Y}}_{1}(t, \theta)\right)} 2 A_{1} \tilde{\mathcal{P}}_{1} \tilde{\mathcal{Y}}_{1, \eta}\left|\tilde{\mathcal{U}}_{2}\right|(t, \theta) d \theta\right) d \eta\left|A_{1}-A_{2}\right| \\
\leqslant & \frac{4 \sqrt{2}}{A^{5} e} \int_{0}^{1}\left|\tilde{\mathcal{U}}_{1}-\tilde{\mathcal{U}}_{2}\right|\left|\tilde{\mathcal{U}}_{1, \eta}\right|(t, \eta)\left(\int_{0}^{\eta} e^{-\frac{1}{A_{2}}\left(\tilde{\mathcal{Y}}_{1}(t, \eta)-\tilde{\mathcal{Y}}_{2}(t, \eta)\right)} \tilde{\mathcal{P}}_{1}^{2} \tilde{\mathcal{Y}}_{1, \eta}(t, \theta) d \theta\right)^{1 / 2} \\
& \times\left(\int_{0}^{\eta} e^{-\frac{1}{2 A_{2}}\left(\tilde{\mathcal{Y}}_{1}(t, \eta)-\tilde{\mathcal{Y}}_{1}(t, \theta)\right)} \tilde{\mathcal{Y}}_{1, \eta}(t, \theta) d \theta\right)^{1 / 2} d \eta\left|A_{1}-A_{2}\right|
\end{aligned}
$$




$$
\begin{aligned}
& \leqslant \frac{4 \sqrt{6}}{A^{2} e} \int_{0}^{1}\left|\tilde{\mathcal{U}}_{1}-\tilde{\mathcal{U}}_{2}\right| \tilde{\mathcal{P}}_{1}^{1 / 2}\left|\tilde{\mathcal{U}}_{1, \eta}\right|(t, \eta) d \eta\left|A_{1}-A_{2}\right| \\
& \leqslant \mathcal{O}(1)\left(\left\|\tilde{\mathcal{U}}_{1}-\tilde{\mathcal{U}}_{2}\right\|^{2}+\left|A_{1}-A_{2}\right|^{2}\right) .
\end{aligned}
$$

The terms $Z_{9}$ and $Z_{10}$ follow this pattern:

$$
\begin{aligned}
& \left|Z_{9}\right|=\frac{1}{A^{7}} \mid \int_{0}^{1}\left(\tilde{\mathcal{U}}_{1}-\tilde{\mathcal{U}}_{2}\right) \tilde{\mathcal{U}}_{2, \eta}(t, \eta)\left(\int_{0}^{\eta}\left(e^{-\frac{1}{a}\left(\tilde{\mathcal{Y}}_{2}(t, \eta)-\tilde{\mathcal{Y}}_{2}(t, \theta)\right)}-e^{-\frac{1}{a}\left(\tilde{\mathcal{Y}}_{1}(t, \eta)-\tilde{\mathcal{Y}}_{1}(t, \theta)\right)}\right)\right. \\
& \left.\times \min _{j}\left(\tilde{\mathcal{D}}_{j}\right) \min _{j}\left(\tilde{\mathcal{U}}_{j}^{+}\right) \tilde{\mathcal{Y}}_{2, \eta} \mathbb{1}_{B(\eta)}(t, \theta) d \theta\right) d \eta \\
& \leqslant \frac{a}{\sqrt{2} A^{7}} \int_{0}^{1}\left|\tilde{\mathcal{U}}_{1}-\tilde{\mathcal{U}}_{2}\right|\left|\tilde{\mathcal{U}}_{2, \eta}\right|(t, \eta) \\
& \times\left(\int_{0}^{\eta}\left(\left|\tilde{\mathcal{Y}}_{2}(t, \eta)-\tilde{\mathcal{Y}}_{1}(t, \eta)\right|+\left|\tilde{\mathcal{Y}}_{2}(t, \theta)-\tilde{\mathcal{Y}}_{1}(t, \theta)\right|\right)\right. \\
& \left.\times e^{-\frac{1}{a}\left(\tilde{\mathcal{Y}}_{2}(t, \eta)-\tilde{\mathcal{Y}}_{2}(t, \theta)\right)} \tilde{\mathcal{D}}_{2} \tilde{\mathcal{Y}}_{2, \eta}(t, \theta) d \theta\right) d \eta \\
& \leqslant \frac{1}{\sqrt{2} A^{6}} \int_{0}^{1}\left|\tilde{\mathcal{U}}_{1}-\tilde{\mathcal{U}}_{2}\right|\left|\tilde{\mathcal{Y}}_{2}-\tilde{\mathcal{Y}}_{1}\right|\left|\tilde{\mathcal{U}}_{2, \eta}\right|(t, \eta) \\
& \times\left(\int_{0}^{\eta} e^{-\frac{1}{A_{2}}\left(\tilde{\mathcal{Y}}_{2}(t, \eta)-\tilde{\mathcal{Y}}_{2}(t, \theta)\right)} \tilde{\mathcal{D}}_{2} \tilde{\mathcal{Y}}_{2, \eta}(t, \theta) d \theta\right) d \eta \\
& +\frac{1}{\sqrt{2} A^{6}} \int_{0}^{1}\left|\tilde{\mathcal{U}}_{1}-\tilde{\mathcal{U}}_{2}\right|\left|\tilde{\mathcal{U}}_{2, \eta}\right|(t, \eta) \\
& \times\left(\int_{0}^{\eta} e^{-\frac{1}{A_{2}}\left(\tilde{\mathcal{Y}}_{2}(t, \eta)-\tilde{\mathcal{Y}}_{2}(t, \theta)\right)} \tilde{\mathcal{D}}_{2} \tilde{\mathcal{Y}}_{2, \eta}\left|\tilde{\mathcal{Y}}_{2}-\tilde{\mathcal{Y}}_{1}\right|(t, \theta) d \theta\right) d \eta \\
& \leqslant \frac{\sqrt{2}}{A^{5}} \int_{0}^{1}\left|\tilde{\mathcal{U}}_{1}-\tilde{\mathcal{U}}_{2}\right|\left|\tilde{\mathcal{Y}}_{2}-\tilde{\mathcal{Y}}_{1}\right|\left|\tilde{\mathcal{U}}_{2, \eta}\right|(t, \eta) \\
& \times\left(\int_{0}^{\eta} e^{-\frac{1}{A_{2}}\left(\tilde{\mathcal{Y}}_{2}(t, \eta)-\tilde{\mathcal{Y}}_{2}(t, \theta)\right)} \tilde{\mathcal{P}}_{2}^{2} \tilde{\mathcal{Y}}_{2, \eta}(t, \theta) d \theta\right)^{1 / 2} \\
& \times\left(\int_{0}^{\eta} e^{-\frac{1}{A_{2}}\left(\tilde{\mathcal{Y}}_{2}(t, \eta)-\tilde{\mathcal{Y}}_{2}(t, \theta)\right)} \tilde{\mathcal{Y}}_{2, \eta}(t, \theta) d \theta\right)^{1 / 2} d \eta \\
& +\left\|\tilde{\mathcal{U}}_{1}-\tilde{\mathcal{U}}_{2}\right\|^{2}+\frac{2}{A^{10}} \int_{0}^{1} \tilde{\mathcal{U}}_{2, \eta}^{2}(t, \eta)\left(\int_{0}^{\eta} e^{-\frac{3}{2 A_{2}}\left(\tilde{\mathcal{Y}}_{2}(t, \eta)-\tilde{\mathcal{Y}}_{2}(t, \theta)\right)} \tilde{\mathcal{P}}_{2} \tilde{\mathcal{Y}}_{2, \eta}(t, \theta) d \theta\right) \\
& \times\left(\int_{0}^{\eta} e^{-\frac{1}{2 A_{2}}\left(\tilde{\mathcal{Y}}_{2}(t, \eta)-\tilde{\mathcal{Y}}_{2}(t, \theta)\right)} \tilde{\mathcal{P}}_{2} \tilde{\mathcal{Y}}_{2, \eta}\left|\tilde{\mathcal{Y}}_{2}-\tilde{\mathcal{Y}}_{1}\right|^{2}(t, \theta) d \theta\right) d \eta \\
& \leqslant \mathcal{O}(1)\left(\left\|\tilde{\mathcal{U}}_{1}-\tilde{\mathcal{U}}_{2}\right\|^{2}+\left\|\tilde{\mathcal{Y}}_{1}-\tilde{\mathcal{Y}}_{2}\right\|^{2}\right) \text {. }
\end{aligned}
$$


The term $Z_{11}$ :

$$
\begin{aligned}
\left|Z_{11}\right|= & \frac{1}{A^{7}} \mid \int_{0}^{1}\left(\tilde{\mathcal{U}}_{1}-\tilde{\mathcal{U}}_{2}\right)\left(\tilde{\mathcal{U}}_{1}-\tilde{\mathcal{U}}_{2}\right)_{\eta}(t, \eta) \\
& \times \min _{k}\left(\int_{0}^{\eta} \min _{j}\left(e^{-\frac{1}{a}\left(\tilde{\mathcal{Y}}_{j}(t, \eta)-\tilde{\mathcal{Y}}_{j}(t, \theta)\right)}\right) \min _{j}\left(\tilde{\mathcal{D}}_{j}\right) \min _{j}\left(\tilde{\mathcal{U}}_{j}^{+}\right) \tilde{\mathcal{Y}}_{k, \eta}(t, \theta) d \theta\right) d \eta \mid \\
\leqslant & \mathcal{O}(1)\left\|\tilde{\mathcal{U}}_{1}-\tilde{\mathcal{U}}_{2}\right\|_{2}^{2},
\end{aligned}
$$

following the estimates employed for the term $\bar{B}_{37}$; see (5.23).

The terms $Z_{12}$ and $Z_{13}$ may be treated as follows:

$$
\begin{aligned}
& \left|Z_{12}\right|=\frac{1}{A^{7}} \mid \int_{0}^{1}\left(\tilde{\mathcal{U}}_{1}-\tilde{\mathcal{U}}_{2}\right) \tilde{\mathcal{U}}_{2, \eta} \mathbb{1}_{D^{c}}(t, \eta)\left(\int_{0}^{\eta} \min _{j}\left(e^{-\frac{1}{a}\left(\tilde{\mathcal{Y}}_{j}(t, \eta)-\tilde{\mathcal{Y}}_{j}(t, \theta)\right)}\right)\right. \\
& \left.\times \min _{j}\left(\tilde{\mathcal{D}}_{j}\right) \min _{j}\left(\tilde{\mathcal{U}}_{j}^{+}\right)\left(\tilde{\mathcal{Y}}_{2, \eta}-\tilde{\mathcal{Y}}_{1, \eta}\right)(t, \theta) d \theta\right) d \eta \mid \\
& =\frac{1}{A^{7}} \mid \int_{0}^{1}\left(\tilde{\mathcal{U}}_{1}-\tilde{\mathcal{U}}_{2}\right) \tilde{\mathcal{U}}_{2, \eta} \mathbb{1}_{D^{c}}(t, \eta) \\
& \times\left[\left.\left(\min _{j}\left(e^{-\frac{1}{a}\left(\tilde{\mathcal{Y}}_{j}(t, \eta)-\tilde{\mathcal{Y}}_{j}(t, \theta)\right)}\right) \min _{j}\left(\tilde{\mathcal{D}}_{j}\right) \min _{j}\left(\tilde{\mathcal{U}}_{j}^{+}\right)\left(\tilde{\mathcal{Y}}_{2}-\tilde{\mathcal{Y}}_{1}\right)(t, \theta)\right)\right|_{\theta=0} ^{\eta}\right. \\
& \left.-\int_{0}^{\eta}\left(\tilde{\mathcal{Y}}_{2}-\tilde{\mathcal{Y}}_{1}\right) \frac{d}{d \theta}\left(\min _{j}\left(e^{-\frac{1}{a}\left(\tilde{\mathcal{Y}}_{j}(t, \eta)-\tilde{\mathcal{Y}}_{j}(t, \theta)\right)}\right) \min _{j}\left(\tilde{\mathcal{D}}_{j}\right) \min _{j}\left(\tilde{\mathcal{U}}_{j}^{+}\right)\right)(t, \theta) d \theta\right] d \eta \mid \\
& \leqslant \frac{1}{A^{7}}\left|\int_{0}^{1}\left(\tilde{\mathcal{U}}_{1}-\tilde{\mathcal{U}}_{2}\right) \tilde{\mathcal{U}}_{2, \eta} \mathbb{1}_{D^{c}} \min _{j}\left(\tilde{\mathcal{D}}_{j}\right) \min _{j}\left(\tilde{\mathcal{U}}_{j}^{+}\right)\left(\tilde{\mathcal{Y}}_{2}-\tilde{\mathcal{Y}}_{1}\right)(t, \eta) d \eta\right| \\
& +\frac{1}{A^{7}} \mid \int_{0}^{1}\left(\tilde{\mathcal{U}}_{1}-\tilde{\mathcal{U}}_{2}\right) \tilde{\mathcal{U}}_{2, \eta} \mathbb{1}_{D^{c}}(t, \eta) \\
& \times\left(\int_{0}^{\eta}\left(\tilde{\mathcal{Y}}_{2}-\tilde{\mathcal{Y}}_{1}\right) \frac{d}{d \theta}\left(\min _{j}\left(e^{-\frac{1}{a}\left(\tilde{\mathcal{Y}}_{j}(t, \eta)-\tilde{\mathcal{Y}}_{j}(t, \theta)\right)}\right) \min _{j}\left(\tilde{\mathcal{D}}_{j}\right) \min _{j}\left(\tilde{\mathcal{U}}_{j}^{+}\right)\right)(t, \theta) d \theta\right) d \eta \mid \\
& \leqslant \frac{A^{2}}{4} \int_{0}^{1}\left|\tilde{\mathcal{U}}_{1}-\tilde{\mathcal{U}}_{2}\right|\left|\tilde{\mathcal{Y}}_{2}-\tilde{\mathcal{Y}}_{1}\right|(t, \eta) d \eta \\
& +\frac{1}{A^{7}} \int_{0}^{1}\left|\tilde{\mathcal{U}}_{1}-\tilde{\mathcal{U}}_{2}\right|\left|\tilde{\mathcal{U}}_{2, \eta}\right|(t, \eta)\left(\int_{0}^{\eta}\left|\tilde{\mathcal{Y}}_{1}-\tilde{\mathcal{Y}}_{2}\right| \mid \frac{d}{d \theta}\left(\min _{j}\left(e^{-\frac{1}{a}\left(\tilde{\mathcal{Y}}_{j}(t, \eta)-\tilde{\mathcal{Y}}_{j}(t, \theta)\right)}\right)\right.\right. \\
& \left.\left.\times \min _{j}\left(\tilde{\mathcal{D}}_{j}\right) \min _{j}\left(\tilde{\mathcal{U}}_{j}^{+}\right)\right) \mid(t, \theta) d \theta\right) d \eta \\
& =\tilde{M}_{1}+\tilde{M}_{2} \text {. }
\end{aligned}
$$

Here we find

$$
\tilde{M}_{1} \leqslant \mathcal{O}(1)\left(\left\|\tilde{\mathcal{U}}_{1}-\tilde{\mathcal{U}}_{2}\right\|^{2}+\left\|\tilde{\mathcal{Y}}_{1}-\tilde{\mathcal{Y}}_{2}\right\|^{2}\right)
$$


while $\tilde{M}_{2}$ requires more care:

$$
\begin{aligned}
& \tilde{M}_{2}=\frac{1}{A^{7}} \int_{0}^{1}\left|\tilde{\mathcal{U}}_{1}-\tilde{\mathcal{U}}_{2}\right| \tilde{\mathcal{U}}_{2, \eta} \mid(t, \eta)\left(\int_{0}^{\eta}\left|\tilde{\mathcal{Y}}_{1}-\tilde{\mathcal{Y}}_{2}\right| \mid \frac{d}{d \theta}\left(\min _{j}\left(e^{-\frac{1}{a}\left(\tilde{\mathcal{Y}}_{j}(t, \eta)-\tilde{\mathcal{Y}}_{j}(t, \theta)\right)}\right)\right.\right. \\
& \left.\left.\times \min _{j}\left(\tilde{\mathcal{D}}_{j}\right) \min _{j}\left(\tilde{\mathcal{U}}_{j}^{+}\right)\right)(t, \theta) \mid d \theta\right) d \eta \\
& \leqslant \frac{1}{A^{7}} \int_{0}^{1}\left|\tilde{\mathcal{U}}_{1}-\tilde{\mathcal{U}}_{2}\right|\left|\tilde{\mathcal{U}}_{2, \eta}\right|(t, \eta)\left(\int_{0}^{\eta}\left|\tilde{\mathcal{Y}}_{1}-\tilde{\mathcal{Y}}_{2}\right|\right. \\
& \times \mid \frac{d}{d \theta}\left(\min _{j}\left(e^{-\frac{1}{a}\left(\tilde{\mathcal{Y}}_{j}(t, \eta)-\tilde{\mathcal{Y}}_{j}(t, \theta)\right)}\right)\right) \min _{j}\left(\tilde{\mathcal{D}}_{j}\right) \min _{j}\left(\tilde{\mathcal{U}}_{j}^{+}\right) \\
& \left.+\min _{j}\left(e^{-\frac{1}{a}\left(\tilde{\mathcal{Y}}_{j}(t, \eta)-\tilde{\mathcal{Y}}_{j}(t, \theta)\right)}\right) \frac{d}{d \theta}\left(\min _{j}\left(\tilde{\mathcal{D}}_{j}\right) \min _{j}\left(\tilde{\mathcal{U}}_{j}^{+}\right)\right) \mid(t, \theta) d \theta\right) d \eta \\
& \leqslant \frac{1}{A^{7}} \int_{0}^{1}\left|\tilde{\mathcal{U}}_{1}-\tilde{\mathcal{U}}_{2}\right|\left|\tilde{\mathcal{U}}_{2, \eta}\right|(t, \eta)\left(\int_{0}^{\eta}\left|\tilde{\mathcal{Y}}_{1}-\tilde{\mathcal{Y}}_{2}\right| \mid \frac{d}{d \theta}\left(\min _{j}\left(e^{-\frac{1}{a}\left(\tilde{\mathcal{Y}}_{j}(t, \eta)-\tilde{\mathcal{Y}}_{j}(t, \theta)\right)}\right)\right)\right. \\
& \left.\times \min _{j}\left(\tilde{\mathcal{D}}_{j}\right) \min _{j}\left(\tilde{\mathcal{U}}_{j}^{+}\right)(t, \theta) \mid d \theta\right) d \eta \\
& +\frac{1}{A^{7}} \int_{0}^{1}\left|\tilde{\mathcal{U}}_{1}-\tilde{\mathcal{U}}_{2}\right|\left|\tilde{\mathcal{U}}_{2, \eta}\right|(t, \eta)\left(\int_{0}^{\eta}\left|\tilde{\mathcal{Y}}_{1}-\tilde{\mathcal{Y}}_{2}\right| \mid \min _{j}\left(e^{-\frac{1}{a}\left(\tilde{\mathcal{Y}}_{j}(t, \eta)-\tilde{\mathcal{Y}}_{j}(t, \theta)\right)}\right)\right. \\
& \left.\times \frac{d}{d \theta}\left(\min _{j}\left(\tilde{\mathcal{D}}_{j}\right) \min _{j}\left(\tilde{\mathcal{U}}_{j}^{+}\right)\right)(t, \theta) \mid d \theta\right) d \eta \\
& \leqslant \frac{1}{a A^{7}} \int_{0}^{1}\left|\tilde{\mathcal{U}}_{1}-\tilde{\mathcal{U}}_{2}\right|\left|\tilde{\mathcal{U}}_{2, \eta}\right|(t, \eta)\left(\int_{0}^{\eta}\left|\tilde{\mathcal{Y}}_{1}-\tilde{\mathcal{Y}}_{2}\right| \min _{j}\left(e^{-\frac{1}{a}\left(\tilde{\mathcal{Y}}_{j}(t, \eta)-\tilde{\mathcal{Y}}_{j}(t, \theta)\right)}\right)\right. \\
& \left.\times \max _{j}\left(\tilde{\mathcal{Y}}_{j, \eta}\right) \min _{j}\left(\tilde{\mathcal{D}}_{j}\right) \min _{j}\left(\tilde{\mathcal{U}}_{j}^{+}\right)(t, \theta) d \theta\right) d \eta \\
& +\mathcal{O}(1) \frac{1}{A^{5 / 2}} \int_{0}^{1}\left|\tilde{\mathcal{U}}_{1}-\tilde{\mathcal{U}}_{2}\right|\left|\tilde{\mathcal{U}}_{2, \eta}\right|(t, \eta) \\
& \times\left(\int_{0}^{\eta}\left|\tilde{\mathcal{Y}}_{1}-\tilde{\mathcal{Y}}_{2}\right| \min _{j}\left(e^{-\frac{1}{a}\left(\tilde{\mathcal{Y}}_{j}(t, \eta)-\tilde{\mathcal{Y}}_{j}(t, \theta)\right)}\right)\left(\min _{j}\left(\tilde{\mathcal{D}}_{j}\right)^{1 / 2}+\left|\tilde{\mathcal{U}}_{2}\right|\right)(t, \theta) d \theta\right) d \eta \\
& =\tilde{M}_{21}+\tilde{M}_{22} \text {, }
\end{aligned}
$$

where estimates for the derivatives come from Lemmas A.2 and A.4. We find for the term $\tilde{M}_{21}$ that

$$
\begin{aligned}
\tilde{M}_{21}= & \frac{1}{a A^{7}} \int_{0}^{1}\left|\tilde{\mathcal{U}}_{1}-\tilde{\mathcal{U}}_{2}\right|\left|\tilde{\mathcal{U}}_{2, \eta}\right|(t, \eta)\left(\int_{0}^{\eta}\left|\tilde{\mathcal{Y}}_{1}-\tilde{\mathcal{Y}}_{2}\right| \min _{j}\left(e^{-\frac{1}{a}\left(\tilde{\mathcal{Y}}_{j}(t, \eta)-\tilde{\mathcal{Y}}_{j}(t, \theta)\right)}\right)\right. \\
& \left.\times \max _{j}\left(\tilde{\mathcal{Y}}_{j, \eta}\right) \min _{j}\left(\tilde{\mathcal{D}}_{j}\right) \min _{j}\left(\tilde{\mathcal{U}}_{j}^{+}\right)(t, \theta) d \theta\right) d \eta
\end{aligned}
$$




$$
\begin{aligned}
& \leqslant\left\|\tilde{\mathcal{U}}_{1}-\tilde{\mathcal{U}}_{2}\right\|^{2}+\frac{1}{a^{2} A^{14}} \int_{0}^{1} \tilde{\mathcal{U}}_{2, \eta}^{2}(t, \eta)\left(\int_{0}^{\eta}\left|\tilde{\mathcal{Y}}_{1}-\tilde{\mathcal{Y}}_{2}\right| \min _{j}\left(e^{-\frac{1}{a}\left(\tilde{\mathcal{Y}}_{j}(t, \eta)-\tilde{\mathcal{Y}}_{j}(t, \theta)\right)}\right)\right. \\
& \left.\times \max _{j}\left(\tilde{\mathcal{Y}}_{j, \eta}\right) \min _{j}\left(\tilde{\mathcal{D}}_{j}\right) \min _{j}\left(\tilde{\mathcal{U}}_{j}^{+}\right)(t, \theta) d \theta\right)^{2} d \eta \\
& \leqslant\left\|\tilde{\mathcal{U}}_{1}-\tilde{\mathcal{U}}_{2}\right\|^{2}+\frac{1}{2} \int_{0}^{1} \tilde{\mathcal{U}}_{2, \eta}^{2}(t, \eta) \\
& \times\left(\int_{0}^{\eta}\left|\tilde{\mathcal{Y}}_{1}-\tilde{\mathcal{Y}}_{2}\right| \min _{j}\left(e^{-\frac{1}{a}\left(\tilde{\mathcal{Y}}_{j}(t, \eta)-\tilde{\mathcal{Y}}_{j}(t, \theta)\right)}\right) d \theta\right)^{2} d \eta \\
& \leqslant\left\|\tilde{\mathcal{U}}_{1}-\tilde{\mathcal{U}}_{2}\right\|^{2}+\frac{A^{3}}{2} \int_{0}^{1} \tilde{\mathcal{Y}}_{2, \eta}(t, \eta) \\
& \times\left(\int_{0}^{\eta}\left(\tilde{\mathcal{Y}}_{1}-\tilde{\mathcal{Y}}_{2}\right)^{2} e^{-\frac{1}{a}\left(\tilde{\mathcal{Y}}_{2}(t, \eta)-\tilde{\mathcal{Y}}_{2}(t, \theta)\right)} d \theta\right)\left(\int_{0}^{\eta} e^{-\frac{1}{a}\left(\tilde{\mathcal{Y}}_{2}(t, \eta)-\tilde{\mathcal{Y}}_{2}(t, \theta)\right)} d \theta\right) d \eta \\
& \leqslant\left\|\tilde{\mathcal{U}}_{1}-\tilde{\mathcal{U}}_{2}\right\|^{2}+\frac{A^{3}}{2} \int_{0}^{1} \tilde{\mathcal{Y}}_{2, \eta}(t, \eta) e^{-\frac{1}{a} \tilde{\mathcal{Y}}_{2}(t, \eta)}\left(\int_{0}^{\eta} e^{\frac{1}{a} \tilde{\mathcal{Y}}_{2}(t, \theta)} d \theta\right) d \eta\left\|\tilde{\mathcal{Y}}_{1}-\tilde{\mathcal{Y}}_{2}\right\|^{2} \\
& \leqslant \mathcal{O}(1)\left(\left\|\tilde{\mathcal{U}}_{1}-\tilde{\mathcal{U}}_{2}\right\|^{2}+\left\|\tilde{\mathcal{Y}}_{1}-\tilde{\mathcal{Y}}_{2}\right\|^{2}\right),
\end{aligned}
$$

where we used the estimate

$$
\max _{j}\left(\tilde{\mathcal{Y}}_{j, \eta}\right) \min _{j}\left(\tilde{\mathcal{D}}_{j}\right) \leqslant 2 \max _{j}\left(\tilde{\mathcal{Y}}_{j, \eta}\right) \min _{j}\left(A_{j} \tilde{\mathcal{P}}_{j}\right) \leqslant 2 A \max _{j}\left(\tilde{\mathcal{P}}_{j} \tilde{\mathcal{Y}}_{j, \eta}\right) \leqslant A^{6}
$$

using (4.15e) and (4.15n), as well as (4.15q) and (4.16a). The term $\tilde{M}_{22}$ reads as

$$
\begin{aligned}
\tilde{M}_{22}= & \mathcal{O}(1) \frac{1}{A^{5 / 2}} \int_{0}^{1}\left|\tilde{\mathcal{U}}_{1}-\tilde{\mathcal{U}}_{2}\right|\left|\tilde{\mathcal{U}}_{2, \eta}\right|(t, \eta) \\
& \times\left(\int_{0}^{\eta}\left|\tilde{\mathcal{Y}}_{1}-\tilde{\mathcal{Y}}_{2}\right| \min _{j}\left(e^{-\frac{1}{a}\left(\tilde{\mathcal{Y}}_{j}(t, \eta)-\tilde{\mathcal{Y}}_{j}(t, \theta)\right)}\right)\left(\min _{j}\left(\tilde{\mathcal{D}}_{j}\right)^{1 / 2}+\left|\tilde{\mathcal{U}}_{2}\right|\right)(t, \theta) d \theta\right) d \eta \\
\leqslant & \left\|\tilde{\mathcal{U}}_{1}-\tilde{\mathcal{U}}_{2}\right\|^{2}+\mathcal{O}(1) \frac{1}{A^{5}} \int_{0}^{1} \tilde{\mathcal{U}}_{2, \eta}^{2}(t, \eta) \\
& \times\left(\int_{0}^{\eta} e^{-\frac{1}{a}\left(\tilde{\mathcal{Y}}_{2}(t, \eta)-\tilde{\mathcal{Y}}_{2}(t, \theta)\right)}\left|\tilde{\mathcal{Y}}_{1}-\tilde{\mathcal{Y}}_{2}\right| \tilde{\mathcal{P}}_{2}^{1 / 2}(t, \theta) d \theta\right)^{2} d \eta \\
\leqslant & \left\|\tilde{\mathcal{U}}_{1}-\tilde{\mathcal{U}}_{2}\right\|^{2}+\mathcal{O}(1) \frac{1}{A^{5}} \int_{0}^{1} \tilde{\mathcal{U}}_{2, \eta}^{2}(t, \eta)\left(\int_{0}^{\eta} e^{-\frac{3}{2 A_{2}}\left(\tilde{\mathcal{Y}}_{2}(t, \eta)-\tilde{\mathcal{Y}}_{2}(t, \theta)\right)} \tilde{\mathcal{P}}_{2}(t, \theta) d \theta\right) \\
& \times\left(\int_{0}^{\eta} e^{-\frac{1}{2 A_{2}}\left(\tilde{\mathcal{Y}}_{2}(t, \eta)-\tilde{\mathcal{Y}}_{2}(t, \theta)\right)}\left(\tilde{\mathcal{Y}}_{1}-\tilde{\mathcal{Y}}_{2}\right)^{2}(t, \theta) d \theta\right) d \eta \\
\leqslant & \left\|\tilde{\mathcal{U}}_{1}-\tilde{\mathcal{U}}_{2}\right\|^{2}+\mathcal{O}(1) \frac{1}{A^{5}} \int_{0}^{1} \tilde{\mathcal{P}}_{2} \tilde{\mathcal{U}}_{2, \eta}^{2}(t, \eta)
\end{aligned}
$$




$$
\begin{aligned}
& \times\left(\int_{0}^{\eta} e^{-\frac{1}{2 A_{2}}\left(\tilde{\mathcal{Y}}_{2}(t, \eta)-\tilde{\mathcal{Y}}_{2}(t, \theta)\right)}\left(\tilde{\mathcal{Y}}_{1}-\tilde{\mathcal{Y}}_{2}\right)^{2}(t, \theta) d \theta\right) d \eta \\
\leqslant & \mathcal{O}(1)\left(\left\|\tilde{\mathcal{U}}_{1}-\tilde{\mathcal{U}}_{2}\right\|^{2}+\left\|\tilde{\mathcal{Y}}_{1}-\tilde{\mathcal{Y}}_{2}\right\|^{2}\right) .
\end{aligned}
$$

Here we employed

$$
\min _{j}\left(\tilde{\mathcal{D}}_{j}\right)^{1 / 2}+\left|\tilde{\mathcal{U}}_{2}\right| \leqslant \sqrt{2 A_{2}} \tilde{\mathcal{P}}_{2}^{1 / 2}+\sqrt{2} \tilde{\mathcal{P}}_{2}^{1 / 2} \leqslant \sqrt{2}\left(1+\sqrt{A_{2}}\right) \tilde{\mathcal{P}}_{2}^{1 / 2}
$$

as well as (4.15q) and (4.16i).

Next, we turn to the term $W_{4}$ :

$$
\begin{aligned}
-W_{4}= & \frac{3}{A^{6}} \int_{0}^{1}\left(\tilde{\mathcal{U}}_{1}-\tilde{\mathcal{U}}_{2}\right)(t, \eta)\left(\tilde{\mathcal{U}}_{2, \eta}(t, \eta) \int_{0}^{\eta} e^{-\frac{1}{A_{2}}\left(\tilde{\mathcal{Y}}_{2}(t, \eta)-\tilde{\mathcal{Y}}_{2}(t, \theta)\right)} \tilde{\mathcal{P}}_{2} \tilde{\mathcal{U}}_{2} \tilde{\mathcal{Y}}_{2, \eta}(t, \theta) d \theta\right. \\
& \left.-\tilde{\mathcal{U}}_{1, \eta}(t, \eta) \int_{0}^{\eta} e^{-\frac{1}{A_{1}}\left(\tilde{\mathcal{Y}}_{1}(t, \eta)-\tilde{\mathcal{Y}}_{1}(t, \theta)\right)} \tilde{\mathcal{P}}_{1} \tilde{\mathcal{U}}_{1} \tilde{\mathcal{Y}}_{1, \eta}(t, \theta) d \theta\right) d \eta \\
= & W_{4}^{+}+W_{4}^{-}
\end{aligned}
$$

with

$$
\begin{aligned}
W_{4}^{ \pm}= & \frac{3}{A^{6}} \int_{0}^{1}\left(\tilde{\mathcal{U}}_{1}-\tilde{\mathcal{U}}_{2}\right)(t, \eta)\left(\tilde{\mathcal{U}}_{2, \eta}(t, \eta) \int_{0}^{\eta} e^{-\frac{1}{A_{2}}\left(\tilde{\mathcal{Y}}_{2}(t, \eta)-\tilde{\mathcal{Y}}_{2}(t, \theta)\right)} \tilde{\mathcal{P}}_{2} \tilde{\mathcal{U}}_{2}^{ \pm} \tilde{\mathcal{Y}}_{2, \eta}(t, \theta) d \theta\right. \\
& \left.-\tilde{\mathcal{U}}_{1, \eta}(t, \eta) \int_{0}^{\eta} e^{-\frac{1}{A_{1}}\left(\tilde{\mathcal{Y}}_{1}(t, \eta)-\tilde{\mathcal{Y}}_{1}(t, \theta)\right)} \tilde{\mathcal{P}}_{1} \tilde{\mathcal{U}}_{1}^{ \pm} \tilde{\mathcal{Y}}_{1, \eta}(t, \theta) d \theta\right) d \eta \\
\leqslant & \mathcal{O}(1)\left(\left\|\tilde{\mathcal{U}}_{1}-\tilde{\mathcal{U}}_{2}\right\|^{2}+\left\|\tilde{\mathcal{P}}_{1}^{1 / 2}-\tilde{\mathcal{P}}_{2}^{1 / 2}\right\|^{2}+\left\|\tilde{\mathcal{Y}}_{1}-\tilde{\mathcal{Y}}_{2}\right\|^{2}+\left|A_{1}-A_{2}\right|^{2}\right),
\end{aligned}
$$

using that $W_{4}^{+}=3 N_{1}$ (see (5.39)) and similarly for $W_{4}^{-}$.

Next, we turn to the term $W_{5}$ :

$$
\begin{aligned}
W_{5}= & \frac{1}{A^{6}} \int_{0}^{1}\left(\tilde{\mathcal{U}}_{1}-\tilde{\mathcal{U}}_{2}\right)(t, \eta)\left(\tilde{\mathcal{U}}_{2, \eta}(t, \eta) \int_{0}^{\eta} e^{-\frac{1}{A_{2}}\left(\tilde{\mathcal{Y}}_{2}(t, \eta)-\tilde{\mathcal{Y}}_{2}(t, \theta)\right)} \tilde{\mathcal{U}}_{2}^{3} \tilde{\mathcal{Y}}_{2, \eta}(t, \theta) d \theta\right. \\
& \left.-\tilde{\mathcal{U}}_{1, \eta}(t, \eta) \int_{0}^{\eta} e^{-\frac{1}{A_{1}}\left(\tilde{\mathcal{Y}}_{1}(t, \eta)-\tilde{\mathcal{Y}}_{1}(t, \theta)\right)} \tilde{\mathcal{U}}_{1}^{3} \tilde{\mathcal{Y}}_{1, \eta}(t, \theta) d \theta\right) d \eta
\end{aligned}
$$

We take positive and negative parts of the term $\tilde{\mathcal{U}}_{j}^{3}$, and thus it suffices to study the term

$$
\begin{aligned}
W_{5}^{+}= & \frac{1}{A^{6}} \int_{0}^{1}\left(\tilde{\mathcal{U}}_{1}-\tilde{\mathcal{U}}_{2}\right)\left[\tilde{\mathcal{U}}_{2, \eta}(t, \eta) \int_{0}^{\eta} e^{-\frac{1}{A_{2}}\left(\tilde{\mathcal{Y}}_{2}(t, \eta)-\tilde{\mathcal{Y}}_{2}(t, \theta)\right)}\left(\tilde{\mathcal{U}}_{2}^{+}\right)^{3} \tilde{\mathcal{Y}}_{2, \eta}(t, \theta) d \theta\right. \\
& \left.-\tilde{\mathcal{U}}_{1, \eta}(t, \eta) \int_{0}^{\eta} e^{-\frac{1}{A_{1}}\left(\tilde{\mathcal{Y}}_{1}(t, \eta)-\tilde{\mathcal{Y}}_{1}(t, \theta)\right)}\left(\tilde{\mathcal{U}}_{1}^{+}\right)^{3} \tilde{\mathcal{Y}}_{1, \eta}(t, \theta) d \theta\right] d \eta .
\end{aligned}
$$


Having a close look at $W_{5}^{+}$one has

$$
W_{5}^{+}=\int_{0}^{1}\left(\tilde{\mathcal{U}}_{1}-\tilde{\mathcal{U}}_{2}\right)\left(J_{1}+J_{2}+J_{3}+J_{4}+J_{5}+J_{6}+J_{7}+J_{8}\right)(t, \eta) d \eta,
$$

where $J_{1}, \ldots, J_{6}$ are defined in (5.33). Thus we can conclude immediately that

$$
\begin{aligned}
\left|W_{5}^{+}\right| \leqslant & \mathcal{O}(1)\left(\left\|\tilde{\mathcal{Y}}_{1}-\tilde{\mathcal{Y}}_{2}\right\|^{2}+\left\|\tilde{\mathcal{U}}_{1}-\tilde{\mathcal{U}}_{2}\right\|^{2}\right. \\
& \left.+\left\|\tilde{\mathcal{P}}_{1}^{1 / 2}-\tilde{\mathcal{P}}_{2}^{1 / 2}\right\|^{2}+\left|A_{1}-A_{2}\right|^{2}\right) .
\end{aligned}
$$

Next, we turn to the term $W_{6}$ :

$$
\begin{aligned}
& 2 W_{6}=\frac{1}{A^{6}} \int_{0}^{1}\left(\tilde{\mathcal{U}}_{1}-\tilde{\mathcal{U}}_{2}\right)(t, \eta)\left(\tilde{\mathcal{U}}_{2, \eta}(t, \eta) \int_{0}^{\eta} e^{-\frac{1}{A_{2}}\left(\tilde{\mathcal{Y}}_{2}(t, \eta)-\tilde{\mathcal{Y}}_{2}(t, \theta)\right)} A_{2}^{5} \tilde{\mathcal{U}}_{2}(t, \theta) d \theta\right. \\
& \left.-\tilde{\mathcal{U}}_{1, \eta}(t, \eta) \int_{0}^{\eta} e^{-\frac{1}{A_{1}}\left(\tilde{\mathcal{Y}}_{1}(t, \eta)-\tilde{\mathcal{Y}}_{1}(t, \theta)\right)} A_{1}^{5} \tilde{\mathcal{U}}_{1}(t, \theta) d \theta\right) d \eta \\
& =\frac{A_{2}^{5}-A_{1}^{5}}{A^{6}} \mathbb{1}_{A_{1} \leqslant A_{2}} \int_{0}^{1}\left(\tilde{\mathcal{U}}_{1}-\tilde{\mathcal{U}}_{2}\right) \tilde{\mathcal{U}}_{2, \eta}(t, \eta) \\
& \times \int_{0}^{\eta} e^{-\frac{1}{A_{2}}\left(\tilde{\mathcal{Y}}_{2}(t, \eta)-\tilde{\mathcal{Y}}_{2}(t, \theta)\right)} \tilde{\mathcal{U}}_{2}(t, \theta) d \theta d \eta \\
& +\frac{A_{2}^{5}-A_{1}^{5}}{A^{6}} \mathbb{1}_{A_{2}<A_{1}} \int_{0}^{1}\left(\tilde{\mathcal{U}}_{1}-\tilde{\mathcal{U}}_{2}\right) \tilde{\mathcal{U}}_{1, \eta}(t, \eta) \\
& \times \int_{0}^{\eta} e^{-\frac{1}{A_{1}}\left(\tilde{\mathcal{Y}}_{1}(t, \eta)-\tilde{\mathcal{Y}}_{1}(t, \theta)\right)} \tilde{\mathcal{U}}_{1}(t, \theta) d \theta d \eta \\
& +\frac{a^{5}}{A^{6}} \mathbb{1}_{A_{1} \leqslant A_{2}} \int_{0}^{1}\left(\tilde{\mathcal{U}}_{1}-\tilde{\mathcal{U}}_{2}\right) \tilde{U}_{2, \eta}(t, \eta) \\
& \times \int_{0}^{\eta}\left(e^{-\frac{1}{A_{2}}\left(\tilde{\mathcal{Y}}_{2}(t, \eta)-\tilde{\mathcal{Y}}_{2}(t, \theta)\right)}-e^{-\frac{1}{A_{1}}\left(\tilde{\mathcal{Y}}_{2}(t, \eta)-\tilde{\mathcal{Y}}_{2}(t, \theta)\right)}\right) \tilde{\mathcal{U}}_{2}(t, \theta) d \theta d \eta \\
& +\frac{a^{5}}{A^{5}} \mathbb{1}_{A_{2}<A_{1}} \int_{0}^{1}\left(\tilde{\mathcal{U}}_{1}-\tilde{\mathcal{U}}_{2}\right) \tilde{\mathcal{U}}_{1, \eta}(t, \eta) \\
& \times \int_{0}^{\eta}\left(e^{-\frac{1}{A_{2}}\left(\tilde{\mathcal{Y}}_{1}(t, \eta)-\tilde{\mathcal{Y}}_{1}(t, \theta)\right)}-e^{-\frac{1}{A_{1}}\left(\tilde{\mathcal{Y}}_{1}(t, \eta)-\tilde{\mathcal{Y}}_{1}(t, \theta)\right)}\right) \tilde{\mathcal{U}}_{1}(t, \theta) d \theta d \eta \\
& +\frac{a^{5}}{A^{6}} \int_{0}^{1}\left(\tilde{\mathcal{U}}_{1}-\tilde{\mathcal{U}}_{2}\right) \tilde{\mathcal{U}}_{2, \eta}(t, \eta) \\
& \times \int_{0}^{\eta}\left(e^{-\frac{1}{a}\left(\tilde{\mathcal{Y}}_{2}(t, \eta)-\tilde{\mathcal{Y}}_{2}(t, \theta)\right)}-e^{-\frac{1}{a}\left(\tilde{\mathcal{Y}}_{1}(t, \eta)-\tilde{\mathcal{Y}}_{1}(t, \theta)\right)}\right) \tilde{\mathcal{U}}_{2} \mathbb{1}_{B(\eta)}(t, \theta) d \theta d \eta \\
& +\frac{a^{5}}{A^{6}} \int_{0}^{1}\left(\tilde{\mathcal{U}}_{1}-\tilde{\mathcal{U}}_{2}\right) \tilde{\mathcal{U}}_{1, \eta}(t, \eta)
\end{aligned}
$$




$$
\begin{aligned}
& \times \int_{0}^{\eta}\left(e^{-\frac{1}{a}\left(\tilde{\mathcal{Y}}_{2}(t, \eta)-\tilde{\mathcal{Y}}_{2}(t, \theta)\right)}-e^{-\frac{1}{a}\left(\tilde{\mathcal{Y}}_{1}(t, \eta)-\tilde{\mathcal{Y}}_{1}(t, \theta)\right)}\right) \tilde{\mathcal{U}}_{1} \mathbb{1}_{B(\eta)^{c}}(t, \theta) d \theta d \eta \\
& +\frac{a^{5}}{A^{6}} \int_{0}^{1}\left(\tilde{\mathcal{U}}_{1}-\tilde{\mathcal{U}}_{2}\right) \tilde{\mathcal{U}}_{2, \eta}(t, \eta) \\
& \times \int_{0}^{\eta} \min _{j}\left(e^{-\frac{1}{a}\left(\tilde{\mathcal{Y}}_{j}(t, \eta)-\tilde{\mathcal{Y}}_{j}(t, \theta)\right)}\right)\left(\tilde{\mathcal{U}}_{2}^{+}-\tilde{\mathcal{U}}_{1}^{+}\right) \mathbb{1}_{\tilde{\mathcal{U}}_{1}^{+} \leqslant \tilde{\mathcal{U}}_{2}^{+}}(t, \theta) d \theta d \eta \\
& +\frac{a^{5}}{A^{6}} \int_{0}^{1}\left(\tilde{\mathcal{U}}_{1}-\tilde{\mathcal{U}}_{2}\right) \tilde{\mathcal{U}}_{2, \eta}(t, \eta) \\
& \times \int_{0}^{\eta} \min _{j}\left(e^{-\frac{1}{a}\left(\tilde{\mathcal{Y}}_{j}(t, \eta)-\tilde{\mathcal{Y}}_{j}(t, \theta)\right)}\right)\left(\tilde{\mathcal{U}}_{2}^{-}-\tilde{\mathcal{U}}_{1}^{-}\right) \mathbb{1}_{\tilde{\mathcal{U}}_{2}^{-} \leqslant \tilde{\mathcal{U}}_{1}^{-}}(t, \theta) d \theta d \eta \\
& +\frac{a^{5}}{A^{6}} \int_{0}^{1}\left(\tilde{\mathcal{U}}_{1}-\tilde{\mathcal{U}}_{2}\right) \tilde{\mathcal{U}}_{1, \eta}(t, \eta) \\
& \times \int_{0}^{\eta} \min _{j}\left(e^{-\frac{1}{a}\left(\tilde{\mathcal{Y}}_{j}(t, \eta)-\tilde{\mathcal{Y}}_{j}(t, \theta)\right)}\right)\left(\tilde{\mathcal{U}}_{2}^{+}-\tilde{\mathcal{U}}_{1}^{+}\right) \mathbb{1}_{\tilde{\mathcal{U}}_{2}^{+}<\tilde{\mathcal{U}}_{1}^{+}}(t, \theta) d \theta d \eta \\
& +\frac{a^{5}}{A^{6}} \int_{0}^{1}\left(\tilde{\mathcal{U}}_{1}-\tilde{\mathcal{U}}_{2}\right) \tilde{\mathcal{U}}_{1, \eta}(t, \eta) \\
& \times \int_{0}^{\eta} \min _{j}\left(e^{-\frac{1}{a}\left(\tilde{\mathcal{Y}}_{j}(t, \eta)-\tilde{\mathcal{Y}}_{j}(t, \theta)\right)}\right)\left(\tilde{\mathcal{U}}_{2}^{-}-\tilde{\mathcal{U}}_{1}^{-}\right) \mathbb{1}_{\tilde{\mathcal{U}}_{1}^{-}<\tilde{\mathcal{U}}_{2}^{-}}(t, \theta) d \theta d \eta \\
& -\frac{a^{5}}{A^{6}} \int_{0}^{1}\left(\tilde{\mathcal{U}}_{1}-\tilde{\mathcal{U}}_{2}\right)\left(\tilde{\mathcal{U}}_{1}-\tilde{\mathcal{U}}_{2}\right)_{\eta}(t, \eta) \\
& \times \int_{0}^{\eta} \min _{j}\left(e^{-\frac{1}{a}\left(\tilde{\mathcal{Y}}_{j}(t, \eta)-\tilde{\mathcal{Y}}_{j}(t, \theta)\right)}\right) \min _{j}\left(\tilde{\mathcal{U}}_{j}^{+}\right)(t, \theta) d \theta d \eta \\
& -\frac{a^{5}}{A^{6}} \int_{0}^{1}\left(\tilde{\mathcal{U}}_{1}-\tilde{\mathcal{U}}_{2}\right)\left(\tilde{\mathcal{U}}_{1}-\tilde{\mathcal{U}}_{2}\right)_{\eta}(t, \eta) \\
& \times \int_{0}^{\eta} \min _{j}\left(e^{-\frac{1}{a}\left(\tilde{\mathcal{Y}}_{j}(t, \eta)-\tilde{\mathcal{Y}}_{j}(t, \theta)\right)}\right) \max _{j}\left(\tilde{\mathcal{U}}_{j}^{-}\right)(t, \theta) d \theta d \eta \\
& =W_{61}+W_{62}+W_{63}+W_{64}+W_{65}+W_{66}+W_{67}^{ \pm}+W_{68}^{ \pm}+W_{69}^{ \pm} \text {. }
\end{aligned}
$$

Here we go again! The terms $W_{61}$ and $W_{62}$ :

$$
\begin{aligned}
\left|W_{61}\right|= & \frac{A_{2}^{5}-A_{1}^{5}}{A^{6}} \mathbb{1}_{A_{1} \leqslant A_{2}} \mid \int_{0}^{1}\left(\tilde{\mathcal{U}}_{1}-\tilde{\mathcal{U}}_{2}\right) \tilde{\mathcal{U}}_{2, \eta}(t, \eta) \\
& \times \int_{0}^{\eta} e^{-\frac{1}{A_{2}}\left(\tilde{\mathcal{Y}}_{2}(t, \eta)-\tilde{\mathcal{Y}}_{2}(t, \theta)\right)} \tilde{\mathcal{U}}_{2}(t, \theta) d \theta d \eta \mid \\
\leqslant & \frac{A_{2}^{5}-A_{1}^{5}}{A^{6}} \mathbb{1}_{A_{1} \leqslant A_{2}} \int_{0}^{1}\left|\tilde{\mathcal{U}}_{1}-\tilde{\mathcal{U}}_{2}\right|\left|\tilde{\mathcal{U}}_{2, \eta}\right|(t, \eta)
\end{aligned}
$$




$$
\begin{aligned}
& \times\left(\int_{0}^{\eta} e^{-\frac{1}{A_{2}}\left(\tilde{\mathcal{Y}}_{2}(t, \eta)-\tilde{\mathcal{Y}}_{2}(t, \theta)\right)} \tilde{\mathcal{U}}_{2}^{2}(t, \theta) d \theta\right)^{1 / 2} \\
& \times\left(\int_{0}^{\eta} e^{-\frac{1}{A_{2}}\left(\tilde{\mathcal{Y}}_{2}(t, \eta)-\tilde{\mathcal{Y}}_{2}(t, \theta)\right)} d \theta\right)^{1 / 2} d \eta \\
\leqslant & \frac{\sqrt{65}}{A^{2}}\left|A_{2}-A_{1}\right| \int_{0}^{1}\left|\tilde{\mathcal{U}}_{1}-\tilde{\mathcal{U}}_{2}\right| \tilde{\mathcal{P}}_{2}^{1 / 2}\left|\tilde{\mathcal{U}}_{2, \eta}\right|(t, \eta) d \eta \\
\leqslant & \sqrt{35} A^{2}\left|A_{2}-A_{1}\right|\left\|\tilde{\mathcal{U}}_{1}-\tilde{\mathcal{U}}_{2}\right\| \\
\leqslant & \mathcal{O}(1)\left(\left|A_{1}-A_{2}\right|^{2}+\left\|\tilde{\mathcal{U}}_{1}-\tilde{\mathcal{U}}_{2}\right\|^{2}\right),
\end{aligned}
$$

using (4.15q) and (4.16a).

The terms $W_{63}$ and $W_{64}$ :

$$
\begin{aligned}
& \left|W_{63}\right| \leqslant \frac{4 a^{4}}{A^{6} e} \int_{0}^{1}\left|\tilde{\mathcal{U}}_{1}-\tilde{\mathcal{U}}_{2}\right|\left|\tilde{\mathcal{U}}_{2, \eta}\right|(t, \eta) \\
& \times \int_{0}^{\eta} e^{-\frac{3}{4 A_{2}}\left(\tilde{\mathcal{Y}}_{2}(t, \eta)-\tilde{\mathcal{Y}}_{2}(t, \theta)\right)}\left|\tilde{\mathcal{U}}_{2}\right|(t, \theta) d \theta d \eta\left|A_{1}-A_{2}\right| \\
& \leqslant \frac{4}{A^{2} e} \int_{0}^{1}\left|\tilde{\mathcal{U}}_{1}-\tilde{\mathcal{U}}_{2}\right|\left|\tilde{\mathcal{U}}_{2, \eta}\right|(t, \eta)\left(\int_{0}^{\eta} e^{-\frac{1}{A_{2}}\left(\tilde{\mathcal{Y}}_{2}(t, \eta)-\tilde{\mathcal{Y}}_{2}(t, \theta)\right)} \tilde{\mathcal{U}}_{2}^{2}(t, \theta) d \theta\right)^{1 / 2} \\
& \times\left(\int_{0}^{\eta} e^{-\frac{1}{2 A_{2}}\left(\tilde{\mathcal{Y}}_{2}(t, \eta)-\tilde{\mathcal{Y}}_{2}(t, \theta)\right)} d \theta\right)^{1 / 2} d \eta\left|A_{1}-A_{2}\right| \\
& \leqslant \frac{4 \sqrt{6}}{A^{2} e} \int_{0}^{1}\left|\tilde{\mathcal{U}}_{1}-\tilde{\mathcal{U}}_{2}\right| \tilde{\mathcal{P}}_{2}^{1 / 2}\left|\tilde{\mathcal{U}}_{2, \eta}\right|(t, \eta) d \eta\left|A_{1}-A_{2}\right| \\
& \leqslant \mathcal{O}(1)\left(\left\|\tilde{\mathcal{U}}_{1}-\tilde{\mathcal{U}}_{2}\right\|^{2}+\left|A_{1}-A_{2}\right|^{2}\right) \text {. }
\end{aligned}
$$

Next comes the terms $W_{65}$ and $W_{66}$ :

$$
\begin{aligned}
\left|W_{65}\right|= & \frac{a^{5}}{A^{6}} \mid \int_{0}^{1}\left(\tilde{\mathcal{U}}_{1}-\tilde{\mathcal{U}}_{2}\right) \tilde{\mathcal{U}}_{2, \eta}(t, \eta) \\
& \times \int_{0}^{\eta}\left(e^{-\frac{1}{a}\left(\tilde{\mathcal{Y}}_{2}(t, \eta)-\tilde{\mathcal{Y}}_{2}(t, \theta)\right)}-e^{-\frac{1}{a}\left(\tilde{\mathcal{Y}}_{1}(t, \eta)-\tilde{\mathcal{Y}}_{1}(t, \theta)\right)}\right) \tilde{\mathcal{U}}_{2} \mathbb{1}_{B(\eta)}(t, \theta) d \theta d \eta \mid \\
\leqslant & \frac{1}{A^{2}} \int_{0}^{1}\left|\tilde{\mathcal{U}}_{1}-\tilde{\mathcal{U}}_{2}\right|\left|\tilde{\mathcal{U}}_{2, \eta}\right|(t, \eta) \\
& \times \int_{0}^{\eta}\left(\left|\tilde{\mathcal{Y}}_{2}(t, \eta)-\tilde{\mathcal{Y}}_{1}(t, \eta)\right|+\left|\tilde{\mathcal{Y}}_{2}(t, \theta)-\tilde{\mathcal{Y}}_{1}(t, \theta)\right|\right) \\
& \times e^{-\frac{1}{a}\left(\tilde{\mathcal{Y}}_{2}(t, \eta)-\tilde{\mathcal{Y}}_{2}(t, \theta)\right)}\left|\tilde{\mathcal{U}}_{2}\right|(t, \theta) d \theta d \eta
\end{aligned}
$$




$$
\begin{aligned}
& \leqslant \frac{1}{A^{2}} \int_{0}^{1}\left|\tilde{\mathcal{U}}_{1}-\tilde{\mathcal{U}}_{2}\right|\left|\tilde{\mathcal{Y}}_{2}-\tilde{\mathcal{Y}}_{1}\right|\left|\tilde{\mathcal{U}}_{2, \eta}\right|(t, \eta) \\
& \times \int_{0}^{\eta} e^{-\frac{1}{a}\left(\tilde{\mathcal{Y}}_{2}(t, \eta)-\tilde{\mathcal{Y}}_{2}(t, \theta)\right)}\left|\tilde{\mathcal{U}}_{2}\right|(t, \theta) d \theta d \eta \\
& +\frac{1}{A^{2}} \int_{0}^{1}\left|\tilde{\mathcal{U}}_{1}-\tilde{\mathcal{U}}_{2}\right|\left|\tilde{\mathcal{U}}_{2, \eta}\right|(t, \eta) \\
& \times \int_{0}^{\eta} e^{-\frac{1}{a}\left(\tilde{\mathcal{Y}}_{2}(t, \eta)-\tilde{\mathcal{Y}}_{2}(t, \theta)\right)}\left|\tilde{\mathcal{Y}}_{2}-\tilde{\mathcal{Y}}_{1}\right|\left|\tilde{\mathcal{U}}_{2}\right|(t, \theta) d \theta d \eta \\
& \leqslant \frac{1}{A^{2}} \int_{0}^{1}\left|\tilde{\mathcal{U}}_{1}-\tilde{\mathcal{U}}_{2}\right|\left|\tilde{\mathcal{Y}}_{2}-\tilde{\mathcal{Y}}_{1}\right|\left|\tilde{\mathcal{U}}_{2, \eta}\right|(t, \eta) \\
& \times\left(\int_{0}^{\eta} e^{-\frac{1}{A_{2}}\left(\tilde{\mathcal{Y}}_{2}(t, \eta)-\tilde{\mathcal{Y}}_{2}(t, \theta)\right)} \tilde{\mathcal{U}}_{2}^{2}(t, \theta) d \theta\right)^{1 / 2} d \eta \\
& +\frac{1}{A^{2}} \int_{0}^{1}\left|\tilde{\mathcal{U}}_{1}-\tilde{\mathcal{U}}_{2}\right|\left|\tilde{\mathcal{U}}_{2, \eta}\right|(t, \eta) \\
& \times\left(\int_{0}^{\eta} e^{-\frac{1}{A_{2}}\left(\tilde{\mathcal{Y}}_{2}(t, \eta)-\tilde{\mathcal{Y}}_{2}(t, \theta)\right)} \tilde{\mathcal{U}}_{2}^{2}(t, \theta), d \theta\right)^{1 / 2} d \eta\left\|\tilde{\mathcal{Y}}_{1}-\tilde{\mathcal{Y}}_{2}\right\| \\
& \leqslant \frac{\sqrt{6}}{A^{2}} \int_{0}^{1}\left|\tilde{\mathcal{U}}_{1}-\tilde{\mathcal{U}}_{2}\right|\left|\tilde{\mathcal{Y}}_{2}-\tilde{\mathcal{Y}}_{1}\right| \tilde{\mathcal{P}}_{2}^{1 / 2}\left|\tilde{\mathcal{U}}_{2, \eta}\right|(t, \eta) d \eta \\
& +\frac{\sqrt{6}}{A^{2}} \int_{0}^{1}\left|\tilde{\mathcal{U}}_{1}-\tilde{\mathcal{U}}_{2}\right| \tilde{\mathcal{P}}_{2}^{1 / 2}\left|\tilde{\mathcal{U}}_{2, \eta}\right|(t, \eta) d \eta\left\|\tilde{\mathcal{Y}}_{1}-\tilde{\mathcal{Y}}_{2}\right\| \\
& \leqslant \mathcal{O}(1)\left\|\tilde{\mathcal{U}}_{1}-\tilde{\mathcal{U}}_{2}\right\|\left\|\tilde{\mathcal{Y}}_{1}-\tilde{\mathcal{Y}}_{2}\right\| \\
& \leqslant \mathcal{O}(1)\left(\left\|\tilde{\mathcal{U}}_{1}-\tilde{\mathcal{U}}_{2}\right\|^{2}+\left\|\tilde{\mathcal{Y}}_{1}-\tilde{\mathcal{Y}}_{2}\right\|^{2}\right) \text {, }
\end{aligned}
$$

using (4.15q), (4.16a), and (5.13).

The terms $W_{67}^{ \pm}$and $W_{68}^{ \pm}$have a similar structure:

$$
\begin{aligned}
\left|W_{67}^{+}\right|= & \frac{a^{5}}{A^{6}} \mid \int_{0}^{1}\left(\tilde{\mathcal{U}}_{1}-\tilde{\mathcal{U}}_{2}\right) \tilde{\mathcal{U}}_{2, \eta}(t, \eta) \\
& \times \int_{0}^{\eta} \min \left(e^{-\frac{1}{a}\left(\tilde{\mathcal{Y}}_{j}(t, \eta)-\tilde{\mathcal{Y}}_{j}(t, \theta)\right)}\right)\left(\tilde{\mathcal{U}}_{2}^{+}-\tilde{\mathcal{U}}_{1}^{+}\right) \mathbb{1}_{\tilde{\mathcal{U}}_{1}^{+} \leqslant \tilde{\mathcal{U}}_{2}^{+}}(t, \theta) d \theta d \eta \mid \\
\leqslant & \left\|\tilde{\mathcal{U}}_{1}-\tilde{\mathcal{U}}_{2}\right\|^{2}+\frac{1}{A^{2}} \int_{0}^{1} \tilde{\mathcal{U}}_{2, \eta}^{2}(t, \eta) \\
& \times\left(\int_{0}^{\eta} e^{-\frac{1}{A_{2}}\left(\tilde{\mathcal{Y}}_{2}(t, \eta)-\tilde{\mathcal{Y}}_{2}(t, \theta)\right)}\left(\tilde{\mathcal{U}}_{2}^{+}-\tilde{\mathcal{U}}_{1}^{+}\right)(t, \theta) d \theta\right)^{2} d \eta
\end{aligned}
$$




$$
\begin{aligned}
\leqslant & \left\|\tilde{\mathcal{U}}_{1}-\tilde{\mathcal{U}}_{2}\right\|^{2}+A \int_{0}^{1} \tilde{\mathcal{Y}}_{2, \eta}(t, \eta) \\
& \times\left(\int_{0}^{\eta} e^{-\frac{1}{A_{2}}\left(\tilde{\mathcal{Y}}_{2}(t, \eta)-\tilde{\mathcal{Y}}_{2}(t, \theta)\right)}\left(\tilde{\mathcal{U}}_{2}-\tilde{\mathcal{U}}_{1}\right)^{2}(t, \theta) d \theta\right) d \eta \\
\leqslant & \left\|\tilde{\mathcal{U}}_{1}-\tilde{\mathcal{U}}_{2}\right\|^{2} \\
& +A \int_{0}^{1} \tilde{\mathcal{Y}}_{2, \eta}(t, \eta) e^{-\frac{1}{A_{2}} \tilde{\mathcal{Y}}_{2}(t, \eta)}\left(\int_{0}^{\eta} e^{\frac{1}{A_{2}} \tilde{\mathcal{Y}}_{2}(t, \theta)}\left(\tilde{\mathcal{U}}_{1}-\tilde{\mathcal{U}}_{2}\right)^{2}(t, \theta) d \theta\right) d \eta \\
\leqslant & \mathcal{O}(1)\left\|\tilde{\mathcal{U}}_{1}-\tilde{\mathcal{U}}_{2}\right\|^{2},
\end{aligned}
$$

using (4.15h).

Finally, the terms $W_{69}^{ \pm}$:

$$
\begin{aligned}
\left|W_{69}^{+}\right|= & \frac{a^{5}}{A^{6}} \mid \int_{0}^{1}\left(\tilde{\mathcal{U}}_{1}-\tilde{\mathcal{U}}_{2}\right)\left(\tilde{\mathcal{U}}_{1}-\tilde{\mathcal{U}}_{2}\right)_{\eta}(t, \eta) \\
& \times \int_{0}^{\eta} \min _{j}\left(e^{-\frac{1}{a}\left(\tilde{\mathcal{Y}}_{j}(t, \eta)-\tilde{\mathcal{Y}}_{j}(t, \theta)\right)}\right) \min _{j}\left(\tilde{\mathcal{U}}_{j}^{+}\right)(t, \theta) d \theta d \eta \mid \\
\leqslant & \frac{1}{2 A}\left|\left(\left(\tilde{\mathcal{U}}_{1}-\tilde{\mathcal{U}}_{2}\right)^{2}(t, \eta) \int_{0}^{\eta} \min _{j}\left(e^{-\frac{1}{a}\left(\tilde{\mathcal{Y}}_{j}(t, \eta)-\tilde{\mathcal{Y}}_{j}(t, \theta)\right)}\right) \min _{j}\left(\tilde{\mathcal{U}}_{j}^{+}\right)(t, \theta) d \theta\right)\right|_{\eta=0}^{1} \\
& -\int_{0}^{1}\left(\tilde{\mathcal{U}}_{1}-\tilde{\mathcal{U}}_{2}\right)^{2}(t, \eta) \frac{d}{d \eta} \int_{0}^{\eta} \min _{j}\left(e^{-\frac{1}{a}\left(\tilde{\mathcal{Y}}_{j}(t, \eta)-\tilde{\mathcal{Y}}_{j}(t, \theta)\right)}\right) \min _{j}\left(\tilde{\mathcal{U}}_{j}^{+}\right)(t, \theta) d \theta d \eta \mid \\
\leqslant & \mathcal{O}(1)\left\|\tilde{\mathcal{U}}_{1}-\tilde{\mathcal{U}}_{2}\right\|^{2} ;
\end{aligned}
$$

see estimates for $\bar{B}_{67}^{ \pm}$(cf. (5.25)) and Lemma A.5.

The terms $M_{2}$ and $M_{3}$ can be treated similarly. More precisely,

$$
\begin{aligned}
\left|M_{2}\right| \leqslant & \mathbb{1}_{A_{2} \leqslant A_{1}}\left(\frac{1}{A_{2}^{6}}-\frac{1}{A_{1}^{6}}\right) \mid \int_{0}^{1}\left(\tilde{\mathcal{U}}_{1}-\tilde{\mathcal{U}}_{2}\right) \tilde{\mathcal{U}}_{2, \eta}(t, \eta) \\
& \times \int_{0}^{\eta} e^{-\frac{1}{A_{2}}\left(\tilde{\mathcal{Y}}_{2}(t, \eta)-\tilde{\mathcal{Y}}_{2}(t, \theta)\right)} \tilde{\mathcal{Q}}_{2} \tilde{\mathcal{U}}_{2, \eta}(t, \theta) d \theta d \eta \mid \\
\leqslant & \mathbb{1}_{A_{2} \leqslant A_{1}} \frac{\left|A_{1}^{6}-A_{2}^{6}\right|}{A^{6} A_{2}^{5}} \int_{0}^{1}\left|\tilde{\mathcal{U}}_{1}-\tilde{\mathcal{U}}_{2}\right|\left|\tilde{\mathcal{U}}_{2, \eta}\right|(t, \eta) \\
& \times \int_{0}^{\eta} e^{-\frac{1}{A_{2}}\left(\tilde{\mathcal{Y}}_{2}(t, \eta)-\tilde{\mathcal{Y}}_{2}(t, \theta)\right)} \tilde{\mathcal{P}}_{2}\left|\tilde{\mathcal{U}}_{2, \eta}\right|(t, \theta) d \theta d \eta \\
\leqslant & \mathbb{1}_{A_{2} \leqslant A_{1}} 6 \frac{\left|A_{1}-A_{2}\right|}{A A_{2}^{5}}\left\|\tilde{\mathcal{U}}_{1}-\tilde{\mathcal{U}}_{2}\right\| \\
& \times\left(\int_{0}^{1} \tilde{\mathcal{U}}_{2, \eta}^{2}(t, \eta)\left(\int_{0}^{\eta} e^{-\frac{1}{A_{2}}\left(\tilde{\mathcal{Y}}_{2}(t, \eta)-\tilde{\mathcal{Y}}_{2}(t, \theta)\right)} \tilde{\mathcal{P}}_{2}\left|\tilde{\mathcal{U}}_{2, \eta}\right|(t, \theta) d \theta\right)^{2} d \eta\right)^{1 / 2}
\end{aligned}
$$




$$
\begin{aligned}
\leqslant & \mathbb{1}_{A_{2} \leqslant A_{1}} 6 \frac{\left|A_{1}-A_{2}\right|}{A A_{2}^{6}}\left\|\tilde{\mathcal{U}}_{1}-\tilde{\mathcal{U}}_{2}\right\| \\
& \times\left(\int_{0}^{1} \tilde{\mathcal{U}}_{2, \eta}^{2}(t, \eta)\left(\int_{0}^{\eta} e^{-\frac{1}{A_{2}}\left(\tilde{\mathcal{Y}}_{2}(t, \eta)-\tilde{\mathcal{Y}}_{2}(t, \theta)\right)} \tilde{\mathcal{P}}_{2}^{2} \tilde{\mathcal{Y}}_{2, \eta}(t, \theta) d \theta\right)\right. \\
& \left.\times\left(\int_{0}^{\eta} e^{-\frac{1}{A_{2}}\left(\tilde{\mathcal{Y}}_{2}(t, \eta)-\tilde{\mathcal{Y}}_{2}(t, \theta)\right)} \tilde{\mathcal{H}}_{2, \eta}(t, \theta) d \theta\right) d \eta\right)^{1 / 2} \\
\leqslant & \mathbb{1}_{A_{2} \leqslant A_{1}} 6 \sqrt{6} \frac{\left|A_{1}-A_{2}\right|}{A A_{2}^{3}}\left\|\tilde{\mathcal{U}}_{1}-\tilde{\mathcal{U}}_{2}\right\|\left(\int_{0}^{1} \tilde{\mathcal{U}}_{2, \eta}^{2} \tilde{\mathcal{P}}_{2}^{2}(t, \eta) d \eta\right)^{1 / 2} \\
\leqslant & \mathcal{O}(1)\left(\left\|\tilde{\mathcal{U}}_{1}-\tilde{\mathcal{U}}_{2}\right\|^{2}+\left|A_{1}-A_{2}\right|^{2}\right) .
\end{aligned}
$$

Here we used

$$
\tilde{\mathcal{P}}_{2}\left|\tilde{\mathcal{U}}_{2, \eta}\right| \leqslant \frac{1}{A_{2}} \tilde{\mathcal{P}}_{2} \sqrt{\tilde{\mathcal{Y}}_{2, \eta} \tilde{\mathcal{H}}_{2, \eta}}
$$

(cf. (4.15m)) as well as (4.15a) and (4.15q). In addition, we applied (4.16b) and (4.16d).

We have shown the anticipated result.

LEMMA 5.4. Let $\tilde{\mathcal{U}}_{i}$ be two solutions of (5.28). Then we have

$$
\begin{aligned}
& \frac{d}{d t}\left\|\tilde{\mathcal{U}}_{1}-\tilde{\mathcal{U}}_{2}\right\|^{2} \\
& \quad \leqslant \mathcal{O}(1)\left(\left\|\tilde{\mathcal{Y}}_{1}-\tilde{\mathcal{Y}}_{2}\right\|^{2}+\left\|\tilde{\mathcal{U}}_{1}-\tilde{\mathcal{U}}_{2}\right\|^{2}+\left\|\tilde{\mathcal{P}}_{1}^{1 / 2}-\tilde{\mathcal{P}}_{2}^{1 / 2}\right\|^{2}+\left|A_{1}-A_{2}\right|^{2}\right),
\end{aligned}
$$

where $\mathcal{O}(1)$ denotes some constant which only depends on $A=\max _{j}\left(A_{j}\right)$ and which remains bounded as $A \rightarrow 0$.

5.3. Lipschitz estimates for $\tilde{\mathcal{P}}$ (or $\left.\tilde{\mathcal{P}}^{1 / 2}\right)$. From the system of differential equations we recall

$$
\left(\tilde{\mathcal{P}}_{i}^{1 / 2}\right)_{t}+\left(\frac{2}{3} \frac{1}{A_{i}^{5}} \tilde{\mathcal{U}}_{i}^{3}+\frac{1}{A_{i}^{6}} \tilde{\mathcal{S}}_{i}\right)\left(\tilde{\mathcal{P}}_{i}^{1 / 2}\right)_{\eta}=\frac{1}{2 A_{i}^{2}} \frac{\tilde{\mathcal{Q}}_{i} \tilde{\mathcal{U}}_{i}}{\tilde{\mathcal{P}}_{i}^{1 / 2}}+\frac{1}{2 A_{i}^{3}} \frac{\tilde{\mathcal{R}}_{i}}{\tilde{\mathcal{P}}_{i}^{1 / 2}}
$$

where

$$
\tilde{\mathcal{Q}}_{i}(t, \eta)=-\frac{1}{4} \int_{0}^{1} \operatorname{sign}(\eta-\theta) e^{-\frac{1}{A_{i}}\left|\tilde{\mathcal{Y}}_{i}(t, \eta)-\tilde{\mathcal{Y}}_{i}(t, \theta)\right|}\left(2\left(\tilde{\mathcal{U}}_{i}^{2}-\tilde{\mathcal{P}}_{i}\right) \tilde{\mathcal{Y}}_{i, \eta}(t, \theta)+A_{i}^{5}\right) d \theta,
$$




$$
\begin{aligned}
\tilde{\mathcal{S}}_{i}(t, \eta)= & \int_{0}^{1} e^{-\frac{1}{A_{i}}\left|\tilde{\mathcal{Y}}_{i}(t, \eta)-\tilde{\mathcal{Y}}_{i}(t, \theta)\right|}\left(\frac{2}{3} \tilde{\mathcal{U}}_{i}^{3} \tilde{\mathcal{Y}}_{i, \eta}-\tilde{\mathcal{Q}}_{i} \tilde{\mathcal{U}}_{i, \eta}-2 \tilde{\mathcal{P}}_{i} \tilde{\mathcal{U}}_{i} \tilde{\mathcal{Y}}_{i, \eta}\right)(t, \theta) d \theta \\
\tilde{\mathcal{R}}_{i}(t, \eta)= & \frac{1}{4} \int_{0}^{1} \operatorname{sign}(\eta-\theta) e^{-\frac{1}{A_{i}}\left|\tilde{\mathcal{Y}}_{i}(t, \eta)-\tilde{\mathcal{Y}}_{i}(t, \theta)\right|}\left(\frac{2}{3} A_{i} \tilde{\mathcal{U}}_{i}^{3} \tilde{\mathcal{Y}}_{i, \eta}+A_{i}^{6} \tilde{\mathcal{U}}_{i}\right)(t, \theta) d \theta \\
& -\frac{1}{2} \int_{0}^{1} e^{-\frac{1}{A_{i}}\left|\tilde{\mathcal{Y}}_{i}(t, \eta)-\tilde{\mathcal{Y}}_{i}(t, \theta)\right|} \tilde{\mathcal{U}}_{i} \tilde{\mathcal{Q}}_{i} \tilde{\mathcal{Y}}_{i, \eta}(t, \theta) d \theta
\end{aligned}
$$

Thus we have

$$
\begin{aligned}
& \frac{d}{d t} \int_{0}^{1}\left(\tilde{\mathcal{P}}_{1}^{1 / 2}-\tilde{\mathcal{P}}_{2}^{1 / 2}\right)^{2}(t, \eta) d \eta \\
& =2 \int_{0}^{1}\left(\tilde{\mathcal{P}}_{1}^{1 / 2}-\tilde{\mathcal{P}}_{2}^{1 / 2}\right)\left(\left(\tilde{\mathcal{P}}_{1}^{1 / 2}\right)_{t}-\left(\tilde{\mathcal{P}}_{2}^{1 / 2}\right)_{t}\right)(t, \eta) d \eta \\
& =-\frac{2}{3} \int_{0}^{1}\left(\tilde{\mathcal{P}}_{1}^{1 / 2}-\tilde{\mathcal{P}}_{2}^{1 / 2}\right)\left(\frac{1}{A_{1}^{5}} \tilde{\mathcal{U}}_{1}^{3}\left(\tilde{\mathcal{P}}_{1}^{1 / 2}\right)_{\eta}-\frac{1}{A_{2}^{5}} \tilde{\mathcal{U}}_{2}^{3}\left(\tilde{\mathcal{P}}_{2}^{1 / 2}\right)_{\eta}\right)(t, \eta) d \eta \\
& -\int_{0}^{1}\left(\tilde{\mathcal{P}}_{1}^{1 / 2}-\tilde{\mathcal{P}}_{2}^{1 / 2}\right)\left(\frac{1}{A_{1}^{6}} \tilde{\mathcal{S}}_{1}\left(\tilde{\mathcal{P}}_{1}^{1 / 2}\right)_{\eta}-\frac{1}{A_{2}^{6}} \tilde{\mathcal{S}}_{2}\left(\tilde{\mathcal{P}}_{2}^{1 / 2}\right)_{\eta}\right)(t, \eta) d \eta \\
& +\frac{1}{2} \int_{0}^{1}\left(\tilde{\mathcal{P}}_{1}^{1 / 2}-\tilde{\mathcal{P}}_{2}^{1 / 2}\right)\left(\frac{1}{A_{1}^{2}} \frac{\tilde{\mathcal{Q}}_{1} \tilde{\mathcal{U}}_{1}}{\tilde{\mathcal{P}}_{1}^{1 / 2}}-\frac{1}{A_{2}^{2}} \frac{\tilde{\mathcal{Q}}_{2} \tilde{\mathcal{U}}_{2}}{\tilde{\mathcal{P}}_{2}^{1 / 2}}\right)(t, \eta) d \eta \\
& +\frac{1}{2} \int_{0}^{1}\left(\tilde{\mathcal{P}}_{1}^{1 / 2}-\tilde{\mathcal{P}}_{2}^{1 / 2}\right)\left(\frac{1}{A_{1}^{3}} \frac{\tilde{\mathcal{P}}_{1}}{\tilde{\mathcal{P}}_{1}^{1 / 2}}-\frac{1}{A_{2}^{3}} \frac{\tilde{\mathcal{R}}_{2}}{\tilde{\mathcal{P}}_{2}^{1 / 2}}\right)(t, \eta) d \eta \\
& =\frac{2}{3} I_{1}+I_{2}+\frac{1}{2} I_{3}+\frac{1}{2} I_{4} \text {. }
\end{aligned}
$$

We will estimate each of these terms, yielding that

$$
\begin{aligned}
& \frac{d}{d t}\left\|\tilde{\mathcal{P}}_{1}^{1 / 2}-\tilde{\mathcal{P}}_{2}^{1 / 2}\right\|^{2} \\
& \quad \leqslant \mathcal{O}(1)\left(\left\|\tilde{\mathcal{Y}}_{1}-\tilde{\mathcal{Y}}_{2}\right\|^{2}+\left\|\tilde{\mathcal{U}}_{1}-\tilde{\mathcal{U}}_{2}\right\|^{2}+\left\|\tilde{\mathcal{P}}_{1}^{1 / 2}-\tilde{\mathcal{P}}_{2}^{1 / 2}\right\|^{2}+\left|A_{1}-A_{2}\right|^{2}\right),
\end{aligned}
$$

where $\mathcal{O}(1)$ denotes some constant which only depends on $A=\max _{j}\left(A_{j}\right)$ and which remains bounded as $A \rightarrow 0$.

The term $I_{1}$ : Here we do as follows:

$$
\begin{aligned}
I_{1} & =\int_{0}^{1}\left(\tilde{\mathcal{P}}_{1}^{1 / 2}-\tilde{\mathcal{P}}_{2}^{1 / 2}\right)\left(\frac{1}{A_{2}^{5}} \tilde{\mathcal{U}}_{2}^{3}\left(\tilde{\mathcal{P}}_{2}^{1 / 2}\right)_{\eta}-\frac{1}{A_{1}^{5}} \tilde{\mathcal{U}}_{1}^{3}\left(\tilde{\mathcal{P}}_{1}^{1 / 2}\right)_{\eta}\right)(t, \eta) d \eta \\
& =\frac{1}{A^{5}} \int_{0}^{1}\left(\tilde{\mathcal{P}}_{1}^{1 / 2}-\tilde{\mathcal{P}}_{2}^{1 / 2}\right)\left(\tilde{\mathcal{U}}_{2}^{3}\left(\tilde{\mathcal{P}}_{2}^{1 / 2}\right)_{\eta}-\tilde{\mathcal{U}}_{1}^{3}\left(\tilde{\mathcal{P}}_{1}^{1 / 2}\right)_{\eta}\right)(t, \eta) d \eta
\end{aligned}
$$




$$
\begin{aligned}
& +\frac{A_{1}^{5}-A_{2}^{5}}{A_{1}^{5} A_{2}^{5}} \int_{0}^{1}\left(\tilde{\mathcal{P}}_{1}^{1 / 2}-\tilde{\mathcal{P}}_{2}^{1 / 2}\right) \\
& \times\left(\tilde{\mathcal{U}}_{2}^{3}\left(\tilde{\mathcal{P}}_{2}^{1 / 2}\right)_{\eta} \mathbb{1}_{A_{2}<A_{1}}+\tilde{\mathcal{U}}_{1}^{3}\left(\tilde{\mathcal{P}}_{1}^{1 / 2}\right)_{\eta} \mathbb{1}_{A_{1} \leqslant A_{2}}\right)(t, \eta) d \eta \\
& =\frac{1}{A^{5}} \int_{0}^{1}\left(\tilde{\mathcal{P}}_{1}^{1 / 2}-\tilde{\mathcal{P}}_{2}^{1 / 2}\right)\left(\tilde{\mathcal{U}}_{2}-\tilde{\mathcal{U}}_{1}\right) \tilde{\mathcal{U}}_{2}^{2}\left(\tilde{\mathcal{P}}_{2}^{1 / 2}\right)_{\eta}(t, \eta) d \eta \\
& +\frac{1}{A^{5}} \int_{0}^{1}\left(\tilde{\mathcal{P}}_{1}^{1 / 2}-\tilde{\mathcal{P}}_{2}^{1 / 2}\right)\left(\tilde{\mathcal{U}}_{2}^{2}\left(\tilde{\mathcal{P}}_{2}^{1 / 2}\right)_{\eta}-\tilde{\mathcal{U}}_{1}^{2}\left(\tilde{\mathcal{P}}_{1}^{1 / 2}\right)_{\eta}\right) \tilde{\mathcal{U}}_{1}(t, \eta) d \eta \\
& +\frac{A_{1}^{5}-A_{2}^{5}}{A_{1}^{5} A_{2}^{5}} \int_{0}^{1}\left(\tilde{\mathcal{P}}_{1}^{1 / 2}-\tilde{\mathcal{P}}_{2}^{1 / 2}\right) \\
& \times\left(\tilde{\mathcal{U}}_{2}^{3}\left(\tilde{\mathcal{P}}_{2}^{1 / 2}\right)_{\eta} \mathbb{1}_{A_{2}<A_{1}}+\tilde{\mathcal{U}}_{1}^{3}\left(\tilde{\mathcal{P}}_{1}^{1 / 2}\right)_{\eta} \mathbb{1}_{A_{1} \leqslant A_{2}}\right)(t, \eta) d \eta \\
& =\frac{1}{A^{5}} \int_{0}^{1}\left(\tilde{\mathcal{P}}_{1}^{1 / 2}-\tilde{\mathcal{P}}_{2}^{1 / 2}\right)\left(\tilde{\mathcal{U}}_{2}-\tilde{\mathcal{U}}_{1}\right) \tilde{\mathcal{U}}_{2}^{2}\left(\tilde{\mathcal{P}}_{2}^{1 / 2}\right)_{\eta}(t, \eta) d \eta \\
& +\frac{1}{A^{5}} \int_{0}^{1}\left(\tilde{\mathcal{P}}_{1}^{1 / 2}-\tilde{\mathcal{P}}_{2}^{1 / 2}\right)\left(\tilde{\mathcal{U}}_{2}^{2}-\tilde{\mathcal{U}}_{1}^{2}\right)\left(\tilde{\mathcal{P}}_{2}^{1 / 2}\right)_{\eta} \tilde{\mathcal{U}}_{1} \mathbb{1}_{\tilde{\mathcal{U}}_{1}^{2} \leqslant \tilde{\mathcal{U}}_{2}^{2}}(t, \eta) d \eta \\
& +\frac{1}{A^{5}} \int_{0}^{1}\left(\tilde{\mathcal{P}}_{1}^{1 / 2}-\tilde{\mathcal{P}}_{2}^{1 / 2}\right)\left(\tilde{\mathcal{U}}_{2}^{2}-\tilde{\mathcal{U}}_{1}^{2}\right)\left(\tilde{\mathcal{P}}_{1}^{1 / 2}\right)_{\eta} \tilde{\mathcal{U}}_{1} \mathbb{1}_{\tilde{\mathcal{U}}_{2}^{2}<\tilde{\mathcal{U}}_{1}^{2}}(t, \eta) d \eta \\
& +\frac{1}{A^{5}} \int_{0}^{1}\left(\tilde{\mathcal{P}}_{1}^{1 / 2}-\tilde{\mathcal{P}}_{2}^{1 / 2}\right)\left(\left(\tilde{\mathcal{P}}_{2}^{1 / 2}\right)_{\eta}-\left(\tilde{\mathcal{P}}_{1}^{1 / 2}\right)_{\eta}\right) \tilde{\mathcal{U}}_{1} \min _{j}\left(\tilde{\mathcal{U}}_{j}^{2}\right)(t, \eta) d \eta \\
& +\frac{A_{1}^{5}-A_{2}^{5}}{A_{1}^{5} A_{2}^{5}} \int_{0}^{1}\left(\tilde{\mathcal{P}}_{1}^{1 / 2}-\tilde{\mathcal{P}}_{2}^{1 / 2}\right) \\
& \times\left(\tilde{\mathcal{U}}_{1}^{3}\left(\tilde{\mathcal{P}}_{1}^{1 / 2}\right)_{\eta} \mathbb{1}_{A_{1} \leqslant A_{2}}+\tilde{\mathcal{U}}_{2}^{3}\left(\tilde{\mathcal{P}}_{2}^{1 / 2}\right)_{\eta} \mathbb{1}_{A_{2}<A_{1}}\right)(t, \eta) d \eta \\
& =I_{11}+I_{12}+I_{13}+I_{14}+I_{15} \text {. }
\end{aligned}
$$

We first estimate

$$
\left|\left(\tilde{\mathcal{P}}_{i}^{1 / 2}\right)_{\eta}\right|=\frac{\left|\tilde{\mathcal{P}}_{i, \eta}\right|}{2 \tilde{\mathcal{P}}_{i}^{1 / 2}}=\frac{\left|\tilde{\mathcal{Q}}_{i} \tilde{\mathcal{Y}}_{i, \eta}\right|}{2 A_{i}^{2} \tilde{\mathcal{P}}_{i}^{1 / 2}} \leqslant \frac{1}{2 A_{i}} \tilde{\mathcal{P}}_{i}^{1 / 2} \tilde{\mathcal{Y}}_{i, \eta}
$$

and thus

$$
\left|\tilde{\mathcal{U}}_{i} \tilde{\mathcal{P}}_{i}^{1 / 2} \tilde{\mathcal{Y}}_{i, \eta}\right| \leqslant \frac{1}{2}\left(\tilde{\mathcal{U}}_{i}^{2} \tilde{\mathcal{Y}}_{i, \eta}+\tilde{\mathcal{P}}_{i} \tilde{\mathcal{Y}}_{i, \eta}\right) \leqslant \frac{3}{4} A_{i}^{5}
$$

We start with the term $I_{11}$ :

$$
\begin{aligned}
\left|I_{11}\right| & \leqslant \mathcal{O}(1) \int_{0}^{1}\left|\tilde{\mathcal{P}}_{1}^{1 / 2}-\tilde{\mathcal{P}}_{2}^{1 / 2}\right|\left|\tilde{\mathcal{U}}_{2}-\tilde{\mathcal{U}}_{1}\right|(t, \eta) d \eta \\
& \leqslant \mathcal{O}(1)\left(\left\|\tilde{\mathcal{P}}_{1}^{1 / 2}-\tilde{\mathcal{P}}_{2}^{1 / 2}\right\|^{2}+\left\|\tilde{\mathcal{U}}_{2}-\tilde{\mathcal{U}}_{1}\right\|^{2}\right) .
\end{aligned}
$$


Subsequently (and similarly for $I_{13}$ ),

$$
\begin{aligned}
\left|I_{12}\right| & \leqslant \frac{1}{A^{5}} \int_{0}^{1}\left|\tilde{\mathcal{P}}_{1}^{1 / 2}-\tilde{\mathcal{P}}_{2}^{1 / 2}\right|\left|\tilde{\mathcal{U}}_{2}-\tilde{\mathcal{U}}_{1}\right|\left|\tilde{\mathcal{U}}_{2}+\tilde{\mathcal{U}}_{1}\right|\left|\left(\tilde{\mathcal{P}}_{2}^{1 / 2}\right)_{\eta} \tilde{\mathcal{U}}_{1}\right| \mathbb{1}_{\tilde{\mathcal{U}}_{1}^{2} \leqslant \tilde{\mathcal{U}}_{2}^{2}}(t, \eta) d \eta \\
& \leqslant \frac{2}{A^{5}} \int_{0}^{1}\left|\tilde{\mathcal{P}}_{1}^{1 / 2}-\tilde{\mathcal{P}}_{2}^{1 / 2}\right|\left|\tilde{\mathcal{U}}_{2}-\tilde{\mathcal{U}}_{1}\right| \tilde{\mathcal{U}}_{2}^{2}\left|\left(\tilde{\mathcal{P}}_{2}^{1 / 2}\right)_{\eta}\right|(t, \eta) d \eta \\
& \leqslant \mathcal{O}(1)\left(\left\|\tilde{\mathcal{P}}_{1}^{1 / 2}-\tilde{\mathcal{P}}_{2}^{1 / 2}\right\|^{2}+\left\|\tilde{\mathcal{U}}_{2}-\tilde{\mathcal{U}}_{1}\right\|^{2}\right)
\end{aligned}
$$

The term $I_{14}$ requires more estimates (see (5.31)):

$$
\begin{aligned}
\left|I_{14}\right|= & \frac{1}{A^{5}}\left|\int_{0}^{1}\left(\tilde{\mathcal{P}}_{1}^{1 / 2}-\tilde{\mathcal{P}}_{2}^{1 / 2}\right)\left(\left(\tilde{\mathcal{P}}_{2}^{1 / 2}\right)_{\eta}-\left(\tilde{\mathcal{P}}_{1}^{1 / 2}\right)_{\eta}\right) \tilde{\mathcal{U}}_{1} \min _{j}\left(\tilde{\mathcal{U}}_{j}^{2}\right)(t, \eta) d \eta\right| \\
= & \left|\frac{1}{2 A^{5}}\left(\tilde{\mathcal{P}}_{1}^{1 / 2}-\tilde{\mathcal{P}}_{2}^{1 / 2}\right)^{2} \tilde{\mathcal{U}}_{1} \min _{j}\left(\tilde{\mathcal{U}}_{j}^{2}\right)(t, \eta)\right|_{\eta=0}^{1} \\
& -\frac{1}{2 A^{5}} \int_{0}^{1}\left(\tilde{\mathcal{P}}_{1}^{1 / 2}-\tilde{\mathcal{P}}_{2}^{1 / 2}\right)^{2}\left(\frac{d}{d \eta} \tilde{\mathcal{U}}_{1} \min _{j}\left(\tilde{\mathcal{U}}_{j}^{2}\right)\right)(t, \eta) d \eta \mid \\
= & \frac{1}{2 A^{5}}\left|\int_{0}^{1}\left(\tilde{\mathcal{P}}_{1}^{1 / 2}-\tilde{\mathcal{P}}_{2}^{1 / 2}\right)^{2}\left(\frac{d}{d \eta} \tilde{\mathcal{U}}_{1} \min _{j}\left(\tilde{\mathcal{U}}_{j}^{2}\right)\right)(t, \eta) d \eta\right| \\
\leqslant & \mathcal{O}(1)\left\|\tilde{\mathcal{P}}_{1}^{1 / 2}-\tilde{\mathcal{P}}_{2}^{1 / 2}\right\|^{2} .
\end{aligned}
$$

The last term can be estimated as follows:

$$
\begin{aligned}
\left|I_{15}\right| \leqslant & \mid \frac{A_{1}^{5}-A_{2}^{5}}{A_{1}^{5} A_{2}^{5}} \int_{0}^{1}\left(\tilde{\mathcal{P}}_{1}^{1 / 2}-\tilde{\mathcal{P}}_{2}^{1 / 2}\right) \\
& \times\left(\tilde{\mathcal{U}}_{1}^{3}\left(\tilde{\mathcal{P}}_{1}^{1 / 2}\right)_{\eta} \mathbb{1}_{A_{1} \leqslant A_{2}}+\tilde{\mathcal{U}}_{2}^{3}\left(\tilde{\mathcal{P}}_{2}^{1 / 2}\right)_{\eta} \mathbb{1}_{A_{2}<A_{1}}\right)(t, \eta) d \eta \mid \\
\leqslant & 5 \frac{\left|A_{1}-A_{2}\right| A^{4}}{A_{1}^{5} A_{2}^{5}} \\
& \times \int_{0}^{1}\left(\tilde{\mathcal{P}}_{1}^{1 / 2}-\tilde{\mathcal{P}}_{2}^{1 / 2}\right)\left(\left|\tilde{\mathcal{U}}_{1}^{3}\left(\tilde{\mathcal{P}}_{1}^{1 / 2}\right)_{\eta}\right| \mathbb{1}_{A_{1} \leqslant A_{2}}+\left|\tilde{\mathcal{U}}_{2}^{3}\left(\tilde{\mathcal{P}}_{2}^{1 / 2}\right)_{\eta}\right| \mathbb{1}_{A_{2}<A_{1}}\right)(t, \eta) d \eta .
\end{aligned}
$$

We consider, in particular, the term

$$
\frac{A^{4}}{A_{1}^{5} A_{2}^{5}}\left|\tilde{\mathcal{U}}_{1}^{3}\left(\tilde{\mathcal{P}}_{1}^{1 / 2}\right)_{\eta}\right| \mathbb{1}_{A_{1} \leqslant A_{2}} \leqslant \frac{A^{4}}{A_{1}^{5} A_{2}^{5}} \frac{3}{16} A_{1}^{8} \mathbb{1}_{A_{1} \leqslant A_{2}} \leqslant \frac{3}{16} A^{2} .
$$


Thus

$$
\begin{aligned}
\left|I_{15}\right| & \leqslant \frac{30}{16} A^{2}\left|A_{1}-A_{2}\right| \int_{0}^{1}\left|\tilde{\mathcal{P}}_{1}^{1 / 2}-\tilde{\mathcal{P}}_{2}^{1 / 2}\right|(t, \eta) d \eta \\
& \leqslant \mathcal{O}(1)\left(\left|A_{1}-A_{2}\right|^{2}+\left\|\tilde{\mathcal{P}}_{1}^{1 / 2}-\tilde{\mathcal{P}}_{2}^{1 / 2}\right\|^{2}\right) .
\end{aligned}
$$

The term $I_{2}$ : Recall that the term reads as follows:

$$
I_{2}=\int_{0}^{1}\left(\tilde{\mathcal{P}}_{1}^{1 / 2}-\tilde{\mathcal{P}}_{2}^{1 / 2}\right)\left(\frac{1}{A_{2}^{6}} \tilde{\mathcal{S}}_{2}\left(\tilde{\mathcal{P}}_{2}^{1 / 2}\right)_{\eta}-\frac{1}{A_{1}^{6}} \tilde{\mathcal{S}}_{1}\left(\tilde{\mathcal{P}}_{1}^{1 / 2}\right)_{\eta}\right)(t, \eta) d \eta
$$

Here we can follow the estimates of the term $I_{3}$; cf. (5.6) and (5.9). Here we go. Define

$$
\begin{aligned}
I_{21}= & \int_{0}^{1}\left(\tilde{\mathcal{P}}_{1}^{1 / 2}-\tilde{\mathcal{P}}_{2}^{1 / 2}\right)(t, \eta)\left(\frac{1}{A_{2}^{6}}\left(\tilde{\mathcal{P}}_{2}^{1 / 2}\right)_{\eta}(t, \eta) \int_{0}^{1} e^{-\frac{1}{A_{2}}\left|\tilde{\mathcal{Y}}_{2}(t, \eta)-\tilde{\mathcal{Y}}_{2}(t, \theta)\right|} \tilde{\mathcal{U}}_{2}^{3} \tilde{\mathcal{Y}}_{2, \eta}(t, \theta) d \theta\right. \\
& \left.-\frac{1}{A_{1}^{6}}\left(\tilde{\mathcal{P}}_{1}^{1 / 2}\right)_{\eta}(t, \eta) \int_{0}^{1} e^{-\frac{1}{A_{1}}\left|\tilde{\mathcal{Y}}_{1}(t, \eta)-\tilde{\mathcal{Y}}_{1}(t, \theta)\right|} \tilde{\mathcal{U}}_{1}^{3} \tilde{\mathcal{Y}}_{1, \eta}(t, \theta) d \theta\right) d \eta \\
I_{22}= & \int_{0}^{1}\left(\tilde{\mathcal{P}}_{1}^{1 / 2}-\tilde{\mathcal{P}}_{2}^{1 / 2}\right)(t, \eta) \\
& \times\left(\frac{1}{A_{2}^{6}}\left(\tilde{\mathcal{P}}_{2}^{1 / 2}\right)_{\eta}(t, \eta) \int_{0}^{1} e^{-\frac{1}{A_{2}}\left|\tilde{\mathcal{Y}}_{2}(t, \eta)-\tilde{\mathcal{Y}}_{2}(t, \theta)\right|} \tilde{\mathcal{P}}_{2} \tilde{\mathcal{U}}_{2} \tilde{\mathcal{Y}}_{2, \eta}(t, \theta) d \theta\right. \\
& \left.-\frac{1}{A_{1}^{6}}\left(\tilde{\mathcal{P}}_{1}^{1 / 2}\right)_{\eta}(t, \eta) \int_{0}^{1} e^{-\frac{1}{A_{1}}\left|\tilde{\mathcal{Y}}_{1}(t, \eta)-\tilde{\mathcal{Y}}_{1}(t, \theta)\right|} \tilde{\mathcal{P}}_{1} \tilde{\mathcal{U}}_{1} \tilde{\mathcal{Y}}_{1, \eta}(t, \theta) d \theta\right) d \eta, \\
I_{23}= & \int_{0}^{1}\left(\tilde{\mathcal{P}}_{1}^{1 / 2}-\tilde{\mathcal{P}}_{2}^{1 / 2}\right)(t, \eta) \\
& \times\left(\frac{1}{A_{2}^{6}}\left(\tilde{\mathcal{P}}_{2}^{1 / 2}\right)_{\eta}(t, \eta) \int_{0}^{1} e^{-\frac{1}{A_{2}}\left|\tilde{\mathcal{Y}}_{2}(t, \eta)-\tilde{\mathcal{Y}}_{2}(t, \theta)\right|} \tilde{\mathcal{Q}}_{2} \tilde{\mathcal{U}}_{2, \eta}(t, \theta) d \theta\right. \\
& \left.-\frac{1}{A_{1}^{6}}\left(\tilde{\mathcal{P}}_{1}^{1 / 2}\right)_{\eta}(t, \eta) \int_{0}^{1} e^{-\frac{1}{A_{1}}\left|\tilde{\mathcal{Y}}_{1}(t, \eta)-\tilde{\mathcal{Y}}_{1}(t, \theta)\right|} \tilde{\mathcal{Q}}_{1} \tilde{\mathcal{U}}_{1, \eta}(t, \theta) d \theta\right) d \eta .
\end{aligned}
$$

Using the definition $(5.41 \mathrm{~b})$ of $\tilde{\mathcal{S}}$, we can write

$$
I_{2}=\frac{2}{3} I_{21}-2 I_{22}-I_{23}
$$

We commence with the estimates for $I_{21}$ :

$$
I_{21}=\int_{0}^{1}\left(\tilde{\mathcal{P}}_{1}^{1 / 2}-\tilde{\mathcal{P}}_{2}^{1 / 2}\right)(t, \eta)
$$




$$
\begin{aligned}
& \times\left(\frac{1}{A_{2}^{6}}\left(\tilde{\mathcal{P}}_{2}^{1 / 2}\right)_{\eta}(t, \eta) \int_{0}^{1} e^{-\frac{1}{A_{2}}\left|\tilde{\mathcal{Y}}_{2}(t, \eta)-\tilde{\mathcal{Y}}_{2}(t, \theta)\right|} \tilde{\mathcal{U}}_{2}^{3} \tilde{\mathcal{Y}}_{2, \eta}(t, \theta) d \theta\right. \\
& \left.-\frac{1}{A_{1}^{6}}\left(\tilde{\mathcal{P}}_{1}^{1 / 2}\right)_{\eta}(t, \eta) \int_{0}^{1} e^{-\frac{1}{A_{1}}\left|\tilde{\mathcal{Y}}_{1}(t, \eta)-\tilde{\mathcal{Y}}_{1}(t, \theta)\right|} \tilde{\mathcal{U}}_{1}^{3} \tilde{\mathcal{Y}}_{1, \eta}(t, \theta) d \theta\right) d \eta \\
& =\int_{0}^{1}\left(\tilde{\mathcal{P}}_{1}^{1 / 2}-\tilde{\mathcal{P}}_{2}^{1 / 2}\right)(t, \eta) \\
& \times\left(\frac{1}{A_{2}^{6}}\left(\tilde{\mathcal{P}}_{2}^{1 / 2}\right)_{\eta}(t, \eta) \int_{0}^{1} e^{-\frac{1}{A_{2}}\left|\tilde{\mathcal{Y}}_{2}(t, \eta)-\tilde{\mathcal{Y}}_{2}(t, \theta)\right|}\left(\tilde{\mathcal{U}}_{2}^{-}\right)^{3} \tilde{\mathcal{Y}}_{2, \eta}(t, \theta) d \theta\right. \\
& \left.-\frac{1}{A_{1}^{6}}\left(\tilde{\mathcal{P}}_{1}^{1 / 2}\right)_{\eta}(t, \eta) \int_{0}^{1} e^{-\frac{1}{A_{1}}\left|\tilde{\mathcal{Y}}_{1}(t, \eta)-\tilde{\mathcal{Y}}_{1}(t, \theta)\right|}\left(\tilde{\mathcal{U}}_{1}^{-}\right)^{3} \tilde{\mathcal{Y}}_{1, \eta}(t, \theta) d \theta\right) d \eta \\
& +\int_{0}^{1}\left(\tilde{\mathcal{P}}_{1}^{1 / 2}-\tilde{\mathcal{P}}_{2}^{1 / 2}\right)(t, \eta) \\
& \times\left(\frac{1}{A_{2}^{6}}\left(\tilde{\mathcal{P}}_{2}^{1 / 2}\right)_{\eta}(t, \eta) \int_{0}^{1} e^{-\frac{1}{A_{2}}\left|\tilde{\mathcal{Y}}_{2}(t, \eta)-\tilde{\mathcal{Y}}_{2}(t, \theta)\right|}\left(\tilde{\mathcal{U}}_{2}^{+}\right)^{3} \tilde{\mathcal{Y}}_{2, \eta}(t, \theta) d \theta\right. \\
& \left.-\frac{1}{A_{1}^{6}}\left(\tilde{\mathcal{P}}_{1}^{1 / 2}\right)_{\eta}(t, \eta) \int_{0}^{1} e^{-\frac{1}{A_{1}}\left|\tilde{\mathcal{Y}}_{1}(t, \eta)-\tilde{\mathcal{Y}}_{1}(t, \theta)\right|}\left(\tilde{\mathcal{U}}_{1}^{+}\right)^{3} \tilde{\mathcal{Y}}_{1, \eta}(t, \theta) d \theta\right) d \eta \text {. }
\end{aligned}
$$

The two terms are similar, and we only discuss the second one. Next, we use trick (5.4). Thus we have to consider the term

$$
\begin{aligned}
& \frac{1}{A_{2}^{6}}\left(\tilde{\mathcal{P}}_{2}^{1 / 2}\right)_{\eta}(t, \eta) \int_{0}^{\eta} e^{-\frac{1}{A_{2}}\left|\tilde{\mathcal{Y}}_{2}(t, \eta)-\tilde{\mathcal{Y}}_{2}(t, \theta)\right|}\left(\tilde{\mathcal{U}}_{2}^{+}\right)^{3} \tilde{\mathcal{Y}}_{2, \eta}(t, \theta) d \theta \\
& \quad-\frac{1}{A_{1}^{6}}\left(\tilde{\mathcal{P}}_{1}^{1 / 2}\right)_{\eta}(t, \eta) \int_{0}^{\eta} e^{-\frac{1}{A_{1}}\left|\tilde{\mathcal{Y}}_{1}(t, \eta)-\tilde{\mathcal{Y}}_{1}(t, \theta)\right|}\left(\tilde{\mathcal{U}}_{1}^{+}\right)^{3} \tilde{\mathcal{Y}}_{1, \eta}(t, \theta) d \theta \\
&=\frac{1}{A^{6}}\left(\left(\tilde{\mathcal{P}}_{2}^{1 / 2}\right)_{\eta}(t, \eta) \int_{0}^{\eta} e^{-\frac{1}{A_{2}}\left(\tilde{\mathcal{Y}}_{2}(t, \eta)-\tilde{\mathcal{Y}}_{2}(t, \theta)\right)}\left(\tilde{\mathcal{U}}_{2}^{+}\right)^{3} \tilde{\mathcal{Y}}_{2, \eta}(t, \theta) d \theta\right. \\
&\left.-\left(\tilde{\mathcal{P}}_{1}^{1 / 2}\right)_{\eta}(t, \eta) \int_{0}^{\eta} e^{-\frac{1}{A_{1}}\left(\tilde{\mathcal{Y}}_{1}(t, \eta)-\tilde{\mathcal{Y}}_{1}(t, \theta)\right)}\left(\tilde{\mathcal{U}}_{1}^{+}\right)^{3} \tilde{\mathcal{Y}}_{1, \eta}(t, \theta) d \theta\right) \\
&+\mathbb{1}_{A_{1} \leqslant A_{2}}\left(\frac{1}{A_{2}^{6}}-\frac{1}{A_{1}^{6}}\right)\left(\tilde{\mathcal{P}}_{1}^{1 / 2}\right)_{\eta}(t, \eta) \int_{0}^{\eta} e^{-\frac{1}{A_{1}}\left(\tilde{\mathcal{Y}}_{1}(t, \eta)-\tilde{\mathcal{Y}}_{1}(t, \theta)\right)}\left(\tilde{\mathcal{U}}_{1}^{+}\right)^{3} \tilde{\mathcal{Y}}_{1, \eta}(t, \theta) d \theta \\
&+\mathbb{1}_{A_{2}<A_{1}}\left(\frac{1}{A_{2}^{6}}-\frac{1}{A_{1}^{6}}\right)\left(\tilde{\mathcal{P}}_{2}^{1 / 2}\right)_{\eta}(t, \eta) \int_{0}^{\eta} e^{-\frac{1}{A_{2}}\left(\tilde{\mathcal{Y}}_{2}(t, \eta)-\tilde{\mathcal{Y}}_{2}(t, \theta)\right)}\left(\tilde{\mathcal{U}}_{2}^{+}\right)^{3} \tilde{\mathcal{Y}}_{2, \eta}(t, \theta) d \theta \\
&= \frac{1}{A^{6}}\left(\tilde{\mathcal{P}}_{2}^{1 / 2}\right)_{\eta}(t, \eta) \\
& \times \int_{0}^{\eta} e^{-\frac{1}{A_{2}}\left(\tilde{\mathcal{Y}}_{2}(t, \eta)-\tilde{\mathcal{Y}}_{2}(t, \theta)\right)}\left(\left(\tilde{\mathcal{U}}_{2}^{+}\right)^{3}-\left(\tilde{\mathcal{U}}_{1}^{+}\right)^{3}\right) \tilde{\mathcal{Y}}_{2, \eta}(t, \theta) \mathbb{1}_{\mathcal{\mathcal { U }}_{1}^{+} \leqslant \tilde{\mathcal{U}}_{2}^{+}}(t, \theta) d \theta
\end{aligned}
$$




$$
\begin{aligned}
& +\frac{1}{A^{6}}\left(\tilde{\mathcal{P}}_{1}^{1 / 2}\right)_{\eta}(t, \eta) \\
& \times \int_{0}^{\eta} e^{-\frac{1}{A_{1}}\left(\tilde{\mathcal{Y}}_{1}(t, \eta)-\tilde{\mathcal{Y}}_{1}(t, \theta)\right)}\left(\left(\tilde{\mathcal{U}}_{2}^{+}\right)^{3}-\left(\tilde{\mathcal{U}}_{1}^{+}\right)^{3}\right) \tilde{\mathcal{Y}}_{1, \eta}(t, \theta) \mathbb{1}_{\tilde{\mathcal{U}}_{2}^{+}<\tilde{\mathcal{U}}_{1}^{+}}(t, \theta) d \theta \\
& +\frac{1}{A^{6}}\left(\tilde{\mathcal{P}}_{2}^{1 / 2}\right)_{\eta}(t, \eta) \int_{0}^{\eta} e^{-\frac{1}{A_{2}}\left(\tilde{\mathcal{Y}}_{2}(t, \eta)-\tilde{\mathcal{Y}}_{2}(t, \theta)\right)} \min _{j}\left(\tilde{\mathcal{U}}_{j}^{+}\right)^{3} \tilde{\mathcal{Y}}_{2, \eta}(t, \theta) d \theta \\
& -\frac{1}{A^{6}}\left(\tilde{\mathcal{P}}_{1}^{1 / 2}\right)_{\eta}(t, \eta) \int_{0}^{\eta} e^{-\frac{1}{A_{1}}\left(\tilde{\mathcal{Y}}_{1}(t, \eta)-\tilde{\mathcal{Y}}_{1}(t, \theta)\right)} \min _{j}\left(\tilde{\mathcal{U}}_{j}^{+}\right)^{3} \tilde{\mathcal{Y}}_{1, \eta}(t, \theta) d \theta \\
& +\mathbb{1}_{A_{1} \leqslant A_{2}}\left(\frac{1}{A_{2}^{6}}-\frac{1}{A_{1}^{6}}\right)\left(\tilde{\mathcal{P}}_{1}^{1 / 2}\right)_{\eta}(t, \eta) \int_{0}^{\eta} e^{-\frac{1}{A_{1}}\left(\tilde{\mathcal{Y}}_{1}(t, \eta)-\tilde{\mathcal{Y}}_{1}(t, \theta)\right)}\left(\tilde{\mathcal{U}}_{1}^{+}\right)^{3} \tilde{\mathcal{Y}}_{1, \eta}(t, \theta) d \theta \\
& +\mathbb{1}_{A_{2}<A_{1}}\left(\frac{1}{A_{2}^{6}}-\frac{1}{A_{1}^{6}}\right)\left(\tilde{\mathcal{P}}_{2}^{1 / 2}\right)_{\eta}(t, \eta) \int_{0}^{\eta} e^{-\frac{1}{A_{2}}\left(\tilde{\mathcal{Y}}_{2}(t, \eta)-\tilde{\mathcal{Y}}_{2}(t, \theta)\right)}\left(\tilde{\mathcal{U}}_{2}^{+}\right)^{3} \tilde{\mathcal{Y}}_{2, \eta}(t, \theta) d \theta \\
& =\frac{1}{A^{6}}\left(\tilde{\mathcal{P}}_{2}^{1 / 2}\right)_{\eta}(t, \eta) \\
& \times \int_{0}^{\eta} e^{-\frac{1}{A_{2}}\left(\tilde{\mathcal{Y}}_{2}(t, \eta)-\tilde{\mathcal{Y}}_{2}(t, \theta)\right)}\left(\left(\tilde{\mathcal{U}}_{2}^{+}\right)^{3}-\left(\tilde{\mathcal{U}}_{1}^{+}\right)^{3}\right) \tilde{\mathcal{Y}}_{2, \eta}(t, \theta) \mathbb{1}_{\tilde{\mathcal{U}}_{1}^{+} \leqslant \tilde{\mathcal{U}}_{2}^{+}}(t, \theta) d \theta \\
& +\frac{1}{A^{6}}\left(\tilde{\mathcal{P}}_{1}^{1 / 2}\right)_{\eta}(t, \eta) \\
& \times \int_{0}^{\eta} e^{-\frac{1}{A_{1}}\left(\tilde{\mathcal{Y}}_{1}(t, \eta)-\tilde{\mathcal{Y}}_{1}(t, \theta)\right)}\left(\left(\tilde{\mathcal{U}}_{2}^{+}\right)^{3}-\left(\tilde{\mathcal{U}}_{1}^{+}\right)^{3}\right) \tilde{\mathcal{Y}}_{1, \eta}(t, \theta) \mathbb{1}_{\tilde{\mathcal{U}}_{2}^{+}<\tilde{\mathcal{U}}_{1}^{+}}(t, \theta) d \theta \\
& +\mathbb{1}_{A_{1} \leqslant A_{2}} \frac{1}{A^{6}}\left(\tilde{\mathcal{P}}_{2}^{1 / 2}\right)_{\eta}(t, \eta) \\
& \times \int_{0}^{\eta}\left(e^{-\frac{1}{A_{2}}\left(\tilde{\mathcal{Y}}_{2}(t, \eta)-\tilde{\mathcal{Y}}_{2}(t, \theta)\right)}-e^{-\frac{1}{A_{1}}\left(\tilde{\mathcal{Y}}_{2}(t, \eta)-\tilde{\mathcal{Y}}_{2}(t, \theta)\right)}\right) \min _{j}\left(\tilde{\mathcal{U}}_{j}^{+}\right)^{3} \tilde{\mathcal{Y}}_{2, \eta}(t, \theta) d \theta d \eta \\
& +\mathbb{1}_{A_{2}<A_{1}} \frac{1}{A^{6}}\left(\tilde{\mathcal{P}}_{1}^{1 / 2}\right)_{\eta}(t, \eta) \\
& \times \int_{0}^{\eta}\left(e^{-\frac{1}{A_{2}}\left(\tilde{\mathcal{Y}}_{1}(t, \eta)-\tilde{\mathcal{Y}}_{1}(t, \theta)\right)}-e^{-\frac{1}{A_{1}}\left(\tilde{\mathcal{Y}}_{1}(t, \eta)-\tilde{\mathcal{Y}}_{1}(t, \theta)\right)}\right) \min _{j}\left(\tilde{\mathcal{U}}_{j}^{+}\right)^{3} \tilde{\mathcal{Y}}_{1, \eta}(t, \theta) d \theta d \eta \\
& +\frac{1}{A^{6}}\left(\tilde{\mathcal{P}}_{2}^{1 / 2}\right)_{\eta}(t, \eta)\left(\int_{0}^{\eta}\left(e^{-\frac{1}{a}\left(\tilde{\mathcal{Y}}_{2}(t, \eta)-\tilde{\mathcal{Y}}_{2}(t, \theta)\right)}-e^{-\frac{1}{a}\left(\tilde{\mathcal{Y}}_{1}(t, \eta)-\tilde{\mathcal{Y}}_{1}(t, \theta)\right)}\right)\right. \\
& \left.\times \min _{j}\left(\tilde{\mathcal{U}}_{j}^{+}\right)^{3} \tilde{\mathcal{Y}}_{2, \eta}(t, \theta) \mathbb{1}_{B(\eta)}(t, \theta) d \theta\right) \\
& -\frac{1}{A^{6}}\left(\tilde{\mathcal{P}}_{1}^{1 / 2}\right)_{\eta}(t, \eta)\left(\int_{0}^{\eta}\left(e^{-\frac{1}{a}\left(\tilde{\mathcal{Y}}_{1}(t, \eta)-\tilde{\mathcal{Y}}_{1}(t, \theta)\right)}-e^{-\frac{1}{a}\left(\tilde{\mathcal{Y}}_{2}(t, \eta)-\tilde{\mathcal{Y}}_{2}(t, \theta)\right)}\right)\right. \\
& \left.\times \min _{j}\left(\tilde{\mathcal{U}}_{j}^{+}\right)^{3} \tilde{\mathcal{Y}}_{1, \eta}(t, \theta) \mathbb{1}_{B^{c}(\eta)}(t, \theta) d \theta\right) \\
& +\frac{1}{A^{6}}\left(\tilde{\mathcal{P}}_{2}^{1 / 2}\right)_{\eta}(t, \eta)\left(\int_{0}^{\eta} \min _{j}\left(e^{-\frac{1}{a}\left(\tilde{\mathcal{Y}}_{j}(t, \eta)-\tilde{\mathcal{Y}}_{j}(t, \theta)\right)}\right) \min _{j}\left(\tilde{\mathcal{U}}_{j}^{+}\right)^{3} \tilde{\mathcal{Y}}_{2, \eta}(t, \theta) d \theta\right)
\end{aligned}
$$




$$
\begin{aligned}
& -\frac{1}{A^{6}}\left(\tilde{\mathcal{P}}_{1}^{1 / 2}\right)_{\eta}(t, \eta)\left(\int_{0}^{\eta} \min _{j}\left(e^{-\frac{1}{a}\left(\tilde{\mathcal{Y}}_{j}(t, \eta)-\tilde{\mathcal{Y}}_{j}(t, \theta)\right)}\right) \min _{j}\left(\tilde{\mathcal{U}}_{j}^{+}\right)^{3} \tilde{\mathcal{Y}}_{1, \eta}(t, \theta) d \theta\right) \\
& +\mathbb{1}_{A_{1} \leqslant A_{2}}\left(\frac{1}{A_{2}^{6}}-\frac{1}{A_{1}^{6}}\right)\left(\tilde{\mathcal{P}}_{1}^{1 / 2}\right)_{\eta}(t, \eta) \int_{0}^{\eta} e^{-\frac{1}{A_{1}}\left(\tilde{\mathcal{Y}}_{1}(t, \eta)-\tilde{\mathcal{Y}}_{1}(t, \theta)\right)}\left(\tilde{\mathcal{U}}_{1}^{+}\right)^{3} \tilde{\mathcal{Y}}_{1, \eta}(t, \theta) d \theta \\
& +\mathbb{1}_{A_{2}<A_{1}}\left(\frac{1}{A_{2}^{6}}-\frac{1}{A_{1}^{6}}\right)\left(\tilde{\mathcal{P}}_{2}^{1 / 2}\right)_{\eta}(t, \eta) \int_{0}^{\eta} e^{-\frac{1}{A_{2}}\left(\tilde{\mathcal{Y}}_{2}(t, \eta)-\tilde{\mathcal{Y}}_{2}(t, \theta)\right)}\left(\tilde{\mathcal{U}}_{2}^{+}\right)^{3} \tilde{\mathcal{Y}}_{2, \eta}(t, \theta) d \theta \\
& =\left(I_{211}+I_{212}+I_{213}+I_{214}+I_{215}+I_{216}+I_{217}+I_{218}+I_{219}+I_{220}\right)(t, \eta) .
\end{aligned}
$$

The same estimate works for $I_{211}$ and $I_{212}$ (cf. (5.34)):

$$
\left|\int_{0}^{1} I_{211}\left(\tilde{\mathcal{P}}_{1}^{1 / 2}-\tilde{\mathcal{P}}_{2}^{1 / 2}\right)(t, \eta) d \eta\right| \leqslant\left\|\tilde{\mathcal{P}}_{1}^{1 / 2}-\tilde{\mathcal{P}}_{2}^{1 / 2}\right\|^{2}+\int_{0}^{1} I_{211}^{2}(t, \eta) d \eta,
$$

which shows that it suffices to estimate

$$
\begin{aligned}
\int_{0}^{1} I_{211}^{2}(t, \eta) d \eta \leqslant & \frac{1}{A^{12}} \int_{0}^{1}\left(\tilde{\mathcal{P}}_{2}^{1 / 2}\right)_{\eta}^{2}(t, \eta)\left(\int_{0}^{\eta} e^{-\frac{1}{A_{2}}\left(\tilde{\mathcal{Y}}_{2}(t, \eta)-\tilde{\mathcal{Y}}_{2}(t, \theta)\right)}\right. \\
& \left.\times\left(\left(\tilde{\mathcal{U}}_{2}^{+}\right)^{3}-\left(\tilde{\mathcal{U}}_{1}^{+}\right)^{3}\right) \tilde{\mathcal{Y}}_{2, \eta}(t, \theta) \mathbb{1}_{\tilde{\mathcal{U}}_{1}^{+} \leqslant \tilde{\mathcal{U}}_{2}^{+}}(t, \theta) d \theta\right)^{2} d \eta \\
\leqslant & \mathcal{O}(1)\left\|\tilde{\mathcal{U}}_{1}-\tilde{\mathcal{U}}_{2}\right\|^{2},
\end{aligned}
$$

where we used that $\left(\left(\tilde{\mathcal{P}}_{2}^{1 / 2}\right)_{\eta}\right)^{2} \leqslant \frac{1}{8} A_{2}^{3} \tilde{\mathcal{Y}}_{2, \eta}$.

Regarding the term $I_{213}$ (and $I_{214}$ ), we find

$$
\begin{aligned}
&\left|\int_{0}^{1} I_{213}\left(\tilde{\mathcal{P}}_{1}^{1 / 2}-\tilde{\mathcal{P}}_{2}^{1 / 2}\right)(t, \eta) d \eta\right| \\
&=\mathbb{1}_{A_{1} \leqslant A_{2}} \frac{1}{A^{6}} \mid \int_{0}^{1}\left(\tilde{\mathcal{P}}_{1}^{1 / 2}-\tilde{\mathcal{P}}_{2}^{1 / 2}\right)\left(\tilde{\mathcal{P}}_{2}^{1 / 2}\right)_{\eta}(t, \eta) \\
& \quad \times \int_{0}^{\eta}\left(e^{-\frac{1}{A_{2}}\left(\tilde{\mathcal{Y}}_{2}(t, \eta)-\tilde{\mathcal{Y}}_{2}(t, \theta)\right)}-e^{-\frac{1}{A_{1}}\left(\tilde{\mathcal{Y}}_{2}(t, \eta)-\tilde{\mathcal{Y}}_{2}(t, \theta)\right)}\right) \min _{j}\left(\tilde{\mathcal{U}}_{j}^{+}\right)^{3} \tilde{\mathcal{Y}}_{2, \eta}(t, \theta) d \theta d \eta \mid \\
& \leqslant \frac{2 \sqrt{2} a}{A^{6} e} \int_{0}^{1}\left|\tilde{\mathcal{P}}_{1}^{1 / 2}-\tilde{\mathcal{P}}_{2}^{1 / 2} \|\left(\tilde{\mathcal{P}}_{2}^{1 / 2}\right)_{\eta}\right|(t, \eta) \\
& \times \int_{0}^{\eta} e^{-\frac{3}{4 A_{2}}\left(\tilde{\mathcal{Y}}_{2}(t, \eta)-\tilde{\mathcal{Y}}_{2}(t, \theta)\right)} \tilde{\mathcal{U}}_{2}^{2} \tilde{\mathcal{Y}}_{2, \eta}(t, \theta) d \theta d \eta\left|A_{1}-A_{2}\right| \\
& \leqslant\left\|\tilde{\mathcal{P}}_{1}^{1 / 2}-\tilde{\mathcal{P}}_{2}^{1 / 2}\right\|^{2} \\
&+\frac{8}{A^{10} e^{2}} \int_{0}^{1}\left(\tilde{\mathcal{P}}_{2}^{1 / 2}\right)_{\eta}^{2}(t, \eta)\left(\int_{0}^{\eta} e^{-\frac{1}{A_{2}}\left(\tilde{\mathcal{Y}}_{2}(t, \eta)-\tilde{\mathcal{Y}}_{2}(t, \theta)\right)} \tilde{\mathcal{U}}_{2}^{2} \tilde{\mathcal{Y}}_{2, \eta}(t, \theta) d \theta\right)
\end{aligned}
$$




$$
\begin{aligned}
& \times\left(\int_{0}^{\eta} e^{-\frac{1}{2 A_{2}}\left(\tilde{\mathcal{Y}}_{2}(t, \eta)-\tilde{\mathcal{Y}}_{2}(t, \theta)\right)} \tilde{\mathcal{U}}_{2}^{2} \tilde{\mathcal{Y}}_{2, \eta}(t, \theta) d \theta\right) d \eta\left|A_{1}-A_{2}\right|^{2} \\
\leqslant & \left\|\tilde{\mathcal{P}}_{1}^{1 / 2}-\tilde{\mathcal{P}}_{2}^{1 / 2}\right\|^{2}+\frac{4}{A e^{2}} \int_{0}^{1} \tilde{\mathcal{P}}_{2} \tilde{\mathcal{Y}}_{2, \eta}(t, \eta) d \eta\left|A_{1}-A_{2}\right|^{2} \\
\leqslant & \mathcal{O}(1)\left(\left\|\tilde{\mathcal{P}}_{1}^{1 / 2}-\tilde{\mathcal{P}}_{2}^{1 / 2}\right\|^{2}+\left|A_{1}-A_{2}\right|^{2}\right) .
\end{aligned}
$$

Regarding the term $I_{215}$ (and $I_{216}$ ), we find

$$
\begin{aligned}
& \left|\int_{0}^{1} I_{215}\left(\tilde{\mathcal{P}}_{1}^{1 / 2}-\tilde{\mathcal{P}}_{2}^{1 / 2}\right)(t, \eta) d \eta\right| \\
& =\frac{1}{A^{6}} \mid \int_{0}^{1}\left(\tilde{\mathcal{P}}_{1}^{1 / 2}-\tilde{\mathcal{P}}_{2}^{1 / 2}\right)\left(\tilde{\mathcal{P}}_{2}^{1 / 2}\right)_{\eta}(t, \eta) \\
& \times\left(\int_{0}^{\eta}\left(e^{-\frac{1}{a}\left(\tilde{\mathcal{Y}}_{2}(t, \eta)-\tilde{\mathcal{Y}}_{2}(t, \theta)\right)}-e^{-\frac{1}{a}\left(\tilde{\mathcal{Y}}_{1}(t, \eta)-\tilde{\mathcal{Y}}_{1}(t, \theta)\right)}\right)\right. \\
& \left.\times \min _{j}\left(\tilde{\mathcal{U}}_{j}^{+}\right)^{3} \tilde{\mathcal{Y}}_{2, \eta}(t, \theta) \mathbb{1}_{B(\eta)}(t, \theta) d \theta\right) d \eta \mid \\
& \leqslant \frac{1}{a A^{6}} \int_{0}^{1}\left|\tilde{\mathcal{P}}_{1}^{1 / 2}-\tilde{\mathcal{P}}_{2}^{1 / 2}\right|\left|\left(\tilde{\mathcal{P}}_{2}^{1 / 2}\right)_{\eta}\right|(t, \eta) \\
& \times\left(\int_{0}^{\eta}\left(\left|\tilde{\mathcal{Y}}_{2}(t, \eta)-\tilde{\mathcal{Y}}_{1}(t, \eta)\right|+\left|\tilde{\mathcal{Y}}_{2}(t, \theta)-\tilde{\mathcal{Y}}_{1}(t, \theta)\right|\right)\right. \\
& \left.\times e^{-\frac{1}{a}\left(\tilde{\mathcal{Y}}_{2}(t, \eta)-\tilde{\mathcal{Y}}_{2}(t, \theta)\right)} \min _{j}\left(\tilde{\mathcal{U}}_{j}^{+}\right)^{3} \tilde{\mathcal{Y}}_{2, \eta}(t, \theta) d \theta\right) d \eta \\
& \leqslant \frac{1}{a A^{6}} \int_{0}^{1}\left|\tilde{\mathcal{P}}_{1}^{1 / 2}-\tilde{\mathcal{P}}_{2}^{1 / 2}\right|\left|\tilde{\mathcal{Y}}_{1}-\tilde{\mathcal{Y}}_{2}\right|\left|\left(\tilde{\mathcal{P}}_{2}^{1 / 2}\right)_{\eta}\right|(t, \eta) \\
& \times \int_{0}^{\eta} e^{-\frac{1}{a}\left(\tilde{\mathcal{Y}}_{2}(t, \eta)-\tilde{\mathcal{Y}}_{2}(t, \theta)\right)} \min _{j}\left(\tilde{\mathcal{U}}_{j}^{+}\right)^{3} \tilde{\mathcal{Y}}_{2, \eta}(t, \theta) d \theta d \eta \\
& +\left\|\tilde{\mathcal{P}}_{1}^{1 / 2}-\tilde{\mathcal{P}}_{2}^{1 / 2}\right\|^{2}+\frac{1}{a^{2} A^{12}} \int_{0}^{1}\left(\tilde{\mathcal{P}}_{2}^{1 / 2}\right)_{\eta}^{2}(t, \eta) \\
& \times\left(\int_{0}^{\eta}\left|\tilde{\mathcal{Y}}_{1}(t, \theta)-\tilde{\mathcal{Y}}_{2}(t, \theta)\right| e^{-\frac{1}{a}\left(\tilde{\mathcal{Y}}_{2}(t, \eta)-\tilde{\mathcal{Y}}_{2}(t, \theta)\right)} \min _{j}\left(\tilde{\mathcal{U}}_{j}^{+}\right)^{3} \tilde{\mathcal{Y}}_{2, \eta}(t, \theta) d \theta\right)^{2} d \eta \\
& \leqslant \mathcal{O}(1)\left(\left\|\tilde{\mathcal{Y}}_{1}-\tilde{\mathcal{Y}}_{2}\right\|^{2}+\left\|\tilde{\mathcal{P}}_{1}^{1 / 2}-\tilde{\mathcal{P}}_{2}^{1 / 2}\right\|^{2}\right)
\end{aligned}
$$

using the estimates in $(5.35)$ and $\left(\left(\tilde{\mathcal{P}}_{2}^{1 / 2}\right)_{\eta}\right)^{2} \leqslant \frac{1}{8} A^{3} \tilde{\mathcal{Y}}_{2, \eta}$.

The next terms are $I_{217}+I_{218}$ :

$$
I_{217}+I_{218}=\frac{1}{A^{6}}\left(\tilde{\mathcal{P}}_{2}^{1 / 2}\right)_{\eta}(t, \eta)
$$




$$
\begin{aligned}
& \times\left(\int_{0}^{\eta} \min _{j}\left(e^{-\frac{1}{a}\left(\tilde{\mathcal{Y}}_{j}(t, \eta)-\tilde{\mathcal{Y}}_{j}(t, \theta)\right)}\right) \min _{j}\left(\tilde{\mathcal{U}}_{j}^{+}\right)^{3} \tilde{\mathcal{Y}}_{2, \eta}(t, \theta) d \theta\right) \\
& -\frac{1}{A^{6}}\left(\tilde{\mathcal{P}}_{1}^{1 / 2}\right)_{\eta}(t, \eta) \\
& \times\left(\int_{0}^{\eta} \min _{j}\left(e^{-\frac{1}{a}\left(\tilde{\mathcal{Y}}_{j}(t, \eta)-\tilde{\mathcal{Y}}_{j}(t, \theta)\right)}\right) \min _{j}\left(\tilde{\mathcal{U}}_{j}^{+}\right)^{3} \tilde{\mathcal{Y}}_{1, \eta}(t, \theta) d \theta\right) \\
& =\frac{1}{A^{6}}\left(\left(\tilde{\mathcal{P}}_{2}^{1 / 2}\right)_{\eta}-\left(\tilde{\mathcal{P}}_{1}^{1 / 2}\right)_{\eta}\right)(t, \eta) \\
& \times \min _{k}\left[\int_{0}^{\eta} \min _{j}\left(e^{-\frac{1}{a}\left(\tilde{\mathcal{Y}}_{j}(t, \eta)-\tilde{\mathcal{Y}}_{j}(t, \theta)\right)}\right) \min _{j}\left(\tilde{\mathcal{U}}_{j}^{+}\right)^{3} \tilde{\mathcal{Y}}_{k, \eta}(t, \theta) d \theta\right] \\
& +\frac{1}{A^{6}}\left(\tilde{\mathcal{P}}_{1}^{1 / 2}\right)_{\eta}(t, \eta) \int_{0}^{\eta} \min _{j}\left(e^{-\frac{1}{a}\left(\tilde{\mathcal{Y}}_{j}(t, \eta)-\tilde{\mathcal{Y}}_{j}(t, \theta)\right)}\right) \\
& \times \min _{j}\left(\tilde{\mathcal{U}}_{j}^{+}\right)^{3}\left(\tilde{\mathcal{Y}}_{2, \eta}-\tilde{\mathcal{Y}}_{1, \eta}\right)(t, \theta) d \theta \mathbb{1}_{E}(t, \eta) \\
& +\frac{1}{A^{6}}\left(\tilde{\mathcal{P}}_{2}^{1 / 2}\right)_{\eta}(t, \eta) \int_{0}^{\eta} \min _{j}\left(e^{-\frac{1}{a}\left(\tilde{\mathcal{Y}}_{j}(t, \eta)-\tilde{\mathcal{Y}}_{j}(t, \theta)\right)}\right) \\
& \times \min _{j}\left(\tilde{\mathcal{U}}_{j}^{+}\right)^{3}\left(\tilde{\mathcal{Y}}_{2, \eta}-\tilde{\mathcal{Y}}_{1, \eta}\right)(t, \theta) d \theta \mathbb{1}_{E^{c}}(t, \eta) \\
& =L_{211}+L_{212}+L_{213} \text {. }
\end{aligned}
$$

The term $L_{211}$ can be estimated as in (5.36), yielding the result

$$
\left|\int_{0}^{1} L_{211}\left(\tilde{\mathcal{P}}_{1}^{1 / 2}-\tilde{\mathcal{P}}_{2}^{1 / 2}\right)(t, \eta) d \eta\right| \leqslant \mathcal{O}(1)\left\|\tilde{\mathcal{P}}_{1}^{1 / 2}-\tilde{\mathcal{P}}_{2}^{1 / 2}\right\|^{2}
$$

The terms $L_{212}$ and $L_{213}$ can be treated similarly; we list $L_{213}$ :

$$
\left|\int_{0}^{1} L_{213}\left(\tilde{\mathcal{P}}_{1}^{1 / 2}-\tilde{\mathcal{P}}_{2}^{1 / 2}\right)(t, \eta) d \eta\right| \leqslant \mathcal{O}(1)\left(\left\|\tilde{\mathcal{Y}}_{1}-\tilde{\mathcal{Y}}_{2}\right\|^{2}+\left\|\tilde{\mathcal{P}}_{1}^{1 / 2}-\tilde{\mathcal{P}}_{2}^{1 / 2}\right\|^{2}\right)
$$

see (5.37). The terms $I_{219}$ and $I_{220}$ share the same behavior. We find, when $A_{1} \leqslant$ $A_{2}$ (otherwise $I_{219}$ vanishes; see (5.38)), that

$$
\begin{aligned}
& \left|\int_{0}^{1} I_{219}\left(\tilde{\mathcal{P}}_{1}^{1 / 2}-\tilde{\mathcal{P}}_{2}^{1 / 2}\right)(t, \eta) d \eta\right| \\
& \quad=\left(\frac{1}{A_{1}^{6}}-\frac{1}{A_{2}^{6}}\right) \mid \int_{0}^{1}\left(\tilde{\mathcal{P}}_{1}^{1 / 2}-\tilde{\mathcal{P}}_{2}^{1 / 2}\right)\left(\tilde{\mathcal{P}}_{1}^{1 / 2}\right)_{\eta}(t, \eta) \\
& \quad \times\left(\int_{0}^{\eta} e^{-\frac{1}{A_{1}}\left(\tilde{\mathcal{Y}}_{1}(t, \eta)-\tilde{\mathcal{Y}}_{1}(t, \theta)\right)}\left(\tilde{\mathcal{U}}_{1}^{+}\right)^{3} \tilde{\mathcal{Y}}_{1, \eta}(t, \theta) d \theta\right) d \eta \mid
\end{aligned}
$$




$$
\leqslant \mathcal{O}(1)\left(\left|A_{1}-A_{2}\right|^{2}+\left\|\tilde{\mathcal{P}}_{1}^{1 / 2}-\tilde{\mathcal{P}}_{2}^{1 / 2}\right\|^{2}\right)
$$

Next, we consider the term $I_{22}$ :

$$
\begin{aligned}
I_{22}= & \int_{0}^{1}\left(\tilde{\mathcal{P}}_{1}^{1 / 2}-\tilde{\mathcal{P}}_{2}^{1 / 2}\right)(t, \eta) \\
& \times\left(\frac{1}{A_{2}^{6}}\left(\tilde{\mathcal{P}}_{2}^{1 / 2}\right)_{\eta}(t, \eta) \int_{0}^{1} e^{-\frac{1}{A_{2}}\left|\tilde{\mathcal{Y}}_{2}(t, \eta)-\tilde{\mathcal{Y}}_{2}(t, \theta)\right|} \tilde{\mathcal{P}}_{2} \tilde{\mathcal{U}}_{2} \tilde{\mathcal{Y}}_{2, \eta}(t, \theta) d \theta\right. \\
& \left.-\frac{1}{A_{1}^{6}}\left(\tilde{\mathcal{P}}_{1}^{1 / 2}\right)_{\eta}(t, \eta) \int_{0}^{1} e^{-\frac{1}{A_{1}}\left|\tilde{\mathcal{Y}}_{1}(t, \eta)-\tilde{\mathcal{Y}}_{1}(t, \theta)\right|} \tilde{\mathcal{P}}_{1} \tilde{\mathcal{U}}_{1} \tilde{\mathcal{Y}}_{1, \eta}(t, \theta) d \theta\right) d \eta
\end{aligned}
$$

We start by replacing the integrals $\int_{0}^{1}(\cdots) d \theta$ with $\int_{0}^{\eta}(\cdots) d \theta$; cf. (5.4). Furthermore, we write $\tilde{\mathcal{U}}_{j}=\tilde{\mathcal{U}}_{j}^{+}+\tilde{\mathcal{U}}_{j}^{-}$, and hence it suffices to consider

$$
\begin{aligned}
& \tilde{I}_{22}=\int_{0}^{1}\left(\tilde{\mathcal{P}}_{1}^{1 / 2}-\tilde{\mathcal{P}}_{2}^{1 / 2}\right)(t, \eta) \\
& \times\left(\frac{1}{A_{2}^{6}}\left(\tilde{\mathcal{P}}_{2}^{1 / 2}\right)_{\eta}(t, \eta) \int_{0}^{\eta} e^{-\frac{1}{A_{2}}\left|\tilde{\mathcal{Y}}_{2}(t, \eta)-\tilde{\mathcal{Y}}_{2}(t, \theta)\right|} \tilde{\mathcal{P}}_{2} \tilde{\mathcal{U}}_{2}^{+} \tilde{\mathcal{Y}}_{2, \eta}(t, \theta) d \theta\right. \\
& \left.-\frac{1}{A_{1}^{6}}\left(\tilde{\mathcal{P}}_{1}^{1 / 2}\right)_{\eta}(t, \eta) \int_{0}^{\eta} e^{-\frac{1}{A_{1}}\left|\tilde{\mathcal{Y}}_{1}(t, \eta)-\tilde{\mathcal{Y}}_{1}(t, \theta)\right|} \tilde{\mathcal{P}}_{1} \tilde{\mathcal{U}}_{1}^{+} \tilde{\mathcal{Y}}_{1, \eta}(t, \theta) d \theta\right) d \eta \\
& =\frac{1}{A^{6}} \int_{0}^{1}\left(\tilde{\mathcal{P}}_{1}^{1 / 2}-\tilde{\mathcal{P}}_{2}^{1 / 2}\right)(t, \eta) \\
& \times\left(\left(\tilde{\mathcal{P}}_{2}^{1 / 2}\right)_{\eta}(t, \eta) \int_{0}^{\eta} e^{-\frac{1}{A_{2}}\left(\tilde{\mathcal{Y}}_{2}(t, \eta)-\tilde{\mathcal{Y}}_{2}(t, \theta)\right)} \tilde{\mathcal{P}}_{2} \tilde{\mathcal{U}}_{2}^{+} \tilde{\mathcal{Y}}_{2, \eta}(t, \theta) d \theta\right. \\
& \left.-\left(\tilde{\mathcal{P}}_{1}^{1 / 2}\right)_{\eta}(t, \eta) \int_{0}^{\eta} e^{-\frac{1}{A_{1}}\left(\tilde{\mathcal{Y}}_{1}(t, \eta)-\tilde{\mathcal{Y}}_{1}(t, \theta)\right)} \tilde{\mathcal{P}}_{1} \tilde{\mathcal{U}}_{1}^{+} \tilde{\mathcal{Y}}_{1, \eta}(t, \theta) d \theta\right) d \eta \\
& +\frac{A_{1}^{6}-A_{2}^{6}}{A_{1}^{6} A_{2}^{6}} \mathbb{1}_{A_{2} \leqslant A_{1}} \int_{0}^{1}\left(\tilde{\mathcal{P}}_{1}^{1 / 2}-\tilde{\mathcal{P}}_{2}^{1 / 2}\right)(t, \eta)\left(\tilde{\mathcal{P}}_{2}^{1 / 2}\right)_{\eta}(t, \eta) \\
& \times\left(\int_{0}^{\eta} e^{-\frac{1}{A_{2}}\left(\tilde{\mathcal{Y}}_{2}(t, \eta)-\tilde{\mathcal{Y}}_{2}(t, \theta)\right)} \tilde{\mathcal{P}}_{2} \tilde{\mathcal{U}}_{2}^{+} \tilde{\mathcal{Y}}_{2, \eta}(t, \theta) d \theta\right) d \eta \\
& +\frac{A_{1}^{6}-A_{2}^{6}}{A_{1}^{6} A_{2}^{6}} \mathbb{1}_{A_{1}<A_{2}} \int_{0}^{1}\left(\tilde{\mathcal{P}}_{1}^{1 / 2}-\tilde{\mathcal{P}}_{2}^{1 / 2}\right)(t, \eta)\left(\tilde{\mathcal{P}}_{1}^{1 / 2}\right)_{\eta}(t, \eta) \\
& \times\left(\int_{0}^{\eta} e^{-\frac{1}{A_{1}}\left(\tilde{\mathcal{Y}}_{1}(t, \eta)-\tilde{\mathcal{Y}}_{1}(t, \theta)\right)} \tilde{\mathcal{P}}_{1} \tilde{\mathcal{U}}_{1}^{+} \tilde{\mathcal{Y}}_{1, \eta}(t, \theta) d \theta\right) d \eta \\
& =N_{1}+N_{2}+N_{3} \text {. }
\end{aligned}
$$


We consider first $N_{1}$, where we get

$$
\begin{aligned}
& N_{1}=\frac{1}{A^{6}} \int_{0}^{1}\left(\tilde{\mathcal{P}}_{1}^{1 / 2}-\tilde{\mathcal{P}}_{2}^{1 / 2}\right)(t, \eta) \\
& \times\left[\left(\tilde{\mathcal{P}}_{2}^{1 / 2}\right)_{\eta}(t, \eta) \int_{0}^{\eta} e^{-\frac{1}{A_{2}}\left(\tilde{\mathcal{Y}}_{2}(t, \eta)-\tilde{\mathcal{Y}}_{2}(t, \theta)\right)} \tilde{\mathcal{P}}_{2} \tilde{\mathcal{U}}_{2}^{+} \tilde{\mathcal{Y}}_{2, \eta}(t, \theta) d \theta\right. \\
& \left.-\left(\tilde{\mathcal{P}}_{1}^{1 / 2}\right)_{\eta}(t, \eta) \int_{0}^{\eta} e^{-\frac{1}{A_{1}}\left(\tilde{\mathcal{Y}}_{1}(t, \eta)-\tilde{\mathcal{Y}}_{1}(t, \theta)\right)} \tilde{\mathcal{P}}_{1} \tilde{\mathcal{U}}_{1}^{+} \tilde{\mathcal{Y}}_{1, \eta}(t, \theta) d \theta\right] d \eta \\
& =\frac{1}{A^{6}} \int_{0}^{1}\left(\tilde{\mathcal{P}}_{1}^{1 / 2}-\tilde{\mathcal{P}}_{2}^{1 / 2}\right)(t, \eta) \\
& \times\left[\left(\tilde{\mathcal{P}}_{2}^{1 / 2}\right)_{\eta}(t, \eta) \int_{0}^{\eta} e^{-\frac{1}{A_{2}}\left(\tilde{\mathcal{Y}}_{2}(t, \eta)-\tilde{\mathcal{Y}}_{2}(t, \theta)\right)}\left(\tilde{\mathcal{P}}_{2}-\tilde{\mathcal{P}}_{1}\right) \tilde{\mathcal{U}}_{2}^{+} \tilde{\mathcal{Y}}_{2, \eta} \mathbb{1}_{\tilde{\mathcal{P}}_{1} \leqslant \tilde{\mathcal{P}}_{2}}(t, \theta) d \theta\right. \\
& +\left(\tilde{\mathcal{P}}_{1}^{1 / 2}\right)_{\eta}(t, \eta) \int_{0}^{\eta} e^{-\frac{1}{A_{1}}\left(\tilde{\mathcal{Y}}_{1}(t, \eta)-\tilde{\mathcal{Y}}_{1}(t, \theta)\right)}\left(\tilde{\mathcal{P}}_{2}-\tilde{\mathcal{P}}_{1}\right) \tilde{\mathcal{U}}_{1}^{+} \tilde{\mathcal{Y}}_{1, \eta} \mathbb{1}_{\tilde{\mathcal{P}}_{2}<\tilde{\mathcal{P}}_{1}}(t, \theta) d \theta \\
& +\left(\tilde{\mathcal{P}}_{2}^{1 / 2}\right)_{\eta}(t, \eta) \\
& \times \int_{0}^{\eta} e^{-\frac{1}{A_{2}}\left(\tilde{\mathcal{Y}}_{2}(t, \eta)-\tilde{\mathcal{Y}}_{2}(t, \theta)\right)} \min _{j}\left(\tilde{\mathcal{P}}_{j}\right)\left(\tilde{\mathcal{U}}_{2}^{+}-\tilde{\mathcal{U}}_{1}^{+}\right) \tilde{\mathcal{Y}}_{2, \eta} \mathbb{1}_{\tilde{\mathcal{U}}_{1}^{+} \leqslant \tilde{\mathcal{U}}_{2}^{+}}(t, \theta) d \theta \\
& +\left(\tilde{\mathcal{P}}_{1}^{1 / 2}\right)_{\eta}(t, \eta) \\
& \times \int_{0}^{\eta} e^{-\frac{1}{A_{1}}\left(\tilde{\mathcal{Y}}_{1}(t, \eta)-\tilde{\mathcal{Y}}_{1}(t, \theta)\right)} \min _{j}\left(\tilde{\mathcal{P}}_{j}\right)\left(\tilde{\mathcal{U}}_{2}^{+}-\tilde{\mathcal{U}}_{1}^{+}\right) \tilde{\mathcal{Y}}_{1, \eta} \mathbb{1}_{\tilde{\mathcal{U}}_{2}^{+}<\tilde{\mathcal{U}}_{1}^{+}}(t, \theta) d \theta \\
& +\left(\tilde{\mathcal{P}}_{2}^{1 / 2}\right)_{\eta}(t, \eta) \\
& \times \int_{0}^{\eta} e^{-\frac{1}{A_{2}}\left(\tilde{\mathcal{Y}}_{2}(t, \eta)-\tilde{\mathcal{Y}}_{2}(t, \theta)\right)} \min _{j}\left(\tilde{\mathcal{P}}_{j}\right) \min _{j}\left(\tilde{\mathcal{U}}_{j}^{+}\right) \tilde{\mathcal{Y}}_{2, \eta}(t, \theta) d \theta \\
& \left.-\left(\tilde{\mathcal{P}}_{1}^{1 / 2}\right)_{\eta}(t, \eta) \int_{0}^{\eta} e^{-\frac{1}{A_{1}}\left(\tilde{\mathcal{Y}}_{1}(t, \eta)-\tilde{\mathcal{Y}}_{1}(t, \theta)\right)} \min _{j}\left(\tilde{\mathcal{P}}_{j}\right) \min _{j}\left(\tilde{\mathcal{U}}_{j}^{+}\right) \tilde{\mathcal{Y}}_{1, \eta}(t, \theta) d \theta\right] d \eta \\
& =N_{11}+N_{12}+N_{13}+N_{14}+N_{15}+N_{16} \text {. }
\end{aligned}
$$

The terms $N_{11}$ and $N_{12}$ can be treated similarly. To that end, we find

$$
\begin{aligned}
\left|N_{11}\right|= & \frac{1}{A^{6}} \mid \int_{0}^{1}\left(\tilde{\mathcal{P}}_{1}^{1 / 2}-\tilde{\mathcal{P}}_{2}^{1 / 2}\right)\left(\tilde{\mathcal{P}}_{2}^{1 / 2}\right)_{\eta}(t, \eta) \\
& \times\left(\int_{0}^{\eta} e^{-\frac{1}{A_{2}}\left(\tilde{\mathcal{Y}}_{2}(t, \eta)-\tilde{\mathcal{Y}}_{2}(t, \theta)\right)}\left(\tilde{\mathcal{P}}_{2}-\tilde{\mathcal{P}}_{1}\right) \tilde{\mathcal{U}}_{2}^{+} \tilde{\mathcal{Y}}_{2, \eta} \mathbb{1}_{\tilde{\mathcal{P}}_{1} \leqslant \tilde{\mathcal{P}}_{2}}(t, \theta) d \theta\right) d \eta \mid \\
\leqslant & \left\|\tilde{\mathcal{P}}_{1}^{1 / 2}-\tilde{\mathcal{P}}_{2}^{1 / 2}\right\|^{2}+\frac{1}{A^{12}} \int_{0}^{1}\left(\left(\tilde{\mathcal{P}}_{2}^{1 / 2}\right)_{\eta}\right)^{2}(t, \eta)
\end{aligned}
$$




$$
\begin{aligned}
& \times\left(\int_{0}^{\eta} e^{-\frac{1}{A_{2}}\left(\tilde{\mathcal{Y}}_{2}(t, \eta)-\tilde{\mathcal{Y}}_{2}(t, \theta)\right)}\left(\tilde{\mathcal{P}}_{2}-\tilde{\mathcal{P}}_{1}\right) \tilde{\mathcal{U}}_{2}^{+} \tilde{\mathcal{Y}}_{2, \eta} \mathbb{1}_{\tilde{\mathcal{P}}_{1} \leqslant \tilde{\mathcal{P}}_{2}}(t, \theta) d \theta\right)^{2} d \eta \\
& \leqslant\left\|\tilde{\mathcal{P}}_{1}^{1 / 2}-\tilde{\mathcal{P}}_{2}^{1 / 2}\right\|^{2} \\
& +\frac{1}{2 A^{9}} \int_{0}^{1} \tilde{\mathcal{Y}}_{2, \eta}(t, \eta)\left(\int_{0}^{\eta} e^{-\frac{1}{A_{2}}\left(\tilde{\mathcal{Y}}_{2}(t, \eta)-\tilde{\mathcal{Y}}_{2}(t, \theta)\right)}\left(\tilde{\mathcal{U}}_{2}^{+}\right)^{2} \tilde{\mathcal{Y}}_{2, \eta}(t, \theta) d \theta\right) \\
& \times\left(\int_{0}^{\eta} e^{-\frac{1}{A_{2}}\left(\tilde{\mathcal{Y}}_{2}(t, \eta)-\tilde{\mathcal{Y}}_{2}(t, \theta)\right)}\left|\tilde{\mathcal{P}}_{2}^{1 / 2}-\tilde{\mathcal{P}}_{1}^{1 / 2}\right|^{2} \tilde{\mathcal{P}}_{2} \tilde{\mathcal{Y}}_{2, \eta}(t, \theta) d \theta\right) d \eta \\
& \leqslant\left\|\tilde{\mathcal{P}}_{1}^{1 / 2}-\tilde{\mathcal{P}}_{2}^{1 / 2}\right\|^{2}+\frac{2}{A^{8}} \int_{0}^{1} \tilde{\mathcal{P}}_{2} \tilde{\mathcal{Y}}_{2, \eta}(t, \eta) \\
& \times\left(\int_{0}^{\eta} e^{-\frac{1}{A_{2}}\left(\tilde{\mathcal{Y}}_{2}(t, \eta)-\tilde{\mathcal{Y}}_{2}(t, \theta)\right)}\left|\tilde{\mathcal{P}}_{2}^{1 / 2}-\tilde{\mathcal{P}}_{1}^{1 / 2}\right|^{2} \tilde{\mathcal{P}}_{2} \tilde{\mathcal{Y}}_{2, \eta}(t, \theta) d \theta\right) d \eta \\
& \leqslant \mathcal{O}(1)\left\|\tilde{\mathcal{P}}_{1}^{1 / 2}-\tilde{\mathcal{P}}_{2}^{1 / 2}\right\|^{2},
\end{aligned}
$$

where we have used (4.15e) and (4.17c). The terms $N_{13}$ and $N_{14}$ follow the same lines. More precisely,

$$
\begin{aligned}
& \left|N_{13}\right|=\frac{1}{A^{6}} \mid \int_{0}^{1}\left(\tilde{\mathcal{P}}_{1}^{1 / 2}-\tilde{\mathcal{P}}_{2}^{1 / 2}\right)\left(\tilde{\mathcal{P}}_{2}^{1 / 2}\right)_{\eta}(t, \eta) \\
& \times \int_{0}^{\eta} e^{-\frac{1}{A_{2}}\left(\tilde{\mathcal{Y}}_{2}(t, \eta)-\tilde{\mathcal{Y}}_{2}(t, \theta)\right)} \min _{j}\left(\tilde{\mathcal{P}}_{j}\right)\left(\tilde{\mathcal{U}}_{2}^{+}-\tilde{\mathcal{U}}_{1}^{+}\right) \tilde{\mathcal{Y}}_{2, \eta} \mathbb{1}_{\tilde{\mathcal{U}}_{1}^{+} \leqslant \tilde{\mathcal{U}}_{2}^{+}}(t, \theta) d \theta d \eta \\
& \leqslant \frac{1}{A^{6}} \int_{0}^{1}\left|\tilde{\mathcal{P}}_{1}^{1 / 2}-\tilde{\mathcal{P}}_{2}^{1 / 2}\right|\left|\left(\tilde{\mathcal{P}}_{2}^{1 / 2}\right)_{\eta}\right|(t, \eta) \\
& \times \int_{0}^{\eta} e^{-\frac{1}{A_{2}}\left(\tilde{\mathcal{Y}}_{2}(t, \eta)-\tilde{\mathcal{Y}}_{2}(t, \theta)\right)} \min _{j}\left(\tilde{\mathcal{P}}_{j}\right)\left|\tilde{\mathcal{U}}_{2}^{+}-\tilde{\mathcal{U}}_{1}^{+}\right| \tilde{\mathcal{Y}}_{2, \eta} \mathbb{1}_{\tilde{\mathcal{U}}_{1}^{+} \leqslant \tilde{\mathcal{U}}_{2}^{+}}(t, \theta) d \theta d \eta \\
& \leqslant\left\|\tilde{\mathcal{P}}_{1}^{1 / 2}-\tilde{\mathcal{P}}_{2}^{1 / 2}\right\|^{2}+\frac{1}{A^{12}} \int_{0}^{1}\left(\left(\tilde{\mathcal{P}}_{2}^{1 / 2}\right)_{\eta}\right)^{2}(t, \eta) \\
& \times\left(\int_{0}^{\eta} e^{-\frac{1}{A_{2}}\left(\tilde{\mathcal{Y}}_{2}(t, \eta)-\tilde{\mathcal{Y}}_{2}(t, \theta)\right)} \min _{j}\left(\tilde{\mathcal{P}}_{j}\right)\left|\tilde{\mathcal{U}}_{2}^{+}-\tilde{\mathcal{U}}_{1}^{+}\right| \tilde{\mathcal{Y}}_{2, \eta}(t, \theta) d \theta\right)^{2} d \eta \\
& \leqslant\left\|\tilde{\mathcal{P}}_{1}^{1 / 2}-\tilde{\mathcal{P}}_{2}^{1 / 2}\right\|^{2}+\frac{1}{A^{12}} \int_{0}^{1}\left(\left(\tilde{\mathcal{P}}_{2}^{1 / 2}\right)_{\eta}\right)^{2}(t, \eta) \\
& \times\left(\int_{0}^{\eta} e^{-\frac{3}{2 A_{2}}\left(\tilde{\mathcal{Y}}_{2}(t, \eta)-\tilde{\mathcal{Y}}_{2}(t, \theta)\right)} \tilde{\mathcal{P}}_{2} \tilde{\mathcal{Y}}_{2, \eta}(t, \theta) d \theta\right) \\
& \times\left(\int_{0}^{\eta} e^{-\frac{1}{2 A_{2}}\left(\tilde{\mathcal{Y}}_{2}(t, \eta)-\tilde{\mathcal{Y}}_{2}(t, \theta)\right)}\left(\tilde{\mathcal{U}}_{2}^{+}-\tilde{\mathcal{U}}_{1}^{+}\right)^{2} \tilde{\mathcal{P}}_{2} \tilde{\mathcal{Y}}_{2, \eta}(t, \theta) d \theta\right) d \eta
\end{aligned}
$$




$$
\begin{aligned}
\leqslant & \left\|\tilde{\mathcal{P}}_{1}^{1 / 2}-\tilde{\mathcal{P}}_{2}^{1 / 2}\right\|^{2}+\frac{2}{A^{11}} \int_{0}^{1} \tilde{\mathcal{P}}_{2}\left(\left(\tilde{\mathcal{P}}_{2}^{1 / 2}\right)_{\eta}\right)^{2}(t, \eta) \\
& \times\left(\int_{0}^{\eta} e^{-\frac{1}{2 A_{2}}\left(\tilde{\mathcal{Y}}_{2}(t, \eta)-\tilde{\mathcal{Y}}_{2}(t, \theta)\right)}\left(\tilde{\mathcal{U}}_{2}-\tilde{\mathcal{U}}_{1}\right)^{2} \tilde{\mathcal{P}}_{2} \tilde{\mathcal{Y}}_{2, \eta}(t, \theta) d \theta\right) d \eta \\
\leqslant & \mathcal{O}(1)\left(\left\|\tilde{\mathcal{P}}_{2}^{1 / 2}-\tilde{\mathcal{P}}_{1}^{1 / 2}\right\|^{2}+\left\|\tilde{\mathcal{U}}_{2}-\tilde{\mathcal{U}}_{1}\right\|^{2}\right),
\end{aligned}
$$

by applying $(4.15 \mathrm{e}),(4.16 \mathrm{e})$, and $(4.17 \mathrm{c})$. The term $N_{15}+N_{16}$ can be estimated as follows:

$$
\begin{aligned}
& N_{15}+N_{16}=\frac{1}{A^{6}} \int_{0}^{1}\left(\tilde{\mathcal{P}}_{1}^{1 / 2}-\tilde{\mathcal{P}}_{2}^{1 / 2}\right)(t, \eta) \\
& \times\left[\left(\tilde{\mathcal{P}}_{2}^{1 / 2}\right)_{\eta}(t, \eta) \int_{0}^{\eta} e^{-\frac{1}{A_{2}}\left(\tilde{\mathcal{Y}}_{2}(t, \eta)-\tilde{\mathcal{Y}}_{2}(t, \theta)\right)} \min _{j}\left(\tilde{\mathcal{P}}_{j}\right) \min _{j}\left(\tilde{\mathcal{U}}_{j}^{+}\right) \tilde{\mathcal{Y}}_{2, \eta}(t, \theta) d \theta\right. \\
& -\left(\tilde{\mathcal{P}}_{1}^{1 / 2}\right)_{\eta}(t, \eta) \int_{0}^{\eta} e^{-\frac{1}{A_{1}}\left(\tilde{\mathcal{Y}}_{1}(t, \eta)-\tilde{\mathcal{Y}}_{1}(t, \theta)\right)} \\
& \left.\times \min _{j}\left(\tilde{\mathcal{P}}_{j}\right) \min _{j}\left(\tilde{\mathcal{U}}_{j}^{+}\right) \tilde{\mathcal{Y}}_{1, \eta}(t, \theta) d \theta\right] d \eta \\
& =\mathbb{1}_{A_{1} \leqslant A_{2}} \frac{1}{A^{6}} \int_{0}^{1}\left(\tilde{\mathcal{P}}_{1}^{1 / 2}-\tilde{\mathcal{P}}_{2}^{1 / 2}\right)(t, \eta)\left(\tilde{\mathcal{P}}_{2}^{1 / 2}\right)_{\eta}(t, \eta) \\
& \times\left(\int_{0}^{\eta}\left(e^{-\frac{1}{A_{2}}\left(\tilde{\mathcal{Y}}_{2}(t, \eta)-\tilde{\mathcal{Y}}_{2}(t, \theta)\right)-} e^{-\frac{1}{A_{1}}\left(\tilde{\mathcal{Y}}_{2}(t, \eta)-\tilde{\mathcal{Y}}_{2}(t, \theta)\right)}\right)\right. \\
& \left.\times \min _{j}\left(\tilde{\mathcal{P}}_{j}\right) \min _{j}\left(\tilde{\mathcal{U}}_{j}^{+}\right) \tilde{\mathcal{Y}}_{2, \eta}(t, \theta) d \theta\right) d \eta \\
& +\mathbb{1}_{A_{2}<A_{1}} \frac{1}{A^{6}} \int_{0}^{1}\left(\tilde{\mathcal{P}}_{1}^{1 / 2}-\tilde{\mathcal{P}}_{2}^{1 / 2}\right)(t, \eta)\left(\tilde{\mathcal{P}}_{1}^{1 / 2}\right)_{\eta}(t, \eta) \\
& \times\left(\int_{0}^{\eta}\left(e^{-\frac{1}{A_{2}}\left(\tilde{\mathcal{Y}}_{1}(t, \eta)-\tilde{\mathcal{Y}}_{1}(t, \theta)\right)}-e^{-\frac{1}{A_{1}}\left(\tilde{\mathcal{Y}}_{1}(t, \eta)-\tilde{\mathcal{Y}}_{1}(t, \theta)\right)}\right)\right. \\
& \left.\times \min _{j}\left(\tilde{\mathcal{P}}_{j}\right) \min _{j}\left(\tilde{\mathcal{U}}_{j}^{+}\right) \tilde{\mathcal{Y}}_{1, \eta}(t, \theta) d \theta\right) d \eta \\
& +\frac{1}{A^{6}} \int_{0}^{1}\left(\tilde{\mathcal{P}}_{1}^{1 / 2}-\tilde{\mathcal{P}}_{2}^{1 / 2}\right)\left(\tilde{\mathcal{P}}_{2}^{1 / 2}\right)_{\eta}(t, \eta) \\
& \times \int_{0}^{\eta}\left(e^{-\frac{1}{a}\left(\tilde{\mathcal{Y}}_{2}(t, \eta)-\tilde{\mathcal{Y}}_{2}(t, \theta)\right)}-e^{-\frac{1}{a}\left(\tilde{\mathcal{Y}}_{1}(t, \eta)-\tilde{\mathcal{Y}}_{1}(t, \theta)\right)}\right) \\
& \times \min _{j}\left(\tilde{\mathcal{P}}_{j}\right) \min _{j}\left(\tilde{\mathcal{U}}_{j}^{+}\right) \tilde{\mathcal{Y}}_{2, \eta}(t, \theta) \mathbb{1}_{B(\eta)}(t, \theta) d \theta \\
& +\frac{1}{A^{6}} \int_{0}^{1}\left(\tilde{\mathcal{P}}_{1}^{1 / 2}-\tilde{\mathcal{P}}_{2}^{1 / 2}\right)\left(\tilde{\mathcal{P}}_{1}^{1 / 2}\right)_{\eta}(t, \eta) \\
& \times \int_{0}^{\eta}\left(e^{-\frac{1}{a}\left(\tilde{\mathcal{Y}}_{2}(t, \eta)-\tilde{\mathcal{Y}}_{2}(t, \theta)\right)}-e^{-\frac{1}{a}\left(\tilde{\mathcal{Y}}_{1}(t, \eta)-\tilde{\mathcal{Y}}_{1}(t, \theta)\right)}\right)
\end{aligned}
$$




$$
\begin{aligned}
& \times \min _{j}\left(\tilde{\mathcal{P}}_{j}\right) \min _{j}\left(\tilde{\mathcal{U}}_{j}^{+}\right) \tilde{\mathcal{Y}}_{1, \eta}(t, \theta) \mathbb{1}_{B^{c}(\eta)}(t, \theta) d \theta \\
& +\frac{1}{A^{6}} \int_{0}^{1}\left(\tilde{\mathcal{P}}_{1}^{1 / 2}-\tilde{\mathcal{P}}_{2}^{1 / 2}\right)(t, \eta)\left[\left(\tilde{\mathcal{P}}_{2}^{1 / 2}\right)_{\eta}(t, \eta) \int_{0}^{\eta} \min _{j}\left(e^{-\frac{1}{a}\left(\tilde{\mathcal{Y}}_{j}(t, \eta)-\tilde{\mathcal{Y}}_{j}(t, \theta)\right)}\right)\right. \\
& \times \min _{j}\left(\tilde{\mathcal{P}}_{j}\right) \min _{j}\left(\tilde{\mathcal{U}}_{j}^{+}\right) \tilde{\mathcal{Y}}_{2, \eta}(t, \theta) d \theta \\
& -\left(\tilde{\mathcal{P}}_{1}^{1 / 2}\right)_{\eta}(t, \eta) \int_{0}^{\eta} \min _{j}\left(e^{-\frac{1}{a}\left(\tilde{\mathcal{Y}}_{j}(t, \eta)-\tilde{\mathcal{Y}}_{j}(t, \theta)\right)}\right) \\
& \left.\times \min _{j}\left(\tilde{\mathcal{P}}_{j}\right) \min _{j}\left(\tilde{\mathcal{U}}_{j}^{+}\right) \tilde{\mathcal{Y}}_{1, \eta}(t, \theta) d \theta\right] d \eta \\
& =N_{151}+N_{152}+N_{153}+N_{154}+N_{155} \text {, }
\end{aligned}
$$

which all, unfortunately, need a special treatment.

The terms $N_{151}$ and $N_{152}$ can be handled as follows:

$$
\begin{aligned}
\left|N_{151}\right| \leqslant & \mathbb{1}_{A_{1} \leqslant A_{2}} \frac{2 \sqrt{2} a}{A^{6} e} \int_{0}^{1}\left|\tilde{\mathcal{P}}_{1}^{1 / 2}-\tilde{\mathcal{P}}_{2}^{1 / 2}\right|\left|\left(\tilde{\mathcal{P}}_{2}^{1 / 2}\right)_{\eta}\right|(t, \eta) \\
& \times\left(\int_{0}^{\eta} e^{-\frac{3}{4 A_{2}}\left(\tilde{\mathcal{Y}}_{2}(t, \eta)-\tilde{\mathcal{Y}}_{2}(t, \theta)\right)} \tilde{\mathcal{P}}_{2} \tilde{\mathcal{Y}}_{2, \eta}(t, \theta) d \theta\right) d \eta\left|A_{1}-A_{2}\right| \\
\leqslant & \frac{2 \sqrt{2} a}{A^{6} e} \int_{0}^{1}\left|\tilde{\mathcal{P}}_{1}^{1 / 2}-\tilde{\mathcal{P}}_{2}^{1 / 2} \|\left(\tilde{\mathcal{P}}_{2}^{1 / 2}\right)_{\eta}\right|(t, \eta) \\
& \times\left(\int_{0}^{\eta} e^{-\frac{3}{2 A_{2}}\left(\tilde{\mathcal{Y}}_{2}(t, \eta)-\tilde{\mathcal{Y}}_{2}(t, \theta)\right)} \tilde{\mathcal{P}}_{2} \tilde{\mathcal{Y}}_{2, \eta}(t, \theta) d \theta\right)^{1 / 2} \\
& \times\left(\int_{0}^{\eta} \tilde{\mathcal{P}}_{2} \tilde{\mathcal{Y}}_{2, \eta}(t, \theta) d \theta\right)^{1 / 2} d \eta\left|A_{1}-A_{2}\right| \\
\leqslant & \frac{2 \sqrt{2} a}{A^{3} e} \int_{0}^{1}\left|\tilde{\mathcal{P}}_{1}^{1 / 2}-\tilde{\mathcal{P}}_{2}^{1 / 2} \|\left(\tilde{\mathcal{P}}_{2}^{1 / 2}\right)_{\eta}\right| \tilde{\mathcal{P}}_{2}^{1 / 2}(t, \eta) d \eta\left|A_{1}-A_{2}\right| \\
\leqslant & \mathcal{O}(1)\left(\left\|\tilde{\mathcal{P}}_{1}^{1 / 2}-\tilde{\mathcal{P}}_{2}^{1 / 2}\right\|^{2}+\left|A_{1}-A_{2}\right|^{2}\right) .
\end{aligned}
$$

The terms $N_{153}$ and $N_{154}$ can be handled as follows:

$$
\begin{aligned}
\left|N_{153}\right|= & \frac{1}{A^{6}} \mid \int_{0}^{1}\left(\tilde{\mathcal{P}}_{1}^{1 / 2}-\tilde{\mathcal{P}}_{2}^{1 / 2}\right)\left(\tilde{\mathcal{P}}_{2}^{1 / 2}\right)_{\eta}(t, \eta) \\
& \times \int_{0}^{\eta}\left(e^{-\frac{1}{a}\left(\tilde{\mathcal{Y}}_{2}(t, \eta)-\tilde{\mathcal{Y}}_{2}(t, \theta)\right)}-e^{-\frac{1}{a}\left(\tilde{\mathcal{Y}}_{1}(t, \eta)-\tilde{\mathcal{Y}}_{1}(t, \theta)\right)}\right) \\
& \times \min _{j}\left(\tilde{\mathcal{P}}_{j}\right) \min _{j}\left(\tilde{\mathcal{U}}_{j}^{+}\right) \tilde{\mathcal{Y}}_{2, \eta}(t, \theta) \mathbb{1}_{B(\eta)}(t, \theta) d \theta d \eta \mid \\
\leqslant & \frac{1}{a A^{6}} \int_{0}^{1}\left|\tilde{\mathcal{P}}_{1}^{1 / 2}-\tilde{\mathcal{P}}_{2}^{1 / 2}\right|\left|\left(\tilde{\mathcal{P}}_{2}^{1 / 2}\right)_{\eta}\right|(t, \eta)
\end{aligned}
$$




$$
\begin{aligned}
& \times \int_{0}^{\eta}\left(\left|\tilde{\mathcal{Y}}_{2}(t, \eta)-\tilde{\mathcal{Y}}_{1}(t, \eta)\right|+\left|\tilde{\mathcal{Y}}_{2}(t, \theta)-\tilde{\mathcal{Y}}_{1}(t, \theta)\right|\right) e^{-\frac{1}{a}\left(\tilde{\mathcal{Y}}_{2}(t, \eta)-\tilde{\mathcal{Y}}_{2}(t, \theta)\right)} \\
& \times \min _{j}\left(\tilde{\mathcal{P}}_{j}\right) \min _{j}\left(\tilde{\mathcal{U}}_{j}^{+}\right) \tilde{\mathcal{Y}}_{2, \eta}(t, \theta) d \theta d \eta \\
& =\frac{1}{a A^{6}} \int_{0}^{1}\left|\tilde{\mathcal{P}}_{1}^{1 / 2}-\tilde{\mathcal{P}}_{2}^{1 / 2}\right|\left|\tilde{\mathcal{Y}}_{2}-\tilde{\mathcal{Y}}_{1}\right|\left|\left(\tilde{\mathcal{P}}_{2}^{1 / 2}\right)_{\eta}\right|(t, \eta) \int_{0}^{\eta} e^{-\frac{1}{a}\left(\tilde{\mathcal{Y}}_{2}(t, \eta)-\tilde{\mathcal{Y}}_{2}(t, \theta)\right)} \\
& \times \min _{j}\left(\tilde{\mathcal{P}}_{j}\right) \min _{j}\left(\tilde{\mathcal{U}}_{j}^{+}\right) \tilde{\mathcal{Y}}_{2, \eta}(t, \theta) d \theta d \eta \\
& +\frac{1}{a A^{6}} \int_{0}^{1}\left|\tilde{\mathcal{P}}_{1}^{1 / 2}-\tilde{\mathcal{P}}_{2}^{1 / 2}\right|\left|\left(\tilde{\mathcal{P}}_{2}^{1 / 2}\right)_{\eta}\right|(t, \eta) \int_{0}^{\eta} e^{-\frac{1}{a}\left(\tilde{\mathcal{Y}}_{2}(t, \eta)-\tilde{\mathcal{Y}}_{2}(t, \theta)\right)}\left|\tilde{\mathcal{Y}}_{2}-\tilde{\mathcal{Y}}_{1}\right| \\
& \times \min _{j}\left(\tilde{\mathcal{P}}_{j}\right) \min _{j}\left(\tilde{\mathcal{U}}_{j}^{+}\right) \tilde{\mathcal{Y}}_{2, \eta}(t, \theta) d \theta d \eta \\
& \leqslant \frac{1}{\sqrt{2} A^{5}} \int_{0}^{1}\left|\tilde{\mathcal{P}}_{1}^{1 / 2}-\tilde{\mathcal{P}}_{2}^{1 / 2}\right|\left|\tilde{\mathcal{Y}}_{2}-\tilde{\mathcal{Y}}_{1}\right|\left|\left(\tilde{\mathcal{P}}_{2}^{1 / 2}\right)_{\eta}\right|(t, \eta) \\
& \times\left(\int_{0}^{\eta} e^{-\frac{1}{a}\left(\tilde{\mathcal{Y}}_{2}(t, \eta)-\tilde{\mathcal{Y}}_{2}(t, \theta)\right)} \tilde{\mathcal{P}}_{2}^{2} \tilde{\mathcal{Y}}_{2, \eta}(t, \theta) d \theta\right)^{1 / 2} \\
& \times\left(\int_{0}^{\eta} e^{-\frac{1}{a}\left(\tilde{\mathcal{Y}}_{2}(t, \eta)-\tilde{\mathcal{Y}}_{2}(t, \theta)\right)} \tilde{\mathcal{Y}}_{2, \eta}(t, \theta) d \theta\right)^{1 / 2} d \eta \\
& +\left\|\tilde{\mathcal{P}}_{1}^{1 / 2}-\tilde{\mathcal{P}}_{2}^{1 / 2}\right\|^{2}+\frac{1}{2 A^{10}} \int_{0}^{1}\left(\left(\tilde{\mathcal{P}}_{2}^{1 / 2}\right)_{\eta}\right)^{2}(t, \eta) \\
& \times\left(\int_{0}^{\eta} e^{-\frac{1}{a}\left(\tilde{\mathcal{Y}}_{2}(t, \eta)-\tilde{\mathcal{Y}}_{2}(t, \theta)\right)}\left|\tilde{\mathcal{Y}}_{2}-\tilde{\mathcal{Y}}_{1}\right| \tilde{\mathcal{P}}_{2} \tilde{\mathcal{Y}}_{2, \eta}(t, \theta) d \theta\right)^{2} d \eta \\
& \leqslant \frac{\sqrt{3}}{2 A^{2}} \int_{0}^{1}\left|\tilde{\mathcal{P}}_{1}^{1 / 2}-\tilde{\mathcal{P}}_{2}^{1 / 2}\right|\left|\tilde{\mathcal{Y}}_{2}-\tilde{\mathcal{Y}}_{1}\right| \tilde{\mathcal{P}}_{2}^{1 / 2}\left|\left(\tilde{\mathcal{P}}_{2}^{1 / 2}\right)_{\eta}\right|(t, \eta) d \eta+\left\|\tilde{\mathcal{P}}_{1}^{1 / 2}-\tilde{\mathcal{P}}_{2}^{1 / 2}\right\|^{2} \\
& +\frac{1}{2 A^{10}} \int_{0}^{1}\left(\left(\tilde{\mathcal{P}}_{2}^{1 / 2}\right)_{\eta}\right)^{2}(t, \eta)\left(\int_{0}^{\eta} e^{-\frac{3}{2 A_{2}}\left(\tilde{\mathcal{Y}}_{2}(t, \eta)-\tilde{\mathcal{Y}}_{2}(t, \theta)\right)} \tilde{\mathcal{P}}_{2} \tilde{\mathcal{Y}}_{2, \eta}(t, \theta) d \theta\right) \\
& \times\left(\int_{0}^{\eta} e^{-\frac{1}{2 a}\left(\tilde{\mathcal{Y}}_{2}(t, \eta)-\tilde{\mathcal{Y}}_{2}(t, \theta)\right)}\left(\tilde{\mathcal{Y}}_{2}-\tilde{\mathcal{Y}}_{1}\right)^{2} \tilde{\mathcal{P}}_{2} \tilde{\mathcal{Y}}_{2, \eta}(t, \theta) d \theta\right) d \eta \\
& \leqslant \mathcal{O}(1)\left(\left\|\tilde{\mathcal{P}}_{1}^{1 / 2}-\tilde{\mathcal{P}}_{2}^{1 / 2}\right\|^{2}+\left\|\tilde{\mathcal{Y}}_{1}-\tilde{\mathcal{Y}}_{2}\right\|^{2}\right),
\end{aligned}
$$

using (4.15e), (4.16b), (4.16e), and (4.17a). We consider now $N_{155}$ :

$$
\begin{aligned}
N_{155}= & \frac{1}{A^{6}} \int_{0}^{1}\left(\tilde{\mathcal{P}}_{1}^{1 / 2}-\tilde{\mathcal{P}}_{2}^{1 / 2}\right)(t, \eta) \\
& \times\left[\left(\tilde{\mathcal{P}}_{2}^{1 / 2}\right)_{\eta}(t, \eta) \int_{0}^{\eta} \min _{j}\left(e^{-\frac{1}{a}\left(\tilde{\mathcal{Y}}_{j}(t, \eta)-\tilde{\mathcal{Y}}_{j}(t, \theta)\right)}\right) \min _{j}\left(\tilde{\mathcal{P}}_{j}\right) \min _{j}\left(\tilde{\mathcal{U}}_{j}^{+}\right) \tilde{\mathcal{Y}}_{2, \eta}(t, \theta) d \theta\right. \\
& \left.-\left(\tilde{\mathcal{P}}_{1}^{1 / 2}\right)_{\eta}(t, \eta) \int_{0}^{\eta} \min _{j}\left(e^{-\frac{1}{a}\left(\tilde{\mathcal{Y}}_{j}(t, \eta)-\tilde{\mathcal{Y}}_{j}(t, \theta)\right)}\right) \min _{j}\left(\tilde{\mathcal{P}}_{j}\right) \min _{j}\left(\tilde{\mathcal{U}}_{j}^{+}\right) \tilde{\mathcal{Y}}_{1, \eta}(t, \theta) d \theta\right] d \eta
\end{aligned}
$$




$$
\begin{aligned}
= & -\frac{1}{A^{6}} \int_{0}^{1}\left(\tilde{\mathcal{P}}_{1}^{1 / 2}-\tilde{\mathcal{P}}_{2}^{1 / 2}\right)\left(\tilde{\mathcal{P}}_{1}^{1 / 2}-\tilde{\mathcal{P}}_{2}^{1 / 2}\right)_{\eta}(t, \eta) \\
& \times \min _{k}\left[\int_{0}^{\eta} \min _{j}\left(e^{-\frac{1}{a}\left(\tilde{\mathcal{Y}}_{j}(t, \eta)-\tilde{\mathcal{Y}}_{j}(t, \theta)\right)}\right) \min _{j}\left(\tilde{\mathcal{P}}_{j}\right) \min _{j}\left(\tilde{\mathcal{U}}_{j}^{+}\right) \tilde{\mathcal{Y}}_{k, \eta}(t, \theta) d \theta\right] d \eta \\
& +\frac{1}{A^{6}} \int_{0}^{1}\left(\tilde{\mathcal{P}}_{1}^{1 / 2}-\tilde{\mathcal{P}}_{2}^{1 / 2}\right)\left(\tilde{\mathcal{P}}_{2}^{1 / 2}\right)_{\eta}(t, \eta) \mathbb{1}_{\tilde{E}^{c}}(t, \eta) \\
& \times\left(\int_{0}^{\eta} \min _{j}\left(e^{-\frac{1}{a}\left(\tilde{\mathcal{Y}}_{j}(t, \eta)-\tilde{\mathcal{Y}}_{j}(t, \theta)\right)}\right) \min _{j}\left(\tilde{\mathcal{P}}_{j}\right) \min _{j}\left(\tilde{\mathcal{U}}_{j}^{+}\right)\left(\tilde{\mathcal{Y}}_{2}-\tilde{\mathcal{Y}}_{1}\right)_{\eta}(t, \theta) d \theta\right) d \eta \\
& +\frac{1}{A^{6}} \int_{0}^{1}\left(\tilde{\mathcal{P}}_{1}^{1 / 2}-\tilde{\mathcal{P}}_{2}^{1 / 2}\right)\left(\tilde{\mathcal{P}}_{1}^{1 / 2}\right)_{\eta}(t, \eta) \mathbb{1}_{\tilde{E}}(t, \eta) \\
& \times\left(\int_{0}^{\eta} \min _{j}\left(e^{-\frac{1}{a}\left(\tilde{\mathcal{Y}}_{j}(t, \eta)-\tilde{\mathcal{Y}}_{j}(t, \theta)\right)}\right) \min _{j}\left(\tilde{\mathcal{P}}_{j}\right) \min _{j}\left(\tilde{\mathcal{U}}_{j}^{+}\right)\left(\tilde{\mathcal{Y}}_{2}-\tilde{\mathcal{Y}}_{1}\right)_{\eta}(t, \theta) d \theta\right) d \eta \\
= & N_{1551}+N_{1552}+N_{1553},
\end{aligned}
$$

which, yet again, requires separate treatment. Here $\tilde{E}$ is defined in (5.16). The term $N_{1551}$ can be handled as the term $\tilde{L}_{31}$ (cf. (5.17)),

$$
\left|N_{1551}\right| \leqslant \mathcal{O}(1)\left\|\tilde{\mathcal{P}}_{1}^{1 / 2}-\tilde{\mathcal{P}}_{2}^{1 / 2}\right\|^{2}
$$

The terms $N_{1552}$ and $N_{1553}$ can be treated in the same manner:

$$
\begin{aligned}
\left|N_{1552}\right| \leqslant & \frac{1}{A^{6}} \int_{0}^{1}\left|\tilde{\mathcal{P}}_{1}^{1 / 2}-\tilde{\mathcal{P}}_{2}^{1 / 2}\right|\left|\left(\tilde{\mathcal{P}}_{2}^{1 / 2}\right)_{\eta}\right|(t, \eta) \mathbb{1}_{\tilde{E}^{c}}(t, \eta) \\
& \times\left|\int_{0}^{\eta} \min _{j}\left(e^{-\frac{1}{a}\left(\tilde{\mathcal{Y}}_{j}(t, \eta)-\tilde{\mathcal{Y}}_{j}(t, \theta)\right)}\right) \min _{j}\left(\tilde{\mathcal{P}}_{j}\right) \min _{j}\left(\tilde{\mathcal{U}}_{j}^{+}\right)\left(\tilde{\mathcal{Y}}_{2}-\tilde{\mathcal{Y}}_{1}\right)_{\eta}(t, \theta) d \theta\right| d \eta \\
\leqslant & \frac{1}{A^{6}} \int_{0}^{1}\left|\tilde{\mathcal{P}}_{1}^{1 / 2}-\tilde{\mathcal{P}}_{2}^{1 / 2}\right|\left|\tilde{\mathcal{Y}}_{1}-\tilde{\mathcal{Y}}_{2}\right| \min _{j}\left(\tilde{\mathcal{P}}_{j}\right) \min _{j}\left(\tilde{\mathcal{U}}_{j}^{+}\right)\left|\left(\tilde{\mathcal{P}}_{2}^{1 / 2}\right)_{\eta}\right|(t, \eta) d \eta \\
& +\frac{1}{A^{6}} \int_{0}^{1}\left|\tilde{\mathcal{P}}_{1}^{1 / 2}-\tilde{\mathcal{P}}_{2}^{1 / 2}\right|\left|\left(\tilde{\mathcal{P}}_{2}^{1 / 2}\right)_{\eta}\right|(t, \eta) \\
& \times \mid \int_{0}^{\eta}\left(\tilde{\mathcal{Y}}_{1}-\tilde{\mathcal{Y}}_{2}\right)\left[\frac{d}{d \theta}\left(\min _{j}\left(e^{-\frac{1}{a}\left(\tilde{\mathcal{Y}}_{j}(t, \eta)-\tilde{\mathcal{Y}}_{j}(t, \theta)\right)}\right)\right) \min _{j}\left(\tilde{\mathcal{P}}_{j}\right) \min _{j}\left(\tilde{\mathcal{U}}_{j}^{+}\right)\right. \\
& \left.+\min _{j}\left(e^{-\frac{1}{a}\left(\tilde{\mathcal{Y}}_{j}(t, \eta)-\tilde{\mathcal{Y}}_{j}(t, \theta)\right)}\right) \frac{d}{d \theta}\left(\min _{j}\left(\tilde{\mathcal{P}}_{j}\right) \min _{j}\left(\tilde{\mathcal{U}}_{j}^{+}\right)\right)\right](t, \theta) d \theta \mid d \eta \\
\leqslant & \mathcal{O}(1)\left(\left\|\tilde{\mathcal{P}}_{1}^{1 / 2}-\tilde{\mathcal{P}}_{2}^{1 / 2}\right\|^{2}+\left\|\tilde{\mathcal{Y}}_{1}-\tilde{\mathcal{Y}}_{2}\right\|^{2}\right) .
\end{aligned}
$$


The term $N_{2}$ (and also $N_{3}$ ) can be treated as follows:

$$
\begin{aligned}
\left|N_{2}\right| \leqslant & \frac{A_{1}^{6}-A_{2}^{6}}{A_{1}^{6} A_{2}^{6}} \int_{0}^{1}\left|\tilde{\mathcal{P}}_{1}^{1 / 2}-\tilde{\mathcal{P}}_{2}^{1 / 2}\right|\left|\left(\tilde{\mathcal{P}}_{2}^{1 / 2}\right)_{\eta}\right|(t, \eta) \\
& \times\left(\int_{0}^{\eta} e^{-\frac{1}{A_{2}}\left(\tilde{\mathcal{Y}}_{2}(t, \eta)-\tilde{\mathcal{Y}}_{2}(t, \theta)\right)} \tilde{\mathcal{P}}_{2}^{2} \tilde{\mathcal{Y}}_{2, \eta}(t, \theta) d \theta\right)^{1 / 2} \\
& \times\left(\int_{0}^{\eta} e^{-\frac{1}{A_{2}}\left(\tilde{\mathcal{Y}}_{2}(t, \eta)-\tilde{\mathcal{Y}}_{2}(t, \theta)\right)} \tilde{\mathcal{U}}_{2}^{2} \tilde{\mathcal{Y}}_{2, \eta}(t, \theta) d \theta\right)^{1 / 2} d \eta \\
\leqslant & \sqrt{6} \frac{A_{1}^{6}-A_{2}^{6}}{A^{6} A_{2}^{3}} \int_{0}^{1}\left|\tilde{\mathcal{P}}_{1}^{1 / 2}-\tilde{\mathcal{P}}_{2}^{1 / 2}\right| \tilde{\mathcal{P}}_{2}\left|\left(\tilde{\mathcal{P}}_{2}^{1 / 2}\right)_{\eta}\right|(t, \eta) d \eta \\
\leqslant & \mathcal{O}(1)\left|A_{1}-A_{2}\right| \int_{0}^{1}\left|\tilde{\mathcal{P}}_{1}^{1 / 2}-\tilde{\mathcal{P}}_{2}^{1 / 2}\right|(t, \eta) d \eta \\
\leqslant & \mathcal{O}(1)\left(\left\|\tilde{\mathcal{P}}_{1}^{1 / 2}-\tilde{\mathcal{P}}_{2}^{1 / 2}\right\|^{2}+\left|A_{1}-A_{2}\right|^{2}\right) .
\end{aligned}
$$

This concludes the discussion of the term $I_{22}$.

The term $I_{23}$ is handled as follows:

$$
\begin{aligned}
I_{23}= & \int_{0}^{1}\left(\tilde{\mathcal{P}}_{1}^{1 / 2}-\tilde{\mathcal{P}}_{2}^{1 / 2}\right)(t, \eta) \\
& \times\left(\frac{1}{A_{2}^{6}}\left(\tilde{\mathcal{P}}_{2}^{1 / 2}\right)_{\eta}(t, \eta) \int_{0}^{1} e^{-\frac{1}{A_{2}}\left|\tilde{\mathcal{Y}}_{2}(t, \eta)-\tilde{\mathcal{Y}}_{2}(t, \theta)\right|} \tilde{\mathcal{Q}}_{2} \tilde{\mathcal{U}}_{2, \eta}(t, \theta) d \theta\right. \\
& \left.-\frac{1}{A_{1}^{6}}\left(\tilde{\mathcal{P}}_{1}^{1 / 2}\right)_{\eta}(t, \eta) \int_{0}^{1} e^{-\frac{1}{A_{1}}\left|\tilde{\mathcal{Y}}_{1}(t, \eta)-\tilde{\mathcal{Y}}_{1}(t, \theta)\right|} \tilde{\mathcal{Q}}_{1} \tilde{\mathcal{U}}_{1, \eta}(t, \theta) d \theta\right) d \eta
\end{aligned}
$$

We start by replacing the integrals $\int_{0}^{1}(\cdots) d \theta$ with $\int_{0}^{\eta}(\cdots) d \theta$; cf. (5.4). Thus

$$
\begin{aligned}
\tilde{I}_{23}= & \int_{0}^{1}\left(\tilde{\mathcal{P}}_{1}^{1 / 2}-\tilde{\mathcal{P}}_{2}^{1 / 2}\right)\left(\frac{1}{A_{2}^{6}}\left(\tilde{\mathcal{P}}_{2}^{1 / 2}\right)_{\eta}(t, \eta) \int_{0}^{\eta} e^{-\frac{1}{A_{2}}\left(\tilde{\mathcal{Y}}_{2}(t, \eta)-\tilde{\mathcal{Y}}_{2}(t, \theta)\right)} \tilde{\mathcal{Q}}_{2} \tilde{\mathcal{U}}_{2, \eta}(t, \theta) d \theta\right. \\
& \left.-\frac{1}{A_{1}}\left(\tilde{\mathcal{P}}_{1}^{1 / 2}\right)_{\eta}(t, \eta) \int_{0}^{\eta} e^{-\frac{1}{A_{1}}\left(\tilde{\mathcal{Y}}_{1}(t, \eta)-\tilde{\mathcal{Y}}_{1}(t, \theta)\right)} \tilde{\mathcal{Q}}_{1} \tilde{\mathcal{U}}_{1, \eta}(t, \theta) d \theta\right) d \eta \\
= & \frac{1}{A^{6}} \int_{0}^{1}\left(\tilde{\mathcal{P}}_{1}^{1 / 2}-\tilde{\mathcal{P}}_{2}^{1 / 2}\right)\left(\left(\tilde{\mathcal{P}}_{2}^{1 / 2}\right)_{\eta}(t, \eta) \int_{0}^{\eta} e^{-\frac{1}{A_{2}}\left(\tilde{\mathcal{Y}}_{2}(t, \eta)-\tilde{\mathcal{Y}}_{2}(t, \theta)\right)} \tilde{\mathcal{Q}}_{2} \tilde{\mathcal{U}}_{2, \eta}(t, \theta) d \theta\right. \\
& \left.-\left(\tilde{\mathcal{P}}_{1}^{1 / 2}\right)_{\eta}(t, \eta) \int_{0}^{\eta} e^{-\frac{1}{A_{1}}\left(\tilde{\mathcal{Y}}_{1}(t, \eta)-\tilde{\mathcal{Y}}_{1}(t, \theta)\right)} \tilde{\mathcal{Q}}_{1} \tilde{\mathcal{U}}_{1, \eta}(t, \theta) d \theta\right) d \eta \\
& +\mathbb{1}_{A_{2} \leqslant A_{1}}\left(\frac{1}{A_{2}^{6}}-\frac{1}{A_{1}^{6}}\right) \int_{0}^{1}\left(\tilde{\mathcal{P}}_{1}^{1 / 2}-\tilde{\mathcal{P}}_{2}^{1 / 2}\right)\left(\tilde{\mathcal{P}}_{2}^{1 / 2}\right)_{\eta}(t, \eta)
\end{aligned}
$$




$$
\begin{aligned}
& \times \int_{0}^{\eta} e^{-\frac{1}{A_{2}}\left(\tilde{\mathcal{Y}}_{2}(t, \eta)-\tilde{\mathcal{Y}}_{2}(t, \theta)\right)} \tilde{\mathcal{Q}}_{2} \tilde{\mathcal{U}}_{2, \eta}(t, \theta) d \theta d \eta \\
& +\mathbb{1}_{A_{1}<A_{2}}\left(\frac{1}{A_{2}^{6}}-\frac{1}{A_{1}^{6}}\right) \int_{0}^{1}\left(\tilde{\mathcal{P}}_{1}^{1 / 2}-\tilde{\mathcal{P}}_{2}^{1 / 2}\right)\left(\tilde{\mathcal{P}}_{1}^{1 / 2}\right)_{\eta}(t, \eta) \\
& \times \int_{0}^{\eta} e^{-\frac{1}{A_{1}}\left(\tilde{\mathcal{Y}}_{1}(t, \eta)-\tilde{\mathcal{Y}}_{1}(t, \theta)\right)} \tilde{\mathcal{Q}}_{1} \tilde{\mathcal{U}}_{1, \eta}(t, \theta) d \theta d \eta \\
& =M_{1}+M_{2}+M_{3} .
\end{aligned}
$$

We estimate, using first that $\tilde{\mathcal{Q}}_{i}=A_{i} \tilde{\mathcal{P}}_{i}-\tilde{\mathcal{D}}_{i}$ (cf. (4.7)):

$$
\begin{aligned}
& M_{1}=\frac{1}{A^{6}} \int_{0}^{1}\left(\tilde{\mathcal{P}}_{1}^{1 / 2}-\tilde{\mathcal{P}}_{2}^{1 / 2}\right)\left(\left(\tilde{\mathcal{P}}_{2}^{1 / 2}\right)_{\eta}(t, \eta) \int_{0}^{\eta} e^{-\frac{1}{A_{2}}\left(\tilde{\mathcal{Y}}_{2}(t, \eta)-\tilde{\mathcal{Y}}_{2}(t, \theta)\right)} \tilde{\mathcal{Q}}_{2} \tilde{\mathcal{U}}_{2, \eta}(t, \theta) d \theta\right. \\
& \left.-\left(\tilde{\mathcal{P}}_{1}^{1 / 2}\right)_{\eta}(t, \eta) \int_{0}^{\eta} e^{-\frac{1}{A_{1}}\left(\tilde{\mathcal{Y}}_{1}(t, \eta)-\tilde{\mathcal{Y}}_{1}(t, \theta)\right)} \tilde{\mathcal{Q}}_{1} \tilde{\mathcal{U}}_{1, \eta}(t, \theta) d \theta\right) d \eta \\
& =\frac{1}{A^{6}} \int_{0}^{1}\left(\tilde{\mathcal{P}}_{1}^{1 / 2}-\tilde{\mathcal{P}}_{2}^{1 / 2}\right)\left(A_{2}\left(\tilde{\mathcal{P}}_{2}^{1 / 2}\right)_{\eta}(t, \eta) \int_{0}^{\eta} e^{-\frac{1}{A_{2}}\left(\tilde{\mathcal{Y}}_{2}(t, \eta)-\tilde{\mathcal{Y}}_{2}(t, \theta)\right)} \tilde{\mathcal{P}}_{2} \tilde{\mathcal{U}}_{2, \eta}(t, \theta) d \theta\right. \\
& \left.-A_{1}\left(\tilde{\mathcal{P}}_{1}^{1 / 2}\right)_{\eta}(t, \eta) \int_{0}^{\eta} e^{-\frac{1}{A_{1}}\left(\tilde{\mathcal{Y}}_{1}(t, \eta)-\tilde{\mathcal{Y}}_{1}(t, \theta)\right)} \tilde{\mathcal{P}}_{1} \tilde{\mathcal{U}}_{1, \eta}(t, \theta) d \theta\right) d \eta \\
& -\frac{1}{A^{6}} \int_{0}^{1}\left(\tilde{\mathcal{P}}_{1}^{1 / 2}-\tilde{\mathcal{P}}_{2}^{1 / 2}\right)\left(\left(\tilde{\mathcal{P}}_{2}^{1 / 2}\right)_{\eta}(t, \eta) \int_{0}^{\eta} e^{-\frac{1}{A_{2}}\left(\tilde{\mathcal{Y}}_{2}(t, \eta)-\tilde{\mathcal{Y}}_{2}(t, \theta)\right)} \tilde{\mathcal{D}}_{2} \tilde{\mathcal{U}}_{2, \eta}(t, \theta) d \theta\right. \\
& \left.-\left(\tilde{\mathcal{P}}_{1}^{1 / 2}\right)_{\eta}(t, \eta) \int_{0}^{\eta} e^{-\frac{1}{A_{1}}\left(\tilde{\mathcal{Y}}_{1}(t, \eta)-\tilde{\mathcal{Y}}_{1}(t, \theta)\right)} \tilde{\mathcal{D}}_{1} \tilde{\mathcal{U}}_{1, \eta}(t, \theta) d \theta\right) d \eta \\
& =\frac{1}{A^{6}} \int_{0}^{1}\left(\tilde{\mathcal{P}}_{1}^{1 / 2}-\tilde{\mathcal{P}}_{2}^{1 / 2}\right)\left(A_{2} \tilde{\mathcal{P}}_{2} \tilde{\mathcal{U}}_{2}\left(\tilde{\mathcal{P}}_{2}^{1 / 2}\right)_{\eta}-A_{1} \tilde{\mathcal{P}}_{1} \tilde{\mathcal{U}}_{1}\left(\tilde{\mathcal{P}}_{1}^{1 / 2}\right)_{\eta}\right)(t, \eta) d \eta \\
& -\frac{1}{A^{6}} \int_{0}^{1}\left(\tilde{\mathcal{P}}_{1}^{1 / 2}-\tilde{\mathcal{P}}_{2}^{1 / 2}\right)\left(\tilde{\mathcal{D}}_{2} \tilde{\mathcal{U}}_{2}\left(\tilde{\mathcal{P}}_{2}^{1 / 2}\right)_{\eta}-\tilde{\mathcal{D}}_{1} \tilde{\mathcal{U}}_{1}\left(\tilde{\mathcal{P}}_{1}^{1 / 2}\right)_{\eta}\right)(t, \eta) d \eta \\
& +\frac{1}{A^{6}} \int_{0}^{1}\left(\tilde{\mathcal{P}}_{1}^{1 / 2}-\tilde{\mathcal{P}}_{2}^{1 / 2}\right) \\
& \times\left(\left(\tilde{\mathcal{P}}_{2}^{1 / 2}\right)_{\eta}(t, \eta) \int_{0}^{\eta} e^{-\frac{1}{A_{2}}\left(\tilde{\mathcal{Y}}_{2}(t, \eta)-\tilde{\mathcal{Y}}_{2}(t, \theta)\right)} \frac{1}{A_{2}} \tilde{\mathcal{D}}_{2} \tilde{\mathcal{U}}_{2} \tilde{\mathcal{Y}}_{2, \eta}(t, \theta) d \theta\right. \\
& \left.-\left(\tilde{\mathcal{P}}_{1}^{1 / 2}\right)_{\eta}(t, \eta) \int_{0}^{\eta} e^{-\frac{1}{A_{1}}\left(\tilde{\mathcal{Y}}_{1}(t, \eta)-\tilde{\mathcal{Y}}_{1}(t, \theta)\right)} \frac{1}{A_{1}} \tilde{\mathcal{D}}_{1} \tilde{\mathcal{U}}_{1} \tilde{\mathcal{Y}}_{1, \eta}(t, \theta) d \theta\right) d \eta \\
& -\frac{3}{A^{6}} \int_{0}^{1}\left(\tilde{\mathcal{P}}_{1}^{1 / 2}-\tilde{\mathcal{P}}_{2}^{1 / 2}\right) \\
& \times\left(\left(\tilde{\mathcal{P}}_{2}^{1 / 2}\right)_{\eta}(t, \eta) \int_{0}^{\eta} e^{-\frac{1}{A_{2}}\left(\tilde{\mathcal{Y}}_{2}(t, \eta)-\tilde{\mathcal{Y}}_{2}(t, \theta)\right)} \tilde{\mathcal{P}}_{2} \tilde{\mathcal{U}}_{2} \tilde{\mathcal{Y}}_{2, \eta}(t, \theta) d \theta\right.
\end{aligned}
$$




$$
\begin{aligned}
& \left.-\left(\tilde{\mathcal{P}}_{1}^{1 / 2}\right)_{\eta}(t, \eta) \int_{0}^{\eta} e^{-\frac{1}{A_{1}}\left(\tilde{\mathcal{Y}}_{1}(t, \eta)-\tilde{\mathcal{Y}}_{1}(t, \theta)\right)} \tilde{\mathcal{P}}_{1} \tilde{\mathcal{U}}_{1} \tilde{\mathcal{Y}}_{1, \eta}(t, \theta) d \theta\right) d \eta \\
& +\frac{1}{A^{6}} \int_{0}^{1}\left(\tilde{\mathcal{P}}_{1}^{1 / 2}-\tilde{\mathcal{P}}_{2}^{1 / 2}\right)\left(\left(\tilde{\mathcal{P}}_{2}^{1 / 2}\right)_{\eta}(t, \eta) \int_{0}^{\eta} e^{-\frac{1}{A_{2}}\left(\tilde{\mathcal{Y}}_{2}(t, \eta)-\tilde{\mathcal{Y}}_{2}(t, \theta)\right)} \tilde{\mathcal{U}}_{2}^{3} \tilde{\mathcal{Y}}_{2, \eta}(t, \theta) d \theta\right. \\
& \left.-\left(\tilde{\mathcal{P}}_{1}^{1 / 2}\right)_{\eta}(t, \eta) \int_{0}^{\eta} e^{-\frac{1}{A_{1}}\left(\tilde{\mathcal{Y}}_{1}(t, \eta)-\tilde{\mathcal{Y}}_{1}(t, \theta)\right)} \tilde{\mathcal{U}}_{1}^{3} \tilde{\mathcal{Y}}_{1, \eta}(t, \theta) d \theta\right) d \eta \\
& +\frac{1}{2 A^{6}} \int_{0}^{1}\left(\tilde{\mathcal{P}}_{1}^{1 / 2}-\tilde{\mathcal{P}}_{2}^{1 / 2}\right)\left(\left(\tilde{\mathcal{P}}_{2}^{1 / 2}\right)_{\eta}(t, \eta) \int_{0}^{\eta} e^{-\frac{1}{A_{2}}\left(\tilde{\mathcal{Y}}_{2}(t, \eta)-\tilde{\mathcal{Y}}_{2}(t, \theta)\right)} A_{2}^{5} \tilde{\mathcal{U}}_{2}(t, \theta) d \theta\right. \\
& \left.-\left(\tilde{\mathcal{P}}_{1}^{1 / 2}\right)_{\eta}(t, \eta) \int_{0}^{\eta} e^{-\frac{1}{A_{1}}\left(\tilde{\mathcal{Y}}_{1}(t, \eta)-\tilde{\mathcal{Y}}_{1}(t, \theta)\right)} A_{1}^{5} \tilde{\mathcal{U}}_{1}(t, \theta) d \theta\right) d \eta \\
& =W_{1}+W_{2}+W_{3}+W_{4}+W_{5}+W_{6} .
\end{aligned}
$$

Here we have used the rewrite employed when manipulating the term $\bar{K}_{1}$ from the expression (5.18) to (5.19). We start by considering the term $W_{1}$ :

$$
\begin{aligned}
W_{1}= & \frac{1}{A^{6}} \int_{0}^{1}\left(\tilde{\mathcal{P}}_{1}^{1 / 2}-\tilde{\mathcal{P}}_{2}^{1 / 2}\right)\left(A_{2} \tilde{\mathcal{P}}_{2} \tilde{\mathcal{U}}_{2}\left(\tilde{\mathcal{P}}_{2}^{1 / 2}\right)_{\eta}-A_{1} \tilde{\mathcal{P}}_{1} \tilde{\mathcal{U}}_{1}\left(\tilde{\mathcal{P}}_{1}^{1 / 2}\right)_{\eta}\right)(t, \eta) d \eta \\
= & \mathbb{1}_{A_{1} \leqslant A_{2}} \frac{A_{2}-A_{1}}{A^{6}} \int_{0}^{1}\left(\tilde{\mathcal{P}}_{1}^{1 / 2}-\tilde{\mathcal{P}}_{2}^{1 / 2}\right) \tilde{\mathcal{P}}_{2} \tilde{\mathcal{U}}_{2}\left(\tilde{\mathcal{P}}_{2}^{1 / 2}\right)_{\eta}(t, \eta) d \eta \\
& +\mathbb{1}_{A_{2}<A_{1}} \frac{A_{2}-A_{1}}{A^{6}} \int_{0}^{1}\left(\tilde{\mathcal{P}}_{1}^{1 / 2}-\tilde{\mathcal{P}}_{2}^{1 / 2}\right) \tilde{\mathcal{P}}_{1} \tilde{\mathcal{U}}_{1}\left(\tilde{\mathcal{P}}_{1}^{1 / 2}\right)_{\eta}(t, \eta) d \eta \\
& +\frac{a}{A^{6}} \int_{0}^{1}\left(\tilde{\mathcal{P}}_{1}^{1 / 2}-\tilde{\mathcal{P}}_{2}^{1 / 2}\right)\left(\tilde{\mathcal{P}}_{2}-\tilde{\mathcal{P}}_{1}\right) \tilde{\mathcal{U}}_{2}\left(\tilde{\mathcal{P}}_{2}^{1 / 2}\right)_{\eta}(t, \eta) \mathbb{1}_{\tilde{\mathcal{P}}_{1} \leqslant \tilde{\mathcal{P}}_{2}}(t, \eta) d \eta \\
& +\frac{a}{A^{6}} \int_{0}^{1}\left(\tilde{\mathcal{P}}_{1}^{1 / 2}-\tilde{\mathcal{P}}_{2}^{1 / 2}\right)\left(\tilde{\mathcal{P}}_{2}-\tilde{\mathcal{P}}_{1}\right) \tilde{\mathcal{U}}_{1}\left(\tilde{\mathcal{P}}_{1}^{1 / 2}\right)_{\eta}(t, \eta) \mathbb{1}_{\tilde{\mathcal{P}}_{2}<\tilde{\mathcal{P}}_{1}}(t, \eta) d \eta \\
& +\frac{a}{A^{6}} \int_{0}^{1}\left(\tilde{\mathcal{P}}_{1}^{1 / 2}-\tilde{\mathcal{P}}_{2}^{1 / 2}\right)\left(\left(\tilde{\mathcal{P}}_{2}^{1 / 2}\right)_{\eta}-\left(\tilde{\mathcal{P}}_{1}^{1 / 2}\right)_{\eta}\right) \min _{j}\left(\tilde{\mathcal{P}}_{j}\right) \tilde{\mathcal{U}}_{2}(t, \eta) d \eta \\
& +\frac{a}{A^{6}} \int_{0}^{1}\left(\tilde{\mathcal{P}}_{1}^{1 / 2}-\tilde{\mathcal{P}}_{2}^{1 / 2}\right)\left(\tilde{\mathcal{U}}_{2}-\tilde{\mathcal{U}}_{1}\right) \min _{j}\left(\tilde{\mathcal{P}}_{j}\right)\left(\tilde{\mathcal{P}}_{1}^{1 / 2}\right)_{\eta}(t, \eta) d \eta \\
= & W_{11}+W_{12}+W_{13}+W_{14}+W_{15}+W_{16} .
\end{aligned}
$$

Using (4.15a), (4.15b), (4.15e), (4.17a), and (4.17b), we find that

$$
\begin{aligned}
& W_{11}+W_{12}+W_{13}+W_{14}+W_{16} \\
& \quad \leqslant \mathcal{O}(1)\left(\left\|\tilde{\mathcal{U}}_{1}-\tilde{\mathcal{U}}_{2}\right\|^{2}+\left\|\tilde{\mathcal{P}}_{1}^{1 / 2}-\tilde{\mathcal{P}}_{2}^{1 / 2}\right\|^{2}+\left|A_{1}-A_{2}\right|^{2}\right) .
\end{aligned}
$$


Regarding the term $W_{15}$ :

$$
\begin{aligned}
\left|W_{15}\right|= & \frac{a}{A^{6}}\left|\int_{0}^{1}\left(\tilde{\mathcal{P}}_{1}^{1 / 2}-\tilde{\mathcal{P}}_{2}^{1 / 2}\right)\left(\left(\tilde{\mathcal{P}}_{2}^{1 / 2}\right)_{\eta}-\left(\tilde{\mathcal{P}}_{1}^{1 / 2}\right)_{\eta}\right) \min _{j}\left(\tilde{\mathcal{P}}_{j}\right) \tilde{\mathcal{U}}_{2}(t, \eta) d \eta\right| \\
= & \left|\frac{a}{2 A^{6}}\left(\tilde{\mathcal{P}}_{1}^{1 / 2}-\tilde{\mathcal{P}}_{2}^{1 / 2}\right)^{2} \min _{j}\left(\tilde{\mathcal{P}}_{j}\right) \tilde{\mathcal{U}}_{2}(t, \eta)\right|_{\eta=0}^{1} \\
& -\frac{a}{2 A^{6}} \int_{0}^{1}\left(\tilde{\mathcal{P}}_{1}^{1 / 2}-\tilde{\mathcal{P}}_{2}^{1 / 2}\right)^{2} \frac{d}{d \eta}\left(\min _{j}\left(\tilde{\mathcal{P}}_{j}\right) \tilde{\mathcal{U}}_{2}\right)(t, \eta) d \eta \mid \\
= & \frac{a}{2 A^{6}}\left|\int_{0}^{1}\left(\tilde{\mathcal{P}}_{1}^{1 / 2}-\tilde{\mathcal{P}}_{2}^{1 / 2}\right)^{2} \frac{d}{d \eta}\left(\min _{j}\left(\tilde{\mathcal{P}}_{j}\right) \tilde{\mathcal{U}}_{2}\right)(t, \eta) d \eta\right| \\
\leqslant & \mathcal{O}(1)\left\|\tilde{\mathcal{P}}_{1}^{1 / 2}-\tilde{\mathcal{P}}_{2}^{1 / 2}\right\|^{2},
\end{aligned}
$$

using the same estimates as for $\bar{B}_{13}$ (cf. (5.20)) and Lemma A.3 (ii).

As for the term $W_{2}$, we find

$$
\begin{aligned}
-W_{2}= & \frac{1}{A^{6}} \int_{0}^{1}\left(\tilde{\mathcal{P}}_{1}^{1 / 2}-\tilde{\mathcal{P}}_{2}^{1 / 2}\right)\left(\tilde{\mathcal{D}}_{2} \tilde{\mathcal{U}}_{2}\left(\tilde{\mathcal{P}}_{2}^{1 / 2}\right)_{\eta}-\tilde{\mathcal{D}}_{1} \tilde{\mathcal{U}}_{1}\left(\tilde{\mathcal{P}}_{1}^{1 / 2}\right)_{\eta}\right)(t, \eta) d \eta \\
= & \frac{1}{A^{6}} \int_{0}^{1}\left(\tilde{\mathcal{P}}_{1}^{1 / 2}-\tilde{\mathcal{P}}_{2}^{1 / 2}\right)\left(\tilde{\mathcal{D}}_{2}-\tilde{\mathcal{D}}_{1}\right) \tilde{\mathcal{U}}_{1}\left(\tilde{\mathcal{P}}_{1}^{1 / 2}\right)_{\eta} \mathbb{1}_{\tilde{\mathcal{D}}_{2} \leqslant \tilde{\mathcal{D}}_{1}}(t, \eta) d \eta \\
& +\frac{1}{A^{6}} \int_{0}^{1}\left(\tilde{\mathcal{P}}_{1}^{1 / 2}-\tilde{\mathcal{P}}_{2}^{1 / 2}\right)\left(\tilde{\mathcal{D}}_{2}-\tilde{\mathcal{D}}_{1}\right) \tilde{\mathcal{U}}_{2}\left(\tilde{\mathcal{P}}_{2}^{1 / 2}\right)_{\eta} \mathbb{1}_{\tilde{\mathcal{D}}_{1}<\tilde{\mathcal{D}}_{2}}(t, \eta) d \eta \\
& +\frac{1}{A^{6}} \int_{0}^{1}\left(\tilde{\mathcal{P}}_{1}^{1 / 2}-\tilde{\mathcal{P}}_{2}^{1 / 2}\right)\left(\left(\tilde{\mathcal{P}}_{2}^{1 / 2}\right)_{\eta}-\left(\tilde{\mathcal{P}}_{1}^{1 / 2}\right)_{\eta}\right) \min _{j}\left(\tilde{\mathcal{D}}_{j}\right) \tilde{\mathcal{U}}_{2}(t, \eta) d \eta \\
& -\frac{1}{A^{6}} \int_{0}^{1}\left(\tilde{\mathcal{P}}_{1}^{1 / 2}-\tilde{\mathcal{P}}_{2}^{1 / 2}\right)\left(\tilde{\mathcal{U}}_{1}-\tilde{\mathcal{U}}_{2}\right) \min _{j}\left(\tilde{\mathcal{D}}_{j}\right)\left(\tilde{\mathcal{P}}_{1}^{1 / 2}\right)_{\eta}(t, \eta) d \eta \\
= & W_{21}+W_{22}+W_{23}+W_{24} .
\end{aligned}
$$

The terms $W_{21}$ and $W_{22}$ can be treated similarly. We need to estimate $\tilde{\mathcal{D}}_{2}-\tilde{\mathcal{D}}_{1}$. Applying Lemma A.9, we have

$$
\begin{aligned}
\left|W_{21}\right|= & \frac{1}{A^{6}}\left|\int_{0}^{1}\left(\tilde{\mathcal{P}}_{1}^{1 / 2}-\tilde{\mathcal{P}}_{2}^{1 / 2}\right)\left(\tilde{\mathcal{D}}_{2}-\tilde{\mathcal{D}}_{1}\right) \tilde{\mathcal{U}}_{1}\left(\tilde{\mathcal{P}}_{1}^{1 / 2}\right)_{\eta}(t, \eta) \mathbb{1}_{\tilde{\mathcal{D}}_{2} \leqslant \tilde{\mathcal{D}}_{1}}(t, \eta) d \eta\right| \\
\leqslant & \frac{2}{A^{9 / 2}} \int_{0}^{1}\left|\tilde{\mathcal{P}}_{1}^{1 / 2}-\tilde{\mathcal{P}}_{2}^{1 / 2}\right|\left|\tilde{\mathcal{Y}}_{1}-\tilde{\mathcal{Y}}_{2}\right| \tilde{\mathcal{D}}_{1}^{1 / 2}\left|\tilde{\mathcal{U}}_{1}\left(\tilde{\mathcal{P}}_{1}^{1 / 2}\right)_{\eta}\right|(t, \eta) d \eta \\
& +\frac{1}{A^{6}} \int_{0}^{1}\left|\tilde{\mathcal{P}}_{1}^{1 / 2}-\tilde{\mathcal{P}}_{2}^{1 / 2}\right|\left|\tilde{\mathcal{Y}}_{1}-\tilde{\mathcal{Y}}_{2}\right|\left(\tilde{\mathcal{U}}_{1}^{2}+\tilde{\mathcal{P}}_{1}\right)\left|\tilde{\mathcal{U}}_{1}\left(\tilde{\mathcal{P}}_{1}^{1 / 2}\right)_{\eta}\right|(t, \eta) d \eta \\
& +\frac{2 \sqrt{2}}{A^{9 / 2}}\left\|\tilde{\mathcal{Y}}_{1}-\tilde{\mathcal{Y}}_{2}\right\| \int_{0}^{1}\left|\tilde{\mathcal{P}}_{1}^{1 / 2}-\tilde{\mathcal{P}}_{2}^{1 / 2}\right| \tilde{\mathcal{D}}_{1}^{1 / 2}\left|\tilde{\mathcal{U}}_{1}\left(\tilde{\mathcal{P}}_{1}^{1 / 2}\right)_{\eta}\right|(t, \eta) d \eta
\end{aligned}
$$




$$
\begin{aligned}
& +\frac{4}{A^{3}} \int_{0}^{1}\left|\tilde{\mathcal{P}}_{1}^{1 / 2}-\tilde{\mathcal{P}}_{2}^{1 / 2}\right| \\
& \times\left(\int_{0}^{\eta} e^{-\frac{1}{A}\left(\tilde{\mathcal{Y}}_{1}(t, \eta)-\tilde{\mathcal{Y}}_{1}(t, \theta)\right)}\left(\tilde{\mathcal{U}}_{1}-\tilde{\mathcal{U}}_{2}\right)^{2}(t, \theta) d \theta\right)^{1 / 2}\left|\tilde{\mathcal{U}}_{1}\left(\tilde{\mathcal{P}}_{1}^{1 / 2}\right)_{\eta}\right|(t, \eta) d \eta \\
& +\frac{2 \sqrt{2}}{A^{3}} \int_{0}^{1}\left|\tilde{\mathcal{P}}_{1}^{1 / 2}-\tilde{\mathcal{P}}_{2}^{1 / 2}\right| \\
& \times\left(\int_{0}^{\eta} e^{-\frac{1}{A}\left(\tilde{\mathcal{Y}}_{1}(t, \eta)-\tilde{\mathcal{Y}}_{1}(t, \theta)\right)}\left(\tilde{\mathcal{P}}_{1}^{1 / 2}-\tilde{\mathcal{P}}_{2}^{1 / 2}\right)^{2}(t, \theta) d \theta\right)^{1 / 2}\left|\tilde{\mathcal{U}}_{1}\left(\tilde{\mathcal{P}}_{1}^{1 / 2}\right)_{\eta}\right|(t, \eta) d \eta \\
& +\frac{3}{\sqrt{2} A^{2}} \int_{0}^{1}\left|\tilde{\mathcal{P}}_{1}^{1 / 2}-\tilde{\mathcal{P}}_{2}^{1 / 2}\right| \\
& \times\left(\int_{0}^{\eta} e^{-\frac{1}{a}\left(\tilde{\mathcal{Y}}_{1}(t, \eta)-\tilde{\mathcal{Y}}_{1}(t, \theta)\right)}\left(\tilde{\mathcal{Y}}_{1}-\tilde{\mathcal{Y}}_{2}\right)^{2}(t, \theta) d \theta\right)^{1 / 2}\left|\tilde{\mathcal{U}}_{1}\left(\tilde{\mathcal{P}}_{1}^{1 / 2}\right)_{\eta}\right|(t, \eta) d \eta \\
& +\frac{3}{2 A^{2}} \int_{0}^{1}\left|\tilde{\mathcal{P}}_{1}^{1 / 2}-\tilde{\mathcal{P}}_{2}^{1 / 2}\right| \\
& \times\left(\int_{0}^{\eta} e^{-\frac{1}{a}\left(\tilde{\mathcal{Y}}_{1}(t, \eta)-\tilde{\mathcal{Y}}_{1}(t, \theta)\right)}\left|\tilde{\mathcal{Y}}_{1}-\tilde{\mathcal{Y}}_{2}\right|(t, \theta) d \theta\right)\left|\tilde{\mathcal{U}}_{1}\left(\tilde{\mathcal{P}}_{1}^{1 / 2}\right)_{\eta}\right|(t, \eta) d \eta \\
& +\frac{6}{A^{2}}\left|A_{1}-A_{2}\right| \int_{0}^{1}\left|\tilde{\mathcal{P}}_{1}^{1 / 2}-\tilde{\mathcal{P}}_{2}^{1 / 2}\right| \\
& \times\left(\int_{0}^{\eta} e^{-\frac{3}{4 A}\left(\tilde{\mathcal{Y}}_{1}(t, \eta)-\tilde{\mathcal{Y}}_{1}(t, \theta)\right)} d \theta\right)\left|\tilde{\mathcal{U}}_{1}\left(\tilde{\mathcal{P}}_{1}^{1 / 2}\right)_{\eta}\right|(t, \eta) d \eta \\
& +\frac{12 \sqrt{2}}{\sqrt{3} e A^{2}}\left|A_{1}-A_{2}\right| \int_{0}^{1}\left|\tilde{\mathcal{P}}_{1}^{1 / 2}-\tilde{\mathcal{P}}_{2}^{1 / 2}\right| \\
& \times\left(\int_{0}^{\eta} e^{-\frac{3}{4 A}\left(\tilde{\mathcal{Y}}_{1}(t, \eta)-\tilde{\mathcal{Y}}_{1}(t, \theta)\right)} d \theta\right)^{1 / 2}\left|\tilde{\mathcal{U}}_{1}\left(\tilde{\mathcal{P}}_{1}^{1 / 2}\right)_{\eta}\right|(t, \eta) d \eta \\
& \leqslant \frac{2}{A^{9 / 2}} \int_{0}^{1}\left|\tilde{\mathcal{P}}_{1}^{1 / 2}-\tilde{\mathcal{P}}_{2}^{1 / 2}\right|\left|\tilde{\mathcal{Y}}_{1}-\tilde{\mathcal{Y}}_{2}\right|(t, \eta) \frac{A^{13 / 2}}{4} d \eta \\
& +\frac{1}{A^{6}} \int_{0}^{1}\left|\tilde{\mathcal{P}}_{1}^{1 / 2}-\tilde{\mathcal{P}}_{2}^{1 / 2}\right|\left|\tilde{\mathcal{Y}}_{1}-\tilde{\mathcal{Y}}_{2}\right|(t, \eta) \frac{3 A^{8}}{8 \sqrt{2}} d \eta \\
& +\frac{2 \sqrt{2}}{A^{9 / 2}}\left\|\tilde{\mathcal{Y}}_{1}-\tilde{\mathcal{Y}}_{2}\right\| \int_{0}^{1}\left|\tilde{\mathcal{P}}_{1}^{1 / 2}-\tilde{\mathcal{P}}_{2}^{1 / 2}\right|(t, \eta) \frac{A^{13 / 2}}{4} d \eta \\
& +\frac{4}{A^{3}} \int_{0}^{1}\left|\tilde{\mathcal{P}}_{1}^{1 / 2}-\tilde{\mathcal{P}}_{2}^{1 / 2}\right|(t, \eta)\left(\int_{0}^{\eta}\left(\tilde{\mathcal{U}}_{1}-\tilde{\mathcal{U}}_{2}\right)^{2}(t, \theta) d \theta\right)^{1 / 2} \frac{A^{4}}{2 \sqrt{2}} d \eta \\
& +\frac{2 \sqrt{2}}{A^{3}} \int_{0}^{1}\left|\tilde{\mathcal{P}}_{1}^{1 / 2}-\tilde{\mathcal{P}}_{2}^{1 / 2}\right|(t, \eta)\left(\int_{0}^{\eta}\left(\tilde{\mathcal{P}}_{1}^{1 / 2}-\tilde{\mathcal{P}}_{2}^{1 / 2}\right)^{2}(t, \theta) d \theta\right)^{1 / 2} \frac{A^{4}}{2 \sqrt{2}} d \eta
\end{aligned}
$$




$$
\begin{aligned}
& +\frac{3}{\sqrt{2} A^{2}} \int_{0}^{1}\left|\tilde{\mathcal{P}}_{1}^{1 / 2}-\tilde{\mathcal{P}}_{2}^{1 / 2}\right|(t, \eta)\left(\int_{0}^{\eta}\left(\tilde{\mathcal{Y}}_{1}-\tilde{\mathcal{Y}}_{2}\right)^{2}(t, \theta) d \theta\right)^{1 / 2} \frac{A^{4}}{2 \sqrt{2}} d \eta \\
& +\frac{3}{2 A^{2}} \int_{0}^{1}\left|\tilde{\mathcal{P}}_{1}^{1 / 2}-\tilde{\mathcal{P}}_{2}^{1 / 2}\right|(t, \eta)\left(\int_{0}^{\eta}\left|\tilde{\mathcal{Y}}_{1}-\tilde{\mathcal{Y}}_{2}\right|(t, \theta) d \theta\right) \frac{A^{4}}{2 \sqrt{2}} d \eta \\
& +\frac{6}{A^{2}}\left|A_{1}-A_{2}\right| \int_{0}^{1}\left|\tilde{\mathcal{P}}_{1}^{1 / 2}-\tilde{\mathcal{P}}_{2}^{1 / 2}\right|(t, \eta) \frac{A^{4}}{2 \sqrt{2}} d \eta \\
& +\frac{12 \sqrt{2}}{\sqrt{3} e A^{2}}\left|A_{1}-A_{2}\right| \int_{0}^{1}\left|\tilde{\mathcal{P}}_{1}^{1 / 2}-\tilde{\mathcal{P}}_{2}^{1 / 2}\right|(t, \eta) \frac{A^{4}}{2 \sqrt{2}} d \eta \\
& \leqslant \mathcal{O}(1)\left(\left\|\tilde{\mathcal{U}}_{1}-\tilde{\mathcal{U}}_{2}\right\|^{2}+\left\|\tilde{\mathcal{P}}_{1}^{1 / 2}-\tilde{\mathcal{P}}_{2}^{1 / 2}\right\|^{2}+\left\|\tilde{\mathcal{Y}}_{1}-\tilde{\mathcal{Y}}_{2}\right\|^{2}+\left|A_{1}-A_{2}\right|^{2}\right),
\end{aligned}
$$

where we have used estimates (4.15a), (4.15b), (4.15n), and (4.17b). Furthermore,

$$
\begin{aligned}
\left|W_{23}\right|= & \frac{1}{A^{6}}\left|\int_{0}^{1}\left(\tilde{\mathcal{P}}_{1}^{1 / 2}-\tilde{\mathcal{P}}_{2}^{1 / 2}\right)\left(\left(\tilde{\mathcal{P}}_{2}^{1 / 2}\right)_{\eta}-\left(\tilde{\mathcal{P}}_{1}^{1 / 2}\right)_{\eta}\right) \min _{j}\left(\tilde{\mathcal{D}}_{j}\right) \tilde{\mathcal{U}}_{2}(t, \eta) d \eta\right| \\
\leqslant & \left|\frac{1}{2 A^{6}}\left(\tilde{\mathcal{P}}_{1}^{1 / 2}-\tilde{\mathcal{P}}_{2}^{1 / 2}\right)^{2} \min _{j}\left(\tilde{\mathcal{D}}_{j}\right) \tilde{\mathcal{U}}_{2}(t, \eta)\right|_{\eta=0}^{1} \\
& -\frac{1}{2 A^{6}} \int_{0}^{1}\left(\tilde{\mathcal{P}}_{1}^{1 / 2}-\tilde{\mathcal{P}}_{2}^{1 / 2}\right)^{2} \frac{d}{d \theta}\left(\min _{j}\left(\tilde{\mathcal{D}}_{j}\right) \tilde{\mathcal{U}}_{2}\right)(t, \eta) d \eta \mid \\
= & \frac{1}{2 A^{6}}\left|\int_{0}^{1}\left(\tilde{\mathcal{P}}_{1}^{1 / 2}-\tilde{\mathcal{P}}_{2}^{1 / 2}\right)^{2} \frac{d}{d \theta}\left(\min _{j}\left(\tilde{\mathcal{D}}_{j}\right) \tilde{\mathcal{U}}_{2}\right)(t, \eta) d \eta\right| \\
\leqslant & \mathcal{O}(1)\left\|\tilde{\mathcal{P}}_{1}^{1 / 2}-\tilde{\mathcal{P}}_{2}^{1 / 2}\right\|^{2},
\end{aligned}
$$

by applying Lemma A.4 (ii). The term $W_{24}$ goes as follows:

$$
\begin{aligned}
\left|W_{24}\right| & =\frac{1}{A^{6}}\left|\int_{0}^{1}\left(\tilde{\mathcal{P}}_{1}^{1 / 2}-\tilde{\mathcal{P}}_{2}^{1 / 2}\right)\left(\tilde{\mathcal{U}}_{1}-\tilde{\mathcal{U}}_{2}\right) \min _{j}\left(\tilde{\mathcal{D}}_{j}\right)\left(\tilde{\mathcal{P}}_{1}^{1 / 2}\right)_{\eta}(t, \eta) d \eta\right| \\
& \leqslant \mathcal{O}(1)\left(\left\|\tilde{\mathcal{U}}_{1}-\tilde{\mathcal{U}}_{2}\right\|^{2}+\left\|\tilde{\mathcal{P}}_{1}^{1 / 2}-\tilde{\mathcal{P}}_{2}^{1 / 2}\right\|^{2}\right)
\end{aligned}
$$

using

$$
\min _{j}\left(\tilde{\mathcal{D}}_{j}\right)\left|\left(\tilde{\mathcal{P}}_{1}^{1 / 2}\right)_{\eta}\right| \leqslant 2 A_{1} \tilde{\mathcal{P}}_{1}\left|\left(\tilde{\mathcal{P}}_{1}^{1 / 2}\right)_{\eta}\right| \leqslant \tilde{\mathcal{P}}_{1}^{1 / 2} \tilde{\mathcal{P}}_{1} \tilde{\mathcal{Y}}_{1, \eta} \leqslant \frac{A^{7}}{4}
$$

from (4.15a), (4.15e), (4.15n), and (4.17a).

Next comes $W_{3}$, namely,

$$
\begin{aligned}
W_{3}= & \frac{1}{A^{6}} \int_{0}^{1}\left(\tilde{\mathcal{P}}_{1}^{1 / 2}-\tilde{\mathcal{P}}_{2}^{1 / 2}\right) \\
& \times\left(\left(\tilde{\mathcal{P}}_{2}^{1 / 2}\right)_{\eta}(t, \eta) \int_{0}^{\eta} e^{-\frac{1}{A_{2}}\left(\tilde{\mathcal{Y}}_{2}(t, \eta)-\tilde{\mathcal{Y}}_{2}(t, \theta)\right)} \frac{1}{A_{2}} \tilde{\mathcal{D}}_{2} \tilde{\mathcal{U}}_{2} \tilde{\mathcal{Y}}_{2, \eta}(t, \theta) d \theta\right.
\end{aligned}
$$




$$
\begin{aligned}
& \left.-\left(\tilde{\mathcal{P}}_{1}^{1 / 2}\right)_{\eta}(t, \eta) \int_{0}^{\eta} e^{-\frac{1}{A_{1}}\left(\tilde{\mathcal{Y}}_{1}(t, \eta)-\tilde{\mathcal{Y}}_{1}(t, \theta)\right)} \frac{1}{A_{1}} \tilde{\mathcal{D}}_{1} \tilde{\mathcal{U}}_{1} \tilde{\mathcal{Y}}_{1, \eta}(t, \theta) d \theta\right) d \eta \\
& =\frac{1}{A^{6}} \int_{0}^{1}\left(\tilde{\mathcal{P}}_{1}^{1 / 2}-\tilde{\mathcal{P}}_{2}^{1 / 2}\right) \\
& \times\left(\left(\tilde{\mathcal{P}}_{2}^{1 / 2}\right)_{\eta}(t, \eta) \int_{0}^{\eta} e^{-\frac{1}{A_{2}}\left(\tilde{\mathcal{Y}}_{2}(t, \eta)-\tilde{\mathcal{Y}}_{2}(t, \theta)\right)} \frac{1}{A_{2}} \tilde{\mathcal{D}}_{2} \tilde{\mathcal{U}}_{2}^{+} \tilde{\mathcal{Y}}_{2, \eta}(t, \theta) d \theta\right. \\
& \left.-\left(\tilde{\mathcal{P}}_{1}^{1 / 2}\right)_{\eta}(t, \eta) \int_{0}^{\eta} e^{-\frac{1}{A_{1}}\left(\tilde{\mathcal{Y}}_{1}(t, \eta)-\tilde{\mathcal{Y}}_{1}(t, \theta)\right)} \frac{1}{A_{1}} \tilde{\mathcal{D}}_{1} \tilde{\mathcal{U}}_{1}^{+} \tilde{\mathcal{Y}}_{1, \eta}(t, \theta) d \theta\right) d \eta \\
& +\frac{1}{A^{6}} \int_{0}^{1}\left(\tilde{\mathcal{P}}_{1}^{1 / 2}-\tilde{\mathcal{P}}_{2}^{1 / 2}\right) \\
& \times\left(\left(\tilde{\mathcal{P}}_{2}^{1 / 2}\right)_{\eta}(t, \eta) \int_{0}^{\eta} e^{-\frac{1}{A_{2}}\left(\tilde{\mathcal{Y}}_{2}(t, \eta)-\tilde{\mathcal{Y}}_{2}(t, \theta)\right)} \frac{1}{A_{2}} \tilde{\mathcal{D}}_{2} \tilde{\mathcal{U}}_{2}^{-} \tilde{\mathcal{Y}}_{2, \eta}(t, \theta) d \theta\right. \\
& \left.-\left(\tilde{\mathcal{P}}_{1}^{1 / 2}\right)_{\eta}(t, \eta) \int_{0}^{\eta} e^{-\frac{1}{A_{1}}\left(\tilde{\mathcal{Y}}_{1}(t, \eta)-\tilde{\mathcal{Y}}_{1}(t, \theta)\right)} \frac{1}{A_{1}} \tilde{\mathcal{D}}_{1} \tilde{\mathcal{U}}_{1}^{-} \tilde{\mathcal{Y}}_{1, \eta}(t, \theta) d \theta\right) d \eta \\
& =W_{31}+W_{32} \text {, }
\end{aligned}
$$

and the two terms can be treated in the same manner. Thus

$$
\begin{aligned}
W_{31}= & \frac{1}{A^{6}} \int_{0}^{1}\left(\tilde{\mathcal{P}}_{1}^{1 / 2}-\tilde{\mathcal{P}}_{2}^{1 / 2}\right) \\
& \times\left(\left(\tilde{\mathcal{P}}_{2}^{1 / 2}\right)_{\eta}(t, \eta) \int_{0}^{\eta} e^{-\frac{1}{A_{2}}\left(\tilde{\mathcal{Y}}_{2}(t, \eta)-\tilde{\mathcal{Y}}_{2}(t, \theta)\right)} \frac{1}{A_{2}} \tilde{\mathcal{D}}_{2} \tilde{\mathcal{U}}_{2}^{+} \tilde{\mathcal{Y}}_{2, \eta}(t, \theta) d \theta\right. \\
& \left.-\left(\tilde{\mathcal{P}}_{1}^{1 / 2}\right)_{\eta}(t, \eta) \int_{0}^{\eta} e^{-\frac{1}{A_{1}}\left(\tilde{\mathcal{Y}}_{1}(t, \eta)-\tilde{\mathcal{Y}}_{1}(t, \theta)\right)} \frac{1}{A_{1}} \tilde{\mathcal{D}}_{1} \tilde{\mathcal{U}}_{1}^{+} \tilde{\mathcal{Y}}_{1, \eta}(t, \theta) d \theta\right) d \eta \\
= & \mathbb{1}_{A_{1} \leqslant A_{2}} \frac{1}{A^{6}}\left(\frac{1}{A_{2}}-\frac{1}{A_{1}}\right) \int_{0}^{1}\left(\tilde{\mathcal{P}}_{1}^{1 / 2}-\tilde{\mathcal{P}}_{2}^{1 / 2}\right)\left(\tilde{\mathcal{P}}_{1}^{1 / 2}\right)_{\eta}(t, \eta) \\
& \times\left(\int_{0}^{\eta} e^{-\frac{1}{A_{1}}\left(\tilde{\mathcal{Y}}_{1}(t, \eta)-\tilde{\mathcal{Y}}_{1}(t, \theta)\right)} \tilde{\mathcal{D}}_{1} \tilde{\mathcal{U}}_{1}^{+} \tilde{\mathcal{Y}}_{1, \eta}(t, \theta) d \theta\right) d \eta \\
& +\mathbb{1}_{A_{2}<A_{1}} \frac{1}{A^{6}}\left(\frac{1}{A_{2}}-\frac{1}{A_{1}}\right) \int_{0}^{1}\left(\tilde{\mathcal{P}}_{1}^{1 / 2}-\tilde{\mathcal{P}}_{2}^{1 / 2}\right)\left(\tilde{\mathcal{P}}_{2}^{1 / 2}\right)_{\eta}(t, \eta) \\
& \times\left(\int_{0}^{\eta} e^{-\frac{1}{A_{2}}\left(\tilde{\mathcal{Y}}_{2}(t, \eta)-\tilde{\mathcal{Y}}_{2}(t, \theta)\right)} \tilde{\mathcal{D}}_{2} \tilde{\mathcal{U}}_{2}^{+} \tilde{\mathcal{Y}}_{2, \eta}(t, \theta) d \theta\right) d \eta \\
& +\frac{1}{A^{7}} \int_{0}^{1}\left(\tilde{\mathcal{P}}_{1}^{1 / 2}-\tilde{\mathcal{P}}_{2}^{1 / 2}\right)\left(\tilde{\mathcal{P}}_{2}^{1 / 2}\right)_{\eta}(t, \eta) \\
& \times\left(\int_{0}^{\eta} e^{-\frac{1}{A_{2}}\left(\tilde{\mathcal{Y}}_{2}(t, \eta)-\tilde{\mathcal{Y}}_{2}(t, \theta)\right)}\left(\tilde{\mathcal{D}}_{2}-\tilde{\mathcal{D}}_{1}\right) \tilde{\mathcal{U}}_{2}^{+} \tilde{\mathcal{Y}}_{2, \eta}(t, \theta) \mathbb{1}_{\tilde{\mathcal{D}}_{1} \leqslant \tilde{\mathcal{D}}_{2}}(t, \theta) d \theta\right) d \eta
\end{aligned}
$$




$$
\begin{aligned}
& +\frac{1}{A^{7}} \int_{0}^{1}\left(\tilde{\mathcal{P}}_{1}^{1 / 2}-\tilde{\mathcal{P}}_{2}^{1 / 2}\right)\left(\tilde{\mathcal{P}}_{1}^{1 / 2}\right)_{\eta}(t, \eta) \\
& \times\left(\int_{0}^{\eta} e^{-\frac{1}{A_{1}}\left(\tilde{\mathcal{Y}}_{1}(t, \eta)-\tilde{\mathcal{Y}}_{1}(t, \theta)\right)}\left(\tilde{\mathcal{D}}_{2}-\tilde{\mathcal{D}}_{1}\right) \tilde{\mathcal{U}}_{1}^{+} \tilde{\mathcal{Y}}_{1, \eta}(t, \theta) \mathbb{1}_{\tilde{\mathcal{D}}_{2}<\tilde{\mathcal{D}}_{1}}(t, \theta) d \theta\right) d \eta \\
& +\frac{1}{A^{7}} \int_{0}^{1}\left(\tilde{\mathcal{P}}_{1}^{1 / 2}-\tilde{\mathcal{P}}_{2}^{1 / 2}\right)\left(\tilde{\mathcal{P}}_{2}^{1 / 2}\right)_{\eta}(t, \eta)\left(\int_{0}^{\eta} e^{-\frac{1}{A_{2}}\left(\tilde{\mathcal{Y}}_{2}(t, \eta)-\tilde{\mathcal{Y}}_{2}(t, \theta)\right)}\right. \\
& \left.\times \min _{j}\left(\tilde{\mathcal{D}}_{j}\right)\left(\tilde{\mathcal{U}}_{2}^{+}-\tilde{\mathcal{U}}_{1}^{+}\right) \tilde{\mathcal{Y}}_{2, \eta}(t, \theta) \mathbb{1}_{\tilde{\mathcal{U}}_{1}^{+} \leqslant \tilde{\mathcal{U}}_{2}^{+}}(t, \theta) d \theta\right) d \eta \\
& +\frac{1}{A^{7}} \int_{0}^{1}\left(\tilde{\mathcal{P}}_{1}^{1 / 2}-\tilde{\mathcal{P}}_{2}^{1 / 2}\right)\left(\tilde{\mathcal{P}}_{1}^{1 / 2}\right)_{\eta}(t, \eta)\left(\int_{0}^{\eta} e^{-\frac{1}{A_{1}}\left(\tilde{\mathcal{Y}}_{1}(t, \eta)-\tilde{\mathcal{Y}}_{1}(t, \theta)\right)}\right. \\
& \left.\times \min _{j}\left(\tilde{\mathcal{D}}_{j}\right)\left(\tilde{\mathcal{U}}_{2}^{+}-\tilde{\mathcal{U}}_{1}^{+}\right) \tilde{\mathcal{Y}}_{1, \eta}(t, \theta) \mathbb{1}_{\tilde{\mathcal{U}}_{2}^{+}<\tilde{\mathcal{U}}_{1}^{+}}(t, \theta) d \theta\right) d \eta \\
& +\mathbb{1}_{A_{1} \leqslant A_{2}} \frac{1}{A^{7}} \int_{0}^{1}\left(\tilde{\mathcal{P}}_{1}^{1 / 2}-\tilde{\mathcal{P}}_{2}^{1 / 2}\right)\left(\tilde{\mathcal{P}}_{2}^{1 / 2}\right)_{\eta}(t, \eta) \\
& \times\left(\int_{0}^{\eta}\left(e^{-\frac{1}{A_{2}}\left(\tilde{\mathcal{Y}}_{2}(t, \eta)-\tilde{\mathcal{Y}}_{2}(t, \theta)\right)}-e^{-\frac{1}{A_{1}}\left(\tilde{\mathcal{Y}}_{2}(t, \eta)-\tilde{\mathcal{Y}}_{2}(t, \theta)\right)}\right)\right. \\
& \left.\times \min _{j}\left(\tilde{\mathcal{D}}_{j}\right) \min _{j}\left(\tilde{\mathcal{U}}_{j}^{+}\right) \tilde{\mathcal{Y}}_{2, \eta}(t, \theta) d \theta\right) d \eta \\
& +\mathbb{1}_{A_{2}<A_{1}} \frac{1}{A^{7}} \int_{0}^{1}\left(\tilde{\mathcal{P}}_{1}^{1 / 2}-\tilde{\mathcal{P}}_{2}^{1 / 2}\right)\left(\tilde{\mathcal{P}}_{1}^{1 / 2}\right)_{\eta}(t, \eta) \\
& \times\left(\int_{0}^{\eta}\left(e^{-\frac{1}{A_{2}}\left(\tilde{\mathcal{Y}}_{1}(t, \eta)-\tilde{\mathcal{Y}}_{1}(t, \theta)\right)}-e^{-\frac{1}{A_{1}}\left(\tilde{\mathcal{Y}}_{1}(t, \eta)-\tilde{\mathcal{Y}}_{1}(t, \theta)\right)}\right)\right. \\
& \left.\times \min _{j}\left(\tilde{\mathcal{D}}_{j}\right) \min _{j}\left(\tilde{\mathcal{U}}_{j}^{+}\right) \tilde{\mathcal{Y}}_{1, \eta}(t, \theta) d \theta\right) d \eta \\
& +\frac{1}{A^{7}} \int_{0}^{1}\left(\tilde{\mathcal{P}}_{1}^{1 / 2}-\tilde{\mathcal{P}}_{2}^{1 / 2}\right)\left(\tilde{\mathcal{P}}_{2}^{1 / 2}\right)_{\eta}(t, \eta) \\
& \times\left(\int_{0}^{\eta}\left(e^{-\frac{1}{a}\left(\tilde{\mathcal{Y}}_{2}(t, \eta)-\tilde{\mathcal{Y}}_{2}(t, \theta)\right)}-e^{-\frac{1}{a}\left(\tilde{\mathcal{Y}}_{1}(t, \eta)-\tilde{\mathcal{Y}}_{1}(t, \theta)\right)}\right)\right. \\
& \left.\times \min _{j}\left(\tilde{\mathcal{D}}_{j}\right) \min _{j}\left(\tilde{\mathcal{U}}_{j}^{+}\right) \tilde{\mathcal{Y}}_{2, \eta}(t, \theta) \mathbb{1}_{B(\eta)}(t, \theta) d \theta\right) d \eta \\
& +\frac{1}{A^{7}} \int_{0}^{1}\left(\tilde{\mathcal{P}}_{1}^{1 / 2}-\tilde{\mathcal{P}}_{2}^{1 / 2}\right)\left(\tilde{\mathcal{P}}_{1}^{1 / 2}\right)_{\eta}(t, \eta) \\
& \times\left(\int_{0}^{\eta}\left(e^{-\frac{1}{a}\left(\tilde{\mathcal{Y}}_{2}(t, \eta)-\tilde{\mathcal{Y}}_{2}(t, \theta)\right)}-e^{-\frac{1}{a}\left(\tilde{\mathcal{Y}}_{1}(t, \eta)-\tilde{\mathcal{Y}}_{1}(t, \theta)\right)}\right)\right. \\
& \left.\times \min _{j}\left(\tilde{\mathcal{D}}_{j}\right) \min _{j}\left(\tilde{\mathcal{U}}_{j}^{+}\right) \tilde{\mathcal{Y}}_{1, \eta}(t, \theta) \mathbb{1}_{B(\eta)^{c}}(t, \theta) d \theta\right) d \eta
\end{aligned}
$$




$$
\begin{aligned}
& +\frac{1}{A^{7}} \int_{0}^{1}\left(\tilde{\mathcal{P}}_{1}^{1 / 2}-\tilde{\mathcal{P}}_{2}^{1 / 2}\right)\left(\left(\tilde{\mathcal{P}}_{2}^{1 / 2}\right)_{\eta}-\left(\tilde{\mathcal{P}}_{1}^{1 / 2}\right)_{\eta}\right)(t, \eta) \\
& \times \min _{k}\left(\int_{0}^{\eta} \min _{j}\left(e^{-\frac{1}{a}\left(\tilde{\mathcal{Y}}_{j}(t, \eta)-\tilde{\mathcal{Y}}_{j}(t, \theta)\right)}\right) \min _{j}\left(\tilde{\mathcal{D}}_{j}\right) \min _{j}\left(\tilde{\mathcal{U}}_{j}^{+}\right) \tilde{\mathcal{Y}}_{k, \eta}(t, \theta) d \theta\right) d \eta \\
& +\frac{1}{A^{7}} \int_{0}^{1}\left(\tilde{\mathcal{P}}_{1}^{1 / 2}-\tilde{\mathcal{P}}_{2}^{1 / 2}\right)\left(\tilde{\mathcal{P}}_{2}^{1 / 2}\right)_{\eta} \mathbb{1}_{D^{c}}(t, \eta) \\
& \times\left(\int_{0}^{\eta} \min _{j}\left(e^{-\frac{1}{a}\left(\tilde{\mathcal{Y}}_{j}(t, \eta)-\tilde{\mathcal{Y}}_{j}(t, \theta)\right)}\right) \min _{j}\left(\tilde{\mathcal{D}}_{j}\right) \min _{j}\left(\tilde{\mathcal{U}}_{j}^{+}\right)\left(\tilde{\mathcal{Y}}_{2, \eta}-\tilde{\mathcal{Y}}_{1, \eta}\right)(t, \theta) d \theta\right) d \eta \\
& +\frac{1}{A^{7}} \int_{0}^{1}\left(\tilde{\mathcal{P}}_{1}^{1 / 2}-\tilde{\mathcal{P}}_{2}^{1 / 2}\right)\left(\tilde{\mathcal{P}}_{1}^{1 / 2}\right)_{\eta} \mathbb{1}_{e}(t, \eta) \\
& \times\left(\int_{0}^{\eta} \min _{j}\left(e^{-\frac{1}{a}\left(\tilde{\mathcal{Y}}_{j}(t, \eta)-\tilde{\mathcal{Y}}_{j}(t, \theta)\right)}\right) \min _{j}\left(\tilde{\mathcal{D}}_{j}\right) \min _{j}\left(\tilde{\mathcal{U}}_{j}^{+}\right)\left(\tilde{\mathcal{Y}}_{2, \eta}-\tilde{\mathcal{Y}}_{1, \eta}\right)(t, \theta) d \theta\right) d \eta \\
& =Z_{1}+Z_{2}+Z_{3}+Z_{4}+Z_{5}+Z_{6}+Z_{7}+Z_{8}+Z_{9}+Z_{10}+Z_{11}+Z_{12}+Z_{13} \text {, }
\end{aligned}
$$

where the set $e$ is given by (5.21). Here there is no alternative but to treat these terms more or less separately. For the terms $Z_{1}$ and $Z_{2}$, we find

$$
\begin{aligned}
\left|Z_{1}\right| \leqslant & \frac{2}{A^{7}}\left|A_{1}-A_{2}\right| \int_{0}^{1}\left|\tilde{\mathcal{P}}_{1}^{1 / 2}-\tilde{\mathcal{P}}_{2}^{1 / 2}\right|\left|\left(\tilde{\mathcal{P}}_{1}^{1 / 2}\right)_{\eta}\right| \\
& \times\left(\int_{0}^{\eta} e^{-\frac{1}{A_{1}}\left(\tilde{\mathcal{Y}}_{1}(t, \eta)-\tilde{\mathcal{Y}}_{1}(t, \theta)\right)} \tilde{\mathcal{Y}}_{1}^{2} \tilde{\mathcal{Y}}_{1, \eta}(t, \theta) d \theta\right)^{1 / 2} \\
& \times\left(\int_{0}^{\eta} e^{-\frac{1}{A_{1}}\left(\tilde{\mathcal{Y}}_{1}(t, \eta)-\tilde{\mathcal{Y}}_{1}(t, \theta)\right)} \tilde{\mathcal{P}}_{1}^{2} \tilde{\mathcal{Y}}_{1, \eta}(t, \theta) d \theta\right)^{1 / 2} d \eta \\
\leqslant & \mathcal{O}(1)\left(\left\|\tilde{\mathcal{P}}_{1}^{1 / 2}-\tilde{\mathcal{P}}_{2}^{1 / 2}\right\|^{2}+\left|A_{1}-A_{2}\right|^{2}\right) .
\end{aligned}
$$

For the terms $Z_{3}$ and $Z_{4}$, we find

$$
\begin{aligned}
\left|Z_{3}\right|= & \frac{1}{A^{7}} \mid \int_{0}^{1}\left(\tilde{\mathcal{P}}_{1}^{1 / 2}-\tilde{\mathcal{P}}_{2}^{1 / 2}\right)\left(\tilde{\mathcal{P}}_{2}^{1 / 2}\right)_{\eta}(t, \eta) \\
& \left.\times\left(\int_{0}^{\eta} e^{-\frac{1}{A_{2}}\left(\tilde{\mathcal{Y}}_{2}(t, \eta)-\tilde{\mathcal{Y}}_{2}(t, \theta)\right)}\left(\tilde{\mathcal{D}}_{2}-\tilde{\mathcal{D}}_{1}\right) \tilde{\mathcal{U}}_{2}^{+} \tilde{\mathcal{Y}}_{2, \eta}(t, \theta) \mathbb{1}_{\tilde{\mathcal{D}}_{1} \leqslant \tilde{\mathcal{D}}_{2}}(t, \theta) d \theta\right) d \eta\right) \\
\leqslant & \mathcal{O}(1)\left(\left\|\tilde{\mathcal{U}}_{2}-\tilde{\mathcal{U}}_{1}\right\|^{2}+\left\|\tilde{\mathcal{Y}}_{2}-\tilde{\mathcal{Y}}_{1}\right\|^{2}+\left\|\tilde{\mathcal{P}}_{2}^{1 / 2}-\tilde{\mathcal{P}}_{1}^{1 / 2}\right\|^{2}+\left|A_{2}-A_{1}\right|^{2}\right)
\end{aligned}
$$

by Lemma A.9.

For the terms $Z_{5}$ and $Z_{6}$, we find

$$
\begin{aligned}
\left|Z_{5}\right|= & \frac{1}{A^{7}} \mid \int_{0}^{1}\left(\tilde{\mathcal{P}}_{1}^{1 / 2}-\tilde{\mathcal{P}}_{2}^{1 / 2}\right)\left(\tilde{\mathcal{P}}_{2}^{1 / 2}\right)_{\eta}(t, \eta) \\
& \times\left(\int_{0}^{\eta} e^{-\frac{1}{A_{2}}\left(\tilde{\mathcal{Y}}_{2}(t, \eta)-\tilde{\mathcal{Y}}_{2}(t, \theta)\right)} \min _{j}\left(\tilde{\mathcal{D}}_{j}\right)\left(\tilde{\mathcal{U}}_{2}^{+}-\tilde{\mathcal{U}}_{1}^{+}\right) \tilde{\mathcal{Y}}_{2, \eta} \mathbb{1}_{\tilde{\mathcal{U}}_{1}^{+} \leqslant \tilde{\mathcal{U}}_{2}^{+}}(t, \theta) d \theta\right) d \eta \mid
\end{aligned}
$$




$$
\begin{aligned}
\leqslant & \left\|\tilde{\mathcal{P}}_{1}^{1 / 2}-\tilde{\mathcal{P}}_{2}^{1 / 2}\right\|^{2}+\frac{1}{A^{14}} \int_{0}^{1}\left(\left(\tilde{\mathcal{P}}_{2}^{1 / 2}\right)_{\eta}\right)^{2}(t, \eta) \\
& \times\left(\int_{0}^{\eta} e^{-\frac{1}{A_{2}}\left(\tilde{\mathcal{Y}}_{2}(t, \eta)-\tilde{\mathcal{Y}}_{2}(t, \theta)\right)} \min _{j}\left(\tilde{\mathcal{D}}_{j}\right)\left(\tilde{\mathcal{U}}_{2}^{+}-\tilde{\mathcal{U}}_{1}^{+}\right) \tilde{\mathcal{Y}}_{2, \eta} \mathbb{1}_{\tilde{\mathcal{U}}_{1}^{+} \leqslant \tilde{\mathcal{U}}_{2}^{+}}(t, \theta) d \theta\right)^{2} d \eta \\
\leqslant & \mathcal{O}(1)\left(\left\|\tilde{\mathcal{P}}_{1}^{1 / 2}-\tilde{\mathcal{P}}_{2}^{1 / 2}\right\|^{2}+\left\|\tilde{\mathcal{U}}_{2}-\tilde{\mathcal{U}}_{1}\right\|^{2}\right),
\end{aligned}
$$

by applying (4.16e), estimating $\tilde{\mathcal{D}}_{2} \leqslant 2 A_{2} \tilde{\mathcal{P}}_{1}$ (cf. (4.15n)), and subsequently (4.17a).

For the terms $Z_{7}$ and $Z_{8}$, we find

$$
\begin{aligned}
& \left|Z_{7}\right|=\mathbb{1}_{A_{1} \leqslant A_{2}} \frac{1}{A^{7}} \mid \int_{0}^{1}\left(\tilde{\mathcal{P}}_{1}^{1 / 2}-\tilde{\mathcal{P}}_{2}^{1 / 2}\right)\left(\tilde{\mathcal{P}}_{2}^{1 / 2}\right)_{\eta}(t, \eta) \\
& \times\left(\int_{0}^{\eta}\left(e^{-\frac{1}{A_{2}}\left(\tilde{\mathcal{Y}}_{2}(t, \eta)-\tilde{\mathcal{Y}}_{2}(t, \theta)\right)}-e^{-\frac{1}{A_{1}}\left(\tilde{\mathcal{Y}}_{2}(t, \eta)-\tilde{\mathcal{Y}}_{2}(t, \theta)\right)}\right)\right. \\
& \left.\times \min _{j}\left(\tilde{\mathcal{D}}_{j}\right) \min _{j}\left(\tilde{\mathcal{U}}_{j}^{+}\right) \tilde{\mathcal{Y}}_{2, \eta}(t, \theta) d \theta\right) d \eta \\
& \leqslant \frac{4}{A_{1} A^{7} e}\left|\int_{0}^{1}\right| \tilde{\mathcal{P}}_{1}^{1 / 2}-\tilde{\mathcal{P}}_{2}^{1 / 2}||\left(\tilde{\mathcal{P}}_{2}^{1 / 2}\right)_{\eta} \mid(t, \eta) \\
& \times\left(\int_{0}^{\eta} e^{-\frac{3}{4 A_{2}}\left(\tilde{\mathcal{Y}}_{2}(t, \eta)-\tilde{\mathcal{Y}}_{2}(t, \theta)\right)} \tilde{\mathcal{D}}_{2}\left|\tilde{\mathcal{U}}_{1}\right| \tilde{\mathcal{Y}}_{2, \eta}(t, \theta) d \theta\right) d \eta|| A_{1}-A_{2} \mid \\
& \leqslant \frac{4 \sqrt{2} a}{A^{6} e} \int_{0}^{1}\left|\tilde{\mathcal{P}}_{1}^{1 / 2}-\tilde{\mathcal{P}}_{2}^{1 / 2} \|\left(\tilde{\mathcal{P}}_{2}^{1 / 2}\right)_{\eta}\right|(t, \eta) \\
& \times\left(\int_{0}^{\eta} e^{-\frac{3}{2 A_{2}}\left(\tilde{\mathcal{Y}}_{2}(t, \eta)-\tilde{\mathcal{Y}}_{2}(t, \theta)\right)} \tilde{\mathcal{P}}_{2} \tilde{\mathcal{Y}}_{2, \eta}(t, \theta) d \theta\right)^{1 / 2} \\
& \times\left(\int_{0}^{\eta} \tilde{\mathcal{P}}_{2} \tilde{\mathcal{Y}}_{2, \eta}(t, \theta) d \theta\right)^{1 / 2} d \eta\left|A_{1}-A_{2}\right| \\
& \leqslant \mathcal{O}(1)\left(\left\|\tilde{\mathcal{P}}_{1}^{1 / 2}-\tilde{\mathcal{P}}_{2}^{1 / 2}\right\|^{2}+\left|A_{1}-A_{2}\right|^{2}\right) \text {. }
\end{aligned}
$$

For the terms $Z_{9}$ and $Z_{10}$, we find

$$
\begin{aligned}
\left|Z_{9}\right|= & \frac{1}{A^{7}} \mid \int_{0}^{1}\left(\tilde{\mathcal{P}}_{1}^{1 / 2}-\tilde{\mathcal{P}}_{2}^{1 / 2}\right)\left(\tilde{\mathcal{P}}_{2}^{1 / 2}\right)_{\eta}(t, \eta) \\
& \times\left(\int_{0}^{\eta}\left(e^{-\frac{1}{a}\left(\tilde{\mathcal{Y}}_{2}(t, \eta)-\tilde{\mathcal{Y}}_{2}(t, \theta)\right)}-e^{-\frac{1}{a}\left(\tilde{\mathcal{Y}}_{1}(t, \eta)-\tilde{\mathcal{Y}}_{1}(t, \theta)\right)}\right)\right. \\
& \left.\times \min _{j}\left(\tilde{\mathcal{D}}_{j}\right) \min _{j}\left(\tilde{\mathcal{U}}_{j}^{+}\right) \tilde{\mathcal{Y}}_{2, \eta}(t, \theta) \mathbb{1}_{B(\eta)}(t, \theta) d \theta\right) d \eta \mid \\
\leqslant & \frac{1}{a A^{7}} \int_{0}^{1}\left|\tilde{\mathcal{P}}_{1}^{1 / 2}-\tilde{\mathcal{P}}_{2}^{1 / 2}\right|\left|\left(\tilde{\mathcal{P}}_{2}^{1 / 2}\right)_{\eta}\right|(t, \eta)
\end{aligned}
$$




$$
\begin{aligned}
& \times\left(\int_{0}^{\eta}\left(\left|\tilde{\mathcal{Y}}_{2}-\tilde{\mathcal{Y}}_{1}\right|(t, \eta)+\left|\tilde{\mathcal{Y}}_{2}-\tilde{\mathcal{Y}}_{1}\right|(t, \theta)\right)\right. \\
& \left.\times e^{-\frac{1}{A_{2}}\left(\tilde{\mathcal{Y}}_{2}(t, \eta)-\tilde{\mathcal{Y}}_{2}(t, \theta)\right)} \tilde{\mathcal{D}}_{2} \min _{j}\left(\tilde{\mathcal{U}}_{j}^{+}\right) \tilde{\mathcal{Y}}_{2, \eta}(t, \theta) d \theta\right) d \eta \\
& \leqslant \frac{1}{\sqrt{2} A^{6}} \int_{0}^{1}\left|\tilde{\mathcal{P}}_{1}^{1 / 2}-\tilde{\mathcal{P}}_{2}^{1 / 2}\right|\left|\tilde{\mathcal{Y}}_{2}-\tilde{\mathcal{Y}}_{1}\right|\left|\left(\tilde{\mathcal{P}}_{2}^{1 / 2}\right)_{\eta}\right|(t, \eta) \\
& \times\left(\int_{0}^{\eta} e^{-\frac{1}{A_{2}}\left(\tilde{\mathcal{Y}}_{2}(t, \eta)-\tilde{\mathcal{Y}}_{2}(t, \theta)\right)} \tilde{\mathcal{D}}_{2} \tilde{\mathcal{Y}}_{2, \eta}(t, \theta) d \theta\right) d \eta \\
& +\frac{1}{\sqrt{2} A^{6}} \int_{0}^{1}\left|\tilde{\mathcal{P}}_{1}^{1 / 2}-\tilde{\mathcal{P}}_{2}^{1 / 2}\right|\left|\left(\tilde{\mathcal{P}}_{2}^{1 / 2}\right)_{\eta}\right|(t, \eta) \\
& \times\left(\int_{0}^{\eta} e^{-\frac{1}{A_{2}}\left(\tilde{\mathcal{Y}}_{2}(t, \eta)-\tilde{\mathcal{Y}}_{2}(t, \theta)\right)}\left|\tilde{\mathcal{Y}}_{2}-\tilde{\mathcal{Y}}_{1}\right| \tilde{\mathcal{D}}_{2} \tilde{\mathcal{Y}}_{2, \eta}(t, \theta) d \theta\right) d \eta \\
& \leqslant \frac{\sqrt{2}}{A^{5}} \int_{0}^{1}\left|\tilde{\mathcal{P}}_{1}^{1 / 2}-\tilde{\mathcal{P}}_{2}^{1 / 2}\right|\left|\tilde{\mathcal{Y}}_{2}-\tilde{\mathcal{Y}}_{1}\right|\left|\left(\tilde{\mathcal{P}}_{2}^{1 / 2}\right)_{\eta}\right|(t, \eta) \\
& \times\left(\int_{0}^{\eta} e^{-\frac{1}{A_{2}}\left(\tilde{\mathcal{Y}}_{2}(t, \eta)-\tilde{\mathcal{Y}}_{2}(t, \theta)\right)} \tilde{\mathcal{P}}_{2}^{2} \tilde{\mathcal{Y}}_{2, \eta}(t, \theta) d \theta\right)^{1 / 2} \\
& \times\left(\int_{0}^{\eta} e^{-\frac{1}{A_{2}}\left(\tilde{\mathcal{Y}}_{2}(t, \eta)-\tilde{\mathcal{Y}}_{2}(t, \theta)\right)} \tilde{\mathcal{Y}}_{2, \eta}(t, \theta) d \theta\right)^{1 / 2} d \eta \\
& +\left\|\tilde{\mathcal{P}}_{1}^{1 / 2}-\tilde{\mathcal{P}}_{2}^{1 / 2}\right\|^{2} \\
& +\frac{2}{A^{10}} \int_{0}^{1}\left(\left(\tilde{\mathcal{P}}_{2}^{1 / 2}\right)_{\eta}\right)^{2}(t, \eta)\left(\int_{0}^{\eta} e^{-\frac{3}{2 A_{2}}\left(\tilde{\mathcal{Y}}_{2}(t, \eta)-\tilde{\mathcal{Y}}_{2}(t, \theta)\right)} \tilde{\mathcal{P}}_{2}^{2} \tilde{\mathcal{Y}}_{2, \eta}^{2}(t, \theta) d \theta\right) \\
& \times\left(\int_{0}^{\eta} e^{-\frac{1}{2 A_{2}}\left(\tilde{\mathcal{Y}}_{2}(t, \eta)-\tilde{\mathcal{Y}}_{2}(t, \theta)\right)}\left(\tilde{\mathcal{Y}}_{2}-\tilde{\mathcal{Y}}_{1}\right)^{2}(t, \theta) d \theta\right) d \eta \\
& \leqslant \mathcal{O}(1)\left(\left\|\tilde{\mathcal{P}}_{1}^{1 / 2}-\tilde{\mathcal{P}}_{2}^{1 / 2}\right\|^{2}+\left\|\tilde{\mathcal{Y}}_{1}-\tilde{\mathcal{Y}}_{2}\right\|^{2}\right),
\end{aligned}
$$

applying the same set of estimates applied when studying $\bar{B}_{35}$; see (5.22). For the term $Z_{11}$, we find

$$
\begin{aligned}
\left|Z_{11}\right|= & \frac{1}{A^{7}} \mid \int_{0}^{1}\left(\tilde{\mathcal{P}}_{1}^{1 / 2}-\tilde{\mathcal{P}}_{2}^{1 / 2}\right)\left(\left(\tilde{\mathcal{P}}_{2}^{1 / 2}\right)_{\eta}-\left(\tilde{\mathcal{P}}_{1}^{1 / 2}\right)_{\eta}\right)(t, \eta) \\
& \quad \times \min _{k}\left(\int_{0}^{\eta} \min _{j}\left(e^{-\frac{1}{a}\left(\tilde{\mathcal{Y}}_{j}(t, \eta)-\tilde{\mathcal{Y}}_{j}(t, \theta)\right)}\right) \min _{j}\left(\tilde{\mathcal{D}}_{j}\right) \min _{j}\left(\tilde{\mathcal{U}}_{j}^{+}\right) \tilde{\mathcal{Y}}_{k, \eta}(t, \theta) d \theta\right) d \eta \mid \\
\leqslant & \mathcal{O}(1)\left\|\tilde{\mathcal{P}}_{1}^{1 / 2}-\tilde{\mathcal{P}}_{2}^{1 / 2}\right\|^{2}
\end{aligned}
$$

see estimates for $\bar{B}_{37}$ (cf. (5.23)). 
For the terms $Z_{12}$ and $Z_{13}$, we find

$$
\begin{aligned}
& \left|Z_{12}\right|=\frac{1}{A^{7}} \mid \int_{0}^{1}\left(\tilde{\mathcal{P}}_{1}^{1 / 2}-\tilde{\mathcal{P}}_{2}^{1 / 2}\right)\left(\tilde{\mathcal{P}}_{2}^{1 / 2}\right)_{\eta} \mathbb{1}_{D^{c}}(t, \eta) \\
& \times\left(\int_{0}^{\eta} \min _{j}\left(e^{-\frac{1}{a}\left(\tilde{\mathcal{Y}}_{j}(t, \eta)-\tilde{\mathcal{Y}}_{j}(t, \theta)\right)}\right) \min _{j}\left(\tilde{\mathcal{D}}_{j}\right) \min _{j}\left(\tilde{\mathcal{U}}_{j}^{+}\right)\left(\tilde{\mathcal{Y}}_{2, \eta}-\tilde{\mathcal{Y}}_{1, \eta}\right)(t, \theta) d \theta\right) d \eta \\
& =\frac{1}{A^{7}} \mid \int_{0}^{1}\left(\tilde{\mathcal{P}}_{1}^{1 / 2}-\tilde{\mathcal{P}}_{2}^{1 / 2}\right)\left(\tilde{\mathcal{P}}_{2}^{1 / 2}\right)_{\eta} \mathbb{1}_{D^{c}}(t, \eta) \\
& \times\left[\left.\left(\min _{j}\left(e^{-\frac{1}{a}\left(\tilde{\mathcal{Y}}_{j}(t, \eta)-\tilde{\mathcal{Y}}_{j}(t, \theta)\right)}\right) \min _{j}\left(\tilde{\mathcal{D}}_{j}\right) \min _{j}\left(\tilde{\mathcal{U}}_{j}^{+}\right)\left(\tilde{\mathcal{Y}}_{2}-\tilde{\mathcal{Y}}_{1}\right)(t, \theta)\right)\right|_{\theta=0} ^{\eta}\right. \\
& \left.-\int_{0}^{\eta}\left(\tilde{\mathcal{Y}}_{2}-\tilde{\mathcal{Y}}_{1}\right) \frac{d}{d \theta}\left(\min _{j}\left(e^{-\frac{1}{a}\left(\tilde{\mathcal{Y}}_{j}(t, \eta)-\tilde{\mathcal{Y}}_{j}(t, \theta)\right)}\right) \min _{j}\left(\tilde{\mathcal{D}}_{j}\right) \min _{j}\left(\tilde{\mathcal{U}}_{j}^{+}\right)\right)(t, \theta) d \theta\right] d \eta \mid \\
& =\mid \frac{1}{A^{7}} \int_{0}^{1}\left(\tilde{\mathcal{P}}_{1}^{1 / 2}-\tilde{\mathcal{P}}_{2}^{1 / 2}\right)\left(\tilde{\mathcal{P}}_{2}^{1 / 2}\right)_{\eta} \mathbb{1}_{D^{c}} \min _{j}\left(\tilde{\mathcal{D}}_{j}\right) \min _{j}\left(\tilde{\mathcal{U}}_{j}^{+}\right)\left(\tilde{\mathcal{Y}}_{2}-\tilde{\mathcal{Y}}_{1}\right)(t, \eta) d \eta \\
& -\frac{1}{A^{7}} \int_{0}^{1}\left(\tilde{\mathcal{P}}_{1}^{1 / 2}-\tilde{\mathcal{P}}_{2}^{1 / 2}\right)\left(\tilde{\mathcal{P}}_{2}^{1 / 2}\right)_{\eta} \mathbb{1}_{D^{c}}(t, \eta)\left(\int_{0}^{\eta}\left(\tilde{\mathcal{Y}}_{2}-\tilde{\mathcal{Y}}_{1}\right)\right. \\
& \left.\times \frac{d}{d \theta}\left(\min _{j}\left(e^{-\frac{1}{a}\left(\tilde{\mathcal{Y}}_{j}(t, \eta)-\tilde{\mathcal{Y}}_{j}(t, \theta)\right)}\right) \min _{j}\left(\tilde{\mathcal{D}}_{j}\right) \min _{j}\left(\tilde{\mathcal{U}}_{j}^{+}\right)\right)(t, \theta) d \theta\right) d \eta \mid \\
& \leqslant \frac{A^{2}}{4 \sqrt{2}} \int_{0}^{1}\left|\tilde{\mathcal{P}}_{1}^{1 / 2}-\tilde{\mathcal{P}}_{2}^{1 / 2}\right|\left|\tilde{\mathcal{Y}}_{2}-\tilde{\mathcal{Y}}_{1}\right|(t, \eta) d \eta \\
& +\frac{1}{A^{7}} \int_{0}^{1}\left|\tilde{\mathcal{P}}_{1}^{1 / 2}-\tilde{\mathcal{P}}_{2}^{1 / 2}\right|\left|\left(\tilde{\mathcal{P}}_{2}^{1 / 2}\right)_{\eta}\right| \mathbb{1}_{D^{c}}(t, \eta)\left(\int_{0}^{\eta}\left|\tilde{\mathcal{Y}}_{2}-\tilde{\mathcal{Y}}_{1}\right|\right. \\
& \left.\times\left|\frac{d}{d \theta}\left(\min _{j}\left(e^{-\frac{1}{a}\left(\tilde{\mathcal{Y}}_{j}(t, \eta)-\tilde{\mathcal{Y}}_{j}(t, \theta)\right)}\right) \min _{j}\left(\tilde{\mathcal{D}}_{j}\right) \min _{j}\left(\tilde{\mathcal{U}}_{j}^{+}\right)\right)\right|(t, \theta) d \theta\right) d \eta \\
& =\tilde{M}_{1}+\tilde{M}_{2} \text {. }
\end{aligned}
$$

We find directly

$$
\left|\tilde{M}_{1}\right| \leqslant \mathcal{O}(1)\left(\left\|\tilde{\mathcal{P}}_{1}^{1 / 2}-\tilde{\mathcal{P}}_{2}^{1 / 2}\right\|^{2}+\left\|\tilde{\mathcal{Y}}_{1}-\tilde{\mathcal{Y}}_{2}\right\|^{2}\right) .
$$

For the other term $\tilde{M}_{2}$, we proceed as follows:

$$
\begin{aligned}
\left|\tilde{M}_{2}\right| \leqslant & \frac{1}{A^{7}} \int_{0}^{1}\left|\tilde{\mathcal{P}}_{1}^{1 / 2}-\tilde{\mathcal{P}}_{2}^{1 / 2}\right|\left|\left(\tilde{\mathcal{P}}_{2}^{1 / 2}\right)_{\eta}\right| \mathbb{1}_{D^{c}}(t, \eta)\left(\int_{0}^{\eta}\left|\tilde{\mathcal{Y}}_{2}-\tilde{\mathcal{Y}}_{1}\right|\right. \\
& \left.\times\left|\frac{d}{d \theta}\left(\min _{j}\left(e^{-\frac{1}{a}\left(\tilde{\mathcal{Y}}_{j}(t, \eta)-\tilde{\mathcal{Y}}_{j}(t, \theta)\right)}\right) \min _{j}\left(\tilde{\mathcal{D}}_{j}\right) \min _{j}\left(\tilde{\mathcal{U}}_{j}^{+}\right)\right)\right|(t, \theta) d \theta\right) d \eta \\
\leqslant & \frac{1}{A^{7}} \int_{0}^{1}\left|\tilde{\mathcal{P}}_{1}^{1 / 2}-\tilde{\mathcal{P}}_{2}^{1 / 2}\right|\left|\left(\tilde{\mathcal{P}}_{2}^{1 / 2}\right)_{\eta}\right| \mathbb{1}_{D^{c}}(t, \eta)
\end{aligned}
$$




$$
\begin{aligned}
& \quad \times\left(\int _ { 0 } ^ { \eta } | \tilde { \mathcal { Y } } _ { 2 } - \tilde { \mathcal { Y } } _ { 1 } | \left[\left|\left(\frac{d}{d \theta} \min _{j}\left(e^{-\frac{1}{a}\left(\tilde{\mathcal{Y}}_{j}(t, \eta)-\tilde{\mathcal{Y}}_{j}(t, \theta)\right)}\right)\right)\right|\left|\min _{j}\left(\tilde{\mathcal{D}}_{j}\right) \min _{j}\left(\tilde{\mathcal{U}}_{j}^{+}\right)\right|\right.\right. \\
& \left.\left.+\left|\min _{j}\left(e^{-\frac{1}{a}\left(\tilde{\mathcal{Y}}_{j}(t, \eta)-\tilde{\mathcal{Y}}_{j}(t, \theta)\right)}\right)\right|\left|\frac{d}{d \theta}\left(\min _{j}\left(\tilde{\mathcal{D}}_{j}\right) \min _{j}\left(\tilde{\mathcal{U}}_{j}^{+}\right)\right)\right|\right](t, \theta) d \theta\right) d \eta \\
& \leqslant \frac{1}{A^{7}} \int_{0}^{1}\left|\tilde{\mathcal{P}}_{1}^{1 / 2}-\tilde{\mathcal{P}}_{2}^{1 / 2}\right|\left|\left(\tilde{\mathcal{P}}_{2}^{1 / 2}\right)_{\eta}\right| \mathbb{1}_{D^{c}}(t, \eta)\left(\int_{0}^{\eta}\left|\tilde{\mathcal{Y}}_{2}-\tilde{\mathcal{Y}}_{1}\right|(t, \theta)\right. \\
& \times\left[\frac{1}{a} \min _{j}\left(e^{-\frac{1}{a}\left(\tilde{\mathcal{Y}}_{j}(t, \eta)-\tilde{\mathcal{Y}}_{j}(t, \theta)\right)}\right) \max _{j}\left(\tilde{\mathcal{Y}}_{j, \eta}\right) \min _{j}\left(\tilde{\mathcal{D}}_{j}\right) \min _{j}\left(\tilde{\mathcal{U}}_{j}^{+}\right)(t, \theta)\right. \\
& \left.\left.+\mathcal{O}(1) A^{9 / 2} \min _{j}\left(e^{-\frac{1}{a}\left(\tilde{\mathcal{Y}}_{j}(t, \eta)-\tilde{\mathcal{Y}}_{j}(t, \theta)\right)}\right)\left(\min _{j}\left(\tilde{\mathcal{D}}_{j}\right)^{1 / 2}+\left|\tilde{\mathcal{U}}_{2}\right|\right)(t, \theta)\right] d \theta\right) d \eta \\
& =\tilde{M}_{21}+\tilde{M}_{22},
\end{aligned}
$$

by using Lemmas A.2 and A.4. For $\tilde{M}_{21}$, we find

$$
\begin{aligned}
& \tilde{M}_{21}=\frac{1}{a A^{7}} \int_{0}^{1}\left|\tilde{\mathcal{P}}_{1}^{1 / 2}-\tilde{\mathcal{P}}_{2}^{1 / 2}\right|\left|\left(\tilde{\mathcal{P}}_{2}^{1 / 2}\right)_{\eta}\right| \mathbb{1}_{D^{c}}(t, \eta)\left(\int_{0}^{\eta} \min _{j}\left(e^{-\frac{1}{a}\left(\tilde{\mathcal{Y}}_{j}(t, \eta)-\tilde{\mathcal{Y}}_{j}(t, \theta)\right)}\right)\right. \\
& \left.\times\left|\tilde{\mathcal{Y}}_{2}-\tilde{\mathcal{Y}}_{1}\right|\left|\max _{j}\left(\tilde{\mathcal{Y}}_{j, \eta}\right) \min _{j}\left(\tilde{\mathcal{D}}_{j}\right) \min _{j}\left(\tilde{\mathcal{U}}_{j}^{+}\right)\right|(t, \theta) d \theta\right) d \eta \\
& \leqslant\left\|\tilde{\mathcal{P}}_{1}^{1 / 2}-\tilde{\mathcal{P}}_{2}^{1 / 2}\right\|^{2}+\frac{1}{a^{2} A^{14}} \int_{0}^{1}\left(\left(\tilde{\mathcal{P}}_{2}^{1 / 2}\right)_{\eta}\right)^{2}(t, \eta)\left(\int_{0}^{\eta} \min _{j}\left(e^{-\frac{1}{a}\left(\tilde{\mathcal{Y}}_{j}(t, \eta)-\tilde{\mathcal{Y}}_{j}(t, \theta)\right)}\right)\right. \\
& \left.\times\left|\tilde{\mathcal{Y}}_{2}-\tilde{\mathcal{Y}}_{1}\right| \max _{j}\left(\tilde{\mathcal{Y}}_{j, \eta}\right) \min _{j}\left(\tilde{\mathcal{D}}_{j}\right) \min _{j}\left(\tilde{\mathcal{U}}_{j}^{+}\right)(t, \theta) d \theta\right)^{2} d \eta \\
& \leqslant\left\|\tilde{\mathcal{P}}_{1}^{1 / 2}-\tilde{\mathcal{P}}_{2}^{1 / 2}\right\|^{2} \\
& +\frac{1}{2} \int_{0}^{1}\left(\left(\tilde{\mathcal{P}}_{2}^{1 / 2}\right)_{\eta}\right)^{2}(t, \eta)\left(\int_{0}^{\eta} e^{-\frac{1}{a}\left(\tilde{\mathcal{Y}}_{2}(t, \eta)-\tilde{\mathcal{Y}}_{2}(t, \theta)\right)}\left|\tilde{\mathcal{Y}}_{2}-\tilde{\mathcal{Y}}_{1}\right|(t, \theta) d \theta\right)^{2} d \eta \\
& \leqslant\left\|\tilde{\mathcal{P}}_{1}^{1 / 2}-\tilde{\mathcal{P}}_{2}^{1 / 2}\right\|^{2} \\
& +\frac{1}{8 A^{2}} \int_{0}^{1} \tilde{\mathcal{P}}_{2} \tilde{\mathcal{Y}}_{2, \eta}^{2}(t, \eta)\left(\int_{0}^{\eta} e^{-\frac{1}{a}\left(\tilde{\mathcal{Y}}_{2}(t, \eta)-\tilde{\mathcal{Y}}_{2}(t, \theta)\right)}\left(\tilde{\mathcal{Y}}_{2}-\tilde{\mathcal{Y}}_{1}\right)^{2}(t, \theta) d \theta\right) \\
& \times\left(\int_{0}^{\eta} e^{-\frac{1}{a}\left(\tilde{\mathcal{Y}}_{2}(t, \eta)-\tilde{\mathcal{Y}}_{2}(t, \theta)\right)} d \theta\right) d \eta \\
& \leqslant\left\|\tilde{\mathcal{P}}_{1}^{1 / 2}-\tilde{\mathcal{P}}_{2}^{1 / 2}\right\|^{2}+\mathcal{O}(1) \int_{0}^{1} \int_{0}^{\eta}\left(\tilde{\mathcal{Y}}_{2}-\tilde{\mathcal{Y}}_{1}\right)^{2}(t, \theta) d \theta d \eta \\
& \leqslant \mathcal{O}(1)\left(\left\|\tilde{\mathcal{P}}_{1}^{1 / 2}-\tilde{\mathcal{P}}_{2}^{1 / 2}\right\|^{2}+\left\|\tilde{\mathcal{Y}}_{2}-\tilde{\mathcal{Y}}_{1}\right\|^{2}\right) \text {, }
\end{aligned}
$$


using

$\max _{j}\left(\tilde{\mathcal{Y}}_{j, \eta}\right) \min _{j}\left(\tilde{\mathcal{D}}_{j}\right) \leqslant 2 \max _{j}\left(\tilde{\mathcal{Y}}_{j, \eta}\right) \min _{j}\left(A_{i} \tilde{\mathcal{P}}_{j}\right) \leqslant 2 \max _{j}\left(A_{i} \tilde{\mathcal{P}}_{j} \tilde{\mathcal{Y}}_{j, \eta}\right) \leqslant A^{6}$, and (4.15e), (4.16a), and (4.17c). For $\tilde{M}_{22}$, we find

$$
\begin{aligned}
& \tilde{M}_{22}=\frac{\mathcal{O}(1)}{A^{5 / 2}} \int_{0}^{1}\left|\tilde{\mathcal{P}}_{1}^{1 / 2}-\tilde{\mathcal{P}}_{2}^{1 / 2}\right|\left|\left(\tilde{\mathcal{P}}_{2}^{1 / 2}\right)_{\eta}\right| \mathbb{1}_{D^{c}}(t, \eta) \\
& \times\left(\int_{0}^{\eta} \min _{j}\left(e^{-\frac{1}{a}\left(\tilde{\mathcal{Y}}_{j}(t, \eta)-\tilde{\mathcal{Y}}_{j}(t, \theta)\right)}\right)\left|\tilde{\mathcal{Y}}_{2}-\tilde{\mathcal{Y}}_{1}\right|\left(\min _{j}\left(\tilde{\mathcal{D}}_{j}\right)^{1 / 2}+\left|\tilde{\mathcal{U}}_{2}\right|\right)(t, \theta) d \theta\right) d \eta \\
& \leqslant\left\|\tilde{\mathcal{P}}_{1}^{1 / 2}-\tilde{\mathcal{P}}_{2}^{1 / 2}\right\|^{2}+\frac{\mathcal{O}(1)}{A^{5}} \int_{0}^{1}\left(\left(\tilde{\mathcal{P}}_{2}^{1 / 2}\right)_{\eta}\right)^{2}(t, \eta) \\
& \times\left(\int_{0}^{\eta} \min _{j}\left(e^{-\frac{1}{a}\left(\tilde{\mathcal{Y}}_{j}(t, \eta)-\tilde{\mathcal{Y}}_{j}(t, \theta)\right)}\right)\left|\tilde{\mathcal{Y}}_{2}-\tilde{\mathcal{Y}}_{1}\right|\left(\min _{j}\left(\tilde{\mathcal{D}}_{j}\right)^{1 / 2}+\left|\tilde{\mathcal{U}}_{2}\right|\right)(t, \theta) d \theta\right)^{2} d \eta \\
& \leqslant\left\|\tilde{\mathcal{P}}_{1}^{1 / 2}-\tilde{\mathcal{P}}_{2}^{1 / 2}\right\|^{2}+\frac{\mathcal{O}(1)}{A^{5}} \int_{0}^{1}\left(\left(\tilde{\mathcal{P}}_{2}^{1 / 2}\right)_{\eta}\right)^{2}(t, \eta) \\
& \times\left(\int_{0}^{\eta} e^{-\frac{1}{a}\left(\tilde{\mathcal{Y}}_{2}(t, \eta)-\tilde{\mathcal{Y}}_{2}(t, \theta)\right)}\left|\tilde{\mathcal{Y}}_{2}-\tilde{\mathcal{Y}}_{1}\right| \tilde{\mathcal{P}}_{2}^{1 / 2}(t, \theta) d \theta\right)^{2} d \eta \\
& \leqslant\left\|\tilde{\mathcal{P}}_{1}^{1 / 2}-\tilde{\mathcal{P}}_{2}^{1 / 2}\right\|^{2} \\
& +\frac{\mathcal{O}(1)}{A^{7}} \int_{0}^{1} \tilde{\mathcal{P}}_{2} \tilde{\mathcal{Y}}_{2, \eta}^{2}(t, \eta)\left(\int_{0}^{\eta} e^{-\frac{3}{2 A_{2}}\left(\tilde{\mathcal{Y}}_{2}(t, \eta)-\tilde{\mathcal{Y}}_{2}(t, \theta)\right)} \tilde{\mathcal{P}}_{2}(t, \theta) d \theta\right) \\
& \times\left(\int_{0}^{\eta} e^{-\frac{1}{2 A_{2}}\left(\tilde{\mathcal{Y}}_{2}(t, \eta)-\tilde{\mathcal{Y}}_{2}(t, \theta)\right)}\left|\tilde{\mathcal{Y}}_{2}-\tilde{\mathcal{Y}}_{1}\right|(t, \theta) d \theta\right) d \eta \\
& \leqslant\left\|\tilde{\mathcal{P}}_{1}^{1 / 2}-\tilde{\mathcal{P}}_{2}^{1 / 2}\right\|^{2} \\
& +\frac{\mathcal{O}(1)}{A^{7}} \int_{0}^{1} \tilde{\mathcal{P}}_{2}^{2} \tilde{\mathcal{Y}}_{2, \eta}^{2}(t, \eta)\left(\int_{0}^{\eta} e^{-\frac{1}{2 A_{2}}\left(\tilde{\mathcal{Y}}_{2}(t, \eta)-\tilde{\mathcal{Y}}_{2}(t, \theta)\right)}\left(\tilde{\mathcal{Y}}_{2}-\tilde{\mathcal{Y}}_{1}\right)^{2}(t, \theta) d \theta\right) d \eta \\
& \leqslant \mathcal{O}(1)\left(\left\|\tilde{\mathcal{P}}_{1}^{1 / 2}-\tilde{\mathcal{P}}_{2}^{1 / 2}\right\|^{2}+\left\|\tilde{\mathcal{Y}}_{2}-\tilde{\mathcal{Y}}_{1}\right\|^{2}\right) \text {, }
\end{aligned}
$$

using that (cf. (4.15d) and (4.15n))

$$
\min _{j}\left(\tilde{\mathcal{D}}_{j}\right)^{1 / 2}+\left|\tilde{\mathcal{U}}_{2}\right| \leqslant \sqrt{2 A_{2}} \tilde{\mathcal{P}}_{2}^{1 / 2}+\left|\tilde{\mathcal{U}}_{2}\right| \leqslant \sqrt{2}\left(1+\sqrt{A_{2}}\right) \tilde{\mathcal{P}}_{2}^{1 / 2}
$$

and (4.15e), (4.16i), and (4.17a).

We now consider the term $W_{4}$ :

$$
\begin{aligned}
W_{4}= & -\frac{3}{A^{6}} \int_{0}^{1}\left(\tilde{\mathcal{P}}_{1}^{1 / 2}-\tilde{\mathcal{P}}_{2}^{1 / 2}\right) \\
& \times\left(\left(\tilde{\mathcal{P}}_{2}^{1 / 2}\right)_{\eta}(t, \eta) \int_{0}^{\eta} e^{-\frac{1}{A_{2}}\left(\tilde{\mathcal{Y}}_{2}(t, \eta)-\tilde{\mathcal{Y}}_{2}(t, \theta)\right)} \tilde{\mathcal{P}}_{2} \tilde{\mathcal{U}}_{2} \tilde{\mathcal{Y}}_{2, \eta}(t, \theta) d \theta\right.
\end{aligned}
$$




$$
\begin{aligned}
& \left.-\left(\tilde{\mathcal{P}}_{1}^{1 / 2}\right)_{\eta}(t, \eta) \int_{0}^{\eta} e^{-\frac{1}{A_{1}}\left(\tilde{\mathcal{Y}}_{1}(t, \eta)-\tilde{\mathcal{Y}}_{1}(t, \theta)\right)} \tilde{\mathcal{P}}_{1} \tilde{\mathcal{U}}_{1} \tilde{\mathcal{Y}}_{1, \eta}(t, \theta) d \theta\right) d \eta \\
= & -\frac{3}{A^{6}} \int_{0}^{1}\left(\tilde{\mathcal{P}}_{1}^{1 / 2}-\tilde{\mathcal{P}}_{2}^{1 / 2}\right) \\
& \times\left(\left(\tilde{\mathcal{P}}_{2}^{1 / 2}\right)_{\eta}(t, \eta) \int_{0}^{\eta} e^{-\frac{1}{A_{2}}\left(\tilde{\mathcal{Y}}_{2}(t, \eta)-\tilde{\mathcal{Y}}_{2}(t, \theta)\right)} \tilde{\mathcal{P}}_{2} \tilde{\mathcal{U}}_{2}^{+} \tilde{\mathcal{Y}}_{2, \eta}(t, \theta) d \theta\right. \\
& \left.-\left(\tilde{\mathcal{P}}_{1}^{1 / 2}\right)_{\eta}(t, \eta) \int_{0}^{\eta} e^{-\frac{1}{A_{1}}\left(\tilde{\mathcal{Y}}_{1}(t, \eta)-\tilde{\mathcal{Y}}_{1}(t, \theta)\right)} \tilde{\mathcal{P}}_{1} \tilde{\mathcal{U}}_{1}^{+} \tilde{\mathcal{Y}}_{1, \eta}(t, \theta) d \theta\right) d \eta \\
& -\frac{3}{A^{6}} \int_{0}^{1}\left(\tilde{\mathcal{P}}_{1}^{1 / 2}-\tilde{\mathcal{P}}_{2}^{1 / 2}\right) \\
& \times\left(\left(\tilde{\mathcal{P}}_{2}^{1 / 2}\right)_{\eta}(t, \eta) \int_{0}^{\eta} e^{-\frac{1}{A_{2}}\left(\tilde{\mathcal{Y}}_{2}(t, \eta)-\tilde{\mathcal{Y}}_{2}(t, \theta)\right)} \tilde{\mathcal{P}}_{2} \tilde{\mathcal{U}}_{2}^{-} \tilde{\mathcal{Y}}_{2, \eta}(t, \theta) d \theta\right. \\
& \left.-\left(\tilde{\mathcal{P}}_{1}^{1 / 2}\right)_{\eta}(t, \eta) \int_{0}^{\eta} e^{-\frac{1}{A_{1}}\left(\tilde{\mathcal{Y}}_{1}(t, \eta)-\tilde{\mathcal{Y}}_{1}(t, \theta)\right)} \tilde{\mathcal{P}}_{1} \tilde{\mathcal{U}}_{1}^{-} \tilde{\mathcal{Y}}_{1, \eta}(t, \theta) d \theta\right) d \eta \\
= & W_{4}^{+}+W_{4}^{-} \cdot
\end{aligned}
$$

Since $W_{4}^{+}=-3 N_{1}(\operatorname{see}(5.43))$, we find that

$$
\left|W_{4}\right| \leqslant \mathcal{O}(1)\left(\left\|\tilde{\mathcal{P}}_{1}^{1 / 2}-\tilde{\mathcal{P}}_{2}^{1 / 2}\right\|^{2}+\left\|\tilde{\mathcal{U}}_{1}-\tilde{\mathcal{U}}_{2}\right\|^{2}+\left\|\tilde{\mathcal{Y}}_{2}-\tilde{\mathcal{Y}}_{1}\right\|^{2}+\left|A_{1}-A_{2}\right|^{2}\right)
$$

The next term $W_{5}$ would be laborious:

$$
\begin{aligned}
W_{5}= & \frac{1}{A^{6}} \int_{0}^{1}\left(\tilde{\mathcal{P}}_{1}^{1 / 2}-\tilde{\mathcal{P}}_{2}^{1 / 2}\right)\left(\left(\tilde{\mathcal{P}}_{2}^{1 / 2}\right)_{\eta}(t, \eta) \int_{0}^{\eta} e^{-\frac{1}{A_{2}}\left(\tilde{\mathcal{Y}}_{2}(t, \eta)-\tilde{\mathcal{Y}}_{2}(t, \theta)\right)} \tilde{\mathcal{U}}_{2}^{3} \tilde{\mathcal{Y}}_{2, \eta}(t, \theta) d \theta\right. \\
& \left.-\left(\tilde{\mathcal{P}}_{1}^{1 / 2}\right)_{\eta}(t, \eta) \int_{0}^{\eta} e^{-\frac{1}{A_{1}}\left(\tilde{\mathcal{Y}}_{1}(t, \eta)-\tilde{\mathcal{Y}}_{1}(t, \theta)\right)} \tilde{\mathcal{U}}_{1}^{3} \tilde{\mathcal{Y}}_{1, \eta}(t, \theta) d \theta\right) d \eta \\
= & \frac{1}{A^{6}} \int_{0}^{1}\left(\tilde{\mathcal{P}}_{1}^{1 / 2}-\tilde{\mathcal{P}}_{2}^{1 / 2}\right)\left(\left(\tilde{\mathcal{P}}_{2}^{1 / 2}\right)_{\eta}(t, \eta) \int_{0}^{\eta} e^{-\frac{1}{A_{2}}\left(\tilde{\mathcal{Y}}_{2}(t, \eta)-\tilde{\mathcal{Y}}_{2}(t, \theta)\right)}\left(\tilde{\mathcal{U}}_{2}^{+}\right)^{3} \tilde{\mathcal{Y}}_{2, \eta}(t, \theta) d \theta\right. \\
& \left.-\left(\tilde{\mathcal{P}}_{1}^{1 / 2}\right)_{\eta}(t, \eta) \int_{0}^{\eta} e^{-\frac{1}{A_{1}}\left(\tilde{\mathcal{Y}}_{1}(t, \eta)-\tilde{\mathcal{Y}}_{1}(t, \theta)\right)}\left(\tilde{\mathcal{U}}_{1}^{+}\right)^{3} \tilde{\mathcal{Y}}_{1, \eta}(t, \theta) d \theta\right) d \eta \\
& +\frac{1}{A^{6}} \int_{0}^{1}\left(\tilde{\mathcal{P}}_{1}^{1 / 2}-\tilde{\mathcal{P}}_{2}^{1 / 2}\right)\left(\left(\tilde{\mathcal{P}}_{2}^{1 / 2}\right)_{\eta}(t, \eta) \int_{0}^{\eta} e^{-\frac{1}{A_{2}}\left(\tilde{\mathcal{Y}}_{2}(t, \eta)-\tilde{\mathcal{Y}}_{2}(t, \theta)\right)}\left(\tilde{\mathcal{U}}_{2}^{-}\right)^{3} \tilde{\mathcal{Y}}_{2, \eta}(t, \theta) d \theta\right. \\
& \left.-\left(\tilde{\mathcal{P}}_{1}^{1 / 2}\right)_{\eta}(t, \eta) \int_{0}^{\eta} e^{-\frac{1}{A_{1}}\left(\tilde{\mathcal{Y}}_{1}(t, \eta)-\tilde{\mathcal{Y}}_{1}(t, \theta)\right)}\left(\tilde{\mathcal{U}}_{1}^{-}\right)^{3} \tilde{\mathcal{Y}}_{1, \eta}(t, \theta) d \theta\right) d \eta \\
= & W_{5}^{+}+W_{5}^{-} .
\end{aligned}
$$

Unfortunately, having a close look at $W_{5}^{+}$one has $W_{5}^{+}=\int_{0}^{1}\left(\tilde{\mathcal{P}}_{1}^{1 / 2}-\tilde{\mathcal{P}}_{2}^{1 / 2}\right)\left(I_{211}+I_{212}+I_{213}+I_{214}+I_{215}+I_{216}+I_{217}+I_{218}\right)(t, \eta) d \eta$, 
where $I_{211}, \ldots, I_{218}$ are defined in (5.42). Thus we can conclude immediately that

$$
\left|W_{5}^{+}\right| \leqslant \mathcal{O}(1)\left(\left\|\tilde{\mathcal{Y}}_{1}-\tilde{\mathcal{Y}}_{2}\right\|^{2}+\left\|\tilde{\mathcal{U}}_{1}-\tilde{\mathcal{U}}_{2}\right\|^{2}+\left\|\tilde{\mathcal{P}}_{1}^{1 / 2}-\tilde{\mathcal{P}}_{2}^{1 / 2}\right\|^{2}+\left|A_{1}-A_{2}\right|^{2}\right) .
$$

For the term $W_{6}$, we find

$$
\begin{aligned}
& W_{6}=\frac{1}{2 A^{6}} \int_{0}^{1}\left(\tilde{\mathcal{P}}_{1}^{1 / 2}-\tilde{\mathcal{P}}_{2}^{1 / 2}\right)\left(\left(\tilde{\mathcal{P}}_{2}^{1 / 2}\right)_{\eta}(t, \eta) \int_{0}^{\eta} e^{-\frac{1}{A_{2}}\left(\tilde{\mathcal{Y}}_{2}(t, \eta)-\tilde{\mathcal{Y}}_{2}(t, \theta)\right)} A_{2}^{5} \tilde{\mathcal{U}}_{2}(t, \theta) d \theta\right. \\
& \left.-\left(\tilde{\mathcal{P}}_{1}^{1 / 2}\right)_{\eta}(t, \eta) \int_{0}^{\eta} e^{-\frac{1}{A_{1}}\left(\tilde{\mathcal{Y}}_{1}(t, \eta)-\tilde{\mathcal{Y}}_{1}(t, \theta)\right)} A_{1}^{5} \tilde{\mathcal{U}}_{1}(t, \theta) d \theta\right) d \eta \\
& =\frac{A_{2}^{5}-A_{1}^{5}}{2 A^{6}} \mathbb{1}_{A_{1} \leqslant A_{2}} \int_{0}^{1}\left(\tilde{\mathcal{P}}_{1}^{1 / 2}-\tilde{\mathcal{P}}_{2}^{1 / 2}\right)\left(\tilde{\mathcal{P}}_{2}^{1 / 2}\right)_{\eta}(t, \eta) \\
& \times \int_{0}^{\eta} e^{-\frac{1}{A_{2}}\left(\tilde{\mathcal{Y}}_{2}(t, \eta)-\tilde{\mathcal{Y}}_{2}(t, \theta)\right)} \tilde{\mathcal{U}}_{2}(t, \theta) d \theta d \eta \\
& +\frac{A_{2}^{5}-A_{1}^{5}}{2 A^{6}} \mathbb{1}_{A_{2}<A_{1}} \int_{0}^{1}\left(\tilde{\mathcal{P}}_{1}^{1 / 2}-\tilde{\mathcal{P}}_{2}^{1 / 2}\right)\left(\tilde{\mathcal{P}}_{1}^{1 / 2}\right)_{\eta}(t, \eta) \\
& \times \int_{0}^{\eta} e^{-\frac{1}{A_{2}}\left(\tilde{\mathcal{Y}}_{2}(t, \eta)-\tilde{\mathcal{Y}}_{2}(t, \theta)\right)} \tilde{\mathcal{U}}_{1}(t, \theta) d \theta d \eta \\
& +\mathbb{1}_{A_{1} \leqslant A_{2}} \frac{a^{5}}{2 A^{6}} \int_{0}^{1}\left(\tilde{\mathcal{P}}_{1}^{1 / 2}-\tilde{\mathcal{P}}_{2}^{1 / 2}\right)\left(\tilde{\mathcal{P}}_{2}^{1 / 2}\right)_{\eta}(t, \eta) \\
& \times\left(\int_{0}^{\eta}\left(e^{-\frac{1}{A_{2}}\left(\tilde{\mathcal{Y}}_{2}(t, \eta)-\tilde{\mathcal{Y}}_{2}(t, \theta)\right)}-e^{-\frac{1}{A_{1}}\left(\tilde{\mathcal{Y}}_{2}(t, \eta)-\tilde{\mathcal{Y}}_{2}(t, \theta)\right)}\right) \tilde{\mathcal{U}}_{2}(t, \theta) d \theta\right) d \eta \\
& +\mathbb{1}_{A_{2}<A_{1}} \frac{a^{5}}{2 A^{6}} \int_{0}^{1}\left(\tilde{\mathcal{P}}_{1}^{1 / 2}-\tilde{\mathcal{P}}_{2}^{1 / 2}\right)\left(\tilde{\mathcal{P}}_{1}^{1 / 2}\right)_{\eta}(t, \eta) \\
& \times\left(\int_{0}^{\eta}\left(e^{-\frac{1}{A_{2}}\left(\tilde{\mathcal{Y}}_{1}(t, \eta)-\tilde{\mathcal{Y}}_{1}(t, \theta)\right)}-e^{-\frac{1}{A_{1}}\left(\tilde{\mathcal{Y}}_{1}(t, \eta)-\tilde{\mathcal{Y}}_{1}(t, \theta)\right)}\right) \tilde{\mathcal{U}}_{1}(t, \theta) d \theta\right) d \eta \\
& +\frac{a^{5}}{2 A^{6}} \int_{0}^{1}\left(\tilde{\mathcal{P}}_{1}^{1 / 2}-\tilde{\mathcal{P}}_{2}^{1 / 2}\right)\left(\tilde{\mathcal{P}}_{2}^{1 / 2}\right)_{\eta}(t, \eta) \\
& \times\left(\int_{0}^{\eta}\left(e^{-\frac{1}{a}\left(\tilde{\mathcal{Y}}_{2}(t, \eta)-\tilde{\mathcal{Y}}_{2}(t, \theta)\right)}-e^{-\frac{1}{a}\left(\tilde{\mathcal{Y}}_{1}(t, \eta)-\tilde{\mathcal{Y}}_{1}(t, \theta)\right)}\right) \tilde{\mathcal{U}}_{2} \mathbb{1}_{B(\eta)}(t, \theta) d \theta\right) d \eta \\
& +\frac{a^{5}}{2 A^{6}} \int_{0}^{1}\left(\tilde{\mathcal{P}}_{1}^{1 / 2}-\tilde{\mathcal{P}}_{2}^{1 / 2}\right)\left(\tilde{\mathcal{P}}_{1}^{1 / 2}\right)_{\eta}(t, \eta) \\
& \times\left(\int_{0}^{\eta}\left(e^{-\frac{1}{a}\left(\tilde{\mathcal{Y}}_{2}(t, \eta)-\tilde{\mathcal{Y}}_{2}(t, \theta)\right)}-e^{-\frac{1}{a}\left(\tilde{\mathcal{Y}}_{1}(t, \eta)-\tilde{\mathcal{Y}}_{1}(t, \theta)\right)}\right) \tilde{\mathcal{U}}_{1} \mathbb{1}_{B(\eta)^{c}}(t, \theta) d \theta\right) d \eta \\
& +\frac{a^{5}}{2 A^{6}} \int_{0}^{1}\left(\tilde{\mathcal{P}}_{1}^{1 / 2}-\tilde{\mathcal{P}}_{2}^{1 / 2}\right)\left(\tilde{\mathcal{P}}_{2}^{1 / 2}\right)_{\eta}(t, \eta)
\end{aligned}
$$




$$
\begin{aligned}
& \times\left(\int_{0}^{\eta} \min _{j}\left(e^{-\frac{1}{a}\left(\tilde{\mathcal{Y}}_{j}(t, \eta)-\tilde{\mathcal{Y}}_{j}(t, \theta)\right)}\right)\left(\tilde{\mathcal{U}}_{2}^{+}-\tilde{\mathcal{U}}_{1}^{+}\right) \mathbb{1}_{\tilde{\mathcal{U}}_{1}^{+} \leqslant \tilde{\mathcal{U}}_{2}^{+}}(t, \theta) d \theta\right) d \eta \\
& +\frac{a^{5}}{2 A^{6}} \int_{0}^{1}\left(\tilde{\mathcal{P}}_{1}^{1 / 2}-\tilde{\mathcal{P}}_{2}^{1 / 2}\right)\left(\tilde{\mathcal{P}}_{2}^{1 / 2}\right)_{\eta}(t, \eta) \\
& \times\left(\int_{0}^{\eta} \min _{j}\left(e^{-\frac{1}{a}\left(\tilde{\mathcal{Y}}_{j}(t, \eta)-\tilde{\mathcal{Y}}_{j}(t, \theta)\right)}\right)\left(\tilde{\mathcal{U}}_{2}^{-}-\tilde{\mathcal{U}}_{1}^{-}\right) \mathbb{1}_{\tilde{\mathcal{U}}_{2}^{-} \leqslant \tilde{\mathcal{U}}_{1}^{-}}(t, \theta) d \theta\right) d \eta \\
& +\frac{a^{5}}{2 A^{6}} \int_{0}^{1}\left(\tilde{\mathcal{P}}_{1}^{1 / 2}-\tilde{\mathcal{P}}_{2}^{1 / 2}\right)\left(\tilde{\mathcal{P}}_{1}^{1 / 2}\right)_{\eta}(t, \eta) \\
& \times\left(\int_{0}^{\eta} \min _{j}\left(e^{-\frac{1}{a}\left(\tilde{\mathcal{Y}}_{j}(t, \eta)-\tilde{\mathcal{Y}}_{j}(t, \theta)\right)}\right)\left(\tilde{\mathcal{U}}_{2}^{+}-\tilde{\mathcal{U}}_{1}^{+}\right) \mathbb{1}_{\tilde{\mathcal{U}}_{2}^{+}<\tilde{\mathcal{U}}_{1}^{+}}(t, \theta) d \theta\right) d \eta \\
& +\frac{a^{5}}{2 A^{6}} \int_{0}^{1}\left(\tilde{\mathcal{P}}_{1}^{1 / 2}-\tilde{\mathcal{P}}_{2}^{1 / 2}\right)\left(\tilde{\mathcal{P}}_{1}^{1 / 2}\right)_{\eta}(t, \eta) \\
& \times\left(\int_{0}^{\eta} \min _{j}\left(e^{-\frac{1}{a}\left(\tilde{\mathcal{Y}}_{j}(t, \eta)-\tilde{\mathcal{Y}}_{j}(t, \theta)\right)}\right)\left(\tilde{\mathcal{U}}_{2}^{-}-\tilde{\mathcal{U}}_{1}^{-}\right) \mathbb{1}_{\tilde{\mathcal{U}}_{1}^{-}<\tilde{\mathcal{U}}_{2}^{-}}(t, \theta) d \theta\right) d \eta \\
& +\frac{a^{5}}{2 A^{6}} \int_{0}^{1}\left(\tilde{\mathcal{P}}_{1}^{1 / 2}-\tilde{\mathcal{P}}_{2}^{1 / 2}\right)\left(\left(\tilde{\mathcal{P}}_{2}^{1 / 2}\right)_{\eta}-\left(\tilde{\mathcal{P}}_{1}^{1 / 2}\right)_{\eta}\right)(t, \eta) \\
& \times\left(\int_{0}^{\eta} \min _{j}\left(e^{-\frac{1}{a}\left(\tilde{\mathcal{Y}}_{j}(t, \eta)-\tilde{\mathcal{Y}}_{j}(t, \theta)\right)}\right) \min _{j}\left(\tilde{\mathcal{U}}_{j}^{+}\right)(t, \theta) d \theta\right) d \eta \\
& +\frac{a^{5}}{2 A^{6}} \int_{0}^{1}\left(\tilde{\mathcal{P}}_{1}^{1 / 2}-\tilde{\mathcal{P}}_{2}^{1 / 2}\right)\left(\left(\tilde{\mathcal{P}}_{2}^{1 / 2}\right)_{\eta}-\left(\tilde{\mathcal{P}}_{1}^{1 / 2}\right)_{\eta}\right)(t, \eta) \\
& \times\left(\int_{0}^{\eta} \max _{j}\left(e^{-\frac{1}{a}\left(\tilde{\mathcal{Y}}_{j}(t, \eta)-\tilde{\mathcal{Y}}_{j}(t, \theta)\right)}\right) \min _{j}\left(\tilde{\mathcal{U}}_{j}^{-}\right)(t, \theta) d \theta\right) d \eta \\
& =W_{61}+W_{62}+W_{63}+W_{64}+W_{65}+W_{66} \\
& +W_{67}^{+}+W_{67}^{-}+W_{68}^{+}+W_{68}^{-}+W_{69}^{+}+W_{69}^{-} \text {. }
\end{aligned}
$$

The terms $W_{61}$ and $W_{62}$ :

$$
\begin{aligned}
\left|W_{61}\right|= & \frac{A_{2}^{5}-A_{1}^{5}}{2 A^{6}} \mathbb{1}_{A_{1} \leqslant A_{2}} \mid \int_{0}^{1}\left(\tilde{\mathcal{P}}_{1}^{1 / 2}-\tilde{\mathcal{P}}_{2}^{1 / 2}\right)\left(\tilde{\mathcal{P}}_{2}^{1 / 2}\right)_{\eta}(t, \eta) \\
& \times \int_{0}^{\eta} e^{-\frac{1}{A_{2}}\left(\tilde{\mathcal{Y}}_{2}(t, \eta)-\tilde{\mathcal{Y}}_{2}(t, \theta)\right)} \tilde{\mathcal{U}}_{2}(t, \theta) d \theta d \eta \mid \\
\leqslant & 5 \frac{A_{2}-A_{1}}{2 A^{2}} \int_{0}^{1}\left|\tilde{\mathcal{P}}_{1}^{1 / 2}-\tilde{\mathcal{P}}_{2}^{1 / 2}\right|\left|\left(\tilde{\mathcal{P}}_{2}^{1 / 2}\right)_{\eta}\right|(t, \eta) \\
& \times\left(\int_{0}^{\eta} e^{-\frac{1}{A_{2}}\left(\tilde{\mathcal{Y}}_{2}(t, \eta)-\tilde{\mathcal{Y}}_{2}(t, \theta)\right)} \tilde{\mathcal{U}}_{2}^{2}(t, \theta) d \theta\right)^{1 / 2}
\end{aligned}
$$




$$
\begin{aligned}
& \times\left(\int_{0}^{\eta} e^{-\frac{1}{A_{2}}\left(\tilde{\mathcal{Y}}_{2}(t, \eta)-\tilde{\mathcal{Y}}_{2}(t, \theta)\right)} d \theta\right)^{1 / 2} d \eta \\
\leqslant & 5 \sqrt{6} \frac{A_{2}-A_{1}}{2 A^{2}} \int_{0}^{1}\left|\tilde{\mathcal{P}}_{1}^{1 / 2}-\tilde{\mathcal{P}}_{2}^{1 / 2}\right| \tilde{\mathcal{P}}_{2}^{1 / 2}\left|\left(\tilde{\mathcal{P}}_{2}^{1 / 2}\right)_{\eta}\right|(t, \eta) d \eta \\
\leqslant & \mathcal{O}(1)\left(\left|A_{1}-A_{2}\right|^{2}+\left\|\tilde{\mathcal{P}}_{1}^{1 / 2}-\tilde{\mathcal{P}}_{2}^{1 / 2}\right\|^{2}\right),
\end{aligned}
$$

using (4.15e), (4.16a), and (4.17a).

The terms $W_{63}$ and $W_{64}$ :

$$
\begin{aligned}
\left|W_{63}\right|= & \mathbb{1}_{A_{1} \leqslant} \frac{a_{2}}{2 A^{6}} \int_{0}^{1}\left|\tilde{\mathcal{P}}_{1}^{1 / 2}-\tilde{\mathcal{P}}_{2}^{1 / 2}\right|\left|\left(\tilde{\mathcal{P}}_{2}^{1 / 2}\right)_{\eta}\right|(t, \eta) \\
& \times\left|\int_{0}^{\eta}\left(e^{-\frac{1}{A_{2}}\left(\tilde{\mathcal{Y}}_{2}(t, \eta)-\tilde{\mathcal{Y}}_{2}(t, \theta)\right)}-e^{-\frac{1}{A_{1}}\left(\tilde{\mathcal{Y}}_{2}(t, \eta)-\tilde{\mathcal{Y}}_{2}(t, \theta)\right)}\right) \tilde{\mathcal{U}}_{2}(t, \theta) d \theta\right| d \eta \\
\leqslant & \frac{4 a^{5}}{2 a A^{6} e} \int_{0}^{1}\left|\tilde{\mathcal{P}}_{1}^{1 / 2}-\tilde{\mathcal{P}}_{2}^{1 / 2}\right|\left|\left(\tilde{\mathcal{P}}_{2}^{1 / 2}\right)_{\eta}\right|(t, \eta) \\
& \times\left(\int_{0}^{\eta} e^{-\frac{3}{4 A_{2}}\left(\tilde{\mathcal{Y}}_{2}(t, \eta)-\tilde{\mathcal{Y}}_{2}(t, \theta)\right)}\left|\tilde{\mathcal{U}}_{2}\right|(t, \theta) d \theta\right) d \eta\left|A_{1}-A_{2}\right| \\
\leqslant & \frac{2}{A^{2} e} \int_{0}^{1}\left|\tilde{\mathcal{P}}_{1}^{1 / 2}-\tilde{\mathcal{P}}_{2}^{1 / 2}\right|\left|\left(\tilde{\mathcal{P}}_{2}^{1 / 2}\right)_{\eta}\right|(t, \eta) \\
& \times\left(\int_{0}^{\eta} e^{-\frac{1}{A_{2}}\left(\tilde{\mathcal{Y}}_{2}(t, \eta)-\tilde{\mathcal{Y}}_{2}(t, \theta)\right)} \tilde{\mathcal{U}}_{2}^{2}(t, \theta) d \theta\right)^{1 / 2} d \eta\left|A_{1}-A_{2}\right| \\
\leqslant & \frac{2 \sqrt{6}}{A^{2} e} \int_{0}^{1}\left|\tilde{\mathcal{P}}_{1}^{1 / 2}-\tilde{\mathcal{P}}_{2}^{1 / 2}\right| \tilde{\mathcal{P}}_{2}^{1 / 2}\left|\left(\tilde{\mathcal{P}}_{2}^{1 / 2}\right)_{\eta}\right|(t, \eta) d \eta\left|A_{1}-A_{2}\right| \\
\leqslant & \mathcal{O}(1)\left(\left\|\tilde{\mathcal{P}}_{1}^{1 / 2}-\tilde{\mathcal{P}}_{2}^{1 / 2}\right\|^{2}+\left|A_{1}-A_{2}\right|^{2}\right) .
\end{aligned}
$$

The terms $W_{65}$ and $W_{66}$ :

$$
\begin{aligned}
\left|W_{65}\right|= & \frac{a^{5}}{2 A^{6}} \mid \int_{0}^{1}\left(\tilde{\mathcal{P}}_{1}^{1 / 2}-\tilde{\mathcal{P}}_{2}^{1 / 2}\right)\left(\tilde{\mathcal{P}}_{2}^{1 / 2}\right)_{\eta}(t, \eta) \\
& \times \int_{0}^{\eta}\left(e^{-\frac{1}{a}\left(\tilde{\mathcal{Y}}_{2}(t, \eta)-\tilde{\mathcal{Y}}_{2}(t, \theta)\right)}-e^{-\frac{1}{a}\left(\tilde{\mathcal{Y}}_{1}(t, \eta)-\tilde{\mathcal{Y}}_{1}(t, \theta)\right)}\right) \tilde{\mathcal{U}}_{2} \mathbb{1}_{B(\eta)}(t, \theta) d \theta d \eta \mid \\
\leqslant & \frac{1}{2 A^{2}} \int_{0}^{1}\left|\tilde{\mathcal{P}}_{1}^{1 / 2}-\tilde{\mathcal{P}}_{2}^{1 / 2}\right|\left|\left(\tilde{\mathcal{P}}_{2}^{1 / 2}\right)_{\eta}\right|(t, \eta) \\
& \times \int_{0}^{\eta}\left(\left|\tilde{\mathcal{Y}}_{2}(t, \eta)-\tilde{\mathcal{Y}}_{1}(t, \eta)\right|+\left|\tilde{\mathcal{Y}}_{2}(t, \theta)-\tilde{\mathcal{Y}}_{1}(t, \theta)\right|\right) \\
& \times e^{-\frac{1}{a}\left(\tilde{\mathcal{Y}}_{2}(t, \eta)-\tilde{\mathcal{Y}}_{2}(t, \theta)\right)}\left|\tilde{\mathcal{U}}_{2}\right|(t, \theta) d \theta d \eta
\end{aligned}
$$




$$
\begin{aligned}
& \leqslant \frac{1}{2 A^{2}} \int_{0}^{1}\left|\tilde{\mathcal{P}}_{1}^{1 / 2}-\tilde{\mathcal{P}}_{2}^{1 / 2}\right|\left|\tilde{\mathcal{Y}}_{2}-\tilde{\mathcal{Y}}_{1} \|\left(\tilde{\mathcal{P}}_{2}^{1 / 2}\right)_{\eta}\right|(t, \eta) \\
& \times \int_{0}^{\eta} e^{-\frac{1}{a}\left(\tilde{\mathcal{Y}}_{2}(t, \eta)-\tilde{\mathcal{Y}}_{2}(t, \theta)\right)}\left|\tilde{\mathcal{U}}_{2}\right|(t, \theta) d \theta d \eta \\
& +\frac{1}{2 A^{2}} \int_{0}^{1}\left|\tilde{\mathcal{P}}_{1}^{1 / 2}-\tilde{\mathcal{P}}_{2}^{1 / 2}\right|\left|\left(\tilde{\mathcal{P}}_{2}^{1 / 2}\right)_{\eta}\right|(t, \eta) \\
& \times \int_{0}^{\eta} e^{-\frac{1}{a}\left(\tilde{\mathcal{Y}}_{2}(t, \eta)-\tilde{\mathcal{Y}}_{2}(t, \theta)\right)}\left|\tilde{\mathcal{Y}}_{2}-\tilde{\mathcal{Y}}_{1}\right|\left|\tilde{\mathcal{U}}_{2}\right|(t, \theta) d \theta d \eta \\
& \leqslant \frac{1}{2 A^{2}} \int_{0}^{1}\left|\tilde{\mathcal{P}}_{1}^{1 / 2}-\tilde{\mathcal{P}}_{2}^{1 / 2}\right|\left|\tilde{\mathcal{Y}}_{2}-\tilde{\mathcal{Y}}_{1} \|\left(\tilde{\mathcal{P}}_{2}^{1 / 2}\right)_{\eta}\right|(t, \eta) \\
& \times\left(\int_{0}^{\eta} e^{-\frac{1}{a}\left(\tilde{\mathcal{Y}}_{2}(t, \eta)-\tilde{\mathcal{Y}}_{2}(t, \theta)\right)} \tilde{\mathcal{U}}_{2}^{2}(t, \theta) d \theta\right)^{1 / 2} d \eta \\
& +\frac{1}{2 A^{2}} \int_{0}^{1}\left|\tilde{\mathcal{P}}_{1}^{1 / 2}-\tilde{\mathcal{P}}_{2}^{1 / 2}\right|\left|\left(\tilde{\mathcal{P}}_{2}^{1 / 2}\right)_{\eta}\right|(t, \eta) \\
& \times\left(\int_{0}^{\eta} e^{-\frac{1}{a}\left(\tilde{\mathcal{Y}}_{2}(t, \eta)-\tilde{\mathcal{Y}}_{2}(t, \theta)\right)} \tilde{\mathcal{U}}_{2}^{2}(t, \theta) d \theta\right)^{1 / 2} d \eta\left\|\tilde{\mathcal{Y}}_{1}-\tilde{\mathcal{Y}}_{2}\right\| \\
& \leqslant \frac{\sqrt{3}}{\sqrt{2} A^{2}} \int_{0}^{1}\left|\tilde{\mathcal{P}}_{1}^{1 / 2}-\tilde{\mathcal{P}}_{2}^{1 / 2}\right|\left|\tilde{\mathcal{Y}}_{2}-\tilde{\mathcal{Y}}_{1}\right| \tilde{\mathcal{P}}_{2}^{1 / 2}\left|\left(\tilde{\mathcal{P}}_{2}^{1 / 2}\right)_{\eta}\right|(t, \eta) d \eta \\
& +\frac{\sqrt{3}}{\sqrt{2} A^{2}} \int_{0}^{1}\left|\tilde{\mathcal{P}}_{1}^{1 / 2}-\tilde{\mathcal{P}}_{2}^{1 / 2}\right| \tilde{\mathcal{P}}_{2}^{1 / 2}\left|\left(\tilde{\mathcal{P}}_{2}^{1 / 2}\right)_{\eta}\right|(t, \eta) d \eta\left\|\tilde{\mathcal{Y}}_{1}-\tilde{\mathcal{Y}}_{2}\right\| \\
& \leqslant \mathcal{O}(1)\left(\left\|\tilde{\mathcal{P}}_{1}^{1 / 2}-\tilde{\mathcal{P}}_{2}^{1 / 2}\right\|^{2}+\left\|\tilde{\mathcal{Y}}_{1}-\tilde{\mathcal{Y}}_{2}\right\|^{2}\right)
\end{aligned}
$$

using (4.15e), (4.16a), (4.17a), and (5.13).

The terms $W_{67}^{ \pm}$and $W_{68}^{ \pm}$:

$$
\begin{aligned}
\left|W_{67}^{+}\right|= & \frac{a^{5}}{2 A^{6}} \mid \int_{0}^{1}\left(\tilde{\mathcal{P}}_{1}^{1 / 2}-\tilde{\mathcal{P}}_{2}^{1 / 2}\right)\left(\tilde{\mathcal{P}}_{2}^{1 / 2}\right)_{\eta}(t, \eta) \\
& \times\left(\int_{0}^{\eta} \min _{j}\left(e^{-\frac{1}{a}\left(\tilde{\mathcal{Y}}_{j}(t, \eta)-\tilde{\mathcal{Y}}_{j}(t, \theta)\right)}\right)\left(\tilde{\mathcal{U}}_{2}^{+}-\tilde{\mathcal{U}}_{1}^{+}\right) \mathbb{1}_{\tilde{\mathcal{U}}_{1}^{+} \leqslant \tilde{\mathcal{U}}_{2}^{+}}(t, \theta) d \theta\right) d \eta \mid \\
\leqslant & \left\|\tilde{\mathcal{P}}_{1}^{1 / 2}-\tilde{\mathcal{P}}_{2}^{1 / 2}\right\|^{2}+\frac{1}{4 A^{2}} \int_{0}^{1}\left(\left(\tilde{\mathcal{P}}_{2}^{1 / 2}\right)_{\eta}\right)^{2}(t, \eta) \\
& \times\left(\int_{0}^{\eta} \min _{j}\left(e^{-\frac{1}{a}\left(\tilde{\mathcal{Y}}_{j}(t, \eta)-\tilde{\mathcal{Y}}_{j}(t, \theta)\right)}\right)\left(\tilde{\mathcal{U}}_{2}^{+}-\tilde{\mathcal{U}}_{1}^{+}\right) \mathbb{1}_{\tilde{\mathcal{U}}_{1}^{+} \leqslant \tilde{\mathcal{U}}_{2}^{+}}(t, \theta) d \theta\right)^{2} d \eta \\
\leqslant & \left\|\tilde{\mathcal{P}}_{1}^{1 / 2}-\tilde{\mathcal{P}}_{2}^{1 / 2}\right\|^{2}
\end{aligned}
$$




$$
\begin{aligned}
& +\frac{1}{16 A^{4}} \int_{0}^{1} \tilde{\mathcal{P}}_{2} \tilde{\mathcal{Y}}_{2}^{2}(t, \eta)\left(\int_{0}^{\eta} e^{-\frac{1}{A_{2}}\left(\tilde{\mathcal{Y}}_{2}(t, \eta)-\tilde{\mathcal{Y}}_{2}(t, \theta)\right)}\left(\tilde{\mathcal{U}}_{2}^{+}-\tilde{\mathcal{U}}_{1}\right)^{2}(t, \theta) d \theta\right) d \eta \\
\leqslant & \left\|\tilde{\mathcal{P}}_{1}^{1 / 2}-\tilde{\mathcal{P}}_{2}^{1 / 2}\right\|^{2} \\
& +\frac{A}{32} \int_{0}^{1} \tilde{\mathcal{Y}}_{2, \eta}(t, \eta)\left(\int_{0}^{\eta} e^{-\frac{1}{A_{2}}\left(\tilde{\mathcal{Y}}_{2}(t, \eta)-\tilde{\mathcal{Y}}_{2}(t, \theta)\right)}\left(\tilde{\mathcal{U}}_{2}-\tilde{\mathcal{U}}_{1}\right)^{2}(t, \theta) d \theta\right) d \eta \\
\leqslant & \mathcal{O}(1)\left(\left\|\tilde{\mathcal{P}}_{1}^{1 / 2}-\tilde{\mathcal{P}}_{2}^{1 / 2}\right\|^{2}+\left\|\tilde{\mathcal{U}}_{1}-\tilde{\mathcal{U}}_{2}\right\|^{2}\right)
\end{aligned}
$$

following the estimates used for $\bar{B}_{65}^{+}$; see (5.24). The term $W_{69}^{ \pm}$:

$$
\begin{aligned}
& \left|W_{69}^{+}\right|=\frac{a^{5}}{2 A^{6}} \mid \int_{0}^{1}\left(\tilde{\mathcal{P}}_{1}^{1 / 2}-\tilde{\mathcal{P}}_{2}^{1 / 2}\right)\left(\left(\tilde{\mathcal{P}}_{2}^{1 / 2}\right)_{\eta}-\left(\tilde{\mathcal{P}}_{1}^{1 / 2}\right)_{\eta}\right)(t, \eta) \\
& \times\left(\int_{0}^{\eta} \min _{j}\left(e^{-\frac{1}{a}\left(\tilde{\mathcal{Y}}_{j}(t, \eta)-\tilde{\mathcal{Y}}_{j}(t, \theta)\right)}\right) \min _{j}\left(\tilde{\mathcal{U}}_{j}^{+}\right)(t, \theta) d \theta\right) d \eta \mid \\
& =\frac{a^{5}}{4 A^{6}}\left|\left(\left(\tilde{\mathcal{P}}_{1}^{1 / 2}-\tilde{\mathcal{P}}_{2}^{1 / 2}\right)^{2} \int_{0}^{\eta} \min _{j}\left(e^{-\frac{1}{a}\left(\tilde{\mathcal{Y}}_{j}(t, \eta)-\tilde{\mathcal{Y}}_{j}(t, \theta)\right)}\right) \min _{j}\left(\tilde{\mathcal{U}}_{j}^{+}\right)(t, \theta) d \theta\right)\right|_{\eta=0}^{1} \\
& -\int_{0}^{1}\left(\tilde{\mathcal{P}}_{1}^{1 / 2}-\tilde{\mathcal{P}}_{2}^{1 / 2}\right)^{2} \frac{d}{d \eta}\left(\int_{0}^{\eta} \min _{j}\left(e^{-\frac{1}{a}\left(\tilde{\mathcal{Y}}_{j}(t, \eta)-\tilde{\mathcal{Y}}_{j}(t, \theta)\right)}\right) \min _{j}\left(\tilde{\mathcal{U}}_{j}^{+}\right)(t, \theta) d \theta\right) d \eta \mid \\
& \leqslant \frac{a^{5}}{4 A^{6}} \int_{0}^{1}\left(\tilde{\mathcal{P}}_{1}^{1 / 2}-\tilde{\mathcal{P}}_{2}^{1 / 2}\right)^{2} \\
& \times\left|\frac{d}{d \eta}\left(\int_{0}^{\eta} \min _{j}\left(e^{-\frac{1}{a}\left(\tilde{\mathcal{Y}}_{j}(t, \eta)-\tilde{\mathcal{Y}}_{j}(t, \theta)\right)}\right) \min _{j}\left(\tilde{\mathcal{U}}_{j}^{+}\right)(t, \theta) d \theta\right)\right| d \eta \\
& \leqslant \mathcal{O}(1)\left\|\tilde{\mathcal{P}}_{1}^{1 / 2}-\tilde{\mathcal{P}}_{2}^{1 / 2}\right\|^{2}
\end{aligned}
$$

see estimates employed for $\bar{B}_{67}$ (cf. (5.25)) and Lemma A.5.

The terms $M_{2}$ and $M_{3}$ can be treated similarly. More precisely,

$$
\begin{aligned}
\left|M_{2}\right| \leqslant & \mathbb{1}_{A_{2} \leqslant A_{1}}\left(\frac{1}{A_{2}^{6}}-\frac{1}{A_{1}^{6}}\right) \mid \int_{0}^{1}\left(\tilde{\mathcal{P}}_{1}^{1 / 2}-\tilde{\mathcal{P}}_{2}^{1 / 2}\right)\left(\tilde{\mathcal{P}}_{2}^{1 / 2}\right)_{\eta}(t, \eta) \\
& \times \int_{0}^{\eta} e^{-\frac{1}{A_{2}}\left(\tilde{\mathcal{Y}}_{2}(t, \eta)-\tilde{\mathcal{Y}}_{2}(t, \theta)\right)} \tilde{\mathcal{Q}}_{2} \tilde{\mathcal{U}}_{2, \eta}(t, \theta) d \theta d \eta \mid \\
\leqslant & \mathbb{1}_{A_{2} \leqslant A_{1}} \frac{\left|A_{1}^{6}-A_{2}^{6}\right|}{A^{6} A_{2}^{5}} \int_{0}^{1}\left|\tilde{\mathcal{P}}_{1}^{1 / 2}-\tilde{\mathcal{P}}_{2}^{1 / 2}\right|\left|\left(\tilde{\mathcal{P}}_{2}^{1 / 2}\right)_{\eta}\right|(t, \eta) \\
& \times \int_{0}^{\eta} e^{-\frac{1}{A_{2}}\left(\tilde{\mathcal{Y}}_{2}(t, \eta)-\tilde{\mathcal{Y}}_{2}(t, \theta)\right)} \tilde{\mathcal{P}}_{2}\left|\tilde{\mathcal{U}}_{2, \eta}\right|(t, \theta) d \theta d \eta \\
\leqslant & \mathbb{1}_{A_{2} \leqslant A_{1}} 6 \frac{\left|A_{1}-A_{2}\right|}{A A_{2}^{5}}\left\|\tilde{\mathcal{P}}_{1}^{1 / 2}-\tilde{\mathcal{P}}_{2}^{1 / 2}\right\| \\
& \times\left(\int_{0}^{1}\left(\left(\tilde{\mathcal{P}}_{2}^{1 / 2}\right)_{\eta}\right)^{2}(t, \eta)\left(\int_{0}^{\eta} e^{-\frac{1}{A_{2}}\left(\tilde{\mathcal{Y}}_{2}(t, \eta)-\tilde{\mathcal{Y}}_{2}(t, \theta)\right)} \tilde{\mathcal{P}}_{2}\left|\tilde{\mathcal{U}}_{2, \eta}\right|(t, \theta) d \theta\right)^{2} d \eta\right)^{1 / 2}
\end{aligned}
$$




$$
\begin{aligned}
\leqslant & \mathbb{1}_{A_{2} \leqslant A_{1}} 6 \frac{\left|A_{1}-A_{2}\right|}{A A_{2}^{6}}\left\|\tilde{\mathcal{P}}_{1}^{1 / 2}-\tilde{\mathcal{P}}_{2}^{1 / 2}\right\| \\
& \times\left(\int_{0}^{1}\left(\left(\tilde{\mathcal{P}}_{2}^{1 / 2}\right)_{\eta}\right)^{2}(t, \eta)\left(\int_{0}^{\eta} e^{-\frac{1}{A_{2}}\left(\tilde{\mathcal{Y}}_{2}(t, \eta)-\tilde{\mathcal{Y}}_{2}(t, \theta)\right)} \tilde{\mathcal{P}}_{2}^{2} \tilde{\mathcal{Y}}_{2, \eta}(t, \theta) d \theta\right)\right. \\
& \left.\times\left(\int_{0}^{\eta} e^{-\frac{1}{A_{2}}\left(\tilde{\mathcal{Y}}_{2}(t, \eta)-\tilde{\mathcal{Y}}_{2}(t, \theta)\right)} \tilde{\mathcal{H}}_{2, \eta}(t, \theta) d \theta\right) d \eta\right)^{1 / 2} \\
\leqslant & \mathbb{1}_{A_{2} \leqslant A_{1}} 6 \sqrt{6} \frac{\left|A_{1}-A_{2}\right|}{A A_{2}^{3}}\left\|\tilde{\mathcal{P}}_{1}^{1 / 2}-\tilde{\mathcal{P}}_{2}^{1 / 2}\right\|\left(\int_{0}^{1}\left(\left(\tilde{\mathcal{P}}_{2}^{1 / 2}\right)_{\eta}\right)^{2} \tilde{\mathcal{P}}_{2}^{2}(t, \eta) d \eta\right)^{1 / 2} \\
\leqslant & \mathcal{O}(1)\left(\left\|\tilde{\mathcal{P}}_{1}^{1 / 2}-\tilde{\mathcal{P}}_{2}^{1 / 2}\right\|^{2}+\left|A_{1}-A_{2}\right|^{2}\right) .
\end{aligned}
$$

Here we used

$$
\tilde{\mathcal{P}}_{2}\left|\tilde{\mathcal{U}}_{2, \eta}\right| \leqslant \frac{1}{A_{2}} \tilde{\mathcal{P}}_{2} \sqrt{\tilde{\mathcal{Y}}_{2, \eta} \tilde{\mathcal{H}}_{2, \eta}},
$$

cf. (4.15m), as well as (4.15c), (4.15e), and (4.17d). In addition, we applied (4.16b) and (4.16d).

The term $I_{3}$ : We have the following estimates:

$$
\begin{aligned}
\left|I_{3}\right| & \leqslant \int_{0}^{1}\left|\tilde{\mathcal{P}}_{1}^{1 / 2}-\tilde{\mathcal{P}}_{2}^{1 / 2}\right|\left|\frac{1}{A_{1}^{2}} \frac{\tilde{\mathcal{Q}}_{1} \tilde{\mathcal{U}}_{1}}{\tilde{\mathcal{P}}_{1}^{1 / 2}}-\frac{1}{A_{2}^{2}} \frac{\tilde{\mathcal{Q}}_{2} \tilde{\mathcal{U}}_{2}}{\tilde{\mathcal{P}}_{2}^{1 / 2}}\right|(t, \eta) d \eta \\
& \leqslant\left\|\tilde{\mathcal{P}}_{1}^{1 / 2}-\tilde{\mathcal{P}}_{2}^{1 / 2}\right\|^{2}+\left\|\frac{1}{A_{1}^{2}} \frac{\tilde{\mathcal{Q}}_{1}}{\tilde{\mathcal{P}}_{1}^{1 / 2}}-\frac{1}{A_{2}^{2}} \frac{\tilde{\mathcal{Q}}_{2}}{\tilde{\mathcal{P}}_{2}^{1 / 2}}\right\|^{2} .
\end{aligned}
$$

We consider the latter term and find

$$
\begin{aligned}
& \left\|\frac{1}{A_{1}^{2}} \frac{\tilde{\mathcal{Q}}_{1} \tilde{\mathcal{U}}_{1}}{\tilde{\mathcal{P}}_{1}^{1 / 2}}-\frac{1}{A_{2}^{2}} \frac{\tilde{\mathcal{Q}}_{2} \tilde{\mathcal{U}}_{2}}{\tilde{\mathcal{P}}_{2}^{1 / 2}}\right\|^{2} \\
& \quad \leqslant 3 \mathbb{1}_{A_{1} \leqslant A_{2}}\left|\frac{1}{A_{1}^{2}}-\frac{1}{A_{2}^{2}}\right|^{2}\left\|\frac{\tilde{\mathcal{Q}}_{1} \tilde{\mathcal{U}}_{1}}{\tilde{\mathcal{P}}_{1}^{1 / 2}}\right\|^{2}+3 \mathbb{1}_{A_{2}<A_{1}}\left|\frac{1}{A_{1}^{2}}-\frac{1}{A_{2}}\right|^{2}\left\|\frac{\tilde{\mathcal{Q}}_{2} \tilde{\mathcal{U}}_{2}}{\tilde{\mathcal{P}}_{2}^{1 / 2}}\right\|^{2} \\
& \quad+3 \frac{1}{A^{4}}\left\|\frac{\tilde{\mathcal{Q}}_{1} \tilde{\mathcal{U}}_{1}}{\tilde{\mathcal{P}}_{1}^{1 / 2}}-\frac{\tilde{\mathcal{Q}}_{2} 2 \tilde{\mathcal{U}}_{2}}{\tilde{\mathcal{P}}_{2}^{1 / 2}}\right\|^{2} \\
& \quad \leqslant \mathcal{O}(1)\left|A_{1}-A_{2}\right|^{2}+3 \frac{1}{A^{4}}\left\|\frac{\tilde{\mathcal{Q}}_{1} \tilde{\mathcal{U}}_{1}}{\tilde{\mathcal{P}}_{1}^{1 / 2}}-\frac{\tilde{\mathcal{Q}}_{2} \tilde{\mathcal{U}}_{2}}{\tilde{\mathcal{P}}_{2}^{1 / 2}}\right\|^{2},
\end{aligned}
$$

where we have used that

$$
\left|\frac{\tilde{\mathcal{Q}}_{i} \tilde{\mathcal{U}}_{i}}{\tilde{\mathcal{P}}_{i}^{1 / 2}}\right| \leqslant \frac{A_{i} \tilde{\mathcal{P}}_{i} \tilde{\mathcal{U}}_{i}}{\tilde{\mathcal{P}}_{i}^{1 / 2}}\left|=A_{i} \tilde{\mathcal{P}}_{i}^{1 / 2}\right| \tilde{\mathcal{U}}_{i} \mid \leqslant \mathcal{O}(1) A_{i}^{5} .
$$


We consider the latter term and find

$$
\begin{aligned}
\frac{1}{A^{4}} \| & \frac{\tilde{\mathcal{Q}}_{1} \tilde{\mathcal{U}}_{1}}{\tilde{\mathcal{P}}_{1}^{1 / 2}}-\left.\frac{\tilde{\mathcal{Q}}_{2} \tilde{\mathcal{U}}_{2}}{\tilde{\mathcal{P}}_{2}^{1 / 2}}\right|^{2} \\
= & \frac{1}{A^{4}} \int_{0}^{1}\left|\frac{\tilde{\mathcal{Q}}_{1} \tilde{\mathcal{U}}_{1}}{\tilde{\mathcal{P}}_{1}^{1 / 2}}-\frac{\tilde{\mathcal{Q}}_{2} \tilde{\mathcal{U}}_{2}}{\tilde{\mathcal{P}}_{2}^{1 / 2}}\right|^{2}\left(\mathbb{1}_{\tilde{\mathcal{P}}_{1} \leqslant \tilde{\mathcal{P}}_{2}}+\mathbb{1}_{\tilde{\mathcal{P}}_{2}<\tilde{\mathcal{P}}_{1}}\right)(t, \eta) d \eta \\
\leqslant & \frac{2}{A^{4}} \int_{0}^{1}\left|\frac{1}{\tilde{\mathcal{P}}_{2}^{1 / 2}}\left(\tilde{\mathcal{Q}}_{1} \tilde{\mathcal{U}}_{1}-\tilde{\mathcal{Q}}_{2} \tilde{\mathcal{U}}_{2}\right)+\left(\frac{1}{\tilde{\mathcal{P}}_{1}^{1 / 2}}-\frac{1}{\tilde{\mathcal{P}}_{2}^{1 / 2}}\right) \tilde{\mathcal{Q}}_{1} \tilde{\mathcal{U}}_{1}\right|^{2} \mathbb{1}_{\tilde{\mathcal{P}}_{1} \leqslant \tilde{\mathcal{P}}_{2}}(t, \eta) d \eta \\
& +\frac{2}{A^{4}} \int_{0}^{1}\left|\frac{1}{\tilde{\mathcal{P}}_{1}^{1 / 2}}\left(\tilde{\mathcal{Q}}_{1} \tilde{\mathcal{U}}_{1}-\tilde{\mathcal{Q}}_{2} \tilde{\mathcal{U}}_{2}\right)+\left(\frac{1}{\tilde{\mathcal{P}}_{1}^{1 / 2}}-\frac{1}{\tilde{\mathcal{P}}_{2}^{1 / 2}}\right) \tilde{\mathcal{Q}}_{2} \tilde{\mathcal{U}}_{2}\right|^{2} \mathbb{1}_{\tilde{\mathcal{P}}_{2}<\tilde{\mathcal{P}}_{1}}(t, \eta) d \eta \\
\leqslant & \frac{4}{A^{4}} \int_{0}^{1}\left|\frac{1}{\max _{i}\left(\tilde{\mathcal{P}}_{i}^{1 / 2}\right)}\left(\tilde{\mathcal{Q}}_{1}\left(\tilde{\mathcal{U}}_{1}-\tilde{\mathcal{U}}_{2}\right)+\tilde{\mathcal{U}}_{2}\left(\tilde{\mathcal{Q}}_{1}-\tilde{\mathcal{Q}}_{2}\right)\right)\right|^{2} \mathbb{1}_{\tilde{\mathcal{P}}_{1} \leqslant \tilde{\mathcal{P}}_{2}}(t, \eta) d \eta \\
& +\frac{4}{A^{4}} \int_{0}^{1}\left|\frac{\tilde{\mathcal{Q}}_{1} \tilde{\mathcal{U}}_{1}}{\tilde{\mathcal{P}}_{1}^{1 / 2} \tilde{\mathcal{P}}_{2}^{1 / 2}}\left(\tilde{\mathcal{P}}_{2}^{1 / 2}-\tilde{\mathcal{P}}_{1}^{1 / 2}\right)\right|^{2} \mathbb{1}_{\tilde{\mathcal{P}}_{1} \leqslant \tilde{\mathcal{P}}_{2}}(t, \eta) d \eta \\
& +\frac{4}{A^{4}} \int_{0}^{1}\left|\frac{1}{\max _{i}\left(\tilde{\mathcal{P}}_{i}^{1 / 2}\right)}\left(\tilde{\mathcal{Q}}_{1}\left(\tilde{\mathcal{U}}_{1}-\tilde{\mathcal{U}}_{2}\right)+\tilde{\mathcal{U}}_{2}\left(\tilde{\mathcal{Q}}_{1}-\tilde{\mathcal{Q}}_{2}\right)\right)\right|^{2} \mathbb{1}_{\tilde{\mathcal{P}}_{2}<\tilde{\mathcal{P}}_{1}}(t, \eta) d \eta \\
& +\frac{4}{A^{4}} \int_{0}^{1}\left|\frac{\tilde{\mathcal{Q}}_{2} \tilde{\mathcal{U}}_{2}}{\tilde{\mathcal{P}}_{1}^{1 / 2} \tilde{\mathcal{P}}_{2}^{1 / 2}}\left(\tilde{\mathcal{P}}_{2}^{1 / 2}-\tilde{\mathcal{P}}_{1}^{1 / 2}\right)\right|^{2} \mathbb{1}_{\tilde{\mathcal{P}}_{2}<\tilde{\mathcal{P}}_{1}}(t, \eta) d \eta \\
= & 4\left(I_{31}+I_{32}+I_{33}+I_{34}\right) .
\end{aligned}
$$

We treat terms $I_{31}$ and $I_{32}$; the others are similar.

$$
\begin{aligned}
I_{31} \leqslant & \frac{2}{A^{4}} \int_{0}^{1}\left|\left(\frac{\tilde{\mathcal{Q}}_{1}}{\max _{i}\left(\tilde{\mathcal{P}}_{i}^{1 / 2}\right)}\right)^{2}\right| \tilde{\mathcal{U}}_{1}-\left.\tilde{\mathcal{U}}_{2}\right|^{2} \\
& +\left(\frac{\tilde{\mathcal{U}}_{2}}{\max _{i}\left(\tilde{\mathcal{P}}_{i}^{1 / 2}\right)}\right)^{2}\left|\tilde{\mathcal{Q}}_{1}-\tilde{\mathcal{Q}}_{2}\right|^{2} \mid \mathbb{1}_{\tilde{\mathcal{P}}_{1} \leqslant \tilde{\mathcal{P}}_{2}}(t, \eta) d \eta \\
\leqslant & \mathcal{O}(1)\left(\left\|\tilde{\mathcal{Y}}_{1}-\tilde{\mathcal{Y}}_{2}\right\|^{2}+\left\|\tilde{\mathcal{U}}_{1}-\tilde{\mathcal{U}}_{2}\right\|^{2}+\left\|\tilde{\mathcal{P}}_{1}^{1 / 2}-\tilde{\mathcal{P}}_{2}^{1 / 2}\right\|^{2}+\left|A_{1}-A_{2}\right|^{2}\right),
\end{aligned}
$$

where we have used (5.29), that

$$
\begin{aligned}
\left|\frac{\tilde{\mathcal{Q}}_{1}}{\max _{i}\left(\tilde{\mathcal{P}}_{i}^{1 / 2}\right)}\right| \mathbb{1}_{\tilde{\mathcal{P}}_{1} \leqslant \tilde{\mathcal{P}}_{2}} & \leqslant \frac{A_{1} \tilde{\mathcal{P}}_{1}}{\max _{i}\left(\tilde{\mathcal{P}}_{i}^{1 / 2}\right)} \mathbb{1}_{\tilde{\mathcal{P}}_{1} \leqslant \tilde{\mathcal{P}}_{2}} \\
& \leqslant A \frac{\max _{i}\left(\tilde{\mathcal{P}}_{i}\right)}{\max _{i}\left(\tilde{\mathcal{P}}_{i}^{1 / 2}\right)} \mathbb{1}_{\tilde{\mathcal{P}}_{1} \leqslant \tilde{\mathcal{P}}_{2}} \leqslant A \max _{i}\left(\tilde{\mathcal{P}}_{i}^{1 / 2}\right) \leqslant \frac{A^{3}}{2},
\end{aligned}
$$


and

$$
\left|\frac{\tilde{\mathcal{U}}_{2}}{\max _{i}\left(\tilde{\mathcal{P}}_{i}^{1 / 2}\right)}\right| \mathbb{1}_{\tilde{\mathcal{P}}_{1} \leqslant \tilde{\mathcal{P}}_{2}} \leqslant \frac{\left|\tilde{\mathcal{U}}_{2}\right|}{\tilde{\mathcal{P}}_{2}^{1 / 2}} \mathbb{1}_{\tilde{\mathcal{P}}_{1} \leqslant \tilde{\mathcal{P}}_{2}} \leqslant \sqrt{2}
$$

Next follows

$$
\begin{aligned}
I_{32} & \leqslant \frac{1}{A^{4}} \int_{0}^{1}\left|\frac{\tilde{\mathcal{Q}}_{1} \tilde{\mathcal{U}}_{1}}{\tilde{\mathcal{P}}_{1}^{1 / 2} \tilde{\mathcal{P}}_{2}^{1 / 2}}\left(\tilde{\mathcal{P}}_{2}^{1 / 2}-\tilde{\mathcal{P}}_{1}^{1 / 2}\right)\right|^{2} \mathbb{1}_{\tilde{\mathcal{P}}_{1} \leqslant \tilde{\mathcal{P}}_{2}}(t, \eta) d \eta \\
& \leqslant \mathcal{O}(1)\left\|\tilde{\mathcal{P}}_{2}^{1 / 2}-\tilde{\mathcal{P}}_{1}^{1 / 2}\right\|^{2}
\end{aligned}
$$

using that

$$
\left|\frac{\tilde{\mathcal{Q}}_{1} \tilde{\mathcal{U}}_{1}}{\tilde{\mathcal{P}}_{1}^{1 / 2} \tilde{\mathcal{P}}_{2}^{1 / 2}}\right| \mathbb{1}_{\tilde{\mathcal{P}}_{1} \leqslant \tilde{\mathcal{P}}_{2}} \leqslant\left|\frac{\tilde{\mathcal{Q}}_{1} \tilde{\mathcal{U}}_{1}}{\tilde{\mathcal{P}}_{1}}\right| \leqslant\left|A_{1} \tilde{\mathcal{U}}_{1}\right| \leqslant \frac{A^{3}}{\sqrt{2}} .
$$

This proves that

$$
\begin{aligned}
& \frac{1}{A^{4}}\left\|\frac{\tilde{\mathcal{Q}}_{1} \tilde{\mathcal{U}}_{1}}{\tilde{\mathcal{P}}_{1}^{1 / 2}}-\frac{\tilde{\mathcal{Q}}_{2} \tilde{\mathcal{U}}_{2}}{\tilde{\mathcal{P}}_{2}^{1 / 2}}\right\|^{2} \\
& \quad \leqslant \mathcal{O}(1)\left(\left\|\tilde{\mathcal{Y}}_{1}-\tilde{\mathcal{Y}}_{2}\right\|^{2}+\left\|\tilde{\mathcal{U}}_{1}-\tilde{\mathcal{U}}_{2}\right\|^{2}+\left\|\tilde{\mathcal{P}}_{1}^{1 / 2}-\tilde{\mathcal{P}}_{2}^{1 / 2}\right\|^{2}+\left|A_{1}-A_{2}\right|^{2}\right)
\end{aligned}
$$

and thus $I_{3}$ has the right form.

The term $\mathrm{I}_{4}$ :

$$
\begin{aligned}
& I_{4}=\int_{0}^{1}\left(\tilde{\mathcal{P}}_{1}^{1 / 2}-\tilde{\mathcal{P}}_{2}^{1 / 2}\right)\left(\frac{1}{A_{1}^{3}} \frac{\tilde{\mathcal{R}}_{1}}{\tilde{\mathcal{P}}_{1}^{1 / 2}}-\frac{1}{A_{2}^{3}} \frac{\tilde{\mathcal{R}}_{2}}{\tilde{\mathcal{P}}_{2}^{1 / 2}}\right)(t, \eta) d \eta \\
& =\mathbb{1}_{A_{1} \leqslant A_{2}}\left(\frac{1}{A_{1}^{3}}-\frac{1}{A_{2}^{3}}\right) \int_{0}^{1}\left(\tilde{\mathcal{P}}_{1}^{1 / 2}-\tilde{\mathcal{P}}_{2}^{1 / 2}\right) \frac{\tilde{\mathcal{R}}_{1}}{\tilde{\mathcal{P}}_{1}^{1 / 2}}(t, \eta) d \eta \\
& +\mathbb{1}_{A_{2}<A_{1}}\left(\frac{1}{A_{1}^{3}}-\frac{1}{A_{2}^{3}}\right) \int_{0}^{1}\left(\tilde{\mathcal{P}}_{1}^{1 / 2}-\tilde{\mathcal{P}}_{2}^{1 / 2}\right) \frac{\tilde{\mathcal{R}}_{2}}{\tilde{\mathcal{P}}_{2}^{1 / 2}}(t, \eta) d \eta \\
& +\frac{1}{A^{3}} \int_{0}^{1}\left(\tilde{\mathcal{P}}_{1}^{1 / 2}-\tilde{\mathcal{P}}_{2}^{1 / 2}\right)\left(\frac{1}{\max _{j}\left(\tilde{\mathcal{P}}_{j}^{1 / 2}\right)}\left(\tilde{\mathcal{R}}_{1}-\tilde{\mathcal{R}}_{2}\right)\right. \\
& \left.-\left(\frac{1}{\tilde{\mathcal{P}}_{2}^{1 / 2}}-\frac{1}{\tilde{\mathcal{P}}_{1}^{1 / 2}}\right)\left(\tilde{\mathcal{R}}_{2} \mathbb{1}_{\tilde{\mathcal{P}}_{2}<\tilde{\mathcal{P}}_{1}}+\tilde{\mathcal{R}}_{1} \mathbb{1}_{\tilde{\mathcal{P}}_{1} \leqslant \tilde{\mathcal{P}}_{2}}\right)\right)(t, \eta) d \eta \\
& =\mathbb{1}_{A_{1} \leqslant A_{2}}\left(\frac{1}{A_{1}^{3}}-\frac{1}{A_{2}^{3}}\right) \int_{0}^{1}\left(\tilde{\mathcal{P}}_{1}^{1 / 2}-\tilde{\mathcal{P}}_{2}^{1 / 2}\right) \frac{\tilde{\mathcal{R}}_{1}}{\tilde{\mathcal{P}}_{1}^{1 / 2}}(t, \eta) d \eta
\end{aligned}
$$




$$
\begin{aligned}
& +\mathbb{1}_{A_{2}<A_{1}}\left(\frac{1}{A_{1}^{3}}-\frac{1}{A_{2}^{3}}\right) \int_{0}^{1}\left(\tilde{\mathcal{P}}_{1}^{1 / 2}-\tilde{\mathcal{P}}_{2}^{1 / 2}\right) \frac{\tilde{\mathcal{R}}_{2}}{\tilde{\mathcal{P}}_{2}^{1 / 2}}(t, \eta) d \eta \\
& +\frac{1}{A^{3}} \int_{0}^{1}\left[\frac{1}{\max _{j}\left(\tilde{\mathcal{P}}_{j}^{1 / 2}\right)}\left(\tilde{\mathcal{P}}_{1}^{1 / 2}-\tilde{\mathcal{P}}_{2}^{1 / 2}\right)\left(\tilde{\mathcal{R}}_{1}-\tilde{\mathcal{R}}_{2}\right)-\left(\tilde{\mathcal{P}}_{1}^{1 / 2}\right.\right. \\
& \left.\left.-\tilde{\mathcal{P}}_{2}^{1 / 2}\right)^{2} \frac{1}{\tilde{\mathcal{P}}_{1}^{1 / 2} \tilde{\mathcal{P}}_{2}^{1 / 2}}\left(\tilde{\mathcal{R}}_{2} \mathbb{1}_{\tilde{\mathcal{P}}_{2}<\tilde{\mathcal{P}}_{1}}+\tilde{\mathcal{R}}_{1} \mathbb{1}_{\tilde{\mathcal{P}}_{1} \leqslant \tilde{\mathcal{P}}_{2}}\right)\right](t, \eta) d \eta \\
& =J_{1}+J_{2}+J_{3}-J_{4}-J_{5},
\end{aligned}
$$

where

$$
\begin{aligned}
& J_{1}=\mathbb{1}_{A_{1} \leqslant A_{2}}\left(\frac{1}{A_{1}^{3}}-\frac{1}{A_{2}^{3}}\right) \int_{0}^{1}\left(\tilde{\mathcal{P}}_{1}^{1 / 2}-\tilde{\mathcal{P}}_{2}^{1 / 2}\right) \frac{\tilde{\mathcal{P}}_{1}}{\tilde{\mathcal{P}}_{1}^{1 / 2}}(t, \eta) d \eta, \\
& J_{2}=\mathbb{1}_{A_{2}<A_{1}}\left(\frac{1}{A_{1}^{3}}-\frac{1}{A_{2}^{3}}\right) \int_{0}^{1}\left(\tilde{\mathcal{P}}_{1}^{1 / 2}-\tilde{\mathcal{P}}_{2}^{1 / 2}\right) \frac{\tilde{\mathcal{P}}_{2}}{\tilde{\mathcal{P}}_{2}^{1 / 2}}(t, \eta) d \eta, \\
& J_{3}=\frac{1}{A^{3}} \int_{0}^{1} \frac{1}{\max _{j}\left(\tilde{\mathcal{P}}_{j}^{1 / 2}\right)}\left(\tilde{\mathcal{P}}_{1}^{1 / 2}-\tilde{\mathcal{P}}_{2}^{1 / 2}\right)\left(\tilde{\mathcal{R}}_{1}-\tilde{\mathcal{R}}_{2}\right)(t, \eta) d \eta, \\
& J_{4}=\frac{1}{A^{3}} \int_{0}^{1}\left(\tilde{\mathcal{P}}_{1}^{1 / 2}-\tilde{\mathcal{P}}_{2}^{1 / 2}\right)^{2} \frac{1}{\tilde{\mathcal{P}}_{1}^{1 / 2} \tilde{\mathcal{P}}_{2}^{1 / 2}} \tilde{\mathcal{R}}_{2} \mathbb{1}_{\tilde{\mathcal{P}}_{2}<\tilde{\mathcal{P}}_{1}}(t, \eta) d \eta, \\
& J_{5}=\frac{1}{A^{3}} \int_{0}^{1}\left(\tilde{\mathcal{P}}_{1}^{1 / 2}-\tilde{\mathcal{P}}_{2}^{1 / 2}\right)^{2} \frac{1}{\tilde{\mathcal{P}}_{1}^{1 / 2} \tilde{\mathcal{P}}_{2}^{1 / 2}} \tilde{\mathcal{R}}_{1} \mathbb{1}_{\tilde{\mathcal{P}}_{1} \leqslant \tilde{\mathcal{P}}_{2}}(t, \eta) d \eta .
\end{aligned}
$$

For $J_{1}$ (and similarly for $J_{2}$ ), we find

$$
\begin{aligned}
\left|J_{1}\right| & \leqslant \frac{A_{2}^{3}-A_{1}^{3}}{a^{3} A^{3}} \int_{0}^{1}\left|\tilde{\mathcal{P}}_{1}^{1 / 2}-\tilde{\mathcal{P}}_{2}^{1 / 2}\right|(t, \eta) \mathcal{O}(1) a^{5} d \eta \\
& \leqslant \mathcal{O}(1)\left(\left\|\tilde{\mathcal{P}}_{1}^{1 / 2}-\tilde{\mathcal{P}}_{2}^{1 / 2}\right\|^{2}+\left|A_{1}-A_{2}\right|^{2}\right),
\end{aligned}
$$

where we used that

$$
\left|\frac{\tilde{\mathcal{R}}_{i}}{\tilde{\mathcal{P}}_{i}^{1 / 2}}\right| \leqslant \mathcal{O}(1) A_{i}^{3} \tilde{\mathcal{P}}_{i}^{1 / 2} \leqslant \mathcal{O}(1) A_{i}^{5} .
$$

For $J_{3}$, we find

$$
\begin{aligned}
J_{3} & =\frac{1}{A^{3}} \int_{0}^{1} \frac{1}{\max _{j}\left(\tilde{\mathcal{P}}_{j}^{1 / 2}\right)}\left(\tilde{\mathcal{P}}_{1}^{1 / 2}-\tilde{\mathcal{P}}_{2}^{1 / 2}\right)\left(\tilde{\mathcal{R}}_{1}-\tilde{\mathcal{R}}_{2}\right)(t, \eta) d \eta \\
& =\frac{1}{6 A^{3}} \int_{0}^{1} \frac{1}{\max _{j}\left(\tilde{\mathcal{P}}_{j}^{1 / 2}\right)}\left(\tilde{\mathcal{P}}_{1}^{1 / 2}-\tilde{\mathcal{P}}_{2}^{1 / 2}\right)(t, \eta)\left(\int_{0}^{1} \operatorname{sign}(\eta-\theta)\right.
\end{aligned}
$$




$$
\begin{aligned}
& \left.\times\left(e^{-\frac{1}{A_{1}}\left|\tilde{\mathcal{Y}}_{1}(t, \eta)-\tilde{\mathcal{Y}}_{1}(t, \theta)\right|} A_{1} \tilde{\mathcal{U}}_{1}^{3} \tilde{\mathcal{Y}}_{1, \eta}-e^{-\frac{1}{A_{2}}\left|\tilde{\mathcal{Y}}_{2}(t, \eta)-\tilde{\mathcal{Y}}_{2}(t, \theta)\right|} A_{2} \tilde{\mathcal{U}}_{2}^{3} \tilde{\mathcal{Y}}_{2, \eta}\right)(t, \theta) d \theta\right) d \eta \\
& +\frac{1}{4 A^{3}} \int_{0}^{1} \frac{1}{\max _{j}\left(\tilde{\mathcal{P}}_{j}^{1 / 2}\right)}\left(\tilde{\mathcal{P}}_{1}^{1 / 2}-\tilde{\mathcal{P}}_{2}^{1 / 2}\right)(t, \eta) \\
& \times\left(\int _ { 0 } ^ { 1 } \operatorname { s i g n } ( \eta - \theta ) \left(e^{-\frac{1}{A_{1}}\left|\tilde{\mathcal{Y}}_{1}(t, \eta)-\tilde{\mathcal{Y}}_{1}(t, \theta)\right|} A_{1}^{6} \tilde{\mathcal{U}}_{1}\right.\right. \\
& \left.\left.-e^{-\frac{1}{A_{2}}\left|\tilde{\mathcal{Y}}_{2}(t, \eta)-\tilde{\mathcal{Y}}_{2}(t, \theta)\right|} A_{2}^{6} \tilde{\mathcal{U}}_{2}\right)(t, \theta) d \theta\right) d \eta \\
& +\frac{1}{2 A^{3}} \int_{0}^{1} \frac{1}{\max _{j}\left(\tilde{\mathcal{P}}_{j}^{1 / 2}\right)}\left(\tilde{\mathcal{P}}_{1}^{1 / 2}-\tilde{\mathcal{P}}_{2}^{1 / 2}\right)(t, \eta) \\
& \times\left(\int _ { 0 } ^ { 1 } \left(e^{-\frac{1}{A_{2}}\left|\tilde{\mathcal{Y}}_{2}(t, \eta)-\tilde{\mathcal{Y}}_{2}(t, \theta)\right|} \tilde{\mathcal{U}}_{2} \tilde{\mathcal{Q}}_{2} \tilde{\mathcal{Y}}_{2, \eta}\right.\right. \\
& \left.\left.-e^{-\frac{1}{A_{1}}\left|\tilde{\mathcal{Y}}_{1}(t, \eta)-\tilde{\mathcal{Y}}_{1}(t, \theta)\right|} \tilde{\mathcal{U}}_{1} \tilde{\mathcal{Q}}_{1} \tilde{\mathcal{Y}}_{1, \eta}\right)(t, \theta) d \theta\right) d \eta \\
& =J_{11}+J_{12}+J_{13} .
\end{aligned}
$$

Next we replace all the inner integrals $\int_{0}^{1} \cdots d \theta$ by $\left(\int_{0}^{\eta}+\int_{\eta}^{1}\right) \cdots d \theta$ (cf. (5.4)), consider only the terms with $\int_{0}^{\eta} \cdots d \theta$, and call the corresponding quantities $\tilde{J}_{11}$, $\tilde{J}_{12}, \tilde{J}_{13}$. Thus

$$
\begin{aligned}
\tilde{J}_{11}= & \frac{1}{6 A^{3}} \int_{0}^{1} \frac{1}{\max _{j}\left(\tilde{\mathcal{P}}_{j}^{1 / 2}\right)}\left(\tilde{\mathcal{P}}_{1}^{1 / 2}-\tilde{\mathcal{P}}_{2}^{1 / 2}\right)(t, \eta)\left(\int _ { 0 } ^ { \eta } \left(e^{-\frac{1}{A_{1}}\left(\tilde{\mathcal{Y}}_{1}(t, \eta)-\tilde{\mathcal{Y}}_{1}(t, \theta)\right)} A_{1} \tilde{\mathcal{U}}_{1}^{3} \tilde{\mathcal{Y}}_{1, \eta}\right.\right. \\
& \left.\left.-e^{-\frac{1}{A_{2}}\left(\tilde{\mathcal{Y}}_{2}(t, \eta)-\tilde{\mathcal{Y}}_{2}(t, \theta)\right)} A_{2} \tilde{\mathcal{U}}_{2}^{3} \tilde{\mathcal{Y}}_{2, \eta}\right)(t, \theta) d \theta\right) d \eta, \\
\tilde{J}_{12}= & \frac{1}{4 A^{3}} \int_{0}^{1} \frac{1}{\max _{j}\left(\tilde{\mathcal{P}}_{j}^{1 / 2}\right)}\left(\tilde{\mathcal{P}}_{1}^{1 / 2}-\tilde{\mathcal{P}}_{2}^{1 / 2}\right)(t, \eta)\left(\int _ { 0 } ^ { \eta } \left(e^{-\frac{1}{A_{1}}\left(\tilde{\mathcal{Y}}_{1}(t, \eta)-\tilde{\mathcal{Y}}_{1}(t, \theta)\right)} A_{1}^{6} \tilde{\mathcal{U}}_{1}\right.\right. \\
& \left.\left.-e^{-\frac{1}{A_{2}}\left(\tilde{\mathcal{Y}}_{2}(t, \eta)-\tilde{\mathcal{Y}}_{2}(t, \theta)\right)} A_{2}^{6} \tilde{\mathcal{U}}_{2}\right)(t, \theta) d \theta\right) d \eta, \\
\tilde{J}_{13}= & \frac{1}{2 A^{3}} \int_{0}^{1} \frac{1}{\max _{j}\left(\tilde{\mathcal{P}}_{j}^{1 / 2}\right)}\left(\tilde{\mathcal{P}}_{1}^{1 / 2}-\tilde{\mathcal{P}}_{2}^{1 / 2}\right)(t, \eta)\left(\int _ { 0 } ^ { \eta } \left(e^{-\frac{1}{A_{2}}\left(\tilde{\mathcal{Y}}_{2}(t, \eta)-\tilde{\mathcal{Y}}_{2}(t, \theta)\right)} \tilde{\mathcal{U}}_{2} \tilde{\mathcal{Q}}_{2} \tilde{\mathcal{Y}}_{2, \eta}\right.\right. \\
& \left.\left.-e^{-\frac{1}{A_{1}}\left(\tilde{\mathcal{Y}}_{1}(t, \eta)-\tilde{\mathcal{Y}}_{1}(t, \theta)\right)} \tilde{\mathcal{U}}_{1} \tilde{\mathcal{Q}}_{1} \tilde{\mathcal{Y}}_{1, \eta}\right)(t, \theta) d \theta\right) d \eta .
\end{aligned}
$$

For the term $\tilde{J}_{11}$ we write $\tilde{\mathcal{U}}_{j}=\tilde{\mathcal{U}}_{j}^{+}+\tilde{\mathcal{U}}_{j}^{-}$, collect terms, and study the positive part and the negative part separately. 
With a slight abuse of notation, we need to consider the term

$$
\begin{aligned}
& \tilde{J}_{11}=\frac{1}{6 A^{3}} \int_{0}^{1} \frac{1}{\max _{j}\left(\tilde{\mathcal{P}}_{j}^{1 / 2}\right)}\left(\tilde{\mathcal{P}}_{1}^{1 / 2}-\tilde{\mathcal{P}}_{2}^{1 / 2}\right)(t, \eta) \\
& \times\left(\int _ { 0 } ^ { \eta } \left(e^{-\frac{1}{A_{1}}\left(\tilde{\mathcal{Y}}_{1}(t, \eta)-\tilde{\mathcal{Y}}_{1}(t, \theta)\right)} A_{1}\left(\tilde{\mathcal{U}}_{1}^{+}\right)^{3} \tilde{\mathcal{Y}}_{1, \eta}\right.\right. \\
& \left.\left.-e^{-\frac{1}{A_{2}}\left(\tilde{\mathcal{Y}}_{2}(t, \eta)-\tilde{\mathcal{Y}}_{2}(t, \theta)\right)} A_{2}\left(\tilde{\mathcal{U}}_{2}^{+}\right)^{3} \tilde{\mathcal{Y}}_{2, \eta}\right)(t, \theta) d \theta\right) d \eta \\
& =\frac{1}{6 A^{3}} \int_{0}^{1} \frac{1}{\max _{j}\left(\tilde{\mathcal{P}}_{j}^{1 / 2}\right)}\left(\tilde{\mathcal{P}}_{1}^{1 / 2}-\tilde{\mathcal{P}}_{2}^{1 / 2}\right)(t, \eta) \\
& \times\left(\mathbb{1}_{A_{2}<A_{1}}\left(A_{1}-A_{2}\right) \int_{0}^{\eta} e^{-\frac{1}{A_{1}}\left(\tilde{\mathcal{Y}}_{1}(t, \eta)-\tilde{\mathcal{Y}}_{1}(t, \theta)\right)}\left(\tilde{\mathcal{U}}_{1}^{+}\right)^{3} \tilde{\mathcal{Y}}_{1, \eta}(t, \theta) d \theta\right. \\
& +\mathbb{1}_{A_{1} \leqslant A_{2}}\left(A_{1}-A_{2}\right) \int_{0}^{\eta} e^{-\frac{1}{A_{2}}\left(\tilde{\mathcal{Y}}_{2}(t, \eta)-\tilde{\mathcal{Y}}_{2}(t, \theta)\right)}\left(\tilde{\mathcal{U}}_{2}^{+}\right)^{3} \tilde{\mathcal{Y}}_{2, \eta}(t, \theta), d \theta \\
& +a \int_{0}^{\eta} e^{-\frac{1}{A_{1}}\left(\tilde{\mathcal{Y}}_{1}(t, \eta)-\tilde{\mathcal{Y}}_{1}(t, \theta)\right)}\left(\left(\tilde{\mathcal{U}}_{1}^{+}\right)^{3}-\left(\tilde{\mathcal{U}}_{2}^{+}\right)^{3}\right) \mathbb{1}_{\tilde{\mathcal{U}}_{2}^{+}<\tilde{\mathcal{U}}_{1}^{+}} \tilde{\mathcal{Y}}_{1, \eta}(t, \theta) d \theta \\
& +a \int_{0}^{\eta} e^{-\frac{1}{A_{2}}\left(\tilde{\mathcal{Y}}_{2}(t, \eta)-\tilde{\mathcal{Y}}_{2}(t, \theta)\right)}\left(\left(\tilde{\mathcal{U}}_{1}^{+}\right)^{3}-\left(\tilde{\mathcal{U}}_{2}^{+}\right)^{3}\right) \mathbb{1}_{\tilde{\mathcal{U}}_{1}^{+} \leqslant \tilde{\mathcal{U}}_{2}^{+}} \tilde{\mathcal{Y}}_{2, \eta}(t, \theta) d \theta \\
& -a \mathbb{1}_{A_{2}<A_{1}} \int_{0}^{\eta}\left(e^{-\frac{1}{A_{2}}\left(\tilde{\mathcal{Y}}_{1}(t, \eta)-\tilde{\mathcal{Y}}_{1}(t, \theta)\right)}-e^{-\frac{1}{A_{1}}\left(\tilde{\mathcal{Y}}_{1}(t, \eta)-\tilde{\mathcal{Y}}_{1}(t, \theta)\right)}\right) \\
& \times \min _{j}\left(\left(\tilde{\mathcal{U}}_{j}^{+}\right)^{3}\right) \tilde{\mathcal{Y}}_{1, \eta}(t, \theta) d \theta \\
& -a \mathbb{1}_{A_{1} \leqslant A_{2}} \int_{0}^{\eta}\left(e^{-\frac{1}{A_{2}}\left(\tilde{\mathcal{Y}}_{2}(t, \eta)-\tilde{\mathcal{Y}}_{2}(t, \theta)\right)}-e^{-\frac{1}{A_{1}}\left(\tilde{\mathcal{Y}}_{2}(t, \eta)-\tilde{\mathcal{Y}}_{2}(t, \theta)\right)}\right) \\
& \times \min _{j}\left(\left(\tilde{\mathcal{U}}_{j}^{+}\right)^{3}\right) \tilde{\mathcal{Y}}_{2, \eta}(t, \theta) d \theta \\
& -a \int_{0}^{\eta}\left(e^{-\frac{1}{a}\left(\tilde{\mathcal{Y}}_{2}(t, \eta)-\tilde{\mathcal{Y}}_{2}(t, \theta)\right)}-e^{-\frac{1}{a}\left(\tilde{\mathcal{Y}}_{1}(t, \eta)-\tilde{\mathcal{Y}}_{1}(t, \theta)\right)}\right) \\
& \times \min _{j}\left(\left(\tilde{\mathcal{U}}_{j}^{+}\right)^{3}\right) \tilde{\mathcal{Y}}_{2, \eta} \mathbb{1}_{B(\eta)}(t, \theta) d \theta \\
& -a \int_{0}^{\eta}\left(e^{-\frac{1}{a}\left(\tilde{\mathcal{Y}}_{2}(t, \eta)-\tilde{\mathcal{Y}}_{2}(t, \theta)\right)}-e^{-\frac{1}{a}\left(\tilde{\mathcal{Y}}_{1}(t, \eta)-\tilde{\mathcal{Y}}_{1}(t, \theta)\right)}\right) \\
& \times \min _{j}\left(\left(\tilde{\mathcal{U}}_{j}^{+}\right)^{3}\right) \tilde{\mathcal{Y}}_{1, \eta} \mathbb{1}_{B(\eta)^{c}}(t, \theta) d \theta
\end{aligned}
$$




$$
\begin{aligned}
& \quad-a \int_{0}^{\eta} \min _{j}\left(e^{-\frac{1}{a}\left(\tilde{\mathcal{Y}}_{j}(t, \eta)-\tilde{\mathcal{Y}}_{j}(t, \theta)\right)}\right) \min _{j}\left(\left(\tilde{\mathcal{U}}_{j}^{+}\right)^{3}\right) \tilde{\mathcal{Y}}_{2, \eta}(t, \theta) d \theta \\
& \left.+a \int_{0}^{\eta} \min _{j}\left(e^{-\frac{1}{a}\left(\tilde{\mathcal{Y}}_{j}(t, \eta)-\tilde{\mathcal{Y}}_{j}(t, \theta)\right)}\right) \min _{j}\left(\left(\tilde{\mathcal{U}}_{j}^{+}\right)^{3}\right) \tilde{\mathcal{Y}}_{1, \eta}(t, \theta) d \theta\right) d \eta \\
& =K_{1}+K_{2}+K_{3}+K_{4}+K_{5}+K_{6}+K_{7}+K_{8}+K_{9}+K_{10} .
\end{aligned}
$$

It never stops, but we need to study the terms in groups.

The term $K_{1}$ can be estimated as follows ( $K_{2}$ is similar):

$$
\begin{aligned}
\left|K_{1}\right|= & \frac{1}{6 A^{3}} \int_{0}^{1} \frac{1}{\max _{j}\left(\tilde{\mathcal{P}}_{j}^{1 / 2}\right)}\left|\tilde{\mathcal{P}}_{1}^{1 / 2}-\tilde{\mathcal{P}}_{2}^{1 / 2}\right|(t, \eta) \\
& \times\left|\mathbb{1}_{A_{2}<A_{1}}\left(A_{1}-A_{2}\right) \int_{0}^{\eta} e^{-\frac{1}{A_{1}}\left(\tilde{\mathcal{Y}}_{1}(t, \eta)-\tilde{\mathcal{Y}}_{1}(t, \theta)\right)}\left(\tilde{\mathcal{U}}_{1}^{+}\right)^{3} \tilde{\mathcal{Y}}_{1, \eta}(t, \theta) d \theta\right| d \eta \\
\leqslant & \frac{\sqrt{2}}{3} \int_{0}^{1} \frac{\tilde{\mathcal{P}}_{1}}{\max _{j}\left(\tilde{\mathcal{P}}_{j}^{1 / 2}\right)}\left|\tilde{\mathcal{P}}_{1}^{1 / 2}-\tilde{\mathcal{P}}_{2}^{1 / 2}\right|(t, \eta) d \eta\left|A_{1}-A_{2}\right| \\
\leqslant & \mathcal{O}(1)\left(\left\|\tilde{\mathcal{P}}_{1}^{1 / 2}-\tilde{\mathcal{P}}_{2}^{1 / 2}\right\|^{2}+\left|A_{1}-A_{2}\right|^{2}\right) .
\end{aligned}
$$

The term $K_{3}$ can be estimated as follows ( $K_{4}$ is similar):

$$
\begin{aligned}
& \left|K_{3}\right|=\frac{a}{6 A^{3}} \mid \int_{0}^{1} \frac{1}{\max _{j}\left(\tilde{\mathcal{P}}_{j}^{1 / 2}\right)}\left(\tilde{\mathcal{P}}_{1}^{1 / 2}-\tilde{\mathcal{P}}_{2}^{1 / 2}\right)(t, \eta) \\
& \times\left(\int_{0}^{\eta} e^{-\frac{1}{A_{1}}\left(\tilde{\mathcal{Y}}_{1}(t, \eta)-\tilde{\mathcal{Y}}_{1}(t, \theta)\right)}\left(\left(\tilde{\mathcal{U}}_{1}^{+}\right)^{3}-\left(\tilde{\mathcal{U}}_{2}^{+}\right)^{3}\right) \mathbb{1}_{\tilde{\mathcal{U}}_{2}^{+}<\tilde{\mathcal{U}}_{1}^{+}} \tilde{\mathcal{Y}}_{1, \eta}(t, \theta) d \theta\right) d \eta \\
& \leqslant \frac{1}{2 A^{2}} \int_{0}^{1} \frac{1}{\max _{j}\left(\tilde{\mathcal{P}}_{j}^{1 / 2}\right)}\left|\tilde{\mathcal{P}}_{1}^{1 / 2}-\tilde{\mathcal{P}}_{2}^{1 / 2}\right|(t, \eta) \\
& \times\left(\int_{0}^{\eta} e^{-\frac{1}{A_{1}}\left(\tilde{\mathcal{Y}}_{1}(t, \eta)-\tilde{\mathcal{Y}}_{1}(t, \theta)\right)}\left(\tilde{\mathcal{U}}_{1}^{+}\right)^{2}\left|\tilde{\mathcal{U}}_{1}^{+}-\tilde{\mathcal{U}}_{2}^{+}\right| \tilde{\mathcal{Y}}_{1, \eta}(t, \theta) d \theta\right) d \eta \\
& \leqslant \frac{1}{2}\left\|\tilde{\mathcal{P}}_{1}^{1 / 2}-\tilde{\mathcal{P}}_{2}^{1 / 2}\right\|^{2}+\frac{1}{2 A^{4}} \int_{0}^{1} \frac{1}{\max _{j}\left(\tilde{\mathcal{P}}_{j}^{1 / 2}\right)^{2}} \\
& \times\left(\int_{0}^{\eta} e^{-\frac{1}{A_{1}}\left(\tilde{\mathcal{Y}}_{1}(t, \eta)-\tilde{\mathcal{Y}}_{1}(t, \theta)\right)}\left(\tilde{\mathcal{U}}_{1}^{+}\right)^{2}\left|\tilde{\mathcal{U}}_{1}^{+}-\tilde{\mathcal{U}}_{2}^{+}\right| \tilde{\mathcal{Y}}_{1, \eta}(t, \theta) d \theta\right)^{2} d \eta \\
& \leqslant \frac{1}{2}\left\|\tilde{\mathcal{P}}_{1}^{1 / 2}-\tilde{\mathcal{P}}_{2}^{1 / 2}\right\|^{2} \\
& +\frac{1}{2 A^{4}} \int_{0}^{1} \frac{1}{\max _{j}\left(\tilde{\mathcal{P}}_{j}^{1 / 2}\right)^{2}}\left(\int_{0}^{\eta} e^{-\frac{1}{A_{1}}\left(\tilde{\mathcal{Y}}_{1}(t, \eta)-\tilde{\mathcal{Y}}_{1}(t, \theta)\right)}\left(\tilde{\mathcal{U}}_{1}^{+}\right)^{2} \tilde{\mathcal{Y}}_{1, \eta}(t, \theta) d \theta\right)
\end{aligned}
$$




$$
\begin{aligned}
& \times\left(\int_{0}^{\eta} e^{-\frac{1}{A_{1}}\left(\tilde{\mathcal{Y}}_{1}(t, \eta)-\tilde{\mathcal{Y}}_{1}(t, \theta)\right)}\left|\tilde{\mathcal{U}}_{1}^{+}-\tilde{\mathcal{U}}_{2}^{+}\right|^{2}\left(\tilde{\mathcal{U}}_{1}^{+}\right)^{2} \tilde{\mathcal{Y}}_{1, \eta}(t, \theta) d \theta\right) d \eta \\
\leqslant & \mathcal{O}(1)\left(\left\|\tilde{\mathcal{P}}_{1}^{1 / 2}-\tilde{\mathcal{P}}_{2}^{1 / 2}\right\|^{2}+\left\|\tilde{\mathcal{U}}_{1}-\tilde{\mathcal{U}}_{2}\right\|^{2}\right),
\end{aligned}
$$

using (4.15g), (4.16c), and (5.3).

As for $K_{5}\left(K_{6}\right.$ is similar), we traverse the following path:

$$
\begin{aligned}
& \left|K_{5}\right|=\frac{1}{6 A^{3}} \mid \int_{0}^{1} \frac{1}{\max _{j}\left(\tilde{\mathcal{P}}_{j}^{1 / 2}\right)}\left(\tilde{\mathcal{P}}_{1}^{1 / 2}-\tilde{\mathcal{P}}_{2}^{1 / 2}\right)(t, \eta) \\
& \times\left(-a \mathbb{1}_{A_{2}<A_{1}} \int_{0}^{\eta}\left(e^{-\frac{1}{A_{2}}\left(\tilde{\mathcal{Y}}_{1}(t, \eta)-\tilde{\mathcal{Y}}_{1}(t, \theta)\right)}-e^{-\frac{1}{A_{1}}\left(\tilde{\mathcal{Y}}_{1}(t, \eta)-\tilde{\mathcal{Y}}_{1}(t, \theta)\right)}\right)\right. \\
& \left.\times \min _{j}\left(\left(\tilde{\mathcal{U}}_{j}^{+}\right)^{3}\right) \tilde{\mathcal{Y}}_{1, \eta}(t, \theta) d \theta\right) d \eta \mid \\
& \leqslant \frac{2}{3 A^{3} e} \int_{0}^{1} \frac{1}{\max _{j}\left(\tilde{\mathcal{P}}_{j}^{1 / 2}\right)}\left|\tilde{\mathcal{P}}_{1}^{1 / 2}-\tilde{\mathcal{P}}_{2}^{1 / 2}\right|(t, \eta) \\
& \times\left(\int_{0}^{\eta} e^{-\frac{3}{4 A_{1}}\left(\tilde{\mathcal{Y}}_{1}(t, \eta)-\tilde{\mathcal{Y}}_{1}(t, \theta)\right)} \min _{j}\left(\left(\tilde{\mathcal{U}}_{j}^{+}\right)^{3}\right) \tilde{\mathcal{Y}}_{1, \eta}(t, \theta) d \theta\right) d \eta\left|A_{1}-A_{2}\right| \\
& \leqslant \frac{2 \sqrt{2} A^{2}}{3} \int_{0}^{1} \frac{\tilde{\mathcal{P}}_{1}^{1 / 2}}{\max _{j}\left(\tilde{\mathcal{P}}_{j}^{1 / 2}\right)}\left|\tilde{\mathcal{P}}_{1}^{1 / 2}-\tilde{\mathcal{P}}_{2}^{1 / 2}\right|(t, \eta) d \eta\left|A_{1}-A_{2}\right| \\
& \leqslant \mathcal{O}(1)\left(\left\|\tilde{\mathcal{P}}_{1}^{1 / 2}-\tilde{\mathcal{P}}_{2}^{1 / 2}\right\|^{2}+\left|A_{1}-A_{2}\right|^{2}\right) .
\end{aligned}
$$

As for $K_{7}\left(K_{8}\right.$ is similar), we traverse the following path:

$$
\begin{aligned}
& \left|K_{7}\right|=\frac{a}{6 A^{3}} \mid \int_{0}^{1} \frac{1}{\max _{j}\left(\tilde{\mathcal{P}}_{j}^{1 / 2}\right)}\left(\tilde{\mathcal{P}}_{1}^{1 / 2}-\tilde{\mathcal{P}}_{2}^{1 / 2}\right)(t, \eta) \\
& \times\left(\int_{0}^{\eta}\left(e^{-\frac{1}{a}\left(\tilde{\mathcal{Y}}_{2}(t, \eta)-\tilde{\mathcal{Y}}_{2}(t, \theta)\right)}-e^{-\frac{1}{a}\left(\tilde{\mathcal{Y}}_{1}(t, \eta)-\tilde{\mathcal{Y}}_{1}(t, \theta)\right)}\right)\right. \\
& \left.\times \min _{j}\left(\left(\tilde{\mathcal{U}}_{j}^{+}\right)^{3}\right) \tilde{\mathcal{Y}}_{2, \eta} \mathbb{1}_{B(\eta)}(t, \theta) d \theta\right) d \eta \\
& \leqslant \frac{1}{6 A^{3}} \int_{0}^{1} \frac{1}{\max _{j}\left(\tilde{\mathcal{P}}_{j}^{1 / 2}\right)}\left|\tilde{\mathcal{P}}_{1}^{1 / 2}-\tilde{\mathcal{P}}_{2}^{1 / 2}\right|(t, \eta)\left(\int_{0}^{\eta} e^{-\frac{1}{a}\left(\tilde{\mathcal{Y}}_{2}(t, \eta)-\tilde{\mathcal{Y}}_{2}(t, \theta)\right)}\right. \\
& \left.\times\left(\left|\tilde{\mathcal{Y}}_{2}(t, \eta)-\tilde{\mathcal{Y}}_{1}(t, \eta)\right|+\left|\tilde{\mathcal{Y}}_{2}(t, \theta)-\tilde{\mathcal{Y}}_{1}(t, \theta)\right|\right)\left(\tilde{\mathcal{U}}_{2}^{+}\right)^{3} \tilde{\mathcal{Y}}_{2, \eta}(t, \theta) d \theta\right) d \eta \\
& \leqslant \frac{1}{6 A^{3}} \int_{0}^{1} \frac{1}{\max _{j}\left(\tilde{\mathcal{P}}_{j}^{1 / 2}\right)}\left|\tilde{\mathcal{P}}_{1}^{1 / 2}-\tilde{\mathcal{P}}_{2}^{1 / 2}\right|\left|\tilde{\mathcal{Y}}_{2}-\tilde{\mathcal{Y}}_{1}\right|(t, \eta) \\
& \times\left(\int_{0}^{\eta} e^{-\frac{1}{A_{2}}\left(\tilde{\mathcal{Y}}_{2}(t, \eta)-\tilde{\mathcal{Y}}_{2}(t, \theta)\right)}\left(\tilde{\mathcal{U}}_{2}^{+}\right)^{3} \tilde{\mathcal{Y}}_{2, \eta}(t, \theta) d \theta\right) d \eta
\end{aligned}
$$




$$
\begin{aligned}
& +\frac{1}{6 A^{3}} \int_{0}^{1} \frac{1}{\max _{j}\left(\tilde{\mathcal{P}}_{j}^{1 / 2}\right)}\left|\tilde{\mathcal{P}}_{1}^{1 / 2}-\tilde{\mathcal{P}}_{2}^{1 / 2}\right|(t, \eta) \\
& \times\left(\int_{0}^{\eta} e^{-\frac{1}{a}\left(\tilde{\mathcal{Y}}_{2}(t, \eta)-\tilde{\mathcal{Y}}_{2}(t, \theta)\right)}\left|\tilde{\mathcal{Y}}_{2}-\tilde{\mathcal{Y}}_{1}\right|\left(\tilde{\mathcal{U}}_{2}^{+}\right)^{3} \tilde{\mathcal{Y}}_{2, \eta}(t, \theta) d \theta\right) d \eta \\
& \leqslant \frac{\sqrt{2}}{3} \int_{0}^{1} \frac{1}{\max _{j}\left(\tilde{\mathcal{P}}_{j}^{1 / 2}\right)}\left|\tilde{\mathcal{P}}_{1}^{1 / 2}-\tilde{\mathcal{P}}_{2}^{1 / 2}\right|\left|\tilde{\mathcal{Y}}_{2}-\tilde{\mathcal{Y}}_{1}\right| \tilde{\mathcal{P}}_{2}(t, \eta) d \eta \\
& +\left\|\tilde{\mathcal{P}}_{1}^{1 / 2}-\tilde{\mathcal{P}}_{2}^{1 / 2}\right\|^{2} \\
& +\frac{1}{36 A^{6}} \int_{0}^{1} \frac{1}{\max _{j}\left(\tilde{\mathcal{P}}_{j}^{1 / 2}\right)^{2}}(t, \eta) \\
& \times\left(\int_{0}^{\eta} e^{-\frac{1}{a}\left(\tilde{\mathcal{Y}}_{2}(t, \eta)-\tilde{\mathcal{Y}}_{2}(t, \theta)\right)}\left|\tilde{\mathcal{Y}}_{2}-\tilde{\mathcal{Y}}_{1}\right|\left(\tilde{\mathcal{U}}_{2}^{+}\right)^{3} \tilde{\mathcal{Y}}_{2, \eta}(t, \theta) d \theta\right)^{2} d \eta \\
& \leqslant \mathcal{O}(1)\left(\left\|\tilde{\mathcal{P}}_{1}^{1 / 2}-\tilde{\mathcal{P}}_{2}^{1 / 2}\right\|^{2}+\left\|\tilde{\mathcal{Y}}_{2}-\tilde{\mathcal{Y}}_{1}\right\|^{2}\right) \\
& +\frac{1}{36 A^{6}} \int_{0}^{1} \frac{1}{\max _{j}\left(\tilde{\mathcal{P}}_{j}^{1 / 2}\right)^{2}}(t, \eta)\left(\int_{0}^{\eta} e^{-\frac{1}{A_{2}}\left(\tilde{\mathcal{Y}}_{2}(t, \eta)-\tilde{\mathcal{Y}}_{2}(t, \theta)\right)}\left(\tilde{\mathcal{U}}_{2}^{+}\right)^{2} \tilde{\mathcal{Y}}_{2, \eta}(t, \theta) d \theta\right) \\
& \times\left(\int_{0}^{\eta} e^{-\frac{1}{A_{2}}\left(\tilde{\mathcal{Y}}_{2}(t, \eta)-\tilde{\mathcal{Y}}_{2}(t, \theta)\right)}\left|\tilde{\mathcal{Y}}_{2}-\tilde{\mathcal{Y}}_{1}\right|^{2}\left(\tilde{\mathcal{U}}_{2}^{+}\right)^{4} \tilde{\mathcal{Y}}_{2, \eta}(t, \theta) d \theta\right) d \eta \\
& \leqslant \mathcal{O}(1)\left(\left\|\tilde{\mathcal{P}}_{1}^{1 / 2}-\tilde{\mathcal{P}}_{2}^{1 / 2}\right\|^{2}+\left\|\tilde{\mathcal{Y}}_{2}-\tilde{\mathcal{Y}}_{1}\right\|^{2}\right) \\
& +\frac{1}{9 A^{5}} \int_{0}^{1} \frac{1}{\max _{j}\left(\tilde{\mathcal{P}}_{j}^{1 / 2}\right)^{2}} \tilde{\mathcal{P}}_{2}(t, \eta) \\
& \times\left(\int_{0}^{\eta} e^{-\frac{1}{A_{2}}\left(\tilde{\mathcal{Y}}_{2}(t, \eta)-\tilde{\mathcal{Y}}_{2}(t, \theta)\right)}\left|\tilde{\mathcal{Y}}_{2}-\tilde{\mathcal{Y}}_{1}\right|^{2}\left(\tilde{\mathcal{U}}_{2}^{+}\right)^{4} \tilde{\mathcal{Y}}_{2, \eta}(t, \theta) d \theta\right) d \eta \\
& \leqslant \mathcal{O}(1)\left(\left\|\tilde{\mathcal{P}}_{1}^{1 / 2}-\tilde{\mathcal{P}}_{2}^{1 / 2}\right\|^{2}+\left\|\tilde{\mathcal{Y}}_{2}-\tilde{\mathcal{Y}}_{1}\right\|^{2}\right) .
\end{aligned}
$$

Here we have applied (4.15b), (4.15g), (4.16c), and (5.13). Lo and behold, we can do $K_{9}$ and $K_{10}$ in one sweep:

$$
\begin{aligned}
K_{9}+K_{10}= & -\frac{a}{6 A^{3}} \int_{0}^{1} \frac{1}{\max _{j}\left(\tilde{\mathcal{P}}_{j}^{1 / 2}\right)}\left(\tilde{\mathcal{P}}_{1}^{1 / 2}-\tilde{\mathcal{P}}_{2}^{1 / 2}\right)(t, \eta) \\
& \times\left(\int_{0}^{\eta} \min _{j}\left(e^{-\frac{1}{a}\left(\tilde{\mathcal{Y}}_{j}(t, \eta)-\tilde{\mathcal{Y}}_{j}(t, \theta)\right)}\right) \min _{j}\left(\left(\tilde{\mathcal{U}}_{j}^{+}\right)^{3}\right) \tilde{\mathcal{Y}}_{2, \eta}(t, \theta) d \theta\right. \\
& \left.-\int_{0}^{\eta} \min _{j}\left(e^{-\frac{1}{a}\left(\tilde{\mathcal{Y}}_{j}(t, \eta)-\tilde{\mathcal{Y}}_{j}(t, \theta)\right)}\right) \min _{j}\left(\left(\tilde{\mathcal{U}}_{j}^{+}\right)^{3}\right) \tilde{\mathcal{Y}}_{1, \eta}(t, \theta) d \theta\right) d \eta \\
= & -\frac{a}{6 A^{3}} \int_{0}^{1} \frac{1}{\max _{j}\left(\tilde{\mathcal{P}}_{j}^{1 / 2}\right)}\left(\tilde{\mathcal{P}}_{1}^{1 / 2}-\tilde{\mathcal{P}}_{2}^{1 / 2}\right)(t, \eta)
\end{aligned}
$$




$$
\begin{aligned}
& \times\left(\int_{0}^{\eta} \min _{j}\left(e^{-\frac{1}{a}\left(\tilde{\mathcal{Y}}_{j}(t, \eta)-\tilde{\mathcal{Y}}_{j}(t, \theta)\right)}\right) \min _{j}\left(\left(\tilde{\mathcal{U}}_{j}^{+}\right)^{3}\right)\left(\tilde{\mathcal{Y}}_{2, \eta}-\tilde{\mathcal{Y}}_{1, \eta}\right)(t, \theta) d \theta\right) d \eta \\
& =-\frac{a}{6 A^{3}} \int_{0}^{1} \frac{1}{\max _{j}\left(\tilde{\mathcal{P}}_{j}^{1 / 2}\right)}\left(\tilde{\mathcal{P}}_{1}^{1 / 2}-\tilde{\mathcal{P}}_{2}^{1 / 2}\right)(t, \eta) \\
& \times\left[\left.\left(\min _{j}\left(e^{-\frac{1}{a}\left(\tilde{\mathcal{Y}}_{j}(t, \eta)-\tilde{\mathcal{Y}}_{j}(t, \theta)\right)}\right) \min _{j}\left(\left(\tilde{\mathcal{U}}_{j}^{+}\right)^{3}\right)\left(\tilde{\mathcal{Y}}_{2}-\tilde{\mathcal{Y}}_{1}\right)\right)(t, \theta)\right|_{\theta=0} ^{\eta}\right. \\
& \left.-\int_{0}^{\eta}\left(\tilde{\mathcal{Y}}_{2}-\tilde{\mathcal{Y}}_{1}\right) \frac{d}{d \theta}\left(\min _{j}\left(e^{-\frac{1}{a}\left(\tilde{\mathcal{Y}}_{j}(t, \eta)-\tilde{\mathcal{Y}}_{j}(t, \theta)\right)}\right) \min _{j}\left(\left(\tilde{\mathcal{U}}_{j}^{+}\right)^{3}\right)\right)(t, \theta) d \theta\right] d \eta \\
& =-\frac{a}{6 A^{3}} \int_{0}^{1} \frac{1}{\max _{j}\left(\tilde{\mathcal{P}}_{j}^{1 / 2}\right)}\left(\tilde{\mathcal{P}}_{1}^{1 / 2}-\tilde{\mathcal{P}}_{2}^{1 / 2}\right)\left(\tilde{\mathcal{Y}}_{2}-\tilde{\mathcal{Y}}_{1}\right) \min _{j}\left(\left(\tilde{\mathcal{U}}_{j}^{+}\right)^{3}\right)(t, \eta) d \eta \\
& +\frac{a}{6 A^{3}} \int_{0}^{1} \frac{1}{\max _{j}\left(\tilde{\mathcal{P}}_{j}^{1 / 2}\right)}\left(\tilde{\mathcal{P}}_{1}^{1 / 2}-\tilde{\mathcal{P}}_{2}^{1 / 2}\right)(t, \eta) \\
& \times\left(\int_{0}^{\eta}\left(\tilde{\mathcal{Y}}_{2}-\tilde{\mathcal{Y}}_{1}\right) \min _{j}\left(\left(\tilde{\mathcal{U}}_{j}^{+}\right)^{3}\right) \frac{d}{d \theta} \min _{j}\left(e^{-\frac{1}{a}\left(\tilde{\mathcal{Y}}_{j}(t, \eta)-\tilde{\mathcal{Y}}_{j}(t, \theta)\right)}\right)(t, \theta) d \theta\right) d \eta \\
& +\frac{a}{6 A^{3}} \int_{0}^{1} \frac{1}{\max _{j}\left(\tilde{\mathcal{P}}_{j}^{1 / 2}\right)}\left(\tilde{\mathcal{P}}_{1}^{1 / 2}-\tilde{\mathcal{P}}_{2}^{1 / 2}\right)(t, \eta) \\
& \times\left(\int_{0}^{\eta} \min _{j}\left(e^{-\frac{1}{a}\left(\tilde{\mathcal{Y}}_{j}(t, \eta)-\tilde{\mathcal{Y}}_{j}(t, \theta)\right)}\right)\left(\tilde{\mathcal{Y}}_{2}-\tilde{\mathcal{Y}}_{1}\right) \frac{d}{d \theta} \min _{j}\left(\left(\tilde{\mathcal{U}}_{j}^{+}\right)^{3}\right)(t, \theta) d \theta\right) d \eta \\
& =L_{1}+L_{2}+L_{3} \text {, }
\end{aligned}
$$

where

$$
\begin{aligned}
L_{1}= & -\frac{a}{6 A^{3}} \int_{0}^{1} \frac{1}{\max _{j}\left(\tilde{\mathcal{P}}_{j}^{1 / 2}\right)}\left(\tilde{\mathcal{P}}_{1}^{1 / 2}-\tilde{\mathcal{P}}_{2}^{1 / 2}\right)\left(\tilde{\mathcal{Y}}_{2}-\tilde{\mathcal{Y}}_{1}\right) \min _{j}\left(\left(\tilde{\mathcal{U}}_{j}^{+}\right)^{3}\right)(t, \eta) d \eta, \\
L_{2}= & \frac{a}{6 A^{3}} \int_{0}^{1} \frac{1}{\max _{j}\left(\tilde{\mathcal{P}}_{j}^{1 / 2}\right)}\left(\tilde{\mathcal{P}}_{1}^{1 / 2}-\tilde{\mathcal{P}}_{2}^{1 / 2}\right)(t, \eta) \\
& \times\left(\int_{0}^{\eta}\left(\tilde{\mathcal{Y}}_{2}-\tilde{\mathcal{Y}}_{1}\right) \min \left(\left(\tilde{\mathcal{U}}_{j}^{+}\right)^{3}\right) \frac{d}{d \theta} \min _{j}\left(e^{-\frac{1}{a}\left(\tilde{\mathcal{Y}}_{j}(t, \eta)-\tilde{\mathcal{Y}}_{j}(t, \theta)\right)}\right)(t, \theta) d \theta\right) d \eta, \\
L_{3}= & \frac{a}{6 A^{3}} \int_{0}^{1} \frac{1}{\max _{j}\left(\tilde{\mathcal{P}}_{j}^{1 / 2}\right)}\left(\tilde{\mathcal{P}}_{1}^{1 / 2}-\tilde{\mathcal{P}}_{2}^{1 / 2}\right)(t, \eta) \\
& \times\left(\int_{0}^{\eta} \min _{j}\left(e^{-\frac{1}{a}\left(\tilde{\mathcal{Y}}_{j}(t, \eta)-\tilde{\mathcal{Y}}_{j}(t, \theta)\right)}\right)\left(\tilde{\mathcal{Y}}_{2}-\tilde{\mathcal{Y}}_{1}\right) \frac{d}{d \theta} \min _{j}\left(\left(\tilde{\mathcal{U}}_{j}^{+}\right)^{3}\right)(t, \theta) d \theta\right) d \eta .
\end{aligned}
$$


We easily find

$$
\begin{aligned}
\left|L_{1}\right| & \leqslant \frac{a}{6 A^{3}} \int_{0}^{1} \frac{1}{\max _{j}\left(\tilde{\mathcal{P}}_{j}^{1 / 2}\right)}\left|\tilde{\mathcal{P}}_{1}^{1 / 2}-\tilde{\mathcal{P}}_{2}^{1 / 2} \| \tilde{\mathcal{Y}}_{2}-\tilde{\mathcal{Y}}_{1}\right| \min _{j}\left(\left(\tilde{\mathcal{U}}_{j}^{+}\right)^{3}\right)(t, \eta) d \eta \\
& \leqslant \mathcal{O}(1)\left(\left\|\tilde{\mathcal{P}}_{1}^{1 / 2}-\tilde{\mathcal{P}}_{2}^{1 / 2}\right\|^{2}+\left\|\tilde{\mathcal{Y}}_{2}-\tilde{\mathcal{Y}}_{1}\right\|^{2}\right),
\end{aligned}
$$

using (4.15b) and (4.15d). Furthermore, applying Lemma A.2,

$$
\begin{aligned}
\left|L_{2}\right| \leqslant & \frac{1}{6 A^{3}} \int_{0}^{1} \frac{1}{\max _{j}\left(\tilde{\mathcal{P}}_{j}^{1 / 2}\right)}\left|\tilde{\mathcal{P}}_{1}^{1 / 2}-\tilde{\mathcal{P}}_{2}^{1 / 2}\right|(t, \eta) \\
& \times\left(\int_{0}^{\eta} \min _{j}\left(e^{-\frac{1}{a}\left(\tilde{\mathcal{Y}}_{j}(t, \eta)-\tilde{\mathcal{Y}}_{j}(t, \theta)\right)}\right)\left|\tilde{\mathcal{Y}}_{2}-\tilde{\mathcal{Y}}_{1}\right|\right. \\
& \left.\times \min _{j}\left(\left(\tilde{\mathcal{U}}_{j}^{+}\right)^{3}\right) \max _{j}\left(\tilde{\mathcal{Y}}_{j, \eta}\right)(t, \theta) d \theta\right) d \eta \\
\leqslant & \frac{1}{6 A^{3}} \int_{0}^{1} \frac{1}{\max _{j}\left(\tilde{\mathcal{P}}_{j}^{1 / 2}\right)}\left|\tilde{\mathcal{P}}_{1}^{1 / 2}-\tilde{\mathcal{P}}_{2}^{1 / 2}\right|(t, \eta) \\
& \times\left(\int_{0}^{\eta} \min _{j}\left(e^{-\frac{1}{A_{j}}\left(\tilde{\mathcal{Y}}_{j}(t, \eta)-\tilde{\mathcal{Y}}_{j}(t, \theta)\right)} \tilde{\mathcal{U}}_{j}^{2}\right)(t, \theta) d \theta\right)^{\frac{1}{2}} \\
& \times\left(\int_{0}^{\eta}\left|\tilde{\mathcal{Y}}_{2}-\tilde{\mathcal{Y}}_{1}\right|^{2} \max _{j}\left(\tilde{\mathcal{U}}_{j}^{4} \tilde{\mathcal{Y}}_{j, \eta}\right)^{2}(t, \theta) d \theta\right)^{\frac{1}{2}} d \eta \\
\leqslant & \mathcal{O}(1) \int_{0}^{1}\left|\tilde{\mathcal{P}}_{1}^{1 / 2}-\tilde{\mathcal{P}}_{2}^{1 / 2}\right|(t, \eta) d \eta\left\|\tilde{\mathcal{Y}}_{1}-\tilde{\mathcal{Y}}_{2}\right\| \\
\leqslant & \mathcal{O}(1)\left(\left\|\tilde{\mathcal{P}}_{1}^{1 / 2}-\tilde{\mathcal{P}}_{2}^{1 / 2}\right\|^{2}+\left\|\tilde{\mathcal{Y}}_{2}-\tilde{\mathcal{Y}}_{1}\right\|^{2}\right),
\end{aligned}
$$

using (4.15g), (4.16a), and

$$
\min _{j}\left(\left(\tilde{\mathcal{U}}_{j}^{+}\right)^{3}\right) \max _{j}\left(\tilde{\mathcal{Y}}_{j, \eta}\right) \leqslant \min _{j}\left(\tilde{\mathcal{U}}_{j}^{+}\right) \max _{j}\left(\tilde{\mathcal{U}}_{j}^{2} \tilde{\mathcal{Y}}_{j, \eta}\right) \leqslant \frac{A^{7}}{\sqrt{2}} .
$$

Another application of Lemma A.2 yields

$$
\begin{aligned}
\left|L_{3}\right| \leqslant & \frac{a}{6 A^{3}} \int_{0}^{1} \frac{1}{\max _{j}\left(\tilde{\mathcal{P}}_{j}^{1 / 2}\right)}\left|\tilde{\mathcal{P}}_{1}^{1 / 2}-\tilde{\mathcal{P}}_{2}^{1 / 2}\right|(t, \eta) \\
& \times\left(\int_{0}^{\eta} \min _{j}\left(e^{-\frac{1}{a}\left(\tilde{\mathcal{Y}}_{j}(t, \eta)-\tilde{\mathcal{Y}}_{j}(t, \theta)\right)}\right)\left|\tilde{\mathcal{Y}}_{2}-\tilde{\mathcal{Y}}_{1}\right|\left|\frac{d}{d \theta} \min _{j}\left(\left(\tilde{\mathcal{U}}_{j}^{+}\right)^{3}\right)\right|(t, \theta) d \theta\right) d \eta \\
\leqslant & \frac{A^{2}}{3} \int_{0}^{1} \frac{1}{\max _{j}\left(\tilde{\mathcal{P}}_{j}^{1 / 2}\right)}\left|\tilde{\mathcal{P}}_{1}^{1 / 2}-\tilde{\mathcal{P}}_{2}^{1 / 2}\right|(t, \eta)
\end{aligned}
$$




$$
\begin{aligned}
& \times\left(\int_{0}^{\eta} \min _{j}\left(e^{-\frac{1}{a}\left(\tilde{\mathcal{Y}}_{j}(t, \eta)-\tilde{\mathcal{Y}}_{j}(t, \theta)\right)}\right)\left|\tilde{\mathcal{Y}}_{2}-\tilde{\mathcal{Y}}_{1}\right| \min _{j}\left(\tilde{\mathcal{U}}_{j}^{+}\right)(t, \theta) d \theta\right) d \eta \\
\leqslant & \frac{A^{2}}{3} \int_{0}^{1} \frac{1}{\max _{j}\left(\tilde{\mathcal{P}}_{j}^{1 / 2}\right)}\left|\tilde{\mathcal{P}}_{1}^{1 / 2}-\tilde{\mathcal{P}}_{2}^{1 / 2}\right|(t, \eta) \\
& \times\left(\int_{0}^{\eta} e^{-\frac{1}{A_{2}}\left(\tilde{\mathcal{Y}}_{2}(t, \eta)-\tilde{\mathcal{Y}}_{2}(t, \theta)\right)} \tilde{\mathcal{U}}_{2}^{2}(t, \theta) d \theta\right)^{1 / 2} \\
& \times\left(\int_{0}^{\eta} \min _{j}\left(e^{-\frac{1}{a}\left(\tilde{\mathcal{Y}}_{j}(t, \eta)-\tilde{\mathcal{Y}}_{j}(t, \theta)\right)}\right)\left|\tilde{\mathcal{Y}}_{2}-\tilde{\mathcal{Y}}_{1}\right|^{2}(t, \theta) d \theta\right)^{1 / 2} d \eta \\
\leqslant & \mathcal{O}(1)\left(\left\|\tilde{\mathcal{P}}_{1}^{1 / 2}-\tilde{\mathcal{P}}_{2}^{1 / 2}\right\|^{2}+\left\|\tilde{\mathcal{Y}}_{2}-\tilde{\mathcal{Y}}_{1}\right\|^{2}\right),
\end{aligned}
$$

using (4.16a). We find

$$
\begin{aligned}
\left|K_{9}+K_{10}\right| & \leqslant\left|L_{1}\right|+\left|L_{2}\right|+\left|L_{3}\right| \\
& \leqslant \mathcal{O}(1)\left(\left\|\tilde{\mathcal{P}}_{1}^{1 / 2}-\tilde{\mathcal{P}}_{2}^{1 / 2}\right\|^{2}+\left\|\tilde{\mathcal{Y}}_{2}-\tilde{\mathcal{Y}}_{1}\right\|^{2}\right)
\end{aligned}
$$

Thus we can sum up the estimates for $\tilde{J}_{11}$, and we find

$$
\left|\tilde{J}_{11}\right| \leqslant \mathcal{O}(1)\left(\left\|\tilde{\mathcal{P}}_{1}^{1 / 2}-\tilde{\mathcal{P}}_{2}^{1 / 2}\right\|^{2}+\left\|\tilde{\mathcal{U}}_{1}-\tilde{\mathcal{U}}_{2}\right\|^{2}+\left\|\tilde{\mathcal{Y}}_{2}-\tilde{\mathcal{Y}}_{1}\right\|^{2}+\left|A_{1}-A_{2}\right|^{2}\right)
$$

We now proceed to the next term from (5.45):

$$
\begin{aligned}
& \tilde{J}_{12}=\frac{1}{4 A^{3}} \int_{0}^{1} \frac{1}{\max _{j}\left(\tilde{\mathcal{P}}_{j}^{1 / 2}\right)}\left(\tilde{\mathcal{P}}_{1}^{1 / 2}-\tilde{\mathcal{P}}_{2}^{1 / 2}\right)(t, \eta) \\
& \times\left(\int_{0}^{\eta}\left(e^{-\frac{1}{A_{1}}\left(\tilde{\mathcal{Y}}_{1}(t, \eta)-\tilde{\mathcal{Y}}_{1}(t, \theta)\right)} A_{1}^{6} \tilde{\mathcal{U}}_{1}-e^{-\frac{1}{A_{2}}\left(\tilde{\mathcal{Y}}_{2}(t, \eta)-\tilde{\mathcal{Y}}_{2}(t, \theta)\right)} A_{2}^{6} \tilde{\mathcal{U}}_{2}\right)(t, \theta) d \theta\right) d \eta \\
& =\frac{1}{4 A^{3}} \int_{0}^{1} \frac{1}{\max _{j}\left(\tilde{\mathcal{P}}_{j}^{1 / 2}\right)}\left(\tilde{\mathcal{P}}_{1}^{1 / 2}-\tilde{\mathcal{P}}_{2}^{1 / 2}\right)(t, \eta) \\
& \times\left(\left(A_{1}^{6}-A_{2}^{6}\right) \mathbb{1}_{A_{1}<A_{2}} \int_{0}^{\eta} e^{-\frac{1}{A_{2}}\left(\tilde{\mathcal{Y}}_{2}(t, \eta)-\tilde{\mathcal{Y}}_{2}(t, \theta)\right)} \tilde{\mathcal{U}}_{2}(t, \theta) d \theta\right. \\
& +\left(A_{1}^{6}-A_{2}^{2}\right) \mathbb{1}_{A_{2} \leqslant A_{1}} \int_{0}^{\eta} e^{-\frac{1}{A_{1}}\left(\tilde{\mathcal{Y}}_{1}(t, \eta)-\tilde{\mathcal{Y}}_{1}(t, \theta)\right)} \tilde{\mathcal{U}}_{1}(t, \theta) d \theta \\
& \left.+a^{6} \int_{0}^{\eta}\left(e^{-\frac{1}{A_{1}}\left(\tilde{\mathcal{Y}}_{1}(t, \eta)-\tilde{\mathcal{Y}}_{1}(t, \theta)\right)} \tilde{\mathcal{U}}_{1}-e^{-\frac{1}{A_{2}}\left(\tilde{\mathcal{Y}}_{2}(t, \eta)-\tilde{\mathcal{Y}}_{2}(t, \theta)\right)} \tilde{\mathcal{U}}_{2}\right)(t, \theta) d \theta\right) d \eta \\
& =M_{1}+M_{2}+M_{3} \text {. }
\end{aligned}
$$


The term $M_{1}\left(M_{2}\right.$ is similar $)$ can be estimated as follows:

$$
\begin{aligned}
\left|M_{1}\right| \leqslant & \frac{1}{4 A^{3}}\left|A_{1}^{6}-A_{2}^{6}\right| \mathbb{1}_{A_{1}<A_{2}} \int_{0}^{1} \frac{1}{\max _{j}\left(\tilde{\mathcal{P}}_{j}^{1 / 2}\right)}\left|\tilde{\mathcal{P}}_{1}^{1 / 2}-\tilde{\mathcal{P}}_{2}^{1 / 2}\right|(t, \eta) \\
& \times\left(\int_{0}^{\eta} e^{-\frac{1}{A_{2}}\left(\tilde{\mathcal{Y}}_{2}(t, \eta)-\tilde{\mathcal{Y}}_{2}(t, \theta)\right)}\left|\tilde{\mathcal{U}}_{2}\right|(t, \theta) d \theta\right) d \eta \\
\leqslant & \frac{3 A^{2}}{2}\left|A_{1}-A_{2}\right| \int_{0}^{1} \frac{1}{\max _{j}\left(\tilde{\mathcal{P}}_{j}^{1 / 2}\right)}\left|\tilde{\mathcal{P}}_{1}^{1 / 2}-\tilde{\mathcal{P}}_{2}^{1 / 2}\right|(t, \eta) \\
& \times\left(\int_{0}^{\eta} e^{-\frac{1}{A_{2}}\left(\tilde{\mathcal{Y}}_{2}(t, \eta)-\tilde{\mathcal{Y}}_{2}(t, \theta)\right)} \tilde{\mathcal{U}}_{2}^{2}(t, \theta) d \theta\right)^{1 / 2}\left(\int_{0}^{\eta} e^{-\frac{1}{A_{2}}\left(\tilde{\mathcal{Y}}_{2}(t, \eta)-\tilde{\mathcal{Y}}_{2}(t, \theta)\right)} d \theta\right)^{1 / 2} d \eta \\
\leqslant & \frac{3 \sqrt{3} A^{2}}{\sqrt{2}}\left|A_{1}-A_{2}\right| \int_{0}^{1}\left|\tilde{\mathcal{P}}_{1}^{1 / 2}-\tilde{\mathcal{P}}_{2}^{1 / 2}\right|(t, \eta) d \eta \\
\leqslant & \mathcal{O}(1)\left(\left\|\tilde{\mathcal{P}}_{1}^{1 / 2}-\tilde{\mathcal{P}}_{2}^{1 / 2}\right\|^{2}+\left|A_{1}-A_{2}\right|^{2}\right),
\end{aligned}
$$

using (4.16a). As for the next term $M_{3}$, we first write $\tilde{\mathcal{U}}_{j}=\tilde{\mathcal{U}}_{j}^{+}+\tilde{\mathcal{U}}_{j}^{-}$, collect terms, and study the positive part and the negative part separately. In the interest of the reader, we do not change the notation. Thus

$$
\begin{aligned}
& M_{3}=\frac{a^{6}}{4 A^{3}} \int_{0}^{1} \frac{1}{\max _{j}\left(\tilde{\mathcal{P}}_{j}^{1 / 2}\right)}\left(\tilde{\mathcal{P}}_{1}^{1 / 2}-\tilde{\mathcal{P}}_{2}^{1 / 2}\right)(t, \eta) \\
& \times \int_{0}^{\eta}\left(e^{-\frac{1}{A_{1}}\left(\tilde{\mathcal{Y}}_{1}(t, \eta)-\tilde{\mathcal{Y}}_{1}(t, \theta)\right)} \tilde{\mathcal{U}}_{1}^{+}-e^{-\frac{1}{A_{2}}\left(\tilde{\mathcal{Y}}_{2}(t, \eta)-\tilde{\mathcal{Y}}_{2}(t, \theta)\right)} \tilde{\mathcal{U}}_{2}^{+}\right)(t, \theta) d \theta d \eta \\
& =\frac{a^{6}}{4 A^{3}} \int_{0}^{1} \frac{1}{\max _{j}\left(\tilde{\mathcal{P}}_{j}^{1 / 2}\right)}\left(\tilde{\mathcal{P}}_{1}^{1 / 2}-\tilde{\mathcal{P}}_{2}^{1 / 2}\right)(t, \eta) \\
& \times\left(\int_{0}^{\eta} e^{-\frac{1}{A_{2}}\left(\tilde{\mathcal{Y}}_{2}(t, \eta)-\tilde{\mathcal{Y}}_{2}(t, \theta)\right)}\left(\mathbb{1}_{\tilde{\mathcal{U}}_{1}^{+}<\tilde{\mathcal{U}}_{2}^{+}}\left(\tilde{\mathcal{U}}_{1}^{+}-\tilde{\mathcal{U}}_{2}^{+}\right)\right)(t, \theta) d \theta\right. \\
& +\int_{0}^{\eta} e^{-\frac{1}{A_{1}}\left(\tilde{\mathcal{Y}}_{1}(t, \eta)-\tilde{\mathcal{Y}}_{1}(t, \theta)\right)}\left(\mathbb{1}_{\tilde{\mathcal{U}}_{2}^{+} \leqslant \tilde{\mathcal{U}}_{1}^{+}}\left(\tilde{\mathcal{U}}_{1}^{+}-\tilde{\mathcal{U}}_{2}^{+}\right)\right)(t, \theta) d \theta \\
& \left.+\int_{0}^{\eta}\left(e^{-\frac{1}{A_{1}}\left(\tilde{\mathcal{Y}}_{1}(t, \eta)-\tilde{\mathcal{Y}}_{1}(t, \theta)\right)}-e^{-\frac{1}{A_{2}}\left(\tilde{\mathcal{Y}}_{2}(t, \eta)-\tilde{\mathcal{Y}}_{2}(t, \theta)\right)}\right) \min _{j}\left(\tilde{\mathcal{U}}_{j}^{+}\right)(t, \theta) d \theta\right) d \eta \\
& =M_{31}+M_{32}+M_{33} \text {. }
\end{aligned}
$$

The terms $M_{31}$ and $M_{32}$ can be treated similarly. Thus

$$
\begin{aligned}
\left|M_{31}\right|= & \frac{a^{6}}{4 A^{3}} \mid \int_{0}^{1} \frac{1}{\max _{j}\left(\tilde{\mathcal{P}}_{j}^{1 / 2}\right)}\left(\tilde{\mathcal{P}}_{1}^{1 / 2}-\tilde{\mathcal{P}}_{2}^{1 / 2}\right)(t, \eta) \\
& \times\left(\int_{0}^{\eta} e^{-\frac{1}{A_{2}}\left(\tilde{\mathcal{Y}}_{2}(t, \eta)-\tilde{\mathcal{Y}}_{2}(t, \theta)\right)}\left(\mathbb{1}_{\tilde{\mathcal{U}}_{1}^{+}<\tilde{\mathcal{U}}_{2}^{+}}\left(\tilde{\mathcal{U}}_{1}^{+}-\tilde{\mathcal{U}}_{2}^{+}\right)\right)(t, \theta) d \theta\right) d \eta \mid
\end{aligned}
$$




$$
\begin{aligned}
\leqslant & \frac{1}{4 A^{2}} \int_{0}^{1} \frac{1}{\max _{j}\left(\tilde{\mathcal{P}}_{j}^{1 / 2}\right)}\left|\tilde{\mathcal{P}}_{1}^{1 / 2}-\tilde{\mathcal{P}}_{2}^{1 / 2}\right|(t, \eta) \\
& \times\left(\int_{0}^{\eta} e^{-\frac{1}{A_{2}}\left(\tilde{\mathcal{Y}}_{2}(t, \eta)-\tilde{\mathcal{Y}}_{2}(t, \theta)\right)} A_{2}^{5}\left|\tilde{\mathcal{U}}_{1}^{+}-\tilde{\mathcal{U}}_{2}^{+}\right|(t, \theta) d \theta\right) d \eta \\
\leqslant & \mathcal{O}(1) \int_{0}^{1} \frac{1}{\max _{j}\left(\tilde{\mathcal{P}}_{j}^{1 / 2}\right)}\left|\tilde{\mathcal{P}}_{1}^{1 / 2}-\tilde{\mathcal{P}}_{2}^{1 / 2}\right| \tilde{\mathcal{P}}_{2}^{1 / 2}(t, \eta)\left\|\tilde{\mathcal{U}}_{1}-\tilde{\mathcal{U}}_{2}\right\| d \eta \\
\leqslant & \mathcal{O}(1) \int_{0}^{1}\left|\tilde{\mathcal{P}}_{1}^{1 / 2}-\tilde{\mathcal{P}}_{2}^{1 / 2}\right|(t, \eta)\left\|\tilde{\mathcal{U}}_{1}-\tilde{\mathcal{U}}_{2}\right\| d \eta \\
\leqslant & \mathcal{O}(1)\left(\left\|\tilde{\mathcal{P}}_{1}^{1 / 2}-\tilde{\mathcal{P}}_{2}^{1 / 2}\right\|^{2}+\left\|\tilde{\mathcal{U}}_{1}-\tilde{\mathcal{U}}_{2}\right\|^{2}\right),
\end{aligned}
$$

using

$$
\begin{aligned}
& a^{5} \int_{0}^{\eta} e^{-\frac{1}{A_{2}}\left(\tilde{\mathcal{Y}}_{2}(t, \eta)-\tilde{\mathcal{Y}}_{2}(t, \theta)\right)}\left|\tilde{\mathcal{U}}_{1}^{+}-\tilde{\mathcal{U}}_{2}^{+}\right|(t, \theta) d \theta \\
& \leqslant \int_{0}^{\eta} e^{-\frac{1}{A_{2}}\left(\tilde{\mathcal{Y}}_{2}(t, \eta)-\tilde{\mathcal{Y}}_{2}(t, \theta)\right)} A_{2}^{5}\left|\tilde{\mathcal{U}}_{1}-\tilde{\mathcal{U}}_{2}\right|(t, \theta) d \theta \\
& =\int_{0}^{\eta} e^{-\frac{1}{A_{2}}\left(\tilde{\mathcal{Y}}_{2}(t, \eta)-\tilde{\mathcal{Y}}_{2}(t, \theta)\right)}\left(\left(2 \tilde{\mathcal{P}}_{2}-\tilde{\mathcal{U}}_{2}^{2}\right) \tilde{\mathcal{Y}}_{2, \eta}+\tilde{\mathcal{H}}_{2, \eta}\right)\left|\tilde{\mathcal{U}}_{1}-\tilde{\mathcal{U}}_{2}\right|(t, \theta) d \theta \\
& =2 \int_{0}^{\eta} e^{-\frac{1}{A_{2}}\left(\tilde{\mathcal{Y}}_{2}(t, \eta)-\tilde{\mathcal{Y}}_{2}(t, \theta)\right)} \tilde{\mathcal{P}}_{2} \tilde{\mathcal{Y}}_{2, \eta}\left|\tilde{\mathcal{U}}_{1}-\tilde{\mathcal{U}}_{2}\right|(t, \theta) d \theta \\
& +\int_{0}^{\eta} e^{-\frac{1}{A_{2}}\left(\tilde{\mathcal{Y}}_{2}(t, \eta)-\tilde{\mathcal{Y}}_{2}(t, \theta)\right)} \tilde{\mathcal{H}}_{2, \eta}\left|\tilde{\mathcal{U}}_{1}-\tilde{\mathcal{U}}_{2}\right|(t, \theta) d \theta \\
& -\int_{0}^{\eta} e^{-\frac{1}{A_{2}}\left(\tilde{\mathcal{Y}}_{2}(t, \eta)-\tilde{\mathcal{Y}}_{2}(t, \theta)\right)} \tilde{\mathcal{U}}_{2}^{2} \tilde{\mathcal{Y}}_{2, \eta}\left|\tilde{\mathcal{U}}_{1}-\tilde{\mathcal{U}}_{2}\right|(t, \theta) d \theta \\
& \leqslant 2 \int_{0}^{\eta} e^{-\frac{1}{A_{2}}\left(\tilde{\mathcal{Y}}_{2}(t, \eta)-\tilde{\mathcal{Y}}_{2}(t, \theta)\right)} \tilde{\mathcal{P}}_{2} \tilde{\mathcal{Y}}_{2, \eta}\left|\tilde{\mathcal{U}}_{1}-\tilde{\mathcal{U}}_{2}\right|(t, \theta) d \theta \\
& +\int_{0}^{\eta} e^{-\frac{1}{A_{2}}\left(\tilde{\mathcal{Y}}_{2}(t, \eta)-\tilde{\mathcal{Y}}_{2}(t, \theta)\right)} \tilde{\mathcal{H}}_{2, \eta}\left|\tilde{\mathcal{U}}_{1}-\tilde{\mathcal{U}}_{2}\right|(t, \theta) d \theta \\
& \leqslant 2\left(\int_{0}^{\eta} e^{-\frac{3}{2 A_{2}}\left(\tilde{\mathcal{Y}}_{2}(t, \eta)-\tilde{\mathcal{Y}}_{2}(t, \theta)\right)} \tilde{\mathcal{P}}_{2} \tilde{\mathcal{Y}}_{2, \eta}(t, \theta) d \theta\right)^{1 / 2} \\
& \times\left(\int_{0}^{\eta} e^{-\frac{1}{2 A_{2}}\left(\tilde{\mathcal{Y}}_{2}(t, \eta)-\tilde{\mathcal{Y}}_{2}(t, \theta)\right)}\left(\tilde{\mathcal{U}}_{1}-\tilde{\mathcal{U}}_{2}\right)^{2} \tilde{\mathcal{P}}_{2} \tilde{\mathcal{Y}}_{2, \eta}(t, \theta) d \theta\right)^{1 / 2} \\
& +\left(\int_{0}^{\eta} e^{-\frac{1}{A_{2}}\left(\tilde{\mathcal{Y}}_{2}(t, \eta)-\tilde{\mathcal{Y}}_{2}(t, \theta)\right)} \tilde{\mathcal{H}}_{2, \eta}(t, \theta) d \theta\right)^{1 / 2}
\end{aligned}
$$




$$
\begin{aligned}
& \times\left(\int_{0}^{\eta} e^{-\frac{1}{A_{2}}\left(\tilde{\mathcal{Y}}_{2}(t, \eta)-\tilde{\mathcal{Y}}_{2}(t, \theta)\right)}\left(\tilde{\mathcal{U}}_{1}-\tilde{\mathcal{U}}_{2}\right)^{2} \tilde{\mathcal{H}}_{2, \eta}(t, \theta) d \theta\right)^{1 / 2} \\
\leqslant & \mathcal{O}(1) A^{3} \tilde{\mathcal{P}}_{2}^{1 / 2}(t, \eta)\left\|\tilde{\mathcal{U}}_{1}-\tilde{\mathcal{U}}_{2}\right\|,
\end{aligned}
$$

using (4.13), (4.15e), (4.15k), (4.16d), and (4.16e). The term $M_{33}$ goes as follows:

$$
\begin{aligned}
& M_{33}=\frac{a^{6}}{4 A^{3}} \int_{0}^{1} \frac{1}{\max _{j}\left(\tilde{\mathcal{P}}_{j}^{1 / 2}\right)}\left(\tilde{\mathcal{P}}_{1}^{1 / 2}-\tilde{\mathcal{P}}_{2}^{1 / 2}\right)(t, \eta) \\
& \times\left(\int_{0}^{\eta}\left(e^{-\frac{1}{A_{1}}\left(\tilde{\mathcal{Y}}_{1}(t, \eta)-\tilde{\mathcal{Y}}_{1}(t, \theta)\right)}-e^{-\frac{1}{A_{2}}\left(\tilde{\mathcal{Y}}_{2}(t, \eta)-\tilde{\mathcal{Y}}_{2}(t, \theta)\right)}\right)\right. \\
& \left.\times \min _{j}\left(\tilde{\mathcal{U}}_{j}^{+}\right)(t, \theta) d \theta\right) d \eta \\
& =\frac{a^{6}}{4 A^{3}} \int_{0}^{1} \frac{1}{\max _{j}\left(\tilde{\mathcal{P}}_{j}^{1 / 2}\right)}\left(\tilde{\mathcal{P}}_{1}^{1 / 2}-\tilde{\mathcal{P}}_{2}^{1 / 2}\right)(t, \eta) \\
& \times \mathbb{1}_{A_{1} \leqslant A_{2}}\left(\int_{0}^{\eta}\left(e^{-\frac{1}{A_{1}}\left(\tilde{\mathcal{Y}}_{2}(t, \eta)-\tilde{\mathcal{Y}}_{2}(t, \theta)\right)}-e^{-\frac{1}{A_{2}}\left(\tilde{\mathcal{Y}}_{2}(t, \eta)-\tilde{\mathcal{Y}}_{2}(t, \theta)\right)}\right)\right. \\
& \left.\times \min _{j}\left(\tilde{\mathcal{U}}_{j}^{+}\right)(t, \theta) d \theta\right) d \eta \\
& +\frac{a^{6}}{4 A^{3}} \int_{0}^{1} \frac{1}{\max _{j}\left(\tilde{\mathcal{P}}_{j}^{1 / 2}\right)}\left(\tilde{\mathcal{P}}_{1}^{1 / 2}-\tilde{\mathcal{P}}_{2}^{1 / 2}\right)(t, \eta) \\
& \times \mathbb{1}_{A_{2}<A_{1}}\left(\int_{0}^{\eta}\left(e^{-\frac{1}{A_{1}}\left(\tilde{\mathcal{Y}}_{1}(t, \eta)-\tilde{\mathcal{Y}}_{1}(t, \theta)\right)}-e^{-\frac{1}{A_{2}}\left(\tilde{\mathcal{Y}}_{1}(t, \eta)-\tilde{\mathcal{Y}}_{1}(t, \theta)\right)}\right)\right. \\
& \left.\times \min _{j}\left(\tilde{\mathcal{U}}_{j}^{+}\right)(t, \theta) d \theta\right) d \eta \\
& +\frac{a^{6}}{4 A^{3}} \int_{0}^{1} \frac{1}{\max _{j}\left(\tilde{\mathcal{P}}_{j}^{1 / 2}\right)}\left(\tilde{\mathcal{P}}_{1}^{1 / 2}-\tilde{\mathcal{P}}_{2}^{1 / 2}\right)(t, \eta) \\
& \times\left(\int_{0}^{\eta}\left(e^{-\frac{1}{a}\left(\tilde{\mathcal{Y}}_{1}(t, \eta)-\tilde{\mathcal{Y}}_{1}(t, \theta)\right)}-e^{-\frac{1}{a}\left(\tilde{\mathcal{Y}}_{2}(t, \eta)-\tilde{\mathcal{Y}}_{2}(t, \theta)\right)}\right) \mathbb{1}_{B(\eta)}\right. \\
& \left.\times \min _{j}\left(\tilde{\mathcal{U}}_{j}^{+}\right)(t, \theta) d \theta\right) d \eta \\
& +\frac{a^{6}}{4 A^{3}} \int_{0}^{1} \frac{1}{\max _{j}\left(\tilde{\mathcal{P}}_{j}^{1 / 2}\right)}\left(\tilde{\mathcal{P}}_{1}^{1 / 2}-\tilde{\mathcal{P}}_{2}^{1 / 2}\right)(t, \eta) \\
& \times\left(\int_{0}^{\eta}\left(e^{-\frac{1}{a}\left(\tilde{\mathcal{Y}}_{1}(t, \eta)-\tilde{\mathcal{Y}}_{1}(t, \theta)\right)}-e^{-\frac{1}{a}\left(\tilde{\mathcal{Y}}_{2}(t, \eta)-\tilde{\mathcal{Y}}_{2}(t, \theta)\right)}\right) \mathbb{1}_{B(\eta)^{c}}\right.
\end{aligned}
$$




$$
\begin{aligned}
& \left.\times \min _{j}\left(\tilde{\mathcal{U}}_{j}^{+}\right)(t, \theta) d \theta\right) d \eta \\
= & M_{331}+M_{332}+M_{333}+M_{334},
\end{aligned}
$$

where $B(\eta)$ is defined by (5.11). The terms $M_{331}$ and $M_{332}$ can be treated in the same manner. More specifically,

$$
\begin{aligned}
\left|M_{331}\right| \leqslant & \frac{A^{2}}{e} \int_{0}^{1} \frac{1}{\max _{j}\left(\tilde{\mathcal{P}}_{j}^{1 / 2}\right)}\left|\tilde{\mathcal{P}}_{1}^{1 / 2}-\tilde{\mathcal{P}}_{2}^{1 / 2}\right|(t, \eta) \\
& \times\left(\int_{0}^{\eta} e^{-\frac{3}{4 A_{2}}\left(\tilde{\mathcal{Y}}_{2}(t, \eta)-\tilde{\mathcal{Y}}_{2}(t, \theta)\right)} \tilde{\mathcal{U}}_{2}^{+}(t, \theta) d \theta\right) d \eta\left|A_{1}-A_{2}\right| \\
\leqslant & \frac{A^{2}}{e} \int_{0}^{1} \frac{1}{\max _{j}\left(\tilde{\mathcal{P}}_{j}^{1 / 2}\right)}\left|\tilde{\mathcal{P}}_{1}^{1 / 2}-\tilde{\mathcal{P}}_{2}^{1 / 2}\right|(t, \eta) \\
& \times\left(\int_{0}^{\eta} e^{-\frac{1}{A_{2}}\left(\tilde{\mathcal{Y}}_{2}(t, \eta)-\tilde{\mathcal{Y}}_{2}(t, \theta)\right)} \tilde{\mathcal{U}}_{2}^{2}(t, \theta) d \theta\right)^{1 / 2} d \eta\left|A_{1}-A_{2}\right| \\
\leqslant & \mathcal{O}(1)\left(\left\|\tilde{\mathcal{P}}_{1}^{1 / 2}-\tilde{\mathcal{P}}_{2}^{1 / 2}\right\|^{2}+\left|A_{1}-A_{2}\right|^{2}\right) .
\end{aligned}
$$

The terms $M_{333}$ and $M_{334}$ can be treated in a similar manner. More specifically,

$$
\begin{aligned}
& \left|M_{333}\right| \leqslant \frac{a^{6}}{4 A^{3}} \int_{0}^{1} \frac{1}{\max _{j}\left(\tilde{\mathcal{P}}_{j}^{1 / 2}\right)}\left|\tilde{\mathcal{P}}_{1}^{1 / 2}-\tilde{\mathcal{P}}_{2}^{1 / 2}\right|(t, \eta) \\
& \times\left(\int_{0}^{\eta}\left|e^{-\frac{1}{a}\left(\tilde{\mathcal{Y}}_{1}(t, \eta)-\tilde{\mathcal{Y}}_{1}(t, \theta)\right)}-e^{-\frac{1}{a}\left(\tilde{\mathcal{Y}}_{2}(t, \eta)-\tilde{\mathcal{Y}}_{2}(t, \theta)\right)}\right| \mathbb{1}_{B(\eta)}\right. \\
& \left.\times \min _{j}\left(\tilde{\mathcal{U}}_{j}^{+}\right)(t, \theta) d \theta\right) d \eta \\
& \leqslant \frac{A^{2}}{4} \int_{0}^{1} \frac{1}{\max _{j}\left(\tilde{\mathcal{P}}_{j}^{1 / 2}\right)}\left|\tilde{\mathcal{P}}_{1}^{1 / 2}-\tilde{\mathcal{P}}_{2}^{1 / 2}\right|(t, \eta)\left(\int_{0}^{\eta} e^{-\frac{1}{A_{2}}\left(\tilde{\mathcal{V}}_{2}(t, \eta)-\tilde{\mathcal{Y}}_{2}(t, \theta)\right)}\right. \\
& \left.\times\left(\left|\tilde{\mathcal{Y}}_{2}(t, \eta)-\tilde{\mathcal{Y}}_{1}(t, \eta)\right|+\left|\tilde{\mathcal{Y}}_{2}(t, \theta)-\tilde{\mathcal{Y}}_{1}(t, \theta)\right|\right) \min _{j}\left(\tilde{\mathcal{U}}_{j}^{+}\right)(t, \theta) d \theta\right) d \eta \\
& \leqslant \frac{A^{2}}{4} \int_{0}^{1} \frac{1}{\max _{j}\left(\tilde{\mathcal{P}}_{j}^{1 / 2}\right)}\left|\tilde{\mathcal{P}}_{1}^{1 / 2}-\tilde{\mathcal{P}}_{2}^{1 / 2}\right|\left|\tilde{\mathcal{Y}}_{2}-\tilde{\mathcal{Y}}_{1}\right|(t, \eta) \\
& \times\left(\int_{0}^{\eta} e^{-\frac{1}{A_{2}}\left(\tilde{\mathcal{Y}}_{2}(t, \eta)-\tilde{\mathcal{Y}}_{2}(t, \theta)\right)} \mathbb{1}_{B(\eta)} \min _{j}\left(\tilde{\mathcal{U}}_{j}^{+}\right)(t, \theta) d \theta\right) d \eta \\
& +\frac{A^{2}}{4} \int_{0}^{1} \frac{1}{\max _{j}\left(\tilde{\mathcal{P}}_{j}^{1 / 2}\right)}\left|\tilde{\mathcal{P}}_{1}^{1 / 2}-\tilde{\mathcal{P}}_{2}^{1 / 2}\right|(t, \eta) \\
& \times\left(\int_{0}^{\eta} e^{-\frac{1}{A_{2}}\left(\tilde{\mathcal{Y}}_{2}(t, \eta)-\tilde{\mathcal{Y}}_{2}(t, \theta)\right)}\left|\tilde{\mathcal{Y}}_{2}-\tilde{\mathcal{Y}}_{1}\right| \min _{j}\left(\tilde{\mathcal{U}}_{j}^{+}\right)(t, \theta) d \theta\right) d \eta
\end{aligned}
$$




$$
\begin{aligned}
\leqslant & \frac{A^{2}}{4} \int_{0}^{1} \frac{1}{\max _{j}\left(\tilde{\mathcal{P}}_{j}^{1 / 2}\right)}\left|\tilde{\mathcal{P}}_{1}^{1 / 2}-\tilde{\mathcal{P}}_{2}^{1 / 2}\right|\left|\tilde{\mathcal{Y}}_{2}-\tilde{\mathcal{Y}}_{1}\right|(t, \eta) \\
& \times\left(\int_{0}^{\eta} e^{-\frac{1}{A_{2}}\left(\tilde{\mathcal{Y}}_{2}(t, \eta)-\tilde{\mathcal{Y}}_{2}(t, \theta)\right)} \tilde{\mathcal{Y}}_{2}^{2}(t, \theta) d \theta\right)^{1 / 2} \\
& \times\left(\int_{0}^{\eta} e^{-\frac{1}{A_{2}}\left(\tilde{\mathcal{Y}}_{2}(t, \eta)-\tilde{\mathcal{Y}}_{2}(t, \theta)\right)} d \theta\right)^{1 / 2} d \eta \\
& +\frac{A^{2}}{4} \int_{0}^{1} \frac{1}{\max _{j}\left(\tilde{\mathcal{P}}_{j}^{1 / 2}\right)}\left|\tilde{\mathcal{P}}_{1}^{1 / 2}-\tilde{\mathcal{P}}_{2}^{1 / 2}\right|(t, \eta) \\
& \times\left(\int_{0}^{\eta} e^{-\frac{1}{A_{2}}\left(\tilde{\mathcal{Y}}_{2}(t, \eta)-\tilde{\mathcal{Y}}_{2}(t, \theta)\right)} \tilde{\mathcal{U}}_{2}^{2}(t, \theta) d \theta\right)^{1 / 2} \\
& \times\left(\int_{0}^{\eta} e^{-\frac{1}{A_{2}}\left(\tilde{\mathcal{Y}}_{2}(t, \eta)-\tilde{\mathcal{Y}}_{2}(t, \theta)\right)}\left(\tilde{\mathcal{Y}}_{2}-\tilde{\mathcal{Y}}_{1}\right)^{2}(t, \theta)\right)^{1 / 2} d \eta \\
\leqslant & \mathcal{O}(1) \int_{0}^{1} \frac{1}{\max _{j}\left(\tilde{\mathcal{P}}_{j}^{1 / 2}\right)}\left|\tilde{\mathcal{P}}_{1}^{1 / 2}-\tilde{\mathcal{P}}_{2}^{1 / 2}\right|\left|\tilde{\mathcal{Y}}_{2}-\tilde{\mathcal{Y}}_{1}\right| \tilde{\mathcal{P}}_{2}^{1 / 2}(t, \eta) d \eta \\
& +\mathcal{O}(1) \int_{0}^{1} \frac{1}{\max _{j}\left(\tilde{\mathcal{P}}_{j}^{1 / 2}\right)}\left|\tilde{\mathcal{P}}_{1}^{1 / 2}-\tilde{\mathcal{P}}_{2}^{1 / 2}\right|\left\|\tilde{\mathcal{Y}}_{2}-\tilde{\mathcal{Y}}_{1}\right\| \tilde{\mathcal{P}}_{2}^{1 / 2}(t, \eta) d \eta \\
& \mathcal{O}(1)\left(\left\|\tilde{\mathcal{P}}_{1}^{1 / 2}-\tilde{\mathcal{P}}_{2}^{1 / 2}\right\|^{2}+\left\|\tilde{\mathcal{Y}}_{2}-\tilde{\mathcal{Y}}_{1}\right\|^{2}\right),
\end{aligned}
$$

using (4.16a) and (5.13).

And the final term from $J_{3}(\mathrm{cf} .(5.46))$ can be estimated as follows:

$$
\begin{aligned}
\tilde{J}_{13}= & \frac{1}{2 A^{3}} \int_{0}^{1} \frac{1}{\max _{j}\left(\tilde{\mathcal{P}}_{j}^{1 / 2}\right)}\left(\tilde{\mathcal{P}}_{1}^{1 / 2}-\tilde{\mathcal{P}}_{2}^{1 / 2}\right)(t, \eta)\left(\int _ { 0 } ^ { \eta } \left(e^{-\frac{1}{A_{2}}\left(\tilde{\mathcal{Y}}_{2}(t, \eta)-\tilde{\mathcal{Y}}_{2}(t, \theta)\right)} \tilde{\mathcal{U}}_{2} \tilde{\mathcal{Q}}_{2} \tilde{\mathcal{Y}}_{2, \eta}\right.\right. \\
& \left.\left.-e^{-\frac{1}{A_{1}}\left(\tilde{\mathcal{Y}}_{1}(t, \eta)-\tilde{\mathcal{Y}}_{1}(t, \theta)\right)} \tilde{\mathcal{U}}_{1} \tilde{\mathcal{Q}}_{1} \tilde{\mathcal{Y}}_{1, \eta}\right)(t, \theta) d \theta\right) d \eta
\end{aligned}
$$

We introduce the positive and negative parts of $\tilde{\mathcal{U}}_{j}$, that is, $\tilde{\mathcal{U}}_{j}=\tilde{\mathcal{U}}_{j}^{+}+\tilde{\mathcal{U}}_{j}^{-}$(see (5.1)) and introduce $\tilde{\mathcal{Q}}_{j}=A_{j} \tilde{\mathcal{P}}_{j}-\tilde{\mathcal{D}}_{j}$. We study the term with $\tilde{\mathcal{P}}_{j}$ first:

$$
\begin{aligned}
\tilde{J}_{131}= & \frac{1}{2 A^{3}} \int_{0}^{1} \frac{1}{\max _{j}\left(\tilde{\mathcal{P}}_{j}^{1 / 2}\right)}\left(\tilde{\mathcal{P}}_{1}^{1 / 2}-\tilde{\mathcal{P}}_{2}^{1 / 2}\right)(t, \eta) \\
& \times\left(\int _ { 0 } ^ { \eta } \left(e^{-\frac{1}{A_{2}}\left(\tilde{\mathcal{Y}}_{2}(t, \eta)-\tilde{\mathcal{Y}}_{2}(t, \theta)\right)} A_{2} \tilde{\mathcal{U}}_{2}^{+} \tilde{\mathcal{P}}_{2} \tilde{\mathcal{Y}}_{2, \eta}\right.\right. \\
& \left.\left.-e^{-\frac{1}{A_{1}}\left(\tilde{\mathcal{Y}}_{1}(t, \eta)-\tilde{\mathcal{Y}}_{1}(t, \theta)\right)} A_{1} \tilde{\mathcal{U}}_{1}^{+} \tilde{\mathcal{P}}_{1} \tilde{\mathcal{Y}}_{1, \eta}\right)(t, \theta) d \theta\right) d \eta
\end{aligned}
$$




$$
\begin{aligned}
& =\frac{1}{2 A^{3}} \int_{0}^{1} \frac{1}{\max _{j}\left(\tilde{\mathcal{P}}_{j}^{1 / 2}\right)}\left(\tilde{\mathcal{P}}_{1}^{1 / 2}-\tilde{\mathcal{P}}_{2}^{1 / 2}\right)(t, \eta) \\
& \times\left[\mathbb{1}_{A_{1} \leqslant A_{2}}\left(A_{2}-A_{1}\right) \int_{0}^{\eta} e^{-\frac{1}{A_{2}}\left(\tilde{\mathcal{Y}}_{2}(t, \eta)-\tilde{\mathcal{Y}}_{2}(t, \theta)\right)} \tilde{\mathcal{U}}_{2}^{+} \tilde{\mathcal{P}}_{2} \tilde{\mathcal{Y}}_{2, \eta}(t, \theta) d \theta\right. \\
& +\mathbb{1}_{A_{2}<A_{1}}\left(A_{2}-A_{1}\right) \int_{0}^{\eta} e^{-\frac{1}{A_{1}}\left(\tilde{\mathcal{Y}}_{1}(t, \eta)-\tilde{\mathcal{Y}}_{1}(t, \theta)\right)} \tilde{\mathcal{U}}_{1}^{+} \tilde{\mathcal{P}}_{1} \tilde{\mathcal{Y}}_{1, \eta}(t, \theta) d \theta \\
& +a \int_{0}^{\eta} e^{-\frac{1}{A_{2}}\left(\tilde{\mathcal{Y}}_{2}(t, \eta)-\tilde{\mathcal{Y}}_{2}(t, \theta)\right)}\left(\tilde{\mathcal{P}}_{2}-\tilde{\mathcal{P}}_{1}\right) \mathbb{1}_{\tilde{\mathcal{P}}_{1<\tilde{\mathcal{P}}_{2}}} \tilde{\mathcal{U}}_{2}^{+} \tilde{\mathcal{Y}}_{2, \eta}(t, \theta) d \theta \\
& +a \int_{0}^{\eta} e^{-\frac{1}{A_{1}}\left(\tilde{\mathcal{Y}}_{1}(t, \eta)-\tilde{\mathcal{Y}}_{1}(t, \theta)\right)}\left(\tilde{\mathcal{P}}_{2}-\tilde{\mathcal{P}}_{1}\right) \mathbb{1}_{\tilde{\mathcal{P}}_{2} \leqslant \tilde{\mathcal{P}}_{1}} \tilde{\mathcal{U}}_{1}^{+} \tilde{\mathcal{Y}}_{1, \eta}(t, \theta) d \theta \\
& +a \int_{0}^{\eta} e^{-\frac{1}{A_{2}}\left(\tilde{\mathcal{Y}}_{2}(t, \eta)-\tilde{\mathcal{Y}}_{2}(t, \theta)\right)} \min _{j}\left(\tilde{\mathcal{P}}_{j}\right)\left(\tilde{\mathcal{U}}_{2}^{+}-\tilde{\mathcal{U}}_{1}^{+}\right) \mathbb{1}_{\tilde{\mathcal{U}}_{1}^{+}<\tilde{\mathcal{U}}_{2}^{+}} \tilde{\mathcal{Y}}_{2, \eta}(t, \theta) d \theta \\
& +a \int_{0}^{\eta} e^{-\frac{1}{A_{1}}\left(\tilde{\mathcal{Y}}_{1}(t, \eta)-\tilde{\mathcal{Y}}_{1}(t, \theta)\right)} \min _{j}\left(\tilde{\mathcal{P}}_{j}\right)\left(\tilde{\mathcal{U}}_{2}^{+}-\tilde{\mathcal{U}}_{1}^{+}\right) \mathbb{1}_{\tilde{\mathcal{U}}_{2}^{+} \leqslant \tilde{\mathcal{U}}_{1}^{+}} \tilde{\mathcal{Y}}_{1, \eta}(t, \theta) d \theta \\
& +a \mathbb{1}_{A_{1} \leqslant A_{2}} \int_{0}^{\eta}\left(e^{-\frac{1}{A_{2}}\left(\tilde{\mathcal{Y}}_{2}(t, \eta)-\tilde{\mathcal{Y}}_{2}(t, \theta)\right)}-e^{-\frac{1}{A_{1}}\left(\tilde{\mathcal{Y}}_{2}(t, \eta)-\tilde{\mathcal{Y}}_{2}(t, \theta)\right)}\right) \\
& \times \min _{j}\left(\tilde{\mathcal{P}}_{j}\right) \min _{j}\left(\tilde{\mathcal{U}}_{j}^{+}\right) \tilde{\mathcal{Y}}_{2, \eta}(t, \theta) d \theta \\
& +a \mathbb{1}_{A_{2}<A_{1}} \int_{0}^{\eta}\left(e^{-\frac{1}{A_{2}}\left(\tilde{\mathcal{Y}}_{1}(t, \eta)-\tilde{\mathcal{Y}}_{1}(t, \theta)\right)}-e^{-\frac{1}{A_{1}}\left(\tilde{\mathcal{Y}}_{1}(t, \eta)-\tilde{\mathcal{Y}}_{1}(t, \theta)\right)}\right) \\
& \times \min _{j}\left(\tilde{\mathcal{P}}_{j}\right) \min _{j}\left(\tilde{\mathcal{U}}_{j}^{+}\right) \tilde{\mathcal{Y}}_{1, \eta}(t, \theta) d \theta \\
& +a \int_{0}^{\eta}\left(e^{-\frac{1}{a}\left(\tilde{\mathcal{Y}}_{2}(t, \eta)-\tilde{\mathcal{Y}}_{2}(t, \theta)\right)}-e^{-\frac{1}{a}\left(\tilde{\mathcal{Y}}_{1}(t, \eta)-\tilde{\mathcal{Y}}_{1}(t, \theta)\right)}\right) \mathbb{1}_{B(\eta)} \\
& \times \min _{j}\left(\tilde{\mathcal{P}}_{j}\right) \min _{j}\left(\tilde{\mathcal{U}}_{j}^{+}\right) \tilde{\mathcal{Y}}_{2, \eta}(t, \theta) d \theta \\
& +a \int_{0}^{\eta}\left(e^{-\frac{1}{a}\left(\tilde{Y}_{2}(t, \eta)-\tilde{\mathcal{Y}}_{2}(t, \theta)\right)}-e^{-\frac{1}{a}\left(\tilde{Y}_{1}(t, \eta)-\tilde{Y}_{1}(t, \theta)\right)}\right) \mathbb{1}_{B(\eta)^{c}} \\
& \times \min _{j}\left(\tilde{\mathcal{P}}_{j}\right) \min _{j}\left(\tilde{\mathcal{U}}_{j}^{+}\right) \tilde{\mathcal{Y}}_{1, \eta}(t, \theta) d \theta \\
& +a \int_{0}^{\eta} \min _{j}\left(e^{-\frac{1}{a}\left(\tilde{\mathcal{Y}}_{j}(t, \eta)-\tilde{\mathcal{Y}}_{j}(t, \theta)\right)}\right) \\
& \left.\times \min _{j}\left(\tilde{\mathcal{P}}_{j}\right) \min _{j}\left(\tilde{\mathcal{U}}_{j}^{+}\right)\left(\tilde{\mathcal{Y}}_{2, \eta}-\tilde{\mathcal{Y}}_{1, \eta}\right)(t, \theta) d \theta\right] d \eta \\
& =K_{1}+K_{2}+K_{3}+K_{4}+K_{5}+K_{6}+K_{7}+K_{8}+K_{9}+K_{10}+K_{11} \text {. }
\end{aligned}
$$


The terms $K_{1}$ and $K_{2}$ can be treated similarly. Thus

$$
\begin{aligned}
\left|K_{1}\right|= & \frac{1}{2 A^{3}} \mid \int_{0}^{1} \frac{1}{\max _{j}\left(\tilde{\mathcal{P}}_{j}^{1 / 2}\right)}\left(\tilde{\mathcal{P}}_{1}^{1 / 2}-\tilde{\mathcal{P}}_{2}^{1 / 2}\right)(t, \eta) \\
& \times\left(\mathbb{1}_{A_{1} \leqslant A_{2}}\left(A_{2}-A_{1}\right) \int_{0}^{\eta} e^{-\frac{1}{A_{2}}\left(\tilde{\mathcal{Y}}_{2}(t, \eta)-\tilde{\mathcal{Y}}_{2}(t, \theta)\right)} \tilde{\mathcal{U}}_{2}^{+} \tilde{\mathcal{P}}_{2} \tilde{\mathcal{Y}}_{2, \eta}(t, \theta) d \theta\right) d \eta \mid \\
\leqslant & \frac{1}{2 A^{3}} \int_{0}^{1} \frac{1}{\max _{j}\left(\tilde{\mathcal{P}}_{j}^{1 / 2}\right)}\left|\tilde{\mathcal{P}}_{1}^{1 / 2}-\tilde{\mathcal{P}}_{2}^{1 / 2}\right|(t, \eta) \\
& \times\left(\int_{0}^{\eta} e^{-\frac{1}{A_{2}}\left(\tilde{\mathcal{Y}}_{2}(t, \eta)-\tilde{\mathcal{Y}}_{2}(t, \theta)\right)} \tilde{\mathcal{P}}_{2}^{2} \tilde{\mathcal{Y}}_{2, \eta}(t, \theta) d \theta\right)^{1 / 2} \\
& \times\left(\int_{0}^{\eta} e^{-\frac{1}{A_{2}}\left(\tilde{\mathcal{Y}}_{2}(t, \eta)-\tilde{\mathcal{Y}}_{2}(t, \theta)\right)} \tilde{\mathcal{U}}_{2}^{2} \tilde{\mathcal{Y}}_{2, \eta}(t, \theta) d \theta\right)^{1 / 2} d \eta\left|A_{1}-A_{2}\right| \\
\leqslant & \mathcal{O}(1)\left(\left\|\tilde{\mathcal{P}}_{1}^{1 / 2}-\tilde{\mathcal{P}}_{2}^{1 / 2}\right\|^{2}+\left|A_{1}-A_{2}\right|^{2}\right) .
\end{aligned}
$$

The terms $K_{3}$ and $K_{4}$ can be treated similarly. Thus

$$
\begin{aligned}
\left|K_{3}\right|= & \frac{a}{2 A^{3}} \mid \int_{0}^{1} \frac{1}{\max _{j}\left(\tilde{\mathcal{P}}_{j}^{1 / 2}\right)}\left(\tilde{\mathcal{P}}_{1}^{1 / 2}-\tilde{\mathcal{P}}_{2}^{1 / 2}\right)(t, \eta) \\
& \times\left(\int_{0}^{\eta} e^{-\frac{1}{A_{2}}\left(\tilde{\mathcal{Y}}_{2}(t, \eta)-\tilde{\mathcal{Y}}_{2}(t, \theta)\right)}\left(\tilde{\mathcal{P}}_{1}-\tilde{\mathcal{P}}_{2}\right) \mathbb{1}_{\tilde{\mathcal{P}}_{1}<\tilde{\mathcal{P}}_{2}} \tilde{\mathcal{U}}_{2}^{+} \tilde{\mathcal{Y}}_{2, \eta}(t, \theta) d \theta\right) d \eta \mid \\
\leqslant & \frac{1}{A^{2}}\left|\int_{0}^{1} \frac{1}{\max _{j}\left(\tilde{\mathcal{P}}_{j}^{1 / 2}\right)}\right| \tilde{\mathcal{P}}_{1}^{1 / 2}-\tilde{\mathcal{P}}_{2}^{1 / 2} \mid(t, \eta) \\
& \times\left(\int_{0}^{\eta} e^{-\frac{1}{A_{2}}\left(\tilde{\mathcal{Y}}_{2}(t, \eta)-\tilde{\mathcal{Y}}_{2}(t, \theta)\right)}\left|\tilde{\mathcal{P}}_{1}^{1 / 2}-\tilde{\mathcal{P}}_{2}^{1 / 2}\right| \tilde{\mathcal{P}}_{2}^{1 / 2} \tilde{\mathcal{U}}_{2}^{+} \tilde{\mathcal{Y}}_{2, \eta}(t, \theta) d \theta\right) d \eta \mid \\
\leqslant & \frac{1}{A^{2}} \int_{0}^{1} \frac{1}{\max _{j}\left(\tilde{\mathcal{P}}_{j}^{1 / 2}\right)}\left|\tilde{\mathcal{P}}_{1}^{1 / 2}-\tilde{\mathcal{P}}_{2}^{1 / 2}\right|(t, \eta) \\
& \times\left(\int_{0}^{\eta} e^{-\frac{1}{A_{2}}\left(\tilde{\mathcal{Y}}_{2}(t, \eta)-\tilde{\mathcal{Y}}_{2}(t, \theta)\right)}\left(\tilde{\mathcal{P}}_{1}^{1 / 2}-\tilde{\mathcal{P}}_{2}^{1 / 2}\right)^{2}(t, \theta) d \theta\right)^{1 / 2} \\
& \times\left(\int_{0}^{\eta} e^{-\frac{1}{A_{2}}\left(\tilde{\mathcal{Y}}_{2}(t, \eta)-\tilde{\mathcal{Y}}_{2}(t, \theta)\right)} \tilde{\mathcal{P}}_{2} \tilde{\mathcal{U}}_{2}^{2} \tilde{\mathcal{Y}}_{2, \eta}^{2}(t, \theta) d \theta\right)^{1 / 2} d \eta \\
\leqslant & \mathcal{O}(1) \int_{0}^{1} \frac{1}{\max _{j}\left(\tilde{\mathcal{P}}_{j}^{1 / 2}\right)}\left|\tilde{\mathcal{P}}_{1}^{1 / 2}-\tilde{\mathcal{P}}_{2}^{1 / 2}\right| \tilde{\mathcal{P}}_{2}^{1 / 2}(t, \eta)
\end{aligned}
$$




$$
\begin{aligned}
& \times\left(\int_{0}^{\eta} e^{-\frac{1}{A_{2}}\left(\tilde{\mathcal{Y}}_{2}(t, \eta)-\tilde{\mathcal{Y}}_{2}(t, \theta)\right)}\left(\tilde{\mathcal{P}}_{1}^{1 / 2}-\tilde{\mathcal{P}}_{2}^{1 / 2}\right)^{2}(t, \theta) d \theta\right)^{1 / 2} d \eta \\
\leqslant & \mathcal{O}(1)\left\|\tilde{\mathcal{P}}_{1}^{1 / 2}-\tilde{\mathcal{P}}_{2}^{1 / 2}\right\|^{2},
\end{aligned}
$$

using (4.15e) and (4.16c). As for $K_{5}$ and $K_{6}$, we find

$$
\begin{aligned}
& \left|K_{5}\right|=\frac{a}{2 A^{3}} \mid \int_{0}^{1} \frac{1}{\max _{j}\left(\tilde{\mathcal{P}}_{j}^{1 / 2}\right)}\left(\tilde{\mathcal{P}}_{1}^{1 / 2}-\tilde{\mathcal{P}}_{2}^{1 / 2}\right)(t, \eta) \\
& +\left(\int_{0}^{\eta} e^{-\frac{1}{A_{2}}\left(\tilde{\mathcal{Y}}_{2}(t, \eta)-\tilde{\mathcal{Y}}_{2}(t, \theta)\right)} \min _{j}\left(\tilde{\mathcal{P}}_{j}\right)\left(\tilde{\mathcal{U}}_{1}^{+}-\tilde{\mathcal{U}}_{2}^{+}\right) \mathbb{1}_{\tilde{\mathcal{U}}_{1}^{+}<\tilde{\mathcal{U}}_{2}^{+}} \tilde{\mathcal{Y}}_{2, \eta}(t, \theta) d \theta\right) d \eta \\
& \leqslant \frac{1}{2 A^{2}} \int_{0}^{1} \frac{1}{\max _{j}\left(\tilde{\mathcal{P}}_{j}^{1 / 2}\right)}\left|\tilde{\mathcal{P}}_{1}^{1 / 2}-\tilde{\mathcal{P}}_{2}^{1 / 2}\right|(t, \eta) \\
& +\left(\int_{0}^{\eta} e^{-\frac{1}{A_{2}}\left(\tilde{\mathcal{Y}}_{2}(t, \eta)-\tilde{\mathcal{Y}}_{2}(t, \theta)\right)} \min _{j}\left(\tilde{\mathcal{P}}_{j}\right)\left|\tilde{\mathcal{U}}_{1}^{+}-\tilde{\mathcal{U}}_{2}^{+}\right| \mathbb{1}_{\tilde{\mathcal{U}}_{1}^{+}<\tilde{\mathcal{U}}_{2}^{+}} \tilde{\mathcal{Y}}_{2, \eta}(t, \theta) d \theta\right) d \eta \\
& \leqslant\left\|\tilde{\mathcal{P}}_{1}^{1 / 2}-\tilde{\mathcal{P}}_{2}^{1 / 2}\right\|^{2}+\frac{1}{4 A^{4}} \int_{0}^{1} \frac{1}{\left(\max _{j}\left(\tilde{\mathcal{P}}_{j}^{1 / 2}\right)\right)^{2}}(t, \eta) \\
& \times\left(\int_{0}^{\eta} e^{-\frac{1}{A_{2}}\left(\tilde{\mathcal{Y}}_{2}(t, \eta)-\tilde{\mathcal{Y}}_{2}(t, \theta)\right)} \min _{j}\left(\tilde{\mathcal{P}}_{j}\right)\left|\tilde{\mathcal{U}}_{1}^{+}-\tilde{\mathcal{U}}_{2}^{+}\right| \mathbb{1}_{\tilde{\mathcal{U}}_{1}^{+}<\tilde{\mathcal{U}}_{2}^{+}} \tilde{\mathcal{Y}}_{2, \eta}(t, \theta) d \theta\right)^{2} d \eta \\
& \leqslant\left\|\tilde{\mathcal{P}}_{1}^{1 / 2}-\tilde{\mathcal{P}}_{2}^{1 / 2}\right\|^{2} \\
& +\frac{1}{4 A^{4}} \int_{0}^{1} \frac{1}{\left(\max _{j}\left(\tilde{\mathcal{P}}_{j}^{1 / 2}\right)\right)^{2}}(t, \eta)\left(\int_{0}^{\eta} e^{-\frac{3}{2 A_{2}}\left(\tilde{\mathcal{Y}}_{2}(t, \eta)-\tilde{\mathcal{Y}}_{2}(t, \theta)\right)} \tilde{\mathcal{P}}_{2} \tilde{\mathcal{Y}}_{2, \eta}(t, \theta) d \theta\right) \\
& \times\left(\int_{0}^{\eta} e^{-\frac{1}{2 A_{2}}\left(\tilde{\mathcal{Y}}_{2}(t, \eta)-\tilde{\mathcal{Y}}_{2}(t, \theta)\right)} \tilde{\mathcal{P}}_{2} \tilde{\mathcal{Y}}_{2, \eta}\left(\tilde{\mathcal{U}}_{1}^{+}-\tilde{\mathcal{U}}_{2}^{+}\right)^{2}(t, \theta) d \theta\right) d \eta \\
& \leqslant\left\|\tilde{\mathcal{P}}_{1}^{1 / 2}-\tilde{\mathcal{P}}_{2}^{1 / 2}\right\|^{2}+\frac{1}{2 A^{3}} \int_{0}^{1} \frac{1}{\left(\max _{j}\left(\tilde{\mathcal{P}}_{j}^{1 / 2}\right)\right)^{2}} \tilde{\mathcal{P}}_{2}(t, \eta) \\
& \times\left(\int_{0}^{\eta} e^{-\frac{1}{2 A_{2}}\left(\tilde{\mathcal{Y}}_{2}(t, \eta)-\tilde{\mathcal{Y}}_{2}(t, \theta)\right)} \tilde{\mathcal{P}}_{2} \tilde{\mathcal{Y}}_{2, \eta}\left(\tilde{\mathcal{U}}_{1}^{+}-\tilde{\mathcal{U}}_{2}^{+}\right)^{2}(t, \theta) d \theta\right) d \eta \\
& \leqslant \mathcal{O}(1)\left(\left\|\tilde{\mathcal{P}}_{1}^{1 / 2}-\tilde{\mathcal{P}}_{2}^{1 / 2}\right\|^{2}+\left\|\tilde{\mathcal{U}}_{1}-\tilde{\mathcal{U}}_{2}\right\|^{2}\right),
\end{aligned}
$$

using (4.15e) and (4.16e). 
The terms $K_{7}$ and $K_{8}$ allow for the following estimates:

$$
\begin{aligned}
& \left|K_{7}\right|=\frac{a}{2 A^{3}} \mid \int_{0}^{1} \frac{1}{\max _{j}\left(\tilde{\mathcal{P}}_{j}^{1 / 2}\right)}\left(\tilde{\mathcal{P}}_{1}^{1 / 2}-\tilde{\mathcal{P}}_{2}^{1 / 2}\right)(t, \eta) \\
& \times \mathbb{1}_{A_{1} \leqslant A_{2}} \int_{0}^{\eta}\left(e^{-\frac{1}{A_{2}}\left(\tilde{\mathcal{Y}}_{2}(t, \eta)-\tilde{\mathcal{Y}}_{2}(t, \theta)\right)}-e^{-\frac{1}{A_{1}}\left(\tilde{\mathcal{Y}}_{2}(t, \eta)-\tilde{\mathcal{Y}}_{2}(t, \theta)\right)}\right) \\
& \times \min _{j}\left(\tilde{\mathcal{P}}_{j}\right) \min _{j}\left(\tilde{\mathcal{U}}_{j}^{+}\right) \tilde{\mathcal{Y}}_{2, \eta}(t, \theta) d \theta d \eta \mid \\
& \leqslant \frac{2}{A^{3} e} \int_{0}^{1} \frac{1}{\max _{j}\left(\tilde{\mathcal{P}}_{j}^{1 / 2}\right)}\left|\tilde{\mathcal{P}}_{1}^{1 / 2}-\tilde{\mathcal{P}}_{2}^{1 / 2}\right|(t, \eta) \\
& \times \int_{0}^{\eta} e^{-\frac{3}{4 A_{2}}\left(\tilde{\mathcal{Y}}_{2}(t, \eta)-\tilde{\mathcal{Y}}_{2}(t, \theta)\right)} \tilde{\mathcal{P}}_{2}\left|\tilde{\mathcal{U}}_{2}\right| \tilde{\mathcal{Y}}_{2, \eta}(t, \theta) d \theta d \eta\left|A_{1}-A_{2}\right| \\
& \leqslant \frac{2}{A^{3} e} \int_{0}^{1} \frac{1}{\max _{j}\left(\tilde{\mathcal{P}}_{j}^{1 / 2}\right)}\left|\tilde{\mathcal{P}}_{1}^{1 / 2}-\tilde{\mathcal{P}}_{2}^{1 / 2}\right|(t, \eta) \\
& \times\left(\int_{0}^{1} e^{-\frac{1}{A_{2}}\left(\tilde{\mathcal{Y}}_{2}(t, \eta)-\tilde{\mathcal{Y}}_{2}(t, \theta)\right)} \tilde{\mathcal{U}}_{2}^{2} \tilde{\mathcal{Y}}_{2, \eta}(t, \theta) d \theta\right)^{1 / 2} \\
& \times\left(\int_{0}^{1} e^{-\frac{1}{2 A_{2}}\left(\tilde{\mathcal{Y}}_{2}(t, \eta)-\tilde{\mathcal{Y}}_{2}(t, \theta)\right)} \tilde{\mathcal{P}}_{2}^{2} \tilde{\mathcal{Y}}_{2, \eta}(t, \theta) d \theta\right)^{1 / 2} d \eta\left|A_{1}-A_{2}\right| \\
& \leqslant \mathcal{O}(1)\left(\left\|\tilde{\mathcal{P}}_{1}^{1 / 2}-\tilde{\mathcal{P}}_{2}^{1 / 2}\right\|^{2}+\left|A_{1}-A_{2}\right|^{2}\right) .
\end{aligned}
$$

The terms $K_{9}$ and $K_{10}$ allow for the following estimates:

$$
\begin{aligned}
\left|K_{9}\right|= & \frac{a}{2 A^{3}} \mid \int_{0}^{1} \frac{1}{\max _{j}\left(\tilde{\mathcal{P}}_{j}^{1 / 2}\right)}\left(\tilde{\mathcal{P}}_{1}^{1 / 2}-\tilde{\mathcal{P}}_{2}^{1 / 2}\right)(t, \eta) \\
& +\int_{0}^{\eta}\left(e^{-\frac{1}{a}\left(\tilde{\mathcal{Y}}_{1}(t, \eta)-\tilde{\mathcal{Y}}_{1}(t, \theta)\right)}-e^{-\frac{1}{a}\left(\tilde{\mathcal{Y}}_{2}(t, \eta)-\tilde{\mathcal{Y}}_{2}(t, \theta)\right)}\right) \mathbb{1}_{B(\eta)} \\
& \times \min _{j}\left(\tilde{\mathcal{P}}_{j}\right) \min _{j}\left(\tilde{\mathcal{U}}_{j}^{+}\right) \tilde{\mathcal{Y}}_{2, \eta}(t, \theta) d \theta d \eta \mid \\
\leqslant & \frac{1}{2 A^{3}} \int_{0}^{1} \frac{1}{\max _{j}\left(\tilde{\mathcal{P}}_{j}^{1 / 2}\right)}\left|\tilde{\mathcal{P}}_{1}^{1 / 2}-\tilde{\mathcal{P}}_{2}^{1 / 2}\right|(t, \eta) \\
& \times \int_{0}^{\eta} e^{-\frac{1}{a}\left(\tilde{\mathcal{Y}}_{2}(t, \eta)-\tilde{\mathcal{Y}}_{2}(t, \theta)\right)}\left(\left|\tilde{\mathcal{Y}}_{2}(t, \eta)-\tilde{\mathcal{Y}}_{1}(t, \eta)\right|+\left|\tilde{\mathcal{Y}}_{2}(t, \theta)-\tilde{\mathcal{Y}}_{1}(t, \theta)\right|\right) \\
& \times \min _{j}\left(\tilde{\mathcal{P}}_{j}\right) \min _{j}\left(\tilde{\mathcal{U}}_{j}^{+}\right) \tilde{\mathcal{Y}}_{2, \eta}(t, \theta) d \theta d \eta
\end{aligned}
$$




$$
\begin{aligned}
& \leqslant \frac{1}{2 A^{3}} \int_{0}^{1} \frac{1}{\max _{j}\left(\tilde{\mathcal{P}}_{j}^{1 / 2}\right)}\left|\tilde{\mathcal{P}}_{1}^{1 / 2}-\tilde{\mathcal{P}}_{2}^{1 / 2}\right|\left|\tilde{\mathcal{Y}}_{2}-\tilde{\mathcal{Y}}_{1}\right|(t, \eta) \\
& \times\left(\int_{0}^{\eta} e^{-\frac{1}{a}\left(\tilde{\mathcal{Y}}_{2}(t, \eta)-\tilde{\mathcal{Y}}_{2}(t, \theta)\right)} \min _{j}\left(\tilde{\mathcal{P}}_{j}\right) \min _{j}\left(\tilde{\mathcal{U}}_{j}^{+}\right) \tilde{\mathcal{Y}}_{2, \eta}(t, \theta) d \theta\right) d \eta \\
& +\frac{1}{2 A^{3}} \int_{0}^{1} \frac{1}{\max _{j}\left(\tilde{\mathcal{P}}_{j}^{1 / 2}\right)}\left|\tilde{\mathcal{P}}_{1}^{1 / 2}-\tilde{\mathcal{P}}_{2}^{1 / 2}\right|(t, \eta) \\
& \times\left(\int_{0}^{\eta} e^{-\frac{1}{a}\left(\tilde{\mathcal{Y}}_{2}(t, \eta)-\tilde{\mathcal{Y}}_{2}(t, \theta)\right)}\left|\tilde{\mathcal{Y}}_{2}-\tilde{\mathcal{Y}}_{1}\right| \min _{j}\left(\tilde{\mathcal{P}}_{j}\right) \min _{j}\left(\tilde{\mathcal{U}}_{j}^{+}\right) \tilde{\mathcal{Y}}_{2, \eta}(t, \theta) d \theta\right) d \eta \\
& \leqslant \frac{1}{2 A^{3}} \int_{0}^{1} \frac{1}{\max _{j}\left(\tilde{\mathcal{P}}_{j}^{1 / 2}\right)}\left|\tilde{\mathcal{P}}_{1}^{1 / 2}-\tilde{\mathcal{P}}_{2}^{1 / 2}\right|\left|\tilde{\mathcal{Y}}_{2}-\tilde{\mathcal{Y}}_{1}\right|(t, \eta) \\
& \times\left(\int_{0}^{\eta} e^{-\frac{1}{A_{2}}\left(\tilde{\mathcal{Y}}_{2}(t, \eta)-\tilde{\mathcal{Y}}_{2}(t, \theta)\right)} \tilde{\mathcal{P}}_{2}^{2} \tilde{\mathcal{Y}}_{2, \eta}(t, \theta) d \theta\right)^{1 / 2} \\
& \times\left(\int_{0}^{\eta} e^{-\frac{1}{A_{2}}\left(\tilde{\mathcal{Y}}_{2}(t, \eta)-\tilde{\mathcal{Y}}_{2}(t, \theta)\right)} \tilde{\mathcal{U}}_{2}^{2} \tilde{\mathcal{Y}}_{2, \eta}(t, \theta) d \theta\right)^{1 / 2} d \eta \\
& +\left\|\tilde{\mathcal{P}}_{1}^{1 / 2}-\tilde{\mathcal{P}}_{2}^{1 / 2}\right\|^{2}+\frac{1}{4 A^{6}} \int_{0}^{1} \frac{1}{\left(\max _{j}\left(\tilde{\mathcal{P}}_{j}^{1 / 2}\right)\right)^{2}}(t, \eta) \\
& \times\left(\int_{0}^{\eta} e^{-\frac{1}{A_{2}}\left(\tilde{\mathcal{Y}}_{2}(t, \eta)-\tilde{\mathcal{Y}}_{2}(t, \theta)\right)}\left|\tilde{\mathcal{Y}}_{2}-\tilde{\mathcal{Y}}_{1}\right| \min _{j}\left(\tilde{\mathcal{P}}_{j}\right) \min _{j}\left(\tilde{\mathcal{U}}_{j}^{+}\right) \tilde{\mathcal{Y}}_{2, \eta}(t, \theta) d \theta\right)^{2} d \eta \\
& \leqslant \mathcal{O}(1)\left(\left\|\tilde{\mathcal{P}}_{1}^{1 / 2}-\tilde{\mathcal{P}}_{2}^{1 / 2}\right\|^{2}+\left\|\tilde{\mathcal{Y}}_{2}-\tilde{\mathcal{Y}}_{1}\right\|^{2}\right)+\frac{1}{4 A^{6}} \int_{0}^{1} \frac{1}{\left(\max _{j}\left(\tilde{\mathcal{P}}_{j}^{1 / 2}\right)\right)^{2}}(t, \eta) \\
& \times\left(\int_{0}^{\eta} e^{-\frac{1}{A_{2}}\left(\tilde{\mathcal{Y}}_{2}(t, \eta)-\tilde{\mathcal{Y}}_{2}(t, \theta)\right)} \tilde{\mathcal{U}}_{2}^{2} \tilde{\mathcal{Y}}_{2, \eta}(t, \theta) d \theta\right) \\
& \times\left(\int_{0}^{\eta} e^{-\frac{1}{A_{2}}\left(\tilde{\mathcal{Y}}_{2}(t, \eta)-\tilde{\mathcal{Y}}_{2}(t, \theta)\right)}\left(\tilde{\mathcal{Y}}_{2}-\tilde{\mathcal{Y}}_{1}\right)^{2} \tilde{\mathcal{P}}_{2}^{2} \tilde{\mathcal{Y}}_{2, \eta}(t, \theta) d \theta\right) d \eta \\
& \leqslant \mathcal{O}(1)\left(\left\|\tilde{\mathcal{P}}_{1}^{1 / 2}-\tilde{\mathcal{P}}_{2}^{1 / 2}\right\|^{2}+\left\|\tilde{\mathcal{Y}}_{2}-\tilde{\mathcal{Y}}_{1}\right\|^{2}\right),
\end{aligned}
$$

using (4.15a), (4.15e), and (4.16c). The last term $K_{11}$ receives special treatment

$$
\begin{aligned}
\left|K_{11}\right|= & \frac{a}{2 A^{3}} \mid \int_{0}^{1} \frac{1}{\max _{j}\left(\tilde{\mathcal{P}}_{j}^{1 / 2}\right)}\left(\tilde{\mathcal{P}}_{1}^{1 / 2}-\tilde{\mathcal{P}}_{2}^{1 / 2}\right)(t, \eta)\left(\int_{0}^{\eta} \min _{j}\left(e^{-\frac{1}{a}\left(\tilde{\mathcal{Y}}_{j}(t, \eta)-\tilde{\mathcal{Y}}_{j}(t, \theta)\right)}\right)\right. \\
& \left.\times \min _{j}\left(\tilde{\mathcal{P}}_{j}\right) \min _{j}\left(\tilde{\mathcal{U}}_{j}^{+}\right)\left(\tilde{\mathcal{Y}}_{1, \eta}-\tilde{\mathcal{Y}}_{2, \eta}\right)(t, \theta) d \theta\right) d \eta \mid \\
= & \frac{a}{2 A^{3}} \mid \int_{0}^{1} \frac{1}{\max _{j}\left(\tilde{\mathcal{P}}_{j}^{1 / 2}\right)}\left(\tilde{\mathcal{P}}_{1}^{1 / 2}-\tilde{\mathcal{P}}_{2}^{1 / 2}\right)(t, \eta)
\end{aligned}
$$




$$
\begin{aligned}
& \times\left[\left.\left(\min _{j}\left(e^{-\frac{1}{a}\left(\tilde{\mathcal{Y}}_{j}(t, \eta)-\tilde{\mathcal{Y}}_{j}(t, \theta)\right)}\right) \min _{j}\left(\tilde{\mathcal{P}}_{j}\right) \min _{j}\left(\tilde{\mathcal{U}}_{j}^{+}\right)\left(\tilde{\mathcal{Y}}_{1}-\tilde{\mathcal{Y}}_{2}\right)(t, \theta)\right)\right|_{\theta=0} ^{1}\right. \\
& -\int_{0}^{\eta}\left(\tilde{\mathcal{Y}}_{1}-\tilde{\mathcal{Y}}_{2}\right) \\
& \left.\times \frac{d}{d \theta}\left(\min _{j}\left(e^{-\frac{1}{a}\left(\tilde{\mathcal{Y}}_{j}(t, \eta)-\tilde{\mathcal{Y}}_{j}(t, \theta)\right)}\right) \min _{j}\left(\tilde{\mathcal{P}}_{j}\right) \min _{j}\left(\tilde{\mathcal{U}}_{j}^{+}\right)\right)(t, \theta) d \theta\right] d \eta \\
& \leqslant \mathcal{O}(1)\left(\left\|\tilde{\mathcal{P}}_{1}^{1 / 2}-\tilde{\mathcal{P}}_{2}^{1 / 2}\right\|^{2}+\left\|\tilde{\mathcal{Y}}_{2}-\tilde{\mathcal{Y}}_{1}\right\|^{2}\right) \\
& +\frac{a}{2 A^{3}} \int_{0}^{1} \frac{1}{\max _{j}\left(\tilde{\mathcal{P}}_{j}^{1 / 2}\right)}\left|\tilde{\mathcal{P}}_{1}^{1 / 2}-\tilde{\mathcal{P}}_{2}^{1 / 2}\right|(t, \eta) \\
& \times \int_{0}^{\eta}\left|\tilde{\mathcal{Y}}_{1}-\tilde{\mathcal{Y}}_{2}\right| \mid\left(\frac{d}{d \theta} \min _{j}\left(e^{-\frac{1}{a}\left(\tilde{\mathcal{Y}}_{j}(t, \eta)-\tilde{\mathcal{Y}}_{j}(t, \theta)\right)}\right)\right) \min _{j}\left(\tilde{\mathcal{P}}_{j}\right) \min _{j}\left(\tilde{\mathcal{U}}_{j}^{+}\right) \\
& +\min _{j}\left(e^{-\frac{1}{a}\left(\tilde{\mathcal{Y}}_{j}(t, \eta)-\tilde{\mathcal{Y}}_{j}(t, \theta)\right)}\right)\left(\frac{d}{d \theta} \min _{j}\left(\tilde{\mathcal{P}}_{j}\right) \min _{j}\left(\tilde{\mathcal{U}}_{j}^{+}\right)\right) \mid(t, \theta) d \theta d \eta \\
& \leqslant \mathcal{O}(1)\left(\left\|\tilde{\mathcal{P}}_{1}^{1 / 2}-\tilde{\mathcal{P}}_{2}^{1 / 2}\right\|^{2}+\left\|\tilde{\mathcal{Y}}_{1}-\tilde{\mathcal{Y}}_{2}\right\|^{2}\right) \\
& +\frac{1}{2 A^{3}} \int_{0}^{1} \frac{1}{\max _{j}\left(\tilde{\mathcal{P}}_{j}^{1 / 2}\right)}\left|\tilde{\mathcal{P}}_{1}^{1 / 2}-\tilde{\mathcal{P}}_{2}^{1 / 2}\right|(t, \eta) \\
& \times \int_{0}^{\eta}\left|\tilde{\mathcal{Y}}_{1}-\tilde{\mathcal{Y}}_{2}\right|\left(\min _{j}\left(e^{-\frac{1}{a}\left(\tilde{\mathcal{Y}}_{j}(t, \eta)-\tilde{\mathcal{Y}}_{j}(t, \theta)\right)}\right) \max _{j}\left(\tilde{\mathcal{Y}}_{j, \eta}\right) \min _{j}\left(\tilde{\mathcal{P}}_{j}\right) \min _{j}\left(\tilde{\mathcal{U}}_{j}^{+}\right)\right. \\
& \left.+2 a A^{4} \min _{j}\left(e^{-\frac{1}{a}\left(\tilde{\mathcal{Y}}_{j}(t, \eta)-\tilde{\mathcal{Y}}_{j}(t, \theta)\right)}\right)\left(\min _{j}\left(\tilde{\mathcal{P}}_{j}^{1 / 2}\right)+\left|\tilde{\mathcal{U}}_{2}\right|\right)\right)(t, \theta) d \theta d \eta \\
& \leqslant \mathcal{O}(1)\left(\left\|\tilde{\mathcal{P}}_{1}^{1 / 2}-\tilde{\mathcal{P}}_{2}^{1 / 2}\right\|^{2}+\left\|\tilde{\mathcal{Y}}_{1}-\tilde{\mathcal{Y}}_{2}\right\|^{2}\right) \\
& +\frac{1}{2 A^{3}} \int_{0}^{1} \frac{1}{\max _{j}\left(\tilde{\mathcal{P}}_{j}^{1 / 2}\right)}\left|\tilde{\mathcal{P}}_{1}^{1 / 2}-\tilde{\mathcal{P}}_{2}^{1 / 2}\right|(t, \eta) \\
& \times\left(\int_{0}^{\eta} e^{-\frac{1}{A_{1}}\left(\tilde{\mathcal{Y}}_{1}(t, \eta)-\tilde{\mathcal{Y}}_{1}(t, \theta)\right)}\left|\tilde{\mathcal{Y}}_{1}-\tilde{\mathcal{Y}}_{2}\right| \tilde{\mathcal{Y}}_{1, \eta} \tilde{\mathcal{P}}_{1} \tilde{\mathcal{U}}_{1}^{+}(t, \theta) d \theta\right) d \eta \\
& +\frac{1}{2 A^{3}} \int_{0}^{1} \frac{1}{\max _{j}\left(\tilde{\mathcal{P}}_{j}^{1 / 2}\right)}\left|\tilde{\mathcal{P}}_{1}^{1 / 2}-\tilde{\mathcal{P}}_{2}^{1 / 2}\right|(t, \eta) \\
& \times\left(\int_{0}^{\eta} e^{-\frac{1}{A_{2}}\left(\tilde{\mathcal{Y}}_{2}(t, \eta)-\tilde{\mathcal{Y}}_{2}(t, \theta)\right)}\left|\tilde{\mathcal{Y}}_{1}-\tilde{\mathcal{Y}}_{2}\right| \tilde{\mathcal{Y}}_{2, \eta} \tilde{\mathcal{P}}_{2} \tilde{\mathcal{U}}_{2}^{+}(t, \theta) d \theta\right) d \eta \\
& +A^{2} \int_{0}^{1} \frac{1}{\max _{j}\left(\tilde{\mathcal{P}}_{j}^{1 / 2}\right)}\left|\tilde{\mathcal{P}}_{1}^{1 / 2}-\tilde{\mathcal{P}}_{2}^{1 / 2}\right|(t, \eta)
\end{aligned}
$$




$$
\begin{aligned}
& \times\left(\int_{0}^{\eta}\left|\tilde{\mathcal{Y}}_{1}-\tilde{\mathcal{Y}}_{2}\right| \min _{j}\left(e^{-\frac{1}{A_{2}}\left(\tilde{\mathcal{Y}}_{2}(t, \eta)-\tilde{\mathcal{Y}}_{2}(t, \theta)\right)}\right)\left(\tilde{\mathcal{P}}_{2}^{1 / 2}+\left|\tilde{\mathcal{U}}_{2}\right|\right)(t, \theta) d \theta\right) d \eta \\
= & \mathcal{O}(1)\left(\left\|\tilde{\mathcal{P}}_{1}^{1 / 2}-\tilde{\mathcal{P}}_{2}^{1 / 2}\right\|^{2}+\left\|\tilde{\mathcal{Y}}_{1}-\tilde{\mathcal{Y}}_{2}\right\|^{2}\right)+K_{71}+K_{72}+K_{73},
\end{aligned}
$$

using Lemmas A.2 and A.3, and

$$
\begin{aligned}
& \min _{j}\left(e^{-\frac{1}{a}\left(\tilde{\mathcal{Y}}_{j}(t, \eta)-\tilde{\mathcal{Y}}_{j}(t, \theta)\right)}\right) \max _{j}\left(\tilde{\mathcal{Y}}_{j, \eta}\right) \min _{j}\left(\tilde{\mathcal{P}}_{j}\right) \min _{j}\left(\tilde{\mathcal{U}}_{j}^{+}\right)(t, \theta) \\
& \quad \leqslant e^{-\frac{1}{A_{1}}\left(\tilde{\mathcal{Y}}_{1}(t, \eta)-\tilde{\mathcal{Y}}_{1}(t, \theta)\right)} \tilde{\mathcal{Y}}_{1, \eta} \tilde{\mathcal{P}}_{1} \tilde{\mathcal{U}}_{1}^{+}(t, \theta)+e^{-\frac{1}{A_{2}}\left(\tilde{\mathcal{Y}}_{2}(t, \eta)-\tilde{\mathcal{Y}}_{2}(t, \theta)\right)} \tilde{\mathcal{Y}}_{2, \eta} \tilde{\mathcal{P}}_{2} \tilde{\mathcal{U}}_{2}^{+}(t, \theta) .
\end{aligned}
$$

Here $K_{71}$ and $K_{72}$ allow for the same treatment:

$$
\begin{aligned}
2 K_{71}= & \frac{1}{A^{3}} \int_{0}^{1} \frac{1}{\max _{j}\left(\tilde{\mathcal{P}}_{j}^{1 / 2}\right)}\left|\tilde{\mathcal{P}}_{1}^{1 / 2}-\tilde{\mathcal{P}}_{2}^{1 / 2}\right|(t, \eta) \\
& \times\left(\int_{0}^{\eta}\left|\tilde{\mathcal{Y}}_{1}-\tilde{\mathcal{Y}}_{2}\right| e^{-\frac{1}{A_{1}}\left(\tilde{\mathcal{Y}}_{1}(t, \eta)-\tilde{\mathcal{Y}}_{1}(t, \theta)\right)} \tilde{\mathcal{Y}}_{1, \eta} \tilde{\mathcal{P}}_{1} \tilde{\mathcal{U}}_{1}^{+}(t, \theta) d \theta\right) d \eta \\
\leqslant & \frac{1}{A^{3}} \int_{0}^{1} \frac{1}{\max _{j}\left(\tilde{\mathcal{P}}_{j}^{1 / 2}\right)}\left|\tilde{\mathcal{P}}_{1}^{1 / 2}-\tilde{\mathcal{P}}_{2}^{1 / 2}\right|(t, \eta) \\
& \times\left(\int_{0}^{\eta} e^{-\frac{1}{A_{1}}\left(\tilde{\mathcal{Y}}_{1}(t, \eta)-\tilde{\mathcal{Y}}_{1}(t, \theta)\right)}\left(\tilde{\mathcal{Y}}_{1}-\tilde{\mathcal{Y}}_{2}\right)^{2}(t, \theta) d \theta\right)^{1 / 2} \\
& \times\left(\int_{0}^{\eta} e^{-\frac{1}{A_{1}}\left(\tilde{\mathcal{Y}}_{1}(t, \eta)-\tilde{\mathcal{Y}}_{1}(t, \theta)\right)} \tilde{\mathcal{Y}}_{1, \eta}^{2} \tilde{\mathcal{P}}_{1}^{2}\left(\tilde{\mathcal{U}}_{1}^{+}\right)^{2}(t, \theta) d \theta\right)^{1 / 2} d \eta \\
\leqslant & A^{2} \sqrt{2} \int_{0}^{1} \frac{1}{\max _{j}\left(\tilde{\mathcal{P}}_{j}^{1 / 2}\right)}\left|\tilde{\mathcal{P}}_{1}^{1 / 2}-\tilde{\mathcal{P}}_{2}^{1 / 2}\right| \tilde{\mathcal{P}}_{1}^{1 / 2}(t, \eta)\left\|\tilde{\mathcal{Y}}_{1}-\tilde{\mathcal{Y}}_{2}\right\| d \eta \\
\leqslant & \mathcal{O}(1)\left(\left\|\tilde{\mathcal{P}}_{1}^{1 / 2}-\tilde{\mathcal{P}}_{2}^{1 / 2}\right\|^{2}+\left\|\tilde{\mathcal{Y}}_{1}-\tilde{\mathcal{Y}}_{2}\right\|^{2}\right),
\end{aligned}
$$

using (4.15g) and (4.16b). Furthermore,

$$
\begin{aligned}
K_{73}= & A^{2} \int_{0}^{1} \frac{1}{\max _{j}\left(\tilde{\mathcal{P}}_{j}^{1 / 2}\right)}\left|\tilde{\mathcal{P}}_{1}^{1 / 2}-\tilde{\mathcal{P}}_{2}^{1 / 2}\right|(t, \eta) \\
& \times\left(\int_{0}^{\eta}\left|\tilde{\mathcal{Y}}_{1}-\tilde{\mathcal{Y}}_{2}\right| \min _{j}\left(e^{-\frac{1}{A_{2}}\left(\tilde{\mathcal{Y}}_{2}(t, \eta)-\tilde{\mathcal{Y}}_{2}(t, \theta)\right)}\right)\left(\tilde{\mathcal{P}}_{2}^{1 / 2}+\left|\tilde{\mathcal{U}}_{2}\right|\right)(t, \theta) d \theta\right) d \eta \\
\leqslant & \mathcal{O}(1) \int_{0}^{1} \frac{1}{\max _{j}\left(\tilde{\mathcal{P}}_{j}^{1 / 2}\right)}\left|\tilde{\mathcal{P}}_{1}^{1 / 2}-\tilde{\mathcal{P}}_{2}^{1 / 2}\right|(t, \eta) \\
& \times\left(\int_{0}^{\eta}\left|\tilde{\mathcal{Y}}_{1}-\tilde{\mathcal{Y}}_{2}\right| \min _{j}\left(e^{-\frac{1}{A_{2}}\left(\tilde{\mathcal{Y}}_{2}(t, \eta)-\tilde{\mathcal{Y}}_{2}(t, \theta)\right)}\right) \tilde{\mathcal{P}}_{2}^{1 / 2}(t, \theta) d \theta\right) d \eta
\end{aligned}
$$




$$
\begin{aligned}
& \leqslant \mathcal{O}(1)\left\|\tilde{\mathcal{P}}_{1}^{1 / 2}-\tilde{\mathcal{P}}_{2}^{1 / 2}\right\|^{2}+\int_{0}^{1} \frac{1}{\left(\max _{j}\left(\tilde{\mathcal{P}}_{j}^{1 / 2}\right)\right)^{2}}(t, \eta) \\
& \times\left(\int_{0}^{\eta}\left|\tilde{\mathcal{Y}}_{1}-\tilde{\mathcal{Y}}_{2}\right| \min _{j}\left(e^{-\frac{1}{A_{2}}\left(\tilde{\mathcal{Y}}_{2}(t, \eta)-\tilde{\mathcal{Y}}_{2}(t, \theta)\right)}\right) \tilde{\mathcal{P}}_{2}^{1 / 2}(t, \theta) d \theta\right)^{2} d \eta \\
& \leqslant \mathcal{O}(1)\left\|\tilde{\mathcal{P}}_{1}^{1 / 2}-\tilde{\mathcal{P}}_{2}^{1 / 2}\right\|^{2} \\
& +\int_{0}^{1} \frac{1}{\left(\max _{j}\left(\tilde{\mathcal{P}}_{j}^{1 / 2}\right)\right)^{2}}(t, \eta)\left(\int_{0}^{\eta} e^{-\frac{1}{2 A_{2}}\left(\tilde{\mathcal{Y}}_{2}(t, \eta)-\tilde{\mathcal{Y}}_{2}(t, \theta)\right)}\left(\tilde{\mathcal{Y}}_{1}-\tilde{\mathcal{Y}}_{2}\right)^{2}(t, \theta) d \theta\right) \\
& \times\left(\int_{0}^{\eta} e^{-\frac{3}{2 A_{2}}\left(\tilde{\mathcal{Y}}_{2}(t, \eta)-\tilde{\mathcal{Y}}_{2}(t, \theta)\right)} \tilde{\mathcal{P}}_{2}(t, \theta) d \theta\right) d \eta \\
& \leqslant \mathcal{O}(1)\left\|\tilde{\mathcal{P}}_{1}^{1 / 2}-\tilde{\mathcal{P}}_{2}^{1 / 2}\right\|^{2}+\mathcal{O}(1) \int_{0}^{1} \frac{1}{\left(\max _{j}\left(\tilde{\mathcal{P}}_{j}^{1 / 2}\right)\right)^{2}} \tilde{\mathcal{P}}_{2}(t, \eta) \\
& \times\left(\int_{0}^{\eta} e^{-\frac{1}{2 A_{2}}\left(\tilde{\mathcal{Y}}_{2}(t, \eta)-\tilde{\mathcal{Y}}_{2}(t, \theta)\right)}\left(\tilde{\mathcal{Y}}_{1}-\tilde{\mathcal{Y}}_{2}\right)^{2}(t, \theta) d \theta\right) d \eta \\
& \leqslant \mathcal{O}(1)\left(\left\|\tilde{\mathcal{P}}_{1}^{1 / 2}-\tilde{\mathcal{P}}_{2}^{1 / 2}\right\|^{2}+\left\|\tilde{\mathcal{Y}}_{1}-\tilde{\mathcal{Y}}_{2}\right\|^{2}\right) \text {, }
\end{aligned}
$$

using (4.15d) and (4.16i). This proves that

$$
\tilde{J}_{131} \leqslant \mathcal{O}(1)\left(\left\|\tilde{\mathcal{P}}_{1}^{1 / 2}-\tilde{\mathcal{P}}_{2}^{1 / 2}\right\|^{2}+\left\|\tilde{\mathcal{U}}_{1}-\tilde{\mathcal{U}}_{2}\right\|^{2}+\left\|\tilde{\mathcal{Y}}_{1}-\tilde{\mathcal{Y}}_{2}\right\|^{2}+\left|A_{1}-A_{2}\right|^{2}\right)
$$

It remains to consider $\tilde{J}_{132}$ :

$$
\begin{aligned}
-\tilde{J}_{132}= & \frac{1}{2 A^{3}} \int_{0}^{1} \frac{1}{\max _{j}\left(\tilde{\mathcal{P}}_{j}^{1 / 2}\right)}\left(\tilde{\mathcal{P}}_{1}^{1 / 2}-\tilde{\mathcal{P}}_{2}^{1 / 2}\right)(t, \eta) \\
& \times\left(\int _ { 0 } ^ { \eta } \left(e^{-\frac{1}{A_{2}}\left(\tilde{\mathcal{Y}}_{2}(t, \eta)-\tilde{\mathcal{Y}}_{2}(t, \theta)\right)} \tilde{\mathcal{U}}_{2}^{+} \tilde{\mathcal{D}}_{2} \tilde{\mathcal{Y}}_{2, \eta}\right.\right. \\
& \left.\left.-e^{-\frac{1}{A_{1}}\left(\tilde{\mathcal{Y}}_{1}(t, \eta)-\tilde{\mathcal{Y}}_{1}(t, \theta)\right)} \tilde{\mathcal{U}}_{1}^{+} \tilde{\mathcal{D}}_{1} \tilde{\mathcal{Y}}_{1, \eta}\right)(t, \theta) d \theta\right) d \eta \\
= & \frac{1}{2 A^{3}} \int_{0}^{1} \frac{1}{\max _{j}\left(\tilde{\mathcal{P}}_{j}^{1 / 2}\right)}\left(\tilde{\mathcal{P}}_{1}^{1 / 2}-\tilde{\mathcal{P}}_{2}^{1 / 2}\right)(t, \eta) \\
& \times \int_{0}^{\eta} e^{-\frac{1}{A_{2}}\left(\tilde{\mathcal{Y}}_{2}(t, \eta)-\tilde{\mathcal{Y}}_{2}(t, \theta)\right)} \tilde{\mathcal{U}}_{2}^{+} \tilde{\mathcal{Y}}_{2, \eta}\left(\tilde{\mathcal{D}}_{2}-\tilde{\mathcal{D}}_{1}\right) \mathbb{1}_{\tilde{\mathcal{D}}_{1}<\tilde{\mathcal{D}}_{2}}(t, \theta) d \theta \\
& +\int_{0}^{\eta} e^{-\frac{1}{A_{1}}\left(\tilde{\mathcal{Y}}_{1}(t, \eta)-\tilde{\mathcal{Y}}_{1}(t, \theta)\right)} \tilde{\mathcal{U}}_{1}^{+} \tilde{\mathcal{Y}}_{1, \eta}\left(\tilde{\mathcal{D}}_{2}-\tilde{\mathcal{D}}_{1}\right) \mathbb{1}_{\tilde{\mathcal{D}}_{2} \leqslant \tilde{\mathcal{D}}_{1}}(t, \theta) d \theta \\
& +\int_{0}^{\eta}\left(e^{-\frac{1}{A_{2}}\left(\tilde{\mathcal{Y}}_{2}(t, \eta)-\tilde{\mathcal{Y}}_{2}(t, \theta)\right)} \tilde{\mathcal{U}}_{2}^{+} \tilde{\mathcal{Y}}_{2, \eta}-e^{-\left(\tilde{\mathcal{Y}}_{1}(t, \eta)-\tilde{\mathcal{Y}}_{1}(t, \theta)\right)} \tilde{\mathcal{U}}_{1}^{+} \tilde{\mathcal{Y}}_{1, \eta}\right)
\end{aligned}
$$




$$
\begin{aligned}
& \left.\times \min _{j}\left(\tilde{\mathcal{D}}_{j}\right)(t, \theta) d \theta\right] d \eta \\
= & \tilde{A}_{1}+\tilde{A}_{2}+\tilde{A}_{3} .
\end{aligned}
$$

The terms $\tilde{A}_{1}$ and $\tilde{A}_{2}$ allow for the same treatment. Specifically, (see Lemma A.9)

$$
\begin{aligned}
\left|\tilde{A}_{1}\right|= & \frac{1}{2 A^{3}} \mid \int_{0}^{1} \frac{1}{\max _{j}\left(\tilde{\mathcal{P}}_{j}^{1 / 2}\right)}\left(\tilde{\mathcal{P}}_{1}^{1 / 2}-\tilde{\mathcal{P}}_{2}^{1 / 2}\right)(t, \eta) \\
& \times\left[\int_{0}^{\eta} e^{-\frac{1}{A_{2}}\left(\tilde{\mathcal{Y}}_{2}(t, \eta)-\tilde{\mathcal{Y}}_{2}(t, \theta)\right)} \tilde{\mathcal{U}}_{2}^{+} \tilde{\mathcal{Y}}_{2, \eta}\left(\tilde{\mathcal{D}}_{2}-\tilde{\mathcal{D}}_{1}\right) \mathbb{1}_{\tilde{\mathcal{D}}_{1}<\tilde{\mathcal{D}}_{2}}(t, \theta) d \theta\right] d \eta \mid \\
\leqslant & \frac{1}{2 A^{3}} \int_{0}^{1} \frac{1}{\max _{j}\left(\tilde{\mathcal{P}}_{j}^{1 / 2}\right)}\left|\tilde{\mathcal{P}}_{1}^{1 / 2}-\tilde{\mathcal{P}}_{2}^{1 / 2}\right|(t, \eta) \\
& \times\left[\int_{0}^{\eta} e^{-\frac{1}{A_{2}}\left(\tilde{\mathcal{Y}}_{2}(t, \eta)-\tilde{\mathcal{Y}}_{2}(t, \theta)\right)} \tilde{\mathcal{U}}_{2}^{+} \tilde{\mathcal{Y}}_{2, \eta}\left(\bar{d}_{11}+\bar{d}_{12}\right) \mathbb{1}_{\tilde{\mathcal{D}}_{1}<\tilde{\mathcal{D}}_{2}}(t, \theta) d \theta\right. \\
& +\int_{0}^{\eta} e^{-\frac{1}{A_{2}}\left(\tilde{\mathcal{Y}}_{2}(t, \eta)-\tilde{\mathcal{Y}}_{2}(t, \theta)\right)} \tilde{\mathcal{U}}_{2}^{+} \tilde{\mathcal{Y}}_{2, \eta} T_{1} \mathbb{1}_{\tilde{\mathcal{D}}_{1}<\tilde{\mathcal{D}}_{2}}(t, \theta) d \theta \\
& +\int_{0}^{\eta} e^{-\frac{1}{A_{2}}\left(\tilde{\mathcal{Y}}_{2}(t, \eta)-\tilde{\mathcal{Y}}_{2}(t, \theta)\right)} \tilde{\mathcal{U}}_{2}^{+} \tilde{\mathcal{Y}}_{2, \eta} T_{2} \mathbb{1}_{\tilde{\mathcal{D}}_{1}<\tilde{\mathcal{D}}_{2}}(t, \theta) d \theta \\
& \left.+\int_{0}^{\eta} e^{-\frac{1}{A_{2}}\left(\tilde{\mathcal{Y}}_{2}(t, \eta)-\tilde{\mathcal{Y}}_{2}(t, \theta)\right)} \tilde{\mathcal{U}}_{2}^{+} \tilde{\mathcal{Y}}_{2, \eta} T_{3} \mathbb{1}_{\tilde{\mathcal{D}}_{1}<\tilde{\mathcal{D}}_{2}}(t, \theta) d \theta\right] d \eta \\
= & \frac{1}{2}\left(A_{11}+A_{12}+A_{13}+A_{14}\right) .
\end{aligned}
$$

Again we are forced to consider individual terms,

$$
\begin{aligned}
A_{11}= & \frac{1}{A^{3}} \int_{0}^{1} \frac{1}{\max _{j}\left(\tilde{\mathcal{P}}_{j}^{1 / 2}\right)}\left|\tilde{\mathcal{P}}_{1}^{1 / 2}-\tilde{\mathcal{P}}_{2}^{1 / 2}\right|(t, \eta) \\
& \times\left[\int_{0}^{\eta} e^{-\frac{1}{A_{2}}\left(\tilde{\mathcal{Y}}_{2}(t, \eta)-\tilde{\mathcal{Y}}_{2}(t, \theta)\right)} \tilde{\mathcal{U}}_{2}^{+} \tilde{\mathcal{Y}}_{2, \eta}\left(\bar{d}_{11}+\bar{d}_{12}\right) \mathbb{1}_{\tilde{\mathcal{D}}_{1}<\tilde{\mathcal{D}}_{2}}(t, \theta) d \theta\right] d \eta \\
\leqslant & \frac{1}{A^{3}} \int_{0}^{1} \frac{1}{\max _{j}\left(\tilde{\mathcal{P}}_{j}^{1 / 2}\right)}\left|\tilde{\mathcal{P}}_{1}^{1 / 2}-\tilde{\mathcal{P}}_{2}^{1 / 2}\right|(t, \eta) \\
& \times\left[\int _ { 0 } ^ { \eta } e ^ { - \frac { 1 } { A _ { 2 } } ( \tilde { \mathcal { Y } } _ { 2 } ( t , \eta ) - \tilde { \mathcal { Y } } _ { 2 } ( t , \theta ) ) } \tilde { \mathcal { U } } _ { 2 } ^ { + } \tilde { \mathcal { Y } } _ { 2 , \eta } \left(2 A^{3 / 2} \tilde{\mathcal{D}}_{2}^{1 / 2}\left|\tilde{\mathcal{Y}}_{2}-\tilde{\mathcal{Y}}_{1}\right|\right.\right. \\
& \left.\left.+2 \sqrt{2} A^{3 / 2} \tilde{\mathcal{D}}_{2}^{1 / 2}\left\|\tilde{\mathcal{Y}}_{2}-\tilde{\mathcal{Y}}_{1}\right\|\right)(t, \theta) d \theta\right] d \eta \\
\leqslant & \left\|\tilde{\mathcal{P}}_{1}^{1 / 2}-\tilde{\mathcal{P}}_{2}^{1 / 2}\right\|^{2}+\frac{4}{A^{3}} \int_{0}^{1} \frac{1}{\left(\max _{j}\left(\tilde{\mathcal{P}}_{j}^{1 / 2}\right)\right)^{2}}(t, \eta)
\end{aligned}
$$




$$
\begin{aligned}
& \times\left(\int_{0}^{\eta} e^{-\frac{1}{A_{2}}\left(\tilde{\mathcal{Y}}_{2}(t, \eta)-\tilde{\mathcal{Y}}_{2}(t, \theta)\right)} \tilde{\mathcal{U}}_{2}^{+} \tilde{\mathcal{Y}}_{2, \eta} \tilde{\mathcal{D}}_{2}^{1 / 2}\left|\tilde{\mathcal{Y}}_{2}-\tilde{\mathcal{Y}}_{1}\right|(t, \theta) d \theta\right)^{2} d \eta \\
& +\frac{2 \sqrt{2}}{A^{3 / 2}}\left\|\tilde{\mathcal{Y}}_{2}-\tilde{\mathcal{Y}}_{1}\right\|\left\|\tilde{\mathcal{P}}_{1}^{1 / 2}-\tilde{\mathcal{P}}_{2}^{1 / 2}\right\|\left(\int_{0}^{1} \frac{1}{\left(\max _{j}\left(\tilde{\mathcal{P}}_{j}^{1 / 2}\right)\right)^{2}}(t, \eta)\right. \\
& \left.\times\left(\int_{0}^{\eta} e^{-\frac{1}{A_{2}}\left(\tilde{\mathcal{Y}}_{2}(t, \eta)-\tilde{\mathcal{Y}}_{2}(t, \theta)\right)} \tilde{\mathcal{U}}_{2}^{+} \tilde{\mathcal{Y}}_{2, \eta} \tilde{\mathcal{D}}_{2}^{1 / 2}(t, \theta) d \theta\right)^{2} d \eta\right)^{1 / 2} \\
& \leqslant\left\|\tilde{\mathcal{P}}_{1}^{1 / 2}-\tilde{\mathcal{P}}_{2}^{1 / 2}\right\|^{2} \\
& +\frac{4}{A^{3}} \int_{0}^{1} \frac{1}{\left(\max _{j}\left(\tilde{\mathcal{P}}_{j}^{1 / 2}\right)\right)^{2}}(t, \eta)\left(\int_{0}^{\eta} e^{-\frac{1}{A_{2}}\left(\tilde{\mathcal{Y}}_{2}(t, \eta)-\tilde{\mathcal{Y}}_{2}(t, \theta)\right)} \tilde{\mathcal{U}}_{2}^{2} \tilde{\mathcal{Y}}_{2, \eta}(t, \theta) d \theta\right) \\
& \times\left(\int_{0}^{\eta} e^{-\frac{1}{A_{2}}\left(\tilde{\mathcal{Y}}_{2}(t, \eta)-\tilde{\mathcal{Y}}_{2}(t, \theta)\right)} \tilde{\mathcal{D}}_{2} \tilde{\mathcal{Y}}_{2, \eta}\left|\tilde{\mathcal{Y}}_{2}-\tilde{\mathcal{Y}}_{1}\right|^{2}(t, \theta) d \theta\right) d \eta \\
& +\frac{2 \sqrt{2}}{A^{3}}\left\|\tilde{\mathcal{Y}}_{2}-\tilde{\mathcal{Y}}_{1}\right\|\left\|\tilde{\mathcal{P}}_{1}^{1 / 2}-\tilde{\mathcal{P}}_{2}^{1 / 2}\right\|\left[\int_{0}^{1} \frac{1}{\left(\max _{j}\left(\tilde{\mathcal{P}}_{j}^{1 / 2}\right)\right)^{2}}(t, \eta)\right. \\
& \times\left(\int_{0}^{\eta} e^{-\frac{1}{A_{2}}\left(\tilde{\mathcal{Y}}_{2}(t, \eta)-\tilde{\mathcal{Y}}_{2}(t, \theta)\right)} \tilde{\mathcal{U}}_{2}^{2} \tilde{\mathcal{Y}}_{2, \eta}(t, \theta) d \theta\right) \\
& \left.\times\left(\int_{0}^{\eta} e^{-\frac{1}{A_{2}}\left(\tilde{\mathcal{Y}}_{2}(t, \eta)-\tilde{\mathcal{Y}}_{2}(t, \theta)\right)} 2 A \tilde{\mathcal{P}}_{2} \tilde{\mathcal{Y}}_{2, \eta}(t, \theta) d \theta\right) d \eta\right]^{1 / 2} \\
& \leqslant \mathcal{O}(1)\left(\left\|\tilde{\mathcal{P}}_{1}^{1 / 2}-\tilde{\mathcal{P}}_{2}^{1 / 2}\right\|^{2}+\left\|\tilde{\mathcal{Y}}_{2}-\tilde{\mathcal{Y}}_{1}\right\|^{2}\right),
\end{aligned}
$$

using (4.15e), (4.15n), and (4.16c). Next we find (see (A.14))

$$
\begin{aligned}
& A_{12}=\frac{1}{A^{3}} \int_{0}^{1} \frac{1}{\max _{j}\left(\tilde{\mathcal{P}}_{j}^{1 / 2}\right)}\left|\tilde{\mathcal{P}}_{1}^{1 / 2}-\tilde{\mathcal{P}}_{2}^{1 / 2}\right|(t, \eta) \\
& \times\left(\int_{0}^{\eta} e^{-\frac{1}{A_{2}}\left(\tilde{\mathcal{Y}}_{2}(t, \eta)-\tilde{\mathcal{Y}}_{2}(t, \theta)\right)} \tilde{\mathcal{U}}_{2}^{+} \tilde{\mathcal{Y}}_{2, \eta} T_{1} \mathbb{1}_{\tilde{\mathcal{D}}_{1}<\tilde{\mathcal{D}}_{2}}(t, \theta) d \theta\right) d \eta \\
& \leqslant \frac{1}{A^{3}} \int_{0}^{1} \frac{1}{\max _{j}\left(\tilde{\mathcal{P}}_{j}^{1 / 2}\right)}\left|\tilde{\mathcal{P}}_{1}^{1 / 2}-\tilde{\mathcal{P}}_{2}^{1 / 2}\right|(t, \eta) \\
& \times\left(\int_{0}^{\eta} e^{-\frac{1}{A_{2}}\left(\tilde{\mathcal{Y}}_{2}(t, \eta)-\tilde{\mathcal{Y}}_{2}(t, \theta)\right)}\left|\tilde{\mathcal{U}}_{2}\right|^{3} \tilde{\mathcal{Y}}_{2, \eta}\left|\tilde{\mathcal{Y}}_{2}-\tilde{\mathcal{Y}}_{1}\right|(t, \theta) d \theta\right) d \eta \\
& +4 \int_{0}^{1} \frac{1}{\max _{j}\left(\tilde{\mathcal{P}}_{j}^{1 / 2}\right)}\left|\tilde{\mathcal{P}}_{1}^{1 / 2}-\tilde{\mathcal{P}}_{2}^{1 / 2}\right|(t, \eta)\left(\int_{0}^{\eta} e^{-\frac{1}{A_{2}}\left(\tilde{\mathcal{Y}}_{2}(t, \eta)-\tilde{\mathcal{Y}}_{2}(t, \theta)\right)}\right. \\
& \left.\times\left(\int_{0}^{\theta} e^{-\frac{1}{A}\left(\tilde{\mathcal{Y}}_{2}(t, \theta)-\tilde{\mathcal{Y}}_{2}(t, l)\right)}\left(\tilde{\mathcal{U}}_{2}-\tilde{\mathcal{U}}_{1}\right)^{2}(t, l) d l\right)^{1 / 2} \tilde{\mathcal{U}}_{2}^{+} \tilde{\mathcal{Y}}_{2, \eta}(t, \theta) d \theta\right) d \eta
\end{aligned}
$$




$$
\begin{aligned}
& +\sqrt{2} A \int_{0}^{1} \frac{1}{\max _{j}\left(\tilde{\mathcal{P}}_{j}^{1 / 2}\right)}\left|\tilde{\mathcal{P}}_{1}^{1 / 2}-\tilde{\mathcal{P}}_{2}^{1 / 2}\right|(t, \eta)\left(\int_{0}^{\eta} e^{-\frac{1}{A_{2}}\left(\tilde{\mathcal{Y}}_{2}(t, \eta)-\tilde{\mathcal{Y}}_{2}(t, \theta)\right)}\right. \\
& \left.\times\left(\int_{0}^{\theta} e^{-\frac{1}{a}\left(\tilde{\mathcal{Y}}_{2}(t, \theta)-\tilde{\mathcal{Y}}_{2}(t, l)\right)}\left(\tilde{\mathcal{Y}}_{2}-\tilde{\mathcal{Y}}_{1}\right)^{2}(t, l) d l\right)^{1 / 2} \tilde{\mathcal{U}}_{2}^{+} \tilde{\mathcal{Y}}_{2, \eta}(t, \theta) d \theta\right) d \eta \\
& +A \int_{0}^{1} \frac{1}{\max _{j}\left(\tilde{\mathcal{P}}_{j}^{1 / 2}\right)}\left|\tilde{\mathcal{P}}_{1}^{1 / 2}-\tilde{\mathcal{P}}_{2}^{1 / 2}\right|(t, \eta)\left(\int_{0}^{\eta} e^{-\frac{1}{A_{2}}\left(\tilde{\mathcal{Y}}_{2}(t, \eta)-\tilde{\mathcal{Y}}_{2}(t, \theta)\right)}\right. \\
& \left.\times\left(\int_{0}^{\theta} e^{-\frac{1}{a}\left(\tilde{\mathcal{Y}}_{2}(t, \theta)-\tilde{\mathcal{Y}}_{2}(t, l)\right)}\left|\tilde{\mathcal{Y}}_{2}-\tilde{\mathcal{Y}}_{1}\right|(t, l) d l\right) \tilde{\mathcal{U}}_{2}^{+} \tilde{\mathcal{Y}}_{2, \eta}(t, \theta) d \theta\right) d \eta \\
& +\frac{8 \sqrt{2} A}{\sqrt{3} e} \int_{0}^{1} \frac{1}{\max _{j}\left(\tilde{\mathcal{P}}_{j}^{1 / 2}\right)}\left|\tilde{\mathcal{P}}_{1}^{1 / 2}-\tilde{\mathcal{P}}_{2}^{1 / 2}\right|(t, \eta)\left(\int_{0}^{\eta} e^{-\frac{1}{A_{2}}\left(\tilde{\mathcal{Y}}_{2}(t, \eta)-\tilde{\mathcal{Y}}_{2}(t, \theta)\right)}\right. \\
& \left.\times\left(\int_{0}^{\theta} e^{-\frac{3}{4 A}\left(\tilde{\mathcal{Y}}_{2}(t, \theta)-\tilde{\mathcal{Y}}_{2}(t, l)\right)} d l\right)^{1 / 2}\left|A_{1}-A_{2}\right| \tilde{\mathcal{U}}_{2}^{+} \tilde{\mathcal{Y}}_{2, \eta}(t, \theta) d \theta\right) d \eta \\
& \leqslant 5\left\|\tilde{\mathcal{P}}_{1}^{1 / 2}-\tilde{\mathcal{P}}_{2}^{1 / 2}\right\|^{2}+\frac{1}{A^{6}} \int_{0}^{1} \frac{1}{\left(\max _{j}\left(\tilde{\mathcal{P}}_{j}^{1 / 2}\right)\right)^{2}}(t, \eta) \\
& \times\left(\int_{0}^{\eta} e^{-\frac{1}{A_{2}}\left(\tilde{\mathcal{Y}}_{2}(t, \eta)-\tilde{\mathcal{Y}}_{2}(t, \theta)\right)}\left|\tilde{\mathcal{U}}_{2}\right|^{3} \tilde{\mathcal{Y}}_{2, \eta}\left|\tilde{\mathcal{Y}}_{2}-\tilde{\mathcal{Y}}_{1}\right|(t, \theta) d \theta\right)^{2} d \eta \\
& +16 \int_{0}^{1} \frac{1}{\left(\max _{j}\left(\tilde{\mathcal{P}}_{j}^{1 / 2}\right)\right)^{2}}(t, \eta)\left(\int_{0}^{\eta} e^{-\frac{1}{A_{2}}\left(\tilde{\mathcal{Y}}_{2}(t, \eta)-\tilde{\mathcal{Y}}_{2}(t, \theta)\right)}\right. \\
& \left.\times\left(\int_{0}^{\theta} e^{-\frac{1}{A}\left(\tilde{\mathcal{Y}}_{2}(t, \theta)-\tilde{\mathcal{Y}}_{2}(t, l)\right)}\left(\tilde{\mathcal{U}}_{2}-\tilde{\mathcal{U}}_{1}\right)^{2}(t, l) d l\right)^{1 / 2} \tilde{\mathcal{U}}_{2}^{+} \tilde{\mathcal{Y}}_{2, \eta}(t, \theta) d \theta\right)^{2} d \eta \\
& +2 A^{2} \int_{0}^{1} \frac{1}{\left(\max _{j}\left(\tilde{\mathcal{P}}_{j}^{1 / 2}\right)\right)^{2}}(t, \eta)\left(\int_{0}^{\eta} e^{-\frac{1}{A_{2}}\left(\tilde{\mathcal{Y}}_{2}(t, \eta)-\tilde{\mathcal{Y}}_{2}(t, \theta)\right)}\right. \\
& \left.\times\left(\int_{0}^{\theta} e^{-\frac{1}{a}\left(\tilde{\mathcal{Y}}_{2}(t, \theta)-\tilde{\mathcal{Y}}_{2}(t, l)\right)}\left(\tilde{\mathcal{Y}}_{2}-\tilde{\mathcal{Y}}_{1}\right)^{2}(t, l) d l\right)^{1 / 2} \tilde{\mathcal{U}}_{2}^{+} \tilde{\mathcal{Y}}_{2, \eta}(t, \theta) d \theta\right)^{2} d \eta \\
& +A^{2} \int_{0}^{1} \frac{1}{\left(\max _{j}\left(\tilde{\mathcal{P}}_{j}^{1 / 2}\right)\right)^{2}}(t, \eta)\left(\int_{0}^{\eta} e^{-\frac{1}{A_{2}}\left(\tilde{\mathcal{Y}}_{2}(t, \eta)-\tilde{\mathcal{Y}}_{2}(t, \theta)\right)}\right. \\
& \left.\times\left(\int_{0}^{\theta} e^{-\frac{1}{a}\left(\tilde{\mathcal{Y}}_{2}(t, \theta)-\tilde{\mathcal{Y}}_{2}(t, l)\right)}\left|\tilde{\mathcal{Y}}_{2}-\tilde{\mathcal{Y}}_{1}\right|(t, l) d l\right) \tilde{\mathcal{U}}_{2}^{+} \tilde{\mathcal{Y}}_{2, \eta}(t, \theta) d \theta\right)^{2} d \eta \\
& +\frac{128 A^{2}}{3 e^{2}} \int_{0}^{1} \frac{1}{\left(\max _{j}\left(\tilde{\mathcal{P}}_{j}^{1 / 2}\right)\right)^{2}}\left(\int_{0}^{\eta} e^{-\frac{1}{A_{2}}\left(\tilde{\mathcal{Y}}_{2}(t, \eta)-\tilde{\mathcal{Y}}_{2}(t, \theta)\right)}\right. \\
& \left.\times\left(\int_{0}^{\theta} e^{-\frac{3}{4 A}\left(\tilde{\mathcal{Y}}_{2}(t, \theta)-\tilde{\mathcal{Y}}_{2}(t, l)\right)} d l\right)^{1 / 2} \tilde{\mathcal{U}}_{2}^{+} \tilde{\mathcal{Y}}_{2, \eta}(t, \theta) d \theta\right)^{2} d \eta\left|A_{1}-A_{2}\right|^{2} \\
& \leqslant 5\left\|\tilde{\mathcal{P}}_{1}^{1 / 2}-\tilde{\mathcal{P}}_{2}^{1 / 2}\right\|^{2}
\end{aligned}
$$




$$
\begin{aligned}
& +\frac{1}{A^{6}} \int_{0}^{1} \frac{1}{\left(\max _{j}\left(\tilde{\mathcal{P}}_{j}^{1 / 2}\right)\right)^{2}}(t, \eta)\left(\int_{0}^{\eta} e^{-\frac{1}{A_{2}}\left(\tilde{\mathcal{Y}}_{2}(t, \eta)-\tilde{\mathcal{Y}}_{2}(t, \theta)\right)} \tilde{\mathcal{U}}_{2}^{2} \tilde{\mathcal{Y}}_{2, \eta}(t, \theta) d \theta\right) \\
& \times\left(\int_{0}^{\eta} e^{-\frac{1}{A_{2}}\left(\tilde{\mathcal{Y}}_{2}(t, \eta)-\tilde{\mathcal{Y}}_{2}(t, \theta)\right)} \tilde{\mathcal{U}}_{2}^{4} \tilde{\mathcal{Y}}_{2, \eta}\left(\tilde{\mathcal{Y}}_{2}-\tilde{\mathcal{Y}}_{1}\right)^{2}(t, \theta) d \theta\right) d \eta \\
& +16 \int_{0}^{1} \frac{1}{\left(\max _{j}\left(\tilde{\mathcal{P}}_{j}^{1 / 2}\right)\right)^{2}}(t, \eta)\left(\int_{0}^{\eta} e^{-\frac{1}{A_{2}}\left(\tilde{\mathcal{Y}}_{2}(t, \eta)-\tilde{\mathcal{Y}}_{2}(t, \theta)\right)} \tilde{\mathcal{U}}_{2}^{2} \tilde{\mathcal{Y}}_{2, \eta} d \theta\right) \\
& \times\left(\int_{0}^{\eta} e^{-\frac{1}{A_{2}}\left(\tilde{\mathcal{Y}}_{2}(t, \eta)-\tilde{\mathcal{Y}}_{2}(t, \theta)\right)}\right. \\
& \left.\times\left(\int_{0}^{\theta} e^{-\frac{1}{A}\left(\tilde{\mathcal{Y}}_{2}(t, \theta)-\tilde{\mathcal{Y}}_{2}(t, l)\right)}\left(\tilde{\mathcal{U}}_{2}-\tilde{\mathcal{U}}_{1}\right)^{2}(t, l) d l\right) \tilde{\mathcal{Y}}_{2, \eta}(t, \theta) d \theta\right) d \eta \\
& +2 A^{2} \int_{0}^{1} \frac{1}{\left(\max _{j}\left(\tilde{\mathcal{P}}_{j}^{1 / 2}\right)\right)^{2}}(t, \eta)\left(\int_{0}^{\eta} e^{-\frac{1}{A_{2}}\left(\tilde{\mathcal{Y}}_{2}(t, \eta)-\tilde{\mathcal{Y}}_{2}(t, \theta)\right)} \tilde{\mathcal{U}}_{2}^{2} \tilde{\mathcal{Y}}_{2, \eta} d \theta\right) \\
& \times\left(\int_{0}^{\eta} e^{-\frac{1}{A_{2}}\left(\tilde{\mathcal{Y}}_{2}(t, \eta)-\tilde{\mathcal{Y}}_{2}(t, \theta)\right)}\right. \\
& \left.\times\left(\int_{0}^{\theta} e^{-\frac{1}{a}\left(\tilde{\mathcal{Y}}_{2}(t, \theta)-\tilde{\mathcal{Y}}_{2}(t, l)\right)}\left(\tilde{\mathcal{Y}}_{2}-\tilde{\mathcal{Y}}_{1}\right)^{2}(t, l) d l\right) \tilde{\mathcal{Y}}_{2, \eta}(t, \theta) d \theta\right) d \eta \\
& +A^{2} \int_{0}^{1} \frac{1}{\left(\max _{j}\left(\tilde{\mathcal{P}}_{j}^{1 / 2}\right)\right)^{2}}(t, \eta)\left(\int_{0}^{\eta} e^{-\frac{1}{A_{2}}\left(\tilde{\mathcal{Y}}_{2}(t, \eta)-\tilde{\mathcal{Y}}_{2}(t, \theta)\right)} \tilde{\mathcal{U}}_{2}^{2} \tilde{\mathcal{Y}}_{2, \eta}(t, \theta) d \theta\right) \\
& \times\left(\int_{0}^{\eta} e^{-\frac{1}{A_{2}}\left(\tilde{\mathcal{Y}}_{2}(t, \eta)-\tilde{\mathcal{Y}}_{2}(t, \theta)\right)}\right. \\
& \left.\times\left(\int_{0}^{\theta} e^{-\frac{1}{a}\left(\tilde{\mathcal{Y}}_{2}(t, \theta)-\tilde{\mathcal{Y}}_{2}(t, l)\right)}\left|\tilde{\mathcal{Y}}_{2}-\tilde{\mathcal{Y}}_{1}\right|(t, l) d l\right)^{2} \tilde{\mathcal{Y}}_{2, \eta}(t, \theta) d \theta\right) d \eta \\
& +\frac{128 A^{2}}{3 e^{2}} \int_{0}^{1} \frac{1}{\left(\max _{j}\left(\tilde{\mathcal{P}}_{j}^{1 / 2}\right)\right)^{2}}(t, \eta)\left(\int_{0}^{\eta} e^{-\frac{1}{A_{2}}\left(\tilde{\mathcal{Y}}_{2}(t, \eta)-\tilde{\mathcal{Y}}_{2}(t, \theta)\right)} \tilde{\mathcal{U}}_{2}^{2} \tilde{\mathcal{Y}}_{2, \eta}(t, \theta) d \theta\right) \\
& \times\left(\int_{0}^{\eta} e^{-\frac{1}{A_{2}}\left(\tilde{\mathcal{Y}}_{2}(t, \eta)-\tilde{\mathcal{Y}}_{2}(t, \theta)\right)}\right. \\
& \left.\times\left(\int_{0}^{\theta} e^{-\frac{3}{4 A}\left(\tilde{\mathcal{Y}}_{2}(t, \theta)-\tilde{\mathcal{Y}}_{2}(t, l)\right)} d l\right) \tilde{\mathcal{Y}}_{2, \eta}(t, \theta) d \theta\right) d \eta\left|A_{1}-A_{2}\right|^{2} \\
& \leqslant \mathcal{O}(1)\left(\left\|\tilde{\mathcal{P}}_{1}^{1 / 2}-\tilde{\mathcal{P}}_{2}^{1 / 2}\right\|^{2}+\left\|\tilde{\mathcal{Y}}_{2}-\tilde{\mathcal{Y}}_{1}\right\|^{2}\right)+\mathcal{O}(1) \int_{0}^{1}\left(\int_{0}^{\eta} e^{-\frac{1}{A_{2}}\left(\tilde{\mathcal{Y}}_{2}(t, \eta)-\tilde{\mathcal{Y}}_{2}(t, \theta)\right)}\right. \\
& \left.\times\left(\int_{0}^{\theta} e^{-\frac{1}{A}\left(\tilde{\mathcal{Y}}_{2}(t, \theta)-\tilde{\mathcal{Y}}_{2}(t, l)\right)}\left(\tilde{\mathcal{U}}_{2}-\tilde{\mathcal{U}}_{1}\right)^{2}(t, l) d l\right) \tilde{\mathcal{Y}}_{2, \eta}(t, \theta) d \theta\right) d \eta \\
& +\mathcal{O}(1) \int_{0}^{1}\left(\int_{0}^{\eta} e^{-\frac{1}{A_{2}}\left(\tilde{\mathcal{Y}}_{2}(t, \eta)-\tilde{\mathcal{Y}}_{2}(t, \theta)\right)}\right.
\end{aligned}
$$




$$
\begin{aligned}
& \left.\times\left(\int_{0}^{\theta} e^{-\frac{1}{a}\left(\tilde{\mathcal{Y}}_{2}(t, \theta)-\tilde{\mathcal{Y}}_{2}(t, l)\right)}\left(\tilde{\mathcal{Y}}_{2}-\tilde{\mathcal{Y}}_{1}\right)^{2}(t, l) d l\right) \tilde{\mathcal{Y}}_{2, \eta}(t, \theta) d \theta\right) d \eta \\
& +\mathcal{O}(1) \int_{0}^{1}\left(\int_{0}^{\eta} e^{-\frac{1}{A_{2}}\left(\tilde{\mathcal{Y}}_{2}(t, \eta)-\tilde{\mathcal{Y}}_{2}(t, \theta)\right)}\right. \\
& \left.\times\left(\int_{0}^{\theta} e^{-\frac{3}{4 A}\left(\tilde{\mathcal{Y}}_{2}(t, \theta)-\tilde{\mathcal{Y}}_{2}(t, l)\right)} d l\right) \tilde{\mathcal{Y}}_{2, \eta}(t, \theta) d \theta\right) d \eta\left|A_{1}-A_{2}\right|^{2} \\
& \leqslant \mathcal{O}(1)\left(\left\|\tilde{\mathcal{P}}_{1}^{1 / 2}-\tilde{\mathcal{P}}_{2}^{1 / 2}\right\|^{2}+\left\|\tilde{\mathcal{Y}}_{2}-\tilde{\mathcal{Y}}_{1}\right\|^{2}\right)+B_{1}+B_{2}+B_{3},
\end{aligned}
$$

using (4.15b), (4.15g), and (4.16c). As for $B_{1}$, we find

$$
\begin{aligned}
& B_{1}=\mathcal{O}(1) \int_{0}^{1}\left(\int_{0}^{\eta} e^{-\frac{1}{A_{2}}\left(\tilde{\mathcal{Y}}_{2}(t, \eta)-\tilde{\mathcal{Y}}_{2}(t, \theta)\right)}\right. \\
& \left.\times\left(\int_{0}^{\theta} e^{-\frac{1}{A}\left(\tilde{\mathcal{Y}}_{2}(t, \theta)-\tilde{\mathcal{Y}}_{2}(t, l)\right)}\left(\tilde{\mathcal{U}}_{2}-\tilde{\mathcal{U}}_{1}\right)^{2}(t, l) d l\right) \tilde{\mathcal{Y}}_{2, \eta}(t, \theta) d \theta\right) d \eta \\
& \leqslant \mathcal{O}(1) \int_{0}^{1}\left(\int_{0}^{\eta} e^{-\frac{1}{2 A}\left(\tilde{\mathcal{Y}}_{2}(t, \eta)-\tilde{\mathcal{Y}}_{2}(t, \theta)\right)}\right. \\
& \left.\times\left(\int_{0}^{\theta} e^{-\frac{1}{A}\left(\tilde{\mathcal{Y}}_{2}(t, \theta)-\tilde{\mathcal{Y}}_{2}(t, l)\right)}\left(\tilde{\mathcal{U}}_{2}-\tilde{\mathcal{U}}_{1}\right)^{2}(t, l) d l\right) \tilde{\mathcal{Y}}_{2, \eta}(t, \theta) d \theta\right) d \eta \\
& =\mathcal{O}(1) \int_{0}^{1}\left(\int_{0}^{\eta} e^{-\frac{1}{2 A} \tilde{\mathcal{Y}}_{2}(t, \theta)} \tilde{\mathcal{Y}}_{2, \eta}(t, \theta)\right. \\
& \left.\times\left(\int_{0}^{\theta} e^{-\left(\frac{1}{2 A} \tilde{\mathcal{Y}}_{2}(t, \eta)-\frac{1}{A} \tilde{\mathcal{Y}}_{2}(t, l)\right)}\left(\tilde{\mathcal{U}}_{2}-\tilde{\mathcal{U}}_{1}\right)^{2}(t, l) d l\right) d \theta\right) d \eta \\
& =\mathcal{O}(1) \int_{0}^{1}\left[\left(-2 A e^{-\frac{1}{2 A} \tilde{\mathcal{Y}}_{2}(t, \theta)}\right.\right. \\
& \left.\times\left(\int_{0}^{\theta} e^{-\left(\frac{1}{2 A} \tilde{\mathcal{Y}}_{2}(t, \eta)-\frac{1}{A} \tilde{\mathcal{Y}}_{2}(t, l)\right)}\left(\tilde{\mathcal{U}}_{2}-\tilde{\mathcal{U}}_{1}\right)^{2}(t, l) d l\right)\right)\left.\right|_{\theta=0} ^{\eta} \\
& \left.+2 A \int_{0}^{\eta} e^{-\frac{1}{2 A} \tilde{\mathcal{Y}}_{2}(t, \theta)} e^{-\left(\frac{1}{2 A} \tilde{\mathcal{Y}}_{2}(t, \eta)-\frac{1}{A} \tilde{\mathcal{Y}}_{2}(t, \theta)\right)}\left(\tilde{\mathcal{U}}_{2}-\tilde{\mathcal{U}}_{1}\right)^{2}(t, \theta) d \theta\right] d \eta \\
& =-2 A \mathcal{O}(1) \int_{0}^{1} \int_{0}^{\eta} e^{-\frac{1}{A}\left(\tilde{\mathcal{Y}}_{2}(t, \eta)-\tilde{\mathcal{Y}}_{2}(t, \theta)\right)}\left(\tilde{\mathcal{U}}_{2}-\tilde{\mathcal{U}}_{1}\right)^{2}(t, \theta) d \theta d \eta \\
& +2 A \mathcal{O}(1) \int_{0}^{1} \int_{0}^{\eta} e^{-\frac{1}{2 A}\left(\tilde{\mathcal{Y}}_{2}(t, \eta)-\tilde{\mathcal{Y}}_{2}(t, \theta)\right)}\left(\tilde{\mathcal{U}}_{2}-\tilde{\mathcal{U}}_{1}\right)^{2}(t, \theta) d \theta d \eta \\
& \leqslant \mathcal{O}(1)\left\|\tilde{\mathcal{U}}_{2}-\tilde{\mathcal{U}}_{1}\right\|^{2} \text {, }
\end{aligned}
$$


while for $B_{2}$, we estimate as follows:

$$
\begin{aligned}
& B_{2}=\mathcal{O}(1) \int_{0}^{1}\left(\int_{0}^{\eta} e^{-\frac{1}{A_{2}}\left(\tilde{\mathcal{Y}}_{2}(t, \eta)-\tilde{\mathcal{Y}}_{2}(t, \theta)\right)}\right. \\
& \left.\times\left(\int_{0}^{\theta} e^{-\frac{1}{a}\left(\tilde{\mathcal{Y}}_{2}(t, \theta)-\tilde{\mathcal{Y}}_{2}(t, l)\right)}\left(\tilde{\mathcal{Y}}_{2}-\tilde{\mathcal{Y}}_{1}\right)^{2}(t, l) d l\right) \tilde{\mathcal{Y}}_{2, \eta}(t, \theta) d \theta\right) d \eta \\
& \leqslant \mathcal{O}(1) \int_{0}^{1}\left(\int_{0}^{\eta} e^{-\frac{1}{2 A_{2}}\left(\tilde{\mathcal{Y}}_{2}(t, \eta)-\tilde{\mathcal{Y}}_{2}(t, \theta)\right)}\right. \\
& \left.\times\left(\int_{0}^{\theta} e^{-\frac{1}{A_{2}}\left(\tilde{\mathcal{Y}}_{2}(t, \theta)-\tilde{\mathcal{Y}}_{2}(t, l)\right)}\left(\tilde{\mathcal{Y}}_{2}-\tilde{\mathcal{Y}}_{1}\right)^{2}(t, l) d l\right) \tilde{\mathcal{Y}}_{2, \eta}(t, \theta) d \theta\right) d \eta \\
& =\mathcal{O}(1) \int_{0}^{1}\left(\int_{0}^{\eta} e^{-\frac{1}{2 A_{2}} \tilde{\mathcal{Y}}_{2}(t, \theta)} \tilde{\mathcal{Y}}_{2, \eta}(t, \theta)\right. \\
& \left.\times\left(\int_{0}^{\theta} e^{-\left(\frac{1}{2 A_{2}} \tilde{\mathcal{Y}}_{2}(t, \eta)-\frac{1}{A_{2}} \tilde{\mathcal{Y}}_{2}(t, l)\right)}\left(\tilde{\mathcal{Y}}_{2}-\tilde{\mathcal{Y}}_{1}\right)^{2}(t, l) d l\right) d \theta\right) d \eta \\
& =\mathcal{O}(1) \int_{0}^{1}\left[\left(-2 A_{2} e^{-\frac{1}{2 A_{2}} \tilde{\mathcal{Y}}_{2}(t, \theta)}\right.\right. \\
& \left.\times\left(\int_{0}^{\theta} e^{-\left(\frac{1}{2 A_{2}} \tilde{\mathcal{Y}}_{2}(t, \eta)-\frac{1}{A_{2}} \tilde{\mathcal{Y}}_{2}(t, l)\right)}\left(\tilde{\mathcal{Y}}_{2}-\tilde{\mathcal{Y}}_{1}\right)^{2}(t, l) d l\right)\right)\left.\right|_{\theta=0} ^{\eta} \\
& \left.+2 A_{2} \int_{0}^{\eta} e^{-\frac{1}{2 A_{2}} \tilde{\mathcal{Y}}_{2}(t, \theta)} e^{-\left(\frac{1}{2 A_{2}} \tilde{\mathcal{Y}}_{2}(t, \eta)-\frac{1}{A_{2}} \tilde{\mathcal{Y}}_{2}(t, \theta)\right)}\left(\tilde{\mathcal{Y}}_{2}-\tilde{\mathcal{Y}}_{1}\right)^{2}(t, \theta) d \theta\right] d \eta \\
& =-2 A_{2} \mathcal{O}(1) \int_{0}^{1} \int_{0}^{\eta} e^{-\frac{1}{A_{2}}\left(\tilde{\mathcal{Y}}_{2}(t, \eta)-\tilde{\mathcal{Y}}_{2}(t, \theta)\right)}\left(\tilde{\mathcal{Y}}_{2}-\tilde{\mathcal{Y}}_{1}\right)^{2}(t, \theta) d \theta d \eta \\
& +2 A_{2} \mathcal{O}(1) \int_{0}^{1} \int_{0}^{\eta} e^{-\frac{1}{2 A_{2}}\left(\tilde{\mathcal{Y}}_{2}(t, \eta)-\tilde{\mathcal{Y}}_{2}(t, \theta)\right)}\left(\tilde{\mathcal{Y}}_{2}-\tilde{\mathcal{Y}}_{1}\right)^{2}(t, \theta) d \theta d \eta \\
& \leqslant \mathcal{O}(1)\left\|\tilde{\mathcal{Y}}_{2}-\tilde{\mathcal{Y}}_{1}\right\|^{2} \text {, }
\end{aligned}
$$

while for $B_{3}$, we estimate as follows:

$$
\begin{aligned}
B_{3}= & \mathcal{O}(1) \int_{0}^{1}\left(\int_{0}^{\eta} e^{-\frac{1}{A_{2}}\left(\tilde{\mathcal{Y}}_{2}(t, \eta)-\tilde{\mathcal{Y}}_{2}(t, \theta)\right)}\right. \\
& \left.\times\left(\int_{0}^{\theta} e^{-\frac{3}{4 A}\left(\tilde{\mathcal{Y}}_{2}(t, \theta)-\tilde{\mathcal{Y}}_{2}(t, l)\right)} d l\right) \tilde{\mathcal{Y}}_{2, \eta}(t, \theta) d \theta\right) d \eta\left|A_{1}-A_{2}\right|^{2} \\
\leqslant & \mathcal{O}(1) \int_{0}^{1}\left(\int_{0}^{\eta} e^{-\frac{3}{8 A}\left(\tilde{\mathcal{Y}}_{2}(t, \eta)-\tilde{\mathcal{Y}}_{2}(t, \theta)\right)}\right. \\
& \left.\times\left(\int_{0}^{\theta} e^{-\frac{3}{4 A}\left(\tilde{\mathcal{Y}}_{2}(t, \theta)-\tilde{\mathcal{Y}}_{2}(t, l)\right)} d l\right) \tilde{\mathcal{Y}}_{2, \eta}(t, \theta) d \theta\right) d \eta\left|A_{1}-A_{2}\right|^{2}
\end{aligned}
$$




$$
\begin{aligned}
& =\mathcal{O}(1) \int_{0}^{1}\left(\int_{0}^{\eta} e^{-\frac{3}{8 A} \tilde{\mathcal{Y}}_{2}(t, \theta)} \tilde{\mathcal{Y}}_{2, \eta}(t, \theta)\right. \\
& \left.\times\left(\int_{0}^{\theta} e^{-\left(\frac{3}{8 A} \tilde{\mathcal{Y}}_{2}(t, \eta)-\frac{3}{4 A} \tilde{\mathcal{Y}}_{2}(t, l)\right)} d l\right) d \theta\right) d \eta\left|A_{1}-A_{2}\right|^{2} \\
& =\mathcal{O}(1) \int_{0}^{1}\left[\left(-\frac{8}{3} A e^{-\frac{3}{8 A} \tilde{\mathcal{Y}}_{2}(t, \theta)}\right.\right. \\
& \left.\times\left(\int_{0}^{\theta} e^{-\left(\frac{3}{8 A} \tilde{\mathcal{Y}}_{2}(t, \eta)-\frac{3}{4 A} \tilde{\mathcal{Y}}_{2}(t, l)\right)} d l\right)\right)\left.\right|_{\theta=0} ^{\eta} \\
& \left.+\frac{8}{3} A \int_{0}^{\eta} e^{-\frac{3}{8 A} \tilde{\mathcal{Y}}_{2}(t, \theta)} e^{-\left(\frac{3}{8 A} \tilde{\mathcal{Y}}_{2}(t, \eta)-\frac{3}{4 A} \tilde{\mathcal{Y}}_{2}(t, \theta)\right)} d \theta\right] d \eta\left|A_{1}-A_{2}\right|^{2} \\
& =-\frac{8}{3} A \mathcal{O}(1) \int_{0}^{1} \int_{0}^{\eta} e^{-\frac{3}{4 A}\left(\tilde{\mathcal{Y}}_{2}(t, \eta)-\tilde{\mathcal{Y}}_{2}(t, \theta)\right)} d \theta d \eta\left|A_{1}-A_{2}\right|^{2} \\
& +\frac{8}{3} A \mathcal{O}(1) \int_{0}^{1} \int_{0}^{\eta} e^{-\frac{3}{8 A}\left(\tilde{\mathcal{Y}}_{2}(t, \eta)-\tilde{\mathcal{Y}}_{2}(t, \theta)\right)} d \theta d \eta\left|A_{1}-A_{2}\right|^{2} \\
& \leqslant \mathcal{O}(1)\left|A_{1}-A_{2}\right|^{2} \text {. }
\end{aligned}
$$

Thus we find that

$$
A_{12} \leqslant \mathcal{O}(1)\left(\left\|\tilde{\mathcal{P}}_{1}^{1 / 2}-\tilde{\mathcal{P}}_{2}^{1 / 2}\right\|^{2}+\left\|\tilde{\mathcal{Y}}_{2}-\tilde{\mathcal{Y}}_{1}\right\|^{2}+\left\|\tilde{\mathcal{U}}_{2}-\tilde{\mathcal{U}}_{1}\right\|^{2}+\left|A_{1}-A_{2}\right|^{2}\right) .
$$

Next we find (see (A.15))

$$
\begin{aligned}
A_{13}= & \frac{1}{A^{3}} \int_{0}^{1} \frac{1}{\max _{j}\left(\tilde{\mathcal{P}}_{j}^{1 / 2}\right)}\left|\tilde{\mathcal{P}}_{1}^{1 / 2}-\tilde{\mathcal{P}}_{2}^{1 / 2}\right|(t, \eta) \\
& \times\left(\int_{0}^{\eta} e^{-\frac{1}{A_{2}}\left(\tilde{\mathcal{Y}}_{2}(t, \eta)-\tilde{\mathcal{Y}}_{2}(t, \theta)\right)} \tilde{\mathcal{U}}_{2}^{+} \tilde{\mathcal{Y}}_{2, \eta} T_{2} \mathbb{1}_{\tilde{\mathcal{D}}_{1}<\tilde{\mathcal{D}}_{2}}(t, \theta) d \theta\right) d \eta \\
\leqslant & \frac{1}{A^{3}} \int_{0}^{1} \frac{1}{\max _{j}\left(\tilde{\mathcal{P}}_{j}^{1 / 2}\right)}\left|\tilde{\mathcal{P}}_{1}^{1 / 2}-\tilde{\mathcal{P}}_{2}^{1 / 2}\right|(t, \eta) \\
& \times\left(\int _ { 0 } ^ { \eta } e ^ { - \frac { 1 } { A _ { 2 } } ( \tilde { \mathcal { Y } } _ { 2 } ( t , \eta ) - \tilde { \mathcal { Y } } _ { 2 } ( t , \theta ) ) } \tilde { \mathcal { U } } _ { 2 } ^ { + } \tilde { \mathcal { Y } } _ { 2 , \eta } ( t , \theta ) \left(\tilde{\mathcal{P}}_{j}\left|\tilde{\mathcal{Y}}_{2}-\tilde{\mathcal{Y}}_{1}\right|(t, \theta)\right.\right. \\
& +2 \sqrt{2} A^{3}\left(\int_{0}^{\theta} e^{-\frac{1}{A}\left(\tilde{\mathcal{Y}}_{2}(t, \theta)-\tilde{\mathcal{Y}}_{2}(t, l)\right)}\left(\tilde{\mathcal{P}}_{1}^{1 / 2}-\tilde{\mathcal{P}}_{2}^{1 / 2}\right)^{2}(t, l) d l\right)^{1 / 2} \\
& +\frac{A^{4}}{\sqrt{2}}\left(\int_{0}^{\theta} e^{-\frac{1}{a}\left(\tilde{\mathcal{Y}}_{2}(t, \theta)-\tilde{\mathcal{Y}}_{2}(t, l)\right)}\left(\tilde{\mathcal{Y}}_{1}-\tilde{\mathcal{Y}}_{2}\right)^{2}(t, l) d l\right)^{1 / 2} \\
& +\frac{A^{4}}{2}\left(\int_{0}^{\theta} e^{-\frac{1}{a}\left(\tilde{\mathcal{Y}}_{2}(t, \theta)-\tilde{\mathcal{Y}}_{2}(t, l)\right)}\left|\tilde{\mathcal{Y}}_{2}-\tilde{\mathcal{Y}}_{1}\right|(t, l) d l\right)
\end{aligned}
$$




$$
\begin{aligned}
& \left.\left.+\frac{4 \sqrt{2} A^{4}}{\sqrt{3} e}\left(\int_{0}^{\eta} e^{-\frac{3}{4 A}\left(\tilde{\mathcal{Y}}_{2}(t, \theta)-\tilde{\mathcal{Y}}_{2}(t, l)\right)} d l\right)^{1 / 2}\left|A_{1}-A_{2}\right|\right) d \theta\right) d \eta \\
& \leqslant 5\left\|\tilde{\mathcal{P}}_{1}^{1 / 2}-\tilde{\mathcal{P}}_{2}^{1 / 2}\right\|^{2}+\frac{1}{A^{6}} \int_{0}^{1} \frac{1}{\left(\max _{j}\left(\tilde{\mathcal{P}}_{j}^{1 / 2}\right)\right)^{2}}(t, \eta) \\
& \times\left(\int_{0}^{\eta} e^{-\frac{1}{A_{2}}\left(\tilde{\mathcal{Y}}_{2}(t, \eta)-\tilde{\mathcal{Y}}_{2}(t, \theta)\right)} \tilde{\mathcal{U}}_{2}^{+} \tilde{\mathcal{Y}}_{2, \eta} \tilde{\mathcal{P}}_{j}\left|\tilde{\mathcal{Y}}_{2}-\tilde{\mathcal{Y}}_{1}\right|(t, \theta) d \theta\right)^{2} d \eta \\
& +8 \int_{0}^{1} \frac{1}{\left(\max _{j}\left(\tilde{\mathcal{P}}_{j}^{1 / 2}\right)\right)^{2}}(t, \eta)\left(\int_{0}^{\eta} e^{-\frac{1}{A_{2}}\left(\tilde{\mathcal{Y}}_{2}(t, \eta)-\tilde{\mathcal{Y}}_{2}(t, \theta)\right)} \tilde{\mathcal{U}}_{2}^{+} \tilde{\mathcal{Y}}_{2, \eta}(t, \theta)\right. \\
& \left.\times\left(\int_{0}^{\theta} e^{-\frac{1}{A}\left(\tilde{\mathcal{Y}}_{2}(t, \theta)-\tilde{\mathcal{Y}}_{2}(t, l)\right)}\left(\tilde{\mathcal{P}}_{1}^{1 / 2}-\tilde{\mathcal{P}}_{2}^{1 / 2}\right)^{2}(t, l) d l\right)^{1 / 2} d \theta\right)^{2} d \eta \\
& +\frac{A^{2}}{2} \int_{0}^{1} \frac{1}{\left(\max _{j}\left(\tilde{\mathcal{P}}_{j}^{1 / 2}\right)\right)^{2}}(t, \eta)\left(\int_{0}^{\eta} e^{-\frac{1}{A_{2}}\left(\tilde{\mathcal{Y}}_{2}(t, \eta)-\tilde{\mathcal{Y}}_{2}(t, \theta)\right)} \tilde{\mathcal{U}}_{2}^{+} \tilde{\mathcal{Y}}_{2, \eta}(t, \theta)\right. \\
& \left.\times\left(\int_{0}^{\theta} e^{-\frac{1}{a}\left(\tilde{\mathcal{Y}}_{2}(t, \theta)-\tilde{\mathcal{Y}}_{2}(t, l)\right)}\left(\tilde{\mathcal{Y}}_{1}-\tilde{\mathcal{Y}}_{2}\right)^{2}(t, l) d l\right)^{1 / 2} d \theta\right)^{2} d \eta \\
& +\frac{A^{2}}{4} \int_{0}^{1} \frac{1}{\left(\max _{j}\left(\tilde{\mathcal{P}}_{j}^{1 / 2}\right)\right)^{2}}(t, \eta)\left(\int_{0}^{\eta} e^{-\frac{1}{A_{2}}\left(\tilde{\mathcal{Y}}_{2}(t, \eta)-\tilde{\mathcal{Y}}_{2}(t, \theta)\right)} \tilde{\mathcal{U}}_{2}^{+} \tilde{\mathcal{Y}}_{2, \eta}(t, \theta)\right. \\
& \left.\times\left(\int_{0}^{\theta} e^{-\frac{1}{a}\left(\tilde{\mathcal{Y}}_{2}(t, \theta)-\tilde{\mathcal{Y}}_{2}(t, l)\right)}\left|\tilde{\mathcal{Y}}_{2}-\tilde{\mathcal{Y}}_{1}\right|(t, l) d l\right) d \theta\right)^{2} d \eta \\
& +\frac{32 A^{2}}{3 e^{2}} \int_{0}^{1} \frac{1}{\left(\max _{j}\left(\tilde{\mathcal{P}}_{j}^{1 / 2}\right)\right)^{2}}(t, \eta)\left(\int_{0}^{\eta} e^{-\frac{1}{A_{2}}\left(\tilde{\mathcal{Y}}_{2}(t, \eta)-\tilde{\mathcal{Y}}_{2}(t, \theta)\right)} \tilde{\mathcal{U}}_{2}^{+} \tilde{\mathcal{Y}}_{2, \eta}(t, \theta)\right. \\
& \left.\times\left(\int_{0}^{\theta} e^{-\frac{3}{4 A}\left(\tilde{\mathcal{Y}}_{2}(t, \theta)-\tilde{\mathcal{Y}}_{2}(t, l)\right)} d l\right)^{1 / 2} d \theta\right)^{2} d \eta\left|A_{1}-A_{2}\right|^{2} \\
& \leqslant 5\left\|\tilde{\mathcal{P}}_{1}^{1 / 2}-\tilde{\mathcal{P}}_{2}^{1 / 2}\right\|^{2} \\
& +\frac{1}{A^{6}} \int_{0}^{1} \frac{1}{\left(\max _{j}\left(\tilde{\mathcal{P}}_{j}^{1 / 2}\right)\right)^{2}}(t, \eta)\left(\int_{0}^{\eta} e^{-\frac{1}{A_{2}}\left(\tilde{\mathcal{Y}}_{2}(t, \eta)-\tilde{\mathcal{Y}}_{2}(t, \theta)\right)} \tilde{\mathcal{P}}_{2}^{2} \tilde{\mathcal{Y}}_{2, \eta}(t, \theta) d \theta\right) \\
& \times\left(\int_{0}^{\eta} e^{-\frac{1}{A_{2}}\left(\tilde{\mathcal{Y}}_{2}(t, \eta)-\tilde{\mathcal{Y}}_{2}(t, \theta)\right)} \tilde{\mathcal{U}}_{2}^{2} \tilde{\mathcal{Y}}_{2, \eta}\left(\tilde{\mathcal{Y}}_{2}-\tilde{\mathcal{Y}}_{1}\right)^{2}(t, \theta) d \theta\right) d \eta \\
& +8 \int_{0}^{1} \frac{1}{\left(\max _{j}\left(\tilde{\mathcal{P}}_{j}^{1 / 2}\right)\right)^{2}}(t, \eta)\left(\int_{0}^{\eta} e^{-\frac{1}{A_{2}}\left(\tilde{\mathcal{Y}}_{2}(t, \eta)-\tilde{\mathcal{Y}}_{2}(t, \theta)\right)} \tilde{\mathcal{U}}_{2}^{2} \tilde{\mathcal{Y}}_{2, \eta}(t, \theta) d \theta\right) \\
& \times\left(\int_{0}^{\eta} e^{-\frac{1}{A_{2}}\left(\tilde{\mathcal{Y}}_{2}(t, \eta)-\tilde{\mathcal{Y}}_{2}(t, \theta)\right)} \tilde{\mathcal{Y}}_{2, \eta}(t, \theta)\right.
\end{aligned}
$$




$$
\begin{aligned}
& \left.\times\left(\int_{0}^{\theta} e^{-\frac{1}{A}\left(\tilde{\mathcal{Y}}_{2}(t, \theta)-\tilde{\mathcal{Y}}_{2}(t, l)\right)}\left(\tilde{\mathcal{P}}_{1}^{1 / 2}-\tilde{\mathcal{P}}_{2}^{1 / 2}\right)^{2}(t, l) d l\right) d \theta\right) d \eta \\
& +\frac{A^{2}}{2} \int_{0}^{1} \frac{1}{\left(\max _{j}\left(\tilde{\mathcal{P}}_{j}^{1 / 2}\right)\right)^{2}}(t, \eta)\left(\int_{0}^{\eta} e^{-\frac{1}{A_{2}}\left(\tilde{\mathcal{Y}}_{2}(t, \eta)-\tilde{\mathcal{Y}}_{2}(t, \theta)\right)} \tilde{\mathcal{U}}_{2}^{2} \tilde{\mathcal{Y}}_{2, \eta}(t, \theta) d \theta\right) \\
& \times\left(\int_{0}^{\eta} e^{-\frac{1}{A_{2}}\left(\tilde{\mathcal{Y}}_{2}(t, \eta)-\tilde{\mathcal{Y}}_{2}(t, \theta)\right)} \tilde{\mathcal{Y}}_{2, \eta}(t, \theta)\right. \\
& \left.\times\left(\int_{0}^{\theta} e^{-\frac{1}{a}\left(\tilde{\mathcal{Y}}_{2}(t, \theta)-\tilde{\mathcal{Y}}_{2}(t, l)\right)}\left(\tilde{\mathcal{Y}}_{1}-\tilde{\mathcal{Y}}_{2}\right)^{2}(t, l) d l\right) d \theta\right) d \eta \\
& +\frac{A^{2}}{4} \int_{0}^{1} \frac{1}{\left(\max _{j}\left(\tilde{\mathcal{P}}_{j}^{1 / 2}\right)\right)^{2}}(t, \eta)\left(\int_{0}^{\eta} e^{-\frac{1}{A_{2}}\left(\tilde{\mathcal{Y}}_{2}(t, \eta)-\tilde{\mathcal{Y}}_{2}(t, \theta)\right)} \tilde{\mathcal{U}}_{2}^{2} \tilde{\mathcal{Y}}_{2, \eta}(t, \theta) d \theta\right) \\
& \times\left(\int_{0}^{\eta} e^{-\frac{1}{A_{2}}\left(\tilde{\mathcal{Y}}_{2}(t, \eta)-\tilde{\mathcal{Y}}_{2}(t, \theta)\right)} \tilde{\mathcal{Y}}_{2, \eta}(t, \theta)\right. \\
& \left.\times\left(\int_{0}^{\theta} e^{-\frac{1}{a}\left(\tilde{\mathcal{Y}}_{2}(t, \theta)-\tilde{\mathcal{Y}}_{2}(t, l)\right)}\left|\tilde{\mathcal{Y}}_{2}-\tilde{\mathcal{Y}}_{1}\right|(t, l) d l\right)^{2} d \theta\right) d \eta \\
& +\frac{32 A^{2}}{3 e^{2}} \int_{0}^{1} \frac{1}{\left(\max _{j}\left(\tilde{\mathcal{P}}_{j}^{1 / 2}\right)\right)^{2}}(t, \eta)\left(\int_{0}^{\eta} e^{-\frac{1}{A_{2}}\left(\tilde{\mathcal{Y}}_{2}(t, \eta)-\tilde{\mathcal{Y}}_{2}(t, \theta)\right)} \tilde{\mathcal{U}}_{2}^{2} \tilde{\mathcal{Y}}_{2, \eta}(t, \theta) d \theta\right) \\
& \times\left(\int_{0}^{\eta} e^{-\frac{1}{A_{2}}\left(\tilde{\mathcal{Y}}_{2}(t, \eta)-\tilde{\mathcal{Y}}_{2}(t, \theta)\right)} \tilde{\mathcal{U}}_{2}^{+} \tilde{\mathcal{Y}}_{2, \eta}(t, \theta)\right. \\
& \left.\times\left(\int_{0}^{\theta} e^{-\frac{3}{4 A}\left(\tilde{\mathcal{Y}}_{2}(t, \theta)-\tilde{\mathcal{Y}}_{2}(t, l)\right)} d l\right) d \theta\right) d \eta\left|A_{1}-A_{2}\right|^{2} \\
& \leqslant \mathcal{O}(1)\left(\left\|\tilde{\mathcal{P}}_{1}^{1 / 2}-\tilde{\mathcal{P}}_{2}^{1 / 2}\right\|^{2}+\left\|\tilde{\mathcal{Y}}_{2}-\tilde{\mathcal{Y}}_{1}\right\|^{2}\right) \\
& +\mathcal{O}(1) \int_{0}^{1}\left(\int_{0}^{\eta} e^{-\frac{1}{A_{2}}\left(\tilde{\mathcal{Y}}_{2}(t, \eta)-\tilde{\mathcal{Y}}_{2}(t, \theta)\right)}\right. \\
& \left.\times\left(\int_{0}^{\theta} e^{-\frac{1}{A}\left(\tilde{\mathcal{Y}}_{2}(t, \theta)-\tilde{\mathcal{Y}}_{2}(t, l)\right)}\left(\tilde{\mathcal{P}}_{1}^{1 / 2}-\tilde{\mathcal{P}}_{2}^{1 / 2}\right)^{2}(t, l) d l\right) \tilde{\mathcal{Y}}_{2, \eta}(t, \theta) d \theta\right) d \eta \\
& +\mathcal{O}(1) \int_{0}^{1}\left(\int_{0}^{\eta} e^{-\frac{1}{A_{2}}\left(\tilde{\mathcal{Y}}_{2}(t, \eta)-\tilde{\mathcal{Y}}_{2}(t, \theta)\right)}\right. \\
& \left.\times\left(\int_{0}^{\theta} e^{-\frac{1}{a}\left(\tilde{\mathcal{Y}}_{2}(t, \theta)-\tilde{\mathcal{Y}}_{2}(t, l)\right)}\left(\tilde{\mathcal{Y}}_{2}-\tilde{\mathcal{Y}}_{1}\right)^{2}(t, l) d l\right) \tilde{\mathcal{Y}}_{2, \eta}(t, \theta) d \theta\right) d \eta \\
& +\mathcal{O}(1)\left(\int_{0}^{\eta} e^{-\frac{1}{A_{2}}\left(\tilde{\mathcal{Y}}_{2}(t, \eta)-\tilde{\mathcal{Y}}_{2}(t, \theta)\right)}\right. \\
& \left.\times\left(\int_{0}^{\theta} e^{-\frac{3}{4 A}\left(\tilde{\mathcal{Y}}_{2}(t, \theta)-\tilde{\mathcal{Y}}_{2}(t, l)\right)} d l\right) \tilde{\mathcal{Y}}_{2, \eta}(t, \theta) d \theta\right) d \eta\left|A_{1}-A_{2}\right|^{2}
\end{aligned}
$$




$$
\begin{aligned}
& \leqslant \mathcal{O}(1)\left(\left\|\tilde{\mathcal{P}}_{1}^{1 / 2}-\tilde{\mathcal{P}}_{2}^{1 / 2}\right\|^{2}+\left\|\tilde{\mathcal{Y}}_{2}-\tilde{\mathcal{Y}}_{1}\right\|^{2}\right)+B_{4}+B_{2}+B_{3} \\
& \leqslant \mathcal{O}(1)\left(\left\|\tilde{\mathcal{P}}_{1}^{1 / 2}-\tilde{\mathcal{P}}_{2}^{1 / 2}\right\|^{2}+\left\|\tilde{\mathcal{Y}}_{2}-\tilde{\mathcal{Y}}_{1}\right\|^{2}+\left|A_{1}-A_{2}\right|^{2}\right),
\end{aligned}
$$

following the approach used for $A_{12}$. As for $B_{4}$, we find

$$
\begin{aligned}
& B_{4}=\mathcal{O}(1) \int_{0}^{1}\left(\int_{0}^{\eta} e^{-\frac{1}{A_{2}}\left(\tilde{\mathcal{Y}}_{2}(t, \eta)-\tilde{\mathcal{Y}}_{2}(t, \theta)\right)}\right. \\
& \left.\times\left(\int_{0}^{\theta} e^{-\frac{1}{A}\left(\tilde{\mathcal{Y}}_{2}(t, \theta)-\tilde{\mathcal{Y}}_{2}(t, l)\right)}\left(\tilde{\mathcal{P}}_{1}^{1 / 2}-\tilde{\mathcal{P}}_{2}^{1 / 2}\right)^{2}(t, l) d l\right) \tilde{\mathcal{Y}}_{2, \eta}(t, \theta) d \theta\right) d \eta \\
& \leqslant \mathcal{O}(1) \int_{0}^{1}\left(\int_{0}^{\eta} e^{-\frac{1}{2 A}\left(\tilde{\mathcal{Y}}_{2}(t, \eta)-\tilde{\mathcal{Y}}_{2}(t, \theta)\right)}\right. \\
& \left.\times\left(\int_{0}^{\theta} e^{-\frac{1}{A}\left(\tilde{\mathcal{Y}}_{2}(t, \theta)-\tilde{\mathcal{Y}}_{2}(t, l)\right)}\left(\tilde{\mathcal{P}}_{1}^{1 / 2}-\tilde{\mathcal{P}}_{2}^{1 / 2}\right)^{2}(t, l) d l\right) \tilde{\mathcal{Y}}_{2, \eta}(t, \theta) d \theta\right) d \eta \\
& =\mathcal{O}(1) \int_{0}^{1}\left(\int_{0}^{\eta} e^{-\frac{1}{2 A} \tilde{\mathcal{Y}}_{2}(t, \theta)} \tilde{\mathcal{Y}}_{2, \eta}(t, \theta)\right. \\
& \left.\times\left(\int_{0}^{\theta} e^{-\left(\frac{1}{2 A} \tilde{\mathcal{Y}}_{2}(t, \eta)-\frac{1}{A} \tilde{\mathcal{Y}}_{2}(t, l)\right)}\left(\tilde{\mathcal{P}}_{1}^{1 / 2}-\tilde{\mathcal{P}}_{2}^{1 / 2}\right)^{2}(t, l) d l\right) d \theta\right) d \eta \\
& =\mathcal{O}(1) \int_{0}^{1}\left[\left(-2 A e^{-\frac{1}{2 A} \tilde{\mathcal{Y}}_{2}(t, \theta)}\right.\right. \\
& \left.\times\left(\int_{0}^{\theta} e^{-\left(\frac{1}{2 A} \tilde{\mathcal{Y}}_{2}(t, \eta)-\frac{1}{A} \tilde{\mathcal{Y}}_{2}(t, l)\right)}\left(\tilde{\mathcal{P}}_{1}^{1 / 2}-\tilde{\mathcal{P}}_{2}^{1 / 2}\right)^{2}(t, l) d l\right)\right)\left.\right|_{\theta=0} ^{\eta} \\
& \left.+2 A \int_{0}^{\eta} e^{-\frac{1}{2 A} \tilde{\mathcal{Y}}_{2}(t, \theta)} e^{-\left(\frac{1}{2 A} \tilde{\mathcal{Y}}_{2}(t, \eta)-\frac{1}{A} \tilde{\mathcal{Y}}_{2}(t, \theta)\right)}\left(\tilde{\mathcal{P}}_{1}^{1 / 2}-\tilde{\mathcal{P}}_{2}^{1 / 2}\right)^{2}(t, \theta) d \theta\right] d \eta \\
& =-2 A \mathcal{O}(1) \int_{0}^{1} \int_{0}^{\eta} e^{-\frac{1}{A}\left(\tilde{\mathcal{Y}}_{2}(t, \eta)-\tilde{\mathcal{Y}}_{2}(t, \theta)\right)}\left(\tilde{\mathcal{P}}_{1}^{1 / 2}-\tilde{\mathcal{P}}_{2}^{1 / 2}\right)^{2}(t, \theta) d \theta d \eta \\
& +2 A \mathcal{O}(1) \int_{0}^{1} \int_{0}^{\eta} e^{-\frac{1}{2 A}\left(\tilde{\mathcal{Y}}_{2}(t, \eta)-\tilde{\mathcal{Y}}_{2}(t, \theta)\right)}\left(\tilde{\mathcal{P}}_{1}^{1 / 2}-\tilde{\mathcal{P}}_{2}^{1 / 2}\right)^{2}(t, \theta) d \theta d \eta \\
& \leqslant \mathcal{O}(1)\left\|\tilde{\mathcal{P}}_{1}^{1 / 2}-\tilde{\mathcal{P}}_{2}^{1 / 2}\right\|^{2}
\end{aligned}
$$

Thus we find that

$$
A_{12} \leqslant \mathcal{O}(1)\left(\left\|\tilde{\mathcal{P}}_{1}^{1 / 2}-\tilde{\mathcal{P}}_{2}^{1 / 2}\right\|^{2}+\left\|\tilde{\mathcal{Y}}_{2}-\tilde{\mathcal{Y}}_{1}\right\|^{2}+\left\|\tilde{\mathcal{U}}_{2}-\tilde{\mathcal{U}}_{1}\right\|^{2}+\left|A_{1}-A_{2}\right|^{2}\right)
$$


Next we find (see (A.16))

$$
\begin{aligned}
& A_{14}=\frac{1}{A^{3}} \int_{0}^{1} \frac{1}{\max _{j}\left(\tilde{\mathcal{P}}_{j}^{1 / 2}\right)}\left|\tilde{\mathcal{P}}_{1}^{1 / 2}-\tilde{\mathcal{P}}_{2}^{1 / 2}\right|(t, \eta) \\
& \times\left(\int_{0}^{\eta} e^{-\frac{1}{A_{2}}\left(\tilde{\mathcal{Y}}_{2}(t, \eta)-\tilde{\mathcal{Y}}_{2}(t, \theta)\right)} \tilde{\mathcal{U}}_{2}^{+} \tilde{\mathcal{Y}}_{2, \eta} T_{3} \mathbb{1}_{\tilde{\mathcal{D}}_{1}<\tilde{\mathcal{D}}_{2}}(t, \theta) d \theta\right) d \eta \\
& \leqslant 12 A\left|A_{1}-A_{2}\right| \int_{0}^{1} \frac{1}{\max _{j}\left(\tilde{\mathcal{P}}_{j}^{1 / 2}\right)}\left|\tilde{\mathcal{P}}_{1}^{1 / 2}-\tilde{\mathcal{P}}_{2}^{1 / 2}\right|(t, \eta) \\
& \times\left(\int_{0}^{\eta} e^{-\frac{1}{A_{2}}\left(\tilde{\mathcal{Y}}_{2}(t, \eta)-\tilde{\mathcal{Y}}_{2}(t, \theta)\right)} \tilde{\mathcal{U}}_{2}^{+} \tilde{\mathcal{Y}}_{2, \eta}(t, \theta)\left(\int_{0}^{\theta} e^{-\frac{3}{4 A}\left(\tilde{\mathcal{Y}}_{2}(t, \theta)-\tilde{\mathcal{Y}}_{2}(t, l)\right)} d l\right) d \theta\right) d \eta \\
& \leqslant 12 A\left|A_{1}-A_{2}\right|\left\|\tilde{\mathcal{P}}_{1}^{1 / 2}-\tilde{\mathcal{P}}_{2}^{1 / 2}\right\|\left(\int_{0}^{1} \frac{1}{\left(\max _{j}\left(\tilde{\mathcal{P}}_{j}^{1 / 2}\right)\right)^{2}}(t, \eta)\right. \\
& \times\left(\int_{0}^{\eta} e^{-\frac{1}{A_{2}}\left(\tilde{\mathcal{Y}}_{2}(t, \eta)-\tilde{\mathcal{Y}}_{2}(t, \theta)\right.} \tilde{\mathcal{U}}_{2}^{+} \tilde{\mathcal{Y}}_{2, \eta}(t, \theta)\right. \\
& \left.\left.\times\left(\int_{0}^{\theta} e^{-\frac{3}{4 A}\left(\tilde{\mathcal{Y}}_{2}(t, \theta)-\tilde{\mathcal{Y}}_{2}(t, l)\right)} d l\right) d \theta\right)^{2} d \eta\right)^{1 / 2} \\
& \leqslant 12 A\left|A_{1}-A_{2}\right|\left\|\tilde{\mathcal{P}}_{1}^{1 / 2}-\tilde{\mathcal{P}}_{2}^{1 / 2}\right\| \\
& \times\left(\int_{0}^{1} \frac{1}{\left(\max _{j}\left(\tilde{\mathcal{P}}_{j}^{1 / 2}\right)\right)^{2}}(t, \eta)\left(\int_{0}^{\eta} e^{-\frac{1}{A_{2}}\left(\tilde{\mathcal{Y}}_{2}(t, \eta)-\tilde{\mathcal{Y}}_{2}(t, \theta)\right)} \tilde{\mathcal{U}}_{2}^{2} \tilde{\mathcal{Y}}_{2, \eta}(t, \theta) d \theta\right)\right. \\
& \left.\times\left(\int_{0}^{\eta} e^{-\frac{1}{A_{2}}\left(\tilde{\mathcal{Y}}_{2}(t, \eta)-\tilde{\mathcal{Y}}_{2}(t, \theta)\right)} \tilde{\mathcal{Y}}_{2, \eta}(t, \theta)\left(\int_{0}^{\theta} e^{-\frac{3}{4 A}\left(\tilde{\mathcal{Y}}_{2}(t, \theta)-\tilde{\mathcal{Y}}_{2}(t, l)\right)} d l\right)^{2} d \theta\right) d \eta\right)^{1 / 2} \\
& \leqslant \mathcal{O}(1)\left|A_{1}-A_{2}\right|\left\|\tilde{\mathcal{P}}_{1}^{1 / 2}-\tilde{\mathcal{P}}_{2}^{1 / 2}\right\|\left(\int _ { 0 } ^ { 1 } \left(\int_{0}^{\eta} e^{-\frac{1}{A_{2}}\left(\tilde{\mathcal{Y}}_{2}(t, \eta)-\tilde{\mathcal{Y}}_{2}(t, \theta)\right)} \tilde{\mathcal{Y}}_{2, \eta}(t, \theta)\right.\right. \\
& \left.\left.\times\left(\int_{0}^{\theta} e^{-\frac{3}{4 A}\left(\tilde{\mathcal{Y}}_{2}(t, \theta)-\tilde{\mathcal{Y}}_{2}(t, l)\right)} d l\right)^{2} d \theta\right) d \eta\right)^{1 / 2} \\
& \leqslant \mathcal{O}(1)\left|A_{1}-A_{2}\right|\left\|\tilde{\mathcal{P}}_{1}^{1 / 2}-\tilde{\mathcal{P}}_{2}^{1 / 2}\right\| \\
& \times\left(\int _ { 0 } ^ { 1 } \left(\int_{0}^{\eta} e^{-\frac{3}{8 A}\left(\tilde{\mathcal{Y}}_{2}(t, \eta)-\tilde{\mathcal{Y}}_{2}(t, \theta)\right)} \tilde{\mathcal{Y}}_{2, \eta}(t, \theta)\right.\right. \\
& \left.\left.\times\left(\int_{0}^{\theta} e^{-\frac{3}{4 A}\left(\tilde{\mathcal{Y}}_{2}(t, \theta)-\tilde{\mathcal{Y}}_{2}(t, l)\right)} d l\right) d \theta\right) d \eta\right)^{1 / 2} \\
& \leqslant \mathcal{O}(1)\left|A_{1}-A_{2}\right|\left\|\tilde{\mathcal{P}}_{1}^{1 / 2}-\tilde{\mathcal{P}}_{2}^{1 / 2}\right\| \\
& \times\left(\int _ { 0 } ^ { 1 } \left(\int_{0}^{\eta} e^{-\frac{3}{8 A} \tilde{\mathcal{Y}}_{2}(t, \theta)} \tilde{\mathcal{Y}}_{2, \eta}(t, \theta)\right.\right. \\
& \left.\left.\times\left(\int_{0}^{\theta} e^{-\left(\frac{3}{8 A} \tilde{\mathcal{Y}}_{2}(t, \eta)-\frac{3}{4 A} \tilde{\mathcal{Y}}_{2}(t, l)\right)} d l\right) d \theta\right) d \eta\right)^{1 / 2}
\end{aligned}
$$




$$
\begin{aligned}
& \leqslant \mathcal{O}(1)\left|A_{1}-A_{2}\right|\left\|\tilde{\mathcal{P}}_{1}^{1 / 2}-\tilde{\mathcal{P}}_{2}^{1 / 2}\right\| \\
& \times\left(\int _ { 0 } ^ { 1 } \left[\left.\left(-\frac{8}{3} A e^{-\frac{3}{8 A} \tilde{\mathcal{Y}}_{2}(t, \theta)} \int_{0}^{\theta} e^{-\left(\frac{3}{8 A} \tilde{\mathcal{Y}}_{2}(t, \eta)-\frac{3}{4 A} \tilde{\mathcal{Y}}_{2}(t, l)\right)} d l\right)\right|_{\theta=0} ^{\eta}\right.\right. \\
& \left.\left.+\frac{8}{3} A \int_{0}^{\eta} e^{-\frac{3}{8 A} \tilde{\mathcal{Y}}_{2}(t, \theta)} e^{-\left(\frac{3}{8 A} \tilde{\mathcal{Y}}_{2}(t, \eta)-\frac{3}{4 A} \tilde{\mathcal{Y}}_{2}(t, \theta)\right)} d \theta\right] d \eta\right)^{1 / 2} \\
& \leqslant \mathcal{O}(1)\left|A_{1}-A_{2}\right|\left\|\tilde{\mathcal{P}}_{1}^{1 / 2}-\tilde{\mathcal{P}}_{2}^{1 / 2}\right\| \\
& \times\left(\int _ { 0 } ^ { 1 } \left[-\frac{8}{3} A \int_{0}^{\eta} e^{-\frac{3}{4 A}\left(\tilde{\mathcal{Y}}_{2}(t, \eta)-\tilde{\mathcal{Y}}_{2}(t, \theta)\right)} d \theta\right.\right. \\
& \left.\left.+\frac{8}{3} A \int_{0}^{\eta} e^{-\frac{3}{8 A}\left(\tilde{\mathcal{Y}}_{2}(t, \eta)-\tilde{\mathcal{Y}}_{2}(t, \theta)\right)} d \theta\right] d \eta\right)^{1 / 2} \\
& \leqslant \mathcal{O}(1)\left(\left|A_{1}-A_{2}\right|^{2}+\left\|\tilde{\mathcal{P}}_{1}^{1 / 2}-\tilde{\mathcal{P}}_{2}^{1 / 2}\right\|^{2}\right) \text {. }
\end{aligned}
$$

Thus we have shown that

$$
\tilde{A}_{1}+\tilde{A}_{2} \leqslant \mathcal{O}(1)\left(\left\|\tilde{\mathcal{P}}_{1}^{1 / 2}-\tilde{\mathcal{P}}_{2}^{1 / 2}\right\|^{2}+\left\|\tilde{\mathcal{Y}}_{2}-\tilde{\mathcal{Y}}_{1}\right\|^{2}+\left\|\tilde{\mathcal{U}}_{2}-\tilde{\mathcal{U}}_{1}\right\|^{2}+\left|A_{1}-A_{2}\right|^{2}\right)
$$

We next consider the term $\tilde{A}_{3}$ :

$$
\begin{aligned}
& \tilde{A}_{3}=\frac{1}{2 A^{3}} \int_{0}^{1} \frac{1}{\max _{j}\left(\tilde{\mathcal{P}}_{j}^{1 / 2}\right)}\left(\tilde{\mathcal{P}}_{1}^{1 / 2}-\tilde{\mathcal{P}}_{2}^{1 / 2}\right)(t, \eta) \int_{0}^{\eta}\left(e^{-\frac{1}{A_{2}}\left(\tilde{\mathcal{Y}}_{2}(t, \eta)-\tilde{\mathcal{Y}}_{2}(t, \theta)\right)} \tilde{\mathcal{U}}_{2}^{+} \tilde{\mathcal{Y}}_{2, \eta}\right. \\
& \left.-e^{-\frac{1}{A_{1}}\left(\tilde{\mathcal{Y}}_{1}(t, \eta)-\tilde{\mathcal{Y}}_{1}(t, \theta)\right)} \tilde{\mathcal{U}}_{1}^{+} \tilde{\mathcal{Y}}_{1, \eta}\right) \min _{j}\left(\tilde{\mathcal{D}}_{j}\right)(t, \theta) d \theta d \eta \\
& =\frac{1}{2 A^{3}} \int_{0}^{1} \frac{1}{\max _{j}\left(\tilde{\mathcal{P}}_{j}^{1 / 2}\right)}\left(\tilde{\mathcal{P}}_{1}^{1 / 2}-\tilde{\mathcal{P}}_{2}^{1 / 2}\right)(t, \eta) \\
& \times\left[\int_{0}^{\eta} e^{-\frac{1}{A_{2}}\left(\tilde{\mathcal{Y}}_{2}(t, \eta)-\tilde{\mathcal{Y}}_{2}(t, \theta)\right)} \min _{j}\left(\tilde{\mathcal{D}}_{j}\right) \tilde{\mathcal{Y}}_{2, \eta}\left(\tilde{\mathcal{U}}_{2}^{+}-\tilde{\mathcal{U}}_{1}^{+}\right) \mathbb{1}_{\tilde{\mathcal{U}}_{1}^{+}<\tilde{\mathcal{U}}_{2}^{+}}(t, \theta) d \theta\right. \\
& +\int_{0}^{\eta} e^{-\frac{1}{A_{1}}\left(\tilde{\mathcal{Y}}_{1}(t, \eta)-\tilde{\mathcal{Y}}_{1}(t, \theta)\right)} \min _{j}\left(\tilde{\mathcal{D}}_{j}\right) \tilde{\mathcal{Y}}_{1, \eta}\left(\tilde{\mathcal{U}}_{2}^{+}-\tilde{\mathcal{U}}_{1}^{+}\right) \mathbb{1}_{\tilde{\mathcal{U}}_{2}^{+} \leqslant \tilde{\mathcal{U}}_{1}^{+}}(t, \theta) d \theta \\
& +\mathbb{1}_{A_{1} \leqslant A_{2}} \int_{0}^{\eta}\left(e^{-\frac{1}{A_{2}}\left(\tilde{\mathcal{Y}}_{2}(t, \eta)-\tilde{\mathcal{Y}}_{2}(t, \theta)\right)}-e^{-\frac{1}{A_{1}}\left(\tilde{\mathcal{Y}}_{2}(t, \eta)-\tilde{\mathcal{Y}}_{2}(t, \theta)\right)}\right) \\
& \times \min _{j}\left(\tilde{\mathcal{D}}_{j}\right) \min _{j}\left(\tilde{\mathcal{U}}_{j}^{+}\right) \tilde{\mathcal{Y}}_{2, \eta}(t, \theta) d \theta \\
& +\mathbb{1}_{A_{2}<A_{1}} \int_{0}^{\eta}\left(e^{-\frac{1}{A_{2}}\left(\tilde{\mathcal{Y}}_{1}(t, \eta)-\tilde{\mathcal{Y}}_{1}(t, \theta)\right)}-e^{-\frac{1}{A_{1}}\left(\tilde{\mathcal{Y}}_{1}(t, \eta)-\tilde{\mathcal{Y}}_{1}(t, \theta)\right)}\right) \\
& \times \min _{j}\left(\tilde{\mathcal{D}}_{j}\right) \min _{j}\left(\tilde{\mathcal{U}}_{j}^{+}\right) \tilde{\mathcal{Y}}_{1, \eta}(t, \theta) d \theta \\
& +\int_{0}^{\eta}\left(e^{-\frac{1}{a}\left(\tilde{\mathcal{Y}}_{2}(t, \eta)-\tilde{\mathcal{Y}}_{2}(t, \theta)\right)}-e^{-\frac{1}{a}\left(\tilde{\mathcal{Y}}_{1}(t, \eta)-\tilde{\mathcal{Y}}_{1}(t, \theta)\right)}\right)
\end{aligned}
$$




$$
\begin{aligned}
& \times \min _{j}\left(\tilde{\mathcal{D}}_{j}\right) \min _{j}\left(\tilde{\mathcal{U}}_{j}^{+}\right) \tilde{\mathcal{Y}}_{2, \eta} \mathbb{1}_{B(\eta)}(t, \theta) d \theta \\
& +\int_{0}^{\eta}\left(e^{-\frac{1}{a}\left(\tilde{\mathcal{Y}}_{2}(t, \eta)-\tilde{\mathcal{Y}}_{2}(t, \theta)\right)}-e^{-\frac{1}{a}\left(\tilde{\mathcal{Y}}_{1}(t, \eta)-\tilde{\mathcal{Y}}_{1}(t, \theta)\right)}\right) \\
& \times \min _{j}\left(\tilde{\mathcal{D}}_{j}\right) \min _{j}\left(\tilde{\mathcal{U}}_{j}^{+}\right) \tilde{\mathcal{Y}}_{1, \eta} \mathbb{1}_{B(\eta)^{c}}(t, \theta) d \theta \\
& \left.+\int_{0}^{\eta} \min _{j}\left(e^{-\frac{1}{a}\left(\tilde{\mathcal{Y}}_{j}(t, \eta)-\tilde{\mathcal{Y}}_{j}(t, \theta)\right)}\right) \min _{j}\left(\tilde{\mathcal{D}}_{j}\right) \min _{j}\left(\tilde{\mathcal{U}}_{j}^{+}\right)\left(\tilde{\mathcal{Y}}_{2, \eta}-\tilde{\mathcal{Y}}_{1, \eta}\right)(t, \theta) d \theta\right] d \eta \\
& =A_{31}+A_{32}+A_{33}+A_{34}+A_{35}+A_{36}+A_{37} .
\end{aligned}
$$

Terms $A_{31}$ and $A_{32}$ allow for the same treatment:

$$
\begin{aligned}
& \left|A_{31}\right|=\frac{1}{2 A^{3}} \mid \int_{0}^{1} \frac{1}{\max _{j}\left(\tilde{\mathcal{P}}_{j}^{1 / 2}\right)}\left(\tilde{\mathcal{P}}_{1}^{1 / 2}-\tilde{\mathcal{P}}_{2}^{1 / 2}\right)(t, \eta) \\
& \times\left(\int_{0}^{\eta} e^{-\frac{1}{A_{2}}\left(\tilde{\mathcal{Y}}_{2}(t, \eta)-\tilde{\mathcal{Y}}_{2}(t, \theta)\right)} \min \left(\tilde{\mathcal{D}}_{j}\right) \tilde{\mathcal{Y}}_{2, \eta}\left(\tilde{\mathcal{U}}_{2}^{+}-\tilde{\mathcal{U}}_{1}^{+}\right) \mathbb{1}_{\tilde{\mathcal{U}}_{1}^{+}<\tilde{\mathcal{U}}_{2}^{+}}(t, \theta) d \theta\right) d \eta \\
& \leqslant \frac{1}{A^{2}} \int_{0}^{1} \frac{1}{\max _{j}\left(\tilde{\mathcal{P}}_{j}^{1 / 2}\right)}\left|\tilde{\mathcal{P}}_{1}^{1 / 2}-\tilde{\mathcal{P}}_{2}^{1 / 2}\right|(t, \eta) \\
& \times\left(\int_{0}^{\eta} e^{-\frac{1}{A_{2}}\left(\tilde{\mathcal{Y}}_{2}(t, \eta)-\tilde{\mathcal{Y}}_{2}(t, \theta)\right)} \tilde{\mathcal{P}}_{2} \tilde{\mathcal{Y}}_{2, \eta}\left|\tilde{\mathcal{U}}_{2}^{+}-\tilde{\mathcal{U}}_{1}^{+}\right|(t, \theta) d \theta\right) d \eta \\
& \leqslant\left\|\tilde{\mathcal{P}}_{1}^{1 / 2}-\tilde{\mathcal{P}}_{2}^{1 / 2}\right\|^{2}+\frac{1}{A^{4}} \int_{0}^{1} \frac{1}{\left(\max _{j}\left(\tilde{\mathcal{P}}_{j}^{1 / 2}\right)\right)^{2}}(t, \eta) \\
& \times\left(\int_{0}^{\eta} e^{-\frac{1}{A_{2}}\left(\tilde{\mathcal{Y}}_{2}(t, \eta)-\tilde{\mathcal{Y}}_{2}(t, \theta)\right)} \tilde{\mathcal{P}}_{2} \tilde{\mathcal{Y}}_{2, \eta}\left|\tilde{\mathcal{U}}_{2}^{+}-\tilde{\mathcal{U}}_{1}^{+}\right|(t, \theta) d \theta\right)^{2} d \eta \\
& \leqslant\left\|\tilde{\mathcal{P}}_{1}^{1 / 2}-\tilde{\mathcal{P}}_{2}^{1 / 2}\right\|^{2}+\frac{1}{A^{4}} \int_{0}^{1} \frac{1}{\left(\max _{j}\left(\tilde{\mathcal{P}}_{j}^{1 / 2}\right)\right)^{2}}(t, \eta) \\
& \times\left(\int_{0}^{\eta} e^{-\frac{3}{2 A_{2}}\left(\tilde{\mathcal{Y}}_{2}(t, \eta)-\tilde{\mathcal{Y}}_{2}(t, \theta)\right)} \tilde{\mathcal{P}}_{2} \tilde{\mathcal{Y}}_{2, \eta}(t, \theta) d \theta\right) \\
& \times\left(\int_{0}^{\eta} e^{-\frac{1}{2 A_{2}}\left(\tilde{\mathcal{Y}}_{2}(t, \eta)-\tilde{\mathcal{Y}}_{2}(t, \theta)\right)} \tilde{\mathcal{P}}_{2} \tilde{\mathcal{Y}}_{2, \eta}\left(\tilde{\mathcal{U}}_{2}-\tilde{\mathcal{U}}_{1}\right)^{2}(t, \theta) d \theta\right) d \eta \\
& \leqslant\left\|\tilde{\mathcal{P}}_{1}^{1 / 2}-\tilde{\mathcal{P}}_{2}^{1 / 2}\right\|^{2} \\
& +\frac{2}{A^{3}} \int_{0}^{1}\left(\int_{0}^{\eta} e^{-\frac{1}{2 A_{2}}\left(\tilde{\mathcal{Y}}_{2}(t, \eta)-\tilde{\mathcal{Y}}_{2}(t, \theta)\right)} \tilde{\mathcal{P}}_{2} \tilde{\mathcal{Y}}_{2, \eta}\left(\tilde{\mathcal{U}}_{2}-\tilde{\mathcal{U}}_{1}\right)^{2}(t, \theta) d \theta\right) d \eta \\
& \leqslant \mathcal{O}(1)\left(\left\|\tilde{\mathcal{P}}_{1}^{1 / 2}-\tilde{\mathcal{P}}_{2}^{1 / 2}\right\|^{2}+\left\|\tilde{\mathcal{U}}_{2}-\tilde{\mathcal{U}}_{1}\right\|^{2}\right),
\end{aligned}
$$

using (4.15e), (4.15n), and (4.16e). 
The terms $A_{33}$ and $A_{34}$ can be treated as follows:

$$
\begin{aligned}
\left|A_{33}\right|= & \frac{1}{2 A^{3}} \mid \int_{0}^{1} \frac{1}{\max _{j}\left(\tilde{\mathcal{P}}_{j}^{1 / 2}\right)}\left(\tilde{\mathcal{P}}_{1}^{1 / 2}-\tilde{\mathcal{P}}_{2}^{1 / 2}\right) \\
& \times \mathbb{1}_{A_{1} \leqslant A_{2}} \int_{0}^{\eta}\left(e^{-\frac{1}{A_{2}}\left(\tilde{\mathcal{Y}}_{2}(t, \eta)-\tilde{\mathcal{Y}}_{2}(t, \theta)\right.}-e^{-\frac{1}{A_{1}}\left(\tilde{\mathcal{Y}}_{2}(t, \eta)-\tilde{\mathcal{Y}}_{2}(t, \theta)\right)}\right) \\
& \times \min _{j}\left(\tilde{\mathcal{D}}_{j}\right) \min _{j}\left(\tilde{\mathcal{U}}_{j}^{+}\right) \tilde{\mathcal{Y}}_{2, \eta}(t, \theta) d \theta d \eta \mid \\
\leqslant & \frac{\sqrt{2} a}{A^{3} e} \int_{0}^{1} \frac{1}{\max _{j}\left(\tilde{\mathcal{P}}_{j}^{1 / 2}\right)}\left|\tilde{\mathcal{P}}_{1}^{1 / 2}-\tilde{\mathcal{P}}_{2}^{1 / 2}\right|(t, \eta) \\
& \times\left(\int_{0}^{\eta} e^{-\frac{3}{4 A_{2}}\left(\tilde{\mathcal{Y}}_{2}(t, \eta)-\tilde{\mathcal{Y}}_{2}(t, \theta)\right)} \tilde{\mathcal{D}}_{2} \tilde{\mathcal{Y}}_{2, \eta}(t, \theta) d \theta\right) d \eta\left|A_{1}-A_{2}\right| \\
\leqslant & \frac{2 \sqrt{2}}{A^{2} e} \int_{0}^{1} \frac{1}{\max _{j}\left(\tilde{\mathcal{P}}_{j}^{1 / 2}\right)}\left|\tilde{\mathcal{P}}_{1}^{1 / 2}-\tilde{\mathcal{P}}_{2}^{1 / 2}\right|(t, \eta) \\
& \times\left(\int_{0}^{\eta} e^{-\frac{3}{2 A_{2}}\left(\tilde{\mathcal{Y}}_{2}(t, \eta)-\tilde{\mathcal{Y}}_{2}(t, \theta)\right)} \tilde{\mathcal{P}}_{2} \tilde{\mathcal{Y}}_{2, \eta}(t, \theta) d \theta\right)^{1 / 2} \\
& \times\left(\int_{0}^{\eta} \tilde{\mathcal{P}}_{2} \tilde{\mathcal{Y}}_{2, \eta}(t, \theta) d \theta\right)^{1 / 2} d \eta\left|A_{1}-A_{2}\right| \\
\leqslant & \mathcal{O}(1)\left(\left\|\tilde{\mathcal{P}}_{1}^{1 / 2}-\tilde{\mathcal{P}}_{2}^{1 / 2}\right\|^{2}+\left|A_{1}-A_{2}\right|^{2}\right) .
\end{aligned}
$$

Furthermore, the terms $A_{35}$ and $A_{36}$ can be estimated like this:

$$
\begin{aligned}
\left|A_{35}\right|= & \frac{1}{2 A^{3}} \mid \int_{0}^{1} \frac{1}{\max _{j}\left(\tilde{\mathcal{P}}_{j}^{1 / 2}\right)}\left(\tilde{\mathcal{P}}_{1}^{1 / 2}-\tilde{\mathcal{P}}_{2}^{1 / 2}\right)(t, \eta) \\
& \times\left(\int_{0}^{\eta}\left(e^{-\frac{1}{a}\left(\tilde{\mathcal{Y}}_{2}(t, \eta)-\tilde{\mathcal{Y}}_{2}(t, \theta)\right)}-e^{-\frac{1}{a}\left(\tilde{\mathcal{Y}}_{1}(t, \eta)-\tilde{\mathcal{Y}}_{1}(t, \theta)\right)}\right)\right. \\
& \left.\times \min _{j}\left(\tilde{\mathcal{D}}_{j}\right) \min _{j}\left(\tilde{\mathcal{U}}_{j}^{+}\right) \tilde{\mathcal{Y}}_{2, \eta} \mathbb{1}_{B(\eta)}(t, \theta) d \theta\right) d \eta \mid \\
\leqslant & \frac{1}{2 a A^{3}} \int_{0}^{1} \frac{1}{\max _{j}\left(\tilde{\mathcal{P}}_{j}^{1 / 2}\right)}\left|\tilde{\mathcal{P}}_{1}^{1 / 2}-\tilde{\mathcal{P}}_{2}^{1 / 2}\right|(t, \eta) \\
& \times\left(\int_{0}^{\eta} e^{-\frac{1}{a}\left(\tilde{\mathcal{Y}}_{2}(t, \eta)-\tilde{\mathcal{Y}}_{2}(t, \theta)\right)}\left(\left|\tilde{\mathcal{Y}}_{2}(t, \eta)-\tilde{\mathcal{Y}}_{1}(t, \eta)\right|+\left|\tilde{\mathcal{Y}}_{2}(t, \theta)-\tilde{\mathcal{Y}}_{1}(t, \theta)\right|\right)\right. \\
& \left.\times \min _{j}\left(\tilde{\mathcal{D}}_{j}\right) \min _{j}\left(\tilde{\mathcal{U}}_{j}^{+}\right) \tilde{\mathcal{Y}}_{2, \eta} \mathbb{1}_{B(\eta)}(t, \theta) d \theta\right) d \eta
\end{aligned}
$$




$$
\begin{aligned}
& \leqslant \frac{a}{2 \sqrt{2} A^{3}} \int_{0}^{1} \frac{1}{\max _{j}\left(\tilde{\mathcal{P}}_{j}^{1 / 2}\right)}\left|\tilde{\mathcal{P}}_{1}^{1 / 2}-\tilde{\mathcal{P}}_{2}^{1 / 2}\right|\left|\tilde{\mathcal{Y}}_{2}-\tilde{\mathcal{Y}}_{1}\right|(t, \eta) \\
& \times\left(\int_{0}^{\eta} e^{-\frac{1}{a}\left(\tilde{\mathcal{Y}}_{2}(t, \eta)-\tilde{\mathcal{Y}}_{2}(t, \theta)\right)} \min _{j}\left(\tilde{\mathcal{D}}_{j}\right) \tilde{\mathcal{Y}}_{2, \eta} \mathbb{1}_{B(\eta)}(t, \theta) d \theta\right) d \eta \\
& +\frac{a}{2 \sqrt{2} A^{3}} \int_{0}^{1} \frac{1}{\max _{j}\left(\tilde{\mathcal{P}}_{j}^{1 / 2}\right)}\left|\tilde{\mathcal{P}}_{1}^{1 / 2}-\tilde{\mathcal{P}}_{2}^{1 / 2}\right|(t, \eta) \\
& \times\left(\int_{0}^{\eta} e^{-\frac{1}{a}\left(\tilde{\mathcal{Y}}_{2}(t, \eta)-\tilde{\mathcal{Y}}_{2}(t, \theta)\right)}\left|\tilde{\mathcal{Y}}_{2}-\tilde{\mathcal{Y}}_{1}\right| \min _{j}\left(\tilde{\mathcal{D}}_{j}\right) \tilde{\mathcal{Y}}_{2, \eta} \mathbb{1}_{B(\eta)}(t, \theta) d \theta\right) d \eta \\
& \leqslant \frac{1}{\sqrt{2} A} \int_{0}^{1} \frac{1}{\max _{j}\left(\tilde{\mathcal{P}}_{j}^{1 / 2}\right)}\left|\tilde{\mathcal{P}}_{1}^{1 / 2}-\tilde{\mathcal{P}}_{2}^{1 / 2}\right|\left|\tilde{\mathcal{Y}}_{2}-\tilde{\mathcal{Y}}_{1}\right|(t, \eta) \\
& \times\left(\int_{0}^{\eta} e^{-\frac{3}{2 A_{2}}\left(\tilde{\mathcal{Y}}_{2}(t, \eta)-\tilde{\mathcal{Y}}_{2}(t, \theta)\right)} \tilde{\mathcal{P}}_{2} \tilde{\mathcal{Y}}_{2, \eta}(t, \theta) d \theta\right)^{1 / 2} \\
& \times\left(\int_{0}^{\eta} e^{-\frac{1}{2 A_{2}}\left(\tilde{\mathcal{Y}}_{2}(t, \eta)-\tilde{\mathcal{Y}}_{2}(t, \theta)\right)} \tilde{\mathcal{P}}_{2} \tilde{\mathcal{Y}}_{2, \eta}(t, \theta) d \theta\right)^{1 / 2} d \eta \\
& +\frac{1}{\sqrt{2} A} \int_{0}^{1} \frac{1}{\max _{j}\left(\tilde{\mathcal{P}}_{j}^{1 / 2}\right)}\left|\tilde{\mathcal{P}}_{1}^{1 / 2}-\tilde{\mathcal{P}}_{2}^{1 / 2}\right|(t, \eta) \\
& \times\left(\int_{0}^{\eta} e^{-\frac{3}{2 A_{2}}\left(\tilde{\mathcal{Y}}_{2}(t, \eta)-\tilde{\mathcal{Y}}_{2}(t, \theta)\right)} \tilde{\mathcal{P}}_{2} \tilde{\mathcal{Y}}_{2, \eta}(t, \theta) d \theta\right)^{1 / 2} \\
& \times\left(\int_{0}^{\eta} e^{-\frac{1}{2 A_{2}}\left(\tilde{\mathcal{Y}}_{2}(t, \eta)-\tilde{\mathcal{Y}}_{2}(t, \theta)\right)} \tilde{\mathcal{P}}_{2} \tilde{\mathcal{Y}}_{2, \eta}\left(\tilde{\mathcal{Y}}_{2}-\tilde{\mathcal{Y}}_{1}\right)^{2}(t, \theta) d \theta\right)^{1 / 2} d \eta \\
& \leqslant \mathcal{O}(1)\left(\left\|\tilde{\mathcal{P}}_{1}^{1 / 2}-\tilde{\mathcal{P}}_{2}^{1 / 2}\right\|^{2}+\left\|\tilde{\mathcal{Y}}_{2}-\tilde{\mathcal{Y}}_{1}\right\|^{2}\right),
\end{aligned}
$$

using (4.15e), (4.15n), and (4.16e). As for $A_{37}$, we follow this path:

$$
\begin{aligned}
\left|A_{37}\right|= & \frac{1}{2 A^{3}} \mid \int_{0}^{1} \frac{1}{\max _{j}\left(\tilde{\mathcal{P}}_{j}^{1 / 2}\right)}\left(\tilde{\mathcal{P}}_{1}^{1 / 2}-\tilde{\mathcal{P}}_{2}^{1 / 2}\right)(t, \eta)\left(\int_{0}^{\eta} \min _{j}\left(e^{-\frac{1}{a}\left(\tilde{\mathcal{Y}}_{j}(t, \eta)-\tilde{\mathcal{Y}}_{j}(t, \theta)\right)}\right)\right. \\
& \left.\times \min _{j}\left(\tilde{\mathcal{D}}_{j}\right) \min _{j}\left(\tilde{\mathcal{U}}_{j}^{+}\right)\left(\tilde{\mathcal{Y}}_{2, \eta}-\tilde{\mathcal{Y}}_{1, \eta}\right)(t, \theta) d \theta\right) d \eta \mid \\
= & \frac{1}{2 A^{3}} \mid \int_{0}^{1} \frac{1}{\max _{j}\left(\tilde{\mathcal{P}}_{j}^{1 / 2}\right)}\left(\tilde{\mathcal{P}}_{1}^{1 / 2}-\tilde{\mathcal{P}}_{2}^{1 / 2}\right)(t, \eta) \\
& \times\left[\left.\left(\min _{j}\left(e^{-\frac{1}{a}\left(\tilde{\mathcal{Y}}_{j}(t, \eta)-\tilde{\mathcal{Y}}_{j}(t, \theta)\right)}\right) \min _{j}\left(\tilde{\mathcal{D}}_{j}\right) \min _{j}\left(\tilde{\mathcal{U}}_{j}^{+}\right)\left(\tilde{\mathcal{Y}}_{2}-\tilde{\mathcal{Y}}_{1}\right)(t, \theta)\right)\right|_{\theta=0} ^{\eta}\right. \\
& -\int_{0}^{\eta}\left(\tilde{\mathcal{Y}}_{2}-\tilde{\mathcal{Y}}_{1}\right)
\end{aligned}
$$




$$
\begin{aligned}
& \left.\times \frac{d}{d \theta}\left(\min _{j}\left(e^{-\frac{1}{a}\left(\tilde{\mathcal{Y}}_{j}(t, \eta)-\tilde{\mathcal{Y}}_{j}(t, \theta)\right)}\right) \min _{j}\left(\tilde{\mathcal{D}}_{j}\right) \min _{j}\left(\tilde{\mathcal{U}}_{j}^{+}\right)\right)(t, \theta) d \theta\right] d \eta \mid \\
& \leqslant \frac{1}{2 A^{3}}\left|\int_{0}^{1} \frac{1}{\max _{j}\left(\tilde{\mathcal{P}}_{j}^{1 / 2}\right)}\left(\tilde{\mathcal{P}}_{1}^{1 / 2}-\tilde{\mathcal{P}}_{2}^{1 / 2}\right) \min _{j}\left(\tilde{\mathcal{D}}_{j}\right) \min _{j}\left(\tilde{\mathcal{U}}_{j}^{+}\right)\left(\tilde{\mathcal{Y}}_{2}-\tilde{\mathcal{Y}}_{1}\right)(t, \eta) d \eta\right| \\
& +\frac{1}{2 A^{3}} \mid \int_{0}^{1} \frac{1}{\max _{j}\left(\tilde{\mathcal{P}}_{j}^{1 / 2}\right)}\left(\tilde{\mathcal{P}}_{1}^{1 / 2}-\tilde{\mathcal{P}}_{2}^{1 / 2}\right)(t, \eta)\left(\int_{0}^{\eta}\left(\tilde{\mathcal{Y}}_{2}-\tilde{\mathcal{Y}}_{1}\right)\right. \\
& \left.\times \frac{d}{d \theta}\left(\min _{j}\left(e^{-\frac{1}{a}\left(\tilde{\mathcal{Y}}_{j}(t, \eta)-\tilde{\mathcal{Y}}_{j}(t, \theta)\right)}\right) \min _{j}\left(\tilde{\mathcal{D}}_{j}\right) \min _{j}\left(\tilde{\mathcal{U}}_{j}^{+}\right)\right)(t, \theta) d \theta\right) d \eta \mid \\
& \leqslant \frac{1}{A^{2}} \int_{0}^{1} \frac{1}{\max _{j}\left(\tilde{\mathcal{P}}_{j}^{1 / 2}\right)}\left|\tilde{\mathcal{P}}_{1}^{1 / 2}-\tilde{\mathcal{P}}_{2}^{1 / 2}\right| \tilde{\mathcal{P}}_{2}\left|\tilde{\mathcal{U}}_{2}\right|\left|\tilde{\mathcal{Y}}_{2}-\tilde{\mathcal{Y}}_{1}\right|(t, \eta) d \eta \\
& +\frac{1}{2 A^{3}} \mid \int_{0}^{1} \frac{1}{\max _{j}\left(\tilde{\mathcal{P}}_{j}^{1 / 2}\right)}\left(\tilde{\mathcal{P}}_{1}^{1 / 2}-\tilde{\mathcal{P}}_{2}^{1 / 2}\right)(t, \eta)\left(\int_{0}^{\eta}\left(\tilde{\mathcal{Y}}_{2}-\tilde{\mathcal{Y}}_{1}\right)\right. \\
& \times\left(\left(\frac{d}{d \theta} \min _{j}\left(e^{-\frac{1}{a}\left(\tilde{\mathcal{Y}}_{j}(t, \eta)-\tilde{\mathcal{Y}}_{j}(t, \theta)\right)}\right)\right) \min _{j}\left(\tilde{\mathcal{D}}_{j}\right) \min _{j}\left(\tilde{\mathcal{U}}_{j}^{+}\right)\right. \\
& \left.\left.+\min _{j}\left(e^{-\frac{1}{a}\left(\tilde{\mathcal{Y}}_{j}(t, \eta)-\tilde{\mathcal{Y}}_{j}(t, \theta)\right)}\right)\left(\frac{d}{d \theta} \min _{j}\left(\tilde{\mathcal{D}}_{j}\right) \min _{j}\left(\tilde{\mathcal{U}}_{j}^{+}\right)\right)\right)(t, \theta) d \theta\right) d \eta \mid \\
& \leqslant \mathcal{O}(1)\left(\left\|\tilde{\mathcal{P}}_{1}^{1 / 2}-\tilde{\mathcal{P}}_{2}^{1 / 2}\right\|^{2}+\left\|\tilde{\mathcal{Y}}_{1}-\tilde{\mathcal{Y}}_{2}\right\|^{2}\right) \\
& +\frac{1}{2 A^{3}} \int_{0}^{1} \frac{1}{\max _{j}\left(\tilde{\mathcal{P}}_{j}^{1 / 2}\right)}\left|\tilde{\mathcal{P}}_{1}^{1 / 2}-\tilde{\mathcal{P}}_{2}^{1 / 2}\right|(t, \eta) \\
& \times\left(\int _ { 0 } ^ { \eta } | \tilde { \mathcal { Y } } _ { 2 } - \tilde { \mathcal { Y } } _ { 1 } | \frac { 1 } { a } \left(\min _{j}\left(e^{-\frac{1}{a}\left(\tilde{\mathcal{Y}}_{j}(t, \eta)-\tilde{\mathcal{Y}}_{j}(t, \theta)\right)}\right)\right.\right. \\
& \times \max _{j}\left(\tilde{\mathcal{Y}}_{j, \eta}\right) \min _{j}\left(\tilde{\mathcal{D}}_{j}\right) \min _{j}\left(\tilde{\mathcal{U}}_{j}^{+}\right) \\
& \left.\left.+\mathcal{O}(1) A^{5} \min _{j}\left(e^{-\frac{1}{a}\left(\tilde{\mathcal{Y}}_{j}(t, \eta)-\tilde{\mathcal{Y}}_{j}(t, \theta)\right)}\right)\left(\min _{j}\left(\tilde{\mathcal{D}}_{j}^{1 / 2}\right)+\left|\tilde{\mathcal{U}}_{2}\right|\right)\right)(t, \theta) d \theta\right) d \eta \\
& \leqslant \mathcal{O}(1)\left(\left\|\tilde{\mathcal{P}}_{1}^{1 / 2}-\tilde{\mathcal{P}}_{2}^{1 / 2}\right\|^{2}+\left\|\tilde{\mathcal{Y}}_{1}-\tilde{\mathcal{Y}}_{2}\right\|^{2}\right)+\frac{1}{2}\left(A_{371}+A_{372}\right),
\end{aligned}
$$

using (4.15a), (4.15b), (4.15n), and Lemmas A.2 and A.4. Here

$$
\begin{aligned}
A_{371}= & \frac{1}{a A^{3}} \int_{0}^{1} \frac{1}{\max _{j}\left(\tilde{\mathcal{P}}_{j}^{1 / 2}\right)}\left|\tilde{\mathcal{P}}_{1}^{1 / 2}-\tilde{\mathcal{P}}_{2}^{1 / 2}\right|(t, \eta) \\
& \times\left(\int _ { 0 } ^ { \eta } | \tilde { \mathcal { Y } } _ { 2 } - \tilde { \mathcal { Y } } _ { 1 } | \left(\min _{j}\left(e^{-\frac{1}{a}\left(\tilde{\mathcal{Y}}_{j}(t, \eta)-\tilde{\mathcal{Y}}_{j}(t, \theta)\right)}\right)\right.\right. \\
& \left.\times \max _{j}\left(\tilde{\mathcal{Y}}_{j, \eta}\right) \min _{j}\left(\tilde{\mathcal{D}}_{j}\right) \min _{j}\left(\tilde{\mathcal{U}}_{j}^{+}\right)(t, \theta) d \theta\right) d \eta
\end{aligned}
$$




$$
\begin{aligned}
\leqslant & \frac{\sqrt{2} a}{A^{2}} \int_{0}^{1} \frac{1}{\max _{j}\left(\tilde{\mathcal{P}}_{j}^{1 / 2}\right)}\left|\tilde{\mathcal{P}}_{1}^{1 / 2}-\tilde{\mathcal{P}}_{2}^{1 / 2}\right|(t, \eta)\left(\int_{0}^{\eta}\left|\tilde{\mathcal{Y}}_{2}-\tilde{\mathcal{Y}}_{1}\right|\right. \\
& \left.\times\left(e^{-\frac{1}{A_{1}}\left(\tilde{\mathcal{Y}}_{1}(t, \eta)-\tilde{\mathcal{Y}}_{1}(t, \theta)\right)} \tilde{\mathcal{P}}_{1} \tilde{\mathcal{Y}}_{1, \eta}+e^{-\frac{1}{A_{2}}\left(\tilde{\mathcal{Y}}_{2}(t, \eta)-\tilde{\mathcal{Y}}_{2}(t, \theta)\right)} \tilde{\mathcal{P}}_{2} \tilde{\mathcal{Y}}_{2, \eta}\right)(t, \theta) d \theta\right) d \eta \\
\leqslant & \frac{\sqrt{2}}{A} \int_{0}^{1} \frac{1}{\max _{j}\left(\tilde{\mathcal{P}}_{j}^{1 / 2}\right)}\left|\tilde{\mathcal{P}}_{1}^{1 / 2}-\tilde{\mathcal{P}}_{2}^{1 / 2}\right|(t, \eta) \\
& \times\left[\left(\int_{0}^{\eta} e^{-\frac{3}{2 A_{1}}\left(\tilde{\mathcal{Y}}_{1}(t, \eta)-\tilde{\mathcal{Y}}_{1}(t, \theta)\right)} \tilde{\mathcal{P}}_{1} \tilde{\mathcal{Y}}_{1, \eta}(t, \theta) d \theta\right)^{1 / 2}\right. \\
& \times\left(\int_{0}^{\eta} e^{-\frac{1}{2 A_{1}}\left(\tilde{\mathcal{Y}}_{1}(t, \eta)-\tilde{\mathcal{Y}}_{1}(t, \theta)\right)} \tilde{\mathcal{P}}_{1} \tilde{\mathcal{Y}}_{1, \eta}\left(\tilde{\mathcal{Y}}_{2}-\tilde{\mathcal{Y}}_{1}\right)^{2}(t, \theta) d \theta\right)^{1 / 2} \\
& +\left(\int_{0}^{\eta} e^{-\frac{3}{2 A_{2}}\left(\tilde{\mathcal{Y}}_{2}(t, \eta)-\tilde{\mathcal{Y}}_{2}(t, \theta)\right)} \tilde{\mathcal{P}}_{2} \tilde{\mathcal{Y}}_{2, \eta}(t, \theta) d \theta\right)^{1 / 2} \\
& \left.\times\left(\int_{0}^{\eta} e^{-\frac{1}{2 A_{2}}\left(\tilde{\mathcal{Y}}_{2}(t, \eta)-\tilde{\mathcal{Y}}_{2}(t, \theta)\right)} \tilde{\mathcal{P}}_{2} \tilde{\mathcal{Y}}_{2, \eta}\left(\tilde{\mathcal{Y}}_{2}-\tilde{\mathcal{Y}}_{1}\right)^{2}(t, \theta) d \theta\right)^{1 / 2}\right] d \eta \\
\leqslant & \mathcal{O}(1)\left(\left\|\tilde{\mathcal{P}}_{1}^{1 / 2}-\tilde{\mathcal{P}}_{2}^{1 / 2}\right\|^{2}+\left\|\tilde{\mathcal{Y}}_{1}-\tilde{\mathcal{Y}}_{2}\right\|^{2}\right),
\end{aligned}
$$

using $\max _{j}\left(a_{j}\right) \leqslant a_{1}+a_{2}$ and $\min _{j}\left(b_{j}\right) \leqslant b_{k}$. Then

$$
\begin{aligned}
A_{372}= & \mathcal{O}(1) \int_{0}^{1} \frac{1}{\max _{j}\left(\tilde{\mathcal{P}}_{j}^{1 / 2}\right)}\left|\tilde{\mathcal{P}}_{1}^{1 / 2}-\tilde{\mathcal{P}}_{2}^{1 / 2}\right|(t, \eta) \\
& \times\left(\int_{0}^{\eta}\left|\tilde{\mathcal{Y}}_{2}-\tilde{\mathcal{Y}}_{1}\right| \min _{j}\left(e^{-\frac{1}{a}\left(\tilde{\mathcal{Y}}_{j}(t, \eta)-\tilde{\mathcal{Y}}_{j}(t, \theta)\right)}\right)\left(\min _{j}\left(\tilde{\mathcal{D}}_{j}^{1 / 2}\right)+\left|\tilde{\mathcal{U}}_{2}\right|\right)(t, \theta) d \theta\right) d \eta \\
\leqslant & \mathcal{O}(1) \int_{0}^{1} \frac{1}{\max _{j}\left(\tilde{\mathcal{P}}_{j}^{1 / 2}\right)}\left|\tilde{\mathcal{P}}_{1}^{1 / 2}-\tilde{\mathcal{P}}_{2}^{1 / 2}\right|(t, \eta) \\
& \times\left(\int_{0}^{\eta}\left|\tilde{\mathcal{Y}}_{2}-\tilde{\mathcal{Y}}_{1}\right| e^{-\frac{1}{A_{2}}\left(\tilde{\mathcal{Y}}_{2}(t, \eta)-\tilde{\mathcal{Y}}_{2}(t, \theta)\right)} \tilde{\mathcal{P}}_{2}^{1 / 2}(t, \theta) d \theta\right) d \eta \\
\leqslant & \mathcal{O}(1) \int_{0}^{1} \frac{1}{\max _{j}\left(\tilde{\mathcal{P}}_{j}^{1 / 2}\right)}\left|\tilde{\mathcal{P}}_{1}^{1 / 2}-\tilde{\mathcal{P}}_{2}^{1 / 2}\right|(t, \eta) \\
& \times\left(\int_{0}^{\eta} e^{-\frac{3}{2 A_{2}}\left(\tilde{\mathcal{Y}}_{2}(t, \eta)-\tilde{\mathcal{Y}}_{2}(t, \theta)\right)} \tilde{\mathcal{P}}_{2}(t, \theta) d \theta\right)^{1 / 2} \\
& \times\left(\int_{0}^{\eta} e^{-\frac{1}{2 A_{2}}\left(\tilde{\mathcal{Y}}_{2}(t, \eta)-\tilde{\mathcal{Y}}_{2}(t, \theta)\right)}\left(\tilde{\mathcal{Y}}_{2}-\tilde{\mathcal{Y}}_{1}\right)^{2}(t, \theta) d \theta\right)^{1 / 2} d \eta \\
\leqslant & \mathcal{O}(1)\left(\left\|\tilde{\mathcal{P}}_{1}^{1 / 2}-\tilde{\mathcal{P}}_{2}^{1 / 2}\right\|^{2}+\left\|\tilde{\mathcal{Y}}_{1}-\tilde{\mathcal{Y}}_{2}\right\|^{2}\right)
\end{aligned}
$$


using (4.15d), (4.15n), and (4.16i). This completes the estimate for $\tilde{A}_{3}$ (see (5.47)):

$$
\tilde{A}_{3} \leqslant \mathcal{O}(1)\left(\left\|\tilde{\mathcal{P}}_{1}^{1 / 2}-\tilde{\mathcal{P}}_{2}^{1 / 2}\right\|^{2}+\left\|\tilde{\mathcal{U}}_{1}-\tilde{\mathcal{U}}_{2}\right\|^{2}+\left\|\tilde{\mathcal{Y}}_{1}-\tilde{\mathcal{Y}}_{2}\right\|^{2}+\left|A_{1}-A_{2}\right|^{2}\right) .
$$

For $J_{4}$ (and similarly for $J_{5}$ ) the estimates read as

$$
\begin{aligned}
\left|J_{4}\right| & \leqslant \frac{1}{A^{3}} \int_{0}^{1}\left|\tilde{\mathcal{P}}_{1}^{1 / 2}-\tilde{\mathcal{P}}_{2}^{1 / 2}\right|^{2} \frac{\left|\tilde{\mathcal{R}}_{2}\right|}{\tilde{\mathcal{P}}_{2}}(t, \eta) d \eta \\
& \leqslant \mathcal{O}(1)\left\|\tilde{\mathcal{P}}_{1}^{1 / 2}-\tilde{\mathcal{P}}_{2}^{1 / 2}\right\|^{2}
\end{aligned}
$$

using (4.15j).

We have shown the anticipated result.

LEMMA 5.5. Let $\tilde{\mathcal{P}}_{i}^{1 / 2}$ be two solutions of (5.40) for $i=1$, 2. Then we have

$$
\begin{aligned}
& \frac{d}{d t}\left\|\tilde{\mathcal{P}}_{1}^{1 / 2}-\tilde{\mathcal{P}}_{2}^{1 / 2}\right\|^{2} \\
& \quad \leqslant \mathcal{O}(1)\left(\left\|\tilde{\mathcal{Y}}_{1}-\tilde{\mathcal{Y}}_{2}\right\|^{2}+\left\|\tilde{\mathcal{U}}_{1}-\tilde{\mathcal{U}}_{2}\right\|^{2}+\left\|\tilde{\mathcal{P}}_{1}^{1 / 2}-\tilde{\mathcal{P}}_{2}^{1 / 2}\right\|^{2}+\left|A_{1}-A_{2}\right|^{2}\right),
\end{aligned}
$$

where $\mathcal{O}(1)$ denotes some constant which only depends on $A=\max _{j}\left(A_{j}\right)$ and which remains bounded as $A \rightarrow 0$.

\section{Acknowledgements}

$\mathrm{KG}$ and $\mathrm{HH}$ were supported by the grants Waves and Nonlinear Phenomena (WaNP) and Wave Phenomena and Stability - a Shocking Combination (WaPheS) from the Research Council of Norway.

JAC was partially supported by the EPSRC grant number EP/P031587/1 and the Advanced Grant Nonlocal-CPD (Nonlocal PDEs for Complex Particle Dynamics: Phase Transitions, Patterns and Synchronization) of the European Research Council Executive Agency (ERC) under the European Union's Horizon 2020 research and innovation programme (grant agreement No. 883363).

JAC, KG, and $\mathrm{HH}$ are very grateful to the Institut Mittag-Leffler, Stockholm for generous support and hospitality during the program Interactions between Partial Differential Equations and Functional Inequalities. HH would like to thank the Isaac Newton Institute for Mathematical Sciences for support and hospitality during the program Nonlinear Water Waves when work on this paper was undertaken. 


\section{Appendix A. Lipschitz continuity and uniformly bounded Lipschitz constants}

We need to establish that a number of complicated functions are Lipschitz continuous with uniformly bounded Lipschitz constants. In a desperate attempt to ease the readability of the main estimates, we collect these results here together with some other estimates that are essential in Section 5.

LEMMA A.1. (i) We have

$$
\begin{aligned}
\left|e^{a_{1}-a_{2}}-e^{b_{1}-b_{2}}\right| \leqslant & \max \left(e^{a_{1}-a_{2}}, e^{b_{1}-b_{2}}\right)\left(\left|b_{1}-a_{1}\right|+\left|b_{2}-a_{2}\right|\right) \\
\leqslant & \left|b_{1}-a_{1}\right|+\left|b_{2}-a_{2}\right| \\
& a_{1}<a_{2}, \quad b_{1}<b_{2} .
\end{aligned}
$$

(ii) Let $0<a \leqslant A_{j} \leqslant A, j=1,2$. Then we have

$$
\left|e^{-\frac{1}{A_{2}} x}-e^{-\frac{1}{A_{1}} x}\right| \leqslant \frac{4}{a e} e^{-\frac{3}{4 A} x}\left|A_{2}-A_{1}\right|, \quad x \in[0, \infty) .
$$

Proof. (i) The result follows from the elementary inequality

$$
\left|e^{a}-e^{b}\right|=\left|\int_{b}^{a} e^{x} d x\right| \leqslant e^{b}\left|\int_{b}^{a} d x\right| \leqslant e^{b}|b-a|, \quad a<b .
$$

(ii) For $x \in[0, \infty)$ one can write

$$
\begin{aligned}
\left|e^{-\frac{1}{A_{2}} x}-e^{-\frac{1}{A_{1}} x}\right| & =\left|\int_{A_{1}}^{A_{2}} \frac{1}{s^{2}} x e^{-\frac{1}{s} x} d s\right| \\
& \leqslant e^{-\frac{3}{4 A} x}\left|\int_{A_{1}}^{A_{2}} \frac{1}{s^{2}} x e^{-\frac{1}{4 s} x} d s\right| \\
& \leqslant \frac{4}{a e} e^{-\frac{3}{4 A} x}\left|A_{2}-A_{1}\right| .
\end{aligned}
$$

Here we used in the last step that for $s \in[a, A]$, the function $f:[0, \infty) \rightarrow[0, \infty)$ with $f(x)=\frac{1}{s^{2}} x e^{-\frac{1}{4 s} x}$ attains its maximum at $x=4 s$ and

$$
0 \leqslant f(4 s)=\frac{4}{s e} \leqslant \frac{4}{a e} .
$$

LEMmA A.2. (i) The function $\theta \mapsto \min _{j}\left(e^{-\frac{1}{a}\left(\tilde{\mathcal{Y}}_{j}(t, \eta)-\tilde{\mathcal{Y}}_{j}(t, \theta)\right)}\right)$ is nondecreasing for almost every $\eta$ and thus differentiable almost everywhere. We have that

$$
\left|\frac{d}{d \theta} \min _{j}\left(e^{-\frac{1}{a}\left(\tilde{\mathcal{Y}}_{j}(t, \eta)-\tilde{\mathcal{Y}}_{j}(t, \theta)\right)}\right)\right| \leqslant \frac{1}{a} \min _{j}\left(e^{-\frac{1}{a}\left(\tilde{\mathcal{Y}}_{j}(t, \eta)-\tilde{\mathcal{Y}}_{j}(t, \theta)\right)}\right) \max _{j}\left(\tilde{\mathcal{Y}}_{j, \eta}(t, \theta)\right) .
$$


(ii) The function $\theta \mapsto \min _{j}\left(\tilde{\mathcal{U}}_{j}^{2}\right)(t, \theta)$ is differentiable almost everywhere with

$$
\left|\frac{d}{d \theta} \min _{j}\left(\tilde{\mathcal{U}}_{j}^{2}(t, \theta)\right)\right| \leqslant A^{4} .
$$

(iii) The function $\theta \mapsto \min _{j}\left(\tilde{\mathcal{U}}_{j}^{+}\right)^{3}(t, \theta)$ is differentiable almost everywhere with

$$
\left|\frac{d}{d \theta} \min _{j}\left(\tilde{\mathcal{U}}_{j}^{+}\right)^{3}(t, \theta)\right| \leqslant 2 A^{4} \min _{j}\left(\tilde{\mathcal{U}}_{j}^{+}\right)(t, \theta) .
$$

Proof. (i): First of all, note that the function $\theta \mapsto \min _{j}\left(e^{-\frac{1}{a}\left(\tilde{\mathcal{Y}}_{j}(t, \eta)-\tilde{\mathcal{Y}}_{j}(t, \theta)\right)}\right)$ is nondecreasing and hence differentiable almost everywhere. Consider in the following $\theta<\eta$. Assume that for fixed $\theta$, such that the given function is differentiable, we have

$$
\min _{j}\left(e^{-\frac{1}{a}\left(\tilde{\mathcal{Y}}_{j}(t, \eta)-\tilde{\mathcal{Y}}_{j}(t, \theta)\right)}\right)=e^{-\frac{1}{a}\left(\tilde{\mathcal{Y}}_{1}(t, \eta)-\tilde{\mathcal{Y}}_{1}(t, \theta)\right)}
$$

and that there exists a sequence $\theta_{n} \uparrow \theta$ such that

$$
\min _{j}\left(e^{-\frac{1}{a}\left(\tilde{\mathcal{Y}}_{j}(t, \eta)-\tilde{\mathcal{Y}}_{j}\left(t, \theta_{n}\right)\right)}\right)=e^{-\frac{1}{a}\left(\tilde{\mathcal{Y}}_{1}(t, \eta)-\tilde{\mathcal{Y}}_{1}\left(t, \theta_{n}\right)\right)} \quad \text { for all } n .
$$

Then we have

$$
\begin{aligned}
& \left|\min _{j}\left(e^{-\frac{1}{a}\left(\tilde{\mathcal{Y}}_{j}(t, \eta)-\tilde{\mathcal{Y}}_{j}(t, \theta)\right)}\right)-\min _{j}\left(e^{-\frac{1}{a}\left(\tilde{\mathcal{Y}}_{j}(t, \eta)-\tilde{\mathcal{Y}}_{j}\left(t, \theta_{n}\right)\right)}\right)\right| \\
& \quad=e^{-\frac{1}{a}\left(\tilde{\mathcal{Y}}_{1}(t, \eta)-\tilde{\mathcal{Y}}_{1}(t, \theta)\right)}-e^{-\frac{1}{a}\left(\tilde{\mathcal{Y}}_{1}(t, \eta)-\tilde{\mathcal{Y}}_{1}\left(t, \theta_{n}\right)\right)} \\
& \quad \leqslant \frac{1}{a} e^{-\frac{1}{a}\left(\tilde{\mathcal{Y}}_{1}(t, \eta)-\tilde{\mathcal{Y}}_{1}(t, \theta)\right)}\left(\tilde{\mathcal{Y}}_{1}(t, \theta)-\tilde{\mathcal{Y}}_{1}\left(t, \theta_{n}\right)\right),
\end{aligned}
$$

since $\tilde{\mathcal{Y}}_{i}(t, \cdot)$ is nondecreasing.

$$
\begin{aligned}
\left|\frac{d}{d \theta} \min _{j}\left(e^{-\frac{1}{a}\left(\tilde{\mathcal{Y}}_{j}(t, \eta)-\tilde{\mathcal{Y}}_{j}(t, \theta)\right)}\right)\right| & \leqslant \lim _{\theta_{n} \uparrow \theta} \frac{1}{a} e^{-\frac{1}{a}\left(\tilde{\mathcal{Y}}_{1}(t, \eta)-\tilde{\mathcal{Y}}_{1}(t, \theta)\right)} \frac{\tilde{\mathcal{Y}}_{1}(t, \theta)-\tilde{\mathcal{Y}}_{1}\left(t, \theta_{n}\right)}{\theta-\theta_{n}} \\
& =\frac{1}{a} e^{-\frac{1}{a}\left(\tilde{\mathcal{Y}}_{1}(t, \eta)-\tilde{\mathcal{Y}}_{1}(t, \theta)\right)} \tilde{\mathcal{Y}}_{1, \eta}(t, \theta) \\
& =\frac{1}{a} \min _{j}\left(e^{-\frac{1}{a}\left(\tilde{\mathcal{Y}}_{j}(t, \eta)-\tilde{\mathcal{Y}}_{j}(t, \theta)\right)}\right) \tilde{\mathcal{Y}}_{1, \eta}(t, \theta) .
\end{aligned}
$$

Assume, on the other hand, that for fixed $\theta$ we have

$$
\min _{j}\left(e^{-\frac{1}{a}\left(\tilde{\mathcal{Y}}_{j}(t, \eta)-\tilde{\mathcal{Y}}_{j}(t, \theta)\right)}\right)=e^{-\frac{1}{a}\left(\tilde{\mathcal{Y}}_{1}(t, \eta)-\tilde{\mathcal{Y}}_{1}(t, \theta)\right)}
$$


and that there exists a sequence $\theta_{n} \downarrow \theta$ such that

$$
\min _{j}\left(e^{-\frac{1}{a}\left(\tilde{\mathcal{Y}}_{j}(t, \eta)-\tilde{\mathcal{Y}}_{j}\left(t, \theta_{n}\right)\right)}\right)=e^{-\frac{1}{a}\left(\tilde{\mathcal{Y}}_{1}(t, \eta)-\tilde{\mathcal{Y}}_{1}\left(t, \theta_{n}\right)\right)} \quad \text { for all } n .
$$

Then we have

$$
\begin{aligned}
& \left|\min _{j}\left(e^{-\frac{1}{a}\left(\tilde{\mathcal{Y}}_{j}(t, \eta)-\tilde{\mathcal{Y}}_{j}(t, \theta)\right)}\right)-\min _{j}\left(e^{-\frac{1}{a}\left(\tilde{\mathcal{Y}}_{j}(t, \eta)-\tilde{\mathcal{Y}}_{j}\left(t, \theta_{n}\right)\right)}\right)\right| \\
& \quad=e^{-\frac{1}{a}\left(\tilde{\mathcal{Y}}_{1}(t, \eta)-\tilde{\mathcal{Y}}_{1}\left(t, \theta_{n}\right)\right)}-e^{-\frac{1}{a}\left(\tilde{\mathcal{Y}}_{1}(t, \eta)-\tilde{\mathcal{Y}}_{1}(t, \theta)\right)} \\
& \quad \leqslant \frac{1}{a} e^{-\frac{1}{a}\left(\tilde{\mathcal{Y}}_{1}(t, \eta)-\tilde{\mathcal{Y}}_{1}\left(t, \theta_{n}\right)\right)}\left(\tilde{\mathcal{Y}}_{1}\left(t, \theta_{n}\right)-\tilde{\mathcal{Y}}_{1}(t, \theta)\right),
\end{aligned}
$$

since $\tilde{\mathcal{Y}}_{i}(t, \cdot)$ is nondecreasing. Thus

$$
\begin{aligned}
\left|\frac{d}{d \theta} \min _{j}\left(e^{-\frac{1}{a}\left(\tilde{\mathcal{Y}}_{j}(t, \eta)-\tilde{\mathcal{Y}}_{j}(t, \theta)\right)}\right)\right| & \leqslant \lim _{\theta_{n} \downarrow \theta} \frac{1}{a} e^{-\frac{1}{a}\left(\tilde{\mathcal{Y}}_{1}(t, \eta)-\tilde{\mathcal{Y}}_{1}\left(t, \theta_{n}\right)\right)} \frac{\tilde{\mathcal{Y}}_{1}\left(t, \theta_{n}\right)-\tilde{\mathcal{Y}}_{1}(t, \theta)}{\theta_{n}-\theta} \\
& =\frac{1}{a} e^{-\frac{1}{a}\left(\tilde{\mathcal{Y}}_{1}(t, \eta)-\tilde{\mathcal{Y}}_{1}(t, \theta)\right)} \tilde{\mathcal{Y}}_{1, \eta}(t, \theta) \\
& =\min _{j}\left(e^{-\frac{1}{a}\left(\tilde{\mathcal{Y}}_{j}(t, \eta)-\tilde{\mathcal{Y}}_{j}(t, \theta)\right)}\right) \tilde{\mathcal{Y}}_{1, \eta}(t, \theta) .
\end{aligned}
$$

Thus in case (A.7) we find

$$
\left|\frac{d}{d \theta} \min _{j}\left(e^{-\frac{1}{a}\left(\tilde{\mathcal{Y}}_{j}(t, \eta)-\tilde{\mathcal{Y}}_{j}(t, \theta)\right)}\right)\right| \leqslant \frac{1}{a} \min _{j}\left(e^{-\frac{1}{a}\left(\tilde{\mathcal{Y}}_{j}(t, \eta)-\tilde{\mathcal{Y}}_{j}(t, \theta)\right)}\right) \tilde{\mathcal{Y}}_{1, \eta}(t, \theta) .
$$

If we instead of (A.7) assume

$$
\min _{j}\left(e^{-\frac{1}{a}\left(\tilde{\mathcal{Y}}_{j}(t, \eta)-\tilde{\mathcal{Y}}_{j}(t, \theta)\right)}\right)=e^{-\frac{1}{a}\left(\tilde{\mathcal{Y}}_{2}(t, \eta)-\tilde{\mathcal{Y}}_{2}(t, \theta)\right)},
$$

a similar argument yields

$$
\left|\frac{d}{d \theta} \min _{j}\left(e^{-\frac{1}{a}\left(\tilde{\mathcal{Y}}_{j}(t, \eta)-\tilde{\mathcal{Y}}_{j}(t, \theta)\right)}\right)\right| \leqslant \min _{j}\left(e^{-\frac{1}{a}\left(\tilde{\mathcal{Y}}_{j}(t, \eta)-\tilde{\mathcal{Y}}_{j}(t, \theta)\right)}\right) \tilde{\mathcal{Y}}_{2, \eta}(t, \theta) .
$$

Thus we conclude that in general we have

$$
\left|\frac{d}{d \theta} \min _{j}\left(e^{-\frac{1}{a}\left(\tilde{\mathcal{Y}}_{j}(t, \eta)-\tilde{\mathcal{Y}}_{j}(t, \theta)\right)}\right)\right| \leqslant \frac{1}{a} \min _{j}\left(e^{-\frac{1}{a}\left(\tilde{\mathcal{Y}}_{j}(t, \eta)-\tilde{\mathcal{Y}}_{j}(t, \theta)\right)}\right) \max _{j}\left(\tilde{\mathcal{Y}}_{j, \eta}\right)(t, \theta) .
$$

(ii): We have (cf. Lemma 5.1) that

$$
\begin{aligned}
& \left|\min _{j}\left(\tilde{\mathcal{U}}_{j}^{2}(t, \eta)\right)-\min _{j}\left(\tilde{\mathcal{U}}_{j}^{2}(t, \theta)\right)\right| \\
& \quad \leqslant \max \left(\left|\tilde{\mathcal{U}}_{1}^{2}(t, \eta)-\tilde{\mathcal{U}}_{1}^{2}(t, \theta)\right|,\left|\tilde{\mathcal{U}}_{2}^{2}(t, \eta)-\tilde{\mathcal{U}}_{2}^{2}(t, \eta)\right|\right) \\
& \quad \leqslant 2 \max _{j}\left\|\tilde{\mathcal{U}}_{j} \tilde{\mathcal{U}}_{j \eta}\right\|_{\infty}|\eta-\theta| \leqslant A^{4}|\eta-\theta|,
\end{aligned}
$$

using (4.15f). 
(iii): Note that one has that for any positive function $m(x)$,

$$
\begin{aligned}
\left|m^{3}(x)-m^{3}(y)\right| & =\left(m^{2}(x)+m(x) m(y)+m^{2}(y)\right)|m(x)-m(y)| \\
& \leqslant\left(m^{2}(x)+2 m(x) m(y)+m^{2}(y)\right)|m(x)-m(y)| \\
& =(m(x)+m(y))^{2}|m(x)-m(y)| \\
& =(m(x)+m(y))\left|m^{2}(x)-m^{2}(y)\right| .
\end{aligned}
$$

If we replace $m(x)$ by $\min _{j}\left(\tilde{\mathcal{U}}_{j}^{+}\right)(t, \eta)$, we have

$$
\begin{aligned}
& \left|\min _{j}\left(\tilde{\mathcal{U}}_{j}^{+}\right)^{3}(t, \eta)-\min _{j}\left(\tilde{\mathcal{U}}_{j}^{+}\right)^{3}(t, \theta)\right| \\
& \quad \leqslant\left(\min _{j}\left(\tilde{\mathcal{U}}_{j}^{+}\right)(t, \eta)+\min _{j}\left(\tilde{\mathcal{U}}_{j}^{+}\right)(t, \theta)\right)\left|\min _{j}\left(\tilde{\mathcal{U}}_{j}^{+}\right)^{2}(t, \eta)-\min _{j}\left(\tilde{\mathcal{U}}_{j}^{+}\right)^{2}(t, \theta)\right| \\
& \quad \leqslant\left(\min _{j}\left(\tilde{\mathcal{U}}_{j}^{+}\right)(t, \eta)+\min _{j}\left(\tilde{\mathcal{U}}_{j}^{+}\right)(t, \theta)\right) \\
& \quad \times \max \left(\left|\left(\tilde{\mathcal{U}}_{1}^{+}\right)^{2}(t, \eta)-\left(\tilde{\mathcal{U}}_{1}^{+}\right)^{2}(t, \theta)\right|,\left|\left(\tilde{\mathcal{U}}_{2}^{+}\right)^{2}(t, \eta)-\left(\tilde{\mathcal{U}}_{2}^{+}\right)^{2}(t, \theta)\right|\right) \\
& \quad \leqslant A^{4}\left(\min _{j}\left(\tilde{\mathcal{U}}_{j}^{+}\right)(t, \eta)+\min _{j}\left(\tilde{\mathcal{U}}_{j}^{+}\right)(t, \theta)\right)|\eta-\theta|
\end{aligned}
$$

(see (A.8)), and hence $\min _{j}\left(\tilde{\mathcal{U}}_{j}^{+}\right)^{3}(t, \eta)$ is differentiable almost everywhere with

$$
\left|\frac{d}{d \theta} \min _{j}\left(\tilde{\mathcal{U}}_{j}^{+}\right)^{3}(t, \theta)\right| \leqslant 2 A^{4} \min _{j}\left(\tilde{\mathcal{U}}_{j}^{+}\right)(t, \theta) .
$$

LemmA A.3. (i) The function $\eta \mapsto \min _{j}\left(\tilde{\mathcal{P}}_{j}\right) \min _{j}\left(\tilde{\mathcal{U}}_{j}^{+}\right)(t, \eta)$ is Lipschitz continuous with a uniformly bounded Lipschitz constant and thus differentiable almost everywhere with

$$
\left|\frac{d}{d \eta}\left(\min _{j}\left(\tilde{\mathcal{P}}_{j}\right) \min _{j}\left(\tilde{\mathcal{U}}_{j}^{+}\right)\right)(t, \eta)\right| \leqslant 2 A^{4}\left(\min _{j}\left(\tilde{\mathcal{P}}_{j}\right)^{1 / 2}+\left|\tilde{\mathcal{U}}_{k}\right|\right)(t, \eta), \quad k=1,2 .
$$

(ii) The function $\eta \mapsto \min _{j}\left(\tilde{\mathcal{P}}_{j}\right) \tilde{\mathcal{U}}_{k}(t, \eta), k=1,2$ is Lipschitz continuous with a uniformly bounded Lipschitz constant and thus differentiable almost everywhere with

$$
\left|\frac{d}{d \eta}\left(\min _{j}\left(\tilde{\mathcal{P}}_{j}\right) \tilde{\mathcal{U}}_{k}\right)(t, \eta)\right| \leqslant \frac{1}{\sqrt{2}} A^{6} .
$$

Proof. (i) We only present the proof for the case $k=2$, since the case $k=1$ is similar. 
Given $0 \leqslant \eta_{1}<\eta_{2} \leqslant 1$, we assume without loss of generality that

$$
0 \leqslant \min _{j}\left(\tilde{\mathcal{P}}_{j}\right) \min _{j}\left(\tilde{\mathcal{U}}_{j}^{+}\right)\left(t, \eta_{2}\right)-\min _{j}\left(\tilde{\mathcal{P}}_{j}\right) \min _{j}\left(\tilde{\mathcal{U}}_{j}^{+}\right)\left(t, \eta_{1}\right) .
$$

To ease the notation, we introduce the function

$$
d(t, \eta)=\min _{j}\left(\tilde{\mathcal{P}}_{j}\right) \min _{j}\left(\tilde{\mathcal{U}}_{j}^{+}\right)(t, \eta) .
$$

We will distinguish several cases:

(1): If $d\left(t, \eta_{2}\right)=0$, then $d\left(t, \eta_{1}\right)=0$ and one has

$$
\begin{aligned}
0 & \leqslant \min _{j}\left(\tilde{\mathcal{U}}_{j}^{+}\right)\left(t, \eta_{2}\right)\left|\min _{j}\left(\tilde{\mathcal{P}}_{j}\right)\left(t, \eta_{2}\right)-\min _{j}\left(\tilde{\mathcal{P}}_{j}\right)\left(t, \eta_{1}\right)\right| \\
& \leqslant \min _{j}\left(\tilde{\mathcal{U}}_{j}^{+}\right)\left(t, \eta_{2}\right) \mid\left(\left|\tilde{\mathcal{P}}_{1}\left(t, \eta_{2}\right)-\tilde{\mathcal{P}}_{1}\left(t, \eta_{1}\right)\right|+\left|\tilde{\mathcal{P}}_{2}\left(t, \eta_{2}\right)-\tilde{\mathcal{P}}_{2}\left(t, \eta_{1}\right)\right|\right) \\
& \leqslant A^{4} \min _{j}\left(\tilde{\mathcal{U}}_{j}^{+}\right)\left(t, \eta_{2}\right)\left|\eta_{2}-\eta_{1}\right| .
\end{aligned}
$$

(2): If $d\left(t, \eta_{2}\right)>0$, then $\min _{j}\left(\tilde{\mathcal{P}}_{j}\right)\left(t, \eta_{2}\right)>0$ and $\min _{j}\left(\tilde{\mathcal{U}}_{j}^{+}\right)\left(t, \eta_{2}\right)>0$ or equivalently

$$
\tilde{\mathcal{P}}_{1}\left(t, \eta_{2}\right)>0, \quad \tilde{\mathcal{P}}_{2}\left(t, \eta_{2}\right)>0, \quad \tilde{\mathcal{U}}_{1}\left(t, \eta_{2}\right)>0, \quad \text { and } \quad \tilde{\mathcal{U}}_{2}\left(t, \eta_{2}\right)>0 .
$$

(2a): Assume that $d\left(t, \eta_{1}\right)=0$ and $\min _{j}\left(\tilde{\mathcal{P}}_{j}\right)\left(t, \eta_{1}\right)=0$. Then

$$
\begin{aligned}
0 & \leqslant d\left(t, \eta_{2}\right)-d\left(t, \eta_{1}\right) \\
& \leqslant\left(\min _{j}\left(\tilde{\mathcal{P}}_{j}\right)\left(t, \eta_{2}\right)-\min _{j}\left(\tilde{\mathcal{P}}_{j}\right)\left(t, \eta_{1}\right)\right) \min _{j}\left(\tilde{\mathcal{U}}_{j}^{+}\right)\left(t, \eta_{2}\right) \\
& \leqslant \min _{j}\left(\tilde{\mathcal{U}}_{j}^{+}\right)\left(t, \eta_{2}\right)\left(\left|\tilde{\mathcal{P}}_{1}\left(t, \eta_{2}\right)-\tilde{\mathcal{P}}_{1}\left(t, \eta_{1}\right)\right|+\left|\tilde{\mathcal{P}}_{2}\left(t, \eta_{2}\right)-\tilde{\mathcal{P}}_{2}\left(t, \eta_{1}\right)\right|\right) \\
& \leqslant A^{4} \min _{j}\left(\tilde{\mathcal{U}}_{j}^{+}\right)\left(t, \eta_{2}\right)\left|\eta_{2}-\eta_{1}\right| .
\end{aligned}
$$

(2b): Assume that $d\left(t, \eta_{1}\right)=0$ and $\min _{j}\left(\tilde{\mathcal{U}}_{j}^{+}\right)\left(t, \eta_{1}\right)=0$. Then one either has that

(i): $\tilde{\mathcal{U}}_{2}\left(t, \eta_{1}\right) \leqslant \tilde{\mathcal{U}}_{2}^{+}\left(t, \eta_{1}\right)=\min _{j}\left(\tilde{\mathcal{U}}_{j}^{+}\right)\left(t, \eta_{1}\right)$, and we can write

$$
\begin{aligned}
0 & \leqslant d\left(t, \eta_{2}\right)-d\left(t, \eta_{1}\right) \\
& \leqslant \tilde{\mathcal{U}}_{2} \min _{j}\left(\tilde{\mathcal{P}}_{j}\right)\left(t, \eta_{2}\right)-\tilde{\mathcal{U}}_{2} \min _{j}\left(\tilde{\mathcal{P}}_{j}\right)\left(t, \eta_{1}\right) \\
& \leqslant \int_{\eta_{1}}^{\eta_{2}}\left(\tilde{\mathcal{U}}_{2, \eta} \min _{j}\left(\tilde{\mathcal{P}}_{j}\right)+\tilde{\mathcal{U}}_{2} \frac{d}{d \theta} \min _{j}\left(\tilde{\mathcal{P}}_{j}\right)\right)(t, s) d s
\end{aligned}
$$




$$
\begin{aligned}
& \leqslant \int_{\eta_{1}}^{\eta_{2}}\left(\left|\tilde{\mathcal{U}}_{2, \eta}\right| \min _{j}\left(\tilde{\mathcal{P}}_{j}\right)+\left|\tilde{\mathcal{U}}_{2}\right|\left(\frac{1}{A_{1}} \tilde{\mathcal{P}}_{1} \tilde{\mathcal{Y}}_{1, \eta}+\frac{1}{A_{2}} \tilde{\mathcal{P}}_{2} \tilde{\mathcal{Y}}_{2, \eta}\right)\right)(t, s) d s \\
& \leqslant \frac{A^{4}}{\sqrt{2}} \int_{\eta_{1}}^{\eta_{2}} \min _{j}\left(\tilde{\mathcal{P}}_{j}\right)^{1 / 2}(t, s) d s+A^{4} \int_{\eta_{1}}^{\eta_{2}}\left|\tilde{\mathcal{U}}_{2}\right|(t, s) d s \\
& \leqslant \frac{3}{2 \sqrt{2}} A^{6}\left|\eta_{2}-\eta_{1}\right|,
\end{aligned}
$$

or

(ii): $\min _{j}\left(\tilde{\mathcal{U}}_{j}^{+}\right)\left(t, \eta_{1}\right)=\tilde{\mathcal{U}}_{1}^{+}\left(t, \eta_{1}\right)$. Then there exists a maximal interval $\left[\eta_{1}\right.$, a] such that $\tilde{\mathcal{U}}_{1}^{+}(t, s)<\tilde{\mathcal{U}}_{2}^{+}(t, s)$ for all $s \in\left[\eta_{1}, a\right)$ and $\tilde{\mathcal{U}}_{1}^{+}(t, a)=\tilde{\mathcal{U}}_{2}^{+}(t, a)$. Moreover, there exists a maximal interval $[b, a] \subset\left[\eta_{1}, a\right]$ such that $\tilde{\mathcal{U}}_{1}^{+}(t, s)>0$ for all $s \in(b, a]$ and $\tilde{\mathcal{U}}_{1}^{+}(t, b)=0$. Hence we can write

$$
\begin{aligned}
0 \leqslant & d\left(t, \eta_{2}\right)-d\left(t, \eta_{1}\right) \\
\leqslant & d\left(t, \eta_{2}\right)-d(t, a)+d(t, a)-d(t, b) \\
\leqslant & \tilde{\mathcal{U}}_{2} \min _{j}\left(\tilde{\mathcal{P}}_{j}\right)\left(t, \eta_{2}\right)-\tilde{\mathcal{U}}_{2} \min _{j}\left(\tilde{\mathcal{P}}_{j}\right)(t, a) \\
& +\tilde{\mathcal{U}}_{1} \min _{j}\left(\tilde{\mathcal{P}}_{j}\right)(t, a)-\tilde{\mathcal{U}}_{1} \min _{j}\left(\tilde{\mathcal{P}}_{j}\right)(t, b) \\
\leqslant & \int_{a}^{\eta_{2}}\left(\tilde{\mathcal{U}}_{2, \eta} \min _{j}\left(\tilde{\mathcal{P}}_{j}\right)+\tilde{\mathcal{U}}_{2} \frac{d}{d \eta} \min _{j}\left(\tilde{\mathcal{P}}_{j}\right)\right)(t, s) d s \\
& +\int_{b}^{a}\left(\tilde{\mathcal{U}}_{1, \eta} \min _{j}\left(\tilde{\mathcal{P}}_{j}\right)+\tilde{\mathcal{U}}_{1} \frac{d}{d \eta} \min _{j}\left(\tilde{\mathcal{P}}_{j}\right)\right)(t, s) d s \\
\leqslant & \int_{a}^{\eta_{2}}\left(\frac{A^{4}}{\sqrt{2}} \min _{j}\left(\tilde{\mathcal{P}}_{j}\right)^{1 / 2}+A^{4}\left|\tilde{\mathcal{U}}_{2}\right|\right)(t, s) d s \\
& +\int_{b}^{a}\left(\frac{A^{4}}{\sqrt{2}} \min _{j}\left(\tilde{\mathcal{P}}_{j}\right)^{1 / 2}+A^{4}\left|\tilde{\mathcal{U}}_{1}\right|\right)(t, s) d s \\
\leqslant & \frac{A^{4}}{\sqrt{2}} \int_{b}^{\eta_{2}} \min _{j}\left(\tilde{\mathcal{P}}_{j}\right)^{1 / 2}(t, s) d s+A^{4} \int_{b}^{\eta_{2}}\left|\tilde{\mathcal{U}}_{2}\right|(t, s) d s \\
\leqslant & \frac{A^{4}}{\sqrt{2}} \int_{\eta_{1}}^{\eta_{2}} \min _{j}\left(\tilde{\mathcal{P}}_{j}\right)^{1 / 2}(t, s) d s+A^{4} \int_{\eta_{1}}^{\eta_{2}}\left|\tilde{\mathcal{U}}_{2}\right|(t, s) d s \\
\leqslant & \frac{3}{2 \sqrt{2}} A^{6}\left|\eta_{2}-\eta_{1}\right| .
\end{aligned}
$$

Note, in the case that $\tilde{\mathcal{U}}_{1}^{+}(t, s)<\tilde{\mathcal{U}}_{2}^{+}(t, s)$ for all $s \in\left[\eta_{1}, \eta_{2}\right]$, the estimate starts with

$$
0 \leqslant d\left(t, \eta_{2}\right)-d\left(t, \eta_{1}\right) \leqslant \tilde{\mathcal{U}}_{1} \min _{j}\left(\tilde{\mathcal{P}}_{j}\right)\left(t, \eta_{2}\right)-\tilde{\mathcal{U}}_{1} \min _{j}\left(\tilde{\mathcal{P}}_{j}\right)(t, b)
$$


where $\left(b, \eta_{2}\right]$ denotes the maximal interval such that $\tilde{\mathcal{U}}_{1}^{+}(t, s)>0$ for all $s \in\left(b, \eta_{2}\right]$.

(2c): Assume that $d\left(t, \eta_{1}\right)>0$; then $\min _{j}\left(\tilde{\mathcal{P}}_{j}\right)\left(t, \eta_{1}\right)>0$ and $\min _{j}\left(\tilde{\mathcal{U}}_{j}^{+}\right)(t$, $\left.\eta_{1}\right)=\min _{j}\left(\tilde{\mathcal{U}}_{j}\right)\left(t, \eta_{1}\right)>0$. Then one either has that

(i): $\min _{j}\left(\tilde{\mathcal{U}}_{j}\right)\left(t, \eta_{1}\right)=\tilde{\mathcal{U}}_{2}\left(t, \eta_{1}\right)$, and we have (as before)

$$
\begin{aligned}
0 & \leqslant d\left(t, \eta_{2}\right)-d\left(t, \eta_{1}\right) \\
& \leqslant \tilde{\mathcal{U}}_{2} \min _{j}\left(\tilde{\mathcal{P}}_{j}\right)\left(t, \eta_{2}\right)-\tilde{\mathcal{U}}_{2} \min _{j}\left(\tilde{\mathcal{P}}_{j}\right)\left(t, \eta_{1}\right) \\
& \leqslant \frac{A^{4}}{\sqrt{2}} \int_{\eta_{1}}^{\eta_{2}} \min _{j}\left(\tilde{\mathcal{P}}_{j}\right)^{1 / 2}(t, s) d s+A^{4} \int_{\eta_{1}}^{\eta_{2}}\left|\tilde{\mathcal{U}}_{2}\right|(t, s) d s \\
& \leqslant \frac{3}{2 \sqrt{2}} A^{6}\left|\eta_{2}-\eta_{1}\right|,
\end{aligned}
$$

or

(ii): $\min _{j}\left(\tilde{\mathcal{U}}_{j}\right)\left(t, \eta_{1}\right)=\tilde{\mathcal{U}}_{1}\left(t, \eta_{1}\right)$. Then there exists a maximal interval $\left[\eta_{1}\right.$, a] such that $\tilde{\mathcal{U}}_{1}^{+}(t, s)<\tilde{\mathcal{U}}_{2}^{+}(t, s)$ for all $s \in\left[\eta_{1}, a\right)$ and $\tilde{\mathcal{U}}_{1}^{+}(t, a)=\tilde{\mathcal{U}}_{2}^{+}(t, a)$. Moreover, there exists a maximal interval $[b, a] \subset\left[\eta_{1}, a\right]$ such that $\tilde{\mathcal{U}}_{1}^{+}(t, s)>0$ for all $s \in(b, a]$ and $\tilde{\mathcal{U}}_{1}^{+}(t, b)=0$. Hence we can write

$$
\begin{aligned}
0 \leqslant & d\left(t, \eta_{2}\right)-d\left(t, \eta_{1}\right) \\
\leqslant & d\left(t, \eta_{2}\right)-d(t, a)+d(t, a)-d(t, b) \\
\leqslant & \tilde{\mathcal{U}}_{2} \min _{j}\left(\tilde{\mathcal{P}}_{j}\right)\left(t, \eta_{2}\right)-\tilde{\mathcal{U}}_{2} \min _{j}\left(\tilde{\mathcal{P}}_{j}\right)(t, a) \\
& +\tilde{\mathcal{U}}_{1} \min _{j}\left(\tilde{\mathcal{P}}_{j}\right)(t, a)-\tilde{\mathcal{U}}_{1} \min _{j}\left(\tilde{\mathcal{P}}_{j}\right)(t, b) \\
\leqslant & \int_{a}^{\eta_{2}}\left(\tilde{\mathcal{U}}_{2, \eta} \min _{j}\left(\tilde{\mathcal{P}}_{j}\right)+\tilde{\mathcal{U}}_{2} \frac{d}{d \eta} \min _{j}\left(\tilde{\mathcal{P}}_{j}\right)\right)(t, s) d s \\
& +\int_{b}^{a}\left(\tilde{\mathcal{U}}_{1, \eta} \min _{j}\left(\tilde{\mathcal{P}}_{j}\right)+\tilde{\mathcal{U}}_{1} \frac{d}{d \eta} \min _{j}\left(\tilde{\mathcal{P}}_{j}\right)\right)(t, s) d s \\
\leqslant & \int_{a}^{\eta_{2}}\left(\frac{A^{4}}{\sqrt{2}} \min _{j}\left(\tilde{\mathcal{P}}_{j}\right)^{1 / 2}+A^{4}\left|\tilde{\mathcal{U}}_{2}\right|\right)(t, s) d s \\
& +\int_{b}^{a}\left(\frac{A^{4}}{\sqrt{2}} \min _{j}\left(\tilde{\mathcal{P}}_{j}\right)^{1 / 2}+A^{4}\left|\tilde{\mathcal{U}}_{1}\right|\right)(t, s) d s \\
\leqslant & \frac{A^{4}}{\sqrt{2}} \int_{b}^{\eta_{2}} \min _{j}\left(\tilde{\mathcal{P}}_{j}\right)^{1 / 2}(t, s) d s+A^{4} \int_{b}^{\eta_{2}}\left|\tilde{\mathcal{U}}_{2}\right|(t, s) d s
\end{aligned}
$$




$$
\begin{aligned}
& \leqslant \frac{A^{4}}{\sqrt{2}} \int_{\eta_{1}}^{\eta_{2}} \min _{j}\left(\tilde{\mathcal{P}}_{j}\right)^{1 / 2}(t, s) d s+A^{4} \int_{\eta_{1}}^{\eta_{2}}\left|\tilde{\mathcal{U}}_{2}\right|(t, s) d s \\
& \leqslant \frac{3}{2 \sqrt{2}} A^{6}\left|\eta_{2}-\eta_{1}\right| .
\end{aligned}
$$

Note, in the case that $\tilde{\mathcal{U}}_{1}^{+}(t, s)<\tilde{\mathcal{U}}_{2}^{+}(t, s)$ for all $s \in\left[\eta_{1}, \eta_{2}\right]$, the estimate starts with

$$
0 \leqslant d\left(t, \eta_{2}\right)-d\left(t, \eta_{1}\right) \leqslant \tilde{\mathcal{U}}_{1} \min _{j}\left(\tilde{\mathcal{P}}_{j}\right)\left(t, \eta_{2}\right)-\tilde{\mathcal{U}}_{1} \min _{j}\left(\tilde{\mathcal{P}}_{j}\right)(t, b),
$$

where $\left(b, \eta_{2}\right]$ denotes the maximal interval such that $\tilde{\mathcal{U}}_{1}^{+}(t, s)>0$ for all $s \in\left(b, \eta_{2}\right]$.

Thus we showed that

$$
\left|d\left(t, \eta_{2}\right)-d\left(t, \eta_{1}\right)\right| \leqslant \frac{3}{2 \sqrt{2}} A^{6}\left|\eta_{2}-\eta_{1}\right|,
$$

or, in other words, $d(t, \cdot)=\min _{j}\left(\tilde{\mathcal{P}}_{j}\right) \min _{j}\left(\tilde{\mathcal{U}}_{j}^{+}\right)(t, \cdot)$ is Lipschitz continuous with Lipschitz constant $\frac{3}{2 \sqrt{2}} A^{6}$, which is independent of time, and thus differentiable almost everywhere. Moreover, a close look reveals that

$$
\begin{aligned}
\left|d\left(t, \eta_{2}\right)-d\left(t, \eta_{1}\right)\right| \leqslant & A^{4} \min _{j}\left(\tilde{\mathcal{U}}_{j}^{+}\right)\left(t, \eta_{2}\right)\left|\eta_{2}-\eta_{1}\right| \\
& +\frac{A^{4}}{\sqrt{2}}\left|\int_{\eta_{1}}^{\eta_{2}} \min _{j}\left(\tilde{\mathcal{P}}_{j}\right)^{1 / 2}(t, s) d s\right|+A^{4}\left|\int_{\eta_{1}}^{\eta_{2}}\right| \tilde{\mathcal{U}}_{2}|(t, s) d s| .
\end{aligned}
$$

Since both $\left|\tilde{\mathcal{U}}_{2}\right|(t, \cdot)$ and $\min _{j}\left(\tilde{\mathcal{P}}_{j}\right)^{1 / 2}(t, \cdot)$ are continuous, the fundamental theorem of calculus implies that

$$
\begin{aligned}
\left|\frac{d\left(t, \eta_{2}\right)-d\left(t, \eta_{1}\right)}{\eta_{2}-\eta_{1}}\right| \leqslant & A^{4} \min _{j}\left(\tilde{\mathcal{U}}_{j}^{+}\right)\left(t, \eta_{2}\right) \\
& +\frac{A^{4}}{\sqrt{2}} \min _{j}\left(\tilde{\mathcal{P}}_{j}\right)^{1 / 2}(t, \tilde{\eta})+A^{4}\left|\tilde{\mathcal{U}}_{2}\right|(t, \tilde{\eta})
\end{aligned}
$$

for some $\tilde{\eta}$ between $\eta_{1}$ and $\eta_{2}$. Letting $\eta_{2} \rightarrow \eta_{1}$ we thus obtain for almost every $\eta$ that

$$
\begin{aligned}
\left|\frac{d}{d \eta} \min _{j}\left(\tilde{\mathcal{P}}_{j}\right) \min _{j}\left(\tilde{\mathcal{U}}_{j}^{+}\right)(t, \eta)\right| & =\left|\frac{d}{d \eta} d(t, \eta)\right| \\
& \leqslant 2 A^{4}\left(\min _{j}\left(\tilde{\mathcal{P}}_{j}\right)^{1 / 2}+\left|\tilde{\mathcal{U}}_{2}\right|\right)(t, \eta) .
\end{aligned}
$$


(ii) We have that

$$
\begin{aligned}
& \left|\min _{j}\left(\tilde{\mathcal{P}}_{j}\right)(t, \eta)-\min _{j}\left(\tilde{\mathcal{P}}_{j}\right)(t, \tilde{\eta})\right| \\
& \quad \leqslant \max \left(\left|\tilde{\mathcal{P}}_{1}(t, \eta)-\tilde{\mathcal{P}}_{1}(t, \tilde{\eta})\right|,\left|\tilde{\mathcal{P}}_{2}(t, \eta)-\tilde{\mathcal{P}}_{2}(t, \tilde{\eta})\right|\right) \\
& \quad \leqslant \frac{A^{4}}{2}|\eta-\tilde{\eta}| .
\end{aligned}
$$

Thus, for almost every $\eta$,

$$
\begin{aligned}
\left|\frac{d}{d \eta}\left(\min _{j}\left(\tilde{\mathcal{P}}_{j}\right) \tilde{\mathcal{U}}_{k}\right)(t, \eta)\right| & =\left|\left(\frac{d}{d \eta} \min _{j}\left(\tilde{\mathcal{P}}_{j}\right)\right) \tilde{\mathcal{U}}_{k}(t, \eta)+\min _{j}\left(\tilde{\mathcal{P}}_{j}\right) \tilde{\mathcal{U}}_{k, \eta}(t, \eta)\right| \\
& \leqslant \frac{A^{4}}{2}\left\|\tilde{\mathcal{U}}_{k}\right\|_{\infty}+\left\|\tilde{\mathcal{P}}_{k} \tilde{\mathcal{U}}_{k, \eta}\right\|_{\infty} \\
& \leqslant \frac{1}{\sqrt{2}} A^{6} .
\end{aligned}
$$

LemmA A.4. (i) The function $\eta \mapsto \min _{j}\left(\tilde{\mathcal{D}}_{j}\right) \min _{j}\left(\tilde{\mathcal{U}}_{j}^{+}\right)(t, \eta)$ is Lipschitz continuous with a uniformly bounded Lipschitz constant and thus differentiable almost everywhere with

$$
\begin{aligned}
& \left|\frac{d}{d \eta}\left(\min _{j}\left(\tilde{\mathcal{D}}_{j}\right) \min _{j}\left(\tilde{\mathcal{U}}_{j}^{+}\right)\right)(t, \eta)\right| \\
& \quad \leqslant \mathcal{O}(1) \sqrt{A} A^{4}\left(\min _{j}\left(\tilde{\mathcal{D}}_{j}\right)^{1 / 2}+\left|\tilde{\mathcal{U}}_{k}\right|\right)(t, \eta), \quad k=1,2 .
\end{aligned}
$$

(ii) The function $\eta \mapsto \min _{j}\left(\tilde{\mathcal{D}}_{j}\right) \tilde{\mathcal{U}}_{k}(t, \eta), k=1,2$, is Lipschitz continuous with a uniformly bounded Lipschitz constant and thus differentiable almost everywhere with

$$
\left|\frac{d}{d \eta}\left(\min _{j}\left(\tilde{\mathcal{D}}_{j}\right) \tilde{\mathcal{U}}_{k}\right)(t, \eta)\right| \leqslant \mathcal{O}(1) A^{7} .
$$

Proof. (i) We only present the proof for the case $k=2$, since the case $k=1$ is similar.

Given $0 \leqslant \eta_{1}<\eta_{2} \leqslant 1$, we assume without loss of generality that

$$
0 \leqslant \min _{j}\left(\tilde{\mathcal{D}}_{j}\right) \min _{j}\left(\tilde{\mathcal{U}}_{j}^{+}\right)\left(t, \eta_{2}\right)-\min _{j}\left(\tilde{\mathcal{D}}_{j}\right) \min _{j}\left(\tilde{\mathcal{U}}_{j}^{+}\right)\left(t, \eta_{1}\right) .
$$

To ease the notation, we introduce the function

$$
\bar{d}(t, \eta)=\min _{j}\left(\tilde{\mathcal{D}}_{j}\right) \min _{j}\left(\tilde{\mathcal{U}}_{j}^{+}\right)(t, \eta) .
$$


We will distinguish several cases:

(1): If $\bar{d}\left(t, \eta_{2}\right)=0$, then $\bar{d}\left(t, \eta_{1}\right)=0$ and one has

$$
\begin{aligned}
0 & \leqslant \min _{j}\left(\tilde{\mathcal{U}}_{j}^{+}\right)\left(t, \eta_{2}\right)\left|\min _{j}\left(\tilde{\mathcal{D}}_{j}\right)\left(t, \eta_{2}\right)-\min _{j}\left(\tilde{\mathcal{D}}_{j}\right)\left(t, \eta_{1}\right)\right| \\
& \leqslant \min _{j}\left(\tilde{\mathcal{U}}_{j}^{+}\right)\left(t, \eta_{2}\right) \mid\left(\left|\tilde{\mathcal{D}}_{1}\left(t, \eta_{2}\right)-\tilde{\mathcal{D}}_{1}\left(t, \eta_{1}\right)\right|+\left|\tilde{\mathcal{D}}_{2}\left(t, \eta_{2}\right)-\tilde{\mathcal{D}}_{2}\left(t, \eta_{1}\right)\right|\right) \\
& \leqslant \mathcal{O}(1) A^{5} \min _{j}\left(\tilde{\mathcal{U}}_{j}^{+}\right)\left(t, \eta_{2}\right)\left|\eta_{2}-\eta_{1}\right| .
\end{aligned}
$$

(2): If $\bar{d}\left(t, \eta_{2}\right)>0$, then $\min _{j}\left(\tilde{\mathcal{D}}_{j}\right)\left(t, \eta_{2}\right)>0$ and $\min _{j}\left(\tilde{\mathcal{U}}_{j}^{+}\right)\left(t, \eta_{2}\right)>0$ or equivalently

$$
\tilde{\mathcal{D}}_{1}\left(t, \eta_{2}\right)>0, \quad \tilde{\mathcal{D}}_{2}\left(t, \eta_{2}\right)>0, \quad \tilde{\mathcal{U}}_{1}\left(t, \eta_{2}\right)>0, \quad \text { and } \quad \tilde{\mathcal{U}}_{2}\left(t, \eta_{2}\right)>0 .
$$

(2a): Assume that $\bar{d}\left(t, \eta_{1}\right)=0$ and $\min _{j}\left(\tilde{\mathcal{D}}_{j}\right)\left(t, \eta_{1}\right)=0$. Then

$$
\begin{aligned}
0 & \leqslant \bar{d}\left(t, \eta_{2}\right)-\bar{d}\left(t, \eta_{1}\right) \\
& \leqslant\left(\min _{j}\left(\tilde{\mathcal{D}}_{j}\right)\left(t, \eta_{2}\right)-\min _{j}\left(\tilde{\mathcal{D}}_{j}\right)\left(t, \eta_{1}\right)\right) \min _{j}\left(\tilde{\mathcal{U}}_{j}^{+}\right)\left(t, \eta_{2}\right) \\
& \leqslant \min _{j}\left(\tilde{\mathcal{U}}_{j}^{+}\right)\left(t, \eta_{2}\right)\left(\left|\tilde{\mathcal{D}}_{1}\left(t, \eta_{2}\right)-\tilde{\mathcal{D}}_{1}\left(t, \eta_{1}\right)\right|+\left|\tilde{\mathcal{D}}_{2}\left(t, \eta_{2}\right)-\tilde{\mathcal{D}}_{2}\left(t, \eta_{1}\right)\right|\right) \\
& \leqslant \mathcal{O}(1) A^{5} \min _{j}\left(\tilde{\mathcal{U}}_{j}^{+}\right)\left(t, \eta_{2}\right)\left|\eta_{2}-\eta_{1}\right| .
\end{aligned}
$$

(2b): Assume that $\bar{d}\left(t, \eta_{1}\right)=0$ and $\min _{j}\left(\tilde{\mathcal{U}}_{j}^{+}\right)\left(t, \eta_{1}\right)=0$. Then one either has that

(i): $\tilde{\mathcal{U}}_{2}\left(t, \eta_{1}\right) \leqslant \tilde{\mathcal{U}}_{2}^{+}\left(t, \eta_{1}\right)=\min _{j}\left(\tilde{\mathcal{U}}_{j}^{+}\right)\left(t, \eta_{1}\right)$ and we can write

$$
\begin{aligned}
0 \leqslant & d\left(t, \eta_{2}\right)-d\left(t, \eta_{1}\right) \\
\leqslant & \tilde{\mathcal{U}}_{2} \min _{j}\left(\tilde{\mathcal{D}}_{j}\right)\left(t, \eta_{2}\right)-\tilde{\mathcal{U}}_{2} \min _{j}\left(\tilde{\mathcal{D}}_{j}\right)\left(t, \eta_{1}\right) \\
\leqslant & \int_{\eta_{1}}^{\eta_{2}} \tilde{\mathcal{U}}_{2, \eta} \min _{j}\left(\tilde{\mathcal{D}}_{j}\right)(t, s)+\tilde{\mathcal{U}}_{2} \frac{d}{d \theta} \min _{j}\left(\tilde{\mathcal{D}}_{j}\right)(t, s) d s \\
\leqslant & \int_{\eta_{1}}^{\eta_{2}}\left|\tilde{\mathcal{U}}_{2, \eta}\right| \min _{j}\left(\tilde{\mathcal{D}}_{j}\right)(t, s) d s \\
& +\int_{\eta_{1}}^{\eta_{2}}\left|\tilde{\mathcal{U}}_{2}\right|\left(\mid \tilde{\mathcal{U}}_{1}^{2}-\tilde{\mathcal{P}}_{1}\right) \tilde{\mathcal{Y}}_{1, \eta}-\frac{1}{A_{1}} \tilde{\mathcal{D}}_{1} \tilde{\mathcal{Y}}_{1, \eta}+\frac{1}{2} A_{1}^{5} \mid \\
& \left.+\left|\left(\tilde{\mathcal{U}}_{2}^{2}-\tilde{\mathcal{P}}_{2}\right) \tilde{\mathcal{Y}}_{2, \eta}-\frac{1}{A_{2}} \tilde{\mathcal{D}}_{2} \tilde{\mathcal{Y}}_{2, \eta}+\frac{1}{2} A_{2}^{5}\right|\right)(t, s) d s
\end{aligned}
$$




$$
\begin{aligned}
& \leqslant \sqrt{A} A^{4} \int_{\eta_{1}}^{\eta_{2}} \min _{j}\left(\tilde{\mathcal{D}}_{j}\right)^{1 / 2}(t, s) d s+\mathcal{O}(1) A^{5} \int_{\eta_{1}}^{\eta_{2}}\left|\tilde{\mathcal{U}}_{2}\right|(t, s) d s \\
& \leqslant \mathcal{O}(1) A^{7}\left|\eta_{2}-\eta_{1}\right|
\end{aligned}
$$

or

(ii): $\min _{j}\left(\tilde{\mathcal{U}}_{j}^{+}\right)\left(t, \eta_{1}\right)=\tilde{\mathcal{U}}_{1}^{+}\left(t, \eta_{1}\right)$. Then there exists a maximal interval $\left[\eta_{1}\right.$, a] such that $\tilde{\mathcal{U}}_{1}^{+}(t, s)<\tilde{\mathcal{U}}_{2}^{+}(t, s)$ for all $s \in\left[\eta_{1}, a\right)$ and $\tilde{\mathcal{U}}_{1}^{+}(t, a)=\tilde{\mathcal{U}}_{2}^{+}(t, a)$. Moreover, there exists a maximal interval $[b, a] \subset\left[\eta_{1}, a\right]$ such that $\tilde{\mathcal{U}}_{1}^{+}(t, s)>0$ for all $s \in(b, a]$ and $\tilde{\mathcal{U}}_{1}^{+}(t, b)=0$. Hence we can write

$$
\begin{aligned}
0 \leqslant & \bar{d}\left(t, \eta_{2}\right)-\bar{d}\left(t, \eta_{1}\right) \\
\leqslant & \bar{d}\left(t, \eta_{2}\right)-\bar{d}(t, a)+\bar{d}(t, a)-\bar{d}(t, b) \\
\leqslant & \tilde{\mathcal{U}}_{2} \min _{j}\left(\tilde{\mathcal{D}}_{j}\right)\left(t, \eta_{2}\right)-\tilde{\mathcal{U}}_{2} \min _{j}\left(\tilde{\mathcal{D}}_{j}\right)(t, a) \\
& +\tilde{\mathcal{U}}_{1} \min _{j}\left(\tilde{\mathcal{D}}_{j}\right)(t, a)-\tilde{\mathcal{U}}_{1} \min _{j}\left(\tilde{\mathcal{D}}_{j}\right)(t, b) \\
\leqslant & \int_{a}^{\eta_{2}}\left(\tilde{\mathcal{U}}_{2, \eta} \min _{j}\left(\tilde{\mathcal{D}}_{j}\right)+\tilde{\mathcal{U}}_{2} \frac{d}{d \eta} \min _{j}\left(\tilde{\mathcal{D}}_{j}\right)\right)(t, s) d s \\
& +\int_{b}^{a}\left(\tilde{\mathcal{U}}_{1, \eta} \min _{j}\left(\tilde{\mathcal{D}}_{j}\right)+\tilde{\mathcal{U}}_{1} \frac{d}{d \eta} \min _{j}\left(\tilde{\mathcal{D}}_{j}\right)\right)(t, s) d s \\
\leqslant & \int_{a}^{\eta_{2}}\left(\sqrt{A} A^{4} \min _{j}\left(\tilde{\mathcal{D}}_{j}\right)^{1 / 2}+\mathcal{O}(1) A^{5}\left|\tilde{\mathcal{U}}_{2}\right|\right)(t, s) d s \\
& +\int_{b}^{a}\left(\sqrt{A} A^{4} \min _{j}\left(\tilde{\mathcal{D}}_{j}\right)^{1 / 2}+\mathcal{O}(1) A^{5}\left|\tilde{\mathcal{U}}_{1}\right|\right)(t, s) d s \\
\leqslant & \sqrt{A} A^{4} \int_{b}^{\eta_{2}} \min _{j}\left(\tilde{\mathcal{D}}_{j}\right)^{1 / 2}(t, s) d s+\mathcal{O}(1) A^{5} \int_{b}^{\eta_{2}}\left|\tilde{\mathcal{U}}_{2}\right|(t, s) d s \\
\leqslant & \sqrt{A} A^{4} \int_{\eta_{1}}^{\eta_{2}} \min _{j}\left(\tilde{\mathcal{D}}_{j}\right)^{1 / 2}(t, s) d s+\mathcal{O}(1) A^{5} \int_{\eta_{1}}^{\eta_{2}}\left|\tilde{\mathcal{U}}_{2}\right|(t, s) d s \\
\leqslant & \mathcal{O}(1) A^{7}\left|\eta_{2}-\eta_{1}\right| .
\end{aligned}
$$

Note, in the case that $\tilde{\mathcal{U}}_{1}^{+}(t, s)<\tilde{\mathcal{U}}_{2}^{+}(t, s)$ for all $s \in\left[\eta_{1}, \eta_{2}\right]$, the estimate starts with

$$
0 \leqslant d\left(t, \eta_{2}\right)-d\left(t, \eta_{1}\right) \leqslant \tilde{\mathcal{U}}_{1} \min _{j}\left(\tilde{\mathcal{D}}_{j}\right)\left(t, \eta_{2}\right)-\tilde{\mathcal{U}}_{1} \min _{j}\left(\tilde{\mathcal{D}}_{j}\right)(t, b),
$$

where $\left(b, \eta_{2}\right]$ denotes the maximal interval such that $\tilde{\mathcal{U}}_{1}^{+}(t, s)>0$ for all $s \in\left(b, \eta_{2}\right]$. 
(2c): Assume that $\bar{d}\left(t, \eta_{1}\right)>0$; then $\min _{j}\left(\tilde{\mathcal{D}}_{j}\right)\left(t, \eta_{1}\right)>0$ and $\min _{j}\left(\tilde{\mathcal{U}}_{j}^{+}\right)(t$, $\left.\eta_{1}\right)=\min _{j}\left(\tilde{\mathcal{U}}_{j}\right)\left(t, \eta_{1}\right)>0$. Then one either has that

(i): $\min _{j}\left(\tilde{\mathcal{U}}_{j}\right)\left(t, \eta_{1}\right)=\tilde{\mathcal{U}}_{2}\left(t, \eta_{1}\right)$, and we have (as before)

$$
\begin{aligned}
0 & \leqslant \bar{d}\left(t, \eta_{2}\right)-\bar{d}\left(t, \eta_{1}\right) \\
& \leqslant \tilde{\mathcal{U}}_{2} \min _{j}\left(\tilde{\mathcal{D}}_{j}\right)\left(t, \eta_{2}\right)-\tilde{\mathcal{U}}_{2} \min _{j}\left(\tilde{\mathcal{D}}_{j}\right)\left(t, \eta_{1}\right) \\
& \leqslant \int_{\eta_{1}}^{\eta_{2}}\left(\frac{1}{2} \sqrt{A} A^{6} \min _{j}\left(\tilde{\mathcal{D}}_{j}\right)^{1 / 2}+\mathcal{O}(1) A^{5}\left|\tilde{\mathcal{U}}_{2}\right|\right)(t, s) d s \\
& \leqslant \mathcal{O}(1) A^{7}\left|\eta_{2}-\eta_{1}\right|,
\end{aligned}
$$

or

(ii): $\min _{j}\left(\tilde{\mathcal{U}}_{j}\right)\left(t, \eta_{1}\right)=\tilde{\mathcal{U}}_{1}\left(t, \eta_{1}\right)$. Then there exists a maximal interval $\left[\eta_{1}\right.$, a] such that $\tilde{\mathcal{U}}_{1}^{+}(t, s)<\tilde{\mathcal{U}}_{2}^{+}(t, s)$ for all $s \in\left[\eta_{1}, a\right)$ and $\tilde{\mathcal{U}}_{1}^{+}(t, a)=\tilde{\mathcal{U}}_{2}^{+}(t, a)$. Moreover, there exists a maximal interval $[b, a] \subset\left[\eta_{1}, a\right]$ such that $\tilde{\mathcal{U}}_{1}^{+}(t, s)>0$ for all $s \in(b, a]$ and $\tilde{\mathcal{U}}_{1}^{+}(t, b)=0$. Hence we can write

$$
\begin{aligned}
0 \leqslant & \bar{d}\left(t, \eta_{2}\right)-\bar{d}\left(t, \eta_{1}\right) \\
\leqslant & \bar{d}\left(t, \eta_{2}\right)-\bar{d}(t, a)+\bar{d}(t, a)-\bar{d}(t, b) \\
\leqslant & \tilde{\mathcal{U}}_{2} \min _{j}\left(\tilde{\mathcal{D}}_{j}\right)\left(t, \eta_{2}\right)-\tilde{\mathcal{U}}_{2} \min _{j}\left(\tilde{\mathcal{D}}_{j}\right)(t, a) \\
& +\tilde{\mathcal{U}}_{1} \min _{j}\left(\tilde{\mathcal{D}}_{j}\right)(t, a)-\tilde{\mathcal{U}}_{1} \min _{j}\left(\tilde{\mathcal{D}}_{j}\right)(t, b) \\
\leqslant & \int_{a}^{\eta_{2}}\left(\tilde{\mathcal{U}}_{2, \eta} \min _{j}\left(\tilde{\mathcal{D}}_{j}\right)+\tilde{\mathcal{U}}_{2} \frac{d}{d \eta} \min _{j}\left(\tilde{\mathcal{D}}_{j}\right)\right)(t, s) d s \\
& +\int_{b}^{a}\left(\tilde{\mathcal{U}}_{1, \eta} \min _{j}\left(\tilde{\mathcal{D}}_{j}\right)+\tilde{\mathcal{U}}_{1} \frac{d}{d \eta} \min _{j}\left(\tilde{\mathcal{D}}_{j}\right)\right)(t, s) d s \\
\leqslant & \int_{a}^{\eta_{2}}\left(\frac{1}{2} \sqrt{A} A^{6} \min _{j}\left(\tilde{\mathcal{D}}_{j}\right)^{1 / 2}+\mathcal{O}(1) A^{5}\left|\tilde{\mathcal{U}}_{2}\right|\right)(t, s) d s \\
& +\int_{b}^{a}\left(\frac{1}{2} \sqrt{A} A^{6} \min _{j}\left(\tilde{\mathcal{D}}_{j}\right)^{1 / 2}+\mathcal{O}(1) A^{5}\left|\tilde{\mathcal{U}}_{1}\right|\right)(t, s) d s \\
\leqslant & \sqrt{A} A^{4} \int_{b}^{\eta_{2}} \min _{j}\left(\tilde{\mathcal{D}}_{j}\right)^{1 / 2}(t, s) d s+\mathcal{O}(1) A^{5} \int_{b}^{\eta_{2}}\left|\tilde{\mathcal{U}}_{2}\right|(t, s) d s \\
\leqslant & \sqrt{A} A^{4} \int_{\eta_{1}}^{\eta_{2}} \min _{j}\left(\tilde{\mathcal{D}}_{j}\right)^{1 / 2}(t, s) d s+\mathcal{O}(1) A^{5} \int_{\eta_{1}}^{\eta_{2}}\left|\tilde{\mathcal{U}}_{2}\right|(t, s) d s \\
\leqslant & \mathcal{O}(1) A^{7}\left|\eta_{2}-\eta_{1}\right| .
\end{aligned}
$$


Note, in the case that $\tilde{\mathcal{U}}_{1}^{+}(t, s)<\tilde{\mathcal{U}}_{2}^{+}(t, s)$ for all $s \in\left[\eta_{1}, \eta_{2}\right]$, the estimate starts with

$$
0 \leqslant d\left(t, \eta_{2}\right)-d\left(t, \eta_{1}\right) \leqslant \tilde{\mathcal{U}}_{1} \min _{j}\left(\tilde{\mathcal{D}}_{j}\right)\left(t, \eta_{2}\right)-\tilde{\mathcal{U}}_{1} \min _{j}\left(\tilde{\mathcal{D}}_{j}\right)(t, b),
$$

where $\left(b, \eta_{2}\right]$ denotes the maximal interval such that $\tilde{\mathcal{U}}_{1}^{+}(t, s)>0$ for all $s \in\left(b, \eta_{2}\right]$.

Thus we showed that

$$
\left|\bar{d}\left(t, \eta_{2}\right)-\bar{d}\left(t, \eta_{1}\right)\right| \leqslant \mathcal{O}(1) A^{7}\left|\eta_{2}-\eta_{1}\right|,
$$

or, in other words, $\bar{d}(t, \cdot)=\min _{j}\left(\tilde{\mathcal{D}}_{j}\right) \min _{j}\left(\tilde{\mathcal{U}}_{j}^{+}\right)(t, \cdot)$ is Lipschitz continuous with Lipschitz constant $\mathcal{O}(1) A^{7}$, which is independent of time, and thus differentiable almost everywhere. Moreover, a close look reveals that

$$
\begin{aligned}
& \mid \bar{d}(t,\left.\eta_{2}\right)-\bar{d}\left(t, \eta_{1}\right) \mid \\
& \leqslant \mathcal{O}(1) A^{5} \min _{j}\left(\tilde{\mathcal{U}}_{j}^{+}\right)\left(t, \eta_{2}\right)\left|\eta_{2}-\eta_{1}\right| \\
& \quad+\sqrt{A} A^{4}\left|\int_{\eta_{1}}^{\eta_{2}} \min _{j}\left(\tilde{\mathcal{D}}_{j}\right)^{1 / 2}(t, s) d s\right|+\mathcal{O}(1) A^{5}\left|\int_{\eta_{1}}^{\eta_{2}}\right| \tilde{\mathcal{U}}_{2}|(t, s) d s| .
\end{aligned}
$$

Since both $\left|\tilde{\mathcal{U}}_{2}\right|(t, \cdot)$ and $\min _{j}\left(\tilde{\mathcal{D}}_{j}\right)^{1 / 2}(t, \cdot)$ are continuous, the fundamental theorem of calculus implies that

$$
\begin{aligned}
\left|\frac{\bar{d}\left(t, \eta_{2}\right)-\bar{d}\left(t, \eta_{1}\right)}{\eta_{2}-\eta_{1}}\right| \leqslant & \mathcal{O}(1) A^{5} \min _{j}\left(\tilde{\mathcal{U}}_{j}^{+}\right)\left(t, \eta_{2}\right) \\
& +\sqrt{A} A^{4} \min _{j}\left(\tilde{\mathcal{D}}_{j}\right)^{1 / 2}(t, \tilde{\eta})+\mathcal{O}(1) A^{5}\left|\tilde{\mathcal{U}}_{2}\right|(t, \tilde{\eta})
\end{aligned}
$$

for some $\tilde{\eta}$ between $\eta_{1}$ and $\eta_{2}$. Letting $\eta_{2} \rightarrow \eta_{1}$ we thus obtain for almost every $\eta$ that

$$
\begin{aligned}
\left|\frac{d}{d \eta} \min _{j}\left(\tilde{\mathcal{D}}_{j}\right) \min _{j}\left(\tilde{\mathcal{U}}_{j}^{+}\right)(t, \eta)\right| & =\left|\frac{d}{d \eta} \bar{d}(t, \eta)\right| \\
& \leqslant \mathcal{O}(1) \sqrt{A} A^{4}\left(\min _{j}\left(\tilde{\mathcal{D}}_{j}\right)^{1 / 2}+\left|\tilde{\mathcal{U}}_{2}\right|\right)(t, \eta) .
\end{aligned}
$$

(ii) We have that

$$
\begin{aligned}
& \left|\min _{j}\left(\tilde{\mathcal{D}}_{j}\right)(t, \eta)-\min _{j}\left(\tilde{\mathcal{D}}_{j}\right)(t, \tilde{\eta})\right| \\
& \quad \leqslant \max \left(\left|\tilde{\mathcal{D}}_{1}(t, \eta)-\tilde{\mathcal{D}}_{1}(t, \tilde{\eta})\right|,\left|\tilde{\mathcal{D}}_{2}(t, \eta)-\tilde{\mathcal{D}}_{2}(t, \tilde{\eta})\right|\right) \\
& \quad \leqslant \mathcal{O}(1) A^{5}|\eta-\tilde{\eta}|,
\end{aligned}
$$


and hence, for almost every $\eta$,

$$
\left|\frac{d}{d \eta} \min _{j}\left(\tilde{\mathcal{D}}_{j}\right)(t, \eta)\right| \leqslant \mathcal{O}(1) A^{5} .
$$

This implies, for almost every $\eta$,

$$
\begin{aligned}
\left|\frac{d}{d \eta}\left(\min _{j}\left(\tilde{\mathcal{D}}_{j}\right) \tilde{\mathcal{U}}_{k}\right)(t, \eta)\right| & =\left|\left(\frac{d}{d \eta} \min _{j}\left(\tilde{\mathcal{D}}_{j}\right)\right) \tilde{\mathcal{U}}_{k}(t, \eta)+\min _{j}\left(\tilde{\mathcal{D}}_{j}\right) \tilde{\mathcal{U}}_{k, \eta}(t, \eta)\right| \\
& \leqslant \mathcal{O}(1) A^{7}+2 A\left\|\tilde{\mathcal{P}}_{k}\left|\tilde{\mathcal{U}}_{k, \eta}\right|\right\| \\
& \leqslant \mathcal{O}(1) A^{7} .
\end{aligned}
$$

LEMMA A.5. The function

$$
\eta \mapsto a \int_{0}^{\eta} \min _{j}\left(e^{-\frac{1}{a}\left(\tilde{\mathcal{Y}}_{j}(t, \eta)-\tilde{\mathcal{Y}}_{j}(t, \theta)\right)}\right) \min _{j}\left(\tilde{\mathcal{U}}_{j}^{+}\right)(t, \theta) d \theta
$$

is Lipschitz continuous with a uniformly bounded Lipschitz constant and thus differentiable almost everywhere with

$$
\left|\frac{d}{d \eta}\left(a \int_{0}^{\eta} \min _{j}\left(e^{-\frac{1}{a}\left(\tilde{\mathcal{Y}}_{j}(t, \eta)-\tilde{\mathcal{Y}}_{j}(t, \theta)\right)}\right) \min _{j}\left(\tilde{\mathcal{U}}_{j}^{+}\right)(t, \theta) d \theta\right)\right| \leqslant \mathcal{O}(1) A^{2} .
$$

Proof. To prove the existence and boundedness of the derivative, we will prove Lipschitz continuity. Let $0 \leqslant \eta_{1}<\eta_{2} \leqslant 1$; then

$$
\begin{aligned}
a \mid \int_{0}^{\eta_{1}} & \min _{j}\left(e^{-\frac{1}{a}\left(\tilde{\mathcal{Y}}_{j}\left(t, \eta_{1}\right)-\tilde{\mathcal{Y}}_{j}(t, \theta)\right)}\right) \min _{j}\left(\tilde{\mathcal{U}}_{j}^{+}\right)(t, \theta) d \theta \\
& -\int_{0}^{\eta_{2}} \min _{j}\left(e^{-\frac{1}{a}\left(\tilde{\mathcal{Y}}_{j}\left(t, \eta_{2}\right)-\tilde{\mathcal{Y}}_{j}(t, \theta)\right)}\right) \min _{j}\left(\tilde{\mathcal{U}}_{j}^{+}\right)(t, \theta) d \theta \mid \\
\leqslant & a \int_{\eta_{1}}^{\eta_{2}} \min _{j}\left(e^{-\frac{1}{a}\left(\tilde{\mathcal{Y}}_{j}\left(t, \eta_{2}\right)-\tilde{\mathcal{Y}}_{j}(t, \theta)\right)}\right) \min _{j}\left(\tilde{\mathcal{U}}_{j}^{+}\right)(t, \theta) d \theta \\
& +a \int_{0}^{\eta_{1}}\left(\min _{j}\left(e^{-\frac{1}{a}\left(\tilde{\mathcal{Y}}_{j}\left(t, \eta_{1}\right)-\tilde{\mathcal{Y}}_{j}(t, \theta)\right)}\right)\right. \\
& \left.-\min _{j}\left(e^{-\frac{1}{a}\left(\tilde{\mathcal{Y}}_{j}\left(t, \eta_{2}\right)-\tilde{\mathcal{Y}}_{j}(t, \theta)\right)}\right)\right) \min _{j}\left(\tilde{\mathcal{U}}_{j}^{+}\right)(t, \theta) d \theta \\
\leqslant & \frac{a A^{2}}{\sqrt{2}}\left|\eta_{2}-\eta_{1}\right| \\
& +a \int_{0}^{\eta_{1}}\left(e^{-\frac{1}{a}\left(\tilde{\mathcal{Y}}_{1}\left(t, \eta_{1}\right)-\tilde{\mathcal{Y}}_{1}(t, \theta)\right)}-e^{-\frac{1}{a}\left(\tilde{\mathcal{Y}}_{1}\left(t, \eta_{2}\right)-\tilde{\mathcal{Y}}_{1}(t, \theta)\right)}\right) \min _{j}\left(\tilde{\mathcal{U}}_{j}^{+}\right)(t, \theta) d \theta
\end{aligned}
$$




$$
\begin{aligned}
& +a \int_{0}^{\eta_{1}}\left(e^{-\frac{1}{a}\left(\tilde{\mathcal{Y}}_{2}\left(t, \eta_{1}\right)-\tilde{\mathcal{Y}}_{2}(t, \theta)\right)}-e^{-\frac{1}{a}\left(\tilde{\mathcal{Y}}_{2}\left(t, \eta_{2}\right)-\tilde{\mathcal{Y}}_{2}(t, \theta)\right)}\right) \min _{j}\left(\tilde{\mathcal{U}}_{j}^{+}\right)(t, \theta) d \theta \\
& \leqslant \frac{a A^{2}}{\sqrt{2}}\left|\eta_{2}-\eta_{1}\right| \\
& +\int_{0}^{\eta_{1}} \int_{\eta_{1}}^{\eta_{2}} e^{-\frac{1}{a}\left(\tilde{\mathcal{Y}}_{1}(t, s)-\tilde{\mathcal{Y}}_{1}(t, \theta)\right)} \tilde{\mathcal{Y}}_{1, \eta}(t, s) d s \min _{j}\left(\tilde{\mathcal{U}}_{j}^{+}\right)(t, \theta) d \theta \\
& +\int_{0}^{\eta_{1}} \int_{\eta_{1}}^{\eta_{2}} e^{-\frac{1}{a}\left(\tilde{\mathcal{Y}}_{2}(t, s)-\tilde{\mathcal{Y}}_{2}(t, \theta)\right)} \tilde{\mathcal{Y}}_{2, \eta}(t, s) d s \min _{j}\left(\tilde{\mathcal{U}}_{j}^{+}\right)(t, \theta) d \theta \\
& \leqslant \frac{a A^{2}}{\sqrt{2}}\left|\eta_{2}-\eta_{1}\right| \\
& +\int_{\eta_{1}}^{\eta_{2}}\left(\int_{0}^{s} e^{-\frac{1}{A_{1}}\left(\tilde{\mathcal{Y}}_{1}(t, s)-\tilde{\mathcal{Y}}_{1}(t, \theta)\right)} \tilde{\mathcal{U}}_{1}^{+}(t, \theta) d \theta\right) \tilde{\mathcal{Y}}_{1, \eta}(t, s) d s \\
& +\int_{\eta_{1}}^{\eta_{2}}\left(\int_{0}^{s} e^{-\frac{1}{A_{2}}\left(\tilde{\mathcal{Y}}_{2}(t, s)-\tilde{\mathcal{Y}}_{2}(t, \theta)\right)} \tilde{\mathcal{U}}_{2}^{+}(t, \theta) d \theta\right) \tilde{\mathcal{Y}}_{2, \eta}(t, s) d s \\
& \leqslant \frac{a A^{2}}{\sqrt{2}}\left|\eta_{2}-\eta_{1}\right|+\frac{1}{A_{1}^{5}} \int_{\eta_{1}}^{\eta_{2}}\left(\int_{0}^{s} e^{-\frac{1}{A_{1}}\left(\tilde{\mathcal{Y}}_{1}(t, s)-\tilde{\mathcal{Y}}_{1}(t, \theta)\right)}\right. \\
& \left.\times\left(\frac{1}{A_{1}} \tilde{\mathcal{P}}_{1}^{2} \tilde{\mathcal{Y}}_{1, \eta}+A_{1} \tilde{\mathcal{U}}_{1}^{2} \tilde{\mathcal{Y}}_{1, \eta}+\tilde{\mathcal{U}}_{1}^{+} \tilde{\mathcal{H}}_{1, \eta}\right)(t, \theta) d \theta\right) \tilde{\mathcal{Y}}_{1, \eta}(t, s) d s \\
& +\frac{1}{A_{2}^{5}} \int_{\eta_{1}}^{\eta_{2}}\left(\int_{0}^{s} e^{-\frac{1}{A_{2}}\left(\tilde{\mathcal{Y}}_{2}(t, s)-\tilde{\mathcal{Y}}_{2}(t, \theta)\right)}\right. \\
& \left.\times\left(\frac{1}{A_{2}} \tilde{\mathcal{P}}_{2}^{2} \tilde{\mathcal{Y}}_{2, \eta}+A_{2} \tilde{\mathcal{U}}_{2}^{2} \tilde{\mathcal{Y}}_{2, \eta}+\tilde{\mathcal{U}}_{2}^{+} \tilde{\mathcal{H}}_{2, \eta}\right)(t, \theta) d \theta\right) \tilde{\mathcal{Y}}_{2, \eta}(t, s) d s \\
& \leqslant \frac{a A^{2}}{\sqrt{2}}\left|\eta_{2}-\eta_{1}\right| \\
& +\mathcal{O}(1) \frac{1}{A_{1}^{3}} \int_{\eta_{1}}^{\eta_{2}} \tilde{\mathcal{P}}_{1} \tilde{\mathcal{Y}}_{1, \eta}(t, s) d s \\
& +\mathcal{O}(1) \frac{1}{A_{2}^{3}} \int_{\eta_{1}}^{\eta_{2}} \tilde{\mathcal{P}}_{2} \tilde{\mathcal{Y}}_{2, \eta}(t, s) d s \\
& \leqslant \mathcal{O}(1) A^{2}\left|\eta_{2}-\eta_{1}\right| \text {. }
\end{aligned}
$$

\section{LEMmA A.6. The function}

$$
\eta \mapsto \min _{k}\left(\int_{0}^{\eta} \min _{j}\left(e^{-\frac{1}{a}\left(\tilde{\mathcal{Y}}_{j}(t, \eta)-\tilde{\mathcal{Y}}_{j}(t, \theta)\right)}\right) \min _{j}\left(\tilde{\mathcal{P}}_{j}\right) \min _{j}\left(\tilde{\mathcal{U}}_{j}^{+}\right) \tilde{\mathcal{Y}}_{k, \eta}(t, \theta) d \theta\right)
$$


is Lipschitz continuous with a uniformly bounded Lipschitz constant and thus differentiable almost everywhere. The derivative satisfies,

$$
\left|\frac{d}{d \eta} \min _{k}\left(\int_{0}^{\eta} \min _{j}\left(e^{-\frac{1}{a}\left(\tilde{\mathcal{Y}}_{j}(t, \eta)-\tilde{\mathcal{Y}}_{j}(t, \theta)\right)}\right) \min _{j}\left(\tilde{\mathcal{P}}_{j}\right) \min _{j}\left(\tilde{\mathcal{U}}_{j}^{+}\right) \tilde{\mathcal{Y}}_{k, \eta}(t, \theta) d \theta\right)\right| \leqslant \mathcal{O}(1) A^{7} .
$$

Proof. Introduce

$$
\begin{aligned}
& \tilde{a}(t, \eta)=\min _{k}\left(\int_{0}^{\eta} \min _{j}\left(e^{-\frac{1}{a}\left(\tilde{\mathcal{Y}}_{j}(t, \eta)-\tilde{\mathcal{Y}}_{j}(t, \theta)\right)}\right) \min _{j}\left(\tilde{\mathcal{P}}_{j}\right) \min _{j}\left(\tilde{\mathcal{U}}_{j}^{+}\right) \tilde{\mathcal{Y}}_{k, \eta}(t, \theta) d \theta\right) \\
& \tilde{b}(t, \eta)=\int_{0}^{\eta} \min _{j}\left(e^{-\frac{1}{a}\left(\tilde{\mathcal{Y}}_{j}(t, \eta)-\tilde{\mathcal{Y}}_{j}(t, \theta)\right)}\right) \min _{j}\left(\tilde{\mathcal{P}}_{j}\right) \min _{j}\left(\tilde{\mathcal{U}}_{j}^{+}\right) \tilde{\mathcal{Y}}_{1, \eta}(t, \theta) d \theta \\
& \tilde{c}(t, \eta)=\int_{0}^{\eta} \min _{j}\left(e^{-\frac{1}{a}\left(\tilde{\mathcal{Y}}_{j}(t, \eta)-\tilde{\mathcal{Y}}_{j}(t, \theta)\right)}\right) \min _{j}\left(\tilde{\mathcal{P}}_{j}\right) \min _{j}\left(\tilde{\mathcal{U}}_{j}^{+}\right) \tilde{\mathcal{Y}}_{2, \eta}(t, \theta) d \theta .
\end{aligned}
$$

Thus

$$
\tilde{a}(t, \eta)=\min (\tilde{b}, \tilde{c})(t, \eta) .
$$

Then we have to show that $\tilde{a}(t, \cdot)$ is Lipschitz continuous with a Lipschitz constant, which only depends on $A$. Clearly, we have that

$$
\left|\tilde{a}\left(t, \eta_{1}\right)-\tilde{a}\left(t, \eta_{2}\right)\right| \leqslant \max \left(\left|\tilde{b}\left(t, \eta_{1}\right)-\tilde{b}\left(t, \eta_{2}\right)\right|,\left|\tilde{c}\left(t, \eta_{1}\right)-\tilde{c}\left(t, \eta_{2}\right)\right|\right),
$$

and it suffices to show that both $\tilde{b}(t, \cdot)$ and $\tilde{c}(t, \cdot)$ are Lipschitz continuous with a Lipschitz constant, which only depends on $A$. We are only going to establish the Lipschitz continuity for $\tilde{b}$, since the argument for $\tilde{c}$ follows the same lines.

Let $0 \leqslant \eta_{1}<\eta_{2} \leqslant 1$. Then we have to consider two cases:

$$
0 \leqslant \tilde{b}\left(t, \eta_{1}\right)-\tilde{b}\left(t, \eta_{2}\right) \quad \text { and } \quad 0 \leqslant \tilde{b}\left(t, \eta_{2}\right)-\tilde{b}\left(t, \eta_{1}\right) .
$$

(i): $0 \leqslant \tilde{b}\left(t, \eta_{2}\right)-\tilde{b}\left(t, \eta_{1}\right):$ By definition, we have

$$
\begin{aligned}
\tilde{b}\left(t, \eta_{2}\right)-\tilde{b}\left(t, \eta_{1}\right)= & \int_{0}^{\eta_{2}} \min _{j}\left(e^{-\frac{1}{a}\left(\tilde{\mathcal{Y}}_{j}\left(t, \eta_{2}\right)-\tilde{\mathcal{Y}}_{j}(t, \theta)\right)}\right) \min _{j}\left(\tilde{\mathcal{P}}_{j}\right) \\
& \times \min _{j}\left(\tilde{\mathcal{U}}_{j}^{+}\right) \tilde{\mathcal{Y}}_{1, \eta}(t, \theta) d \theta \\
& -\int_{0}^{\eta_{1}} \min _{j}\left(e^{-\frac{1}{a}\left(\tilde{\mathcal{Y}}_{j}\left(t, \eta_{1}\right)-\tilde{\mathcal{Y}}_{j}(t, \theta)\right)}\right) \min _{j}\left(\tilde{\mathcal{P}}_{j}\right) \\
& \times \min _{j}\left(\tilde{\mathcal{U}}_{j}^{+}\right) \tilde{\mathcal{Y}}_{1, \eta}(t, \theta) d \theta \\
= & \int_{\eta_{1}}^{\eta_{2}} \min _{j}\left(e^{-\frac{1}{a}\left(\tilde{\mathcal{Y}}_{j}\left(t, \eta_{2}\right)-\tilde{\mathcal{Y}}_{j}(t, \theta)\right)}\right) \min _{j}\left(\tilde{\mathcal{P}}_{j}\right)
\end{aligned}
$$




$$
\begin{aligned}
& \times \min _{j}\left(\tilde{\mathcal{U}}_{j}^{+}\right) \tilde{\mathcal{Y}}_{1, \eta}(t, \theta) d \theta \\
& +\int_{0}^{\eta_{1}}\left(\min _{j}\left(e^{-\frac{1}{a}\left(\tilde{\mathcal{Y}}_{j}\left(t, \eta_{2}\right)-\tilde{\mathcal{Y}}_{j}(t, \theta)\right)}\right)\right. \\
& \left.-\min _{j}\left(e^{-\frac{1}{a}\left(\tilde{\mathcal{Y}}_{j}\left(t, \eta_{1}\right)-\tilde{\mathcal{Y}}_{j}(t, \theta)\right)}\right)\right) \\
& \times \min _{j}\left(\tilde{\mathcal{P}}_{j}\right) \min _{j}\left(\tilde{\mathcal{U}}_{j}^{+}\right) \tilde{\mathcal{Y}}_{1, \eta}(t, \theta) d \theta \\
\leqslant & \int_{\eta_{1}}^{\eta_{2}} \min _{j}\left(e^{-\frac{1}{a}\left(\tilde{\mathcal{Y}}_{j}\left(t, \eta_{2}\right)-\tilde{\mathcal{Y}}_{j}(t, \theta)\right)}\right) \min \left(\tilde{\mathcal{P}}_{j}\right) \\
& \times \min _{j}\left(\tilde{\mathcal{U}}_{j}^{+}\right) \tilde{\mathcal{Y}}_{1, \eta}(t, \theta) d \theta,
\end{aligned}
$$

where we used in the last step that $0 \leqslant \min _{j}\left(\tilde{\mathcal{P}}_{j}\right) \min _{j}\left(\tilde{\mathcal{U}}_{j}^{+}\right) \tilde{\mathcal{Y}}_{1, \eta}(t, \eta)$ and that $\tilde{\mathcal{Y}}_{1}(t, \eta)$ is increasing, which implies

$$
\min _{j}\left(e^{-\frac{1}{a}\left(\tilde{\mathcal{Y}}_{j}\left(t, \eta_{1}\right)-\tilde{\mathcal{Y}}_{j}(t, \theta)\right)}\right) \geqslant \min _{j}\left(e^{-\frac{1}{a}\left(\tilde{\mathcal{Y}}_{j}\left(t, \eta_{2}\right)-\tilde{\mathcal{Y}}_{j}(t, \theta)\right)}\right) .
$$

Moreover, note that $\min _{j}\left(e^{-\frac{1}{a}\left(\tilde{\mathcal{Y}}_{j}\left(t, \eta_{2}\right)-\tilde{\mathcal{Y}}_{j}(t, \theta)\right)}\right) \leqslant 1$, for $0 \leqslant \eta_{1} \leqslant \theta \leqslant \eta_{2}$, and that

$$
0 \leqslant 2 \sqrt{2} \min _{j}\left(\tilde{\mathcal{P}}_{j}\right) \min _{j}\left(\tilde{\mathcal{U}}_{j}^{+}\right) \tilde{\mathcal{Y}}_{1, \eta}(t, \theta) \leqslant A_{1}^{7} \leqslant A^{7}
$$

by (4.15b) and (4.15e). Thus

$$
\begin{aligned}
0 & \leqslant \tilde{b}\left(t, \eta_{2}\right)-\tilde{b}\left(t, \eta_{1}\right) \\
& \leqslant \int_{\eta_{1}}^{\eta_{2}} \min _{j}\left(e^{-\frac{1}{a}\left(\tilde{\mathcal{Y}}_{j}\left(t, \eta_{2}\right)-\tilde{\mathcal{Y}}_{j}(t, \theta)\right)}\right) \min _{j}\left(\tilde{\mathcal{P}}_{j}\right) \min _{j}\left(\tilde{\mathcal{U}}_{j}^{+}\right) \tilde{\mathcal{Y}}_{1, \eta}(t, \theta) d \theta \\
& \leqslant \frac{A^{7}}{2 \sqrt{2}}\left|\eta_{2}-\eta_{1}\right| .
\end{aligned}
$$

(ii): $0 \leqslant \tilde{b}\left(t, \eta_{1}\right)-\tilde{b}\left(t, \eta_{2}\right)$ : By definition, we have

$$
\begin{aligned}
0 \leqslant & \tilde{b}\left(t, \eta_{1}\right)-\tilde{b}\left(t, \eta_{2}\right) \\
= & \int_{0}^{\eta_{1}} \min _{j}\left(e^{-\frac{1}{a}\left(\tilde{\mathcal{Y}}_{j}\left(t, \eta_{1}\right)-\tilde{\mathcal{Y}}_{j}(t, \theta)\right)}\right) \min _{j}\left(\tilde{\mathcal{P}}_{j}\right) \min _{j}\left(\tilde{\mathcal{U}}_{j}^{+}\right) \tilde{\mathcal{Y}}_{1, \eta}(t, \theta) d \theta \\
& -\int_{0}^{\eta_{2}} \min _{j}\left(e^{-\frac{1}{a}\left(\tilde{\mathcal{Y}}_{j}\left(t, \eta_{2}\right)-\tilde{\mathcal{Y}}_{j}(t, \theta)\right)}\right) \min _{j}\left(\tilde{\mathcal{P}}_{j}\right) \min _{j}\left(\tilde{\mathcal{U}}_{j}^{+}\right) \tilde{\mathcal{Y}}_{1, \eta}(t, \theta) d \theta \\
= & \int_{0}^{\eta_{1}}\left(\min _{j}\left(e^{-\frac{1}{a}\left(\tilde{\mathcal{Y}}_{j}\left(t, \eta_{1}\right)-\tilde{\mathcal{Y}}_{j}(t, \theta)\right)}\right)-\min _{j}\left(e^{-\frac{1}{a}\left(\tilde{\mathcal{Y}}_{j}\left(t, \eta_{2}\right)-\tilde{\mathcal{Y}}_{j}(t, \theta)\right)}\right)\right)
\end{aligned}
$$




$$
\begin{aligned}
& \times \min _{j}\left(\tilde{\mathcal{P}}_{j}\right) \min _{j}\left(\tilde{\mathcal{U}}_{j}^{+}\right) \tilde{\mathcal{Y}}_{1, \eta}(t, \theta) d \theta \\
& -\int_{\eta_{1}}^{\eta_{2}} \min _{j}\left(e^{-\frac{1}{a}\left(\tilde{\mathcal{Y}}_{j}\left(t, \eta_{2}\right)-\tilde{\mathcal{Y}}_{j}(t, \theta)\right)}\right) \min _{j}\left(\tilde{\mathcal{P}}_{j}\right) \min _{j}\left(\tilde{\mathcal{U}}_{j}^{+}\right) \tilde{\mathcal{Y}}_{1, \eta}(t, \theta) d \theta \\
\leqslant & \int_{0}^{\eta_{1}}\left(\min _{j}\left(e^{-\frac{1}{a}\left(\tilde{\mathcal{Y}}_{j}\left(t, \eta_{1}\right)-\tilde{\mathcal{Y}}_{j}(t, \theta)\right)}\right)-\min _{j}\left(e^{-\frac{1}{a}\left(\tilde{\mathcal{Y}}_{j}\left(t, \eta_{2}\right)-\tilde{\mathcal{Y}}_{j}(t, \theta)\right)}\right)\right) \\
& \times \min _{j}\left(\tilde{\mathcal{P}}_{j}\right) \min _{j}\left(\tilde{\mathcal{U}}_{j}^{+}\right) \tilde{\mathcal{Y}}_{1, \eta}(t, \theta) d \theta .
\end{aligned}
$$

Now we have to be much more careful than before. Namely, we have (as before)

$$
\begin{aligned}
0 & \leqslant \min _{j}\left(e^{-\frac{1}{a}\left(\tilde{\mathcal{Y}}_{j}\left(t, \eta_{1}\right)-\tilde{\mathcal{Y}}_{j}(t, \theta)\right)}\right)-\min _{j}\left(e^{-\frac{1}{a}\left(\tilde{\mathcal{Y}}_{j}\left(t, \eta_{2}\right)-\tilde{\mathcal{Y}}_{j}(t, \theta)\right)}\right) \\
& \leqslant \frac{1}{a} \int_{\eta_{1}}^{\eta_{2}}\left(e^{\frac{1}{a}\left(\tilde{\mathcal{Y}}_{1}(t, \theta)-\tilde{\mathcal{Y}}_{1}(t, s)\right)} \tilde{\mathcal{Y}}_{1, \eta}(t, s)+e^{\frac{1}{a}\left(\tilde{\mathcal{Y}}_{2}(t, \theta)-\tilde{\mathcal{Y}}_{2}(t, s)\right)} \tilde{\mathcal{Y}}_{2, \eta}(t, s)\right) d s .
\end{aligned}
$$

Hence

$$
\begin{aligned}
& \tilde{b}\left(t, \eta_{1}\right)-\tilde{b}\left(t, \eta_{2}\right) \\
& \leqslant \int_{0}^{\eta_{1}}\left(\min _{j}\left(e^{-\frac{1}{a}\left(\tilde{\mathcal{Y}}_{j}\left(t, \eta_{1}\right)-\tilde{\mathcal{Y}}_{j}(t, \theta)\right)}\right)-\min _{j}\left(e^{-\frac{1}{a}\left(\tilde{\mathcal{Y}}_{j}\left(t, \eta_{2}\right)-\tilde{\mathcal{Y}}_{j}(t, \theta)\right)}\right)\right) \\
& \times \min _{j}\left(\tilde{\mathcal{P}}_{j}\right) \min _{j}\left(\tilde{\mathcal{U}}_{j}^{+}\right) \tilde{\mathcal{Y}}_{1, \eta}(t, \theta) d \theta \\
& \leqslant \frac{1}{a} \int_{0}^{\eta_{1}} \int_{\eta_{1}}^{\eta_{2}} e^{-\frac{1}{a}\left(\tilde{\mathcal{Y}}_{1}(t, s)-\tilde{\mathcal{Y}}_{1}(t, \theta)\right)} \tilde{\mathcal{Y}}_{1, \eta}(t, s) d s \min _{j}\left(\tilde{\mathcal{P}}_{j}\right) \min _{j}\left(\tilde{\mathcal{U}}_{j}^{+}\right) \tilde{\mathcal{Y}}_{1, \eta}(t, \theta) d \theta \\
& +\frac{1}{a} \int_{0}^{\eta_{1}} \int_{\eta_{1}}^{\eta_{2}} e^{-\frac{1}{a}\left(\tilde{\mathcal{Y}}_{2}(t, s)-\tilde{\mathcal{Y}}_{2}(t, \theta)\right)} \tilde{\mathcal{Y}}_{2, \eta}(t, s) d s \min _{j}\left(\tilde{\mathcal{P}}_{j}\right) \min _{j}\left(\tilde{\mathcal{U}}_{j}^{+}\right) \tilde{\mathcal{Y}}_{1, \eta}(t, \theta) d \theta \\
& =\frac{1}{a} \int_{\eta_{1}}^{\eta_{2}} \tilde{\mathcal{Y}}_{1, \eta}(t, s)\left(\int_{0}^{\eta_{1}} e^{-\frac{1}{a}\left(\tilde{\mathcal{Y}}_{1}(t, s)-\tilde{\mathcal{Y}}_{1}(t, \theta)\right)} \min _{j}\left(\tilde{\mathcal{P}}_{j}\right) \min _{j}\left(\tilde{\mathcal{U}}_{j}^{+}\right) \tilde{\mathcal{Y}}_{1, \eta}(t, \theta) d \theta\right) d s \\
& +\frac{1}{a} \int_{\eta_{1}}^{\eta_{2}} \tilde{\mathcal{Y}}_{2, \eta}(t, s)\left(\int_{0}^{\eta_{1}} e^{-\frac{1}{a}\left(\tilde{\mathcal{Y}}_{2}(t, s)-\tilde{\mathcal{Y}}_{2}(t, \theta)\right)} \min _{j}\left(\tilde{\mathcal{P}}_{j}\right) \min _{j}\left(\tilde{\mathcal{U}}_{j}^{+}\right) \tilde{\mathcal{Y}}_{1, \eta}(t, \theta) d \theta\right) d s \\
& \leqslant \frac{1}{a} \int_{\eta_{1}}^{\eta_{2}} \tilde{\mathcal{Y}}_{1, \eta}(t, s)\left(\int_{0}^{s} e^{-\frac{1}{a}\left(\tilde{\mathcal{Y}}_{1}(t, s)-\tilde{\mathcal{Y}}_{1}(t, \theta)\right)} \min _{j}\left(\tilde{\mathcal{P}}_{j}\right) \min _{j}\left(\tilde{\mathcal{U}}_{j}^{+}\right) \tilde{\mathcal{Y}}_{1, \eta}(t, \theta) d \theta\right) d s \\
& +\frac{1}{a} \int_{\eta_{1}}^{\eta_{2}} \tilde{\mathcal{Y}}_{2, \eta}(t, s)\left(\int_{0}^{s} e^{-\frac{1}{a}\left(\tilde{\mathcal{Y}}_{2}(t, s)-\tilde{\mathcal{Y}}_{2}(t, \theta)\right)} \min _{j}\left(\tilde{\mathcal{P}}_{j}\right) \min _{j}\left(\tilde{\mathcal{U}}_{j}^{+}\right) \tilde{\mathcal{Y}}_{1, \eta}(t, \theta) d \theta\right) d s \\
& =\int_{\eta_{1}}^{\eta_{2}} \tilde{B}_{1}(t, s) d s+\int_{\eta_{1}}^{\eta_{2}} \tilde{B}_{2}(t, s) d s
\end{aligned}
$$


As far as $\tilde{B}_{1}(t, s)$ is concerned, we have

$$
\begin{aligned}
\int_{\eta_{1}}^{\eta_{2}} & \tilde{B}_{1}(t, s) d s \\
= & \frac{1}{a} \int_{\eta_{1}}^{\eta_{2}}\left(\int_{0}^{s} e^{-\frac{1}{a}\left(\tilde{\mathcal{Y}}_{1}(t, s)-\tilde{\mathcal{Y}}_{1}(t, \theta)\right)} \min _{j}\left(\tilde{\mathcal{P}}_{j}\right) \min _{j}\left(\tilde{\mathcal{U}}_{j}^{+}\right) \tilde{\mathcal{Y}}_{1, \eta}(t, \theta) d \theta\right) \tilde{\mathcal{Y}}_{1, \eta}(t, s) d s \\
\leqslant & \frac{1}{\sqrt{2}} \int_{\eta_{1}}^{\eta_{2}}\left(\int_{0}^{s} e^{-\frac{1}{A_{1}}\left(\tilde{\mathcal{Y}}_{1}(t, s)-\tilde{\mathcal{Y}}_{1}(t, \theta)\right)} \tilde{\mathcal{P}}_{1}^{3 / 4} \tilde{\mathcal{U}}_{1}^{+} \tilde{\mathcal{Y}}_{1, \eta}(t, \theta) d \theta\right) \tilde{\mathcal{Y}}_{1, \eta}(t, s) d s \\
\leqslant & \frac{1}{\sqrt{2}} \int_{\eta_{1}}^{\eta_{2}}\left(\int_{0}^{s} e^{-\frac{1}{A_{1}}\left(\tilde{\mathcal{Y}}_{1}(t, s)-\tilde{\mathcal{Y}}_{1}(t, \theta)\right)} \tilde{\mathcal{P}}_{1}^{3 / 2} \tilde{\mathcal{Y}}_{1, \eta}(t, \theta) d \theta\right)^{1 / 2} \\
& \times\left(\int_{0}^{s} e^{-\frac{1}{A_{1}}\left(\tilde{\mathcal{Y}}_{1}(t, s)-\tilde{\mathcal{Y}}_{1}(t, \theta)\right)} \tilde{\mathcal{U}}_{1}^{2} \tilde{\mathcal{Y}}_{1, \eta}(t, \theta) d \theta\right)^{1 / 2} \tilde{\mathcal{Y}}_{1, \eta}(t, s) d s \\
\leqslant & 3 A^{2} \int_{\eta_{1}}^{\eta_{2}} \tilde{\mathcal{P}}_{1} \tilde{\mathcal{Y}}_{1, \eta}(t, s) d s \\
\leqslant & \frac{3}{2} A^{7}\left|\eta_{2}-\eta_{1}\right|
\end{aligned}
$$

using (4.16k).

As far as $\tilde{B}_{2}(t, s)$ is concerned, we have to be more careful. Therefore recall that we have (cf. (4.13)) that

$$
A_{2}^{5}=2 \tilde{\mathcal{P}}_{2} \tilde{\mathcal{Y}}_{2, \eta}(t, \eta)-\tilde{\mathcal{U}}_{2}^{2} \tilde{\mathcal{Y}}_{2, \eta}(t, \eta)+\tilde{\mathcal{H}}_{2, \eta}(t, \eta) \leqslant 2 \tilde{\mathcal{P}}_{2} \tilde{\mathcal{Y}}_{2, \eta}(t, \eta)+\tilde{\mathcal{H}}_{2, \eta}(t, \eta) .
$$

Therefore we can write

$$
\begin{aligned}
& \frac{1}{a} \int_{0}^{s} e^{-\frac{1}{a}\left(\tilde{\mathcal{Y}}_{2}(t, s)-\tilde{\mathcal{Y}}_{2}(t, \theta)\right)} \min _{j}\left(\tilde{\mathcal{P}}_{j}\right) \min _{j}\left(\tilde{\mathcal{U}}_{j}^{+}\right) \tilde{\mathcal{Y}}_{1, \eta}(t, \theta) d \theta \\
& \quad \leqslant \frac{\sqrt{2}}{a} \int_{0}^{s} e^{-\frac{1}{A_{2}}\left(\tilde{\mathcal{Y}}_{2}(t, s)-\tilde{\mathcal{Y}}_{2}(t, \theta)\right)} \min _{j}\left(\tilde{\mathcal{P}}_{j}\right)^{3 / 2} \tilde{\mathcal{Y}}_{1, \eta}(t, \theta) d \theta \\
& \quad \leqslant \int_{0}^{s} e^{-\frac{1}{A_{2}}\left(\tilde{\mathcal{Y}}_{2}(t, s)-\tilde{\mathcal{Y}}_{2}(t, \theta)\right)} \tilde{\mathcal{P}}_{2}^{1 / 4} \tilde{\mathcal{P}}_{1} \tilde{\mathcal{Y}}_{1, \eta}(t, \theta) d \theta \\
& \leqslant \frac{A_{1}^{5}}{2 A_{2}^{5}} \int_{0}^{s} e^{-\frac{1}{A_{2}}\left(\tilde{\mathcal{Y}}_{2}(t, s)-\tilde{\mathcal{Y}}_{2}(t, \theta)\right)} \tilde{\mathcal{P}}_{2}^{1 / 4}\left(2 \tilde{\mathcal{P}}_{2} \tilde{\mathcal{Y}}_{2, \eta}+\tilde{\mathcal{H}}_{2, \eta}\right)(t, \theta) d \theta \\
& \leqslant \frac{A_{1}^{5}}{2 A_{2}^{5}} \int_{0}^{s} e^{-\frac{1}{A_{2}}\left(\tilde{\mathcal{Y}}_{2}(t, s)-\tilde{\mathcal{Y}}_{2}(t, \theta)\right)}\left(2 \tilde{\mathcal{P}}_{2}^{5 / 4} \tilde{\mathcal{Y}}_{2, \eta}+\frac{A_{2}}{\sqrt{2}} \tilde{\mathcal{H}}_{2, \eta}\right)(t, \theta) d \theta \\
& \leqslant \frac{A_{1}^{5}}{2 A_{2}^{5}}\left(2 \sqrt{2} A_{2}^{2} \tilde{\mathcal{P}}_{2}(t, s)+2 \int_{0}^{s} e^{-\frac{1}{A_{2}}\left(\tilde{\mathcal{Y}}_{2}(t, s)-\tilde{\mathcal{Y}}_{2}(t, \theta)\right)} \tilde{\mathcal{P}}_{2}^{5 / 4} \tilde{\mathcal{Y}}_{2, \eta}(t, \theta) d \theta\right) .
\end{aligned}
$$


Note that the integral term can be bounded by $\mathcal{O}(1) \tilde{\mathcal{P}}_{2}(t, s)$ since

$$
\int_{0}^{s} e^{-\frac{1}{A_{2}}\left(\tilde{\mathcal{Y}}_{2}(t, s)-\tilde{\mathcal{Y}}_{2}(t, \theta)\right)} \tilde{\mathcal{P}}_{2}^{5 / 4} \tilde{\mathcal{Y}}_{2, \eta}(t, \theta) d \theta \leqslant \frac{15}{\sqrt{2}} A_{2}^{2} \tilde{\mathcal{P}}_{2}(t, s)
$$

by (4.16k). We end up with

$$
\begin{aligned}
\int_{\eta_{1}}^{\eta_{2}} \tilde{B}_{2}(t, s) d s= & \frac{1}{a} \int_{\eta_{1}}^{\eta_{2}} \tilde{\mathcal{Y}}_{2, \eta}(t, s) \\
& \times\left(\int_{0}^{s} e^{-\frac{1}{a}\left(\tilde{\mathcal{Y}}_{2}(t, s)-\tilde{\mathcal{Y}}_{2}(t, \theta)\right)} \min _{j}\left(\tilde{\mathcal{P}}_{j}\right) \min _{j}\left(\tilde{\mathcal{U}}_{j}^{+}\right) \tilde{\mathcal{Y}}_{1, \eta}(t, \theta) d \theta\right) d s \\
\leqslant & \frac{17}{\sqrt{2}} \frac{A_{1}^{5}}{A_{2}^{3}} \int_{\eta_{1}}^{\eta_{2}} \tilde{\mathcal{P}}_{2} \tilde{\mathcal{Y}}_{2, \eta}(t, s) d s \\
\leqslant & \frac{17}{2 \sqrt{2}} A^{7}\left|\eta_{2}-\eta_{1}\right| .
\end{aligned}
$$

Moreover,

$$
\begin{aligned}
\tilde{b}\left(t, \eta_{1}\right)-\tilde{b}\left(t, \eta_{2}\right) & \leqslant \int_{\eta_{1}}^{\eta_{2}} \tilde{B}_{1}(t, s) d s+\int_{\eta_{1}}^{\eta_{2}} \tilde{B}_{2}(t, s) d s \\
& \leqslant \mathcal{O}(1) A^{7}\left|\eta_{2}-\eta_{1}\right|,
\end{aligned}
$$

where $\mathcal{O}(1)$ denotes some constant, which only depends on $A$ and which remains bounded as $A \rightarrow 0$.

Finally combining both cases yields that there exists a constant $\mathcal{O}(1)$, which only depends on $A$ and which remains bounded as $A \rightarrow 0$, such that

$$
\left|\tilde{b}\left(t, \eta_{2}\right)-\tilde{b}\left(t, \eta_{1}\right)\right| \leqslant \mathcal{O}(1) A^{7}\left|\eta_{2}-\eta_{1}\right|
$$

and subsequently

$$
\left|\tilde{a}\left(t, \eta_{2}\right)-\tilde{a}\left(t, \eta_{1}\right)\right| \leqslant \mathcal{O}(1) A^{7}\left|\eta_{2}-\eta_{1}\right| .
$$

This proves that the derivative exists for almost every $\eta$ and is bounded by (A.9).

\section{LEMMA A.7. The function}

$$
\eta \mapsto \min _{k}\left(\int_{0}^{\eta} \min _{j}\left(e^{-\frac{1}{a}\left(\tilde{\mathcal{Y}}_{j}(t, \eta)-\tilde{\mathcal{Y}}_{j}(t, \theta)\right)}\right) \min _{j}\left(\tilde{\mathcal{D}}_{j}\right) \min _{j}\left(\tilde{\mathcal{U}}_{j}^{+}\right) \tilde{\mathcal{Y}}_{k, \eta}(t, \theta) d \theta\right)
$$

is Lipschitz continuous with uniformly bounded Lipschitz constant and thus differentiable almost everywhere. The derivative satisfies,

$$
\left|\frac{d}{d \eta} \min _{k}\left(\int_{0}^{\eta} \min _{j}\left(e^{-\frac{1}{a}\left(\tilde{\mathcal{Y}}_{j}(t, \eta)-\tilde{\mathcal{Y}}_{j}(t, \theta)\right)}\right) \min _{j}\left(\tilde{\mathcal{D}}_{j}\right) \min _{j}\left(\tilde{\mathcal{U}}_{j}^{+}\right) \tilde{\mathcal{Y}}_{k, \eta}(t, \theta) d \theta\right)\right| \leqslant \mathcal{O}(1) A^{8} .
$$


Proof. We present the following argument. Introduce

$$
\begin{aligned}
& \bar{a}(t, \eta)=\min _{k}\left(\int_{0}^{\eta} \min _{j}\left(e^{-\frac{1}{a}\left(\tilde{\mathcal{Y}}_{j}(t, \eta)-\tilde{\mathcal{Y}}_{j}(t, \theta)\right)}\right) \min _{j}\left(\tilde{\mathcal{D}}_{j}\right) \min _{j}\left(\tilde{\mathcal{U}}_{j}^{+}\right) \tilde{\mathcal{Y}}_{k, \eta}(t, \theta) d \theta\right), \\
& \bar{b}(t, \eta)=\int_{0}^{\eta} \min _{j}\left(e^{-\frac{1}{a}\left(\tilde{\mathcal{Y}}_{j}(t, \eta)-\tilde{\mathcal{Y}}_{j}(t, \theta)\right)}\right) \min _{j}\left(\tilde{\mathcal{D}}_{j}\right) \min _{j}\left(\tilde{\mathcal{U}}_{j}^{+}\right) \tilde{\mathcal{Y}}_{1, \eta}(t, \theta) d \theta, \\
& \bar{c}(t, \eta)=\int_{0}^{\eta} \min _{j}\left(e^{-\frac{1}{a}\left(\tilde{\mathcal{Y}}_{j}(t, \eta)-\tilde{\mathcal{Y}}_{j}(t, \theta)\right)}\right) \min _{j}\left(\tilde{\mathcal{D}}_{j}\right) \min _{j}\left(\tilde{\mathcal{U}}_{j}^{+}\right) \tilde{\mathcal{Y}}_{2, \eta}(t, \theta) d \theta .
\end{aligned}
$$

Thus

$$
\bar{a}(t, \eta)=\min (\bar{b}, \bar{c})(t, \eta) .
$$

We have to show that $\bar{a}(t, \cdot)$ is Lipschitz continuous with a Lipschitz constant, which only depends on $A$. Clearly, we have that

$$
\left|\bar{a}\left(t, \eta_{1}\right)-\bar{a}\left(t, \eta_{2}\right)\right| \leqslant \max \left(\left|\bar{b}\left(t, \eta_{1}\right)-\bar{b}\left(t, \eta_{2}\right)\right|,\left|\bar{c}\left(t, \eta_{1}\right)-\bar{c}\left(t, \eta_{2}\right)\right|\right),
$$

and it suffices to show that both $\bar{b}(t, \cdot)$ and $\bar{c}(t, \cdot)$ are Lipschitz continuous with a Lipschitz constant, which only depends on $A$. We are only going to establish the Lipschitz continuity for $\bar{b}$ since the argument for $\bar{c}$ follows the same lines. Let $0 \leqslant \eta_{1}<\eta_{2} \leqslant 1$. Then we have to consider two cases:

$$
0 \leqslant \bar{b}\left(t, \eta_{1}\right)-\bar{b}\left(t, \eta_{2}\right) \quad \text { and } \quad 0 \leqslant \bar{b}\left(t, \eta_{2}\right)-\bar{b}\left(t, \eta_{1}\right) .
$$

(i): $0 \leqslant \bar{b}\left(t, \eta_{2}\right)-\bar{b}\left(t, \eta_{1}\right):$ By definition, we have

$$
\begin{aligned}
\bar{b}(t, & \left.\eta_{2}\right)-\bar{b}\left(t, \eta_{1}\right) \\
= & \int_{0}^{\eta_{2}} \min _{j}\left(e^{-\frac{1}{a}\left(\tilde{\mathcal{Y}}_{j}\left(t, \eta_{2}\right)-\tilde{\mathcal{Y}}_{j}(t, \theta)\right)}\right) \min _{j}\left(\tilde{\mathcal{D}}_{j}\right) \min _{j}\left(\tilde{\mathcal{U}}_{j}^{+}\right) \tilde{\mathcal{Y}}_{1, \eta}(t, \theta) d \theta \\
& -\int_{0}^{\eta_{1}} \min _{j}\left(e^{-\frac{1}{a}\left(\tilde{\mathcal{Y}}_{j}\left(t, \eta_{1}\right)-\tilde{\mathcal{Y}}_{j}(t, \theta)\right)}\right) \min _{j}\left(\tilde{\mathcal{D}}_{j}\right) \min _{j}\left(\tilde{\mathcal{U}}_{j}^{+}\right) \tilde{\mathcal{Y}}_{1, \eta}(t, \theta) d \theta \\
= & \int_{\eta_{1}}^{\eta_{2}} \min _{j}\left(e^{-\frac{1}{a}\left(\tilde{\mathcal{Y}}_{j}\left(t, \eta_{2}\right)-\tilde{\mathcal{Y}}_{j}(t, \theta)\right)}\right) \\
& \times \min _{j}\left(\tilde{\mathcal{D}}_{j}\right) \min _{j}\left(\tilde{\mathcal{U}}_{j}^{+}\right) \tilde{\mathcal{Y}}_{1, \eta}(t, \theta) d \theta \\
& +\int_{0}^{\eta_{1}}\left(\min _{j}\left(e^{-\frac{1}{a}\left(\tilde{\mathcal{Y}}_{j}\left(t, \eta_{2}\right)-\tilde{\mathcal{Y}}_{j}(t, \theta)\right)}\right)\right. \\
& \left.-\min _{j}\left(e^{-\frac{1}{a}\left(\tilde{\mathcal{Y}}_{j}\left(t, \eta_{1}\right)-\tilde{\mathcal{Y}}_{j}(t, \theta)\right)}\right)\right) \\
& \times \min _{j}\left(\tilde{\mathcal{D}}_{j}\right) \min _{j}\left(\tilde{\mathcal{U}}_{j}^{+}\right) \tilde{\mathcal{Y}}_{1, \eta}(t, \theta) d \theta
\end{aligned}
$$




$$
\begin{aligned}
\leqslant & \int_{\eta_{1}}^{\eta_{2}} \min _{j}\left(e^{-\frac{1}{a}\left(\tilde{\mathcal{Y}}_{j}\left(t, \eta_{2}\right)-\tilde{\mathcal{Y}}_{j}(t, \theta)\right)}\right) \\
& \times \min _{j}\left(\tilde{\mathcal{D}}_{j}\right) \min _{j}\left(\tilde{\mathcal{U}}_{j}^{+}\right) \tilde{\mathcal{Y}}_{1, \eta}(t, \theta) d \theta,
\end{aligned}
$$

where we used in the last step that $0 \leqslant \min _{j}\left(\tilde{\mathcal{D}}_{j}\right) \min _{j}\left(\tilde{\mathcal{U}}_{j}^{+}\right) \tilde{\mathcal{Y}}_{1, \eta}(t, \eta)$ and that $\tilde{\mathcal{Y}}_{i}(t, \eta)$ is increasing, which implies

$$
\min _{j}\left(e^{-\frac{1}{a}\left(\tilde{\mathcal{Y}}_{j}\left(t, \eta_{1}\right)-\tilde{\mathcal{Y}}_{j}(t, \theta)\right)}\right) \geqslant \min _{j}\left(e^{-\frac{1}{a}\left(\tilde{\mathcal{Y}}_{j}\left(t, \eta_{2}\right)-\tilde{\mathcal{Y}}_{j}(t, \theta)\right)}\right) .
$$

Moreover, note that $\min _{j}\left(e^{-\frac{1}{a}\left(\tilde{\mathcal{Y}}_{j}\left(t, \eta_{2}\right)-\tilde{\mathcal{Y}}_{j}(t, \theta)\right)}\right) \leqslant 1$ for $0 \leqslant \eta_{1} \leqslant \theta \leqslant \eta_{2}$ and that

$$
0 \leqslant \min _{j}\left(\tilde{\mathcal{D}}_{j}\right) \min _{j}\left(\tilde{\mathcal{U}}_{j}^{+}\right) \tilde{\mathcal{Y}}_{1, \eta}(t, \theta) \leqslant \frac{1}{\sqrt{2}} A_{1}^{8} \leqslant \frac{1}{\sqrt{2}} A^{8}
$$

by (4.15b), (4.15e), and (4.15n). Thus

$$
0 \leqslant \bar{b}\left(t, \eta_{2}\right)-\bar{b}\left(t, \eta_{1}\right) \leqslant \frac{1}{\sqrt{2}} A^{8}\left|\eta_{2}-\eta_{1}\right|
$$

(ii) $0 \leqslant \bar{b}\left(t, \eta_{1}\right)-\bar{b}\left(t, \eta_{2}\right)$ : By definition, we have

$$
\begin{aligned}
0 \leqslant & \bar{b}\left(t, \eta_{1}\right)-\bar{b}\left(t, \eta_{2}\right) \\
= & \int_{0}^{\eta_{1}} \min _{j}\left(e^{-\frac{1}{a}\left(\tilde{\mathcal{Y}}_{j}\left(t, \eta_{1}\right)-\tilde{\mathcal{Y}}_{j}(t, \theta)\right)}\right) \min _{j}\left(\tilde{\mathcal{D}}_{j}\right) \min _{j}\left(\tilde{\mathcal{U}}_{j}^{+}\right) \tilde{\mathcal{Y}}_{1, \eta}(t, \theta) d \theta \\
& -\int_{0}^{\eta_{2}} \min _{j}\left(e^{-\frac{1}{a}\left(\tilde{\mathcal{Y}}_{j}\left(t, \eta_{2}\right)-\tilde{\mathcal{Y}}_{j}(t, \theta)\right)}\right) \min _{j}\left(\tilde{\mathcal{D}}_{j}\right) \min _{j}\left(\tilde{\mathcal{U}}_{j}^{+}\right) \tilde{\mathcal{Y}}_{1, \eta}(t, \theta) d \theta \\
= & \int_{0}^{\eta_{1}}\left(\min _{j}\left(e^{-\frac{1}{a}\left(\tilde{\mathcal{Y}}_{j}\left(t, \eta_{1}\right)-\tilde{\mathcal{Y}}_{j}(t, \theta)\right)}\right)-\min _{j}\left(e^{-\frac{1}{a}\left(\tilde{\mathcal{Y}}_{j}\left(t, \eta_{2}\right)-\tilde{\mathcal{Y}}_{j}(t, \theta)\right)}\right)\right) \\
& \times \min _{j}\left(\tilde{\mathcal{D}}_{j}\right) \min _{j}\left(\tilde{\mathcal{U}}_{j}^{+}\right) \tilde{\mathcal{Y}}_{1, \eta}(t, \theta) d \theta \\
& -\int_{\eta_{1}}^{\eta_{2}} \min _{j}\left(e^{-\frac{1}{a}\left(\tilde{\mathcal{Y}}_{j}\left(t, \eta_{2}\right)-\tilde{\mathcal{Y}}_{j}(t, \theta)\right)}\right) \min _{j}\left(\tilde{\mathcal{D}}_{j}\right) \min _{j}\left(\tilde{\mathcal{U}}_{j}^{+}\right) \tilde{\mathcal{Y}}_{1, \eta}(t, \theta) d \theta \\
\leqslant & \int_{0}^{\eta_{1}}\left(\min _{j}\left(e^{-\frac{1}{a}\left(\tilde{\mathcal{Y}}_{j}\left(t, \eta_{1}\right)-\tilde{\mathcal{Y}}_{j}(t, \theta)\right)}\right)-\min _{j}\left(e^{-\frac{1}{a}\left(\tilde{\mathcal{Y}}_{j}\left(t, \eta_{2}\right)-\tilde{\mathcal{Y}}_{j}(t, \theta)\right)}\right)\right) \\
& \times \min _{j}\left(\tilde{\mathcal{D}}_{j}\right) \min _{j}\left(\tilde{\mathcal{U}}_{j}^{+}\right) \tilde{\mathcal{Y}}_{1, \eta}(t, \theta) d \theta .
\end{aligned}
$$


Now we have to be much more careful than before. Namely, we have (as before)

$$
\begin{aligned}
0 & \leqslant \min _{j}\left(e^{-\frac{1}{a}\left(\tilde{\mathcal{Y}}_{j}\left(t, \eta_{1}\right)-\tilde{\mathcal{Y}}_{j}(t, \theta)\right)}\right)-\min _{j}\left(e^{-\frac{1}{a}\left(\tilde{\mathcal{Y}}_{j}\left(t, \eta_{2}\right)-\tilde{\mathcal{Y}}_{j}(t, \theta)\right)}\right) \\
& \leqslant \frac{1}{a} \int_{\eta_{1}}^{\eta_{2}}\left(e^{-\frac{1}{a}\left(\tilde{\mathcal{Y}}_{1}(t, s)-\tilde{\mathcal{Y}}_{1}(t, \theta)\right)} \tilde{\mathcal{Y}}_{1, \eta}(t, s)+e^{-\frac{1}{a}\left(\tilde{\mathcal{Y}}_{2}(t, s)-\tilde{\mathcal{Y}}_{2}(t, \theta)\right)} \tilde{\mathcal{Y}}_{2, \eta}(t, s)\right) d s .
\end{aligned}
$$

Hence

$$
\begin{aligned}
& \bar{b}\left(t, \eta_{1}\right)-\bar{b}\left(t, \eta_{2}\right) \\
& \leqslant \int_{0}^{\eta_{1}}\left(\min _{j}\left(e^{-\frac{1}{a}\left(\tilde{\mathcal{Y}}_{j}\left(t, \eta_{1}\right)-\tilde{\mathcal{Y}}_{j}(t, \theta)\right)}\right)-\min _{j}\left(e^{-\frac{1}{a}\left(\tilde{\mathcal{Y}}_{j}\left(t, \eta_{2}\right)-\tilde{\mathcal{Y}}_{j}(t, \theta)\right)}\right)\right) \\
& \times \min _{j}\left(\tilde{\mathcal{D}}_{j}\right) \min _{j}\left(\tilde{\mathcal{U}}_{j}^{+}\right) \tilde{\mathcal{Y}}_{1, \eta}(t, \theta) d \theta \\
& \leqslant \frac{1}{a} \int_{0}^{\eta_{1}} \int_{\eta_{1}}^{\eta_{2}} e^{-\frac{1}{a}\left(\tilde{\mathcal{Y}}_{1}(t, s)-\tilde{\mathcal{Y}}_{1}(t, \theta)\right)} \tilde{\mathcal{Y}}_{1, \eta}(t, s) d s \min _{j}\left(\tilde{\mathcal{D}}_{j}\right) \min _{j}\left(\tilde{\mathcal{U}}_{j}^{+}\right) \tilde{\mathcal{Y}}_{1, \eta}(t, \theta) d \theta \\
& +\frac{1}{a} \int_{0}^{\eta_{1}} \int_{\eta_{1}}^{\eta_{2}} e^{-\frac{1}{a}\left(\tilde{\mathcal{Y}}_{2}(t, s)-\tilde{\mathcal{Y}}_{2}(t, \theta)\right)} \tilde{\mathcal{Y}}_{2, \eta}(t, s) d s \\
& \times \min _{j}\left(\tilde{\mathcal{D}}_{j}\right) \min _{j}\left(\tilde{\mathcal{U}}_{j}^{+}\right) \tilde{\mathcal{Y}}_{1, \eta}(t, \theta) d \theta \\
& =\frac{1}{a} \int_{\eta_{1}}^{\eta_{2}} \tilde{\mathcal{Y}}_{1, \eta}(t, s) \\
& \times\left(\int_{0}^{\eta_{1}} e^{-\frac{1}{a}\left(\tilde{\mathcal{Y}}_{1}(t, s)-\tilde{\mathcal{Y}}_{1}(t, \theta)\right)} \min _{j}\left(\tilde{\mathcal{D}}_{j}\right) \min _{j}\left(\tilde{\mathcal{U}}_{j}^{+}\right) \tilde{\mathcal{Y}}_{1, \eta}(t, \theta) d \theta\right) d s \\
& +\frac{1}{a} \int_{\eta_{1}}^{\eta_{2}} \tilde{\mathcal{Y}}_{2, \eta}(t, s) \\
& \times\left(\int_{0}^{\eta_{1}} e^{-\frac{1}{a}\left(\tilde{\mathcal{Y}}_{2}(t, s)-\tilde{\mathcal{Y}}_{2}(t, \theta)\right)} \min _{j}\left(\tilde{\mathcal{D}}_{j}\right) \min _{j}\left(\tilde{\mathcal{U}}_{j}^{+}\right) \tilde{\mathcal{Y}}_{1, \eta}(t, \theta) d \theta\right) d s \\
& \leqslant \frac{1}{a} \int_{\eta_{1}}^{\eta_{2}} \tilde{\mathcal{Y}}_{1, \eta}(t, s) \\
& \times\left(\int_{0}^{s} e^{-\frac{1}{a}\left(\tilde{\mathcal{Y}}_{1}(t, s)-\tilde{\mathcal{Y}}_{1}(t, \theta)\right)} \min _{j}\left(\tilde{\mathcal{D}}_{j}\right) \min _{j}\left(\tilde{\mathcal{U}}_{j}^{+}\right) \tilde{\mathcal{Y}}_{1, \eta}(t, \theta) d \theta\right) d s \\
& +\frac{1}{a} \int_{\eta_{1}}^{\eta_{2}} \tilde{\mathcal{Y}}_{2, \eta}(t, s) \\
& \times\left(\int_{0}^{s} e^{-\frac{1}{a}\left(\tilde{\mathcal{Y}}_{2}(t, s)-\tilde{\mathcal{Y}}_{2}(t, \theta)\right)} \min _{j}\left(\tilde{\mathcal{D}}_{j}\right) \min _{j}\left(\tilde{\mathcal{U}}_{j}^{+}\right) \tilde{\mathcal{Y}}_{1, \eta}(t, \theta) d \theta\right) d s \\
& =\int_{\eta_{1}}^{\eta_{2}} \bar{B}_{1}(t, s) d s+\int_{\eta_{1}}^{\eta_{2}} \bar{B}_{2}(t, s) d s
\end{aligned}
$$


As far as $\bar{B}_{1}(t, s)$ is concerned, we have

$$
\begin{aligned}
\int_{\eta_{1}}^{\eta_{2}} & \bar{B}_{1}(t, s) d s \\
= & \frac{1}{a} \int_{\eta_{1}}^{\eta_{2}}\left(\int_{0}^{s} e^{-\frac{1}{a}\left(\tilde{\mathcal{Y}}_{1}(t, s)-\tilde{\mathcal{Y}}_{1}(t, \theta)\right)} \min _{j}\left(\tilde{\mathcal{D}}_{j}\right) \min _{j}\left(\tilde{\mathcal{U}}_{j}^{+}\right) \tilde{\mathcal{Y}}_{1, \eta}(t, \theta) d \theta\right) \tilde{\mathcal{Y}}_{1, \eta}(t, s) d s \\
\leqslant & \sqrt{2} A \int_{\eta_{1}}^{\eta_{2}}\left(\int_{0}^{s} e^{-\frac{1}{A_{1}}\left(\tilde{\mathcal{Y}}_{1}(t, s)-\tilde{\mathcal{Y}}_{1}(t, \theta)\right)} \tilde{\mathcal{P}}_{1}^{3 / 4} \tilde{\mathcal{U}}_{1}^{+} \tilde{\mathcal{Y}}_{1, \eta}(t, \theta) d \theta\right) \tilde{\mathcal{Y}}_{1, \eta}(t, s) d s \\
\leqslant & \sqrt{2} A \int_{\eta_{1}}^{\eta_{2}}\left(\int_{0}^{s} e^{-\frac{1}{A_{1}}\left(\tilde{\mathcal{Y}}_{1}(t, s)-\tilde{\mathcal{Y}}_{1}(t, \theta)\right)} \tilde{\mathcal{P}}_{1}^{3 / 2} \tilde{\mathcal{Y}}_{1, \eta}(t, \theta) d \theta\right)^{1 / 2} \\
& \times\left(\int_{0}^{s} e^{-\frac{1}{A_{1}}\left(\tilde{\mathcal{Y}}_{1}(t, s)-\tilde{\mathcal{Y}}_{1}(t, \theta)\right)} \tilde{\mathcal{Y}}_{1}^{2} \tilde{\mathcal{Y}}_{1, \eta}(t, \theta) d \theta\right)^{1 / 2} \tilde{\mathcal{Y}}_{1, \eta}(t, s) d s \\
\leqslant & 6 A^{3} \int_{\eta_{1}}^{\eta_{2}} \tilde{\mathcal{P}}_{1} \tilde{\mathcal{Y}}_{1, \eta}(t, s) d s \\
\leqslant & 3 A^{8}\left|\eta_{2}-\eta_{1}\right|
\end{aligned}
$$

where we used (4.15n).

As far as $\bar{B}_{2}(t, s)$ is concerned, we have to be more careful. Therefore recall that we have (cf. (4.13)) that

$$
A_{2}^{5}=2 \tilde{\mathcal{P}}_{2} \tilde{\mathcal{Y}}_{2, \eta}(t, \eta)-\tilde{\mathcal{U}}_{2}^{2} \tilde{\mathcal{Y}}_{2, \eta}(t, \eta)+\tilde{\mathcal{H}}_{2, \eta}(t, \eta) \leqslant 2 \tilde{\mathcal{P}}_{2} \tilde{\mathcal{Y}}_{2, \eta}(t, \eta)+\tilde{\mathcal{H}}_{2, \eta}(t, \eta) .
$$

Therefore we can write

$$
\begin{aligned}
& \frac{1}{a} \int_{0}^{s} \mathrm{e}^{-\frac{1}{a}\left(\tilde{\mathcal{Y}}_{2}(t, s)-\tilde{\mathcal{Y}}_{2}(t, \theta)\right)} \min _{j}\left(\tilde{\mathcal{D}}_{j}\right) \min _{j}\left(\tilde{\mathcal{U}}_{j}^{+}\right) \tilde{\mathcal{Y}}_{1, \eta}(t, \theta) d \theta \\
& \quad \leqslant \frac{2 \sqrt{2} A}{a} \int_{0}^{s} e^{-\frac{1}{A_{2}}\left(\tilde{\mathcal{Y}}_{2}(t, s)-\tilde{\mathcal{Y}}_{2}(t, \theta)\right)} \min _{j}\left(\tilde{\mathcal{P}}_{j}\right)^{3 / 2} \tilde{\mathcal{Y}}_{1, \eta}(t, \theta) d \theta \\
& \quad \leqslant 2 A \int_{0}^{s} e^{-\frac{1}{A_{2}}\left(\tilde{\mathcal{Y}}_{2}(t, s)-\tilde{\mathcal{Y}}_{2}(t, \theta)\right)} \tilde{\mathcal{P}}_{2}^{1 / 4} \tilde{\mathcal{P}}_{1} \tilde{\mathcal{Y}}_{1, \eta}(t, \theta) d \theta \\
& \quad \leqslant A \frac{A_{1}^{5}}{A_{2}^{5}} \int_{0}^{s} e^{-\frac{1}{A_{2}}\left(\tilde{\mathcal{Y}}_{2}(t, s)-\tilde{\mathcal{Y}}_{2}(t, \theta)\right)} \tilde{\mathcal{P}}_{2}^{1 / 4}\left(2 \tilde{\mathcal{P}}_{2} \tilde{\mathcal{Y}}_{2, \eta}+\tilde{\mathcal{H}}_{2, \eta}\right)(t, \theta) d \theta \\
& \quad \leqslant A \frac{A_{1}^{5}}{A_{2}^{5}} \int_{0}^{s} e^{-\frac{1}{A_{2}}\left(\tilde{\mathcal{Y}}_{2}(t, s)-\tilde{\mathcal{Y}}_{2}(t, \theta)\right)}\left(2 \tilde{\mathcal{P}}_{2}^{5 / 4} \tilde{\mathcal{Y}}_{2, \eta}+\frac{A_{2}}{\sqrt{2}} \tilde{\mathcal{H}}_{2, \eta}\right)(t, \theta) d \theta \\
& \quad \leqslant A \frac{A_{1}^{5}}{A_{2}^{5}}\left(2 \sqrt{2} A_{2}^{2} \tilde{\mathcal{P}}_{2}(t, s)+2 \int_{0}^{s} e^{-\frac{1}{A_{2}}\left(\tilde{\mathcal{Y}}_{2}(t, s)-\tilde{\mathcal{Y}}_{2}(t, \theta)\right)} \tilde{\mathcal{P}}_{2}^{5 / 4} \tilde{\mathcal{Y}}_{2, \eta}(t, \theta) d \theta\right) .
\end{aligned}
$$


Note that the integral term can be bounded by $\mathcal{O}(1) \tilde{\mathcal{P}}_{2}(t, s)$ since

$$
\int_{0}^{s} e^{-\frac{1}{a}\left(\hat{\mathcal{Y}}_{2}(t, s)-\tilde{\mathcal{Y}}_{2}(t, \theta)\right)} \tilde{\mathcal{P}}_{2}^{5 / 4} \tilde{\mathcal{Y}}_{2, \eta}(t, \theta) d \theta \leqslant \frac{15}{\sqrt{2}} A_{2}^{2} \tilde{\mathcal{P}}_{2}(t, s)
$$

by (4.16k). We end up with

$$
\begin{aligned}
& \int_{\eta_{1}}^{\eta_{2}} \bar{B}_{2}(t, s) d s \\
& \quad=\frac{1}{a} \int_{\eta_{1}}^{\eta_{2}} \tilde{\mathcal{Y}}_{2, \eta}(t, s)\left(\int_{0}^{s} e^{-\frac{1}{a}\left(\tilde{\mathcal{Y}}_{2}(t, s)-\tilde{\mathcal{Y}}_{2}(t, \theta)\right)} \min _{j}\left(\tilde{\mathcal{D}}_{j}\right) \min _{j}\left(\tilde{\mathcal{U}}_{j}^{+}\right) \tilde{\mathcal{Y}}_{1, \eta}(t, \theta) d \theta\right) d s \\
& \quad \leqslant \frac{17 \sqrt{2} A^{6}}{A_{2}^{3}} \int_{\eta_{1}}^{\eta_{2}} \tilde{\mathcal{P}}_{2} \tilde{\mathcal{Y}}_{2, \eta}(t, s) d s \\
& \quad \leqslant \frac{17 A^{8}}{\sqrt{2}}\left|\eta_{2}-\eta_{1}\right| .
\end{aligned}
$$

Moreover,

$$
\begin{aligned}
\bar{b}\left(t, \eta_{1}\right)-\bar{b}\left(t, \eta_{2}\right) & \leqslant \int_{\eta_{1}}^{\eta_{2}} \bar{B}_{1}(t, s) d s+\int_{\eta_{1}}^{\eta_{2}} \bar{B}_{2}(t, s) d s \\
& \leqslant \mathcal{O}(1) A^{8}\left|\eta_{2}-\eta_{1}\right|,
\end{aligned}
$$

where $\mathcal{O}(1)$ denotes some constant, which only depends on $A$ and which remains bounded as $A \rightarrow 0$.

Finally, combining both cases yields that there exists a constant $\mathcal{O}(1)$, which only depends on $A$ and which remains bounded as $A \rightarrow 0$, such that

$$
\left|\bar{b}\left(t, \eta_{2}\right)-\bar{b}\left(t, \eta_{1}\right)\right| \leqslant \mathcal{O}(1) A^{8}\left|\eta_{2}-\eta_{1}\right|
$$

and subsequently

$$
\left|\bar{a}\left(t, \eta_{2}\right)-\bar{a}\left(t, \eta_{1}\right)\right| \leqslant \mathcal{O}(1) A^{8}\left|\eta_{2}-\eta_{1}\right|
$$

This proves that the derivative exists for almost every $\eta$ and is bounded by (A.10).

LEMMA A.8. The function

$$
\eta \mapsto \min _{k}\left[\int_{0}^{\eta} \min _{j}\left(e^{-\frac{1}{a}\left(\tilde{\mathcal{Y}}_{j}(t, \eta)-\tilde{\mathcal{Y}}_{j}(t, \theta)\right)}\right) \min _{j}\left(\tilde{\mathcal{U}}_{j}^{+}\right)^{3} \tilde{\mathcal{Y}}_{k, \eta}(t, \theta) d \theta\right]
$$


is Lipschitz continuous with a uniformly bounded Lipschitz constant and thus differentiable almost everywhere. The derivative satisfies

$$
\left|\frac{d}{d \eta} \min _{k}\left[\int_{0}^{\eta} \min _{j}\left(e^{-\left(\tilde{\mathcal{Y}}_{j}(t, \eta)-\tilde{\mathcal{Y}}_{j}(t, \theta)\right)}\right) \min _{j}\left(\tilde{\mathcal{U}}_{j}^{+}\right)^{3} \tilde{\mathcal{Y}}_{k, \eta}(t, \theta) d \theta\right]\right| \leqslant \mathcal{O}(1) A^{6},
$$

where $\mathcal{O}(1)$ denotes a constant, which only depends on $A$ and which remains bounded as $A \rightarrow 0$.

Proof. To that end, we present the following argument. Introduce

$$
\begin{aligned}
& a(t, \eta)=\min _{k}\left[\int_{0}^{\eta} \min _{j}\left(e^{-\frac{1}{a}\left(\tilde{\mathcal{Y}}_{j}(t, \eta)-\tilde{\mathcal{Y}}_{j}(t, \theta)\right)}\right) \min _{j}\left(\tilde{\mathcal{U}}_{j}^{+}\right)^{3} \tilde{\mathcal{Y}}_{k, \eta}(t, \theta) d \theta\right], \\
& b(t, \eta)=\int_{0}^{\eta} \min _{j}\left(e^{-\frac{1}{a}\left(\tilde{\mathcal{Y}}_{j}(t, \eta)-\tilde{\mathcal{Y}}_{j}(t, \theta)\right)}\right) \min _{j}\left(\tilde{\mathcal{U}}_{j}^{+}\right)^{3} \tilde{\mathcal{Y}}_{1, \eta}(t, \theta) d \theta, \\
& c(t, \eta)=\int_{0}^{\eta} \min _{j}\left(e^{-\frac{1}{a}\left(\tilde{\mathcal{Y}}_{j}(t, \eta)-\tilde{\mathcal{Y}}_{j}(t, \theta)\right)}\right) \min _{j}\left(\tilde{\mathcal{U}}_{j}^{+}\right)^{3} \tilde{\mathcal{Y}}_{2, \eta}(t, \theta) d \theta .
\end{aligned}
$$

Thus

$$
a(t, \eta)=\min (b, c)(t, \eta) .
$$

Then we have to show that $a(t, \cdot)$ is Lipschitz continuous with a Lipschitz constant, which only depends on $A$ and hence on $C$. Clearly, we have that

$$
\left|a\left(t, \eta_{1}\right)-a\left(t, \eta_{2}\right)\right| \leqslant \max \left(\left|b\left(t, \eta_{1}\right)-b\left(t, \eta_{2}\right)\right|,\left|c\left(t, \eta_{1}\right)-c\left(t, \eta_{2}\right)\right|\right),
$$

and it suffices to show that both $b(t, \cdot)$ and $c(t, \cdot)$ are Lipschitz continuous with a Lipschitz constant, which only depends on $A$. We are only going to establish the Lipschitz continuity for $b$, since the argument for $c$ follows the same lines. Let $0 \leqslant \eta_{1}<\eta_{2} \leqslant 1$. Then we have to consider two cases:

$$
0 \leqslant b\left(t, \eta_{1}\right)-b\left(t, \eta_{2}\right) \quad \text { and } \quad 0 \leqslant b\left(t, \eta_{2}\right)-b\left(t, \eta_{1}\right) .
$$

(i): $0 \leqslant b\left(t, \eta_{2}\right)-b\left(t, \eta_{1}\right)$ : By definition, we have

$$
\begin{aligned}
b(t, & \left.\eta_{2}\right)-b\left(t, \eta_{1}\right) \\
= & \int_{0}^{\eta_{2}} \min _{j}\left(e^{-\frac{1}{a}\left(\tilde{\mathcal{Y}}_{j}\left(t, \eta_{2}\right)-\tilde{\mathcal{Y}}_{j}(t, \theta)\right)}\right) \min _{j}\left(\tilde{\mathcal{U}}_{j}^{+}\right)^{3} \tilde{\mathcal{Y}}_{1, \eta}(t, \theta) d \theta \\
& -\int_{0}^{\eta_{1}} \min _{j}\left(e^{-\frac{1}{a}\left(\tilde{\mathcal{Y}}_{j}\left(t, \eta_{1}\right)-\tilde{\mathcal{Y}}_{j}(t, \theta)\right)}\right) \min _{j}\left(\tilde{\mathcal{U}}_{j}^{+}\right)^{3} \tilde{\mathcal{Y}}_{1, \eta}(t, \theta) d \theta \\
= & \int_{\eta_{1}}^{\eta_{2}} \min _{j}\left(e^{-\frac{1}{a}\left(\tilde{\mathcal{Y}}_{j}\left(t, \eta_{2}\right)-\tilde{\mathcal{Y}}_{j}(t, \theta)\right)}\right) \min _{j}\left(\tilde{\mathcal{U}}_{j}^{+}\right)^{3} \tilde{\mathcal{Y}}_{1, \eta}(t, \theta) d \theta
\end{aligned}
$$




$$
\begin{aligned}
& +\int_{0}^{\eta_{1}}\left(\min _{j}\left(e^{-\frac{1}{a}\left(\tilde{\mathcal{Y}}_{j}\left(t, \eta_{2}\right)-\tilde{\mathcal{Y}}_{j}(t, \theta)\right)}\right)\right. \\
& \left.-\min _{j}\left(e^{-\frac{1}{a}\left(\tilde{\mathcal{Y}}_{j}\left(t, \eta_{1}\right)-\tilde{\mathcal{Y}}_{j}(t, \theta)\right)}\right)\right) \\
& \times \min _{j}\left(\tilde{\mathcal{U}}_{j}^{+}\right)^{3} \tilde{\mathcal{Y}}_{1, \eta}(t, \theta) d \theta \\
& \leqslant \int_{\eta_{1}}^{\eta_{2}} \min _{j}\left(e^{-\frac{1}{a}\left(\tilde{\mathcal{Y}}_{j}\left(t, \eta_{2}\right)-\tilde{\mathcal{Y}}_{j}(t, \theta)\right)}\right) \min _{j}\left(\tilde{\mathcal{U}}_{j}^{+}\right)^{3} \tilde{\mathcal{Y}}_{1, \eta}(t, \theta) d \theta
\end{aligned}
$$

where we used in the last step that $0 \leqslant \min _{j}\left(\tilde{\mathcal{U}}_{j}^{+}\right)^{3} \tilde{\mathcal{Y}}_{1, \eta}(t, \eta)$ and that $\tilde{\mathcal{Y}}_{1}(t, \cdot)$ is increasing, which implies

$$
\begin{aligned}
\min _{j}\left(e^{-\frac{1}{a}\left(\tilde{\mathcal{Y}}_{j}\left(t, \eta_{1}\right)-\tilde{\mathcal{Y}}_{j}(t, \theta)\right)}\right) \\
\quad=\min _{j}\left(e^{-\frac{1}{a}\left(\tilde{\mathcal{Y}}_{j}\left(t, \eta_{1}\right)-\tilde{\mathcal{Y}}_{j}\left(t, \eta_{2}\right)+\tilde{\mathcal{Y}}_{j}\left(t, \eta_{2}\right)-\tilde{\mathcal{Y}}_{j}(t, \theta)\right)}\right) \\
\quad \geqslant \min _{j}\left(e^{-\frac{1}{a}\left(\tilde{\mathcal{Y}}_{j}\left(t, \eta_{2}\right)-\tilde{\mathcal{Y}}_{j}(t, \theta)\right)}\right) \min _{j}\left(e^{-\frac{1}{a}\left(\tilde{\mathcal{Y}}_{j}\left(t, \eta_{1}\right)-\tilde{\mathcal{Y}}_{j}\left(t, \eta_{2}\right)\right)}\right) \\
\geqslant \min _{j}\left(e^{-\frac{1}{a}\left(\tilde{\mathcal{Y}}_{j}\left(t, \eta_{2}\right)-\tilde{\mathcal{Y}}_{j}(t, \theta)\right)}\right) .
\end{aligned}
$$

Moreover, note that $\min _{j}\left(e^{-\frac{1}{a}\left(\tilde{\mathcal{Y}}_{j}\left(t, \eta_{2}\right)-\tilde{\mathcal{Y}}_{j}(t, \theta)\right)}\right) \leqslant 1$ since $\eta_{1} \leqslant \theta \leqslant \eta_{2}$, and that

$$
0 \leqslant \min _{j}\left(\tilde{\mathcal{U}}_{j}^{+}\right)^{3} \tilde{\mathcal{Y}}_{1, \eta}(t, \eta) \leqslant\left|\tilde{\mathcal{U}}_{1}^{3}\right| \tilde{\mathcal{Y}}_{1, \eta}(t, \eta) \leqslant \frac{1}{\sqrt{2}} A_{1}^{7} \leqslant \frac{1}{\sqrt{2}} A^{7}
$$

by (4.15b) and (4.15g). Thus

$$
\begin{aligned}
0 & \leqslant b\left(t, \eta_{2}\right)-b\left(t, \eta_{1}\right) \\
& \leqslant \int_{\eta_{1}}^{\eta_{2}} \min _{j}\left(e^{-\frac{1}{a}\left(\tilde{\mathcal{Y}}_{j}\left(t, \eta_{2}\right)-\tilde{\mathcal{Y}}_{j}(t, \theta)\right)}\right) \min _{j}\left(\tilde{\mathcal{U}}_{j}^{+}\right)^{3} \tilde{\mathcal{Y}}_{1, \eta}(t, \theta) d \theta \\
& \leqslant \frac{1}{\sqrt{2}} A^{7}\left|\eta_{2}-\eta_{1}\right| .
\end{aligned}
$$

(ii): $0 \leqslant b\left(t, \eta_{1}\right)-b\left(t, \eta_{2}\right):$ By definition, we have

$$
\begin{aligned}
b(t, & \left.\eta_{1}\right)-b\left(t, \eta_{2}\right) \\
= & \int_{0}^{\eta_{1}} \min _{j}\left(e^{-\frac{1}{a}\left(\tilde{\mathcal{Y}}_{j}\left(t, \eta_{1}\right)-\tilde{\mathcal{Y}}_{j}(t, \theta)\right)}\right) \min _{j}\left(\tilde{\mathcal{U}}_{j}^{+}\right)^{3} \tilde{\mathcal{Y}}_{1, \eta}(t, \theta) d \theta \\
& -\int_{0}^{\eta_{2}} \min _{j}\left(e^{-\frac{1}{a}\left(\tilde{\mathcal{Y}}_{j}\left(t, \eta_{2}\right)-\tilde{\mathcal{Y}}_{j}(t, \theta)\right)}\right) \min _{j}\left(\tilde{\mathcal{U}}_{j}^{+}\right)^{3} \tilde{\mathcal{Y}}_{1, \eta}(t, \theta) d \theta
\end{aligned}
$$




$$
\begin{aligned}
= & \int_{0}^{\eta_{1}}\left(\min _{j}\left(e^{-\frac{1}{a}\left(\tilde{\mathcal{Y}}_{j}\left(t, \eta_{1}\right)-\tilde{\mathcal{Y}}_{j}(t, \theta)\right)}\right)\right. \\
& \left.-\min _{j}\left(e^{-\frac{1}{a}\left(\tilde{\mathcal{Y}}_{j}\left(t, \eta_{2}\right)-\tilde{\mathcal{Y}}_{j}(t, \theta)\right)}\right)\right) \\
& \times \min _{j}\left(\tilde{\mathcal{U}}_{j}^{+}\right)^{3} \tilde{\mathcal{Y}}_{1, \eta}(t, \theta) d \theta \\
& -\int_{\eta_{1}}^{\eta_{2}} \min _{j}\left(e^{-\frac{1}{a}\left(\tilde{\mathcal{Y}}_{j}\left(t, \eta_{2}\right)-\tilde{\mathcal{Y}}_{j}(t, \theta)\right)}\right) \min _{j}\left(\tilde{\mathcal{U}}_{j}^{+}\right)^{3} \tilde{\mathcal{Y}}_{1, \eta}(t, \theta) d \theta \\
\leqslant & \int_{0}^{\eta_{1}}\left(\min _{j}\left(e^{-\frac{1}{a}\left(\tilde{\mathcal{Y}}_{j}\left(t, \eta_{1}\right)-\tilde{\mathcal{Y}}_{j}(t, \theta)\right)}\right)\right. \\
& \left.-\min _{j}\left(e^{-\frac{1}{a}\left(\tilde{\mathcal{Y}}_{j}\left(t, \eta_{2}\right)-\tilde{\mathcal{Y}}_{j}(t, \theta)\right)}\right)\right) \\
& \times \min _{j}\left(\tilde{\mathcal{U}}_{j}^{+}\right)^{3} \tilde{\mathcal{Y}}_{1, \eta}(t, \theta) d \theta .
\end{aligned}
$$

Now we have to be much more careful than before. Namely, if $\min _{j}\left(\tilde{\mathcal{Y}}_{j}(t, \theta)-\right.$ $\left.\tilde{\mathcal{Y}}_{j}\left(t, \eta_{2}\right)\right)=\tilde{\mathcal{Y}}_{i}(t, \theta)-\tilde{\mathcal{Y}}_{i}\left(t, \eta_{2}\right)$, we have

$$
\begin{aligned}
0 & \leqslant \min _{j}\left(e^{-\frac{1}{a}\left(\tilde{\mathcal{Y}}_{j}\left(t, \eta_{1}\right)-\tilde{\mathcal{Y}}_{j}(t, \theta)\right)}\right)-\min _{j}\left(e^{-\frac{1}{a}\left(\tilde{\mathcal{Y}}_{j}\left(t, \eta_{2}\right)-\tilde{\mathcal{Y}}_{j}(t, \theta)\right)}\right) \\
& =\min _{j}\left(e^{-\frac{1}{a}\left(\tilde{\mathcal{Y}}_{j}\left(t, \eta_{1}\right)-\tilde{\mathcal{Y}}_{j}(t, \theta)\right)}\right)-e^{\frac{1}{a}\left(\tilde{\mathcal{Y}}_{i}(t, \theta)-\tilde{\mathcal{Y}}_{i}\left(t, \eta_{2}\right)\right)} \\
& \leqslant e^{\frac{1}{a}\left(\tilde{\mathcal{Y}}_{i}(t, \theta)-\tilde{\mathcal{Y}}_{i}\left(t, \eta_{1}\right)\right)}-e^{\frac{1}{a}\left(\tilde{\mathcal{Y}}_{i}(t, \theta)-\tilde{\mathcal{Y}}_{i}\left(t, \eta_{2}\right)\right)} \\
& =\frac{1}{a} \int_{\eta_{1}}^{\eta_{2}} e^{\frac{1}{a}\left(\tilde{\mathcal{Y}}_{i}(t, \theta)-\tilde{\mathcal{Y}}_{i}(t, s)\right)} \tilde{\mathcal{Y}}_{i, \eta}(t, s) d s \\
& \leqslant \frac{1}{a} \int_{\eta_{1}}^{\eta_{2}}\left(e^{\frac{1}{a}\left(\tilde{\mathcal{Y}}_{1}(t, \theta)-\tilde{\mathcal{Y}}_{1}(t, s)\right)} \tilde{\mathcal{Y}}_{1, \eta}(t, s)+e^{\frac{1}{a}\left(\tilde{\mathcal{Y}}_{2}(t, \theta)-\tilde{\mathcal{Y}}_{2}(t, s)\right)} \tilde{\mathcal{Y}}_{2, \eta}(t, s)\right) d s .
\end{aligned}
$$

\section{Hence}

$$
\begin{aligned}
b(t, & \left.\eta_{1}\right)-b\left(t, \eta_{2}\right) \\
\leqslant & \int_{0}^{\eta_{1}}\left(\min _{j}\left(e^{-\frac{1}{a}\left(\tilde{\mathcal{Y}}_{j}\left(t, \eta_{1}\right)-\tilde{\mathcal{Y}}_{j}(t, \theta)\right)}\right)\right. \\
& \left.-\min _{j}\left(e^{-\frac{1}{a}\left(\tilde{\mathcal{Y}}_{j}\left(t, \eta_{2}\right)-\tilde{\mathcal{Y}}_{j}(t, \theta)\right)}\right)\right) \\
& \times \min _{j}\left(\tilde{\mathcal{U}}_{j}^{+}\right)^{3} \tilde{\mathcal{Y}}_{1, \eta}(t, \theta) d \theta \\
\leqslant & \frac{1}{a} \int_{0}^{\eta_{1}} \int_{\eta_{1}}^{\eta_{2}} e^{-\frac{1}{a}\left(\tilde{\mathcal{Y}}_{1}(t, s)-\tilde{\mathcal{Y}}_{1}(t, \theta)\right)} \tilde{\mathcal{Y}}_{1, \eta}(t, s) d s \min _{j}\left(\tilde{\mathcal{U}}_{j}^{+}\right)^{3} \tilde{\mathcal{Y}}_{1, \eta}(t, \theta) d \theta \\
& +\frac{1}{a} \int_{0}^{\eta_{1}} \int_{\eta_{1}}^{\eta_{2}} e^{-\frac{1}{a}\left(\tilde{\mathcal{Y}}_{2}(t, s)-\tilde{\mathcal{Y}}_{2}(t, \theta)\right)} \tilde{\mathcal{Y}}_{2, \eta}(t, s) d s \min _{j}\left(\tilde{\mathcal{U}}_{j}^{+}\right)^{3} \tilde{\mathcal{Y}}_{1, \eta}(t, \theta) d \theta
\end{aligned}
$$




$$
\begin{aligned}
= & \frac{1}{a} \int_{\eta_{1}}^{\eta_{2}} \tilde{\mathcal{Y}}_{1, \eta}(t, s)\left(\int_{0}^{\eta_{1}} e^{-\frac{1}{a}\left(\tilde{\mathcal{Y}}_{1}(t, s)-\tilde{\mathcal{Y}}_{1}(t, \theta)\right)} \min _{j}\left(\tilde{\mathcal{U}}_{j}^{+}\right)^{3} \tilde{\mathcal{Y}}_{1, \eta}(t, \theta) d \theta\right) d s \\
& +\frac{1}{a} \int_{\eta_{1}}^{\eta_{2}} \tilde{\mathcal{Y}}_{2, \eta}(t, s)\left(\int_{0}^{\eta_{1}} e^{-\frac{1}{a}\left(\tilde{\mathcal{Y}}_{2}(t, s)-\tilde{\mathcal{Y}}_{2}(t, \theta)\right)} \min _{j}\left(\tilde{\mathcal{U}}_{j}^{+}\right)^{3} \tilde{\mathcal{Y}}_{1, \eta}(t, \theta) d \theta\right) d s \\
\leqslant & \frac{1}{a} \int_{\eta_{1}}^{\eta_{2}} \tilde{\mathcal{Y}}_{1, \eta}(t, s)\left(\int_{0}^{s} e^{-\frac{1}{a}\left(\tilde{\mathcal{Y}}_{1}(t, s)-\tilde{\mathcal{Y}}_{1}(t, \theta)\right)} \min _{j}\left(\tilde{\mathcal{U}}_{j}^{+}\right)^{3} \tilde{\mathcal{Y}}_{1, \eta}(t, \theta) d \theta\right) d s \\
& +\frac{1}{a} \int_{\eta_{1}}^{\eta_{2}} \tilde{\mathcal{Y}}_{2, \eta}(t, s)\left(\int_{0}^{s} e^{-\frac{1}{a}\left(\tilde{\mathcal{Y}}_{2}(t, s)-\tilde{\mathcal{Y}}_{2}(t, \theta)\right)} \min _{j}\left(\tilde{\mathcal{U}}_{j}^{+}\right)^{3} \tilde{\mathcal{Y}}_{1, \eta}(t, \theta) d \theta\right) d s \\
= & \int_{\eta_{1}}^{\eta_{2}} B_{1}(t, s) d s+\int_{\eta_{1}}^{\eta_{2}} B_{2}(t, s) d s .
\end{aligned}
$$

As far as $B_{1}(t, s)$ is concerned, recall that

$$
\begin{aligned}
\tilde{\mathcal{P}}_{i}(t, s) & =\frac{1}{4 A_{i}} \int_{0}^{1} e^{-\frac{1}{A_{i}}\left|\tilde{\mathcal{Y}}_{i}(t, s)-\tilde{\mathcal{Y}}_{i}(t, \theta)\right|}\left(2\left(\tilde{\mathcal{U}}_{i}^{2}-\tilde{\mathcal{P}}_{i}\right) \tilde{\mathcal{Y}}_{i, \eta}(t, \theta)+A_{i}^{5}\right) d \theta \\
& =\frac{1}{4 A_{i}} \int_{0}^{1} e^{-\frac{1}{A_{i}}\left|\tilde{\mathcal{Y}}_{i}(t, s)-\tilde{\mathcal{Y}}_{i}(t, \theta)\right|}\left(\tilde{\mathcal{U}}_{i}^{2} \tilde{\mathcal{Y}}_{i, \eta}+\tilde{\mathcal{H}}_{i, \eta}\right)(t, \theta) d \theta,
\end{aligned}
$$

which implies that

$$
\begin{aligned}
& \int_{\eta_{1}}^{\eta_{2}} B_{1}(t, s) d s \\
& =\frac{1}{a} \int_{\eta_{1}}^{\eta_{2}} \tilde{\mathcal{Y}}_{1, \eta}(t, s)\left(\int_{0}^{s} e^{-\frac{1}{a}\left(\tilde{\mathcal{Y}}_{1}(t, s)-\tilde{\mathcal{Y}}_{1}(t, \theta)\right)} \min _{j}\left(\tilde{\mathcal{U}}_{j}^{+}\right)^{3} \tilde{\mathcal{Y}}_{1, \eta}(t, \theta) d \theta\right) d s \\
& \leqslant \frac{1}{a} \int_{\eta_{1}}^{\eta_{2}} \tilde{\mathcal{Y}}_{1, \eta}(t, s)\left(\int_{0}^{s} e^{-\frac{1}{a}\left(\tilde{\mathcal{Y}}_{1}(t, s)-\tilde{\mathcal{Y}}_{1}(t, \theta)\right)} \min _{j}\left(\tilde{\mathcal{U}}_{j}^{+}\right) \tilde{\mathcal{U}}_{1}^{2} \tilde{\mathcal{Y}}_{1, \eta}(t, \theta) d \theta\right) d s \\
& \leqslant \frac{a}{\sqrt{2}} \int_{\eta_{1}}^{\eta_{2}} \tilde{\mathcal{Y}}_{1, \eta}(t, s)\left(\int_{0}^{s} e^{-\frac{1}{a}\left(\tilde{\mathcal{Y}}_{1}(t, s)-\tilde{\mathcal{Y}}_{1}(t, \theta)\right)} \tilde{\mathcal{U}}_{1}^{2} \tilde{\mathcal{Y}}_{1, \eta}(t, \theta) d \theta\right) d s \\
& \leqslant \frac{4}{\sqrt{2}} A^{2} \int_{\eta_{1}}^{\eta_{2}} \tilde{\mathcal{P}}_{1} \tilde{\mathcal{Y}}_{1, \eta}(t, s) d s \\
& \leqslant \sqrt{2} A^{7}\left|\eta_{2}-\eta_{1}\right| \text {. }
\end{aligned}
$$

As far as $B_{2}(t, s)$ is concerned, we have to be more careful. Therefore recall that we have

$$
A_{2}^{5}=2 \tilde{\mathcal{P}}_{2} \tilde{\mathcal{Y}}_{2, \eta}(t, \eta)-\tilde{\mathcal{U}}_{2}^{2} \tilde{\mathcal{Y}}_{2, \eta}(t, \eta)+\tilde{\mathcal{H}}_{2, \eta}(t, \eta) \leqslant 2 \tilde{\mathcal{P}}_{2} \tilde{\mathcal{Y}}_{2, \eta}(t, \eta)+\tilde{\mathcal{H}}_{2, \eta}(t, \eta)
$$

Therefore we can write

$$
\frac{1}{a} \int_{0}^{s} e^{-\frac{1}{a}\left(\tilde{\mathcal{Y}}_{2}(t, s)-\tilde{\mathcal{Y}}_{2}(t, \theta)\right)} \min _{j}\left(\tilde{\mathcal{U}}_{j}^{+}\right)^{3} \tilde{\mathcal{Y}}_{1, \eta}(t, \theta) d \theta
$$




$$
\begin{aligned}
\leqslant & \frac{1}{a} \int_{0}^{s} e^{-\frac{1}{a}\left(\tilde{\mathcal{Y}}_{2}(t, s)-\tilde{\mathcal{Y}}_{2}(t, \theta)\right)}\left|\tilde{\mathcal{U}}_{2}\right| \tilde{\mathcal{U}}_{1}^{2} \tilde{\mathcal{Y}}_{1, \eta}(t, \theta) d \theta \\
\leqslant & \frac{A_{1}^{5}}{a A_{2}^{5}} \int_{0}^{s} e^{-\frac{1}{a}\left(\tilde{\mathcal{Y}}_{2}(t, s)-\tilde{\mathcal{Y}}_{2}(t, \theta)\right)}\left|\tilde{\mathcal{U}}_{2}\right|\left(2 \tilde{\mathcal{P}}_{2} \tilde{\mathcal{Y}}_{2, \eta}+\tilde{\mathcal{H}}_{2, \eta}\right)(t, \theta) d \theta \\
\leqslant & \frac{A_{1}^{5}}{a A_{2}^{5}} \int_{0}^{s} e^{-\frac{1}{a}\left(\tilde{\mathcal{Y}}_{2}(t, s)-\tilde{\mathcal{Y}}_{2}(t, \theta)\right)}\left(A_{2} \tilde{\mathcal{U}}_{2}^{2} \tilde{\mathcal{Y}}_{2, \eta}+\frac{1}{A_{2}} \tilde{\mathcal{P}}_{2}^{2} \tilde{\mathcal{Y}}_{2, \eta}+A_{2}^{2} \tilde{\mathcal{H}}_{2, \eta}\right)(t, \theta) d \theta \\
\leqslant & \frac{A_{1}^{5}}{a A_{2}^{5}} \int_{0}^{1} e^{-\frac{1}{A_{2}}\left|\tilde{\mathcal{Y}}_{2}(t, s)-\tilde{\mathcal{Y}}_{2}(t, \theta)\right|}\left(A_{2} \tilde{\mathcal{U}}_{2}^{2} \tilde{\mathcal{Y}}_{2, \eta}+A_{2}^{2} \tilde{\mathcal{H}}_{2, \eta}+\frac{1}{A_{2}} \tilde{\mathcal{P}}_{2}^{2} \tilde{\mathcal{Y}}_{2, \eta}\right)(t, \theta) d \theta \\
\leqslant & \frac{A_{1}^{5}}{a A_{2}^{5}}\left(4 A_{2}^{2} \tilde{\mathcal{P}}_{2}(t, s)+4 A_{2}^{3} \tilde{\mathcal{P}}_{2}(t, s)\right. \\
& \left.+\frac{1}{A_{2}} \int_{0}^{1} e^{-\frac{1}{A_{2}}\left|\tilde{\mathcal{Y}}_{2}(t, s)-\tilde{\mathcal{Y}}_{2}(t, \theta)\right|} \tilde{\mathcal{P}}_{2}^{2} \tilde{\mathcal{Y}}_{2, \eta}(t, \theta) d \theta\right) .
\end{aligned}
$$

Note that the integral term can be bounded by $\mathcal{O}(1) \tilde{\mathcal{P}}_{2}(t, s)$ since

$$
\begin{aligned}
& \int_{0}^{1} e^{-\frac{1}{A_{2}}\left|\tilde{\mathcal{Y}}_{2}(t, s)-\tilde{\mathcal{Y}}_{2}(t, \theta)\right|} \tilde{\mathcal{P}}_{2}^{2} \tilde{\mathcal{Y}}_{2, \eta}(t, \theta) d \theta \\
& \quad=A_{2}^{7} \int_{0}^{1} e^{-\left|\mathcal{Y}_{2}\left(t, A_{2}^{2} s\right)-\mathcal{Y}_{2}\left(t, A_{2}^{2} \theta\right)\right|} \mathcal{P}_{2}^{2} \mathcal{Y}_{2, \eta}\left(t, A_{2}^{2} \theta\right) d \theta \\
& \quad=A_{2}^{5} \int_{0}^{A_{2}^{2}} e^{-\left|\mathcal{Y}_{2}\left(t, A_{2}^{2} s\right)-\mathcal{Y}_{2}(t, \theta)\right|} \mathcal{P}_{2}^{2} \mathcal{Y}_{2, \eta}(t, \theta) d \theta \\
& \quad \leqslant \mathcal{O}(1) A_{2}^{5} \mathcal{P}_{2}\left(t, A_{2}^{2} s\right)=\mathcal{O}(1) A_{2}^{3} \tilde{\mathcal{P}}_{2}(t, s)
\end{aligned}
$$

by (3.34). We end up with

$$
\begin{aligned}
\int_{\eta_{1}}^{\eta_{2}} & B_{2}(t, s) d s \\
= & \frac{1}{a} \int_{\eta_{1}}^{\eta_{2}} \tilde{\mathcal{Y}}_{2, \eta}(t, s)\left(\int_{0}^{s} e^{-\frac{1}{a}\left(\tilde{\mathcal{Y}}_{2}(t, s)-\tilde{\mathcal{Y}}_{2}(t, \theta)\right)} \min _{j}\left(\tilde{\mathcal{U}}_{j}^{+}\right)^{3} \tilde{\mathcal{Y}}_{1, \eta}(t, \theta) d \theta\right) d s \\
\leqslant & \frac{A_{1}^{5}}{a A_{2}^{5}} \int_{\eta_{1}}^{\eta_{2}} \tilde{\mathcal{Y}}_{2, \eta}(t, s) \\
& \times\left(A_{2}^{2} \mathcal{O}(A) \tilde{\mathcal{P}}_{2}(t, s)+\frac{1}{A_{2}} \int_{0}^{1} e^{-\frac{1}{A_{2}}\left|\tilde{\mathcal{Y}}_{2}(t, s)-\tilde{\mathcal{Y}}_{2}(t, \theta)\right|} \tilde{\mathcal{P}}_{2}^{2} \tilde{\mathcal{Y}}_{2, \eta}(t, \theta) d \theta\right) d s \\
\leqslant & \frac{\mathcal{O}(1) A_{1}^{5} A_{2}^{2}}{a A_{2}^{5}} \int_{\eta_{1}}^{\eta_{2}} \tilde{\mathcal{P}}_{2} \tilde{\mathcal{Y}}_{2, \eta}(t, s) d s \\
\leqslant & \mathcal{O}(1) A^{6}\left|\eta_{2}-\eta_{1}\right| .
\end{aligned}
$$


Moreover,

$$
\begin{aligned}
b\left(t, \eta_{1}\right)-b\left(t, \eta_{2}\right) & \leqslant \int_{\eta_{1}}^{\eta_{2}} B_{1}(t, s) d s+\int_{\eta_{1}}^{\eta_{2}} B_{2}(t, s) d s \\
& \leqslant \mathcal{O}(1) A^{6}\left|\eta_{2}-\eta_{1}\right|,
\end{aligned}
$$

where $\mathcal{O}(1)$ only depends on $A$ and hence on $C=\frac{A^{2}}{2}$, and remains bounded as $A \rightarrow 0$.

Finally, combining both cases yields that

$$
\left|b\left(t, \eta_{2}\right)-b\left(t, \eta_{1}\right)\right| \leqslant \mathcal{O}(1) A^{6}\left|\eta_{2}-\eta_{1}\right|
$$

and subsequently

$$
\left|a\left(t, \eta_{2}\right)-a\left(t, \eta_{1}\right)\right| \leqslant \mathcal{O}(1) A^{6}\left|\eta_{2}-\eta_{1}\right|,
$$

where $\mathcal{O}(1)$ remains bounded as $A \rightarrow 0$. This proves that the derivative exists for almost every $\eta$ and is bounded by (A.11).

We need to estimate the pointwise difference between two functions $\tilde{\mathcal{D}}_{j}, j=1$, 2. This is the content of the following lemma.

LEMMA A.9. We have that

$$
\begin{aligned}
\left|\tilde{\mathcal{D}}_{1}(t, \eta)-\tilde{\mathcal{D}}_{2}(t, \eta)\right| \leqslant & 2 A^{3 / 2} \max _{j}\left(\tilde{\mathcal{D}}_{j}^{1 / 2}\right)(t, \eta)\left|\tilde{\mathcal{Y}}_{1}(t, \eta)-\tilde{\mathcal{Y}}_{2}(t, \eta)\right| \\
& +2 \sqrt{2} A^{3 / 2} \max _{j}\left(\tilde{\mathcal{D}}_{j}^{1 / 2}\right)(t, \eta)\left\|\tilde{\mathcal{Y}}_{1}-\tilde{\mathcal{Y}}_{2}\right\| \\
& +4 A^{3}\left(\int_{0}^{\eta} e^{-\frac{1}{A}\left(\tilde{\mathcal{Y}}_{j}(t, \eta)-\tilde{\mathcal{Y}}_{j}(t, \theta)\right)}\left(\tilde{\mathcal{U}}_{1}-\tilde{\mathcal{U}}_{2}\right)^{2}(t, \theta) d \theta\right)^{1 / 2} \\
& +2 \sqrt{2} A^{3}\left(\int_{0}^{\eta} e^{-\frac{1}{A}\left(\tilde{\mathcal{Y}}_{j}(t, \eta)-\tilde{\mathcal{Y}}_{j}(t, \theta)\right)}\left(\tilde{\mathcal{P}}_{1}^{1 / 2}-\tilde{\mathcal{P}}_{2}^{1 / 2}\right)^{2}(t, \theta) d \theta\right)^{1 / 2} \\
& +\frac{3 A^{4}}{\sqrt{2}}\left(\int_{0}^{\eta} e^{-\frac{1}{a}\left(\tilde{\mathcal{Y}}_{j}(t, \eta)-\tilde{\mathcal{Y}}_{j}(t, \theta)\right)}\left(\tilde{\mathcal{Y}}_{1}-\tilde{\mathcal{Y}}_{2}\right)^{2}(t, \theta) d \theta\right)^{1 / 2} \\
& +\frac{12 \sqrt{2} A^{4}}{\sqrt{3} e}\left(\int_{0}^{\eta} e^{-\frac{3}{4 A}\left(\tilde{\mathcal{Y}}_{j}(t, \eta)-\tilde{\mathcal{Y}}_{j}(t, \theta)\right)} d \theta\right)^{1 / 2}\left|A_{1}-A_{2}\right| \\
& +\tilde{\mathcal{U}}_{j}^{2}\left|\tilde{\mathcal{Y}}_{1}-\tilde{\mathcal{Y}}_{2}\right|(t, \eta) \\
& +\tilde{\mathcal{P}}_{j}\left|\tilde{\mathcal{Y}}_{1}-\tilde{\mathcal{Y}}_{2}\right|(t, \eta) \\
& +\frac{3 A^{4}}{2} \int_{0}^{\eta} e^{-\frac{1}{a}\left(\tilde{\mathcal{Y}}_{j}(t, \eta)-\tilde{\mathcal{Y}}_{j}(t, \theta)\right)}\left|\tilde{\mathcal{Y}}_{1}-\tilde{\mathcal{Y}}_{2}\right|(t, \theta) d \theta
\end{aligned}
$$




$$
+6 A^{4} \int_{0}^{\eta} e^{-\frac{3}{4 A}\left(\tilde{\mathcal{Y}}_{j}(t, \eta)-\tilde{\mathcal{Y}}_{j}(t, \theta)\right)} d \theta\left|A_{1}-A_{2}\right|
$$

for any value of $j=1,2$.

Proof. Direct calculations yield

$$
\begin{aligned}
\mid \tilde{\mathcal{D}}_{1}( & t, \eta)-\tilde{\mathcal{D}}_{2}(t, \eta) \mid \\
= & \mid \int_{0}^{\eta} e^{-\frac{1}{A_{1}}\left(\tilde{\mathcal{Y}}_{1}(t, \eta)-\tilde{\mathcal{Y}}_{1}(t, \theta)\right)}\left(\left(\tilde{\mathcal{U}}_{1}^{2}-\tilde{\mathcal{P}}_{1}\right) \tilde{\mathcal{Y}}_{1, \eta}(t, \theta)+\frac{1}{2} A_{1}^{5}\right) d \theta \\
& -\int_{0}^{\eta} e^{-\frac{1}{A_{2}}\left(\tilde{\mathcal{Y}}_{2}(t, \eta)-\tilde{\mathcal{Y}}_{2}(t, \theta)\right)}\left(\left(\tilde{\mathcal{U}}_{2}^{2}-\tilde{\mathcal{P}}_{2}\right) \tilde{\mathcal{Y}}_{2, \eta}(t, \theta)+\frac{1}{2} A_{2}^{5}\right) d \theta \mid \\
\leqslant & \mid \int_{0}^{\eta}\left(e^{-\frac{1}{A_{1}}\left(\tilde{\mathcal{Y}}_{1}(t, \eta)-\tilde{\mathcal{Y}}_{1}(t, \theta)\right)}-e^{-\frac{1}{A_{1}}\left(\tilde{\mathcal{Y}}_{2}(t, \eta)-\tilde{\mathcal{Y}}_{2}(t, \theta)\right)}\right) \\
& \left.\times\left(\tilde{\mathcal{U}}_{1}^{2}-\tilde{\mathcal{P}}_{1}\right) \tilde{\mathcal{Y}}_{1, \eta}(t, \theta)+\frac{1}{2} A_{1}^{5}\right) \mathbb{1}_{B(\eta)}(t, \theta) d \theta \mid \\
+ & \mid \int_{0}^{\eta}\left(e^{-\frac{1}{A_{2}}\left(\tilde{\mathcal{Y}}_{1}(t, \eta)-\tilde{\mathcal{Y}}_{1}(t, \theta)\right)}-e^{-\frac{1}{A_{2}}\left(\tilde{\mathcal{Y}}_{2}(t, \eta)-\tilde{\mathcal{Y}}_{2}(t, \theta)\right)}\right) \\
& \times\left(\left(\tilde{\mathcal{U}}_{2}^{2}-\tilde{\mathcal{P}}_{2}\right) \tilde{\mathcal{Y}}_{2, \eta}(t, \theta)+\frac{1}{2} A_{2}^{5}\right) \mathbb{1}_{B(\eta)}(t, \theta) d \theta \mid \\
+ & \left.\mid \int_{0}^{\eta} \min _{j}\left(e^{-\frac{1}{A_{1}}\left(\tilde{\mathcal{Y}}_{j}(t, \eta)-\tilde{\mathcal{Y}}_{j}(t, \theta)\right)}\right)\left(\tilde{\mathcal{U}}_{1}^{2}-\tilde{\mathcal{P}}_{1}\right) \tilde{\mathcal{Y}}_{1, \eta}(t, \theta)+\frac{1}{2} A_{1}^{5}\right) d \theta \\
= & \left.\left.\int_{11}^{\eta} \min _{j}(t, \eta)+e^{-\frac{1}{A_{2}}\left(\tilde{\mathcal{Y}}_{j}(t, \eta)-\tilde{\mathcal{Y}}_{j}(t, \theta)\right)}\right)\left(\tilde{\mathcal{U}}_{2}^{2}-\tilde{\mathcal{P}}_{2}\right) \tilde{\mathcal{Y}}_{2, \eta}(t, \theta)+\frac{1}{2} A_{2}^{5}\right) d \theta \mid \\
& -\eta(t, \eta)+\bar{d}_{13}(t, \eta),
\end{aligned}
$$

where $B(\eta)$ is defined in (5.11).

For $\bar{d}_{11}(t, \eta)$ we immediately obtain

$$
\begin{aligned}
\bar{d}_{11}(t, \eta) \leqslant & \frac{1}{A_{1}} \int_{0}^{\eta}\left(\left|\tilde{\mathcal{Y}}_{1}(t, \eta)-\tilde{\mathcal{Y}}_{2}(t, \eta)\right|+\left|\tilde{\mathcal{Y}}_{1}(t, \theta)-\tilde{\mathcal{Y}}_{2}(t, \theta)\right|\right) \\
& \times e^{-\frac{1}{A_{1}}\left(\tilde{\mathcal{Y}}_{1}(t, \eta)-\tilde{\mathcal{Y}}_{1}(t, \theta)\right)}\left(\left(\tilde{\mathcal{U}}_{1}^{2}-\tilde{\mathcal{P}}_{1}\right) \tilde{\mathcal{Y}}_{1, \eta}(t, \theta)+\frac{1}{2} A_{1}^{5}\right) d \theta \\
\leqslant & \frac{\tilde{\mathcal{D}}_{1}(t, \eta)}{A_{1}}\left|\tilde{\mathcal{Y}}_{1}(t, \eta)-\tilde{\mathcal{Y}}_{2}(t, \eta)\right|+\sqrt{2} A_{1}^{3 / 2} \tilde{\mathcal{D}}_{1}^{1 / 2}(t, \eta)\left\|\tilde{\mathcal{Y}}_{1}-\tilde{\mathcal{Y}}_{2}\right\| \\
\leqslant & A_{1}^{3 / 2} \tilde{\mathcal{D}}_{1}^{1 / 2}(t, \eta)\left|\tilde{\mathcal{Y}}_{1}(t, \eta)-\tilde{\mathcal{Y}}_{2}(t, \eta)\right|+\sqrt{2} A_{1}^{3 / 2} \tilde{\mathcal{D}}_{1}^{1 / 2}(t, \eta)\left\|\tilde{\mathcal{Y}}_{1}-\tilde{\mathcal{Y}}_{2}\right\| .
\end{aligned}
$$


Following the same lines, we end up with

$$
\begin{aligned}
\bar{d}_{12}(t, \eta) \leqslant & \frac{1}{A_{2}} \int_{0}^{\eta}\left(\left|\tilde{\mathcal{Y}}_{1}(t, \eta)-\tilde{\mathcal{Y}}_{2}(t, \eta)\right|+\left|\tilde{\mathcal{Y}}_{1}(t, \theta)-\tilde{\mathcal{Y}}_{2}(t, \theta)\right|\right) \\
& \times e^{-\frac{1}{A_{2}}\left(\tilde{\mathcal{Y}}_{2}(t, \eta)-\tilde{\mathcal{Y}}_{2}(t, \theta)\right)}\left|\left(\tilde{\mathcal{U}}_{2}^{2}-\tilde{\mathcal{P}}_{2}\right) \tilde{\mathcal{Y}}_{2, \eta}(t, \theta)+\frac{1}{2} A_{2}^{5}\right| d \theta \\
\leqslant & A_{2}^{3 / 2} \tilde{\mathcal{D}}_{2}^{1 / 2}(t, \eta)\left|\tilde{\mathcal{Y}}_{1}(t, \eta)-\tilde{\mathcal{Y}}_{2}(t, \eta)\right|+\sqrt{2} A_{2}^{3 / 2} \tilde{\mathcal{D}}_{2}^{1 / 2}(t, \eta)\left\|\tilde{\mathcal{Y}}_{1}-\tilde{\mathcal{Y}}_{2}\right\| .
\end{aligned}
$$

The last term $\bar{d}_{13}(t, \eta)$ needs a bit more work. Indeed,

$$
\begin{aligned}
& \bar{d}_{13}(t, \eta)=\mid \int_{0}^{\eta} \min _{j}\left(e^{-\frac{1}{A_{1}}\left(\tilde{\mathcal{Y}}_{j}(t, \eta)-\tilde{\mathcal{Y}}_{j}(t, \theta)\right)}\right)\left(\left(\tilde{\mathcal{U}}_{1}^{2}-\tilde{\mathcal{P}}_{1}\right) \tilde{\mathcal{Y}}_{1, \eta}(t, \theta)+\frac{1}{2} A_{1}^{5}\right) d \theta \\
& -\int_{0}^{\eta} \min _{j}\left(e^{-\frac{1}{A_{2}}\left(\tilde{\mathcal{Y}}_{j}(t, \eta)-\tilde{\mathcal{Y}}_{j}(t, \theta)\right)}\right)\left(\left(\tilde{\mathcal{U}}_{2}^{2}-\tilde{\mathcal{P}}_{2}\right) \tilde{\mathcal{Y}}_{2, \eta}(t, \theta)+\frac{1}{2} A_{2}^{5}\right) d \theta \mid \\
& \leqslant \mid \int_{0}^{\eta}\left(\min _{j}\left(e^{-\frac{1}{A_{1}}\left(\tilde{\mathcal{Y}}_{j}(t, \eta)-\tilde{\mathcal{Y}}_{j}(t, \theta)\right)}\right) \tilde{\mathcal{U}}_{1}^{2} \tilde{\mathcal{Y}}_{1, \eta}(t, \theta)\right. \\
& \left.-\min _{j}\left(e^{-\frac{1}{A_{2}}\left(\tilde{\mathcal{Y}}_{j}(t, \eta)-\tilde{\mathcal{Y}}_{j}(t, \theta)\right)}\right) \tilde{\mathcal{U}}_{2}^{2} \tilde{\mathcal{Y}}_{2, \eta}(t, \theta)\right) d \theta \mid \\
& +\mid \int_{0}^{\eta}\left(\min _{j}\left(e^{-\frac{1}{A_{1}}\left(\tilde{\mathcal{Y}}_{j}(t, \eta)-\tilde{\mathcal{Y}}_{j}(t, \theta)\right)}\right) \tilde{\mathcal{P}}_{1} \tilde{\mathcal{Y}}_{1, \eta}(t, \theta)\right. \\
& \left.-\min _{j}\left(e^{-\frac{1}{A_{2}}\left(\tilde{\mathcal{Y}}_{j}(t, \eta)-\tilde{\mathcal{Y}}_{j}(t, \theta)\right)}\right) \tilde{\mathcal{P}}_{2} \tilde{\mathcal{Y}}_{2, \eta}(t, \theta)\right) d \theta \mid \\
& +\mid \int_{0}^{\eta}\left(\min _{j}\left(e^{-\frac{1}{A_{1}}\left(\tilde{\mathcal{Y}}_{j}(t, \eta)-\tilde{\mathcal{Y}}_{j}(t, \theta)\right)}\right) \frac{1}{2} A_{1}^{5}\right. \\
& \left.-\min _{j}\left(e^{-\frac{1}{A_{2}}\left(\tilde{\mathcal{Y}}_{j}(t, \eta)-\tilde{\mathcal{Y}}_{j}(t, \theta)\right)}\right) \frac{1}{2} A_{2}^{5}\right) d \theta \\
& =\bar{T}_{1}(t, \eta)+\bar{T}_{2}(t, \eta)+\bar{T}_{3}(t, \eta) .
\end{aligned}
$$

To estimate $\bar{T}_{1}(t, \eta)$, recall (3.40), (A.4), (A.5), and Lemma A.1 (ii), which imply

$$
\begin{aligned}
\bar{T}_{1}(t, \eta)= & \mid \int_{0}^{\eta}\left(\min _{j}\left(\mathrm{e}^{-\frac{1}{A_{1}}\left(\tilde{\mathcal{Y}}_{j}(t, \eta)-\tilde{\mathcal{Y}}_{j}(t, \theta)\right)}\right) \tilde{\mathcal{U}}_{1}^{2} \tilde{\mathcal{Y}}_{1, \eta}(t, \theta)\right. \\
& \left.-\min _{j}\left(e^{-\frac{1}{A_{2}}\left(\tilde{\mathcal{Y}}_{j}(t, \eta)-\tilde{\mathcal{Y}}_{j}(t, \theta)\right)}\right) \tilde{\mathcal{U}}_{2}^{2} \tilde{\mathcal{Y}}_{2, \eta}(t, \theta)\right) d \theta \mid \\
\leqslant & \left|\int_{0}^{\eta} \min _{j}\left(e^{-\frac{1}{A_{1}}\left(\tilde{\mathcal{Y}}_{j}(t, \eta)-\tilde{\mathcal{Y}}_{j}(t, \theta)\right)}\right)\left(\tilde{\mathcal{U}}_{1}^{2}-\tilde{\mathcal{U}}_{2}^{2}\right) \tilde{\mathcal{Y}}_{1, \eta}(t, \theta) \mathbb{1}_{\tilde{\mathcal{U}}_{2}^{2} \leqslant \tilde{\mathcal{U}}_{1}^{2}}(t, \theta) d \theta\right|
\end{aligned}
$$




$$
\begin{aligned}
& +\left|\int_{0}^{\eta} \min _{j}\left(e^{-\frac{1}{A_{2}}\left(\tilde{\mathcal{Y}}_{j}(t, \eta)-\tilde{\mathcal{Y}}_{j}(t, \theta)\right)}\right)\left(\tilde{\mathcal{U}}_{1}^{2}-\tilde{\mathcal{U}}_{2}^{2}\right) \tilde{\mathcal{Y}}_{2, \eta}(t, \theta) \mathbb{1}_{\tilde{\mathcal{U}}_{1}^{2}<\tilde{\mathcal{U}}_{2}^{2}}(t, \theta) d \theta\right| \\
& +\mathbb{1}_{A_{1} \leqslant A_{2}} \mid \int_{0}^{\eta}\left(\min _{j}\left(e^{-\frac{1}{A_{1}}\left(\tilde{\mathcal{Y}}_{j}(t, \eta)-\tilde{\mathcal{Y}}_{j}(t, \theta)\right)}\right)\right. \\
& \left.-\min _{j}\left(e^{-\frac{1}{A_{2}}\left(\tilde{\mathcal{Y}}_{j}(t, \eta)-\tilde{\mathcal{Y}}_{j}(t, \theta)\right)}\right)\right) \min _{j}\left(\tilde{\mathcal{U}}_{j}^{2}\right) \tilde{\mathcal{Y}}_{2, \eta}(t, \theta) d \theta \mid \\
& +\mathbb{1}_{A_{2}<A_{1}} \mid \int_{0}^{\eta}\left(\min _{j}\left(e^{-\frac{1}{A_{1}}\left(\tilde{\mathcal{Y}}_{j}(t, \eta)-\tilde{\mathcal{Y}}_{j}(t, \theta)\right)}\right)\right. \\
& \left.-\min _{j}\left(e^{-\frac{1}{A_{2}}\left(\tilde{\mathcal{Y}}_{j}(t, \eta)-\tilde{\mathcal{Y}}_{j}(t, \theta)\right)}\right)\right) \min _{j}\left(\tilde{\mathcal{U}}_{j}^{2}\right) \tilde{\mathcal{Y}}_{1, \eta}(t, \theta) d \theta \\
& +\left|\int_{0}^{\eta} \min _{j}\left(e^{-\frac{1}{a}\left(\tilde{\mathcal{Y}}_{j}(t, \eta)-\tilde{\mathcal{Y}}_{j}(t, \theta)\right)}\right) \min _{j}\left(\tilde{\mathcal{U}}_{j}^{2}\right)\left(\tilde{\mathcal{Y}}_{1, \eta}-\tilde{\mathcal{Y}}_{2, \eta}\right)(t, \theta) d \theta\right| \\
& \leqslant 2 \int_{0}^{\eta} \min _{j}\left(e^{-\frac{1}{A_{1}}\left(\tilde{\mathcal{Y}}_{j}(t, \eta)-\tilde{\mathcal{Y}}_{j}(t, \theta)\right)}\right)\left|\tilde{\mathcal{U}}_{1}\right| \tilde{\mathcal{Y}}_{1, \eta}\left|\tilde{\mathcal{U}}_{1}-\tilde{\mathcal{U}}_{2}\right|(t, \theta) d \theta \\
& +2 \int_{0}^{\eta} \min _{j}\left(e^{-\frac{1}{A_{2}}\left(\tilde{\mathcal{Y}}_{j}(t, \eta)-\tilde{\mathcal{Y}}_{j}(t, \theta)\right)}\right)\left|\tilde{\mathcal{U}}_{2}\right| \tilde{\mathcal{Y}}_{2, \eta}\left|\tilde{\mathcal{U}}_{1}-\tilde{\mathcal{U}}_{2}\right|(t, \theta) d \theta \\
& +\mathbb{1}_{A_{1} \leqslant A_{2}} \frac{4}{a e}\left|\int_{0}^{\eta} \min _{j}\left(e^{-\frac{3}{4 A}\left(\tilde{\mathcal{Y}}_{j}(t, \eta)-\tilde{\mathcal{Y}}_{j}(t, \theta)\right)}\right) \min _{j}\left(\tilde{\mathcal{U}}_{j}^{2}\right) \tilde{\mathcal{Y}}_{2, \eta}(t, \theta) d \theta\right|\left|A_{1}-A_{2}\right| \\
& +\mathbb{1}_{A_{2}<A_{1}} \frac{4}{a e}\left|\int_{0}^{\eta} \min _{j}\left(e^{-\frac{3}{4 A}\left(\tilde{\mathcal{Y}}_{j}(t, \eta)-\tilde{\mathcal{Y}}_{j}(t, \theta)\right)}\right) \min _{j}\left(\tilde{\mathcal{U}}_{j}^{2}\right) \tilde{\mathcal{Y}}_{1, \eta}(t, \theta) d \theta\right|\left|A_{1}-A_{2}\right| \\
& +\left|\min _{j}\left(e^{-\frac{1}{a}\left(\tilde{\mathcal{Y}}_{j}(t, \eta)-\tilde{\mathcal{Y}}_{j}(t, \theta)\right)}\right) \min _{j}\left(\tilde{\mathcal{U}}_{j}^{2}\right)\left(\tilde{\mathcal{Y}}_{1}-\tilde{\mathcal{Y}}_{2}\right)\right|_{\theta=0}^{\eta} \\
& -\int_{0}^{\eta} \frac{d}{d \theta}\left(\min _{j}\left(e^{-\frac{1}{a}\left(\tilde{\mathcal{Y}}_{j}(t, \eta)-\tilde{\mathcal{Y}}_{j}(t, \theta)\right)}\right) \min _{j}\left(\tilde{\mathcal{U}}_{j}^{2}\right)\right)\left(\tilde{\mathcal{Y}}_{1}-\tilde{\mathcal{Y}}_{2}\right)(t, \theta) d \theta \\
& \leqslant 2\left(\int_{0}^{\eta} e^{-\frac{1}{A_{1}}\left(\tilde{\mathcal{Y}}_{1}(t, \eta)-\tilde{\mathcal{Y}}_{1}(t, \theta)\right)} \tilde{\mathcal{U}}_{1}^{2} \tilde{\mathcal{Y}}_{1, \eta}^{2}(t, \theta) d \theta\right)^{1 / 2} \\
& \times\left(\int_{0}^{\eta} e^{-\frac{1}{A_{1}}\left(\tilde{\mathcal{Y}}_{j}(t, \eta)-\tilde{\mathcal{Y}}_{j}(t, \theta)\right)}\left(\tilde{\mathcal{U}}_{1}-\tilde{\mathcal{U}}_{2}\right)^{2}(t, \theta) d \theta\right)^{1 / 2} \\
& +2\left(\int_{0}^{\eta} e^{-\frac{1}{A_{2}}\left(\tilde{\mathcal{Y}}_{2}(t, \eta)-\tilde{\mathcal{Y}}_{2}(t, \theta)\right)} \tilde{\mathcal{U}}_{2}^{2} \tilde{\mathcal{Y}}_{2, \eta}^{2}(t, \theta) d \theta\right)^{1 / 2} \\
& \times\left(\int_{0}^{\eta} e^{-\frac{1}{A_{2}}\left(\tilde{\mathcal{Y}}_{j}(t, \eta)-\tilde{\mathcal{Y}}_{j}(t, \theta)\right)}\left(\tilde{\mathcal{U}}_{1}-\tilde{\mathcal{U}}_{2}\right)^{2}(t, \theta) d \theta\right)^{1 / 2} \\
& +\frac{2 \sqrt{2} A}{e}\left(\int_{0}^{\eta} e^{-\frac{3}{4 A_{2}}\left(\tilde{\mathcal{Y}}_{2}(t, \eta)-\tilde{\mathcal{Y}}_{2}(t, \theta)\right)} \tilde{\mathcal{U}}_{2}^{2} \tilde{\mathcal{Y}}_{2, \eta}^{2}(t, \theta) d \theta\right)^{1 / 2} \\
& \times\left(\int_{0}^{\eta} e^{-\frac{3}{4 A}\left(\tilde{\mathcal{Y}}_{j}(t, \eta)-\tilde{\mathcal{Y}}_{j}(t, \theta)\right)} d \theta\right)^{1 / 2}\left|A_{1}-A_{2}\right|
\end{aligned}
$$




$$
\begin{aligned}
& +\frac{2 \sqrt{2} A}{e}\left(\int_{0}^{\eta} e^{-\frac{3}{4 A_{1}}\left(\tilde{\mathcal{Y}}_{1}(t, \eta)-\tilde{\mathcal{Y}}_{1}(t, \theta)\right)} \tilde{\mathcal{U}}_{1}^{2} \tilde{\mathcal{Y}}_{1, \eta}^{2}(t, \theta) d \theta\right)^{1 / 2} \\
& \times\left(\int_{0}^{\eta} e^{-\frac{3}{4 A}\left(\tilde{\mathcal{Y}}_{j}(t, \eta)-\tilde{\mathcal{Y}}_{j}(t, \theta)\right)} d \theta\right)^{1 / 2}\left|A_{1}-A_{2}\right| \\
& +\tilde{\mathcal{U}}_{j}^{2}\left|\tilde{\mathcal{Y}}_{1}-\tilde{\mathcal{Y}}_{2}\right|(t, \eta) \\
& +\frac{1}{a} \int_{0}^{\eta} e^{-\frac{1}{a}\left(\tilde{\mathcal{Y}}_{j}(t, \eta)-\tilde{\mathcal{Y}}_{j}(t, \theta)\right)} \min _{j}\left(\tilde{\mathcal{U}}_{j}^{2}\right)\left(\tilde{\mathcal{Y}}_{1, \eta}+\tilde{\mathcal{Y}}_{2, \eta}\right)\left|\tilde{\mathcal{Y}}_{1}-\tilde{\mathcal{Y}}_{2}\right|(t, \theta) d \theta \\
& +A^{4} \int_{0}^{\eta} e^{-\frac{1}{a}\left(\tilde{\mathcal{Y}}_{j}(t, \eta)-\tilde{\mathcal{Y}}_{j}(t, \theta)\right)}\left|\tilde{\mathcal{Y}}_{1}-\tilde{\mathcal{Y}}_{2}\right|(t, \theta) d \theta \\
& \leqslant 4 A^{3}\left(\int_{0}^{\eta} e^{-\frac{1}{A}\left(\tilde{\mathcal{Y}}_{j}(t, \eta)-\tilde{\mathcal{Y}}_{j}(t, \theta)\right)}\left(\tilde{\mathcal{U}}_{1}-\tilde{\mathcal{U}}_{2}\right)^{2}(t, \theta) d \theta\right)^{1 / 2} \\
& +\frac{8 \sqrt{2} A^{4}}{\sqrt{3} e}\left(\int_{0}^{\eta} e^{-\frac{3}{4 A}\left(\tilde{\mathcal{Y}}_{j}(t, \eta)-\tilde{\mathcal{Y}}_{j}(t, \theta)\right)} d \theta\right)^{1 / 2}\left|A_{1}-A_{2}\right| \\
& +\tilde{\mathcal{U}}_{j}^{2}\left|\tilde{\mathcal{Y}}_{1}-\tilde{\mathcal{Y}}_{2}\right|(t, \eta) \\
& +\sqrt{2} A^{4}\left(\int_{0}^{\eta} e^{-\frac{1}{a}\left(\tilde{\mathcal{Y}}_{j}(t, \eta)-\tilde{\mathcal{Y}}_{j}(t, \theta)\right)}\left(\tilde{\mathcal{Y}}_{1}-\tilde{\mathcal{Y}}_{2}\right)^{2}(t, \theta) d \theta\right)^{1 / 2} \\
& +A^{4} \int_{0}^{\eta} e^{-\frac{1}{a}\left(\tilde{\mathcal{Y}}_{j}(t, \eta)-\tilde{\mathcal{Y}}_{j}(t, \theta)\right)}\left|\tilde{\mathcal{Y}}_{1}-\tilde{\mathcal{Y}}_{2}\right|(t, \theta) d \theta \text {. }
\end{aligned}
$$

Following the same lines, one has

$$
\begin{aligned}
& \bar{T}_{2}(t, \eta)=\mid \int_{0}^{\eta}\left(\min _{j}\left(\mathrm{e}^{-\frac{1}{A_{1}}\left(\tilde{\mathcal{Y}}_{j}(t, \eta)-\tilde{\mathcal{Y}}_{j}(t, \theta)\right)}\right) \tilde{\mathcal{P}}_{1} \tilde{\mathcal{Y}}_{1, \eta}(t, \theta)\right. \\
& \left.-\min _{j}\left(e^{-\frac{1}{A_{2}}\left(\tilde{\mathcal{Y}}_{j}(t, \eta)-\tilde{\mathcal{Y}}_{j}(t, \theta)\right)}\right) \tilde{\mathcal{P}}_{2} \tilde{\mathcal{Y}}_{2, \eta}(t, \theta)\right) d \theta \mid \\
& \leqslant\left|\int_{0}^{\eta} \min _{j}\left(e^{-\frac{1}{A_{1}}\left(\tilde{\mathcal{Y}}_{j}(t, \eta)-\tilde{\mathcal{Y}}_{j}(t, \theta)\right)}\right)\left(\tilde{\mathcal{P}}_{1}-\tilde{\mathcal{P}}_{2}\right) \tilde{\mathcal{Y}}_{1, \eta}(t, \theta) \mathbb{1}_{\tilde{\mathcal{P}}_{2} \leqslant \tilde{\mathcal{P}}_{1}}(t, \theta) d \theta\right| \\
& +\left|\int_{0}^{\eta} \min _{j}\left(e^{-\frac{1}{A_{2}}\left(\tilde{\mathcal{Y}}_{j}(t, \eta)-\tilde{\mathcal{Y}}_{j}(t, \theta)\right)}\right)\left(\tilde{\mathcal{P}}_{1}-\tilde{\mathcal{P}}_{2}\right) \tilde{\mathcal{Y}}_{2, \eta}(t, \theta) \mathbb{1}_{\tilde{\mathcal{P}}_{1}<\tilde{\mathcal{P}}_{2}}(t, \theta) d \theta\right| \\
& +\mathbb{1}_{A_{1} \leqslant A_{2}} \mid \int_{0}^{\eta}\left(\min _{j}\left(e^{-\frac{1}{A_{1}}\left(\tilde{\mathcal{Y}}_{j}(t, \eta)-\tilde{\mathcal{Y}}_{j}(t, \theta)\right)}\right)\right. \\
& \left.-\min _{j}\left(e^{-\frac{1}{A_{2}}\left(\tilde{\mathcal{Y}}_{j}(t, \eta)-\tilde{\mathcal{Y}}_{j}(t, \theta)\right)}\right)\right) \min _{j}\left(\tilde{\mathcal{P}}_{j}\right) \tilde{\mathcal{Y}}_{2, \eta}(t, \theta) d \theta \\
& +\mathbb{1}_{A_{2}<A_{1}} \mid \int_{0}^{\eta}\left(\min _{j}\left(e^{-\frac{1}{A_{1}}\left(\tilde{\mathcal{Y}}_{j}(t, \eta)-\tilde{\mathcal{Y}}_{j}(t, \theta)\right)}\right)\right. \\
& \left.-\min _{j}\left(e^{-\frac{1}{A_{2}}\left(\tilde{\mathcal{Y}}_{j}(t, \eta)-\tilde{\mathcal{Y}}_{j}(t, \theta)\right)}\right)\right) \min _{j}\left(\tilde{\mathcal{P}}_{j}\right) \tilde{\mathcal{Y}}_{1, \eta}(t, \theta) d \theta
\end{aligned}
$$




$$
\begin{aligned}
& +\left|\int_{0}^{\eta} \min _{j}\left(e^{-\frac{1}{a}\left(\tilde{\mathcal{Y}}_{j}(t, \eta)-\tilde{\mathcal{Y}}_{j}(t, \theta)\right)}\right) \min _{j}\left(\tilde{\mathcal{P}}_{j}\right)\left(\tilde{\mathcal{Y}}_{1, \eta}-\tilde{\mathcal{Y}}_{2, \eta}\right)(t, \theta) d \theta\right| \\
& \leqslant 2 \int_{0}^{\eta} \min _{j}\left(e^{-\frac{1}{A_{1}}\left(\tilde{\mathcal{Y}}_{j}(t, \eta)-\tilde{\mathcal{Y}}_{j}(t, \theta)\right)}\right)\left|\tilde{\mathcal{P}}_{1}^{1 / 2}\right| \tilde{\mathcal{Y}}_{1, \eta}\left|\tilde{\mathcal{P}}_{1}^{1 / 2}-\tilde{\mathcal{P}}_{2}^{1 / 2}\right|(t, \theta) d \theta \\
& +2 \int_{0}^{\eta} \min _{j}\left(e^{-\frac{1}{A_{2}}\left(\tilde{\mathcal{Y}}_{j}(t, \eta)-\tilde{\mathcal{Y}}_{j}(t, \theta)\right)}\right)\left|\tilde{\mathcal{P}}_{2}^{1 / 2}\right| \tilde{\mathcal{Y}}_{2, \eta}\left|\tilde{\mathcal{P}}_{1}^{1 / 2}-\tilde{\mathcal{P}}_{2}^{1 / 2}\right|(t, \theta) d \theta \\
& +\mathbb{1}_{A_{1} \leqslant A_{2}} \frac{4}{a e}\left|\int_{0}^{\eta} \min _{j}\left(e^{-\frac{3}{4 A}\left(\tilde{\mathcal{Y}}_{j}(t, \eta)-\tilde{\mathcal{Y}}_{j}(t, \theta)\right)}\right) \min _{j}\left(\tilde{\mathcal{P}}_{j}\right) \tilde{\mathcal{Y}}_{2, \eta}(t, \theta) d \theta\right|\left|A_{1}-A_{2}\right| \\
& +\mathbb{1}_{A_{2}<A_{1}} \frac{4}{a e}\left|\int_{0}^{\eta} \min _{j}\left(e^{-\frac{3}{4 A}\left(\tilde{\mathcal{Y}}_{j}(t, \eta)-\tilde{\mathcal{Y}}_{j}(t, \theta)\right)}\right) \min _{j}\left(\tilde{\mathcal{P}}_{j}\right) \tilde{\mathcal{Y}}_{1, \eta}(t, \theta) d \theta\right|\left|A_{1}-A_{2}\right| \\
& +\left|\min _{j}\left(e^{-\frac{1}{a}\left(\tilde{\mathcal{Y}}_{j}(t, \eta)-\tilde{\mathcal{Y}}_{j}(t, \theta)\right)}\right) \min _{j}\left(\tilde{\mathcal{P}}_{j}\right)\left(\tilde{\mathcal{Y}}_{1}-\tilde{\mathcal{Y}}_{2}\right)\right|_{\theta=0}^{\eta} \\
& -\int_{0}^{\eta} \frac{d}{d \theta}\left(\min _{j}\left(e^{-\frac{1}{a}\left(\tilde{\mathcal{Y}}_{j}(t, \eta)-\tilde{\mathcal{Y}}_{j}(t, \theta)\right)}\right) \min _{j}\left(\tilde{\mathcal{P}}_{j}\right)\right)\left(\tilde{\mathcal{Y}}_{1}-\tilde{\mathcal{Y}}_{2}\right)(t, \theta) d \theta \\
& \leqslant 2\left(\int_{0}^{\eta} e^{-\frac{1}{A_{1}}\left(\tilde{\mathcal{Y}}_{1}(t, \eta)-\tilde{\mathcal{Y}}_{1}(t, \theta)\right)} \tilde{\mathcal{P}}_{1} \tilde{\mathcal{Y}}_{1, \eta}^{2}(t, \theta) d \theta\right)^{1 / 2} \\
& \times\left(\int_{0}^{\eta} e^{-\frac{1}{A_{1}}\left(\tilde{\mathcal{Y}}_{j}(t, \eta)-\tilde{\mathcal{Y}}_{j}(t, \theta)\right)}\left(\tilde{\mathcal{P}}_{1}^{1 / 2}-\tilde{\mathcal{P}}_{2}^{1 / 2}\right)^{2}(t, \theta) d \theta\right)^{1 / 2} \\
& +2\left(\int_{0}^{\eta} e^{-\frac{1}{A_{2}}\left(\tilde{\mathcal{Y}}_{2}(t, \eta)-\tilde{\mathcal{Y}}_{2}(t, \theta)\right)} \tilde{\mathcal{P}}_{2} \tilde{\mathcal{Y}}_{2, \eta}^{2}(t, \theta) d \theta\right)^{1 / 2} \\
& \times\left(\int_{0}^{\eta} e^{-\frac{1}{A_{2}}\left(\tilde{\mathcal{Y}}_{j}(t, \eta)-\tilde{\mathcal{Y}}_{j}(t, \theta)\right)}\left(\tilde{\mathcal{P}}_{1}^{1 / 2}-\tilde{\mathcal{P}}_{2}^{1 / 2}\right)^{2}(t, \theta) d \theta\right)^{1 / 2} \\
& +\frac{2 A}{e}\left(\int_{0}^{\eta} e^{-\frac{3}{4 A_{2}}\left(\tilde{\mathcal{Y}}_{2}(t, \eta)-\tilde{\mathcal{Y}}_{2}(t, \theta)\right)} \tilde{\mathcal{P}}_{2} \tilde{\mathcal{Y}}_{2, \eta}^{2}(t, \theta) d \theta\right)^{1 / 2} \\
& \times\left(\int_{0}^{\eta} e^{-\frac{3}{4 A}\left(\tilde{\mathcal{Y}}_{j}(t, \eta)-\tilde{\mathcal{Y}}_{j}(t, \theta)\right)} d \theta\right)^{1 / 2}\left|A_{1}-A_{2}\right| \\
& +\frac{2 A}{e}\left(\int_{0}^{\eta} e^{-\frac{3}{4 A_{1}}\left(\tilde{\mathcal{Y}}_{1}(t, \eta)-\tilde{\mathcal{Y}}_{1}(t, \theta)\right)} \tilde{\mathcal{P}}_{1} \tilde{\mathcal{Y}}_{1, \eta}^{2}(t, \theta) d \theta\right)^{2} \\
& \times\left(\int_{0}^{\eta} e^{-\frac{3}{4 A}\left(\tilde{\mathcal{Y}}_{j}(t, \eta)-\tilde{\mathcal{Y}}_{j}(t, \theta)\right)} d \theta\right)^{1 / 2}\left|A_{1}-A_{2}\right| \\
& +\tilde{\mathcal{P}}_{j}\left|\tilde{\mathcal{Y}}_{1}-\tilde{\mathcal{Y}}_{2}\right|(t, \eta) \\
& +\frac{1}{a} \int_{0}^{\eta} e^{-\frac{1}{a}\left(\tilde{\mathcal{Y}}_{j}(t, \eta)-\tilde{\mathcal{Y}}_{j}(t, \theta)\right)} \min _{j}\left(\tilde{\mathcal{P}}_{j}\right)\left(\tilde{\mathcal{Y}}_{1, \eta}+\tilde{\mathcal{Y}}_{2, \eta}\right)\left|\tilde{\mathcal{Y}}_{1}-\tilde{\mathcal{Y}}_{2}\right|(t, \theta) d \theta \\
& +\frac{A^{4}}{2} \int_{0}^{\eta} e^{-\frac{1}{a}\left(\tilde{\mathcal{Y}}_{j}(t, \eta)-\tilde{\mathcal{Y}}_{j}(t, \theta)\right)}\left|\tilde{\mathcal{Y}}_{1}-\tilde{\mathcal{Y}}_{2}\right|(t, \theta) d \theta
\end{aligned}
$$




$$
\begin{aligned}
\leqslant & 2 \sqrt{2} A^{3}\left(\int_{0}^{\eta} e^{-\frac{1}{A}\left(\tilde{\mathcal{Y}}_{j}(t, \eta)-\tilde{\mathcal{Y}}_{j}(t, \theta)\right)}\left(\tilde{\mathcal{P}}_{1}^{1 / 2}-\tilde{\mathcal{P}}_{2}^{1 / 2}\right)^{2}(t, \theta) d \theta\right)^{1 / 2} \\
& +\frac{4 \sqrt{2} A^{4}}{\sqrt{3} e}\left(\int_{0}^{\eta} e^{-\frac{3}{4 A}\left(\tilde{\mathcal{Y}}_{j}(t, \eta)-\tilde{\mathcal{Y}}_{j}(t, \theta)\right)} d \theta\right)^{1 / 2}\left|A_{1}-A_{2}\right| \\
& +\tilde{\mathcal{P}}_{j}\left|\tilde{\mathcal{Y}}_{1}-\tilde{\mathcal{Y}}_{2}\right|(t, \eta) \\
& +\frac{A^{4}}{\sqrt{2}}\left(\int_{0}^{\eta} e^{-\frac{1}{a}\left(\tilde{\mathcal{Y}}_{j}(t, \eta)-\tilde{\mathcal{Y}}_{j}(t, \theta)\right)}\left(\tilde{\mathcal{Y}}_{1}-\tilde{\mathcal{Y}}_{2}\right)^{2}(t, \theta) d \theta\right)^{1 / 2} \\
& +\frac{A^{4}}{2} \int_{0}^{\eta} e^{-\frac{1}{a}\left(\tilde{\mathcal{Y}}_{j}(t, \eta)-\tilde{\mathcal{Y}}_{j}(t, \theta)\right)}\left|\tilde{\mathcal{Y}}_{1}-\tilde{\mathcal{Y}}_{2}\right|(t, \theta) d \theta
\end{aligned}
$$

For the last term $\bar{T}_{3}(t, \eta)$, we have

$$
\begin{aligned}
2 \bar{T}_{3}(t, \eta)= & \mid \int_{0}^{\eta}\left(\min _{j}\left(e^{-\frac{1}{A_{1}}\left(\tilde{\mathcal{Y}}_{j}(t, \eta)-\tilde{\mathcal{Y}}_{j}(t, \theta)\right)}\right) A_{1}^{5}-\left(\min _{j}\left(e^{-\frac{1}{A_{2}}\left(\tilde{\mathcal{Y}}_{j}(t, \eta)-\tilde{\mathcal{Y}}_{j}(t, \theta)\right)}\right) A_{2}^{5}\right) d \theta \mid\right. \\
\leqslant & \mathbb{1}_{A_{1} \leqslant A_{2}}\left|\int_{0}^{\eta}\left(e^{-\frac{1}{A_{2}}\left(\tilde{\mathcal{Y}}_{j}(t, \eta)-\tilde{\mathcal{Y}}_{j}(t, \theta)\right)}\right)\left(A_{1}^{5}-A_{2}^{5}\right) d \theta\right| \\
& +\mathbb{1}_{A_{2}<A_{1}}\left|\int_{0}^{\eta}\left(e^{-\frac{1}{A_{1}}\left(\tilde{\mathcal{Y}}_{j}(t, \eta)-\tilde{\mathcal{Y}}_{j}(t, \theta)\right)}\right)\left(A_{1}^{5}-A_{2}^{5}\right) d \theta\right| \\
& +a^{5} \int_{0}^{\eta}\left|\min _{j}\left(e^{-\frac{1}{A_{1}}\left(\tilde{\mathcal{Y}}_{j}(t, \eta)-\tilde{\mathcal{Y}}_{j}(t, \theta)\right)}\right)-\min _{j}\left(e^{-\frac{1}{A_{2}}\left(\tilde{\mathcal{Y}}_{j}(t, \eta)-\tilde{\mathcal{Y}}_{j}(t, \theta)\right)}\right)\right| d \theta \\
\leqslant & 10 A^{4} \int_{0}^{\eta} e^{-\frac{1}{A}\left(\tilde{\mathcal{Y}}_{j}(t, \eta)-\tilde{\mathcal{Y}}_{j}(t, \theta)\right)} d \theta\left|A_{1}-A_{2}\right| \\
& +\frac{4 a^{4}}{e} \int_{0}^{\eta} e^{-\frac{3}{4 A}\left(\tilde{\mathcal{Y}}_{j}(t, \eta)-\tilde{\mathcal{Y}}_{j}(t, \theta)\right)} d \theta\left|A_{1}-A_{2}\right| \\
\leqslant & 12 A^{4} \int_{0}^{\eta} e^{-\frac{3}{4 A}\left(\tilde{\mathcal{Y}}_{j}(t, \eta)-\tilde{\mathcal{Y}}_{j}(t, \theta)\right)} d \theta\left|A_{1}-A_{2}\right| .
\end{aligned}
$$

Thus we end up with

$$
\begin{aligned}
\left|\tilde{\mathcal{D}}_{1}(t, \eta)-\tilde{\mathcal{D}}_{2}(t, \eta)\right| \leqslant & 2 A^{3 / 2} \max _{j}\left(\tilde{\mathcal{D}}_{j}^{1 / 2}\right)(t, \eta)\left|\tilde{\mathcal{Y}}_{1}(t, \eta)-\tilde{\mathcal{Y}}_{2}(t, \eta)\right| \\
& +2 \sqrt{2} A^{3 / 2} \max _{j}\left(\tilde{\mathcal{D}}_{j}^{1 / 2}\right)(t, \eta)\left\|\tilde{\mathcal{Y}}_{1}-\tilde{\mathcal{Y}}_{2}\right\| \\
& +4 A^{3}\left(\int_{0}^{\eta} e^{-\frac{1}{A}\left(\tilde{\mathcal{Y}}_{j}(t, \eta)-\tilde{\mathcal{Y}}_{j}(t, \theta)\right)}\left(\tilde{\mathcal{U}}_{1}-\tilde{\mathcal{U}}_{2}\right)^{2}(t, \theta) d \theta\right)^{1 / 2} \\
& +2 \sqrt{2} A^{3}\left(\int_{0}^{\eta} e^{-\frac{1}{A}\left(\tilde{\mathcal{Y}}_{j}(t, \eta)-\tilde{\mathcal{Y}}_{j}(t, \theta)\right)}\left(\tilde{\mathcal{P}}_{1}^{1 / 2}-\tilde{\mathcal{P}}_{2}^{1 / 2}\right)^{2}(t, \theta) d \theta\right)^{1 / 2} \\
& +\frac{3 A^{4}}{\sqrt{2}}\left(\int_{0}^{\eta} e^{-\frac{1}{a}\left(\tilde{\mathcal{Y}}_{j}(t, \eta)-\tilde{\mathcal{Y}}_{j}(t, \theta)\right)}\left(\tilde{\mathcal{Y}}_{1}-\tilde{\mathcal{Y}}_{2}\right)^{2}(t, \theta) d \theta\right)^{1 / 2}
\end{aligned}
$$




$$
\begin{aligned}
& +\frac{12 \sqrt{2} A^{4}}{\sqrt{3} e}\left(\int_{0}^{\eta} e^{-\frac{3}{4 A}\left(\tilde{\mathcal{Y}}_{j}(t, \eta)-\tilde{\mathcal{Y}}_{j}(t, \theta)\right)} d \theta\right)^{1 / 2}\left|A_{1}-A_{2}\right| \\
& +\tilde{\mathcal{Y}}_{j}^{2}\left|\tilde{\mathcal{Y}}_{1}-\tilde{\mathcal{Y}}_{2}\right|(t, \eta) \\
& +\tilde{\mathcal{P}}_{j}\left|\tilde{\mathcal{Y}}_{1}-\tilde{\mathcal{Y}}_{2}\right|(t, \eta) \\
& +\frac{3 A^{4}}{2} \int_{0}^{\eta} e^{-\frac{1}{a}\left(\tilde{\mathcal{Y}}_{j}(t, \eta)-\tilde{\mathcal{Y}}_{j}(t, \theta)\right)}\left|\tilde{\mathcal{Y}}_{1}-\tilde{\mathcal{Y}}_{2}\right|(t, \theta) d \theta \\
& +6 A^{4} \int_{0}^{\eta} e^{-\frac{3}{4 A}\left(\tilde{\mathcal{Y}}_{j}(t, \eta)-\tilde{\mathcal{Y}}_{j}(t, \theta)\right)} d \theta\left|A_{1}-A_{2}\right|,
\end{aligned}
$$

which proves the lemma.

LEMMA A.10. We have the following estimates:

$$
\begin{aligned}
& \int_{0}^{\eta} e^{-\frac{3}{2 A}(\tilde{\mathcal{Y}}(t, \eta)-\tilde{\mathcal{Y}}(t, \theta))} \tilde{\mathcal{P}} \tilde{\mathcal{Y}}_{\eta}(t, \theta) d \theta \leqslant 2 A \tilde{\mathcal{P}}(t, \eta) \\
& \int_{0}^{\eta} e^{-\frac{3}{2 A}(\tilde{\mathcal{Y}}(t, \eta)-\tilde{\mathcal{Y}}(t, \theta))} \tilde{\mathcal{H}}_{\eta}(t, \theta) d \theta \leqslant 4 A \tilde{\mathcal{P}}(t, \eta) \\
& \int_{0}^{\eta} e^{-\frac{1}{A}(\tilde{\mathcal{Y}}(t, \eta)-\tilde{\mathcal{Y}}(t, \theta))} \tilde{\mathcal{U}}^{2}(t, \theta) d \theta \leqslant 6 \tilde{\mathcal{P}}(t, \eta) \\
& \int_{0}^{\eta} e^{-\frac{5}{4 A}(\tilde{\mathcal{Y}}(t, \eta)-\tilde{\mathcal{Y}}(t, \theta))} \tilde{\mathcal{P}}_{\eta} \tilde{\mathcal{Y}}_{\eta}(t, \theta) d \theta \leqslant 4 A \tilde{\mathcal{P}}(t, \eta), \\
& \int_{0}^{\eta} e^{-\frac{3}{2 A}(\tilde{\mathcal{Y}}(t, \eta)-\tilde{\mathcal{Y}}(t, \theta))} \tilde{\mathcal{P}}(t, \theta) d \theta \leqslant 7 \tilde{\mathcal{P}}(t, \eta), \\
& \int_{0}^{\eta} e^{-\frac{1}{2 A}(\tilde{\mathcal{Y}}(t, \eta)-\tilde{\mathcal{Y}}(t, \theta))} \tilde{\mathcal{P}}^{2} \tilde{\mathcal{Y}}_{\eta}(t, \theta) d \theta \leqslant A \mathcal{O}(1) \tilde{\mathcal{P}}^{1 / 2}(t, \eta), \\
& \int_{0}^{\eta} e^{-\frac{1}{A}(\tilde{\mathcal{Y}}(t, \eta)-\tilde{\mathcal{Y}}(t, \theta))} \tilde{\mathcal{P}}^{1+\beta} \tilde{\mathcal{Y}}_{\eta}(t, \theta) d \theta \leqslant 3 \frac{1+\beta}{\beta} \frac{A^{1+4 \beta}}{4^{\beta}} \tilde{\mathcal{P}}(t, \eta), \quad \beta>0 .
\end{aligned}
$$

Proof. The proof of (A.17) goes as follows:

$$
\begin{aligned}
\int_{0}^{\eta} & e^{-\frac{3}{2 A}(\tilde{\mathcal{Y}}(t, \eta)-\tilde{\mathcal{Y}}(t, \theta))} \tilde{\mathcal{P}} \tilde{\mathcal{Y}}_{\eta}(t, \theta) d \theta \\
\quad= & \frac{2}{3} A \tilde{\mathcal{P}}(t, \eta)-\frac{2}{3 A} \int_{0}^{\eta} e^{-\frac{3}{2 A}(\tilde{\mathcal{Y}}(t, \eta)-\tilde{\mathcal{Y}}(t, \theta))} \tilde{\mathcal{Q}} \tilde{\mathcal{Y}}_{\eta}(t, \theta) d \theta \\
\quad & \frac{2}{3} A \tilde{\mathcal{P}}(t, \eta)+\frac{2}{3} \int_{0}^{\eta} e^{-\frac{3}{2 A}(\tilde{\mathcal{Y}}(t, \eta)-\tilde{\mathcal{Y}}(t, \theta))} \tilde{\mathcal{P}} \tilde{\mathcal{Y}}_{\eta}(t, \theta) d \theta
\end{aligned}
$$


which implies

$$
\int_{0}^{\eta} e^{-\frac{3}{2 A}(\tilde{\mathcal{Y}}(t, \eta)-\tilde{\mathcal{Y}}(t, \theta))} \tilde{\mathcal{P}}_{\eta}(t, \theta) d \theta \leqslant 2 A \tilde{\mathcal{P}}(t, \eta) .
$$

Next, we use that

$$
\begin{aligned}
\int_{0}^{\eta} e^{-\frac{3}{2 A}(\tilde{\mathcal{Y}}(t, \eta)-\tilde{\mathcal{Y}}(t, \theta))} \tilde{\mathcal{H}}_{\eta}(t, \theta) d \theta & \leqslant \int_{0}^{\eta} e^{-\frac{1}{A}(\tilde{\mathcal{Y}}(t, \eta)-\tilde{\mathcal{Y}}(t, \theta))} \tilde{\mathcal{H}}_{\eta}(t, \theta) d \theta \\
& \leqslant 4 A \tilde{\mathcal{P}}(t, \eta)
\end{aligned}
$$

(see (4.16d)), showing (A.18).

For (A.19) we find

$$
\begin{aligned}
\int_{0}^{\eta} & e^{-\frac{1}{A}(\tilde{\mathcal{Y}}(t, \eta)-\tilde{\mathcal{Y}}(t, \theta))} \tilde{\mathcal{U}}^{2}(t, \theta) d \theta \\
= & \left.\theta e^{-\frac{1}{A}(\tilde{\mathcal{Y}}(t, \eta)-\tilde{\mathcal{Y}}(t, \theta))} \tilde{\mathcal{U}}^{2}(t, \theta)\right|_{\theta=0} ^{\eta} \\
& -\int_{0}^{\eta} \theta e^{-\frac{1}{A}(\tilde{\mathcal{Y}}(t, \eta)-\tilde{\mathcal{Y}}(t, \theta))}\left(\frac{1}{A} \tilde{\mathcal{U}}^{2} \tilde{\mathcal{Y}}_{\eta}+2 \tilde{\mathcal{U}} \tilde{\mathcal{U}}_{\eta}\right)(t, \theta) d \theta \\
= & \eta \tilde{\mathcal{U}}^{2}(t, \eta)-\int_{0}^{\eta} \theta e^{-\frac{1}{A}(\tilde{\mathcal{Y}}(t, \eta)-\tilde{\mathcal{Y}}(t, \theta))}\left(\frac{1}{A} \tilde{\mathcal{U}}^{2} \tilde{\mathcal{Y}}_{\eta}+2 \tilde{\mathcal{U}} \tilde{\mathcal{U}}_{\eta}\right)(t, \theta) d \theta \\
\leqslant & \tilde{\mathcal{U}}^{2}(t, \eta)+\int_{0}^{\eta} e^{-\frac{1}{A}(\tilde{\mathcal{Y}}(t, \eta)-\tilde{\mathcal{Y}}(t, \theta))}\left(\frac{1}{A} \tilde{\mathcal{U}}^{2} \tilde{\mathcal{Y}}_{\eta}+2\left|\tilde{\mathcal{U}} \tilde{\mathcal{U}}_{\eta}\right|\right)(t, \theta) d \theta \\
\leqslant & \tilde{\mathcal{U}}^{2}(t, \eta)+4 \tilde{\mathcal{P}}(t, \eta) \\
\leqslant & 6 \tilde{\mathcal{P}}(t, \eta) .
\end{aligned}
$$

The proof of (A.20),

$$
\int_{0}^{\eta} e^{-\frac{5}{4 A}(\tilde{\mathcal{Y}}(t, \eta)-\tilde{\mathcal{Y}}(t, \theta))} \tilde{\mathcal{P}} \tilde{\mathcal{Y}}_{\eta}(t, \theta) d \theta \leqslant 4 A \tilde{\mathcal{P}}(t, \eta),
$$

follows in the same manner as (A.17).

Furthermore, for (A.21) we find

$$
\begin{aligned}
\int_{0}^{\eta} & e^{-\frac{3}{2 A}(\tilde{\mathcal{Y}}(t, \eta)-\tilde{\mathcal{Y}}(t, \theta))} \tilde{\mathcal{P}}(t, \theta) d \theta \\
= & \left.\theta e^{-\frac{3}{2 A}(\tilde{\mathcal{Y}}(t, \eta)-\tilde{\mathcal{Y}}(t, \theta))} \tilde{\mathcal{P}}(t, \theta)\right|_{\theta=0} ^{\eta} \\
& -\int_{0}^{\eta} \theta e^{-\frac{3}{2 A}(\tilde{\mathcal{Y}}(t, \eta)-\tilde{\mathcal{Y}}(t, \theta))}\left(\frac{3}{2 A} \tilde{\mathcal{P}} \tilde{\mathcal{Y}}_{\eta}+\frac{1}{A^{2}} \tilde{\mathcal{Y}} \tilde{\mathcal{Y}}_{\eta}\right)(t, \theta) d \theta
\end{aligned}
$$




$$
\begin{aligned}
& \leqslant \eta \tilde{\mathcal{P}}(t, \eta)+\frac{3}{A} \int_{0}^{\eta} e^{-\frac{3}{2 A}(\tilde{\mathcal{Y}}(t, \eta)-\tilde{\mathcal{Y}}(t, \theta))} \tilde{\mathcal{P}} \tilde{\mathcal{Y}}_{\eta}(t, \theta) d \theta \\
& \leqslant 7 \tilde{\mathcal{P}}(t, \eta)
\end{aligned}
$$

which follows from (A.17).

In order to prove (A.22), we do as follows:

$$
\begin{aligned}
\int_{0}^{\eta} & e^{-\frac{1}{2 A}(\tilde{\mathcal{Y}}(t, \eta)-\tilde{\mathcal{Y}}(t, \theta))} \tilde{\mathcal{P}}^{2} \tilde{\mathcal{Y}}_{\eta}(t, \theta) d \theta \\
= & 2 A \tilde{\mathcal{P}}^{2}(t, \eta)-8 \tilde{\mathcal{P}} \tilde{\mathcal{Q}}(t, \eta) \\
& +8 \int_{0}^{\eta} e^{-\frac{1}{2 A}(\tilde{\mathcal{Y}}(t, \eta)-\tilde{\mathcal{Y}}(t, \theta))}\left(\tilde{\mathcal{P}}^{2}+\frac{1}{A^{2}} \tilde{\mathcal{Q}}^{2}\right) \tilde{\mathcal{Y}}_{\eta}(t, \theta) d \theta \\
& +8 \int_{0}^{\eta} e^{-\frac{1}{2 A}(\tilde{\mathcal{Y}}(t, \eta)-\tilde{\mathcal{Y}}(t, \theta))}\left(\left(\tilde{\mathcal{P}}-\tilde{\mathcal{U}}^{2}\right) \tilde{\mathcal{Y}}_{\eta}(t, \theta)-\frac{1}{2} A^{5}\right) \tilde{\mathcal{P}}(t, \theta) d \theta
\end{aligned}
$$

and hence

$$
\begin{aligned}
& \int_{0}^{\eta} e^{-\frac{1}{2 A}(\tilde{\mathcal{Y}}(t, \eta)-\tilde{\mathcal{Y}}(t, \theta))} \tilde{\mathcal{P}}^{2} \tilde{\mathcal{Y}}_{\eta}(t, \theta) d \theta \\
& \leqslant 8 \tilde{\mathcal{P}} \tilde{\mathcal{Q}}(t, \eta)+8 \int_{0}^{\eta} e^{-\frac{1}{2 A}(\tilde{\mathcal{Y}}(t, \eta)-\tilde{\mathcal{Y}}(t, \theta))}\left(\left(\tilde{\mathcal{U}}^{2}-\tilde{\mathcal{P}}\right) \tilde{\mathcal{Y}}_{\eta}(t, \theta)+\frac{1}{2} A^{5}\right) \tilde{\mathcal{P}}(t, \theta) d \theta \\
& \leqslant 8 A \tilde{\mathcal{P}}^{2}(t, \eta)+8\left(\int_{0}^{\eta} e^{-\frac{1}{A}(\tilde{\mathcal{Y}}(t, \eta)-\tilde{\mathcal{Y}}(t, \theta))}\left(\left(\tilde{\mathcal{U}}^{2}-\tilde{\mathcal{P}}\right) \tilde{\mathcal{Y}}_{\eta}(t, \theta)+\frac{1}{2} A^{5}\right) d \theta\right)^{1 / 2} \\
& \quad \times\left(\int_{0}^{\eta}\left(\left(\tilde{\mathcal{U}}^{2}-\tilde{\mathcal{P}}\right) \tilde{\mathcal{Y}}_{\eta}(t, \theta)+\frac{1}{2} A^{5}\right) \tilde{\mathcal{P}}^{2}(t, \theta) d \theta\right)^{1 / 2} \\
& \leqslant \\
& \quad A \mathcal{O}(1) \tilde{\mathcal{P}}^{1 / 2}(t, \eta) .
\end{aligned}
$$

As for the proof of (A.23), we proceed as follows:

$$
\begin{aligned}
\int_{0}^{\eta} & e^{-\frac{1}{A}(\tilde{\mathcal{Y}}(t, \eta)-\tilde{\mathcal{Y}}(t, \theta))} \tilde{\mathcal{P}}^{1+\beta} \tilde{\mathcal{Y}}_{\eta}(t, \theta) d \theta \\
= & \left.A e^{-\frac{1}{A}(\tilde{\mathcal{Y}}(t, \eta)-\tilde{\mathcal{Y}}(t, \theta))} \tilde{\mathcal{P}}^{1+\beta}(t, \theta)\right|_{\theta=0} ^{\eta} \\
& -A(1+\beta) \int_{0}^{\eta} e^{-\frac{1}{A}(\tilde{\mathcal{Y}}(t, \eta)-\tilde{\mathcal{Y}}(t, \theta))} \tilde{\mathcal{P}}^{\beta} \tilde{\mathcal{P}}_{\eta}(t, \theta) d \theta \\
= & A \tilde{\mathcal{P}}^{1+\beta}(t, \eta)-\frac{1}{A}(1+\beta) \int_{0}^{\eta} e^{-\frac{1}{A}(\tilde{\mathcal{Y}}(t, \eta)-\tilde{\mathcal{Y}}(t, \theta))} \tilde{\mathcal{P}}^{\beta} \tilde{\mathcal{Q}}_{\eta} \tilde{\mathcal{Y}}_{\eta}(t, \theta) d \theta \\
= & A \tilde{\mathcal{P}}^{1+\beta}(t, \eta)-\left.(1+\beta) e^{-\frac{1}{A}(\tilde{\mathcal{Y}}(t, \eta)-\tilde{\mathcal{Y}}(t, \theta))} \tilde{\mathcal{P}}^{\beta} \tilde{\mathcal{Q}}(t, \theta)\right|_{\theta=0} ^{\eta} \\
& +(1+\beta) \int_{0}^{\eta} e^{-\frac{1}{A}(\tilde{\mathcal{Y}}(t, \eta)-\tilde{\mathcal{Y}}(t, \theta))}\left(\beta \tilde{\mathcal{P}}^{\beta-1} \tilde{\mathcal{P}}_{\eta} \tilde{\mathcal{Q}}+\tilde{\mathcal{P}}^{\beta} \tilde{\mathcal{Q}}_{\eta}\right)(t, \theta) d \theta
\end{aligned}
$$




$$
\begin{aligned}
= & A \tilde{\mathcal{P}}^{1+\beta}(t, \eta)-(1+\beta) \tilde{\mathcal{P}}^{\beta} \tilde{\mathcal{Q}}(t, \eta) \\
& +(1+\beta) \int_{0}^{\eta} e^{-\frac{1}{A}(\tilde{\mathcal{Y}}(t, \eta)-\tilde{\mathcal{Y}}(t, \theta))}\left(\beta \tilde{\mathcal{P}}^{\beta-1} \tilde{\mathcal{Q}}^{2} \tilde{\mathcal{Y}}_{\eta} \frac{1}{A^{2}}+\tilde{\mathcal{P}}^{\beta} \tilde{\mathcal{Q}}_{\eta}\right)(t, \theta) d \theta .
\end{aligned}
$$

Recall (4.6), (4.9), and (4.10), which together imply

$$
\tilde{\mathcal{Q}}_{\eta}(t, \eta)=\frac{1}{2}\left(\tilde{\mathcal{E}}_{\eta}-\tilde{\mathcal{D}}_{\eta}\right)(t, \eta)=-\left(\tilde{\mathcal{U}}^{2}-\tilde{\mathcal{P}}\right) \tilde{\mathcal{Y}}_{\eta}(t, \eta)-\frac{1}{2} A^{5}+\tilde{\mathcal{P}}_{\eta}(t, \eta)
$$

Thus

$$
\begin{aligned}
& \beta \tilde{\mathcal{P}}^{\beta-1} \tilde{\mathcal{Q}}^{2} \tilde{\mathcal{Y}}_{\eta} \frac{1}{A^{2}}+\tilde{\mathcal{P}}^{\beta} \tilde{\mathcal{Q}}_{\eta} \\
& =\frac{\beta}{A^{2}} \tilde{\mathcal{Q}}^{2} \tilde{\mathcal{P}}^{\beta-1} \tilde{\mathcal{Y}}_{\eta}+\tilde{\mathcal{P}}^{\beta+1} \tilde{\mathcal{Y}}_{\eta}+\tilde{\mathcal{P}}^{\beta}\left(-\left(\tilde{\mathcal{U}}^{2}-\tilde{\mathcal{P}}\right) \tilde{\mathcal{Y}}_{\eta}-\frac{1}{2} A^{5}\right) .
\end{aligned}
$$

Inserting this expression in (A.24) and re-ordering the terms, we find

$$
\begin{aligned}
& \beta \int_{0}^{\eta} e^{-\frac{1}{A}(\tilde{\mathcal{Y}}(t, \eta)-\tilde{\mathcal{Y}}(t, \theta))} \tilde{\mathcal{P}}^{1+\beta} \tilde{\mathcal{Y}}_{\eta}(t, \theta) d \theta \\
& \quad+\frac{1+\beta}{A^{2}} \int_{0}^{\eta} e^{-\frac{1}{A}(\tilde{\mathcal{Y}}(t, \eta)-\tilde{\mathcal{Y}}(t, \theta))} \tilde{\mathcal{P}}^{\beta-1} \tilde{\mathcal{Q}}^{2} \tilde{\mathcal{Y}}_{\eta}(t, \theta) d \theta \\
& =(1+\beta) \tilde{\mathcal{P}}^{\beta} \tilde{\mathcal{Q}}(t, \eta)-A \tilde{\mathcal{P}}^{1+\beta}(t, \eta) \\
& \quad+(1+\beta) \int_{0}^{\eta} e^{-\frac{1}{A}(\tilde{\mathcal{Y}}(t, \eta)-\tilde{\mathcal{Y}}(t, \theta))} \tilde{\mathcal{P}}^{\beta}\left(\left(\tilde{\mathcal{U}}^{2}-\tilde{\mathcal{P}}\right) \tilde{\mathcal{Y}}_{\eta}+\frac{1}{2} A^{5}\right)(t, \theta) d \theta .
\end{aligned}
$$

Estimating this, using (4.5a), we find

$$
\begin{aligned}
\beta \int_{0}^{\eta} & e^{-\frac{1}{A}(\tilde{\mathcal{Y}}(t, \eta)-\tilde{\mathcal{Y}}(t, \theta))} \tilde{\mathcal{P}}^{1+\beta} \tilde{\mathcal{Y}}_{\eta}(t, \theta) d \theta \\
\leqslant & (1+\beta) \tilde{\mathcal{P}}^{\beta}|\tilde{\mathcal{Q}}|(t, \eta) \\
& +(1+\beta) \int_{0}^{\eta} e^{-\frac{1}{A}(\tilde{\mathcal{Y}}(t, \eta)-\tilde{\mathcal{Y}}(t, \theta))} \tilde{\mathcal{P}}^{\beta}\left(\left(\tilde{\mathcal{U}}^{2}-\tilde{\mathcal{P}}\right) \tilde{\mathcal{Y}}_{\eta}+\frac{1}{2} A^{5}\right)(t, \theta) d \theta \\
\leqslant & (1+\beta)\|\tilde{\mathcal{P}}\|_{\infty}^{\beta} A \tilde{\mathcal{P}}(t, \eta) \\
& +(1+\beta)\|\tilde{\mathcal{P}}\|_{\infty}^{\beta} \int_{0}^{\eta} e^{-\frac{1}{A}(\tilde{\mathcal{Y}}(t, \eta)-\tilde{\mathcal{Y}}(t, \theta))}\left(\left(\tilde{\mathcal{U}}^{2}-\tilde{\mathcal{P}}\right) \tilde{\mathcal{Y}}_{\eta}+\frac{1}{2} A^{5}\right)(t, \theta) d \theta \\
\leqslant & (1+\beta)\|\tilde{\mathcal{P}}\|_{\infty}^{\beta} A \tilde{\mathcal{P}}(t, \eta)+(1+\beta) \| \tilde{\mathcal{P}}_{\infty}^{\beta} \tilde{\mathcal{D}}(t, \eta) \\
\leqslant & 3(1+\beta)\left(\frac{A^{4}}{4}\right)^{\beta} A \tilde{\mathcal{P}}(t, \eta),
\end{aligned}
$$

where we used (4.8), (4.15a), and (4.15c). 

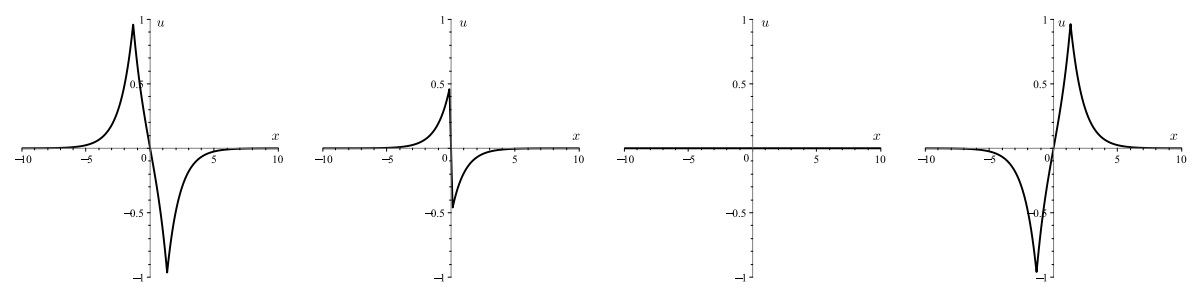

Figure B.3. Time evolution of $u(t, x)$ with $C=E^{2}=4$ and $t_{0}=2$ at $t=0,1.5$, 2,4 .
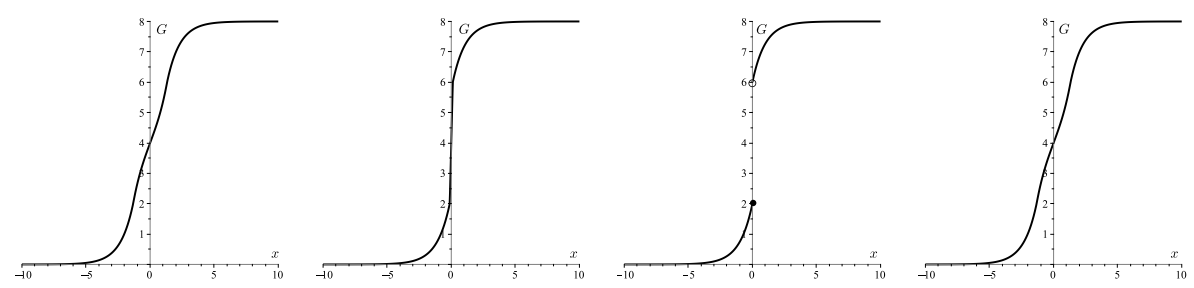

Figure B.4. Time evolution of $G(t, x)$ with $C=E^{2}=4$ and $t_{0}=2$ at $t=0,1.5$, 2,4 .
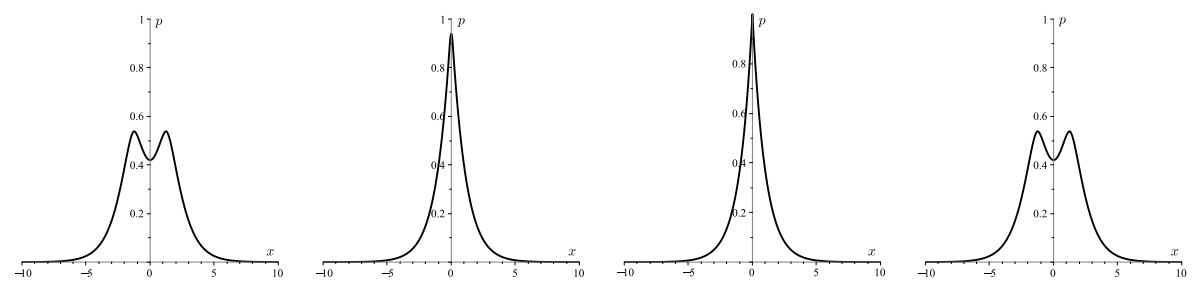

Figure B.5. Time evolution of $p(t, x)$ with $C=E^{2}=4$ and $t_{0}=2$ at $t=0,1.5$, 2,4 .

\section{Appendix B. The antisymmetric peakon-antipeakon example}

Fortunately, one can compute explicitly the quantities described in this paper in the important case of an antisymmetric peakon-antipeakon solution. The various functions are depicted on Figures B.3-B.14.

More precisely, consider the function [41]

$$
u(t, x)= \begin{cases}\beta(t) \sinh (x), & |x| \leqslant \gamma(t), \\ \operatorname{sign}(x) \alpha(t) e^{-|x|}, & |x| \geqslant \gamma(t),\end{cases}
$$



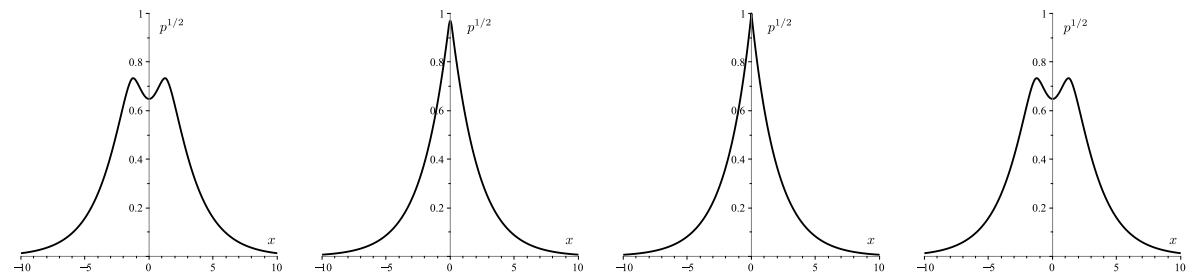

Figure B.6. Time evolution of $p^{1 / 2}(t, x)$ with $C=E^{2}=4$ and $t_{0}=2$ at $t=0$, 1.5, 2, 4 .
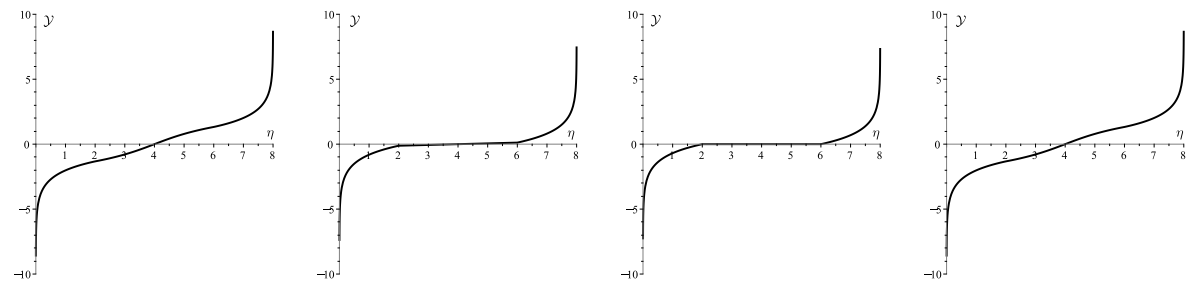

Figure B.7. Time evolution of $\mathcal{Y}(t, \eta)$ with $C=E^{2}=4$ and $t_{0}=2$ at $t=0,1.5$, 2, 4 .
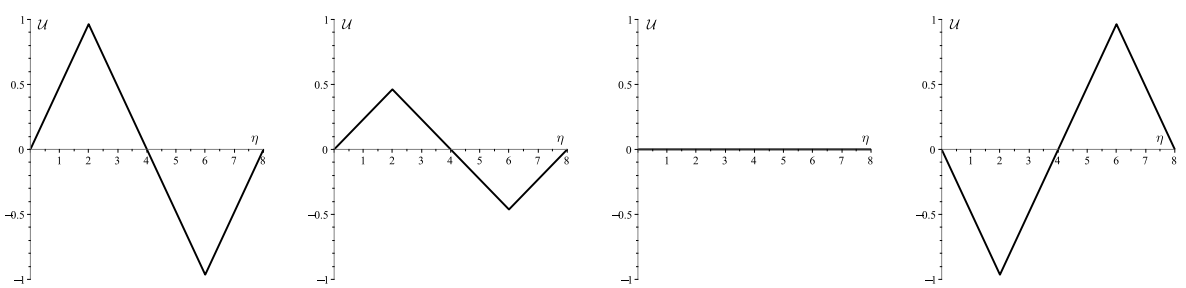

Figure B.8. Time evolution of $\mathcal{U}(t, \eta)$ with $C=E^{2}=4$ and $t_{0}=2$ at $t=0,1.5$, 2, 4 .

$$
= \begin{cases}-\alpha(t) e^{x}, & x \leqslant-\gamma(t), \\ \beta(t) \sinh (x), & -\gamma(t) \leqslant x \leqslant \gamma(t), \\ \alpha(t) e^{-x}, & \gamma(t) \leqslant x,\end{cases}
$$

where

$$
\begin{gathered}
\alpha(t)=\frac{E}{2} \sinh \left(\frac{E}{2}\left(t-t_{0}\right)\right), \quad \beta(t)=E \frac{1}{\sinh \left(\frac{E}{2}\left(t-t_{0}\right)\right)}, \\
\gamma(t)=\ln \left(\cosh \left(\frac{E}{2}\left(t-t_{0}\right)\right)\right) .
\end{gathered}
$$



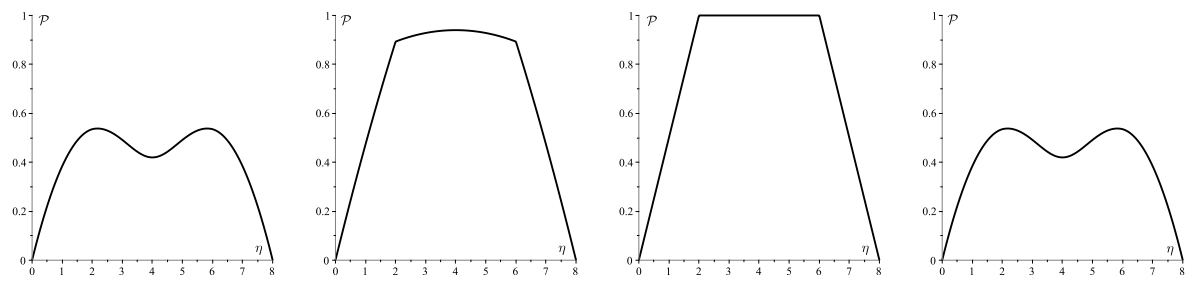

Figure B.9. Time evolution of $\mathcal{P}(t, \eta)$ with $C=E^{2}=4$ and $t_{0}=2$ at $t=0,1.5$, 2, 4 .
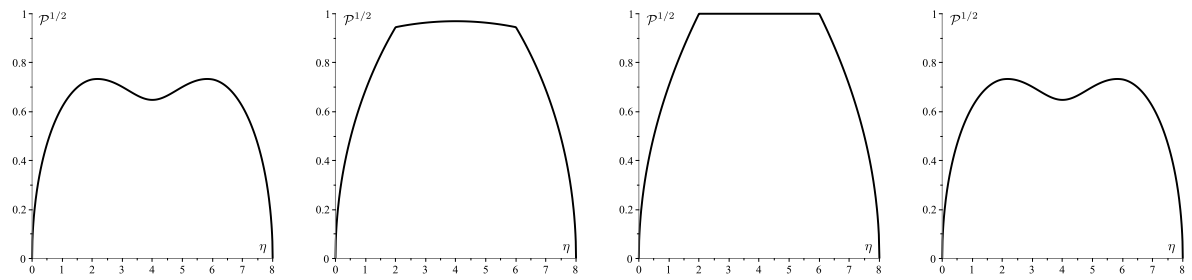

Figure B.10. Time evolution of $\mathcal{P}^{1 / 2}(t, \eta)$ with $C=E^{2}=4$ and $t_{0}=2$ at $t=0$, 1.5, 2, 4 .
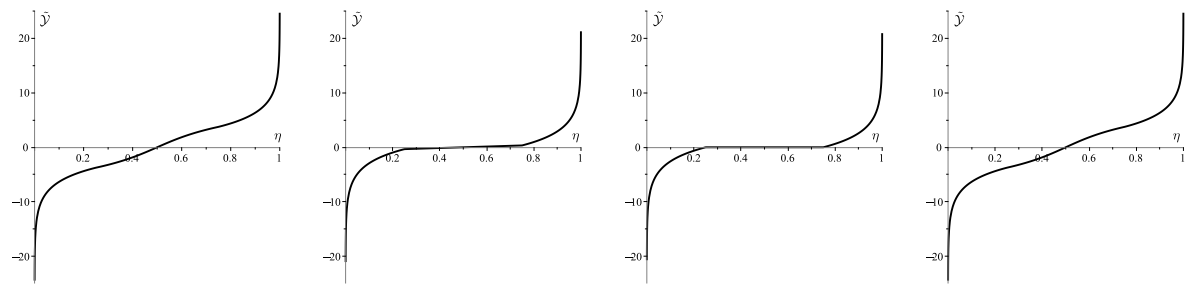

Figure B.11. Time evolution of $\tilde{\mathcal{Y}}(t, \eta)$ with $C=E^{2}=4$ and $t_{0}=2$ at $t=0$, 1.5, 2, 4 .

Here $E=\|u(t)\|_{H^{1}}, t \neq t_{0}$, denotes the total energy. The corresponding energy density is given by

$$
\mu(t, x)=\left(u^{2}+u_{x}^{2}\right)(t, x)= \begin{cases}2 \alpha^{2}(t) e^{2 x}, & x \leqslant-\gamma(t), \\ \beta^{2}(t) \cosh (2 x), & -\gamma(t) \leqslant x \leqslant \gamma(t), \quad t \neq t_{0}, \\ 2 \alpha^{2}(t) e^{-2 x}, & \gamma(t) \leqslant x,\end{cases}
$$



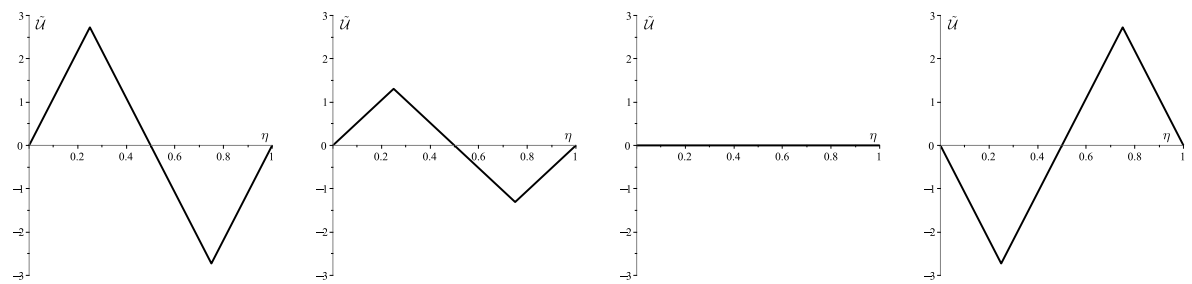

Figure B.12. Time evolution of $\tilde{\mathcal{U}}(t, \eta)$ with $C=E^{2}=4$ and $t_{0}=2$ at $t=0$, 1.5, 2, 4 .
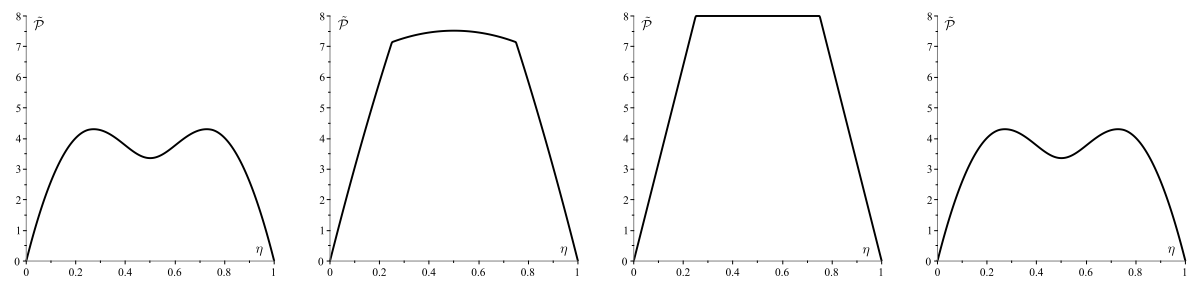

Figure B.13. Time evolution of $\tilde{\mathcal{P}}(t, \eta)$ with $C=E^{2}=4$ and $t_{0}=2$ at $t=0$, 1.5, 2, 4.
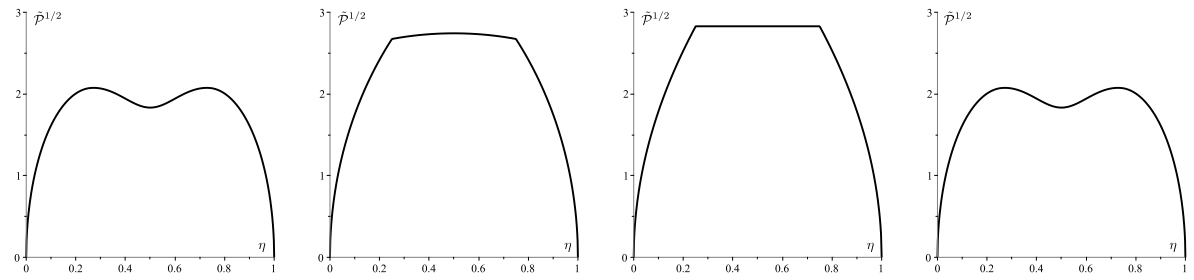

Figure B.14. Time evolution of $\tilde{\mathcal{P}}^{1 / 2}(t, \eta)$ with $C=E^{2}=4$ and $t_{0}=2$ at $t=0$, 1.5, 2, 4.

with $\mu\left(t_{0}, x\right)=E^{2} \delta_{0}(x)$ for $t=t_{0}$. Hence $C=\mu(t, \mathbb{R})=E^{2}$, and

$$
F(t, x)= \begin{cases}\alpha(t)^{2} e^{2 x}, & x<-\gamma(t), \\ \frac{E^{2}}{4} \tanh ^{2}\left(\frac{E}{2}\left(t-t_{0}\right)\right), & x=-\gamma(t), \\ \frac{1}{2} E^{2}+\frac{1}{2} \beta(t)^{2} \sinh (2 x), & -\gamma(t)<x<\gamma(t), \\ E^{2}-\frac{E^{2}}{4} \tanh ^{2}\left(\frac{E}{2}\left(t-t_{0}\right)\right), & x=\gamma(t), \\ E^{2}-\alpha(t)^{2} e^{-2 x}, & \gamma(t)<x .\end{cases}
$$


In particular, this solution experiences wave breaking at time $t=t_{0}$, that is, $u_{x}(t$, $0)$ tends to $-\infty$ as $t \rightarrow t_{0}-$ and

$$
F\left(t_{0}-, x\right)= \begin{cases}0, & x \leqslant 0 \\ E^{2}, & 0<x\end{cases}
$$

The corresponding function $p_{x}(t, x)$, which can be computed using $p_{x}(t, x)=$ $-u_{t}(t, x)-u u_{x}(t, x)$, is given by

$$
p_{x}(t, x)= \begin{cases}\alpha^{\prime}(t) e^{x}-\alpha(t)^{2} e^{2 x}, & x<-\gamma(t), \\ \frac{E^{2}}{4}-\frac{E^{2}}{4} \tanh ^{2}\left(\frac{E}{2}\left(t-t_{0}\right)\right), & x=-\gamma(t), \\ -\beta^{\prime}(t) \sinh (x)-\frac{1}{2} \beta(t)^{2} \sinh (2 x), & -\gamma(t)<x<\gamma(t), \\ -\frac{E^{2}}{4}+\frac{E^{2}}{4} \tanh ^{2}\left(\frac{E}{2}\left(t-t_{0}\right)\right), & \gamma(t)=x, \\ -\alpha^{\prime}(t) e^{-x}+\alpha(t)^{2} e^{-2 x}, & \gamma(t)<x .\end{cases}
$$

In particular, one obtains at wave breaking time $t=t_{0}$ that

$$
p_{x}\left(t_{0}-, x\right)= \begin{cases}\frac{E^{2}}{4} e^{x}, & x<0, \\ -\frac{E^{2}}{4} e^{-x}, & 0<x .\end{cases}
$$

Here it is important to note that $p_{x}\left(t_{0}-, x\right)$ has a (negative) jump of height $-\frac{E^{2}}{2}$ at $x=0$ at time $t=t_{0}$. For all other points $x \in \mathbb{R}$, the function $p_{x}\left(t_{0}-, x\right)$ is continuous.

Thus the function $G(t, x)=2 p_{x}(t, x)+2 F(t, x)$ is given by

$$
G(t, x)= \begin{cases}2 \alpha^{\prime}(t) e^{x}, & x<-\gamma(t), \\ \frac{E^{2}}{2}, & x=-\gamma(t), \\ E^{2}-2 \beta^{\prime}(t) \sinh (x), & -\gamma(t)<x<\gamma(t), \\ \frac{3 E^{2}}{2}, & x=\gamma(t), \\ 2 E^{2}-2 \alpha^{\prime}(t) e^{-x}, & \gamma(t)<x .\end{cases}
$$


In particular, one observes that $G_{x}(t, x)>0$ for all $x \in \mathbb{R}$,

$$
\lim _{x \rightarrow-\infty} G(t, x)=0 \quad \text { and } \quad \lim _{x \rightarrow \infty} G(t, x)=2 E^{2}=2 C .
$$

Thus the limits at $\pm \infty$ are independent of time. Moreover, one has

$$
G\left(t_{0}-, x\right)= \begin{cases}\frac{E^{2}}{2} e^{x}, & x \leqslant 0 \\ 2 E^{2}-\frac{E^{2}}{2} e^{-x}, & 0<x\end{cases}
$$

Here it is important to note that $G\left(t_{0}-, x\right)$ has a jump of size $E^{2}$ due to the wave breaking at $t=t_{0}$, that is, $\mu\left(t_{0}, x\right)=E^{2} \delta_{0}(x)$.

Direct computations using $p=p_{x x}+\frac{1}{2} u^{2}+\frac{1}{2} d \mu$ yield

$$
p(t, x)= \begin{cases}\alpha^{\prime}(t) e^{x}-\frac{1}{2} \alpha(t)^{2} e^{2 x}, & x \leqslant-\gamma(t), \\ -\beta^{\prime}(t) \cosh (x)-\frac{1}{2} \beta(t)^{2} \cosh ^{2}(x), & -\gamma(t) \leqslant x \leqslant \gamma(t), \\ \alpha^{\prime}(t) e^{-x}-\frac{1}{2} \alpha(t)^{2} e^{-2 x}, & \gamma(t) \leqslant x .\end{cases}
$$

In the new coordinates, using $G(t, \mathcal{Y}(t, \eta))=\eta$, the solution reads as

$$
\mathcal{Y}(t, \eta)= \begin{cases}\ln \left(\frac{\eta}{2 \alpha^{\prime}(t)}\right), & 0<\eta \leqslant \frac{E^{2}}{2}, \\ \sinh ^{-1}\left(\frac{E^{2}-\eta}{2 \beta^{\prime}(t)}\right), & \frac{E^{2}}{2} \leqslant \eta \leqslant \frac{3 E^{2}}{2}, \\ \ln \left(\frac{2 \alpha^{\prime}(t)}{2 E^{2}-\eta}\right), & \frac{3 E^{2}}{2} \leqslant \eta<2 E^{2},\end{cases}
$$

and, applying $\mathcal{U}(t, \eta)=u(t, \mathcal{Y}(t, \eta))$,

$$
\mathcal{U}(t, \eta)= \begin{cases}-\frac{\alpha(t)}{2 \alpha^{\prime}(t)} \eta, & 0<\eta \leqslant \frac{E^{2}}{2}, \\ \frac{\beta(t)}{2 \beta^{\prime}(t)}\left(E^{2}-\eta\right), & \frac{E^{2}}{2} \leqslant \eta \leqslant \frac{3 E^{2}}{2}, \\ \frac{\alpha(t)}{2 \alpha^{\prime}(t)}\left(2 E^{2}-\eta\right), & \frac{3 E^{2}}{2} \leqslant \eta<2 E^{2},\end{cases}
$$


and, by invoking $\mathcal{P}(t, \eta)=p(t, \mathcal{Y}(t, \eta))$,

$$
\mathcal{P}(t, \eta)= \begin{cases}\frac{1}{2} \eta-\frac{\alpha(t)^{2}}{8 \alpha^{\prime}(t)^{2}} \eta^{2}, & 0<\eta \leqslant \frac{E^{2}}{2}, \\ -\beta^{\prime}(t) \sqrt{1+\frac{\left(E^{2}-\eta\right)^{2}}{4 \beta^{\prime}(t)^{2}}} & \\ -\frac{1}{2} \beta(t)^{2}\left(1+\frac{\left(E^{2}-\eta\right)^{2}}{4 \beta^{\prime}(t)^{2}}\right), & \frac{E^{2}}{2} \leqslant \eta \leqslant \frac{3 E^{2}}{2}, \\ \frac{1}{2}\left(2 E^{2}-\eta\right)-\frac{\alpha(t)^{2}}{8 \alpha^{\prime}(t)^{2}}\left(2 E^{2}-\eta\right)^{2}, & \frac{3 E^{2}}{2} \leqslant \eta<2 E^{2},\end{cases}
$$

where we used the convention that $\sinh ^{-1}(x)$ denotes the inverse of $\sinh (x)$.

For the scaled quantities we find, when we introduce $A=\sqrt{2 C}=\sqrt{2} E$, that

$$
\begin{aligned}
& \tilde{\mathcal{Y}}(t, \eta)=A \mathcal{Y}\left(t, A^{2} \eta\right)=\sqrt{2 C} \begin{cases}\ln \left(\frac{C \eta}{\alpha^{\prime}(t)}\right), & 0<\eta \leqslant \frac{1}{4}, \\
\sinh ^{-1}\left(\frac{C(1-2 \eta)}{2 \beta^{\prime}(t)}\right), & \frac{1}{4} \leqslant \eta \leqslant \frac{3}{4}, \\
\ln \left(\frac{\alpha^{\prime}(t)}{C(1-\eta)}\right), & \frac{3}{4} \leqslant \eta<1,\end{cases} \\
& \tilde{\mathcal{U}}(t, \eta)=A \mathcal{U}\left(t, A^{2} \eta\right)=\sqrt{2 C} \begin{cases}-\frac{\alpha(t)}{\alpha^{\prime}(t)} C \eta, & 0<\eta \leqslant \frac{1}{4}, \\
\frac{\beta(t)}{2 \beta^{\prime}(t)} C(1-2 \eta), & \frac{1}{4} \leqslant \eta \leqslant \frac{3}{4}, \\
\frac{\alpha(t)}{\alpha^{\prime}(t)} C(1-\eta), & \frac{3}{4} \leqslant \eta<1,\end{cases} \\
& \tilde{\mathcal{P}}(t, \eta)=A^{2} \mathcal{P}\left(t, A^{2} \eta\right)
\end{aligned}
$$$$
=2 C \begin{cases}C \eta-\frac{\alpha(t)^{2}}{2 \alpha^{\prime}(t)^{2}} C^{2} \eta^{2}, & 0<\eta \leqslant \frac{1}{4}, \\ -\beta^{\prime}(t) \sqrt{1+\frac{C^{2}(1-2 \eta)^{2}}{4 \beta^{\prime}(t)^{2}}} & \\ -\frac{1}{2} \beta(t)^{2}\left(1+\frac{C^{2}(1-2 \eta)^{2}}{4 \beta^{\prime}(t)^{2}}\right), & \frac{1}{4} \leqslant \eta \leqslant \frac{3}{4}, \\ C(1-\eta)-\frac{\alpha(t)^{2}}{2 \alpha^{\prime}(t)^{2}} C^{2}(1-\eta)^{2}, & \frac{3}{4} \leqslant \eta<1 .\end{cases}
$$ 


\section{Conflict of Interest: None.}

\section{References}

[1] L. Ambrosio, N. Gigli and G. Savaré, Gradient Flows in Metric Spaces and in the Space of Probability Measures, Lectures in Mathematics ETH Zürich (Birkhäuser Verlag, Basel, 2008).

[2] A. Blanchet, V. Calvez and J. A. Carrillo, 'Convergence of the mass-transport steepest descent scheme for the subcritical Patlak-Keller-Segel model', SIAM J. Numer. Anal. 46 (2008), 691-721.

[3] F. Bolley, J. A. Cañizo and J. A. Carrillo, 'Stochastic mean-field limit: non-Lipschitz forces and swarming', Math. Models Methods Appl. Sci. 21 (2011), 2179-2210.

[4] G. A. Bonaschi, J. A. Carrillo, M. Di Francesco and M. A. Peletier, 'Equivalence of gradient flows and entropy solutions for singular nonlocal interaction equations in 1D', ESAIM Control Optim. Calc. Var. 21 (2015), 414-441.

[5] W. Braun and K. Hepp, 'The Vlasov dynamics and its fluctuations in the $1 / N$ limit of interacting classical particles', Comm. Math. Phys. 56 (1977), 101-113.

[6] A. Bressan and A. Constantin, 'Global conservative solutions of the Camassa-Holm equation', Arch. Ration. Mech. Anal. 183 (2007), 215-239.

[7] A. Bressan and A. Constantin, 'Global dissipative solutions of the Camassa-Holm equation', Anal. Appl. (Singap.) 5 (2007), 1-27.

[8] A. Bressan and M. Fonte, 'An optimal transportation metric for solutions of the CamassaHolm equation', Methods Appl. Anal. 12 (2005), 191-219.

[9] A. Bressan, H. Holden and X. Raynaud, 'Lipschitz metric for the Hunter-Saxton equation', J. Math. Pures Appl. (9) 94 (2010), 68-92.

[10] R. Camassa and D. D. Holm, 'An integrable shallow water equation with peaked solutions', Phys. Rev. Lett. 71 (1993), 1661-1664.

[11] R. Camassa, D. D. Holm and J. Hyman, 'A new integrable shallow water equation', Adv. Appl. Mech. 31 (1994), 1-33.

[12] J. A. Cañizo, J. A. Carrillo and J. Rosado, 'A well-posedness theory in measures for some kinetic models of collective motion', Math. Models Methods Appl. Sci. 21 (2011), 515-539.

[13] J. A. Carrillo, Y.-P. Choi, M. Hauray and S. Salem, 'Mean-field limit for collective behavior models with sharp sensitivity regions', J. Eur. Math. Soc. (JEMS) 21 (2019), 121-161.

[14] J. A. Carrillo, M. Di Francesco, A. Figalli, T. Laurent and D. Slepčev, 'Global-in-time weak measure solutions and finite-time aggregation for nonlocal interaction equations', Duke Math. J. 156 (2011), 229-271.

[15] J. A. Carrillo, M. Di Francesco and G. Toscani, 'Strict contractivity of the 2-Wasserstein distance for the porous medium equation by mass-centering', Proc. Amer. Math. Soc. 135 (2007), 353-363.

[16] J. A. Carrillo, K. Grunert and H. Holden, 'A Lipschitz metric for the Hunter-Saxton equation', Comm. Partial Differential Equations 44 (2019), 309-334.

[17] J. A. Carrillo and J. S. Moll, 'Numerical simulation of diffusive and aggregation phenomena in nonlinear continuity equations by evolving diffeomorphisms', SIAM J. Sci. Comput. 31 (2009/10), 4305-4329.

[18] J. A. Carrillo, H. Ranetbauer and M.-T. Wolfram, 'Numerical simulation of nonlinear continuity equations by evolving diffeomorphisms', J. Comput. Phys. 327 (2016), 186-202. 
[19] J. A. Carrillo and G. Toscani, 'Wasserstein metric and large-time asymptotics of non-linear diffusion equations', in New Trends in Mathematical Physics (World Sci. Publ., Hackensack, NJ, 2004), 234-244.

[20] J. A. Carrillo and G. Toscani, 'Contractive probability metrics and asymptotic behavior of dissipative kinetic equations', Riv. Mat. Univ. Parma (7) 6 (2007), 75-198.

[21] R. M. Chen and Y. Liu, 'Wave breaking and global existence for a generalized two-component Camassa-Holm system', Int. Math. Res. Not. IMRN 2011(6) (2011), 1381-1416.

[22] M. Chen, S.-Q. Liu and Y. Zhang, 'A two-component generalization of the Camassa-Holm equation and its solutions', Lett. Math. Phys. 75 (2006), 1-15.

[23] A. Chertock, J.-G. Liu and T. Pendleton, 'Convergence of a particle method and global weak solutions for a family of evolutionary PDEs', SIAM J. Numer. Anal. 50 (2012), 1-21.

[24] G. M. Coclite, H. Holden and K. H. Karlsen, 'Global weak solutions to a generalized hyperelastic-rod wave equation', SIAM J. Math. Anal. 37 (2005), 1044-1069.

[25] A. Constantin and J. Escher, 'Wave breaking for nonlinear nonlocal shallow water equations', Acta Math. 181 (1998), 229-243.

[26] A. Constantin and B. Kolev, 'Geodesic flow on the diffeomorphism group of the circle', Comment. Math. Helv. 78 (2003), 787-804.

[27] A. Constantin and D. Lannes, 'The hydrodynamical relevance of the Camassa-Holm and Degasperis-Procesi equations', Arch. Ration. Mech. Anal. 192 (2009), 165-186.

[28] H.-H. Dai and Y. Huo, 'Solitary shock waves and other travelling waves in a general compressible hyperelastic rod', Proc. R. Soc. Lond. Ser. A Math. Phys. Eng. Sci. 456 (2000), 331-363.

[29] R. L. Dobrushin, 'Vlasov equations', Funct. Anal. Appl. 13 (1979), 115-123.

[30] B. Düring, D. Matthes and J. P. Milišić, 'A gradient flow scheme for nonlinear fourth order equations', Discrete Contin. Dyn. Syst. Ser. B 14 (2010), 935-959.

[31] J. Escher and B. Kolev, 'Geodesic completeness for Sobolev $H^{s}$-metrics on the diffeomorphism group of the circle', J. Evol. Equ. 14 (2014), 949-968.

[32] J. Escher, O. Lechtenfeld and Z. Yin, 'Well-posedness and blow-up phenomena for the 2component Camassa-Holm equation', Discrete Contin. Dyn. Syst. 19 (2007), 493-513.

[33] Y. Fu and C. Qu, 'Well posedness and blow-up solution for a new coupled Camassa-Holm equations with peakons', J. Math. Phys. 50 (2009), 012906.

[34] T. Gallouët and F.-X. Vialard, 'The Camassa-Holm equation as an incompressible Euler equations: a geometric point of view', J. Differential Equations 264 (2018), 4199-4234.

[35] F. Golse, 'The mean-field limit for the dynamics of large particle systems', J. Équ. aux Dérivées Partielles, 9, Univ. Nantes, Nantes, 2003, 47pp.

[36] F. Golse, 'On the dynamics of large particle systems in the mean-field limit', in Macroscopic and Large Scale Phenomena: Coarse Graining, Mean Field Limits and Ergodicity, Lecture Notes in Applied and Mathematics and Mechanics, 3 (Springer, Cham, 2016), 1-144.

[37] L. Gosse and G. Toscani, 'Lagrangian numerical approximations to one-dimensional convolution-diffusion equations', SIAM J. Sci. Comput. 28 (2006), 1203-1227.

[38] L. Gosse and G. Toscani, 'Identification of asymptotic decay to self-similarity for onedimensional filtration equations', SIAM J. Numer. Anal. 43 (2006), 2590-2606.

[39] M. Grasmair, K. Grunert and H. Holden, 'On the equivalence of Eulerian and Lagrangian variables for the two-component Camassa-Holm system', in Current Research in Nonlinear Analysis: In Honor of Haim Brezis and Louis Nirenberg, (ed. Th. M. Rassias) Springer Optimization and Its Applications, 135 (Springer, Cham, Switzerland, 2018), 157-201. 
[40] K. Grunert, 'Solutions of the Camassa-Holm equation with accumulating breaking times', Dyn. Partial Differ. Equ. 13 (2016), 91-105.

[41] K. Grunert and H. Holden, 'The general peakon-antipeakon solution for the Camassa-Holm equation', J. Hyperbolic Differ. Equ. 13 (2016), 353-380.

[42] K. Grunert, H. Holden and X. Raynaud, 'Lipschitz metric for the periodic Camassa-Holm equation', J. Differential Equations 250 (2011), 1460-1492.

[43] K. Grunert, H. Holden and X. Raynaud, 'Lipschitz metric for the Camassa-Holm equation on the line', Discrete Contin. Dyn. Syst. 33 (2013), 2809-2827.

[44] K. Grunert, H. Holden and X. Raynaud, 'Global conservative solutions to the CamassaHolm equation for initial data with nonvanishing asymptotics', Discrete Contin. Dyn. Syst. 32 (2012), 4209-4227.

[45] K. Grunert, H. Holden and X. Raynaud, 'Global dissipative solutions of the two-component Camassa-Holm system for initial data with nonvanishing asymptotics', Nonlinear Anal. Real World Appl. 17 (2014), 203-244.

[46] K. Grunert, H. Holden and X. Raynaud, 'Global solutions for the two-component CamassaHolm system', Comm. Partial Differential Equations 37 (2012), 2245-2271.

[47] K. Grunert, H. Holden and X. Raynaud, 'A continuous interpolation between conservative and dissipative solutions for the two-component Camassa-Holm system', Forum Math. Sigma 3 (2014), e1, 73 pages.

[48] C. Guan, K. H. Karlsen and Z. Yin, 'Well-posedness and blow-up phenomenal for a modified two-component Camassa-Holm equation', in Nonlinear Partial Differential Equations and Hyperbolic Wave Phenomena, (eds. H. Holden and K. H. Karlsen) Contemporary Mathematics, 526 (American Mathematical Society, 2010), 199-220.

[49] C. Guan and Z. Yin, 'Global existence and blow-up phenomena for an integrable twocomponent Camassa-Holm shallow water system', J. Differential Equations 248 (2010), 2003-2014.

[50] C. Guan and Z. Yin, 'Global weak solutions for a two-component Camassa-Holm shallow water system', J. Funct. Anal. 260 (2011), 1132-1154.

[51] G. Gui and Y. Liu, 'On the global existence and wave breaking criteria for the two-component Camassa-Holm system', J. Funct. Anal. 258 (2010), 4251-4278.

[52] G. Gui and Y. Liu, 'On the Cauchy problem for the two-component Camassa-Holm system', Math. Z. 268 (2011), 45-66.

[53] Z. Guo and Y. Zhou, 'On solutions to a two-component generalized Camassa-Holm equation', Stud. Appl. Math. 124 (2010), 307-322.

[54] H. Holden and X. Raynaud, 'Global conservative solutions for the Camassa-Holm equation - a Lagrangian point of view', Comm. Partial Differential Equations 32 (2007), 1511-1549.

[55] H. Holden and X. Raynaud, 'Global conservative solutions of the generalized hyperelasticrod wave equation', J. Differential Equations 233 (2007), 448-484.

[56] H. Holden and X. Raynaud, 'Dissipative solutions for the Camassa-Holm equation', Discrete Contin. Dyn. Syst. 24 (2009), 1047-1112.

[57] H. Holden and X. Raynaud, 'Global dissipative multipeakon solutions of the Camassa-Holm equation', Comm. Partial Differential Equations 33 (2008), 2040-2063.

[58] R. S. Johnson, 'Camassa-Holm, Korteweg-de Vries and related models for water waves', J. Fluid Mech. 455 (2002), 63-82.

[59] O. Junge, D. Matthes and H. Osberger, 'A fully discrete variational scheme for solving nonlinear Fokker-Planck equations in multiple space dimensions', SIAM J. Numer. Anal. 55 (2017), 419-443. 
[60] S. Kouranbaeva, 'The Camassa-Holm equation as a geodesic flow on the diffeomorphism group', J. Math. Phys. 40 (1999), 857-868.

[61] H. Li and G. Toscani, 'Long-time asymptotics of kinetic models of granular flows', Arch. Ration. Mech. Anal. 172 (2004), 407-428.

[62] D. Matthes and H. Osberger, 'Convergence of a variational Lagrangian scheme for a nonlinear drift diffusion equation', ESAIM Math. Model. Numer. Anal. 48 (2014), 697-726.

[63] H. Neunzert, 'An introduction to the nonlinear Boltzmann-Vlasov equation', in Kinetic Theories and the Boltzmann Equation (Montecatini, 1981), Lecture Notes in Mathematics, 1048 (Springer, Berlin, 1984), 60-110.

[64] H. Spohn, Large Scale Dynamics of Interacting Particles, Texts and Monographs in Physics (Springer, Berlin, 1991).

[65] A.-S. Sznitman, 'Topics in propagation of chaos', in Ecole d'Eté de Probabilités de SaintFlour XIX 1989, Lecture Notes in Mathematics, 1464 (Springer, Berlin, 1991), 165-251.

[66] G. Toscani, 'One-dimensional kinetic models of granular flows', M2AN Math. Model. Numer. Anal. 34 (2000), 1277-1291.

[67] C. Villani, Topics in Optimal Transportation, Graduate Studies in Mathematics, 58 (American Mathematical Society, Providence, RI, 2003).

[68] M. Westdickenberg and J. Wilkening, 'Variational particle schemes for the porous medium equation and for the system of isentropic Euler equations', M2AN Math. Model. Numer. Anal. 44 (2010), 133-166. 